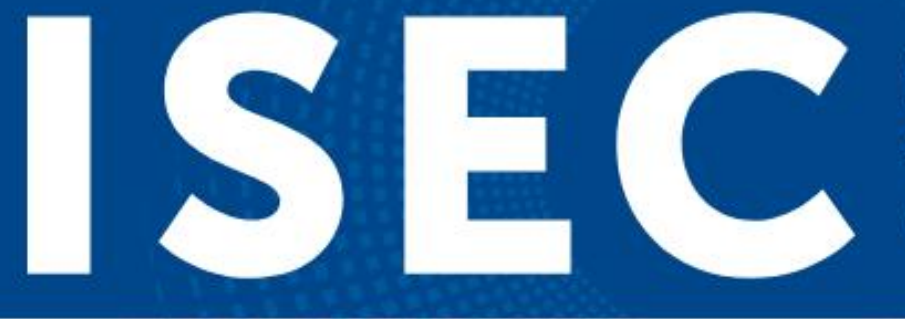

INTERNATIONAL SUSTAINABLE ENERGY CONFERENCE 2018

www.aee-intec-events.org

\title{
Renewable
}

\section{Heating and Cooling}

e 20

u 18

- a t

Official Event of the Austrian Presidency of the Council of the European Union

\section{in Integrated Urban and Industrial Energy Systems}

3 - 5 October 2018

Congress Graz

Austria

\section{Proceedings}




\title{
LCC ANALYSIS OF A SWEDISH NET ZERO ENERGY BUILDING - INCLUDING CO- BENEFITS
}

\author{
Björn Berggren1,2*, Maria Wall2, Tobias Weiss3, Federico Garzia4, Roberta Pernetti4 \\ 1Skanska Sverige AB, Sweden \\ 2Lund University, Energy and Building Design, Sweden \\ 3AEE - Institute for Sustainable Technologies, Austria \\ ${ }_{4}$ EURAC Research, Italy \\ * Corresponding author: Phone: +46 104483023 \\ E-Mail: bjorn.berggren@skanska.se
}

\section{SUMMARY}

An increasing population with the need of new buildings in combination with global warming is important issues ahead. Hence, for defining a clear path towards a low and zero-emission building stock in the EU by 2050, as recently stated by the new EPBD recast, Nearly Zero Energy Buildings are one of many necessary measures for climate change mitigation. Finding cost optimal solutions are important, where a short time perspective and narrow concept for evaluation may be wrong. This study presents a Net Zero Energy Building in Sweden, with verified plus energy performance in the operation phase.

Furthermore, it presents an economic analysis, based on life cycle costing (LCC), where additional cobenefits are included. The study shows that the discounted, cumulative annual cost reductions due to energy savings may exceed the initial extra costs after more than 20 years. However, when including additional green values and increased property value, breakeven may occur already after roughly five years.

Key words: Net Zero Energy Building; Life Cycle Costing; Net ZEB, LCC

\section{INTRODUCTION}

Buildings account for over $40 \%$ of the primary energy use worldwide and almost $25 \%$ of its greenhouse gas emissions (International Energy Agency (IEA), 2013). The world's population and need for buildings is growing. Hence, for defining a clear path towards a low and zero-emission building stock in the EU by 2050, as recently stated by the new EPBD recast (European Parliament, 2018), Nearly Zero Energy Buildings (nZEBs) are one of many necessary measures for climate change mitigation. Net Zero Energy Buildings (Net ZEBs) and Nearly Zero Energy Buildings (nZEBs) are usually also defined as "green buildings", which here are referred to as buildings with high performance within the aspects of energy, thermal comfort, indoor air quality, building materials etc.

A NetZEB, is a building with zero net energy consumption, where the weighted energy demand is equal or less than the weighted energy supply (Sartori, Napolitano \& Voss, 2012). Another concept approved and implemented by the European Union is nearly Zero Energy Building (nZEB), with the goal of having all buildings in the member states of the European Union reaching nZEB standards by 2020 (European Parliament, 2010). The wording 'nearly' underlines the fact that this concept is less ambitious compared to the NetZEB ones.

Cost optimal solutions using concepts as Net NEBs and nZEBs will be major drivers in the construction sector in the next few years, as all new buildings in the EU from 2021 onwards are expected to be nZEBs (European Parliament, 2010).

In Sweden, energy tariffs are relatively low today and it may be difficult to justify investments in NetZEBs and/or nZEBs solely based on cost savings related to energy savings. A narrow concept and a short time perspective for evaluating profit, only focusing on increased investment costs and decreased energy costs, may be wrong. Both from a strict business perspective and from a socio economic perspective. 
This paper presents a verified NetZEB in Sweden including LCC analysis when other values in "green buildings" are taken into account, such as increased productivity, improved health, publicity value, etc. The estimation of the Life Cycle Costs is based on the LCC as adopted within the H2020 project CRAVEZero, aimed at identifying and reducing extra-costs of nZEBs during the whole life cycle.

\section{ADDED VALUE IN GREEN BUILDINGS}

It is important to quantify added value in green buildings in monetary terms, communicating and presenting business opportunities in a business language that potential investors are familiar with, as technical performance is less likely to attract their interest (Bleyl et al., 2017). I.e. co-benefits such as increased productivity, improved health, publicity value, etc. need to be quantified. The calculation procedures may not be complex; the challenge is to gather well proven input data for the calculations. However, examples exist where increased productivity, higher revenue, reduced employee turnover, reduced absenteeism, etc. have been quantified (Bleyl et al., 2017; Brew, 2017). Furthermore, studies do exist which may be used as a basis for analysing added values.

Studies show that employees in green buildings perceive a positive effect of their work environment and productivity (Bleyl et al., 2017; Hedge, Miller \& Dorsey, 2014; Singh, Syal, Grady \& Korkmaz, 2010; Thatcher \& Milner, 2014). In one case, a $10000 \mathrm{~m}_{2}$ office building, an increased productivity of $0.3 \%$ percent were reported, equal to $8 € / \mathrm{m} 2 \mathrm{a}$.

An American study showed that roughly 20-25\% of 534 tenants/companies reported higher employee morale, more effective client meetings and easier to recruit employees (Miller, Pogue, Gough \& Davis, 2009). Furthermore, $19 \%$ reported lower employee turnover.

In two studies, reduced absenteeism was also found (Singh et al., 2010; Thatcher \& Milner, 2014). However, in relation to green buildings and productivity and wellbeing, a recent study pointed out that social factors may have a greater impact, in monetary terms, compared to environmental factors (Hugh \& Eziaku Onyeizu, 2016).

In addition to well-being and productivity, higher revenues from rent or sales may be expected. Bleyl et al reviewed previous studies and concluded that higher rent income may range roughly in between 5 $\%$ and $20 \%$. Furthermore, higher market valuations may range from below $10 \%$ to up to $30 \%$ (Bleyl et al., 2017).

The value of a positive news article about a specific building or a specific project could also be comparable to advertising costs in the specific source, in which the article is published (Berggren, Wall \& Togerö, 2017).

One way to discuss the importance to investigate different co-benefits may be to rank them as presented in Figure 1. The classification is a subjective judgement, highlighting the relevance and the difficulty to value the co-benefits discussed above.

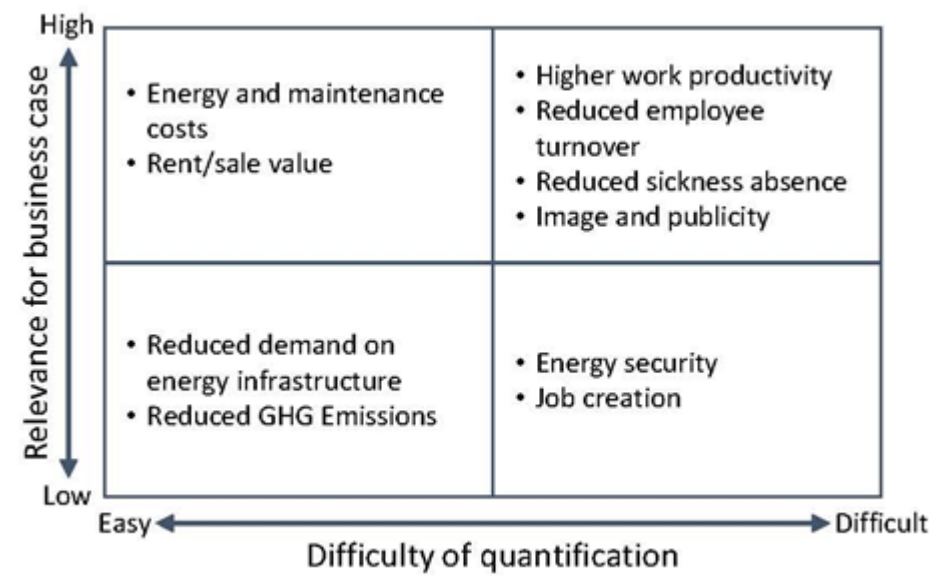

Figure 1 Co-benefits classifications, based on (Bleyl et al., 2017). 


\section{METHOD}

The case study and costs related to the building construction and operation is presented and analysed including co-benefits expressed in monetary terms. The focus is on benefits with high relevance for a business case as classified in Figure 1.

Reduced energy use and exported energy, reduced energy costs (REC) is valued according to Eq. 1. $R E C=(\Sigma E I \cdot \alpha+E E \cdot \beta) /(1+(r-i-\gamma) /(1+i+\gamma))^{\wedge} t(\mathrm{Eq} .1)$

where $E I$ is the reduced imported energy, $\alpha$ is its energy tariff, $E E$ is the increased exported energy, $\beta$ is its energy tariff , $r$ is the nominal discount rate, $i$ is the inflation rate, $\gamma$ is the increase in energy tariffs and $t$ is time.

Increased productivity value (IPV) is valued according to Eq. 2.

$I P V=\Sigma(E m p \cdot S C \cdot I P) /(1+R)^{\wedge} t($ Eq. 2$)$

where Emp is the quantity of employees, $S C$ is the average salary costs per employee, IP is the increased productivity per employee and $R$ is the discount rate as presented in Eq. 6 .

Reduced employee turnover costs (RETC) is valued according to Eq. 3.

$R E T C=\Sigma(\varepsilon \cdot E m p(R C+I C+R P C+L I+D C)) /(1+R)^{\wedge} t($ Eq. 3$)$

where $\varepsilon$ is the reduced employee turnover, $R C$ is the recruitment cost per employee, $I C$ is the introduction course for new employee, $R P C$ is the reduced productivity cost (new employee and supervisor), $L I$ is the lost income during vacancy and $D C$ is the decommissioning cost.

Reduced sickness absence salary (RSAC) is valued according to Eq. 4.

$R S A C=\Sigma(E m p \cdot 0.8 S C \cdot \phi \cdot \kappa) /(1+R)^{\wedge} t($ Eq. 4$)$

where $\phi$ is the average sickness absence and $\kappa$ is the reduced sickness absence.

Public publicity value (PPV) is valued according to Eq. 5.

$P P V=\Sigma A I P \cdot A C$ (Eq. 5)

where $A I P$ is article in press and $A C$ is the advertising costs in the specific source (paper, internet, etc.).

$R=(r-i) /(1+i)($ Eq. 6$)$

The considered time interval for the LCC calculation is 40 years in order to include in the analysis the maintenance occurrence of most of the building components. The calculation is based on the technical standard EN ISO 15686 (International Organization for Standardization, 2011).

\section{THE CASE STUDY}

The case study is located in the south of Sweden, see Figure 2. The building is a Net ZEB office building completed in 2012, with verified plus energy performance in the user phase (Kempe, 2014). The building is designed according to the passive house design principles; an airtight and wellinsulated building envelope and balanced mechanical ventilation with heat recovery. Heat is supplied via a ground source heat pump, GSHP, connected to boreholes. During summer, the boreholes are used as a natural heat sink, free cooling is extracted by circulating the working fluid for the heat pump in the boreholes. The building's roof sides facing south-west are equipped with PV panels. A summary of technical description is given in Table 1. More technical information and results from measurements and verification may also be found in other publications (Berggren, Dokka \& Lassen, 2015; Berggren, Kempe \& Togerö, 2014; Berggren, Wall, Flodberg \& Sandberg, 2012; Kempe, 2014). 

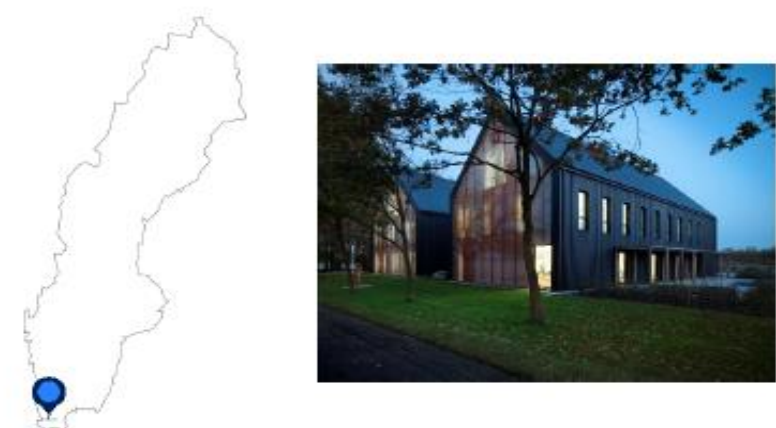

Figure 2 Left: Location of the case study in Sweden. Right: Photo of the case study

The building was taken into use in 2012 and the energy performance has been monitored, see Table 2 . The measurements have not been normalized for deviating boundary conditions (e.g. external climate, deviating use of building, etc.).

Table 1 Summary of technical description, Väla Gård

\begin{tabular}{|l|c|}
\hline Technical description & Data \\
\hline Conditioned floor area & $1670 \mathrm{~m}^{2}$ \\
\hline U-average, building envelope & $\mathrm{U}=0.26 \mathrm{~W} / \mathrm{m}^{2} \mathrm{~K}$ \\
\hline Air tightmess, assumed (qso/nso) & $0.31 / \mathrm{s}, \mathrm{m}^{2} / 1.0 \mathrm{~h}^{-1}$ \\
\hline Ventilation heat recovery & $82 \%$ \\
\hline COP (heating/cooling) & $3 / 20$ \\
\hline PV-panels (area/kWp) & $455 \mathrm{~m}^{2} / 67.5 \mathrm{kWp}$ \\
\hline
\end{tabular}

Table 2 Summarized energy performance, Vāla Gảrd

(Sim = Simulated results, Meas. $=$ Measured results $)$

\begin{tabular}{|l|c|c|}
\hline & \multicolumn{2}{|c|}{$k W h / m^{2} \mathbf{a}$} \\
Energy use & Sim. & Meas. \\
\hline Heating, cooling \& auxiliary energy & 19 & 14 \\
\hline Plug loads and lighting & 29 & 26 \\
\hline PV-panels & 38 & 41 \\
\hline Imported electricity & 29 & 23 \\
\hline Exported electricity & 19 & 24 \\
\hline
\end{tabular}




\section{RESULTS AND DISCUSSION}

Boundary conditions regarding nominal discount rate, inflation, energy tariffs, changes in energy tariffs and period of analysis are presented in Table 3. Regarding nominal discount rate, governmental and municipal organizations usually have rather low requirements, 4-6\% (Offentliga fastigheter, 2017). However, private actors may have higher requirements. In this study, we have chosen to set 7 $\%$ as the baseline. The inflation is constantly changing. In Sweden, the national target is $2 \%$ (Swedish monetary department, 2017). Hence, $2 \%$ is chosen as a baseline. Regarding energy tariffs, data show that the increase of energy prices over time in Sweden has been almost $4 \%$, not adjusted for inflation (Nils Holgersson Gruppen, 2016). I.e. a lower value, $2 \%$ is chosen. Energy tariffs are set to reflect Swedish conditions.

Table 3 Boundary conditions

\begin{tabular}{|ll|r|}
\hline Boundary condition & Input \\
\hline Nominal discount rate, $r$ & {$[\%]$} & 7 \\
\hline Inflation, $i$ & {$[\%]$} & 2 \\
\hline Tariff for imported energy, $\alpha$ & {$[€ / \mathrm{kWh}]$} & 0.12 \\
\hline Tariff for exported energy, $\beta$ & {$[€ / \mathrm{kWh}]$} & 0.10 \\
\hline Increase in energy tariff, $\gamma$ & {$[\%]$} & 2 \\
\hline Period of analysis & [years] & 40 \\
\hline
\end{tabular}

Accounting for 40 years, the LCC for Väla Gård accounts for almost $4000000 €$, corresponding to 2 $352 € / \mathrm{m}_{2}$ and $59 € /\left(\mathrm{m}_{2}\right.$, year). All costs excluding VAT. In the LCC, cost for land, site enabling and planning fees are excluded.

The main impacting phase is the construction phase (including cost of materials and labour), which accounts for $74 \%$ of the LCC. The operation- and maintenance costs are $18 \%$, while the design is around $8 \%$.

It is important to point out that the energy consumed, considering the balance with the production of the RES installed, impacts for $0.3 \%$ of the overall LCC, while the impact of maintenance, calculated by adopting the estimations proposed by the standard UNI EN 15459, is roughly $17 \%$. Figure 3 reports the breakdown of LCC cost for each phase (design, construction and labour, maintenance), also distinguishing the costs for envelope and services.
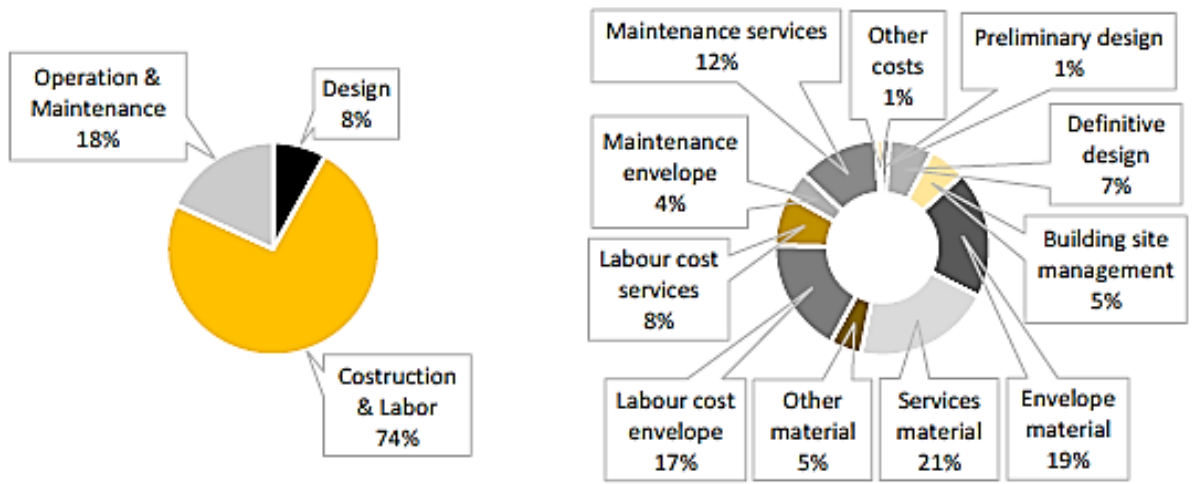

Figure 3 Left: Overall breakdown of LCC. Right: Detailed breakdown of LCC

Väla Gård reported increased costs amounting to almost $450000 €$ or $268 € / \mathrm{m} 2$ compared to if the office would have been a "normal office". Increased production costs, consultants and certifications costs are included. Regarding investment costs, a state grant was given for the PV-panels, amounting to roughly $82000 €$ or $49 € / \mathrm{m}^{2}$. 
The profitability of the increased costs related to increased energy efficiency and green values of the building are based on Eq 1-6. Reduced energy costs are based on measured values (Table 2) and boundary conditions given in Table 3 .

Salary costs, SC, are based on average salaries in Sweden (Statistiska centralbyrån, 2017c), which is roughly $3765 € /$ month. Including costs for employers, the salary costs amount to $6325 € /$ month. No differences in salary for managers and other employees have been included. In total 70 persons are employed to work at Väla Gård.

Basis for calculation of reduced employee turnover costs are given in Table 4. The average employee turnover in Sweden is $3.5 \%$ (Statistiska centralbyrån, 2017b). Based on previous findings in reduced employee turnover (Miller et al., 2009), we assume that a reduced employee turnover of $0.5 \%$ to 3.0 $\%$ is reasonable.

Based on an estimation of roughly two days of work, per recruited employee, and costs for advertising for new staff; the recruitment cost is summarised to $6500 €$ per new employee. Furthermore an introduction course for each employee is expected to cost $2000 €$.

In order to summarise reduced productivity cost, a reduced productivity of $20 \%$ for two persons is expected for 6 months. One person is the new employee the other person is one experienced co-worker who helps and guides the new employee.

Lost income during vacancy is based on a vacancy of 3 months, salary costs and nominal discount rate.

The decommissioning cost is based on an assumption of reduced productivity of the employee by 50 $\%$ after the person resigns for the remaining time of the employment.

Table 4 Basis for quantification of employee tumover costs

\begin{tabular}{|c|c|}
\hline Data & Input \\
\hline Reduced employee tumover, $\varepsilon[\%]$ & 0.5 \\
\hline Recruitment cost, $\mathrm{RC}$ & 6.5 \\
\hline Introduction course, $I C$ & 2.0 \\
\hline Reduced productivity cost, $R P C\left[€ \times 10^{3} / \mathrm{p}\right]$ & 15.1 \\
\hline Lost income during vacancy, $L I\left[€ \times 10^{3} / \mathrm{p}\right]$ & 1.3 \\
\hline Decommissioning cost, $D C \quad\left[\epsilon_{\mathrm{x}} 10^{3} / \mathrm{p}\right]$ & 9.5 \\
\hline
\end{tabular}

Average sick absences in Sweden were six days per year in Sweden 2016 (Statistiska centralbyrån, 2017a). Based on previous findings of reductions of absenteeism (Singh et al., 2010; Thatcher \& Milner, 2014), we assume a reduced sickness absence of $10 \%$, see Table 5.

Table 5 Basis for quantification of sick absence costs

\begin{tabular}{|l|r|}
\hline Data & \multicolumn{1}{|c|}{ Input } \\
\hline Average sickness absence, $\phi \quad[\mathrm{d}]$ & 6.0 \\
\hline Reduced sickness absence, $x \quad[\%]$ & 10 \\
\hline
\end{tabular}

The quantification of increased productivity is based on the reduction of share of time were an employee does not perform value creating work. I.e. increased productivity. Based on previous findings (Hedge et al., 2014; Singh et al., 2010; Thatcher \& Milner, 2014), we estimate that the productivity may increase by $0.5 \%$.

Numerous articles were published about Väla Gård. In total, ten publications about Väla Gård were considered to have such a positive value that it could be considered to be equal to advertising. The corresponding cost were estimated to $3500 €$ per article.

Based on the input data presented, the recurring cost reductions for Väla Gård (REC, RETC, RSAC and IPV) amount to roughly $69000 € /$ year or $42 € / \mathrm{m}_{2}$ a.

The distribution of the summarised green values for the first ten years and the accumulated discounted value for the cost reductions, normalised by conditioned area, are presented in Figure 4. As can be seen, the value of energy savings, compared to the performances that the building would have reached if built according to current energy requirements in building regulations, amounts to $25 \%$ of the total 
green values for the first ten years. The assumed increased productivity has the largest relative impact. Hence, these effects should be prioritized in future research.

The accumulated green values exceed the initial costs after roughly four years for Väla Gård. If only reduced costs due to energy use and PV grant would be considered, the breaking point is after 26 years.
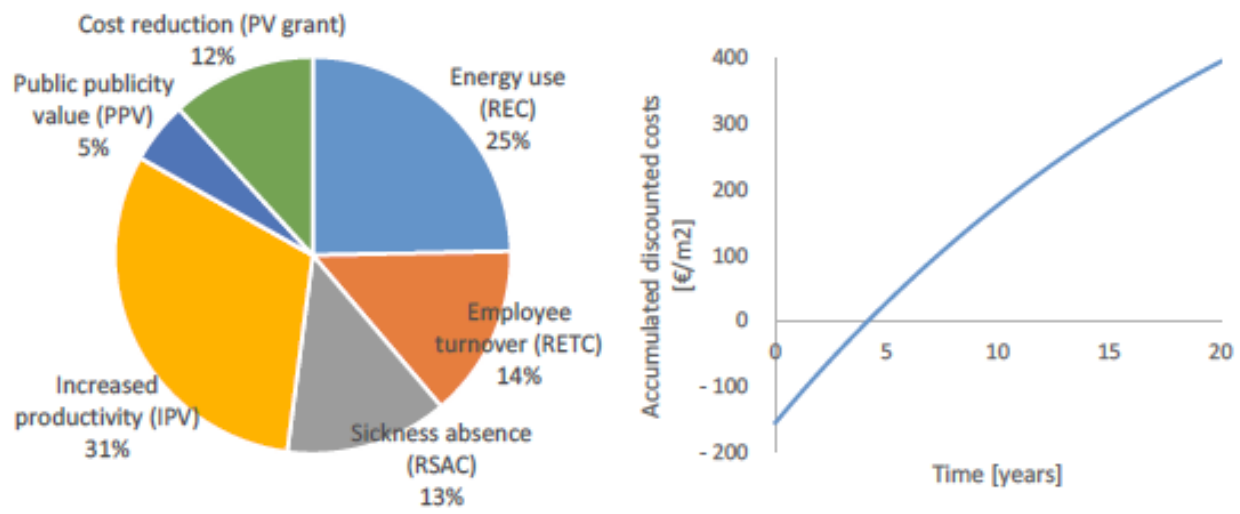

Figure 4 Left: Distribution of summarised green values for ten years. Right: Accumulated costs for investments and for green values

\section{CONCLUSIONS}

In this study we show how green values could be quantified in monetary terms. The study shows that it may be very profitable to build green buildings if one accounts for green values. In fact, the business plan is significantly affected by further values than energy savings, which that cannot balance the initial extra-investment for reaching the target nZEB or Net ZEB if a short time perspective for evaluating profit is applied.

However, more research should be done in order to further develop these methods and to gain more knowledge regarding reduced employee turnover, reduced sick absence, increased productivity, etc. in green buildings, in order to provide more reliable results to be applied in the design phase, for defining an effective business plan.

\section{AKNOWLEDGEMENTS}

This work has been co-funded by the Horizon 2020 Framework Programme of the European Union within the project CRAVEzero - Grant Agreement No. 741223 (www.cravezero.eu).

The authors wishes to thank the project manager in the case study for sharing information regarding investment costs. 


\section{REFERENCES}

Berggren, B., Dokka, T. H. \& Lassen, N. (2015). Comparison of five zero and plus energy projects in Sweden and Norway - A technical review. Paper presented at the Passivhus Norden - Sustainable Cities and Buildings, Copenhagen. http://passivhus.dk/wp-content/uploads/7PHN_proceedings/110.pdf

Berggren, B., Kempe, P. \& Togerö, Å. (2014). Väla Gård - a Net Zero Office Building in Sweden. REHVA Journal.

Berggren, B., Wall, M., Flodberg, K. \& Sandberg, E. (2012). Net ZEB office in Sweden - A case $\quad$ study, testing the Swedish Net ZEB definition. International Journal of Sustainable Built Environment, 1(2), 217-226. doi: https://doi.org/10.1016/j.ijsbe.2013.05.002

Berggren, B., Wall, M. \& Togerö, ̊. (2017). Profitable Net ZEBs - How to break the traditional LCC analysis. Paper presented at the International Conference on Energy, Environment and Economics (ICEEE), Edinburgh.

Bleyl, J., Bareit, M., Casas, M., Coolen, J., Bruyn, B. D., Hulshoff, A., . . . Robertson, M. (2017). Building deep energy retroft: Using dynamic cash fow analysis and multiple benefts to convince investors. Paper presented at the eceee 2017 Summer Study on energy efficiency: Consumption, efficiency and limits, Hyères.

Brew, J. (2017). Finding the Value in Deep Energy Retrofits. Paper presented at the World Sustainable Built Environment Conference 2017, Hong Kong.

European Parliament. (2010). Directive 2010/31/EU of the European Parliament and the Council of 19 May 2010 on the energy perfromance of buildings. Official Journal of the European Union.

European Parliament. (2018). Directive (EU) 2018/844 of the European Parliament and of the Council of 30 May 2018 amending Directive 2010/31/EU on the energy performance of buildings and Directive 2012/27/EU on energy efficiency. Official Journal of the European Union.

Hedge, A., Miller, L. \& Dorsey, J. A. (2014). Occupant comfort and health in green and conventional university buildings. Work, 49(3), 363-372. doi: 10.3233/WOR-141870

Hugh, B. \& Eziaku Onyeizu, R. (2016). The Productivity Paradox in Green Buildings. Sustainability, Vol 8, Iss 4, p 347 (2016)(4), 347. doi: 10.3390/su8040347

International Energy Agency (IEA). (2013). SHC Task 40/ECBCS Annex 52 Towards Net-Zero Energy Solar Buildings, 2013 Highlights. In S. H. C. Programme (Ed.), (pp. 2): IEA.

International Organization for Standardization. (2011). ISO 15686 Buildings and Constructed Assets Service Life Planning. Geneva: ISO Central Secretariat.

Kempe, P. (2014). Väla Gård i Helsingborg. LÅGAN Bygg.

Miller, N., Pogue, D., Gough, Q. \& Davis, S. (2009). Green Buildings and Productivity. Journal of Sustainable Real Estate, 1(1), 65-89. doi: 10.5555/jsre.1.1.6402637n11778213

Nils Holgersson Gruppen. (2016). Nils Holgersson Gruppen. 2016, from www.nilsholgersson.nu

Offentliga fastigheter. (2017). LCC-kalkyler för en hållbar förvaltning.

Sartori, I., Napolitano, A. \& Voss, K. (2012). Net zero energy buildings: A consistent definition framework. Energy and Buildings, 48, 220-232. doi: http://dx.doi.org/10.1016/j.enbuild.2012.01.032

Singh, A., Syal, M., Grady, S. C. \& Korkmaz, S. (2010). Effects of Green Buildings on Employee Health and Productivity. American Journal of Public Health, 100(9), 1665-1668. doi: 10.2105/AJPH.2009.180687

Statistiska centralbyrån. (2017a). Antal sjukdagar per anställd efter kön, sektor och kvartal. Retrieved June 13, 2017, from http://www.statistikdatabasen.scb.se/pxweb/sv/ssd/START_AM_AM0209/Sjuklone periodSektor/table/tableViewLayout1/?rxid=15a28834-1e96-4178-b307-31291 dbdb80b

Statistiska centralbyrån. (2017b). Kortperiodisk sysselsättningsstatistik 1:a kvartalet 2017 - Short-term employment 1 st quarter of 2017.

Statistiska centralbyrån. (2017c). Löneutveckling 1992-2016. Retrieved June 13, 2017, from http://www.scb.se/ hitta-statistik/statistik-efter-amne/arbetsmarknad/loner-ocharbetskostnader/lonestrukturstatistik-hela-ekonomin/pong/tabell-och-diagram/loneutveckling/

Swedish monetary department. (2017). The inflation target. Retrieved June 13, 2017, from http://www.riksbank.se/en/Monetary-policy/Inflation/Adoption-of-the-inflation-target/

Thatcher, A., \& Milner, K. (2014). Changes in productivity, psychological wellbeing and physical wellbeing from working in a 'green' building. Work, 49(3), 381-393. doi: 10.3233/WOR141876 


\section{CREATION OF HYBRID SIMULATION MODEL}

DI Mike Lagler

DI Dr. Ernst Schmautzer

Univ.-Prof. Robert Schürhuber

Institute of Electrical Power Systems

Inffeldgasse 18, $8010 \mathrm{Graz}$, Austria

Phone: +43 316 / 873-7567

E-Mail: lagler@tugraz.at
DI Werner Lerch

DI Dr. Richard Heimrath

DI Dr. Thomas Mach

Institute of Thermal Engineering

Inffeldgasse 25/B, $8010 \mathrm{Graz}$, Austria

Phone: +43316 / 873-7318

E-Mail: werner.lerch@tugraz.at

\section{SUMMARY}

The aim of this paper is to present a universal and transferable simulation model for modular hybrid energy systems using the example of a complex real-world industrial facility. By bundling the competences of two institutes of the Graz University of Technology, the Institute of Thermal Engineering (IWT) and the Institute of Electrical Power Systems (IEAN), two existing independent models, a MATLAB simulation model for the electrical system of buildings including control logic and a TRNSYS model for the thermal system were coupled together. For the hybrid simulation model, a parallel interface was chosen. In contrast to the serial variant, the level of detail of the thermal model in TRNSYS is completely retained, which means that the thermal system no longer has to be replicated in MATLAB in detail. However, the parallel variant results in an increased simulation time.

\section{INTRODUCTION}

A hybrid energy system is a system that intelligently combines energy demand and generation processes as well as energy storage systems for electricity, heating and cooling. By combining thermal and electrical systems resources can be spared, energy costs can be saved, and also the perceived comfort can be increased.

As part of the research project "Regelungsstrategien zur Effizienzsteigerung komplexer hybrider Energiesysteme (REsys)", a hybrid simulation model for economic and ecologic optimization of an industrial company is developed and the transferability to other hybrid energy systems is investigated.

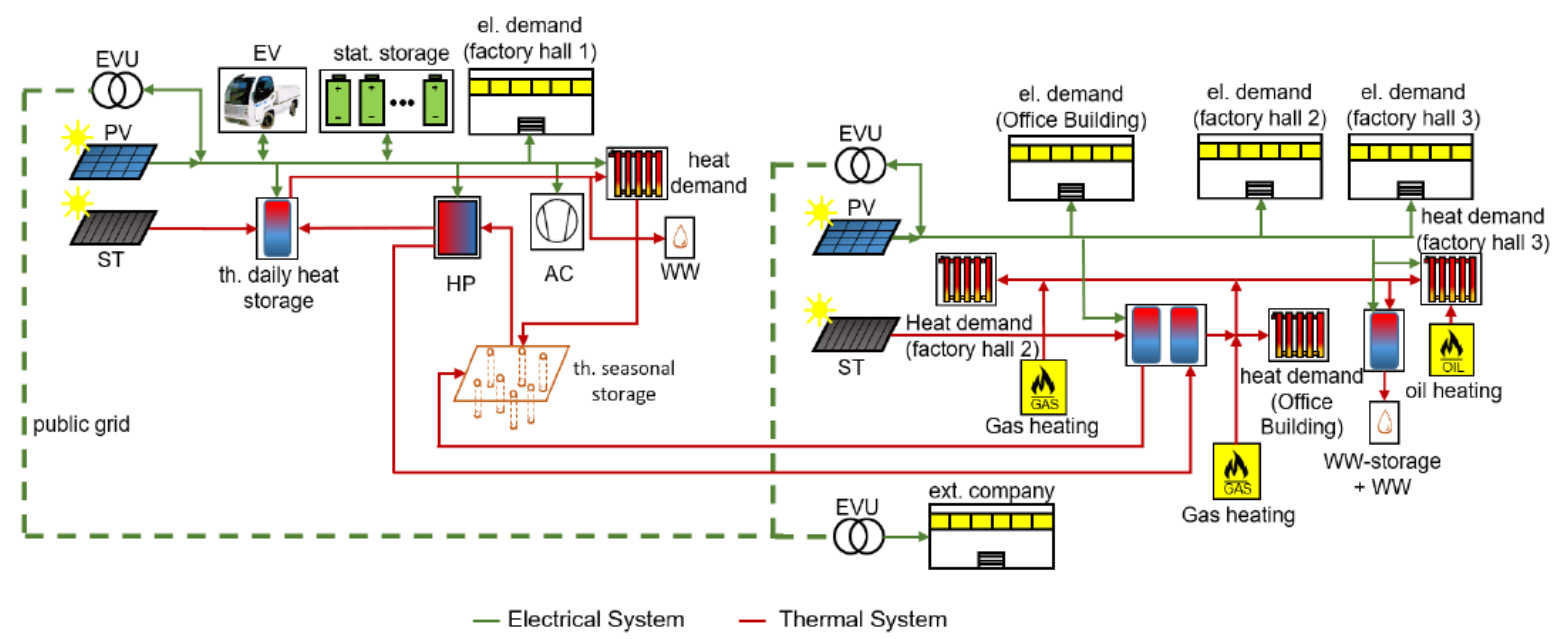

Fig 1: Schematic representation of the industrial facility 
The hybrid simulation model is controlled by the means of MATLAB, where both systems - electrical and thermal systems - are modeled and simulated. The detailed thermal system is modeled using the software TRNSYS [3], which is a tool for dynamic thermal energy building and system simulation. For the overall hybrid simulation model an interface between MATLAB and TRNSYS is necessary, which is realized by the Type 155 in TRNSYS.

\section{METHODS}

The model coupling (TRNSYS and MATLAB) takes place through TRNSYS Type 155. The connection uses the MATLAB engine, which is launched as a separate process. The Fortran routine communicates with the MATLAB engine through a Component Object Model (COM) interface. The Type 155 can have different calling modes (e.g. iterative component or real-time controller). There are no specific limits on the number of inputs, outputs, or instances of Type 155 in a simulation which makes him universally applicable [4]. The following Fig 2 shows the schematic representation of the implemented interface.

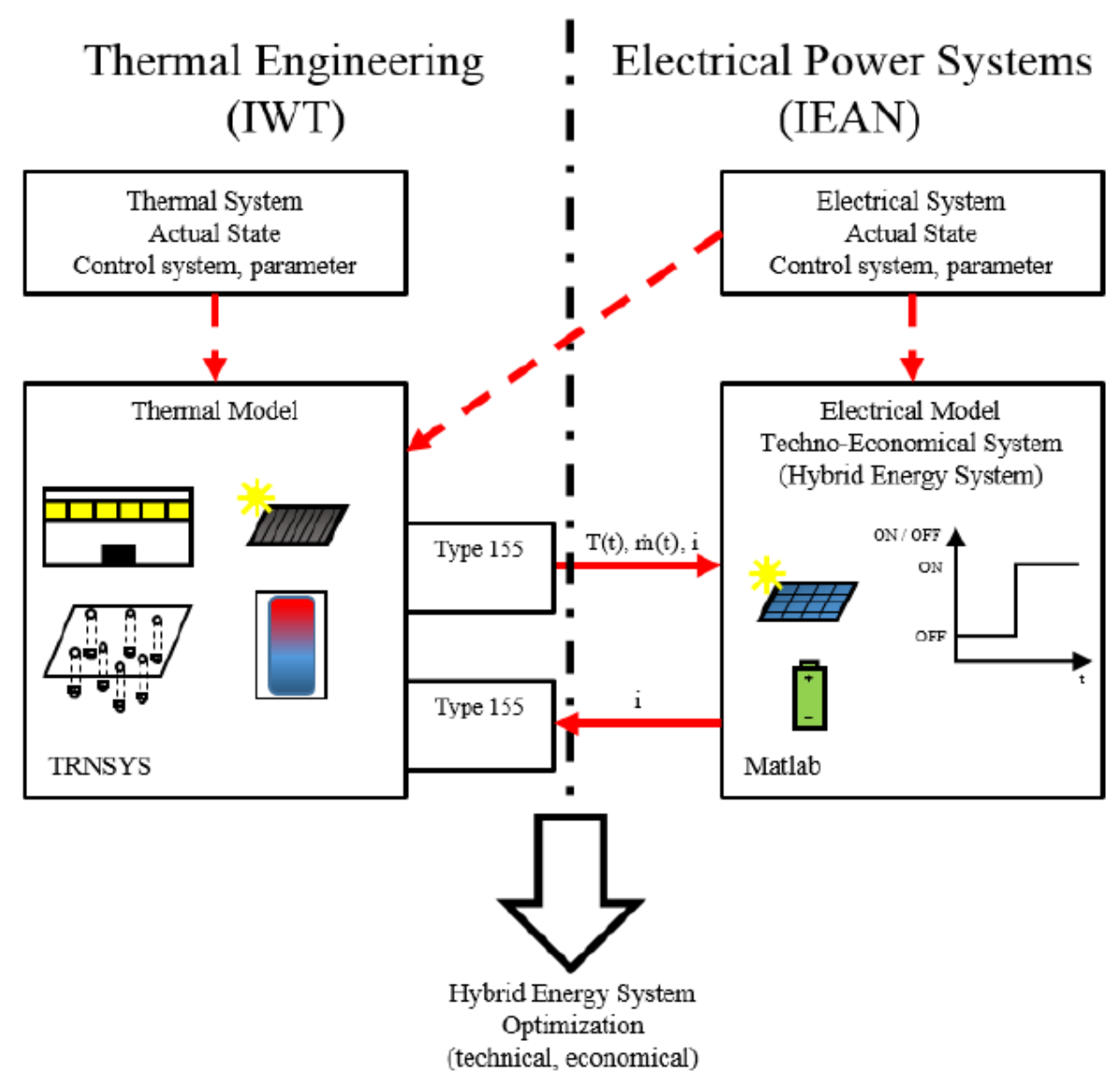

Fig 2: Schematic representation of the interface (Type 155)

There are two ways to implement the interface, the serial and the parallel interface. The parallel interface has advantages over the serial interface as well as disadvantages. Thus, the simulation time increases significantly in the parallel interface due to the constant exchange between the two models. Furthermore, the collaboration between the two institutes is becoming more intense, creating mutual dependencies on the creation of the models, which have a temporal and organizational impact. This does not apply to the serial interface where additional validations would have to be performed. The coupled model (TRNSYS and MATLAB) is optimized using a linear optimization algorithm (MILP - Mixed Integer Linear Programming), where MATLAB acts as a so called "Master" and transfers the resulting control parameters (e.g. heat pump 1 "on") to TRNSYS via the implemented 
interface (Type 155). The methodology of the implemented optimization is shown in the following Fig 3.

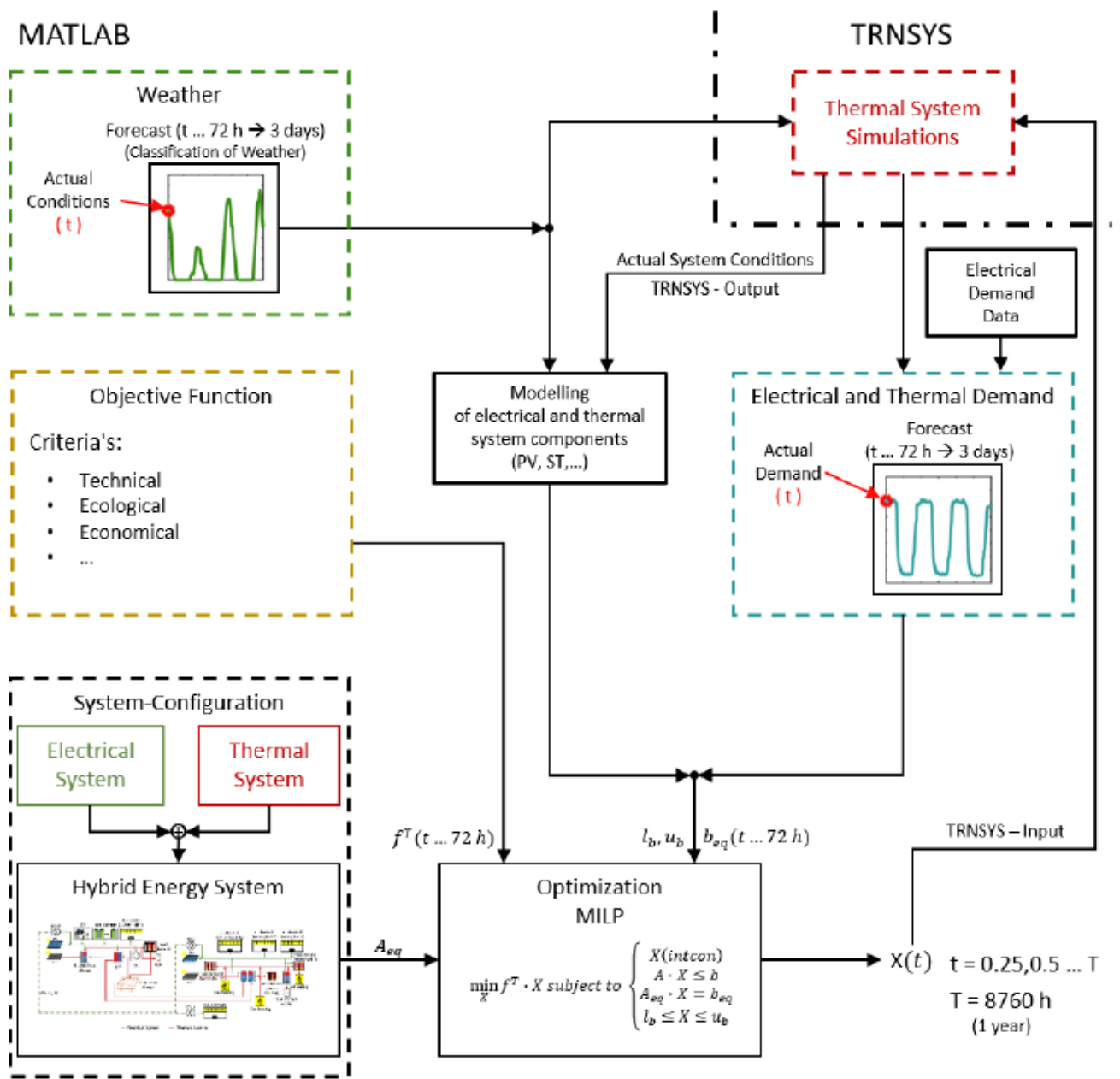

Fig 3: Methodology of optimization

The optimization essentially consists of the following five parts:

- System configuration

- Objective function

- Weather and demand

- Modelling of system components

- Optimization algorithm

The entire system is represented by the system matrix Aeq. This Matrix represents the physical connections of all system components to each other, i.e. which technologies are interconnected (see Fig 1). The objective function defines the goal of optimization, e.g. minimize the cost of the system or increase the degree of autonomy. The weather data is important for modeling (e.g. season, global radiation and ambient temperature). These have a significant impact on the operation and energy demand of the industrial facility (e.g. heating behavior) and power generation (e.g. photovoltaic or solar thermal generation).

To account long-term effects, the optimization is performed in a temporal time resolution of 15 minutes over a period of at least one year. Since long-term forecasts of weather and demand, especially the electricity demand, have a high degree of uncertainty, the optimization algorithm always takes into account the upcoming 3 days (72 hours). 
The chosen optimization algorithm determines for a given objective function $\mathrm{f}_{\mathrm{T}} \cdot \mathrm{X}$, under the specification of linear constraints $\left(A_{e q} \cdot X=b_{e q}\right)$, for each selected scenario an optimal result (see (eq. $1)$ ).

$$
\begin{aligned}
& \min _{x} f^{T} \cdot x \text { subject to }\left\{\begin{array}{c}
x(\text { intcon }) \\
A \cdot x \leq b \\
A_{e q} \cdot x=b_{e q} \\
l_{b} \leq x \leq u_{b}
\end{array}\right. \\
& X \quad \quad \ldots \text { system variables (energy flow) }
\end{aligned}
$$

The objective function can be based on economic (most economical use of system components), technical (optimal use of decentralized energy sources, optimal autonomy), given scenarios (e.g. gas heating disabled), and, in this project optional, ecological criteria $\left(\mathrm{CO}_{2}\right.$ equivalence).

\section{RESULTS}

At the beginning of the results chapter an overview of the thermal conversion and demand side for the considered industrial company is shown. The thermal balance for the heat sources and consumers are shown in Fig 4 for the period 01.08.2016 - 31.07.2017. All thermal heat sources are stacked in the left monthly column (marked with a plus sign in the legend of the figure) and the thermal heat sinks (marked with a minus sign) are stacked on the right monthly column. The description of the legend for Fig 4 is shown in Table 1. In Fig 4 the heat sources consist of the four heat pumps, the two solar thermal collector fields and a gas boiler. The heat sinks on the other hand contains four different buildings, the heat losses of the water storages, the losses of the district heating system and the thermal heat for the regeneration of the boreholes. The thermal balance shows the exact composition of the heat sources and sinks and their monthly course. It is particularly worth mentioning that at the peak of the heating load in the winter months of December, January and February, a very large proportion of the required heat must be contributed by the gas boiler. For example, for the month of January the gas boiler supplies a heat quantity of $137.5 \mathrm{MWh}$ of a total demand of $261.4 \mathrm{MWh}$, which corresponds to a share of $52.6 \%$.

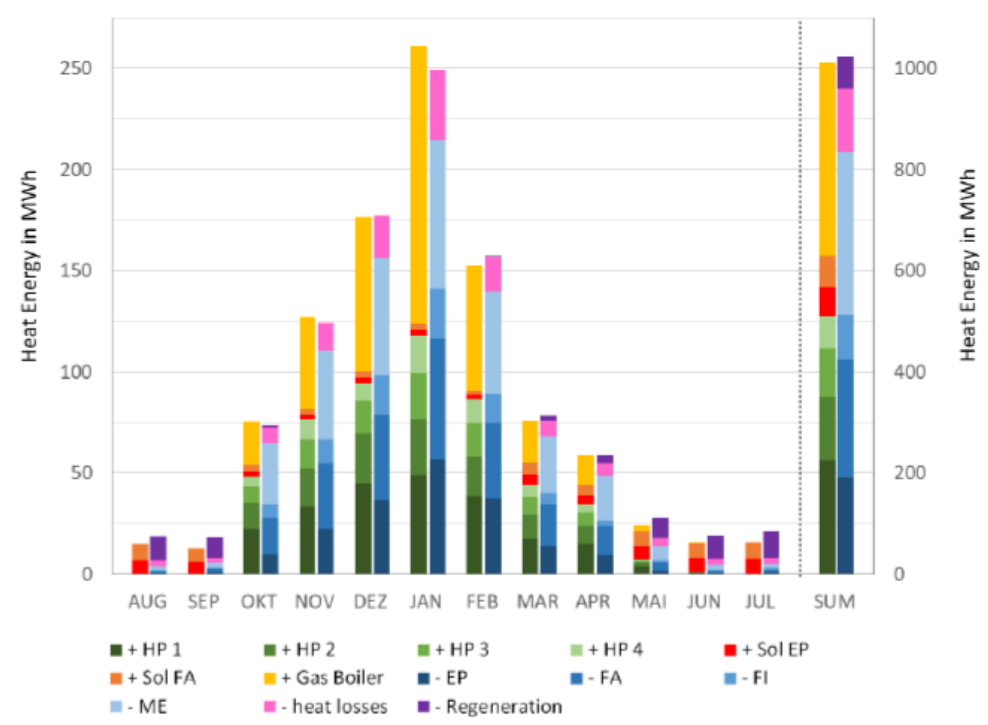

Fig 4: Heat balance (01.08.2016-31.07.2017) (See Table 1 for abbreviations) 
Table 1: Legend to Figure 2

\begin{tabular}{|c|c|}
\hline Abbreviation & \\
\hline +HP 1 & Converted heat on the condenser of HP1 \\
\hline+ HP 2 & Converted heat on the condenser of HP2 \\
\hline +HP 3 & Converted heat on the condenser of HP3 \\
\hline + HP 4 & Converted heat on the condenser of HP4 \\
\hline + Sol EP & Supplied solar heat (E-Production) \\
\hline + Sol FA & Supplied solar heat (Fassadenbau) \\
\hline+ Gas Boiler & Heat supply by gas boiler to the district heating system \\
\hline -EP & Heat demand building E-Production \\
\hline -FA & Heat demand building Fassadenbau \\
\hline -FI & Heat demand building Fibag \\
\hline -MA & Heat demand building Metallbau \\
\hline heatlosses & Heat losses storages and district heating system \\
\hline Regeneration & Solar heat supplied to the boreholes (regeneration) \\
\hline
\end{tabular}

Furthermore, the balance shows that the regeneration of the geothermal boreholes occurs mainly in the months of May to September and a total amount of heat of $63.8 \mathrm{MWh}$ could be used for regeneration of a total yield of solar systems of $121.0 \mathrm{MWh}$, which represents a share of $52.7 \%$. Finally, it should be noted that the significant differences between the heat sources and sinks of the individual month is caused due to different charge level of the storages. This means a temporal shift of the heat conversion and the heat demand due to the storage capacity of the entire system. Looking at the balance sheet for the full year, however, this effect can be almost completely compensated. A cumulative amount of heat from 1010.8 MWh was generated for the entire period on the heat source side. On the other side, there is a heat demand of the sinks of $1023.9 \mathrm{MWh}$, which corresponds to a deviation of about $1.3 \%$ based on the heat sources.

Due to the historical growth of the industrial company, the electrical system is separated into two parts (EP; FA, FI, ME) and another external company location connected via the public electricity grid. The public electricity grid is also part of the optimization.

The photovoltaic system (PV-systems) is divided into two subsystems, with an installed capacity of $345 \mathrm{kWp}$ of the first and $660 \mathrm{kWp}$ of the second subsystem, with an orientation of the modules varying from west to east. Fig. 5 (right columns) shows the monthly electrical energy demand of the industrial company and Fig. 5 (left columns) shows the calculated electrical energy converted by photovoltaic systems. 


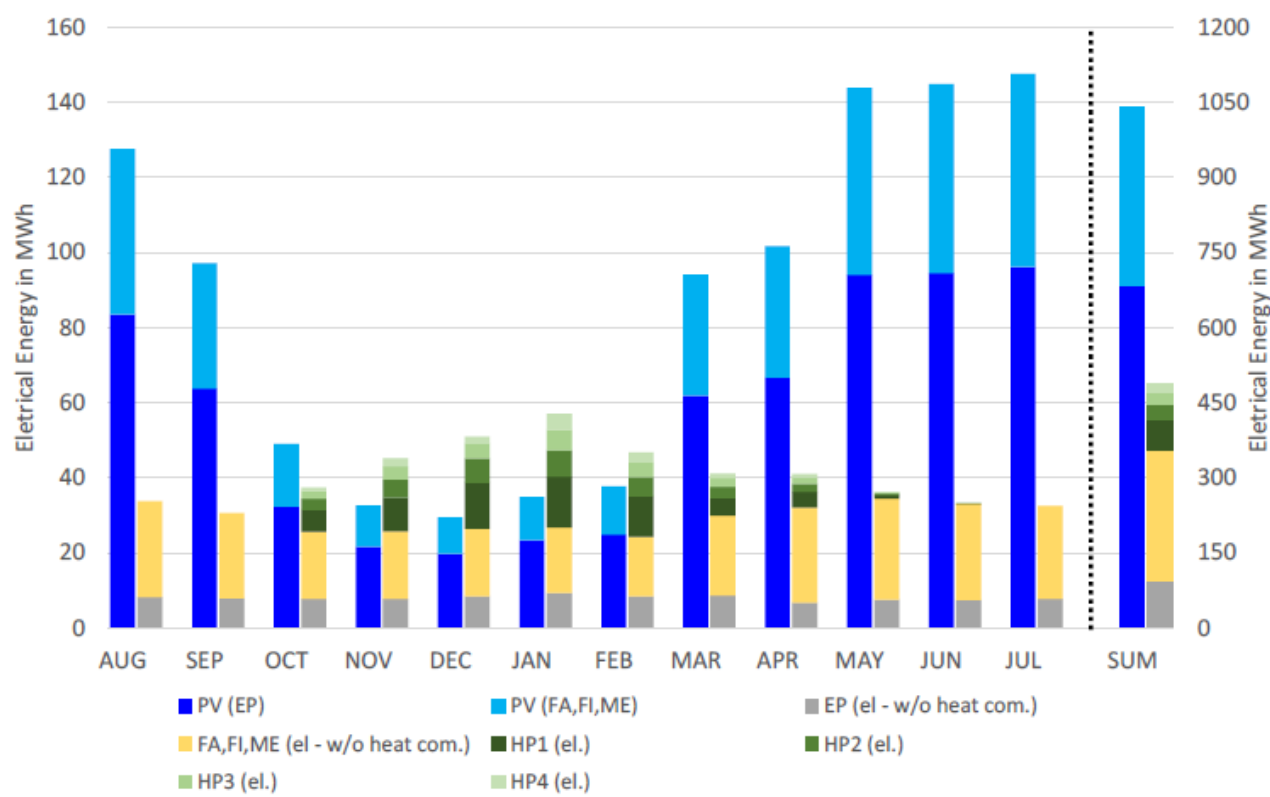

Fig. 5: Monthly electrical energy generation (left columns) and demand (right columns / w/o el. demand of the heating system)

The electrical energy demand varies over the year from 24 MWh to $34 \mathrm{MWh}$ per month, with an average demand of about $29 \mathrm{MWh}$ per month, without the electrical demand of the heating system. The four heat pumps together have an electrical energy demand of $131 \mathrm{MWh}$ per year. The generation of the photovoltaic system cumulatively amounts to $1040 \mathrm{MWh}$. This would correspond to a percentage share of the electrical energy demand (excluding electrical energy demand of the heating systems) of $293 \%$. Further Fig. 5 shows, that there is a significant phase shift of about 6 months between maximum PV yield and maximum electricity consumption. As a result, there is a significant deficit of supply on the PV side during the winter months and a significant oversupply in the summer months.

\section{CONCLUSION}

At the beginning of the project the system components at the industrial plant were collected with the associated integrated control strategy on the thermal and on the electrical side. In the next step, the system was modeled and tested by simulation models in TRNSYS and MATLAB. At the same time, the plant was equipped with measuring instruments and a monitoring was started. Based on the obtained measurement data, the simulation models were validated for a complete measurement year. The next step was to couple the separately validated models (thermal and electrical). The choice fell on a parallel solution, because the simulation time increases significantly in the parallel interface due to the constant exchange of the two models and a simulation time step interaction is present. The coupling of TRNSYS and MATLAB has been implemented and tested by Type 155 . The thermal control was also handed over to MATLAB, as a result of which the regulation of the thermal system components is now activated or deactivated as a function of the optimization algorithm. With the optimization algorithm different target functions can be defined. For example, economic $(€ / \mathrm{kWh})$ and ecological (CO2 emissions) target functions can be defined and, as a result, dynamic building and system simulations can be evaluated through different indicators (heat demand MWh/a, solar fraction, share of the energy of the gas boiler). A validated coupled simulation model is currently available with which optimization calculations can be carried out, whereby an additional 72-hour forecast (weather and demand side) can be taken into account. 


\section{ACKNOWLEDGEMENTS}

This research was supported / funded by the "Klima- und Energiefonds" (KLIEN) and is associated with the project "Regelungsstrategien zur Effizienzsteigerung komplexer hybrider Energiesysteme (REsys)" (Project No. 848936).

\section{REFERENCES}

Lagler, M., Schmautzer, E., Grobbauer, M., Gratzer, J., Michtner, M. (2017), Modellierung eines industriellen hybriden Energiesystems unter Einbeziehung dezentraler Energieerzeugung und speicherung, IEWT 2017, Wien, Österreich.

Lagler, M., Schmautzer, E., Forsthofer, S. (2016), Modellierung eines hybriden Energiesystems unter Berücksichtigung dezentraler Energieerzeugung und -speicherung am Beispiel eines

Einfamilienhauses mit Anbindung an das öffentliche Elektrizitätsnetz, Forum Econogy 2016, Linz, Österreich.

MATLAB (2014), MATLAB R2014b, MathWorks

TRNSYS (2012), A Transient System Simulation Program: V17.02.0004, Solar Energy Lab, University of Wisconsin - Madison, USA.

TRNSYS17 (2009), a TRaNsient System Simulation program, Volume 4, Mathematical Reference, Solar Energy Laboratory, University of Wisconsin-Madison. 


\section{VITALITY - DESIGNRULES FOR BUILDING INTEGRATED PHOTOVOLTAICS IN THE EARLY PROJECT DEVELOPMENT STAGE}

Tim Selke1*, Marcus Rennhofer1, Thomas Schlager1, Sebastian Sautter2, Martin Kaftan2, Anita Preisler3, Julia Wenin 4

1 AIT Austrian Institute of Technology GmbH, Giefinggasse 4, 1210 Vienna, Austria

2 Institute for Energy and Buildings, Graz University of Technology, Rechbauerstraße 12/III, 8010 Graz, Austria 3teamgmi Ingenieurbüro GmbH, Schönbrunnerstraße 44/10, 1050 Wien;

4 Master's program (Master thesis in progress)/ University of Natural Resources and Life Sciences, Vienna *Phone: +436648251008

*E-Mail: tim.selke@ ait.ac.at

\section{SUMMARY}

For achieving the ambitious Climate and Energy Targets of the European Union (2009, 2011 and 2015) the area-wide implementation of integrated photovoltaics (BIPV) is key. Therefore, these objectives require strong efforts in terms of simplifying and enabling the consideration of BIPV in the planning process of buildings and urban districts. For developing building projects, the integral planning approach supports the successful implementation of BIPV. One obstacle is the lack of both tools and easy-to-use rules for planning support, especially by non-PV specialists in the early planning stage. Therefore, the aim of the Austrian research project VITALITY is to find a) design rules and b) to indicate technically meaningful planning parameter, c) to show up exemplary use cases and d) to analyze the influence of BIPV on further planning parameters of buildings (such as thermal comfort, electrical yield). The usability or relevance for BIM systems should play an essential role. Within this 20 paper first findings based on modelling and simulation are presented.

Keywords: building integrated photovoltaic (BIPV), integral planning, design rules, simplified models

\section{INTRODUCTION}

In the planning process of buildings, an integral planning guideline is required to successfully implement Building-Integrated Photovoltaics (BIPV). Currently existing shortcomings are a lack of both tools and a set of rules that can be easily applied in order to support especially those planners at an early stage who are not experienced in PV. VITALITY aims at developing design rules and key indicators of technically meaningful planning parameter to be applied in exemplary use-cases for urban context. Further, the influence of BIPV on further planning parameters of buildings (like thermal comfort, electrical yield) are evaluated. The usability and relevance for Building Information Modelling (BIM systems) will play an essential role in the project. According to the definitions of Climate Targets of the European Union (2009, 2011 and 2015) the area-wide implementation of BIPV is key. At the same time the European Building Directive [1] foresees the compulsory implementation of BIPV or similar active energy generation measures. As well the 'Technology-Roadmap for Austria' [2] highlights that photovoltaic will play a major role in the future energy system and continued research fosters the Austrian BIPV market. These developments require strong efforts in terms of simplifying and enabling the consideration of BIPV in the planning process of buildings and urban districts.

\section{METHOD}

VITALITY addresses the elaboration of tools for an integrative planning process. The results of the project are a set of numerical but simplified rules that allow a refined BIPV planning already during the design process. The project analyses design criteria and standardization and transforms them into simplified models. Prototype buildings are planned in parametric studies, simulated in terms of thermal performance, and they are examined with regards to their thermal, electrical and BIPV expert planning. Planning interfaces and planning information flows are considered, and the relevance for BIM-systems is displayed. 


\section{RESULTS}

\section{PV for a 5-storey building in the city}

Vitality addresses the integration of photovoltaics as well in cities, i.e. building envelopes are shaded by neighbouring buildings, construction, vegetation etc. For cities like Vienna, surfaces of building envelopes with the orientation south and a slope of around 30 degrees achieve highest annual yields of solar irradiation. All other surface orientations have lower values of annual solar irradiation. For south oriented façades only $70 \%$ of the maximal irradiation values can be achieved.

A parametric study has been applied for getting findings or rules of thumb for generated solar electricity for buildings in the cities which are partially shaded by the urban environment. The parametric study is based on simulation results by using the commercial software $\mathrm{PV} * \mathrm{SOL}$ premium (R5) [3]. Exemplary, a five-storey multi-family building was modelled and the street facing façade is turned virtually to five different azimuths. With respect to shading effects by neighbouring buildings, a vis-à-vis building is modelled with same height and three different distances from façade to façade. Finally, the defined parametric study generates in total fifteen times five annual solar irradiation values for different building surfaces with different orientation. In summary the parameters that have been varied and analysed are:

a) Five different orientation of the street facing façade (Azimuth/Slope): $\left(\right.$ East $\left./ 90^{\circ}\right),\left(\right.$ East-South $\left./ 90^{\circ}\right)$, South $\left./ 90^{\circ}\right)$, West $/$ South $\left./ 90^{\circ}\right)$, West $\left./ 90^{\circ}\right)$.

b) Three different distances from façade to façade: 15, 20 and 30-meter street wide

c) Five storeys multi-family house: First level up to the fifth level

The simulation based parametric study applies the weather data set WIEN/CITY(AUT) with degree of latitude $48^{\circ} 12^{\prime} 0^{\prime \prime}$ and degree of longitude $16^{\circ} 21^{\prime} 35^{\prime \prime}$ of the PV*Sol software. The weather data set for Vienna contain hourly values that represent mean values from 2000 till 2009. The annual solar irradiation on the horizontal surface is around $1,200 \mathrm{kWh} / \mathrm{m}^{2}$. For modelling the photovoltaic system, modules that have been selected are made from polycrystalline silicon and the performance is modelled by the technical data taken from the product KPV PE NEC $270 \mathrm{Wp}$.

Fig. 1 displays the simulation results of 75 runs. The colourful patterns indicate relative values, where the $100 \%$ value corresponds to the maximum yield of solar electricity from a south oriented and 30 degree sloped module.

For indicating the PV electricity yield as a function of the same set of parameters, an analysis of the economics of several PV system designs were carried out. According to the standards VDI 2067 and ÖNORM M 7140 a dynamic economic approach was applied and two economic parameters are indicated by calculation: a) Levelized Cost Of Electricity (LCOE) and b) the payback period. With regards to the economics the ratio of self-consumed PV electricity and the cost for PV System is crucial. Generally speaking, the more PV electricity can directly be consumed in house and the lower the front up cost of the PV system the better the economics. The total annual electricity demand of the five-storey multi-family house is around 48.08 MWhel based on the specific value of $38,4 \mathrm{kWh}$ per m² useful area. The standard electricity profile "H0 Household general" [4] from electricity providers has been taken for the investigation.

Two cases for the investigation were defined and set up:

1) Four different PV system capacities in $10 \mathrm{kWpeak}$ steps were modelled (10, 20, 30 and 40 $\mathrm{kWpeak}$ ), the PV system is rooftop mounted with an azimuth of south and slope of $30^{\circ}$. Technical performance data from polycrystalline modules are taken - see above.

2) Five different orientation of a $40 \mathrm{kWpeak} P V$ system were modelled, i.e. the PV modules are oriented to i) South/30; ii) West/30; iii) East/30; iv) South/90 and v) West and East/30) 
Fig. 2 displays and lists the results of the parametric study in terms of energy and economic performance.

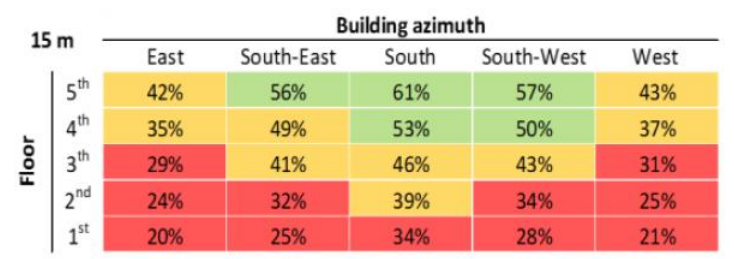

\begin{tabular}{|c|c|c|c|c|c|c|}
\hline \multirow{2}{*}{\multicolumn{2}{|c|}{$30 \mathrm{~m}$}} & \multicolumn{5}{|c|}{ Building azimuth } \\
\hline & & East & South-East & South & South-West & West \\
\hline \multirow{5}{*}{ ๖ั } & $5^{\text {th }}$ & $47 \%$ & $62 \%$ & $66 \%$ & $63 \%$ & $49 \%$ \\
\hline & $4^{\text {th }}$ & $44 \%$ & $58 \%$ & $63 \%$ & $59 \%$ & $45 \%$ \\
\hline & $3^{\text {th }}$ & $39 \%$ & $55 \%$ & $58 \%$ & $55 \%$ & $41 \%$ \\
\hline & $2^{\text {nd }}$ & $35 \%$ & $48 \%$ & $52 \%$ & $46 \%$ & $38 \%$ \\
\hline & $1^{\text {st }}$ & $32 \%$ & $29 \%$ & $48 \%$ & $30 \%$ & $33 \%$ \\
\hline
\end{tabular}

\begin{tabular}{|c|c|c|c|c|c|}
\hline \multirow{2}{*}{$20 \mathrm{~m}$} & \multicolumn{5}{|c|}{ Building azimuth } \\
\hline & East & South-East & South & South-West & West \\
\hline $5^{\text {th }}$ & $42 \%$ & $58 \%$ & $63 \%$ & $60 \%$ & $43 \%$ \\
\hline $4^{\text {th }}$ & $35 \%$ & $53 \%$ & $57 \%$ & $54 \%$ & $37 \%$ \\
\hline $3^{\text {th }}$ & $29 \%$ & $47 \%$ & $51 \%$ & $47 \%$ & $31 \%$ \\
\hline $2^{\text {nd }}$ & $24 \%$ & $40 \%$ & $46 \%$ & $41 \%$ & $25 \%$ \\
\hline $1^{\text {st }}$ & $20 \%$ & $34 \%$ & $40 \%$ & $34 \%$ & $21 \%$ \\
\hline
\end{tabular}

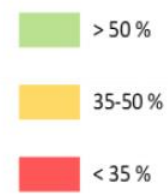

Fig. 1: Results of the parametric studies for indicating solar irradiation potential on the façade of a five-storey building. Façade shaded by vis-à-vis buildings in 15, 20 and 30-meter distance (Source: Wenin)
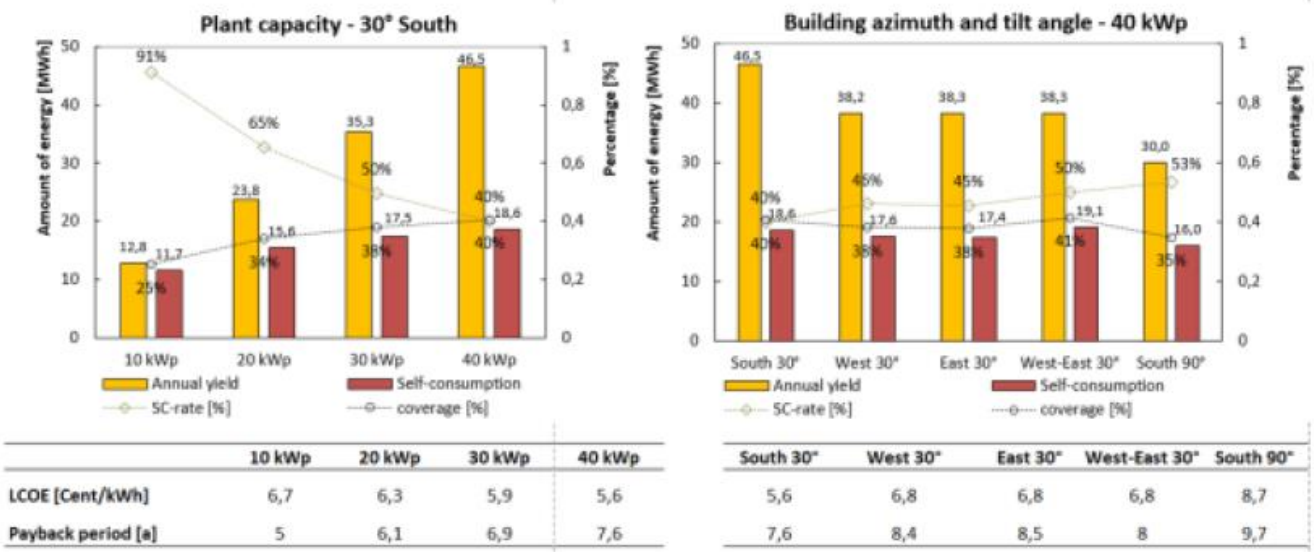

Fig. 2: Parametric study of PV yield calculation and energy performance indicators: (left) different PV system capacities rooftop mounted (south $/ 30^{\circ}$ ) and (right) different module position (rooftop, façade) of a $40 \mathrm{kWp}$ capacity (Source: Wenin)

\section{Indication of the solar potential of different building typologies (rotated $360^{\circ}$ )}

As a result, the simplified models and parametrical simulations lead to establish design rules for an early planning stage, respecting the typical needs of planners for both planning flexibility and precision in terms of the expected BIPV-yield. First studies and visualization of the solar potentials on building surface were carried out for defined building typologies. In order to visualize the solar potential for different building orientations, the building was rotated in $1^{\circ}$ steps. The modelling work and calculations were performed by the software interface Rhino/Grasshopper® with Accelerad [5]. A specially written interactive tool was used to evaluate the results (Fig. 3). 

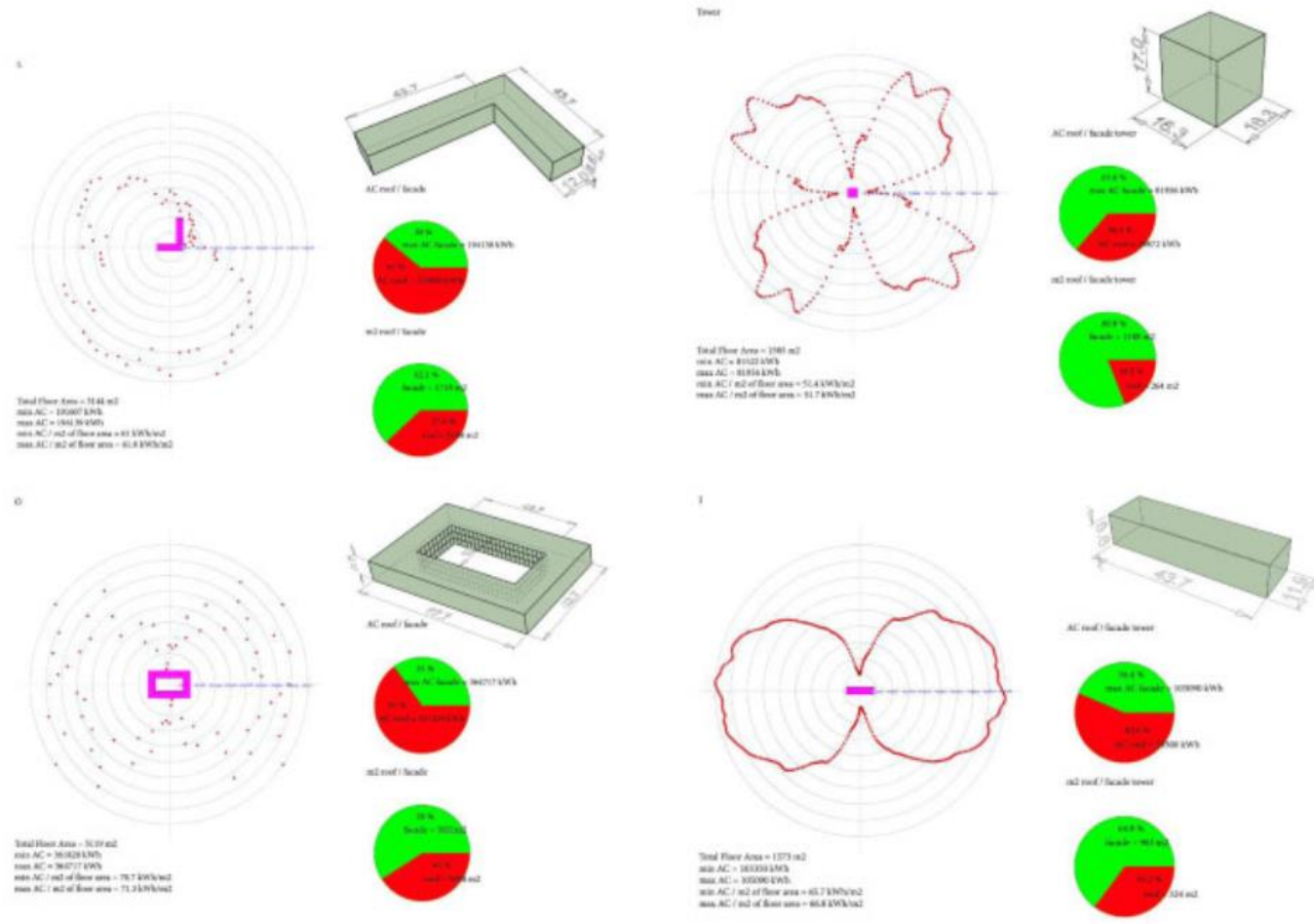

Fig. 3: Solar potential of different building topologies presented via interactive tool

\section{Method of suitable PV module placement on the building shell}

A fast way to determine the effective positions of PVs is to divide the building envelope into a grid and calculate the solar radiation on it. According to the selectable threshold of minimum yearly radiation $(\mathrm{kWh} / \mathrm{m} 2)$ the calculation result can be visualized building's area suitable for PV placement (Fig.4). The advantage of this approach is the reduced complexity of data processing due to using only daily values. Therefore, the solar analysis can be coupled with fast analysis of energy demand, such as AugP [6].
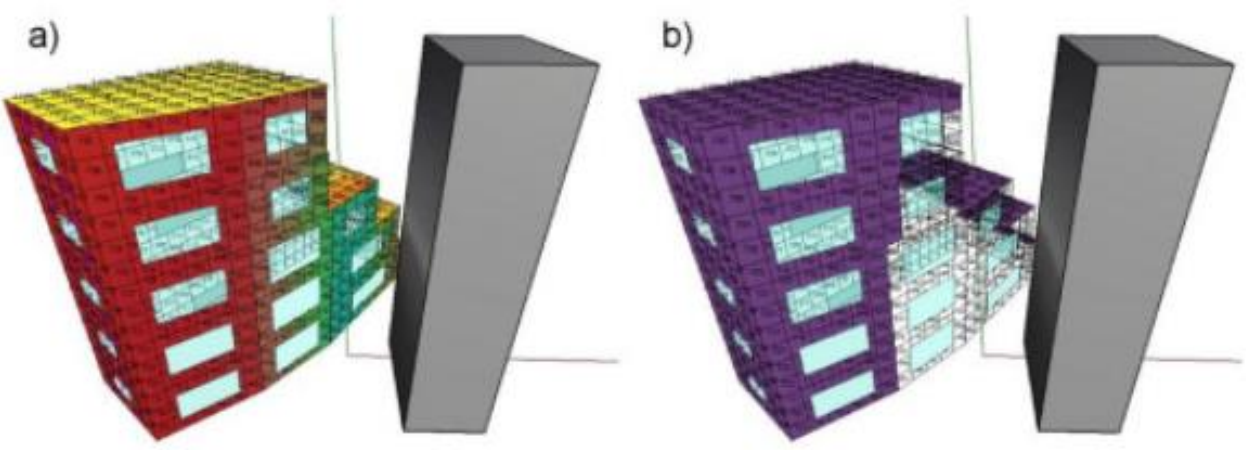

Fig. 4: a) solar radiation grid, b) PV effective distribution $>6 \mathrm{kWh} /$ year 
However, for more thorough evaluation of the PV potential it is necessary to work in hourly steps. For this, the MIT tool Accelerad was used again with custom script accessing the database with hourly values. For generating energy demand profiles EnergyPlus [7] was used. Two different approaches were used to determine the best positions of PV panels on façade according to the energy demand. In the first approach for each hour in a year were PV panels sorted according to the actual production. The place in the list gave the panel a value of importance. The yearly sum gave each PV panel its absolute importance value. However, this approach provides abstract evaluation plus panels with smaller sizes are penalized (Fig. 5a). Therefore, a second approach was used. In this case PV panel's hourly production was sorted again, but now only the best performing panels that covered the energy demand in that particular hour were calculated as valuable. The yearly sum gave each PV panel its production value that it would contribute to cover the total energy demand. This was then converted to the cost savings and compered to the investment cost which gave each panel its estimation of investment returns in years. Therefore, this analysis can be used as a threshold (Fig. 5b).

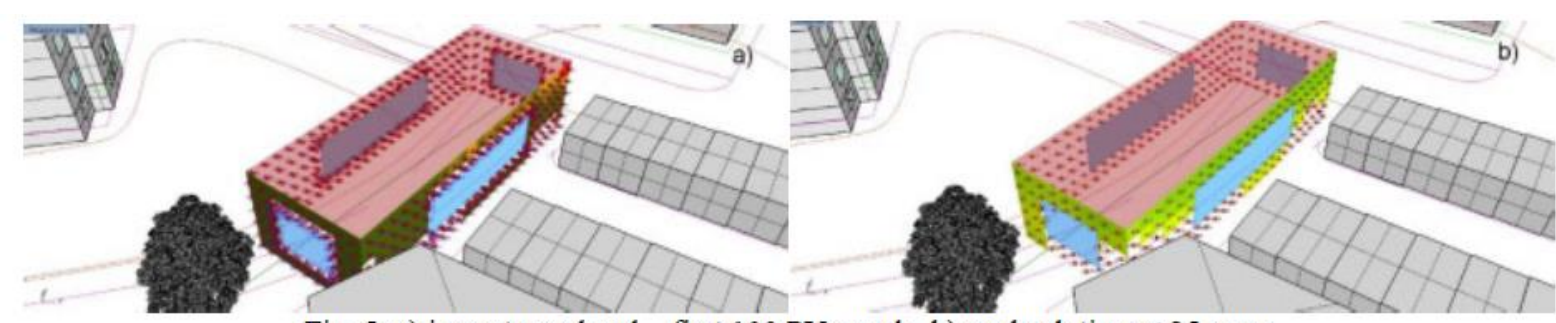

Fig. 5: a) importance level - first $100 \mathrm{PV}$ panels, b) payback time $<25$ years

\section{Evaluation of the simplified PV yield calculation and validation}

To enable an initial evaluation of PV energy yield in a short calculation time a simplified calculation method was implemented in the Grasshopper environment. Fig.6 shows the method of the yield calculation.

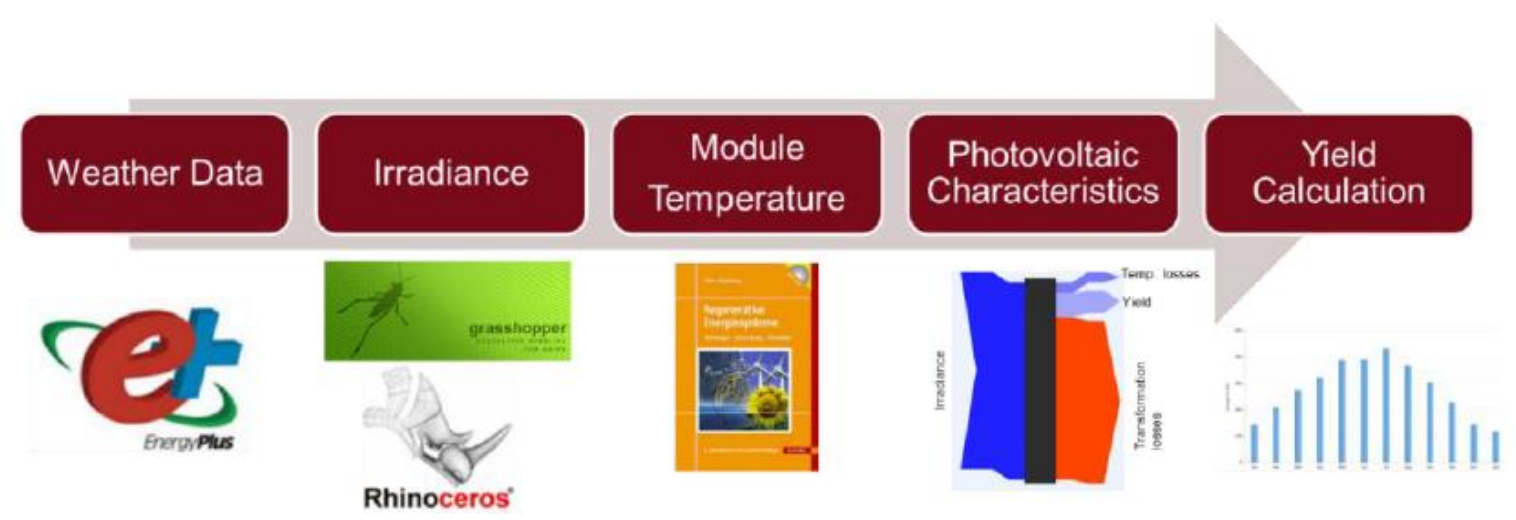

The basis of the yield calculation is the weather data provided by the EnergyPlus database. A building model can be created or loaded in the computer-aided design (CAD) application software

Rhinoceros ${ }^{\circledR}$. The integrated programming module of grasshopper enables to connect the weather data with the building model, what leads to the calculation of the irradiance on every surface of the building envelope. As the focus of the project is on building integrated PV, the irradiance on the surface of the building envelope is the same as the irradiance in plane of the PV modules (EPV). The module temperature $(\vartheta \mathrm{M})$ is calculated as a function of irradiation, ambient temperature and installation situation. Standard characteristic values for different PV components, like module efficiency $(\eta \mathrm{PV})$, inverter efficiency ( $\eta \mathrm{INV})$ and temperature coefficient $(\alpha \mathrm{P})$ are used to respect the energy losses in the yield calculation. Finally, the PV yield is calculated as follows:

$$
\mathrm{PV} \underset{\text { Yield }}{ }=\mathrm{E} \underset{\mathrm{PV}}{\eta_{\mathrm{PV}}} \eta_{\operatorname{Inv}}\left(1+\alpha_{\mathrm{P}}\left(\vartheta_{\mathrm{M}}-25^{\circ} \mathrm{C}\right)\right)
$$


To validate the simplified calculation method, a comparison of the yearly energy yield for north, east, south and west façade with the commercial PV*SOL simulation tool was done. The deviation of results is shown in Fig.7. On the east and west façade, the yearly energy yield simulated is about 2\% higher than the calculated yield, whereas on the south façade it is about $7 \%$ less. Most significant deviations occur on the north facade with more than $14 \%$.

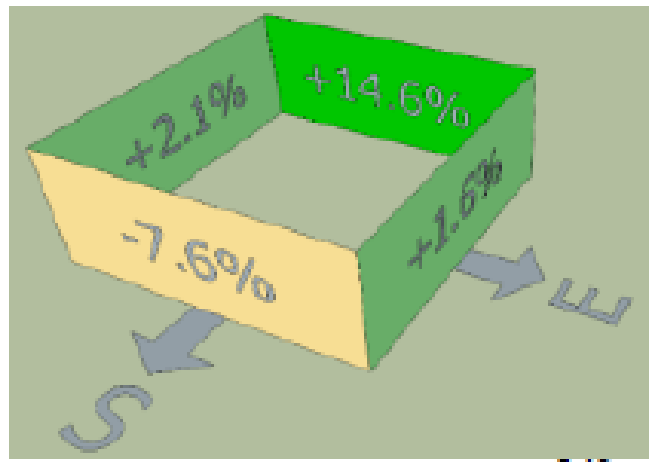

Fig. 7: Validation of the simplified yield calculation with the commercial simulation tool PV*SOL.

The simplified PV yield calculation depend on irradiation, module type, inverter type and installation situation, but it does not respect cable losses, partial shading, low irradiance behaviour of the modules, mismatch losses, soiling, startstop behaviour of MPP-trackers, power cut-off of the inverter, partial load behaviour and stand by consumption. Deviations are caused by these simplifications.

The main goal of the simplified yield calculation is to find a compromise between calculation time and accuracy. A simplified calculation routine was created. Results show that the yearly energy yield can be calculated with a deviation of less than $8 \%$ in areas with the highest energy output. This accuracy meets the requirements of this tool. The calculation speed is high as the PV yield calculation is done with a simple equation.

\section{Thermal building simulation and electrical load file generation}

In a first step three typical building shapes and sizes are defined to provide representative specific values for heating and cooling. The chosen building types are a) office buildings and b) residential buildings for the location Vienna. In a next step, the building envelope quality and shading devices are varied, to verify their influence on the energy demand. The building envelope quality is assessed by the characteristic length (lc-value) and the average u-value. With these basic parameters the buildings are modeled in TRNSYS 17 [8], see Fig. 8.

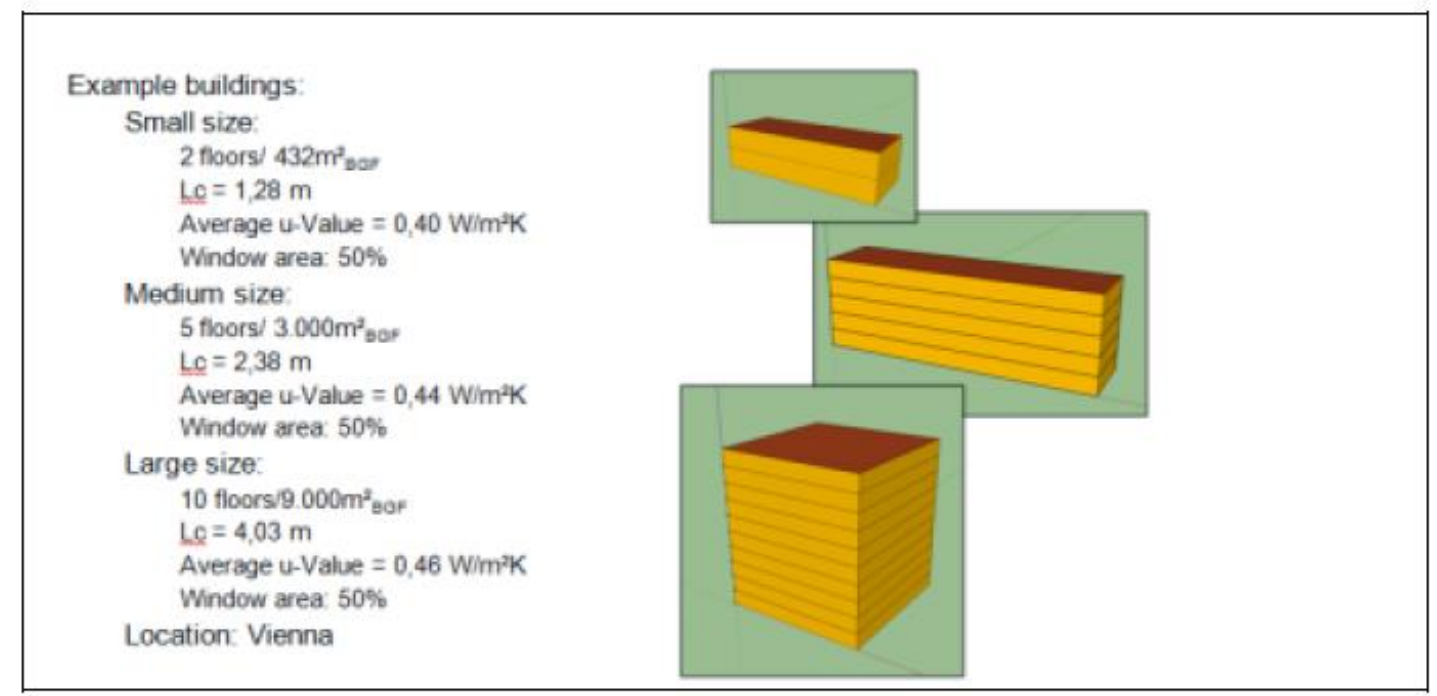

Fig. 8: Basic parameters for building simulation (source: teamgmi, 2018)

Based on the thermal energy demand for heating and cooling for each building model, electrical hourly load files are generated by post processing. The load files are related to the strategies of the 
energy concept matrix in Fig. 9. Therefore, specific Performance-Indicators (PI) are fixed, for example seasonal performance factors for heat pumps in combination with different heat sources. The electrical load files are generated using those PI for hourly values.

\begin{tabular}{|c|c|c|c|}
\hline & Strategy 1 & Strategy 2 & Strategy 3 \\
\hline $\begin{array}{l}\text { Heating/ DHW } \\
\text { supply }\end{array}$ & $\begin{array}{l}\text { Thermal source } \\
\text { (District heating, } \\
\text { Biomass, Gas) }\end{array}$ & $\begin{array}{l}\text { water-water-heat } \\
\text { pump with a ground } \\
\text { water well }\end{array}$ & air-water-heat pump \\
\hline Cooling supply & $\begin{array}{l}\text { Compression } \\
\text { Cooling with dry } \\
\text { heat rejection }\end{array}$ & $\begin{array}{l}\text { Free-cooling by } \\
\text { ground water well / } \\
\text { Rev. heat pump } \\
\text { Peak load: } \\
\text { Compression } \\
\text { Cooling with dry heat } \\
\text { rejection }\end{array}$ & $\begin{array}{l}\text { Rev. heat pump } \\
\text { Peak load: } \\
\text { Compression } \\
\text { Cooling with dry heat } \\
\text { rejection }\end{array}$ \\
\hline $\begin{array}{l}\text { Central } \\
\text { mechanical } \\
\text { ventilation for } \\
\text { fresh air supply }\end{array}$ & $35 \mathrm{~m}^{3} / \mathrm{hP}$ & $35 \mathrm{~m}^{3} / \mathrm{hP}$ & $35 \mathrm{~m}^{3} / \mathrm{hP}$ \\
\hline
\end{tabular}

Fig. 9: Energy concepts matrix (Source: teamgmi, 2018)
Fig. 10 shows the influence of the building envelope quality and shading devices on the specific electricity demand in an office building by using the same energy concept. The results show that the electricity demand for artificial lighting and equipment are the highest ones, then the electricity demand for cooling follows. The electricity demand for heating, mechanical ventilation and domestic hot water preparation (DHW) are quite low. The influence of the compactness of the building is recognizable, but not dominant. Furthermore, the results show that with a high-quality building envelope (passive house) and intelligent shading devices (external shading, daylight controlled) the cooling demand can be reduced significantly.
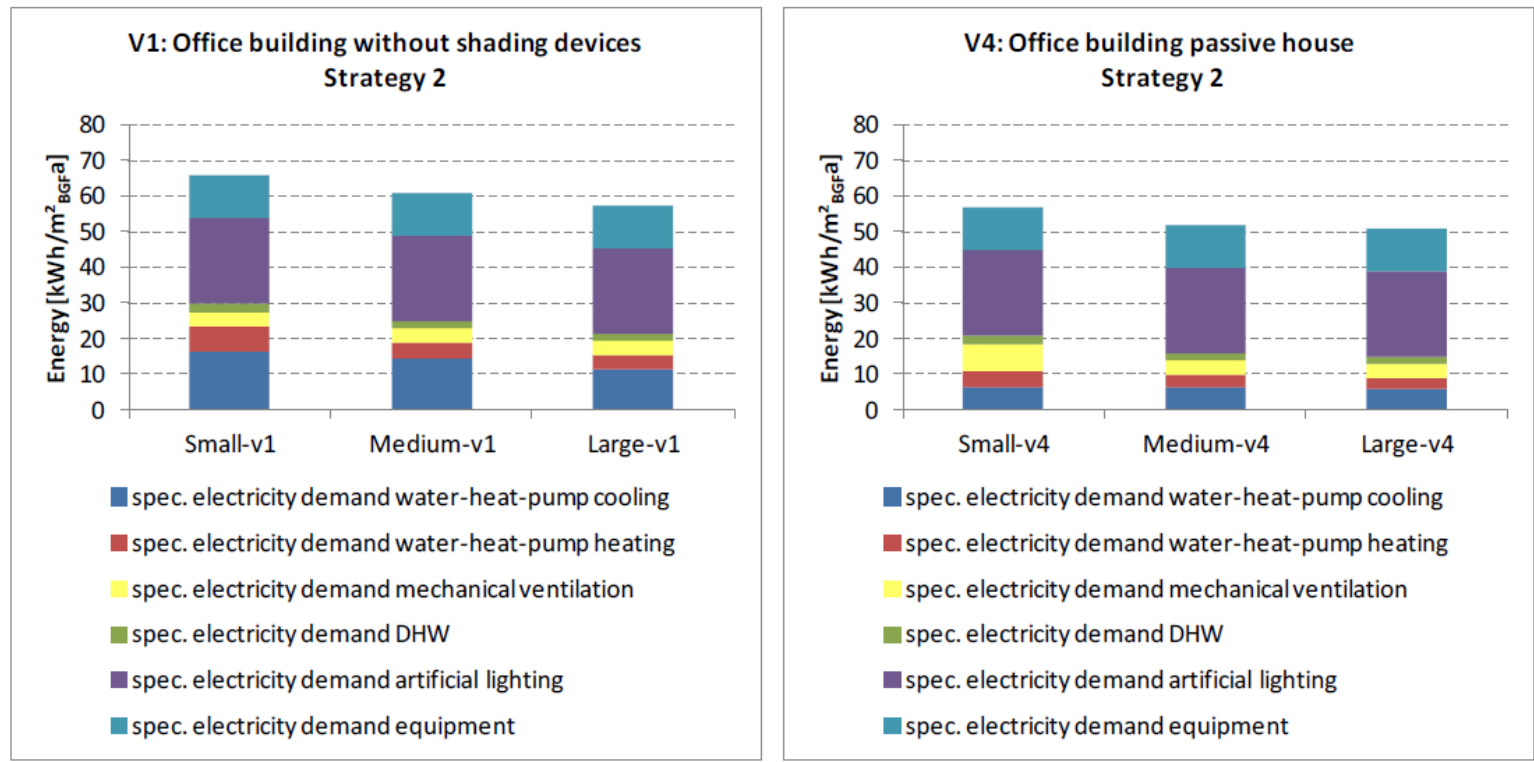

Fig. 10: Specific electricity demand for a standard office building (left) and for a high energy efficient office building (right), (source: teamgmi, 2018)

The electrical load files show us clearly that the electricity demand of the users - including artificial lighting and equipment - are interesting for PV-systems with high direct usage of the produced electricity in the building. The electricity demands for the other categories (heat-pump heating/cooling, mechanical ventilation and DHW) are comparatively low.

\section{KEY FINDINGS AND OUTLOOK}

Parametric studies by applying simulation tools were carried out to indicate the solar potential, PV yield and first design rules for BIPV. Based on the parametric study results of the USE CASE urban building following findings can be extracted:

- The placement of the façade integrated PV is relevant, the calculation indicates a range from 20 up to $66 \%$ of solar electricity yield generated by a south oriented and $30^{\circ}$ sloped PV module 
- In case of a multi-family house with $48 \mathrm{MWh}$ electricity demand per year; a $40 \mathrm{kWpeak}$ PV system (South $/ 30^{\circ}$ ) covers $40 \%$ of the overall electricity demand and $40 \%$ of the PV electricity production is directly consumed

- All investigated PV system designs lead to payback periods less than 10 year. The LCOE is in a range of 5.6 and 8.7 EUR Cent per kWh. Austrian PV funding schemes are considered.

- The impact of the orientation of a $40 \mathrm{kWp}$ rooftop PV system on the coverage of solar electricity is not significant, all orientation causes a coverage of almost $40 \%$

Findings of the parametric study of the $360^{\circ}$ rotated different building typologies

- Horizontal roof areas indicate the highest solar potential

- For L-shaped buildings the orientation does not have significant influence on potential solar electricity production (AC) by photovoltaics.

- External shading by adjacent building has a significant influence on the solar potential

Simplification is key for the VITALITY project and the project team aims to develop a simple and accurate approach for fast generation of electric load profiles of buildings in operation. In a previous step thermal transient building simulation were applied to get the annual heating and cooling load profiles. A post-processing routine has been set up for transforming thermal into electricity demand profiles. For the upcoming research period it is foreseen to develop a method to generated accurate electric profiles when relevant building parameters are indicated.

The individual urban location is key when assessing the solar potential for BIPV, especially on façades. Therefore, a VITALTIY method indicating optimal placements for PV modules with best yield performance on the building surface were set up and investigated. The next improvement is to optimize the PV placement for minimizing the deviation between the profile of energy demand and profile of PV production. However, this requires implementation of nonlinear multicriterial solving algorithms and its currently in the process of investigation.

\section{ACKNOWLEDGEMENT}

This research project 'VITALITY' is funded by the Austrian Ministry for Transport, Innovation and Technology with the programme 'Stadt der Zukunft' 3rd Call in 2016.

\section{REFERENCES}

[1] EU-Directive 2010/31: The Energy Performance of Buildings Directive (2010/31/EU) recast Oct 2010

[2] Fechner et. Al., Technologie-Roadmap für Photovoltaik in Österreich in Berichte aus Energieund Umweltforschung 15/2016, Hrsg. bmvit 2016

[3] Valentin Software PV Sol Premium. url: https://www.valentin-

software.com/produkte/photovoltaik/57/pvsol-premium (access 19th July 2018)

[4] APCS Synthetische Lastprofile. url: https://www.apcs.at/de/clearing/technisches-

clearing/lastprofile (access 19th July 2018)

[5] http://web.mit.edu/sustainabledesignlab/projects/Accelerad/ (access 19th July 2018)

[6] https://iam.tugraz.at/augmented-parametrics/ (access 19th July 2018)

[7] https://energyplus.net/ (access 19th July 2018)

[8] TRNSYS. (Version 17). Transient System Simulation Tool, Version 17. Madison: Thermal Energy System Specialists, LLC. 


\section{URBAN BUILDING ENERGY MODELING - METHOD AND SCENARIO CASE STUDY SCHALLMOOS}

Peter Nageler1, Richard Heimrath1, Thomas Mach1, Oskar Mair am Tinkhof2, Ingrid Schardinger3, Franz Mauthner4, Hermann Schranzhofer1, Christoph Hochenauer1

Institute of Thermal Engineering, Graz University of Technology1

Inffeldgasse 25/B, $8010 \mathrm{Graz}$, Austria

E-Mail: peter.nageler@tugraz.at

Phone: +43 $316873 / 7310$

Salzburger Institut für Raumordnung und Wohnen2

Schillerstraße 25, 5020 Salzburg, Austria

Research Studios Austria Forschungsgesellschaft mbH - Studio iSPACE3

Schillerstraße 25, 5020 Salzburg, Austria

AEE - Institute for Sustainable Technologies4

Feldgasse 19, 8200 Gleisdorf, Austria

\section{SUMMARY}

Dynamic thermal modeling has been gaining increasing importance in the context of the transformation of existing energy systems into smart energy systems because of growing environmental consciousness and urbanization. This study presents a validated method for fully automated building modeling within urban districts, which is applied to a case study concerning the city district Schallmoos in Salzburg (Austria). Based on the current status, several realistic future scenarios for the years 2025 and 2050 are created and compared with the status quo, regarding heat requirement, heating load as well as the $\mathrm{CO}_{2}$ equivalent emissions by energy carriers.

Keywords: dynamic urban energy simulation; spatial energy planning; IDA ICE; automation

\section{INTRODUCTION}

International energy and climate policies are aiming at a reduction of global energy demand and carbon dioxide emissions to limit global warming (UN, 2015). Energy needs continue to increase through urbanization (Pérez-Lombard, 2008). Statistics make it clear that cities are currently responsible for over two-thirds of the world's energy consumption and account for more than $70 \%$ of global carbon dioxide emissions (IEA, 2008). In this context, the energy consumption of a building therefore plays a crucial role in climate change. Retrofitting the existing building stock and integrating the buildings in future energy systems are therefore key strategies for cities to reduce energy demand and greenhouse gas emissions.

An analysis of the building energy demand is thus essential for city planners to analyze the energy demand of the actual building stock or evaluate restructuring measures, densifications of urban development areas or the expansion potential of district heating networks (DHN). The underlying approaches, challenges and opportunities for urban energy system models are presented by Keirstead et al. (2012) and Kavgic et al. (2010). State-of-the-art building models for heating and cooling energy demand at city scale are discussed by Frayssinet et al. (2018). Reinhart et al. (2016) noticed the importance of dynamic building modeling and discussed the existing dynamic urban energy modeling tools. An important point in the modeling process is the data acquisition, since the collection of all the necessary data for the energy analysis of a building can be a difficult process owing to the data protection rules that apply for individuals and corporations. This results in data gaps in the building modeling process. One approach to close these gaps was presented by Loga et al. (2016) (TABULA project), which used the building age as an indicator for the building energy consumption and assigned age-related building archetypes for the residential building stock. The 3D geometry can also be obtained through 3D laser scanning point clouds (Kedzierski, 2015). Nageler et al. (2017) used these building archetypes and presented an automated dynamic building modeling method at urban scale based on publicly available data. Fuchs et al. (2016) developed an automated workflow for combined modeling of buildings (resistance and capacity models) and DHN. Dogan and Reinhart (2017) presented an automated multi-zone method to perform urban simulations. All these methods have used building model generation tools to reduce the considerable time needed for manual input, and have minimized the error rate caused by typographical errors.

Furthermore, software tools are required in the process of data acquisition and visualization of the simulation results. Geographical information systems (GIS) are thus applied to collect, manipulate, 
organize and visualize geographical data and these systems are already widely used for potential analysis in urban areas. The usefulness of a combination of GIS and energy simulation programs has already been recognized (Fuchs et al. 2016). The collected and manipulated data is stored in a database and an interface produces a simulation model for the simulation tool (Nageler et al. 2017a) (Nageler et al. 2017b) (Zucker et al. 2016). These building models can be used to calculate the thermal energy demand of buildings or to capture the dynamic interactions between buildings, DHN and energy supply unit using co-simulation (Nageler et al. 2018).

This study presents a validated methodology for fully automated building modeling within urban districts based on publicly available data, which is applied to a case study for Schallmoos in Salzburg (Austria). Based on 60 the current state, several realistic future scenarios from 2025 and 2050 are created and compared with the status quo.

\section{METHOD}

This paper presents a physical approach for thermal analysis of urban districts. This chapter describes the workflow from the raw data to the visualization and evaluation of the simulation results. The approach is divided 65 into three main steps (see Fig. 1): the scenario definition, the thermal simulation of urban districts and the scenario evaluation.

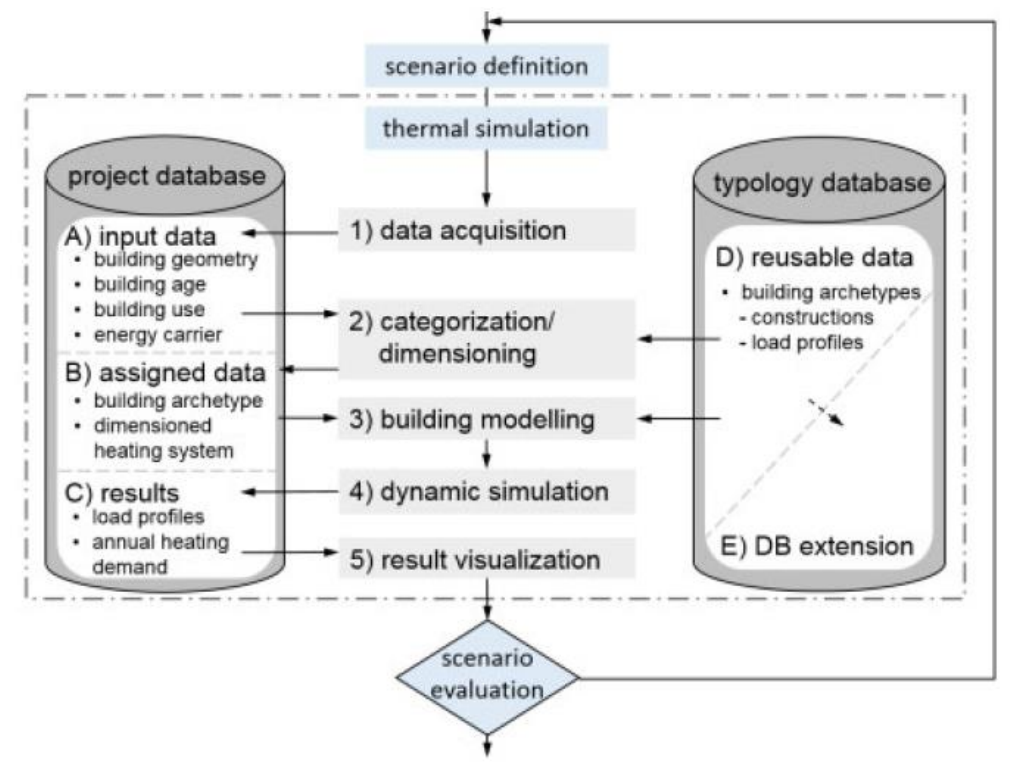

Fig. 1: Workflow of the physical approach for thermal analysis of urban districts.

\section{Scenario definition}

The urban development process starts with the development of possible courses of action or planning options, see Fig. 1. The energy-technical definition of a planning option is termed a scenario. First, the scenario definition procedure is explained in general, and then the simulated scenarios are presented for the case study.

For the estimation of the future energy demand, the definition of the refurbishment rate and the refurbishment quality as well as the determination of the densification rate and the quality of the construction are necessary. Refurbishment rates for residential and non-residential housing were derived from records for recent years. Therefore, an average life cycle is derived based on the refurbishment rate. Starting from the assumption that the older building will be renovated first, each individual building can be assigned a refurbishment status based on the year of construction. As with the refurbishment rate, the densification rates for residential and non-residential buildings are derived from the records for recent years. Gross floor areas GFA can be derived using average sizes for residential buildings and non-residential buildings. These densifications are assigned to estates in development areas. The following assumption is taken into account: the existing buildings on an estate are added another stories aliquot according to the additional GFA and buildings with at least one floor 
are created on undeveloped estates. The choice of climate data is another option to evaluate future scenarios. Here, future weather data can be selected to take into account the effects of climate change.

The realistic allocation of energy carriers to buildings in a future scenario depends on two factors: the potential and the prioritization of energy carriers. The potential depends strongly on the heat requirement and the spatial condition of each building. For example, buildings in areas of low-density housing cannot be supplied by grid-bound energy carriers such as district heating or gas networks, or if heat pumps are not allowed due to environmental or acoustic considerations. If potential for more than one energy carrier exists, the selection of the energy carrier is made according to a priority list. Prioritization depends mainly on the economic and political situation. The population can be guided by regulations and promotion in a desired direction.

Furthermore, it is assumed that a building only changes its energy carriers if it is renovated.

Since the allocation of the energy carriers depends on the heat demand, it is advantageous to calculate the energy demand for hot water and space heating independently of the energy sources. The heating system is thus calculated on a simplified basis by means of an ideal heater.

Based on the analysis of the simulation results from the status quo and potential analyses of the case study, realistic future-oriented scenarios for 2025 and 2050 were defined in Section 4.2.

\section{Thermal simulation}

The physical modeling of entire neighborhoods proves to be extremely time-consuming, because every single building has to be geometrically modeled and parameterized with wall constructions, glazing parameters, heating systems (if necessary) and internal gains. An automation process is required to shorten the considerable time needed for manual input. This study thus uses a validated method for automated building modeling (Nageler et al. 2017a).

First, a database (DB) system is created, which allows the user to manage multiple sources for these data sets. The DB system consists of a project-related DB and a reusable typology DB (see Fig. 1), which are both object-relational PostgreSQL DB. The typology database stores non-geometrical project independent data, which are for example wall constructions for typical buildings or load profiles for domestic hot water $(\mathrm{DWH})$ and internal loads. These data are reusable for the next project (see Fig.1 D). So-called archetypes were created with all non-geometric data of a building such as wall and window constructions. These archetypes can be subdivided, for example, into eight building age classes for Austrian residential buildings (Loga et al. 2016). Data that has not yet been implemented in the database can be added and new building archetypes can be created from these data (see Fig.1 $\mathbf{E}$ ). The project database stores the relevant project data with a spatial reference, and these data are the required input data (see Fig.1 A), the assigned data (see Fig.1 B) by means of a categorization/dimensioning process (see Fig.1 2) and the dynamic simulation results (see Fig.1 C).

The workflow of the approach is divided into the following five process steps (see Fig. 1). The first step (see Fig.1 1) deals with the acquisition of the input data, which are the building geometry, the building age, the building use and the energy carrier used for the building. In the second step (see Fig.1 2) the buildings are categorized according to the archetypes and the heating system is dimensioned if this is necessary. In the third step (see Fig.1 3), a building model with heating system is automatically generated from the input data and the assigned data using a building model generation tool (Nageler et al. 2017b). In the fourth step (see Fig.1 4) the automatically created model is simulated with the dynamic building simulation environment IDA ICE. In the fifth step (see Fig.1 5), the results are graphically prepared in form of heat maps, 3D visualizations or spatio-temporal animations of building`s heating load by means of GIS.

\section{Scenario evaluation}

Based on the graphical representation of the results, the individual scenarios can be evaluated by stakeholders and/or decision makers, which are for example municipalities, energy suppliers, urban planners and investors. Further, the results are evaluated by comparing the results with previously set benchmarks like $\mathrm{CO}_{2}$ or heating demand reduction goals. The evaluation determines whether the results for this scenario meet the requirements and benchmarks. In this case the process is finished. If the result is considered insufficient, the process starts again. 


\section{CASE STUDY SCHALLMOOS}

\section{Study area}

A method for GIS based dynamic urban energy simulation is tested on the basis of a case study. The investigated area is the building stock of Schallmoos, which is a district in the City of Salzburg $\left(47^{\circ} 48^{\prime}\right.$ N 13 $3^{\prime}$ E 429 m ASL) in Austria, see Fig. 2a. The examined building stock consists of 2321 buildings, which are spread over an area of approximately two square kilometers. The use, age and geometry of these buildings are well known, which suggests that 1335 buildings are heated. Schallmoos is a residential area with a large number of commercial businesses, see Fig $2 \mathrm{~b}$. The average year of construction of the heated buildings is 1955 and the buildings are mainly heated by gas and district heating. All the facts of this case were collected for the reference year 2014.
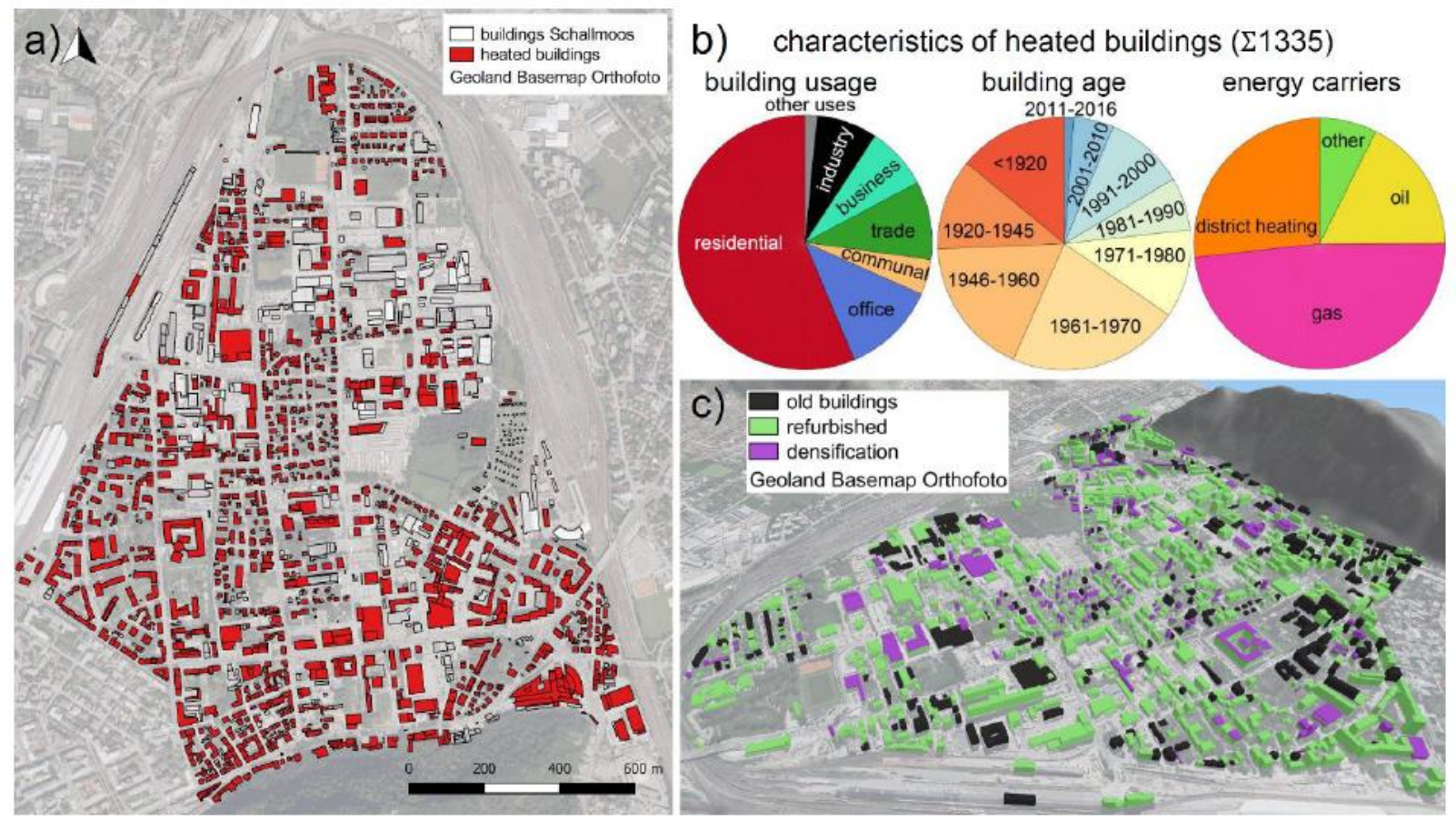

Fig. 2: a) Schallmoos - study area; 2321 buildings (white), 1335 heated buildings (red), b) characteristics of Schallmoos, c) densification (pink) and refurbishment (green) of Schallmoos for the year 2050.

\section{Simulation scenarios}

The following six example scenarios were defined in the case study Schallmoos respectively for 2025 and 2050:

- Conservative: today's standard for refurbishment and construction quality; no comprehensive structural measures are being taken in the field of energy infrastructure

- Ambitious: ambitious refurbishment and construction quality; no comprehensive structural measures are being taken in the field of energy infrastructure

- Renewable: today's standard for refurbishment and construction quality; the energy provided is of higher quality, as centralized energy suppliers are increasingly supplied with renewable energy sources

- Ambitious/renewable: ambitious refurbishment and construction quality; the energy provided is of higher quality, as centralized energy suppliers are increasingly supplied with renewable energy sources

In these scenarios, oil and electrical heating systems are replaced by an alternative system in the case of renovation or new construction depending on their potential. Fig. $2 \mathrm{c}$ shows refurbished buildings and the densification for the year 2050 . 
Another two scenarios arise based on the conventional scenario by substituting natural gas systems with an alternative system:

- Substitution gas with DHN: requirement local availability of DHN

- Acceleration DHN: requirement mean heat demand density $\geq 40 \mathrm{kWh} / \mathrm{m}^{2} \mathrm{a}$

\section{RESULTS AND DISCUSSION}

This chapter presents the results of the applied modeling and simulation method (see Chapter 3). First, the simulation results of the status quo are presented and compared to the measurement data for Schallmoos. Second, the heating demand and the equivalent $\mathrm{CO}_{2}$ emissions of the scenarios were compared to the status quo.

\section{Status quo 2014}

Fig. 3 shows the comparison of the simulation results of the status quo (2014) with measurement data from the local energy utility Salzburg AG. The consumption data (total values) of the district heating demand, the gas consumption and the electricity demand are available from Salzburg AG. In sum, the simulated thermal energy consumption is $91 \mathrm{GWh}$ and the electrical consumption is $41 \mathrm{GWh}$. The figure shows a good agreement with the measurement data with a percentage error of $4.9 \%$ for district heating, $12.7 \%$ for gas and $-20.6 \%$ for electricity. The electrical demand consists of usage-dependent load profiles for lighting and equipment and serves as input for the thermal building simulation. The deviation of the electrical demand can be explained by the fact that in addition to lighting and equipment there are other energy meters such as heating systems, etc. Heat pumps, electricity and biomass heaters are grouped under "other" energy meters, which are responsible for $6 \mathrm{GWh}$ thermal consumption. The simulated energy demand for the energy carrier "oil" is $13 \mathrm{kWh}$, for which no measured data are available.

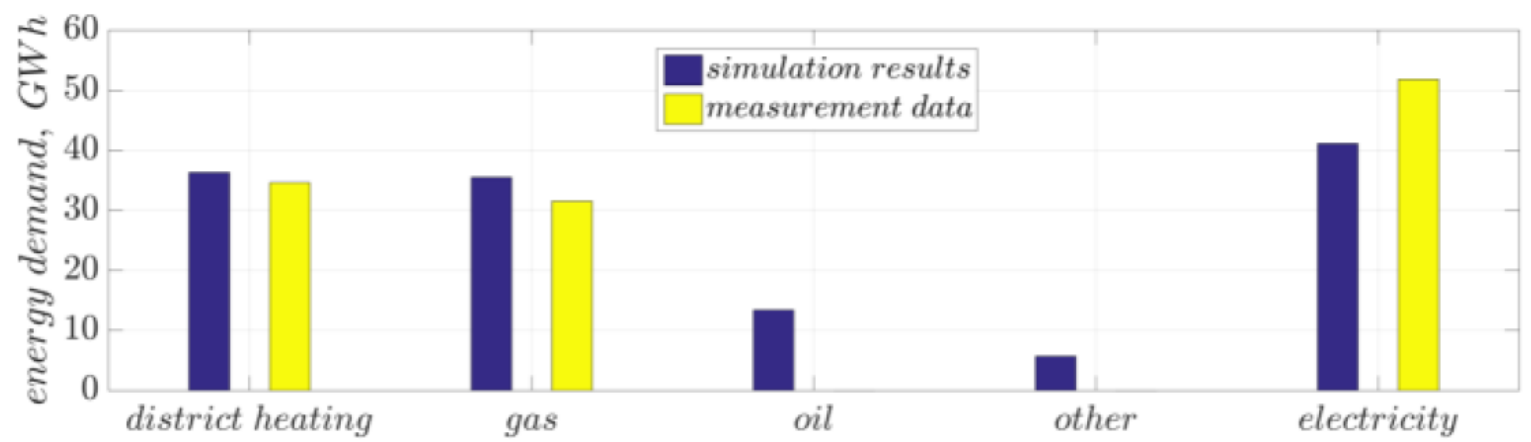

Fig. 3: Simulated energy demand of the status quo (2014) by energy carriers compared with the measurement data.

Fig. 4 shows the heating load for domestic hot water and space heating of the status quo (2014) by energy carriers. The maximum heating load of all energy carriers together is about $60 \mathrm{MW}$, with gas and district heating accounting for the largest share. In the winter months, the heat demand is made up of the demand for space heating and hot water, in which the heating system is modeled with a night setback $\left(17^{\circ} \mathrm{C}\right)$ between $6 \mathrm{pm}$ and $4 \mathrm{am}$. The heating load during the summer months is characterized by stochastically distributed hot water tapping. The required power is about $7 \mathrm{MW}$ for oil and $4 \mathrm{MW}$ for "other" energy carriers. 


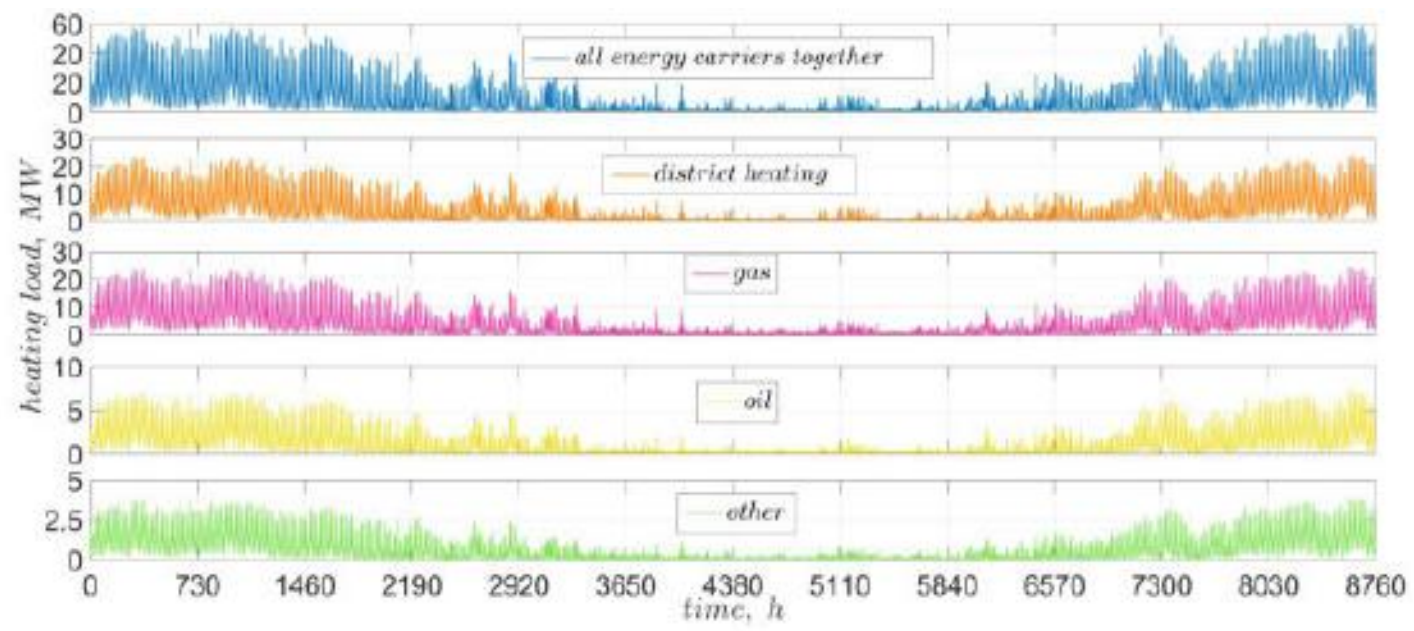

Fig. 4: Heating load for domestic hot water and room heating of the status quo (2014) by energy carriers.

\section{Scenario comparison}

The heating demand of the scenarios 2025 and 2050 can be calculated using the assumptions for densification and refurbishment. By 2050 (from 2014 levels), the densification leads to an increase in the GFA of $94600 \mathrm{~m}_{2}(9 \%)$ and $766000 \mathrm{~m}_{2}(75 \%)$ GFA is assumed to become renovated. Fig. 5 shows heat maps of the conservative scenarios 2025 and 2050. The heating demand drops to $81 \mathrm{GWh}$ (2025) or rather $55 \mathrm{GWh}$ (2050) per year due to renovation measures. The same climate data was used in all scenarios to ensure the comparability of refurbishment and densification measures. A heat map shows the areas with high and less energy consumption. A heat map of this kind can be used to identify suitable areas for an extension of the existing district heating.

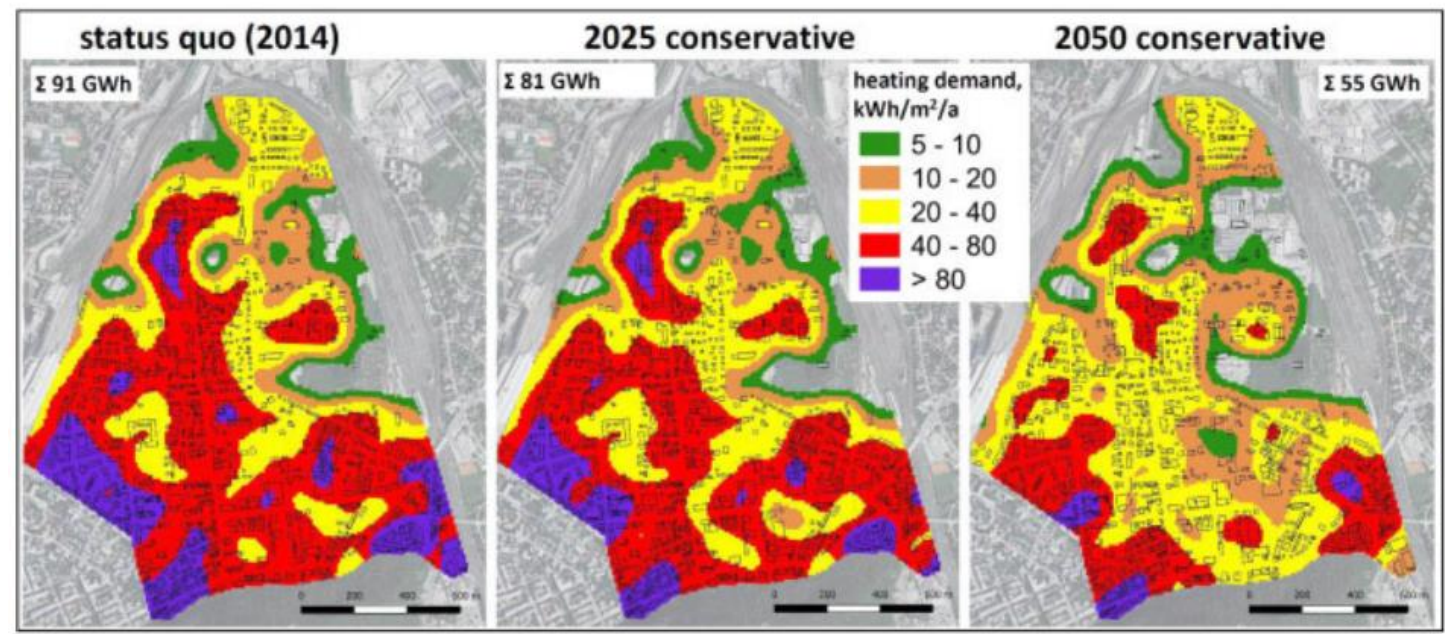

Fig.

Scenario comparison: heating demand of the status quo and conservative scenarios 2025 and 2050.

Fig. 6 shows the comparison of six scenarios (see Section 4.2) for 2050 according to heating demand and the equivalent $\mathrm{CO} 2$ emissions. The heating demand for the ambitious scenario drops from 55 GWh to $52 \mathrm{GWh}$ due to stricter building regulations. The $\mathrm{CO} 2$ equivalent emissions can be reduced from 19900 t CO2 (status quo) to 11100 t CO2 (conservative 2050) or 10600 t CO2 (ambitious 2050) per year due to energy savings. Another significant part of the $\mathrm{CO} 2$ equivalent emissions can be reduced by a "greener" or more sustainable heat supply. Here, the conversion factors of the energy carriers are changed according to the regional conditions. In this case 205 another $4000 \mathrm{t}$ of $\mathrm{CO} 2$ (renewable 2050) per year can be saved (ambitious 2050). Further scenarios imply a redistribution of the energy carriers but no significant changes in the total values (see acceleration DHN or substitute gas). 


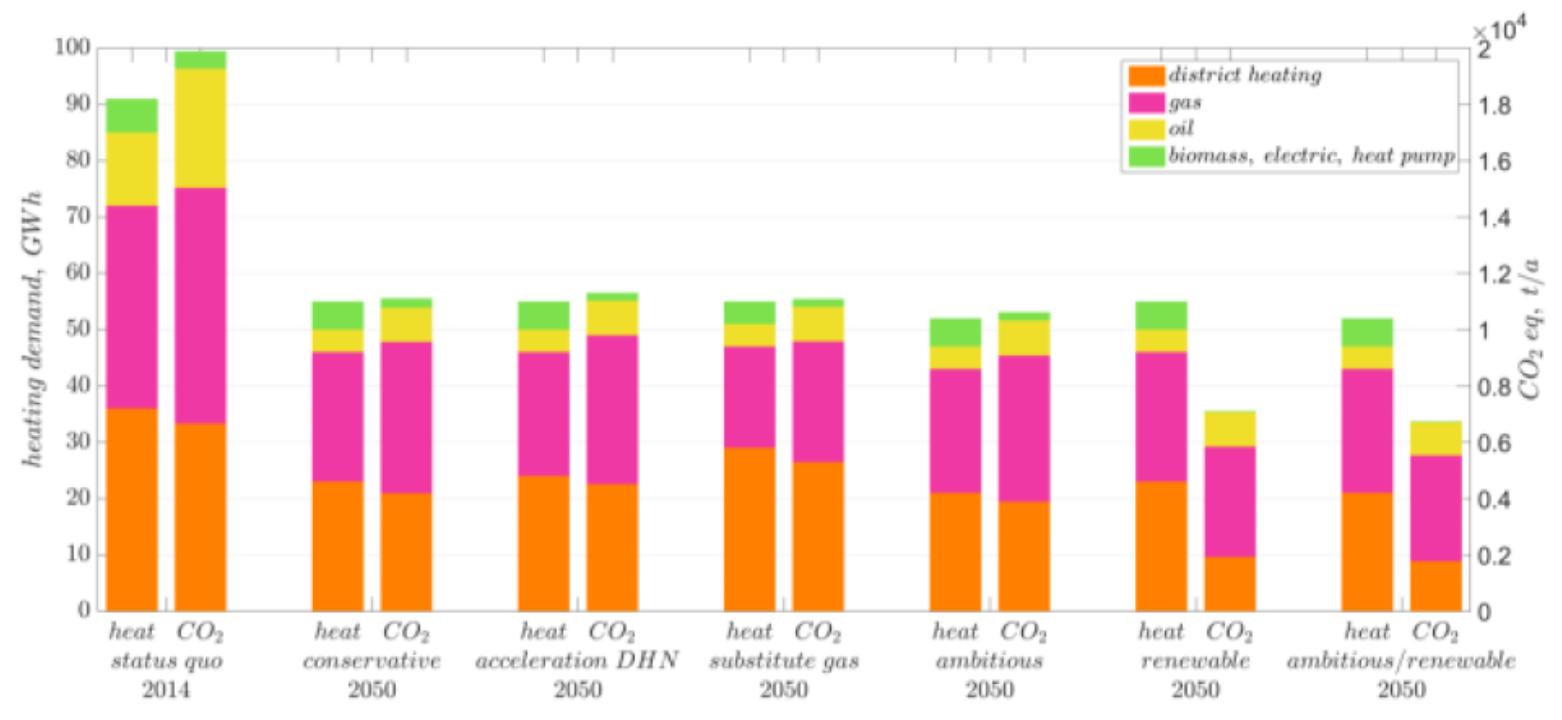

Fig. 6: Scenario comparison according to the heating demand and the equivalent $\mathrm{CO} 2$ emissions.

\section{CONCLUSION AND OUTLOOK}

This paper presents a method for automated building modeling within urban districts, which was successfully applied to a case study concerning the city district Schallmoos in Salzburg (Austria). The simulation results show a good agreement with the measured data with a percent error of $4.9 \%$ for district heating, $12.7 \%$ for gas and $-20.6 \%$ for electricity. The deviation can be explained by assumptions regarding age-typical wall constructions.

Furthermore, the heating demand and $\mathrm{CO}_{2}$ equivalent emissions of six realistic scenarios are discussed. The results show that $\mathrm{CO}_{2}$ equivalent emissions are best reduced by a combination of heat demand reduction and "greener", more sustainable heat supply. In detail, the results indicate that $\mathrm{CO}_{2}$ reduction potential for the district of Schallmoos in 2050 (compared to 2014) is in the range between $-43.1 \%$ and $-66.1 \%$. This range considers different scenario assumptions for building refurbishment and construction quality as well as future densification of the existing building stock. Moreover, a more environmental friendly energy supply considering both locally available energy sources as well as "greener" grid energy supply is assumed. For the district of Schallmoos it can be concluded from the results, that zero carbon emission targets for 2050 may not be met by means of technical measures according to the status quo (2014) or even not if ambitious measures - as assumed in the scenarios together with local stakeholders - are going to be realized. In a next step, the set-up of a possible future energy supply system for the district of Schallmoos that meets local low to zero carbon requirements should be identified based on assumptions for full exploitation of locally available renewable energy resources (geothermal, solar irradiation, waste heat, etc.), more ambitious facing out of local fossilbased thermal energy supply, introduction of decentralized renewable energy supply systems and further "greening" of grid- connected energy supply.

\section{REFERENCES}

UN, (2015), UN Climate Change Conference Paris 2015. [Online]. Available: https://www.un.org/sustainabledevelopment/cop21/ [accessed 16-Jul-2018].

IEA, (2008), World Energy Outlook 2008, Paris, International Energy Agency.

Dogan, T., Reinhart, C., (2017), Shoeboxer: An algorithm for abstracted rapid multi-zone urban building energy model generation and simulation, Energy and Buildings:140-153.

Frayssinet, L., Merlier, L., Kuznik, F., Hubert, J.L., Milliez, M., Roux, J.J., (2018), Modeling the heating and cooling energy demand of urban buildings at city scale, Renewable and Sustainable Energy Reviews 81(2):2318-2327. 
Fuchs, M., Teichmann, J., Lauster, M., Remmen, P., Streblow, R., Müller, D., (2016), Workflow automation for combined modeling of buildings and district energy systems, Energy 117(2):478-484.

Kavgic, M., Mavrogianni, A., Mumovic, D., Summerfield, A., Stevanovic, Z., Djurovic-Petrovic, M., (2010), A review of bottom-up building stock models for energy consumption in the residential sector, Building and Environment 45(7):1683-1697.

Kedzierski, M., Fryskowska A., (2015), Methods of laser scanning point clouds integration in precise 3D building modelling, Measurement 74:221-232.

Keirstead, J., Jennings, M., Sivakumar, A., (2012), A review of urban energy system models: Approaches, challenges and opportunities, Renewable and Sustainable Energy Reviews 16(6):38473866.

Loga, T., Stein, B., Diefenbach, N., (2016), TABULA building typologies in 20 European countriesMaking energy-related features of residential building stocks comparable, Energy and Buildings 132:4-12.

Nageler, P., Zahrer, G., Heimrath, R., Mach, T., Mauthner, F., Leusbrock, I., Schranzhofer, H., Hochenauer, C., (2017a), Novel validated method for GIS based automated dynamic urban building energy simulations, Energy 139:142-154.

Nageler, P., Mach, T., Heimrath, R., Schranzhofer, H., Hochenauer, C., (2017b), Generation tool for automated thermal city modelling. Applied Mechanics and Materials, accepted.

Nageler, P., Schweiger, G., Schranzhofer, H., Mach, T., Heimrath, R., Hochenauer, C., (2018), Novel method to simulate large-scale thermal city models, Energy 157:633-646.

Pérez-Lombard, L., Ortiz, J., Pout, C., (2008), A review on buildings energy consumption information, Energy and Buildings 40(3):394-398.

Reinhart, C.F., Davila, C.C., (2016), Urban building energy modeling - A review of a nascent field, Building and Environment 97:196-202.

Zucker, G., Judex, F., Blöchle, M., Köstl, M., Widl, E., Hauer, S., Bres, A., Zeilinger, J., (2016), A new method for optimizing operation of large neighborhoods of buildings using thermal simulation, Energy and Buildings:153-160.

\section{ACKNOWLEDGEMENTS}

This research was supported / funded by the Austrian Federal Ministry of Transport, Innovation and Technology (bmvit) and is associated with the projects "EnergySimCity-Ganzheitliche Analyse und Simulation von Energiesystemen und Ressourcenverbünden in Städten und Stadtquartieren" (Project No. 844732) and "EnergyCityConcepts - method and concept development for the implementation of sustainable energy systems in cities and communities" (Project No. 850129). 


\section{EVALUATION OF BUSINESS MODELS FOR THE LARGE-SCALE IMPLEMENTATION OF NEARLY ZERO-ENERGY BUILDINGS IN EUROPE}

Benjamin Köhlerı, Anneke Quast1, Annalisa Andaloro2, Arnulf Dinkelı

Fraunhofer Institute for Solar Energy Systems ISE1

Heidenhofstraße 2, 79110 Freiburg, Germany

Phone: +49 761 / 4588-5308

Eurac Research - Institute for Renewable Energy2

Via A. Volta 13/A, 39100 Bozen/Bolzano, Italy

E-Mail: benjamin.koehler@ise.fraunhofer.de

\section{SUMMARY}

Nearly zero-energy buildings (nZEBs) will be the standard for new buildings in Europe from 2019 (public buildings) and 2021 (private buildings). However, even though the technologies for realizing nZEBs are already available, their market share in Europe is still very low. One reason is the high initial investment for nZEBs and missing adequate business models for all stakeholders involved in buildings' lifecycle. The aim of this paper is to present the exemplary evaluation of an existing nZEB business model for nZEBs in Austria. The work presented in this paper is developed in the EU funded project CRAVEzero - Cost Reduction and market Acceleration for Viable nearly zero-Energy buildings. In the course of the project additional business models are analysed qualitatively and quantitatively. A detailed evaluation of success factors, critical factors and inconsistencies (qualitatively and quantitatively) is essential for developing new business models for a market uptake of nZEBs ensuring a cost-optimal nZEB implementation and profits for all stakeholders involved.

Keywords: Nearly zero-energy buildings, Business Models, Lifecycle, Multilevel Analysis

\section{INTRODUCTION}

Climate change is one of the major challenges for humanity in the future. In order to reduce the risks of climate change, almost all countries in the world signed the Paris Convention from 2015 with the goal of limiting global warming to below 2 Kelvin. This sets the frame for European and national greenhouse gas (GHG) emission reduction of $80-95 \%$ compared to 1990 and the related energy efficiency targets. In Europe, the building sector accounts for approximately $40 \%$ of the annual end energy consumption. In order to achieve the GHG emission reduction targets it is essential to decarbonise the building sector. Nearly zero-energy buildings (nZEBs) are seen as one central possibility for achieving the energy and GHG emission reduction targets. Therefore, European legislation set nZEBs as the standard for all new buildings by 2021 and new public buildings by 2019 in the European Performance of Buildings Directive (EPBD). However, according to (Paoletti, Ramón, and Anagnostopoulos 2016) nZEBs still have a comparably low market share in the construction sector, even though technologies needed for realising nZEBs are already available today. Possible reasons for the current struggle in market uptake are comparably high efforts for planning and constructing, high initial investments and the lack of adequate business models for accelerating the nZEB market.

A central goal of the European project CRAVEzero is the analysis of existing business models of different stakeholders in the lifecycle of an nZEB as well as the development of new nZEB business models. In this paper, authors present the detailed exemplary evaluation of an existing business model in order to identify their strengths and weaknesses as well as success, critical factors and inconsistencies. The results will be the basis for new enhanced and innovative business models developed in the course of the project.

\section{SCOPE AND METHODOLOGY}

The business model analysis is developed through a multilevel approach, addressing the following dimensions: (i) analysis of the macro- and micro-environment; (ii) Business Model Canvas (BMC; Delphi-Survey); (iii)

Problem solution fit; (iv) Consistency Check; and (v) MICMAC analysis. The overall methodology has the aim to highlight business models profitability. However, detailed quantitative information about the revenue streams and cost structure and thereby the profitability is not yet available from the 
surveys filled in by the stakeholders. Therefore, this step of the methodology is not part of the results presented in this work. In the following, steps of business model analysis and ranking applied to the nZEB demo cases involved in the CRAVEzero project are outlined.

The analysis of the macro- and micro-environment of the business model provides information regarding the markets and industries attractiveness. Based on the concept of the PESTE analysis a limited number of market specific political, economic, socio-cultural, technical and environmental factors are analysed (Schallmo, 2013: p. 32). Following Porters Five Forces analysis additional questions concerning the threat of new entries and substitutes, the bargaining power of buyers and suppliers and the general competition in the industry, are answered in order to determine the industry's attractiveness. A further requirement for a business success is the fit of the offered value proposition and the needs of the customer (Osterwalder \& Pigneur, 2010: p. 132). The chosen customer segment is analysed in terms of pains, gains and 'jobs-to-get-done'. The information is then compared to the solutions, pain relievers and gain creators of the business models' value proposition to evaluate the level of fit. In the Delphi-survey, industry partners in the CRAVEzero consortium filled in several BMCs as proposed by A. Osterwalder (see Osterwalder \& Pigneur, 2010). Such templates were compiled from their companies' perspective and from other stakeholders' perspectives related to their country of origin. Additionally, further business models of European markets external to the project consortium will be assessed using the BMC in order to collect a representative quantity of different nZEB business models. The Delphi-survey provides an objective outcome with regard to business models and evaluation criteria.

The collected business models undergo a simple consistency check based on a pairwise comparison carried out through a matrix. This is a useful step to identify substantial internal inconsistencies (Miller, 1996, p. 510; Casadesus-Masanell \& Ricart, 2010, p. 204). The consistency check is based on morphology, which includes a set of dimensions allowing to compare the influence of BMC components on each other (Eriksson \& Ritchey, 2002). The first step is choosing variables (here: components of BMC), the second is the attribution of different shapes to each component - here: information received from project partners. Identified variables and their shapes are then transferred to the consistency matrix.

In a following step the MICMAC analysis developed by Godet (Godet et al. 1999) is conducted. The structural analysis describes the value of the relationship between different parameters of the BMC. The identified critical success factors are converted to another matrix in which the level of contradiction and/ or promotion between the parameters is evaluated using a scale from -2 to 2 . By calculating the eigenvector of each parameter the relative weight can be determined describing the level of influence and dependence of each parameter on the overall system. The calculated values are the basis for an influence-dependence-chart (IDC; see Fig. 1) showing the stability, importance and potential of the different parameters and the overall business model. The chart also indicates the level of efficiency, lock-in, innovation and complementarity which are described to be the four design themes that contribute to a business success according to (Amit \& Zott 2001). Moreover, so-called feedback loops are identified. They describe a chain of interrelated factors that lead back to the origin and reinforce their impact with each loop which can enhance the value of the business model and protect it from being imitated by competitors. The first sector contains the influent variables or determinants, characterised by high influence on the overall system and comparably low dependence on it. Even the slightest changes in these factors can have a big influence on the system. The second sector represents relay variables, characterized by both high influence and dependence on other factors and the overall system. Changes in these factors influence the factors themselves and the overall systems (feedback loops). Factors situated close to the so-called stake-diagonal subdividing the relay sector are extremely instable and critical (Arcade et al., 1999: p. 17 ff.). Result variables contain variables that are strongly influenced by the system while autonomous variables are both independent and not influential. Regulating variables can be found in the upper right corner of the fourth sector 


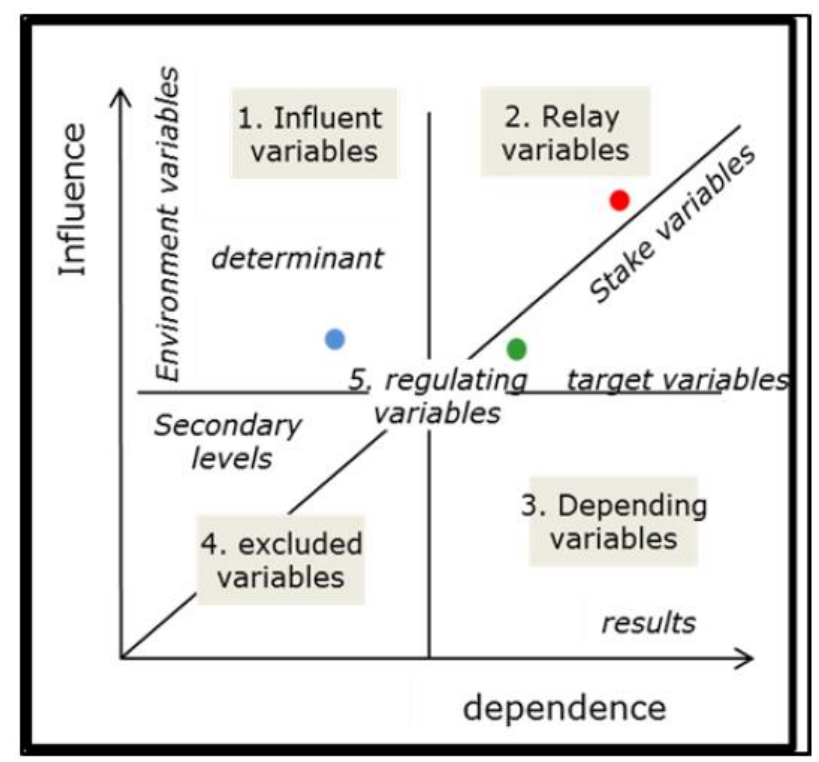

Fig. 1: Influence-dependence-chart

\section{ANALYZED NZEB-BUSINESS MODELS - RESULTS OF THE DELPHI-SURVEY}

For the analysis of the business models the partners of the CRAVEzero project provided their own and additional business models, which represent several stakeholder perspectives. Currently available are 13 business models from the perspective of Real Estate Developers, Planners, General Contractors, Engineering and Construction, Facility Management and Urban Planning. The basis of the analysis are the filled in BMCs describing the major components of a business model according to (Osterwalder \& Pigneur 2010), which are the Value Proposition, Customer Segment, Customer Relationship, Distribution Channels, Revenue Streams, Key Activities, Key Resources, Key Partners and the Cost Structure. However, most partners had difficulties to determine the costs and revenues that can specifically be tracked back to the described business model. Due to that fact, the evaluation of the profitability is not possible with the data acquired so far. In the following, the developed methodology for business model analysis is shown exemplarily for one project stakeholder based in Germany and Austria. The building (office building) is located in Austria and the business model is called Lifecycle cost and $\mathrm{CO}_{2}$ optimisation in early design stage. It basically describes the development of energy concepts for nZEBs that allow the reduction of energy demand and usage of efficient technologies (renewable energies and passive solutions). The energy demand has actively been lowered by measures in the design of the building, orientation and envelope leading to an undercut of the Austrian building regulation by $55 \%$.

The target customers are building owners, operators and users, who are reached via direct contact and personal assistance and have a contract for the consultation and evaluation of the building project. Accordingly, the company earns money for the concept and consultation offered. The prices are thereby highly dependent on the contract and project (size, number of variants and effort of investigation). Key activities are simulation and evaluation of energy related performance indicators and thermal and daylight simulations, for which especially personnel know-how is essential. Therefore, the highest costs are the personnel expenditures for experts followed by general office costs.

\section{RESULTS OF THE BUSINESS MODEL ANALYSIS}

In the following, the results of the exemplary evaluation of the business model described above, are presented. 


\section{External environment - Austria}

The building is located in a stable and democratic political system. Due to being a member state of the European Union, the political framework is aligned and closely connected to European legislation. Therefore, a pathway for the market acceleration of nZEBs needs to be built in order to make it the standard until 2021. Therefore, the market is generally open and attractive for the acceleration of nZEBs. The building sector was growing in the past years with a growth rate of the investment in the built sector of around 4\% in 2017.The economic situation is stable with an unemployment rate below $5 \%$ and an average income above the OECD average. However, the top $20 \%$ of the population in terms of income earn around four times as much as the bottom $20 \%$ indicating a considerable gap (OECD, n.d.). Around $21 \%$ of the income is spent on housing, which is a comparably high share (Organisation for Economic Co-Operation and Development [OECD], 2018a).

Around $30 \%$ of total energy demand is already met by renewable energies (Organisation for Economic Co-Operation and Development [OECD], 2018b). The Degree of stringency of environmental policies (degree of stringency of 14 environmental policy instruments primarily related to climate and air pollution) is 2. out of 6 . Therefore, making an internal comparison, Austria seems to have a rather high environmental awareness and responsibility. Austria can be described as a politically and economically stable country that has a comparably high environmental awareness and stringency.

Concerning the industry attractiveness it can be summarized that the threat of new entries is rather high due to low entry barriers like access to distribution channels, governmental restrictions and height of start-up costs. Due to the fact that in the built sector, usually services requiring a high level of specific knowledge that cannot be easily acquired by companies from foreign industries are offered, the threat of substitutes can be predicted as low despite low switching costs. The bargaining power of suppliers is neutral due to balanced concentrations of construction companies and suppliers. Low switching costs are balanced by a bigger threat of forward integration. As a result of a high concentration on the buyer side, a high value of orders for the supplier side and a high level of standardization, the bargaining power of buyers is high. This is also supported by low switching costs and a low threat of backward integration (Pekrul, 2006: p. 51-60).

\subsection{Problem-solution-fit}

\begin{tabular}{|l|l|l|}
\hline \multicolumn{1}{|c|}{ Customer pains } & \multicolumn{1}{c|}{ Customer gains } & \multicolumn{1}{c|}{ Jobs-to-get-done } \\
\hline Rising costs for energy & Saving energy costs & $\begin{array}{l}\text { Investment for future (price } \\
\text { stability) }\end{array}$ \\
\hline High investment costs for building & Reasonable investment costs & Independency of fossil fuels \\
\hline $\begin{array}{l}\text { Energy costs staying equal } \\
\text { compared to standard house }\end{array}$ & High durability of technologies & $\begin{array}{l}\text { Building environmental friendly } \\
\text { house }\end{array}$ \\
\hline $\begin{array}{l}\text { Slow amortisation of investments } \\
\text { Inefficient energy system }\end{array}$ & $\begin{array}{l}\text { Less risk of obsolescence } \\
\text { savings } \\
\text { sources }\end{array}$ \\
\hline $\begin{array}{l}\text { Spending much time and effort on } \\
\text { finding } \\
\text { subsidies for additional costs }\end{array}$ & $\begin{array}{l}\text { Carefree planning process due to } \\
\text { experienced planners }\end{array}$ & $\begin{array}{l}\text { Being perceived as 'one-step- } \\
\text { ahead' }\end{array}$ \\
\hline $\begin{array}{l}\text { Incompetent consultancy } \\
\text { Lack of awareness }\end{array}$ & Easy usability of technologies & $\begin{array}{l}\text { Taking responsibilities for future } \\
\text { generations }\end{array}$ \\
\hline $\mathbf{4 / 8}$ & Being a role model to others & $\mathbf{6 / 7}$ \\
\hline & $\mathbf{4 / 8}$ & \\
\hline
\end{tabular}

Fig. 2: Analysis of Problem-solution-fit 
The analyzed customer segment was "building owners with wish to build nearly Zero-Energy Buildings". The customer profile is illustrated with the collected pains, gains and jobs-to-get-done in Fig. 2.

The factors colored in green are met or taken care off by the value proposition given in the BMC, the ones in red aren't - or the information of the BMC is not giving indications. With 14 of 23 possible fits the business model covers around $61 \%$ of the customers' problems with the solutions it offers. Taking into account that no business model can solve all problems this can be seen as a rather good problem-solution-fit.

\section{Consistency check}

For the consistency check the parameters of the BMC have been transferred to the matrix in Fig. 3. A pairwise comparison shows whether the parameter contradict (called trade off; marked with ' $x$ '), support one another (called fit; marked with ' $\mathrm{o}$ ') or don't influence each other at all (marked with '/').

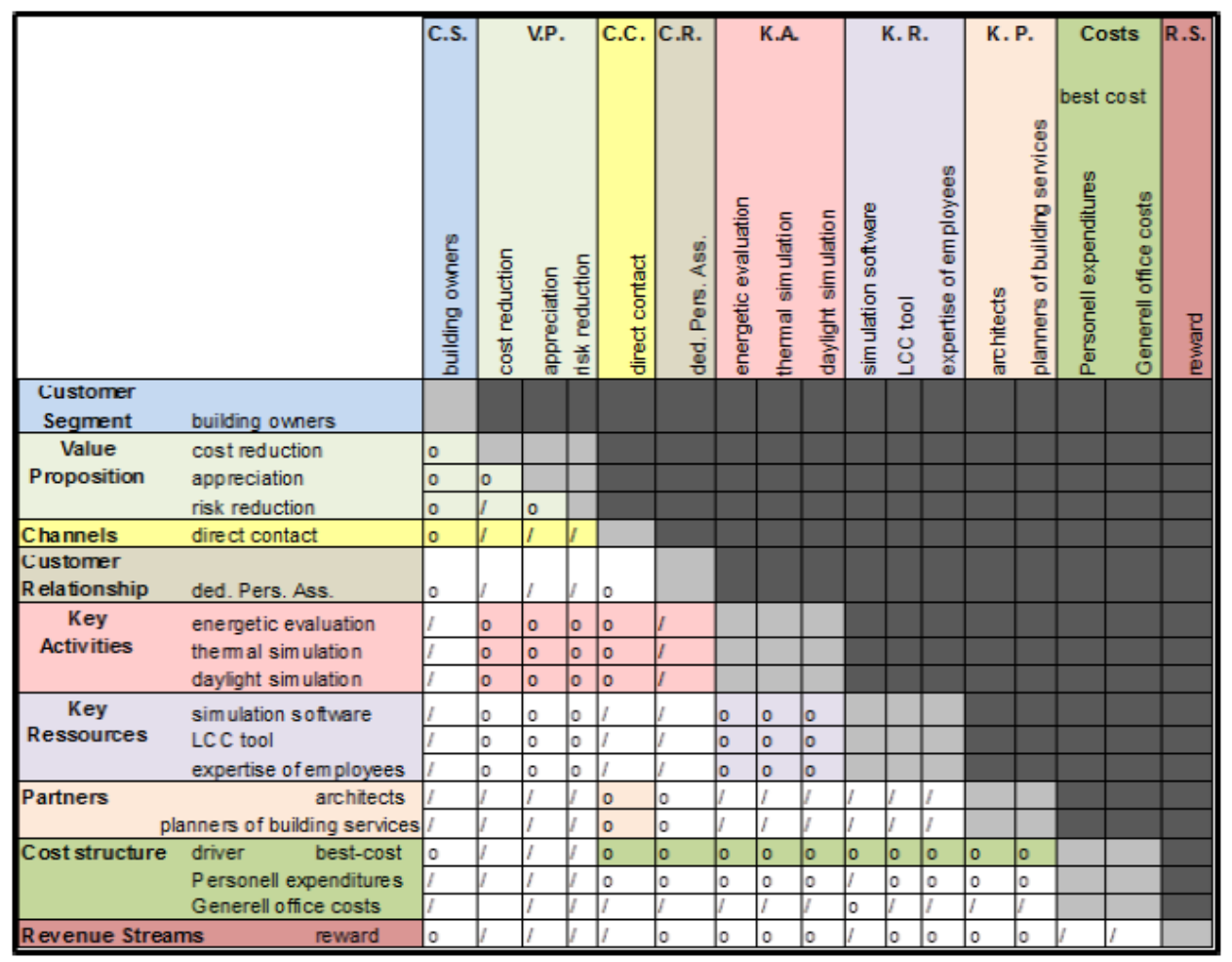

Fig. 3: Business Model consistency check

With the information given, no hidden inconsistencies could be identified. Moreover, areas of special interest indicated fits. Overall, the business model has a consistent inner logic. Furthermore, the lacking focus on channels and customer relationships results in less costs and the strong focus on quality and the differentiation of using self-developed tools indicate the strategy of being a best-cost provider. Regarding the design themes, the mentioned parameters indicate no focus on lock-in (lack of channels and customer relationships) but on innovation (self-developed tools and new softwares).

\section{MICMAC analysis}

For the MICMAC analysis several critical success factors have been identified (both factors by choice and consequence). The factors are optimized energetic concept, low costs and efforts for marketing, abundance of expensive channels, usage of new software, reduced lifecycle costs, enhanced building quality and worth, costs for personal expenditures, self-developed LCC Tool, skilled employees, few orders per year, reduced $\mathrm{CO}_{2}$ emissions and high degree of customization. These factors were transferred to the Vestersche Matrix for another pairwise comparison of how much a certain line 
influences the according column. A high supporting influence is a 2, a high contradicting one is a -2 . In order to reveal also indirect impacts the matrix is afterwards potentiated until the active and passive sums become stable in terms of hierarchy. They can then be normalized and transferred to an influence-dependence-chart as can be seen in Fig. 4.

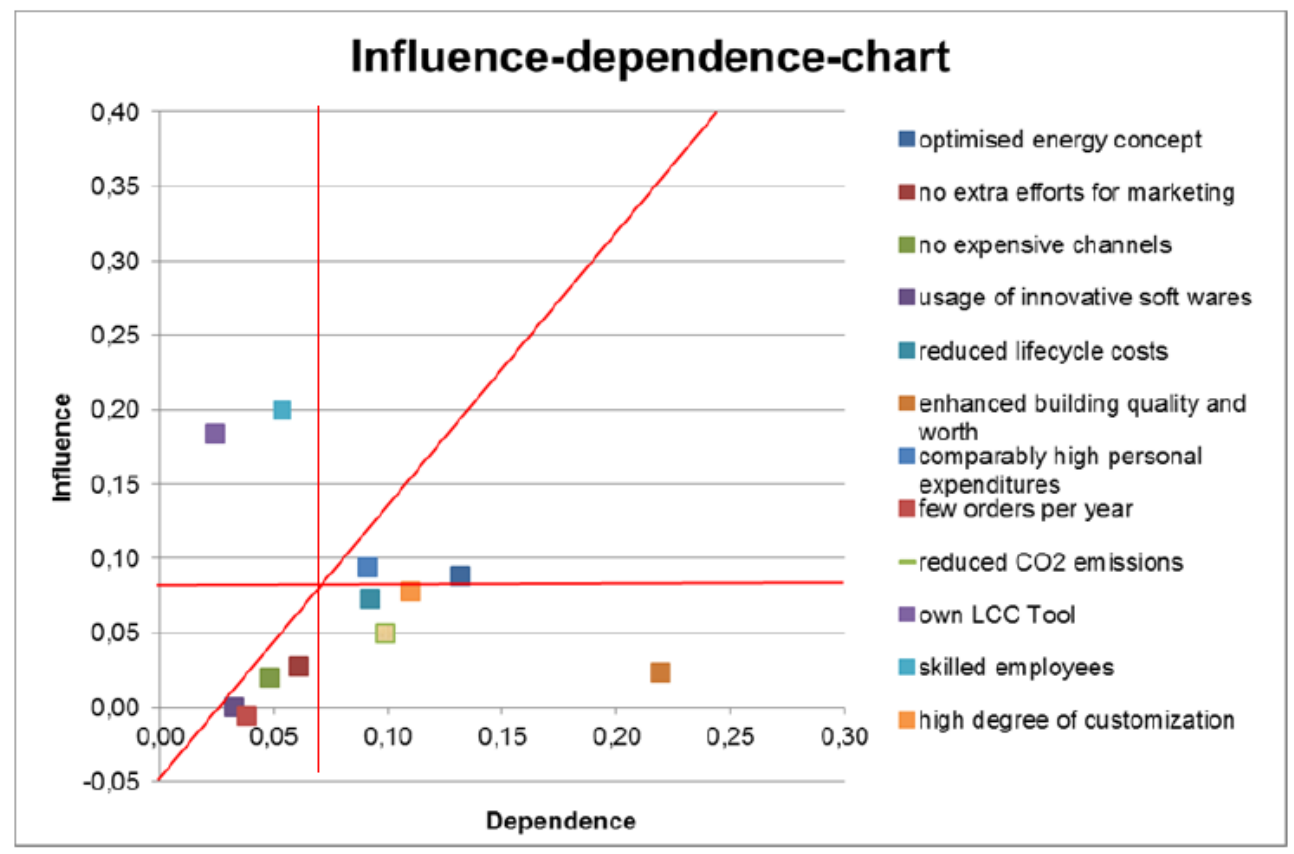

Fig. 4: Influence-Dependence-Chart of the Business Model

The horizontal and vertical red lines divide the chart in four sectors. The upper left one includes socalled influent variables which are characterized by having a high influence but low dependence on the overall system business model. The parameters in the upper right sector have both high influence and dependence and are called relay variables. In the bottom right sector the parameters have a rather low influence but high dependence on the system. They are called depending variables. Those close to the horizontal line between the depending and relay variables are the target variables. The last sector includes the autonomous variables, which cannot be seen as key success factors. The fact that this business model has very few relay variables indicates that it is easy to control. Adaption in the parameters can be done without endangering the stability of the system. However, it also implicates that few positive effects are to expect that create an exceptional competitive advantage. Besides the influence-dependence-chart, two feedback loops were identified based on the MICMAC method (see Fig. 5). The profitability could not be analyzed quantitatively as originally planned. The reason is the information provided was only of qualitative nature.
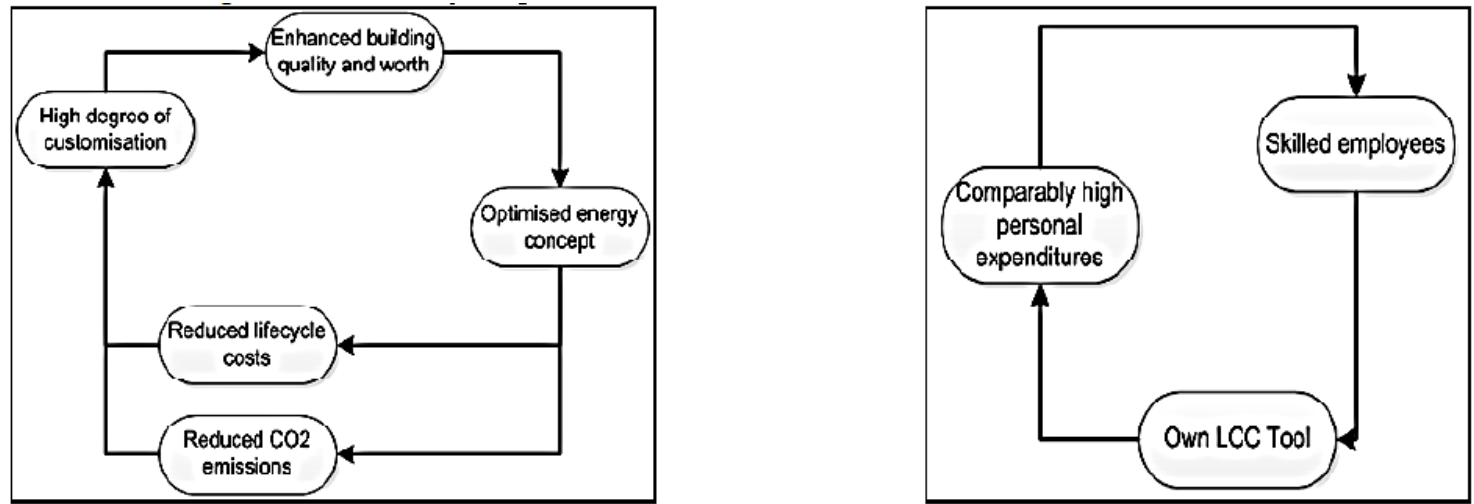

Fig. 5: Feedback loop 1 (left) and feedback loop 2 (right). 


\section{Attractiveness Portfolio}

In order to evaluate whether a business model is worth pursuing or adapting the attractiveness portfolio can be used as an indicator and illustrated in a diagram showing the level of internal (profitability) and external attractiveness (customer value). Therefore, project partners were asked to evaluate the business models based on the information given in the BMC and place them in one of the four sectors of the attractiveness portfolio. The pursue-sector contains business models with strong inand external attractiveness, the two adapt sectors have either a strong external or a strong internal attractiveness and need configurations to enhance the other, and the discard sector contains those models that lack performance in both areas and can therefore be dismissed (Schallmo, 2013: p. 167). To date, three partners gave their feedback. In Fig. 6 the averaged feedback of the partners for the business model $L C C \& \mathrm{CO}_{2}$ optimization in early design stage is indicated. The result is that the BMC should be pursed.

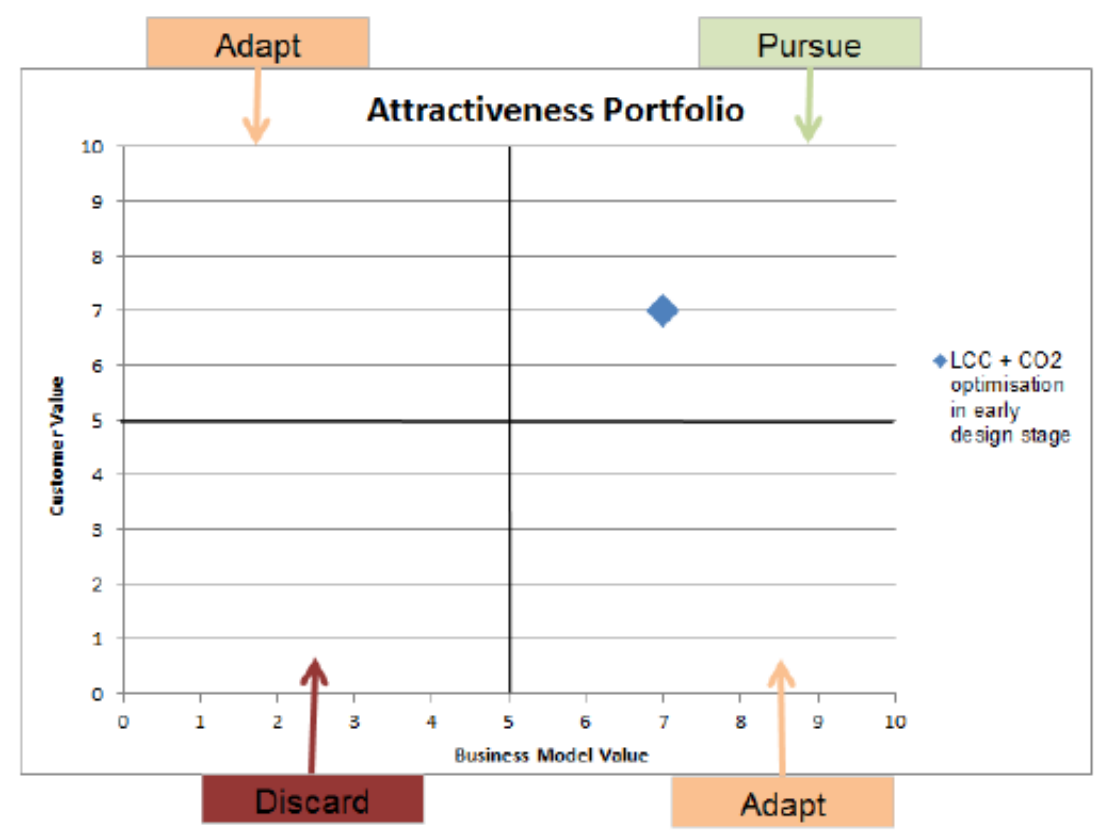

Fig. 6: Attractiveness portfolio of business model LCC \& CO2 optimization in early design stage as rated by project partners.

\section{REFERENCES}

Amit, R. \& Zott, C. (2001). Value creation in E-business. Strategic Management Journal, 22 (6-7), 493-520.

Bergmann, P. N. (March 2014). Business Model Check. Evaluation von Geschäftsmodellen unter Ungewissheit. Master Thesis, Universität Koblenz Landau. Koblenz. Accessed 30th May 2018.

Casadesus-Masanell, R. \& Ricart, J. E. (2009). From strategy to Business Models and Tactics. Working Paper, Havard Business School. Accessed 14th March 2018 under http://www.hbs.edu/faculty/Publication\%20Files/ 10-036.Pdf

Eriksson, T. \& Ritchey, T. (2002). Scenario Development using Computerised Morphological Analysis. Accessed 15th June 2018 under http://citeseerx.ist.psu.edu/viewdoc/download?doi=10.1.1.469.9096\&rep=rep 1\&type=pdf Godet, M. (1994). From anticipation to action. A handbook of strategic prospective. UNSECO Publishing. Accessed 26th March 2018 under http://unesdoc.unesco.org/images/0009/000970/097082eb.pdf Godet, M.; Arcade, J.; Meunier, F. and Roubelat, F. (1999). Structural analysis with the MICMAC method \& Actor's strategy with MACTOR method. Laboratory for Investigation in Prospective and Strategy (LIPS). Paris, FR. In: Glenn, J. and Gordon, T. (Publisher). The Millennium Project - Futures Research Methodology V2.0. 
Miller, D. (1996). Configurations revisited. Strategic Management Journal, 17, 505-512.

OECD (OECD, Hrsg.). (n.d.). Austria. How is life? Zugriff am 27.07.2018. Verfügbar unter http://www.oecdbetterlifeindex.org/countries/austria/

Organisation for Economic Co-Operation and Development (Organisation for Economic Co-Operation and Development (OECD), Hrsg.). (2018a). Housing. Zugriff am 12.07.2018. Verfügbar unter http://www.oecdbetterlifeindex.org/topics/housing/

Organisation for Economic Co-Operation and Development (Organisation for Economic Co-Operation and Development (OECD), Hrsg.). (2018b). Renewable energy. Zugriff am 12.07.2018. Verfügbar unter https://data.oecd.org/energy/renewable-energy.htm

Osterwalder, A. and Pigneur, Y. (2010). Business Model Generation. Campus Verlag; Frankfurt, DE; New York, USA.

Paoletti, G.; Ramón, P. and Anagnostopoulos, F. (2016), Zebra2020: Nearly Zero-Energy Building Strategy 2020 - D5.1: Nearly Zero-Energy Building (nZEB) technology solutions, cost assessment and performance. Bolzano, IT; Brussels, BE.

Pekrul, S. (2006). Strategien und Maßnahmen zur Steigerung der Wettbewerbsfähigkeit deutscher Bauunternehmen. Ein Branchenvergleich mit dem Anlagenbau (Univ.-Prof. Dr.-Ing. Bernd Kochendörfer, Hrsg.). : Technische Universität Berlin.

Schallmo, D. R. A. (2013). Geschäftsmodelle erfolgreich entwickeln und implementieren. Mit Aufgaben und Kontrollfragen (Lehrbuch, 1., 2013). Berlin: Springer Berlin.

\section{CONCLUSION}

The developed methodology presented in this paper allows the qualitative and quantitative evaluation of business models. However, it is strongly depending on the quality of the data collected in the Business Model Canvas. The major challenge is to collect quantitative data to evaluate the profitability of a business model; to date, no partner was able to provide all data needed to evaluate the profitability of one specific business model. One major reason is that companies usually do not strategically evaluate and plan their activities according to the evaluation of single business models; it is essential that the whole company works and different business models (of different departments) work together and generate value for the company as a whole as well as the customers. The exemplary application of the developed methodology has shown that critical factors of a business model have the following characteristics in regard to impact relationships: (i) high influence on the system (both positive and negative), (ii) high influence and dependence on the system and/ or (iii) embedment in feedback loops. A successful business model includes the characteristics (i) High amount of fits between its activities which at least means a simple consistency, (ii) a high amount of fits between customer needs and solutions offered with the value proposition and (Iii) prevention of trade-offs. For the analysis of the profitability of business models, additional and more detailed information about revenue streams and other (financial) parameters as well as more research in this regard is needed.

\section{ACKNOLEDGEMENT}

The presented work is co-funded by the Horizon 2020 Framework Programme of the European Union. The information and views set out in this paper are those of the authors and do not necessarily reflect the official opinion of the European Union. Neither the EASME nor the European Commission are responsible for any use that may be made of the information contained therein. 


\section{CONCENTRATED SOLAR POWER COMBINED WITH FLAT SOLAR PANELS IN DENMARK}

Jes Donneborg, Andreas Zourellis and Jelica Matoricz

Aalborg CSP A/S

Hjulmagervej 55, 9000 Aalborg, Denmark

Phone: +4588168847

E-Mail: jem@aalborgcsp.com

\section{SUMMARY}

This paper presents an advanced 6.8 MWth solar district heating system in Denmark which combines $4,039 \mathrm{~m}_{2}$ concentrated solar power (CSP) with $5,972 \mathrm{~m}_{2}$ flat solar-thermal collectors. The combination allows the achievement of the highest solar energy share (in this case up to $30 \%$ of the annual heat demand) and better daily energy distribution compared to a stand-alone flat panel system. Monitored by the Technical University of Denmark, performance shows a perfect production curve.

\section{CONCENTRATED SOLAR POWER IN DENMARK}

Concentrated solar power (CSP) plants have so far mainly been built to produce electricity for export

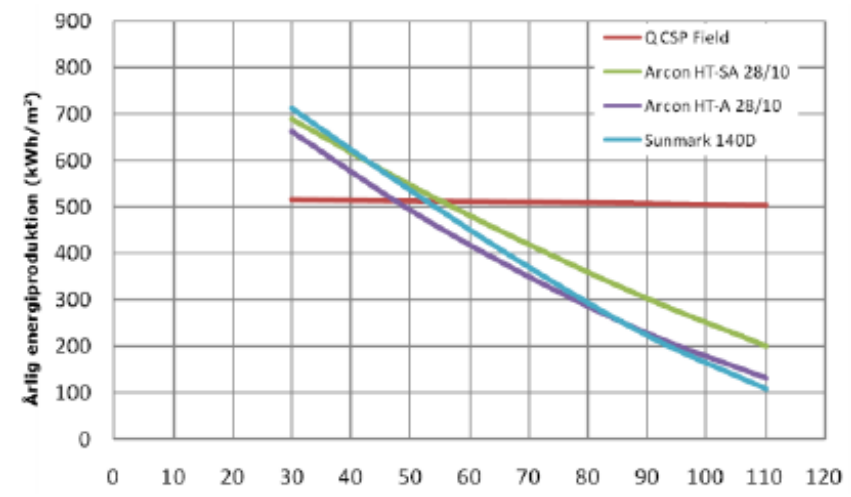

to the grid, however, numerous advantages have been identified in industrial setting as well. Due to the technology's flexibility to produce high and mid temperature heat, it provides an ideal solar-thermal solution for industrial purposes. Parabolic trough CSP plants are typically found in countries around the solar belt, but economic viability has also been proven in a place with limited solar resources, in Denmark, where efficiency of the system was monitored and compared with flat solar-thermal panels. The report concluded that CSP produces more energy above $50{ }^{\circ} \mathrm{C}$, provides a better

Figure 1: CSP surpasses performance of flat panels in Danish climate economy over the system's 25-years lifetime and ensures a year-round energy production even in Nordic climate conditions compared to flat panel systems [1].

\section{CSP COMBINED WITH FLAT PANELS - AN OPTIMAL MATCH}

In August 2015, a globally-unique solar district heating system went into operation in the northern part of Denmark, in the municipality of Taars. The system delivered by Aalborg CSP A/S is one of a kind because it combines the CSP technology with flat solar-thermal panels to supply around 850 households as well as some small-scale industries with sustainable heating.

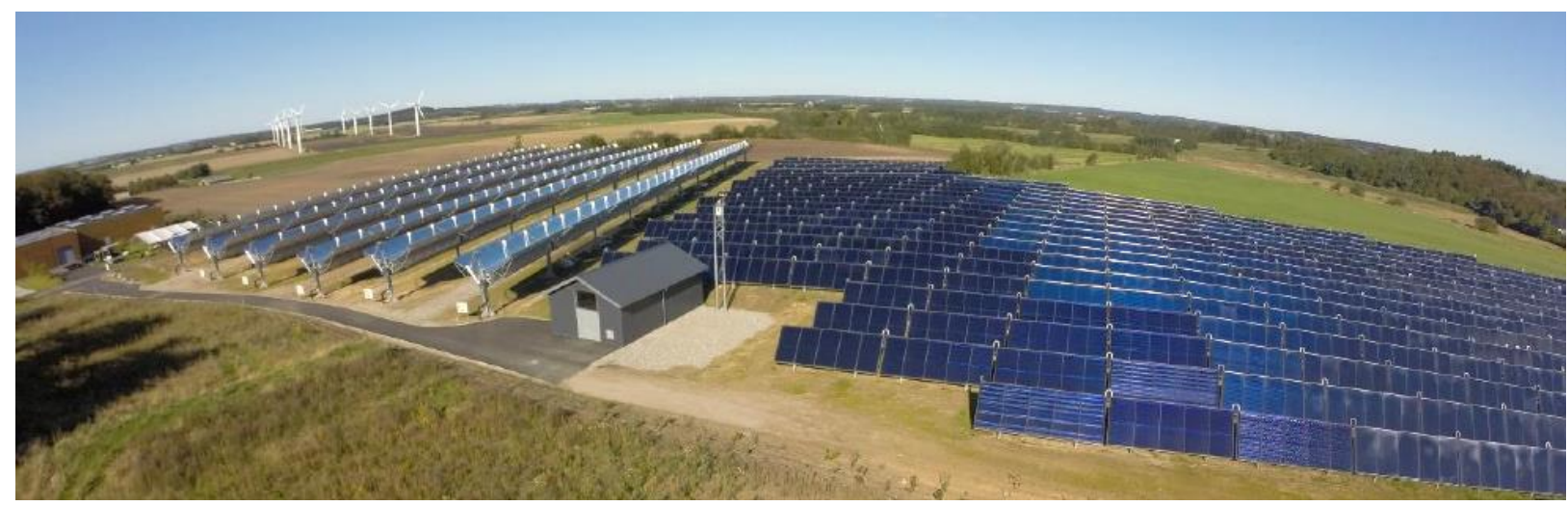

Figure 2: CSP combines with flat panels in the city of Taars, Denmark 
The combination of these two solar-thermal technologies is an optimal match: flat collectors have a higher performance at lower temperatures and produce more heat around midday, whereas CSP is most efficient at higher temperatures and provides a more balanced heat production throughout the day. The mix of the two technologies also allows better daily energy distribution. The output temperature from the flat panels can be kept at $70-75^{\circ} \mathrm{C}$ degrees and the output in $\mathrm{MWh}$ is thereby maximized. The CSP trough is then used to reach the maximum temperature for an unpressurised system of $98-99^{\circ} \mathrm{C}$. Any surplus of heat produced is directed to the client's accumulation tanks for utilization during night time and during overcast days.

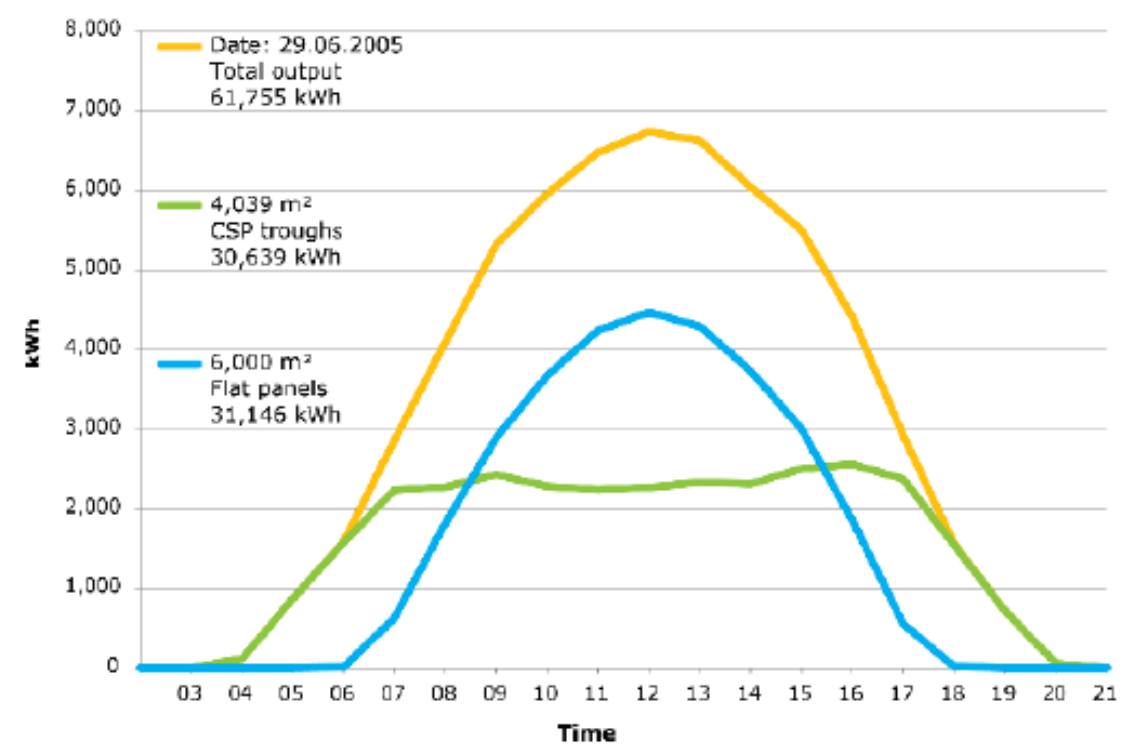

Figure 3: Energy production throughout the day

Normally the maximum utilization of flat panel systems without seasonal storage lies around $20 \%$. By investing in a combination plant with $60 \%$ flat panels $\left(5,972 \mathrm{~m}_{2}\right)$ and $40 \%$ CSP parabolic troughs $\left(4,039 \mathrm{~m}_{2}\right)$ a production of $6,082 \mathrm{MWh}$ is achieved - covering close to $30 \%$ of the city's heat demand. This is the largest solar energy share achieved compared to a conventional plant consisting of flat panels only. Even if the cost per $\mathrm{m}_{2}$ of CSP is higher, it shows cost-effectiveness and the overall system is cost-competitive to present natural gas boiler heat production costs [2].

The 30\% solar energy share is also supported by the fact, that the CSP troughs can be defocused when the energy storage is full, in contrast to flat panels that always deliver heat when the sun is shining.

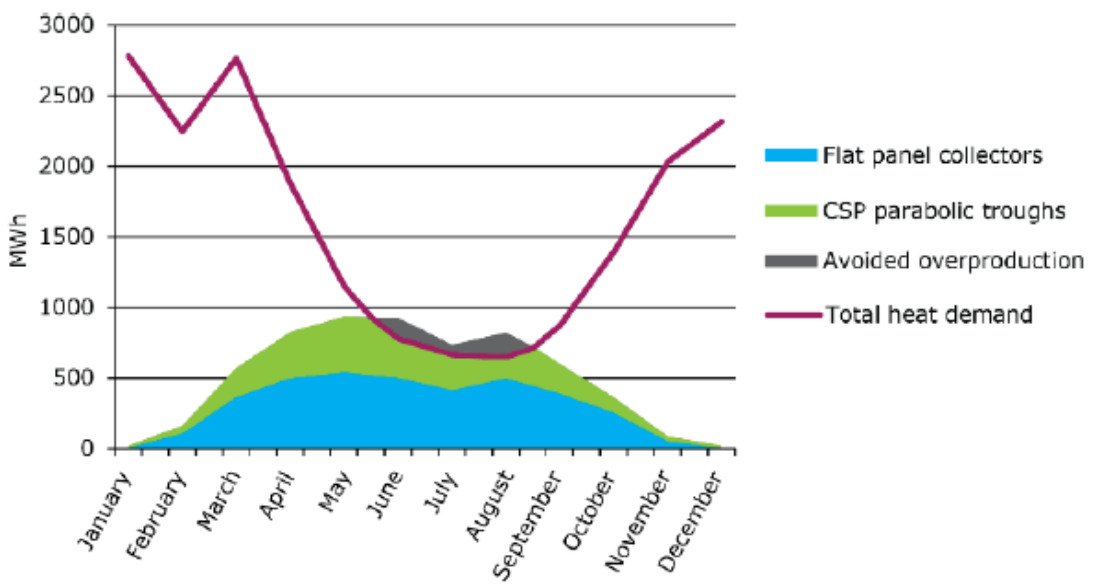

Figure 4: Solar energy output projection based on DMI DRY 2012 weather data 
Besides maximizing heat production, the combi-plant also achieves $15 \%$ price reduction for the end users.

\section{CONCLUSION}

The efficiency of the system has been monitored closely by the Technical University of Denmark and performance shows a perfect production curve. The output measured compared with the actual output is almost perfectly correlated until the system is turned off focus.

\section{REFERENCES}

[1] B. Perers, S. Furbo and D. Janne , "Thermal performance of concentrating tracking solar collectors," Technical Univeristy of Denmark (DTU) Civil Engineering Report R-292 (UK) Department of Civil Engineering, Denmark, 2013.

[2]. B. Perers, S. Furbo, Z. Tian, J. Egelwisse, F. Bava and J. Fan, "Tårs $10000 \mathrm{~m} 2$ CSP + Flat Plate Solar Collector Plant - Cost-Performance Optimization," Energy Procedia, vol. 91, pp. 312-316, 2016.

\section{CONFERENCE TOPIC}

- Renewable Heating and Cooling in a future energy system

- Future role of buildings and industry for the flexibility and stability of thermal and electric grids

- Hybridization of energy sectors

- Urban District Heating and Cooling Technologies

- Energy Efficiency, Process Intensification and Renewables in Industrial Processes 


\section{THE POTENTIAL OF LARGE-SCALE IMPLEMENTATION OF SOLAR THERMALTECHNOLOGIES IN SOUTH AFRICAN HOSPITALS}

Mr Angelo Ian Buckley1, Ms Karin Kritzinger2

Centre for Renewable and Sustainable Energy Studies 1

Stellenbosch University, Corner of Banghoek \& Joubert Streets, Stellenbosch, 7600

South Africa

Phone: +21218083605

E-Mail: buckley@ sun.ac.za

Centre for Renewable and Sustainable Energy Studies2

Stellenbosch University, Corner of Banghoek \& Joubert Streets, Stellenbosch, 7600

South Africa

Phone: +21 218083605

E-Mail: karink@sun.ac.za

\section{SUMMARY}

This study investigates the potential for the large-scale roll-out and implementation of solar thermal (ST) technologies in South African hospitals to offset conventional fuel use and identifies challenges relating to this potential uptake. There are a total of 696 hospitals in South Africa (SA) with a total thermal energy demand for hot water of $370 \mathrm{GWh}$ per year. A total installed collector area of 73972 $\mathrm{m}_{2}$ to $147944 \mathrm{~m}_{2}$ across all hospitals in SA would increase the overall ST capacity of all these hospitals to compensate for 20 to $40 \%$ of their combined annual thermal energy demand. The major limiting factor for the uptake of ST technologies in South African hospitals is the current wide spread use of coal fired boilers as well as heat-pumps, making the adoption of ST technologies less feasible from a financial perspective.

\section{INTRODUCTION}

This study investigates the potential for the large-scale roll-out and implementation of solar thermal (ST) technologies in hospitals in SA specifically relating to the use of solar water heating for the low temperature applications. The study focuses on public and privately owned, large and smaller hospitals in the country.

Hospitals in SA typically have hot water temperature requirement of $60{ }^{\circ} \mathrm{C}$, used for staff and patient ablution purposes and laundry. The study specifically investigates the penetration levels of ST technologies across the hospital sector in SA, assuming realistic solar fractions to identify the potential energy offset from conventional fuel sources, currently used by hospitals for producing hot water. The study also identifies the challenges relating to the uptake of ST technologies in South African hospitals.

\section{OVERVIEW}

The potential for ST systems varies across the world, depending on the irradiation. SA experiences an abundance of solar irradiance and exhibits great potential for the application of large-scale ST systems in industrial applications. This is clear from Figure 1 (left), illustrating the distribution of the average annual sum of global horizontal irradiance (GHI) in SA. A database that identifies all public and privately owned hospitals in SA was developed to establish the total number of hospital beds in the country. This database identified a total of 696 hospitals. This list excludes small clinics and healthcare facilities. The map in Figure 1 (right) displays the geographical location of the listed hospitals. The location of most of the hospitals in the country are in regions with the highest population density, such as Cape Town, Johannesburg and Durban, as expected. 


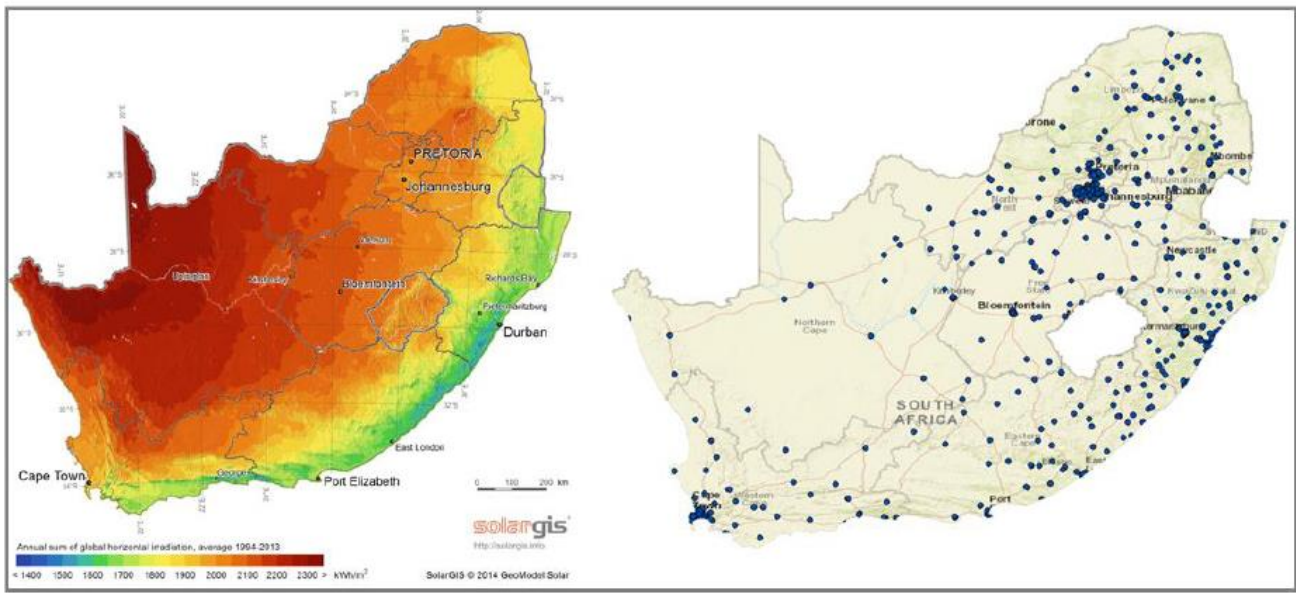

Figure 2:Solar resource map (GHI) of SA (left) and the GIS map of hospital locations in SA (right)

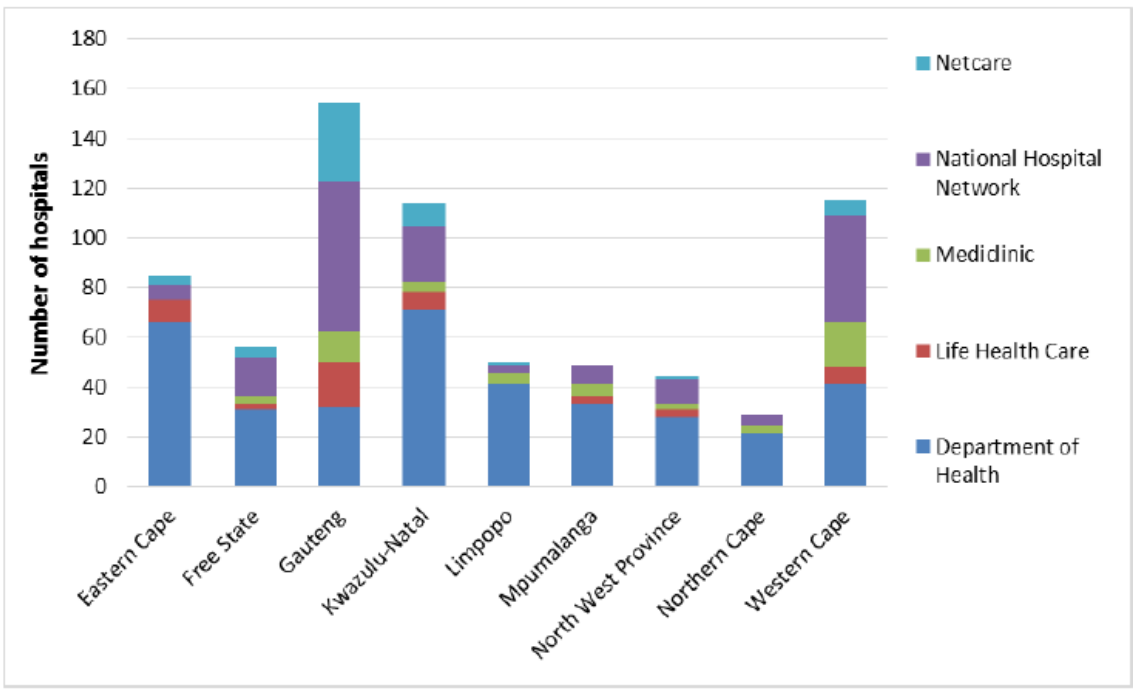

Figure 1: Number of hospitals per province in South Africa based on management

It is estimated that these 696 hospitals have approximately 126490 beds in total, with a maximum bed count of 3200 in a single hospital. The location of these hospitals can be seen in Figure 2. These include large provincial hospitals that are managed by the provincial health departments on a district level, as well as private hospitals owned by companies such as Netcare, Mediclinic, Life Healthcare and other hospital groups affiliated with the National Hospital Network.

Most hospitals throughout SA are owned and managed by the respective provincial health departments (Department of Health), as seen in Figure 2. Privately owned hospitals make up approximately $48 \%$ of the total hospitals in the country based on the data sourced, and form the majority of the hospitals in the Western Cape (WC) and Gauteng. Provincial hospitals are in the majority in other provinces of SA.

\section{CURRENT HEATING TECHNOLOGIES USED IN SOUTH AFRICAN HOSPITALS}

The database of hospitals managed by the WC Department of Health was used to get an overview of current thermal technologies used by hospitals in SA. This database identifies the current heating technologies 


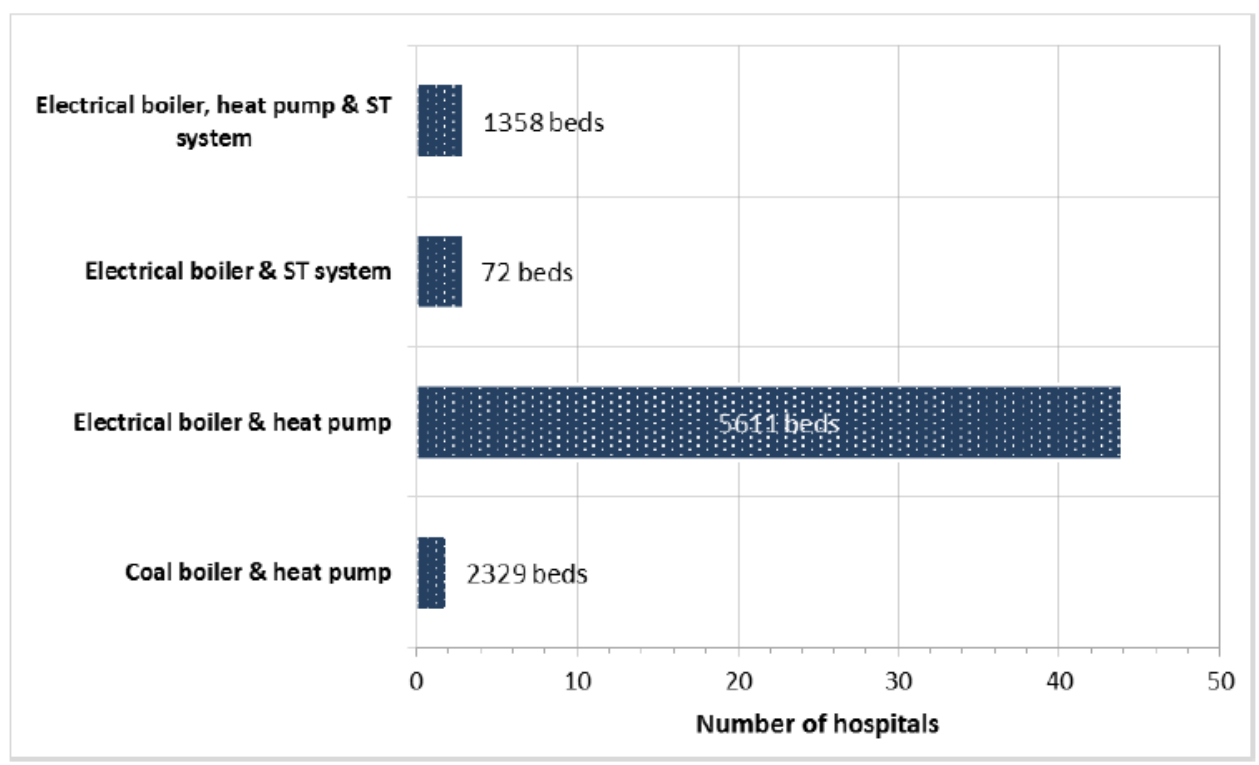

Figure 3: Thermal technologies used by hospitals managed by the WC Department of Health

installed, number of beds and water consumption. Figure 3 shows the type of heating technologies employed by the Department of Health hospitals in the WC. These hospitals make use of either coalfired or electrical boilers, including electrical heat pumps and/or ST systems.

Only two of the hospitals managed by the WC Department of Health makes use of coal boilers. See Figure 3. However, these two hospitals consists of the most beds per hospital, accounting for 2320 beds (25\%) of the total 9370 beds managed by the department. Both of these hospitals have heat pumps installed as well. Fifty of the hospitals make use of electrical boilers. Of these, 44 also have heat pumps installed, 3 also have ST technologies installed, and 3 also have a ST system and heat pumps installed. Figure 3 shows that 49 of the hospitals managed by the WC Department of Health have heat pumps installed.

The large number of heat pumps in provincial hospitals makes the adoption of ST technologies at these

hospitals challenging. The financial feasibility of ST technologies for the larger hospitals that make use of coal to meet their heat demand is also a challenge, since coal is a low-cost fuel source in SA, significantly cheaper than ST solutions when compared to the levelised cost of heat (LCOH) for each thermal energy source.

If this is the case for most provincial hospitals, which account for $52 \%$ of hospitals in SA, it will be difficult to prove the feasibility of ST systems for provincial hospitals. In cases where large heat pumps have been installed, much smaller ST systems would be the most sensible solution, although this option still carries the risk of presenting longer payback periods than normal, when compared to hospitals that solely depend on conventional electrical and coal boiler technologies.

On the other hand, privately owned facilities, such as hospitals owned by Mediclinic and Netcare, have installed renewable energy technologies, including ST and solar PV solutions. In many cases, these hospitals have installed combined solar and heat-pump systems, using electrical resistive heating as a back-up heat source. These systems demonstrate how the different technologies can effectively work together to supply the hot water needs of hospitals.

\section{CURRENT SOLAR THERMAL APPLICATIONS AT SOUTH AFRICAN HOSPITALS}

The large-scale ST database created during this study was redefined to identify all large-scale ST installations at hospitals in SA. It should be noted that this database was created through extensive market research and online information and its inclusion is subject to information being available to the researchers. Based on the information gathered in the database, there are 23 large-scale ST systems installed at hospitals within SA, equating to a total ST collector area of $1333 \mathrm{~m}^{2}$. Figure 4 
displays the total collector area and number of systems per province, based on the specific collector area range of each system. These systems range in a gross collector area from 10 to $250 \mathrm{~m}^{2}$.

Most of the ST systems installed at hospitals in SA are in the WC, with 11 of the 23 recorded systems. See Figure 4. These WC systems account for $547 \mathrm{~m} 2$, or $41 \%$, of the total collector area installed in the country at hospitals. Gauteng has a total of seven recorded systems, amounting to a total installed collector area of $546 \mathrm{~m}^{2}$. Other provinces, including the Free State, KwaZulu-Natal and the Northern Cape, have five recorded systems with a total collector area of $240 \mathrm{~m} 2$, amounting to $18 \%$ of the total collector area installed at hospitals throughout the country. Furthermore, it is noticeable that most of these systems have a total gross collector area ranging from $50 \mathrm{~m}_{2}$ to $125 \mathrm{~m}^{2}$.

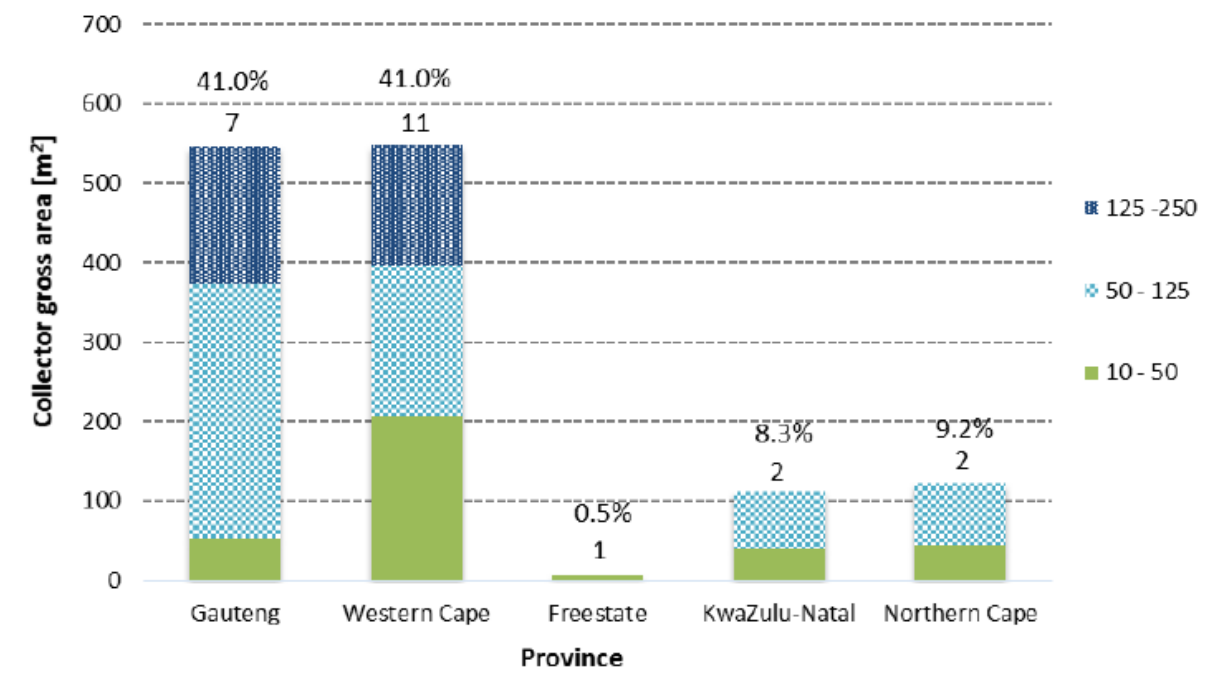

Figure 4: Large-scale ST system installed at hospitals in South Africa, per province

\section{ANNUAL HEAT DEMAND OF HOSPITALS}

Large hospitals around the world require thermal energy for a variety of purposes, including central heating, staff and patient ablutions, and in certain cases process steam. Some larger and older public hospitals in SA make use of process steam, generated in larger-scale boilers, to operate autoclaves for sterilisation, humidification, heating, and generating hot water for domestic purposes. Most of the newly built public and private hospitals in the country do not depend on these steam cycles and make use of more efficient technologies and cycles. This section of the report focuses on the hot water demand in hospitals in SA for domestic applications, including staff and patient ablutions.

The South African National Standard (SANS) 10252-1 states that hospitals in SA can be expected to have a daily water demand of $450 \mathrm{l} / \mathrm{bed}$ and $550 \mathrm{l} / \mathrm{bed}$ based on design considerations and general hospitals are expected to consume 130 to $140 \mathrm{l} / \mathrm{bed}$ of hot water per day. Considering the hot water temperature requirement of $60{ }^{\circ} \mathrm{C}$ for most hospitals in SA, general hospitals can thus be expected to have a thermal energy requirement of 6,77 to $7,29 \mathrm{kWh} / \mathrm{bed} / \mathrm{day}$.

To get an overview of the expected annual thermal energy demand of all 696 hospitals identified in SA, based on daily hot water usage per bed as set out in SANS 10252-1, an extrapolation was done using the total number of beds accounted for in the database. The results are shown in Figure 5. It should be noted that this estimation assumes that all 696 hospitals consume hot water within the range that is set out in SANS 10252-1 for general hospitals and does not compensate for the variation in hot water usage based on different functions of hospitals. 


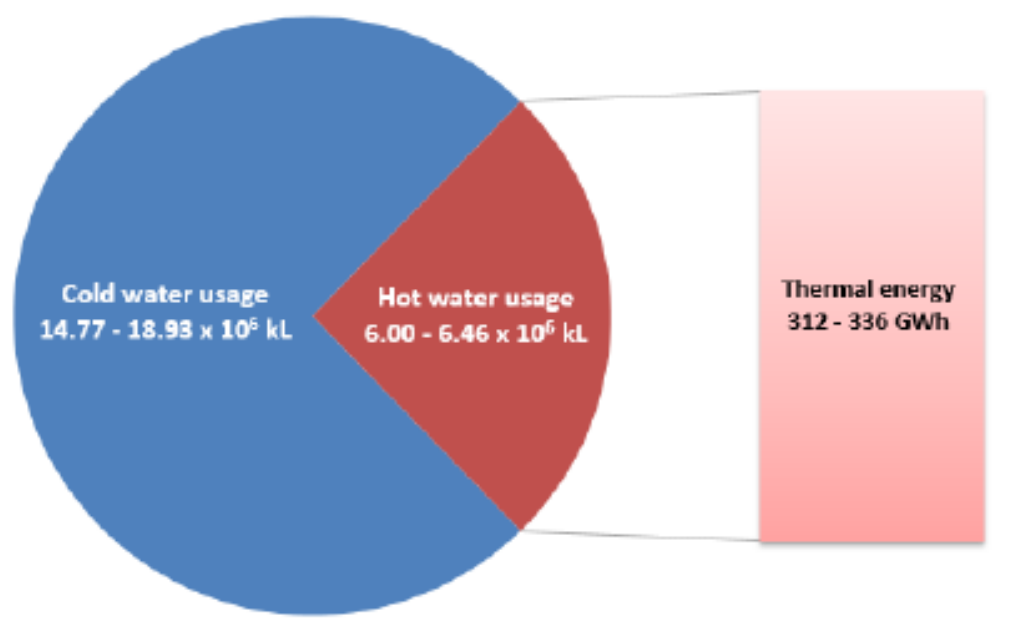

Figure 5: Annual heat demand of all hospitals for hot water production in South Africa

It is approximated that all 696 hospitals in SA have a combined thermal energy requirement of 312 to $336 \mathrm{GWh} /$ year to heat the total hot water demand range to $60{ }^{\circ} \mathrm{C}$ for domestic purposes. This excludes heat requirements attributed to steam to operate autoclaves for sterilisation and humidification, and space heating.

As mentioned, the WC Department of health hospital database identifies key information, such as the average daily cold water consumption and electricity usage per bed, the number of beds and current heating technologies installed at each hospital. This database lists 52 hospitals managed by the WC Department of Health, including small and large hospitals. The average daily cold water consumption of each of the 52 hospitals is shown in Figure 6.

Most provincial hospitals in the WC fall well below the lower limit specified presented in SANS 10252-1 (450l/bed/day). See Figure 6. However, there are a number of hospitals that consume more than the $550 \mathrm{l} / \mathrm{bed} / \mathrm{day}$. This variation in cold water usage could be related to the variation in services offered by each hospital, patient capacity, age of the infrastructure, geographical location, and whether it is situated in a rural or urban area. This makes estimating the cold and hot water demand difficult, as shown in Figure 5, as the heat demand will vary significantly for each hospital.

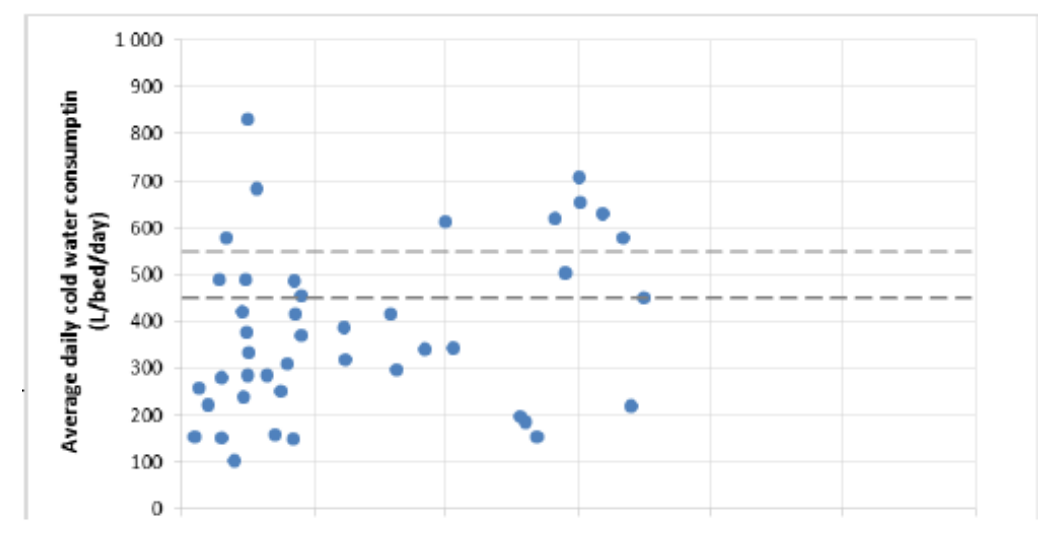

Figure 6: Average daily cold water consumption per bed for hospitals managed by the Western Cape Department of Health 
The annual cold water consumption of these hospitals and the linear relationship with the number of beds is shown in Figure 7 (left). Garcia-Sanz-Calcedo et al (2017) provides a quantification of cold and hot water consumption for human consumption and domestic use in hospitals, based on the number of beds as opposed to using a fixed ratio. The study makes use of the

Figure 7: Annual cold water consumption of Western Cape Department of Health hospitals and linear relationship with number of beds (left) and relationship between the annual cold and hot water consumption in hospitals based on number of beds (right) (Source: Garcia-Sanz-Calcedo et al, 2017)

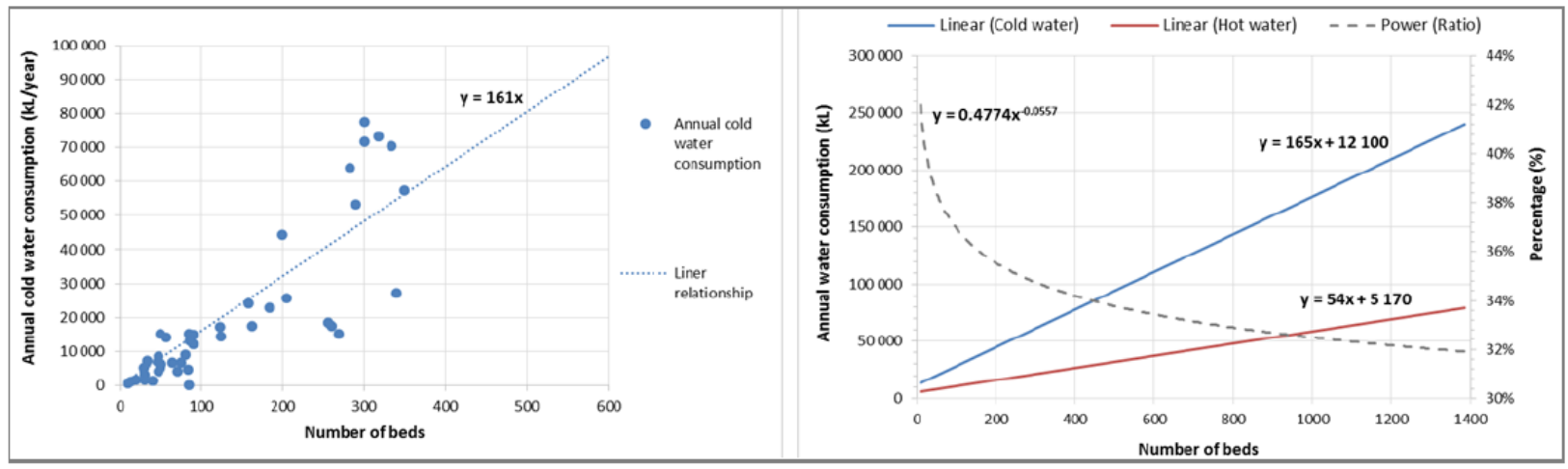

measured cold and hot water consumption of 13 public hospitals in Spain. The relationship identified for the average annual cold and hot water consumption for domestic use in hospitals based on the number of beds is shown in Figure 7 (right)

The linear relationship developed for the annual cold water consumption of hospitals in SA, shown in Figure 7 (left), and the relationship developed between the cold and hot water consumption of hospitals based on Garcia-Sanz-Calcedo et al. (2017), shown in Figure 7 (right), was used to develop a relation to the number of beds and the hot water consumption, shown in Figure 8. Furthermore, it was used to more accurately estimate the annual cold water, hot water and heat demand of the 696 hospitals identified in SA and, given in Table 1.

Table 1: Results of cold and hot water consumption and thermal energy requirements for the 696 hospitals in SA

\begin{tabular}{|l|l|c|}
\hline Hot water & Annual consumption & $7099964 \mathrm{k \ell}$ \\
\hline & Average daily hot water consumption per bed & $154 \mathrm{c} / \mathrm{bed} / \mathrm{day}$ \\
\hline Thermal energy & Annual thermal energy demand & $370 \mathrm{GWh}$ \\
\hline
\end{tabular}

Thus, based on a detailed assessment of existing cold water consumption and literature, the earlier estimation of the annual thermal energy demand for all hospitals in SA, which was 312 to $336 \mathrm{GWh}$ (see Figure 5), was recalculated as $370 \mathrm{GWh}$. This represents a more accurate and realistic approach for determining the total thermal energy needs of hospitals and is used to investigate the potential for the large-scale application of ST technologies at hospitals in SA. 


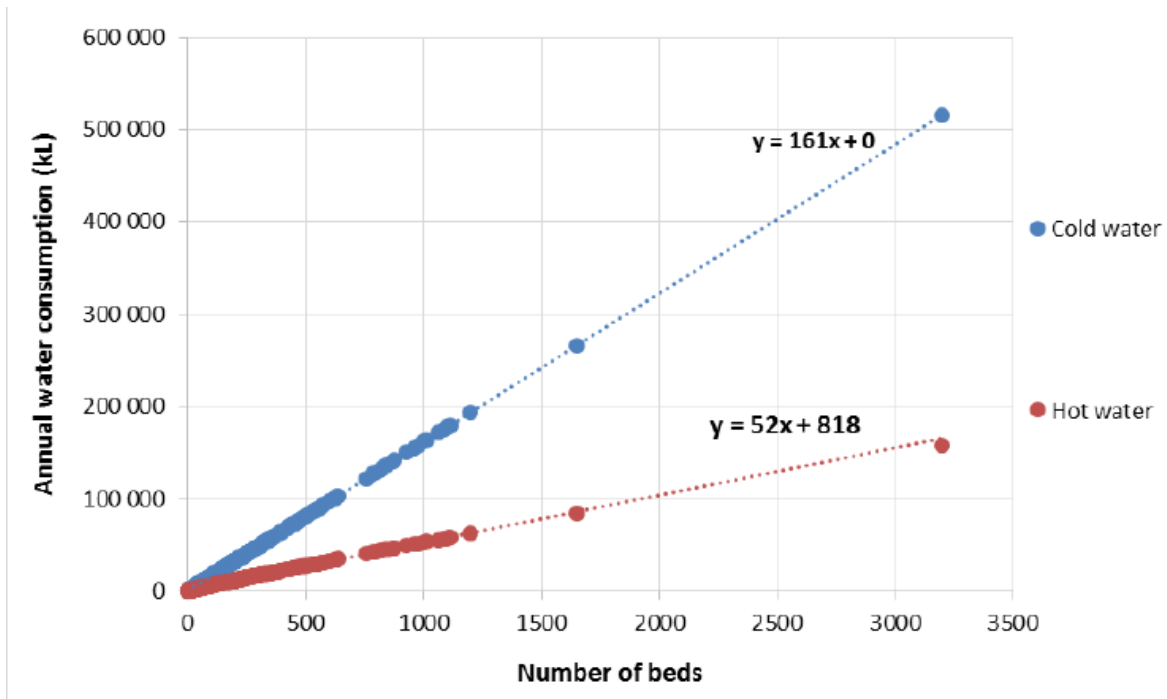

Figure 8: Annual cold and hot water consumption of 696 hospitals identified in South Africa

\section{FINANCIAL CONSIDERATIONS}

When considering the findings of this study and the large uptake of heat-pump systems in most of the provincial hospitals in the WC, ST projects may present extended amortisation times compared to those presented in Joubert et al. (2016). In Joubert et al. (2016), the amortisation times and LCOH of large-scale ST systems in SA for a system cost of $€ 603 / \mathrm{m}_{2}$ and at an exchange rate of R15.30/€ was estimated. See Figure 9. The study shows the payback period for ST that can be expected when replacing conventional energy sources, based on different annual increases of 0 to $12 \%$ on the cost of these energy sources, which are commonly coal and electricity in South African hospitals.
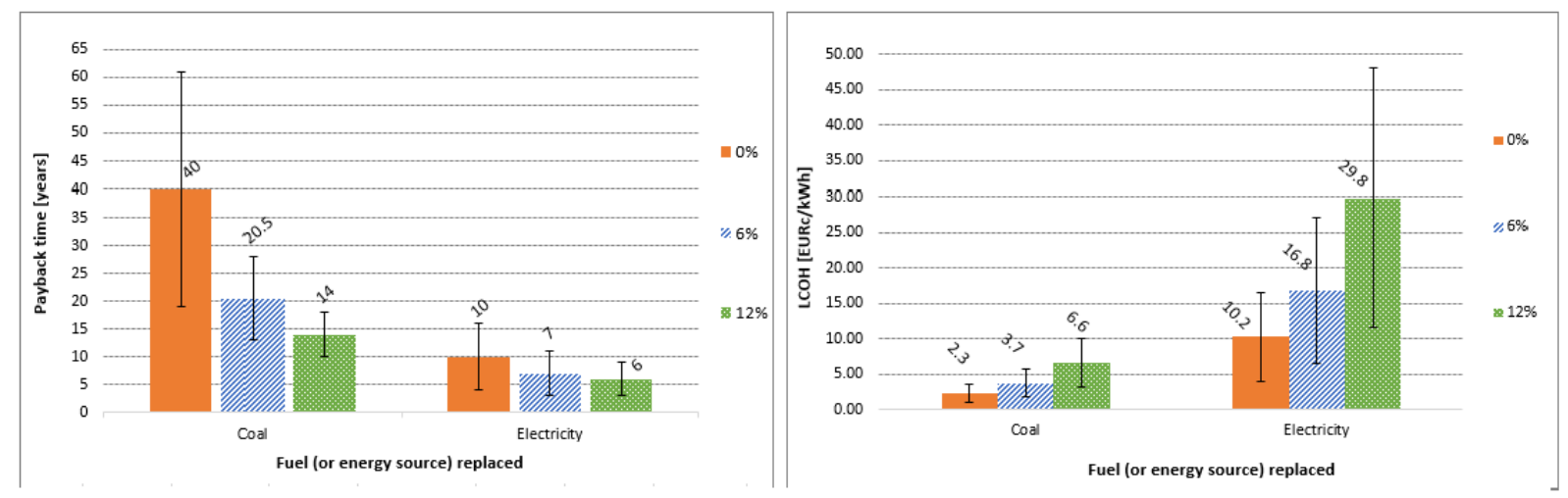

Figure 9: Amortisation times (left) and LCOH (right) of a current large-scale ST system

It can be assumed that most hospitals rely on electrical boilers for generating hot water in cases where heat pumps are not installed. In these cases, hospitals can be expected to invest in ST projects presenting simple payback periods ranging from 6 to 10 years. In the case of older, larger hospitals that still rely on coal for heating, projects with a simple payback period of 14 to 40 years can be expected, making the investment unfeasible.

Figure 9 shows that the LCOH of coal and electricity is 2,3 to 6,6 EUROc/kWh and 10,2 to 29,8 EUROc/kWh, respectively, based on the annual fuel increase of 0 to $12 \%$ and discount rate of $6 \%$ over 20 years (Joubert et al, 2016). 


\section{RESULTS}

Figure 10 depicts the scale and size to which ST technologies would have to be implemented at hospitals in SA to achieve a different solar fraction of the total annual thermal energy demand of 370 $\mathrm{GWh}$, as well as the current total collector area installed at hospitals in the country. Figure 10 was generated assuming an average specific gain of $1000 \mathrm{kWh} / \mathrm{m} 2 /$ year throughout the country where hospitals are located.

Currently, South African hospitals have a total installed collector area of $1333 \mathrm{~m} 2$, as indicated in Figure 4 . This collector area only accounts for approximately $0,4 \%$ of the total annual thermal energy needs for producing domestic hot water in all hospitals the country, estimated at $370 \mathrm{GWh}$.

It can be realistically assumed that hospitals can feasibly increase their overall ST capacities to compensate for 20 to $40 \%$ of their combined annual thermal energy needs by way of enhancing the performance of installed heat-pump systems and also by reducing the industry's dependence on boilers and conventional fuel sources.

This would require the installation of approximately $73972 \mathrm{~m} 2$ to $147944 \mathrm{~m} 2$ of collector area throughout the 696 hospitals identified in SA.

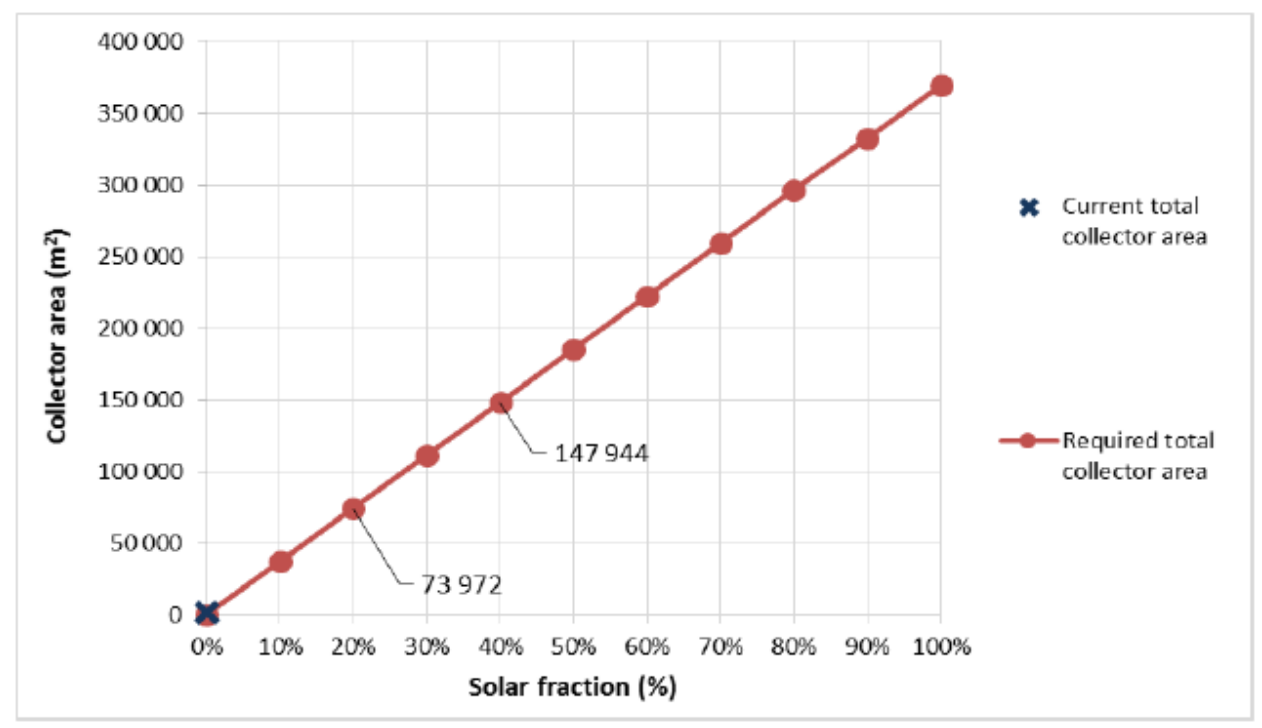

Figure 10: Relationship of total collector area and solar fraction of the total thermal energy needs of hospitals in SA

\section{CHALLENGES FACING SOLAR THERMAL APPLIACATIONS IN HOSPITALS}

There are a number of challenges impacting the uptake of ST technologies in hospitals in SA, including; most hospitals are not equipped with measuring instrumentation to record the hot water consumption, essential to characterise the hot water demand; A number of hospitals have installed solar photovoltaic (PV) systems which may reduce interest in investing in the use of ST technologies, even if these offer a further reduction in electricity consumption. Many hospitals have urgent problems with the maintenance of existing water systems, including corrosion of pipes and ineffective maintenance of leaks, outdated and less efficient heating technologies such as electrical and coal boilers in older hospitals and non-existent or poor insulation of piping and storage tanks, leading to preventable heat losses.

Some hospitals do not have enough space to install the large storage tanks that are required for ST systems. In some large hospitals, large storage tanks and boilers are located on the top floors of the building, making the removal and addition of these tanks very costly and labour intensive. Large and 
older hospital buildings have been constructed with asbestos roofs. Installers may be reluctant to mount solar collectors on asbestos roof areas owing to asbestos legislation and the serious health risks associated with asbestos work; and many public and private hospitals in the country have installed heat-pump systems in conjunction with conventional boiler technologies to reduce dependence on boilers and become more energy efficient.

\section{CONCLUSION}

Currently there are 23 ST systems installed at hospitals in SA, accounting for a total installed collector area of $1333 \mathrm{~m}$. Most of these systems are installed in Gauteng and the WC. Most hospitals currently make use of a combination of electric boiler and heat pump technologies for producing hot water. The use of coal boilers is seen in large, older hospitals in the country. A total installed collector area of 73 $972 \mathrm{~m}_{2}$ to $147944 \mathrm{~m}_{2}$ across all hospitals in SA could feasibly increase the overall ST capacity to compensate for 20 to $40 \%$ of the combined annual thermal energy needs for hot water. The major limiting factors for the uptake of ST technologies in hospitals are the current large-scale usage of heatpumps and coal fired boiler technologies, with resultant challenges in making a financial feasibility argument. Other challenges include existing problems with hot and cold water supply systems in older hospitals, lack of understanding and awareness of ST technologies, competiveness and preference for other renewable energy and energy efficient technologies such as solar PV and heat-pumps. The prevalence of asbestos roofs and limited roof space were also identified as challenges.

\section{REFERENCES}

Anon., 2012. Standards South African National. Water supply installations for buildings. Pretoria: (SANS 205 10252-1: 2012).

Garcia-Sanz-Calcedo, J., Lopez-Rodriguez, F., Yusaf, T. \& Al-Kassir, A., 2017. Analysis of the average annual consumption in the hospitals of Extremadura (Spain). Energies, 10(479), pp. 1 - 10

Joubert, E. C., Hess, S. \& Van Niekerk, J. L., 2016. Large-scale solar water heating in South Africa: Status, barriers and recommendations. Renewable Energy Volume, Volume 97, pp. 809 - 822 


\title{
A COMPARATIVE STUDY OF SOLAR WATER HEATER AND PHOTOVOLTAIC WATER HEATER IN WINDHOEK
}

\author{
Mr Senior Shimhanda, Mrs Helvi Ileka \& Ms Fenni Shidhika \\ Namibia Energy Institute \\ 13 Storch Street, Windhoek \\ Phone: +264816460744 \\ E-Mail: shimzenior@gmail.com
}

\begin{abstract}
SUMMARY
The Namibia Energy Institute (NEI), at the Namibia University of Science and Technology (NUST), together with AEE-Intec, have installed the first ELWA photovoltaic water heater (PWH) in Namibia. The ELWA PV system is an emerging "hot water from photovoltaics (PV)" made in Austria for domestic water heating. The PWH has been installed for research purposes under the Southern African Solar Thermal Training and Demonstration Initiative (SOLTRAIN) project. The PWH was compared with the solar water heater (SWH) in terms of energy performance and techno-economic benefits. PVsizing was done with the "PV Dimensioning for ELWA (Excel Sheet)." It was concluded that PWH does not require antifreeze protection which reduces maintenance costs and it has simple installation as no additional hydraulic pipe network is needed but it is payback period is greater than SWH's due to high initial capital.
\end{abstract}

Keywords: solar water heater (SWH), photovoltaic water heater $(\mathrm{PWH})$, techno-economic, costeffective.

\section{INTRODUCTION}

Through SOLTRAIN, a regional project funded by the Austrian Development Agency (ADA) and OPEC Fund for International Development (OFID), NEI installed 62 solar water heaters (SWH) at selected houses in Windhoek's Otjomuise residential suburb under the National Housing Enterprise (NHE) program between December 2015 and March 2016. Data logging systems were also installed at six houses of which four houses are equipped with SWH and two houses equipped with electrical geysers. Hence, data has been collected and analysed at these houses since 2016. However, NEI recently installed a $1.59 \mathrm{~kW}$ PV array in 2018 to power an ELWA system at one of the monitored houses which previously used a standard electrical geyser. The ELWA PWH is an emerging solarpowered technology made in Austria for domestic water heating. It consists of a photovoltaic array, a back-up electrical heating element and a water storage tank. For this research, the ELWA heating element immersed in a $300 \mathrm{~L}$ vertical tank is directly powered by a $1.59 \mathrm{~kW} \mathrm{PV}$ array without an inverter. At another house which was using an electrical geyser before, a 300L SWH with $4 \mathrm{~m}_{2}$ collector area was installed to conduct a comprehensive study comparing the SWH and the PWH systems in Namibia. The systems were designed based on the hot water demand per person in the respective houses as analysed over a period of one year.

\section{RESEARCH QUESTIONS}

The techno-economic analysis of SWH and PWH systems in the Namibian context aimed to answer the following research questions:

- Whether the water heating systems are viable technically and economically?

- Their possible impacts on the environment

- The initial capital costs and operation costs of each water heating system

- Which technology is more cost-effective in terms of energy performance and economic benefits?

- And which technology is more efficient and less prone to intermittent weather fluctuations?

\section{RESULTS}

The PWH system shown in Fig.1 below heats water in a boiler using solar power and optimizes the energy consumption of the household with professional controls. It consists of a photovoltaic array, a back-up electrical heating element and a water storage tank. Compared to solar thermal systems, a large number of expensive elements such as pipes, pumps, valves, expansion vessels, antifreeze liquid, 
insulation, cabling for sensors and control unit are eliminated from the "ELWA hot water from PV system."

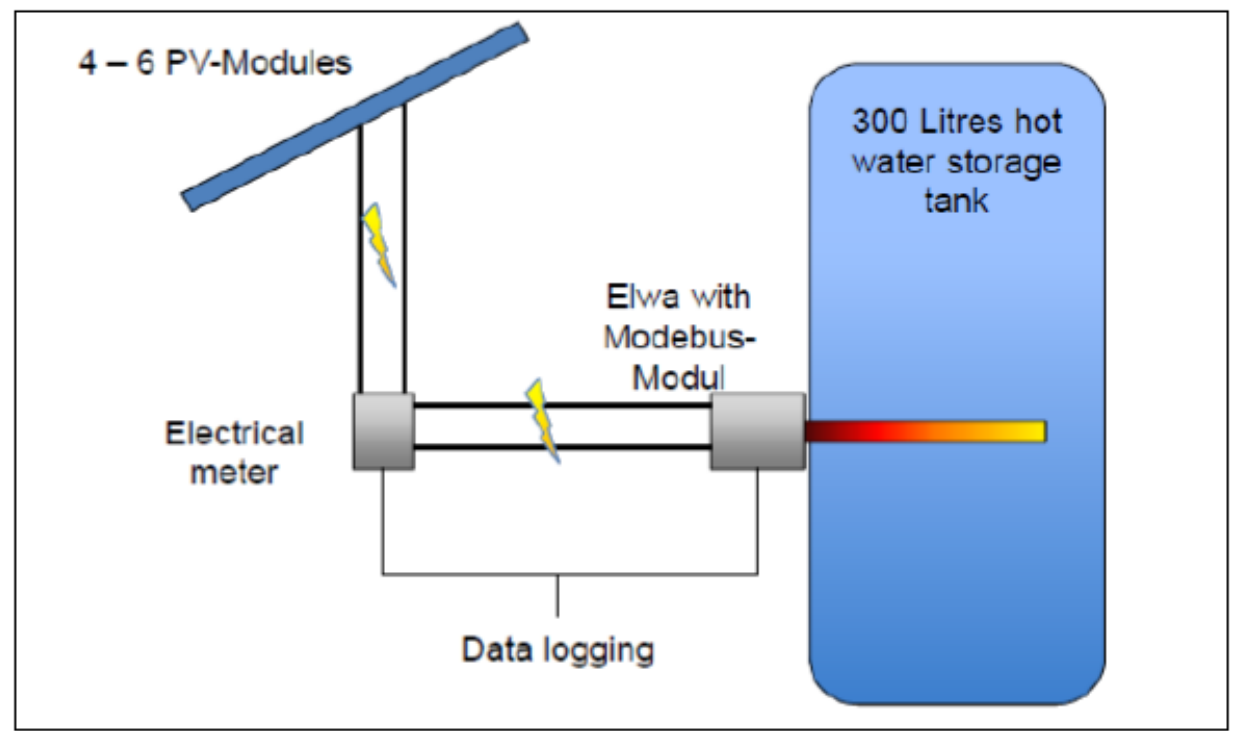

Fig.1: A schematic of the first ELWA photovoltaic water heater installed in Windhoek, Namibia

\section{CONCLUSION}

Heating with PV is feasible as demonstrated by the ELWA PV heater. PWH involves easy installation whereas SWH requires hydraulic pipe network including a heat transfer fluid which leads to higher transfer and conversion losses in the heat exhanger. Moreover, no freezing protection is needed with PWH and system efficiency is independent regarding to the operating temperature in the storage tank. A minimum of four PV modules are required to reach sufficient current to switch on the heating element. The mimimum required PV area is $6.5 \mathrm{~m}_{2}$ whereas the required solar thermal collector area to reach same capacity is $2 \mathrm{~m}^{2}$.

\section{REFERENCES}

Moschik, R, \& Buckley, A., (2017), PV water heating system - first experiences in South Africa and Austria

Rimpler, G., (2017), ELWA Assembly and Operation Manual., Neuzeug, Austria. http://www.mypv.com/download/elwa/Assembly-Operation\%20Manual\%20ELWA\%20EN170504.pdf

\section{TOPIC}

Renewable Heating and Cooling in a future energy system 


\section{LEGAL ANALYSIS OF HEAT FEED-IN IN AUSTRIAN DISTRICT HEATING NETWORKS}

Marie-Theres Holzleitner / Simon Moser

Energieinstitut an der Johannes Kepler Universität

Altenberger Straße 69, 4040 Linz, Austria

Phone: +43732 24685675

E-Mail: holzleitner@energieinstitut-linz.at

\section{SUMMARY}

The feed-in of waste heat into an existing district heating network is hindered by technical, economic and also legal obstacles. In this paper we analyze the currently available legal opportunities for a heat feed-in by a third party. On the one hand, we differentiate between the possibility of feed-in and the purchase to the district heating network operator and, on the other hand, the possibility of transmission and thus of direct sale by the third party to a specific customer. In addition, a hypothetical concept describing an obligation to accept a feed-in demand is elaborated from the Austrian perspective, whereby this concept is designed in accordance with the Swedish District Heating Law. On the basis of the antitrust law analysis, we come to the conclusion that district heating networks are designed very individually, which implies that the legal claim would have to be assessed on a case-by-case basis. Objectively justified reasons such as economic and technical unacceptability lead to the exclusion of the antitrust claim to feed-in. A general statutory obligation to accept would not consider the specificities of the individual networks and would likely cause significant administrative burdens.

\section{INTRODUCTION: THE PROJECT “OPEN HEAT GRID”}

The first call of the "City of Tomorrow" funding program of the Austrian Federal Ministry of Transport, Innovation and Technology raised the question about the impacts of different market designs and the different network charges and prices between the electricity, gas and heating networks. In order to find answers, the Open Heat Grid project [1] was funded within this program. The project primarily examined the barriers to the feed-in of industrial waste heat into district heating networks. Subsequently, it was analyzed how policy instruments can and should be used to accelerate the integration of waste heat. In order to do so, first, a literature analysis was conducted summarizing theoretical concepts and existing country-specific solutions. Based on the literature review, the most promising concepts of regulation have been selected. These possible concepts of the legal organization of the waste heat feed-in were chosen because they represent scientifically interesting, polar concepts or frequently discussed concepts. Second, a stakeholder analysis, an economic analysis and a legal analysis were conducted.

In this paper, the focus is on the legal analysis. Due to the relevance and practicability of the concept of a regulation (obligation to accept), only this concept is dealt within this paper. A summary of the economic analysis at the end of the paper completes the results. Note that the legal analysis was conducted for the Austrian case. However, many results may be generalized.

\section{LEGAL ANALYSIS}

At first two possible scenarios within existing legislation were considered and analyzed: in scenario (1a), the third party heat generator wants to feed in heat and the district heating (DH) network operator acts as the buyer. It is examined whether there is a legal possibility to feed in against the will of the operator. In second scenario (1b), the heat generator wants to feed in heat and "transit" it through the DH network. He already has a concrete customer for the heat.

Additionally, scenario (2) was designed that is based on the assumption that the DH network operator is obliged to accept feed-in from other generators. Network connection costs are covered by the heat generator. The DH network operator is obliged to pay the costs not incurred (i.e. primary energy) as feed-in tariff to the generator. Accordingly, in this scenario the DH network operator is obliged to grant access and allow feed-in in a cost-neutral manner. A critical aspect of this concept is that DH networks have limited capacities. 


\section{OPTIONS IN THE CURRENT LEGAL FRAMEWORK}

In the current situation, the DH network operator is like a price-regulated monopoly to the local district heating end-user. That is why the DH network operator is usually the only DH supplier with significant customer access. For an industry with waste heat potentials, the construction of its own DH network is therefore not lucrative and negotiations are required with the DH network operator. The latter has thus a strong negotiating position due to its position as sole option for feed-in. If the DH network operator agrees to feed third party heat into his network, there are two options. Within the first option the operation of the DH network and the supply of end customers remain with the previous DH network operator. The operator integrates the third party heat source as another generation unit in the operator's portfolio. Within the second option the operator is only responsible for operating the network and the third party has its own costumers.

The contracting parties are free to make provisions regarding backup capacities, load and generation profiles, entry points, temperatures, etc.

Depending on the overall contract design (i.e. defining the partner who bears the costs of the components of the waste heat feed-in as well as the definition of feed-in profiles, backup capacities, etc.) between the industry and the DH network operator, the use of waste heat must prove to be economically more favourable for the DH network operator than the using the own generation units.

\section{Elaboration of option 1a "heat feed-in and purchase by the operator}

The heat generator wants to feed heat into the local DH network. At this point, it is crucial to distinguish whether the third party heat generator intends to act as a competitor who wants to sell heat to end-users (scenario 1b) or he just wants to feed-in the heat into the network (expecting a remuneration; scenario 1a) [2]. Scenario (1a) was analysed within existing legislation. Here, antitrust regulations are to be analyzed if they are to be applied concerning a feed-in option into existing DH networks.

First of all, it is necessary to clarify whether the operator of the respective geographically limited DH network has a dominant market position acc. to the antitrust act ( $\$ 4$ KartG 2005). If there already exists a DH network in a certain area, there is usually only one, which is run by a single operator. Unlike the electricity and gas networks, DH networks are not interconnected supra-regionally. The operator is a vertically integrated company that not only operates the DH network, but also generates heat and delivers it to consumers. However, consumers are not able to switch the DH operator. Consequently, in the absence of competition, this operator is a monopolist holding a dominant position in its network operation, especially as the creation of competition through the establishment of parallel DH networks would be economically questionable. This dominant position will most likely be maintained by long-term supply contracts.

For the DH market, there are no special provisions that go beyond the general civil provisions (Civil Code ABGB, Consumer Protection Act KSchG etc.), which would prohibit or limit long contract periods. Consequently, as the operator of a local DH network has a dominant position, the question arises whether he can be compelled to grant third parties access to his network for the purpose of supply. There may be an obligation to contract resulting from the antitrust act if one party has enough power to determine the decision of others, so in particular when holding a monopoly position. If the conclusion of the contract is reasonable, the owner of a monopoly position must have a good objective reason for refusing to conclude a contract. The DH network operator as a monopolist could be obliged to allow the feed-in of third parties, since according to the antitrust act ( $\$ 5 \mathrm{Abs} .1 \mathrm{KartG} 2005$ ) the abuse of a dominant position is prohibited.

However, it must be taken into account that this paragraph ( $\$ 5$ para. 1 it.1 KartG 2005) of the antitrust act can only be applied if someone wants to act as a competitor to the DH network operator on the upstream or downstream market. That means, i.e. if the third party heat generator seeks to supply other consumers with its generated heat. However, due to the lack of other options to feed-in and transport the heat, the use of the existing DH network is necessary to act as competitor on the market. In any case, the heat generator is an actor in the upstream market and thus a market participant. The DH network operator is to be regarded as a dominant company with regard to the integration of heat sources, since the potential heat generator that wants to feed-in usually has no other possibility to sell his heat. 
In principle, there is no obligation to contract even for dominant companies. In spite of that, under certain circumstances, a monopolist may be required to open its facilities to competitors. Here it is to be examined whether the feed-in of the DH represents a business operation, which is usually accessible to other enterprises. An already open market is required. When examining an open market, it can be determined whether the company has already taken heat from other third parties or may even do so on a regular basis. In that case, a dominant position acc. to the antitrust act ( $\$ 5 \mathrm{KartG}$ ) would be given.

However, the antitrust act ( $\$ 5$ para. $1 \mathrm{KartG}$ ) prohibits the abuse of the dominant position. A company acts abusively when it refuses access to products or services that are indispensable and that are in demand. Acc, to the antitrust act, dominant position is abused if there is no justification for refusal of access. In any case, the refusal of access is abusive only if the abusive behavior leads to excluding any competition in the relevant product market without justification. For analysing possible justification for the refusal of access the different interests of the parties must be considered.The DH network operator is only obliged to open its network to other market participants if that is technically possible. He does not have to promote another competitor to his own disadvantage. [3] In addition, technical or economic reasons regarding the impossibility or unreasonableness of the feed-in of third parties are to be investigated individually.

\section{Impossibility of feeding-in}

The technical possibilities are considered differently in the literature. One part of the literature [3] considers the connection of heat generators to an existing DH network as technically possible if the necessary financial effort is done. Different feed-in temperatures as well as missing capacities do not represent a reason for a technical impossibility. The network operator is to be expected to reduce its own use or to raise capacity by efficiency increase.

Another part of the literature $[4 ; 5]$ sees the technical impossibility as given when the heat generator wants to feed in a pressure, temperature or aggregate state, which does not correspond to the condition of the conduit pipe of the DH network. It may also be "impossible" if the access to the DH networks is not technically possible at the desired local site. In terms of lack of capacity, there is a technical impossibility if all objectively available capacity has already been allocated to third parties in order to supply their own customers and if capacity cannot easily be expanded. [5] In the DH sector an increase in efficiency is not possible simply by temperature monitoring or anything similar. Usually network extension is necessary, which often fails due to lack of space or high investment costs. [4]

Due to the strong necessary conjunction of the heat generation and the DH network, technical impossibility for the operator may also be given if the third party heat cannot go along with the heat already in the network - because of different pressure, temperature or physical state. Impossibility is given if this obstacle cannot be overcome with an economically feasible effort. [4] Geographical limitations and the lack of space for a further expansion of the DH pipes also lead to technical impossibility. However, this decision on the technical possibility must be considered individually for each DH network and must be decided on a case-by-case basis.

With the necessary financial effort, a technical disability can be solved in many cases and then a lack of technical possibility is difficult to be argued. In any case, the effort that is necessary for the implementation of technical solutions to grant access must be assessed. This effort is to be included in the economic possibility and subsequently the reasonableness of these changes for the DH network operator are to be evaluated [4].

\section{Unreasonableness of the feed-in}

It should also be taken into account that the DH network operator, who also acts as a supplier for his own customers, must secure his long-term relationship and for that very reason has already created the corresponding generation capacities himself. Due to the closed heat cycle, the additional heat would mean that the own generation of the DH network operator would have to be throttled in order to balance out the total quantity. Other conceivable reasons of unreasonableness would be, for example, the amortization interest [3] (elimination of customers limited calculated revenues, endangering the 
profitability of the supply), a possible threat to the supply of the own customers through the opening of the DH network or even ecological reasons. [2]

\section{Conclusions with regard to heat feed-in:}

Due to frequently lacking technical and economic reasonability, a claim of the heat generator according to the antitrust act ( $\$ 5$ para $1 \mathrm{KartG}$ ) on the feed-in of generated heat (for a fee) into an existing DH network is likely to fail due to existing justification reasons.

Since there is no legal claim in the heat sector to access the network, private-contractual agreements between the heat generator and the DH network operator are possible. Those agreements regulate the purchase of heat generated by third parties if this is technically feasible (among others with regard to pressure and temperature). However, due to missing regulation, the DH network operator does not have to get involved in this. Because of the policy of freedom of contract, the DH network operator can decide on its own from whom he buys heat and under what conditions he does so.

\section{Elaboration of option $1 \mathrm{~b}$,heat transit“"}

Scenario (1b) was also analysed within existing legislation. The heat generator aims to enter the DH market in order to pass generated heat to a specific customer via the existing DH network of the operator. For this network usage the generator would pay a usage fee.

Again, it has to be examined whether or under which conditions the refusal of network access by the heat network operator is abusive according to the antrust act. Objectively justified reasons for refusal of the monopolist must again be observed.

As the heat generator seeks to supply other consumers with its generated heat, he intends to act as a competitor to the DH network operator in the downstream market. For this purpose, the use of the existing DH network may be required, especially as it is inefficient to duplicate the existing DH network by the establishment of a parallel infrastructure [4]. Due to the fact that heat transportation is only possible within a heat network, another distribution possibility is not presentable. [5] As far as the third party heat generator does not want to substitute the entire DH network, the existing DH network is to be classified as essential and thus the only way for the heat generator to be able to supply its customers. Therefore, the third party heat generator may be dependent on a claim for transit.

Thus, a refusal of the DH network operator leads to the exclusion of competition in the market. However, the DH network operator may not immediately be forced to conclude a feed in contract desired by a third party. Instead, he may refuse to conclude a contract for objectively justified reasons. It is therefore necessary to examine whether or under which conditions the refusal of network access by the operator is legitimated or abusive.

While the reasons for impossibility do not deviate significantly from scenario $1 \mathrm{a}$, the reasons for the unreasonableness of the DH network access may be more specific to the operator's own business interests.

For the DH network operator, the high entrepreneurial risks as well as the high investment costs in the local DH network have to be taken into account. This justifies an interest of the DH network operator in the amortization, which requires long contract periods as well as reliable pricing.[5]

Such long-term contracts, which may use all available DH network capacity, will help the operator to maintain its dominant position, but also to create incentives to invest in the service. It is not reasonable for the DH network operator to terminate these long-term contracts in order to free capacities needed by the competitor. Thus, only the amount of remaining capacity in the DH network is free for third party requests. However, the long-term supply contracts protect the operator's interests only with existing customers, but not with new customers or with expired contractual relationships. Due to the strong connection between heat generation and the DH network, it is also unreasonable for the DH network operator to throttle its own generating plants for the purpose of "heat transit" by third parties. It is also not reasonable for the operator having the sudden need to buy heat from elsewhere because of an unexpected missing or reduced heat feed-in by the third party (e.g. production downtime). The reason that the operator would incur massive customer losses as a result of a new entrant is not an objectively justified reason for excluding the feed-in request, as this would be contrary to the very purpose of the law. [6] In addition, due to further feed-in from third parties, efficient DH network 
control and system operation could be required - that would also be a justified reason for the refusal of network access.

Heat generation systems, which rely on weather circumstances, cannot produce regularly and thus cannot cover the entire heat demand of the customer. This would mean that the DH network operator or another third party would need to provide backup, meaning that the operator has another "additional" DH network user. However, it is not reasonable for the DH network operator to reserve capacity for third parties or to buy missing heat. At this point, it can be assumed that it is not per se economically feasible for the DH network operator to provide reserve capacity.

\section{Conclusions with regard to heat transit:}

Although it can be assumed that the resident DH network operator holds a dominant position, he does comply with the requirements of the law. The antitrust act ( $\$ 5$ KartG 2005) is complied, if a transit is not possible or not reasonable. This could be due to a technical reason, but also some economic reasons are eligible.

In individual cases, if there are no technical or economic justifications, there may be a right to transit the heat from a third party heat generator.

Thus, for the purpose of passing the heat through the existing DH network by a third party generator, there is only the possibility of implementation by private-law agreements. The DH network operator and the third party heat generator can make private-law agreements for feeding the heat into the local DH network. However, since the DH network was created through investment, it is up to the operator's private-autonomous decision whether and with whom he concludes such contracts.

\section{FICTIOUS OBLIGATION TO ACCEPT A FEED-IN DEMAND}

Scenario (2) is a fictive scenario, i.e. it is not based on current jurisdiction but on a hypothetical act.

\section{Scenario description}

In this scenario, basically, the situation is the same for a private contract: Third party generators and DH network operators can agree on the feed-in or transit of heat at any time, free of any specific legal requirements. But: When a potential third party heat generator seeks to set up a feed-in contract, he appeals to the DH network operator for contract negotiations. If the operator does not agree to the feed-in request of the third party generator, in this scenario a legal process is started..

First, the act schedules binding contract negotiations. All technical and economic aspects (quantity, profile, temperatures, location of the feed-in, price of heat, backup capacities, securities, seasonality, etc.) are to be discussed. This requirement aims to reach an agreement on the access with the requesting actor on mutually accepted terms.

Second, if no agreement is reached, the DH network operator must grant the (still) requesting third party generator a so-called regulated access. Feed-in is required to take place at network temperature. The operator must offer a draft contract, in which the next possible location of the feed-in as well as further technical requirements are listed.

In this scenario, network connection costs are borne by the heat generator. The DH network operator is obliged to pay the costs not incurred (i.e. primary energy not used in the generation plants) as feed-in tariff to the generator. Accordingly, in this scenario the DH network operator is obliged to grant access and allow feed-in but has to do so in a cost-neutral manner.

\section{Elaborations}

Scenario (2) is based on the assumption that the DH operator is obliged to accept DH from third party generators. DH network connection costs are borne by the heat generator. The DH operator only pays a feed-in tariff in relation to the amount he saves himself at own expense. However that may be, the operator is rising at neutral cost.

The operator would be obliged to purchase third party heat within this scenario. A critical aspect is that DH networks have limited capacities. In the electricity and gas sector, statutory provisions on the network expansion obligation are within the scope of economic reasonableness. In the DH sector, there is no statutory provision on the network expansion obligation. A statutory provision would be 
required if the operator had to purchase externally generated heat. [4] A purchase obligation on the operator would thus also indicate an expansion obligation.

When implementing this scenario, it would be necessary to specify exactly how the heat is to be fed in. It requires precise specifications of the DH network operator (individually for each DH network) regarding the amount of heat to be fed in, the location, the timing and the nature of the heat (pressure, temperature, etc.). In addition, it would have to be determined by law how to deal with the abovementioned objectively justified reasons. These would, as already described, be present in many cases and thus preclude the feed-in obligation.

Also, a supervisory authority would have to be provided for disputes, supervisory functions or establishment of framework conditions by law.

In addition, there are some open economic issues that cannot be fully addressed here. Since the heat generators have to pay the investment costs for the network connection themselves, it is an open question whose amortization interest should be satisfied first, if there are several requests for feeding in: Who gets network access first, if the capacities are already exhausted or the expansion is not yet completed? How to do if another heat generator could feed in cheaper, but the capacities are exhausted?

\section{Conclusions with regard to an obligation to grant access:}

Due to the conceptual unity of heat generation and the DH network, the local boundaries and closed nature of the DH network, there is no interconnected system such as electricity and gas. Therefore, it is questionable whether a purchase obligation for the DH network operator is technically possible. In any case, some legal rules have to be adopted when implementing the concept:

- Acceptance obligation for the DH network operator

- Determination of a cost-neutral feed-in tariff

- Obligation to expand the DH network, if required (on the third party's costs)

- Clear (individual) specifications for each DH network operator regarding the status, quantity, time, location, etc. of the heat to be fed in

- Establishment of a kind of supervisory authority.

However, the technical characteristics of DH networks make it difficult to issue a network access right for "all heat generators" due to the limited network capacity and the local limitation of the networks. A law that regulates DH network access and an acceptance obligation of the DH network operator would have to be either very complex or very unspecific due to the particularities of the individual regional DH networks.

\section{ECONOMIC ANALYSIS}

In the current situation, the DH network operator is usually the only DH supplier with significant reginal customer access. At first sight, the strong bargaining position and the status of the operator as the sole customer indicate a monopsony concerning feed-in. However, we find that the monopsony approach derived from economic theory and its recommended regulatory approaches are not correct. Rather, it is a bilateral basis for negotiation, which is also gaining more and more attention in the competitive economy: Inderst and Wey show that a private-sector solution is reached if the situation brings a positive net present value for the involved players. Thus, the realization of the feed-in of industrial waste heat into the existing DH network has to result in a positive present value for the two actors (this includes the possibility of one actor compensating the other). [7]

In contrast to electricity networks, for example, which have to fulfil certain technical conditions (voltage, frequency), DH networks differ from network to network - but there are also differences within the network in terms of pressure, temperatures, capacities, etc. Thus, there need to be negotiation about different, interrelated parameters. Negotiating the determination of one parameter automatically influences the others. This often leads to a high complexity, resulting in frustration and perplexity of the negotiators and, finally, in a stop of negotiations. However, at the same time, a bilateral agreement gives contractors maximum flexibility in determining the technical and financial parameters of the feed-in. In order to decrease complexity, it is recommended to develop guidelines to 
structure goal-oriented negotiations. An essential part of the guidelines is the implementation of a cost-benefit analysis. [1]

Due to the high complexity of feed-in negotiations, it is shown that the benchmark bilateral contract model is to be preferred as it allows a flexible adoption to the circumstances of the local network. Based on the economic analysis, we also find that an optimal regulation of feed-in would lead to the same result as are to be expected on the free market. However, often personal or political barriers prevent the implementation of "rational" economic measures. Regulation may help to overcome personal or political hindrances. In that case of the "accept a feed-in" regulation, the waste heat producing industry has the right to feed in, but must ensure economic feasibility of the project on its own. The costs saved by the heating network operator (primary energy costs) have to be forwarded as revenues to the waste heat provider. Then, the access is cost-neutral for the heat network operator and its final customers, while the investment pays back for the industry. [1]

\section{CONCLUSION}

The concept of a "private contract", which reflects the current legal situation in both explained scenarios (1a) and (1b), is to be regarded as an appropriate choice, since it requires a longer-term and clear agreement in order to ensure investment security. The private contract creates acceptance, being a prerequisite for a positive and longer-term cooperation. A private agreement gives contractors maximum flexibility in financing and determining the technical parameters of the feed-in. An obligation to accept feed-in demands may have positive effects on the integration of waste heat. However, the technical conditions of existing DH networks often make mandatory network access difficult. That is why such an obligation would have to be either very complex or very unspecific due to the particularities of the individual regional DH networks. Thus, the implementation of a regulation which includes the obligation of the network operator to accept a feed-in can therefore not be clearly recommended.

\section{REFERENCES}

1 Energieinstitut an der JKU Linz. Open Heating Grid. Offene Wärmenetze in urbanen Hybridsystemen, abzurufen unter. https://nachhaltigwirtschaften.at/de/sdz/projekte/open-heatgrid-offene-waermenetze-in-urbanen-hybridsystemen.php\#publications (26 June 2018, date last accessed).

2 Schett G. Europäisches Klimaschutzrecht und Erneuerbare Energien. RdU;2014:215-59.

3 Säcker FJ, Wolf $M$. Wettbewerbsrechtliche Bindung der Fernwärmenetzbetreiber. $R d E ; 2011: 277-86$.

4 Greb K, Böcker L. Wettbewerbliche Öffnung der letzten Bastionen? Netzzugangsansprüche und Regulierungsdiskussion in Fernwärme und Wassersektor. RdE;2013:15-21.

5 Körber T. Die Fernwärmenetze zwischen Wettbewerbs- und Klimaschutz. RdE;2012:372-82.

6 Bundeskartellamt. Sektoruntersuchung Fernwärme. Abschlussbericht gem. § 32e GWB, 2012. https://www.bundeskartellamt.de/SharedDocs/Publikation/DE/Sektoruntersuchungen/Sektorunter suchung\%20Fernwaerme\%20-\%20Abschlussbericht.html?nn=3591568 (26 June 2018, date last accessed).

7 Inderst R, Wey C. Die Wettbewerbsanalyse von Nachfragemacht aus verhandlungstheoretischer Sicht. Korrigierte Fassung. https://www.diw.de/documents/publikationen/73/72132/rn25.pdf. 


\title{
METHOD FOR INTEGRATED STRATEGIC HEATING AND COOLING PLANNING ON REGIONAL LEVEL - THE CASE OF BRASOV
}

\author{
Richard Büchele, Lukas Kranzl, Marcus Hummel \\ Institute of Energy Systems and Electrical Drives \\ Gusshausstr. 25-29/370-3, 1040 Wien, Austria \\ Phone: +43-1-58801/370 368 \\ E-Mail: buechele@eeg.tuwien.ac.at
}

\section{SUMMARY}

In this work a method for integrated strategic heating and cooling planning on regional level is presented which is applied for the case study city of Brasov. The overall methodology comprises the calculation of the cost-optimal combination of heat savings with either district heating or individual supply technologies for different building groups located in different areas according to the availability of a current district heating network and the calculation of different indicators like total system costs, total $\mathrm{CO} 2$ emissions, share of renewables etc. for different scenarios and for different interest rates and energy tax assumptions to analyse the economic efficiency as well as the $\mathrm{CO} 2$ reduction potentials of various options to save heat and supply heat in the buildings. The results of the assessment show that at least a certain amount of heat savings is cheaper than all assessed heat supply options for all building groups but that renewable supply options are not the most economical alternatives per se in the assessed case study under current conditions.

\section{INTRODUCTION}

Decarbonising the heating sector is essential to reach the climate goals agreed on 2015 United Nations Climate Change Conference held in Paris (COP21). Because heating and cooling cannot be transported over too long distances its issues mainly appear on local and regional level. In former times there was no planning effort given to heating and cooling supply and the sector developed according to pure economics, availability and outdated technology preferences without taking into account climate targets and long-term issues. But to exploit the decarbonisation potential of the heating and cooling sector, integrated methods are needed on how to perform strategic heating and cooling planning on local and regional level. This planning process should include long term targets and the assessment of different heat saving and heat supply options accompanied by intensive and target-group oriented information campaigns and involvement of all relevant stakeholders in order to ensure the achievement of the desired objectives. For example, district heating (DH) in general is seen as an important technology to decarbonise the heating sector especially in urban areas but especially this technology needs an integrated planning approach to include future development of heat demand into the assessment and to ensure a sufficient heat density with enough customers making DH an economic effective solution. Therefore in this paper, the method and the results of an integrated strategic heating and cooling planning process performed for the case of Brasov is shown.

\section{METHOD}

The integrated strategic heating and cooling planning process presented in this paper was developed within the Horizon 2020 project progRESsHEAT and then the methodology was adapted to the case study municipality of Brasov, located in the centre of Romania.

The overall methodology comprises the calculation of the cost-optimal combination of heat savings with either district heating or individual supply technologies for different building groups located in different areas according to the availability of a current district heating network and included following steps: 
(1) Calculation of costs and potentials for heat savings for ten different building types with three different construction periods with the Invert/EE-Lab model (Müller, 2015). (2) Calculation of costs for heat supply with five different individual heating technologies for the before mentioned buildings. (3) Modelling of the existing district heating system and possible alternative supply portfolios for the future of the district heating system in energyPRO (EMD International, n.d.) to obtain the district heating generation costs and the sensitivity of the costs to disconnection or to additional costumers. (4) GIS based analysis to divide the municipality into four different types of areas according to the availability of a current district heating network or the feasibility and costs of expanding the network into adjacent areas. (5) For all building groups and all areas within the municipality the cheapest combination of heat saving level and the supply with district heating or individual technologies is calculated. This is done for a reference scenario and for a technical alternative scenario depicting a desirable future regarding the heat supply portfolio of the district heating system and for different interest rates and energy tax assumptions. Indicators like total system costs, total $\mathrm{CO} 2$ emissions, share of renewables etc. are calculated both for the reference and for the alternative scenario to analyse the economic efficiency as well as the $\mathrm{CO} 2$ reduction potentials of various options to save heat and supply heat in the buildings. All these steps are carried out along with a wide stakeholder integration process where all the strategic issues and long term targets are discussed to find realistic assumptions and coherent scenarios for the whole heating and cooling planning process.

\section{RESULTS}

The results of the assessment show that at least a certain amount of heat savings, if performed when maintenance work is needed anyhow, is cheaper than all assessed heat supply options for all building categories also in absence of energy taxes and with low interest rates. This applies especially for an old building stock but also for newer buildings with construction period between 1995 and 2008.

In course of the heat supply options chosen in combination with the most economic heat saving the situation looks different for the future scenario: When not taking into account energy taxes and assuming depreciation times in the range of the lifetime of the supply systems, the cheapest combination with heat savings are natural gas boiler followed by air source heat pumps (assuming annual COP of 2.8). When taking into account energy taxes and shorter depreciation times, biomass boilers are the cheapest supply option in combination with heat savings followed by air-source heat pumps. In densely populated areas with limited potential for individual biomass boiler and air source heat pumps again individual natural gas boiler are the cheapest option. Nevertheless, all assessed supply technologies have heat generation costs close to each other and their economic feasibility depends on assumed taxes, lifetimes and other framework conditions. Especially the economic efficiency of district heating highly depends on the achieved connection of customers within the network area. This shows the importance of integrated strategic heating and cooling planning to evaluate the needed framework conditions facilitating the implementation of cost optimal combination of heat savings with renewable and low carbon heating technologies.

\section{REFERENCES}

EMD International, (n.d.), EnergyPRO modelling Tool, http://www.emd.dk/energypro

Müller, A., (2015), Energy Demand Assessment for Space Conditioning and Domestic Hot Water: A Case Study for the Austrian Building Stock, PhD Thesis, TU Wien, Vienna 2015. http://www.invert.at/

\section{CONFERENCE TOPIC}

Spatial energy planning with focus on renewable energies 


\section{DIGITAL APPROACH FOR SPATIAL ENERGY PLANNING - BEST PRACTICE IN SWITZERLAND}

Gabriel RUIZ

Navitas Consilium SA, Rue Marconi 19

1920 Martigny, Switzerland

Phone +4127 72219 62, Cell +4179506 2215

gabriel.ruiz@ncsa.ch

Political authorities worldwide are setting increasingly ambitious and binding energy-climate goals, with drastic CO2-reductions. The achievement of such objectives requires (i) to develop effective strategies, to plan the desirable development of their energy consumptions and productions, and (ii) to follow the energy evolution and to measure the effect of the implementation of these strategies on their territory.

To serve the first requirement, the regional energy planning tool PlanETer was developed by the CREM (Centre de Recherches Energétiques et Municipales located in Martigny, $\mathrm{CH}$ ) and today applied by Navitas Consilium to achieve territorial energy planning for more than 40 municipalities in Switzerland. The approach is to consider the city as a set of multiple and different consumers. Calculation of heat and electrical consumptions and needs of each territorial element such as building is made through the use of several data bases. The potential of local renewable energy resources is determined based on resources availability, regulations and technological models. Geo-referencing all of these data provides a multi-scale vision of the energy situation. Furthermore, the simulation of several future scenarios allows to represent and to analyse the local energy situation with different temporalities [1].

To address the need of following the territory's evolution and to help deciding on corrective measures towards the set objectives, the energyCity platform was developed. Based on a development involving cities, multi-energy utilities and academic partners, the energyCity platform offers Visual Energy Analytics for an Efficient Digital Energy Transition. Developed by Navitas Consilium and enersis, two swiss start-up companies, the platform is inspired by the MEU research project [2]. Today, energyCity is a professional territorial energy management tool which uniquely details a city's energy picture and allows visualizing its evolution by measuring the impact of the realized actions. energyCity encompasses all aspects of the energy complexity of the city through a detailed and flexible bottom-up data model. Measured consumptions are primarily used but the tool also provides estimations in case of missing information, in a data sustainability approach which includes data quality and tracing management. energyCity gives access to detailed energy balances and multiple indicators based on user-created scenarios.

energyCity also foresees to offer a decision-making support by creating scenarios on "how to bring renewable energy at the right place and at the right moment". Local authorities and utilities can thus rely on a tool to engage in a dialogue with all stakeholders. Fostering the dialogue among all city stakeholders represents a unique shared foundation upon which a sustainable "Smart Energy City" can be built. Thanks to energyCity, the energy transition can be assessed, monitored, and sustainably achieved.

[1] G. Cherix, M. Cappezzali, J. Rager, "Territorial energy systems: A methodological approach and case study", Proceedings of the 10th Conference on Sustainable Development of Energy, Water and Environment Systems, SDEWES2015. SDEWES2015.0700, 1-20 (2014).

[2] P. Puerto, M. Pernet, M. Capezzali, L. Darmayan and G. Cherix, "The MEU web platform: A tool dedicated to urban energy management”, CISBAT 2015, Lausanne Switzerland, 2015. 


\section{HOW COMBINED SPATIAL ENERGY PLANNING, SIMULATION AND STAKEHOLDER INTEGRATION LEAD TO SUSTAINABLE DISTRICT HEATING SYSTEMS}

Ingo Leusbrock, Harald Schrammel, Franz Mauthner, Keith O’Donovan, Basak Falay

AEE - Institute for Sustainable Technologies

A-8200 Gleisdorf, Feldgasse 19, Austria

phone: +43 (0)3112 5886-261

email: i.leusbrock@aee.at

\section{SUMMARY}

Energy utility companies are more and more favoring local renewable energy sources (e.g., solar thermal, waste heat) and flexibility measures such as heat pumps and central / decentral storage to actively achieve climate and sustainability targets. District heating systems are considered in this regard as cornerstone for our future energy systems and sustainable heat supply, especially in urban areas. Their further development requires long-term implementation strategies considering mutually social, technical and economic aspects. To highlight latest advancements in spatial energy planning, simulation and stakeholder integration we will present the results of the latest efforts to modernize and extend the district heating system of Gleisdorf.

\section{INTRODUCTION}

Energy utility companies are more and more favoring local renewable energy sources (e.g., solar thermal, waste heat) and flexibility measures such as heat pumps and central / decentral storage to actively achieve climate and sustainability targets. This process requires long-term implementation strategies considering mutually social, technical and economic aspects. From a (spatial) energy planning perspective, long-term strategies for renewable district heating extensions need to be developed and implemented developed in the framework of other ongoing research projects. Here, recommendations for local stakeholders (local government, city and landscape planning departments, energy utilities, etc.) need to be derived enabling long-term infrastructural changes in the community based on combined technical simulation and spatial planning knowhow. From a simulation and control point of view, the increased number of central / decentral supply and storage units and an increased amount of available monitoring lead to new options in conceptualization and operation, yet also pose a significant challenge due to increased complexity and data volume.

\section{RESEARCH OBJECTIVE}

To highlight latest advancements in combined spatial energy planning, simulation and stakeholder integration we will present the results of the latest efforts to modernize and extend the district heating system of Gleisdorf.

We will discuss the following aspects using this case:

- Establishment of energy databases for spatial energy planning

- Integration of spatial energy planning methods and results in DH extension planning

- Evaluation and optimization of the unit commitment of existing and future supply units and central / decentral storage

- Analysis and optimization of the thermal merit order of the conversion units including storage management

- Assessment of current and future district heating operation characteristics

- Optimization of overall district heating system performance by means of implementation of advanced control strategies and adapted system hydraulics 


\section{BACKGROUND}

The local multi-utility company Stadtwerke Gleisdorf $\mathrm{GmbH}$ has strong ambitions towards implementing a renewable district heating system as a substitute for local natural gas and other fossil based heating systems. Following this premises, the utility company started in 2009 with the construction of a biomass (two $850 \mathrm{kWth}$ biomass boilers) and solar thermal (300 $\mathrm{m}^{2}$ gross collector area, $191 \mathrm{kWp}$,th) based district heating system although a natural gas infrastructure was already established in large parts of the city. In the course of ongoing

extensions, several larger consumers have been hydraulically connected, which were initially supplied by natural gas boilers via micro-grids. Moreover, further feed-in points for distributed solar thermal systems have been identified to increase the overall share of renewables. In 2015, the total district heating system had a length of $5.1 \mathrm{~km}$ supplying 70 consumers with a load of $5.2 \mathrm{MWth}$ and a yearly consumption of 5,750 MWh (heat losses: 1,630 MWh, 22 \%) were connected. Due to the hydraulic set-up and current operation strategy, natural gas boilers were used for base and peak load supply leading to around $30 \%$ of the final energy demand being supplied by natural while the remainder was supplied by biomass and solar thermal (68\% biomass / $2 \%$ solar thermal).

\section{METHOD AND RESULTS}

The status quo of the district heating system in 2015 as well as future scenarios were analyzed and optimized applying (spatial) analysis of monitoring and simulation results (QGIS), energy modeling (EnergyPLAN) and thermo-hydraulic simulations (simplex, STANET, Dymola). Different measures to increase both energy efficiency and the share of renewables were identified, investigated and partially already implemented:

- Reduction of thermal losses in the distribution network by implementation of a weatherdepended supply temperature control

- Adaptation / optimization of hydraulic set-up and control strategy of distributed boilers and pumps to enable base load district heating supply via biomass boilers and solar thermal and

- Installation of a roof-mounted solar thermal collector field with a gross collector area of 496 $\mathrm{m}^{2}(316 \mathrm{kWp}$, th) and impact-analysis for another $250 \mathrm{kWth}$ base load pellets boiler

- Assessment of customer substations and identification of critical operating conditions (above average supply and / or return temperatures)

We could show by simulation that the overall share of renewables in the system can be increased from $70 \%$ to $85 \%$ by optimized hydraulic integration of the wood-chip boilers (already implemented) and even increased up to $92 \%$ if additional renewables are integrated (solar thermal system already realized, pellets boiler pending). A total cost assessment based on real cost data from the utility company shows that levelized costs of heat may be

decreased by 4-6\%. A major outcome and success story of the project is linked to improvements to the existing control system. A virtual controller based-on real-time district heating simulations was developed and programmatically linked to the existing control system via a standardized interface protocol. The virtual controller is already implemented and currently tested in actual operation.

\section{OUTLOOK}

Further improvement potential has been identified for several customer substations (mainly high return temperatures) and is going to be further analyzed. Consequently, higher solar thermal yields are expected if network temperatures are being decreased and thermal storage management further improved. Moreover, from a spatial energy planning perspective long-term strategies for renewable district heating extensions are developed in the framework of other ongoing research projects. Here, recommendations for local stakeholders (local government, city and landscape planning departments, energy utilities, etc.) are derived enabling long-term infrastructural changes in the community based on combined technical simulation and spatial planning knowhow. These recommendations are jointly developed with the local stakeholders in Gleisdorf and are part of the current efforts for a new spatial development and infrastructure plan for Gleisdorf. 


\title{
Grid based energy system setup optimisation with Rivus in dedicated regions
}

\author{
$\underline{\text { Fabian Hofsäß }}^{1}$, Maurizio Santin ${ }^{2}$, Massimiliano Condotta $^{3}$, Markus Biberacher $^{1}$, Damiana Chinese ${ }^{2}$, Giovanni \\ Borga $^{3}$ \\ ${ }^{1}$ Research Studio iSPACE \\ Schillerstraße 25, 5020 Salzburg, Austria \\ Phone: +43662908585222 \\ E-Mail: fabian.hofsaess @ researchstudio.at \\ ${ }^{2}$ DPIA, Università di Udine \\ Via delle Scienze, 206, 33100 Udine \\ Phone: +390432558024 \\ E-Mail: damiana.chinese@uniud.it \\ ${ }^{3}$ Università Iuav di Venezia \\ Santa croce Dorsoduro 2196, Cotonificio veneziano,30123 Venezia, Italy \\ Phone: +390412571271 \\ E-Mail: condotta@iuav.it
}

\section{SUMMARY}

Within the project IDEE (Integrated Design Efficient Energy systems in urban regions) the expertise of four cross-border (Italia \& Austria) research centres and one public authority is bundled up to support the planning of new setups or the extension of existing setups in grid based sustainable energy systems for pilot regions inside the project areas. A special focus within the project is the optimization of network topologies in district heating setups. First scenarios on possible system setups for the pilot regions have been calculated outlining the topology of optimal pipe setups as well as the load of (Heat-) pipes at different time steps with the objective to minimise overall system costs.

Keywords: District heating, Optimization, Network calculation, MILP

\section{INTRODUCTION}

The heating systems of residential and commercial buildings in urban areas are mainly supplied from grid based energy systems: natural gas grids typically feed boilers, power grids may feed different types of heat pumps and resistance heating, and district energy systems, directly supply users with heat carriers. District heating systems, in particular, are generally recognized (Lund et al., 2016, Hast et al., 2018) to be an essential part of decarbonisation and renewable energy strategies, at least in continental Europe (Finney et al., 2012).

However:

- the market penetration of different grids is different in cities, in urban and rural areas (Soltero and Chartegui, 2018), as well as across Europe (Werner, 2017), particularly on the two sides of the Alps;

- it is recognized (Köfinger et al., 2016, Lund, 2017) that, with decreasing heating demand of building stock, district heating networks need to evolve into so called $4^{\text {th }}$ generation district energy, i.e. into smart and low temperature systems, to remain competitive with other technology options.

In this context, the INTERREG project IDEE aims to develop integrative planning tools, which allow the quantification of complex correlations appearing in grid based systems. In particular, the project objective is to identify least costs systems for achieving emission reduction and energy efficiency improvements in Austrian and Italian program areas and to the dedicated pilot regions Maniago, Feltre and Salzburger Seenland.

Optimization based on mixed integer linear programming (MILP) has been recognized as the most widely applicable approach to support district heating systems planning (Sameti and Haghighat, 2017). In the last decade, GIS have also been increasingly used for managing all data relevant for district 
energy, such as heating demand (Finney et al., 2012) and costs of connecting different urban areas with different grids (Möller and Lund, 2010, Nielsen and Möller, 2013).

Integrating GIS and MILP has thus emerged as a state of the art methodology for grid based energy systems planning; however, only a few recent models are reported in literature.

Delmastro et al. (2016) integrate GIS and optimization by using a modified version of the Steiner algorithm, typically used for power grids, in order to produce optimized layouts for district heating grids taking into account demand as a quali-quantitative importance factor.

Unternährer et al. (2017) focus on large urban areas with a high number of buildings and propose a combination of preliminary clustering algorithms and minimum spanning trees.

While previous models mainly aim at network layout optimization, the model RIVUS, developed by Dorfner (2016) adds the sizing of energy conversion processes, and thus allows a more comprehensive evaluation of alternative urban energy systems. Moreover, unlike previous models, RIVUS is an open source model, implemented in Python, and it enables simultaneous modelling of different energy grids (gas, power and district energy).

For these reasons, RIVUS was chosen as a starting point for the development of IDEE methodological framework and its application in the project pilot areas.

\section{METHODOLOGY}

Preliminary panels and discussions with stakeholder of pilot areas highlighted a strong interest in district heating which is perceived as a means to promote energy and economic savings and, most evidently, to reduce local air pollution. Interest for $\mathrm{CO} 2 \mathrm{eq}$ emission reduction has been mainly expressed in Austrian pilot regions, which belong to the Climate and Energy Model Regions of the Klimafonds program. Besides economic information, decision makers thus mainly require information on air emissions. However, a literature review performed at the beginning of the project (Chinese et al., 2018) has highlighted that in district energy planning models greenhouse gas emissions are commonly taken as a proxy for overall environmental impact. Just a few studies take other air pollutants (Genon et al., 2009, Fang et al., 2013, Li et al., 2016) and environmental impact categories into account (Oliver-Sola et al., 2009, Ghafghazi et al., 2011, Bartolozzi et al., 2017): such studies show that sometimes trade-offs arise between reducing global emissions, i.e. greenhouse gases, and local emissions, such as NOx, SOx and particulate matter. RIVUS structure is suitable to evaluate several energy and material flows (commodities), up to now, however, only calculations of CO2eq has been reported for some German case studies (Dorfner, 2016).

\section{RIVUS MODEL IMPLEMENTATION FOR ECONOMIC AND ENVIRONMENTAL ANALYSIS}

RIVUS has the objective to identify minimum cost scenarios for grid based heating infrastructures through Mixed-Integer-Linear-Programming (MILP). The cost function is build up out of investment, fix und variable costs. Costs have to be paid for the network grid, for the supplied energy and for technical processes.

$\sum \cos t=\sum i n v+\sum f i x+\sum v a r$

There are two types of technical processes, domestic (hub) and central processes. Each one has a specific investment cost $(€ / \mathrm{kW})$, but only the central process has an additional fixed investment cost $(€)$, which accounts for size-independent investment costs. The network grid has fixed investment costs $(€ / \mathrm{m})$ and variable investment costs $(€ / \mathrm{kW} / \mathrm{m})$. Each purchase energy unit is also part of the cost function $(€ / \mathrm{kWh})$, and additional specific fixed costs $(€ / \mathrm{kW})$ and variable costs $(€ / \mathrm{kWh})$ can be introduced to account for further operational expenses such as maintenance. 
In RIVUS, the complete process chain with all relevant commodity transformation processes, related efficiencies and costs are considered. All estimated demands are either satisfied through a grid based supply system (e.g. district heating, natural gas or electricity) which is tied to the topology of the (advanced road) infrastructure or satisfied through an alternative predefined option. The status-quo of the existing grid based energy infrastructure is considered as starting point for any optimized system setup. The model identifies the least cost system setup which satisfies all geo-localized demands by either through a connection to the district heating grid or local supply options (etc. heat pumps).

The RIVUS model provide as output the optimal system setup as snapshot for one specific year; to opportunely weigh long term investment costs stretching over $n$ years with the interest rate $i$, the equivalent annual cost method is used, multiplying present investment costs by the annuity factor $A N F$ :

$A N F(n, i)=\frac{(1+i)^{n_{* i}}}{(1+i)^{n}-1}$

RIVUS separates the energy demand in different time scopes. For each declared time scope, the amount of hours and the fraction of the energy demand has to be declared. In the upcoming scenarios, the four original time scopes specified in RIVUS have been used for Salzburger Seenland; for northern Italy, the duration curves calculated in Chinese et al. (2018) have been used.

The outcome of the RIVUS is a cost optimal system setup for the energy supply. Related emissions are not part of the objective function but part of user set absolute constraints in the model. Emission values for $\mathrm{CO}_{2} \mathrm{eq}, \mathrm{NOx}, \mathrm{SO}_{2}$ and particulate (PP) in combustion processes have been incorporated into the model within the IDEE project. Emission factors related to net heat generation based on the IINAS database (2018) have been assumed. For local pollutants, such as $\mathrm{NOx}, \mathrm{SO}_{2}$ and particulates, only direct emissions were accounted for. Since emissions of greenhouse gases are global, a life cycle oriented approach was taken, providing also an assessment of life cycle $\mathrm{CO}_{2}$ emissions as reported in Chinese et al. (2018). Credits for avoided remote electricity production have been assigned, and subtracted from direct $\mathrm{CO} 2 \mathrm{eq}$ emissions, in case cogeneration systems are chosen as an energy source for municipal systems. Emission factors for the national energy mix of Italy and Austria were obtained from Chinese et al. (2017).

Centralized woodchip boilers and woodchip based ORC - CHP systems have been considered as sources for district energy systems. Heat recovery from nearby process industries was also considered for Italian case studies: in that case, it was assumed that no additional emissions occurred when connecting the system to district heating.

Based on local conditions, it was chosen to examine electric resistance heating as alternative to district heating in the Austrian case study, while natural gas heating has been taken as the alternative option for Italy.

Electricity domestic heating does not produce local pollution and only the $\mathrm{CO}_{2}$ equivalents of the Austrian energy mix are hence considered. For natural gas, direct emissions for boilers up to $1 \mathrm{MW}$ are evaluated. Indirect life cycle emissions were also evaluated for $\mathrm{CO} 2 \mathrm{eq}$, but bounds are always set to direct emissions only.

\section{GIS PREPROCESSING AND NETWORK GRID MODELLING}

For Austrian pilot regions, the location and capacity of existing power plants are derived from Open Government Data (SAGIS, 2018), for Italian regions information on existing power plants is obtained from the RSE Atlas (GSE atlas, 2018) and integrated with local information on available energy or waste heat sources.

All data are collected and administrated in a database, which is linked to the RIVUS model (Havasi, 2017). 


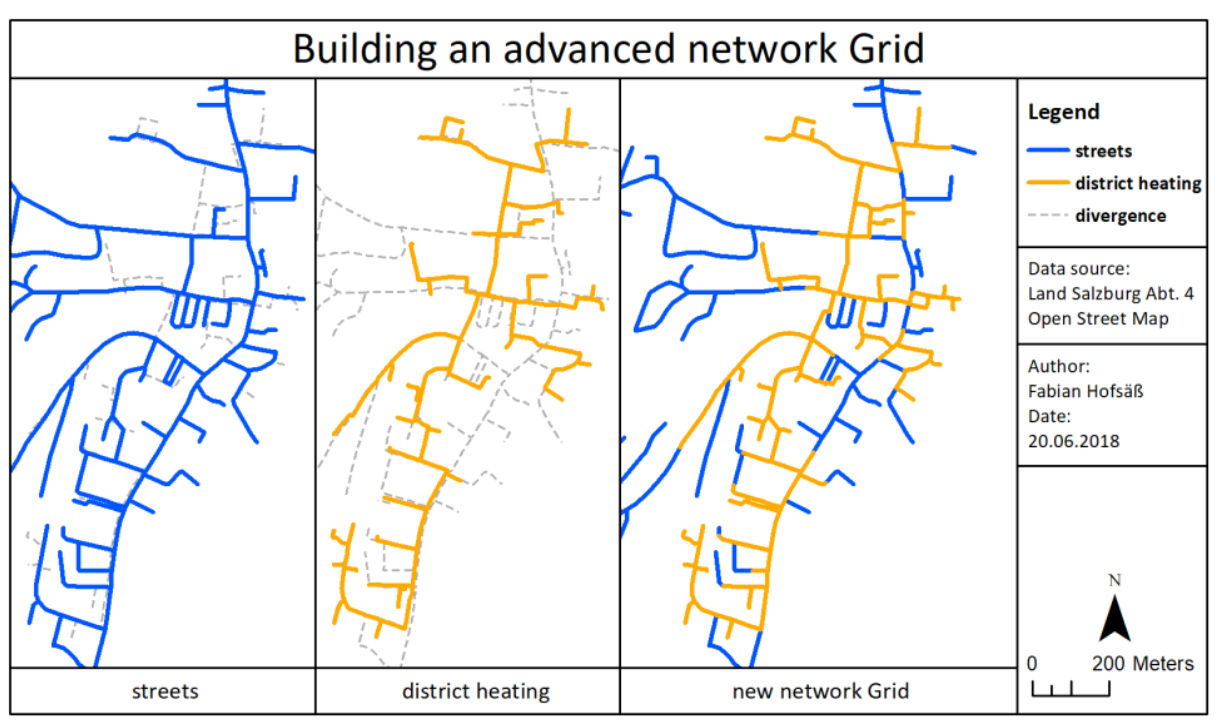

Figure 1"progress of fitting two networks together"
Open Street Map data have been proven an appropriate data source to serve as base layers for describing the building and infrastructure stock. The existing road network is considered as indicator dataset for future grid based supply infrastructure and is therefore the foundations of the grid based solution.

Embedding the current supply infrastructure like heat pipes to the existing road network has been made by a semi-automated progress. The status-quo of the existing district heating network for the Austrian project area has been provided by Land Salzburg. The selected heat pipes are snapped to the road network. Thus, an advanced road network with new major grid lines is built.

\section{ESTIMATING ENERGY DEMAND}

One of the key aspect in Designing Efficient Energy systems in urban regions is to estimate, with accuracy, the energy demand of building stock i.e. the energy flows to be delivered to each single building.

Generally, for the assessment of the annual energy use for space heating and cooling of a residential or non-residential building is used the European standard formula (European Committee for Standardization, 2008):

$\mathrm{QH}, \mathrm{nd}=(\mathrm{QH}, \mathrm{tr}+\mathrm{QH}, \mathrm{ve})-\eta \mathrm{H}, \mathrm{gn}(\mathrm{Qin}+\mathrm{Qsol})$

This method includes the calculation of the heat transfer by transmission and ventilation of the building and the contribution of internal and solar heat gains to the building heat balance. The result is the annual energy that is required by a building.

We decided to use this method to calculate the energy demand of each single building to be implemented in RIVUS. Of course, it is not possible to run a simulation for each and every building in a city. The solution is to apply a characteristic transmission loss, ventilation loss, internal gain and solar gain value to each edifice of the city, which depends on the building functional typology, dimension and technological features that are related to building age. The characteristic energy demand value of each single building is then calculated according to the formula (3) by multiplying a characteristic energy loss or energy gain (in $\mathrm{kWh} / \mathrm{m}^{2} \mathrm{y}$ ) by a geometric parameter which is specific for the building (in $\mathrm{m}^{2}$ ). It is done for each individual building in considered area with regard to the specific geometry of each single building.

The characteristic energy loss or energy gain values (in $\mathrm{kWh} / \mathrm{m}^{2} \mathrm{y}$ ) are deduced from a series of dynamic energy simulation runs on a set of typical buildings selected among different building typologies. The dynamic simulations have been done using the software Energy Plus (2018) developed by the US Department of Energy and the graphic interface Design Builder Software Limited. 
The geometric parameters of each single building are based on land registry data and national census databases and processed with GIS tools. Geometric information on buildings layouts and information about floors numbers and functional typology is derived from the cadastre. Information about buildings age come from elaboration of national census data, where available, integrated with the results of local surveys performed during the project.

A detailed description of the methodology applied to the Italian case studies can be found in (Condotta, 2018)

For the Austrian side, a similar modelling of typical buildings using the method described is still under development. For the present analysis, the estimates of building energy demand in the project area are calculated using indicators based on gross floor area, which are based on building age and building type (Rehbogen et al., 2018).

\section{MODEL APPLICATION AND SCENARIO DEFINITION}

A showcase of the described integrative approach has been performed for Feltre (Italy) and Seeham (Austria). Both Scenarios have similar settings, but different demands, sources and topology.

\section{Scenario "Feltre"}

As many Italian towns, Feltre currently hasn't any district heating system and relies almost $90 \%$ on natural gas. The current analysis for Feltre takes into account two technology options to meet domestic heat demand: domestic boilers fuelled with gas and district heating. In particular, district heating is assumed to be connected to a biomass boiler using woodchips and/or to a waste heat recovery from a metallurgical company located in the industrial area. The base scenario set up for Feltre aims at system cost minimization with no additional constraint: in this case, RIVUS identifies the existing natural gas network, whose infrastructure costs are assumed to be sunk, plus a small district heating network as the least cost option, due to the low cost of biomass. In terms of total $\mathrm{CO} 2$ emissions, this probably corresponds to the current situation of Feltre, since wood combustion in domestic ovens is the second energy source after natural gas.

Two follow-up scenarios are hence evaluated with the additional constraint of reducing total $\mathrm{CO} 2$ equivalent emissions from the entire system by $20 \%$ and $40 \%$, respectively. A set of potential locations for new biomass boilers was defined based on the availability of land to build new plants, leaving the selection of the minimum cost layout (including combinations of existing natural gas and new district energy systems) to the optimization procedure implemented in RIVUS.

\section{$\underline{\text { Scenario "Seeham" }}$}

The Base scenario, for the village "Seeham", represents a notional status quo of the district heating network. In a pre-processing step, the energy supply through the current district heating network was identified for the first Scenario. The follow-up scenarios are limited by a decrease output of the emitted $\mathrm{CO} 2$ equivalents of the Base scenario. The reduction steps of the $\mathrm{CO} 2$ are set, as for Feltre, to 20 and 40 percent. Electric resistance heating is assumed to be the only alternative to biomass based district heating in Seeham: as a result, the model outcome shows a high potential in upgrading the district heating network. To limit the enlargement of the district heating network in the scenarios to similar targets as in Feltre, costs of the heat pipes were first artificially increased. Once corresponding layouts had been obtained, regular market prices of heat pipes were then assumed to evaluate actual costs of modelled scenarios. 


\section{RESULTS}

For a first evaluation of the results obtained in combining GIS-tools with MILP, a simplified plot showing only the layout of DH networks is provided in Figure 3.

For Feltre, two new DH-boilers built in the base case scenario would be sufficient to supply most buildings with district heat. However, there is still a lot of natural gas used for domestic heating especially in times where the demand is significantly high, as it is in "peak" and "high"-load timesteps. By decreasing the $\mathrm{CO} 2$ limit the Waste-Heat recovery in the south of Feltre is the best price option for the heat peak demand. As soon as the $\mathrm{CO} 2$ limit is further decreased the $\mathrm{CO} 2$-free WasteHeat recovery is chosen to meet energy demand all over the year, completely substituting biomass boilers and thereby decreasing also local pollutants such as particulate and SOx, as shown in Figure 2.

The Seeham Base scenario is reflected by the current installed DH-network. By decreasing the CO2 limit some branches are installed in the near distance to the current network. The output of last scenario shows an ambitious build of DH into the west of Seeham. As soon as a breakpoint is exceeded the DH-network supplies a large part of west Seeham. As shown in Figure 3, this gives an image of the potential of the housings for DH in the west.

\begin{tabular}{|c|c|c|c|c|c|c|c|c|}
\hline Scenario & $\begin{array}{l}\text { System } \\
\text { costs } \\
{[k €]}\end{array}$ & $\begin{array}{l}\text { Percentage } \\
{[\%]}\end{array}$ & $\begin{array}{l}\text { District } \\
\text { Heating } \\
\text { Supply } \\
\text { [MWh] }\end{array}$ & $\begin{array}{l}\text { Waste } \\
\text { Heat } \\
\text { Supply } \\
\text { [MWh] }\end{array}$ & $\begin{array}{l}\text { Domestic } \\
\text { Heating } \\
{[\mathrm{MWh}]}\end{array}$ & $\begin{array}{l}\text { District } \\
\text { Heating } \\
\text { pipes } \\
\text { length [m] } \\
\end{array}$ & $\begin{array}{l}\text { Percent of } \\
\text { supplied } \\
\text { buildings in } \\
\text { the area [\%] }\end{array}$ & $\begin{array}{l}\text { Energy } \\
\text { loss } \\
\text { [\%] }\end{array}$ \\
\hline Feltre Base & 2069 & 100 & 41892 & 0 & 2488 & 16813 & 99 & 6.46 \\
\hline Feltre $80 \% \mathrm{CO} 2$ & 2305 & 111 & 42399 & 1436 & 950 & 17673 & 99 & 7.09 \\
\hline Feltre $60 \%$ CO2 & 2388 & 115 & 37325 & 7493 & 71 & 17673 & 99 & 7.14 \\
\hline Seeham Base & 1495 & 100 & 3869 & 0 & 6729 & 4002 & 35 & 10.52 \\
\hline Seeham 80\% CO2 & 1307 & 87 & 5874 & 0 & 4932 & 4971 & 50 & 9.75 \\
\hline Seeham 60\% CO2 & 1135 & 76 & 7941 & 0 & 3074 & 6235 & 66 & 10.10 \\
\hline
\end{tabular}

Table 1 "RIVUS Output and Benchmark numbers for the scenario Seeham"

In Table 1 the System cost of Feltre compared to Seeham are cheap if the amount of energy is considered. The costs for DH is for both the same, but the cost for the alternative domestic heat supply is not, in fact electric heating is three times more expensive than gas heating. As a result, the system costs in Seeham decrease when the DH system is expanded. In all scenarios, the amount of heat supplied through DH-networks is increasing, however, this requires higher investments in Feltre. 

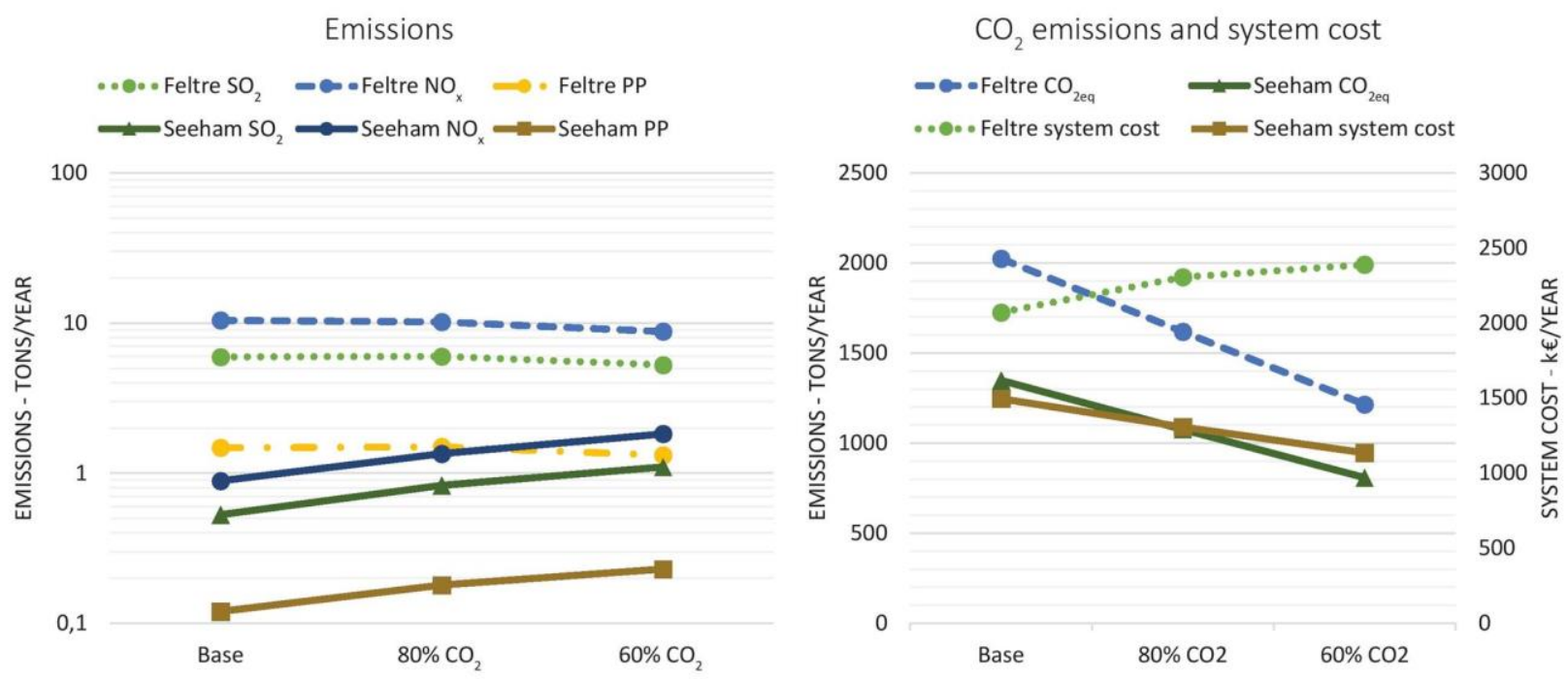

Figure 2 "Summary chart for Feltre and Seeham with all the pollutants evaluated and the systems cost"

In Figure 2 the environmental performance of energy systems in Feltre and Seeham is compared. Regarding the results about Feltre, it can be noted that with the increase of the $\mathrm{CO} 2$ reduction constraints a reduction of the air pollutants is reached, in all cases thanks to the incorporation of Industrial Waste Heat into the system, with a more extensive use in the $60 \%$ scenario.

For Seeham, the substitution of electric heating with biomass based DH results in higher local pollution ( $\mathrm{SO} 2, \mathrm{PP}$ and $\mathrm{NOx}$ ) when more ambitious $\mathrm{CO} 2$ reduction targets are set. From the economic point of view, contrary to Feltre, the system cost is decreasing with the reduction of $\mathrm{CO} 2$ emissions.

\section{CONCLUSIONS}

In this paper, scenarios on possible system setups for Feltre and Seeham have been calculated outlining the topology of optimal pipe setups as well as the load of heat pipes at different time steps with the objective to minimize overall system costs and setting also $\mathrm{CO} 2$ emission reduction constraints. It has been shown that district heating is an opportunity to reduce air pollutants especially if coupled with industrial heat recovery. On the other hand, based on available data, biomass boilers reduce the total amount of $\mathrm{CO}_{2}$ emitted but the emissions of other pollutants such as $\mathrm{SO}_{2}, \mathrm{NOx}$ and $\mathrm{PP}$ are increased. From the economic point of view, it has been shown that the reduction of emissions of $\mathrm{CO}_{2}$ leads to an increase of the system cost in the Italian case study, due to higher costs of low emission options, while costs decrease in the Austrian case study, mainly due to the high costs of resistance heating, which was taken as only alternative. Considering the current state of the market, more realistic results are expected to be obtained for both cases if low temperature alternatives are incorporated into the system, such as heat pumps, e.g. instead of resistance heating, or solar heating, as an alternative or complement to industrial waste heat recovery. Further work in this direction, as well as more detailed sensitivity analysis, is planned to be performed in the scope of the IDEE project.

\section{ACKNOWLEDGEMENTS}

This research was funded by the European Regional Development Fund - Interreg V-A Italia Österreich 2014-2020 - Axis 1.1 project IDEE ITAT1007 - CUP G22F16000860007 


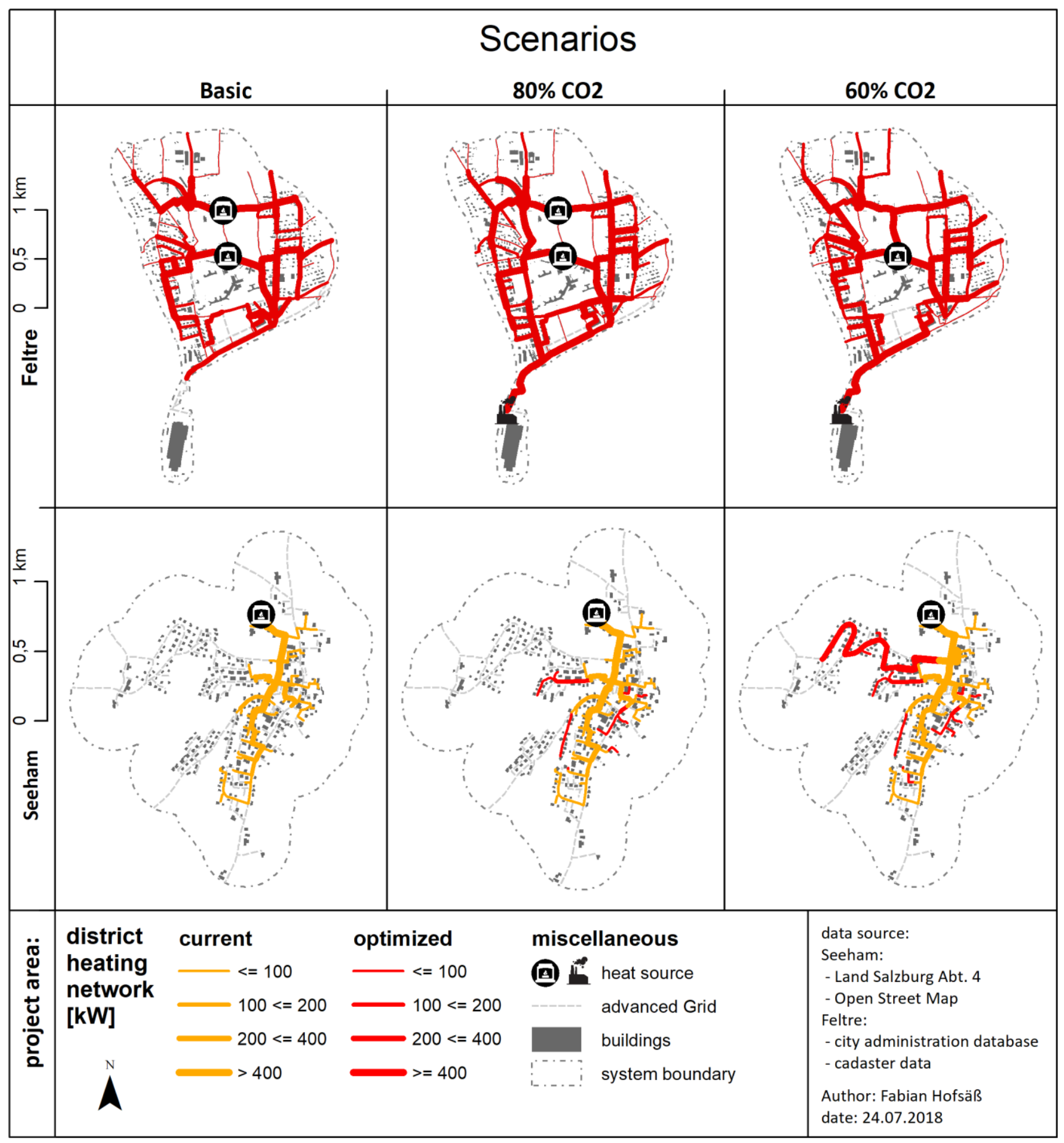

Figure 2"Scenario Feltre \& Seeham"

\section{REFERENCES}

Bartolozzi I., Rizzi F., Frey M., Are district heating systems and renewable energy sources always an environmental win-win solution? A life cycle assessment case study in Tuscany, Italy, Renewable and Sustainable Energy Reviews, Volume 80, 2017, Pages 408-420

Chinese, D., Santin, M., Saro, O. (2017) Water-energy and GHG nexus assessment of alternative heat recovery options in industry: A case study on electric steelmaking in Europe, Energy, Volume 141,2017,Pages 2670-2687

Chinese, D., Santin, M., Angelis, A., Saro, O., Biberacher, M. (2018), What to do with industrial waste heat considering a water-energy nexus perspective, Proceedings of the eceee Industrial Efficiency Conference, Berlin, 2018 
Condotta, M., Borga, G. Urban energy performance monitoring for Smart City decision support environment. In Techne special Issue 01/2018, Firenze University Press, Firenze, Italy.

Delmastro, C., Mutani, G. \& Schranz, L. (2016), The evaluation of buildings energy consumption and the optimization of district heating networks: a GIS-based model, International Journal of Energy and Environmental Engineering 7: 343

Dorfner, Johannes; Open source modelling and optimization of Energy Infrastructure at urban scale. Ph.D. Thesis, Technical University of Munich, 2016,

European Committee for Standardization (2008). ISO - "International Organization for Standardization, Standard ISO EN 13790/2008: Energy performance of buildings - Calculation of energy use for space heating and cooling".

Fang H., Xia J., Zhu K., Su Y., Jiang Y., Industrial waste heat utilization for low temperature district heating, Energy Policy, Volume 62, 2013, Pages 236-246

Karen N. Finney, Vida N. Sharifi, Jim Swithenbank, Andy Nolan, Simon White, Simon Ogden, Developments to an existing city-wide district energy network - Part I: Identification of potential expansions using heat mapping, Energy Conversion and Management, Volume 62, 2012, Pages 165175

Genon, G., Torchio, M. F., Poggio, A., \& Poggio, M. (2009). Energy and environmental assessment of small district heating systems: Global

Ghafghazi, S., Sowlati, T., Sokhansanj, S. et al. Life cycle assessment of base-load heat sources for district heating system options, The International Journal of Life Cycle Assessment (2011) 16: 212

Aira Hast, Sanna Syri, Vidas Lekavičius, Arvydas Galinis, District heating in cities as a part of lowcarbon energy system, Energy, Volume 152, 2018, Pages 627-639

Havasi, K., (2017), Open Source Project Advancement for Spatial Energy Infrastructure Optimization, Master thesis, Technical University of Munich

M. Köfinger, D. Basciotti, R.R. Schmidt, E. Meissner, C. Doczekal, A. Giovannini, Low temperature district heating in Austria: Energetic, ecologic and economic comparison of four case studies, Energy, Volume 110, 2016, Pages 95-104

Li Y., Xia J., Fang H., Su Y., Jiang Y., Case study on industrial surplus heat of steel plants for district heating in Northern China, Energy, Volume 102, 2016, Pages 397-405

Mohammad Sameti, Fariborz Haghighat, (2017), Optimization approaches in district heating and cooling thermal network, Energy and Buildings, Volume 140,2017,Pages 121-130

Bernd Möller, Henrik Lund, Conversion of individual natural gas to district heating: Geographical studies of supply costs and consequences for the Danish energy system, Applied Energy, Volume 87, Issue 6, 2010, Pages 1846-1857

Steffen Nielsen, Bernd Möller, GIS based analysis of future district heating potential in Denmark, Energy, Volume 57, 2013, Pages 458-468

Oliver-Solà, J., Gabarrell, X., \& Rieradevall, J. (2009). Environmental impacts of the infrastructure for district heating in urban neighbourhoods. Energy Policy, 37(11), 4711-4719.

Rehbogen, Strasser, Koblmüller, Mostegl, Schardinger, Biberacher, Zach, (2018) Integrierter Wärmeplan Zentralraum Salzburg - Umsetzungsplanung für die Wärmewende der EnergieVorzeigeregion Salzburg Endbericht heatswap_Salzburg Energieforschungsprogramm FFG

Mohammad Sameti, Fariborz Haghighat, Optimization approaches in district heating and cooling thermal network, Energy and Buildings, Volume 140, 2017, Pages 121-130

V.M. Soltero, R. Chacartegui, C. Ortiz, R. Velázquez, Potential of biomass district heating systems in rural areas,

Energy, Volume 156, 2018, Pages 132-143

Unternährer, J., Moret, S., Joost, S., Maréchal F., (2017) Spatial clustering for district heating integration in urban energy systems: Application to geothermal energy, Applied Energy, Pages 749762

Sven Werner, International review of district heating and cooling, Energy, Volume 137, 2017, Pages 617-631 
Internet based sources:

https://energyplus.net (EnergyPlus, requested on 26.07.2018)

https://atla.gse.it/atlaimpianti/project/Atlaimpianti_Internet.html (GSE atlas, requested on 26.07.2018)

http://www.iinas.org/gemis.htm (IINAS, requested on 26.07.2018)

http://www.SAGIS.at (SAGIS, requested on 26.07.2018) 


\section{SMART CITY MICRO QUARTERS}

Jens Leibold, Veronika Huemer-Kals, Thomas Zelger, IBO - Österreichisches Institut für Bauen und Ökologie GmbH

Alserbachstraße 5/8, 1090 Wien, Austria Phone: + 43 (0) 131920 05-50

FH Technikum Wien- Institut für Erneuerbare Energie Höchstädtplatz 6, 1200 Wien, Austria E-Mail: jens.leibold@ibo.at

\section{SUMMARY}

The research project "Smart City Micro Quarters" had the goal of simply and quickly exploring the urban renewal potential (regarding densification, efficiency, use of renewable energies etc.) of a city area and thus making city planning viable and capable of action. For four selected micro quarters, which typically occur in Austrian urban and rural areas, various densification possibilities were analysed. Whole city areas can be represented by multiplying and putting together the micro quarters. One focus was the solar potential in comparison with the energy demand of the variants to reach a high share of local energy autonomy. By means of this method the improvement potential of a district, in terms of energy supply, ecological, social, economic and urban planning criteria, can be determined at the early planning stage with little financial and temporal effort.

\section{INTRODUCTION}

To counteract the scarcity of housing and rapid urban sprawl in cities and their surrounding areas, densification is essential. A horizontal compaction increases the surface sealing, potential green space and other open spaces are getting lost. Vertical densification can lead to increased shading and a worse daylight situation for existing buildings. Therefore, densification in general often leads to resistance from residents.

In addition, if the ratio of roof area to Gross Floor Area (GFA) is lower, it is more difficult to achieve a high local coverage of the energy consumption with renewable energies. But what could a good compromise look like, that creates housing space, increases the subjectively perceived quality of life and protects the climate by a high rate of local renewable energies?

\section{METHOD}

\section{Micro quarters approach in general}

In the method developed within the project SC Micro Quarters, possibilities of future city planning for a development towards low-carbon districts are demonstrated. For this purpose, urban areas are abstracted into micro quarters (MQ). These MQ include important urban genetics that characterize the specific city district, like building structure (time of origin of the buildings, way of construction and thermal behaviour, dimension, mix of uses etc.), typical street cross sections, qualities of the public space, construction distances and technical and social infrastructure.

The four MQ that are used can be assigned to the types block, row and single-family housing. For the type single-family housing two variants are used, one open and one closed structure, like it is common in Lower Austria. In Figure 1 the considered types of micro quarters are shown. If there are larger deviations between the so called "Basis- MQ" and the considered one, they should be adapted for each location. Thereby local specifics can be considered like respective building heights, building distance, open space area, gaps between buildings etc.

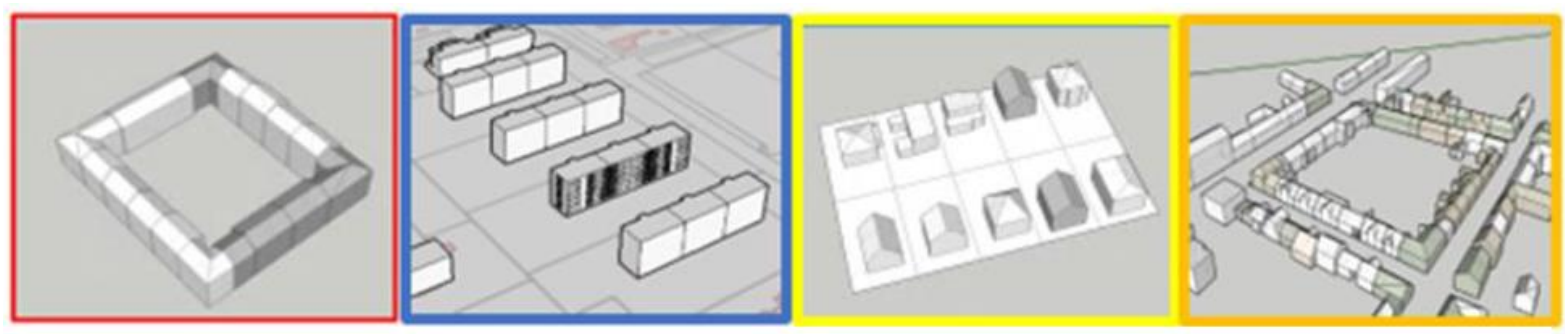

Figure 1: Used types of micro quarters "block" (red), "row" (blue) and "single-family housing" open (yellow) and closed structure (orange) 
In a next step variants of densification are developed for each MQ. In the research project, a pool of up to 20 variants per each micro quarter was developed. These were analysed, and strengths and weaknesses displayed. In the following figure, four possibilities of the construction extensions for MQ "Block" are presented.

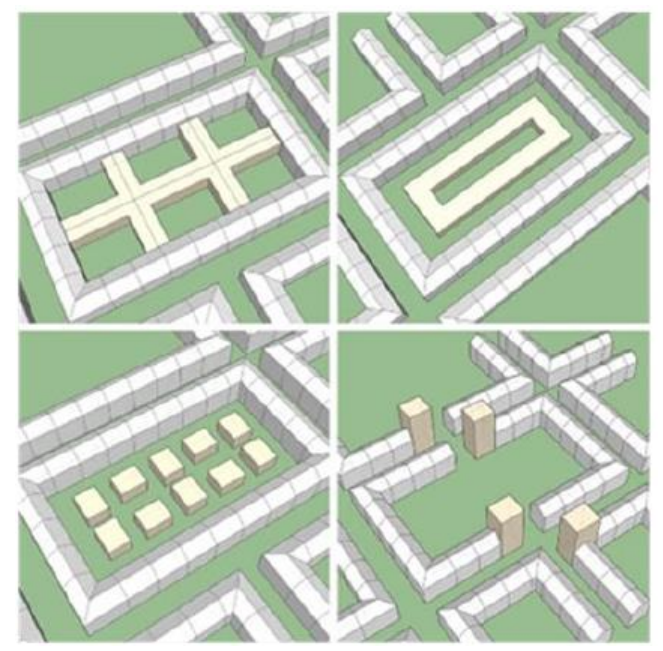

Figure 2: Examples for densification variants for the MQ „Block“

By multiplying the different MQ, whole city areas can be represented. According to the project partners a majority of city areas in Austria can be reproduced by the MQ approach. In Figure 3 it is demonstrated how a chosen city area is replicated by three different MQs.

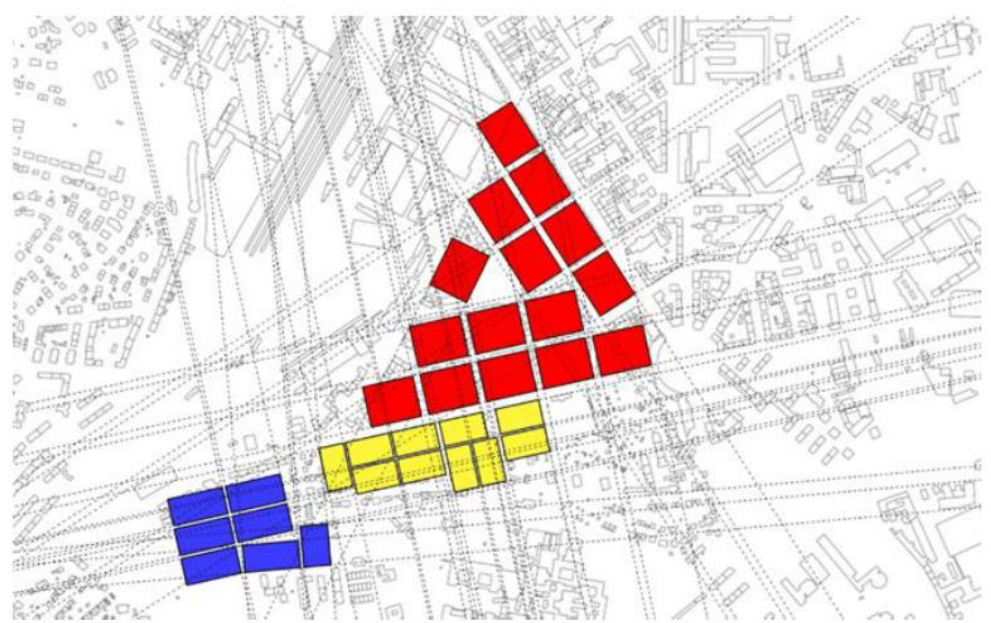

Figure 3: Composition of a city area example by multiplying each of the 3 different MQs

\section{MQ energy demand and supply calculation}

Since there is currently on the market no simulation software available that outputs for a city area or MQ all the desired energy and quality of life parameters, different software-based simulation tools are used. The MQ variants are drawn in SketchUp and the simulation tools should be compatible with this format to limit the amount of work. During the project various simulation tools were checked. In the end for the energy demand and HVAC system TRNSYS were used. For the passive and active solar potential PVsites were chosen.

The simulation results show the effects of different densification scenarios on the resulting energy demand, the examined quality of life parameters and the local potential of renewable energy production.

For each MQ at least one basis building is selected, for the closed structures (Block) a longitudinal and a corner building are analysed. Simulations and calculations to determine the solar potential, energy demand as well as life cycle costs (LCC) will be done for these basis buildings and projected for the 
whole MQ. Thereby the influence of shadow from neighbouring houses and different orientations are taken into consideration.

Dynamic simulations to determine energy demand are carried out with TRNSYS multi-zone model. Different construction standards (inventory, OIB minimum requirements for building components and passive house standard) as well as four different orientations are considered. The calculation of the energy requirement for the entire micro-district is made by extrapolation through the GFA.

The locally available renewable generation potential (PV, solar thermal, geothermal, groundwater heat / cold, outdoor air etc.) is simulated in different variants and compared to the respective energy requirements. The local renewable energy potential for the MQ within the dense urban context suggests a primary use of solar energy. Therefore, the individual designs are examined for their active solar potential. Figure 4 shows the yearly cumulative solar irradiance of a selected variant. The graphical evaluation serves to pre-select suitable roof areas which should have at least an annual solar irradiation of $700 \mathrm{kWh} / \mathrm{m}^{2}$.

The active solar potential is considered in two variants, from standard roof to a maximum version, including balconies, canopies and suitable facades (see Figure 6). In a first step, the roof surfaces were covered with PV modules. Thus, PV yields will be calculated with PVsites on an hourly basis (important for self-consumption determination). PVsites, a software available for free in its current state (beta version) allows users to model and evaluate BIPV projects. Figure 5 shows the PV coverage of the roofs for a considered variant. Due to the different colour design, or more accurate through specific selection of individual PV strings and modules, the resulting solar yield can be optimized.

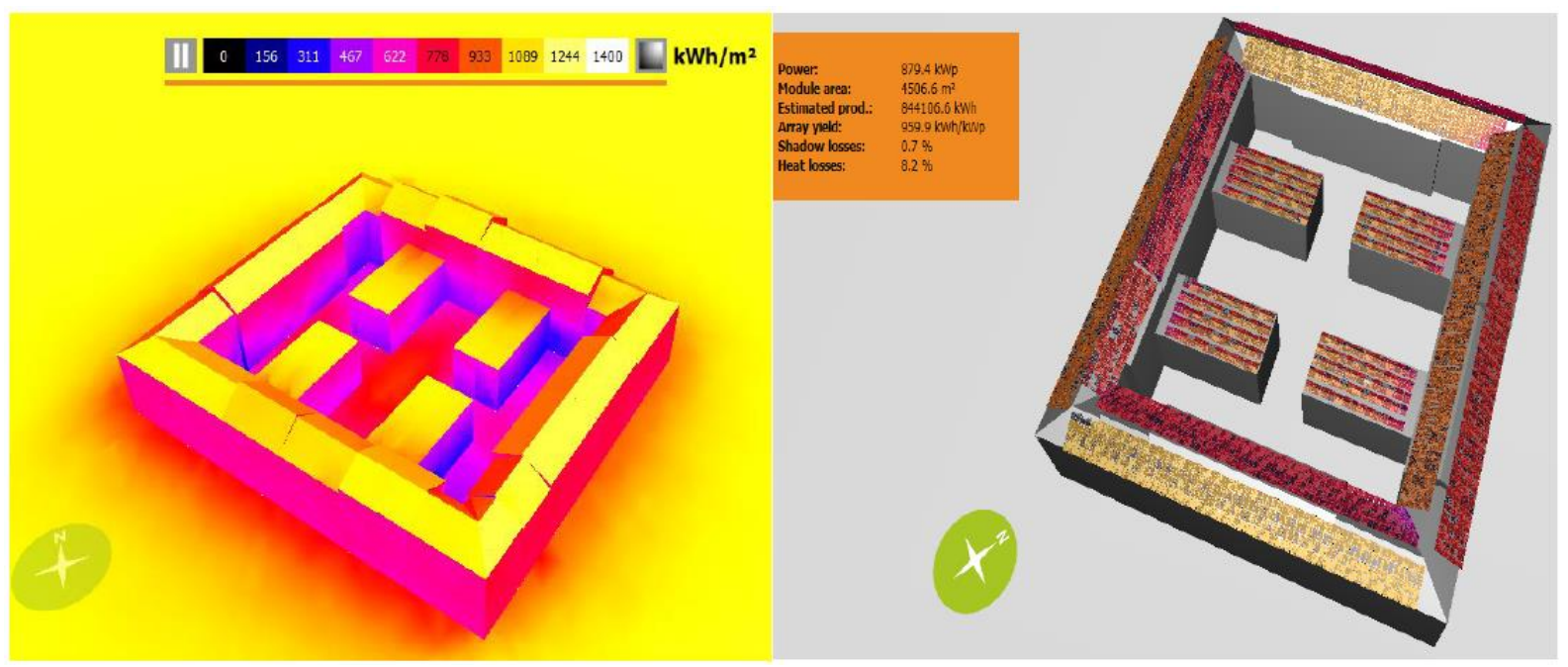

Figure 4: Annual solar irradiation for MQ Block variant "double teeth" (PVsites)
Figure 5: PV allocation "only roof" for MQ Block variant "double teeth" (PVsites)

\section{RESULTS}

As a central result for each MQ, ideal variants were determined and their strengths and weaknesses were identified. Elaborated Smart City indicators make it possible to compare and evaluate the different variants at the micro and city quarter level. The compression of the chosen 90 indicators from established city area rating systems (DGNB Stadtquartiere, LEED 2009 for Neighbourhood Development, BREEAM Communities, Urban Area Parameters) into 23 essential indicators is graphically processed in a sun chart. The sun chart shows clearly the focus and strengths of each variant. An example is shown in Figure 6 below, on the right side the category "energy" is displayed in detail. 


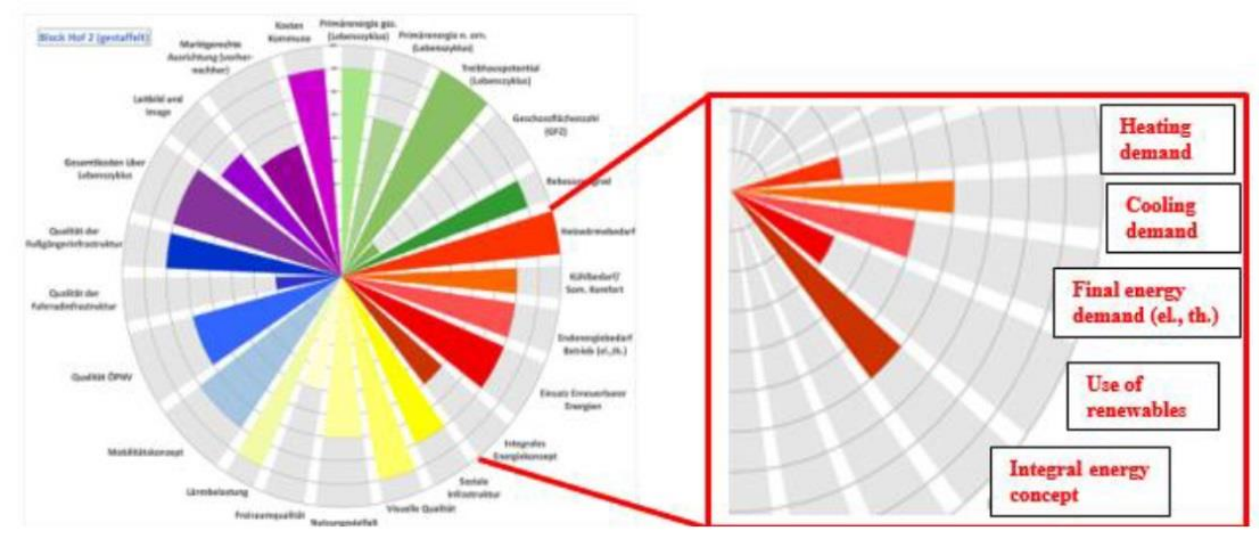

Figure 6: Sun chart with smart city indicators to evaluate variants and the focus "energy" (right side)

Models and guidelines for energy-optimized MQ, in terms of densification, energy efficiency, use of renewable energies and quality of life were developed. For this purpose, a workflow was planned, that determines the effects of the different variants on the energy demand, potential of renewable energy production and quality of life parameters in the concept phase of a MQ. For energy space planning, a workflow with coordinated simulation tools was developed to determine the effects of the different variants on energy demand and potential of renewable energy production. To combine the dynamic results of the energy demand with energy supply and excel tool was developed, which enables determinations regarding degree of autonomy and self-consumption rate. Figure 7 shows on the left side an example of the PV occupancy for the base buildings of MQ Block and Single-family house. On the right-hand side, the resulting autonomy and autarchy degrees as a function of the considered $\mathrm{PV}$ and e-mobility scenario are shown. The results clearly show that it is also possible to achieve a plus-energy MQ block with the PVmax variant. However, if future e-mobility shares are considered things get more challenging. In the case emob, where $75 \%$ of households are considered to have an electric car, only the MQ Single family house can achieve a plus in the annual balance. These surpluses at city area level is necessary to balance out deficits in the dense MQ "Block" area. For the MQ Single family house also an additional variant was considered, with $1.25 \mathrm{e}$-cars per household. The result of $99 \%$ autonomy shows why the advanced PVmax variants will become more important in the future to reach at least a yearly zero energy balance.

The energy outputs for the different MQ were put into a simulation tool for the whole city districts to optimize the future energy grid regarding costs and $\mathrm{CO}_{2}$ - emissions. As a result, the electrification for the future energy grid is forecasted (Fleischhacker 2018), which underlines the importance of a high local renewable production to reduce $\mathrm{CO}_{2}$ - emissions, costs and grid capacity. 

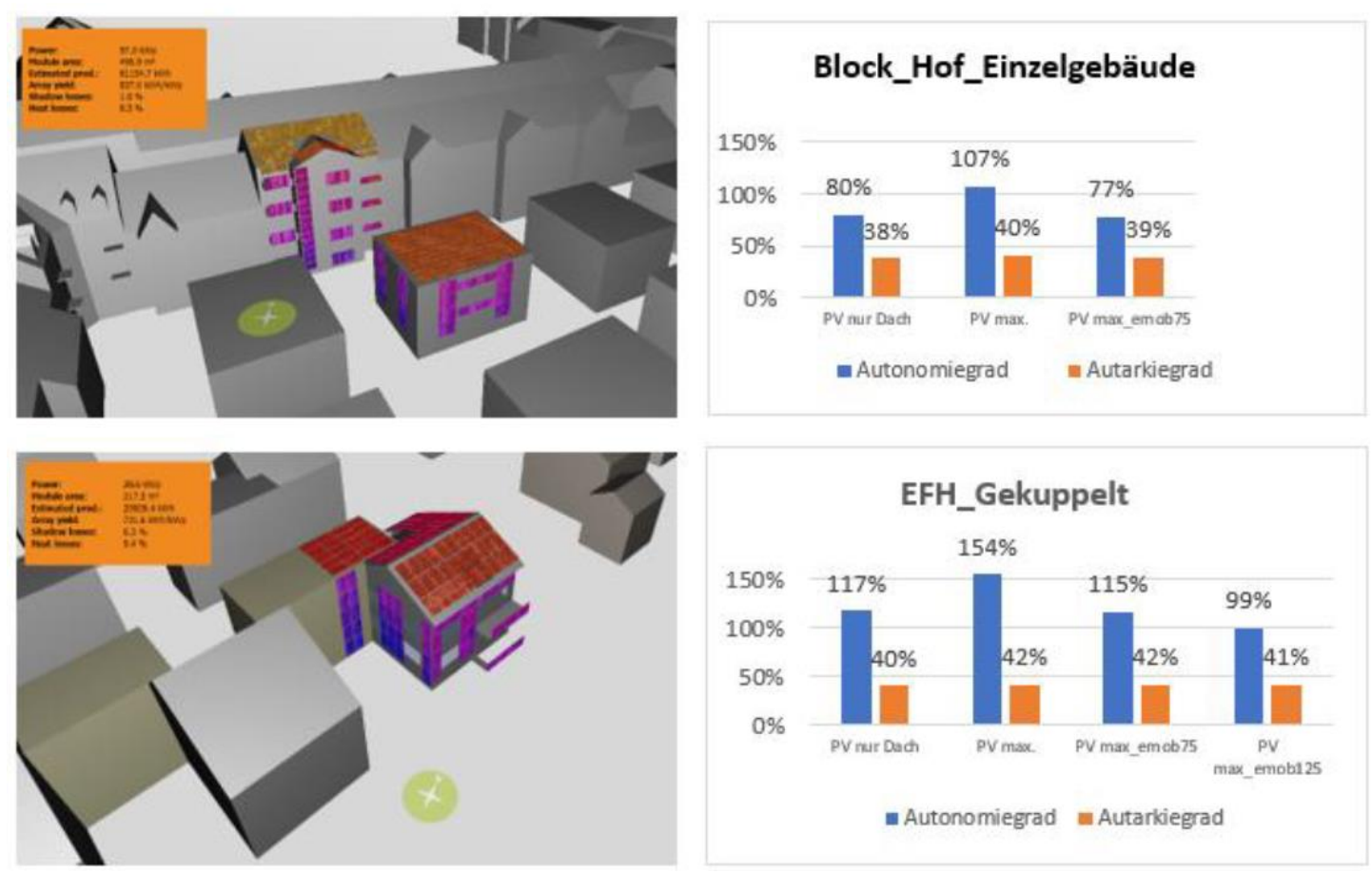

Figure 7: PVmax variants for base buildings MQ Block and Single- family house and the resulting degrees of autonomy and autarchy, depending on the selected PV and e-mobility scenario

\section{DISCUSSION}

By the MQ method a quick clarification of the potential of a planning area with a reasonable degree of accuracy is possible. The approach requires little preparatory work (low data collection effort), which leads to a short project duration and therefore low cost.

At the beginning of the project, there was the idea to identify an ideal development for each MQ.

During the course of the project, several attempts to get an overall rating were carried out, the presented sun chart is one of them. In the end the project partners recognized that the rating within the MQ variants strongly depended on the package of measures (building standard, use of renewables...) and not so much on the differences of the building structures. Therefore, the method in the future will be used as a modular system, which provides for each MQ a pool of construction variants, as well as several measures to guarantee the sustainability and quality of a MQ. The choice of the preferred MQ will take place in a guided process between stakeholders, urban planners and - depending on project priorities energy and/ or mobility planners. This could be a workshop where the different MQ will be developed together with the decision makers and stakeholders by means of the modular system.

\section{REFERENCES}

Mair am Tinkhof, O. et al., (2017), Richt- und Zielwerte für Siedlungen, zur integralen Bewertung der Klimaverträglichkeit von Gebäuden und Mobilitätsinfrastruktur in Neubausiedlungen.

Bundesministerium für Verkehr, Innovation und Technologie (Berichte aus Energie- und Umweltforschung), Wien, Austria

Fleischhacker, A. (2018), Sektorenkopplung führt zu einer Elektrifizierung des Systems, Blogeintrag https://smartcity-mikroquartiere.at, TU Wien 


\title{
POTENTIAL STUDY ON DEMAND SIDE MANAGEMENT IN DISTRICT HEATING AND COOLING NETWORKS WITH DECENTRALIZED HEAT PUMPS
}

\author{
$\underline{\text { Simone Buffa }}{ }^{1,2}$, Marco Cozzini ${ }^{1}$, Gregor P. Henze ${ }^{3}$, Chiara Dipasquale ${ }^{1}$, Marco Baratieri ${ }^{2}$, Roberto Fedrizzi ${ }^{1}$ \\ ${ }^{1}$ Eurac Research, Institute for Renewable Energy \\ Viale Druso 1, 39100 Bolzano, Italy \\ ${ }^{2}$ Free University of Bolzano, Faculty of Science and Technology \\ Piazza Università 5, Bolzano 39100, Italy \\ ${ }^{3}$ University of Colorado Boulder, Department of Civil, Environmental and Architectural Engineering \\ UCB 428, Boulder, CO 80309-0428 \\ Phone: +390471055636 \\ SUMMARY \\ E-Mail: simone.buffa@eurac.edu
}

The present study describes the implementation of a time-of-use demand response program as applied to fifth generation district heating and cooling networks. This technology involves the exploitation of decentralised water source heat pumps coupled with thermal energy storage. The latter can be charged at higher temperature storing electricity as thermal energy. The benefit of the process depends on the economic and physical boundary conditions imposed. The scenarios presented herein compare a constant and a variable thermal network temperature control strategies adopted during the demand response program with a reference case. The results show that an increase of the thermal network temperature avoids the drop of heat pump performance during the extra-charge process and suggests that such a shift of energy input to the heat pump from the electric power grid to the thermal distribution network can achieve savings in the total bill.

\section{INTRODUCTION}

Demand side management (DSM) strategies include a portfolio of practices aiming at modifying the demand side of an energy system. Among these practices, demand response (DR) programs applied in electric power grids include short-term dynamic incentives to push customers to reshape their usual consumption patterns. These changes can be conceived to shift the electrical load over time (load shifting) or to decrease/increase the energy demand (e.g. peak shaving/valley filling) (Lund et al., 2015). Demand and supply need to be balanced continuously to operate a power grid properly. DR measures add flexibility to a power system by making the demand more elastic. Reshaping the load reduces curtailment of electricity from variable renewable energy sources (VRESs) allowing the achievement of high penetration of VRES without the need of significant investments to upgrade the distribution and transmission grids.

DR strategies can be divided into incentive-based programs (IBDRPs) and price-based programs (PBDRPs). IBDRPs consist of contractual arrangements with grid operators or utility companies that include a reward to the end users for the reduction of their electricity consumptions for a certain amount of time upon request or with the remote control of their equipment. PBDRPs aim to change consumption patterns by influencing end-user choices with a time-dependent purchase price signal. A review of IBDRPs and PBDRPs can be found in Faria and Vale (2011) and in Alasseri et al. (2017). Among PBDRPs the most prevalent strategies in which the end-user takes part voluntarily are time-ofuse (TOU) rates and real-time pricing (RTP). TOU rates consist of a predefined variation of electricity prices generally within the day and the week. This solution intends to influence the end-users to shift their consumptions from peak hours to off-peak hours. In RTP rates vary dynamically along time 
reflecting the trend of the wholesale electricity price. It can be formulated as hour-ahead or day-ahead programs and requires a reliable communication system between utility companies and end-users.

The implementation of DR in the residential sector is very relevant because it can also help in balancing decentralised electricity produced from VRES at the household level (e.g. from rooftop PV fields). Residential buildings that have not installed batteries can contribute in DR programs mainly with two kinds of devices, classified according to Kok et al. (1998), into "shiftable operation" ones (e.g. washing machines, dryers and dishwashers) and "external resource buffering" ones (e.g. freezers, heat pumps, etc.).

Heat pumps (HPs) can participate in DR programs exploiting the passive thermal mass of buildings or/and dedicated thermal energy storage (TES). The first solution entails the increase (in heating mode) or the reduction (in cooling mode) of the room temperature set-point from typical values, potentially affecting the user comfort. In general, the second solution consists in water-based TES tanks that can be charged in a short time without affecting directly the indoor temperature. Waterbased "buffer" TES can be useful to limit the number of ON-OFF cycles of the HP in space heating (SH) or space cooling (SC) modes allowing the operation of the unit with a minimum run-time period. Moreover, in residential buildings a domestic hot water (DHW) TES tank is always needed allowing for a separation in time of thermal energy production from utilisation.

The implementation of DR programs with air source heat pumps (ASHP) can be found in several studies. Miara et al. (2014) developed different scenarios of TOU programs where the DSM signal has been derived from the daily normalized residual load (DNRL): the difference between grid's load and VRES electricity generation. The results of the load shifting strategies show an increase of electricity consumption up to 19\%. Schibuola et al. (2015) applied an RTP program for an ASHP adopting three different control strategies to use the price series on the electricity stock market as a signal. Moreover, Pau et al. (2018) implemented a mixed integer linear programming (MILP) optimization problem to schedule the optimal operation of a pool of HPs to achieve power peak shaving.

Fifth generation district heating and cooling (5GDHC) networks employ decentralised water source heat pumps (WSHPs) at the building site, allowing the coupling of power and thermal grids as shown in Fig. 1. Unlike traditional high-temperature district heating, 5GDHC systems exploit a network operating at a temperature close to the ground $\left(10-25^{\circ} \mathrm{C}\right)$ achieving negligible thermal losses and allowing for bidirectional thermal energy flow. The installation of decentralised HPs in DHC substations is needed to lift the temperature to meet occupant needs satisfying both heating and cooling demand of buildings. 5GDHC projects are growing in Europe, yet solutions of a smart coupling between the thermal and power grids are hard to find. In the framework of the EU H2020 project Sim4Blocks, the implementation of DR programs is anticipated in the 5GDHC systems of the

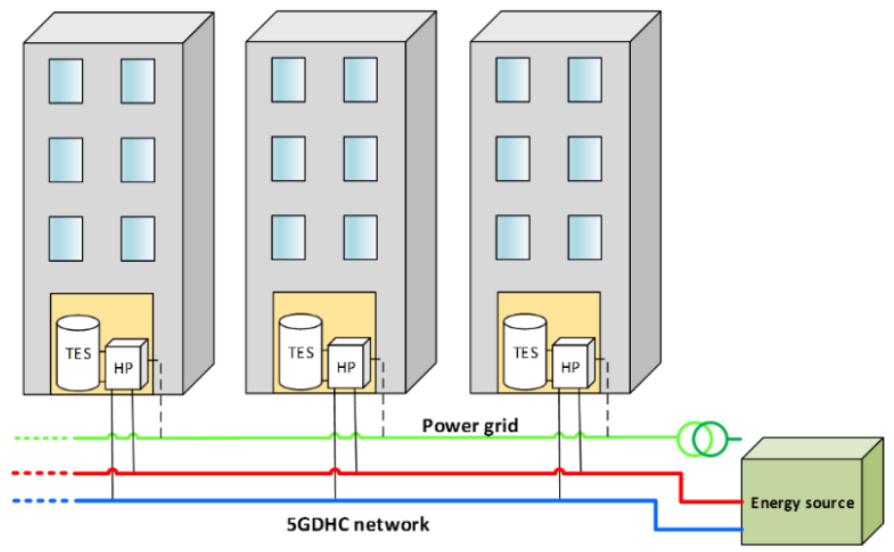

Fig. 1: Scheme of a 5GDHC network concept. 
communities of Wüstenrot (Germany) and Naters (Switzerland).

The present study, conducted within the H2020 project FLEXYNETS, evaluates the potential of DR strategies that engage WSHPs connected to a 5GDHC system. Here, different from ASHP systems, the network temperature can be treated as an additional free variable that can be controlled by the DHC network operator to maintain high performance of the substations during the participation in the DR programs. The work aims at assessing the exploitation of the DHW TES capacity of a single substation that is charged to a higher temperature during a daily TOU DR program. Different scenarios have been developed comparing the energetic and economic performance under different control strategies and different prices of the electricity and thermal energy. In this framework DR strategies that exploit the electricity price as signal could have two goals: to reduce the bill for the end customer or to decrease the operational costs of the system for the DHC manager by allowing the latter to remotely control the 5GDHC substations.

\section{METHODS}

To assess the behaviour of a HP connected to a 5GDHC network participating in DR programs different numerical simulations have been performed in TRNSYS. The aim of the developed control strategies is to shift the DHW production from high-cost (peak) to low-cost (off-peak) hours exploiting the capacity of the DHW TES. The following sections include a description of the numerical model implemented, DR strategies and scenarios considered.

\section{MODEL DESCRIPTION}

The present study takes into considerations a 5GDHC substation that supplies a residential building. The latter consists of a small multi-family house with a total of 10 apartments divided into 5 floors. The single apartments have a floor area of $50 \mathrm{~m}^{2}$ and can be considered a new or a refurbished building with an energy level of about $45 \mathrm{kWh} /\left(\mathrm{m}^{2}\right.$ year) for space heating ( $\mathrm{SH}$ ) according to the study performed in (Fedrizzi et al., 2015). The climate conditions are the ones of Rome (Italy). It has 1415 heating degree-days and it is classified as a Csa climate (Mediterranean climate) type according to the Köppen classification. The DHW draw profile has been generated by means of the software tool DHWcalc V.2.02b with a time resolution of 1 minute. The occupancy level considered is 2 people/apartment with a DHW demand of 40 1/(person day). This results in a specific DHW load of 24 $\mathrm{kWh} / \mathrm{m}^{2}$. The SH and space cooling (SC) demands of the building have been assessed by means of dynamic simulations using TRNSYS Type 56. The peak thermal power for SH results in $22.7 \mathrm{~kW}_{\text {th }}$ whereas the peak thermal power for SC results in $18.4 \mathrm{~kW}_{\mathrm{th}}$.

The building heat emission system consists of ceiling radiant panels which are supplied by a central 5GDHC substation. Fig. 2 shows the hydraulic scheme of the substation considered where all the components included in the red boundary can be considered as part of a compact unit. It includes mainly an electrically driven WSHP, two TESs, temperature sensors, hydraulic valves and pumps. These components have been sized to satisfy completely the building energy needs. In particular, the centralised DHW TES consists of a stratified water tank and it has been sized according to the Italian standard UNI 9182 (2010) resulting in a volume of 450 litres. The on-off controlled single-stage WSHP operates in monovalent mode and has been oversized to fully cover both SH and SC demand. Its capacity results in about $25 \mathrm{~kW}_{\text {th }}$ and its performance has been evaluated using a performance map derived from laboratory tests (Menegon and Fedrizzi, 2016). The buffer TES has been sized considering the reference value of 15 litres $/ \mathrm{kW}_{\text {th }}$ with respect to the WSHP thermal power capacity. It results in a volume of 375 litres. Both TES have two ports and have been simulated using the Type 
534 of the TESS library. The buffer tank has been divided into three nodes of equal volume whereas the DHW tank has been divided into five nodes to reproduce the effect of thermal stratification.

The DHW distribution system has been conceived with decentralised substations equipped with heat exchangers for the instantaneous production. For these simulations, 40 meters of distribution circuit has been considered, the decentralised substations have been simplified concentrating the total drawoff in in only one oversized heat exchanger. To decrease the delay in hot water supply during a draw event, a continuous recirculation of water is needed in the DHW distribution circuit. This leads to a continuous extraction of thermal energy from the DHW TES and to notable thermal losses. To restrict these losses during the extra-charge process of the DHW TES, the mixing valve MV3 has been introduced, limiting the DHW distribution supply temperature to $50^{\circ} \mathrm{C}$. The DHC network has been considered an ideal thermal energy source that is able to supply the single substation considered at the desired temperature set-point neglecting time delays and storage effects. During the DR program, the DHC network temperature can be increased in order to limit the drop of the HP efficiency during the DHW extra-charge process.

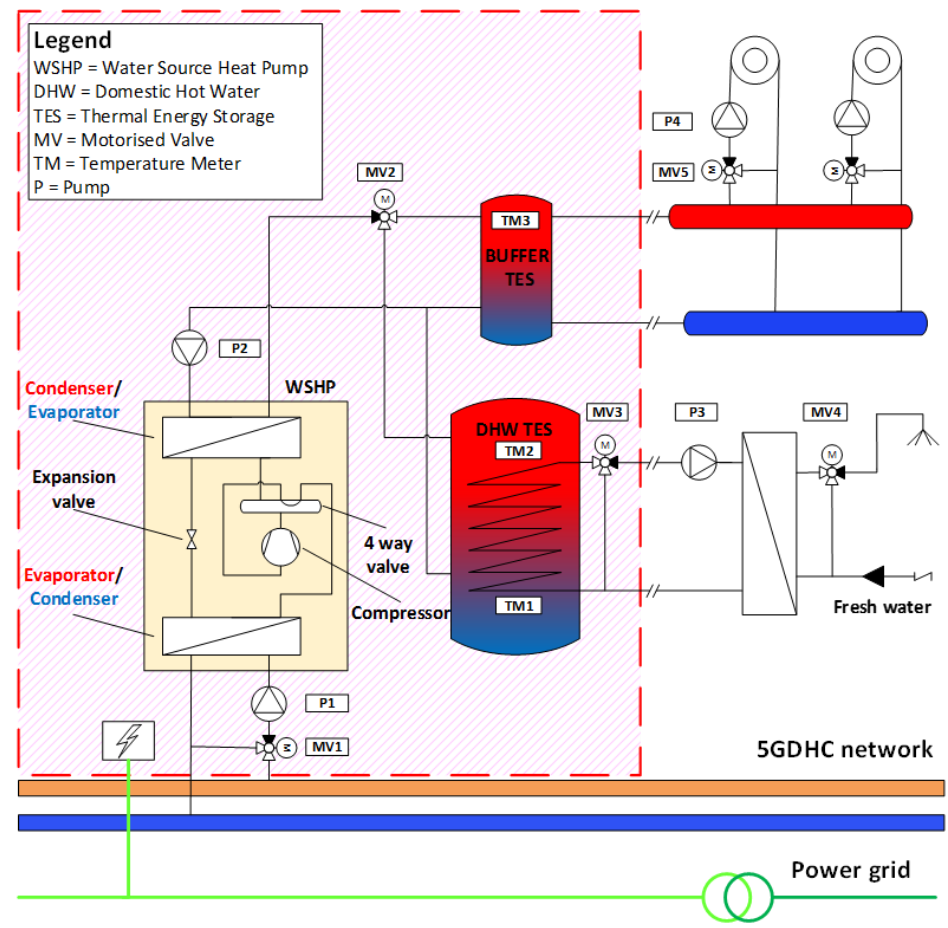

Fig. 2: Detailed hydraulic scheme of a 5GDHC substation.

\section{DEMAND RESPONSE STRATEGY DEFINITION}

The operation of the substation has been performed through the implementation of rule-based control (RBC) strategies in a supervisory controller. These consist of a series of operating schemes that combine logically the output signals of hysteresis controllers (on-off feedback controllers). The goal of the control is to maintain the proper temperatures of each TES tank to satisfy both heating and cooling demand according to the signals and set points listed in Table 1. The logic rules have been conceived such that charging the DHW TES has priority over charging the buffer TES tank. 
Table 1: List of output signals from hysteresis controller logically combined.

\begin{tabular}{clccc}
\hline $\begin{array}{c}\text { Hysteresis } \\
\text { name }\end{array}$ & \multicolumn{1}{c}{ Acquisition signal } & $\begin{array}{c}\text { Set point } \\
\text { value }\end{array}$ & $\begin{array}{c}\text { Upper } \\
\text { dead band }\end{array}$ & $\begin{array}{c}\text { Lower } \\
\text { dead band }\end{array}$ \\
\hline Signal A & $\begin{array}{l}\text { Indoor sensor temperature determines if there is a need for } \\
\text { SH/SC demand (Winter/Summer operation modes). }\end{array}$ & $22^{\circ} \mathrm{C}$ & $0{ }^{\circ} \mathrm{C}$ & $0{ }^{\circ} \mathrm{C}$ \\
Signal B & $\begin{array}{l}\text { Bottom temperature of the DHW TES (TM1) to assess if the } \\
\text { energy content is sufficiently high to cover the DHW request. }\end{array}$ & $45{ }^{\circ} \mathrm{C}$ & $5{ }^{\circ} \mathrm{C}$ & $0{ }^{\circ} \mathrm{C}$ \\
Signal C & $\begin{array}{l}\text { Top temperature of the buffer TES (TM3) to charge it with a } \\
\text { set-point value defined according to SH/SC operation modes. }\end{array}$ & $\begin{array}{l}35^{\circ} \mathrm{C}(\mathrm{SH}) \\
15^{\circ} \mathrm{C} \\
\text { (SC) }\end{array}$ & $3{ }^{\circ} \mathrm{C}$ & $-3^{\circ} \mathrm{C}$ \\
\hline
\end{tabular}

The DR strategies applied in this study aim at exploiting the capacity of the DHW TES tank only. Unlike SH/SC demand, the DHW load is not affected by seasonality allowing the participation in DR programs year-round. The control of the DHW TES temperature operates so that only the upper dead band of the hysteresis (signal B) is increased from $5^{\circ} \mathrm{C}$ to $15^{\circ} \mathrm{C}$ when an extra-charge process occurs. In this way, the DHW storage is not charged instantaneously but only after its total discharge. This solution allows limiting the TES thermal losses and to add diversity to the system. Indeed, since the start of the charging process depends on the current TES state-of-charge (SOC) and DHW load, which is different among the substations, the HPs do not start their operation in a synchronised fashion. In this way, unwanted power peaks are mitigated both on the electrical and thermal grids. Charging the TES beyond the currently needed value involves increasing the tank set point temperature. This introduces a drawback: with a higher temperature, the HP coefficient of performance $(C O P)$ and heating capacity drops and additional thermal losses $\left(E_{t h, a d d . ~ l o s s e s}\right)$ occur.

For the sake of simplicity, considering only the cost for electricity consumption $\left(c_{e l}\right.$ is the specific cost in EUR/kWh), the driving concept of the analysis is the following: Producing the thermal energy needed $\left(E_{t h}\right)$ during the peak hour at the minimum storage temperature would cost $c_{e l, p e a k} E_{t h} /$ $\mathrm{COP}_{\text {max }}$ while producing it in advance at a higher temperature would $\operatorname{cost} c_{\text {el,off-peak }}\left(E_{t h}+\right.$ $\left.E_{t h, a d d . \text { losses }}\right) /$ COP. To achieve savings in the electricity bill, the following inequalities must be satisfied:

$$
\begin{aligned}
& \frac{c_{\text {el,off-peak }}\left(E_{t h}+E_{t h, \text { add. losses }}\right)}{C O P}- \\
& C O P>\left(\frac{c_{\text {el,off-peak }}}{c_{\text {el,peak }}}\right)\left(1+\frac{E_{t h, \text { add. losses }}}{E_{t h}}\right) C O P_{\text {max }}
\end{aligned}
$$

According to Eq. 2 there is a lower limit of the COP for the TES extra-charge process that corresponds to a limit in the difference between HP sink and source temperatures. It depends on the electricity price ratio between off-peak hours and peak hours and the ratio between the additional thermal losses introduced with respect to the thermal energy produced $\left(E_{t h}\right)$. Charging the DHW TES at higher temperature is advantageous only if the electricity costs ratio compensates at least the additional thermal losses introduced. In other words, considering the TOU DR program shown in Fehler! Verweisquelle konnte nicht gefunden werden., there exists a relationship between the price variation from peak-hours to off-peak hours and the maximum charging temperature of TES to make the TOU program economically feasible. 


\section{DEMAND RESPONSE SCENARIOS}

The TOU DR program investigated in this study, based on the Italian tariff D1 taken as a reference, distinguishes peak hours (from 8:00 until 19:00) from the remaining off-peak hours. During weekend days, only the off-peak tariff is applied. The DR signal is activated only once a day for the two hours preceding the increment of tariff.

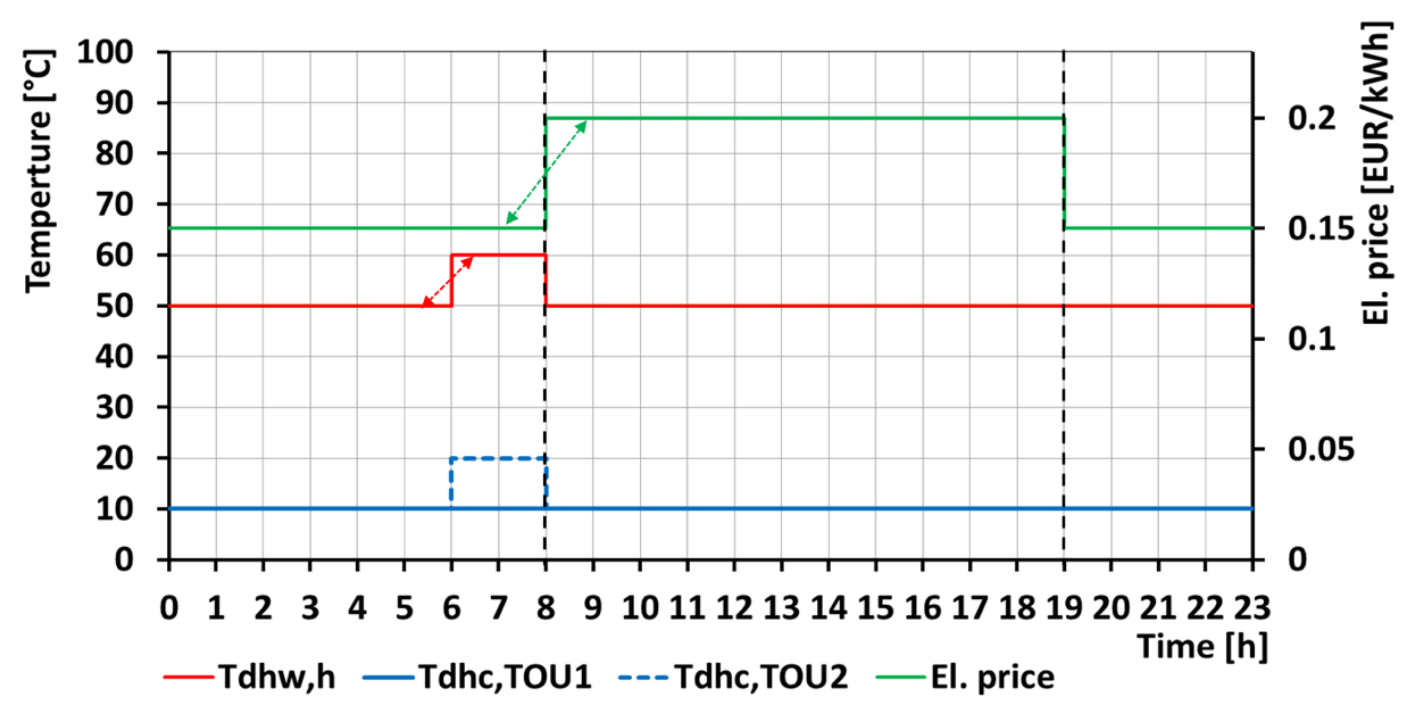

Fig. 3: Description of the TOU DR program considered.

During this period the DHW TES hysteresis upper dead band (Tdhw,h) is shifted from $50^{\circ} \mathrm{C}$ to $60^{\circ} \mathrm{C}$ so that as much as thermal energy is stored after a discharge. Two different approaches have been implemented for the management of the thermal grid. In scenario TOU1, the supply temperature of the DHC network is considered constant at $10^{\circ} \mathrm{C}$ all year-round as it generally occurs in 5GDHC systems that exploit groundwater as its thermal energy source. In scenario TOU2, the DHC operator raises the network temperature from $10^{\circ}$ to $20^{\circ} \mathrm{C}$ when the DR signal is activated so that the drop of HP performance during the extra-charge of the TES is limited. From the two TOU DR scenarios described above a parametric study has been conducted. In particular, the economic assessments described in Table 3 have been performed considering an off-peak electricity price of $0.15 \mathrm{EUR} / \mathrm{kWh}$ and a peak electricity price of $0.17 \mathrm{EUR} / \mathrm{kWh}$ (case A) and $0.20 \mathrm{EUR} / \mathrm{kWh}$ (case B), respectively. Moreover, these scenarios have been combined with two different prices of the thermal energy purchased from the DHC network (respectively of $0.05 \mathrm{EUR} / \mathrm{kWh}$ and $0.10 \mathrm{EUR} / \mathrm{kWh}$ ) obtaining eight different results of the total bill for the DHW demand only. Since the DR strategies do not exploit the buffer TES capacity the variation in SH/SC bill has been neglected.

\section{RESULTS}

In this analysis, for a single 5GDHC substation the energy performance and the economic benefits have been assessed for the different scenarios presented in Section 3.3. 


\section{ENERGY ASSESSMENTS}

In this section, the energy evaluations of the two TOU DR strategies implemented are compared with the reference case as summarised in Table 2. The seasonal HP COP for the DHW preparation $\left(\mathrm{SCOP}_{\mathrm{DHW}}\right)$, in scenario TOU1 decreases from 2.36 (reference scenario) to 2.32 due to the extracharge process of the TES. On the other hand, the higher DHC network temperature during the DR program TOU2 raises the $\mathrm{SCOP}_{\mathrm{DHW}}$ to 2.42 .

Table 2: Energy assessments of the different DR scenarios.

\begin{tabular}{|c|c|c|c|c|}
\hline & & Unit & $\begin{array}{c}\text { Scenario TOU1 } \\
\text { Tdhe const. } \\
\left(1^{\circ} \mathrm{C}\right)\end{array}$ & $\begin{array}{l}\text { Scenario TOU2 } \\
\text { Tdhe var. } \\
\left(10 \div 20^{\circ} \mathrm{C}\right)\end{array}$ \\
\hline \multirow{13}{*}{ 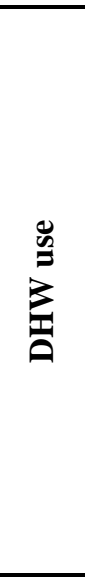 } & $\mathrm{SCOP}_{\mathrm{DHW}}$ ref. & {$[-]$} & 2.36 & 2.36 \\
\hline & $\mathrm{SCOP}_{\mathrm{DHW}} \mathrm{TOU}$ & {$[-]$} & 2.32 & 2.42 \\
\hline & Qdhw,load ref. & {$[\mathrm{MWh}]$} & 11.96 & 11.96 \\
\hline & Qdhw,load TOU & {$[\mathrm{MWh}]$} & 11.96 & 11.96 \\
\hline & Qdhw,in ref. & {$[\mathrm{MWh}]$} & 16.20 & 16.20 \\
\hline & Qdhw,in TOU & {$[\mathrm{MWh}]$} & 16.21 & 16.21 \\
\hline & $\Delta$ Qdhw,in & {$[\mathrm{MWh}]$} & 0.01 & 0.01 \\
\hline & Qloss ref. & {$[\mathrm{MWh}]$} & 4.24 & 4.24 \\
\hline & Qloss TOU & [MWh] & 4.25 & 4.25 \\
\hline & $\Delta$ Qloss & {$[\mathrm{MWh}]$} & $0.01(+0.24 \%)$ & $0.01(+0.24 \%)$ \\
\hline & Qstored,DR ref. & {$[\mathrm{MWh}]$} & 0.38 & 0.38 \\
\hline & Qstored,DR TOU & [MWh] & 1.36 & 1.27 \\
\hline & $\Delta$ Qstored,DR & [MWh] & $0.98(+258 \%)$ & $0.89(+234 \%)$ \\
\hline \multirow{9}{*}{ 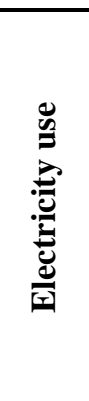 } & Eel,dhw peak hours ref. & [MWh] & 1.64 & 1.64 \\
\hline & Eel,dhw peak hours TOU & [MWh] & 1.30 & 1.36 \\
\hline & $\Delta$ Eel,dhw peak hours TOU & {$[\mathrm{MWh}]$} & $-0.33(-20 \%)$ & $-0.28(-17 \%)$ \\
\hline & Eel,dhw off-peak hours ref. & {$[\mathrm{MWh}]$} & 5.25 & 5.25 \\
\hline & Eel,dhw off-peak hours TOU & [MWh] & 5.72 & 5.36 \\
\hline & $\Delta$ Eel,dhw off-peak hours TOU & [MWh] & $0.46(+8.7 \%)$ & $0.11(+2.1 \%)$ \\
\hline & Tot Eel,dhw ref. & [MWh] & 6.89 & 6.89 \\
\hline & Tot Eel,dhw TOU & [MWh] & 7.02 & 6.72 \\
\hline & $\Delta$ Eel,dhw & {$[\mathrm{MWh}]$} & $0.13(+1.8 \%)$ & $-0.17(-3.2 \%)$ \\
\hline \multirow{3}{*}{ 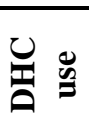 } & Qdhc,dhw ref. & [MWh] & 9.53 & 9.53 \\
\hline & Qdhc TOU & [MWh] & 9.41 & 9.70 \\
\hline & $\Delta$ Qdhe & [MWh] & $-0.12(-1.3 \%)$ & $0.17(+1.8 \%)$ \\
\hline
\end{tabular}

The yearly DHW load on the user side is 11.96 MWh. To meet this load, 16.20 MWh of thermal energy have been supplied by the HP to the DHW TES. The thermal losses of the TES and DHW distribution circuit amount to $4.24 \mathrm{MWh}$ that corresponds to about $26 \%$ of the energy input to the TES. During the TOU DR scenarios, the thermal losses increase about $0.1 \mathrm{MWh}$, or $+0.24 \%$, of the total TES losses compared to the reference condition. During the TOU programs, the energy input to the TES grows by the same quantity to cover the additional thermal losses due to the extra-charge process of the TES. Concerning the thermal energy stored during the DR program hours, the TOU strategy permits to store of about $1 \mathrm{MWh}$ more than the reference case. This is almost two and a half times the energy stored in the reference case. In the scenario TOU2, this value is a bit less because the DHW TES was not able to reach a full charge during some DR program periods. In scenario TOU1, the DR program is able to shift $0.33 \mathrm{MWh}$ of electricity used from peak-hours to off-peak hours. On one hand, this shift is paid with higher total electricity consumptions of $1.8 \%$ because of the lower 
$\mathrm{COP}$ with respect to the reference scenario. On the other hand, the same amount of thermal energy has not been used from the DHC network (-0.12 MWh). In TOU2 scenario, only $17 \%$ of the peak hours electricity consumption is shifted to off-peak hours. Moreover, the higher DHC network temperature permits to operate at higher COP limiting the total electricity consumption to about $0.17 \mathrm{MWh}$. Unlike scenario TOU1, in scenario TOU2 the thermal energy used from the DHC network increases by the same amount as the electricity saved.

\section{ECONOMIC ASSESSMENTS}

Table 3 reports the evaluation of the electrical, thermal and total bill for the different scenarios according to the energy prices presented in Section 3.3.

Table 3: Economic assessments of the different DR scenarios.

\begin{tabular}{|c|c|c|c|c|c|c|}
\hline & Unit & $\begin{array}{c}\text { Scenario } \\
\text { TOU1A } \\
\text { Tdhe const. } \\
\left(10^{\circ} \mathrm{C}\right) \\
\end{array}$ & $\begin{array}{c}\text { Scenario } \\
\text { TOU1B } \\
\text { Tdhe const. } \\
\left(10^{\circ} \mathrm{C}\right) \\
\end{array}$ & $\begin{array}{l}\text { Scenario } \\
\text { TOU2A } \\
\text { Tdhc var. } \\
(\mathbf{1 0} \div \mathbf{2 0} \text { C })\end{array}$ & $\begin{array}{c}\text { Scenario } \\
\text { TOU2B } \\
\text { Tdhc var. } \\
\left(10 \div 20^{\circ} \mathrm{C}\right)\end{array}$ \\
\hline & Peak hour Eel Price increment & {$[\%]$} & $15 \%$ & $30 \%$ & $15 \%$ & $30 \%$ \\
\hline & Cel off-peak /Cel peak & {$[-]$} & 0.87 & 0.77 & 0.87 & 0.77 \\
\hline & Eel prices off-peak ho & {$[\mathrm{EUR} / \mathrm{kWh}]$} & 0.15 & 0.15 & 0.15 & 0.15 \\
\hline & Eel prices peak hours & {$[\mathrm{EUR} / \mathrm{kWh}]$} & 0.17 & 0.20 & 0.17 & 0.20 \\
\hline & Tot Eel costs ref. & [EUR] & 1070.5 & 1107.3 & 1070.5 & 1107.3 \\
\hline & Tot Eel costs TOU & [EUR] & 1082.2 & 1111.5 & 1038.7 & 1069.3 \\
\hline & $\Delta$ Tot Eel costs & [EUR] & $11.7(+1.1 \%)$ & $4.2(+0.4 \%)$ & $-31.8(-3 \%)$ & $-38.0(-3.4 \%)$ \\
\hline \multirow{6}{*}{ 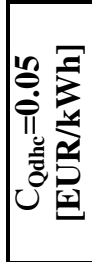 } & Total costs Qdhc ref. & [EUR] & 476.6 & 476.6 & 476.6 & 476.6 \\
\hline & Total costs Qdhc TOU & [EUR] & 470.5 & 470.5 & 485.2 & 485.2 \\
\hline & $\Delta$ Tot Qdhe costs & [EUR] & $-6.1(-1.3 \%)$ & $-6.1(-1.3 \%)$ & $8.6(+1.8 \%)$ & $8.6(+1.8 \%)$ \\
\hline & Total costs ref. & [EUR] & 1547.0 & 1583.9 & 1547.0 & 1583.9 \\
\hline & Total costs TOU & [EUR] & 1552.7 & 1582.0 & 1523.8 & 1554.5 \\
\hline & $\Delta$ Tot costs & [EUR] & $5.7(+0.4 \%)$ & $-1.9(-0.1 \%)$ & $-23.2(-1.5 \%)$ & $-29.4(-1.9 \%)$ \\
\hline \multirow{6}{*}{ 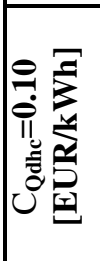 } & Total costs Qdhc & [EUR] & 953.1 & 953.1 & 953.1 & 953.1 \\
\hline & Total costs Qdhc TOU & [EUR] & 941.0 & 941.0 & 970.4 & 970.4 \\
\hline & $\Delta$ Tot Qdhe costs & [EUR] & $-12.1(-1.3 \%)$ & $-12.1(-1.3 \%)$ & $17.2(+1.8 \%)$ & $17.2(+1.8 \%)$ \\
\hline & Total costs ref. & [EUR] & 2023.6 & 2060.4 & 2023.6 & 2060.4 \\
\hline & Total costs TOU & [EUR] & 2023.2 & 2052.5 & 2009.0 & 2039.6 \\
\hline & $\Delta$ Tot costs & [EUR] & $-0.4(-0.02 \%)$ & $-7.9(-0.4 \%)$ & $-14.6(-0.7 \%)$ & $-20.8(-1 \%)$ \\
\hline
\end{tabular}

In scenarios TOU1A and TOU1B the ratio between off-peak and peak price of electricity is not low enough so that the shift of energy consumptions compensates the increment of total electricity used according with the concept described in section 3.2. This results in a higher electricity bill in comparison with the reference case. Considering an off-peak electricity price of $0.15 \mathrm{EUR} / \mathrm{kWh}$, the ratio of 0.72 permit to eliminate the difference between the electricity bills resulted from the DR program and the reference case. Savings in the total bill can be achieved through the savings in the thermal bill. In Scenario TOU1A, the savings obtained from the thermal bill with a thermal energy costs of 0.05 EUR/kWh are not enough to compensate the increment in the electricity bill. The resulting higher total bill under the TOU1A scenario shows that the economic boundaries chosen make the DR program economically unfavourable. In scenario TOU2, the savings obtained in the electrical bill are always higher than the additional costs in the thermal bill making this approach always advantageous. Nevertheless, according to the economic boundaries fixed above only a cost saving of $1.8 \%$ in the total bill is achieved in the best scenario due to the limit of the TES capacity and TOU program. 


\section{CONCLUSION}

Demand response programs in fifth-generation district heating and cooling networks achieve economic savings only under properly selected boundary conditions. The increase of the network temperature during the DR program hours permits to operate the heat pump at a higher COP. Saving between 1-2\% are achieved because the energy input to the 5GDHC substation is shifted from the electrical to the thermal bill. Nevertheless, the strategy implemented allows for shifting of electricity consumptions from peak to off-peak hours of about $20 \%$.

The modest savings achieved are due to the limited capacity of the DHW thermal energy storage and of the TOU scheme that activates the demand response program only once a day during working days. Further research is needed to explore the benefit of a real-time pricing demand response programs as well as higher capacities of the thermal energy storage under the same circumstances.

\section{ACKNOWLEDGEMENTS}

This work is part of the research activities of the project FLEXYNETS, funded by the European Union's Horizon 2020 research and innovation programme under grant agreement No 649820.

\section{REFERENCES}

Alasseri, R., Tripathi, A., Joji Rao, T., and Sreekanth, K.J. (2017), A review on implementation strategies for demand side management (DSM) in Kuwait through incentive-based demand response programs. Renew. Sustain. Energy Rev. 77, 617-635.

Faria, P., and Vale, Z. (2011), Demand response in electrical energy supply: An optimal real time pricing approach. Energy 36, 5374-5384.

Fedrizzi, R., Dipasquale, C., Bellini, A., Gustafsson, M., Bales, C., Ochs, F., Demerzentzis, G., Nouvel, R., and Cotrado, M. (2015), FP7 INSPIRE project D6.3c: Performance of the Studied Systemic Renovation Packages - Multifamily Houses.

J.K. Kok, C.J. Warmer, and I.G Kamphuis (1998), PowerMatcher: Multiagent Control in the Electricity Infrastructure. Proceedings of the fourth international joint conference on Autonomous agents and multiagent systems AAMAS '05, Utrecht, NL.

Lund, P.D., Lindgren, J., Mikkola, J., and Salpakari, J. (2015), Review of energy system flexibility measures to enable high levels of variable renewable electricity. Renew. Sustain. Energy Rev. 45, 785-807.

Menegon, D., and Fedrizzi, R. (2016), FP7 INSPIRE project D4.7: Report on laboratory dynamic tests.

Miara, M., Günther, D., Leitner, Z.L., and Wapler, J. (2014), Simulation of an Air-to-Water Heat Pump System to Evaluate the Impact of Demand-Side-Management Measures on Efficiency and Load-Shifting Potential. Energy Technol. 2, 90-99.

Pau, M., Cunsolo, F., Vivian, J., Ponci, F., and Monti, A. (2018), Optimal Scheduling of Electric Heat Pumps combined with Thermal Storage for Power Peak Shaving. Proceedings of the 18th International Conference on Environmentand Electrical Engineering EEEIC, Palermo, IT.

Schibuola, L., Scarpa, M., and Tambani, C. (2015), Demand response management by means of heat pumps controlled via real time pricing. Energy Build. 90, 15-28.

UNI Ente Nazionale Italiano di Unificazione (2010), UNI 9182. Impianti di alimentazione e distribuzione d'acqua fredda e calda - Criteri di progettazione, collaudo e gestione, Milano, IT.

EU H2020 project Sim4Blocks: http://www.sim4blocks.eu/.

EU H2020 FLEXYNETS project: http://www.flexynets.eu/. 


\section{TECHNICAL AND POTENTIAL ANALYSIS OF THERMAL COOLING DISTRICTS IN COLOMBIA}

Carlos Mario Ceballos, Carlos Andrés Gómez, Farid Chejne*, Juan Pablo Rios, Santiago Arango, Ricardo Smith Group of Applied Thermodynamics and Alternative Energies (TAYEA)

Facultad de Minas, Universidad Nacional de Colombia

Carrera 80 \# 65 - 223, M3-214. Medellín, Colombia

Phone: +5744255333

E-Mail: fchejne@unal.edu.co

\section{SUMMARY}

In this work, an analysis of technologies and potential demand for the implementation of Cooling Districts (CD) in Colombia was developed. Simulations of demand sceneries were developed with the LEAP tool. Five configurations for CDs were analysed, and proposals for implementation in different cities were considered. Those configurations considered cogeneration (with natural gas or flare gas), solar PV and Eolic as the main technologies to improve the CDs impacts. One or more technologies were proposed for each one of the thirteen cities studied in this paper, according to the energy basket locally available in each case. From the analysis, it was determined that the largest potentials for the implementation of CDs are in the cities of Barranquilla and Cartagena, as well as in the island of San Andres and Medellin city. It was estimated a total demand in the analysed cities close to 2,300 Million $\mathrm{BTU} / \mathrm{h}$ and an associated potential of decrease of accumulated emissions to the year 2030 of 132,000 tCO2 eq, which represents emission reductions close to $50 \%$ in the last year evaluated.

Keywords: Cooling District, Renewable energy, LEAP, Cooling demand.

\section{INTRODUCTION}

Colombia has an energy basket mainly composed of hydro (70\%) and thermal sources $(29.5 \%)$, and a small portion of renewable sources $(0.5 \%)$ (XM, 2016). According to the Mining and Energy Planning Unit (UPME) report in Colombia, only $13 \%$ of the energy consumed is electric power, whereas $87 \%$ corresponds to thermal energy in various sectors such as the transportation, which is the largest energy consumer (UPME, 2015). Likewise, the industrial and commercial sectors are the second and third largest energy consumers in the country. It is in these two sectors where the highest consumption of cold is observed, whether for air conditioning or for other applications (INCOMBUSTION, 2014).

In this work, they were only considered cooling requirements for air conditioning used in public buildings, hotels, industrial parks (including free trade zones), convention centres, colleges, and universities, as well as urban equipment (airports and sports centres). For this study, cities with more than 200,000 habitants and an average annual temperature above $20^{\circ} \mathrm{C}$ were considered. To calculate the environmental benefits, it was supposed that the current demand for cold is covered with conventional air conditioning systems, either through individual units (room air conditioner or minisplit) or centralized-type air conditioning systems.

In this scenario, the CDs are just beginning to be considered as a real alternative to supply cold and heat in the Colombian energy system. The first CD project developed in Colombia is located at "La Alpujarra" sector in Medellín. It has a capacity of 3,700 tons of refrigeration [RT] and operates with a cogeneration system based on natural gas, which leads to energy savings between $15 \%$ to $20 \%$ (EPM/WB, 2016). 
This type of system (CD) is one of the main alternatives that may allow attaining the commitments acquired by Colombia at COP21. This paper analyses the technological options (equipment and configurations) for the development of CDs in other cities. For this purpose, it will be considered the energy resources available in each region (both renewable and non-renewable), the potential cold demand in 13 cities of the country, and the possible configurations proposed for each combination of local resources and demand. From these evaluations, several configurations applicable in each analysed city are proposed.

\section{POTENTIAL AND ENERGY BASKET}

In the first stage of the project, the supply side of available energy sources in Colombia was analysed. Thus, the installed generation capacity was considered by using the division of regions proposed by the UPME. For this purpose, market information including both conventional (coal, crude, natural gas and hydraulic energy) and non-conventional sources (solar, wind, geothermal, marine, biomass, and small hydroelectric projects) was used. Likewise, the availability of self-generation and cogeneration was analysed. Table 4 presents a historic of the composition of the Colombian energy basket from 2006 to 2016.

Table 4. Historical of installed capacity in electricity generation in Colombia 2006 - 2016 (XM, 2016)

\begin{tabular}{|l|l|c|c|c|c|c|c|c|c|c|c|c|}
\hline SOURCE & COMBUST & $\mathbf{2 0 0 6}$ & $\mathbf{2 0 0 7}$ & $\mathbf{2 0 0 8}$ & $\mathbf{2 0 0 9}$ & $\mathbf{2 0 1 0}$ & $\mathbf{2 0 1 1}$ & $\mathbf{2 0 1 2}$ & $\mathbf{2 0 1 3}$ & $\mathbf{2 0 1 4}$ & $\mathbf{2 0 1 5}$ & $\mathbf{2 0 1 6}$ \\
\hline HYDRIC & Water & 9,368 & 8,947 & 8,991 & 8,997 & 8,997 & 9,044 & 9,718 & 9,777 & 9,875 & 10,900 & 11,533 \\
\hline THERMAL & Coal & 694 & 700 & 700 & 700 & 851 & 851 & 701 & 997 & 997 & 1,003 & 1,353 \\
\hline THERMAL & Diesel & - & - & - & - & - & - & - & 726 & 1,122 & 1,023 & 1,247 \\
\hline THERMAL & Fuel Oil & 187 & 130 & 130 & 187 & 314 & 314 & 187 & 307 & 307 & 297 & 299 \\
\hline THERMAL & Natural Gas & 3,496 & 3,459 & 3,549 & 3,552 & 3,293 & 3,020 & 3,746 & 2,484 & 1,850 & 1,848 & 1,650 \\
\hline THERMAL & JET A-1 & - & - & - & - & - & - & - & 46 & 322 & 322 & 322 \\
\hline THERMAL & Others & - & - & - & - & - & - & - & - & - & - & 6 \\
\hline EOLIC & Wind & 10 & 18 & 18 & 18 & 18 & 18 & 18 & 18 & 18 & 18 & 18 \\
\hline COGEN & Bagasse & 20 & 22 & 22 & 22 & 33 & 53 & 55 & 57 & 61 & 72 & 84 \\
\hline COGEN & Biomass & - & 1 & 1 & 1 & - & - & - & - & - & - & - \\
\hline COGEN & Coal & - & 2 & 2 & 2 & 2 & 2 & 2 & - & 5 & 5 & 9 \\
\hline COGEN & Natural Gas & 1 & 1 & 1 & - & - & - & - & - & - & - & - \\
\hline & TOTAL & & & & & & & & & & & \\
& IMW] & $\mathbf{1 3 , 7 7 5}$ & $\mathbf{1 3 , 2 8 0}$ & $\mathbf{1 3 , 4 1 4}$ & $\mathbf{1 3 , 4 7 9}$ & $\mathbf{1 3 , 5 0 9}$ & $\mathbf{1 3 , 3 0 3}$ & $\mathbf{1 4 , 4 2 7}$ & $\mathbf{1 4 , 4 1 3}$ & $\mathbf{1 4 , 5 5 9}$ & $\mathbf{1 5 , 4 8 9}$ & $\mathbf{1 6 , 5 2 2}$ \\
\hline & Hydric & $68.01 \%$ & $67.37 \%$ & $67.03 \%$ & $66.75 \%$ & $66.60 \%$ & $67.99 \%$ & $67.36 \%$ & $67.83 \%$ & $67.83 \%$ & $70.37 \%$ & $69.81 \%$ \\
\cline { 2 - 10 }$y$ & Thermal & $31.77 \%$ & $32.29 \%$ & $32.64 \%$ & $32.93 \%$ & $33.00 \%$ & $31.46 \%$ & $32.12 \%$ & $31.64 \%$ & $31.59 \%$ & $29.01 \%$ & $29.51 \%$ \\
\cline { 2 - 10 }$y$ & Others & $0.22 \%$ & $0.33 \%$ & $0.33 \%$ & $0.32 \%$ & $0.40 \%$ & $0.55 \%$ & $0.52 \%$ & $0.53 \%$ & $0.58 \%$ & $0.62 \%$ & $0.68 \%$ \\
\hline
\end{tabular}

In addition to the installed capacity, there are projects that will increase the electric power in about 3,622 MW including thermal and hydraulic (XM, 2015), and projects approved by the UPME in nonconventional sources for a total of 2,998 MW (UPME, 2016). All the installed capacity to the date together with new projects that take advantage of primary energy potentials may be exploited by CDs.

In a second component, the potential of CDs in Colombia was evaluated for cities of the country that met two criteria: more than 200,000 habitants, and average annual temperature larger than $20{ }^{\circ} \mathrm{C}$. Then, the cities belonging to the metropolitan areas (or are in a conurbated region) were grouped under the main city that constitutes the area. Some cities were discarded due to structural difficulties for the 
implementation of this type of systems. Finally, a list of 13 cities was obtained: Cartagena, Medellin, Santa Marta, Barranquilla, Monteria, Bucaramanga, Cali, Pereira, Ibagué, Neiva, Villavicencio, Cúcuta and its respective metropolitan areas, as well as the island of San Andrés.

After selecting the cities, strategic sectors demanding cold in large quantities and that can be attached to a CD configuration were defined. These sectors are the following:

- Industrial: includes industrial and commercial parks, as well as free trade zones. In these spaces, the administrative areas (offices) that may demand refrigeration are considered.

- Public buildings: in general, in this category have been grouped the buildings where the local or regional government offices are located.

- Hotel: the areas to be cooled correspond to the rooms and common covered areas, such as lobbies, corridors and restaurants. It only includes hotels duly registered.

- Educational: includes the buildings of educational centres such as schools and universities. The specific areas to be cooled correspond to the classrooms and administrative offices.

- Hospital: a set of hospitals and clinics (in terms of the number of offices). The areas to be cooled correspond to the rooms, operating rooms, intensive care units (ICU), corridors, and common indoors areas.

- Convention centres: are all those places that are used for events, congresses, conferences or meetings.

- Urban equipment: includes sports centres such as stadiums, sports units and transport terminals (airports and bus terminals). The spaces to be refrigerated in each of these enclosures are administrative offices.

The geographical areas where the demand potentials for each sector are concentrated are determined from maps by using criteria of continuity, proximity and density. Both the average temperature of the different geographical areas and the constructed area to be cooled were estimated from public and secondary information sources. From this information, estimates of cold demand were made for each selected city. The estimation of the cooling power in each sector, according to the built area, is made considering the following equation

$P_{\text {Total }}=P_{(T)} \times A+C$

where $\boldsymbol{P}_{\text {Total }}$ is the total energy that must be supplied to a space per unit of time $[B T U / h] . \boldsymbol{P}_{(\boldsymbol{T})}$ is the cooling power that must be supplied to a space per unit of area and time, which varies according to the ambient temperature, $\left[B T U / \mathrm{hm}^{2}\right]$. $\boldsymbol{A}$ is the area to be cooled in $\left[\mathrm{m}^{2}\right]$, and $\mathbf{C}[B T U / h]$ is the thermal load, that is, it is the additional power that must be supplied to the space to be cooled due to the presence of people or objects in it.

The cooling power is defined according to the climate and the temperature range. In cities with temperatures below $18{ }^{\circ} \mathrm{C}$, the cooling capacity is $500 \mathrm{BTU} / \mathrm{h} \mathrm{m}^{2}$. In this way, power is established for the other climates: warm, hot and very hot, according to Fehler! Verweisquelle konnte nicht gefunden werden.. It should be noted that these ranges and powers are defined considering aspects to guarantee the comfort temperature, according to ASHRAE recommendations. The thermal load is the additional power that must be supplied to a space for compensating the irradiation of heat by the people or objects that are in there. For calculations, the thermal load will be considered as in Table 6 (Air Shop, 2011) 
Table 5. Cooling power per unit area and time in each temperature range

\begin{tabular}{|c|c|c|c|}
\hline Climate & $\operatorname{Min}\left({ }^{\circ} \mathbf{C}\right)$ & $\operatorname{Max}\left({ }^{\circ} \mathbf{C}\right)$ & $\mathbf{B T U} / \mathbf{h m}^{2}$ \\
\hline Cold & - & 18 & 500 \\
\hline Warm & 19 & 25 & 550 \\
\hline Hot & 26 & 33 & 600 \\
\hline Very hot & 34 & - & 650 \\
\hline
\end{tabular}

Table 6. Thermal load [BTU / h] associated with different objects in the spaces to be cooled

\begin{tabular}{|l|c|}
\hline Object & BTU/h \\
\hline Person & 500 \\
\hline Computer & 400 \\
\hline Conventional TV & 600 \\
\hline Halogen bulb & 400 \\
\hline Flat TV & 400 \\
\hline
\end{tabular}

Additional assumptions were made to determine the estimated cooling power (demand) of each sector. In the hotel sector, it was made an estimate of $35 \mathrm{~m}^{2}$ of area per room plus $20 \%$ related to the common areas of the hotel (corridors and lobby); and a thermal load per room of 3 people, 3 bulbs, 0.5 computers, and $1.5 \mathrm{TVs}$. For the hospitals, areas of $25 \mathrm{~m}^{2}$ per room, $37 \mathrm{~m}^{2}$ per operating room, and 15 $\mathrm{m}^{2}$ per ICU were used. For the industrial parks, it was estimated that only $10 \%$ of the total areas of the industrial parks or free trade zones are considered for cooling. Data from building plans, offered rooms, news and others were used to estimate the areas and the required cold power. From this information, the cooling demand per sector was calculated in each city, which is shown in Table 7. It was calculated a total demand for the 13 cities of 196,900 RT (2,360 MBTU/h).

Table 7. Summary by sectors of the cooling capacity (in thousands of tons of refrigeration) per city

\begin{tabular}{|c|c|c|c|c|c|c|c|c|}
\hline City & Industrial & Government & $\begin{array}{c}\text { Conventions } \\
\text { Centres }\end{array}$ & $\begin{array}{c}\text { Urban } \\
\text { Equipment }\end{array}$ & Hotels & Education & Hospitals & TOTAL \\
\hline Barranquilla & 8.8 & 1.4 & 0.8 & 0.2 & 9.7 & 3.6 & 2.2 & 26.7 \\
\hline Bucaramanga & 1.5 & 1.5 & 0.2 & 0.5 & 5.4 & 1.1 & 2 & 12.2 \\
\hline Cali & 1 & 1.7 & 0.8 & 1.7 & 9.2 & 6.3 & 3.3 & 24.2 \\
\hline Cartagena & 2.1 & 1.4 & 1 & 0.2 & 13.5 & 5 & 2.8 & 25.9 \\
\hline Cúcuta & 0.2 & 0.7 & 0.1 & 2.2 & 2.7 & 3 & 1.3 & 10.3 \\
\hline Ibagué & 0.1 & 0.8 & 0 & 1.1 & 2.7 & 3.7 & 2.5 & 10.9 \\
\hline Medellín & 1.3 & 1 & 1.3 & 3.5 & 13 & 9.9 & 7.2 & 37.2 \\
\hline Montería & 0 & 0.3 & 0.1 & 0.2 & 2.6 & 1.5 & 0.3 & 5.1 \\
\hline Neiva & 0.3 & 1.1 & 0.4 & 0.8 & 2.4 & 0.8 & 1.8 & 7.6 \\
\hline Pereira & 0.6 & 0.5 & 0.3 & 0.7 & 2.1 & 3 & 0.8 & 7.9 \\
\hline San Andrés & - & 0.3 & 0.1 & 0.3 & 6.9 & 0.1 & 0.9 & 8.5 \\
\hline Santa Marta & 0.6 & 0.2 & 0.3 & 0.1 & 7.9 & 1 & 3.9 & 13.9 \\
\hline Villavicencio & 0.2 & 0.3 & 0 & 0.8 & 2.9 & 2 & 0.3 & 6.5 \\
\hline
\end{tabular}

The areas and cooling demands calculated for each potential district are used in the projection models developed with the LEAP tool. LEAP is a tool allowing to simulate models with "bottom-up" kind technical coefficients. Two scenarios were simulated: the trend scenario known as BAU (Business as Usual), and the CD scenario where the different thermal districts for each city are tested. A different model was performed for each city, i.e., each city has a BAU scenario and a CD scenario. The results of the simulation in the $\mathrm{CD}$ scenario show the projection of cold demand for the cities of the Caribbean region (Santa Marta, Barranquilla, Monteria and Cartagena), intermediate cities (Villavicencio, Neiva, Pereira, Ibagué, Cúcuta and Bucaramanga), for the big cities (Cali and 
Medellín) and for San Andrés Island. This information is used as input data to calculate the capacity that must be installed in each thermal district belonging to each city.

In order to model the offer, the projection of demand was taken into account, and two basic CDS packages were designed to facilitate the calculations. The first one was designed for systems demanding 6,800 RT per hour or more, whereas the second package is designed to meet demands between 1,200 RT and 6,800 RT. The first package consists of a Taurus T60 Solar turbine and eight units of Carrier electric compression chiller. The second package has an SDMO GXC1200 with MTU internal combustion engine 12V4000L62 and two units of Carrier electric compression chiller Aquaforce series.

These two configurations were designed for particular cases, implying that it is possible to obtain configurations with higher performances or efficiencies. The proposed technologies consume electricity and natural gas. Regarding GEI emissions, it should be noted that it was specified for the model that conventional refrigeration systems use R11, R22 and HFC 134 as the refrigerant, whereas the model for CDs employ R134a. According to the results obtained from the LEAP model (see Figure 3 ), the possible thermal districts by city appear aggregated in circles due to that it is necessary that the users be near for the conformation of the $\mathrm{CD}$. It is estimated that for a user to be profitable on a $\mathrm{CD}$, he must demand at least $1 \mathrm{RT}$ every $800 \mathrm{~m}$ of distance from the CD.

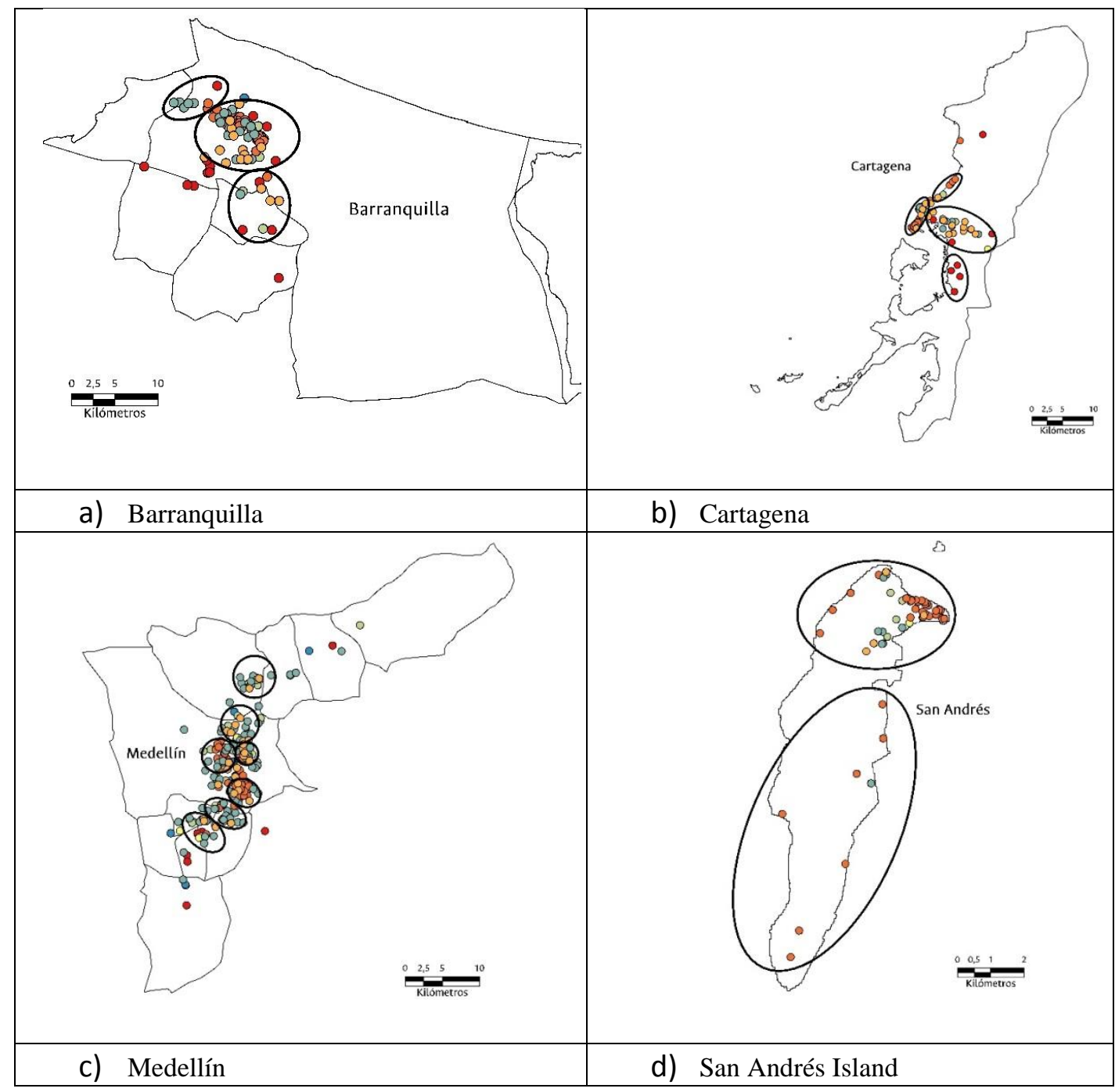

Figure 3. Demand distribution and groupings for CD design in cities. 
Considering the geographic distribution of the demand, as well as the distance restrictions of the CDs, it was found that the cities with the largest potential for implementing profitable CDs are Barranquilla (North and Puerto Colombia - 19 RT), Bucaramanga (central - 9 RT), Cali (North Cali - 8 RT), Cartagena (Boca Grande and historic centre - 14 RT), Medellín (The town - 10 RT), and San Andrés island (North - 6 RT). Although the latter is small in comparison to the others, San Andrés island has some special characteristics because it is the only city analysed that is not connected to the Interconnected National System (SIN for its acronym in Spanish) and represents a significant potential for saving energy as well as an important solution for the island. Illustratively, Figure 3 shows the geographical distribution analysed for Barranquilla, Cartagena, Medellín, and San Andrés island.

\section{TECHNOLOGICAL ANALYSIS}

The analysis of resources and technological configurations were made simultaneously. Two technologies are considered for refrigeration: steam compression equipment with an electric motor, and absorption refrigeration equipment allowing the use of thermal energy. In CDs, cogeneration is used to generate electricity either for sale or to be used in electric chillers, and the heat can be used for the generation of either steam or hot water that feeds an absorption chiller. Those technologies were related to the available resources using the sunshine and wind maps form IDEAM and UPME, and five possible configurations were proposed.

\begin{tabular}{|l|l|}
\hline A. SEA WATER AIR CONDITIONING (SWAC) & $\begin{array}{l}\text { Option A considers the use of deep ocean seawater for } \\
\text { air conditioning. Water located } 80 \text { meters deep is } \\
\text { approximately at } 4{ }^{\circ} \mathrm{C} \text {. This system comprises to pump } \\
\text { the deep water and use it directly as a cooling fluid for } \\
\text { the CD. Then, the water is deposited back to the sea } \\
\text { over the surface. The operating cost of this system is } \\
\text { mainly related to the water pumping. }\end{array}$ \\
\hline B. COOLING WITH RENEWABLE ENERGIES & $\begin{array}{l}\text { Option B considers a system using steam compression } \\
\text { chillers and absorption chillers. This configuration } \\
\text { works by using electricity from wind energy and solar } \\
\text { PV, and with energy from SIN as the backup. For } \\
\text { absorption chillers, the use of hot water from thermal } \\
\text { solar energy or hot water coming from industrial } \\
\text { processes is proposed. }\end{array}$ \\
\hline C. NG COGENERATION AND SOLAR PV
\end{tabular}




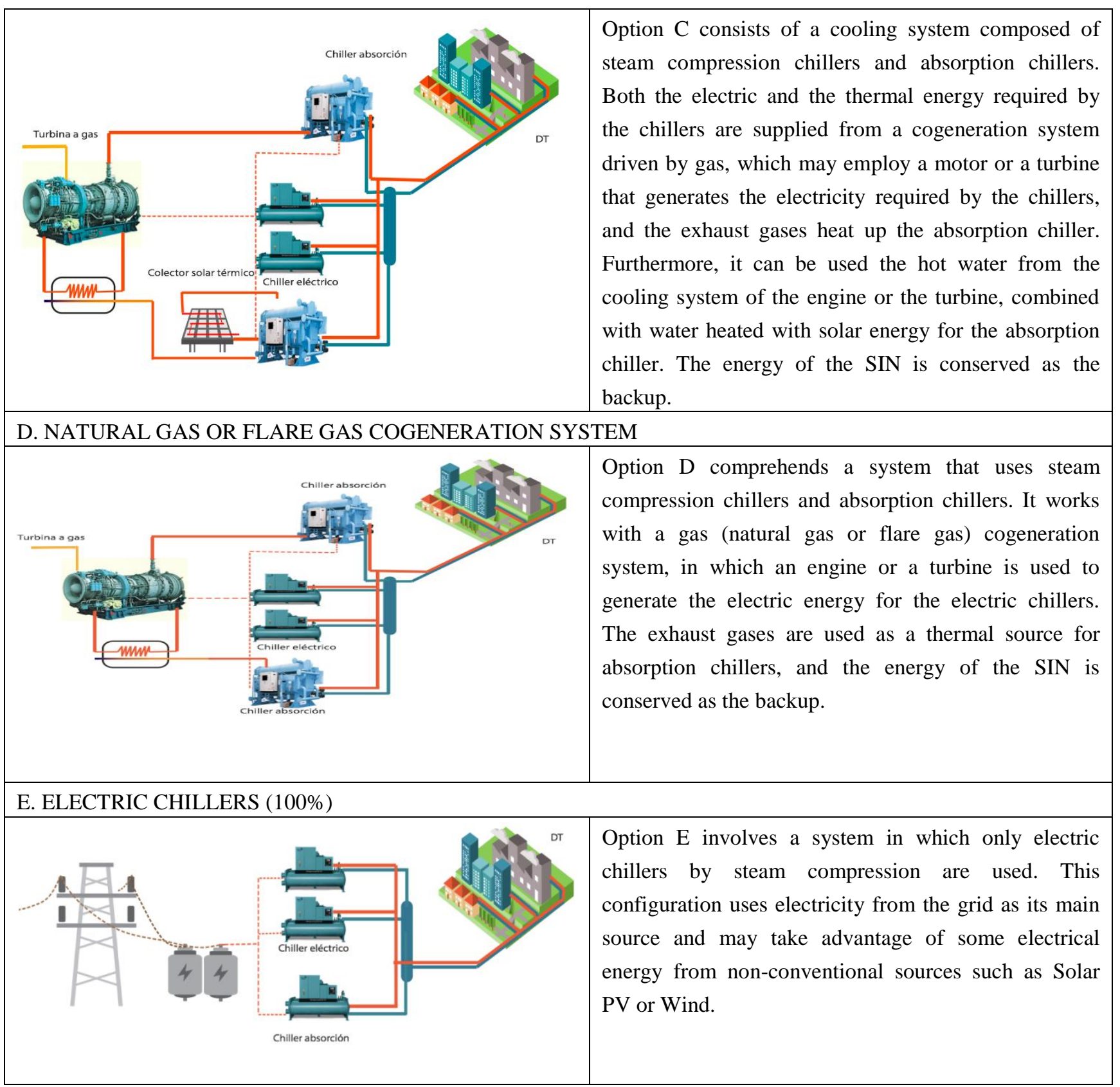

Figure 4. Configuration options for the cooling production system in the cooling districts.

According to the cross-analysis, it was found that for each region there are more interesting options according to the available energy. Thus, the following options were defined for each city as the most interesting to analyse in the implementation of CDs. A, B, C and E for Barranquilla; D and E; Cali D and $\mathrm{E}$ for Bucaramanga; $\mathrm{C}$ and $\mathrm{E}$ for Cartagena; $\mathrm{C}$ and $\mathrm{E}$ for Cúcuta; $\mathrm{C}$ and $\mathrm{E}$ for Ibagué; $\mathrm{D}$ and $\mathrm{E}$ for Medellín; C and E for Montería; C and E for Neiva; A, C and E for Santa Marta, C and E for Villavicencio, and A, B and E for San Andrés island.

Another fundamental equipment is the Transfer Station (TS). In order to deliver cold, TS can be a heat exchanger or Air Conditioned Handling Units (AHU). In a direct connection system, the cooling district water is distributed to the user building directly to terminal equipment such as AHU, "fan coil" units, induction units, etc. An indirect connection system uses one or multiple heat exchangers between the CD distribution systems and the building. In the analysis of this component, there was a significant deficiency in the supply chain for Colombia, since there are no direct representatives of the 
most recognized brands worldwide. Therefore, it is necessary an international search of the required systems or using local workshops with tailored designs; however, both of them are a risk for CDs projects in Colombia.

\section{ANALYSIS OF ENVIRONMENTAL IMPACTS}

By quantifying demand and supply, it is possible to determine the potential for emissions reduction. To do this, the results of the trend scenario (BAU) must be compared with the mitigation scenario. With the help of the LEAP tool, the potential for emissions avoided by introducing thermal districts in the country was analysed. Figure 5 describes the results of the BAU scenario in contrast to the DT scenario.

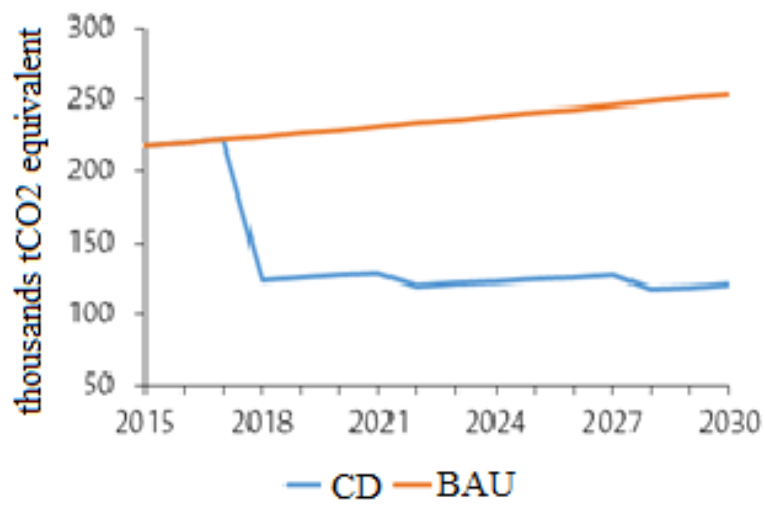

Figure 5: Projection of the equivalent $\mathrm{CO}_{2}$ emissions in the BAU Vs CD scenarios in Colombia 2015-2030.

To sum up, the CD scenario presents a reduction in greenhouse gas emissions with respect to the BAU: an accumulated reduction of 1,500 kTon of $\mathrm{CO}_{2}$ equivalent for 2030 was estimated. Additionally, by comparing both scenarios at the end of the simulation, it was found that the specific reduction for the last year of the CD scenario compared to the BAU scenario is larger than $50 \%$.

The implications of this reduction in emissions are: (i) contribute to achieving the goal set by the government with COP 21-2030 (Government of Colombia, 2015); (ii) contribute to the reduction of ozone depleting substances, which is one of the commitments acquired from Colombia in the Montreal Protocol; and (iii) to promote the international support due to the positive impacts on the environment.

\section{CONCLUSIONS}

There is a calculated potential of $196,900 \mathrm{RT}(2,360 \mathrm{MBTU} / \mathrm{h})$ for the implementation of CDs in Colombia. Barranquilla, Cartagena, and Santa Marta were the cities of the north coast with higher potential and the favourable conditions to implement cooling districts. Furthermore, the cooling district in San Andrés island implies extra benefits related to the energy efficiency, due the island energy is produced from diesel, and the CD would diminish its requirements. Medellín is recognised worldwide as the most innovator city, and the potential keeps being higher to install at least four CDs with about 24,000 RT.

The large potential for cooling districts in most of the cities implies that both the use of renewable resources and cogeneration are the main technologies to improve the environmental effect of CDs implementation. It was estimated for the two configurations (cogeneration) of CDs evaluated an 
accumulated diminution of $1,500,000 \mathrm{tCO}_{2}$ equivalent for 2030, and a diminution for the last year (2030) larger than 50\% respect to the BAU scenario. The use of renewable resources would improve these estimations.

The island of San Andrés is one of the cities for prioritizing the execution of CDs due to the special characteristics that it presents. For instance, nowadays energy in San Andrés island is generated from fossil fuels and there is a significant potential for non-conventional energy sources (solar and wind energy), and the possible use of deep sea waters for its implementation in CDs.

\section{REFERENCIAS}

Air Shop. (2011). Cómo Calcular su Aire Acondicionado adecuado. Adapted from: https://www.youtube.com/watch?v=-iKuGo NhIg

EPM/WB. (2016). District cooling for efficient energy. Medellín: World Bank Group.

INCOMBUSTION. (2014). Report of project "Determinación del potencial de reducción del consumo energético en los subsectores manufactureros códigos CIIU 10 a 18 en Colombia”. Medellín

UPME. (2015). Plan energético nacional Colombia: Ideario Energético 2050. From: https://goo.gl/UjQTFZ

UPME. (June 2016). Registro de Proyectos de Generación. Visited 20 ${ }^{\text {th }}$ July 2016, on http://goo.gl/9o9Gdd

XM. (2015). Capacidad efectiva neta. (XM) Visited $10^{\text {th }}$ July 2016, from http://goo.gl/i263Ef

XM. (2016). Capacidad efectiva por tipo de generación. (XM) Visited $18^{\text {th }}$ Aug 2016, from http://goo.gl/sdzdwW

\section{ACKNOWLEDGEMENT}

The authors which to thank to "Empresas Públicas de Medellín - EPM" who founded the project "Characterize the supply and demand of thermal energy services in Colombia; and evaluate the technical and economic substitution options from the perspective of implementing Thermal Districts projects" and to the project "Feasibility study of water heating through energy integration of solar energy and heating by submerged pipes in the National Chocolates Company" developed by U.T. INCOMBUSTION and financed by COLCIENCIAS, contract No. FOP44842-474-2016. 


\title{
SMALL HEATING GRIDS FOR COMMUNITIES IN BALKAN COUNTRIES
}

\author{
DI(FH) DI Christian Doczekal \\ Güssing Energy Technologies GmbH \\ Wiener Straße 49, 7540 Güssing, Austria \\ Phone: +43 332242606331 \\ E-Mail: c.doczekal@get.ac.at \\ co-author: DI Dominik Rutz, M.Sc., WIP Renewable Energies, Germany
}

\section{SUMMARY}

The CoolHeating project promotes the implementation of "small modular renewable heating and cooling grids" (SMDHC) for communities in South-Eastern Europe. CoolHeating transfers knowledge from partners in countries where renewable district heating and cooling examples exist (Austria, Denmark, Germany) to countries where there are less examples in the sector (Croatia, Slovenia, Macedonia, Serbia, Bosnia-Herzegovina). Core activities, besides techno-economical assessments, include measures to stimulate the interest of communities and citizens to set-up renewable district heating systems (DH) as well as the capacity building about financing and business models. The outcome is the initiation of new small renewable district heating and cooling grids in 5 target communities up to the investment stage. This abstract presents 7 technical concepts for the implementation of SMDHC in Balkan countries, as well as their economy and the feasibility.

\section{TECHNICAL CONCEPTS}

The rural settlement of Cven in Slovenia has 226 households and a few larger public. All public buildings should be connected to the DH grid, as well as $90 \%$ of the households. The technical concept considers a small biomass CHP (combined heat and power) unit with $112 \mathrm{~kW}$ el for the baseload, a $800 \mathrm{~kW}$ biomass boiler and a $2.2 \mathrm{MW}$ natural gas peak load boiler. A buffer storage tank could decrease the peaks after night setback time in the morning, when most households start heating again. Biomass (e.g. wood chips) is available in this region.

The municipality of Ljutomer in Slovenia selected the industrial zone for developing a DHC project. A biomass CHP with $448 \mathrm{kWel}$, a $2 \mathrm{MW}$ biomass boiler, a $4 \mathrm{MW}$ natural gas peak load boiler, a $90 \mathrm{~m}^{3}$ buffer storage tank and a 1.2 MW biomass steam boiler is considered. This scenario also covers the cooling needs from a dairy with an absorption chiller, operated with the DH grid.

The technical concept for the city of Ozalj in Croatia includes a $25 \mathrm{MW}$ biomass boiler, $30,000 \mathrm{~m}^{2}$ flat plate solar collectors and a fuel oil peak load boiler. The solar collectors are in combination with a $40,000 \mathrm{~m}^{3}$ seasonal thermal storage, the biomass boiler with a $300 \mathrm{~m}^{3}$ thermal storage.

Air quality during the heating season is quite bad in Visoko (Bosnia and Herzegovina) due to heavy use of coal for heating. Existing heating systems are mainly individual and currently dominated by coal as the cheapest energy source on the market. Therefore, the concept plans a new DH grid using a $6.3 \mathrm{MW}$ heat pump (from the river) as well as $5,000 \mathrm{~m}^{2}$ solar thermal collectors in combination with a $13,500 \mathrm{~m}^{3}$ seasonal storage, plus a natural gas peak load boiler. 150 private houses, 30 collective housing facilities and 6 public buildings could be connected.

The concept for Letnjikovac in Šabac (Serbia) could include a biomass boiler with $1.5 \mathrm{MW}$ and a 3.5 MW fuel oil boiler to connect public buildings and about 248 households. The feasibility study could show if this DH grid is economic, due to the low grid density.

Šabac in Serbia has an existing district heating system, using natural gas boilers. The concept for the implementation of renewable energy in the DH grid Šabac could include three biomass boilers with 4.5 MW nominal capacity each. This could lead to about $61 \%$ coverage of the annual heat demand with renewable energy.

The new area Zajcev Rid in Karposh (Macedonia) could be connected to a DH grid, using a $15 \mathrm{MW}_{\text {th }}$ ground water heat pump, $5,000 \mathrm{~m}^{2}$ solar thermal collectors, in combination with a $55,000 \mathrm{~m}^{3}$ seasonal storage, plus a fuel oil peak load boiler. Using the DH grid for cooling in summer (15 $\mathrm{MW}$ th electr. chiller) could also be an option.

Table 1 gives an overview of the 7 calculated technical concepts. 
Table 1: Technical data of the 7 technical DHC concepts in the CoolHeating target municipalities

\begin{tabular}{|c|c|c|c|c|c|c|c|}
\hline & 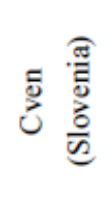 & 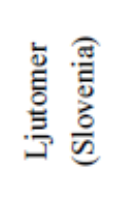 & 预 & 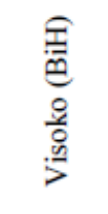 & 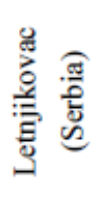 & 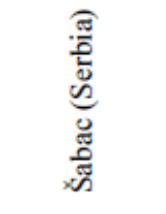 & 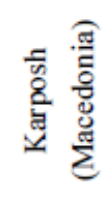 \\
\hline $\begin{array}{l}\text { DH grid length incl. consumer connections } \\
{[\mathrm{m}]}\end{array}$ & 3,400 & 4,175 & 16,586 & 5,500 & 7,656 & existing & 9,500 \\
\hline Grid density [kWh/m/a] & 934 & 3,524 & 3,873 & 3,482 & 462 & & 4,467 \\
\hline Annual heat losses of the $\mathrm{DH}$ grid [\%] & 19 & 6 & 5 & 6 & 17 & & 11 \\
\hline Annual heat losses of the DH grid [MWh/a] & 745 & 915 & 3,029 & 1,129 & 738 & & 5,400 \\
\hline Consumer needs heat $[\mathrm{GWh} / \mathrm{a}]$ & 2.24 & 10.94 & & 17 & 3.54 & & 42.44 \\
\hline $\begin{array}{l}\text { Heat for wood drying for CHP operation } \\
\text { [GWh/a] }\end{array}$ & 0.94 & 3.77 & & & & & \\
\hline Total heat for $\mathrm{DH}$ grid incl. losses [GWh/a] & 3.93 & 15.63 & 66.41 & 18.13 & 4.28 & $\begin{array}{c}37.69 \text { from } \\
\text { biomass }\end{array}$ & 47.84 \\
\hline $\begin{array}{l}\text { Temperature level for } \mathrm{DH} \text { grid, flow/return } \\
{\left[{ }^{\circ} \mathrm{C}\right]}\end{array}$ & $90 / 65$ & $100 / 70$ & $90 / 65$ & $80 / 60$ & $90 / 65$ & $110 / 60$ & $70 / 40$ \\
\hline DH grid operation in summertime & yes & yes & yes & no & no & no & cooling \\
\hline $\begin{array}{l}\text { CHP gross electr. production }\left[\mathrm{kW}_{\mathrm{p}} /\right. \\
\mathrm{MWh} / \mathrm{a}]\end{array}$ & $\begin{array}{c}112 / \\
918 \\
\end{array}$ & $\begin{array}{l}448 / \\
3,673 \\
\end{array}$ & & & & & \\
\hline Share of total heat for DH from CHP [\%] & 48 & 48 & & & & & \\
\hline $\begin{array}{l}\text { Share of total heat for } \mathrm{DH} \text { from biomass } \\
\text { boiler [\%] }\end{array}$ & 49 & 51 & 72 & & 91 & 61 & \\
\hline $\begin{array}{l}\text { Share of total heat for } \mathrm{DH} \text { from solar } \\
\text { thermal }[\%]\end{array}$ & & & 24 & 16 & & & 11 \\
\hline $\begin{array}{l}\text { Share of total heat for } \mathrm{DH} \text { from heat pump } \\
{[\%]}\end{array}$ & & & & 79 & & & 88 \\
\hline $\begin{array}{l}\text { Share of total heat for DH from fossil peak } \\
\text { boiler [\%] }\end{array}$ & 3 & 2 & 4 & 5 & 9 & 39 & 1 \\
\hline Thermal storage $\left[\mathrm{m}^{3}\right]$ & 50 & 90 & 40,000 & 13,500 & 60 & 200 & 55,000 \\
\hline
\end{tabular}

\section{REFERENCES}

Doczekal, C. et al. (2018), Heating/cooling demand and technical concepts for district heating/cooling - Report elaborated in the CoolHeating project (Project-No. 691679), www.coolheating.eu

\section{CONCLUSION}

Seven technical concepts for the implementation of SMDHC in South-Eastern Europe were developed with different types of heat generation units and fuels, based on the local border conditions. The calculated total share of renewable energy of these grids is $61 \%$ to $99 \%$. Three concepts consider seasonal storage with up to $55,000 \mathrm{~m}^{3}$. 


\title{
FEASIBILITY OF HEAT PUMPS SUPPLYING DISTRICT HEATING SYSTEMS - CASE STUDY FOR AUSTRIA AND DENMARK
}

\author{
Wiebke Meesenburg1, Roman Geyer2, Olatz Terreros2, Henrik Pieper1, Torben Ommen1, Brian Elmegaard 1 \\ 1 Technical University of Denmark, Department of Mechanical Engineering, Section of Thermal Energy \\ Nils Koppels Allé 403, 2800 Kongens Lyngby \\ E-mail:wmeese@mek.dtu.dk \\ 2 AIT Austrian Institute of Technology GmbH, Integrated Energy Systems Group, Giefinggasse 4, 1210 Wien, \\ E-Mail: Roman.Geyer@ait.ac.at
}

\section{SUMMARY}

The frame conditions for large-scale heat pumps (HPs) in district heating (DH) systems were studied for Denmark and Austria. While large-scale HPs are becoming more and more often implemented as DH supply units in Denmark, examples from Austria are rare. An economic analysis was conducted for both countries, comparing DH solutions based on either large-scale heat pumps or wood-fired heat only boilers to individual HPs. The results showed that large-scale HPs were beneficial compared to individual units down to linear heat demand densities of $0.85 \mathrm{MWh} / \mathrm{m} / \mathrm{a}$ for Denmark and $0.97 \mathrm{MWh} / \mathrm{m} / \mathrm{a}$ for Austria. The levelized cost of energy of central HPs could compete with wood-fired boilers especially for low DH temperatures $\left(60{ }^{\circ} \mathrm{C} / 30{ }^{\circ} \mathrm{C}\right)$. From a socioeconomic perspective HPs were beneficial compared to woodfired

boilers. In Austria the private economic feasibility of wood-fired boilers benefits from subsidies, which showed to decrease the competitiveness of large-scale HPs.

\section{INTRODUCTION}

Recently, DH has been in the focus of research as it is expected to play a key role in the transition to more energy-efficient and fossil fuel free energy supply systems [1]. DH enables exploitation of large-scale heat sources that cannot be exploited using individual units. Further, DH has also been identified as a key element in integrated energy systems [2]. This integration can be achieved using large-scale power-toheat units, such as HPs which use electricity to supply heat and might also help to balance the power grid [3]. Large-scale HPs represent an efficient way of heat supply using electricity in combination with low temperature sources, such as groundwater, seawater or air. Centrally supplied systems are well suited for areas with high heat demand densities, i.e. densely built areas and houses with space heating (SH) and domestic hot water (DHW) demand. The feasibility of centrally supplied systems is less certain in new areas with low energy demand buildings as well as in less dense areas.

Austria and Denmark both have high shares of renewable power production [4]-[5] and the use of DH is well established in urban and rural areas [6]-[7]. However, the boundary conditions and the current policies in place are very different. Despite the fact that both countries have high shares of renewable power production, the generation mix of both power and heating are different, compare Figure 1.
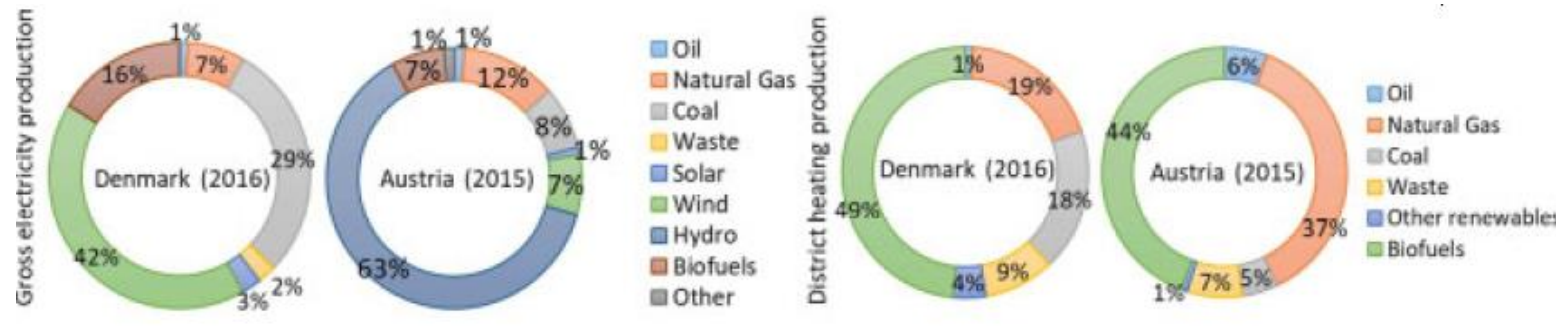

Figure 1 Composition of electricity generation and DH supply in Denmark and Austria [4], [5], [36]

This is mainly related to the geographical and meteorological conditions being different. The mountainous region in Austria provides favourable conditions for hydro power plants and just below half of the Austrian area is forestland, i.e. large resources of biomass are available. The Danish geography is especially suited for power generation from wind. The main source of biomass in Denmark is municipal waste, by-products from agricultural activities, like straw and manure, and wood-based biomass, of which ca. $50 \%$ is imported [8]. An increase of biomass usage in Denmark would result in higher shares of imported biomass [9], which implies the risk of promoting non-sustainable land usage in other countries. 
As Denmark aims to achieve independency of fossil fuels, the available biomass resources might be needed in other sectors. Renewable electricity generation from non-biomass-based sources is thus expected to play a key role in the transition towards renewable supply of heat and electricity. To accommodate high shares of transient electricity generation in the energy system, the idea of an integrated energy system, utilizing synergies between different energy sectors is promoted in Denmark (e.g. [2]). This strategy has been widely acknowledged and current research and demonstration projects, and recent policy changes [10] undergird the vision of an integrated energy system. The integrated energy strategy for Austria is still in the green paper phase [11].

In both countries DH systems are common in cities and in rural areas. In Austria biomass-based DH systems are most common in rural areas. Biomass-based small-scale DH systems were supported as a subsidy for farmers, who often also own forestland in Austria, thereby exploiting synergies of sustainable forest management and creating an additional income to the farmers [12]. Nowadays, the funding has been harmonized and is available to communities and companies under the domestic environmental support scheme [13] and a quality management scheme is in place [14]. Financial support is available for heating networks and for industrial waste heat utilization for DH purposes, which can lead to indirect support of large-scale HPs. HP-specific subsidies are only available for self-supply and the use of biomass is still strongly promoted. A lack of information and dissemination has been identified as a further barrier for large-scale HPs for DH in Austria [15].

In Denmark, most rural DH networks were developed after the first oil crisis and were usually based on natural gas-fired units. DH companies in Denmark are mostly cooperatives or municipality-owned and so directly or indirectly owned by the customers [16]. They are obliged to work on a non-profit basis and a national benchmark system is in place, ensuring that the most cost-effective and environmental friendly solutions are promoted [17]. In combination with dedicated policy measures [17]-[18] this has led to high penetration of DH in Denmark (64 \% in 2016 [4], compared to $25 \%$ in Austria [19]). Today, many of the original natural gas units have been replaced by biomass-fired plants, and increasingly solar thermal plants and HPs. The diffusion of large-scale HPs benefits from research and demonstration projects, collection and dissemination of knowledge [20], and targeted tax-reduction and financial support, e.g. [10], [21].

Within this work the private and socio-economic feasibility of large-scale heat pumps supplying DH was analysed compared to supply from individual units and to wood-fired heat only boilers in Austria and Denmark. The aim was to identify beneficial boundary conditions and barrier for the implementation of large-scale HPs as supply units for DH.

\section{METHOD}

The feasibility of different integration possibilities of HPs into DH systems was assessed for Denmark and Austria using the district heating assessment tool (DHAT) published by the Danish Energy agency [22]. The DHAT is an MS Excel based tool that can be used to calculate the economic feasibility of establishing DH in areas currently supplied by individual units. The tool includes technical data and price projections that are adjustable for different countries. It includes a cost-benefit analysis for the local society, customers, and the DH company. It calculates the socioeconomic feasibility of the project according to Danish guidelines [23] and the levelized cost of energy (LCOE), including the total DH network cost, and investment, fuel and O\&M cost for the supply units.

The economic evaluation for the local society refers to all entities involved in the project, while the socioeconomic evaluation refers to the respective country [22]. The analysis takes the socioeconomic cost of fuels, investment and emissions into consideration, while assuming that the project itself does not have an impact on the electricity or fuel price level or the labour market. The socioeconomic as well as the local society net present value (NPV) were calculated as the difference between the net present value of the DH solution and the best individual supply solution.

The DHAT as published by the Danish Energy Agency was extended to represent the HP coefficient of performance (COP) and cost in more detail to account for the characteristics of different heat sources. An estimation of the network cost and the heat losses were included to account for different DH temperatures.

The analyses focussed on newly developed areas without the possibility to be connected to an existing grid. The feasibility was assessed for varying plot ratios, i.e. the ratio between the built space area and the land area supplied by DH, and thus linear heat demand densities (LHDD). The supplied area was fixed to $340,000 \mathrm{~m}$. Five central unit types were considered - air source HP, groundwater HP, sewage water HP, wood pellet and wood chips boiler. Different DH forward and return temperature cases $\left(80 / 50{ }^{\circ} \mathrm{C}\right.$, 
$70 / 40^{\circ} \mathrm{C}, 60 / 30{ }^{\circ} \mathrm{C}$ ) were analysed for two heat demand scenarios. The $\mathrm{DH}$ solutions were compared to air-to-water HP, brine-to-water (ground source) HPs and electric boilers.

\section{Estimation of DH network cost}

To identify beneficial boundary conditions for the economic feasibility of central HPs, the network layout was not predefined. Instead the total network cost was estimated based on the plot ratio, the expected specific heat demand, the effective width and an estimated average pipe diameter, as proposed by Persson and Werner [24]. The plot ratio was varied in this analysis between 0.1 (very sparsely populated) to 1.4 (urban area). The expected specific heat demand was based on the Danish 2020 building regulation, according to which the total heat demand has to be reduced to the DHW demand only [25]. But it was expected that there still was a small SH demand due to the behaviour of the inhabitants (NB-medium). A second scenario with higher SH demand was examined to account for less energy efficient buildings (NBhigh)[26], see Table 1. The piping cost were based on estimates given by Rambøll in DHAT [22]. The necessary electricity grid investment was disregarded.

Table 1 Specific heat demand scenarios

\begin{tabular}{lrrrr}
\hline Scenario & & SH demand & DHW demand & Total demand \\
\hline New built (NB) medium & {$\left[W / m^{2} / a\right]$} & 3 & 20 & 23 \\
New built (NB) high & {$\left[W / m^{2} / a\right]$} & 22.5 & 20 & 42.5 \\
\hline
\end{tabular}

\section{Heat supply units data}

The central HPs for the analysis were sized to have a heat capacity which was $80 \%$ and $65 \%$ of the maximum demand for the NB-medium and NB-high case, respectively. The sizing criteria for the two scenarios were chosen to be different, as the scenario with very low space heating demand was dominated by daily variations of heat demand rather than seasonal variations. A restriction to less than $80 \%$ of the maximum demand would lead to increased utilization of peak units, in this case an electric boiler, and thus a considerably reduced system efficiency. Accordingly, the HPs' thermal capacities varied between $0.2 \mathrm{MW}$ and 3.2 MW depending on the heat demand scenario and the plot ratio. Investments into a backup natural gas boiler and a heat storage were considered. The COP of the central and individual HPs was calculated from the sink and source mean logarithmic temperatures and estimated exergy efficiencies [27]. Exergy efficiencies of 0.5 for the central HPs and 0.3 for individual HPs were assumed. The source temperatures for air-source HPs were hourly values from the Danish Design Reference Year [28] and the standard temperature development for Vienna [29]. The sewage water temperature was assumed to be constant at $12.5^{\circ} \mathrm{C}$. The groundwater temperature was assumed to be $10{ }^{\circ} \mathrm{C}$. Weather compensation was applied to the DH supply temperature for ambient air temperatures below $5^{\circ} \mathrm{C}$ [30]. Cost functions and assumed lifetime of all technologies are depicted in Table 2. As the cost functions were based on only few data and many of the HPs considered in this study have capacities lower than the values given in [31], an additional power function was fitted to the data to account for insecurities.

Table 2 Investment cost and lifetime for central and decentral units

\begin{tabular}{|c|c|c|c|c|}
\hline Technology & Investment $[\mathrm{M} € / \mathrm{MW}]$ & r/power function & Lifetime [a] & Sources \\
\hline HP Groundwater & $1.1117 \dot{Q}_{\text {nom }}^{-0.23105}$ & $1.236 \dot{Q}_{\text {nom }}^{-0.2599}$ & 25 & [31] \\
\hline HP Sewage water & $1.2166 \dot{Q}_{\text {nom }}^{-0.33122}$ & $1.1038 \dot{Q}_{\text {nom }}^{-0.2435}$ & 25 & [31] \\
\hline HP Air & $0.9366 \dot{Q}_{n=0}^{-0.1418}$ & $1.0503 \dot{Q}_{\text {nom }}^{-0.3078}$ & 25 & [31] \\
\hline Wood chips boiler & \multicolumn{2}{|c|}{0.9} & 20 & [32] \\
\hline Wood pellet boiler & \multicolumn{2}{|c|}{0.48} & 20 & [32] \\
\hline Electric boiler & \multicolumn{2}{|c|}{0.11} & 20 & [32] \\
\hline Gas boiler & \multicolumn{2}{|c|}{0.06} & 25 & [32] \\
\hline Indv. Air to water HP & \multicolumn{2}{|c|}{0.95} & 15 & [32] \\
\hline Indv. Brine to water HP & \multicolumn{2}{|c|}{1.52} & 20 & [32] \\
\hline Indv. Electric heater & \multicolumn{2}{|c|}{0.86} & 30 & [32] \\
\hline
\end{tabular}




\section{RESULTS}

\section{Levelized cost of energy}
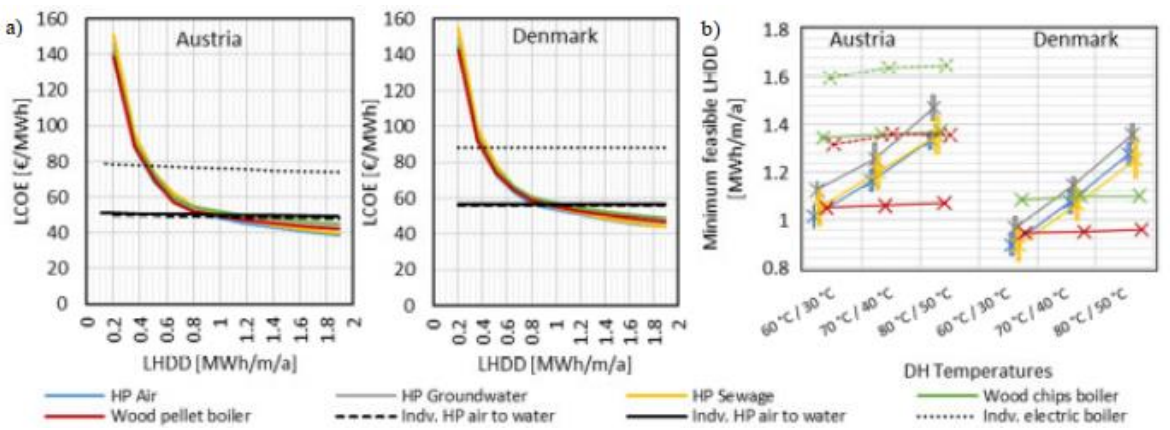

Figure 2 a) LCOE for central and individual units for Austria and Denmark, DH temperatures of $60 / 30{ }^{\circ} \mathrm{C}$, NB-medium and linear HP cost functions , b) Range of minimum feasible LHDD for central solutions for different DH temperature cases, dashed lines refer to cases without subsidies

Figure 2 a) the LCOE of all central units are compared to the three decentral units for the NB-medium heat demand scenario and distribution temperature of $60{ }^{\circ} \mathrm{C}$ forward and $30{ }^{\circ} \mathrm{C}$ return for Austria and Denmark. The cheapest individual solution was using air to water HPs. The LCOE development for decreasing LHDD was similar for all central generation units. It was lower than the LCOE from individual units for LHDD higher than 1.34 (1.08) MWh/m/a for wood chips boiler to $0.97(0.85)$ $\mathrm{MWh} / \mathrm{m} / \mathrm{a}$ for air-source HPs for Austria and Denmark (in brackets), respectively. These LHDD translate to plot ratios of 0.5-0.6 with NB-medium demand buildings, this could e.g. be in suburban areas with 1-2 family houses. For lower LHDD the LCOE increase drastically. This had two major reasons, firstly the share of network cost of the total cost increased with lower LHDD. Secondly, the heat losses in the system increased as LHDD decreased. The LCOE of all technologies was lower in Austria due to differences in electricity taxation. In both cases, central heat pumps were in in the same LCOE range as wood-fired boilers.

The level of LCOE and thereby the minimum feasible LHDD depended on various factors. Figure $2 b$ ) shows the minimum feasible LHDD for different DH temperature cases. The minimum feasible LHDD was lower in Denmark compared to Austria. The representation as bars accounts for the uncertainties in the HP cost functions, which is largest for sewage water HPs. In the Austrian case also the cost for woodfired boilers without subsidies is shown. Compared to these all HP types would have lower minimum feasible LHDD and thus LCOE than the wood-fired boilers for DH temperatures of $70{ }^{\circ} \mathrm{C} / 40{ }^{\circ} \mathrm{C}$ and $60{ }^{\circ} \mathrm{C} / 30^{\circ} \mathrm{C}$. The implementation of HPs seemed especially beneficial for low DH temperatures, where the HPs benefited from better COPs due to the lower temperature lift. The calculated HP COPs can be seen in Table 3. Air-source HPs were cheaper than groundwater HPs for NB-medium. This was due to new buildings considered, i.e. there were no large demand peaks in winter. Accordingly, the advantage from a better COP in winter for the groundwater HPs was lower and the cheaper investment cost for air source HPs made the difference. For the NB-high case the seasonal variation was considerable and the air-source HP could not compete with groundwater and sewage water HP. 


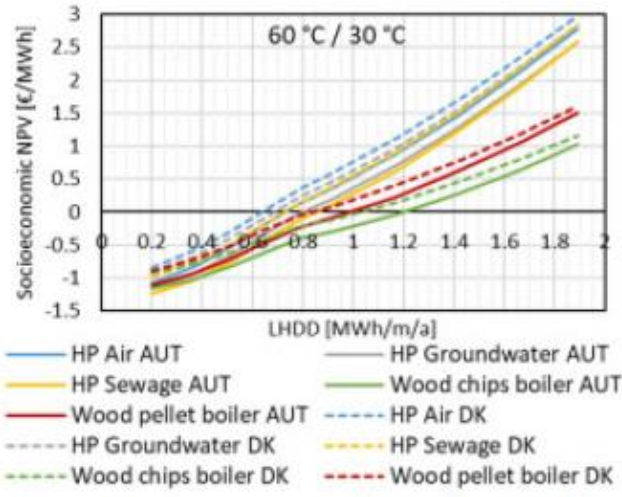

Figure 3 socioeconomic NPV for DK and AUT, $\mathrm{DH}$ temperatures of $60^{\circ} \mathrm{C} / 30^{\circ} \mathrm{C}, \mathrm{NB}$ medium, lin. cost function

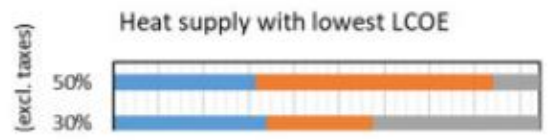

Figure 4 Solution with lowest $\mathrm{LCOE}$ for varying LHDD and electricity price, $\mathrm{DH}$ temperatures of $60^{\circ} \mathrm{C} / 30^{\circ} \mathrm{C}, \mathrm{NB}$ medium, lin. cost function

The influence of the electricity and wood price (excl. taxes) on the economic feasibility of the different solutions was assessed. Figure 4 shows that the minimum feasible LHDD for central solutions increased for decreasing electricity price. This was due to the reduction in production cost in the individual unit. As the LCOE for central solutions also included the investment in the DH network, the effect of a reduction in fuel cost was less pronounced than for individual units. For increasing electricity prices wood-fired boilers became cheapest, especially for low LHDD. The variation of wood prices showed that a reduction in wood prices would decrease the minimum feasible LHDD and increase the feasibility for wood-fired boilers also for low DH forward and return temperatures. It was further studied how a variation in network cost, central HP COP and central unit investment cost influenced the minimum feasible LHDD. The results showed that the LCOE for heat sparse networks was most sensitive to a change in distribution network investment.

Table 3 COP of central HPs for different DH temperature cases and HD scenarios for Austria / Denmark

\begin{tabular}{lccccccccc}
\hline & \multicolumn{3}{c}{ HP air } & \multicolumn{3}{c}{ HP groundwater } & \multicolumn{3}{c}{ HP sewage water } \\
\hline DH-Temp. $\left[{ }^{\circ} \mathrm{C}\right]$ & $60 / 30$ & $70 / 40$ & $80 / 50$ & $60 / 30$ & $70 / 40$ & $80 / 50$ & $60 / 30$ & $70 / 40$ & $80 / 50$ \\
\hline NB-medium & $4.26 / 4.10$ & $3.47 / 3.37$ & $2.96 / 2.89$ & 4.31 & 3.50 & 2.98 & 4.62 & 3.70 & 3.11 \\
NB-high & $4.15 / 3.99$ & $3.40 / 3.29$ & $2.91 / 2.83$ & 4.31 & 3.50 & 2.98 & 4.62 & 3.70 & 3.11 \\
\hline
\end{tabular}

\section{Socioeconomic net present value}

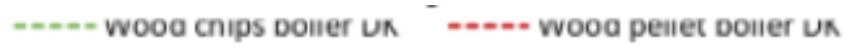

Figure 3shows the socioeconomic NPV of a DH project for different central solutions compared to supply by individual air-to-water HPs in Austria and Denmark. The NPV of the HP cases obtained for Denmark were slightly higher than for Austria. From a socioeconomic perspective the DH solutions based on HPs were clearly better than the wood-fired boilers due to the emission cost caused. It has to be noted that this result was based on the assumption that the realization of the respective project will not change the countries' electricity generation portfolio.

When varying wood and electricity price it was observed that the wood-fired boilers became socioeconomically more feasible than the best HP solution, only for a reduction in wood price of $50 \%$.

And even for an increase of $50 \%$ in electricity price the heat pumps were socioeconomically more feasible than the boilers. Varying the DH temperature sets, it was observed that the increase in NPV with decreasing temperature was much more pronounced for HPs than for wood-fired boiler. This was due to the assumption of a constant boiler efficiency, while the HP COP changed with DH temperatures.

\section{DISCUSSION}

Comparing the results to current values from Denmark it was found that distribution cost, LCOE and LHDD showed to be in good agreement with the values from realised projects [33].

In areas with very low LHDD central DH solutions based on HPs have to compete with heat supply from 
individual units and DH supply based on biomass-fired units. The feasibility of HPs supplying DH in heat sparse areas was better in Denmark than in Austria. Lower DH-temperatures and reductions in distribution costs were beneficial. These may be obtained by new piping types, and cost efficient digging [34].

However, the case of heat sparse areas is a very special case and it needs to be mentioned that the economic feasibility of heat pumps supplying DH in terms of LCOE and socioeconomic NPV improved for larger LHDD compared to the studied alternatives. Further, the heat pump cost functions used were based on very few built heat pumps all larger than $800 \mathrm{~kW}$ and thus showed insecurities for smaller HP

capacities. It should be kept in mind that the study presented aimed at identifying overarching trends but cannot replace a detailed planning for a specific project in question.

The feasibility of the different solutions depended on the boundary conditions in place. The available subsidies for wood-fired boilers in Austria made this type of heat supply units especially attractive for small-scale networks. As shown would central HPs be a better option for low temperature networks $\left(60{ }^{\circ} \mathrm{C} / 30^{\circ} \mathrm{C}\right.$ and $\left.70{ }^{\circ} \mathrm{C} / 70{ }^{\circ} \mathrm{C}\right)$ in Austria, without the subsidies for wood-fired boilers. As the wood used as a fuel in Austria is often produced in close vicinity to the heating plant, it can be assumed that lower prices than the assumed market prices can be obtained. As showed this results in cheaper LCOE than central heat pump units can provide, even for low DH forward and return temperatures.

Within this study ambient heat sources were considered for the HPs. However, if industrial waste heat, even at low temperature levels was available, heat source exploitation cost could be lower compared to e.g. groundwater HPs and subsidies would be available for waste heat utilization in Austria, which would improve the economic feasibility of HPs [35]. There is however no financial support available for ambient heat sources or waste heat sources from communal sewage water. The implementation of large-scale HPs in Denmark benefits from their socioeconomic feasibility, as heat supply projects in Denmark must prove private- and socioeconomically beneficial. Further, the Danish owner structure and regulation provides stable conditions for long-term investment into DH systems. Finally, from an energy system perspective, HPs could play an important role in terms of system integration and thereby provide flexibility to the power system. Providing regulation power might generate further income opportunities for HPs and thereby increase the economic feasibility, also in heat sparse areas.

\section{CONCLUSION}

It was shown that DH supplied by central HPs compared to individual heat supply from air-to-water HPs was economically feasible in Denmark and Austria down to LHDD of 0.85 and 0.97 , respectively, depending on the DH supply temperatures and the type of HP. The economic benefit increased with

increasing LHDD, i.e. less energy efficient buildings or higher plot ratios. The reduction of seasonal peaks in low energy buildings benefited the economic feasibility of ambient source HPs. A barrier largescale

HPs in Austria is the promotion of wood-fired boilers as an alternative heat supply solution, while financial support to ambient source HPs is currently not available. A reduction in electricity cost would as well reduce the feasibility of central HP solution in heat-sparse areas (without a priori existing DH network), as this would decrease the LCOE of individual units more than of central units. Apart from recent tax reductions on electricity, research and dissemination activities the non-profit regulation and the condition of socioeconomic feasibility enhance the feasibility of large-scale heat pumps for DH supply in Denmark.

\section{ACKNOWLEDGEMENT}

This research project is financially funded by EUDP (Energy Technology Development and Demonstration) under the project "EnergyLab Nordhavn - New Urban Energy Infrastructures" (project number: 64014-0555).

\section{BIBLIOGRAPHY}

[1] H. Lund et al., "4th Generation District Heating (4GDH): Integrating smart thermal grids into future sustainable energy systems," Energy, vol. 68, pp. 1-11, 2014.

[2] P. A. Østergaard, H. Lund, and B. V. Mathiesen, "Smart energy systems and 4th generation district heating," Int. J. Sustain. Energy Plan. Manag., vol. 10, pp. 1-2, 2016.

[3] A. David, B. V. Mathiesen, H. Averfalk, S. Werner, and H. Lund, "Heat Roadmap Europe: LargeScale Electric Heat Pumps in District Heating Systems," Energies, vol. 10, no. 4, p. 578, Apr.

2017.

[4] Danish Energy Agency, Energy statistics 2016. Danish Energy Agency, 2018.

[5] Energie-Control Austria, "Key Statistics 2016," 2016. 
[6] M. G. Fernández, C. Roger-Lacan, U. Gährs, and V. Aumaitre, Efficient district heating and cooling systems in the EU, no. December. 2016.

[7] R. Büchle et al., "Bewertung des Potenzials für den Einsatz der hocheffizienten KWK und effizienter Fernwärme- und Fernkälteversorgung,” no. September 2015, p. 147, 2015.

[8] Danish Energy Agency, "Biomass | Energistyrelsen." [Online]. Available: https://ens.dk/en/ourresponsibilities/

bioenergy/solid-biomass. [Accessed: 13-Jun-2018].

[9] Danish Energy Agency, "Analysis of Bioenergy in Denmark (Analyse af bioenergi i Danmark)," no. March 2012, p. 160, 2014.

[10] Energi- Forsynings- og Klimaministeriet, "Forslag til Lov om ændring af lov om fremme af vedvarende energi, lov om tilskud til fremme af vedvarende energi i virksomheders

produktionsprocesser og lov om elforsyning," no. 1756, pp. 11-13, 2016.

[11] C. Maurer et al., "Grünbuch für eine integrierte Energie- und Klimastrategie," p. 106, 2016.

[12] R. Madlener, "Innovation diffusion, public policy, and local initiative: The case of wood-fuelled district heating systems in Austria," Energy Policy, vol. 35, no. 3, pp. 1992-2008, 2007.

[13] RIS, "Gesamte Rechtsvorschrift für Umweltförderungsgesetz.” 2018.

[14] "QM Holzheizwerke." [Online]. Available: www.qmholzheizwerke.ch [Accessed: 25-Jul-2018].

[15] M. Hartl, P. Biermayr, A. Schneeberger, and P. Schöfmann, "Österreichische Technologie-

Roadmap für Wärmepumpen,” 2016.

[16] Dansk Fjernvarme, "Heat generation in Denmark," 2013.

[17] Energi- Forsynings- og Klimaministeriet, "Bekendtgørelse af lov om varmeforsyning," vol. 2017, no. 523, p. 24, 2014.

[18] Danish Government, "Reformopfølgning Regulering af fjernvarmesektoren," no. 7. april, p. 4, 2016.

[19] FGW - Fachverband der Gas- und Wärmeversorgungsunternehmungen, “Zahlenspiegel 2017 Erdgas und Fernwärme in Österreich,” Wien, 2017.

[20] Danish Energy Agency, "Rejsehold for store varmepumper | Energistyrelsen.” [Online]. Available: https://ens.dk/ansvarsomraader/varme/rejsehold-store-varmepumper. [Accessed: 21-Jun-2018].

[21] C. E. Fich, P. Bentzen, and S. S. Birkbak, "Tilskud til eldrevne varmepumper og rådgivning til grundbeløbsværker," no. september 2017, pp. 2017-2019, 2018.

[22] Rambøll, "District Heating Assessment Tool." Danish Energy Agency, 2017.

[23] Energistyrelsen, "Vejledning i samfundsøkonomiske analyser på energiområdet," 2005.

[24] U. Persson and S. Werner, "Heat distribution and the future competitiveness of district heating,"

Appl. Energy, 2011.

[25] C. F. Hansen and M. L. Hansen, "Executive Order on the Publication of the Danish Building Regulations 2015 (BR15)," vol. 2015, no. 1185, 2015.

[26] HOFOR, "Fjernvarmeforsyning af nordhavn," no. 6210, 2013.

[27] J. K. Jensen, T. Ommen, W. B. Markussen, and B. Elmegaard, "Design of serially connected district heating heat pumps utilising a geothermal heat source," Energy, 2017.

[28] P. G. Wang, M. Scharling, K. B. Wittchen, and C. Kern-Hansen, "Technical Report 13-19 2001 2010 Danish Design Reference Year - Reference Climate Dataset for Technical Dimensioning in Building , Construction and other Sectors," 2013.

[29] Meteotest AG, "Meteonorm: Irradiation data for every place on Earth." [Online]. Available: http://www.meteonorm.com/en/. [Accessed: 22-Jun-2018].

[30] X. Yang ,S. Svendsen, "Ultra-low temperature district heating system with central heat pump and local boosters for low-heat-density area: Analyses on a real case in Denmark," Energy, Jun. 2018.

[31] H. Pieper, T. Ommen, F. Bühler, and B. Lava, "Allocation of investment costs for large-scale heat pumps supplying district heating Contact information :," vol. 00, p. 64014, 2017.

[32] Energinet.dk, "Technology Data for Energy Plants," 2016. [Online]. Available: www.ens.dk.

[Accessed: 12-Oct-2017].

[33] Dansk Fjernvarme, "Dansk Fjernvarmes statistik 2017," 2018. [Online]. Available:

http://www.danskfjernvarme.dk/viden-om/statistik-subsection/aarsstatistik/statistik-2017.

[Accessed: 20-Jun-2018].

[34] H. Zinko, B. Bøhm, H. Kristjansson, U. Ottosson, M. Rämä, and K. Sipilä, "District heating distribution in areas with low heat demand density," 2008.

[35] Kommunal Kredit Public consulting, "Informationsblatt Abwärmeauskopplung," no. 113, pp. 1-5, 2018.

[36] Bundesministerium für Wissenschaft Forschung undWirtschaft, "Energie in Österreich - Zahlen,

Daten, Fakten,” 2017. 


\title{
District Heating by Heat Recovery from the Brewing Process of the Brewery Puntigam
}

\author{
Dr. Gerald Koglbauer $^{1}$, Dr. Gerald Zanker ${ }^{2}$ \\ KELAG Energie \& Wärme $\mathrm{GmbH}^{1}$ \\ St. Magdalener Strasse 81, 9524 Villach, Austria \\ Phone: +43 (0) 67687802887 \\ E-Mail: gerald.koglbauer@kew.at \\ BRAU UNION ÖSTERREICH AG, Brauerei Puntigam² \\ Triesterstrasse 357-359, 8055 Graz, Austria \\ Phone: +43 (0) 3165023263 \\ E-Mail: g.zanker@brauunion.com
}

\section{SUMMARY}

Three project partners, Puntigamer brewery, C \& P Immobilien AG, and KELAG Energie \& Wärme $\mathrm{GmbH}$ have realised a unique use of exhaust heat from the brewing process in the Graz district of Puntigam. The application of heat recovery from the present ammonia refrigeration circle in combination with two heat pumps provides the entire district heat of the "Brauquartier Puntigam". The overall coefficient of performance (COP) of the heat pumps was optimized thru the using of a threepipe-system. This enables the district heat transport with two temperature levels, maximum $46^{\circ} \mathrm{C}$ and maximum $70^{\circ} \mathrm{C}$.

\section{INTRODUCTION}

Puntigamer, part of Brau Union Österreich AG, is one of the largest breweries in Austria with a beer out-put of more than 1 million hectolitres per year. The fermentation of beer wort by cultured yeast creates exhaust heat. Therefore was centuries long beer brewed only in the winter time. First time the invention of the cooling machine by Carl von Linde made possible a continuous production.

KELAG Energie \& Wärme GmbH, Austria's largest inter-regional district heating company, has implemented a process together with the brewery Puntigam to use that heat for a district heating system. An integral part of this process is the application of district heat transport using a three-pipesystem. A heat exchanger recovers the fermentation exhaust heat out of the ammonia refrigeration circle of Puntigamer. Two heat pumps raise this fermentation heat to the required flow temperatures of maximum $46^{\circ} \mathrm{C}$ and maximum $70^{\circ} \mathrm{C}$. Average $\mathrm{COP}$ values of 4.7 for the temperature $46^{\circ} \mathrm{C}$ and 3.5 for the temperature $70^{\circ} \mathrm{C}$ are archived in the previous operation of both heat pumps.

The $\mathrm{C} \& \mathrm{P}$ Immobilien AG as the leading Austrian provider of investment apartments in the residential segment, has developed close to the brewery to 4.2 hectares, the project "Brauquartier Puntigam", which is already largely realized. Since December 2017, KELAG Wärme GmbH already supplies the future 65,000 square meters of effective area of the Brauquartier Puntigam with district heat. This development project includes around 800 apartments between 35 and 75 square meters, as well as offices, business premises and a kindergarten. The $\mathrm{C} \& \mathrm{P}$ Immobilien AG provided an important precondition for economical heat pump operation; the entire usable space in the Brauquartier is designed for operation with a low-temperature heating system. In the final phase, around 3.8 million $\mathrm{kWh}$ of heat will be supplied to the customers in the Brauquartier per year; almost $\mathrm{CO}_{2}$ neutral and $100 \%$ sustainable. 


\section{THE PUNTIGAMER BREWERY AS A HEATING PLANT}

Puntigamer, part of Brau Union Österreich AG, is one of the largest breweries in Austria with a beer out-put of more than 1 million hectolitres per year. According to this production output, naturally large amounts of raw materials such as malt, hops, water and yeast are processed. The exhaust heat released later in fermentation is still stored at the beginning of the brewing process in the form of malt carbohydrates. During mashing the carbohydrates are broken down by the malt own enzymes to fermentable sugars such as maltose and glucose. As a result, a sugar-rich beer wort is produced. This is an energy-rich substrate to the cultured yeasts - Saccharomyces carlsbergensis (syn. S. pastorianus) - enabling them to do the alcoholic fermentation. The dissolved glucose in the wort has a physiological calorific value of $16.2 \mathrm{~kJ} / \mathrm{g}$. In comparison, the calorific value of air-dry wood is around $15 \mathrm{~kJ} / \mathrm{g}$.

During the fermentation, each glucose molecule is broken down into two pyruvate molecules, $\mathrm{CH}_{3} \mathrm{COCOO}^{-}$, in a process known as glycolysis. Glycolysis is summarized by equation 1 .

$$
\begin{aligned}
& \mathrm{C}_{6} \mathrm{H}_{12} \mathrm{O}_{6}+2 \mathrm{ADP}+2 \mathrm{P}_{\mathrm{i}}+2 \mathrm{NAD}^{+} \rightarrow \\
& 2 \mathrm{CH}_{3} \mathrm{COCOO}^{-}+2 \mathrm{ATP}+2 \mathrm{NADH}+2 \mathrm{H}_{2} \mathrm{O}+2 \mathrm{H}^{+}
\end{aligned}
$$

During this reaction the "phosphate based energy storage molecule" adenosine diphosphate, ADP, is regenerated to adenosine triphosphate ATP. And the "hydrogen based energy storage molecule" $\mathrm{NAD}^{+}$ is regenerated to $\mathrm{NADH}$, too. Finally, pyruvate is converted to ethanol, $\mathrm{C}_{2} \mathrm{H}_{5} \mathrm{OH}$, and $\mathrm{CO}_{2}$ in two steps, regenerating oxidized $\mathrm{NAD}^{+}$needed for glycolysis. The first step is catalysed by the enzyme pyruvate decarboxylase (eq. 2), and creates the "sparkling" - carbon dioxide. The second reaction (eq.3) is catalysed by alcohol dehydrogenase, creating the typical alcohol content of beer.

$$
\mathrm{CH}_{3} \mathrm{COCOO}^{-}+\mathrm{H}^{+} \rightarrow \mathrm{CH}_{3} \mathrm{CHO}+\mathbf{C O}_{2}
$$

$$
\mathrm{CH}_{3} \mathrm{CHO}+\mathrm{NADH}+\mathrm{H}^{+} \rightarrow \mathbf{C}_{2} \mathbf{H}_{5} \mathbf{O H}+\mathrm{NAD}^{+}
$$

After all, the amount of energy released from these reactions is $\Delta \mathrm{G}^{0 \prime}=-218 \mathrm{~kJ} / \mathrm{mole}$ of glucose or 1.2 $\mathrm{kJ} / \mathrm{g}$ glucose. Reducing that amount by the chemical energy directly consumed by the yeast of about $20 \%$ the remaining energy is released as heat into the wort. Therefore was centuries long beer brewed only in the winter time.

First time the invention of compression chiller by Carl von Linde made possible a continuous beer production. Since that time, it was possible to remove the resulting waste heat in a continuous and controlled manner from the fermentation process. The principle of the compression chiller used at that time has not changed to this day very much. Even the original used refrigerant ammonia $-\mathrm{NH}_{3}-$ is still the most used in breweries today. On the one hand, ammonia has excellent thermophysical properties, on the other hand $\mathrm{NH}_{3}$, despite undisputed disadvantages, is a very environmentally friendly refrigerant.

In order to ensure the quality of the beer, the fermentation heat typically has to be removed from the wort at temperatures around $0^{\circ} \mathrm{C}$. In the brewery Puntigam $\mathrm{NH}_{3}$ direct evaporators do most of the cooling. That is, $\mathrm{NH}_{3}$ evaporates at low pressure (about 3.6 bar abs.) in the heat exchanger and absorbs the fermentation heat as latent heat of the $\mathrm{NH}_{3}$ vapour. In this process, the temperature of $\mathrm{NH}_{3}$ does not change as long as liquid $\mathrm{NH}_{3}$ is present; this is about $-4.5^{\circ} \mathrm{C}$. In order for the vaporized $\mathrm{NH}_{3}$ to

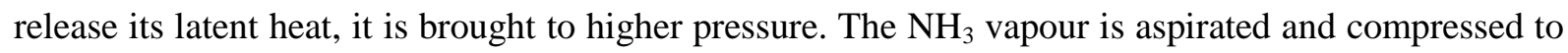
the compression pressure by means of electrically driven compressors. This is on average 9.8 bar abs., 
corresponding to a condensation temperature of about $24.5^{\circ} \mathrm{C}$. During liquefaction, $\mathrm{NH}_{3}$ often releases its latent heat to the environment, but not in this case. Here, the heat released is transferred to a heat transfer medium and thus recovered for heating purposes.

\section{HEAT SUPPLY OF THE BRAUQUARTIER PUNTIGAM}

In the planning phase of the heating supply of the Brauquartier it was necessary to find clear answers to the most important questions of the technical implementation possibilities. In particular, a number of technical constraints had to be given or generated.

The following boundary conditions had to be prepared to be in the brewery:

a) Continuous heat advent of the brewery: A continuous heat volume is absolutely necessary for year continuous heat supply. This requirement is satisfied through the years continuous brewing operation of the brewery Puntigam.

b) Central extraction point of the exhaust heat: A central extraction point ensures the heat transport by the shortest route for heat treatment. Here the selected outcoupling point is located near the central $\mathrm{NH}_{3}$ recooler. Thus, this requirement is satisfied.

c) Balancing heat system for exhaust heat-independent heat supply: In the event of faults, maintenance or short-term demand peaks, the heat balancing system must be able to take over the heat supply. The brewery has a sufficiently high steam generating capacity reserve for the brewing operation, so that the heat balancing system in the form of a steam converter can also be operated without problems. Thus, this requirement is satisfied, too.

The following boundary conditions had to be prepared to be in the objects of the Brauquartier:

a) Low temperature heating operation of the objects: The developer(s) has opted for the design of the heating surfaces as a low-temperature variant, so this requirement is satisfied.

b) Object supply via a three-pipe heating network: The developer(s) has provided the route paths required for a three-pipe system. Furthermore, the heating centers of the objects were prepared for the heat transfer executed from a three-pipe system. Thus, this requirement is satisfied, too. In parallel, the heat demand situation of the future service area Brauquartier was determined. For this purpose, a time-resolved simulation based on artificial demand profiles was created. Derived therefrom again, a production profile could be calculated (Figure 1). The generation profile shows the share of waste heat after heat pumps as well as the demand for load balancing energy. Here, around $93 \%$ of the heat demand would come from the waste heat of the brewery and just $7 \%$ would have to be covered through Brewery steam or natural gas, respectively.

By providing two temperature levels, a further breakdown of the amount of energy is necessary. The heating takes place at a maximum of $46^{\circ} \mathrm{C}$, while for domestic hot water production about $70^{\circ} \mathrm{C}$ are required. The technical solution for this is the design of two heat pump units, a heat pump with around $800 \mathrm{~kW}$ for the temperature range of the heating operation, and a heat pump with around $420 \mathrm{~kW}$ for the temperature range of the demand hot water operation. The applied refrigerant of both heat pumps is R134a. In addition, due to the compression of the $\mathrm{NH}_{3}$ this overheats, and thus part of the heat from the $\mathrm{NH}_{3}$ can be used directly. A Sankey diagram (Figure 2) provides a summary of the actual distribution of energy flows.

Figure 3 shows a simplified diagram of the exhaust heat-extraction and the heat processing by the heat pumps. The exhaust heat extraction takes place as a boundary condition mentioned before in front of the central recooler; here executed as evaporation condensator. For this purpose a $\mathrm{NH}_{3}$ bypass was built and a welded hermetically sealed plate heat exchanger installed. In this $\mathrm{NH}_{3}$ is liquefied against the heat carrier "brine", which is composed of glycol and water. The bypass line ensures that in the event of a malfunction in the exhaust heat extraction, the system can again be routed directly via the 
recooler. In addition, the exhaust heat exchanger and the recooler are connected in series. Thereby, any residual $\mathrm{NH}_{3}$ vapor from the extraction heat exchanger is liquefied in the recooler anyway.

Both heat pumps have been designed and provided by the Austrian Company FRIGOPOL Kälteanlagen $\mathrm{GmbH}$ (Frauental an der Lassnitz), which has also built the exhaust heat extraction system.

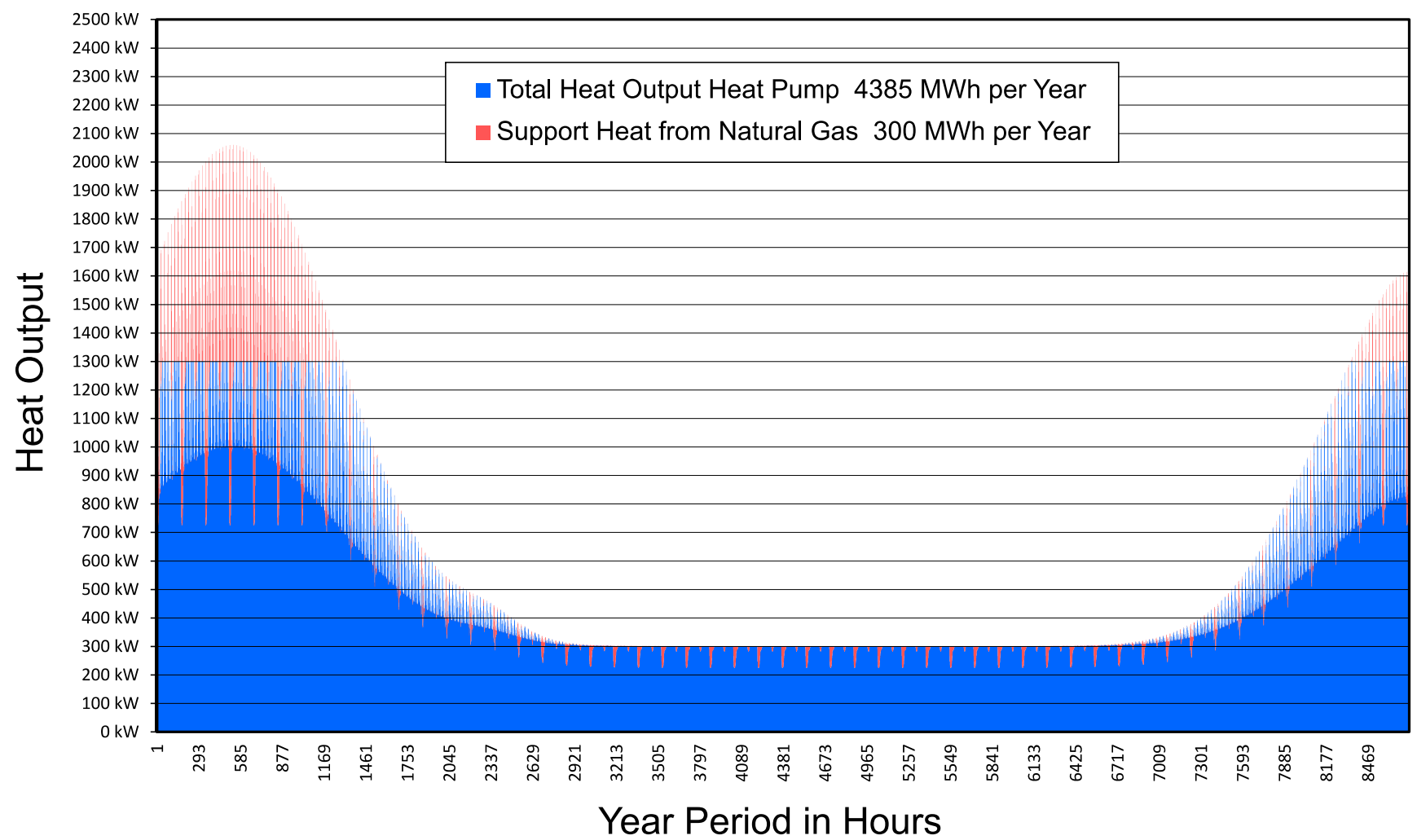

Fig. 1: Result diagram of the simulation of the possible heat output of the brewery for district heating 


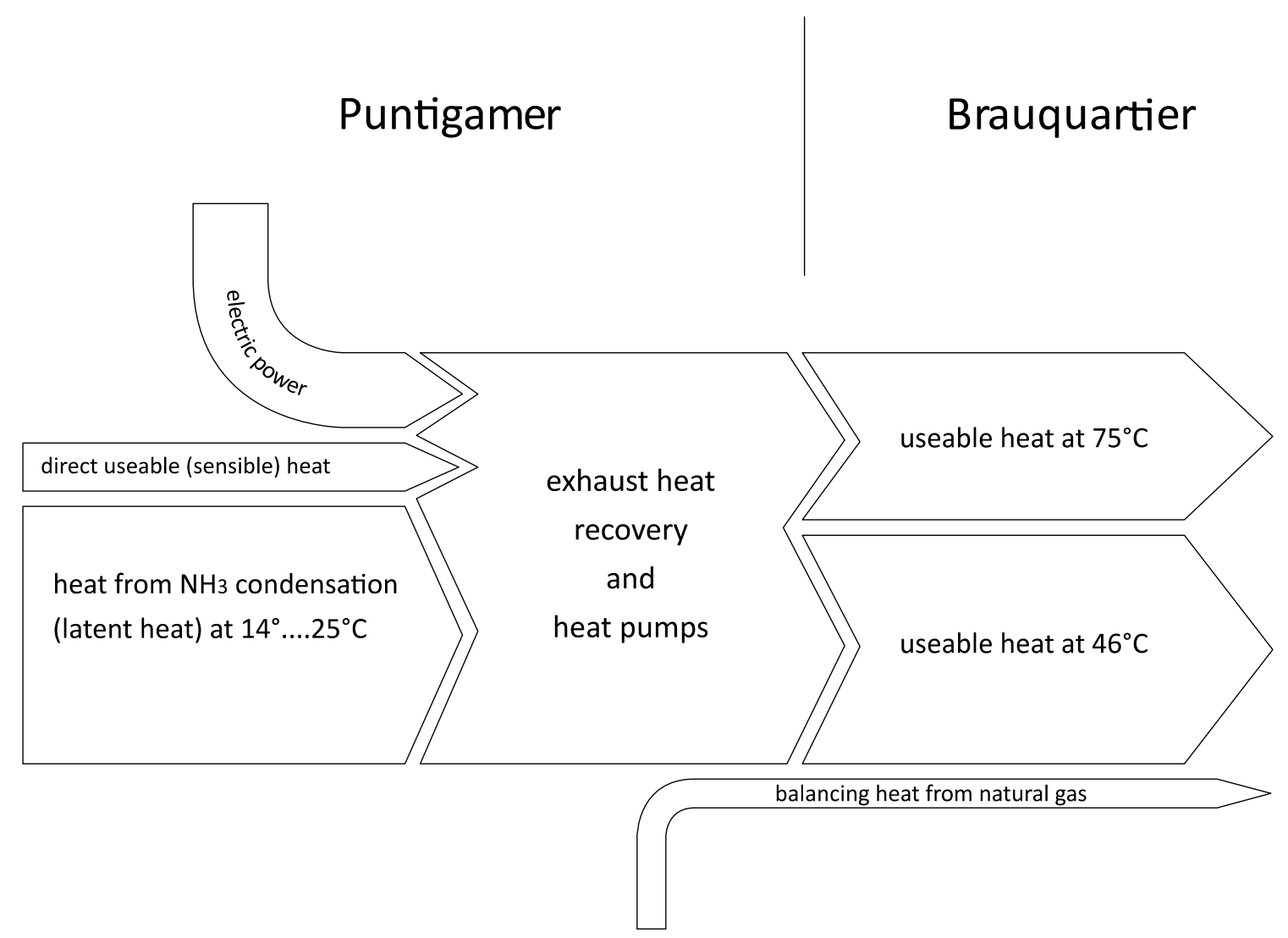

Fig. 2: Sankey diagram of the exhaust heat transfer from the Puntigamer brewery to the Brauquartier

The brine serves both heat pumps as primary heat source. With the prevailing conditions, an average COP of 4.7 is achieved on the medium-temperature heat pump. The high temperature heat pump achieves an average COP of 3.5. These are preliminary values, since the plant went into operation in January 2018. The first full year of operation is expected to be reached in mid-2019 and only then can concrete statements be made on the Seasonal Performance Factors (SPF) of the heat pumps. 


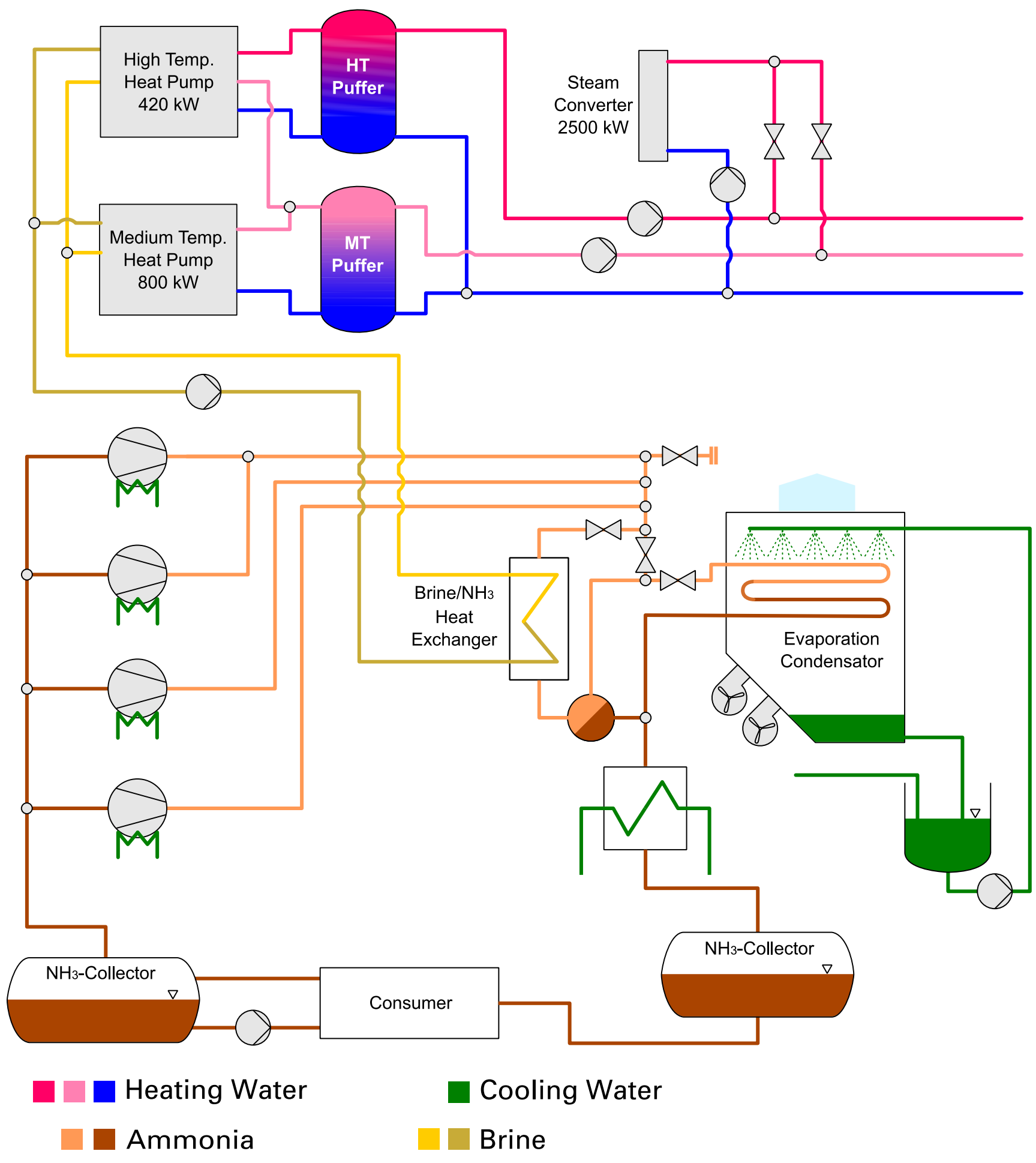

Fig. 3: Simplified scheme of the exhaust heat extraction process by an intermediate brine circuit 


\section{CONCLUSION}

By now, all exhaust heat projects face the challenge that the exhaust heat would be available in large quantities, but the temperature level of the exhaust heat is too low for any direct application. The project Brauquartier has shown very clearly, what is important in such projects. In essence, the exhaust heat provider, the heat processor, and the heat consumer must be willing to make technical and economic compromises. Only then, the most optimal solution for all parties is feasible.

The project Brauquartier is to be seen as part of a series of waste heat projects with district solutions, which always form a close link between industrial and residential areas. Not to mention that the resulting energetic synergy leads to a considerable saving of greenhouse gases, thus improving the life quality of all inhabitants.

\section{REFERENCES}

Voet D., and Voet J. G. (2004), Biochemistry 3rd Edition, New York, John Wiley \& Sons, Inc. Chris Boulton (20 May 2013), Encyclopaedia of Brewing, New York John Wiley \& Sons, Inc.

P.J. Linstrom and W.G. Mallard, Eds., NIST Chemistry WebBook, NIST Standard Reference Database Number 69, National Institute of Standards and Technology, Gaithersburg MD, 20899 


\title{
Decarbonizing Industry: Extending the Scope of Mitigation Options
}

\author{
Dr Andrea Herbst, Dr Tobias Fleiter, Matthias Rehfeldt \\ Fraunhofer Institute for Systems and Innovation Research ISI \\ Breslauer Str. 48, 76139 Karlsruhe, Germany \\ Phone: +49 $7216809-439$ \\ E-Mail: andrea.herbst@isi.fraunhofer.de
}

\section{SUMMARY}

Industry is responsible for about $25 \%$ of EU final energy demand and uses gas, electricity, coal, and oil as the dominant energy carriers making industry critical for the achievement of European climate goals. Analyses show that the industry sector is unlikely to meet ambitious climate mitigation targets without a major change in technologies used and the accompanying policy frame. This contribution presents a transition scenario for the EU28 that achieves an ambitious reduction in GHG emissions of $70 \%$ by 2050 compared to 2015 for the industrial sector. The shown transition scenario contains different mitigation options: incremental and BAT energy efficiency improvements, advanced energy- and resource efficient processes, fuel and feedstock switching (e.g. towards renewable electricity or hydrogen), recycling and re-use, as well as material efficiency and substitution.

\section{INTRODUCTION}

The industrial sector ${ }^{1}$ accounts for about $25 \%$ of EU final energy demand and uses gas, electricity, coal, and oil as the dominant energy carriers. This high share in final energy demand is mainly due to energy-intensive industries such as iron and steel or the chemical sector. Within these industries, specific energy-intensive products/processes (e.g. steel, cement, ammonia) are particularly relevant for the future achievement of European climate targets. Some sectors already use a high share of electricity and biomass but industry still needs to make substantial further efforts to reduce the use of fossil fuels in the next decades. Reducing process emissions appears to be a particular challenge for the industrial sector, as these types of emissions can only be reduced by radical changes in the production process, product mix or by the use of carbon capture and storage. In terms of end-uses, most industrial GHG emissions are from high-temperature process heat, either in the form of steam or hot water, or from the direct firing of various types of furnaces. The high temperatures and the specific requirements of furnaces limit the use of renewable energies here to biomass or secondary energy carriers. Process-related emissions account for about $21 \%$ of all direct emissions and it is technically difficult or even impossible to mitigate them in the processes used at present. In the following, we assess a potential transition pathway towards a low-carbon industry sector including a broad variety of mitigation options.

\section{METHODOLOGY}

The FORECAST modelling platform (www.forecast-model.eu) aims to develop long-term scenarios for future energy demand of individual countries and world regions until 2050. It is based on a bottom-up modelling approach considering the dynamics of technologies and socio-economic drivers. The model allows addressing various research questions related to energy demand including scenarios for the future demand of individual energy carriers like electricity or natural gas, calculating energy saving potentials and the impact on greenhouse gas (GHG) emissions as well as abatement cost curves

\footnotetext{
${ }^{1}$ The definition of industry in this article follows Eurostat final energy definitions and excludes the refinery sector as well as electricity onsite generation.
} 
and ex-ante policy impact assessments. Energy-intensive processes are explicitly considered, while other technologies and energy-using equipment are modelled as cross-cutting technologies. FORECAST is a simulation model used to support investment decisions, taking into consideration barriers to the adoption of energy efficient technologies as well as various policy instruments. Different approaches are used to simulate technology diffusion, including diffusion curves, vintage stock models and discrete choice simulation. Main macro-economic drivers are industrial production for over $\mathbf{7 0}$ individually modelled basic materials products, gross value added, and employment for less energy-intensive sub-sectors. Five sub-modules cover: basic materials processes, space heating, electric motor systems, furnaces and steam systems. Saving options (incremental changes, radically new production processes) unfold their impact on energy consumption and GHG emissions by diffusing through the modelled technology stock and, thus, reducing the specific energy consumption or specific process related emissions of individual production processes. (Fleiter et al. 2012, Fleiter et al. 2013, Biere et al. 2014, Rehfeldt et al. 2016, Herbst et al. 2018b)

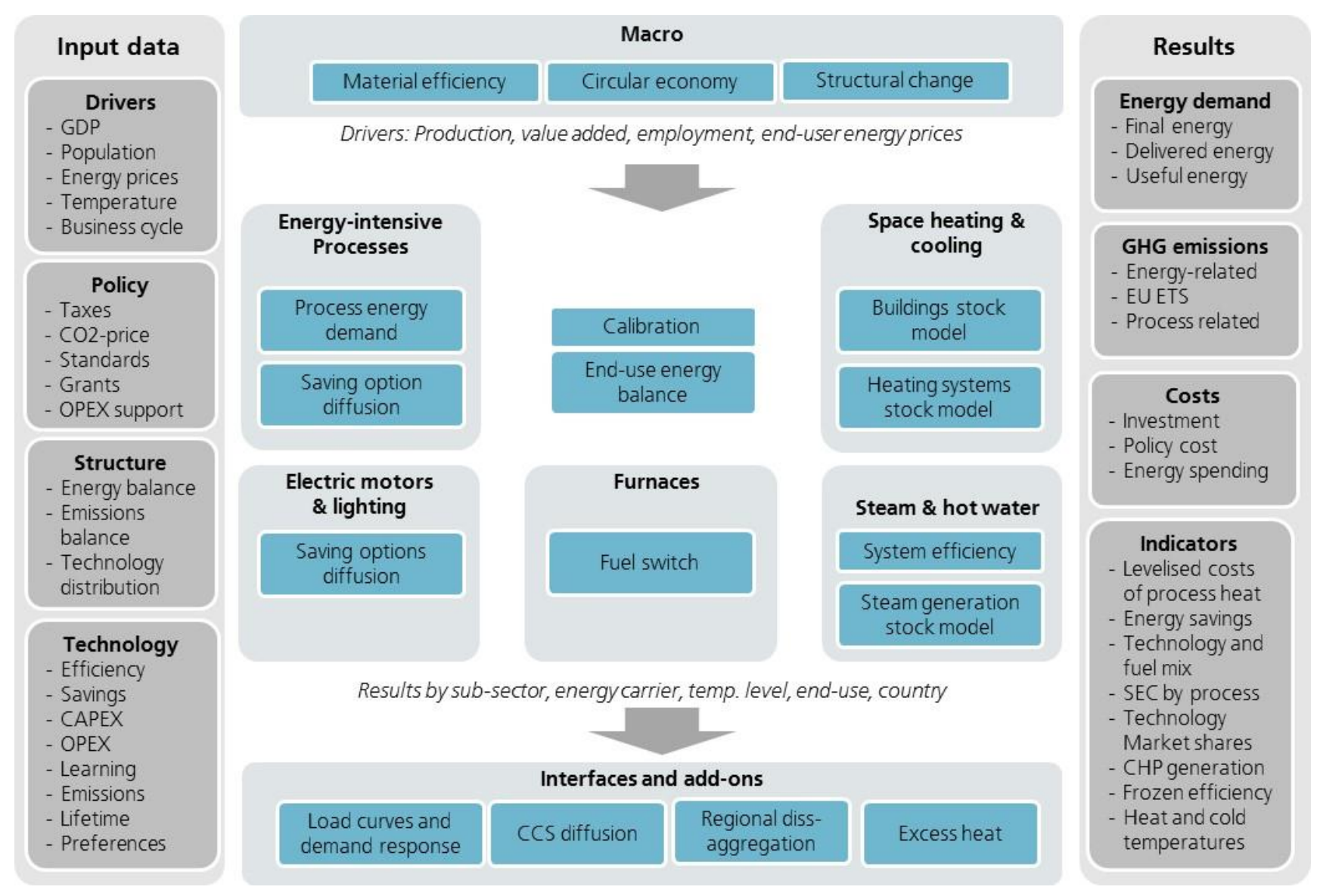

Fig. 1: FORECAST-Industry model overview 


\section{SCENARIO DEFINITION}

For the model-based analysis, we define two scenarios (Herbst et al. 2018a):

A Reference scenario $(R E F)$, which reflects the effects of current policies on the future energy system and serves as a benchmark to compare the more ambitious scenarios.

The Transition scenario including innovative process technologies in industry (TRANS-IPT) describes an industrial decarbonisation pathway aiming to reduce European industrial greenhouse gas emissions in 2050 using innovative production technologies and new products but excluding CCS.

\section{Framework conditions}

The macroeconomic framework data (gross domestic product, gross value added, population) for the model-based analysis is taken from the European Reference Scenario 2016 (Capros et al. 2016) and stays the same across scenarios to allow better comparability of changes in policy parameters and assumptions between scenarios. The same holds true for the assumptions on wholesale fossil fuel price development. Electricity prices differ between scenarios, assuming a stronger increase in the TRANS-IPT scenario due to the higher share of renewables expected/necessary in the energy system. In addition, financial support for RES is assumed in the TRANS-IPT scenario.

\section{Level of ambition and mitigation options}

In the TRANS-IPT scenario any remaining energy efficiency potentials are almost completely exploited implying that effective policies are in place to overcome barriers to improved energy efficiency (e.g. EMS, audits, minimum standards). In addition, financial support for RES is assumed to support fuel switching to biomass, power-to-heat and power to gas. Furthermore, $\mathrm{a} \mathrm{CO}_{2}$ price increase to 150 euros/t $\mathrm{CO}_{2}$ in 2050 is assumed. Companies in the TRANS-IPT scenario can anticipate increasing prices ten years in advance, implying a stringent and well-communicated commitment to the EU ETS or even a $\mathrm{CO}_{2}$ floor price path.

The most important assumption, however, in this scenario is the assumed paradigm shift in industry, which allows radical changes to take place and new technologies to enter the market (e.g. H2-plasma steel, low carbon cement sorts).

Table 1: Scenario characterization by mitigation option

\begin{tabular}{|c|c|c|}
\hline $\begin{array}{l}\text { Clusters of mitigation } \\
\text { options }\end{array}$ & REF & TRANS-IPT \\
\hline $\begin{array}{l}\text { Incremental efficiency } \\
\text { improvement }\end{array}$ & $\begin{array}{l}\text { Energy efficiency progress } \\
\text { according to current policy } \\
\text { framework and historical } \\
\text { trends. }\end{array}$ & $\begin{array}{l}\text { Faster diffusion of incremental process improvements } \\
\text { (BAT \& INNOV } \geq T R L 5 \text { ). }\end{array}$ \\
\hline $\begin{array}{l}\text { Fundamental processes } \\
\text { improvement } \\
\text { energy efficiency, process } \\
\text { emissions }\end{array}$ & - & $\begin{array}{l}\text { Radical process changes } \\
\text { (INNOV } \geq T R L 5 \text { ) }\end{array}$ \\
\hline
\end{tabular}




\begin{tabular}{|l|l|l|}
\hline $\begin{array}{l}\text { Fuel switching to RES } \\
\text { towards RES, decarbonized } \\
\text { electricity and/or hydrogen }\end{array}$ & $\begin{array}{l}\text { Fuel switching driven by } \\
\text { energy prices and assumed } \\
\mathrm{CO}_{2} \text {-price increase. }\end{array}$ & $\begin{array}{l}\text { High financial support for RES technologies: } \\
\text { Stronger fuel switching to biomass, power-to-heat and } \\
\text { power-to-gas technologies. } \\
\text { Radical changes in industrial process technologies drive } \\
\text { fuel switch (e.g. switch to hydrogen). }\end{array}$ \\
\hline $\begin{array}{l}\text { Carbon capture and } \\
\text { storage (CCS) }\end{array}$ & - & - \\
\hline Recycling and re-use & $\begin{array}{l}\text { Slow increase in recycling } \\
\text { rates based on historical } \\
\text { trends. }\end{array}$ & $\begin{array}{l}\text { Stronger switch to secondary production } \\
\text { (e.g. electric steel, secondary aluminium). }\end{array}$ \\
\hline $\begin{array}{l}\text { Material efficiency and } \\
\text { substitution }\end{array}$ & Based on historic trends. & $\begin{array}{l}\text { Increase in material efficiency \& substitution. } \\
\text { Decrease in clinker factor. }\end{array}$ \\
\hline
\end{tabular}

\section{RESULTS}

In the reference scenario (REF), the direct emissions of the industry sector (direct energy and process related) decrease from 630 to $560 \mathrm{Mt} \mathrm{CO}_{2}$-eq. between 2015 and 2050. This reflects a future

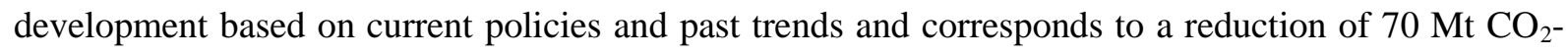
eq. or $11 \%$. For the TRANS-IPT scenario results show that RES, power-to-heat, power-to-gas and energy efficiency have high potentials towards decarbonisation. GHG emissions are continuously decreasing in the TRANS-IPT scenario to $192 \mathrm{Mt} \mathrm{CO}_{2}$-eq. in 2050 see figure 2). This corresponds to a $70 \%$ reduction compared to 2015 and an $83 \%$ reduction compared to 1990.

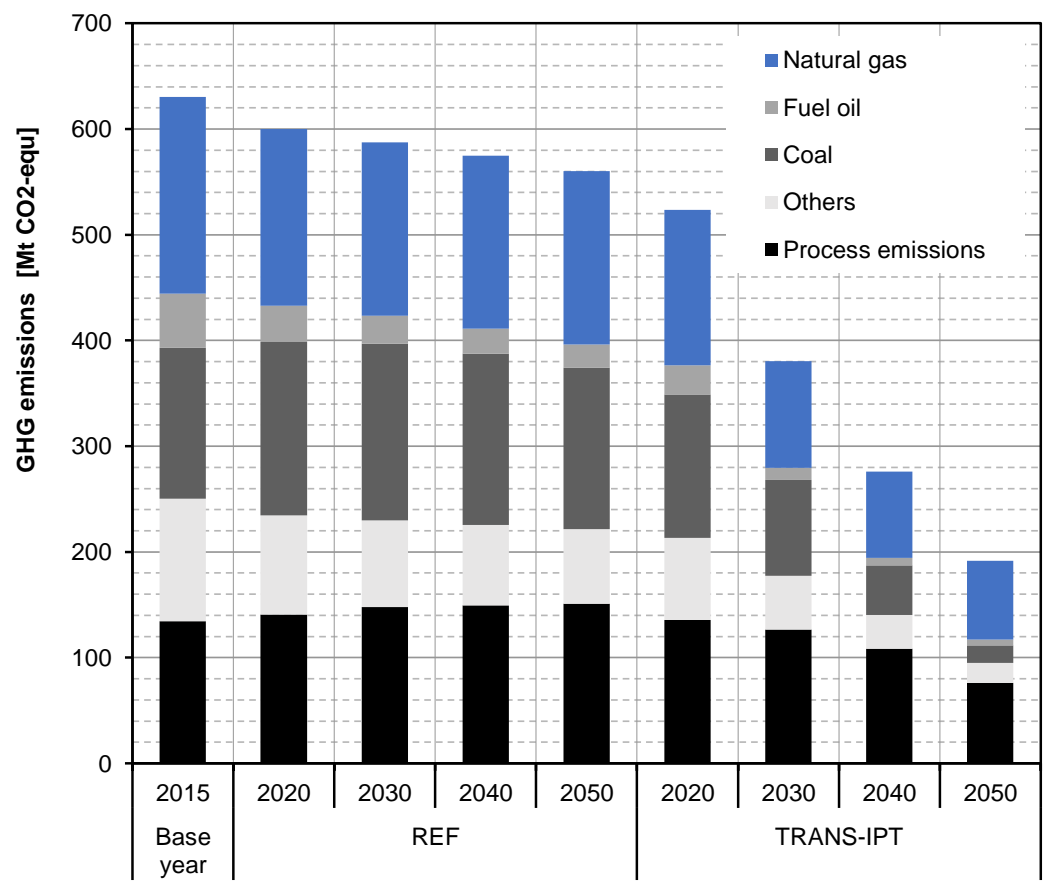

Fig.2: EU28 industrial $\mathrm{CO}_{2}$ emissions by scenario and energy carrier (2015-2050) 
However, this TRANS-IPT scenario also results in a higher demand for secondary energy carriers like electricity and hydrogen and depends on the successful market introduction of as yet unproven technologies.

In the TRANS-IPT scenario, emissions from the iron and steel industry are reduced by almost $90 \%$ in 2050 compared to 2015 by replacing oxygen steel with electric steel and substituting the remaining blast furnace route with renewable-hydrogen-based steel. Conventional cement production is partly substituted in the TRANS-IPT scenario by innovative types of cement using new binders and reducing the specific energy- and process-related cement emissions by between -30 and $-70 \%$. Additional potentials in the non-metallic minerals sector are tapped using electric melting processes in the glass industry as well as incremental process improvements (e.g. oxyfuel combustion incl. waste heat recovery) and fuel switching. Overall, the direct emission reductions in the non-metallic minerals sector amount to $-54 \%$ in 2050 compared to 2015.

In the reference scenario (REF) industrial final energy demand (FED) for the EU28 is only slightly decreasing as efficiency effects are nearly equalled out by activity effects (e.g. gross value added growth) from $3233 \mathrm{TWh}$ in 2015 to $2928 \mathrm{TWh}$ in 2050. In the TRANS-IPT scenario, FED is decreasing, however a lot slower than GHG emissions by 25\% compared to 2015 to $2430 \mathrm{TWh}$ in 2050 (figure 3). Two trends can be observed in the transition scenario: a decrease in conventional demand due to integrated process improvements and fuel switch to biomass and an additional need for large volumes of renewable electricity due to fundamental process changes. This need for additional renewable electricity leads to a demand of 1144 TWh of electricity in 2050 in the TRANS-IPT scenario, which is an increase of $15 \%$ compared to 2015 . In general, renewable energies substitute a large part of industry's demand for natural gas in the transition scenario. The use of biomass more than doubles in 2050 compared to 2015 in the TRANS-IPT scenario (658 TWh in 2050). Coal and oil are replaced almost completely in the TRANS-IPT scenario - particularly in the areas of fuel demand for low-temperature heat and the use of waste heat in combination with heat pumps.

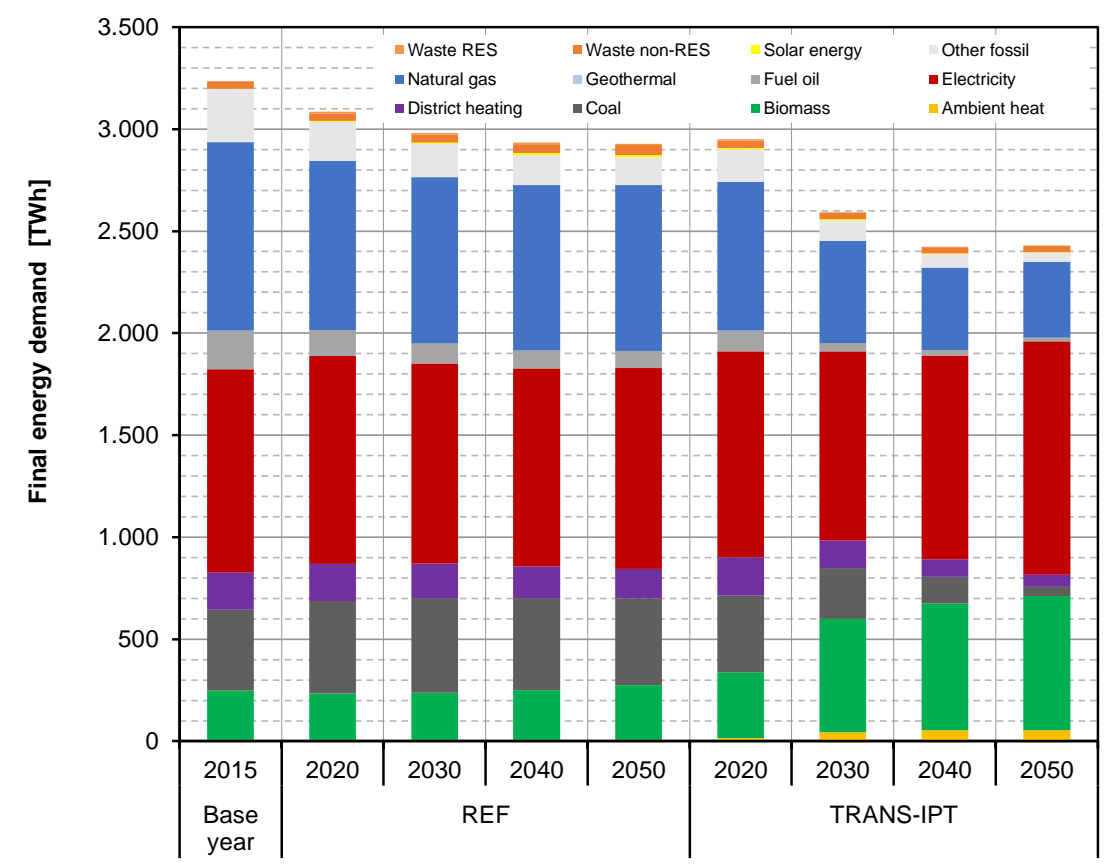

Fig.3: EU28 final energy demand by scenario and energy carrier (2015-2050) 


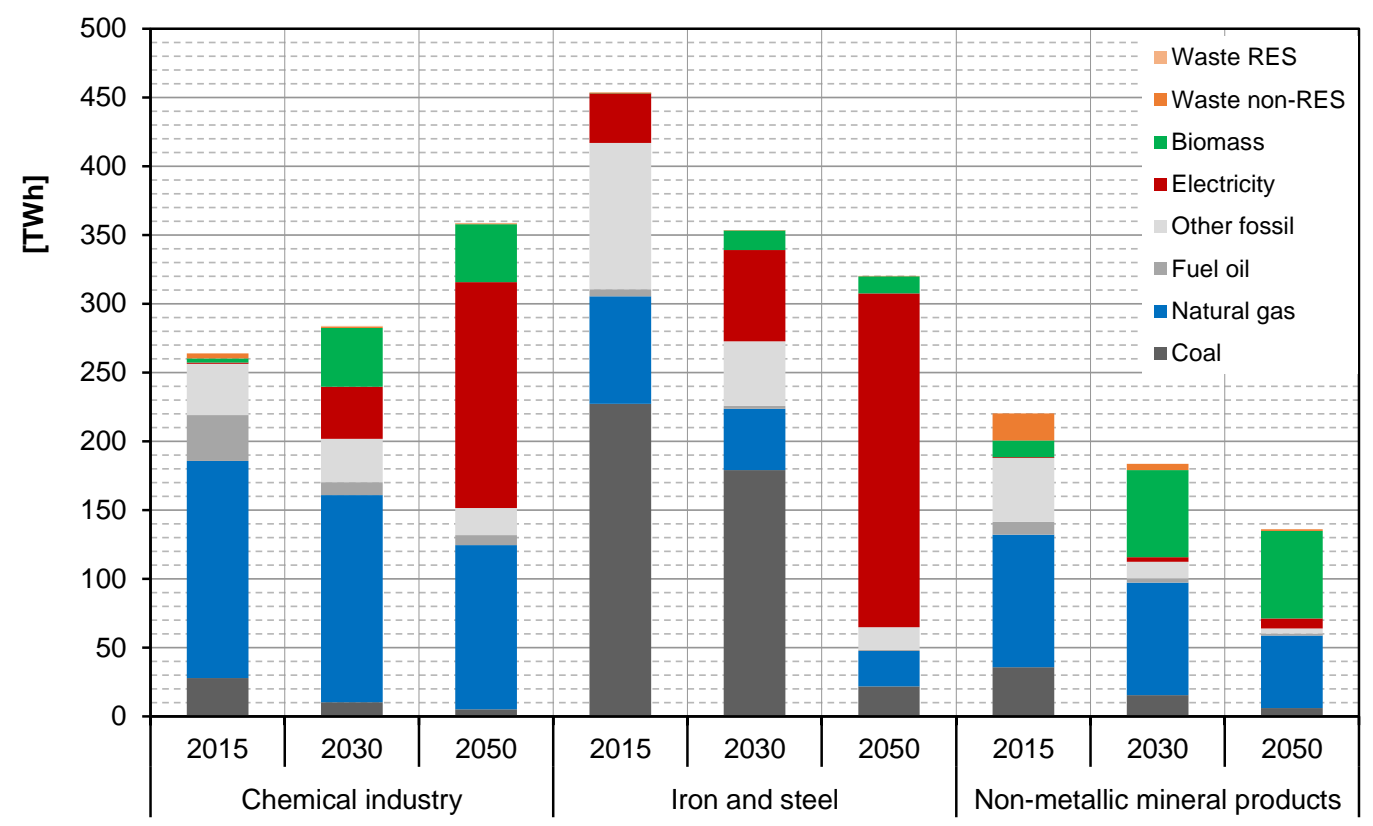

Fig.4: EU28 fuel switch in furnaces by energy carrier (2015-2050)

Another important field for fuel switching are industrial furnaces (figure 4). Comparted to steam systems, furnaces are very diverse and specific to the related production process. They often work at high temperatures above $1000^{\circ} \mathrm{C}$, e.g. in the cement, glass and steel production. Fuel switching is possible, but the use of energy carriers experiences more technical restrictions and RES are sometimes difficult to integrate. In the TRANS-IPT scenario a strong shift towards biomass and electricity takes place. Financial support for biomass is high in this scenario, leading to a more comprehensive use where technically possible like in the cement and lime production. The increase in electricity demand is driven by fundamental process changes that include e.g. the use of H2-plasma steel production, completely replacing the conventional oxygen steel route. However, across all sectors also in 2050 still a substantial amount of natural gas is used.

\section{CONCLUSIONS}

The results presented discuss the impacts of different mitigation options, such as incremental and BAT energy efficiency improvements, advanced energy and resource efficient processes, fuel switching, recycling and re-use, as well as material efficiency and substitution on EU industrial energy demand and $\mathrm{CO}_{2}$ emissions.

It is shown that the mentioned mitigation options can lead to a significant reduction of industrial $\mathbf{C O}_{2}$ emissions $(-70 \%)$ and final energy demand $(-25 \%)$ in the TRANS-IPT scenario in 2050 compared to 2015 which reflects a $83 \%$ emissions reduction compared to 1990.

However, the processes currently used to produce energy-intensive basic material products have been optimised over many decades. The remaining energy efficiency potentials due to applying the best available technology (BAT) are limited. For example, even the most efficient clinker furnace will not be able to reduce its energy demand by much more than $10 \%$ compared to today's average. In addition, fuel switching from fossil fuels like natural gas to renewable sources is limited due to the high temperature levels required in industrial furnaces and the competition for biomass with other sectors. Although incremental improvements of energy efficiency and fuel switching are important 
pillars of industrial decarbonisation pathways, these two options alone will not suffice to achieve a low-carbon industry sector by 2050 .

Deep emission cuts require substantial changes in the iron and steel, cement and chemicals industries, but also support for RES and energy efficiency in other sectors and companies. Biomass is the most important RES in industry, particularly in the medium term. However, biomass resource potentials and their sustainability are limited. In the long-term, RES-based electricity (power-to-heat) can play a more important role, particularly if electricity generation has very low emission levels. However, electricity is not yet competitive with biomass even in the most ambitious transition policy scenario, meaning that replacing biomass by electricity would require policies that are more specific. Improved material efficiency and the circular economy have a huge mitigation potential. However, it is still unclear what an effective policy mix would look like and this probably encompasses a wide range of individual measures.

The scenarios envisage radical changes to industrial production systems like innovative processes and large-scale power-to-heat for steam generation mainly in the time horizon after 2030. Before 2030, energy efficiency improvements combined with fuel switching to biomass and progress towards a circular economy are the main mitigation options that drive $\mathrm{CO}_{2}$ emissions downward. However, in order to have new process technologies and innovations ready by 2030, substantial research, development and innovation activities need to take place in the coming decade. Pilot and demonstration plants need to be built to prepare for market introduction. It might easily take 10 years for new processes in the materials industry to progress from lab-scale to market. Certification processes such as those needed for new cement types can prolong the time taken even more.

Consequently, the current policy mix needs to be adjusted in order to effectively support $R \& D$ activities directed at the decarbonisation of industrial production. This includes the following elements:

EU Emissions Trading Scheme (ETS): At the current level of certificate prices (EUAs), the ETS is not effective in reducing industrial emissions. For investments in low-carbon technologies, companies' expectations of future prices are even more important than the current price levels. If companies cannot rely on rising EUA prices, they will not invest in $\mathrm{CO}_{2}$ abatement technologies. Extending the ETS with a minimum price path (i.e. a floor price) could provide more long-term clarity and the certainty needed for investors in low-carbon innovations.

In the context of a highly uncertain environment and large potential investments, public RD\&I funding can play an important role in accelerating the market introduction of innovative low-carbon processes. The current proposal of the European Commission to establish an EU Innovation Fund as a follow-up to the ongoing NER300 programme is an important step in this direction.

In addition, targeted public procurement can support the market introduction of low-carbon products by establishing niche markets. For example, considering life-cycle $\mathrm{CO}_{2}$ emissions when procuring building materials might encourage the cement industry to develop and provide more lowcarbon alternatives.

Although a major share of industrial GHG emissions is covered under the EU ETS emissions cap, a high amount of industrial $\mathrm{CO}_{2}$ emissions remains outside the ETS and thus does not receive a $\mathrm{CO}_{2}$ price signal. These companies currently have no incentive to switch to renewable or low-carbon fuels

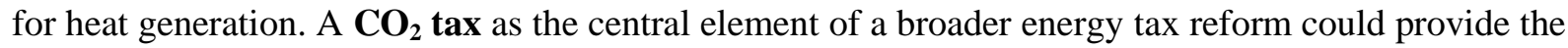
incentives needed for fuel switching. This must avoid any double burden on companies inside the ETS. 
Boosting material efficiency and a circular economy approach along the value chain requires a broad policy mix. Examples of individual policies include:

Re-evaluation of value added tax according to the carbon-footprint of products and a lower value added tax for repair services.

Reform the EU ETS to keep $\mathrm{CO}_{2}$ price signals along the value chain visible for downstream consumers and companies.

Evaluate building codes and the regulative framework in the construction industry to facilitate the use of sustainable building products and the efficient use of materials.

Sector-specific measures to increase recycling rates where these are still very low like in plastics or concrete.

Implementing policies to overcome barriers to energy efficiency (energy management schemes, audits, soft loans, and energy service market) is a prerequisite for other (price-based) policies to work effectively as well. On the EU level, the Energy Efficiency Directive already provides important incentives by requiring regular energy audits for large enterprises, asking for national measures to support audits in small companies and setting up national energy efficiency obligation schemes. Some countries go beyond the Directive and implement additional measures. For instance, in Germany, companies receive tax discounts for using a certified energy management system, which has led to a drastic increase in ISO 50001 certifications here. Further, the country supports so-called learning energy efficiency networks. Both measures could be used as a blueprint for EU initiatives.

Energy-intensive industries can also help other sectors to decarbonise, e.g. by providing excess heat to nearby district heating networks. While large potentials are available here throughout Europe, various barriers are preventing its uptake. Policies can support the uptake by e.g. hedging high risks in individual projects, engaging top management by offering adequate incentives, regulating excess heat release in national immission control acts, strengthening local heat planning and providing investment grants.

In general, it is necessary to set incentives towards a low-carbon industry as early as possible to accelerate the market entry of efficient and innovative processes as increases of $\mathrm{CO}_{2}$ price probably take place after 2040 and consequently affect only a small share of investment decisions taken.

\section{REFERENCES}

Biere, D.; Fleiter, T.; Hirzel, S.; Sontag, B., (2014), Industry - more than just processes: acombined stock-model approach to quantify the energy saving potential for space heating in European industry,Arnhem (eceee industrial summer study 2014, June 2-5, Arnhem).

Capros et al., (2016), EU Reference Scenario 2016, Energy, transport and GHG emissions Trends to 2050, Luxembourg, Publications Office of the European Union.

Fleiter, T., Fehrenbach, D., Worrell, E., Eichhammer, W., (2012), Energy efficiency in the German pulp and paper industry - A model-based assessment of saving potentials, In Energy 40 (1), pp. 84-99.

Fleiter, T., Schlomann, B., Eichhammer, W., (Eds.) (2013), Energieverbrauch und CO2 Emissionen industrieller Prozesstechniken - Einsparpotenziale, Hemmnisse und Instrumente, Fraunhofer Institut für System- und Innovationsforschung, Stuttgart: Fraunhofer Verlag. 
Herbst et al., (2018a), D.5.5: Summary report on case study: The contribution of innovative technologies to decarbonise industrial process heat, A report compiled within the H2020 project SETNav. www.set-nav.eu.

Herbst et al., (2018b), Scenario analysis of a low-carbon transition of the EU industry by 2050: Extending the scope of mitigation options, In: European council for an energy efficient economy (eceee), Berlin: eceee 2018 Industrial Summer Study on Energy Efficiency, Proceedings. Panel: 4. Technology, products and system optimisation.

Rehfeldt, M., Rohde, C., Fleiter, T., Toro, F., Reitze, F., (2016), A bottom-up estimation of heating and cooling demand in the European industry, ECEEE Industrial Summer Study Proceedings 2016 (103-16 Rehfeldt et al).

\section{ACKNOWLEDGEMENTS}

The analysis was executed within the EU project SET-Nav (Navigating the Roadmap for Clean, Secure and Efficient Energy Innovation), which received funding from the European Union's Horizon 2020 research and innovation programme [GA-No. 691843]. For further information, see: http://www.set-nav.eu/. 


\title{
OSCILLATORY FLOW BIOREACTOR FOR BIO-PROCESSING WITH LOW TEMPERATURE HEAT SUPPLY
}

\author{
Bettina Muster ${ }^{1}$, Judith Buchmaier ${ }^{1}$, Ulrich Griesbacher ${ }^{1}$, Bernd Nidetzky ${ }^{2}$, Rama Gudumichi ${ }^{2}$, Safaa Ahmed $^{3}$, \\ Anh Phan ${ }^{3}$, Adam Harvey ${ }^{3}$, Christoph Brunner ${ }^{1}$ \\ ${ }^{1}$ Industrial Processes and Energy Systems \\ AEE - Institute for Sustainable Technologies \\ A-8200 Gleisdorf, Feldgasse 19 \\ Tel.: +43 (0)3112 5886-452 \\ b.muster@aee.at \\ ${ }^{2}$ Austrian Center of Industrial Biotechnology \\ Petersgasse 14, 8010 Graz, Austria \\ ${ }^{3}$ School of Engineering \\ Newcastle University \\ Newcastle upon Tyne, UK
}

NE1 7RU

\section{SUMMARY}

In this work we introduce a new "oscillatory flow bioreactor" (OFB), specifically designed for treating bio-slurries with high solid loading. The feasibility of the reactor is proven in terms of mixing and oscillatory power requirement. It is shown that uniform mixing of cellulose slurries with up to $15 \%$ solids can be maintained along the reactor length and energy input is minimally influenced by the slurry density and slurry viscosity. The oscillation amplitude and reactor length have minimal influence, while the main dictating factor for energy demand is the oscillation frequency. These results prove that the reactor enables efficient mixing with low energy intensity. Heat transfer coefficients are increased by oscillatory mixing, allowing for better low temperature heat supply in comparison to standard tubular reactors. The reactor was evaluated for enzymatic hydrolysis, with reference to stirred tank reactors (STR). The OFB system operated at $3.5 \mathrm{~Hz}$ and $10 \mathrm{~mm}$ oscillation amplitude exhibited similar conversion efficiency after 6 hours, despite operating at only $12 \%$ of the STR's energy intensity $\left(\mathrm{W} / \mathrm{m}^{3}\right)$. Future work will focus on continuous operation of the plant, including product separation and efficient pumping at low net flows.

\section{INTRODUCTION}

Processing of biogenic resources and extraction of valuables from biogenic solids, such as starch, ligno-cellulose or lignin, has tremendous importance in industry ranging from the food industry to pharmaceutical industry, the pulp and paper industry up to the biobased industry. Additionally, lignocellulosic waste (agricultural, forest residues and food waste etc.) is abundant and considered an important source for producing high-value chemicals and commodities ${ }^{1}$. The amount of lignocellulosic waste is tremendous and using this waste as raw material would be one huge step in making our society more resilient towards the devastating resource depletion we are facing.

The bioconversion of ligno-cellulosic materials, however, still faces problems in developing an economic process ${ }^{2}$. Among the many challenges encountered during development of lignocellulosebased value chains, one pervasive and often dominant problem is the effective extraction of soluble sugars (xylose, glucose, pentose etc.) from the polysaccharides cellulose and hemicellulose, which together make up for about $60-70 \%$ of total mass of the lignocellulose ${ }^{1,3}$. Despite enormous efforts over decades to improve the enzymatic saccharification, the actual costs of this core step of any 
bioconversion process still remain high ${ }^{4}$. It is recognized that the high costs of lignocellulose hydrolysis are due to two strongly intertwined factors: the high enzyme loading required (to achieve a certain degree of conversion within an acceptable time) and inefficient process performance due to a rather slow reaction. The slow reaction kinetics, true for all enzymatic hydrolysis processes based on starch or ligno-cellulose, are currently dealt with via batch processing in classic stirred tank reactors (STR). This reactor concept poses not only problems in term of potential inhomogeneous mixing and unstructured mass transfer, but also in terms of power consumption, with high required energy intensity due to heating large batches and necessary large energy transfer areas if low temperature heat, such as waste heat should be used for process heating. To address these problems, a new reactor concept for the (ligno) cellulose hydrolysis is proposed in this work. It is based on the design of a baffled tubular reactor with oscillating flow. This type of reactor, the "OBR" (oscillatory baffled reactor), offers very good mixing and plug flow properties. A core characteristic of OBRs is their ability to decouple flow velocity and residence time. This enables continuous processing for processes with long residence times. This is achieved via oscillations inducing turbulent flow of process media (oscillatory Reynolds number, Re, $\mathrm{o}=1000-2000$ ) between baffles, while the net flow velocity remains in the laminar regime $(\mathrm{Re}, \mathrm{n}=50-200)$. Oscillatory flow reactors are thus perfectly suited for processes in which long residence times, uniform mixing and plug flow are required. Oscillations are induced into the fluid via a piston, the reactor itself remains stationary. The area between the baffles is turbulent and ideally mixed.

\section{MATERIAL AND METHODS OFB plant design}

For biogenic slurries, a specific Oscillating Flow Bioreactor has been developed to allow for a continuous enzymatic hydrolysis process using a plug-flow reactor for the first time with substantial effects mixing possibilities at high solid loadings. The plant concept is shown in Figure 1. The continuous process aims to allow much lower energy demand peaks and the potential integration of waste heat without the enlargement of heat exchanger surfaces.

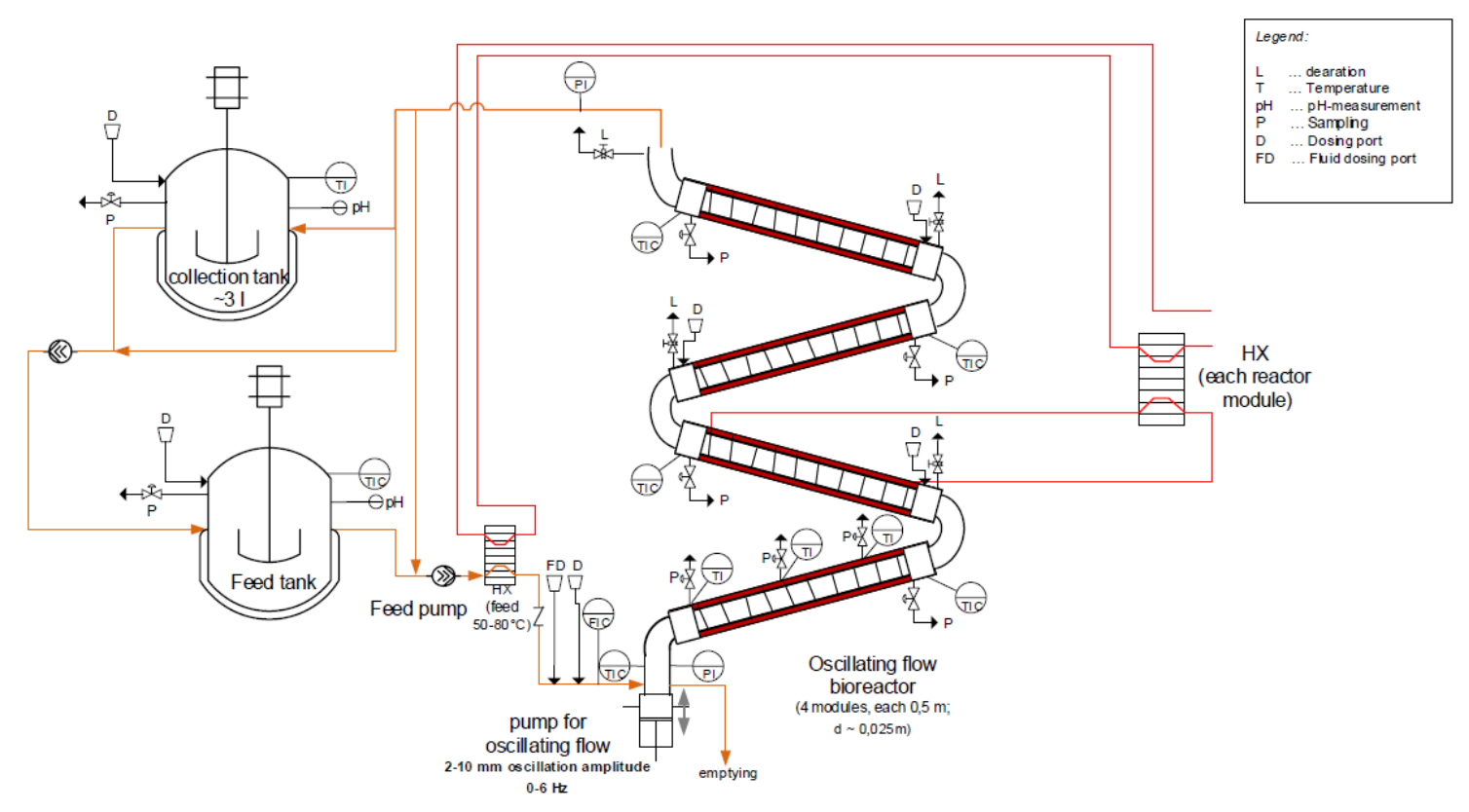

The OFB laboratory plant has been conceptually designed based on pre-tests performed at existing OBR modules at the Newcastle University, UK and was constructed at Möstl Anlagenbau. The 
modular set-up consists of a core reactor of 4 modules, each $1 \mathrm{~m}$ long with $25 \mathrm{~mm}$ inner diameter. The glass tubes are double-walled to allow for hot water heat supply (Heatboy Type E-Tech W 09). Modular connections allow various configurations with varying reactor positions (angles from vertical to horizontal) and integration of measurement points. In this work, mixing and power requirement measurements have been evaluated in a 1 module set-up, positioned either horizontally, inclined to $19 \%$ or fully vertical. One feed and one CIP tank have been included in the plant. A preliminary design tool has been set-up in the conceptual phase which is currently validated with the experimental results.

\section{Testing procedure and analysis}

Specific tests have been carried out for analysing the mixing performance, as well as the energy requirement and heat transfer enhancement in the new reactor design. To reduce the amount of treated mass flows, the biobased industry aims to treat slurries at high solid loadings. Therefore, tests focussed on $5,9,12$ and $15 \%$ of cellulose concentration.

The OFB plant is referenced with lab-scale STRs (1.5-L fedbatch-pro® bioreactor system equipped with a six-bladed Rushton turbine impeller, DASGIP AG, Juelich, Germany) to evaluate the intensification potential of enzymatic hydrolysis. In this paper, we focus on the results of batch hydrolysis. In preliminary tests, a well-defined input stream of $\alpha$-cellulose powder (Sigma Aldrich) was used.

For evaluating the mixing performance, samples were drawn along the reactor length and TS content as well as glucose conversion at the end of a 6 hour saccharification period was measured. Additionally mixing was qualitatively monitored via cameras. Power requirement is recorded with power meters (Acean power meters, DDS 353) connected to the oscillator as well as the stirrers of the feed and CIP tank. For reference tests in lab scale STRs, Energy logger 4000, Voltacraft, Hirschau, Germany was used for power measurements.

Heat transfer coefficients can be evaluated based on flow and temperature conditions of the heated double-wall reactor modules monitored with Flexim ultrasonic measurement equipment.

For assessing the conversion rate, an analysis procedure based on DMA 35 from Anton Paar has been set-up for glucose measurement. DNS assays were used for detailed evaluation.

\section{RESULTS}

\section{Mixing performance}

Test results show that alpha-cellulose cellulose in the OFB lab plant remains uniformly mixed. Figure 8 shows cellulose concentrations at various mixing times and reactor positions in a vertical set-up at $3.5 \mathrm{~Hz}$ oscillation frequency and $6 \mathrm{~mm}$ amplitude. The uniform mixing results here agree with the visual observation of the uniform cellulose slurry $(\mathrm{SL}=12 \%)$ as shown in Figure 7. This indicates that the effect of the tube length on the mixing of cellulose slurry is insignificant.

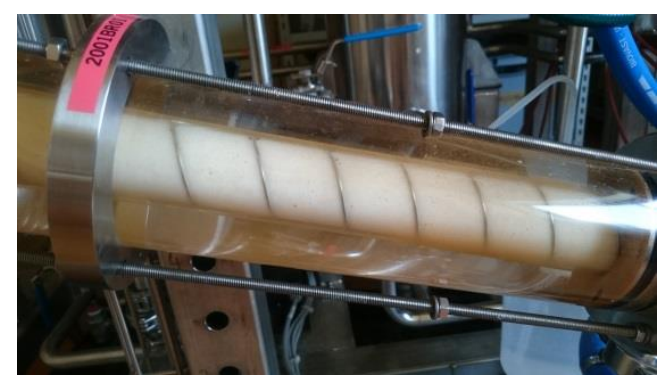

Figure 7: Picture of $12 \%$ cellulose slurry mixed at $3.5 \mathrm{~Hz}$. 

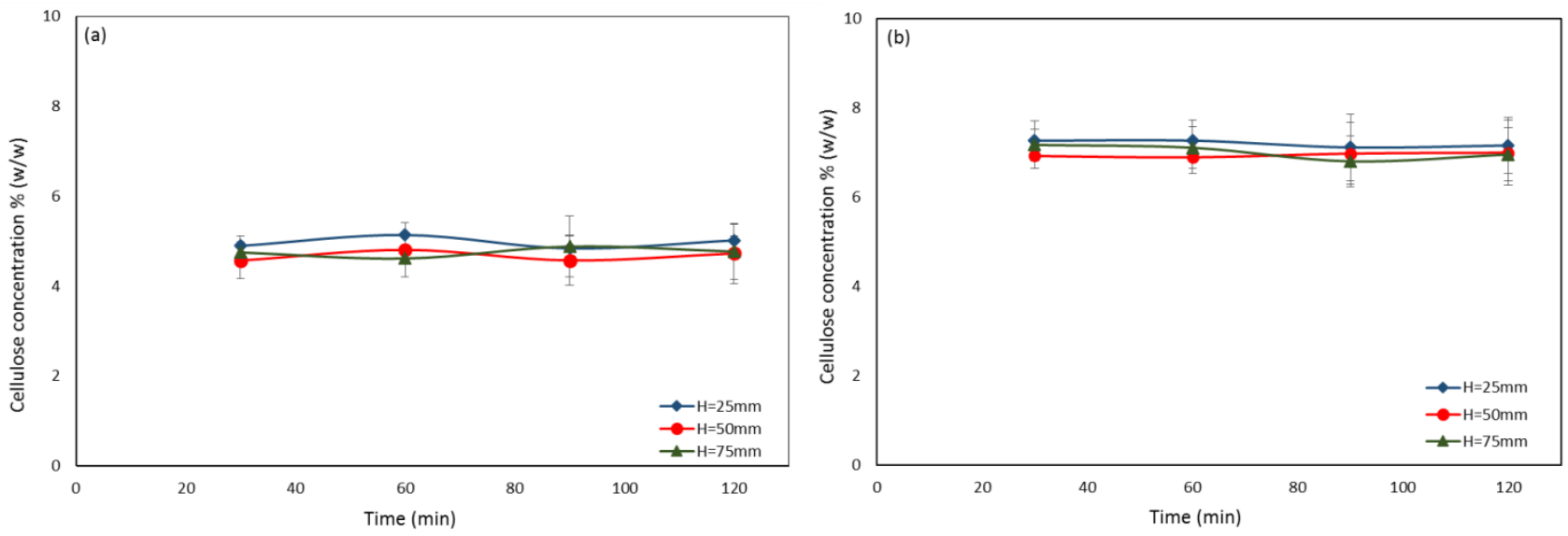

Figure 8: Variation of cellulose concentration along the reactor within 2 hours at $f=3.5 \mathrm{~Hz}$ and $\mathrm{x}_{0}=6 \mathrm{~mm}$, varying height from inlet

As alpha-cellulose powder swells in water, a 15\% SL becomes a pasty mixture, like potato puree. While this cannot be stirred effectively in a stirred tank reactor, as dead zones occur, it is uniformly mixed in the OFB reactor. This becomes visible in Figure 9Fehler! Verweisquelle konnte nicht gefunden werden., showing a picture of the reactor and glucose concentration (enzymatically converted from a cellulose slurry with $12 \%$ cellulose concentration) along the reactor length in horizontal set-up. At a $1.5 \mathrm{~Hz}$ oscillation frequency, there are still slight differences between the glucose measurements along the reactor (Figure 9) indicating insufficient mixing. Mixing is uniform with constant conversion of cellulose to glucose along the reactor length at any frequency higher than $2 \mathrm{~Hz}$.

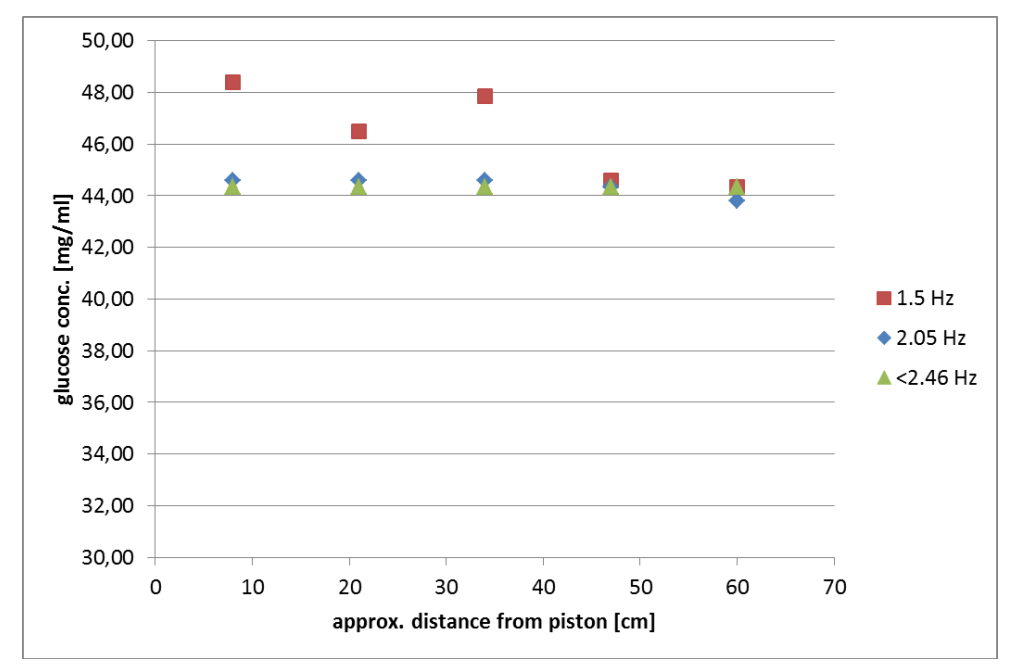

Figure 9: Variation of glucose concentration along the reactor length after 6 hours of enzymatic hydrolysis with $12 \%$ cellulose at varying oscillation frequencies (left)

\section{Power requirement}

Power requirement for oscillation interestingly does not correlate with feed viscosity, allowing the treatment of viscous slurries at low energy intensity. Figure 10 shows the power requirement for the oscillator at $6 \mathrm{~mm}$ oscillation amplitude for various fluids. Tests with this rig showed that neither density nor viscosity have a direct influence on oscillatory power requirement. In fact, water and $15 \%$ cellulose slurry show only minor deviations. The oscillation frequency has a direct influence on power 
requirement in all tests. The mixing results proved that frequencies $>2 \mathrm{~Hz}$ will be sufficient for mixing, which means power requirement of the OFB can be minimsed by optimizing the required frequency at around $2 \mathrm{~Hz}$.

The effect of reactor length and oscillation amplitude is shown in Figure 11 for $10 \%$ cellulose slurry. Doubling the reactor length only increased the power requirement by $\sim 2 \%$ for $3.5 \mathrm{~Hz}$ and $15.8 \%$ for 5 Hz. These results will be proven in further measurements with longer measurement periods, but they give a promising picture for the lower frequency range. The change of oscillation amplitude from 6 $\mathrm{mm}$ to $10 \mathrm{~mm}$ influences power requirement by $7 \%$ for $1 \mathrm{~Hz}$ and by $17-18 \%$ for 3.5 and $5 \mathrm{~Hz}$ respectively.

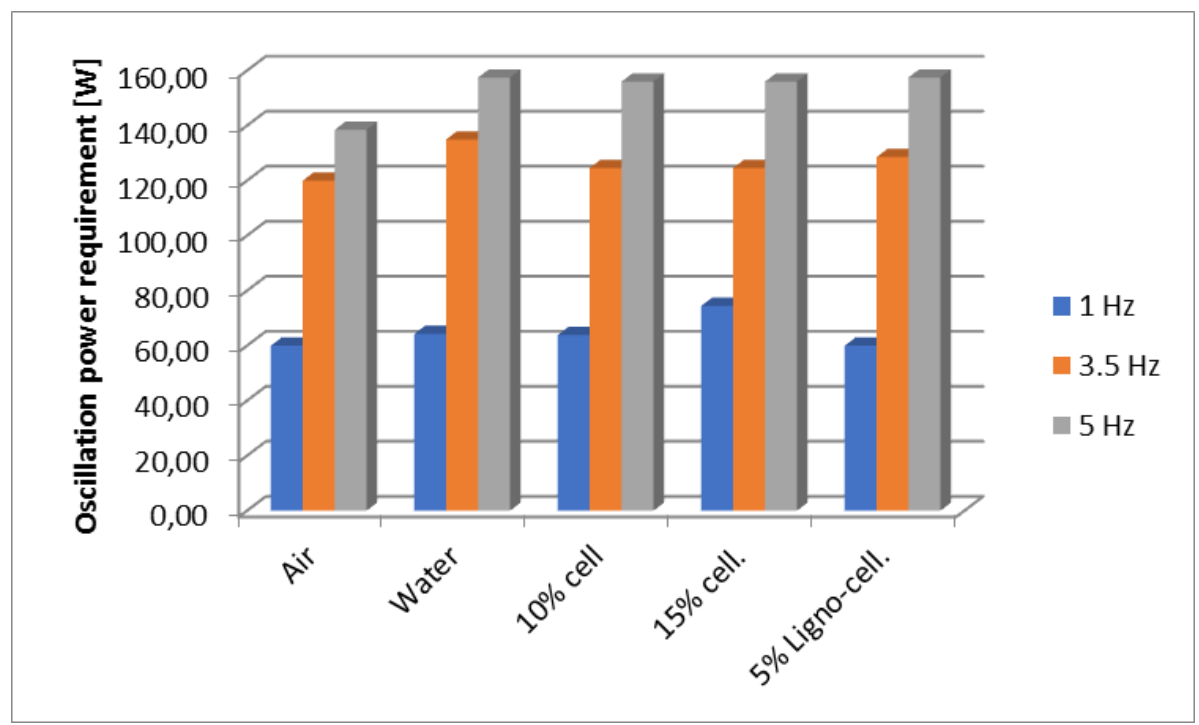

Figure 10: Energy Input at various oscillation frequencies for different media
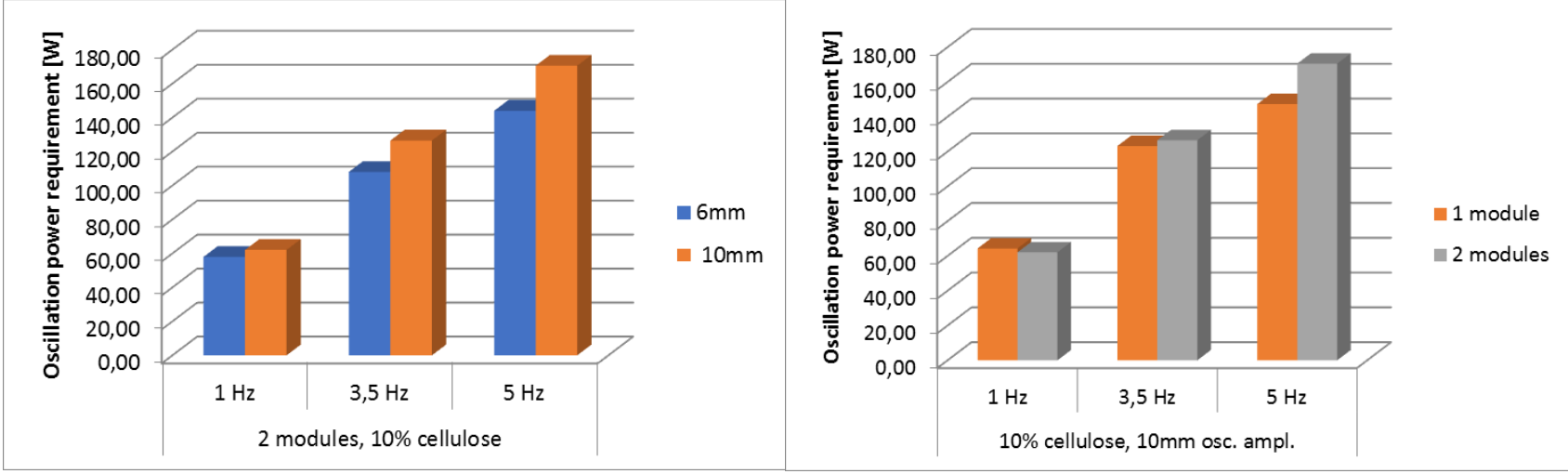

Figure 11: Oscillation power requirement for varying reactor length (left) for $10 \%$ cellulose concentration, $10 \mathrm{~mm}$ oscillation amplitude and varying oscillation amplitude (right) for $10 \%$ cellulose concentration for 2 reactor modules

\section{Enhancement of heat transfer coefficients}

In terms of potential waste heat usage, calculations have shown that the continuous process set-up for enzymatic starch hydrolysis (such as in mashing in breweries) reduces the required area for heat transfer by $1 / 3$ and energy demand peaks can be reduced by $80 \%{ }^{5}$. In that study, this is mainly due to smooth heating profiles implemented in the "continuous process".

The oscillatory mixing increases the radial transport of the fluid within the reactor, increasing heat transfer coefficients, for the same net flow Reynolds numbers. Figure 12 shows that a change in 
frequency from 1 to $3 \mathrm{~Hz}$ (and thus higher mixing intensity) leads to 30-40\% higher heat transfer coefficients for $9 \%$ cellulose slurry. The influence of the SL, however, is minor. Tests were additionally performed with water, showing an increase in heat transfer coefficient of $+23 \%$. The lower increase for water in comparison to biomass slurry indicates that specifically heat transfer of biogenic slurries can be improved by the reactor.

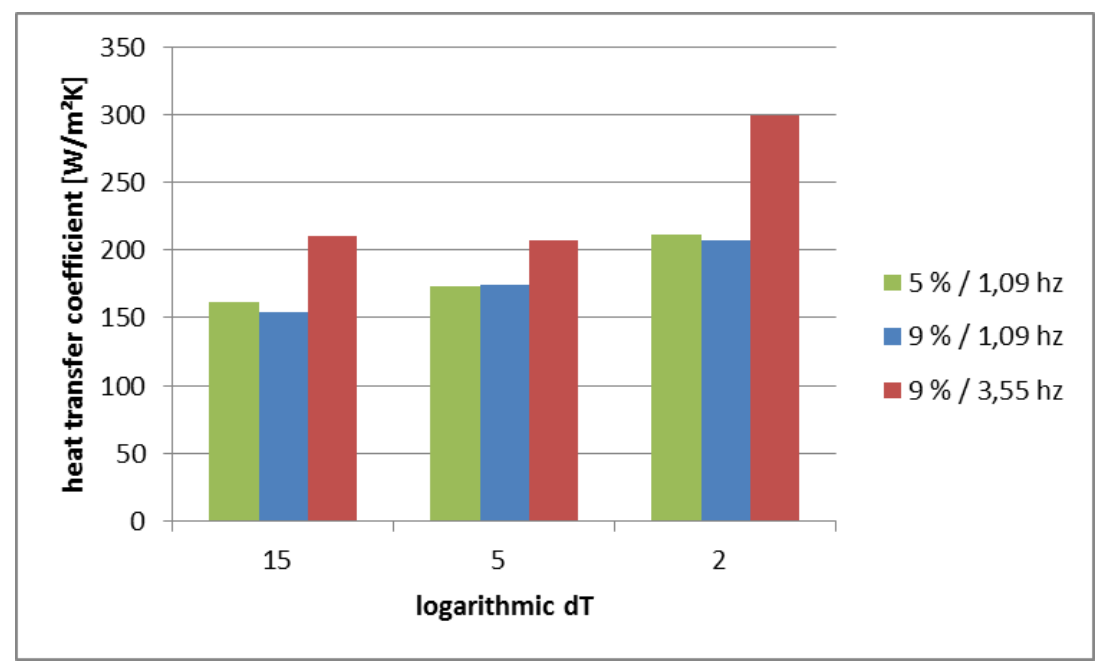

Figure 12: Heat transfer ceofficients measured for 5\% and 9\% SL at different frequencies in OFB lab plant

\section{Enzymatic hydrolysis in batch mode}

Enzymatic hydrolysis was performed in stirred tank reactors and in OFB modules in batch mode and glucose concentration increase was measured over the course of the first 6 hours. It was expected that highest benefits of the OFB can be achieved in the beginning of the reaction course, when mixing is decisive. However, the OFB lab plant did have difficulties in remaining a constant temperature profile along the reactor due to missing insulation and unheated reactor parts (measurement points, sample ports etc). The highest conversion could be achieved for 15\% SL, where mixing of the OFB module outperforms the STR mixing efficiency. The OFB system operating at 3,5 Hz and $10 \mathrm{~mm}$ oscillation amplitude shows similar conversion efficiency after 6 hours, although operating at only $12 \%$ of the STR's power density. Increasing the frequency and mixing intensity beyond this level does not lead to better enzymatic conversion, leading to the conclusion that this level of mixing is high enough for the system to be operating in the mixing-independent regime.

Further tests for 24 hours will be necessary to evaluate the effects of shear along the whole reaction course of enzymatic hydrolysis. In addition, continuous tests are foreseen to overcome the effects of product inhibition in batch reactors. Here, the greatest intensification potential is expected with the OFB plant, as it should allow a practical design of continuous reactor for slow enzymatic hydrolysis reaction, without mixing limitations, operating in true plug flow (which should minimize the size of the reactor). 


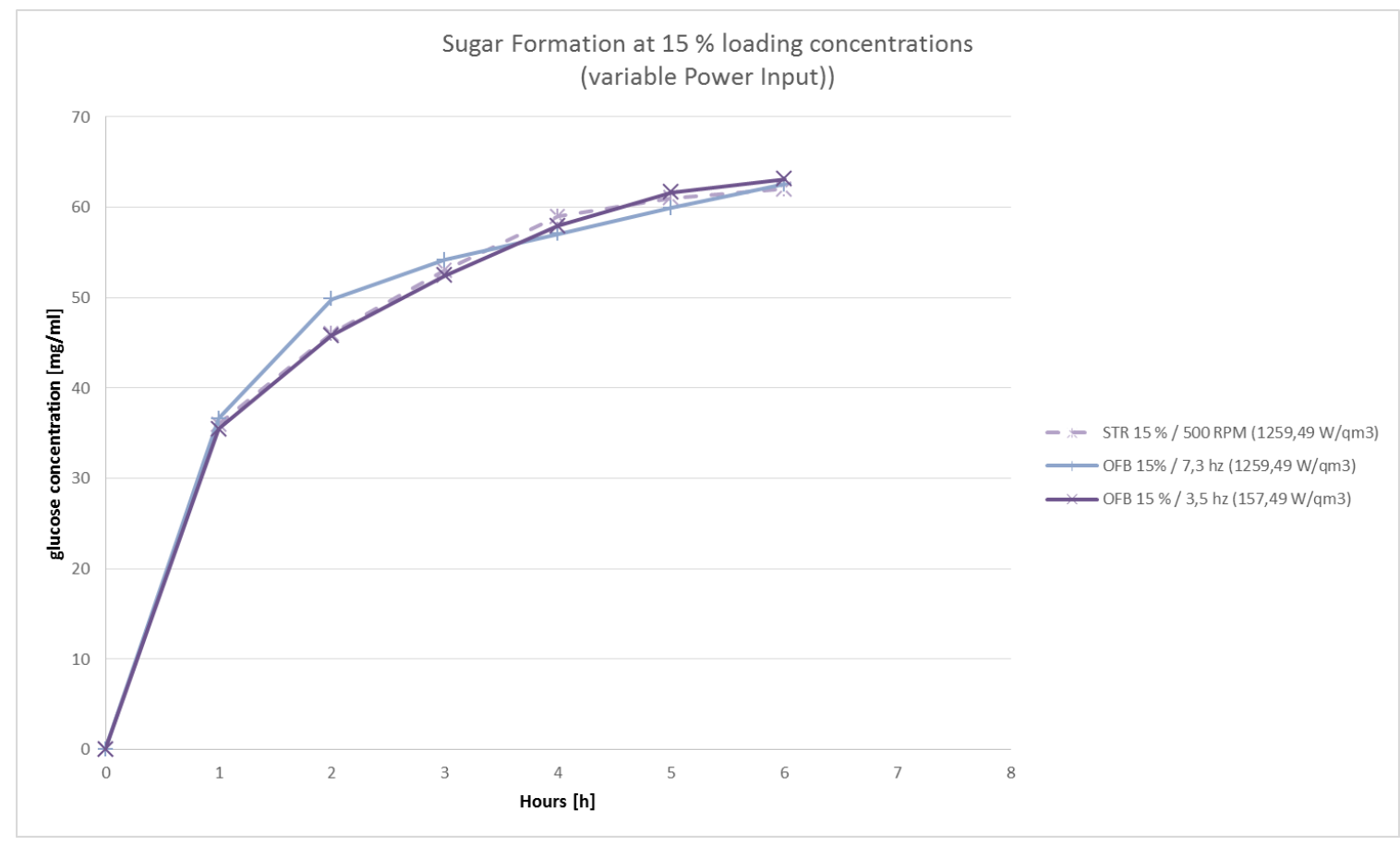

Figure 13: Evolution of glucose concentration over the first 6 hours of enzymtic hydrolysis comparison of STR and OFB performance at different energy intensities $\left(\mathrm{W} / \mathrm{m}^{3}\right)$

\section{CONCLUSIONS}

In this work, a novel design of "OFB" (Oscillatory Flow Bioreactor) was evaluated for enzymatic hydrolysis of alpha cellulose. So far it is clear that at small scale it can reproduce the results achieved in an STR. Here, we have identified the conditions necessary for operating the OFB in the mixingindependent regime, and the agreement with STR results at the same scale is promising. This is very encouraging, as this was achieved at $12 \%$ of the power density of the STR.

Importantly, the performance of OBRs can be reproduced at larger scales, whereas that of STRs typically cannot. Uniform mixing is notoriously difficult to achieve in large STRs in the chemical industry. Here, this problem is magnified by the use of biomass, as, firstly, the scale of biomass processing tends to be significantly greater than (batch processes in) the chemical industry and the physical properties of the feedstock are generally more challenging, as they are necessarily heterogeneous, and often highly viscous. Furthermore, the OFB would be operated in a truly continuous plug flow manner, with the various advantages of this mode of operation.

Based on the results so far, it appears that the newly developed OFB module could be the basis for a hydrolysis reactor concept. This could be applicable in the biobased industries, food industry, chemical industry, biodiesel production or pulp and paper industry to save energy, resources and time. Any other slow biogenic reaction, currently performed in batch, could potentially be enhanced by the reactor concept. 


\section{REFERENCES}

1. S. Chundawat, G. Beckham, M. Himmel and B. Dale, Deconstruction of lignocellulosic biomass to fuels and chemicals, Annu Rev Chem Biomol Eng, 2011, 2, 121-145.

2. M. Taha, M. Foda, E. Shahsavari, A. Aburto-Medina, E. Adetutu and A. Ball, Commercial feasibility of lignocellulose biodegradation: possibilities and challenges, Curr Opin Biotechnol. , 2016, 38, 190-197.

3. L. Jönsson and C. Martín, Pretreatment of lignocellulose: Formation of inhibitory byproducts and strategies for minimizing their effects, Bioresour Technol., 2016, 199.

4. H. Blanch, B. Simmons and D. Klein-Marcuschamer, Biomass deconstruction to sugars, Biotechnol J., 2011, 6, 1086-1102.

5. B. Muster-Slawitsch, Dissertation, Technical University Graz, 2014. 


\title{
ENERGY EFFICIENCY AND FLEXIBILITY FOR URBAN INDUSTRIAL PRODUCTION SITES THROUGH INTEGRATION OF GROUND SOURCE HEAT PUMPS (GSHP)
}

\author{
Dipl.-Ing. Ivan Bogdanov, M.Sc. ${ }^{1}$, Prof. Dr.-Ing. Dipl.-Kfm. Alexander Sauer ${ }^{1}$ \\ Fraunhofer Institute for Manufacturing Engineering and Automation IPA ${ }^{1}$ \\ Nobelstr. 12, 70569 Stuttgart, Germany \\ Phone: +49-711-9701338 \\ E-Mail: ivan.bogdanov@ipa.fraunhofer.de
}

\section{SUMMARY}

The present paper provides an insight into the current research field of heating and cooling in the industry, with focus on industrial processes as part of urban production sites. An intelligent and sustainable energetic coupling of production sites with their urban surroundings is a key solution to increase the energy efficiency and flexibility. With this objective, an approach is described, which pursues the integration of ground source heat pumps (GSHP) into energy systems of urban industrial production sites. Besides the methodology and its assessment model for manufacturing processes, the paper presents energy efficiency and flexibility scenarios in the automotive industry, as well as some existing isolated applications as best-practice examples.

\section{INTRODUCTION}

One of the current challenges of our society consists in the reduction of fossil fuel consumption and greenhouse gas emissions, aiming a sustainable life support of future generations. The German government has therefore issued the so called "Energiewende", a vast national project of energy transition to a low-carbon economy.

Besides the sectors building, transport and commerce, trade and services, the manufacturing sector accounts for a large proportion of the negative environmental impact. It caused in 2015 about 61.5 million tons of $\mathrm{CO}_{2}$ emissions in Germany solely due to production processes [Umweltbundesamt 2015]. In turn, approx. $28 \%$ of the German final energy consumption is attributable to the industry, of which approx. $75 \%$ represent process heat and cooling applications [Arbeitsgemeinschaft Energiebilanzen 2013].

Other important challenges of today's social and economic sectors occur due to the trend of urbanisation. Forecasts like the high increase of urban population or the doubling of resource needs by 2050 are forcing a sustainable life and economy, with urban space to be particularly regarded by manufacturing companies related to their production operations - the vision of "urban production". This vision allows a production, which is indulgent and compatible and can take place even in urban areas, to the advantage of all stakeholders: companies and employees, but also city and citizens. The basis for an urban production thereby represents a symbiosis between production sites and their urban surroundings, by optimising the positive contribution of production sites to these surroundings, instead of "merely" minimising their negative impact. [Lentes 2016]

In view of the above, manufacturing companies must respond on the one hand to external challenges and constraints, such as increasing energy efficiency, implementing energy flexibility, but also assuring the security of energy supply as well as integrating production sites in the urban surroundings. On the other hand, it is important to adhere to internal challenges and constraints, such as cost reduction, greenhouse gas emissions reduction, demand side management (DSM) and decentralised energy supply (figure 1). Specific challenges are thereby e.g. a high primary energy 
consumption and high energy costs for the provision of heating and cooling at production sites, but also the lack of closed energy circuits (e.g. energy cascades - waste heat recovery), an efficient energy storage (including heat and cold) or the exploitation of renewable energy sources.

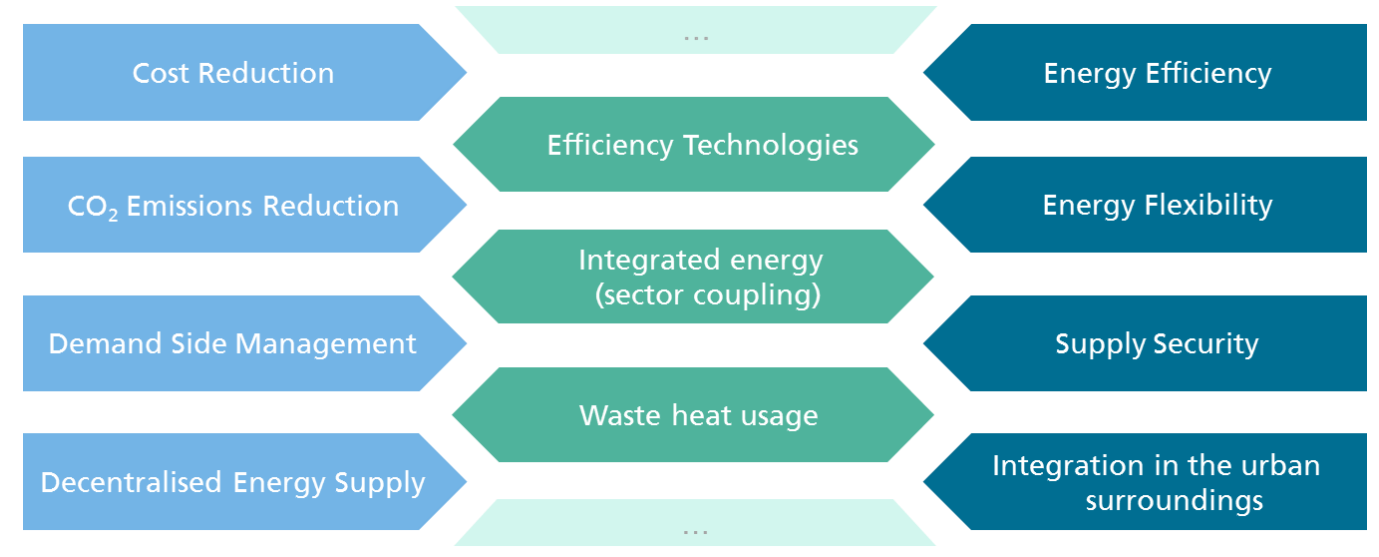

Fig. 1: Field of tension between internal (left) and external (right) constraints for manufacturing companies

In this field of tension, it is important to develop holistic concepts as a solution for the realisation of the energy transition in the factory. In order to create optimal synergies, the entire energy function chain should be considered: from efficient and sustainable energy generation, via intelligent energy distribution and storage, to efficient and flexible consumption and effective recovery. Furthermore, an intelligent and sustainable energetic coupling of production sites with their urban surroundings leads to the creation of energetic symbioses to the advantage of all stakeholders.

In view of the above, such an energetic coupling is a key solution to increase the energy efficiency and energy flexibility of production sites. In order to accomplish these goals, technologies must be used, among others, for exploiting, but also storing industrial waste heat and renewable heat sources. Specifically, the use of efficiency technologies plays a major role also for implementing the concept of integrated energy - sector coupling.

One particular efficiency technology, which can be used to achieve these targets, is the ground source heat pump (GSHP). In the case of industrial applications, the high temperature heat pump can be deployed. This technology, which enables both the usage and storage of industrial waste heat, as well as the exploitation of base load capable renewable energy sources, can be implemented at the interface between (residential) buildings and manufacturing processes, building a symbiosis between factory and urban surroundings.

\section{THE HEAT PUMP - AN EFFICIENCY TECHNOLOGY FOR URBAN PRODUCTION SITES}

In a current study carried out with the participation of the University of Stuttgart [Kube et al. 2017] approx. 150 innovative and commercially available technologies have been examined, which are still largely unexploited, but have on the long-term a high potential to become economical and to increase the energy efficiency in the manufacturing industry. After a classification in technology categories, an assessment by means of quantitative and qualitative indicators, as well as an analysis on the resilience and scalability of the researched efficiency potentials, a remaining short-list of ten technologies was established. These technologies were then analysed on their technical and economical saving potentials by means of a literature review, but also through a survey among manufacturers and users of the respective technologies. 
Based on this data, application examples were described, upon which the technical potential at the federal level was scaled up in selected industrial sectors in Germany. According to their respective technical potential, the following descending ranking resulted [Kube et al. 2017]:

High temperature heat pump (HTW)

Optimisation software for energy network systems (OfE)

Intelligent drive solutions (IA)

Automatic shutting-off of stand-by compressed air networks (AASBD)

Water as refrigerant $(\mathrm{WaK})$

Magnetic cooling (MK)

Microwave technology (MWT)

Intelligent DC networks (IG)

Honeycombed ceramic heat exchanger $(\mathrm{WkW})$

Energy efficient control cabinet cooling (ESK)

The savings that have been considered in the estimation of the technical potential are those that arise when the respective technology is used wherever it is technically possible. The extrapolation of the technical potential at the level of the Federal Republic of Germany was carried out based on the respective data from specific applications for each technology. The savings potential of the hightemperature heat pump refers to the total industrial heat demand in Germany. The other technologies were estimated respectively for the so-called top-industry-sector, in which the respective technology can reach the highest efficiency potential. As a result, Figure 1 shows the projected final energy consumption, both with and without the use of the respective technology in the corresponding topindustry-sector in Germany [Kube et al. 2017]. 


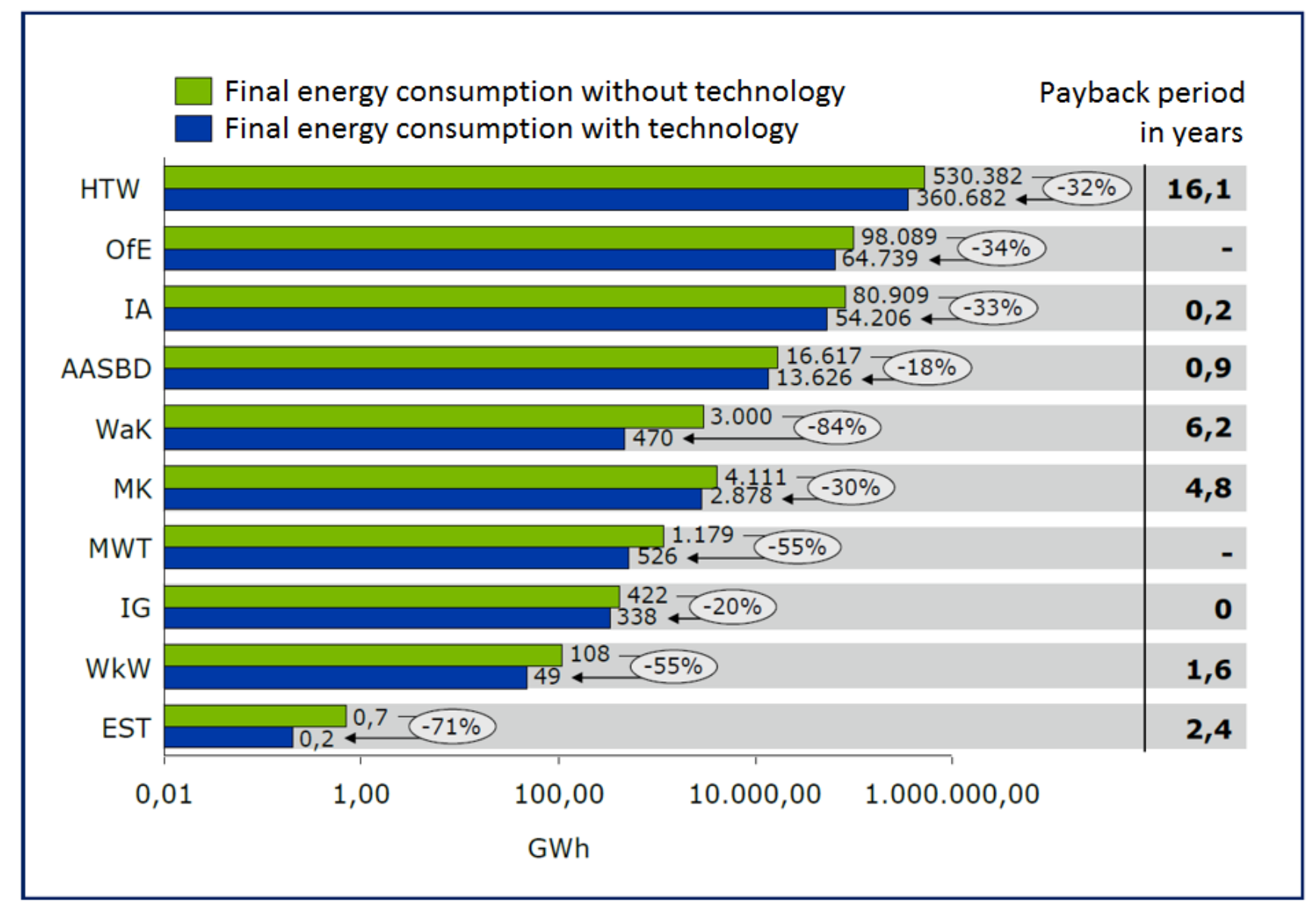

Fig. 1: Final energy consumption with and without the use of the respective technologies, scaled up on federal level in Germany [Kube et al. 2017]

With a technical energy saving potential of approx. $120 \mathrm{TWh} / \mathrm{a}\left(<100^{\circ} \mathrm{C}\right)$ or even approx. $170 \mathrm{TWh} / \mathrm{a}$ $\left(<140{ }^{\circ} \mathrm{C}\right)$ in the German industry [Wolf et al. 2014], the high-temperature heat pump represents the most promising energy efficiency technology [Kube et al. 2017]. Furthermore, an analysis of [Naegler et al. 2015] shows that approx. $25 \%$ of the final energy consumption in the European industry can be attributed to the generation of process heat, space heating and hot water at temperatures below $100{ }^{\circ} \mathrm{C}$, which leads to even higher potentials for this technology at the interface between industrial processes and building infrastructures.

However, the high-temperature heat pump is accompanied by high investment costs, which leads to an average payback period of over 16 years. Thus, the economic potential of this technology is estimated at $5 \%$ of the technical potential, resulting in the possible realisation of $8.5 \mathrm{TWh}$ energy savings. [Kube et al. 2017]

High-temperature heat pumps can generate heating and water temperatures of up to $140{ }^{\circ} \mathrm{C}$ in the flow using special compressor technology, multistage cycle processes and specially developed refrigerants [Bobelin 2012]. The various refrigerants or mixed refrigerants used include, for example, ECO3, HFO-1336mzz-Z, LG6 or R744 $\left(\mathrm{CO}_{2}\right)$ [Wolf et al. 2014]. An economic and efficient operation of heat pumps in industrial environments requires the presence of a suitable heat source. If this requirement is met, a high-temperature heat pump often achieves a COP of 3.0 or more, merely in the heating operation.

This very efficient technology, however, is in direct competition with conventional technologies based on combustion (e.g. gas firing) or with other technologies for waste heat recovery and thus often 
represents only a supplementary measure to these technologies in industry [Kube et al. 2017]. Nevertheless, the use of heat pumps can still reduce the fuel demand and thus contribute to the decarbonisation of the industry. This technology can be used efficiently, especially in the food, paper, chemical, pharmaceutical, plastics or metalworking industries. Particularly in the first three sectors mentioned, there is great potential for heat pump applications, also for heat demand beyond $80{ }^{\circ} \mathrm{C}$ [Kube et al. 2017]. However, depending on the particular industry, the existing infrastructure, and the availability of suitable heat sources and heat sinks in operation, the relative energy savings are broadbased. For example, according to Wolf et al., the use of a high-temperature heat pump by a prefabricated house manufacturer, while using the waste heat of the on-site power plant, could reduce the heat demand of the wood drying plant by $73 \%$ [Wolf et al. 2014].

Furthermore, a particular application area of high-temperature heat pumps is the exploitation of the environment as heat source or heat sink. Specifically, the particular case of the exploitation of the shallow geothermal energy source $(<400 \mathrm{~m}$ depth) represents, from human perspective, an inexhaustible and practically ubiquitous, environment-friendly and thus very sustainable energy source. While in the building heating and cooling sector it stands for the state of the art since many years, industrial applications using this particular energy source remain, however, so far hardly considered. The reasons for this are numerous, including the already mentioned unusual payback periods due to high (exploitation) equipment and investment costs, but also the lack of information and widespread expertise, as well as the absence of a systematic approach for the integration of ground source heat pumps (GSHP) into energy systems of production companies.

These companies often face the current challenge of urbanisation of population and hence of manufacturing sites. Either existing factories are being surrounded by residential areas, or, in some cases, companies even deliberately tend to develop greenfield sites in the vicinity of residential estates.

In the first, historically developed case, the particularities of the urban vicinity mostly do not find any further consideration, therefore the factory or company is at best neutral to its urban surroundings. Furthermore, if an active involvement of citizens or partner companies takes place in the factory or the process and product design, the plant is considered participatory. On the other hand, the case of deliberate setup of manufacturing sites in the proximity of residential areas emphasises the use of the potential of the city or the exploitation of the corresponding markets. Depending on the adopted business model, companies either maintain existing business models or establish new ones, for example by using the emerging "sharing economy". Consequently, the basis of an "urban production" is a symbiosis between production sites and urban surroundings in the sense of optimising the positive contribution of the production to the city and the environment. [Lentes 2016]

Such symbioses can be established by the optimisation and integration of various factors at the interface between industrial sites and urban surroundings, especially in the area of energy. The wide base for energetic symbioses represents an interconnected handling of energy by considering two specific chain links of the energy function chain: efficient and sustainable, renewable energy generation as well as effective waste energy recovery, especially regarding heating and cooling, which count for a high rate of energy consumption, both in industry and residential. Therefore, a sustainable energetic coupling of production sites with their urban surroundings by mutual heat and cold generation as well as waste heat recovery, e.g. integrated compound heat utilisation, leads to the creation of symbioses for all stakeholders.

Against this background, a systematic approach for the efficient and economic integration of shallow geothermal energy using ground source heat pumps (GSHP) into energy systems of urban production sites is being developed and presented hereinafter. 


\section{INTEGRATION OF GSHP IN URBAN PRODUCTION SITES}

Aiming at the overriding goal of sustainable, cost-stable and $\mathrm{CO}_{2}$-free supply of heating and cooling in industry and residential, a systematic approach for the integration of GSHP in the industry is presented, which simultaneously leads to increased energy efficiency by sustainably providing and storing renewable as well as waste heat and cold.

In a first step in the preparation of this approach, as a pre-investigation to a technical potential analysis, an assessment model for manufacturing processes was developed. The aim is to identify, from the variety of these processes, those whose heating and/or cooling demands can be technically and economically (largely) covered by the use of GSHP. The basis for this model represents a catalogue of criteria and a weighting scale, which allow the assessment of most manufacturing processes - see Table 1 .

Table 1: Criteria catalogue and weighting scale as the basis of the assessment model of manufacturing processes

\begin{tabular}{|l|l|l|}
\hline Criteria category & Criteria & $\boldsymbol{W}_{\text {criteria }}$ \\
\hline \multirow{5}{*}{ Location criteria } & Subsurface conditions & 3 \\
\cline { 2 - 4 } & Environmental effects of heating and cooling supply & 2 \\
\cline { 2 - 4 } & Security of supply & 2 \\
\hline \multirow{5}{*}{ Process criteria } & Energy-related applicability (temperatures, required output) & 3 \\
\cline { 2 - 4 } & Snvestment, maintenance and operating costs & 3 \\
\cline { 2 - 4 } & Share of heating and/or cooling demand on the total energy demand of the process & 2 \\
\cline { 2 - 4 } & Influence of energetic process parameters on product quality & 1 \\
\cline { 2 - 4 } & Influence on production and process layout design & 1 \\
\hline \multirow{2}{*}{ Total } & & $\mathbf{1 7}$ \\
\hline
\end{tabular}

The weighting of these criteria ( $W_{\text {criteria }}$ ) is based on a three-level scale, whereby the largest weighting factor (3) was assigned to the constraints. Non-inevitable factors, but to which a high importance is attributed, are weighted by the factor 2. A weighting factor of 1 is used for criteria corresponding to secondary aspects regarding the integration of this energy supply approach.

Each criterion can be assigned a score value $\left(S_{\text {criteria, },}\right)$ of 0 or 1 , depending on whether the criterion is fulfilled or not. The final assessment score $\left(S_{\text {process }}\right)$ is formed from the sum of the respective products between each criteria score value $\left(S_{\text {criteria }, i}\right)$ and the corresponding criteria weighting $\left(W_{\text {criteria }, i}\right)$, according to (eq. 1):

$S_{\text {process }}=\sum_{i=1}^{n} S_{\text {criteria }, i} \times W_{\text {criteria }, i}$

Whereby: $\quad S_{\text {criteria }, i} \in\{0,1\}$

$W_{\text {criteria }, i} \in\{1,2,3\}$, according to Table 1

$n=$ Number of considered criteria, according to Table 1 
The proposed constraints include subsurface conditions, energy-related applicability and costs. The proposals for the weighting factors 2 with regard to environmental impacts and security of supply should generally be valid for the location Germany. The low-estimated interaction between the energy supply approach discussed herein and production aspects like product quality and production layout design is reflected in the weighting of these criteria, numbered 1. However, the proposed weighting scale may be adjusted accordingly, depending on the industry sector or local constraints (e.g. environmental impact, security of supply).

The extent of the energetic use of the soil is determined by existing subsurface conditions, e.g. high thermal conductivity, low borehole resistance and the existence of groundwater facilitate the integration of GSHP. Depending on the needed process flow temperature and the respective COP, but also on the required energy output in order to cover the demand, the applicability of GSHP for industrial processes can be given or not. Other crucial aspects are the investment, maintenance and operating costs. While the latter two are less significant, the investment costs for GSHP are often decisive. Regarding the energy demand of manufacturing processes, a higher share of heating and/or cooling on the total energy demand leads to more favourable conditions for the integration of GSHP into manufacturing sites. Another aspect, the environmental effect of heating and cooling supply, is basically being improved by the integration of GSHP, especially through lower emissions. However, it has to be analysed for both the phases of construction and operation of a GSHP system. The security of energy supply is also a very important factor in industry. Although the temperature of the shallow geothermal energy is a function of subsurface conditions, it remains constant over the year, which ensures a uniform heat flow throughout the time. Therefore, this renewable energy source is capable of providing baseload heating and cooling. Various energetic process parameters, e.g. heat transfer and temperature tolerance may have also an influence on the product quality and therefore have to be considered as well. Another factor to be regarded relates to the influence on the production and process layout design, as this heating and cooling technology is spatially bound.

By means of this assessment model, over 250 manufacturing processes according to the norm DIN 8580 - Manufacturing Processes, as well as process engineering processes, partly under certain conditions (e.g. specific materials), were analysed. Therefor the most relevant parameters were used, namely the required process temperatures, as well as the specific energy demand per functional unit of manufactured products or processed materials. Therefore, the identified manufacturing processes were evaluated (see Figure 2) and grouped according to the suitability of their energy supply by use of GSHP. As a result, Table 2 presents manufacturing processes, which are, according to the presented assessment, best suited for the considered thermal energy supply concept. 


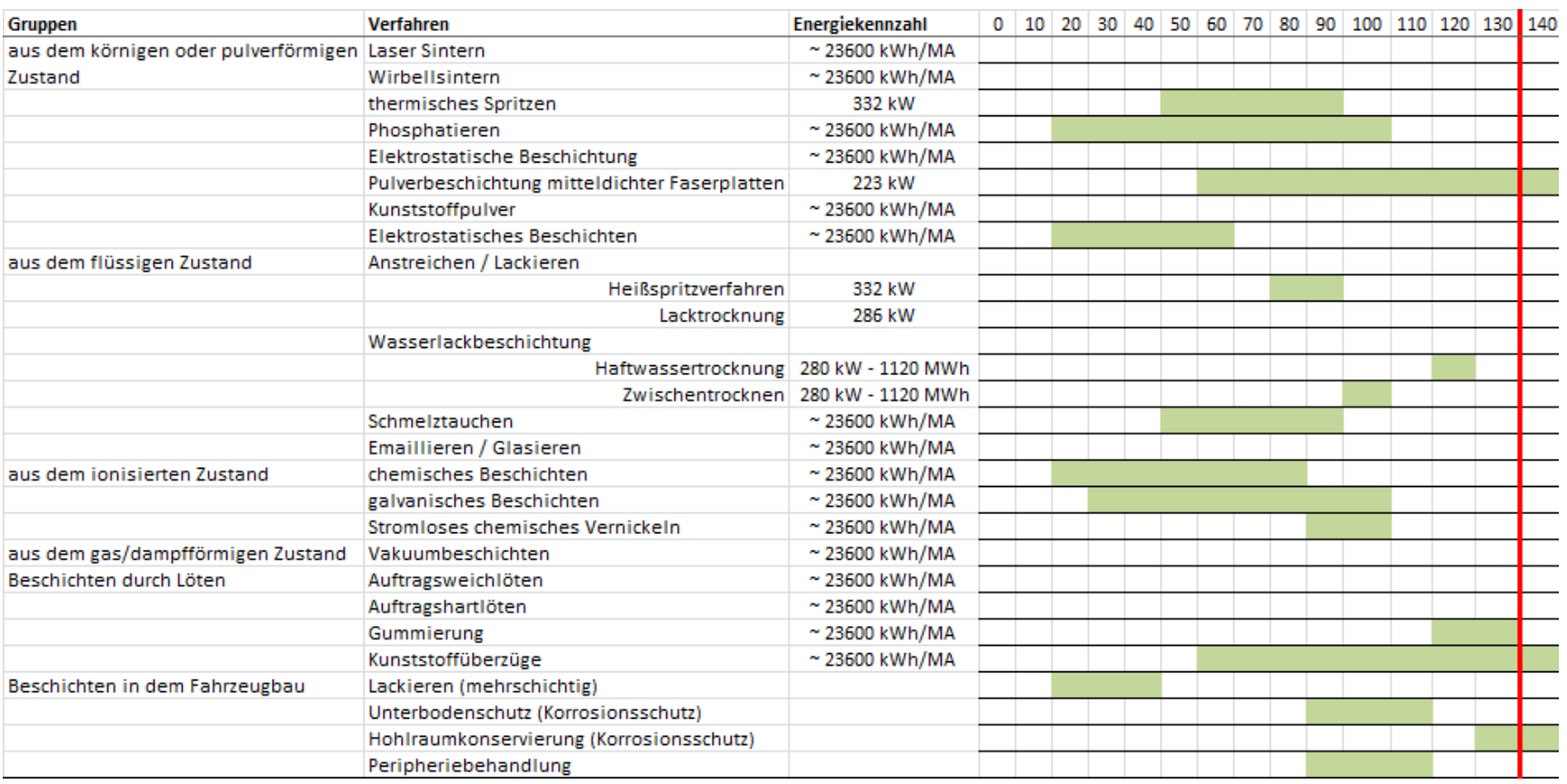

Fig. 2: Result of the energetic analysis of manufacturing processes (excerpt - German)

Table 2: Manufacturing processes best suited for energy supply by use of GSHP (excerpt)

\begin{tabular}{|c|c|c|c|}
\hline Manufacturing process & \begin{tabular}{|l} 
Classification according to \\
DIN 8580 or industry sector
\end{tabular} & $\begin{array}{l}\text { Temperature } \\
\text { level }\end{array}$ & $\begin{array}{l}S_{\text {process }}(\text { only } \\
\text { process criteria })\end{array}$ \\
\hline Bonding with chemically setting adhesives & Main group 4, Subgroup 4.8.2 & $80^{\circ} \mathrm{C}-180^{\circ} \mathrm{C}$ & 6 \\
\hline $\begin{array}{l}\text { Application of anti-corrosion agent } \\
\text { (underbody protection) }\end{array}$ & Main group 5 & $90^{\circ} \mathrm{C}-110^{\circ} \mathrm{C}$ & 7 \\
\hline Gumming & Main group 5 & $120^{\circ} \mathrm{C}-130^{\circ} \mathrm{C}$ & 8 \\
\hline Tempering & Main group 6, Subgroup 6.2 & $110^{\circ} \mathrm{C}-120^{\circ} \mathrm{C}$ & 9 \\
\hline Etching & Woodworking industry & $50^{\circ} \mathrm{C}-80^{\circ} \mathrm{C}$ & 9 \\
\hline Boiling & Chemical industry & $100^{\circ} \mathrm{C}-110^{\circ} \mathrm{C}$ & 10 \\
\hline Production of plastics (LDPE, HDPE, PVC) & Plastics industry & $80^{\circ} \mathrm{C}-100^{\circ} \mathrm{C}$ & 7 \\
\hline Processing of plastics (LDPE) & Plastics industry & $120^{\circ} \mathrm{C}-140^{\circ} \mathrm{C}$ & 8 \\
\hline Drying & Ceramics industry & $20^{\circ} \mathrm{C}-120^{\circ} \mathrm{C}$ & 10 \\
\hline Bleaching & Textile industry & $60{ }^{\circ} \mathrm{C}-100^{\circ} \mathrm{C}$ & 9 \\
\hline Pad-Roll-Process & Textile industry & $40^{\circ} \mathrm{C}-60^{\circ} \mathrm{C}$ & 7 \\
\hline Desizing and Powertex-Process & Textile industry & $40^{\circ} \mathrm{C}-60^{\circ} \mathrm{C}$ & 7 \\
\hline Washing and drying & Textile industry & $30^{\circ} \mathrm{C}-100^{\circ} \mathrm{C}$ & 10 \\
\hline
\end{tabular}

The next steps in further developing this systematic approach of integrating GSHP into urban manufacturing sites will include the integration of additional criteria regarding the urban perspective, 
e.g. energy supply structure and energy demand of the adjoining residential areas. On this basis, a decision support tool is planned, which should enable the analysis of certain categories of urban production site profiles suitable for the integration of GSHP, aiming to create a symbiosis with the urban surroundings.

With the objective of validating this approach by this point, energy efficiency and flexibility scenarios based on key industrial processes in the automotive industry were established and are further shown in chapter 5, besides other best-practice examples identified in industry.

\section{BEST-PRACTICE EXAMPLES AND SCENARIOS IN INDUSTRY}

To underpin the approach presented, some existing isolated applications, as well as two scenarios especially established in industry are presented hereinafter.

The EU-funded project "Integration of Geothermal Energy into Industrial Applications - IGEIA" (2006 - 2009) aimed to support the development of geothermal heating and cooling at industrial sites. In a practical case study within the project, the waste heat of a foundry in Sweden was stored by GSHP, recovering about 3,600 MWh per year and feeding it into the district heating network of the adjoining city. [EC 2008]

Another application example shows the cooling of production equipment in the automotive industry, i.e. welding guns, by means of 3,300 energy piles. The construction of this worldwide largest shallow geothermal field in its time (2012) and the associated sustainable energy supply system with a capacity of $2 \mathrm{MW}$ led to the decision against the construction of a cooling tower and thus to further resource savings, such as the consumption of $25,000 \mathrm{~m}^{3}$ of water per year. [Bundesverband Geothermie 2012]

One of the most innovative and well-developed industry sectors is the automotive industry. Within a current research project carried out with the participation of Fraunhofer IPA, two GSHP application scenarios for the heating and cooling supply of an automotive original equipment manufacturer (OEM) were established.

The first scenario includes the extensive use of three existing GSHP, not only for heating and cooling of production spaces, but also for the cooling of production equipment in the car body shop, i.e. welding guns. The cooling demand of $2.1 \mathrm{GW}_{\text {th }} \mathrm{h} / \mathrm{a}$ can be covered by the existing GSHP at a COP of approx. 4.0. Depending on whether the scenario is used as an energy efficiency measure (permanent implementation) or as an energy flexibility measure (implementation depending on the energy market), savings of up to $315 \mathrm{MW}_{\text {el. }} \mathrm{h} / \mathrm{a}$ compared to the use of conventional cooling technology are possible.

The second scenario regards the integration of GSHP in the energy subsystem of the paint shop. The goal is efficient and flexible thermal energy supply for the processes pre-treatment and electro-coating. The first process has a low-temperature heat demand of $10.3 \mathrm{GW}_{\text {th }} \mathrm{h} / \mathrm{a}$, the second one a cooling demand of $1.2 \mathrm{GW}_{\text {th. }} \mathrm{h} / \mathrm{a}$. Therefore, an energetic coupling by use of a heat pump was evaluated. The ground coupling of the heat pump enables moreover subsurface waste heat storage and thus a flexible and renewable thermal energy supply of both processes. Based on COP values of 4.0 for cooling and 3.0 for heating, the savings from using GSHP over conventional technologies amount to $180 \mathrm{MW}_{\text {el. }} \mathrm{h} / \mathrm{a}$ for cooling and 5.900 MWh/a for heating. 


\section{REFERENCES}

Arbeitsgemeinschaft Energiebilanzen (2013): Anwendungsbilanzen für die Endenergiesektoren in Deutschland in den Jahren 2010 und 2011. AGEB, Berlin.

Bobelin, D., Bourig, A. (2012): Experimental Results of a Newly Developed Very High Temperature Industrial Heat Pump $\left(140^{\circ} \mathrm{C}\right)$ Equipped With Scroll Compressors and Working With a New Blend Refrigerant. In: International Refrigeration and Air Conditioning Conference, Paper 1299. Purdue University, West Lafayette.

Bundesverband Geothermie e.V. (2012): VW-Werk in Emden wird „grün“ durch Geothermie

European Commission - EC (2008): IGEIA - Integration of Geothermal Energy into Industrial Applications; Deliverable 8 - Report on technical, environmental and economic system of actual energy use under defined conditions for three industrial sites. Saunier \& Associés, SWECO, UBeG $\mathrm{GbR}$

Kube, M. et al. (2017), Marktverfügbare Innovationen mit hoher Relevanz für die Energieeffizienz in der Industrie. Ecofys, adelphi, Universität Stuttgart, Prognos, Berlin.

Lentes J., (2016), Urbane Produktion. In: D. Spath, E. Westkämper (ed.), Handbuch Unternehmensorganisation Springer Reference Technik, Springer Berlin Heidelberg.

Naegler, T., Simon, S., Klein, M., Gils, H. C. (2015): Quantification of the European industrial heat demand by branch and temperature level. In: International Journal of Energy Research, Wiley Online Library.

Umweltbundesamt, ed., (2015), Berichterstattung unter der Klimarahmenkonvention der Vereinten Nationen und dem Kyoto-Protokoll 2017 - Nationaler Inventarbericht zum Deutschen Treibhausgasinventar 1990 - 2015. Umweltbundesamt, Berlin, Germany.

Wolf, S., Fahl, U., Blesl, M., Voß, A., Jakobs, R., (2014): Analyse des Potenzials von Industriewärmepumpen in Deutschland - Forschungsbericht. Universität Stuttgart, Institut für Energiewirtschaft und Rationelle Energieanwendung (IER), Stuttgart, Germany.

\section{CONCLUSION}

A promising technology for increasing energy efficiency in industry by enabling the use of renewable heating and cooling sources and waste heat is the high-temperature heat pump. Although it is commercially available, it is still largely unexploited, but has a technical energy-saving potential of more than $120 \mathrm{TWh} / \mathrm{a}$ in the German industry, while the actual corresponding economic potential is currently $5 \%$ [Kube et al. 2017].

This paper presents a systematic approach for the integration of GSHP in the industry, aiming the supply with process heat and cooling, with the overall objective of increasing energy efficiency and flexibility in the industry. The advantages of the approach lie in the various application areas: exploitation of renewable energy sources and waste heat recovery, but also in the possibility of efficient combination with actually competing technologies based on fossil fuels (e.g. combined heat and power plants) or with heat storage technologies.

The basis of this approach is a model for the technical analysis of the use of this technology in the industry. Based on the evaluation scheme established, over 250 manufacturing processes were 
subsequently analysed, evaluated and grouped according to the suitability of their energy supply by GSHP.

In order to validate this approach, two scenarios were put in place in the automotive industry. The results of the analyses of these scenarios show potential energy savings between 37.5 and $63 \%$.

The next steps in the development of the approach are to establish energy-related, urban-perspective criteria of production that will complement the assessment model, and to create a decision support tool to enable the analysis of different categories of urban production site profiles suitable for the integration of GSHP.

Looking ahead, the overarching goal of this approach is to support sustainable, cost-effective and lowcarbon concepts for the supply of energy-autonomous urban factories of the future. In doing so, it must be examined on a case-by-case basis whether independence from the volatile energy markets or, on the contrary, cooperation with these markets in the future should be sought, in line with the energyflexibility approach. 


\title{
PROCESS INTEGRATION IN A DAIRY FACTORY CONSIDERING THERMAL ENERGY STORAGES - A COMPARISON OF TWO DIFFERENT APPROACHES
}

\author{
$\underline{\text { Anton Beck }}^{\mathrm{a}}$, Wolfgang Glatzl ${ }^{\mathrm{b}}$, Jürgen Fluch ${ }^{\mathrm{b}}$, René Hofmann*,a,c. \\ ${ }^{a}$ Austrian Institute of Technology GmbH, Center for Energy, Giefinggasse 2, A-1210 Vienna, Austria \\ ${ }^{\mathrm{b}}$ AEE - INTEC, Industrial Processes and Energy Systems, Feldgasse 19, A-8200 Gleisdorf, Austria \\ ${ }^{\mathrm{c}}$ TU Wien, Institute for Energy Systems and Thermodynamics, Getreidemarkt 9/BA, A-1060 Vienna, Austria \\ *E-Mail: rene.hofmann@tuwien.ac.at / rene.hofmann@ait.ac.at
}

\begin{abstract}
In this paper, heat integration for a dairy factory is performed using two different approaches with storage integration capabilities. The use-case consists of 36 process streams which can be considered large-scale. Stream data is available for a period of three weeks with high temporal resolution. Even though a slightly different data basis is needed, both approaches yield comparable results which are analyzed in this paper. The first approach considered in this work is the software tool SOCO which is based on pinch-analysis for time-dependent process streams. It incorporates a flowsheet simulation environment for detailed storage simulation. The second approach is based on mathematical programming and cost-driven superstructure optimization using simple storage models. The best results obtained using both approaches are compared regarding heat exchanger networks, storage integration and economic data. Also, potential synergies between the two approaches are identified that could further improve solutions.
\end{abstract}

\section{INTRODUCTION}

Heat integration with the goal of reducing energy consumption and costs has been a research topic for several decades now (Kemp, 2007). Since process integration became a major topic in process engineering, two distinct approaches were intensively studied. On the one hand graphical approaches based on composite curves and the idea of the pinch point were developed and on the other hand mathematical optimization formulations were used to find optimal process integration solutions by minimization of either energy consumption or costs. Heat integration in batch and time-dependent processes has been a topic of scientific interest since the 1980s but only since the turn of the century storage integration has been intensively studied.

For solving time-dependent problems, complex optimization principles are needed. There are two major approaches to overcome non-simultaneous heating and cooling demands - rescheduling of process tasks and heat integration using thermal energy storages (TES). Generally, TES are indispensable and necessary for significantly increasing the resource and energy efficiencies in complex and volatile energy systems. Anastasovski (2017) used Pinch Technology for the integration of two-tank storage systems under economic considerations. Storage costs are optimized by variation of storage parameters and temperature differences. Another approach for storage integration using Pinch Analysis methods was proposed by Krummenacher (2001). A method was proposed that allows to identify the minimum number of storage tanks for a given heat recovery. This method was later on implemented in the process integration software PinCH (Olsen, et al., 2016). Opposed to integration methods based on Pinch Analysis Chen and Ciou (2008) proposed a MINLP superstructure formulation for cost-efficient indirect heat recovery using TES. Also, a sequential design procedure for heat exchanger network synthesis (HENS) with selection of an optimal utility system and storage integration was proposed by Mian et al. (2016). They tried to overcome the drawbacks of the sequential approach by applying a derivative-free hybrid algorithm.

PROBLEM STATEMENT 
For the integration of TES several approaches with different capabilities have been presented in the past, which have not been thoroughly compared. Within the framework of the IEA IETS Annex $15^{2}$ the project partners AIT and AEE INTEC have developed methods for heat integration with storage capabilities. These methods should be compared and potential synergies should be demonstrated based on a real dairy factory. This use-case can be considered large-scale as it consists of 14 cold and 22 hot process streams. Most of the process streams are discontinuous and some also change start and target temperatures as well as mass flows when active, resulting in a difficult problem for HENS with storage integration.

\section{STORAGE INTEGRATION METHODS}

The two approaches compared in this paper are AEE INTEC's Storage Optimisation Concepts (SOCO) (Muster-Slawitsch, et al., 2014) and the mathematical programming (MP) framework for heat integration developed by the AIT which are presented in the following. Although these approaches are inherently different, both yield comparable energy recovery systems. A flowchart representation for the individual steps and the corresponding input data is presented in Figure 1.

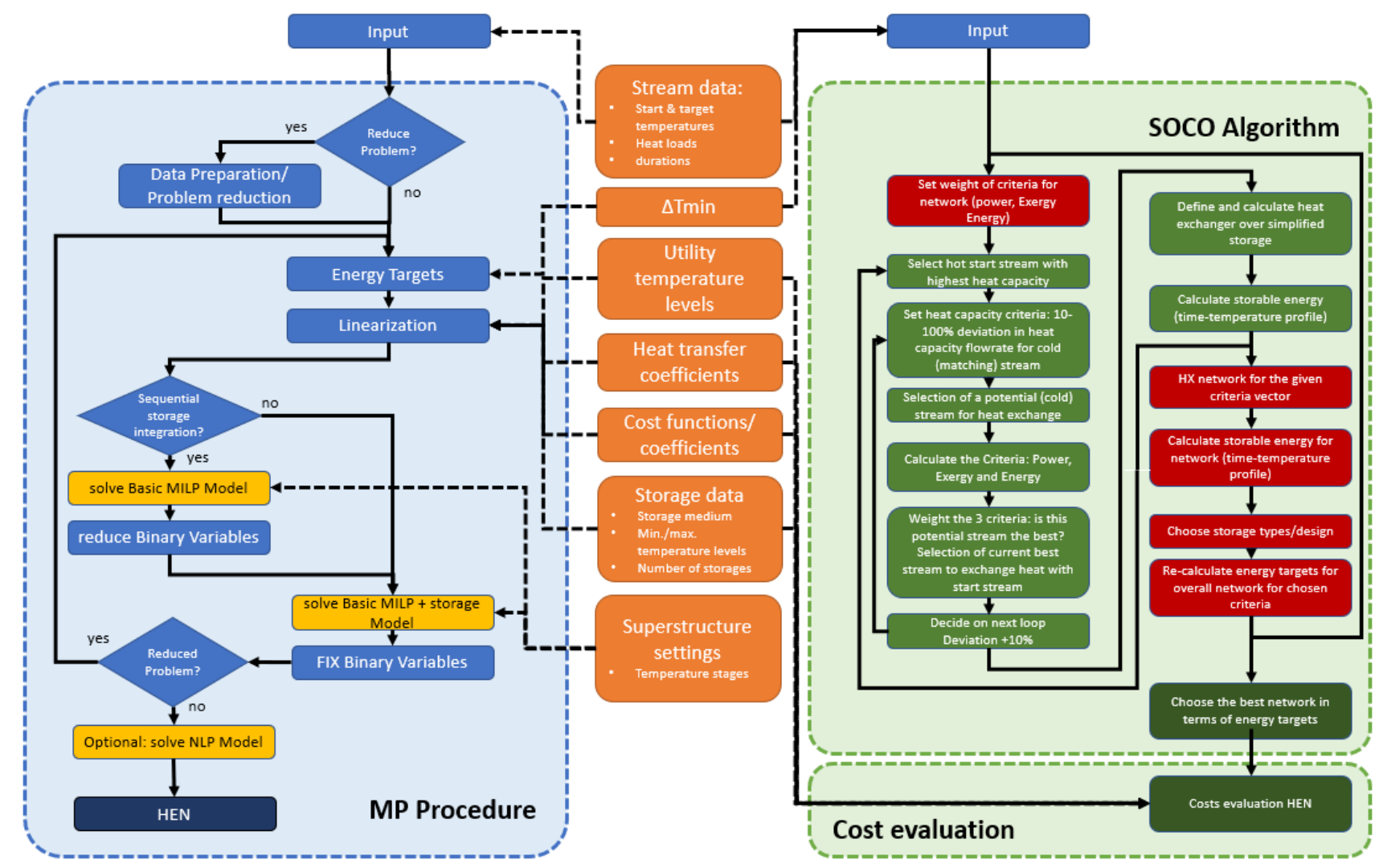

Figure 14: Flowchart SOCO-algorithm and MP procedure

\section{SOCO}

SOCO is a compiled planning tool for optimization and design of heat exchanger and thermal storage systems in complex thermal energy systems that allows to perform heat integration based on Pinch Analysis for time-dependent streams. The SOCO algorithm has been presented by Fluch et al. (2012). The workflow for SOCO can be divided into the following steps:

\footnotetext{
${ }^{2}$ The IETS (Industrial Energy-Related Technologies and Systems) program unites the IEA (International Energy Agency) activities in this area. The work is organized in Annexes. Annex 15 Phase 2 is the second task on "Industrial Excess Heat Recovery".
} 
1. Stream data import: Stream data can be prepared in a suitable spreadsheet template and then imported to SOCO. Temperatures and mass flow can vary in each time step and thus provide full flexibility in the definition of all streams.

2. HEN Proposal Based on a deterministic Pinch algorithm (Fluch, et al., 2012) all possible stream combinations are evaluated with and without heat storage. The procedure is re-iterated and the best HEN in terms of total energy recovery, heat exchanger power installed and exegetic use of available streams is selected. The HEN proposal allows the definition of boundary conditions for equipment like minimal and maximal storage size.

3. HX and heat storage modification: The proposed heat exchanger and heat storages can be modified manually by the user. The heat storage can be parameterized with volume, insulation (top, mantle, bottom), wall thickness and up to 20 ports with height, shape and diameter.

4. HEN Design: SOCO has a build-in flowsheet which allows the design of the HEN as proposed (in step 2) and modified (in step 3). All limitations that were not included in HEN proposal algorithm can now be considered by the planner (e.g. actual location of streams; combinations of multiple proposed heat storages with similar temperature profiles to one heat storage)

5. HEN Simulation: The designed HEN in the flowsheet can be simulated. By going back to step 3, the HX and heat storages can be further modified and again simulated. Data of all streams and equipment can be viewed directly in the flowsheet and also exported for further post-processing.

The SOCO storage model allows the definition of two types (fixed level with loading and unloading HX and variable level), detailed insulations definition, ambient and initial temperatures, stratified charging or ports with fixed height.

\section{Mathematical Programming framework}

The second approach is based on an extended MINLP (Mixed Integer Non-Linear Programming) superstructure formulation for cost-optimal heat exchanger network design and storage integration. Both heat exchanger network (HEN) and storage systems are optimized simultaneously allowing for cost-optimal grassroot and retrofit designs. A multi-period extension for the superstructure formulation proposed by Yee \& Grossmann (1990) and a simple 0-D model for the two-tank system are used in this paper (Beck \& Hofmann, 2018a). The formulation allows to specify forbidden stream matches and also to specify streams as soft-streams, which means that reaching the specified target temperatures is optional. Simplifications for the problem such as reduction of operation periods and reduction of considered process streams are often necessary to handle it rigorously. As these types of combinatorial problems scale poorly with problem size, a linearization approach and tighter model formulations are used as presented by Beck \& Hofmann (2017, 2018b). The workflow can be divided into several consecutive steps with different data inputs:

1. Stream data must be provided by the user in an appropriate form. Inlet and target temperatures for each process stream and heat capacity flow rates need to be set as well as the duration of each operating period.

2. Problem reduction: Depending on the problem size, the problem might need to be reduced due to the poor scaling of simultaneous MI(N)LP problems for HENS. This can be done by reduction of the process streams considered in the HENS problem and by merging consecutive operating periods with equal stream parameters.

3. Energy targets: Using the stream data and a user-defined minimum temperature difference $\Delta T$ min, energy targets are calculated. These targets are then used for linearization of the 
original MINLP formulation and to tighten the resulting MILP problem. Also, the energy targets yield information about theoretically possible heat recovery for both storage integration and direct heat recovery.

4. Linearization: In the linearization step, the original MINLP superstructure formulation is linearized. Insights from the energy targeting are used to find good approximations of the nonconvex constraints used in the original formulation. The user needs to provide heat transfer coefficients, cost coefficients, utilities and storage parameters such as bounds for storage temperatures and storage materials for this step.

5. Simultaneous/sequential storage integration: Next, the user can decide whether storages should be introduced simultaneously with the HEN or if the storage systems should be integrated after an optimal HEN was found. The simultaneous approach might yield a slightly better solution. However, computation time increases significantly.

6. Solution for original problem: In the case, that the problem was reduced, the MILP superstructure for the total problem is solved with fixed binary variables which reduces the problem to an LP formulation.

7. Optional NLP stage: The user can then decide if an additional NLP problem should be solved, which might yield slight improvements on the LP solution by shifting heat loads and adjusting storage sizes and heat exchanger areas.

With the mathematical programming approach, (sub)optimal heat recovery systems for timedependent processes can be obtained. However, the solution process is time-consuming and thus thorough preparation of the optimization model is needed to avoid solutions that are infeasible for real implementation. This infeasibility might result from stream matches that cannot be allowed for safety reasons or that are economically infeasible due to excessive piping costs that are not considered in the optimization procedure.

\section{PROCESS DESCRIPTION}

The present dairy process has 36 process streams, of which 22 have cooling demand and 14 must be heated. The process streams are active at different time intervals. Some process streams are also subject to changes in process stream parameters such as mass flow, inlet and outlet temperature and specific heat capacity. Data for the process streams are available for a representative period of three weeks (including weekends) with a time resolution of 10 minutes. The stream parameters are periodic with a period of one week. The Gantt chart is shown in Fig. 2.

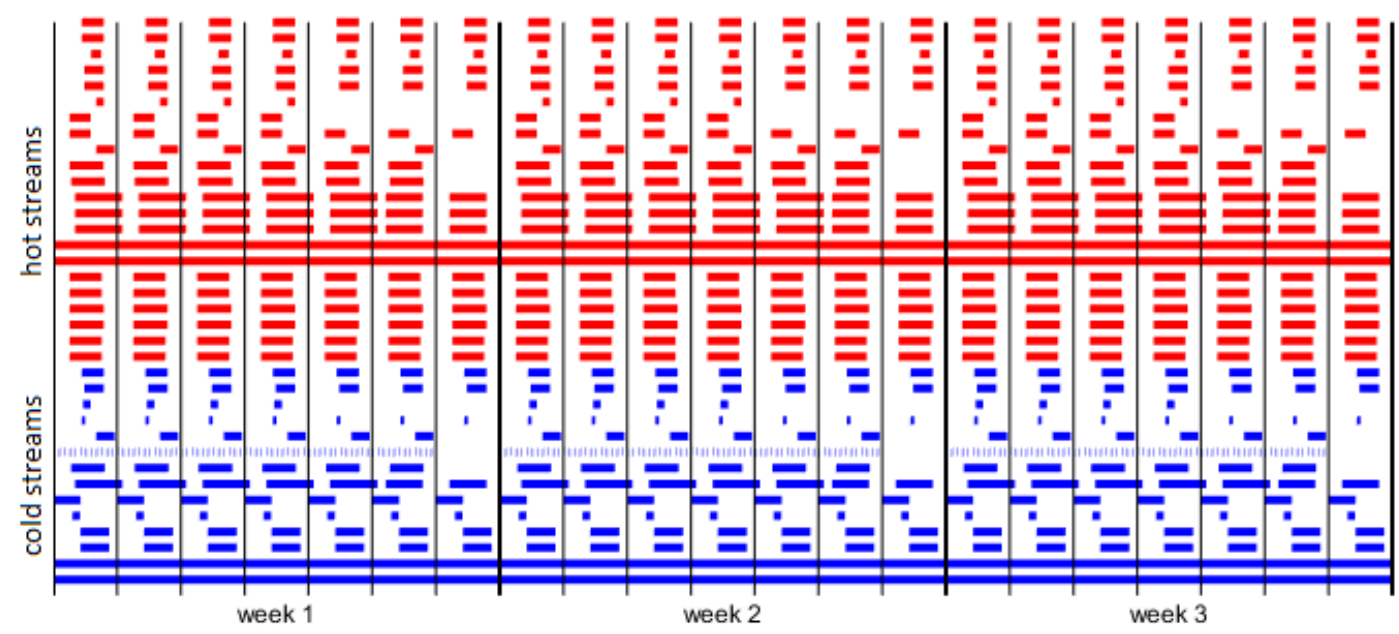

Figure 2: Gantt-chart for the period of available data (all three weeks identical) 
The days Tuesday to Thursday are identical. On Mondays process streams H12-H14 are not active in the morning. From Friday until Sunday process streams H6, H7 and C3 are inactive. At the weekend the mass flows of the waste heat streams H17-H22 are reduced and the process streams H12-H14 are active only during the day and are not operated as on weekdays until about midnight. On Sunday, process streams H9-H11 and C5-C7 are also inactive. The stream data for the case-study are presented in Table 1. Cooling for hot process streams with a target temperature of $20^{\circ} \mathrm{C}$ or higher are cooled using cooling water taken from the nearby river.

Table 1: Stream data and cost parameters for use case

\begin{tabular}{|c|c|c|c|c|c|c|c|c|c|}
\hline & $\operatorname{Tin}\left({ }^{\circ} \mathbf{C}\right)$ & Tout $\left({ }^{\circ} \mathbf{C}\right)$ & $m[\mathrm{~kg} / \mathrm{s}]$ & cp $[\mathrm{kJ} / \mathrm{kgK}]$ & & $\operatorname{Tin}\left({ }^{\circ} \mathbf{C}\right)$ & $\operatorname{Tout}\left({ }^{\circ} \mathbf{C}\right)$ & $\mathrm{m}[\mathrm{kg} / \mathrm{s}]$ & cp [kJ/kgK] \\
\hline H1 & 8 & 4 & 11.11 & 3.77 & H21 & 44.9 & $>30$ & $0.02-0.38$ & 4.19 \\
\hline H2 & 11 & 4 & 9.89 & 3.77 & H22 & 30 & $>10$ & $0.07-1.47$ & 4.19 \\
\hline H3 & 5 & 3 & 6.94 & 3.77 & $\mathrm{C1}$ & $33-87$ & $40-94$ & 11.11 & 3.77 \\
\hline H4 & 55 & 8 & 1.39 & 2.88 & $\mathrm{C2}$ & 105 & 110 & 1.39 & 2.88 \\
\hline H5 & 13 & 8 & 1.39 & 2.88 & C3 & 8 & 21 & 3.33 & 2.88 \\
\hline H6 & 21 & 11 & 3.33 & 2.88 & $\mathrm{C4}$ & 11 & 28 & 8.52 & 3.77 \\
\hline H7 & 11 & 6 & 2.05 & 3.77 & C5 & 6 & 70 & 6.68 & 3.77 \\
\hline H8 & 28 & 6 & 0.32 & 3.91 & C6 & 10 & 40 & $0.74 / 1.48$ & 4.19 \\
\hline H9 & 70 & 32 & 6.68 & 3.77 & C7 & 8 & 50 & 3.94 & 4.09 \\
\hline H10 & 42 & 8 & 3.94 & 4.09 & C8 & 8 & 81.5 & 3.03 & 4.09 \\
\hline H11 & 50 & 8 & 3.94 & 4.09 & C9 & 25 & 90 & 0.33 & 3.00 \\
\hline H12 & 70 & 5 & 0.92 & 4.19 & $\mathrm{C10}$ & 50 & 77 & 0.39 & 4.19 \\
\hline H13 & 48 & 5 & 2.22 & 4.19 & C11 & 15 & 65 & 0.23 & 4.19 \\
\hline H14 & 53 & $5 / 25$ & $0.22 / 0.4$ & $3.8 / 3$ & $\mathrm{C12}$ & 15 & 65 & 0.54 & 4.19 \\
\hline H15 & 140 & $>60$ & 6.18 & 1.11 & $\mathrm{C13}$ & 12 & 102 & 0.93 & 4.19 \\
\hline H16 & 216 & $>60$ & 0.29 & 1.11 & C14 & 90 & 102 & 0.26 & 4.19 \\
\hline H17 & 62.9 & $>30$ & $0.01-0.3$ & 4.19 & & & & & \\
\hline H18 & 30 & $>10$ & $0.12-2.58$ & 4.19 & HU & 150 & 150 & & \\
\hline H19 & 75 & $>15$ & $0.06-0.55$ & 4.19 & CU1 & 15 & 18 & & \\
\hline H20 & 15 & $>10$ & $3.85-34.69$ & 4.19 & CU2 & -5 & 0 & & \\
\hline
\end{tabular}

Overall heat transfer coefficient: 4kW/m²K; Utility costs: HU $262.8 € / \mathrm{kW} / \mathrm{y}, \mathrm{CU} 110 € / \mathrm{kW} / \mathrm{y}, \mathrm{CU} 2292 € / \mathrm{kW} / \mathrm{y}$, Heat exchanger costs

$6133.5^{*}[\text { Area }]^{0.219} / \mathrm{y}$; Storage costs: $(756.2 *[$ Volume $]+1760) / \mathrm{y} ;$ Annualization factor $\mathrm{y}=3$; $\mathrm{dTmin}=2^{\circ} \mathrm{C}$

Process streams with lower target temperatures are cooled by means of liquid chillers with an energy efficiency ratio of 3 ( $3 \mathrm{~kW}$ thermal equivalent to $1 \mathrm{~kW}$ electric). The hot utility power is steam at $150{ }^{\circ} \mathrm{C}$. The cost coefficients used for operating costs (costs for hot and cold utility) and investment costs (heat exchangers fixed costs and variable costs) and the cost exponent for declining costs for heat transfer surfaces are also summarized in Table 1. A uniform heat transfer coefficient $k$ was chosen for all utility and process flows.

\section{PROBLEM SET UP}

\section{Mathematical Programming procedure}

Taking into account active/inactive and variable process flow parameters, 118 different operating states result. In order to carry out a simultaneous design of a cost-efficient HEN, the problem complexity must be reduced for this case-study. 

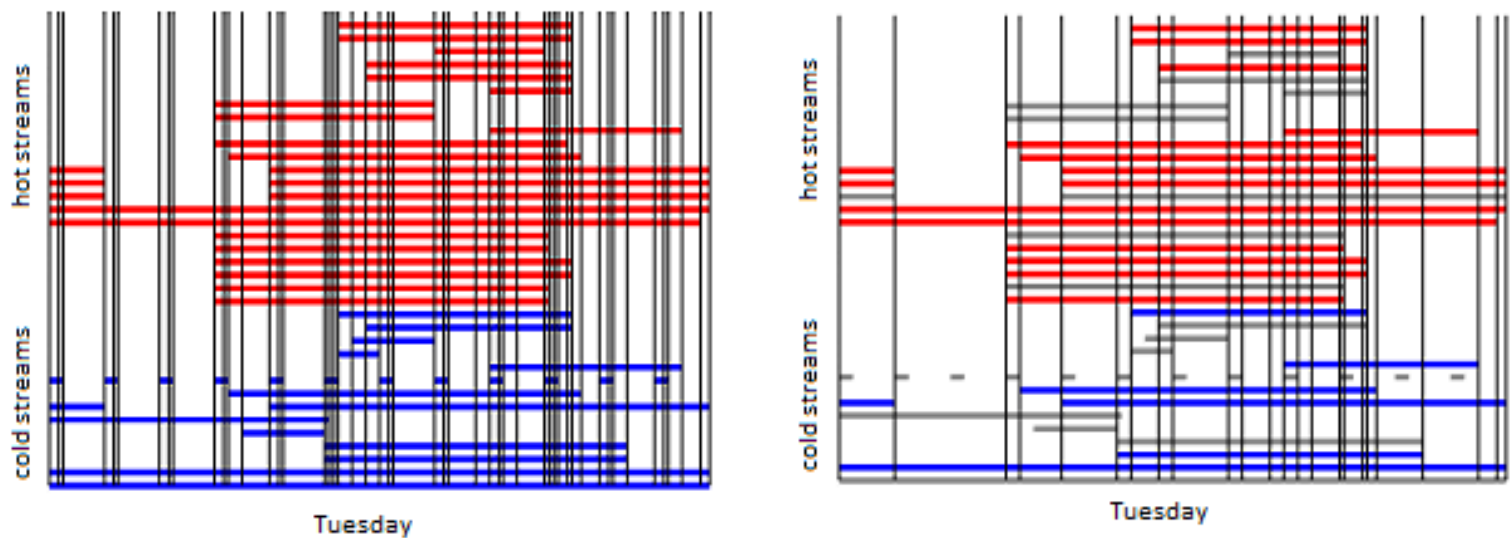

Figure 3: Gantt-chart for Tuesday with time intervals; all streams (left) and reduced streams (right)

Tuesday is selected as a representative day for the entire week, since all process streams are active on this day. This reduces the number of different operating states to 45 . The 20 process streams with the highest energy content account for about $95 \%$ of the total energy content. An analysis is shown in Figure 3. By reducing the problem to 20 process streams, the different operating states are reduced to 23 intervals with constant operating conditions. The Gantt chart for Tuesday is shown in Figure 2 with individual operating periods for the original and the reduced problem.

To further simplify the problem, the sequential approach is used for storage integration. This means, that first a cost optimal HEN is synthesized and then the storage is introduced to the superstructure. Also, within the superstructure each stream pair may only use one heat exchanger for heat transfer. Three temperature stages were used for the superstructure.

SOCO

SOCO can use the variable stream data directly for the HEN proposal and HEN simulation. No problem reduction and no exclusion of stream combinations are necessary. Figure 4 shows two examples of the variety of streams.

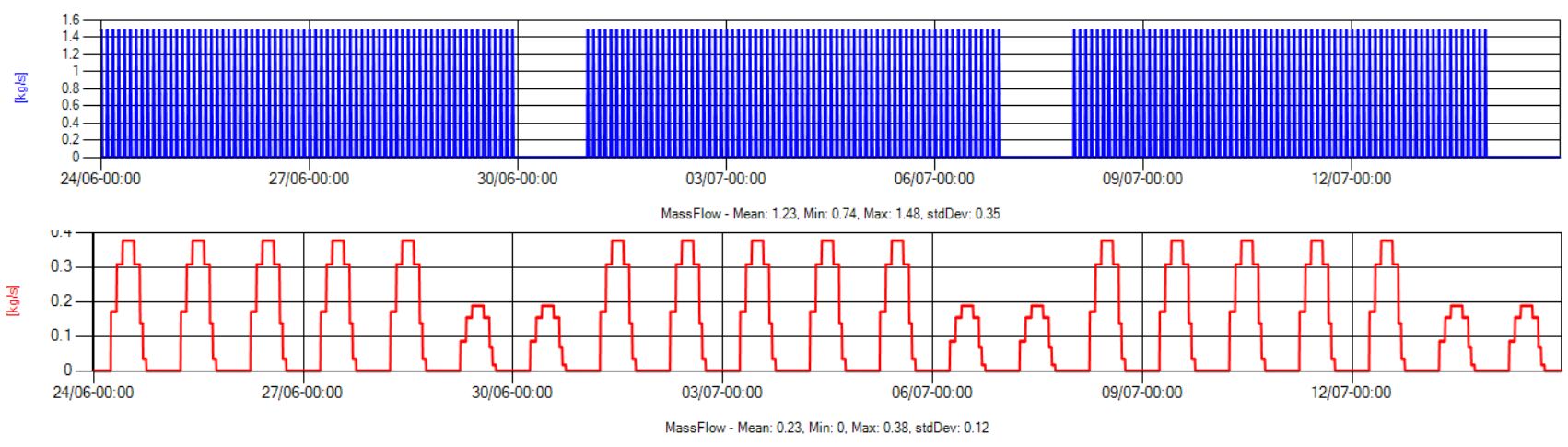

Figure 4: (Top): Cold stream (C6) is active 10 minutes every 2 hours for 6 days a week. (Bottom): Hot stream (H19) is waste heat from a chiller that varies over production day and has reduced load at weekends

\section{HEAT EXCHANGER NETWORK AND HEAT RECOVERY}

It needs to be mentioned that, although one strength of SOCO is the possibility to adapt the HEN proposal in manifold way in the flowsheet, the HEN proposal was converted directly to the built-in flowsheet area of SOCO to investigate the economic performance of the HEN proposal. The only adaption was the merging of storages with the same hot stream as source. Thereby, 9 proposed storages with $620 \mathrm{~m}^{3}$ volume were merged to 5 storages with $449 \mathrm{~m}^{3}$. The results for the number of 
$\mathrm{HX}$ and the heat recovery achieved with the networks obtained using SOCO and the MP procedure is shown in Table 2. Also, the number of storage units suggested and the approximate computation time for the solution are shown. The results presented for SOCO shows the results of the HEN simulation without further adaptions of the HEN proposal.

The solution found by using the MP framework achieves approximately $83.6 \%$ of the heat recovery possible through heat integration by means of heat exchangers and the use of TES compared to $72.3 \%$ for the HEN proposed by SOCO. A big difference between the solutions is the use of storages. The MP solution uses one storage subsystem with a $43.14 \mathrm{~m}^{3}$ stratified tank whereas with SOCO five storages were proposed. As explained in 4.3, the HEN proposal suggests a set of HX and heat storages depending on energy criteria. Each storage is designed for the combination of two streams. The merging of storages with similar temperature profiles is not performed in the HEN proposal.

Table 2: Number of heat exchangers, storage sizes, heat recovery and computation time for both approaches

\begin{tabular}{lrrrrrrr} 
& $\begin{array}{r}\text { HX } \\
\text { utility }\end{array}$ & $\begin{array}{r}\text { HX } \\
\text { streams }\end{array}$ & $\begin{array}{r}\text { HX } \\
\text { storage }\end{array}$ & $\begin{array}{r}\text { Storages / } \\
\text { Vol. }\end{array}$ & $\begin{array}{r}\text { Heat recovery } \\
\text { MWh/a }\end{array}$ & $\begin{array}{r}\text { Max. direct/mixed } \\
\text { MWh/a }\end{array}$ & $\begin{array}{r}\text { Computation } \\
\text { time }\end{array}$ \\
\hline MP & 26 & 10 & 3 & $1 / 43.14$ & $13,975.4$ & $14,894.13 / 16,725.58$ & rd. 24 hours \\
& & & & $\mathrm{m}^{3}$ & & & \\
SOCO & 28 & 9 & 15 & $5 / 449 \mathrm{~m}^{3}$ & $12,088.0$ & $14,894.13 / 16,725.58$ & rd. 1 second \\
\hline
\end{tabular}

The number of heat exchangers proposed by SOCO with $52 \mathrm{HX}$ is also higher than the number of HX obtained using rigorous optimization which yields a HEN with $39 \mathrm{HX}$.

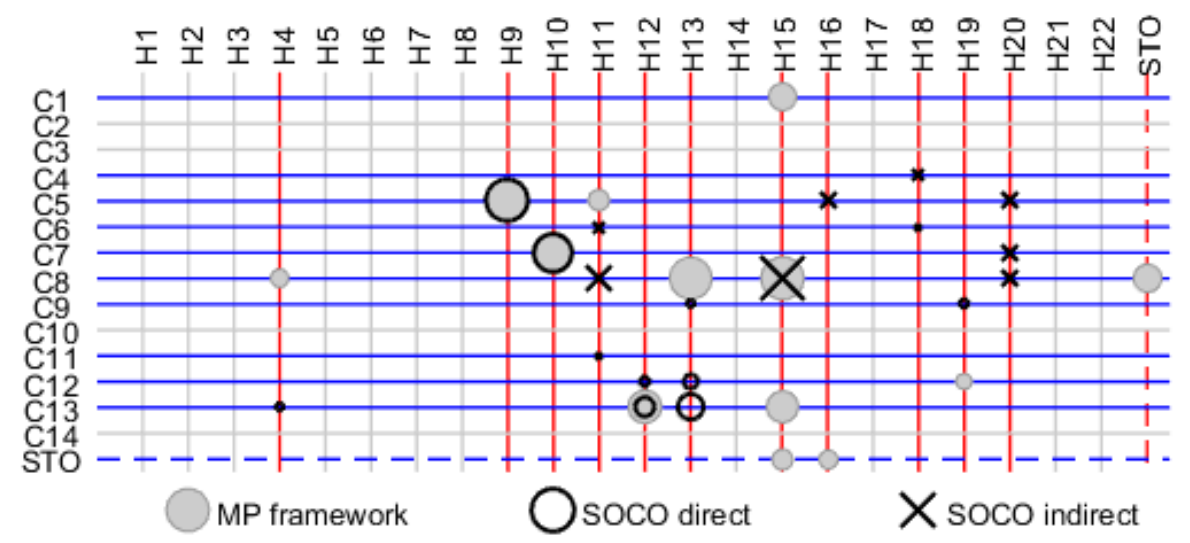

Figure 5: Stream combinations with storage integration; MP framework (gray), SOCO direct (dark circle), SOCO indirect (dark X), marker size represents the amount of energy transferred

Comparison of the stream combinations proposed by SOCO and the combinations found to be costefficient using the MP framework (Figure 5) shows that only four streams combinations are common. Two of them (H9/C5 and H10/C7) transfer approximately the same energy. Using SOCO more heat exchangers with lower total transferred energy were proposed compared to the solution found by the MP framework.

In this case study, only 11 of 22 hot streams and 10 of 14 cold streams where considered by either approach. 8 out of these 11 hot streams and 5 out of these 10 cold streams were considered by both approaches. However, 5 out of 6 cold streams and 8 out of 8 hot streams considered by the MP framework were also considered by the SOCO algorithm for heat recovery.

Comparison of the annual costs obtained by both approaches (Table 3) shows that rigorous optimization of costs yields lower total annual costs (TAC). This result was expected as in the SOCO proposal cost drivers such as utility costs and heat exchanger costs are not considered but HX are 
selected by energy criteria. In this case-study it was assumed that process streams with a target temperature lower than $20^{\circ} \mathrm{C}$ need to be cooled using chillers (CU2 in Table 1) with very high utility costs and thus heat recovery of low grade waste heat is crucial to achieve low operating costs. The focus of SOCO to use rather more storages leads to significantly higher costs in storage and HX for storage.

Table 3: Annual Costs

\begin{tabular}{lrrrrrr} 
& OP & HX streams & HX utility & HX storage & Storages & Total \\
\hline \multirow{2}{*}{ MP } & $229.6 \mathrm{k} €$ & $35.3 \mathrm{k} €$ & $48.7 \mathrm{k} €$ & $8.9 \mathrm{k} €(2.6 \%)$ & $14.9 \mathrm{k} €(4.4 \%)$ & 337.3 \\
& $(68.1 \%)$ & $(10.5 \%)$ & $(14.4 \%)$ & & & $\mathrm{k} €$ \\
\multirow{3}{*}{ SOCO } & $355.7 \mathrm{k} €$ & $28.6 \mathrm{k} €(4.2 \%)$ & $74.4 \mathrm{k} €$ & $107.6 \mathrm{k} €$ & $116.2 \mathrm{k} €$ & 682.5 \\
& $(52.1 \%)$ & & $(10.9 \%)$ & $(15.8 \%)$ & $(17.0 \%)$ & $\mathrm{k} €$ \\
\hline
\end{tabular}

\section{POTENTIAL SYNERGIES}

Even though SOCO does not consider costs in its algorithm for HENS, it selected all but one process streams for heat recovery that were also used in the solution of the MP framework. The SOCO solution process is very fast (seconds) compared to rigorous mathematical optimization (hours/days, depending on problem size) and thus could be used to find a good initial guess for the MP approach. This might speed up the MP approach significantly. Furthermore, the SOCO approach could be used to generate a number of HEN proposals to identify process streams that are likely to be used within the network. The MP model could then be restricted to these process streams for faster solution. The MP solution can then be considered as a global optimum, which cannot be guaranteed by the HEN proposal of SOCO. The MP solution could be drawn in the flowsheet of SOCO to simulate and fine tune the HEN with special attention towards individual storage design.

\section{CONCLUSION}

In this paper, HENs including TES for a large-scale dairy plant were generated using SOCO which is a compiled planning tool for optimization and design of heat exchanger and thermal storage systems in complex thermal energy systems developed by AEE INTEC and a mathematical programming framework developed by AIT. The workflows for both approaches were presented and advantages and disadvantages were discussed. It became clear that rigorous optimization of TAC is very timeconsuming and requires thorough data preparation but can find very good solutions from an economic point of view. SOCO does not require economic data which might be hard to get in the first place, does not require time-consuming problem setup and can find solutions for both direct and indirect heat recovery using storages in seconds. However, as it does not consider costs, the HENs obtained yield higher payback time compared to the rigorous cost-optimization.

Also, possible synergies for both approaches were identified. SOCO might be used to initialize optimization models with good initial guesses and could also be used for problem reduction which can potentially speed up optimization significantly.

\section{REFERENCES}

Anastasovski, A., 2017. Design of Heat Storage Units for use in repeatable Time Slices. Applied Thermal Engineering, Band 112, p. 1590-1600.

Beck, A. \& Hofmann, R., 2017. Tightening of MINLP Superstructure Relaxation for Faster Solution of Heat Exchanger Network Synthesis Problems. Dubrovnik, - .

Beck, A. \& Hofmann, R., 2018. A Novel Approach for Linearization of a MINLP Stage-Wise Superstructure Formulation. Computers \& Chemical Engineering, Band 112, p. 17-26. 
Beck, A. \& Hofmann, R., 2018a. Extensions for Multi-Period MINLP Superstructure Formulation for Integration of Thermal Energy Storages in Industrial Processes. Graz, Austria, Elsevier.

Beck, A. \& Hofmann, R., 2018b. How to tighten a commonly used MINLP superstructure formulation for simultaneous heat exchanger network synthesis. Computers \& Chemical Engineering, Band 112, p. $48-56$.

Chaturvedi, N. D. \& Bandyopadhyay, S., 2012. Minimization of storage requirement in a batch process using pinch analysis. In: 11th International Symposium on Process Systems Engineering. s.1.:Elsevier, p. 670-674.

Chen, C.-L. \& Ciou, Y.-J., 2008. Design and Optimization of Indirect Energy Storage Systems for Batch Process Plants. Industrial \& Engineering Chemistry Research, 47(14), p. 4817-4829.

Fluch, J., Brunner, C. \& Muster-Slawitsch, B., 2012. Storage Optimisation Concept in Industries, Commerce and District Heating Businesses. Chemical Engineering Transactions, pp. 493-498.

Kemp, I. C., 2007. Pinch Analysis and Process Integration. s.1.:Elsevier.

krummenacher, p., 2001. CONTRIBUTION TO THE HEAT INTEGRATION OF BATCH PROCESSES (WITH OR WITHOUT HEAT STORAGE), PhD Thesis Nr 2480 (2001). EPFL: s.n.

Mian, A., Martelli, E. \& Maréchal, F., 2016. Multi-period Sequential Synthesis of Heat Exchanger Networks and Utility Systems including storages. In: 26th European Symposium on Computer Aided Process Engineering. s.1.:Elsevier, p. 967-972.

Muster-Slawitsch, B., Brunner, C. \& Fluch, J., 2014. Application of an advanced pinch methodology for the food and drink production. Wiley Interdisciplinary Reviews: Energy and Environment, 3(6), p. 561-574.

Olsen, D., Abdelouadouda, Y., Wellig, B. \& Krummenacher, P., 2016. Systematic thermal energy storage integration in industry using pinch analysis. Portoroz, PROCEEDINGS OF ECOS 2016. 


\title{
RECOVERY OF VALUABLE SUBSTANCES LIKE GOLD AND PALLADIUM BY TREATMENT OF LIQUIDS FROM THE PRINTED-CIRCUIT-BOARD INDUSTRY WITH MEMBRANE DISTILLATION
}

\author{
C. Platzer*, S. Meitz**, E. Guillen, A. A. J. Tahir, C. Brunner, J. Buchmaier, B. Muster \\ AEE - Institut für Nachhaltige Technologien \\ Feldgasse 19, 8200 Gleisdorf, Austria \\ Phone: +43 3112 / 5886- 522 \\ E-Mail: c.platzer@aee.at
}

\section{SUMMARY}

There are several energy- and resource intensive process steps in the printed circuit board (PCB) industry such as the widespread (hard) gold -nickel process for surface treatment. In the hard gold plating process the plated circuit board needs to be rinsed after exposure to the active bath. By rinsing the work pieces in an external pool valuable active gold bath liquid is removed and lost in large amounts in gold cyanid "contaminated" wastewater. Test results showed around $30 \%$ of the added gold in the active gold bath is being lost in the rinsing bath. A possible process adaption is, to rinse the work pieces directly above the gold bath. Thereby the loss of gold and other ingredients as well as the amount of waste water is greatly reduced. However by following this new approach an efficient concentration technology for the thereby diluted active bath is required.

\section{INTRODUCTION}

As conventional concentration technologies like thermal processes (e.g. evaporation) or pressuredriven membrane technologies (e.g. reverse osmosis and micro-, ultra- and nanofiltration) need a high amount of thermal or electrical energy [1] [2], recovery strategies for chemical solutions are rarely applied. In this approach the feasibility of membrane distillation (MD) for the continuous treatment of gold baths was investigated. The MD offers a promising alternative since available low temperature waste heat from other processes on site or solar thermal heat can be used as the driving force for the separation technology.
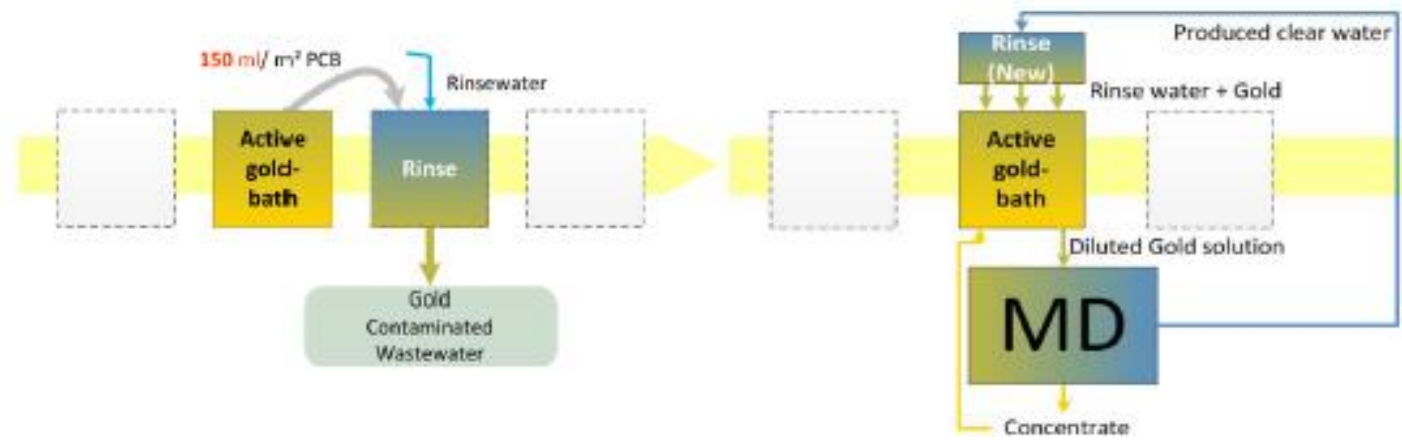

Fig. 1: Status Quo (left), The new approach; recovering gold bath liquid and closing the water cycle by integration of membrane distillation (MD) (right)

\section{MATERIAL AND METHODS}

In this study lab-scale experiments with real process water from one of the world's leading and Europe's largest PCB manufacturers AT\&S - Austria Technologie\&Systemtechnik AG - were conducted. In a first step, the stability, in terms of the chemical resistance, of a poly tetra fluor ethylene (PTFE) membrane was proven by conducting liquid entry pressure (LEP) tests as well as contact angle (CA) measurements with membranes that have been exposed to the respective liquid before. The dynamic experiments were performed on a direct contact membrane distillation (DCMD) unit using a flat-sheet test cell (membrane area $0,0375 \mathrm{~m}^{2}$ ). Further a DCMD flat-plate module (membrane area $0,91 \mathrm{~m}^{2}$ ) designed by AEE INTEC was used. The goal was to remain a constant gold 
and cobalt concentration in the active bath. The separated water had to meet given quality requirements for its reuse in the rinsing process.

\section{RESULTS AND DISCUSSION}

Evidence of permanent compliance with the required target concentration / concentration levels were detected in laboratory tests. The effects of varying operating parameters like absolute temperatures, trans-membrane temperature difference and circulation velocity were evaluated. Furthermore, the operational capability of the MD for concentrating and reusing waste water in the gold processes was tested. In order to depict the efficient system integration of the membrane distillation in the PCB industry a scale-up concept was elaborated. The scale-up concept is based on real data of the industrial partner for covering the thermal energy demand (heating and cooling) by the production process itself. Based on the scale-up concept a monetary evaluation of the technology, has been developed.

\section{CONCLUSIONS}

Through the adaptation and development of the membrane distillation (MD) process to the chemical, physical and process-technical characteristics of the surface treatment processes, an energy-efficient and sustainable method for the recovery of the valuable raw materials gold and palladium from processes of the printed circuit board production has been developed. These results laid the foundation for the further development of the process to a real-scale facility. The installation of a first real scale demo MD-Plant at AT\&S Fehring is planned for the second half of the year 2018.

\section{ACKNOWLEDGMENTS}

This work has been funded by the Austrian Federal Ministry for Transport, Innovation and Technology within the project "MD-Gold" (Innovative membrane distillation for gold and palladium recovery in the printed circuit board industry) - Production of the future program.

The Demo-Plant will funded by EU within the "ReWaCEM" project (Resource recovery from industrial waste water by cutting edge membrane technologies) - Horizon 2020 program, Grant Agreement no. 723729.

\section{REFERENCES}

[1] A. Alkhudhiri, N. Darwish und N. Hilal, (2012), „Membrane distillation: A comprehensive review,"Science Direct - Desalination

[2] K. W. Lawson and D. R. Lloyd, (1997), Membrane distillation,” J. Membr. Sci., vol. 124, no. 1, pp. $1-25,1$

\section{CONFERENCE TOPIC}

Energy and Resource Recovery from Low-grade Sources 


\section{PARTICLE SOLAR TOWER FOR HIGH TEMPERATURE PROCESS HEAT}

\author{
Dr.-Ing. Lars Amsbeck \\ Institute for Solar Research \\ German Aerospace Center (DLR) \\ Pfaffenwaldring 38-40, 70569 \\ Stuttgart, Germany \\ Phone: +49 711/ 6862306 \\ E-Mail: lars.amsbeck@dlr.de
}

\author{
Dr.-Ing. Reiner Buck \\ Institute for Solar Research \\ German Aerospace Center (DLR) \\ Pfaffenwaldring 38-40, 70569 \\ Stuttgart, Germany \\ Phone: +49 711/ 6862602 \\ E-Mail: reiner.buck@dlr.de
}

\author{
Dipl.-Ing. (FH) Miriam Ebert \\ Institute for Solar Research \\ German Aerospace Center \\ (DLR)Pfaffenwaldring 38-40, \\ 70569 Stuttgart, Germany \\ Phone: +49 711/ 6862626 \\ E-Mail: miriam.ebert@dlr.de
}

\section{SUMMARY}

The paper presents the Concentrated Solar Power (CSP) particle tower technology currently developed by DLR for high temperature process heat, combined heat and power and electricity generation applications. In this technology ceramic particles act as heat carrier and also as storage medium for temperatures up to $1000^{\circ} \mathrm{C}$. A first test campaign of the so called centrifugal particle receiver with a capacity of up to $2.5 \mathrm{MW}_{\text {th }}$ at the test facility "Solar Tower Jülich" demonstrated already $965^{\circ} \mathrm{C}$ particle outlet temperature and nearly $1000^{\circ} \mathrm{C}$ particle temperature in the receiver. An envisioned 50 $\mathrm{MW}_{\text {th }}$ receiver is expected to achieve heat collection costs of only $0.013 € / \mathrm{kWh}_{\text {th }}$ for a location with one of the best worldwide solar resources, a value competitive to power to heat from PV or wind and in the range of the actual world coal price. In an updated case study to partly substitute electric heating in a Brazilian foundry a payback time $<3$ years is estimated.

Keywords: Solar tower, CSP, particle receiver, process heat

\section{INTRODUCTION}

Concentrated solar tower plants with molten salt as the heat carrier in the receiver and storage with a maximum temperature of $565^{\circ} \mathrm{C}$ make up already about $50 \%$ of the CSP capacity under construction. Even higher temperatures up to $1000^{\circ} \mathrm{C}$ can be achieved with ceramic particles. These ceramic particles allow further cost reductions due to more efficient turbines, higher storage densities and lower component costs. Furthermore high temperature process heat applications for example in the steel industry can now be targeted. Storage related properties for the used sintered bauxite and as a reference the salt mixture used in commercial solar tower plants like the Crescent Dunes Solar Tower Plant in the USA are given in Table 8.

\begin{tabular}{|c|c|c|c|c|}
\hline & $\begin{array}{c}\text { Specific } \\
\text { heat } \\
{[\mathrm{kJ} / \mathrm{kg}]}\end{array}$ & $\begin{array}{c}\text { Mass per } \\
\mathrm{kWh} \\
{[\mathrm{kg} / \mathrm{kWh}]}\end{array}$ & $\begin{array}{c}\text { Volumetric storage } \\
\text { capacity }[\mathrm{kWh} / \mathrm{m} 3]\end{array}$ & $\begin{array}{c}\text { Specific } \\
\text { material costs at } \\
1 € / \mathrm{kg}[€ / \mathrm{kWh}]\end{array}$ \\
\hline $\begin{array}{c}\text { Solar Salt } \\
(60 \% \text { NaNO3,40\% KNO3) } \\
\mathrm{T}=288^{\circ} \mathrm{C}-565^{\circ} \mathrm{C}\end{array}$ & 446 & 8.1 & 204 & 8.1 \\
\hline $\begin{array}{c}\text { Sintered bauxite particles } \\
\mathrm{T}=288^{\circ} \mathrm{C}-565^{\circ} \mathrm{C}\end{array}$ & 314 & 11.5 & 175 & 11.5 \\
\hline $\begin{array}{c}\text { Sintered bauxite particles } \\
\mathrm{T}=400^{\circ} \mathrm{C}-1000^{\circ} \mathrm{C}\end{array}$ & 735 & 4.9 & 408 & 4.9 \\
\hline
\end{tabular}

Table 8: Storage related properties of sintered bauxite particles and solar salt 
The principle of direct absorption receiceiver with dark ceramic particlses was first followed in a large research program in the 1980 's by Sandia in the USA. In this program a falling particle receiver concept was favored [1-4], but also general subjects of particles as a heat carrier and storage medium [5] and other components of the particle loop like direct contact heat exchangers were investigated [6]. The centrifugal particle receiver concept $(\operatorname{CentRec} \AA)$ is a rotating receiver that allows the uniform heating of a thin but closed and opaque particle film with a very high efficiency for the complete load range. This particle film is achieved by a superposition of gravitational force and centrifugal force. With a tilted receiver axis the gravitational force and the centrifugal force push the particles together against the wall in the lower half of the receiver during each turn. In the upper part of the receiver the centrifugal force counteracts the gravitation. This results in a step-wise movement of the particles towards the aperture during each turn. The centrifugal force and with it the step-size and therefore retention time of the particles in the receiver is controlled through the rotational speed which can be easily changed through the inverter of the electric drive. Together with a particle mass flow control into the receiver the desired outlet temperature can be achieved under any load conditions [7].

The movement of the particles in the receiver and especially the deceleration of the particles in the collector ring after the rotating part produce attrition. But laboratory tests of particle attrition in the receiver indicate negligible particle replacement costs of only $0.32 € / \mathrm{MWh}_{\mathrm{th}}[8]$.

The low cost of the particles allows a direct storage of the collected heat in a two tank configuration as shown in Fig. 1. High temperature heat as steam or hot gas can then be delivered from the storage on demand up to $24 \mathrm{~h}$ per day for:

1. industrial process heat applications up to $1000^{\circ} \mathrm{C}$

2. pure electricity production with steam, $\mathrm{sCO}_{2}$ and potentially also gas turbines

3. combined heat and power

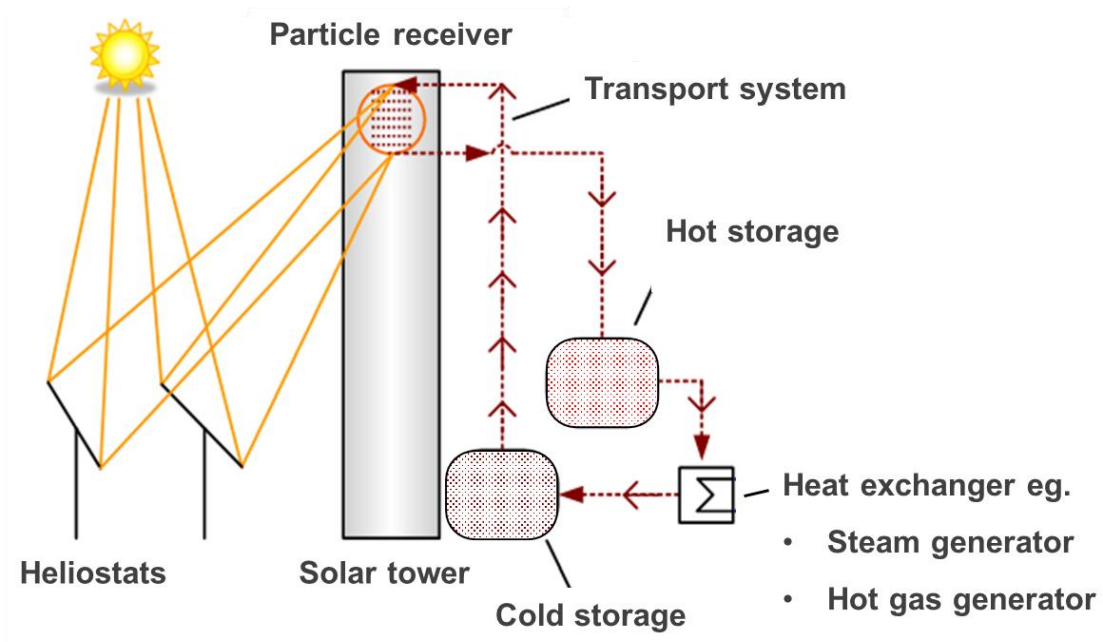

Fig. 1: Scheme of a particle solar tower system for process heat, electricity production or CHP

The possible direct contact heat exchange between the particles and gases allows also a simple hybridization with e.g. natural gas, biomass, green $\mathrm{H}_{2}$ or surplus wind energy. With this a weather independent energy supply is guaranteed which can achieve the same availability than the conventional fossil energy supply. 


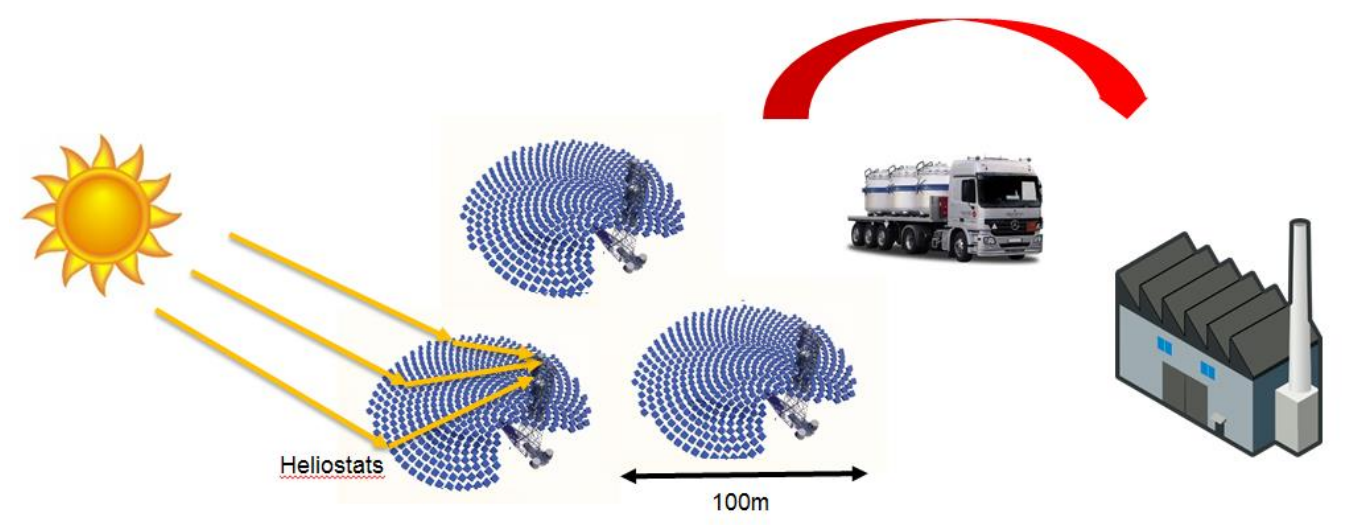

Fig. 2: Scheme of a particle solar tower system for process heat, electricity production or CHP

Furthermore a horizontal particle transport by truck (electric and autonomous if possible) or rail enables multi-tower systems to achieve higher capacities and locating the solar plant some $\mathrm{km}$ away from the location of use. This transport system as shown in Figure 2 is inspired by the road transport of molten aluminium at $>700^{\circ} \mathrm{C}$ until distances of $100 \mathrm{~km}$ in Germany. This solves the problem of often unavailable or expensive land and potential dust problems next to industrial heat users.

\section{TESTS RESULTS}

With $900^{\circ} \mathrm{C}$ particle outlet generation a $10 \mathrm{~kW}_{\text {th }}$ power level proof-of-concept was successfully operated in 2012 [9]. As a next step a receiver with a capacity up to $2.5 \mathrm{MW}_{\text {th }}$ was constructed and first tested

with

a

$100 \mathrm{~kW}_{\mathrm{el}}$ infrared radiator [10]. In 2017 the receiver was installed at the test facility "Solar Tower Jülich" and a first test campaign reached particle outlet temperatures up to $775^{\circ} \mathrm{C}$ as shown in Figure 3. Particle temperatures in the receiver of $>900^{\circ} \mathrm{C}$ and a particle temperature homogeneity of $\pm 25^{\circ} \mathrm{C}$ was demonstrated [11]. In the second test campaign in 2018 a particle outlet temperature of $965^{\circ} \mathrm{C}$ and nearly $1000^{\circ} \mathrm{C}$ particle temperature in the receiver was achieved [12].

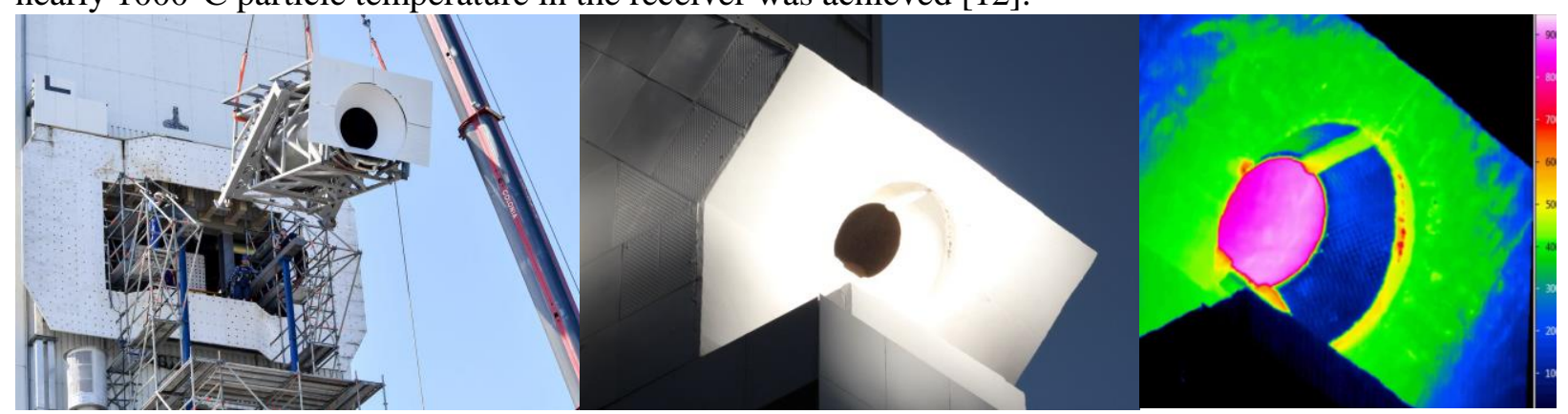

Fig. 3: Receiver during installation (left), solar operation (middle) and in an infrared image (right)

\section{HEAT PRICE COMPARISON}

A $50 \mathrm{MW}_{\text {th }}$ particle solar tower system in [13] showed heat costs delivered from the storage of only $0.0146 € / \mathrm{kWh}_{\mathrm{th}}$ for a location with nearly perfect atmospheric conditions in Chile with an annual direct irradiation of $3583 \mathrm{kWh} / \mathrm{m}^{2}$ a. The collection part (heliostat field, tower, receiver, particle transport system) without the thermal storage system and the heat exchanger to the process contribute only $0.013 € / \mathrm{kWh}_{\text {th. }}$. This compares still well with the actual world record PV price of $0.0153 € / \mathrm{kWh}$ $\left(0.0179 \$ / \mathrm{kWh}_{\mathrm{e}}\right.$ at $\left.0.854 € / \$[14]\right)$ which could be used for power to heat. But this is still a rough 
comparison as important data like solar resource and financing conditions for the PV plant are not known.

Furthermore these heat prices get even in the actual world coal price range of $0.0106 € / \mathrm{kWh}_{\text {th }}(86.6 \$ / \mathrm{t}$ for South African coal, 6.978 MWh/t, 03/2018 [15]).

\section{CASE STUDY HIGH TEMPERATURE SOLAR PROCESS HEAT IN THE STEEL INDUSTRY}

One of the most economic applications of the particle technology is the partly substitution of high temperature process heat generated with electricity. Electric heating happens in the metal industries in induction furnaces and partly in electric arc furnaces, which have often supplementary chemical energy inputs from the burning of gas, added carbon materials and alloyed carbon. In [16] a case study of a $70000 \mathrm{t} / \mathrm{a}$ grey iron foundry in Brazil is presented where the technology achieves a payback time of 4.1 years. Preheating technology from electric arc furnaces is used to integrate the solar energy into the induction furnace by preheating scrap to $600^{\circ} \mathrm{C}$, saving $1 / 3$ of the electric energy. With recent reductions in heliostat costs to $100 € / \mathrm{m}^{2}$ installed costs and an upscaled system [13] we estimate payback time now to be $<3$ years.

\section{CONCLUSIONS AND OUTLOOK}

CSP particle tower technology has the potential to achieve significant market share in the areas of high temperature process heat, electricity production and combined heat and power. The receiver as the key component was successfully demonstrated on the Solar Tower Jülich and the economic performance of the technology looks also promising. Therefore the next aspired development step for the technology is a semi-commercial demonstration project.

\section{ACKNOWLEDGEMENTS}

This work was supported by the Technology Marketing of the German Aerospace Center and DLR Energy Programme Directorate (grant no. LRV 16/113) and the Helmholtz Validation Fund (HVF) of the Helmholtz Association (grant no. HVF-0028).

\section{REFERENCES}

[1] J. Martin et al., (1982), Ascuas: A solar central receiver utilizing a solid thermal carrier. Technical report, Sandia National Laboratories Paper No. SAND 82-8203

[2] P.K. Falcone et al., (1985) Assessment of a solid particle receiver for a high temperature solar central receiver system. Technical report, Sandia National Laboratories Paper No. SAND85-8208

[3] J. M. Hruby, (1986), A technical feasibility study of a solid particle solar central receiver for high temperature applications. Technical report, Sandia National Laboratories Paper No. SAND 86-8211

[4] J. M. Hruby et al., (1988), An experimental and numerical study of flow and convective heat transfer in freely falling curtain of particles. Technical report, Sandia National Laboratories Paper No. SAND 868714

[5] J.W. Griffin and K.A. Stahl, (1986), Optical properties of solid particle receiver materials i,ii. Solar Energy Materials, 14:395 \{425

[6] Green, H.J. et al., (1986), Technical and Economic Evaluation of a solid-particle/air direct contact heat exchanger, Solar Energy Research Institute, Colorado 
[7] Amsbeck, L. et al.,(2017), First Tests of a Centrifugal Particle Receiver with a 1m2 Aperture, proceedings of the Solarpaces conference

[8] Gobereit, B. et al., (2016), Abrasion, Corrosion and Erosion of Particles and Metallic Structure in Solid Particle Receivers, proceedings of the Solarpaces conference

[9] Wu, W. (2015), A centrifugal Particle Receiver for High-Temperature Solar Applications, PhD RWTH Aachen 2014, Logos Verlag Berlin, ISBN 978-3-8325-3882-8

[10] Ebert M. et al. (2016), Upscaling, Manufacturing and Test of a Centrifugal Particle Receiver, ASME. Energy Sustainability, V001T04A007. doi:10.1115/ES2016-59252.

[11] Ebert M. et al. (2018), First on-sun tests of a centrifugal particle receiver system, ASME. Energy Sustainability

[12] Ebert, M. et al., (2018), Operational Experience of a $500 \mathrm{kWth}$ Centrifugal Particle Receiver System, proceedings of the Solarpaces conference

[13] Buck, R. et al., (2018), Impact of CSP Design Parameters on $\mathrm{sCO}_{2}$-Based Solar Tower Plants, $2^{\text {nd }}$ European supercritical CO2 Conference, August 30-31, Essen, Germany

[14] https://reneweconomy.com.au/stunning-new-low-for-solar-pv-as-even-iea-hails-age-of-solar$\underline{43509 /}$

[15] World Bank Commodities Price Data (The Pink Sheet), 6 April 2018, http://pubdocs.worldbank.org/en/664371522786567489/CMO-Pink-Sheet-April-2018.pdf

[16] Amsbeck, L. et al., (2016), Particle tower technology applied to metallurgic plants and peak-time boosting of steam power plants, AIP Conference Proceedings 1734, 070001 (2016); https://doi.org/10.1063/1.4949148 


\title{
GREEN AUTOMOTIVE INDUSTRY- FACING CHALLENGES AND OPPORTUNITIES OF SOLAR HEAT ON THE WAY TOWARDS "GREEN" PRODUCTION
}

\author{
Juergen Fluch, Christoph Brunner, Thomas Ramschak, François Veynandt, Christian Fink \\ AEE - Institute for Sustainable Technologies \\ Feldgasse 19, 8200 Gleisdorf, Austria \\ Phone: +433112 / 58860 \\ E-Mail: j.fluch@aee.at \\ Bastian Schmitt, Felix Pag \\ University of Kassel, Institute of Thermal Engineering \\ Kurt-Wolters-Straße 3, 34109 Kassel \\ Phone: +49 561 / 8043890 \\ E-Mail: solar@uni-kassel.de
}

\section{SUMMARY}

Solar process heat has a huge potential for supporting the automotive and the automotive supplier industry on the way towards shifting into a low carbon, environment-friendly sector. The high potential for solar heat is determined by the temperature level of process heat required. Results of 29 case studies conducted within the project "SolarAutomotive" in Europe and worldwide show different integration possibilities for different sub-branches and typical collector field sizes as well as country specific environmental and economic conditions for the integration. According to a survey, performed by the project consortium most enterprises surveyed state the existence of environmental goals. Although the willingness of the sector and the potential for the use of "green" technologies seem to be high, there is a lack of implementations due to barriers along the decision-making chain. Therefore, a guideline fostering market uptake of solar process heat is being conducted dealing with country specific challenges.

Keywords: green production, solar process heat, automotive, SHIP, processes, depreciation, market uptake

\section{INTRODUCTION}

According to ACEA (2017), the automotive and supply industry is the most important industry in the European Union, accounting for more than $10 \%$ of manufacturing employment in the European Union and about $4 \%$ of European gross domestic product. Due to the process requirements, typical processes in the industry are very well suited to be supplied with solar process heat. As the industry is known as a leader in innovation, switching to "green" production could serve as a model for the entire European manufacturing industry and be the key to market acceptance of sustainable technologies such as solar thermal.

The branch of industry is characterized by its inhomogeneity, but therefore offers great potential for transferring new insights into other industries. As part of the project, 12 sub-sectors of the automotive and supplier industry were identified and considered within the German-Austrian cooperation project "SolarAutomotive". The project was launched in May 2016. The transnational project consortium comprising the project coordinators AEE - Institute for Sustainable Technologies (AEE INTEC) and Department of Solar- and Systems Engineering from University of Kassel are completed by the experienced solar companies S.O.L.I.D. GmbH and KPV Solar GmbH in Austria and by the Foundation for Resource Efficiency and Climate Protection (STREKS) and the Department of Sustainable Products and Processes (University of Kassel) in Germany. The three-year research project funded by the Austrian Climate and Energy Fund and the German Federal Ministry for Economic Affairs and Energy has set itself the goal of facilitating the entry of solar process heat into the automotive industry and its upstream supplier industry. Through innovative applications, potentials are identified and new impulses are set by the realization of lighthouse projects, which are supposed to show that the decarbonization of heat demand is possible. 


\section{POTENTIAL, CHALLENGES AND SOLUTIONS}

Solar thermal energy offers great potential in industrial process heat supply, especially in the low temperature range up to $100{ }^{\circ} \mathrm{C}$ as well as in the middle temperature range up to $250{ }^{\circ} \mathrm{C}$. Assuming that theoretically the entire industrial heat requirement below $200{ }^{\circ} \mathrm{C}$ can be supplied by solar thermal energy, an installed collector area of 4.2 million $\mathrm{m}^{2}$ with a capacity of 2.4 gigawatts SHIP (Solar Heat for Industrial Processes) would lead to a $\mathrm{CO} 2$ avoidance potential of 25 million metric tons per year. In order to exploit the potential in concrete concepts and implementations for the industry, those processes of the project were identified that are particularly well suited for solar thermal supply due to their process requirements (temperature, load profiles). These key processes in the 12 identified subsectors of the automotive industry (including the supply industry) include washing and dyeing processes in the textile industry as well as electroplating processes in metal surface treatment and air conditioning steps in the automotive industry.

The presented potential for SHIP is currently not nearly tapped. The reasons for this are a general information deficit with regard to the potentials and possible uses of solar process heat, reservations about the technology as well as the lack of know-how about simple integration possibilities and the cost-effectiveness of the implementation. As a result, there are few concrete implementations and lighthouse projects with which these barriers can be addressed. SolarAutomotive approaches this challenge and, in addition to information on possible applications and integration concepts, also provides tools for quick and detailed design as well as economic analysis in a guideline. Results from literature studies, concrete case studies and planned implementations, surveys and "lessons learned" of the solar companies are incorporated here.

\section{CASE STUDIES}

Together with the project partners in Germany, almost 30 detailed case studies were conducted in different countries, based on the indicated potentials of the various industries. Figure 1 shows the locations of these case studies.

The audits were carried out starting with the evaluation of the existing energy supply up to detailed analyses of the production processes. Based on this, appropriate efficiency measures were identified and evaluated in a first step in order to take account not only of process optimization possibilities but also of internal heat recovery potentials. Only after taking these points into account, it is possible to present a reliable description of the potential for the integration of solar thermal energy both at the process and at the supply level.

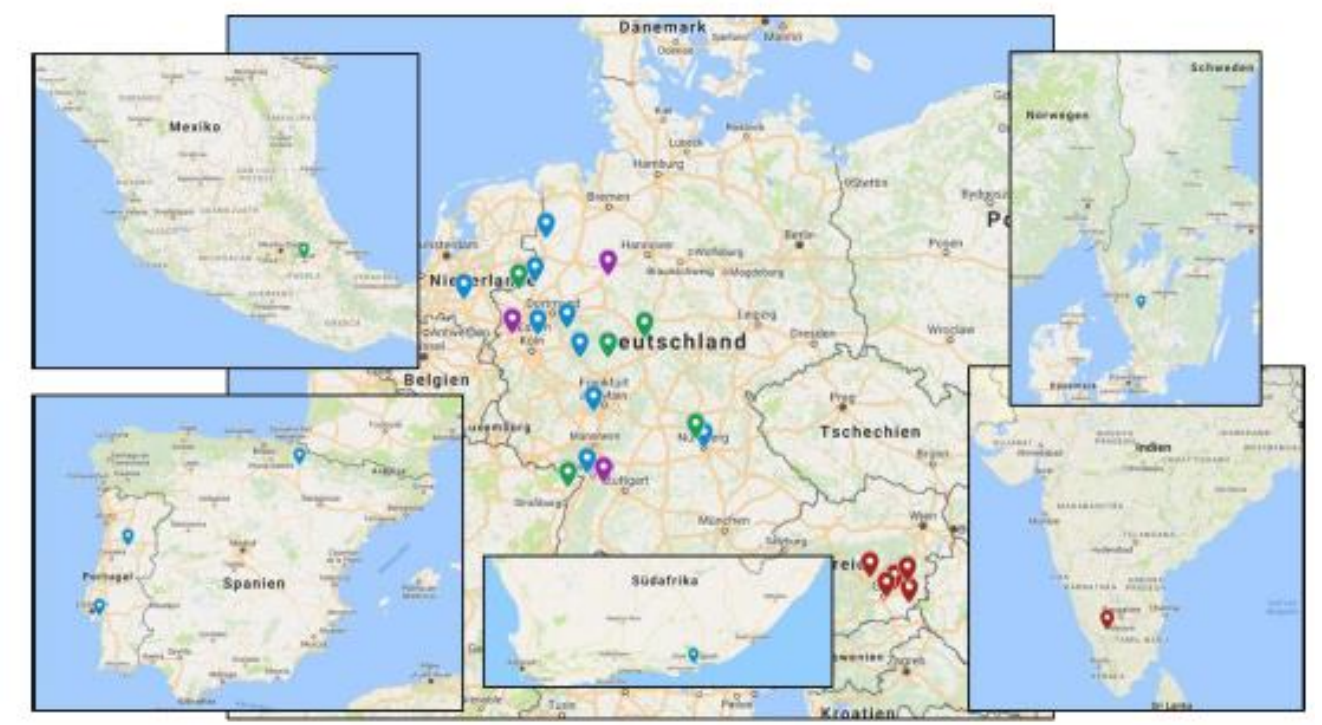

Figure 1: Case studies within different countries performed within SolarAutomotive 
Exemplary methodology and steps for an energy audit and SHIP concept development:

- General data on the company: company size, economic conditions

- Used energy sources and energy consumption: typically mainly electricity and gas

- Energy supply and distribution

- Heat demand per production process: relevant process parameters such as temperature, load profiles, mass flows, operating and heating times, etc. are crucial for the optimized integration of solar process heat

- Presentation of mass and energy balancing status quo (including Sankey diagrams)

- Process optimization to reduce energy consumption (insulation, bath covers, etc.)

- System optimization (heat recovery) with the help of pinch analysis for efficient use of waste heat dryers, exhaust air, waste water, etc., taking into account technical feasibility and local characteristics

- Evaluation of the measures according to technical, ecological and economic criteria

Based on this, the identification and selection of possible integration points for the solar thermal supply takes place. Process parameters (temperature level, spread, heating rates, etc.) and processspecific features and product quality are included in the evaluation. The result is a sensible choice of collector technology, collector area, storage, location and orientation as a result of detailed design and simulation. The highest possible yield of the plant faces economic optimizations. Heat recovery and solar thermal energy are not in competition with each other in an optimized energy concept, but in combination with existing technology are part of a hybrid and secure industrial energy supply.

The conducted case studies of the project show both technically and economically feasible plants of different sizes and yields (plants with solar thermal areas between $50 \mathrm{~m}^{2}$ and 3,200 $\mathrm{m}^{2}$ ). In a next step, after positive internal evaluation and approval, the concepts are envisaged to be implemented. Particularly important is the involvement of the company in all steps of the data collection up to the concept development and evaluation, in order to clear possible reservations and doubts in advance and also to take into account the company-specific knowledge on conditions such as space requirements or feasibility of measures.

\section{SURVEY}

In addition to the technical hard facts, it is ultimately crucial for companies whether an investment can be represented economically. For this, companies have different evaluation criteria and internal requirements that have to be addressed in concept development. Small businesses are different to large companies and use completely other economic criteria. Above all, it was important to find out the parameters for the evaluation of a possible investment decision. For this purpose, a survey has been carried out, which has raised corporate policy framework conditions, drivers and barriers to investments in the field of renewable energies, as well as internal guidelines. This should be used to find specific leverage points for an investment decision and to address them accordingly. The questionnaire was answered by 45 manufacturing companies from 14 different subsectors of the automotive and automotive supply industry and evaluated accordingly. 


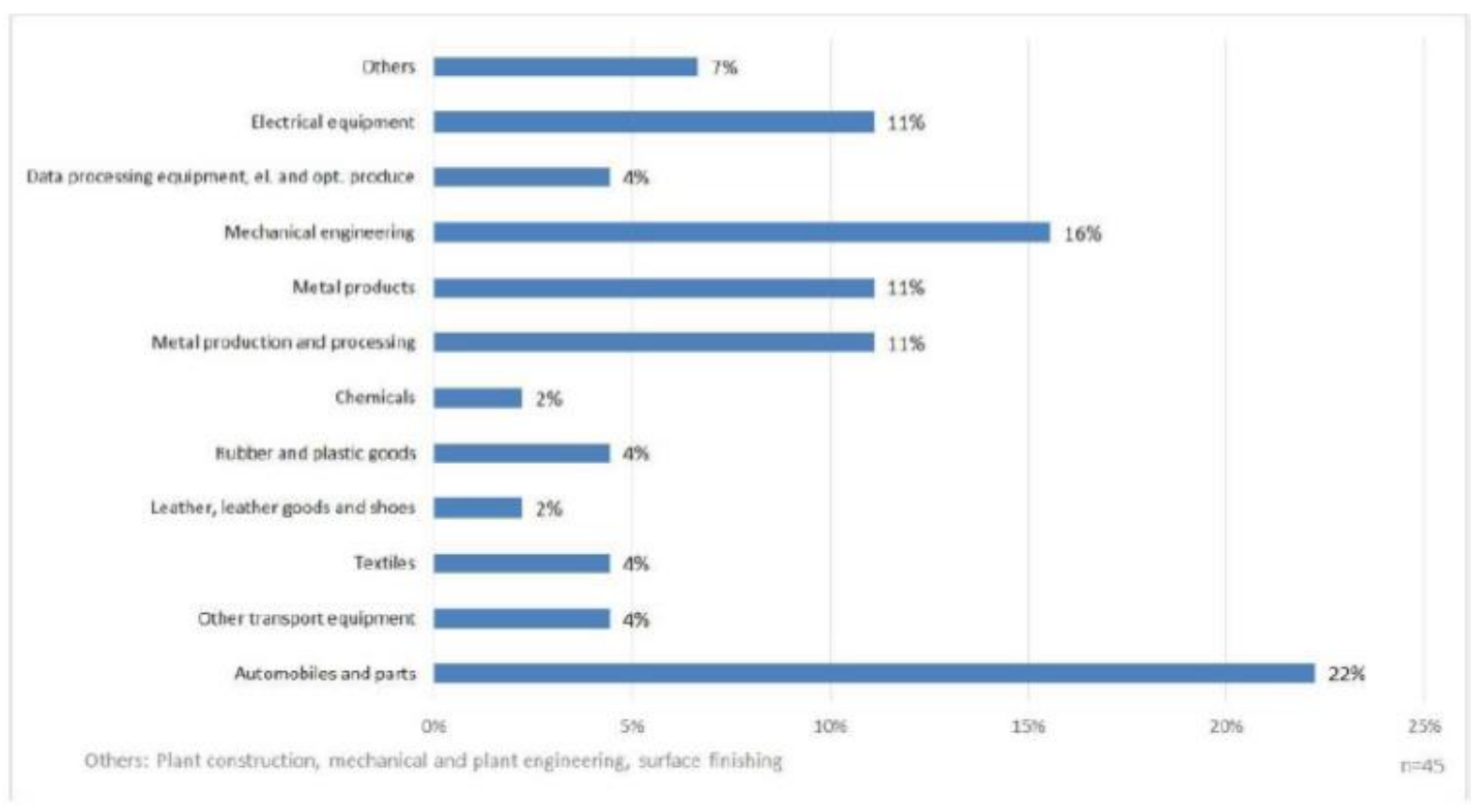

Figure 2: Distribution of sub-sectors on the answered survey

The participating companies are made up of companies with different sizes and numbers of employees ranging from less than 10 to more than 10,000, with locations in Europe and around the world. Striking are the different employees according to company size, the decision-makers involved and the associated decision-making channels. The importance of energy efficiency and renewable energies also shows considerable differences in the different positions in the company. Thus, it can be seen that the fundamental awareness of sustainable production or its energy supply in the top management is indeed present, but there are major hurdles to the implementation. The technical understanding is more likely to be in the area of internal environmental officers or energy managers, where the economic evaluation of the concepts is considered too optimistic (too high acceptable ROIs).

Companies that have already implemented energy efficiency and renewable energy initiatives have identified short payback periods and environmental considerations as key drivers for their implementation. The result of the evaluation underlines the objective of the project, to reduce planning costs, to identify key processes for the solar thermal supply and thus to contribute to the reduction of the payback period. Furthermore, the results show that external conditions e.g. by clients does not play an important role so far regarding the decisions for an investment in renewable energy or energy efficiency. 


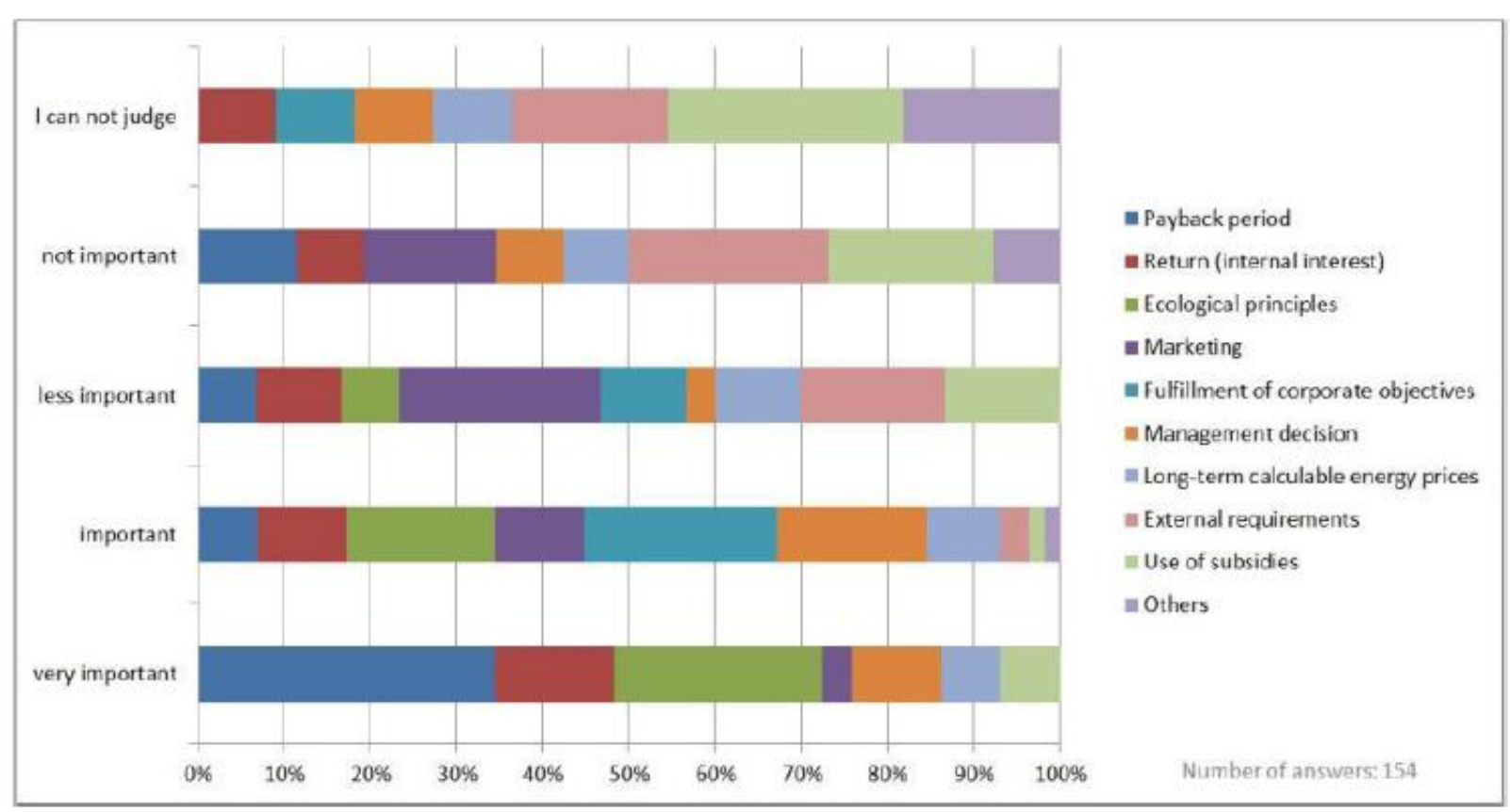

Figure 3: Weighting of the factors for implementation of measures in the field of energy efficiency / renewable energies

\section{ECONOMIC CONSIDERATION}

When presenting solar thermal projects, the solar industry often does not use the same language as investors or banks do. An economic view is of crucial strategic importance for investment decisions with high initial costs for long-term projects for the companies. The investment costs for solar process heat plants vary greatly depending on the type of collector used, the system hydraulics including energy storage system, the process connection, local conditions.

In order to be able to offer solar companies an opportunity in direct conversation with their customers to react to local conditions (e.g. reduced space requirements of freely available roof areas for collectors) or company policy requirements, methods and striking representations (nomograms) for evaluating the economic consideration of solar process heat plants have been developed. These allow to respond fast to the needs and objectives of the enterprise and to find the optimal system configuration for the enterprise. "Easy-to-use" graphs and parameters are necessary to break down the hard to understand interaction of e.g. solar yield, collector area, specific storage volume and heat production costs. Figure 4 is an example to show the relationship between these parameters. Targeting minimized heat production costs influence the design and size of the solar collector field as well as the specific storage volume and by this the achieved solar coverage, what is not a maximized value. Nomograms like this help the designers and the industry to understand the optimized design of a SHIP plant. 


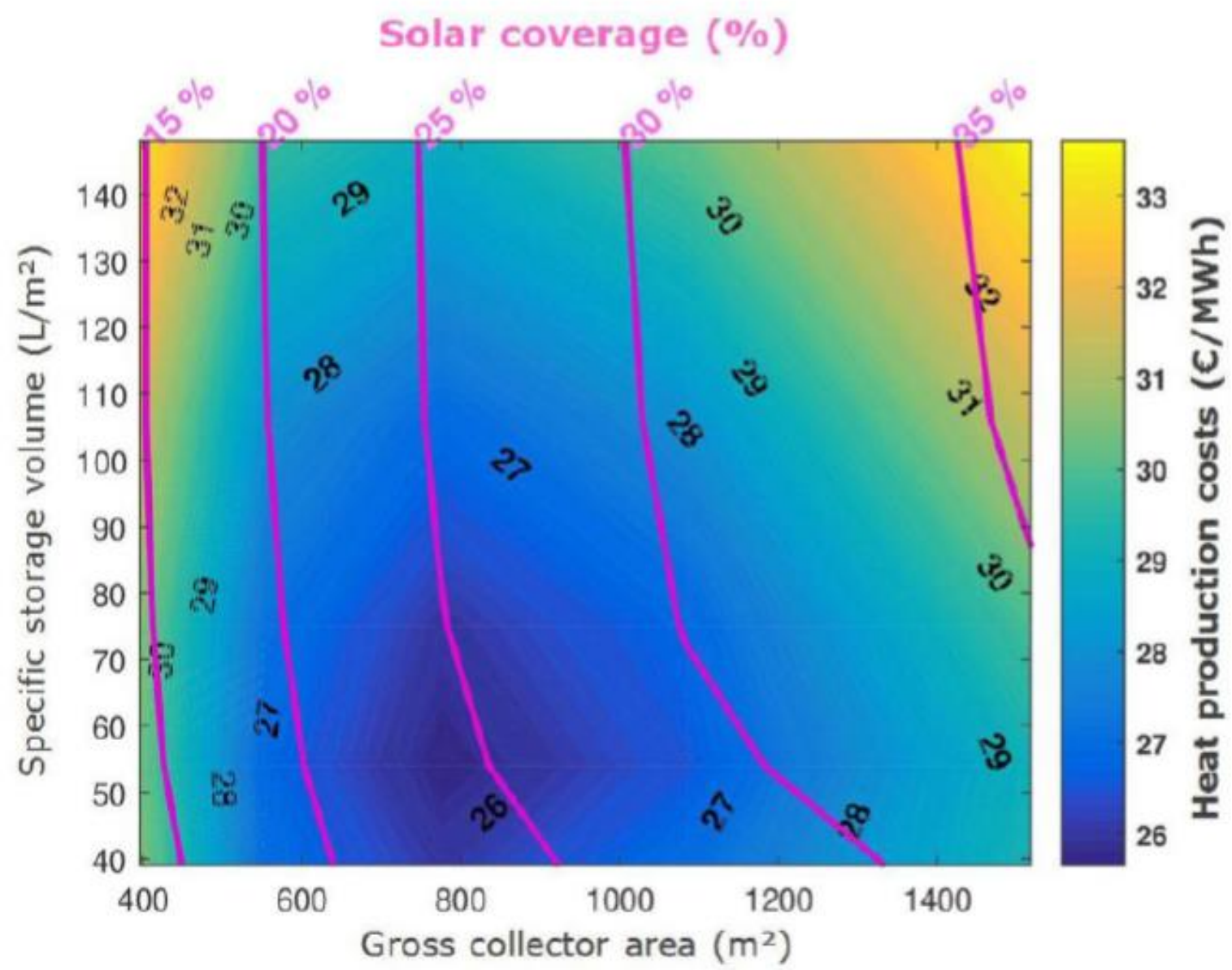

Figure 4: Exemplary nomogram on heat production costs (over a period of observation of 25 years) and solar coverage for different combinations of collector area and storage volume supplying a cleaning water process in Austria

\section{SUPPORTING TOOLS}

Key to the identification and evaluation of solar thermal concepts are simple and tailor-made tools that provide important information and evaluations of a solar thermal system depending on the target group. According to their requirements, target groups are industrial companies (internal energy managers), energy consultants, solar thermal providers, etc. As part of the project, these requirements were addressed in two different tools - a preliminary design tool and a detailed simulation tool, SolarSOCO. The different levels of detail enable the distribution to different target groups.

\section{Preliminary design tool}

The created preliminary design tool offers a quick-check of the potential of solar thermal energy, that is especially interesting for solar companies, industrial companies and energy consultants. The target is to identify very user-friendly a first idea of SHIP based on few input parameters leading to key output criteria. Part of this tool is a first yield assessment, that will be further extended by an economic evaluation. Particular attention is paid to the ease of use and adaptability to changing economic conditions and the different requirements of companies. The tool is available free of charge at http://designtool.solar4industry.info/.

\section{SolarSOCO}

This tool, which was developed based on preliminary work, enables a design in a much higher degree of detail. In a first step, heat recovery potentials are identified with the aid of the pinch analysis and, based on this, adetailed solar thermal integration is simulated. At the same time, necessary storage systems are integrated into the overall concept in order to find a total exergetic optimum. The core of the optimization algorithm as well as the integrated identification, simulation and evaluation of the 
solar integration is a temporally resolved load profile that is linked to available solar radiation (weather data) and calculates the yield of the plant at any time depending on the collector efficiency (process temperature, orientation, losses, etc.). Through the combination in the system design with a possible heat recovery and possible storage as well as their detailed simulation, concrete statements are made regarding the technical and economic evaluation of the system. Currently (as of May 2018), SolarSOCO is in a beta testing phase and will be evaluated in terms of usability and simulation results to be completed in a final step.

\section{REFERENCES}

ACEA, (2017), The Automobile Industry Pocket Guide 2017-2018.

EUROSTAT, (2017), Statistics Explained, Energieverbrauch, EU-28, 2015

\section{CONCLUSION}

If we want to achieve the defined climate goals in the EU, industry as a "consumer" of $25 \%$ of the final energy input (EUROSTAT, 2017) and thus the automotive and supplier industry as an innovation leader, must make a contribution. In order to remove barriers and from an economic point of view existing reservations against sustainable and renewable energy sources, lighthouse projects must be initiated and standardized solutions and concepts derived from them, which accordingly find many imitators. Carried out analyses and case studies clearly confirm the potential of solar process heat in this industrial sector due to the process technologies and parameters used there.

The great expected potential of the automotive and supplier industry for the use of solar process heat could be confirmed by the case studies carried out. The results of the audits and other surveys on the production processes in the industry are used to identify key processes with particularly high potential and to develop standardized integration concepts from them. This addresses raised barriers and reservations about solar process heat (too expensive, too complicated, lack of know-how, etc.) and the often high planning effort is reduced. In addition to the planned direct implementations and their side effects within the project, the results are incorporated in a guideline and tools in order to facilitate and promote the further dissemination and implementation of solar process heat in the automotive and supplier industry.

The promotion of solar process heat in the automotive and supplier industry ensures that two areas of strength of the Austrian economy, the automotive and supplier industry and the solar thermal industry are combined, thus contributing to technological leadership in this area and to the development of the industry's exemplary character. The project eliminates the implementation barriers of SHIP, increases the affordability of the technology and initiates concrete flagship projects. At the same time, standardized concepts, tools and a guideline will help to ensure that green technology is given the corresponding importance in industrial energy supply over the next few years. The project SolarAutomotive can be an initial spark for this development, if it succeeds to publicize the project results, to make projects affordable and thus to decisively reduce the barriers. 


\title{
EXPERIMENTAL ASSESSMENT OF SOLAR PROCESS HEAT POTENTIAL OF GERMAN PLASTIC INJECTION MOULDERS
}

\author{
Florian Schlossera, Heiko Dunkelberga, Frank Veitengrubera, Henning Meschedea, Felix Pagb, Bastian \\ Schmittb \\ University of Kassel, aSustainable Products and Processes, bSolar and Systems Engineering \\ Kurt-Wolters-Straße 3, 34125 Kassel, Germany \\ Phone: +49 561/804 3442, E-Mail: schlosser@upp-kassel.de
}

\section{SUMMARY}

One challenge of the German heat energy transition is the substitution of fossil burned fuels by renewable heat sources like solar thermal for the industrial process heat supply. Especially in the plastics processing industry, there is an information deficit about possible utilisation of this technology. For that purpose, a potential study is being conducted focussing on the implementation of solar process heat. This paper identifies thermal production sinks which are principally suitable for solar thermal applications regarding the temperature level of their heat supply. By means of experimental test series, technical implementations for the drying of granules are evaluated. Based on that, relative energy savings regarding to the status quo as well as solar fractions and yields for different collector types are quantified. The integration of solar heat at $60-70{ }^{\circ} \mathrm{C}$ for drying polyamide and $70-80{ }^{\circ} \mathrm{C}$ for polypropylene is suitable for flat plate collector and vacuum tube collector. Additionally, this work characterizes the German injection moulding industry and identifies 128 enterprises with an aggregated thermal drying energy demand of $1.8 \mathrm{GWh} / \mathrm{a}$ which, due to their associated property structure, are particularly suitable for solar process heat applications.

\section{INTRODUCTION}

Germany formulates ambitious targets regarding the reduction of greenhouse gas emissions and the saving of primary energy. In addition to increase energy efficiency, the implementation of renewable energy is a possibility on the way to a decarbonised energy supply. A significant number of studies are related to energy efficiency and process optimization potentials for the plastic processing industry (Weiß (2016), Matarrese et al. (2017), Mianehrow and Abbasian (2017)). As Lauterbach et al. (2012) have already observed, in many cases the electricity consumption, but not industrial heat consumption, is in the focus of efficiency measures. According to the BMWi (2017), the demand of industrial process heat, which is largely generated by fossil energy sources, is about $500 \mathrm{TWh}$, viz just under one-fifth of the total German final energy consumption. In this context, the heat sector has a significant potential to provide industrial process heat by solar thermal systems, which would reduce greenhouse gas emissions more drastically. Besides others, one obstacle to the implementation and use of solar heat in industrial companies is a very widespread information deficit about possible applications of this technology, especially in the plastics processing industry. Both Lauterbach et al. (2010) and Müller et al. (2004) have already conducted general potential studies for the use of solar process heat in various industrial sectors based on temperature level and heat demand. The plastics industry is reported with a comparatively low potential. This is not least due to the high temperature level in the central plastic processing processes, as shown in Fig. 1, and to the predominantly electrical heating.

Compared to other forming processes, the German injection moulding industry generates almost three times the annual turnover with about 55 billion euros, which is why this branch of industry has become more important (Destatis, 2016). A general description of the plastic industry in Germany can be found in Görlitz (2017) and Dispan and Vassiliadis (2014) summarising key industry parameters, 
such as employment, sales or production. Internationally, the authors of the RECIPE study (euRECIPE, 2006) deal with the plastics industry in Europe. Elaborations or studies containing the classification and summary of companies according to their characteristics in an industry cannot be found in the literature.

With respect to the mentioned targets and the lack of knowledge, this study aims on both, the identification of meaningful application fields as well as implementation possibilities for the integration of solar process heat in the plastics processing industry. Based on this, the potential is to be identified and quantified, using experimental as well as empirical research methods. Therefore, a technical integration concept is demonstrated with an experimental test series using the example of granule drying, and associated energy savings will be determined. Further results of the investigation are recommended actions for the collector types flat plate collector and vacuum tube collector as well as assessments of their solar fraction based on a solar annual simulation. In a final step, the mediumsized injection moulding industry in Germany is classified regarding to their property structure. Based on that, a target function identifies companies that are particularly suitable for the implementation of solar process heat due to certain characteristics.

\section{HEAT SINKS IN THE PLASTICS INDUSTRY}

Measures for the integration of low-temperature heat in industrial processes are investigated in various papers. Schumm et al. (2017) describes a system that partially substitutes the conventional steam supply in the food industry by hot water from low temperature heat sources (e.g. solar thermal, waste heat, CHP, heat pumps) using a hybrid heating system-technology. Schmitt (2016) classifies the industrial heat sinks according to process and supply integration level. Schmitt et al. (2015a and $2015 \mathrm{~b}$ ) also examines various concepts for the integration of solar heat in the food industry and the fast feasibility assessment for solar thermal systems based on various collector types.

In addition to extrusion, injection moulding as the primary moulding process is widely used in plastics processing and consumes about $777 \mathrm{GWh} / \mathrm{a}$ of energy per year (Statista, 2011). Most of the plastics processing processes consist of the three main process steps conditioning, moulding and posttreatment. Suitable heat sinks for the integration of solar process heat are characterized by a constant possible load profile during summer season and low integration temperatures. The lower the integration temperatures, the lower are heat losses at the collector and the collector efficiency increases (Lauterbach et al., 2011). Fig. 1 assigns a potential assessment to the essential processes of the three main processing steps based on their temperature requirement. 


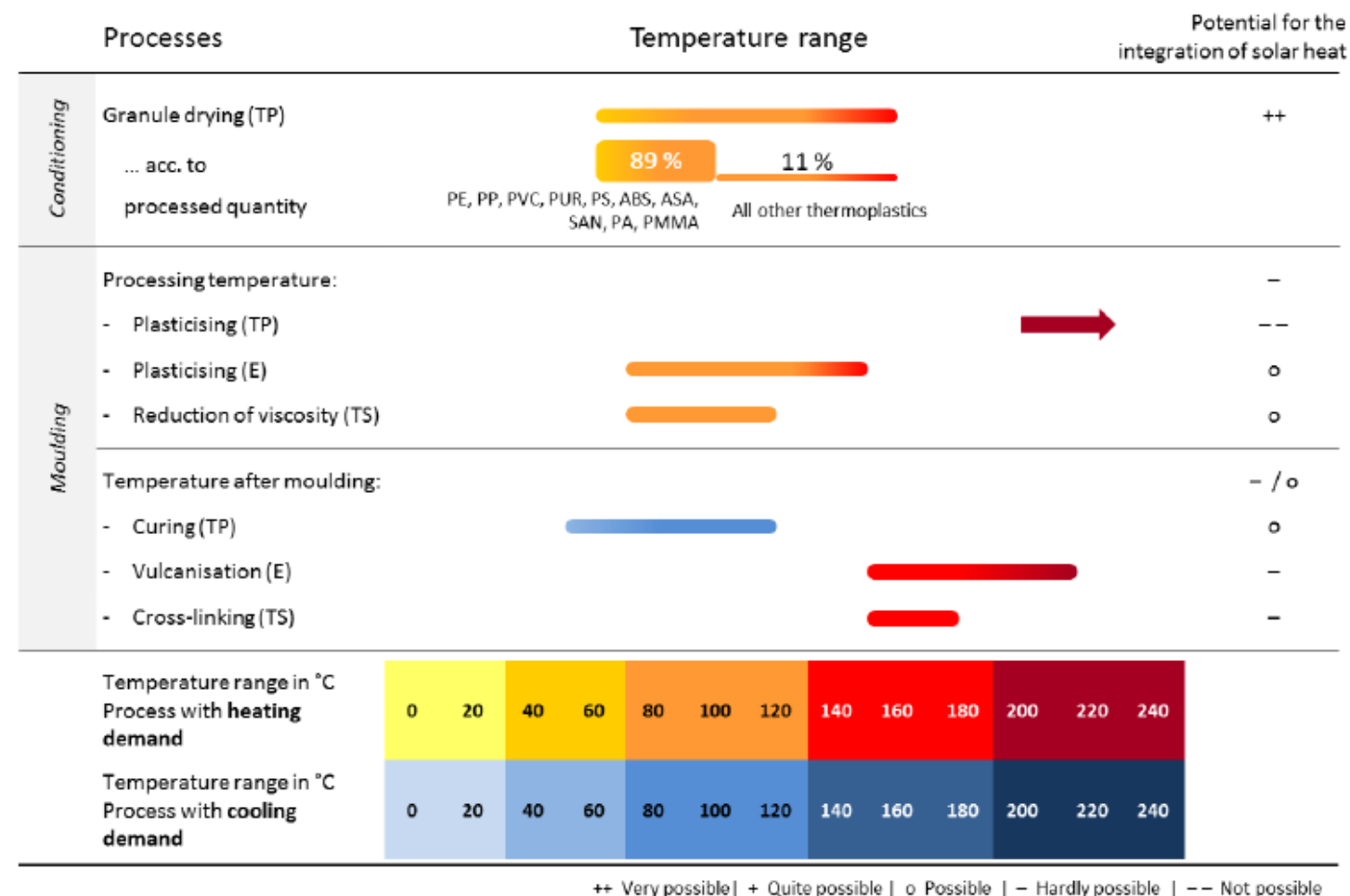

Thermoplastics (TP), Elastomers (E), Thermosetting plastics (TS), Polyethylene (PE), Polyvinyl Chloride (PVC), Polystyrene (PS), Acrylonitrile Butadiene Styrene (ABS), Styrene Acrylonitrile (SAN), Polyurethane (PUR), Acrylonitrile Styrene Acrylate (ASA), Polymethylmethacrylate (PMMA)

Fig. 1: Overview of the plastics processing processes with temperature requirements and assessment of the solar heat integration potential

The processing temperature for plasticising of thermoplastics (TP), with $85 \%$ of the total processed amount, the central process of injection moulding is above $200{ }^{\circ} \mathrm{C}$. This temperature range proves to be unsuitable. Furthermore, about $15 \%$ of the total processing amount accounts for the group of elastomers (E) and thermosets (TS). Their processing needs significantly lower temperatures between $70{ }^{\circ} \mathrm{C}$ and $150{ }^{\circ} \mathrm{C}$. At a generally suitable temperature level, the integration potential for solar process heat is classified as conditionally possible due to predominantly electrical heating. In addition to shortterm heating requirements in mould temperature control (Curing of TP), the granule drying of thermoplastics is most promising as a pretreatment step with drying temperatures for $89 \%$ of the whole plastic amount below $100{ }^{\circ} \mathrm{C}$ and a relative constant heat demand. The latter is especially true for companies with central dryer units. The most widely used plastics are polyamide (PA), polypropylene (PP) and polycarbonate (PC) with characteristic drying temperatures of 80, 90 and 120 ${ }^{\circ} \mathrm{C}$. PP must be dried only for hygroscopic reasons. PE has no drying requirement.

\section{METHODS}

As drying processes in the plastics industry predominantly need temperatures that are suitable for the use of solar process heat, a drying process is analysed experimentally to find the most promising integration point and to assess the solar potential. 


\section{Experimental investigation of integration points}

A customary granule dryer consists of an air dryer for dehumidification, a fan and an electrical auxiliary heater, 90 as seen in Fig. 2. The object of the investigation is a decentralized granule dryer of the type Thermolift 100-2 from Arburg. In addition to the (dry) fresh-air operation, there is still the recirculation mode. During recirculation mode the dryer operates at high conveying air temperatures, which reduces the solar efficiency significantly. For the dry fresh-air mode, the suction air (1) is first dehumidified (2). In the case of hygroscopic plastics, this is switched on so that the fresh air is predried by means of a desiccant dehumidifier (DH). Many granule dryers already have a cross-flow heat exchanger (HEX) for the integration of external heat sources $(4,5)$, such as solar thermal. The transport of the air is ensured by a fan. The compression of the air results in a temperature increase (3-6). A subsequent electric heater sets the desired target temperature (7) or acts as a redundant heater.

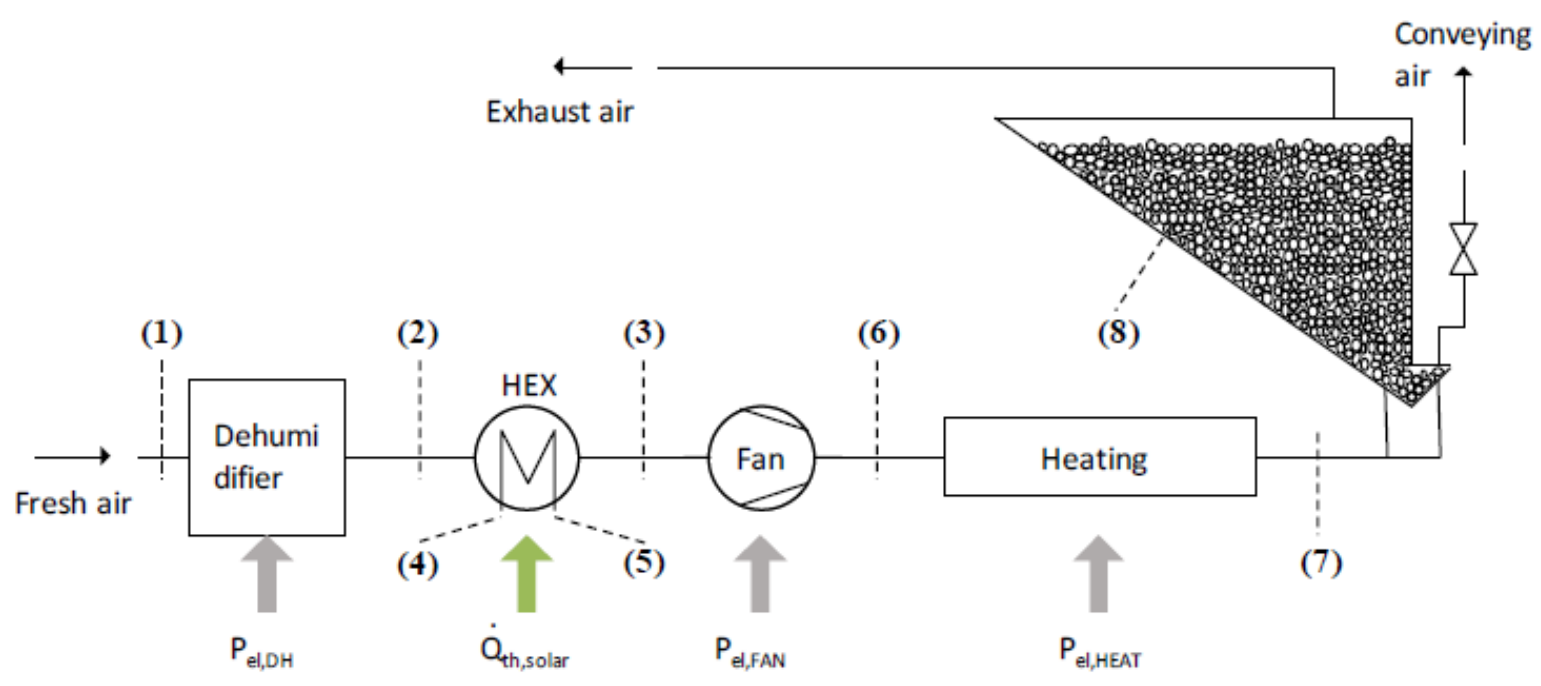

Fig. 2: Schematic structure of a granule dryer

As stated above, many granule dryers already have a heat exchanger for the integration of external heat sources. During on a laboratory scale test by integrating the solar HEX before (3) and after the fan (6) and for different temperatures, suitable integration concepts for the different types of collectors are identified and compared regarding their solar fraction and energy savings.

For this purpose, two test series are carried out. The first test examines the effect of HEX integration before and after the fan for PA. Apart from that, the electric energy savings for drying PA, PP and PC are measured. Regarding Fig. 1, eleven thermoplastic materials have a majority share of about $89 \%$ of the total German processing volume and furthermore, are in a drying temperature range between $60{ }^{\circ} \mathrm{C}$ and $100{ }^{\circ} \mathrm{C}$, which results on average a weighted drying temperature of about $82{ }^{\circ} \mathrm{C}$ for these materials. This temperature defines the requirement for the process air temperature to be achieved in that test series.

In the context of the second experiment, the solar integration temperature (5) for constant mass flow was increased over the experimental time in $10 \mathrm{~K}$ steps. For technical implementation, a water-air HEX is used in the laboratory tests. The primary-side temperature level of the solar process heat is adjusted and simulated by means of a temperature control device. The degree of heat integration and the collector efficiency depending on the integration temperature defines technical collector 
integration setups for PA, PP and PC. A solar annual simulation study for a typical plastic processor with a processed quantity of in each case $10 \mathrm{t} / \mathrm{d}$ of PA, PP and PC shows possible integration concepts.

\section{Empirical Assessment of the solar heat potential}

Based on the experimental investigations, the national potential is empirically quantified. The study has two objectives: First, similarities regarding the property structure, used material and company size should be analysed. Second, suitable companies for utilising solar process heat should be figured out and the total potential should be estimated. Therefore, different variables describing all Germany-wide injection moulding companies are chosen. In addition, the results of the calculated energy indices for granule drying from Table 1 are included in the investigation.

The data basis for this study forms a data set of the English market research company AMI (2016) of about 2,349 enterprises of the German injection moulding industry. The companies are described based on numerical and binary characteristics from the following six categories: processed polymers, supplied industries, services, machine park, production type and further data. The assigned numerical and binary characteristics can be seen in Fig. 3 .

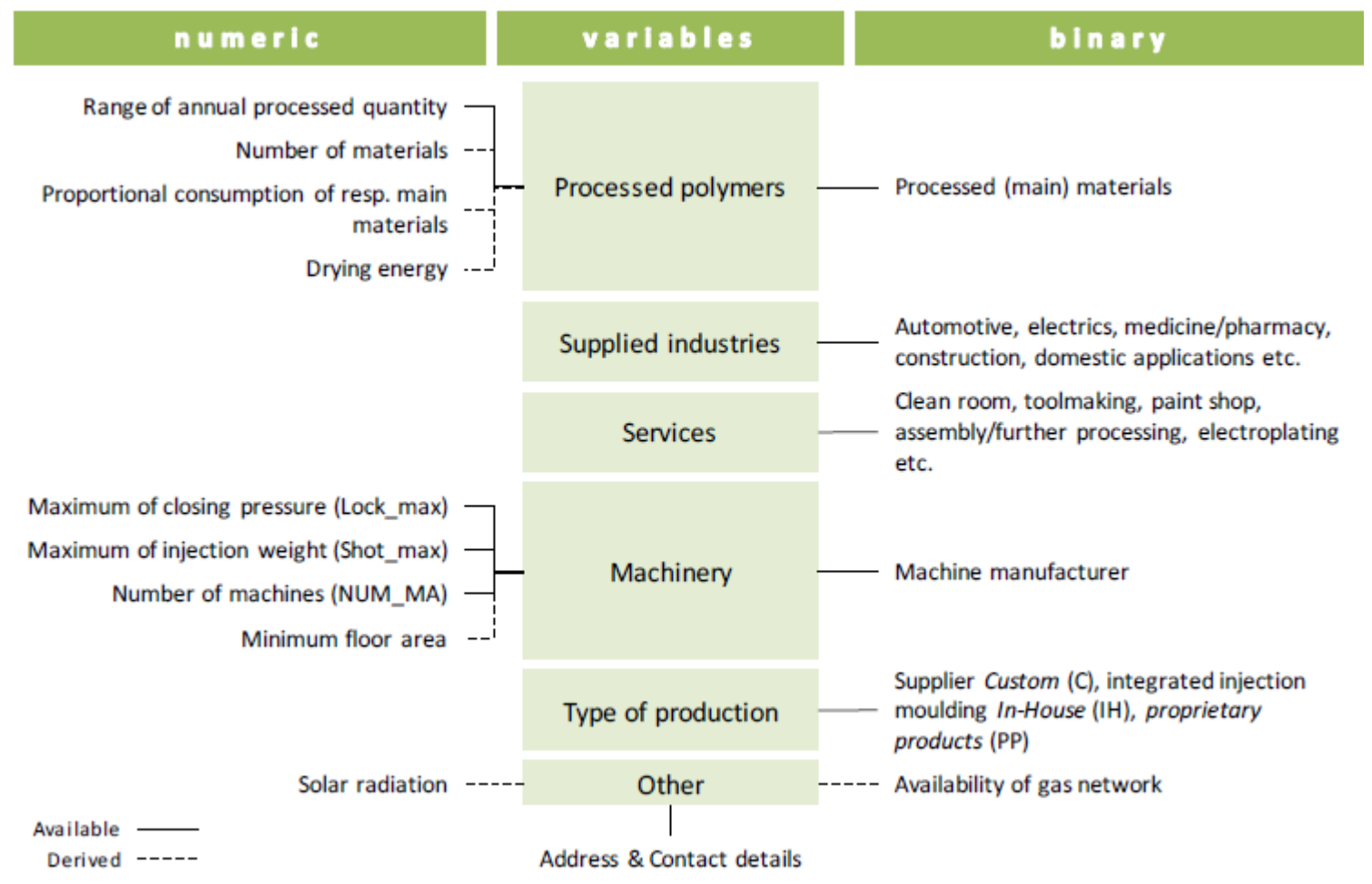

Fig. 3: Numeric and binary characteristics of the six main categories

In addition to a required temperature level and a constant load profile, further process-independent framework conditions favour solar process heat integration: The basic requirement for the implementation of solar thermal systems is a sufficient available surface area and the amount of annual irradiation on the inclined surface. Ideally, the modules should be shading-free with an orientation between south-east and south-west and, depending on the intended use, with an inclination angle in the range of $20^{\circ}$ to $60^{\circ}$ to the horizontal (Kaltschmitt et al., 2014). The focus of this study is therefore on processed polymers, which defines the amount and occurrence of the drying energy demand as well as 
their temperature requirements. Furthermore, the machinery variables (number, floor area) represent the available collector surface area.

Before conducting the numeric-binary analysis, the raw data set of 2,349 companies was first adjusted from missing values and from distorting companies to 1,377 companies. Based on this dataset, companies are identified due to positive characteristic values for solar process heat. Apart from that, the German injection moulders are classified by their property structures.

\section{RESULTS}

\section{Integration concepts}

In the following, the evaluations for tests with PA are described. PA is a hygroscopic plastic, which is often used for technical parts. Further plastics and their effects on the solar fraction and collector temperatures will be discussed below, in the context of a case study. Since a low integration temperature increases the solar efficiency, a (dry) fresh-air operation with lower supply air temperatures is more suitable than for recirculation mode. For reasons of more constant load profiles and the lower technical equipment effort, an integration point at central drying plants is to be preferred. In principle, the solar load can be integrated by means of HEX before or after the fan. Fig. 4 (a) shows the course of the air temperatures for heat exchanging after the fan. The air temperature entering the heat exchanger or leaving the fan is about $52{ }^{\circ} \mathrm{C}$. The temperature increase in the heat exchanger is approx. $24 \mathrm{~K}$.

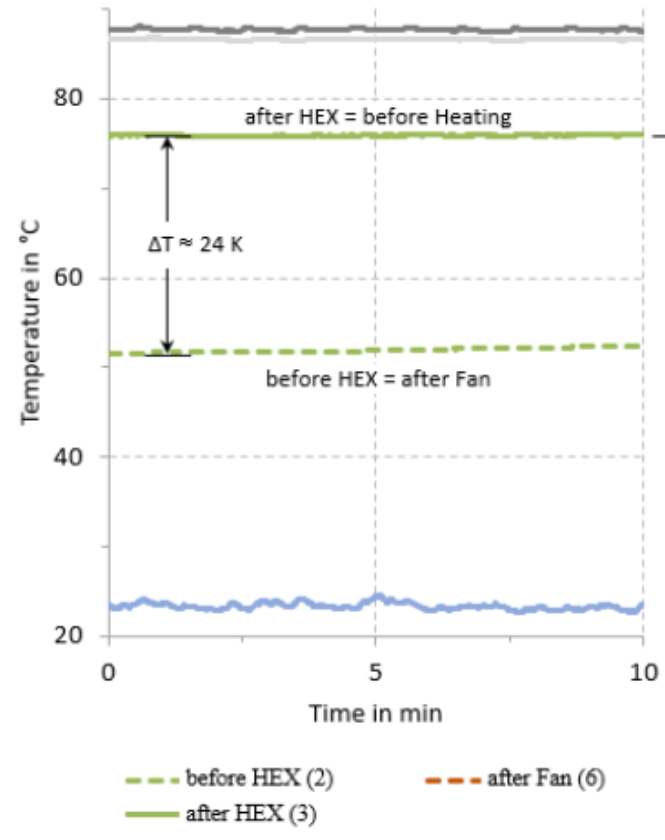

(a) Heat exchanger after fan

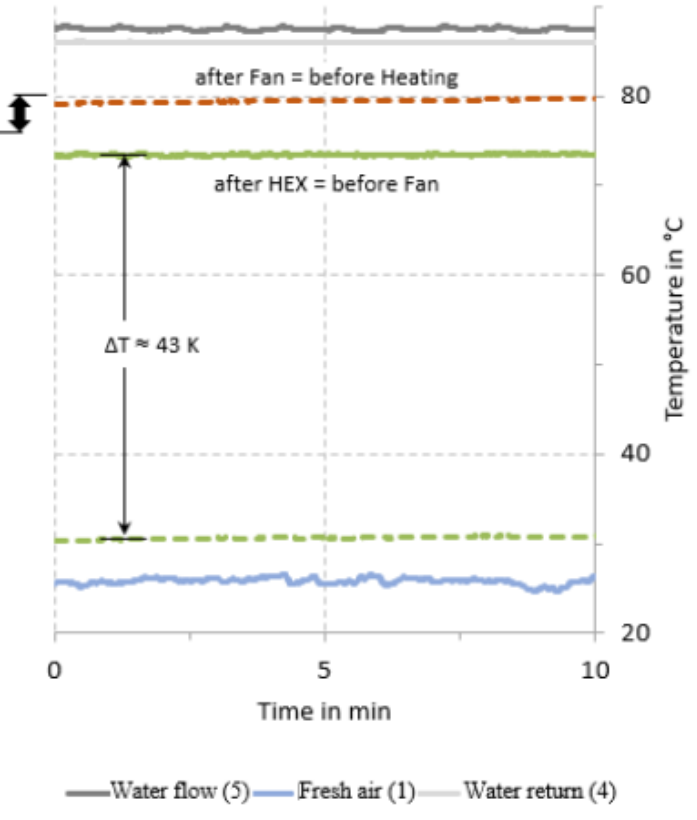

(b) Heat exchanger before fan

Fig. 4: Identification of the solar integration point: (a) after fan and (b) before fan

Fig. 4 (b) illustrates the scenario with the heat exchanger before the fan. Compared to Fig. 4 (a), the air inlet temperature in the heat exchanger at approx. $31{ }^{\circ} \mathrm{C}$ is significantly lower, while the medium is simultaneously heated considerably more by approx. $43 \mathrm{~K}$. The heat input of the fan causes an 
additional air temperature increase from $74{ }^{\circ} \mathrm{C}$ to $80{ }^{\circ} \mathrm{C}$ after the fan. It is obvious that for almost the same initial conditions the scenario with the heat exchanger before the fan achieves improved system temperatures. The air temperatures entering the heater are increased by about $3.5 \mathrm{~K}$, and by about $7 \mathrm{~K}$ when the heater is inactive. Despite an increased electric fan demand due to the decreased density and a resulting higher volume flow, the process heat provided by solar energy integrated before the fan is used more efficiently. In view of this, the test series and integration concepts are carried out using the example of this variant.

As part of the second test series, different collector temperatures were simulated, and the temperature measured in the granule dryer at the appropriate locations. In addition to the temperatures, Fig. 5 also shows the solar heat input $Q_{t h}$. The collector temperature, starting at about $48{ }^{\circ} \mathrm{C}$ was increased by 10 $\mathrm{K}$ steps. Fig. 5 shows the correlating solar load. The peaks result by means of the not-stationary heating up. For the evaluation, only the stationary sections with constant solar integration are considered.

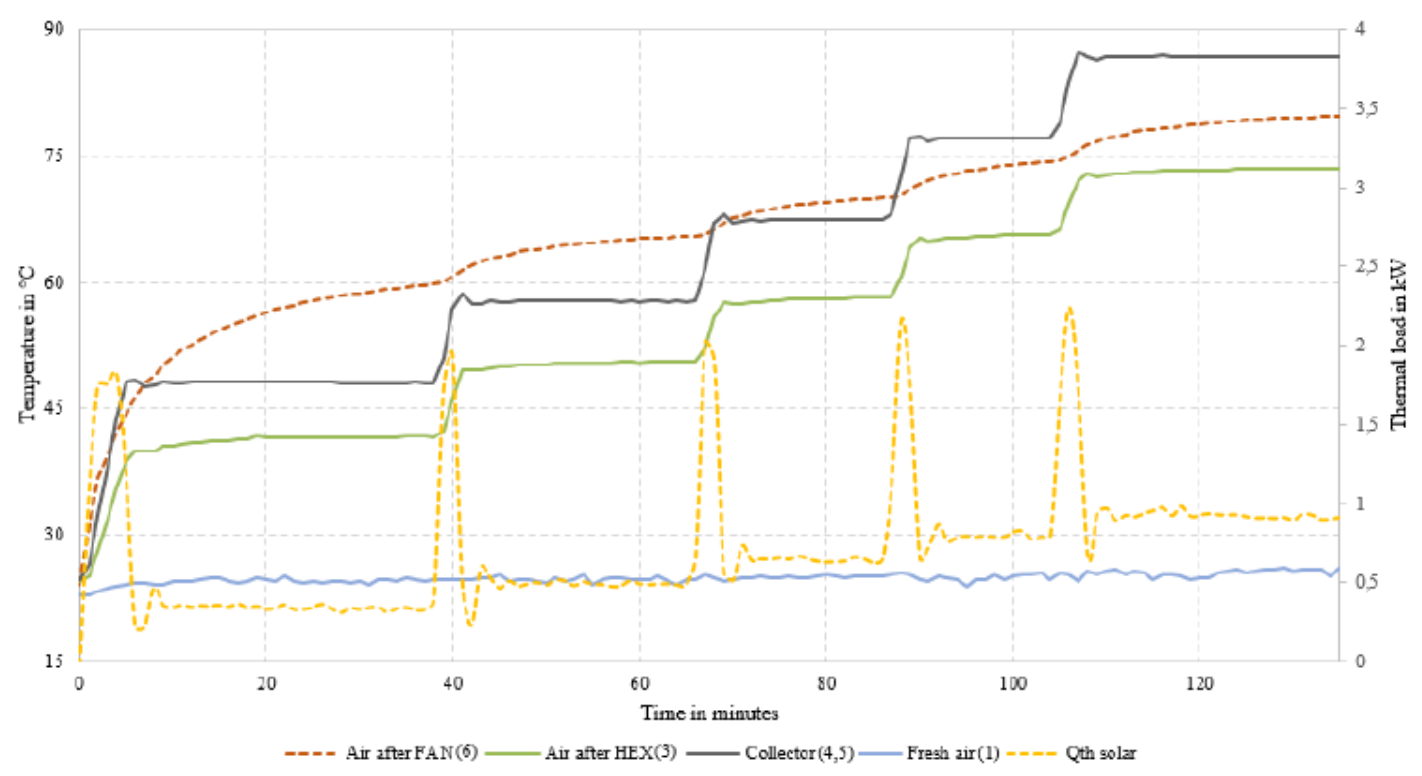

Fig. 5: Solar heat input for various collector temperature levels

As the collector temperature rises, both the usable temperature of the process air and the rate of the solar input increase $Q_{t h}$. For every $10 \mathrm{~K}$ decrease of integration temperature, the integration potential also decreases by about $15 \%$. Based on the lab-scale heat integration before the fan, the following primary energy savings can be achieved by (partial) substitution of the electrical heating (Heat demand) depending on the drying temperature level. Furthermore, the granule dryer has a high remaining electric demand for the $\mathrm{DH}$, the fan and the solar pump (SP). 


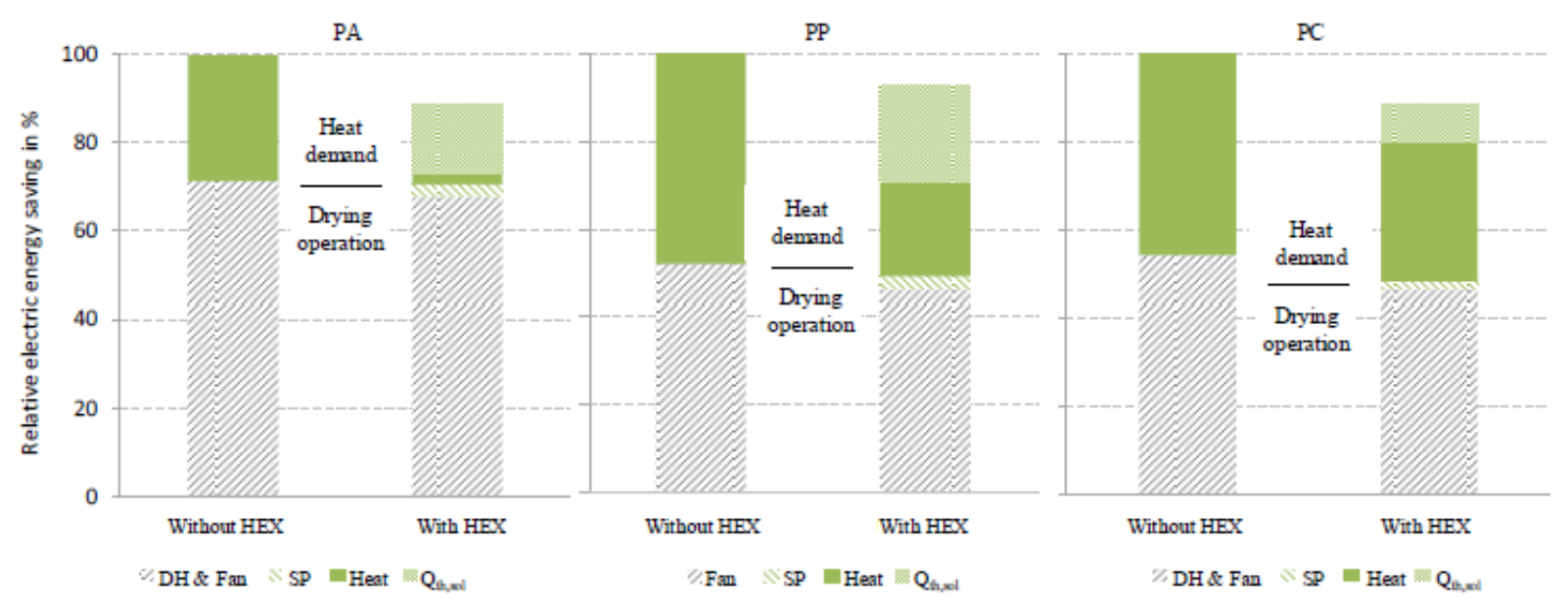

Fig. 6: Relative electric energy demand with(out) solar HEX resulting from test series with PA, PP and $\mathrm{PC}$ and a drying temperature of $82^{\circ} \mathrm{C}$

For three main plastics PA, PP and PC at an average drying temperature of $82{ }^{\circ} \mathrm{C}$, there are thermal energy savings (green share) regarding the electrical heat demand over $90 \%$ for PA, approx. $55 \%$ for PP and $32 \%$ for PC. Further electric demand for the dehumidifier and fan is remaining.

Based on the described test evaluation, it becomes clear that the drying energy demand varies and is material-specific. Regardless of the drying method or the drying operation the following factors influence the drying energy demand for granule drying: Ambient conditions (temperature, humidity), characteristics of the plastic material (bulk density, water content, hygroscopic properties) as well as target drying temperature and duration. According to Schroer and Wortberg (2002), the specific thermal energy demand qтот for granule drying according to eq. 1 is equal to the sum of the enthalpies for material heating $q M A T$ and the evaporation energy qEVA of the water content to be removed. Here, the energy required to heat the amount of water is neglected. Fundamentally, the thermal drying energy demand is of interest. Other consumers and peripherals, which require additional energy demand are not included in the calculations as they vary depending on the system. This ensures a system-independent and comparable energy ratio.

$q T O T=q M A T+q E V A=c \cdot \Delta T+\Delta \varphi \cdot \Delta H v$

Table 1 lists the specific energy indices of the main granule drying materials in the form of the thermal drying energy requirement per ton of plastic granule. While the specific energy values for their drying target temperatures for PP, PA, PC and PE are based on practical experiments by Veitengruber (2018) and Wolff (2015), the calculation of the values for PVC, PS, ABS / SAN bases on references according to Beitl (2007) and Johannaber and Michaeli (2004). 
Table 1: Specific energy ratios of main materials for granule drying: Thermal drying energy per ton of plastic granules.

\begin{tabular}{|c|c|c|c|c|c|c|c|c|}
\hline & \multirow[b]{2}{*}{ Unit } & \multicolumn{3}{|c|}{ Veitengruber (2018) } & \multirow{2}{*}{$\begin{array}{l}\text { Wolff } \\
\text { (2015) } \\
\text { PE* }\end{array}$} & \multicolumn{3}{|c|}{ Determined theoretically } \\
\hline & & PP* & $\mathrm{PA}$ & $\mathrm{PC}$ & & PVC & PS & $\mathrm{ABS} / \mathrm{SAN}$ \\
\hline \multicolumn{9}{|c|}{ Drying temperature: } \\
\hline$\underline{T}$ & ${ }^{\circ} \mathrm{C}$ & 90 & 80 & 120 & 90 & 70 & 80 & 80 \\
\hline \multicolumn{9}{|c|}{ Specific drying energy demand: } \\
\hline qTOT & $\mathrm{kWh} / \mathrm{t}$ & 33,2 & 30,1 & 31,6 & 46,6 & 12,6 & 22,3 & 23,0 \\
\hline
\end{tabular}

As a case study, one typical Top 10 (Fig. 8) injection moulder dries $10 \mathrm{t} / \mathrm{d}$ of PA, PP and PC in a oneshift operation. Regarding the degree of solar heat integration (Fig. 5) for different integration temperatures, the partial substitution of electric heating by solar heat (green share in Fig. 6) and the specific drying energy demand (eq. 1, Table 1) solar annual simulations define the following solar fraction rates and specific solar yields of the two collector types flat plate collector (FPC) and vacuum tube collector (VTC) for the given summerlike daily process heat demand and the plastics PA, PP and PC (Fig. 7). Solar fraction refers to the share of solar useful heat output compared to the process heat demand and is thus a measure of the substitution of conventional energy sources, in this case electricity. By contrast, the solar yield evaluates the efficiency of the integration of solar process heat in relation to the collector surface. The consideration of both key indicators results in a compromise between economic and ecological assessment criteria. Due to increasing drying temperatures (Table 1) and relating heat losses as well as decreasing proportion of solar integration (Fig. 6) in the order of PA, PP and PC, both the overall level of solar fraction and the solar yield decrease in that order. Because the higher efficiency of vacuum tube collectors, the solar fraction is always above the flat plate collectors. This effect regarding to the solar yield comes only from $60{ }^{\circ} \mathrm{C}$ into effect. The most appropriate integration temperatures are for PA between 70 and $80^{\circ} \mathrm{C}$ and for 
PP between 80 and $90{ }^{\circ} \mathrm{C}$. Due to the high drying temperatures, the low solar fraction and the low solar yield, only limited reasonable integration concepts are available for PC drying.

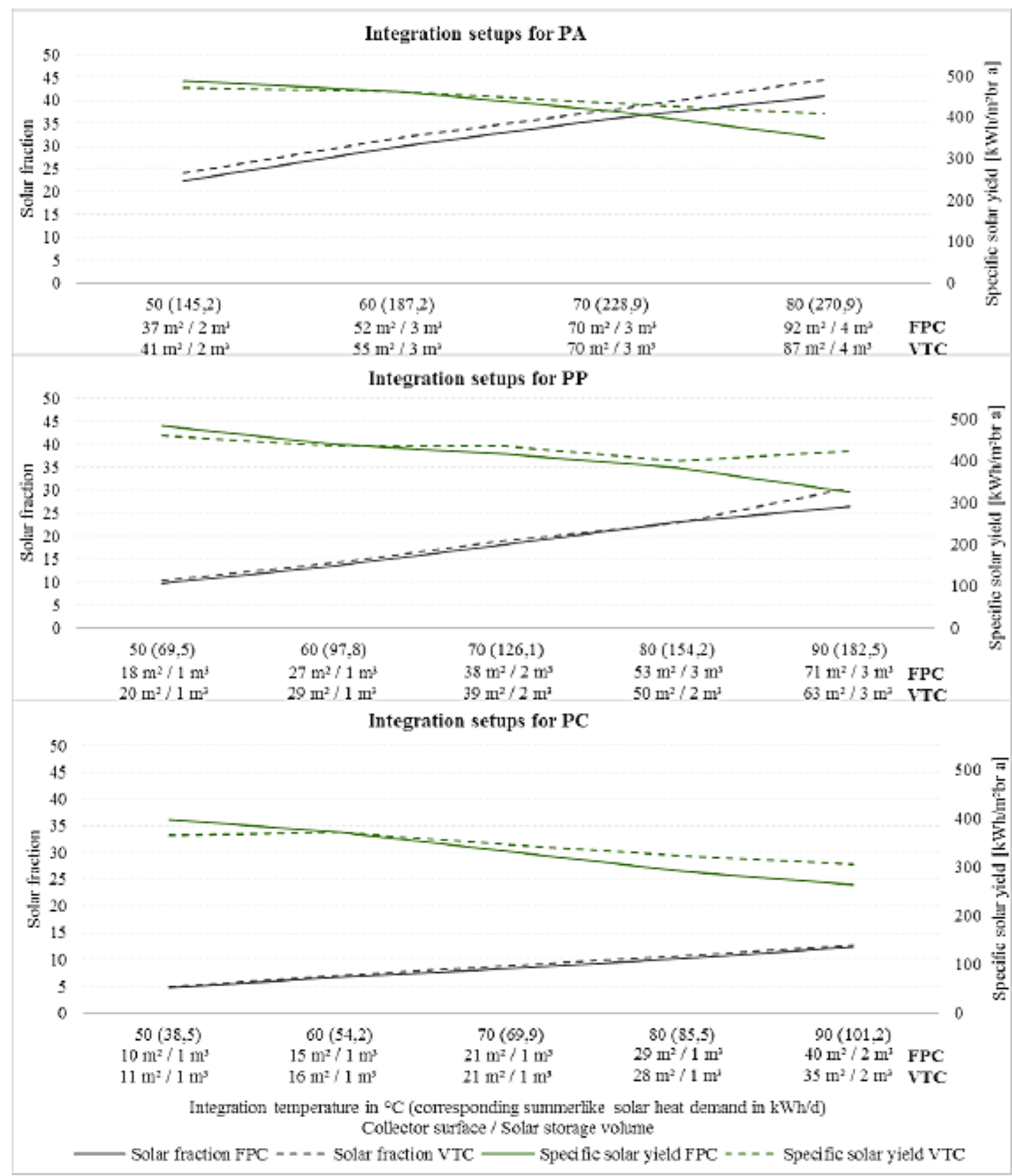

Fig. 7: Assessment of integration setups for meeting the summerlike process heat demand of PA, PP. PC drvina

\section{Property structures of German Injection Moulders}

Overall, for all 2,349 injection moulding companies in Germany, there is a total theoretical drying energy demand for plastics with drying temperatures of up to $90{ }^{\circ} \mathrm{C}$ of approx. $15.7 \mathrm{GWh} / \mathrm{a}$. Using 
numerical as well as binary analysis, this results in two general types of plastic companies. They can typically be understood as either all-230 round processors or specialized processors. The latter are characterized by a higher drying demand and a more constant load profile since they process less different materials and therefore have a higher processing volume and fewer mould changes. A high drying demand below $90{ }^{\circ} \mathrm{C}$ and a large resulting roof area are positive characteristic values for the integration of solar process heat. In total, 128 or approx. $10 \%$ of the 1,377 injection moulding companies with a drying demand of $1.8 \mathrm{GWh} / \mathrm{a}$ show a significant potential for the integration of solar 235 process heat.

The respective locations with evidenced potential can be found in the map of Germany in Fig. 8 (a). It is noticeable that there are three geographic agglomeration clusters with plastic processors, especially for the conurbations in Baden-Württemberg and North Rhine-Westphalia, as well as the Nuremberg region, to which integration potential is attributed.

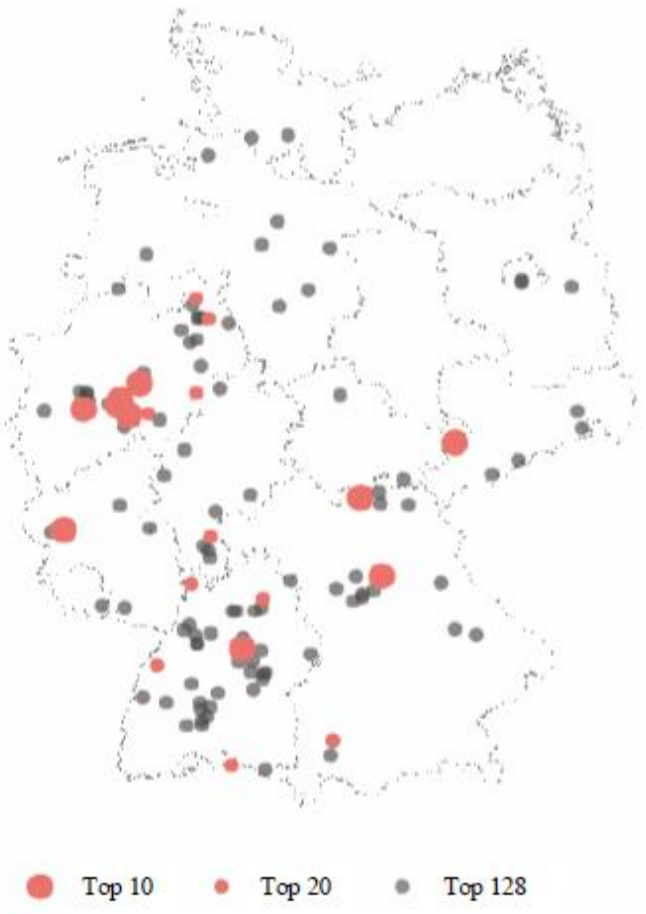

(a) Proposed potential

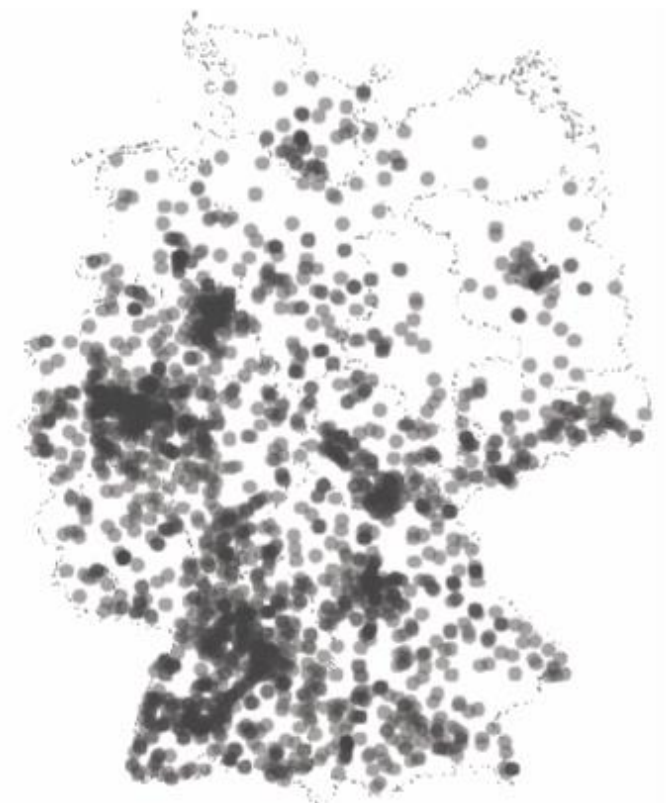

(b) All 2349 Processors

Fig. 8: Locations of the German Injection Moulders (BKG, 2011)

\section{CONCLUSION}

Despite the evident potential deficit, promising niche processes for the integration of solar process heat, such as granule drying, could be identified based on this study, not least because of $89 \%$ of the thermoplastics are in a 245 drying range of $60-100{ }^{\circ} \mathrm{C}$. The experimental investigations showed that the integration point before the blower of a drying plant is the energetically more favorable variant. The simulation of different integration concepts shows suitable integrations for the plastics PA (60 $\left.70{ }^{\circ} \mathrm{C}\right)$ and $\mathrm{PP}\left(70-80{ }^{\circ} \mathrm{C}\right)$ due to their lower drying temperature and the higher share of substitution of conventional energy sources. Overall, the study identifies 128 companies with a thermal drying energy demand of $1.8 \mathrm{GWh} / \mathrm{a}$ for materials with drying temperatures up to $25090{ }^{\circ} \mathrm{C}$. Thereby, positive characteristic values for the integration of solar process heat are a high drying demand below 
$90{ }^{\circ} \mathrm{C}$ and a large resulting roof area. Apart from that, the findings of the study can be transferred to the integration of other low-temperature heat sources such as heat pumps or CHPs.

\section{ACKNOWLEDGMENTS}

The contents of this paper have been acquired within the cooperation project „Smart Consumer Energy efficiency through systemic coupling of energy flows by means of intelligent measurement and control technology". The Project is funded by the German Federal Ministry for Economic Affairs and Energy (FKZ: 03ET1180).

This research has been supported by the project "SolarAutomotive - Solar process heat for the automotive and supplier industry". This project (FKZ: 0325863A) is funded by Federal Ministry for Economic Affairs and 260 Energy on the basis of a decision by the German Bundestag.

\section{REFERENCES}

Applied Market Information Ltd., (2016), Injection moulders in Germany - AMI's directory <ami.international/pubs/prod.aspx?catalog=Publishing\&product=p341 accessed 25.10.2017.

Beitl, F., (2007), 1000 Tipps zum Spritzgießen, Band 1: Maschineneinstellung und Prozessparameter, 2., überarb. Auflage, Hüthig, Heidelberg.

BKG, (2011), Verwaltungsgrenzen von Deutschland <arcgis.com/home/item.html?id=ae25571c60d94ce5b7fcbf74e27c00e0 accessed 04.07.2018.

Bundesministerium für Wirtschaft und Energie (BMWi), (2017), Energiedaten: Gesamtausgabe $<$ bmwi.de/Redaktion/DE/Downloads/Energiedaten/energiedaten-gesamt-pdf-grafiken.pdf accessed 07.01.2018.

Destatis, (2016), Produzierendes Gewerbe, Beschäftigung und Umsatz der Betriebe des Verarbeitenden Gewerbes sowie des Bergbaus und der Gewinnung von Steinen und Erden <de.statista.com/statistik/daten/studie/200623/umfrage/umsatz-der-deutschen-kunststoffindustrienach-275 branchensektoren/ accessed 19.03.2018.

Dispan, J., Vassiliadis, M., (2014), Kunststoffverarbeitung in Deutschland, Eine Branchenanalyse, Hannover <igbce.de/vanity/renderDownloadLink/8222/71002 accessed 23.03.2018.

euRECIPE, (2006), Low Energy Plastics Processing, European Best Practice Guide.

Görlitz, P., (2017), The Plastics Industry in Germany, Industry Overview, Berlin 280 <gtai.de/GTAI/Content/EN/Invest/_SharedDocs/Downloads/GTAI/Industry-overviews/industryoverview-plastics-industry-in-germany-en.pdf accessed 23.03.2018.

Johannaber, F., Michaeli, W., (2004), Handbuch Spritzgießen, 2. Auflage, Carl Hanser Verlag, München.

Kaltschmitt, M., Streicher, W., Wiese, A. (Eds.), (2014), Erneuerbare Energien, Systemtechnik, Wirtschaftlichkeit, Umweltaspekte, 5., erweiterte Auflage, korrigierter Nachdruck, Springer Vieweg, Berlin.

KEMA, (2012), Industrial Sectors Market Characterization, Plastics Industry, Oakland, California.

Lauterbach, C., Schmitt, B., Jordan, U., Vajen, K., (2012), The potential of solar heat for industrial processes in Germany, Renewable and Sustainable Energy Reviews 16, 5121-5130. 
Lauterbach, C., Schmitt, B., Vajen, K., (2011), Feasibility Assessment of Solar Process Heat Applications, 1-10.

Lauterbach, C., Schmitt, B., Vajen, K., Jordan, U., (2010), Potential for Solar Process Heat in Germany - Suitable Industrial Sectors and Processes, in: Chwieduk, D., Streicher, W., Weiss, W. (Eds.), Proceedings of the EuroSun 2010 Conference, International Solar Energy Society, Freiburg, Germany, pp. 1-8.

Matarrese, P., Fontana, A., Sorlini, M., Diviani, L., Specht, I., Maggi, A., (2017), Estimating energy consumption of injection moulding for environmental-driven mould design, Journal of Cleaner Production 168, 295 1505-1512.

Meschede, H., Holzapfel, P., Kadelbach, F., Hesselbach, J., (2016), Classification of global island regarding the opportunity of using RES, Applied Energy 175, 251-258.

Mianehrow, H., Abbasian, A., (2017), Energy monitoring of plastic injection molding process running with hydraulic injection molding machines, Journal of Cleaner Production 148, 804-810.

Müller, T., Weiß, W., Schnitzer, H., Brunner, C., Begander, U., Themel, O., (2004), Produzieren mit Sonnenenergie, Potenzialstudie zur thermischen Solarenergienutzung in österreichischen Gewerbeund Industriebetrieben, Berichte aus Energie- und Umweltforschung, 01/2004, Gleisdorf (AT).

Schmitt, B., (2016), Classification of Industrial Heat Consumers for Integration of Solar Heat, Energy Procedia 91, 650-660.

Schmitt, B., Lauterbach, C., Ritter, D., Meyers, S., Vajen, K., (2015a), Fast Feasibility Assessment for Solar Thermal Systems in Industry, in: Frank, E., Papillon, P. (Eds.), Proceedings of the EuroSun 2014 Conference, International Solar Energy Society, Freiburg, Germany, pp. 1-10.

Schmitt, B., Pag, F., Wimmer, L., Best, I., Ritter, D., Vajen, K., (2015b), SolFood, Leitfaden zur Nutzung solarer Prozesswärme in der Ernährungsindustrie, Kassel.

Schroer, T., Wortberg, J., (2002), Granulat richtig trocknen, Ein wichtiger Verfahrensschritt für hohe Produktqualität, Kunststoffe 92, 44-49.

Schumm, G., Philipp, M., Schlosser, F., Hesselbach, J., Walmsley, T.G., Atkins, M.J., (2017), Hybrid heating system for increased energy efficiency and flexible control of low temperature heat, Energy Efficiency 6, 685.

Statista, (2011), Umsatz der deutschen Kunststoffindustrie nach Sektoren im Jahr 2016 (in Millionen Euro) $\quad 315<$ de.statista.com/statistik/daten/studie/200623/umfrage/umsatz-der-deutschenkunststoffindustrie-nach-branchensektoren/ accessed 19.03.2018.

Veitengruber, F.P., (2018), Potenzialstudie zur Implementierung solarer Prozesswärme in branchentypische Verfahrensschritte der deutschen Kunststoffindustrie, Masterthesis.

Weiß, P., (2016), Energieeffizienz und Abwärmenutzung in der Kunststoffverarbeitung, Dissertation.

Wolff, A., (2015), Energetische Analyse eines Kunststoff-Granulattrockners, Bachelorthesis.

Zhu, X., Liu, Y., He, M., Luo, D., Wu, Y., (2018), Entrepreneurship and industrial clusters, Evidence from China industrial census, Small Bus Econ 41, 757. 


\section{SOLID OXIDE FUEL CELL COMBINED COOLING HEAT AND POWER USING RENEWABLE FUELS FOR A SUSTAINABLE AND HIGHLY EFFICIENT ENERGY SUPPLY}

$\underline{\text { Michael Seidl, Martin Hauth }}$

AVL List GmbH

Hans-List-Platz 1, 8020 Graz, Austria

Phone: +43316/787 5585

E-Mail: michael.seidl@avl.com

\section{SUMMARY}

The coupling of AVLs $6 \mathrm{~kW}_{\mathrm{el}}$ SOFC CHP system using an IKTS stack module with a $5 \mathrm{~kW}_{\text {cold }}$ absorption chiller developed by TU Graz will be presented. The exhaust gas heat from the SOFC which is available at $>200{ }^{\circ} \mathrm{C}$ will be used to operate the thermally driven heat pump. The SOFC's fuel capability was extended towards renewable fuels such as biogas, and synthetic diesel using a novel gas cleaning concept. For buildings, a heat driven operation mode leads to low operating hours per year for conventional CHP systems during summer season. Generating cooling power in addition to heat will increase the annual operating hours per year and thus economic efficiency significantly. This analysis presents the capability of the SOFC CCHP system for a flexible and demand-oriented generation of electricity, heat and cooling power and shows how cold and cooling water temperatures influence the overall system size. This work was done within the project "SOFCool" in cooperation with Plansee, TU Graz, Institute of Thermal Engineering and Fraunhofer IKTS.

\section{INTRODUCTION}

For modern energy generators, a sustainable operation combined with a high efficiency is absolutely necessary in order to reduce or avoid problems caused by increased $\mathrm{CO}_{2}$ emissions and by shortages of fossil fuels. Within this project a combination of an SOFC (Solid Oxide Fuel Cell) with an absorption chiller (AC) was considered to achieve electrical efficiencies above $50 \%$ and total efficiencies above $90 \%$. This technology is especially attractive for instance for buildings in urban districts and hospitals where a seasonal (winter/summer) or spatial (surgery room/patient room) distribution for heating and cooling is desired. In this context, it is the aim to increase the operating hours per year of the SOFC system by using its off gas heat to create cooling power, heating power or both at a time. Example applications for an SOFC CCHP (Combined Cooling, Heating and Power) system with cooling power

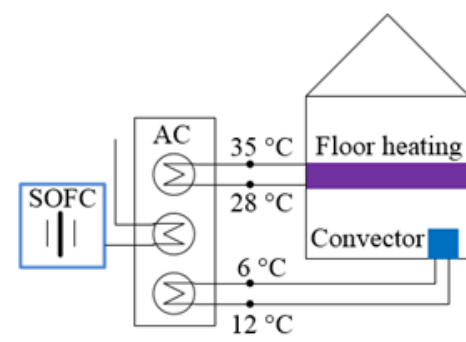

hospital

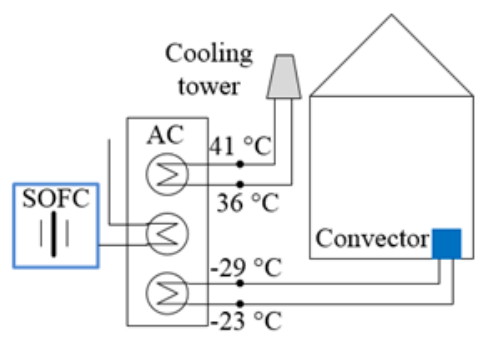

cold storage house

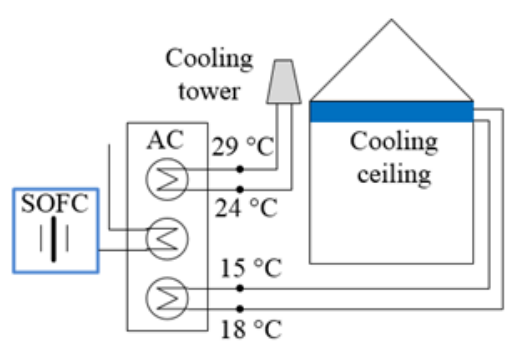

office building

Fig. 4: Example applications for an SOFC CCHP. The scenarios only differ by the temperature demand of the absorption chiller. Different applications lead to different sizes of SOFC system and absorption chiller powers. The SOFC exhaust gas temperature is in the range of $220-260^{\circ} \mathrm{C}$. (Hauth et al.)

consumers are given in Figure 1.

Various renewable energy sources were investigated to allow for sustainable operation. Depending on the utilized fuel which will be converted in the SOFC, different requirements for the gas cleaning unit 
had to be taken into account. Due to the planned multi fuel ability of the system an overall concept for the gas cleaning and gas processing unit had to be developed to achieve a high degree of standardization.

One of the major challenges in the operation of SOFCs with renewable fuels is the preparation of these fuels. Biogas is a versatile, renewable, widely available, and potentially sustainable energy source and besides that, it is almost carbon neutral (Aravind and Jong). Operation with biogas becomes increasingly crucial since it is a renewable and clean form of energy which is able to replace the conventional sources of energy, e.g. fossil fuels (Andriani et al.).

Furthermore, the absorption chiller had to be designed specifically to match the temperature level of the SOFC exhaust gas, which is utilized in the absorption chillers generator. Due to the size of the SOFC with $6 \mathrm{~kW}_{\mathrm{el}}$ and its resulting exhaust gas heat, it was aimed at an absorption chiller with low cooling power to allow realistic testing when both systems are coupled. The design of an absorption heat pump requires a minimum size which was determined to be about $5 \mathrm{~kW}_{\text {cold. }}$. However, this would require an SOFC with at least $19 \mathrm{~kW}_{\mathrm{el}}$. Therefore, while testing the system the missing SOFC exhaust gas heat was generated by an extra gas heat source to simulate the exhaust gas heating power of the real scale SOFC. Figure 2 shows the schematic energy flow diagram of the SOFC CCHP system in which the SOFC's off gas heat is transferred and used within the subsequently positioned absorption

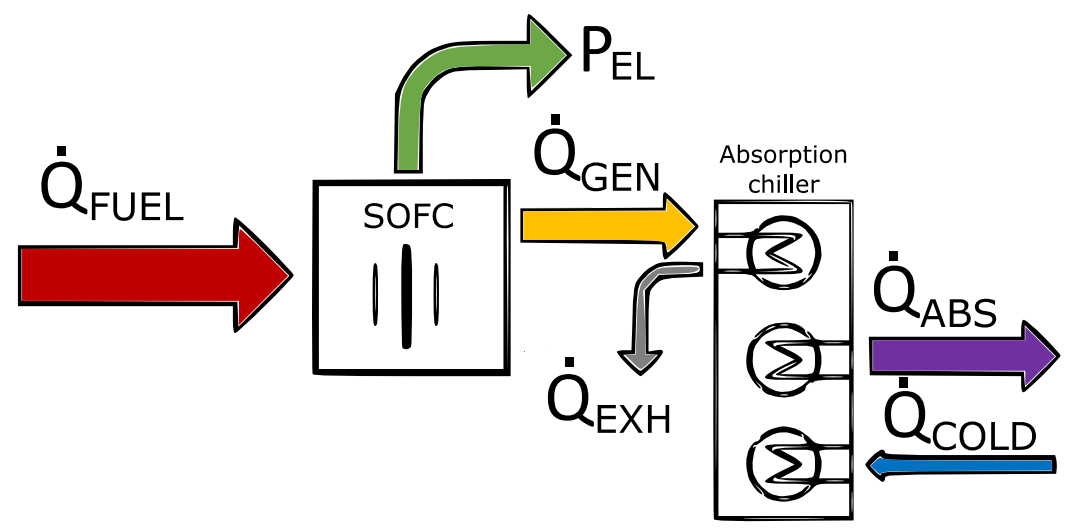

Fig. 5: Energy flow diagram of the SOFC CCHP system. In the SOFC system, fuel power is converted into electric energy $(>50 \%)$ and heat, which is lead into the absorption chiller's generator heat exchanger. There it powers the absorption process that creates cooling power. (Hauth et al.)

chiller.

An optimization of the absorption process towards the exhaust gas temperature allows very high coefficients of performance. Moreover, the required cold and cooling water temperatures determine the type of the refrigerant as well as the cooling process itself. Another challenging aspect is the handling of transient operations of a combined system with two different thermal inertias. This requires special attention when it comes to load following operation.

The target was to run a fully functioning SOFC CCHP experimental plant to investigate the interaction between the SOFC system and the absorption chiller. First, the SOFC system and the absorption chiller were built and tested separately. Afterwards the two sub-systems were integrated to one system for complete system tests. This work reports final test results of the complete SOFC CCHP system carried out at AVL. 


\section{EXPERIMENTAL SETUP}

The SOFC sub system of the CCHP is based on AVL's SOFC CHP system powered by natural gas. The natural gas is converted by steam reforming to a $\mathrm{H}_{2}$ rich reformate gas to operate the SOFC. The switch from a gaseous to a liquid fuel requires several modifications regarding the fuel processing such as the steam reforming process parameters, design of the start-up burner and the implementation of a diesel evaporator to feed the system with vaporized diesel. Therefore, the key balance of plant components of the SOFC system were further developed towards diesel operation. The aim was to develop a multi-fuel system which can use liquid or gaseous fuel without the need for system modifications or the need to stop the system when changing fuel (also known as dual fuel capability). Hydrotreated vegetable oil (HVO) was taken as the fuel to represent synthetic or renewable diesel, respectively for the performance analysis. After testing both sub-systems individually, the fully automated absorption chiller and the appendant thermal management unit developed by Albert (Albert and Rieberer) were shipped to AVL and integrated into the CCHP system. For combined stationary operation, the absorption chillers cooling- and cold water temperatures were set to $36{ }^{\circ} \mathrm{C}$ and $12{ }^{\circ} \mathrm{C}$, which could be used e.g. in a typical hospital application. For a given electrical power and efficiency of the SOFC system a certain exhaust gas stream will be generated. The challenge is to convert as much exhaust gas heat as possible into cooling power in the absorption heat pumping process and minimize the exhaust gas power after the absorption heat pump. Figure 3 shows photographs of the CCHP plant as it was set up in the fuel cell test container at AVL.

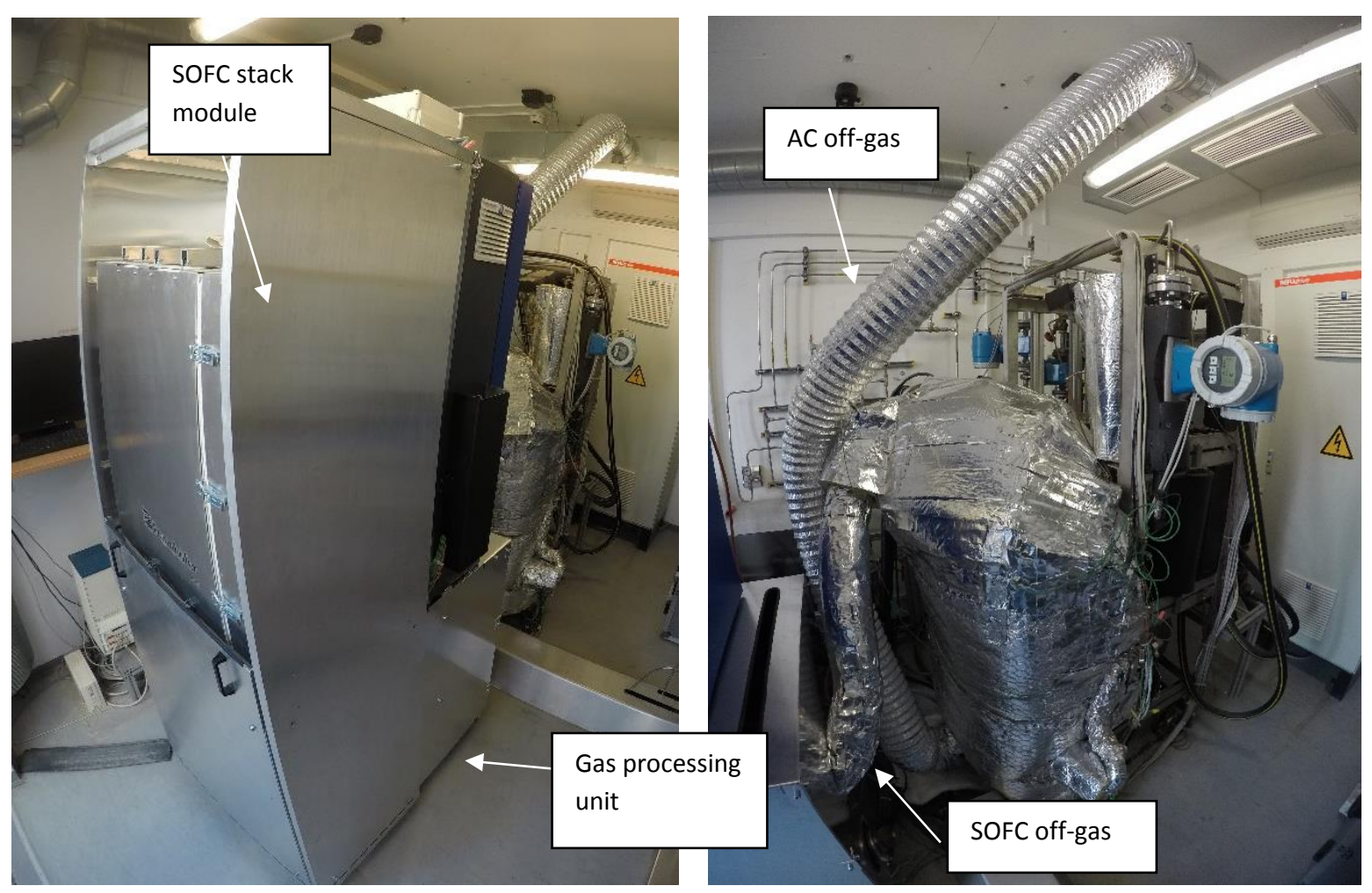

Fig. 3: SOFC CCHP system prototype including an IKTS stack module. The picture on the left side shows the front of the system with the connected absorption chiller in the background. The right picture shows the absorption chiller from TU Graz as it is connected to the SOFC system off-gas. The prototype system was tested within an AVL fuel cell test container. 


\section{RESULTS}

The SOFC sub-system was simulated with different fuels including anode gas recirculation to see the influence of the fuel type on the system performance and the off gas temperature. Key simulation results can be seen in Table 1. The anode gas recirculation ratio was determined in order to avoid carbon deposition at the reforming catalyst considering the targeted reforming temperature of 450 $550{ }^{\circ} \mathrm{C}$. The anode inlet composition was provided to IKTS to determine the stack module performance. Tests of the stack module on the test bench at IKTS showed stack module DC power outputs of $\sim 6.3 \mathrm{~kW}$ for diesel reformate. This performance value was implemented into the SOFC process simulation by calibrating the value for the area specific resistance (ASR). The ASR is used to model the fuel cell performance in 1D process simulations. Therefore, the system DC electrical efficiency was determined to be $>60-62 \%$. The ideal exhaust gas temperature of the SOFC system which is further used in the absorption chiller without considering heat losses was calculated to be 250 $-300^{\circ} \mathrm{C}$

Table 1: Comparison of SOFC system performance for different fuels. $\mathrm{P}_{\mathrm{DC}, \text { gross }}$ is the system's gross DC power, eta $a_{\mathrm{DC}, \text { gross }}$ the electric efficiency, $\mathrm{t}_{\mathrm{EG}, \mathrm{SOFC}}$ the off gas temperature and $\mathrm{U}_{\mathrm{f}, \mathrm{system}}$ is the SOFC system's fuel utilizsation.

\begin{tabular}{lllll}
\hline & Unit & Natural gas & Biogas & Diesel \\
\hline$P_{\mathrm{DC}, \text { gross }}$ & {$[\mathrm{W}]$} & 6334 & 6209 & 6248 \\
eta $_{\mathrm{DC}, \text { gross }}$ & {$[\%]$} & 64.8 & 63.7 & 62.9 \\
$t_{\mathrm{EG}, \mathrm{SOFC}}$ & {$\left[{ }^{\circ} \mathrm{C}\right]$} & 256 & 267 & 300 \\
$\mathrm{i}$ & {$\left[\mathrm{mA} / \mathrm{cm}^{2}\right]$} & 278 & 277 & 277 \\
$\mathrm{U}_{\mathrm{f}, \text { system }}$ & {$[\%]$} & 90 & 90 & 90 \\
\hline
\end{tabular}

In experiments, $6.9 \mathrm{~kW}_{\mathrm{el}}$ full load operation with natural gas and a gross electric efficiency of $60 \%$ lead to an SOFC off-gas temperature of around $220^{\circ} \mathrm{C}$, which was used to operate the absorption chiller. Figure 4 shows key performance indicators and the off-gas temperature of a natural gas full load operating point. A respective operating point of the absorption chiller with $220{ }^{\circ} \mathrm{C}$ generator inlet temperature can be seen in Figure 5.

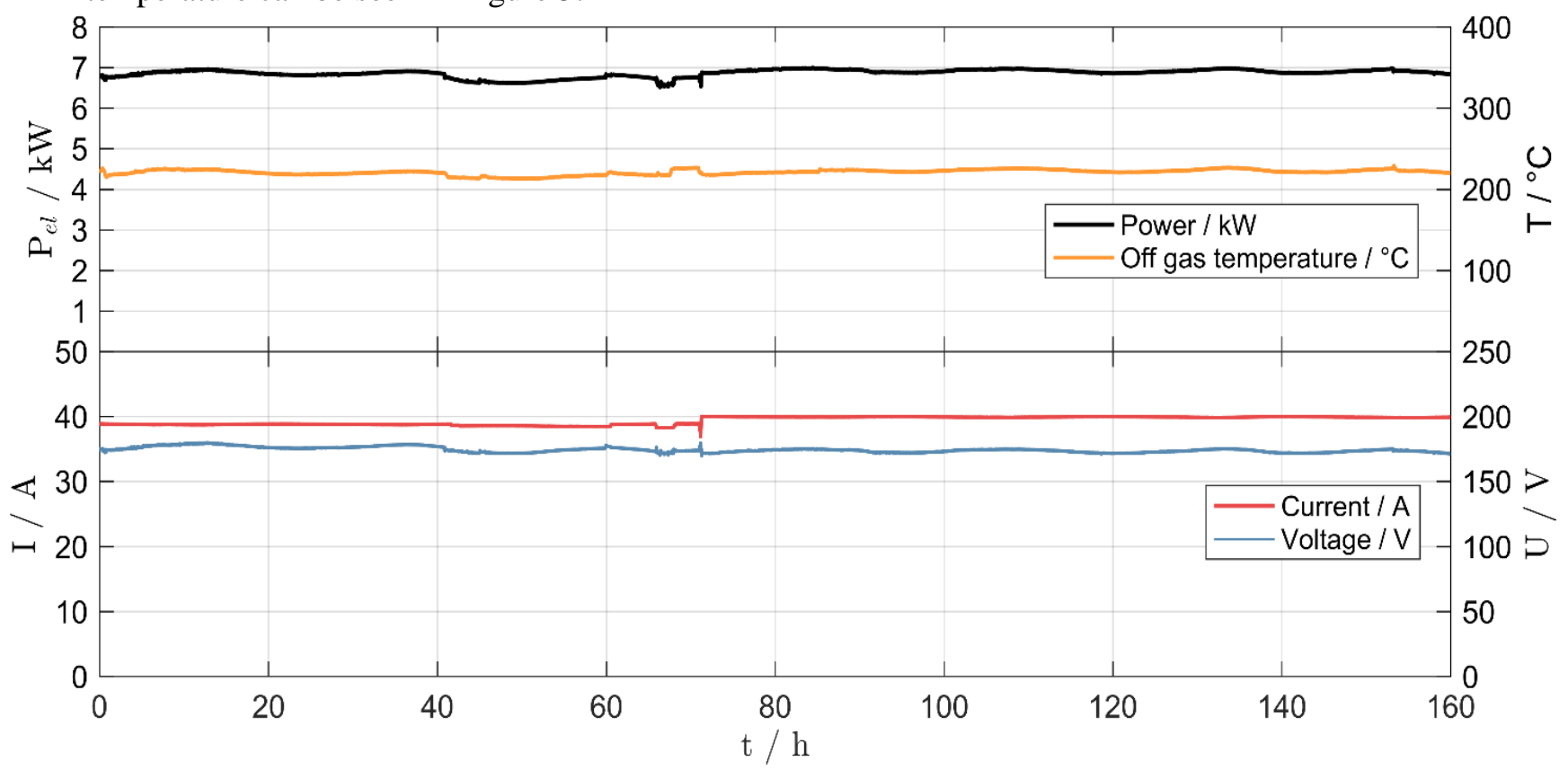

Figure 4: SOFC system operation with natural gas. The system was equipped with an 8 x 30 cell IKTS stack module with $127 \mathrm{~cm}^{2}$ cell area. In the $6.9 \mathrm{~kW}_{\mathrm{EL}}$ operating point the off-gas temperature is around $220{ }^{\circ} \mathrm{C}$. 


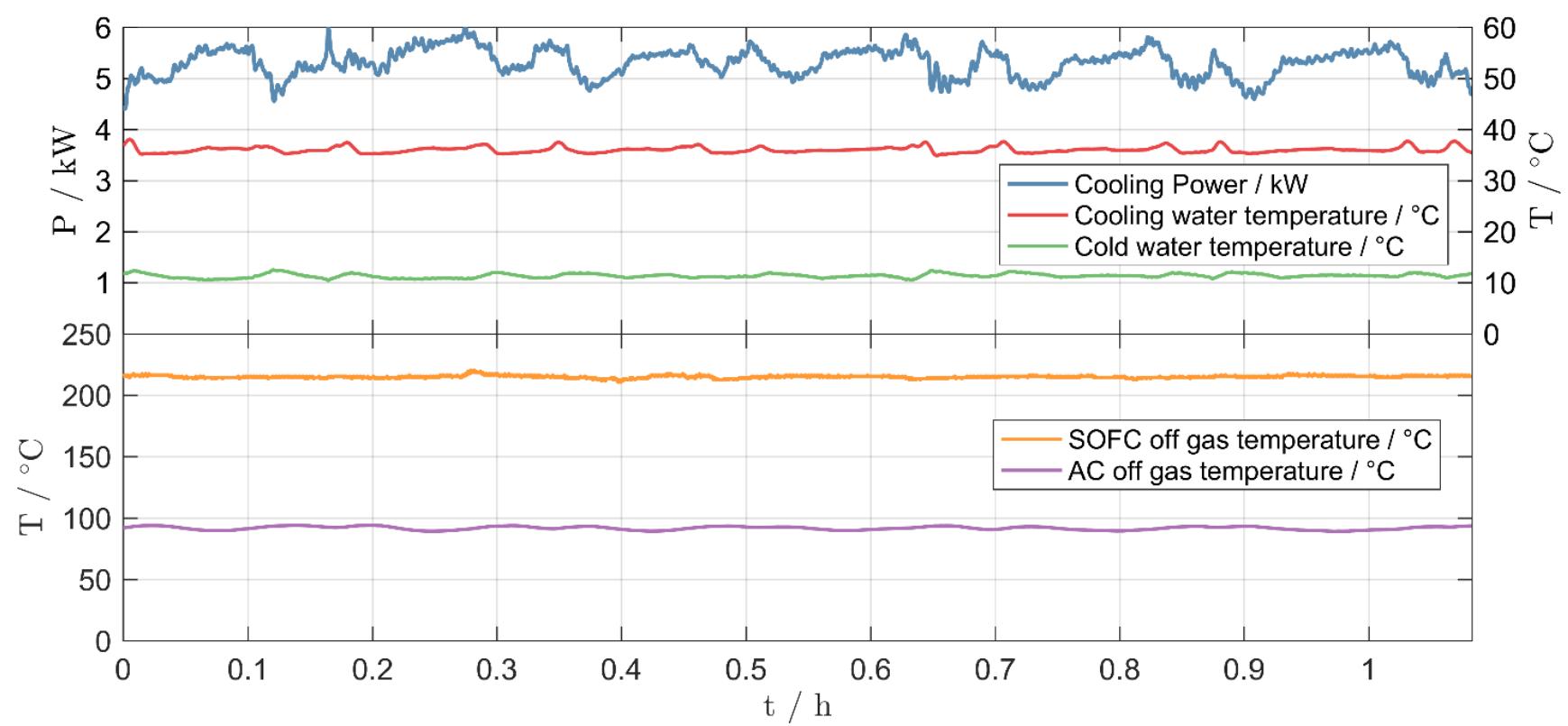

Figure 5: Operation of the absorption chiller with $220^{\circ} \mathrm{C}$ generator inlet temperature. The top chart shows the evaporator power (cooling power) and the temperatures of cooling- and cold water. Cooling water is set to $36{ }^{\circ} \mathrm{C}$, cold water is $12{ }^{\circ} \mathrm{C}$. In the bottom chart the corresponding gas temperatures of the generator inlet and outlet can be seen. Fluctuations in the cooling power were due to a slightly too large mass flow of refrigerant in the absorption heat pump.

Full scale long term tests of the diesel steam reformer were performed on a test rig under laboratory conditions. Steam to carbon ratios (S/C) of $2-4$ were investigated in a temperature range of $400-$ $600{ }^{\circ} \mathrm{C}$ and compared with equilibrium calculations. As the temperature in the reformer is not homogenous the equilibrium was calculated for a certain temperature range to evaluate the measured gas composition. As can be seen in Figure 15 the measured composition matches the equilibrium very well within a temperature window of $10 \mathrm{~K}$ which is an indication that the steam reforming works properly. Besides that, the reformate gas composition at the reformer outlet was stable for the whole

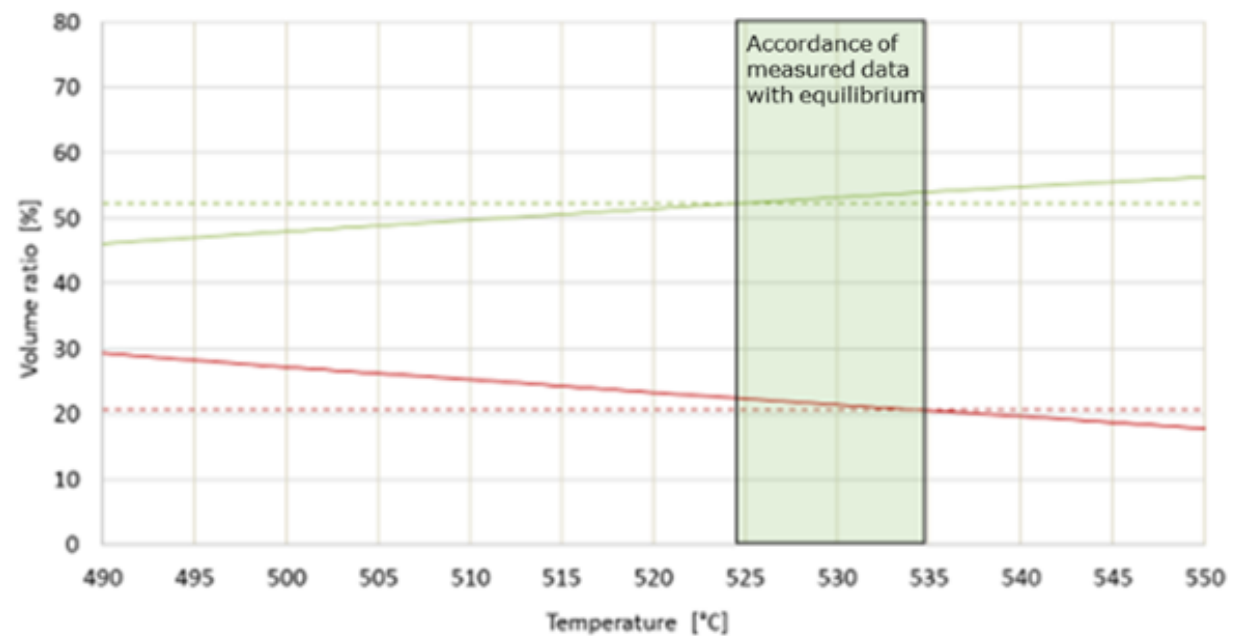

Figure 15: Comparison of measured (dotted curves) and equilibrium (full curves) $\mathrm{H} 2$ and $\mathrm{CH} 4$ concentrations for a test with a steam to carbon ratio of 2 .

testing period once the temperature in the reformer has levelled off. 
The long term test of $\sim 200 \mathrm{~h}$ also showed that the pressure drop can be kept constant which suggests that severe carbon deposition can be excluded.

Besides long term test bench experiments, reforming tests within the hot anode gas recirculation cycle of the SOFC system including a used stack module at higher temperatures were performed (see Figure 16). First, a used stack module was taken for the tests to avoid any damage of the new stack module due to diesel operation. The stack module was operated at open circuit conditions (OCV). Therefore, the reformed diesel fuel which leaves the reformer is not further electrochemically converted in the SOFC. Multiple test runs in a period of several days showed no sign of carbon deposition or degradation. Therefore, the steam reforming concept was proven to be suitable for system operation with the stack module. Figure 16 compares the measured reformer outlet composition with the theoretical equilibrium. The good accordance suggests a correct reforming behavior without carbon

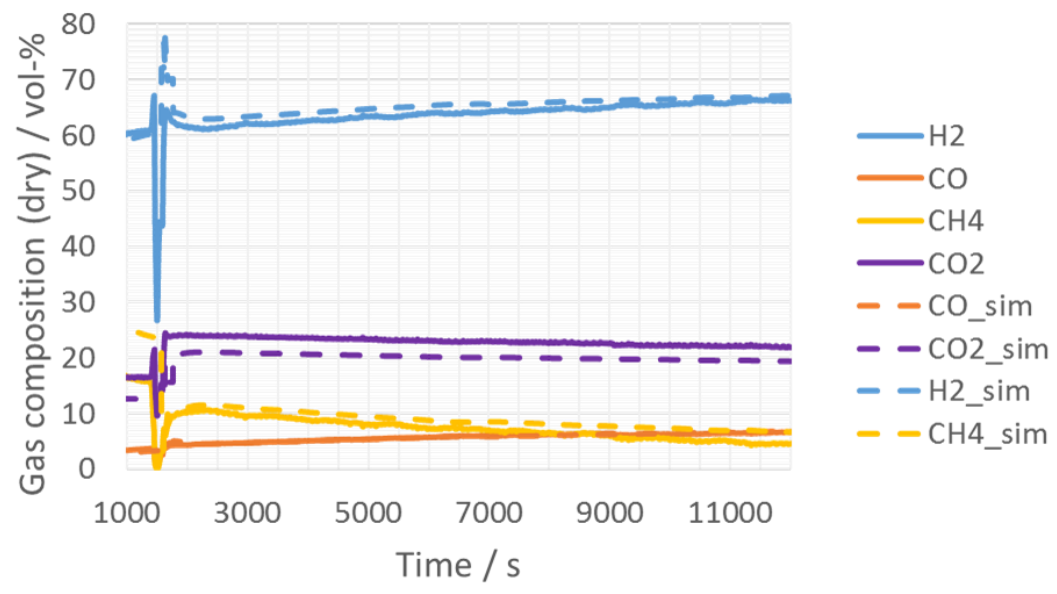

Figure 16: Comparison of diesel reforming composition between theoretical equilibrium (simulated) and measurement under system operating conditions. (Hauth et al.)

deposition.

\section{CONCLUSION}

The coupling of AVL's SOFC CHP platform with an absorption heat pump from TU Graz for CCHP applications provides a very promising and demand flexible technology to serve a large variety of applications. The analysis of three different application scenarios showed that hospitals and office buildings are attractive options for SOFC CCHP. Tests of the prototype CCHP system showed that the temperature levels of the two sub systems match perfectly to generate both electric and cooling power at high efficiencies. Adaptions to the SOFC system have been implemented for the operation with biogas and (renewable) diesel as fuel.

\section{ACKNOWLEDGMENTS}

This work was supported by the project "SOFCool", which was supported by funding from the Austrian "Climate and Energy Fund" and carried out in the program „e!Mission.at". Many thanks to all our project partners for the wonderful cooperation.

\section{REFERENCES}

Albert, J., Rieberer, R., (2016), Entwicklung einer Absorptionskältemaschine für die Nutzung der Abwärme einer Festoxidbrennstoffzelle, DKV, Kassel, Germany

Albert, J., Rieberer, R., (2017), Experimentelle und simulationsgestützte Analyse einer Absorptionskältemaschine für KWKK-Anlagen, DKV, Bremen, Germany 
Hauth, M., Seidl, M., Postl, A., Sallai, C., Soukup, N., Albert, J., Weinländer, C., Rieberer, R., Hochenauer, C., (2017) Development of a Highly Flexible SOFC CCHP System towards DemandOrientated Power Generation from Renewable Fuels, SOFC XV, Hollywood, Florida

P. V. Aravind and W. de Jong, (2012), Evaluation of high temperature gas cleaning options for biomass gasification product gas for Solid Oxide Fuel Cells, Progress in Energy and Combustion Science 38(6): 737-764.

D. Andriani, A. Wresta, T. D. Atmaja and A. A. Saepudin, (2014), Review on optimization production and upgrading biogas through $\mathrm{CO} 2$ removal using various techniques, Applied Biochemistry and Biotechnology 172(4): 1909-1928. 


\title{
FAÇADE-INTEGRATED DECENTRALIZED COOLING SYSTEM - EVALUATION IN AN OUTDOOR TEST FACILITY
}

\author{
Daniel Brandl ${ }^{1, *}$, Andreas Heinz ${ }^{1}$, Thomas Mach ${ }^{1}$, Oliver Schmiedbauer ${ }^{1}$, Simon Zimmerberger ${ }^{1}$, Marcus \\ Rennhofer ${ }^{2}$, Thomas Schlager ${ }^{2}$, Tim Selke ${ }^{2}$ \\ ${ }^{1}$ Institute of Thermal Engineering, Graz University of Technology \\ Inffeldgasse 25/B, 8010 Graz, Austria \\ ${ }^{2}$ AIT Austrian Institute of Technology GmbH \\ Giefinggasse 2, 1210 Vienna, Austria \\ *Phone: +433168737306 \\ E-Mail: daniel.brandl@tugraz.at
}

\section{SUMMARY}

The objective of the research project COOLSKIN is the development, assessment and functionality approval of a decentralised, façade-integrated energy system for cooling of interior spaces. Façade integrated photovoltaic modules convert the solar irradiation into electricity in order to operate a heat pump system for controlling the indoor temperature of the adjacent interior room. The system concept addresses decentralization of the energy supply and energy autarky.

This paper is focusing on the description of the sophisticated heat pump system, which was designed for testing of different cooling strategies. Alternatively, this system can also be operated in heating mode for colder climate conditions. Furthermore, the heat pump cycle can be connected either to a water or an air cooling cycle. In this paper the authors present first results and findings from the integrated cooling system in operation in an outdoor test facility.

Keywords: solar cooling, façade integrated heat pump system, building integrated photovoltaic (BIPV)

\section{INTRODUCTION}

Based on the elaboration of a comprehensively documented system and component knowledge on building-integrated cooling systems supplied by photovoltaics, the project COOLSKIN will deliver descriptions and analyses of decentralized cooling systems to be integrated in façades. Analyses cover the energetic system behaviour, economic aspects, the ability of integration into façades and the architectural design. At the end of the project a functional model of a photovoltaic façade-integrated cooling system will be available, that has been optimized by means of simulations and experimental work and that has been tested under real conditions in a test façade.

The project results will be directly reported in Task 53 of the International Energy Agency Program 'Solar Heating and Cooling' (IEA-SHC).

A number of experiments with different photovoltaic modules as well as the commissioning and first tests of the heat pump system were performed under laboratory conditions, before the whole system was installed in an outdoor test facility. This study presents the description of the façade integrated cooling system as well as exemplay results and findings from long term tests in an outdoor test facility.

\section{FAÇADE-INTEGRATED HEATING AND COOLING SYSTEM}

This chapter is presenting the design of the façade-integrated cooling (COOLSKIN) system and the functionality of its most important components. The three dimensional CAD model which is shown in Fig. 1 is supporting the description of the system setup. The core unit of the whole system is the heat pump cycle which ensures the energy, which is required for the temperature conditioning of the interior room. 
The required electric power is provided by a façade integrated (BIPV) photovoltaic plant. All components of the electric circuit except the PV modules are positioned inside the façade. The heat pump cycle as well as the electric circuit are described in detail in the chapters 3.1 and 3.2.
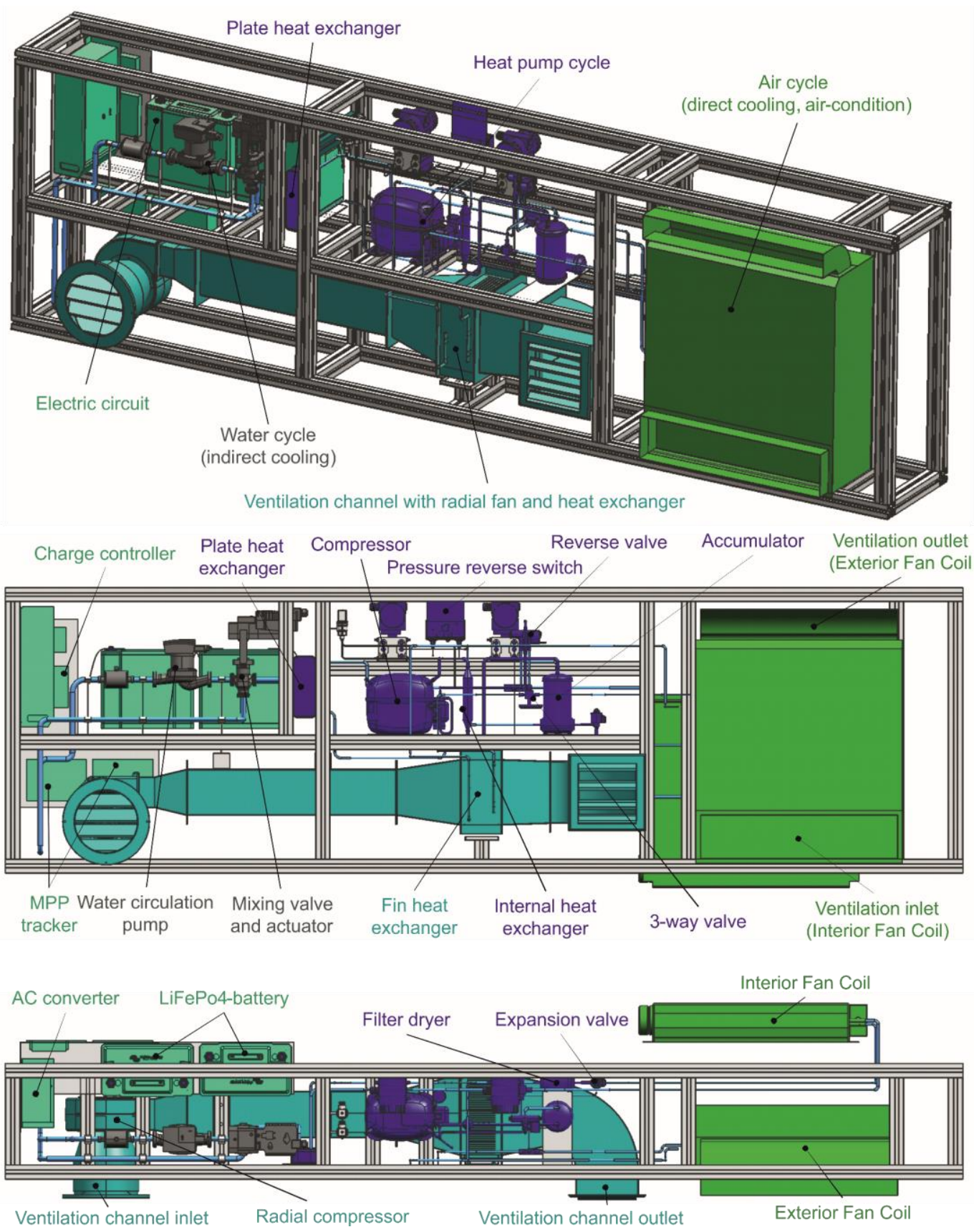

Fig. 1: CAD model of the façade-integrated temperature conditioning (COOLSKIN) system

Furthermore, the system contains a ventilation channel with an integrated heat exchanger, where the refrigerant is either condensed or evaporated depending on the operating mode. The ventilation channel was specially designed for this system and includes an integrated finned heat exchanger as well as a fan in order to establish an air flow of approximately $360 \mathrm{~m}^{3} / \mathrm{h}$ from the ambient (exterior environment), over the heat exchanger and back to the ambient. A water cycle is connected to the heat pump cycle via a plate heat exchanger in order to supply the room's ceiling (thermal component activation) or floor integrated pipework with warm or cold water (indirect conditioning). Additionally, the heat pump cycle is connected to a fan coil unit to enable a direct cooling of the indoor air (direct 
conditioning). All components of the system were assembled at the Institute of Thermal Engineering within an aluminum framework, which has the main dimensions of $2.97 \times 0.89 \times 0.36 \mathrm{~m}$.

\section{Heat pump cycle}

This chapter includes a detailed description of the heat pump cycle, therefor the schematics for cooling and heating modes are shown in Fig. 2. Primarily, the heat pump cycle was designed for cooling of the interior space (left schematic and T-h diagram in Fig 2.). The refrigerant leaves the compressor (3), passes the reverse valve and can either be sent to the heat exchanger (3-5) in the ventilation channel or in the exterior fan coil, which act as condenser in the cooling mode. Subsequently, the refrigerant flows through the internal heat exchanger (5-6) in order to enable a superheated refrigerant state at the compressor inlet. Then the refrigerant is expanded in the electronic expansion valve (6-7) and is then evaporated either in the plate heat exchanger of the water cycle or in the heat exchanger of the interior fan coil (7). Finally, the refrigerant enters the accumulator (1") via the reverse valve, and the refrigerant vapour (1) flows through the internal heat exchanger (1-2) and back to the compressor inlet (2). Within the compressor, the pressure of the refrigerant is increased from 3.5 to 10.2 bar (2-3) in this exemplary operation point. A Coefficient of Performance (COP) of 4.3 can be achieved.
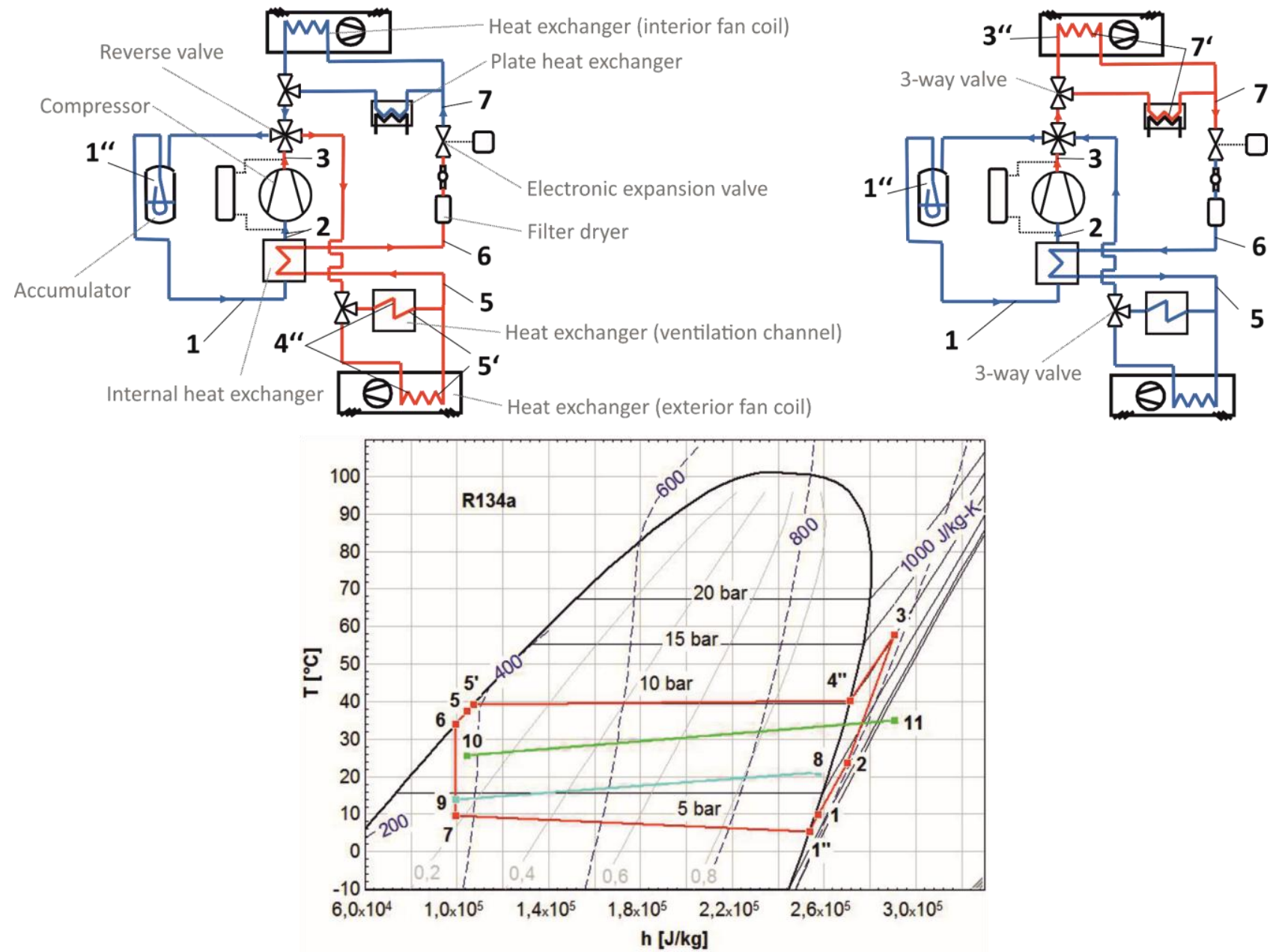

Fig. 2: The schematic and the T-h diagram on the left show the working principle for the cooling mode whereas the heating mode is illustrated on the right.

With the installed (4-way) reverse valve after the compressor, the refrigerant flow can be redirected so that the interior room can be heated at cold climate conditions (right schematic and T-h diagram in Fig. 2). In this case the refrigerant is evaporated in one of the exterior heat exchangers, while it is 
condensed either in the plate heat exchanger or the interior fan coil's heat exchanger by transferring heat for heating of the interior room.

The core element of the heat pump cycle is a reciprocating compressor, which has an electric consumption between 200 and $450 \mathrm{~W}$ (depending on the operation mode). The compressor's speed can be varied between 2000 and $4500 \mathrm{~min}^{-1}$ in order to enable an energy efficient operation.

\section{Electric circuit}

In order to realize the decentralized heating and cooling of the interior space, a photovoltaic plant is attached to the building's façade. All components of the electric circuit are integrated in the façade as it is illustrated in the CAD model in Fig. 1 and the photo in Fig. 3. Furthermore, Fig. 3 includes a schematic of the electric circuit containing all main components. The photovoltaic plant contains four glass-glass modules with a cumulative nominal power of approximately $1170 \mathrm{~W}$. The maximum power point (MPP) tracker is regulating the battery charging and provides the most efficient operating mode for the whole photovoltaic system. Two lithium iron phosphate batteries $\left(\mathrm{LiFePO}_{4}\right)$ are connected in series and act as an electric storage with a capacity of $2300 \mathrm{Wh}$. With the help of the inverter the battery voltage of $25.6 \mathrm{~V}$ (DC) is transformed to $230 \mathrm{~V}$ and $50 \mathrm{~Hz} \mathrm{AC}$ in order to supply the compressor (max. $450 \mathrm{~W}$ ), the radial fan inside the ventilation channel (max. $140 \mathrm{~W}$ ) or the exterior fan coil (max. $63 \mathrm{~W}$ ), the water cycle pump (max. $75 \mathrm{~W}$ ) or the interior fan coil (max. $17.1 \mathrm{~W}$ ) and the electronic expansion valve $(\max 3.6 \mathrm{~W})$ with electricity. At peak load the whole electric circuit has an energy conversion efficiency of $85 \%$, it is lower in partial load operation.
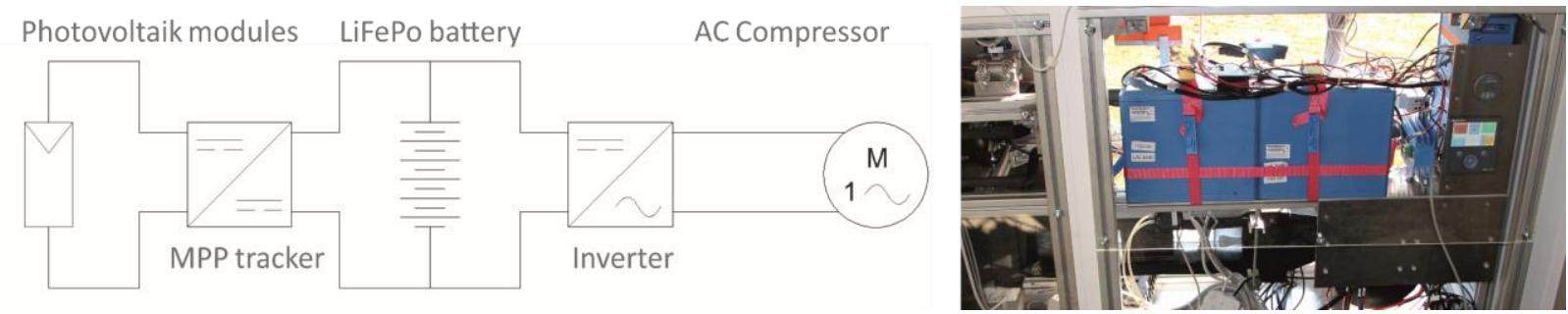

Fig. 3: Schematic of the electric circuit and photo of the electrical components

\section{OUTDOOR TEST FACILITY AND FIELD TESTS}

After assembly and functionality tests in the laboratory, the COOLSKIN system was installed in a test box for long-term tests under real climate conditions. At the campus of Graz University of Technology two identical boxes with an effective floor area of $13.49 \mathrm{~m}^{2}$ were available for the upcoming field tests (shown in Fig. 4). A container was set up behind these boxes in order to supply the boxes with electricity and energy for heating and cooling. The boxes' ceiling and floor are made of concrete with an integrated water (heating) circuit in the floor as well as two water (cooling) circuits in the ceiling. The boxes are equipped with a $200 \mathrm{~mm}$ thick thermal insulation made of EPS and have windows which are oriented to the south. The COOLSKIN system is implemented in the eastern box (cooled/heated box) below the windows, in the western box (reference box) an empty aluminium framework is installed. Both frameworks are thermally insulated with $50 \mathrm{~mm}$ XPS to prevent freezing of water filled pipes inside the system in winter.

The test boxes are equipped with a multitude of sensors for monitoring temperatures and humidity of the interior space as well as the temperature at the interior and exterior surfaces and furthermore inside the concrete floor and ceiling. The COOLSKIN system also contains a large number of sensors in order to determine the system's cooling/heating power, the refrigerant states in the heat pump cycle, electricity consumption and much more. 
In the field test the differences of the interior temperatures and the resulting thermal comfort between the box with the integrated COOLSKIN system and the reference box without cooling will be analysed over the period of a year. The reference box is either supplied with warm water when the interior temperature drops below $12{ }^{\circ} \mathrm{C}$ in order to prevent damage due to freezing or for ensuring a comfortable temperature (between 20 and $23{ }^{\circ} \mathrm{C}$ ) of the interior space alternatively. Both boxes are equipped with an internal heat load (dummy) in order to simulate the internal thermal loads over the day due to persons and equipment of an office room. Therefore, the internal heat load of $300 \mathrm{~W}$ is activated on working days between 8:00 and 16:00. Also a mechanical ventilation is installed in each room (room volume app. $30 \mathrm{~m}^{3}$ ) ensuring an air exchange of $60 \mathrm{~m}^{3} / \mathrm{h}$ during the working periods mentioned before. First test runs have been already performed, exemplary results are presented in section 5 of this paper.

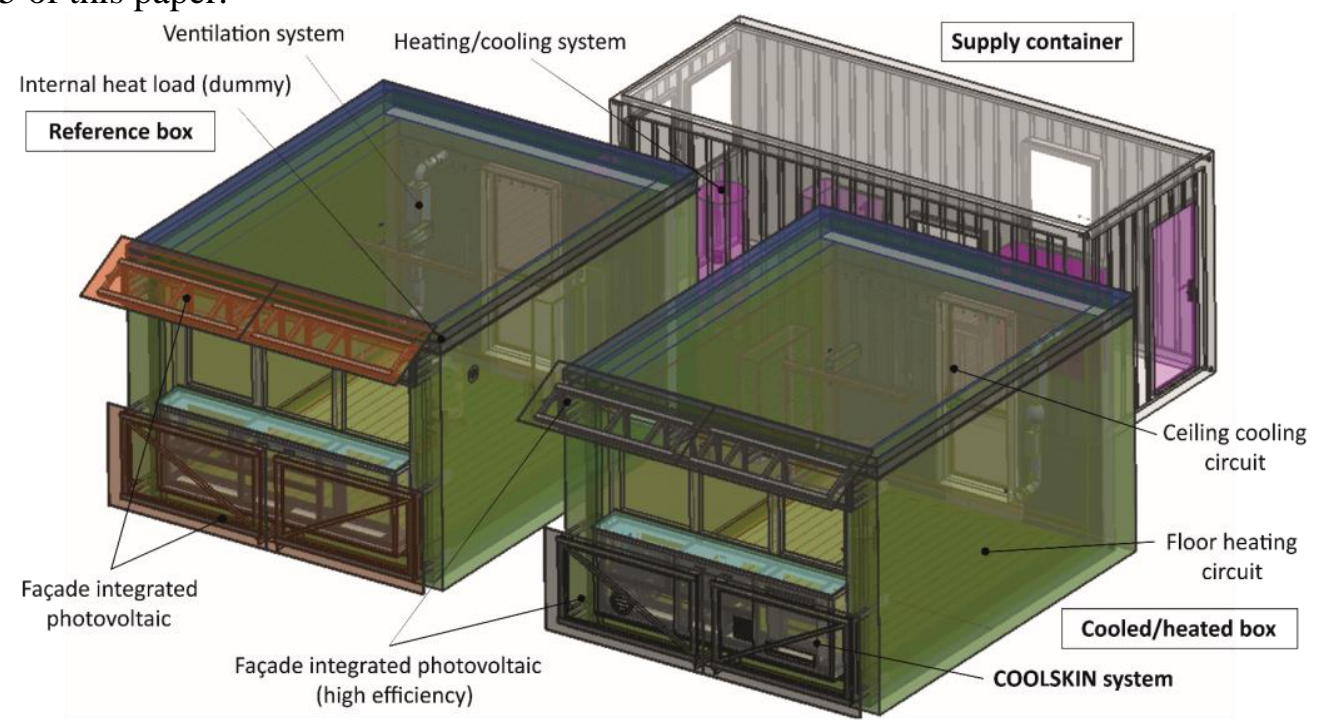

Fig. 4: View on the 3D CAD model of the test boxes and the energy supply container.

Two PV modules per box are mounted in front of the COOLSKIN system with a distance of $150 \mathrm{~mm}$ while two more modules are mounted with an adjustable angle above the window. The modules below the window have an area of $2.39 \mathrm{~m}^{2}$, while the modules above the window are slightly smaller with an area of $1.75 \mathrm{~m}^{2}$. Two different types of PV modules with different colours are used, black coloured modules with a high solar energy conversion efficiency and grey coloured modules with app. $78 \%$ of the efficiency of the black modules but favourable from the architectural point of view. The electric circuit was designed in order to enable to use either the black or the grey or both modules as energy source for the system.

\section{FIRST FIELD TEST RESULTS}

The COOLSKIN system was attached to the test facilities south oriented façade in January, from the end of January to the end of May the functionality and regulation of all involved units, in particular the compressor, was tested regarding heating and cooling of the interior room with the direct conditioning system (fan coil). In the sections 5.2 and 5.3 first results of the heating and cooling characteristic are presented. The start of the long run tests is planned for the end of July when the assembly of the water cycle (indirect cooling system) is finished. Furthermore, the characteristic of the PV system was analysed in this period which is presented in section 5.1 of this paper. 


\section{PV system characteristic}

Between January and April 2018 the system operates in heating mode. The energy yield of the $1170 \mathrm{~W}$ PV system is used to drive the $500 \mathrm{~W}$ speed controlled compressor. Fig. 5 shows typical yield- and load- profiles. Due to the façade integration of all components, a balance must be achieved between available PV power and needed battery size. Measurement results show the importance of component dimensioning and how they determine the system behavior. Especially in winter months, the energy demand can only be covered with PV in times of ideal weather conditions. Operating states shown in Fig. 5 are described below:

(1) Load is equal to the PV yield: Battery voltage is stable and load can be covered.

(2) Load is temporarily switched off: Battery is charged or discharged according to the PV yield.

(3) Low PV yield with load: Battery voltage decreases

(4) PV yield without load: Battery is fully charged

(5) High irradiation: Load is covered by the PV yield

(6) Low irradiation: Load is disconnected when the battery voltage decreases to the cut off voltage.

(7) PV yield higher than load: Battery is charged while the load is covered

(8) Fluctuating load: continuous operation in charging and discharging mode.
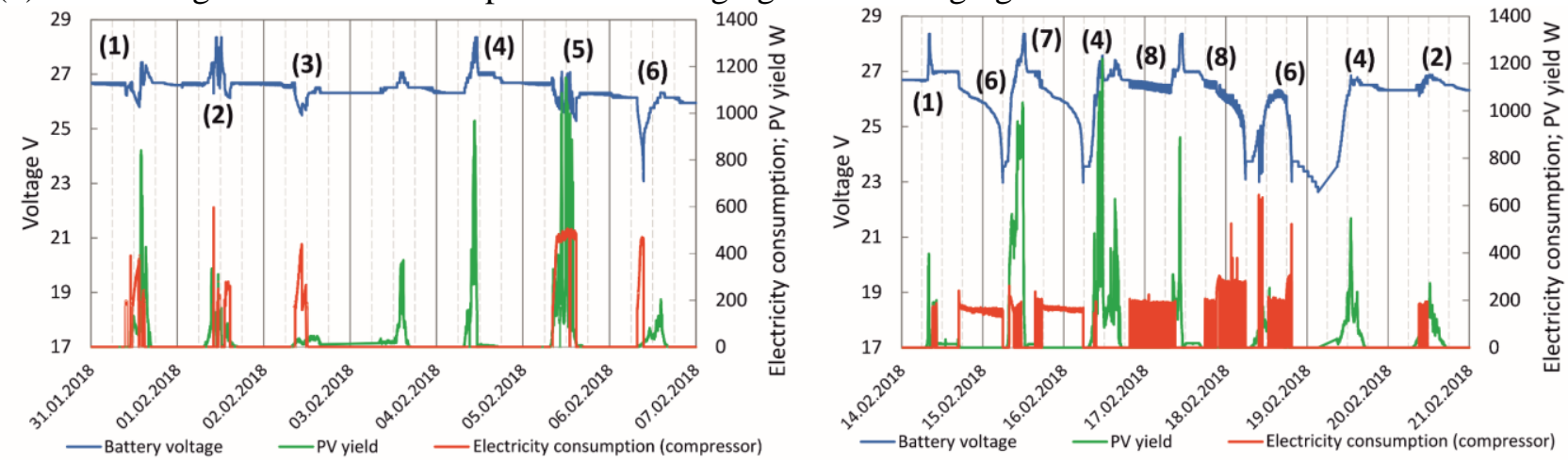

Fig. 5: Real-Setup at the test boxes in Graz from 31.01.2018 to 07.02.2018 (left diagram) and from 14.02.2018 to 21.02.2018 (right diagram). The numbers define different operating states described in the text above.

\section{Heating characteristic}

Fig. 6 shows two diagrams with monitoring data over 24 hours starting on $15^{\text {th }}$ of February 2018 at 7:00. The upper diagram contains the monitored electricity consumption of the compressor of the heat pump cycle and the measured global radiation as well as the PV yield. In this test phase only the compressor was connected to the batteries of the PV system, the electricity consumption of other components was not monitored. The lower diagram shows the temperature profiles of the most important sensors of the outdoor test facility, the exterior temperature from the ZAMG climate station, the sensor which is positioned in front of the interior fan coil ventilation inlet as well as the operative room temperature at two different positions for both boxes.

In the observed period the exterior temperature varied between -6.0 and $3.2^{\circ} \mathrm{C}$, solar (global) radiation onto the horizontal was available between 7:00 and 16:00 with a maximum value of $550 \mathrm{~W} / \mathrm{m}^{2}$. In the period between 8:00 and 13:00 the PV modules were harvesting solar radiation. At 8:00 the internal heat loads were activated, one hour later the COOLSKIN system started in the heating mode.

During this day, different operating characteristics of the heating system were observed:

(1) Between 9:00 and 11:30 the heat pump was running in the alternating mode (on/off). The temperature sensor in front of the interior ventilation inlet controlled the system. The heat pump was activated when the temperature drops below $19^{\circ} \mathrm{C}$ and deactivated when it exceeds a temperature of $21^{\circ} \mathrm{C}$. It was observed that the heating periods are decreasing (from 14 to $3 \mathrm{~min}$ ) with each step while 
the off-periods are increasing (from 20 to $40 \mathrm{~min}$ ). The electricity consumption of the compressor was varying between 150 and $200 \mathrm{~W}$.

(2) Between 11:30 and 15:30 the heating system was inactive, because the control temperature was above $19^{\circ} \mathrm{C}$.

(3) Between 15:30 and 17:30 the heating system showed again an alternating characteristic, stepwise the heating periods were increasing and the off-periods decreasing. The electricity consumption of the compressor in the heat pump cycle was varying between 170 and $250 \mathrm{~W}$. At 16:30 the operative room temperature dropped below the interior fan coil inlet temperature for the first time that day.

(4) From 17:30 to 5:00 on the next day the heating system was continuously in operation. During this period the operative room temperature was always lower than the temperature at the interior fan coil inlet. Generally, a decrease of temperature (with a rate of $-0.075 \mathrm{~K} / \mathrm{h}$ ) occurred at all sensor positions inside the test box. The compressor's electricity consumption was continuously decreasing from 180 to $160 \mathrm{~W}$ after a peak of $220 \mathrm{~W}$ at the beginning of this heating period.

(5) After 12 hours continuous heating of the interior room the battery was discharged and the system stopped. The temperature inside the box rapidly fell below $19{ }^{\circ} \mathrm{C}$ at all sensor positions in the room. In the considered period the reference box was accidentally heated to higher temperatures than intended (as described in section 4). The days before the concrete ceiling was supplied with warm water from $13^{\text {th }}$ Feb. 20:50 to $14^{\text {th }}$ Feb. 8:40 (790 W, on average), from $14^{\text {th }}$ Feb. 23:50 to $15^{\text {th }}$ Feb. 8:40 (895 W, avg.) and on $16^{\text {th }}$ Feb. from 2:20 to 9:10 (948 W, avg.). The energy was stored in the concrete of the reference box, which has the effect of a higher operative room temperature due to higher wall temperatures compared to the box heated by the COOLSKIN system, which was heated via the fan coil.

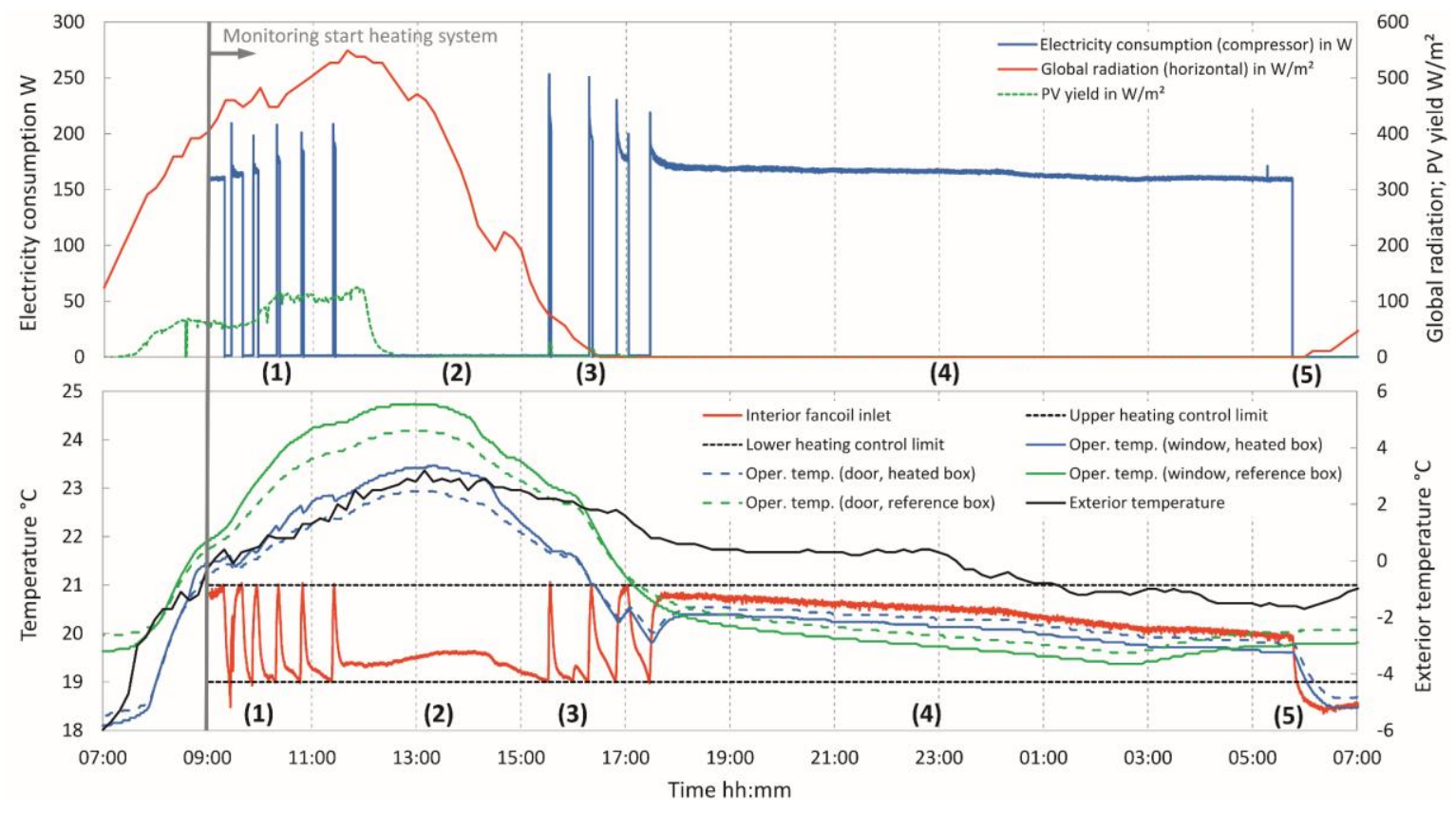

Fig. 6: Diagrams with monitored data from outdoor tests over 24 hours starting from $15^{\text {th }}$ of February, 2018 at 7:00.

\section{Cooling characteristic}

Fig. 7 shows two diagrams with monitoring data between $10^{\text {th }}$ of June 2018 7:00 and $11^{\text {th }}$ of June 1:00. The upper diagram contains measured global radiation and the PV yield. In this test run in cooling mode not only the compressor but also the electronic expansion valve, the radial fan of the ventilation channel and the interior fan coil were connected to the electric circuit of the PV system. For this 
reason, the upper diagram in Fig. 7 shows the electricity consumption of the compressor only as well as the sum of all electricity consumers of the cooling system (plant) mentioned before.

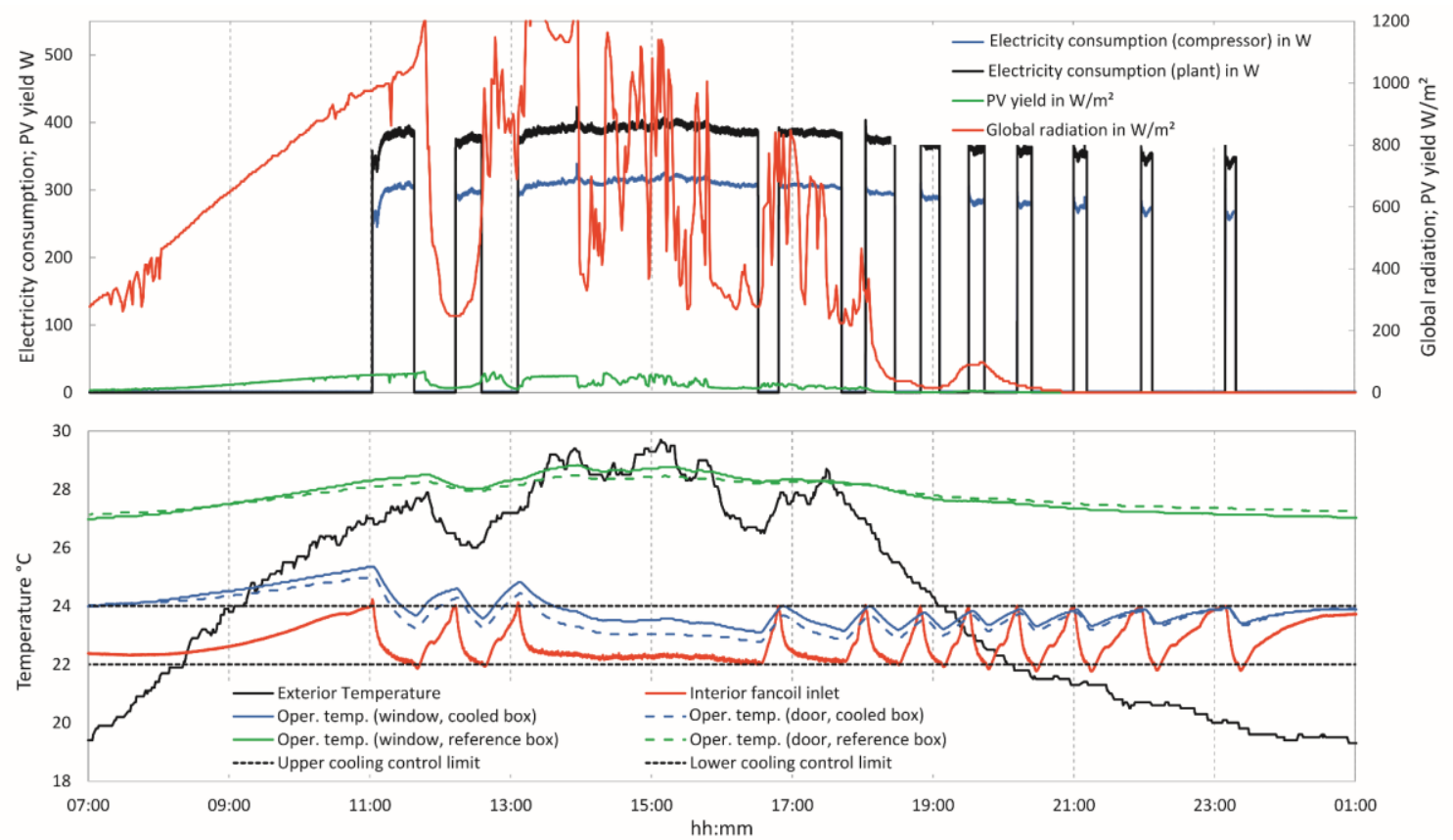

Fig. 7: Diagrams with monitored data from the outdoor test facility from $10^{\text {th }}$ of June, 7:00 to $11^{\text {th }}$ of June, 1:00.

The lower diagram shows the temperature profiles of the same sensors as in Fig. 6 in section 5.2. The exterior temperature increased from $19.4{ }^{\circ} \mathrm{C}$ to a maximum of $29.6{ }^{\circ} \mathrm{C}$ between 7:00 and 15:00 and decreased back to $19.4{ }^{\circ} \mathrm{C}$ until 1:00, solar radiation occurred between 6:00 and 21:00.

The following characteristic behaviour was observed at this day:

(1) Between 7:00 and 11:00 the exterior temperature and the solar radiation were steadily increasing which was heating up the test box. The temperature of the sensor used for controlling the system (interior fan coil inlet) was lower than the measured operative room temperature.

(2) At 11:00 the temperature measured by this sensor exceeded the upper cooling control limit, which automatically activated the cooling system, which operated in on/off mode until 12:30. In this period the compressor had an electricity consumption between 240 and $310 \mathrm{~W}$ when it was running, the whole plant's electricity consumption amounted to $330-390 \mathrm{~W}$. The operative room temperature was reduced between 1.3 and $1.9 \mathrm{~K}$ per cooling step.

(3) During the hottest period of the day (from 12:30 to 16:30) the cooling system was always on because the temperature close before the fan coil inlet did not drop below the lower cooling control limit. The operative room temperature was decreasing to $22.9{ }^{\circ} \mathrm{C}$ in this period and the highest electricity consumption of the compressor $(290-340 \mathrm{~W})$ and the plant $(370-425 \mathrm{~W})$ was observed.

(4) In the period between 16:30 and 0:00 the cooling system was operating in the on/off mode again, the interior room temperature did not exceed a value of $24{ }^{\circ} \mathrm{C}$. The electricity consumption of the compressor varied between $260-330 \mathrm{~W}$ and between $340-410 \mathrm{~W}$ of the plant during this period.

(5) After 0:00 the temperature in front of the interior fan coil inlet did no more exceed the upper cooling limit.

Finally, over this time period it was observed that the operative temperature in the cooled box was significantly lower than in the reference box (with a difference varying between 3 and $5.5 \mathrm{~K}$ ). 


\section{CONCLUSION}

A facade-integrated heating and cooling system called "COOLSKIN" was developed, built and successfully put into operation at the campus of Graz University of technology.

Results from first field tests showed that the system works well in both heating and cooling mode, where the expected power of $1 \mathrm{~kW}$ was achieved and partly exceeded. In these tests only the ventilation channel (with the radial fan and the fin heat exchanger) was used at the exterior side and the fan coil unit to temperate the interior room. Probably, the efficiency of the system can be improved by heating or cooling via the water circuits in the floor or the ceiling because of the activation of thermal storages. Furthermore, the electricity consumption of the plant can be reduced by use of the less electricity consuming exterior fan coil. This assumption should be confirmed by the long-term tests, which will start soon. The long-term tests are planned from the end of July 2018 to the end of August 2019.

Concerning the electric circuit of the COOLSKIN system, the first results imply that the battery capacity and PV power is undersized for a continuous heating operation in winter times, as it was expected from the beginning. An increase of PV size and significant increase of battery capacity would be needed to cover the high energy demand in winter. Both is unprofitable in real operation. Nevertheless, a part of the heat energy demand can be covered. Whether the system can provide enough electricity in order to ensure comfortable conditions in the room at any time will be figured out by the upcoming long-term tests.

\section{ACKNOWLEDGMENT}

The FFG project 848871 'COOLSKIN - Autarkes Kühlen über Gebäudehüllen' (2015 - 2018) is funded by the Austrian 'Klima- und Energiefonds' within the programme,e!MISSION' $1^{\text {st }}$ Call 2014. 


\section{PERFORMANCE INVESTIGATION OF A DESICCANT ASSISTED SOLAR AND GEOTHERMAL AIR CONDITIONING SYSTEM DURING WINTER AND SUMMER}

Peter Niemann1, Finn Richter1, Arne Speerforck1, Gerhard Schmitz1

Hamburg University of Technology, Institute of Engineering Thermodynamics 1

Denickestrasse 17, 21073 Hamburg, Germany

Phone: +49 (0)40 42878-3273

E-mail: peter.niemann@tuhh.de

\section{SUMMARY}

The objective of this work is to analyse the performance of a ground coupled and desiccant assisted air conditioning system during cooling and heating operation. Experimental data measured during summer 2016 an winter 2017 is presented. In summer, cooling demand is covered by a borehole heat exchanger; in winter, heating demand is supplied by a ground coupled heat pump. During summer, an electrical Energy Efficiency Ratio of 4.77 is achieved for the investigated system. A Seasonal Performance Factor of 153 is found for the geothermal system. Compared to a conventional air conditioning system the power consumption is reduced by $66 \%$ for the investigated period. During winter, moisture recovery efficiency of the desiccant wheel is 0.75 . On a cold winter day the test facility reduces power consumption by $30 \%$ compared with a reference system relying on isothermal

air humidification.

Key-words: Air conditioning, borehole heat exchanger, desiccant wheel, system evaluation.

\section{INTRODUCTION}

In general, air conditioning systems are used to provide comfortable indoor air conditions. In summer it is usually necessary to remove sensible and latent loads from outside air. Especially moisture removal accounts for peak loads of conventional air conditioning systems since it requires cooling air below dew point temperature.

Required cooling capacities are often provided by electrical driven vapor compression cycles. Cooling and dehumidification are coupled within the process. In a desiccant assisted air conditioning system removal of sensible and latent loads can be separated within the process, leading to a reduction of required cooling capacities. Moisture is removed using a solid or liquid desiccant. Thus, sensible loads can be removed by a heat sink working at a higher temperature level, e. g. geothermal energy. With regard to full-year operation, an equalized energy balance of the soil using a ground coupled heat pump for heat supply during winter is meaningful. Furthermore, low indoor air humidity ratio is a common problem in winter. To achieve sufficient humidity ratios, air humidification requires additional components within conventional systems. In a desiccant assisted system moisture recovery by means of the existing hygroscopic materials is possible. Electricity demand is reduced to auxiliary energies during summer and winter mode.

Even though several studies investigated desiccant assisted air conditioning systems during summer mode experimentally or numerically (e. g. Fong et al. (2011), Mazzei et al. (2002), Angrisani et al. (2015), Speerforck et al. (2016)), there is still a lack of studies investigating winter operation as well as full-year operation. Nevertheless, there are a few studies investigating winter operation (e. g. Antonellis et al. (2015), Kawamoto et al. (2016), La et al. (2011)). None of these studies investigated 
winter operation of a desiccant and geothermal assisted air conditioning system for temperate and heating dominated climate conditions. In Speerforck and Schmitz (2016) the considered system is evaluated for summer operation including cooling and dehumidification.

Furthermore, Speerforck et al. (2017) have shown that the system is applicable to different locations by means of a system simulation model using modelling language Modelica ${ }^{\circledR}$. In this study the system is investigated during summer as well as winter operation experimentally based on measured data during summer 2016 and winter 2017. The performance and limitations of the system are analyzed. To evaluate the energy consumption the

system is compared with reference systems relying on vapor compression chillers for summer operation. For winter operation the reference systems are equipped with electric isothermal or adiabatic humidification. Additionally, details about the geothermal system are presented.

\section{TEST FACILITY}

The test facility is located on the campus of Hamburg University of Technology. Some impressions of the pilot installation and relevant components are shown in Fig. 1. In total, the test facility consists of eight $20 \mathrm{ft}$. containers. The four containers on the lower floor contain the air handling unit and further technical installations. The four upper containers serve as office and conference room (area: $56 \mathrm{~m}^{2}$ ) and are used as reference room for the air conditioning system.

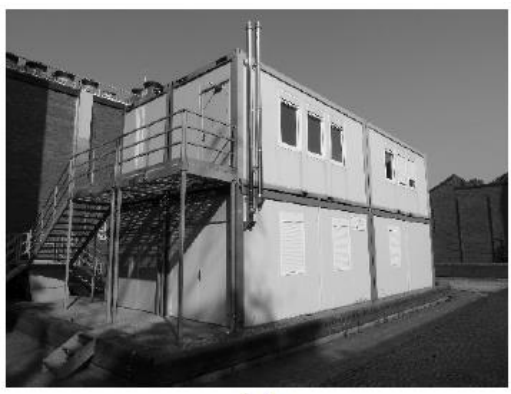

(a)

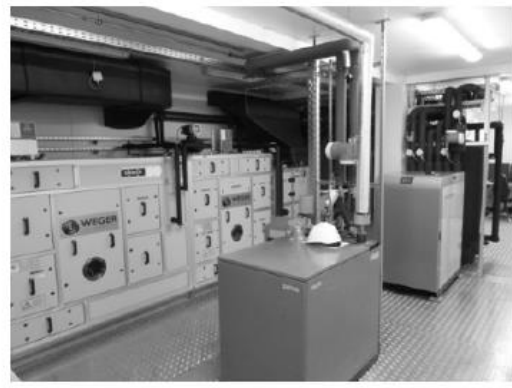

(b)

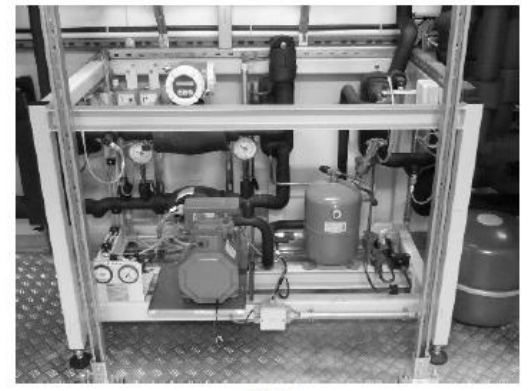

(c)

Fig. 1: Test facility (a), air handling unit (b) and ground coupled heat pump (c)

\subsection{Test Facility}

As shown in Fig. 2, the schematic layout of the pilot installation can be divided into the following three subsystems: the reference room, the air handling unit as well as the hot and cold water circuit. The air handling unit is designed as hybrid system, an open desiccant assisted ventilation process is combined with a closed cooling/heating loop similar to the system presented by Speerforck and Schmitz (2016).

A short description of system operation in summer and winter mode is given in the following. During summer operation mode, outside air (oda) is dehumidified within a desiccant wheel $(1 \rightarrow 2)$ and precooled by a sensible rotating heat exchanger $(2 \rightarrow 3)$. Water vapor is accumulated at the hygroscopic coating of the desiccant wheel (DW); Lithium chloride ( $\mathrm{LiCl}$ ) is used as desiccant. Afterwards, process air is finally cooled to the desired supply air (sup) temperature within a sensible water to air heat exchanger $(3 \rightarrow 4)$. Extract air (eta) is preheated $(5 \rightarrow 6)$ by the heat recovery wheel and further heated to the required regeneration air temperature within another sensible heat exchanger $(6 \rightarrow 7)$. Finally, eta is used to regenerate the desiccant $(7 \rightarrow 8)$ before it is emitted to the environment (exa). To achieve efficient operation, different components of the air handling unit can be bypassed as shown in Fig. 2. Thus, the electricity demand of the fans is reduced for demand-orientated air conditioning. 
Both wheels have a diameter of $0.6 \mathrm{~m}$. Regarding winter operation the desiccant wheel is operated as enthalpy wheel at higher rotational speed for coupled heat and mass transfer $(1 \rightarrow 2)$. Oda is remoistened and reheated within this process using the eta stream. If oda humidity is within comfort limits regarding humidity ratio, it is preheated using the regenerative heat exchanger $(2 \rightarrow 3)$; the desiccant wheel is bypassed then. Otherwise, the heat recovery wheel is not utilized. The reheater $(3 \rightarrow$ 4 ) is used to adjust the air to the desired sup temperature. Eta is either used for sensible heat recovery $(5 \rightarrow 6)$ or coupled heat and moisture recovery $(7 \rightarrow 8)$. The heater $(6 \rightarrow 7)$ was not operated in winter operation mode. The reference room is connected to the air handling unit on the supply and extract air

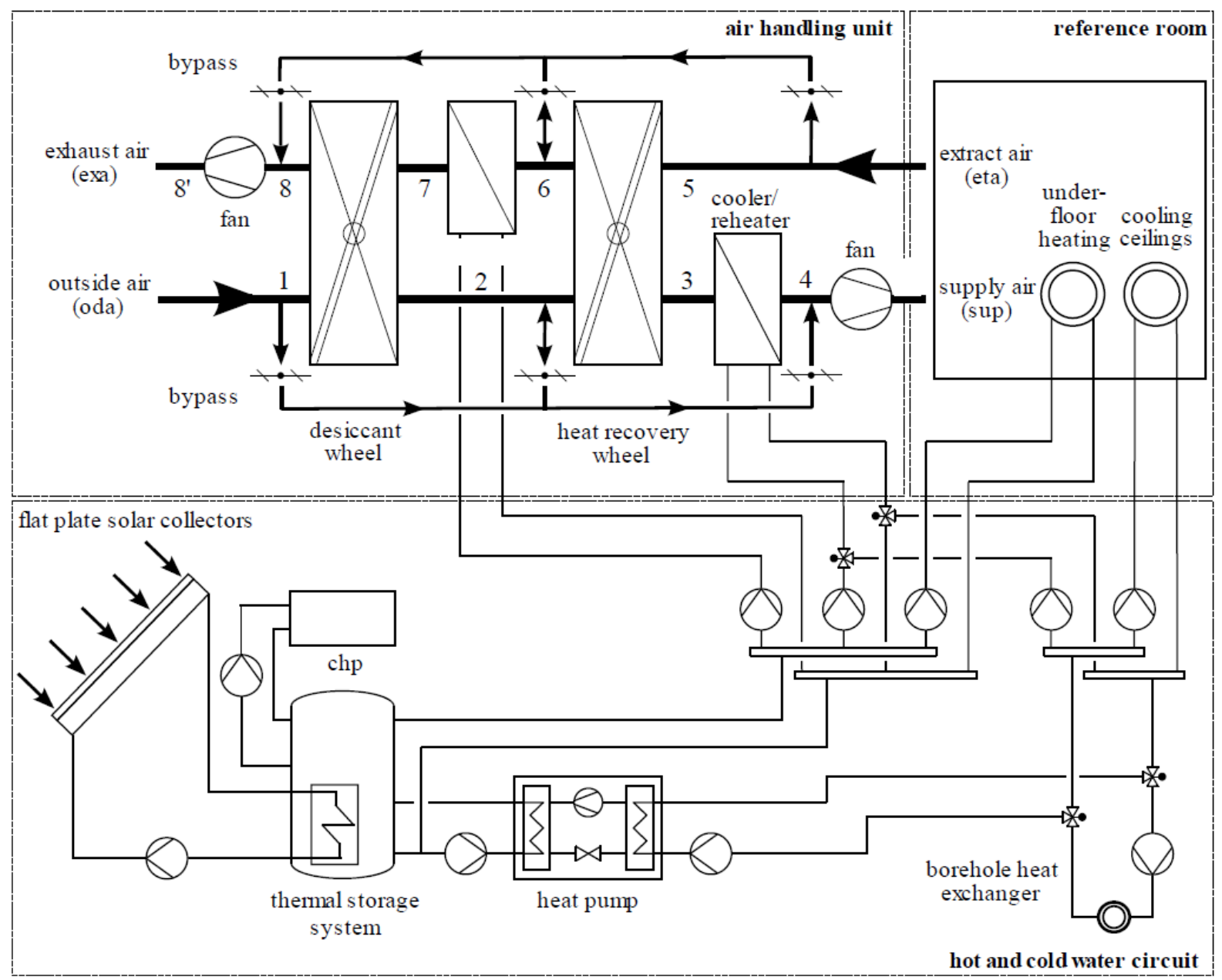

side. Furthermore, to cover sensible heat and cooling loads directly, it is equipped with underfloor heating and cooling ceilings.

Fig. 2: System layout of the test facility

Desiccant assisted air conditioning enables the integration of shallow geothermal energy for cooling in summer. Utilizing the geothermal system during full year operation as heat sink and heat source is promising for the reason of improving the annual energy balance of the soil as well as for the reason of maximizing the use of renewable energies around a year. Thus, heat supply during winter is primarily based on a ground coupled heat pump $\left(\dot{Q}_{\mathrm{GHP}, \text { nom }}=5.1 \mathrm{~kW}_{\mathrm{th}}\right.$ at BW5/W30). Solar thermal energy is utilized as primary heat source during summer mode $\left(A_{\mathrm{STU}}=20 \mathrm{~m}^{2}\right)$. A small scale gas driven CHP unit $\left(\dot{Q}_{\mathrm{CHP}, \text { nom }}=12.5 \mathrm{~kW}\right.$ th,$\left.P_{\mathrm{CHP}, \mathrm{nom}}=5 \mathrm{~kW}_{\mathrm{el}}\right)$ is used as backup system and to cover peak loads throughout the year. Integrating a stratified thermal storage system $\left(V=1 \mathrm{~m}^{3}\right)$ into the hot water circuit enables heat supply and heat demand to be decoupled temporally. A mixture of water and $22 \%$ ethylene glycol is used as heat transfer fluid within the hot and cold water circuit. 


\subsection{Geothermal System}

As shown in Fig. 2, the geothermal system is based on a single double U-tube borehole heat exchanger (BHE), utilized as heat sink of the cold water circuit (summer) and heat source to supply the GHP system (winter). Fig. 3 shows the structure of the soil surrounding the BHE. A reference BHE of the same type is used to analyze the impact of utilizing geothermal energy on the surrounding soil. The soil at the drilling location primarily consists of fine, medium and coarse sand until $18 \mathrm{~m}$ below ground surface. A layer of till and silt is located underneath. For the reamaining length below $30 \mathrm{~m}$ the BHE is surrounded by micaceous clay. The final drilling depth is $80 \mathrm{~m}$ below ground surface. There are no relevant ground water flows at the drilling location in general. A grouting material with a thermal conductivity of $\lambda=2 \mathrm{Wm}^{-1} \mathrm{~K}^{-1}$ is used inside the borehole for thermal connection between BHE and surrounding soil.

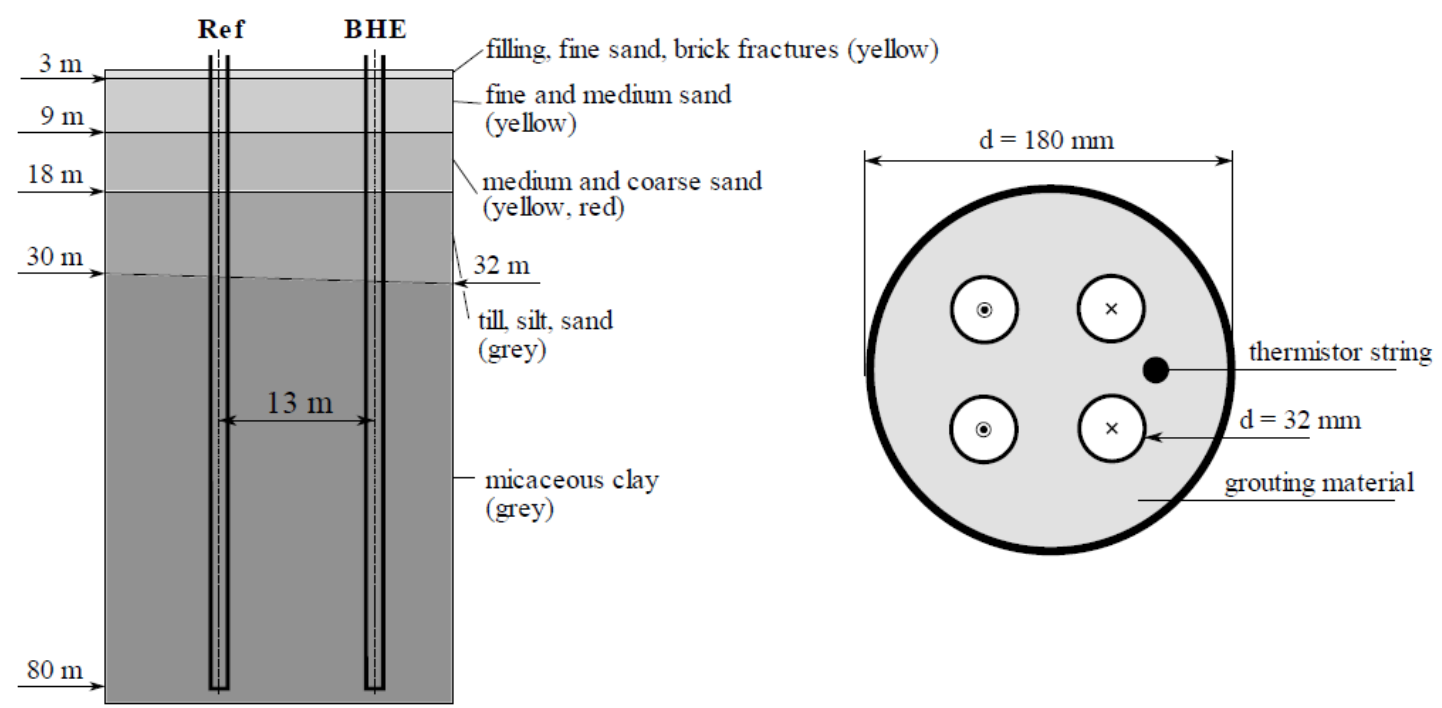

Fig. 3: Underlying geology of the soil and structure of the borehole heat exchangers

\section{Data Acquisition}

Relevant parameters characterizing the status of working fluids within the overall process are measured and recorded. Air temperature and relative humidity are measured at the inlet and outlet of each component of the air handling unit. Labeling of each air state is shown in Fig. 2. Flow averaging is applied as proposed by Slayzak and Ryan (2000) to take into account inhomogeneity within the air streams. Airside pressure drop is acquired across each component. Volume flow of supply and extract air is measured at positions 4 and 8 . Each hydraulic

circuit is evaluated measuring volume flow as well as fluid temperature at the inlet and outlet. Thermistor strings are embedded in the boreholes next to the pipe system for temperature measurement in different depths $(10,15,20,40,60$ and $80 \mathrm{~m})$ below ground surface. The electrical energy demand of each relevant component according to Fig. 2 is measured as well as further parameters characterizing thermal comfort within the reference room.

Measurement devices in use and the related uncertainties are listed in Table 1. All measured data are recorded every minute. A National Instruments data acquisition system and LabVIEW software in connection with Siemens S7 controlling is used to control and regulate the entire system.

Capacitive humidity sensors are used to measure relative humidity in the presented air handling unit. Drifting effects of these sensors (typical $1 \%$ r.h. per year) limit the resilience of derived quantities, 
energy and mass balances as time goes by. Thus, the sensors are recalibrated onsite every year. The calibration method is based on 36 set points of temperature and relative humidity. According to the principle of differential pressure, the pressure difference at the fan's joint is measured to determine the volume flow of supply and extract air indirectly.

Table 1: Measurement devices used within the test facility and related uncertainties

\begin{tabular}{llll}
\hline Measured property & & Sensor type / measuring principle & Measurement uncertainty \\
\hline Air and water temperature & $\vartheta$ & Pt100 (accuracy class W 0.1$)$ & $\pm 1 / 3 \cdot(0.3+0.005 \cdot \vartheta)$ \\
Soil temperature & $\vartheta$ & Thermistor string & $\pm 0.5 \mathrm{~K}$ \\
Relative humidity & $\varphi$ & Capacitive humidity sensor & $\pm 2 \% \mathrm{r}$.h. for $10 \ldots 90 \% \mathrm{r} . \mathrm{h}$. \\
Volume flow (air) & $\dot{V}$ & Differential pressure & $\pm 10 \%$ \\
Volume flow (water) & $\dot{V}$ & Electromagnetic flow meter & $\pm 0.5 \% \pm 1 \mathrm{~mm} / \mathrm{s}$ \\
Pressure difference & $\Delta p$ & Ceramic fulcrum lever technology & $\pm 2 \%$ of full scale (range: \\
& & & $0 \ldots 300 \mathrm{~Pa}$ and $0 \ldots 1000 \mathrm{~Pa})$ \\
Electric power & $P$ & AC energy meter & $\pm 2 \%$ of reading \\
\hline
\end{tabular}

\section{MEASUREMENT RESULTS}

The results presented in this study are based on measured data of the cooling period in 2016 from June till September and the following heating period in 2017 from January till March. During the considered periods the test facility was operated from 7 am to $10 \mathrm{pm}$ every day of the week. Volume flow of supply air is controlled to be constant at $(950 \pm 95) \mathrm{m}^{3} \mathrm{~h}$; mass flow rates of supply and extract air are controlled to be equal. The following evaluation is subdivided into three parts. First, the system itself is evaluated regarding relevant performance parameters of summer and winter operation. Afterwards, the investigated system is compared with different reference systems regarding electrical and thermal energy demands. Finally, the performance of the geothermal system is evaluated.

\section{System Performance}

The evaluation of system performance is based on the investigated periods. During summer the electrical energy demand of the entire system is in the range of $750 \ldots 780 \mathrm{~W}$. This is primarily caused by the fans (about $85 \%$ ), whereas the remaining part is divided equally between other auxiliary energies (drive of the wheels, circulation pumps). An electrical Energy Efficiency Ratio

$$
\operatorname{EER}_{\mathrm{el}}=\frac{\int_{\mathrm{p}} \dot{Q}_{\mathrm{C}} \mathrm{d} t}{\int_{\mathrm{p}} P_{\mathrm{el}} \mathrm{d} t}
$$

of $\mathrm{EER}_{\mathrm{el}}=4.77$ is achieved for the overall system during summer period. The average dehumidification efficiency of the desiccant wheel is 0.93 . For the considered winter period an average moisture and heat recovery efficiency of 0.75 is achieved for the desiccant wheel. Thus, humidity ratio of process air is increased by $1.1 \mathrm{~g}_{\mathrm{w}} \mathrm{kg}_{\mathrm{air}}$ on average.

Comfort requirements according to cat. II in DIN EN 15251 (2012) were maintained during $96 \%$ of operation time within cooling period. This share accounts for $75 \%$ during the considered heating period. 


\section{System Comparison}

To further evaluate the investigated system it is compared with two reference systems relying on an electrical powered vapor compression chiller during summer operation. For winter operation moistening of process air is realized by adiabatic or isothermal humidification for the reference systems. The reference systems are simulated based on measured data of the investigated system (SGEO); oda and sup conditions as well as sup mass flow rate are assumed to be equal (sup: $22^{\circ} \mathrm{C}, 8$ $\mathrm{g}_{\mathrm{w}} / \mathrm{kg}_{\mathrm{air}}$ ). For the conventional reference system (DP-VC) a constant electrical Energy Efficiency Ratio of $\mathrm{EER}_{\mathrm{el}} 3$ for the chiller is assumed; the corresponding supply air temperature during summer is $18^{\circ} \mathrm{C}$. The second reference system for the cooling period (DW-VC) is a hybrid air conditioning system. The BHE is replaced by a vapor compression chiller $\left(\mathrm{EER}_{\mathrm{el}} 3.2\right)$, while the rest of the investigated system remains unchanged. The first reference system for winter operation (HP-AH) is relying on adiabatic humidification using an electrical powered impeller humidifier and the second reference system (HPIH) is equipped with isothermal humidification relying on an electrical powered steam humidifier, respectively. Fig. 4 shows the results of the system comparison for considered periods. Significant reductions in electrical energy demand can be achieved by the investigated system compared to the reference systems in summer. The savings sum up to $50 \%$ compared to the conventional system (DP-VC) and $34 \%$ compared to the hybrid system (DW-VC). The chiller unit (black bar) requires $61 \%$ of the total electrical energy demand for the system DP-VC, whereas approx. $1 \%$ of the system S-GEO are required for the BHE circulation pump. The thermal energy demand of the desiccant assisted systems (S-GEO, DW-VC) is increased by a factor of 1.2 compared to the conventional system (DP-VC), caused by the regeneration air heater.

During winter operation, the electricity demand for ventilation and distribution of the system HP-IH is increased by factor 1.8 compared with the investigated system. 

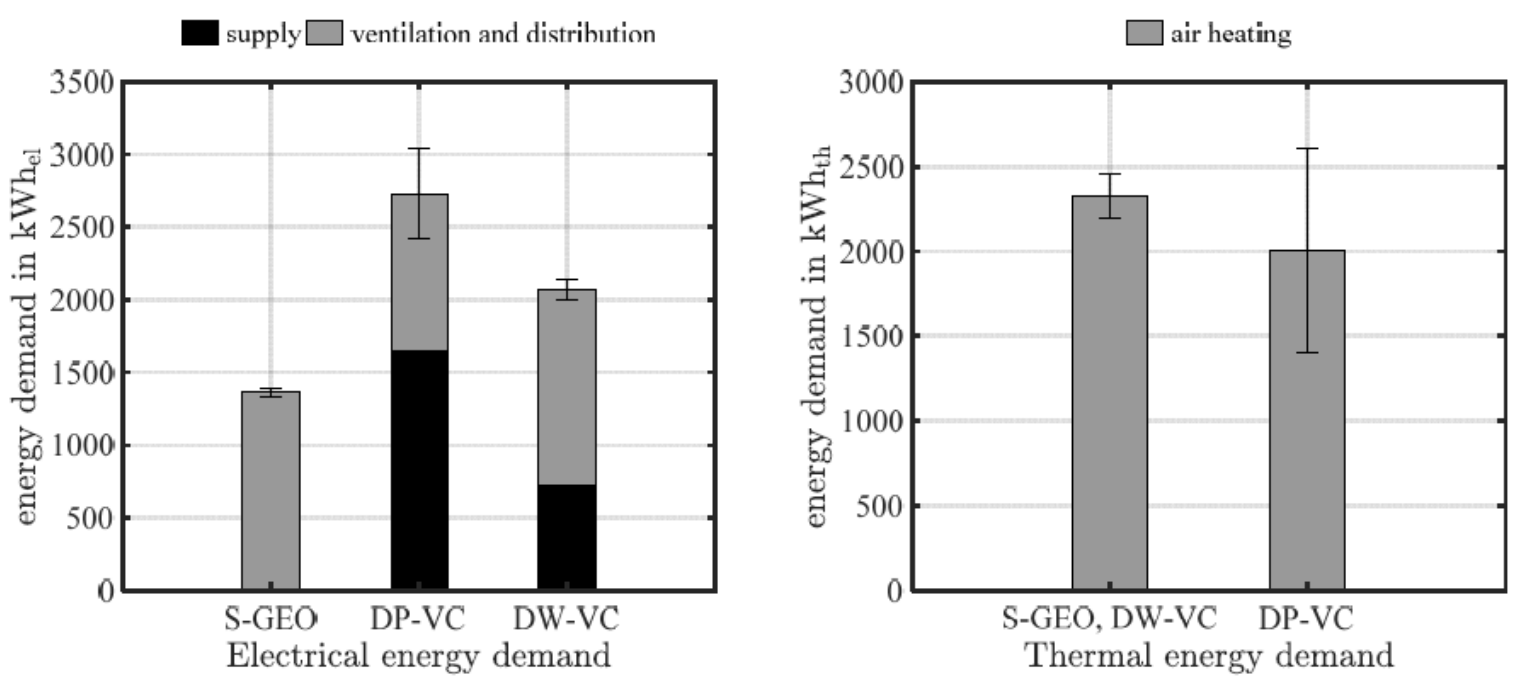

(a) cooling period

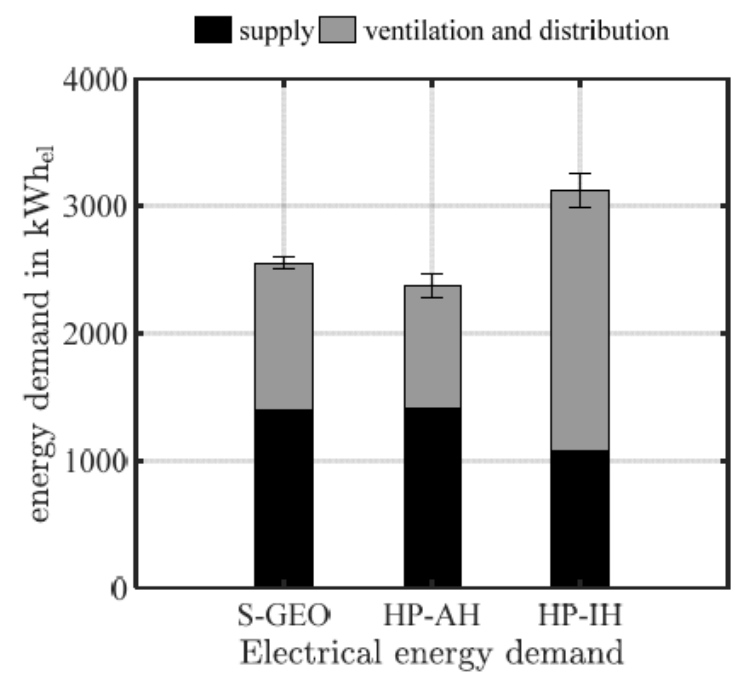

(b) heating period

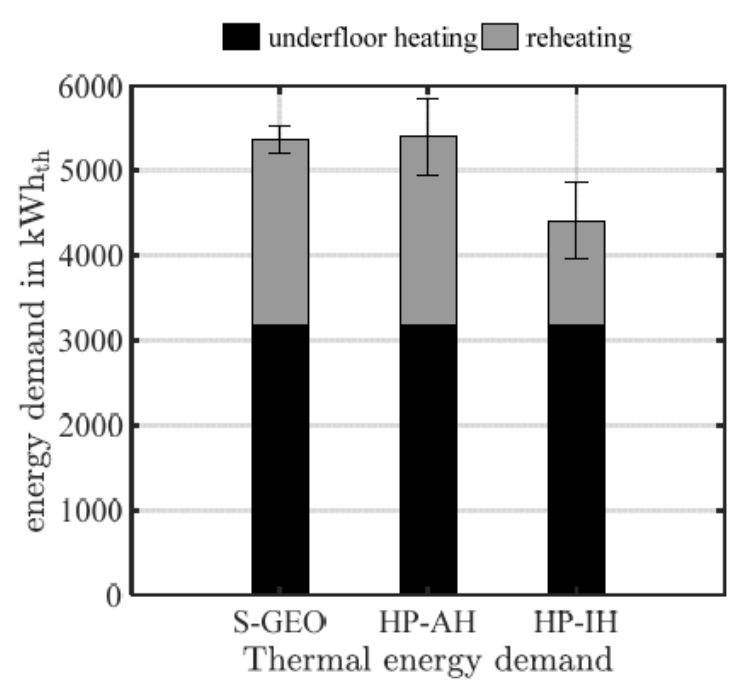

Fig. 4: Electrical and thermal energy demands of the investigated system (S-GEO) and reference systems (summer: DP-VC, DW-VC; winter: HP-AH, HP-IH) for the entire periods under consideration

Nearly $50 \%$ of this demand is associated with the electrical steam humidifier. Regarding the system HP-AH, the corresponding electricity demand is reduced by $16 \%$ due to the reduced pressure drop within the air handling unit. In total, the electricity demand of S-GEO is reduced by $18 \%$ compared with the isothermal assisted system (HP-IH); it is increased by $7 \%$ compared with the adiabatic assisted system. Thermal energy required for heating of process air to the desired supply air temperature is substituted by the steam humidifier (HP-IH). Therefore, the thermal energy demand for reheating within the other systems (S-GEO, HP-AH) is about $44 \%$ higher.

\section{Geothermal System}

The soil temperature $15 \mathrm{~m}$ below ground surface is found to be independent of seasonal related temperature fluctuations. Fig. 5 shows the temperature profile of the BHE in use (grey line) as well as the reference BHE (black line) for the period of around one year including summer and winter 
operation. The curves result from temperature averaging below $15 \mathrm{~m}$ for each BHE. Soil temperature at the BHE is significantly influenced by system operation in cooling and heating mode. The maximum peak temperature within cooling period is $18.1^{\circ} \mathrm{C}$ and the lowest temperature is $4.5^{\circ} \mathrm{C}$. The overall temperature increase of the soil during summer mode is crucial to maintain comfort indoor air conditions; during winter operation the overall temperature decrease is crucial to operate the GHP system efficiently. Balancing summer and winter operation in combination with natural regeneration, an equalized energy balance of the soil is achieved. The temperature of the undisturbed soil is found

to be $9.8^{\circ} \mathrm{C}$ before summer operation. A slight temperature influence of using the soil as primary heat sink and heat source is visible for the undisturbed soil at the reference BHE. Maximum soil temperature of $10.0^{\circ} \mathrm{C}$ is achieved at the beginning of winter operation and lowest temperature of the undisturbed soil $\left(9.7^{\circ} \mathrm{C}\right)$ occurs at the end of winter operation, respectively. With respect to measurement uncertainties, the present temperature difference is within the corresponding uncertainty of the thermistor strings. Therefore, no influence on the surrounding soil of the BHE in use can be detected.

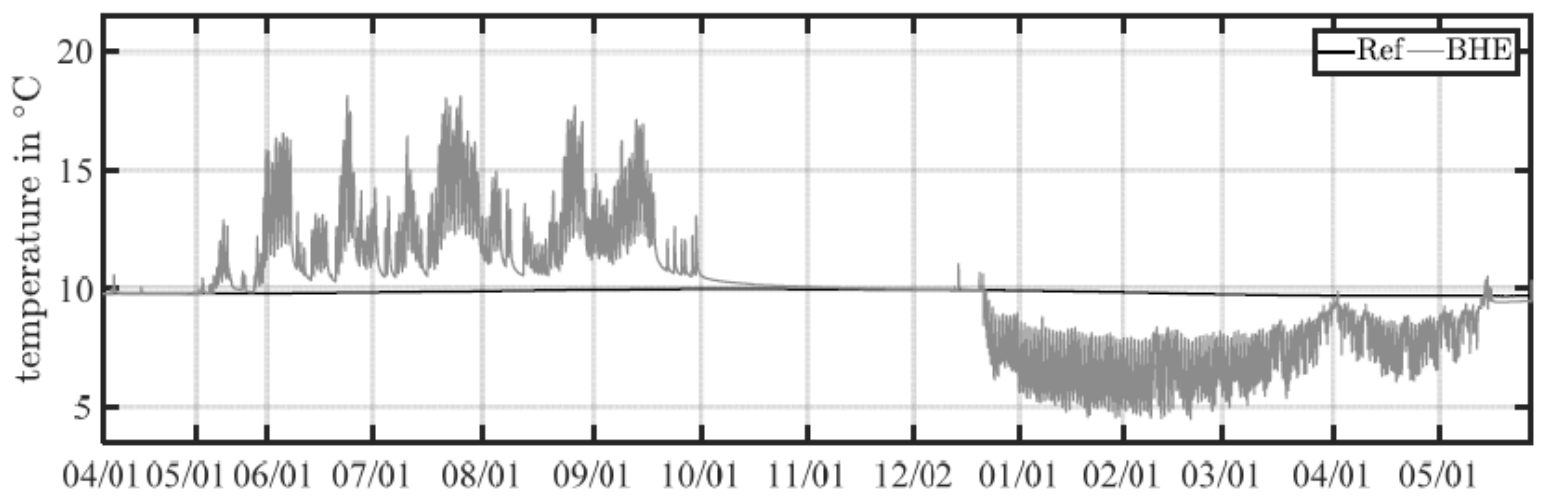

Fig. 5: Temperature profile for the operated BHE and the reference BHE

To further analyze the performance of the geothermal system, Fig. 6 shows the amount of energy transferred at the BHE as well as related performance parameters during summer (a) and winter (b) operation mode. Even though the BHE is only utilizable in conjunction with a heat pump for heat supply, both systems are evaluated separately for a direct comparison of summer and winter operation.

The energetic evaluation of the BHE is based on key figures as defined by Speerforck and Schmitz 2016), relating the amount of transferred thermal energy with the electrical energy demand for energy distribution 195 (pump). These are the Monthly Performance Factor (MPF) and the Seasonal Performance Factor (SPF): 
$\mathrm{MPF}=\frac{\int_{\mathrm{m}}\left|\dot{Q}_{\mathrm{BHE}}\right| \mathrm{d} t}{\int_{\mathrm{m}} P_{\mathrm{PU}} \mathrm{d} t} \quad \mathrm{SPF}=\frac{\int_{\mathrm{p}}\left|\dot{Q}_{\mathrm{BHE}}\right| \mathrm{d} t}{\int_{\mathrm{p}} P_{\mathrm{PU}} \mathrm{d} t}$
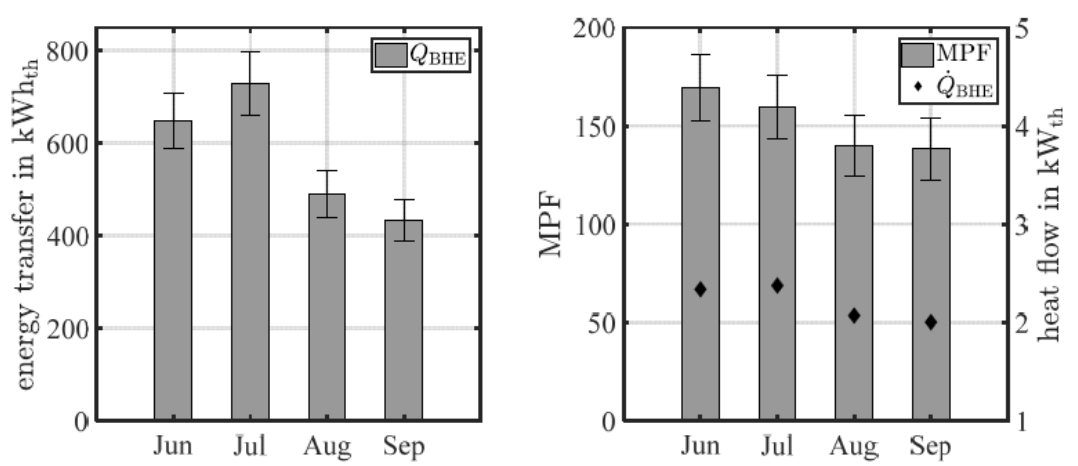

(a) cooling period
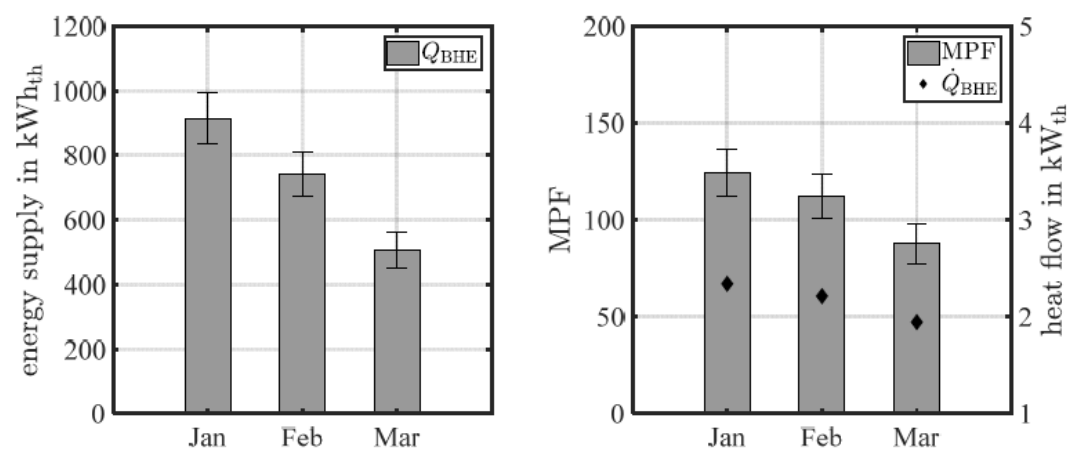

(b) heating period

Fig. 6: Amount of energy transferred at the BHE (left) and performance parameters of the BHE (right)

The monthly amount of thermal energy transferred at the BHE as shown in Fig 6 is a result of outside air conditions and the related cooling or heating loads. Even though in July the most thermal energy was transferred to the soil, the highest MPF was achieved in June $\left(\mathrm{MPF}_{\mathrm{su}, \max }=170 \pm 17\right)$, primarily caused by lower temperature increase of the soil, see Fig. 6 (a). The MPF exhibited the lowest value at the end of the cooling period in September $\left(\mathrm{MPF}_{\mathrm{su}, \min }=138 \pm 15\right)$. Evaluating the entire cooling period, a seasonal performance of $\mathrm{SPF}_{\mathrm{su}}=153 \pm 15$ is achieved, indicating a high efficiency of the geothermal heat sink. With regard to the investigated heating period, resulting BHE performance is shown in Fig. 6 (b). Decreasing monthly performance from $M_{P F} F_{w, m a x}=124 \pm 12$ in January to $\mathrm{MPF}_{\mathrm{wi}, \text { min }}=89 \pm 10 \mathrm{in}$ March is caused by decreasing heat flow as a result of decreasing average soil temperature level around the BHE. The resulting seasonal performance is $\mathrm{SPF}_{\mathrm{wi}}=110 \pm 11$.

Compared with cooling operation the performance during winter mode is lower in general due to higher volume flow of heat transfer medium to supply the evaporator of the heat pump. The electrical $\mathrm{SPF}$ of the ground coupled heat pump $\left(\mathrm{SPF}_{\mathrm{GHP}}=\mathrm{Q}_{\mathrm{GHP}}=\mathrm{W}_{\mathrm{GHP}}\right)$ is $\mathrm{SPF}_{\mathrm{GHP}} 3$, limiting the capacity of the geothermal assisted heat supply.

\section{CONCLUSIONS}

In this study experimental investigations of a desiccant and geothermal assisted air conditioning system during summer and winter mode are presented. The presented system is beneficial compared with reference systems. In combination with a ground coupled heat pump the geothermal system can be operated efficiently resulting in high seasonal performance factors for the BHE during the considered periods. Furthermore, the thermal energy balance is equalized throughout full year operation. 


\section{REFERENCES}

Angrisani, G., Roselli, C., Sasso, M., (2015), Experimental assessment of the energy performance of a hybrid desiccant cooling system and comparison with other air-conditioning technologies. Applied Energy, 138, 533-545.

Antonellis, S. D., Intini, M., Joppolo, C. M., Molinaroli, L., Romano, F., (2015), Desiccant wheels for air humidification: An experimental and numerical analysis. Energy Conversion and Management, 106, 355-364.

DIN EN 15251, (2012), Indoor environmental input for design and assessment of energy performance of buildings addressing indoor air quality, thermal environment, lighting and acoustics. Beuth, Berlin, Germany.

Fong, K. F. et al. (2011), Investigation on solar hybrid desiccant cooling system for commercial premises with high latent cooling load in subtropical Hong Kong. Applied Thermal

Engineering, 31, 3393-3401.

Kawamoto, K., Cho, W., Kohno, H., Koganei, M., Ooka, R., Kato, S., (2016), Field Study on Humidification Performance of a Desiccant Air-Conditioning System Combined with a Heat Pump. Energies, 9, 89.

La, D., Dai, Y., Li, H., Li, Y., Kiplagat, J. K., Wang, R., (2011), Experimental investigation and theoretical analysis of solar heating and humidification system with desiccant rotor. Energy and Buildings, 43, 1113-1122.

Mazzei, P., Minichiello, D., Palma, D., (2002), Desiccant HVAC systems for commercial buildings. Applied Thermal Engineering, 22, 545-560.

Slayzak, S. J., Ryan, J. P., (2000), Desiccant Dehumidification Wheel Test Guide: Technical Report. NREL/TP-235 550-26131, National Renewable Energy Laboratory, Golden (Colorado), USA.

Speerforck, A., Schmitz, G., (2016), Experimental investigation of a ground-coupled desiccant assisted airconditioning system. Applied Energy, 181, 575-585.

Speerforck, A., Ling, J., Aute, V., Radermacher, R., Schmitz, G., (2017), Modeling and simulation of a desiccant assisted solar and geothermal air conditioning system. Energy, 141, 2321-2336.

\section{ACKNOWLEDGEMENT}

This work is being conducted in the frame of a project funded by the Federal Ministry for Economic Affairs and Energy (www.bmwi.de), cf. project funding reference number 03ET1421A. 


\title{
NEW GENERATION SOLAR COOLING AND HEATING - EXPERIENCES FOR SUCCESFULL DESIGN AND OPERATION
}

\author{
Daniel Neyer1,2, Rebekka Köll3, Daniel Mugnier4 \\ danielneyerbrainworks 5 \\ Oberradin 50, 6700 Bludenz, Austria \\ Phone: +43 664 / 2826529, E-mail: daniel@ neyer-brainworks.at \\ University of Innsbruck, Unit of Energy Efficient Buildings, Innsbruck, Austria2 \\ AEE INTEC, Gleisdorf, Austria3 \\ 10 TECSOL, Perpignan, France 4
}

\section{SUMMARY}

Solar thermal and photovoltaic supported heating and cooling systems have an enormous potential to serve as environmental friendly technology for future cooling needs in buildings and industry. However, solar cooling 15 still faces barriers to emerge as an economically competitive solution. In past and ongoing IEA SHC Tasks findings of existing installations are collected and technical and economic solutions that are efficient, reliable and cost competitive are explored and evaluated.

Main findings are merged into key principles for successful design and operation. Further, twenty-nine plants are analysed and compared in a comprehensive format. The systems represent a wide mix of technologies, 20 applications and locations. All plants are analysed regarding their technical and economic performance and analysed trend wise under certain boundary conditions. In addition, a sensitivity analysis is performed varying certain boundary conditions to indicate their influence on the technical and economic performance of the SHC systems.

Several solar heating and cooling systems can be cost competitive and show levelized costs of energy below 25 those of reference systems while most of the plants can reach non-renewable primary energy savings greater than $50 \%$.

Key words: Principles for successful design, technical and economic solutions, IEA SHC Task

\section{INTRODUCTION}

On global level the energy demand for space cooling is rising rapidly. Actual 2'000 TWh electricity are used to drive air-conditioner or fans. This demand could rise up to three times till 2050 if energy efficiency is not increased strongly. Even with this increased efficiency the demand will still increase by a factor of roughly 1.5. Main reason for that rising demand are the economic and population growth and thus rising living standards 35 (OECD/IEA 2018). In the last decade this trend was indicated by the increase of sales of air-conditioners already (JRAI, 2017).

Solar driven heating and cooling systems are considered to be promising technical solutions to cover parts of this rising demand of air-conditioning with renewable energy. The IEA strategic plan focuses on solar thermal driven systems as key technologies, due to their potential to significantly reduce greenhouse gas emissions worldwide. The number of commercial deployments has increased steadily, and interest in solar airconditioning (SAC) has grown over the last years. A survey by Mugnier and Jakob (2015) has estimated the number of worldwide installations at nearly 1,350 systems in 2015. Although the number of commercial deployment has increased steadily in the last decades two main barriers are evident. (i) the lack of economic competitiveness and (ii) the lack of proven long-term energy performance and reliability.

To boost a sustainable market for solar thermal and photovoltaic driven new innovative cooling systems the IEA SHC Task 53 (T53) was conducted (Mugnier et al., 2014). It is based on IEA SHC work carried out in the last decade e.g. Task 38 (Henning, 2006) and Task 48 (Mugnier, 2011). Aim of this cooperative work is to develop solar driven heating and cooling systems which are reliable, efficient and cost competitive.

The work performed in SHC Task 38 is focusing on the monitoring and analysis of small (50 preengineered) and large-scale (customized) systems. It draws a bow from availability and performance of components, development of monitoring procedure, the deep analysis of the monitored and simulated systems. The lack of performance on component but especially on system level was identified as major issue. Thus, the focus of follow up IEA SHC Task 48 is on quality on component and system level as well as on market support 55 measures. 
In IEA SHC Task 48 focusing on Quality Assurance for solar cooling, the state of the art, characterization and future developments are discussed in a great detail on component level. Whereas the focus of the system level is on best practice and its technical and economic assessment. Activities for market support are assisted by labelling, financing models and policy advices.

60 The focus of the latest and ongoing IEA SHC Task 53 is on the performance, rating and benchmarking of demonstration project. It aims to analyze performance of monitored new generation solar thermal and PV supported cooling and heating systems. 29 systems were analyzed and summarized in comprehensive graphs showing trend of solar thermal and PV driven systems. A short summary of its results as well as key principles for successful design are presented in this paper.

\section{KEY PRINCIPLES FOR SUCCESSFUL AIR-CONDITIONING DESIGN}

Merging the quintessence of these IEA Task 48 activities led to the verbalization of 10 key principles. These qualitative principles are the core of a compendium including three build, monitored and optimized systems in a Guide called "The Solar Cooling Design Guide: Case Studies of Successful Solar Air Conditioning Design" 70 (Mugnier et al. 2017). The design guide is intended as a companion to the IEA Solar Cooling Handbook (Henning et al. 2013). Three selected examples are used to explained step by step the design in different ranges of scale and technology. Nevertheless, it should be noted that there are many other attractive solar cooling technology solutions.

75 A first overview of the 10 key principles (Mugnier et al., 2017) is given in the following list; more details on the scientific background and links to past and latest research results are published by Neyer et. al (2018b).

- Principle 0: Reduce energy demand before applying renewables

- Principle 1: Choose applications where high annual solar utilization can be achieved

- Principle 2: Avoid using fossil fuels as a backup for single effect ab-/adsorption chillers

- Principle 3: Design the ab-/adsorption chiller for relatively constant operation at near full load

- Principle 4: Use wet (or hybrid) cooling towers whenever possible

- Principle 5: Design the solar collectors for operation at average (not peak) solar radiation levels

- Principle 6: Keep the process flowsheet simple and compact

- Principle 7: Provide thermal storage capacity and hydraulics in a form that matches the thermal requirements of each application

- Principle 8: Minimise parasitic power

- Principle 9: Minimise heat losses

- Principle 10: Apply appropriate resources to designing, monitoring and commissioning

A survey was conducted, intending to review these Principles in the light of additional case studies, and to provide opportunity for the solar cooling expert community to further elucidate on their solar cooling experiences. The survey was sent to 33 solar cooling experts, they were asked to answer five open- and closed ended questions. The main interest is on the rating and ranking of the key principles and is reconsidered with the following two questions.

- How important do you consider each of the 10 design principles $(5=$ very high, $951=$ very low)?

- Rank the four most important principles $(1=$ the single most important principle, $2=$ the second most important principle, etc to 4 )

The results are separated into the origin of the experts and their profession (Neyer et al., 2018b). In Fig. 1 the 100 specific average and its standard deviation is shown as bar diagram. The respective number of counts (\#counts 1-4) to be under the main four principles is displayed as marker. 


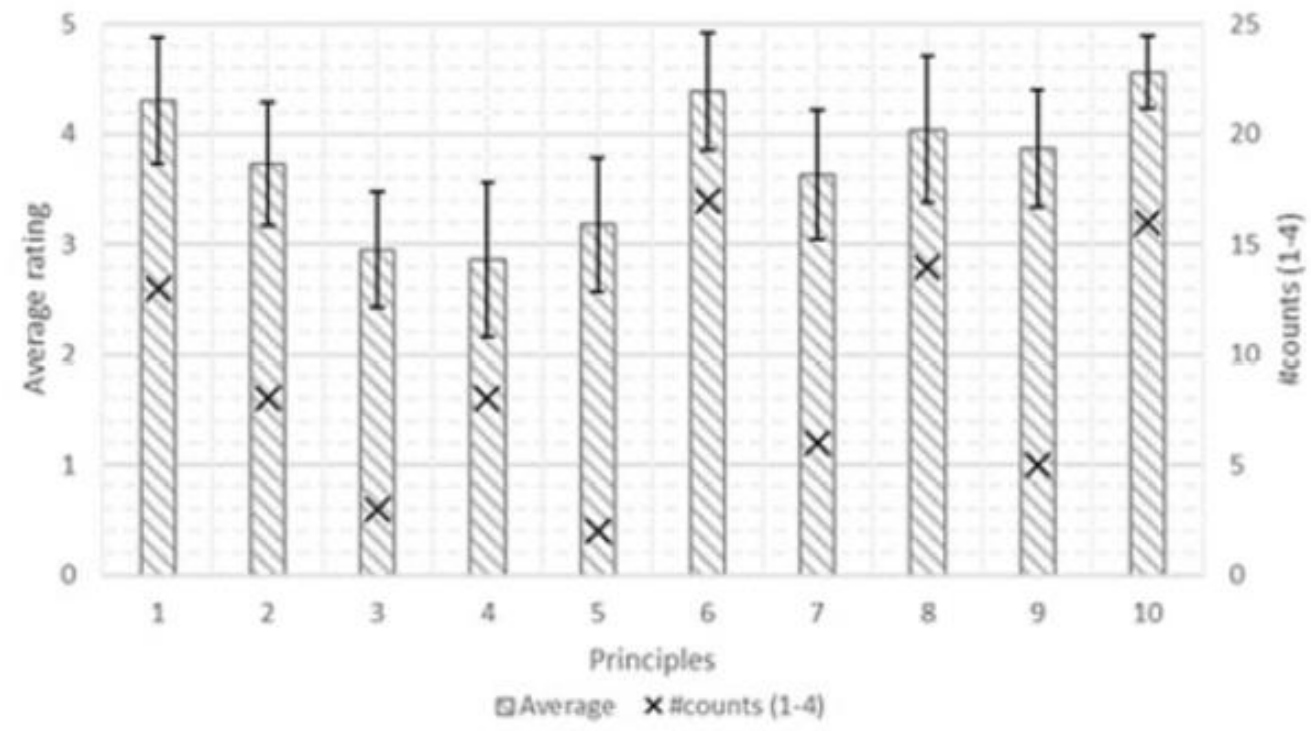

Fig. 1: Average rating of 10 key principles by the interviewed solar cooling experts (Neyer et al., 2018b)

The mean average rating yields between 2.9 and 4.6; the total average is $3.78 \pm 0.62$. Four of the principles show values above 4 (principles $1,6,8,10$ ), three principles recline between 3 and 4 (principles 2, 7, 9) and three principles can be considered to be equal 3 (principles 3, 4, 5). From number of counts three principles gather more than 15 counts (principles $6,8,10$ ), three principles recline between 5 and 15 counts (principles 1,2,4) and four principles gather less than 5 counts (principles 3, 5, 6, 9). This result emphasizes three categories of principles. (i) essentials, being undisputed under the experts (ii) important ones, still high in ranking but from second attention and (iii) controversial, that can be solved in different ways, if certain knowledge is available.

\section{TECHNICAL AND ECONOMIC ASSESSMENT OF NEW GENERATION SYSTEMS}

Within the IEA SHC Task 53 "New Generation of Solar Cooling" 29 solar heating and cooling systems are analyzed based on their economic and technical performance. The aim is to show if and under which conditions SHC systems can be cost competitive. The assessment is performed by means of the T53E4-Tool (Neyer et al., 2016) and is based on monthly energy balances provided for each of the entire systems. The Tool provides a wide range of fundamental data for several technologies and components such as 120 efficiencies, investment and maintenance cost but also country specific information like energy prices and primary energy conversion factors. Based on some basic information of the system, technical, environmental and economic Key Performance Indicators (KPI) are calculated on system level.

Main technical key figures are the non-renewable primary energy ratio (PERNRE), the non-renewable primary energy savings ( $f_{\text {sav.NRE }}$ - comparison of PERNRE of SHC System with PERNRE of a reference system) as well as an electrical equivalent seasonal performance factor $\left(\mathrm{SPF}_{\text {equ }}\right)$. The standard predefined reference 125 system is integrating a natural gas boiler for DHW and space heating purposes and an air-cooled vapor compression chiller for cooling.

Main economic key figure is defined and expressed as CostRatio (CR). Under the consideration of specific investment, replacement, operation- and consumption-based costs, the annualized costs for the entire SHC 130 system and the defined reference system are calculated by using the annuity method. Based on these costs the levelized life cycle cost (levelized cost of energy: LCOE) are calculated and used to express the additional costs of an entire SHC system. The CostRatio is the ratio of the LCOE of the SHC system to the LCOE of the predefined and standardized reference system.

135 The analysed SHC systems present a great variety of different system designs and applications. The technologies are clustered according to the main component (i) PV: electrical driven and photovoltaic supported systems, (ii) ST: heat driven and solar thermal supported systems, (ii) ST+HP: electrical driven and solar thermal supported systems and (iv) ST+PV: systems supported by photovoltaic and solar thermal. The applications are clustered according to the energy demands of 
space heating (SH), cooling (C) and domestic hot water (DHW) and different combinations. Fig 2 gives an overview on the distribution of the 28 analysed systems.
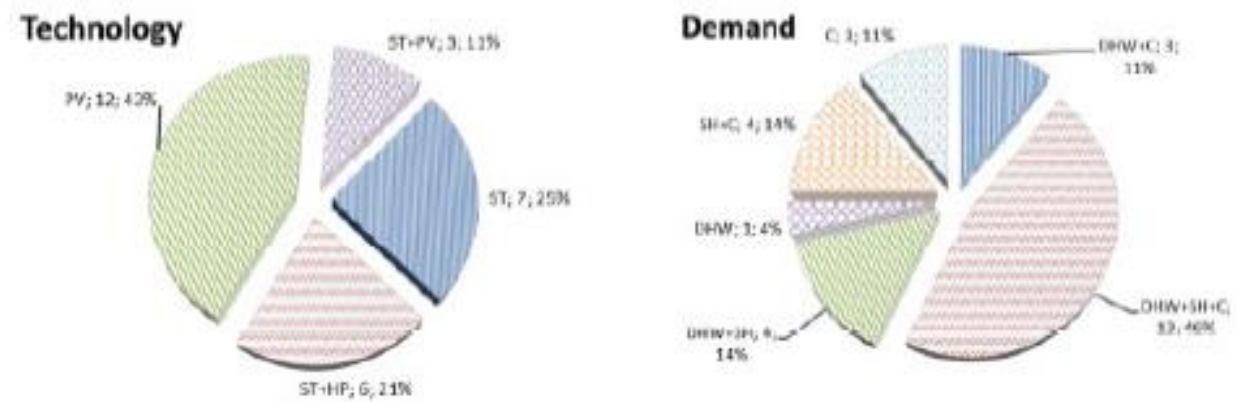

Fig. 2: Overview of chosen SHC systems for the assessment summarized by the used technology (left) and the application / energy demand (right)

The analysed systems are dominated by small scale systems with a total heating/cooling capacity of below $10 \mathrm{~kW}$ (c.f. Fig 3) and hence also deliver rather small amount of energy over the year. The smaller systems in the assessment are mainly PV systems, whereas most of the solar thermal systems have an energy production of more than $100 \mathrm{MWh}$. The medium sized systems are dominated by systems using heat pump in combination 150 with solar thermal collectors and PV systems.
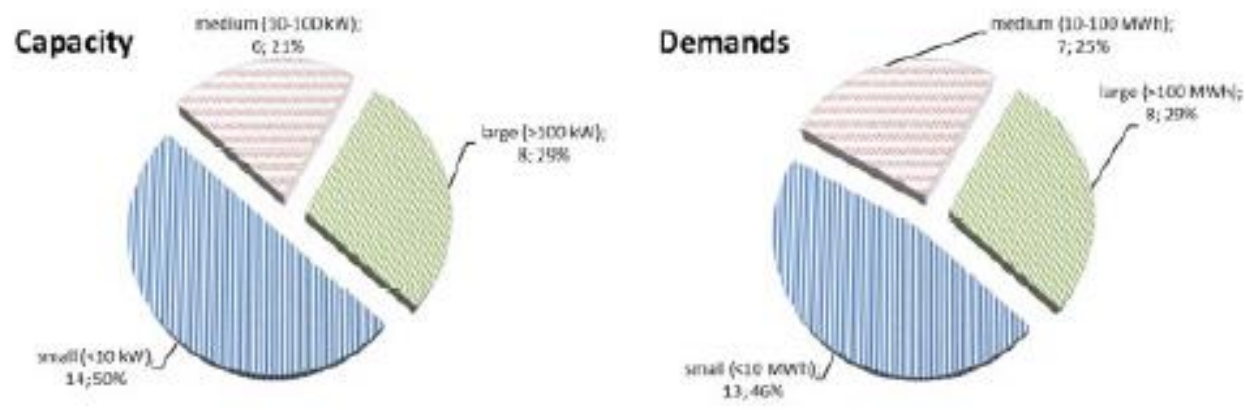

Fig. 3: Plant distribution categorized by the system heating and cooling capacity (left) and by the total yearly energy consumption (right)

A detailed description of each configuration is presented in Köll 155 and Neyer (2018).

Fig. 4 shows the comparison of the non-renewable primary energy savings ( $\left.f_{\text {sav.NRE}}\right)$ on the $\mathrm{x}$-axis and the cost ratio (CR) of levelized costs of energy on the $y$-Axis. The higher the $f_{\text {sav.NRE, the more primary }}$ energy savings and the lower the cost ratio the cheaper the renewable system. A CRof one refers to the reference system 160 (REF), thus if the value is below one the entire SHC system is more economic than the reference system. The trend analysis of all plants (Fig. 4) shows that both, solar thermal as well as PV driven SHC systems are cost competitive at lower solar fraction and lower primary energy savings respectively. The cost ratio increases with the increase of primary energy savings. Nevertheless, it can be seen that the variation in this area is much higher and there are several examples showing also cost savings at high solar fraction. 


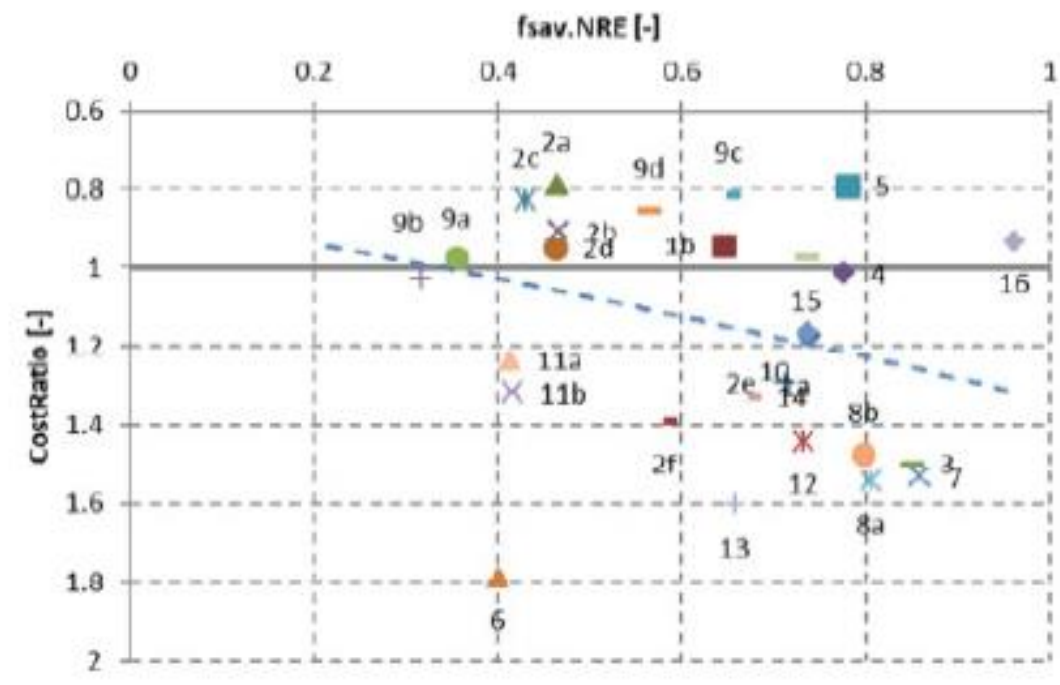

Fig. 4: Comparison of levelized costs of energy as cost ratio and non-renewable primary energy savings for the SHC systems in relation to the reference system. (Köll \& Neyer, 2018)

These results are based on predefined boundary conditions. If one of these boundaries is changing the results might change as well. Thus, the crucial boundary conditions are evaluated with a sensitivity analysis. Accordingly, six boundaries are changed in a wide range and the results are summarized for the overall trend, and the trends for northern and southern location separated according to the solar technology (PV or ST). The six parameters are the investment cost, electricity and natural gas price, the auxiliary demand and energy 175 output as well as the conversion factor for electricity. Each parameter is varied seven times in a selected range to represent a reasonable and market relevant series. Representative results for the variation of investment cost are shown in Fig. 5. The effect is larger in northern located SHC systems as they are more investment dominant compared to the southern location, were the 180 (cooling) demand and thus the fuel costs are more important. The blue $100 \%$ lines indicate the initial state. The southern origin trend (left) starts at a CR of roughly 0.8 and reaches 1.1 for ST supported and 1.05 for PV supported systems. If the investment costs can be decreased this small advantage of PV is equalized. The trends for ST and PV gets equal at $-15 \%$, ST shows a small advantage at $-30 \%$. This change is pointing on the fact that ST is slightly more investment dominated compared to PV driven systems at the same level of savings.

The northern trend is representing a much stronger gradient compared to the southern locations. 185 Its original trend starts at a CR of roughly 0.9 with savings of $30 \%$ but is ending at higher savings at a $\mathrm{CR}$ of 1.6. In the northern locations the PV is showing slightly higher CostRatio's. If the costs are reduced accordingly, the CR drops and a large part of the trendline is ending at CRs smaller than 1. The change in trendlines shows that for northern location the PV supported systems are much more investment dominated than the ST supported ones. 190 This is especially driven by the demands (heating and cooling) and its coincidence of solar irradiation but also due to the design of the systems
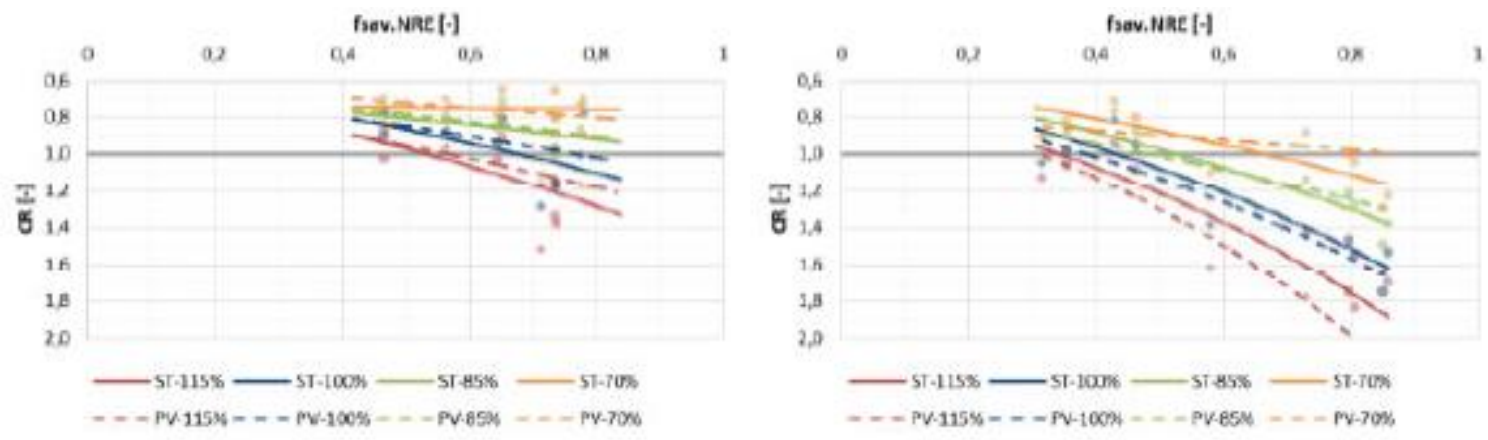

Fig. 5: Sensitivity analysis on investment cost for the southern (left) and northern (right) location 195 separated for PV and ST (Köll \& Neyer, 2018) 
The most significant influence on economics (other sensitivity results are not shown here) is driven by the investment costs. With standard investment costs parity of levelized costs of energy $(C R=1)$ is reached by systems designed for less than $30 \%$ non-renewable primary energy savings. With an investment cost reduction 200 of $15 \%$ already systems achieving $65 \%$ savings can reach parity. If a cost reduction of $30 \%$ (not unrealistic, c.f. ROCOCO (Preißler, 2008) can be reached the trend line considerably undermatches a CostRatio of 1 . Thus, the SHC systems can provide an economic benefit over its life time and can possibly assure more than high non-renewable primary energy savings.

Furthermore, a significant influence is occurring due to changes of natural gas costs used for the references 205 systems but also in some SHC plants for backup heater. The standard price is defined to be $5 € \mathrm{ct} / \mathrm{kWh}$, future changes in the prices depend a lot on political, economic and exploration boundary conditions and are hardly possible to be foreseen. Thus, the price is only varied in a range that is already possible due to the change from commercial to private consumers. When the natural gas costs are increased by $50 \%$ to $7.5 € \mathrm{\epsilon t} / \mathrm{kWh}$ the parity can be achieved by systems with up to $60 \%$ savings instead of $30 \%$.

\section{CONCLUSION}

Summing up, both technologies, solar thermal and PV, can be integrated to support a HVAC system accordingly and both systems can be competitive against reference systems when they are well designed and boundary conditions are favorable. The background of the collected best practice examples and its expressed 10 key principles can help to find a proper design and support with general qualitive hints and question to successfully design new generation solar heating and cooling systems. The presented technical and economic assessment of 28 plants and configurations shows that 9 plants were able to reach cost parity or CR even lower than 1 under the present boundary conditions. If boundaries are changing according to the sensitivity analysis already up to 16 plants would reach CR lower than one. Under these conditions best cases come up with CR of roughly 0.7 , presenting $30 \%$ lower levelized cost of energy for the entire systems compared to the reference system!

In general, economics of SHC systems are mainly investment cost driven whereas the reference systems are dominated by the fuel costs. Therefore, SHC systems can be considered as cost efficient if they are integrated for covering baseload and in combination with conventional system for covering peak demands. 225 Although from environmental point of view solar autonomous systems should be from highest interest, they come up with higher costs but also with higher primary energy savings. Thus, future R\&D priority should focus on investment cost reduction (materials, mass production, simplification, etc.). Minor priority, but only from an economic point of view, is required on efficiency 230 measures. However, efficiency and respective auxiliary demand reduction can get more significant if the first priority was successful and investment costs are getting lower.

\section{REFERENCES}

Henning, Hans-Martin (2006): IEA SHC Task 38. Solar Air Conditioning and Refrigeration. Edited by SHC Solar Heating \& Cooling Programme. Germany.

Henning, Hans-Martin; Mugnier, Daniel; Motta, Mario (Eds.) (2013): Solar Cooling Handbook. A Guide to Solar Assisted Cooling and Dehumidification Processes. Basel/Berlin/Boston: Ambra.

JRAIA (2017): World Air Conditioner Demand by Region. Edited by The Japan Refrigeration and Air Conditiong Industry Association.

OECD/IEA (2018), The Future of Cooling. Opportunities for energy efficient air conditioning, Edited by IEA Publications, International Energy Agency

Köll, R.; Neyer, D. (2018): Monitoring data analysis on technical issues \& on performances. Deliverable C3, IEA SHC Task 53. Edited by International Energy Agency.

Mugnier, D. (2011): Quality Assurance \& Support Measures for Solar Cooling Systems - IEA SHC Task 48.

Edited by SHC Solar Heating \& Cooling Programme. http://task48.iea-shc.org/

Mugnier, D. (2014): New Generation Solar Cooling \& Heating Systems (PV or solar thermally driven systems).

IEA SHC Task 53. Edited by SHC Solar Heating \& Cooling Programme. http://task53.iea-shc.org/ Mugnier, D.; Jakob, U. (2015): Status of solar cooling in the World. Markets and available products. In WIREs Energy Environ 4 (3) 
Mugnier, D.; Neyer, D.; White, S. (Eds.) (2017): The Solar Cooling Design Guide - Case Studies of Successful

Solar Air Conditioning Design. Berlin, Germany: Wilhelm Ernst \& Sohn.

Neyer, Daniel; Neyer, Jacqueline; Stadler, Katharina; Thür, Alexander (2016): Energy-EconomyEcology-Evaluation Tool, T53E4-Tool, Tool Description and introductory Manual. Deliverable C3-1

Neyer, D.; Ostheimer, M.; Dipasquale, C.; Köll, R. (2018a): Technical and economic assessment of solar heating and cooling - Methodology and examples of IEA SHC Task 53. In Solar Energy.

Neyer, D.; Ostheimer, M.; Mugnier, D.; White, S. (2018b): 10 key principles for successful solar air conditioning design - A compendium of IEA SHC Task 48 experiences. In Solar Energy.

Preißler A., Selke T., Sisó A., LeDenn, Ungerböck R. Rococo - Reduction of costs of solar cooling systems,

European Project ; Project No. TREN/05/FP6EN/S07.54855/020094, Specific Support Action, Vienna 2008

\section{ACKNOWLEDGE}

The funding of Austrian participation at the IEA SHC Task53 through the Federal Ministry for Transport, Innovation and Technology (BMVIT) is gratefully acknowledged.

The funding of French participation at the IEA SHC Task 53 through the French Agency of Environment and Energy management (ADEME) is gratefully acknowledged. 


\title{
HOW HEAT AND COOLING STORAGES BENEFITS FROM ECONOMY OF SCALE
}

\author{
B. Sc. Flemming Ulbjerg, B. Sc. Patrick Durup Thomsen, M.Sc. Anders Dyrelund \\ Ramboll Energy. \\ Englandsgade 25, 5100 Odense C. Denmark \\ Phone +45 51611000 \\ E-Mail: FU@ramboll.com PDT@ramboll.com AD@ramboll.com
}

\section{SUMMARY}

Almost all energy sources, which can replace fossil fuels, are of low quality or not always available when needed. We have excess heat in summer and too much free cooling during the winter. Likewise, we have surplus of electricity in some hours, days and weeks and deficits in others. In the future there will be increasing demand for energy storage on a system basis - in particular thermal storages for storing heat and cooling directly, stimulating district heating (DH) and district cooling (DC) while shaving uncontrolled peaks in the power systems. Thus, smart integration of storage in the thermal systems can replace and eliminate the need for expensive electric batteries.

The bigger, the better is often used as a guiding term describing different topics, where size or scale matters. In the case with thermal storage technology, this is absolutely true.

The two main drivers for investing in thermal storages are:

1. The marginal capacity investment goes down, when size goes up.

2. The energy losses in pct. of the volume from a thermal storage decreases, as size is increased. All energy losses from the thermal storage can be attributed to the construction surfaces. When lengths increase, surface increase by second order, whereas the storage volume increase by third order.

Investments in large cost-effective thermal storages will stimulate development of DH and secure that all available surplus heat is collected. That will in turn stimulate development of DC showing the way for an efficient and cost-effective transformation to a low carbon society.

\section{INTRODUCTION}

The integrated energy system, including the 4 energy carriers: electricity, DH, DC and gas, is vital for cost-effective and efficient integration of renewable energy sources. The thermal storage technology is one of the key-technologies in this transformation, ensuring maximal utilization of fluctuating renewables such as solar, wind and surplus heat, e.g. from cooling and CHP plants.

Almost half of all end-use energy demand in our modern society is used to deliver thermal comfort, heating in winter and cooling in summer. Moreover, many industrial processes need cooling, or they deliver surplus heat constantly during the year. A good and recent example is the development of projects where excess low temperature heat from some of the world largest data centers is being harnessed, upgraded and utilized in large DH systems.

\section{WHERE TO APPLY LARGE THERMAL STORAGES}

In most parts of the world there is surplus of cheap heat but expensive cooling in summer and the opposite in winter. Therefore, it is an old idea to establish seasonal storage for balancing production and demand of heat and cooling. Fortunately, this idea materialized thanks to economy of scale. Whereas it would not be feasible for one individual building alone to store heat or cold on a seasonal basis due to energy losses, it is possible and cost-effective in large scale, if many buildings are interconnected, e.g. at campus or city level. In mild climates, e.g. Denmark, DH (including thermal storages) is the most cost effective low carbon solution in almost all towns, but DC is only cost effective in clusters of commercial buildings. In warmer climate both DH and DC are the most competitive solution in most cities and at all campuses. In warm climate, DH will only be competitive in very densely residential areas, whereas DC will be competitive in most places. Thus, where will be a market for large thermal storages for DH and/or DC in most urban areas and all campuses.

We will compare costs and temperature losses for small and large storage tanks and not least for our newly developed heat storage pit. 


\section{THE HEAT STORAGE BENEFITS FROM ECONOMY OF SCALE}

In the following we look at the concrete benefits of increasing capacity and geometric scales of a large thermal storage. The figure below shows the economy of scale comparatively for a small one family house (steel tank) going up to larger storages for $\mathrm{DH}$.

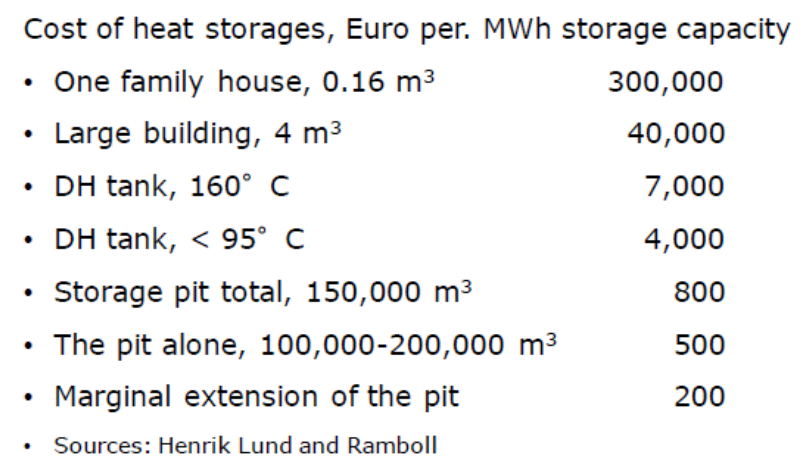

Fig. 1: Economy of scale for heat storages

In the figure below, we compare costs of storages for the $\mathrm{DH}$, taking into account temperature and size.

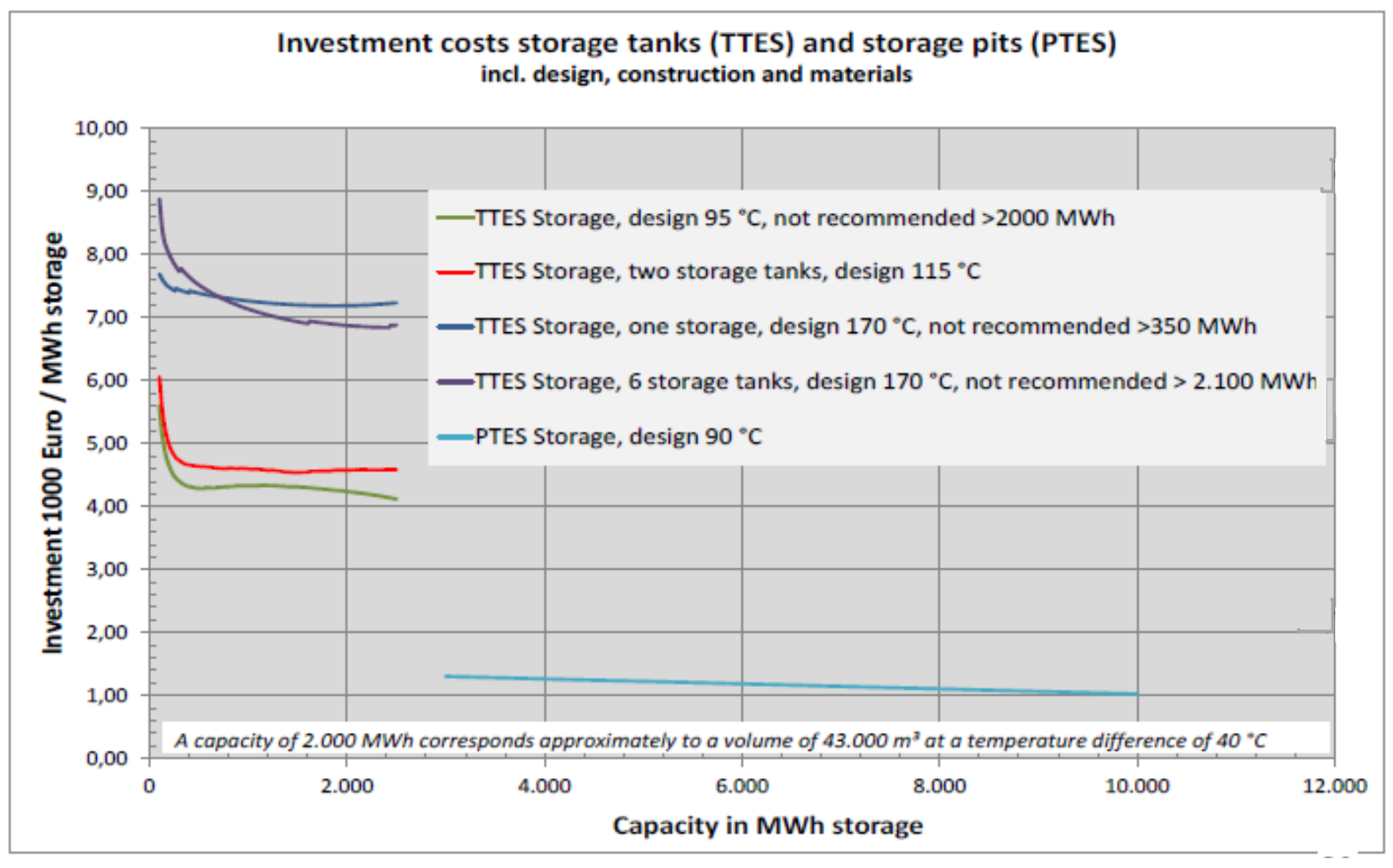

Fig. 2: Unit costs for large thermal storages

The cost break-down is also very much depending on the total size of the storage. In the charts below example is given on a size basis when establishing pit storage of various sizes. 

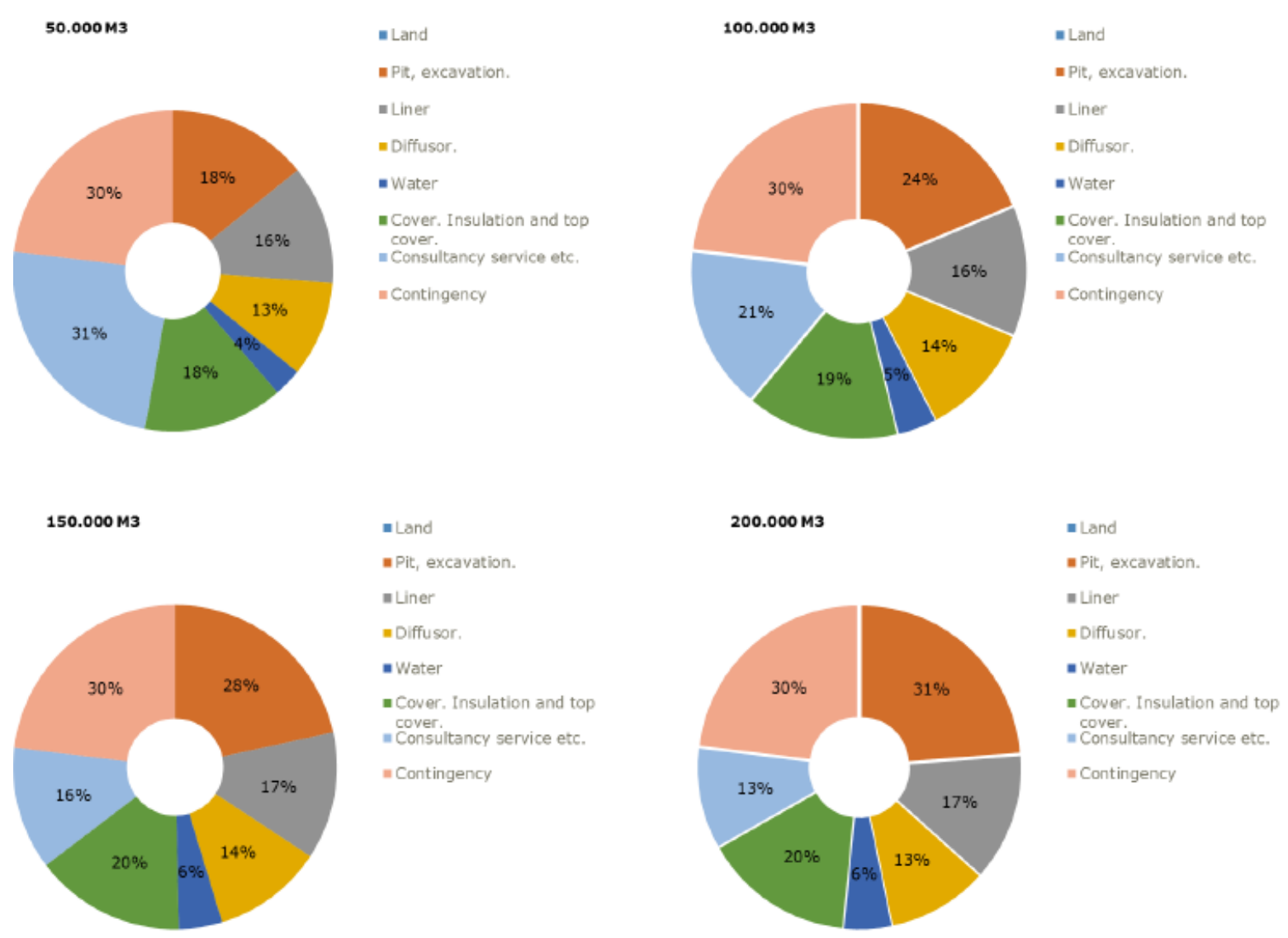

Fig. 3: Cost break down of the pit storage on a size basis

In order to compare the costs of operation, the heat losses and the number of annual load cycles have to be taken into account. The annual heat loss in pct. of the stored volume is several hundred pct. for small tanks, whereas the annual load cycles typically can be 365 . For the large heat storage pits, the annual heat loss, e.g. at the newest plant in Toftlund, Denmark, is estimated at approximately $15 \%$ of the storage volume in stable 95 operation. In case the storage only stores solar heat from summer to winter to cover up to $60 \%$ of the heat production, the storage will almost have two load cycles per year, and thus a relatively stand-by heat loss of less than $10 \%$. In case the storage can be used to store other fluctuating energy sources, e.g. from CHP plants and electric boilers in the winter period, the relatively heat loss will be even lower due to the higher degree of utilization. In the first years after construction the losses from a pit storage has shown larger as the soil around 100 the storage has to be heated to be part of the storage capacity. Also, unexpected water in the insulation material, e.g. from leaks during the construction phase reduces the insulation capability compared to the theoretic design loss calculations until the insulation material is completely dry.

\section{THE HEAT STORAGE PIT}

The heat storage pit is a simple innovative combination of the storage tank technology and the technology of modern protected landfills sealed by a water tight plastic liner. The only new element is the floating insulating cover. We have taken part in development of this technology since the first test projects at Marstal District Heating in Denmark and can present the lessons learned and the newest design concepts which are tested in full scale.

- Test plants with subsidy 
- $10,000 \mathrm{~m} 3$ Test plant in 2010 in Marstal

- 70,000 m3 Full-scale test plant 2012 in Marstal

- 62,000 m3 Full-scale test plant 2014 in Dronninglund

- Commercially, without subsidy, new floating cover

- $125,000 \mathrm{~m} 3$ Gram district heating 2015

- $200,000 \mathrm{~m} 3$ in Vojens district heating 2015

- $70,000 \mathrm{~m} 3$ in Toftlund district heating 2017

- $150,000 \mathrm{~m} 3$ in Løgumkloster district heating 2017/18

Several more in the pipeline, may be 100 in 2025

Fig. 3: Development of heat storage pits in Denmark

In the picture below, we see a heat storage pit under construction. In case an old sand pit is available, this can be used as a basis for the pit. In case the top layer of sand is filtered there is no need for other materials. In case there is no pit available, the pit can be constructed with an embankment around the pit, thereby achieving soil balance. Only a thin layer of fine sand is necessary to protect the liner.

The diffuser tower is the same construction, as the ones used inside the smaller steel storage tanks and are as such considered fully tested, except that corrosion protection is necessary. One might think that a wide heat storage pit would require several diffuser towers, but surprisingly one tower is enough to ensure stratification of the water.

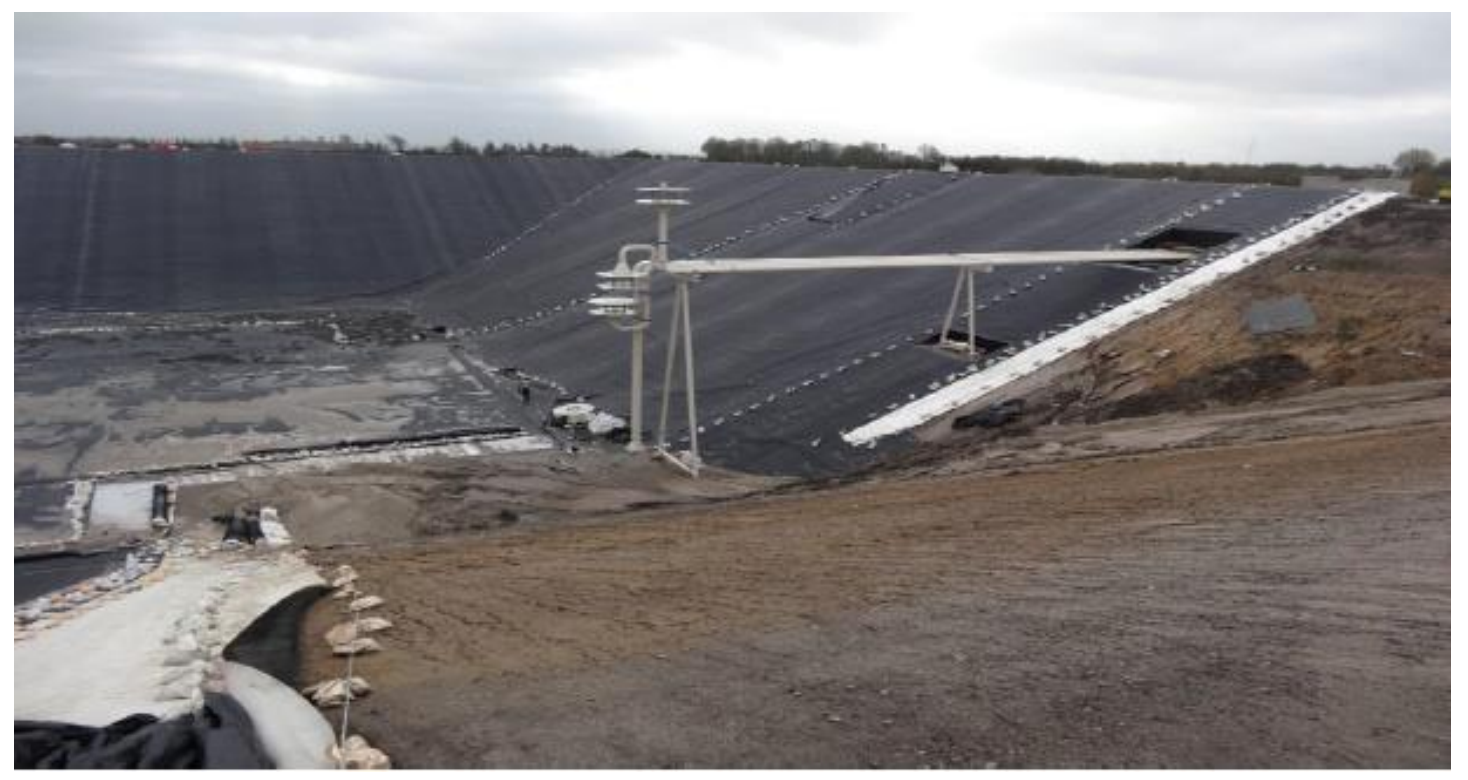

Fig. 4: Heat storage pit under construction

The floating cover, which replaces a traditional construction with pillars and decks, as we see in the old ancient cisterns, is vital in order to reduce costs. In the first plants, two main principles have been tested. Both rely on a top liner which shall prevent the water in the pit from penetrating into the insulation and a top liner on top of the insulation which shall drain off rain water and water from melting snow.

It has been a challenge to drain water from the almost horizontal surface efficiently and it has also been a challenge to avoid holes in the top liner, implying that special attention must be applied during the liner assembly phase. Execution of laying and welding of liners should be carried out using proven and standardized methods with extensive testing and under strong supervision during the entire phase. 
As with regards to the insulation material used in the lid construction, there has so far been used two different types of material: plastic foam and ceramic clay (LECA).

As it is impossible to prevent oxygen in the water it is necessary to separate the water in the pit from the district heating water as well as from the antifreeze in the solar heating system, in case solar is one of the energy sources.

\section{THE COLD STORAGE PIT}

Storing cooling from winter to summer is not that difficult. For centuries, it was e.g. big business to use ice from our lakes in large cold storage cellars for cooling, but the electrical refrigerator took over. In the old days, 145 buildings were designed to store cooling directly from the cold underground, but modern buildings need active cooling and air conditioning, which in most summer scenarios is supplied by electrical chillers. They are expensive and in some cases, we can observe that the uncontrolled use of electricity leads to brown-outs or worst case a black-out in the electrical grid.

Today we combine the good old traditions with new technologies to save cost and energy. Ground source 150 cooling is an efficient way of storing cooling from winter to summer. This can of course only be applied in regions where hydrological conditions allow it and the temperature is cold enough. Ice storages and chilled water tanks can level the daily fluctuations and save expensive electricity, but not for days and weeks. The storage technologies mentioned in this paper is not temperature specific as such, and can therefore be applied in cooling applications as well as the perhaps more acknowledged purpose in various heating systems. The 155 concept does not change, switching from hot to cold water, only the design parameters change. Large-scale thermal storages are cost effective regardless of temperature levels. The storage pit technology can therefore be used for storing cold water. So far, this idea is only in the conceptual design phase. The biggest problem seems to be that land is expensive in districts with large cooling demands.

An alternative solution is to utilize underground caverns where the underground is solid rock or limestone at the given geographical location. which could be cost effective in case the value of the gravel or rock could compensate for the construction cost. There is e.g. established a chilled water storage in an underground cavern in Finland.

\section{CONCLUSION}

The concept for the large-scale thermal storage tanks and pits, have proved to be a cost effective and energy efficient way to integrate fluctuating and cheap sources of hot water into our energy supply. However, the presence of a distribution network is essential in order to benefit from economy scale.

Traditional tanks for district heating, storing daily or weekly fluctuations can have between 50 and 365 load cycles a year and has proven to be the most cost-effective storage technology for volumes between 1,000 and $100,000 \mathrm{~m} 3$.

For integrating monthly and seasonal variations, the number of load cycles per year goes down, and the cost per stored energy goes up. Therefore, the heat storage pit can be an interesting solution. If cheap land is available and the main purpose is seasonal storage, e.g. to store solar heat to cover up to $50 \%$ of the annual heat demand, heat storage tanks are too expensive and pits the only cost-effective solution at the moment. In case the storage can benefit from daily and weekly fluctuations from e.g. wind, one or two more load cycles can be added and 180 the costs per unit will be reduced further. 


\section{OPPORTUNITIES AND BARRIERS FOR ASSET MANAGERS INTEGRATING ENERGY FLEXIBILITY}

Dr. Civ. Eng.-arch. Erwin Mlecnik

TU Delft, Faculty of Architecture and the Built Environment, Department of Management in the Built Environment,

P.O. Box 5043, 2600 GA Delft, The Netherlands

Phone: +31152789869

E-Mail: e.mlecnik@tudelft.nl

Yin, R.K., (2014), Case study research: design and methods, 5th edition, SAGE Publications, London.

\section{SUMMARY}

It is expected that future buildings will be able to manage their demand and generation not only according to local climate conditions and user needs, but also related to changing grid requirements. This development will introduce new challenges regarding asset management, which so far have not been explored.

This innovation adoption research identifies key opportunities and barriers for asset managers from a case study that considers changing grid conditions on a university campus in the Netherlands. The case illustrates that implementing energy flexibility is certainly not only a problem of data communication and transfer. The paper sets the scene that asset management for energy flexibility also requires detailed consideration and planning of required technical changes in buildings and grids.

\section{INTRODUCTION}

With its "Clean Energy For All Europeans" package the European Commission (2016) proposed new ways to deal with energy efficiency and renewable energy, with an outlook towards new business development in the energy market. While energy efficiency is put first, smart grid development now also come in the picture, as well as market changes to accelerate competitive renewable energy production. Connected to smart grids, buildings can becoming micro energy hubs consuming, producing, storing and supplying energy more flexibly than before. From literature it is expected that in the near future, there will be a need for "Energy Flexible Buildings"; buildings that are able to manage their demand and generation not only according to local climate conditions and user needs, but also related to changing grid requirements (Jensen et al., 2017). Energy Flexible Buildings might aid demand side management/load control and thereby demand response based on the requirements of the surrounding grids.

Based on the agenda "Energy Flexible Buildings" of the EBC Annex 67 research group of the International Energy Agency, there appears to be a need to explore if users find the concept of energy flexibility suitable for their daily practice or business development. So far, current social studies are limited to understanding the perception of end-users, such as homeowners (Li et al., 2017) and office workers (ongoing IEA EBC Annex 67 activity). There is however also a need to better understand the implications for facility and asset managers, who can potentially play a pivotal role in the acceptance of energy flexible services. Therefore this paper looks into the barriers and opportunities that asset managers encounter that can either hinder or facilitate adoption of energy flexibility.

\section{RESEARCH APPROACH}

As energy flexibility is perceived as a new concept by asset managers, it can be investigated from the viewpoint of adoption of innovation. Innovation theories were already introduced during the 60'ies and have been applied on hundreds of adoption problems (Rogers, 2003), including the adoption of energy-saving and environmental technologies, concepts and demonstration buildings (Mlecnik, 2013), and provides relevant research models.

In-depth knowledge from demonstrations is needed for the future development of asset management strategies. The aim of this research is to better understand experiences from a real-world case study, assuming that this is likely to unravel important contextual conditions for the adoption of energy flexibility. Methodologically, the research uses a case study approach (Yin, 2014), supported by literature search, stakeholder interviews and (procurement) action research to investigate adoption barriers and opportunities related to the adoption of the concept of "Energy Flexibility" and its effect on asset management strategy, using the experiences from asset managers from a university campus in the Netherlands. 


\section{CASE STUDY}

The Technical University of Delft has developed ambitious targets regarding the implementation of energy efficiency and renewable energy systems on its campus. Amongst other, its facility managers are looking for ways to lower the heat supply temperature in the local (heat) grid on the campus. The changing grid conditions imply needed changes in connected buildings. The transition from a high to a medium supply temperature on the TU Delft campus appears to have far-reaching consequences on the facility management of the buildings 55 and the redevelopment of the heat grid. Simulations and tests show that a smart control system can lower the heat network supply temperature, which can support the implementation of renewable energy systems. However, such control strategies imply a time shift in the energy use of individual buildings and technical modifications of hardware, devices, buildings and systems. The lack of interoperability of building management, control and data transfer systems is an important practical barrier.

\section{CONCLUSION}

New challenges lie ahead to be able to manage building portfolios more effectively, taking into account the need of buildings being able to respond to changing grid requirements. This paper provides insights to better understand asset managers' perceived opportunities and barriers for introducing 'energy flexibility' and lessons from the development of energy management practices on the TU Delft campus.

In theory older buildings can be made suitable for low supply temperature with fast and - compared to renovations - relatively small investments. When user-friendly, the implementation of control systems and optimization algorithms can allow to save energy and monitor comfort. However, the implementation in practice can result in high innovation risks due to testing in a working environment and additional transaction and hardware costs which might hinder adoption. Furthermore, the adoption of energy flexibility is challenged by data transfer and communication difficulties and conflicting views on the implementation of sustainable portfolios. The research identifies a need for future research regarding 'energy flexible' asset management strategies, which includes the development of suitable policy instruments, new business models and procurement and end-user guidelines.

\section{REFERENCES}

European Commission, (2016), Clean Energy For All Europeans, European Commission, Brussels, 30.11 .2016

$\operatorname{COM}(2016) 860$ final.

Jensen, S.Ø., Marszal-Pomianowska, A.J., Lollini, R., Pasut, W., Knotzer, A., Engelmann, P., Stafford, A., Reynders, G., (2017), IEA EBC Annex 67 Energy Flexible Buildings, Energy and Buildings 155, 25-34.

Li, R., Dane, G., Finck, C., Zeiler, W., (2017), Are building users prepared for energy flexible buildings? A large-scale survey in the Netherlands, Applied Energy 203, 623-634.

Mlecnik, E., (2013), Innovation development for highly energy-efficient housing. Opportunities and challenges related to the adoption of passive houses, PhD dissertation, TU Delft, Delft, The Netherlands.

Rogers, E.M., (2003), Diffusion of Innovations, 5th ed., Free Press, New York, NY.

Yin, R.K., (2014), Case study research: design and methods, 5th edition, SAGE Publications, London.

\section{CONFERENCE TOPIC}

Future role of buildings and industry for the flexibility and stability of thermal and electric grids. 


\section{HIGH SOLAR FRACTION BY THERMALLY ACTIVATED COMPONENTS}

Thomas Ramschak1, Walter Becke1, Christian Fink1, Werner Lerch2, Richard Heimrath2, Thomas Mach2 AEE - Institute for Sustainable Technologies (AEE INTEC)1

Feldgasse 19, 8200 Gleisdorf, Austria

Phone: +43 (0) 3112 - $5886-0$

Institute of Thermal Engineering (TU-Graz)2

Infeldgasse 25b, $8010 \mathrm{Graz}$, Austria

E-Mail: thomas.ramschak@aee.at

\section{SUMMARY}

Thermally activated building elements (TABs) are employed successfully in the building sector in recent years. From an energetic point of view, such low temperature systems are especially beneficial when combined with solar thermal installations or heat pumps. Within the scope of national funding programs and $R \& D$ projects the intensive use of thermally activated building elements is investigated, as main thermal storage in different buildings, with focus on the combination with solar technologies (thermal, photovoltaic) for energy supply.

Investigations based on measuring data from realised buildings and on a theoretical approach, various technical system concepts with solar thermal or photovoltaic in combination with thermally activated components for single- (SFH) and multifamily houses (MFH) were analysed under technical, economic and ecological criteria. The results show that through the activation and use of the thermal storage potential contained in the building mass, high solar fractions up to $100 \%$ of the building's heat demand can be achieved.

\section{INTRODUCTION}

The Renewable Energy Directive of the EU requires buildings to fulfil at least $20 \%$ of its total energy needs with renewables by 2020. An efficient integration of fluctuating renewable energy from solar thermal and photovoltaic systems into the heating infrastructure of a building is a big challenge. However, compensating for these fluctuations with the assistance of batteries and big thermal storages at the level of the heat supply is very cost-intensive. On the other hand, thermally activated building systems (TABs) have been shown to enable an efficient and cost effective integration of renewable energy. With TABs, the large thermal capacities of the building structure - such as massive floors and ceilings - are used as heat storage and they are as such integrated into the overall energy system of the building. By absorbing heat from internal or external passive gains through radiation, conduction and convection or by releasing stored energy, the slabs provide respectively cooling and heating to the rooms. The thermal activation of the building mass enables it to store more heat, providing inertia against temperature fluctuations and allowing it to be heated or cooled in hours with renewable energy production. Moreover, the large areas of the thermo-active surfaces allow for substantial heat flux between room and structure, even with relatively low temperature differences. For these reasons, TABs are predestined for the application of solar thermal and low temperature heating sources, such as near-surface geothermal, ground water and outside air.

The goal of the research project solSPONGEhigh is the detailed analysis of this approach. On the basis of numerical models and several case studies the project and its results contribute to a better understanding of the energy-related processes in and the design of such systems.

\section{METHOD}

For two prototype buildings, a one-family house and an apartment building, different system concepts for heating and preparation of domestic hot water are being developed. In addition to small buffer storage tanks, the system concepts mainly include thermally activated components (storey ceilings) as heat storage and heat dissipation system. The energy source is provided by building-integrated solarthermal or solar-electric systems combined with heat pumps. The system concepts are numerically modelled in different configurations, supplemented with control strategies and used to cover the heat requirements of different heat demand levels of the considered buildings. 
In the first step, the single-family house $\left(120 \mathrm{~m}^{2}\right.$ floor space $)$ and the multi-family house $\left(540 \mathrm{~m}^{2}\right.$ floor space), in each case with two different heat demand levels, were modelled in the simulation environment TRNSYS.

The heat demand level "Low Energy Building" (LEB) meets the current state of thermal protection in new buildings in Austria and the heat demand level "Nearly Zero Energy" is equivalent to the heat consumption of a passive house. With regard to the four different heat demand profiles, different heat supply systems were developed in the second step (see chapter 4). The system A is supplied in a monovalent way via an air-water heat pump, the systems B, C, D are additionally equipped with building-integrated thermal solar systems and the systems $\mathrm{E}$ and $\mathrm{F}$ include a photovoltaic system. In addition, variations in the type of heat pump and the thermal charging of the storey ceiling are defined and modelled in TRNSYS. In the case of "solar overheating", an increase of the upper temperature limit by 2 Kelvin is allowed in the component (Fig. 2). Systems B to F are equipped with three differently sized solar systems (SFH: $20 \mathrm{~m}^{2}$ (a), $40 \mathrm{~m}^{2}$ (b), $60 \mathrm{~m}^{2}$ (c); MFH: $25 \mathrm{~m}^{2}$ (a), $125 \mathrm{~m}^{2}$ (b) $200 \mathrm{~m}^{2}$ (c)).

Table 1: Overview of the established systems for the heat supply of the considered buildings

\begin{tabular}{|c|c|c|c|c|c|}
\hline & solar plant & heatpump & storage tank & solar charging & $\begin{array}{l}\text { activated structural } \\
\text { elements }\end{array}$ \\
\hline system A & - & air-water & domestic hot water & - & ceiling slab \\
\hline system C & solar thermal & air-water & domestic hot water & +2 Kelvin & ceiling slab \\
\hline system D & solar thermal & soil-water & domestic hot water & +2 Kelvin & ceiling slab \\
\hline system E & photovoltaic & air-water & domestic hot water & - & ceiling slab \\
\hline
\end{tabular}

In the third step, the models of the systems (A to F) were combined with the building models in both heat demand levels, equipped with control systems and simulated as overall system models on an annual basis. Stepfour was the definition of characteristic figures for the assessment of the systems, with the aim of being able to assess both the energy-related performance, the economic and the ecological performance. In the fifth step, the simulation results were evaluated, compared and analyzed with regard to the defined energetic (see chapter 2.3), economic and ecological assessment figures (see chapter 2.4).

\section{CONSIDERED SYSTEMS}

At the start of the project, different heat supply concepts were defined to cover as much heat as possible through the solar thermal and solar electrical system by the additional activation of the storage mass in the building (primarily concrete). The considered Systems are described in Table 2.

Table 2: Considered systems for the heat supply of the buildings

\begin{tabular}{|l|l|} 
An air/water HP system (System A) was selected as the first heat supply \\
system. In the case of the monovalent heating system, in the buffer \\
storage only a hot water volume is available. Space heating can be \\
directly done with the HP (without water storage). This means that for \\
System A volume is only available in the buffer store for DHW heating.
\end{tabular}




\begin{tabular}{|c|c|}
\hline$\underbrace{4}_{\infty}$ & $\begin{array}{l}\text { In System B the HP is coupled to a solar thermal system, whereby solar } \\
\text { heat is only stored in the buffer storage. With System B the building can } \\
\text { be heated by the additional solar system with the corresponding } \\
\text { temperature in the storage from the water storage or directly with the HP } \\
\text { (if no solar heat is available). }\end{array}$ \\
\hline $\begin{array}{l}0 \\
=\infty\end{array}$ & $\begin{array}{l}\text { On the solar side of System C, different hydraulic connections are being } \\
\text { considered in the case of the direct or indirect integration in the heat } \\
\text { supply systems. This means the solar thermal heat can be stored in the } \\
\text { buffer store or directly in the building (TABS), but also serial charging } \\
\text { (water storage and TABS) is possible if the required temperature levels } \\
\text { are available. }\end{array}$ \\
\hline$\square$ क्षा & $\begin{array}{l}\text { In System D a soil-water heat pump with a soil storage under the building } \\
\text { is coupled to a solar thermal system. In that case the solar thermal heat } \\
\text { can be stored same as in System C and additionally regenerate the soil } \\
\text { storage. }\end{array}$ \\
\hline$\sum_{\infty}^{\infty}$ & $\begin{array}{l}\text { System } \mathrm{E} \text { and } \mathrm{F} \text { is equal with to System } \mathrm{A} \text { including an additional } \\
\text { photovoltaic system. In System } \mathrm{F} \text { the control strategy has been } \\
\text { adapted, that depending on the heat pump design performance and } \\
\text { the current photovoltaic power, the building mass is loaded to a } \\
\text { higher temperature level ( }+2 \text { Kelvin). }\end{array}$ \\
\hline
\end{tabular}

\section{BUILDING}

Concerning the climatic conditions the city of Graz (Austria) was chosen for the building location. For the thermal building and system simulations a mean climatic data set (over 10 years) is used (Tmean ambient: $10.7^{\circ} \mathrm{C}$, heating degree days: $3102 \mathrm{Kd}$, global hor. radiation: $1206 \mathrm{kWh} / \mathrm{m}^{2} \mathrm{a}$, diffuse hor. radiation: $616 \mathrm{kWh} / \mathrm{m}^{2} \mathrm{a}$ ). The building designs (insulation standards) are based on the building guidelines OIB-RL 6 for the minimum requirement and for a very ambitious case a nearly zero energy building (including controlled ventilation with air heat recovery) was defined. The specific head demand of the two different considered buildings amounts for the low energy building (LEB) 37 $\mathrm{kWh} /\left(\mathrm{m}^{2} \mathrm{a}\right)$ and for the net zero energy building (NZEB) $17 \mathrm{kWh} /\left(\mathrm{m}^{2} \mathrm{a}\right)$. The dimensioning of the required heating capacity of the backup system was based on a heating load calculation. For the nearly zero energy building the same design criteria were chosen for the backup systems as for the low energy building. The dynamic heat load calculation (simulations in TRNSYS) for the low energy

buildings for a room air temperature of $22{ }^{\circ} \mathrm{C}$ during the heating period are summarized in Table 3 . Since the solar thermal systems are compared with an air heat pump system (System A), the backup system has to be dimensioned to ensure the coverage of the heat requirements for the DHW heating and space heating without solar support.

Table 3: Heating load of the LEB and heating capacity for heat pumps

\begin{tabular}{cccc}
\hline LEB & Heating load & air-water heat pump (A-12W35) & soil-water heat pump (S0W35) \\
\hline SFH & $4.3 \mathrm{~kW}$ & $5.4 \mathrm{~kW}$ & $5.8 \mathrm{~kW}$ \\
MFH & $20.4 \mathrm{~kW}$ & $19.8 \mathrm{~kW}$ & - \\
\hline
\end{tabular}

In addition to the various heat-supply systems, the heat dissipation system is also considered and analyzed. The systems and ceiling constructions is shown in Fig. 1 for the ground floor of the building. The largest difference to an underfloor heating system $(\mathrm{FH})$ is the positioning of the insulation layer. In the case of the underfloor heating system, the heat dissipation system is installed in the screed, and 
the concrete is separated by the insulation layer in order to limit its activation. If the building is heated through the ceiling (TABS), where the heat transfer system is installed directly in the concrete, more storage mass can be activated. The next difference is that the pipes of the heat dissipation system are positioned much deeper in the concrete in the TABS system than in the FH system and the heat transfer coefficients of the heat output surfaces (ceiling or floor) are also be different and calculated through the building model in TRNSYS dependent on the room air temperature and the surface temperature of the heat transfer surface.

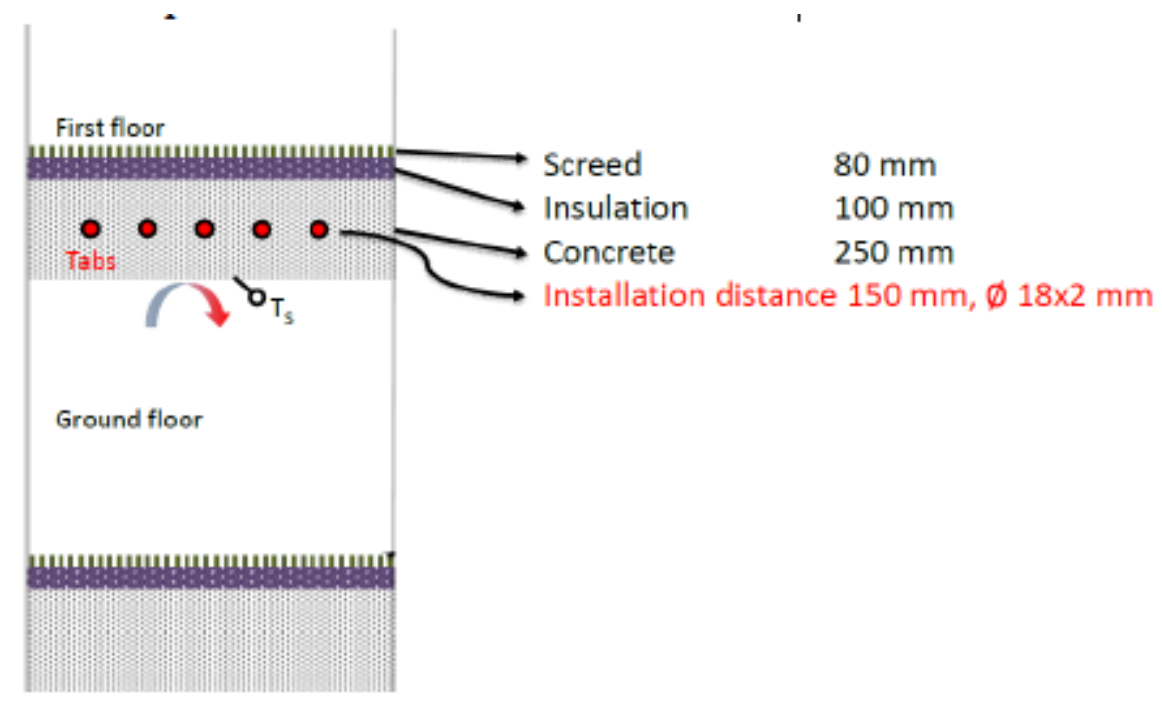

Fig. 1 Ceiling construction for the concrete core activation (TABS)

\section{CONTROL STRATEGY}

On the basis of the evaluations of the different simulation data of the various types of control temperatures that were tested, the surface temperature (Ts) was chosen for the control variable for the room heating. The building model of the considered building consists of multiple thermal zones. Each thermal zone can be charged independently of one another, whereby the control variable (surface temperature of the thermally activated component) is dependent on the outside air temperature. The required control surface temperature changes with the outside air temperature. In addition, depending on the heat source (solar or HP), the temperature hysteresis for the charge controller changes (Fig. 2). If heat is required to charge the thermal zone and no solar heat is available, the backup system is charging to a surface temperature hysteresis of $2 \mathrm{~K}$ (Tsurf Heat ON, Tsurf HP OFF). If solar heat is available, the hysteresis is increased by $2 \mathrm{~K}$ to $4 \mathrm{~K}$, so that the storage mass is charged to a higher average temperature level.

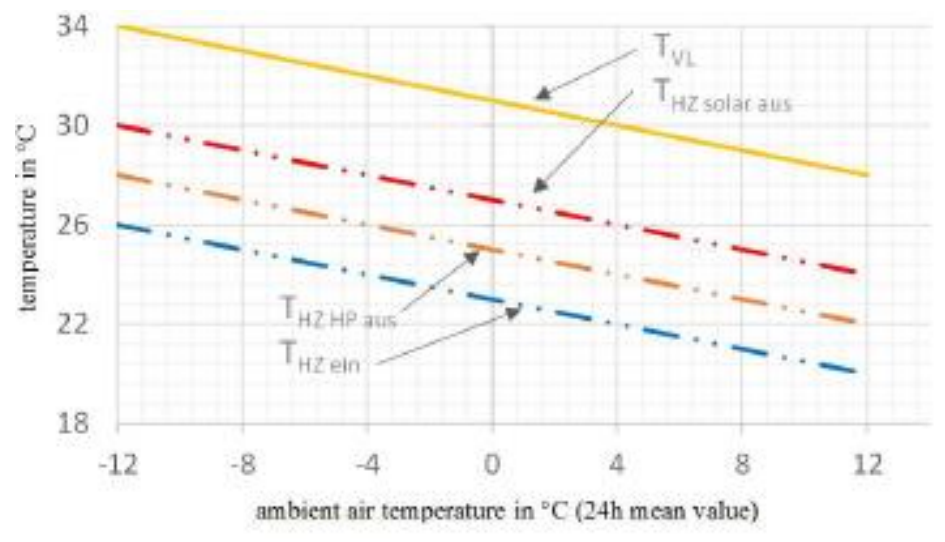

Fig. 2: Control strategy 


\section{RESULTS}

At the centre of the considerations is the question of the achievable degree of self-sufficiency with heat for the reference buildings. The solar coverage SD, as a measure of the self-sufficiency, depends both on the ratio of solar heat provided to the heat consumption, and on the possibilities for storing heat.

Due to the storage capacity of the storey ceilings, a single-family house in low energy level can achieve a solar fraction of approx. SD $=50 \%$ even with the smallest considered solar thermal collector area $20 \mathrm{~m}^{2}$ (a) and a small buffer storage $\left(1.5 \mathrm{~m}^{3}\right)$. With using all considered possibilities for improvement, reduction of heat consumption to the level Nearly Zero Energy, plus solar overheating, plus use of the soil, plus covering of the entire roof area and the entire south façade with thermal collectors $60 \mathrm{~m}^{2}$ (c), the solar coverage is $\mathrm{SD}=91 \%$. Thus, over $90 \%$ of the heat demand in the assumed single-family home can be covered in a decentral way and the heat pump has to contribute only about $10 \%$ to a full thermal supply.

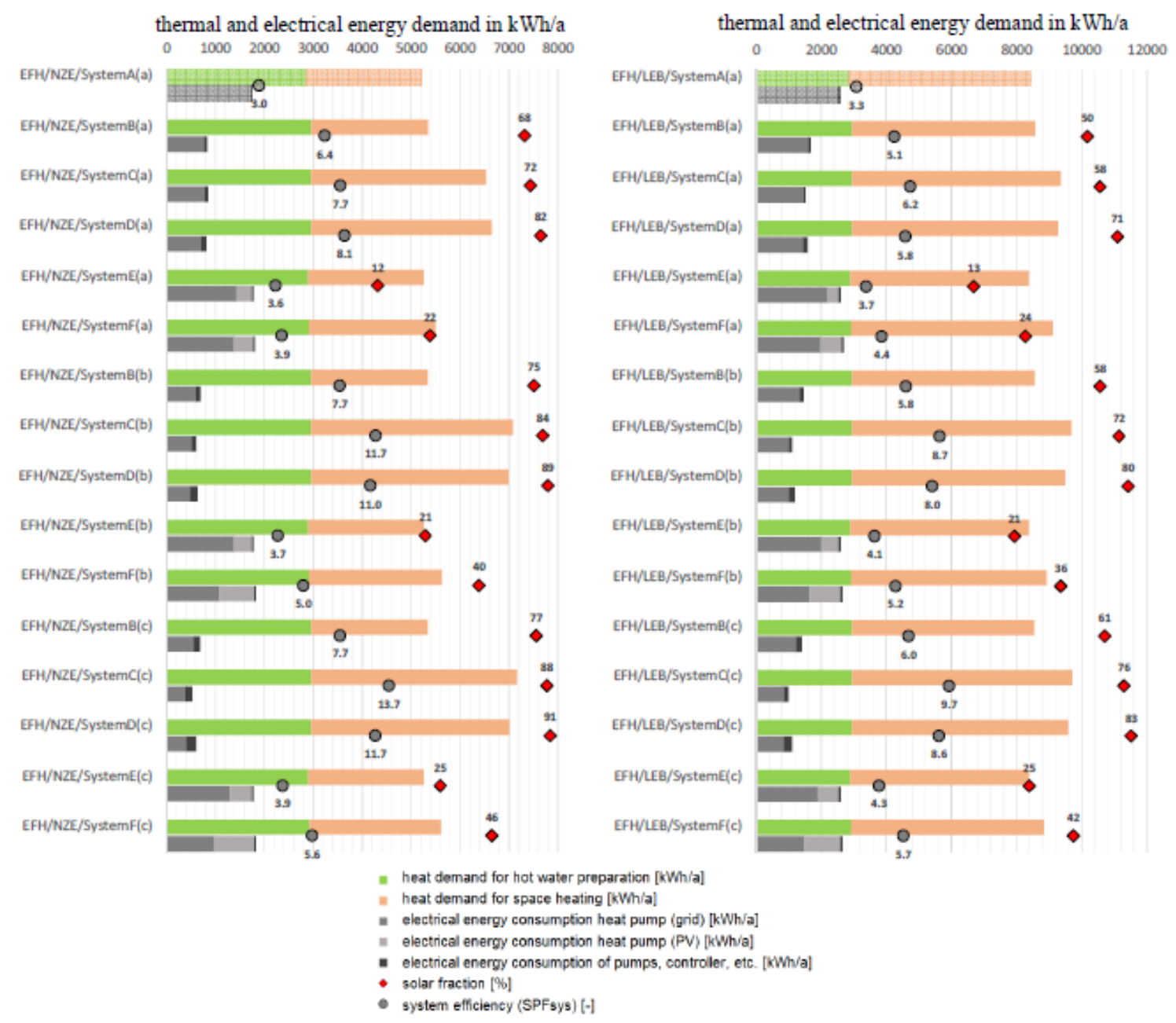

Fig. 3: Energetic results for the single-family house

For the multi-family building, the prerequisites for high solar fractions are less pronounced than for singlefamily dwellings. Due to the higher compactness of the building the specific heating demand is slightly lower, but in relation to the heated volume significantly less external surfaces are available for solar use. These circumstances are evident in the achievable solar fractions. In the heat demand level Low Energy Building with a compact energy storage $\left(301 / \mathrm{m}^{2}\right.$ collector area) and with the smallest considered collector area of $25 \mathrm{~m}^{2}$, a solar fraction of approx. SD $=25 \%$ can be achieved. The full utilization of all examined possibilities of improvement leads to a maximum achievable solar fraction of approx. $\mathrm{SD}=75 \%$. 


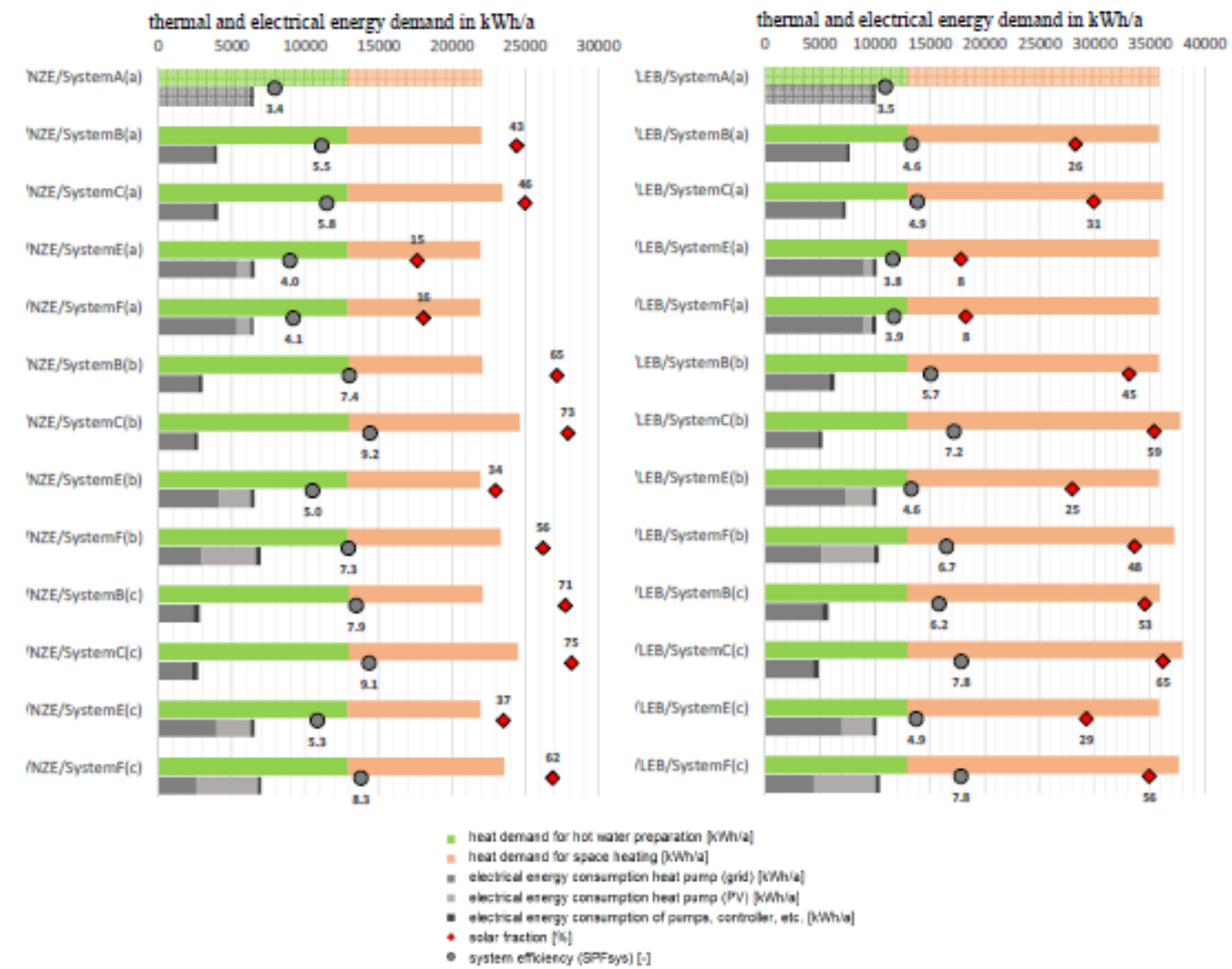

Fig. 4: Energetic results for the multi-family house

Besides the solar fraction a number of additional assessment parameters were analyzed for the considered scenarios. With regard to the costs of the energy concepts, it can be seen that the solar thermal concepts cause high additional investment costs. However, even small collector areas cause a significant reduction of the energy demand and thus also the operating costs. The solar-electric concepts have lower additional investment costs, whereby the solar electricity yields that cannot be directly utilized can be offset against the operating costs considering a feed-in tariff into the electricity grid.
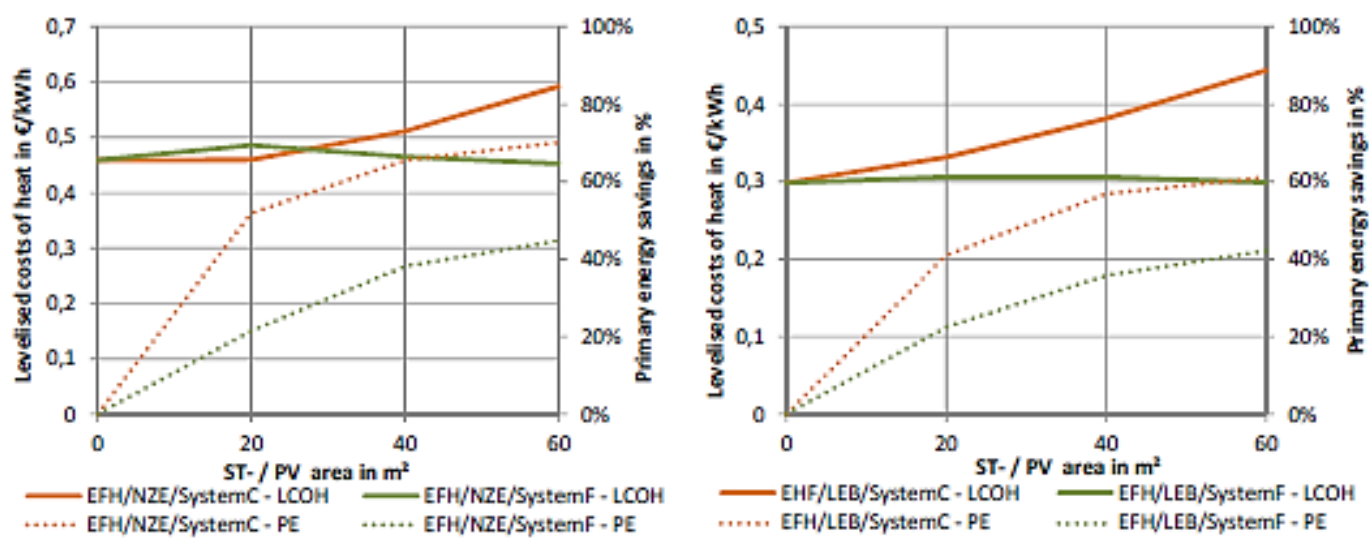

Fig. 5: Economical and ecological results for the single-family house 

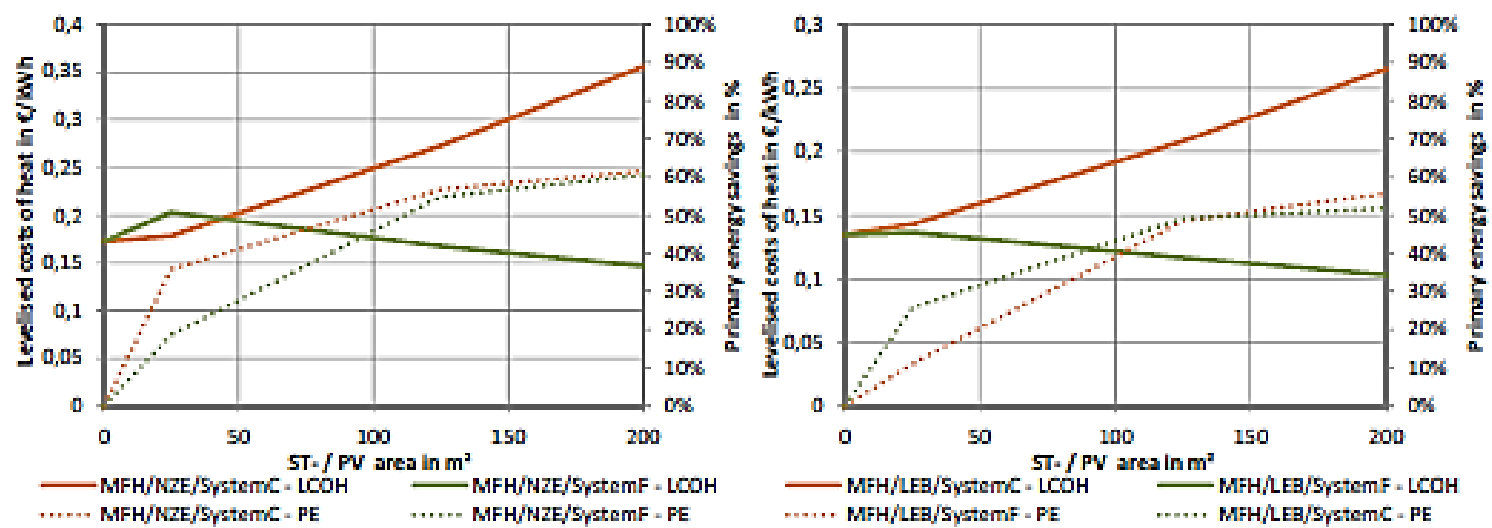

Fig. 6: Economical and ecological results for the multi-family house

When interpreting the presented results, it should be considered that the heat supply systems combined with the reference buildings are simulated using extensive and complex models based on a number of assumptions. The presented results are valid only in relation to these assumptions.

\section{ACKNOWLEDGEMENTS}

The reported research has been conducted within the framework of the Austrian project solSPONGEhigh" (FFG Project-No.: 845182), funded by the Austrian Ministry for Transport, Innovation and Technology.

\section{REFERENCES}

Heimrath et al. (2018): Final Project Report: Hohe solare Deckungsgrade durch thermisch aktivierte Bauteile im urbanen Umfeld (in German), Austrian Klima- und Energiefond, Project number: 845182

\section{CONCLUSION}

Due to the mismatch in time between the solar supply and the energy demand, usually only a part of the solar energy can be used to cover the energy needs of a building. The larger the solar energy system is dimensioned, the more important is the integration of an energy storage. In order to achieve high solar fractions, the short to medium term storage of larger amounts of heat is essential. This raises the question of alternative solutions for heat storage in buildings.

One way to increase the heat storage capacity of a building has been analyzed in the research project solSPONGEhigh. The thermal activation of solid components such as ceilings and foundations (used as sensible heat storage elements), with solar-thermal or solar-electric systems shows a huge energy saving potential. The results show that through the activation and use of the thermal storage potential contained in the building mass, high solar fractions up to $100 \%$ of the building's heat demand can be achieved.

A general picture across the analysis of the different systems, buildings and insulation standards showed that that a solar thermal concepts shows a better system efficiency and thereby a higher primary energy- and $\mathrm{CO} 2$-reduction potential in comparison with solar-electrical concepts but also higher levelised costs of heat for bigger collector areas. For solar thermal concepts it makes sense to realize to realize rather small collector areas and for solar-electric concepts a larger solar-electric area should be preferred. In general terms, it can be said that the use of renewable energy sources and the building as thermal storage makes heat supply systems with adequate investment costs, low operating costs, a significant reduction in the dependency on energy sources and minimal primary energy use possible. 


\title{
A NEW CONTROL STRATEGY FOR THE EXPLOITATION OF SOLAR ENERGY
}

\author{
Dr. Matthias Gladt, Dr. Simon Handler, DI Sebastian Zilles, Prof. Dr. Thomas Bednar \\ Forschungsbereich für Bauphysik und Schallschutz1 \\ Karlsplatz 206.02, 1040 Wien, Austria \\ Phone: +43 3112/34567 \\ Institut für Hochbau und Technologie 2 \\ Karlsplatz 206, 1040 Wien, Austria \\ E-Mail: Sebastian.zilles@tuwien.ac.at
}

\section{SUMMARY}

The total heating and cooling demand in the context of building operations makes up an important share of the overall worldwide energy consumption. Thus efforts have been made to increase the amount of renewable energy like solar radiation. One of the means to reach this goal is to accumulate the energy emitted via solar radiation within thermal mass. An effective means to raise thermal mass is to add buffer tanks next to or inside a building. The costs of these buffer tanks and the space they use grow with the size of the tanks. Thus the tanks are to be kept as small as possible.

This is where the paper outlines an alternative way to exploit existing thermal mass the best possible way. It demonstrates a new method to increase the accumulation of energy within thermal mass rather by overheating heavy construction elements than by raising the size of water storage tanks.

\section{INTRODUCTION}

Research regarding the effect of thermal building mass has been intensified during the last years. Dominiković et al. (2018) have investigated the effect of thermal building mass on the thermal behavior with respect to potential heat supply cut-offs.

Reilly and Kinnane (2017) focus on the effects of thermal mass compared with steady state calculations. They demonstrate that thermal mass can have a negative effect on the heating energy demand in case a building is not permanently occupied.

Research regarding thermal component activation can be based either on measurements within existing buildings or on the results of thermal simulations. Measurements of existing buildings are rare and can even more rarely be compared with measurements from other buildings in order to investigate the effects of differences in geometry, building stock etc. Therefore the present paper is based on the results of thermal simulations.

The simulations focus on the effect of thermal mass of construction elements in the context of a new control technology that ensures that the biggest possible amount of solar energy is accumulated. The effect of the control technology on the overall solar coverage will be investigated in the following. The results are compared with simulations of traditional control technologies with varying thermal masses.

\section{METHOD}

This work focuses on the exploitation of thermal mass. The total amount of thermal energy that can be stored within a particular construction element reads:

$$
Q=c \cdot m \cdot \Delta T \text { (eq. } 1)
$$

$c$ denotes mass specific heat capacitance, $m$ mass and $\Delta T$ the temperature difference between two states. Obviously the amount can be increased either if thermal mass $(c \cdot m)$ is augmented or if the temperature difference ( $\Delta T)$ is raised. An increase of thermal mass of a building's construction elements causes higher thermal inertia which helps to dampen temperature changes. Thermal mass of water storage tanks allows to accumulate solar energy and to consume it as heating energy at a later time. Frequently an increase of thermal mass is reached by increasing the water storage tank that provides the water for thermal component activation and/or hot water.

However due to the costs of storage tanks and due to the space they consume within a building alternative solutions are worth investigating. One of these solutions is to increase the temperature difference between two states on the right hand side of eq. 1 in order to increase the thermal energy on the left hand side of eq. 1.

Applying the idea to a particular case study means to use as much solar energy as possible to activate existing construction elements and to accept that the operational temperature rises beyond a set point that is normally relevant for heating control systems. 


\section{Control strategy}

Usually there is a single set point relevant for the heating control system. Below the set point (minus a certain gap) the heating is switched on and above the set point plus a certain gap the heating is switched off. In both cases the complete available solar energy is accumulated by heating the buffer tank.

With the new control strategy a second set point is introduced which is above the first (original) set point. Below the original lower set point the heating system operates in the same way as described before. But between the first and the second set point any available solar energy is used to activate the selected construction elements rather than to heat up the water storage tank. Only when the inside operational temperature rises above the second set point the water storage tank is heated with the available solar energy. Also below the first set point all available solar energy is used to heat the water buffer tank in order to make sure that the required supply temperature can be reached also when no solar energy is available. If the inside operational temperature drops below the first set point, heating energy might be needed in order to keep the buffer tank warm enough to deliver water with supply temperature for activating construction elements.

Fig. 1 displays a flow chart that visualizes the new control strategy.

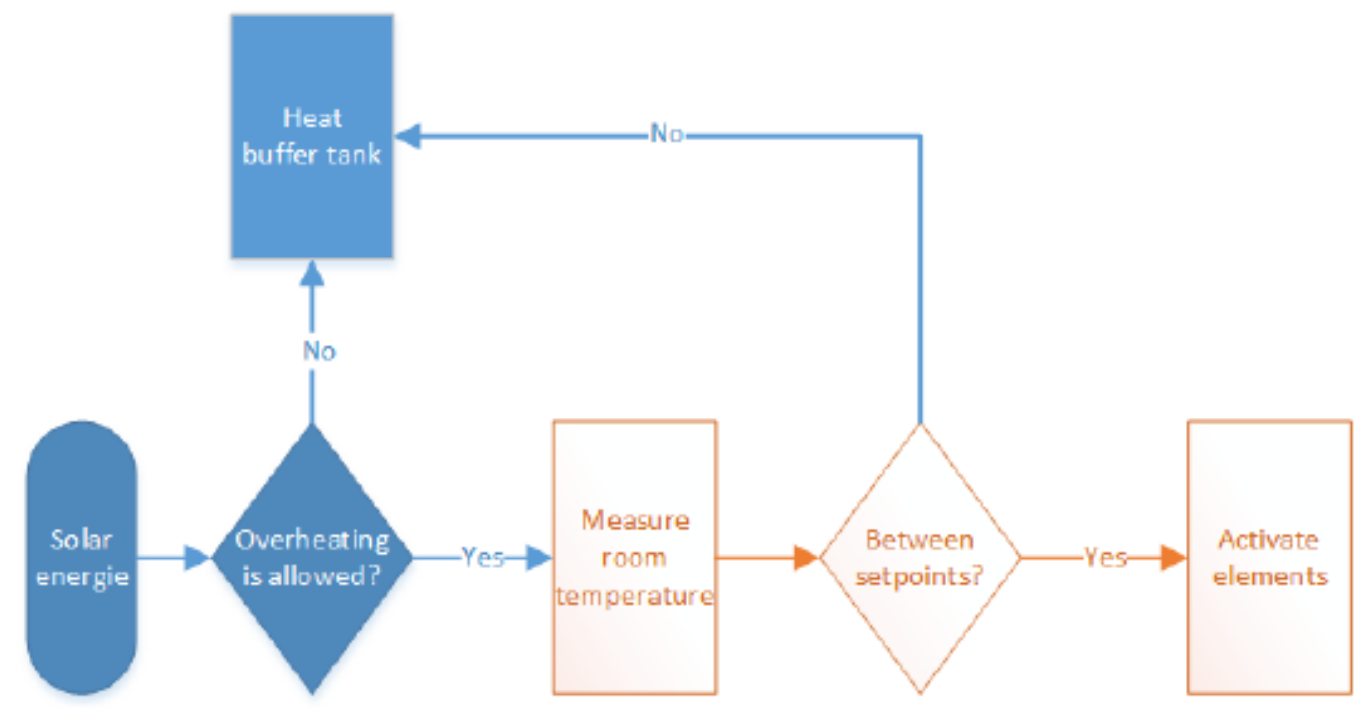

Fig. 1: Flow chart of the „overheat" control strategy

\section{Model}

The implementation of the model represents a particular challenge because it has to allow for different control strategies and easy adaptation between individual simulation runs at the same time. Thus the choice of a suitable implementation was of predominant interest. Kramer et al. (2012) have written a literature review on building models suited for implementation purposes. They classify models into black box models, grey box and white box models. Black box models are neural network models, white box models are lumped capacitance models and grey box models are based partially on physical knowledge and partially on empiricism. The overwhelming majority of building models is made up of white box models. Lumped capacitance (LC) models have been investigated as early as 1985 (Achterbosch et al.) and ever since been object to further scientific research. (e.g. Gouda et al., 2002, Dobbs and Hencey, 2012 or Kircher and Max Zhang 2015) 
The model that was chosen for all thermal simulations with respect to the present paper is based on Gladt's 80 implementation (2014) which represents a classical LC model and on Friembichler et al. (2014) where the basic idea of the implementation is outlined.

The flat plate solar collector and the construction element activation are modelled according to Handler (2014). The characteristic curve is displayed in Fig. 2

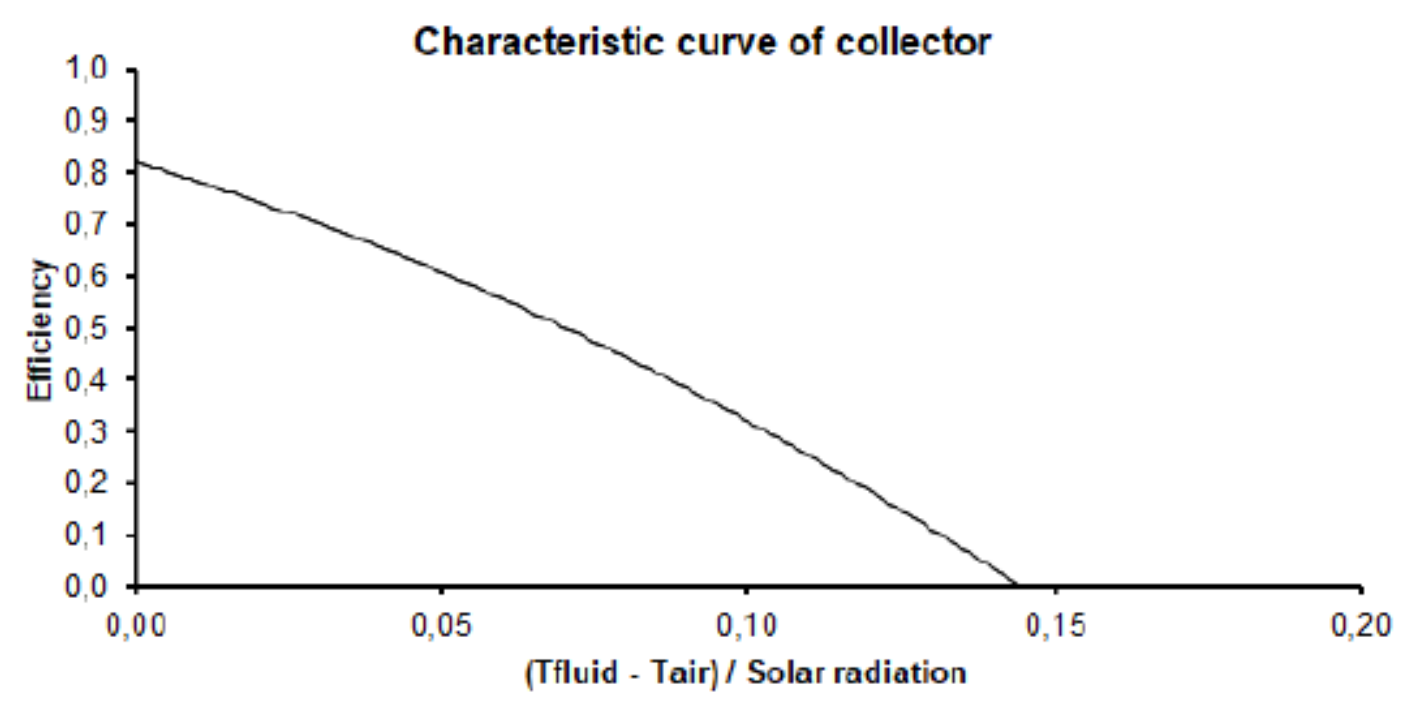

Fig. 2: The characteristic curve of the solar collector used in the simulations. Tfluid represents the temperature of the collector and Tair the exterior air temperature.

\section{Case study}

The model that is used for testing purposes presents a one family house with two floors and a living space of about $250 \mathrm{~m} 2$.

In order to test the control strategy presented in 3.1 two sets of models ("overheat" and "buffer tanks") are created. In all cases the model of the building is based on Fig. 3. The space heating is achieved solely via the activation of the three largest concrete construction elements. The temperature of the water storage tank is kept at least at $45^{\circ} \mathrm{C}$ at all times. If there is a lack of solar energy as much heating energy is supplied as to prevent the two top layers of the storage tank from cooling down below $45^{\circ} \mathrm{C}$. The storage tank is modelled with five equally sized isothermal layers.

The first set of models follows the control strategy presented in 3.1. The size of the temperature gap that is allowed to exceed the first set point is varied between 0 (no overheat at all) and $4 \mathrm{~K}$. The size of the water storage tank in contrast is kept constant at $1 \mathrm{~m} 3$.

The second set of models is set up in the opposite way. The temperature gap is kept constant at $0 \mathrm{~K}$ and the size of the water buffer tank is varied between $1 \mathrm{~m}_{3}$ and $15 \mathrm{~m}_{3}$. (See also Fig. 3.) 


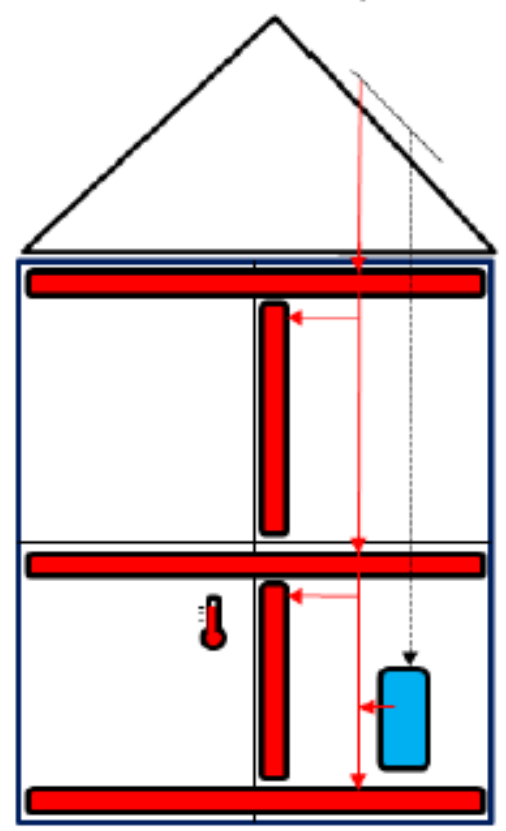

Amount of overheat gap is varied between 0 and $4 \mathrm{~K}$ (72 simulations)

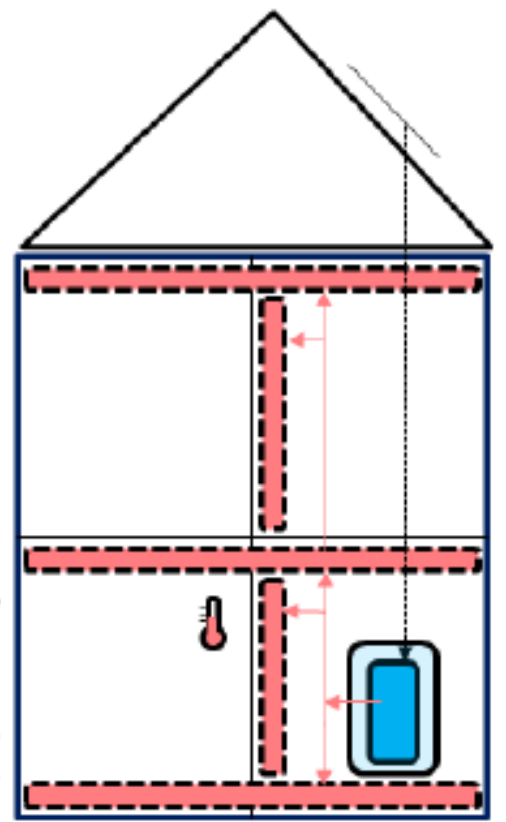

\section{Buffer tank}

\section{$\square$ Activated overheated I= Activated not overheated}

Fig. 3: Two sets of simulations are run in order to evaluate the new control strategy

For both sets the quality of the building envelope is varied synchronously such that the ventilation is augmented gradually and the insulation is reduced. In particular between two simulations the insulation of the exterior walls is reduced by $1 \mathrm{~cm}$ and the ventilation is increased according to the scale in the charts. For the ventilation no heat recovery is assumed. The size of the solar collector was assumed with $25 \mathrm{~m}^{2}$. Some average Austrian meteorological data series were used to model the climatic conditions. For further details please contact the authors.

Now all models of the two sets have undergone a simulation over a period of one year and the results were printed out and aggregated.

\section{RESULTS}

The following pages hold plots of some selected aggregated simulation results.
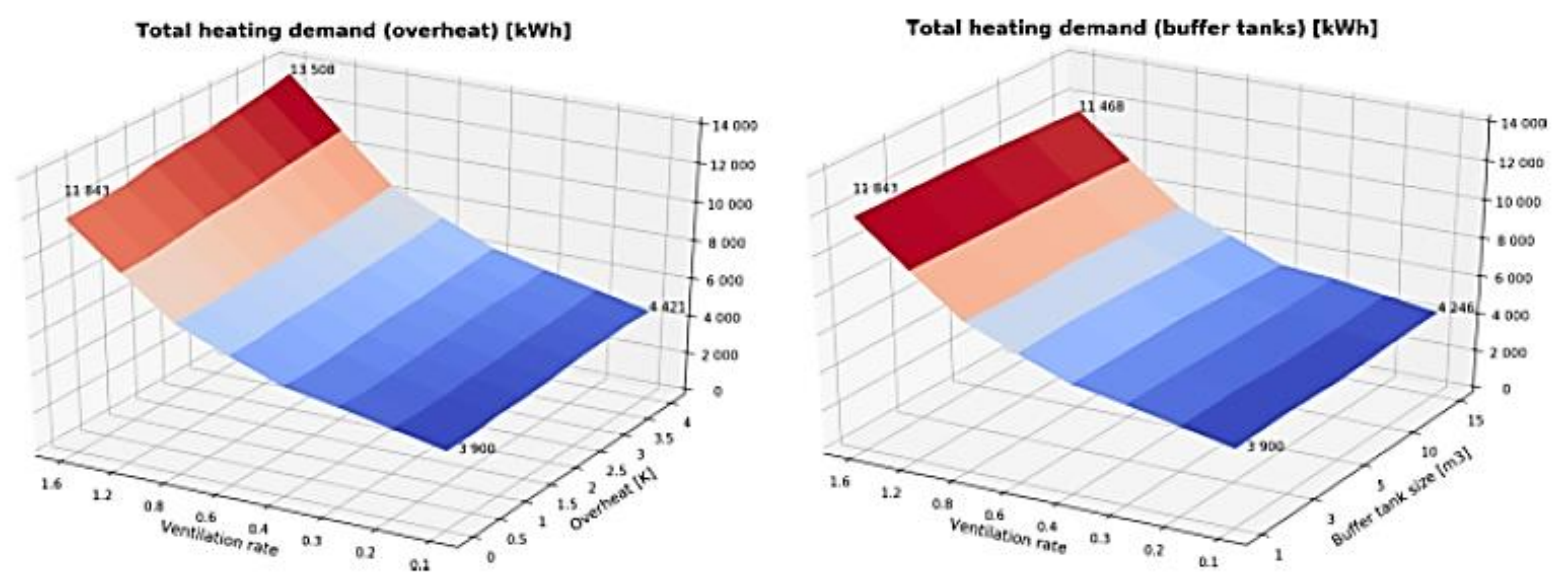

Fig. 4: Total heating demand with respect to quality of building envelope and to overheat gap (left) and to buffer tank size respectively (right) 
Fig. 4 displays the total heating demand for both sets of models. The ventilation rate on the $\mathrm{x}$-axis can also be interpreted as quality of the building envelope with lower ventilation rate representing higher quality. The y-axis displays the size of the temperature gap that is allowed to be reached while overheating with solar energy (left-hand side) and the size of the storage water tank respectively (right-hand side).

Whereas the characteristic of the two grids is similar for both sets of models the rise of the heating demand with a growing overheat gap is plausible because higher room temperatures require more heating energy.
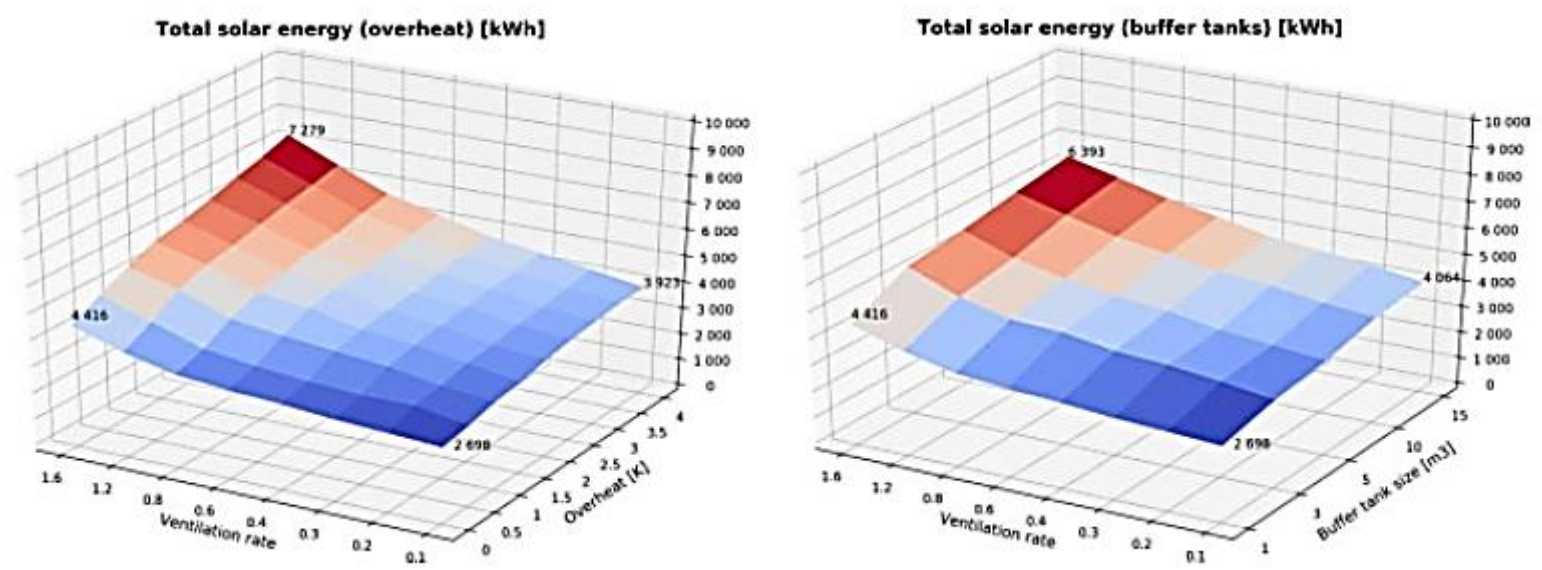

Fig. 5: Total solar energy gained via collector with respect to quality of building envelope and to overheat gap (left) and to buffer tank size respectively (right)

Fig. 5 displays the total amount of accumulated solar energy. For the "overheat" set of models the total of the accumulated solar energy is made up by the portion accumulated in the water storage tank and by the portion accumulated by activating the relevant concrete construction elements. For the "buffer tanks" set of models all solar energy is accumulated in the buffer tank.

The total rises for lower quality buildings since the accumulation of solar energy is more efficient for cooler supply fluid temperatures.
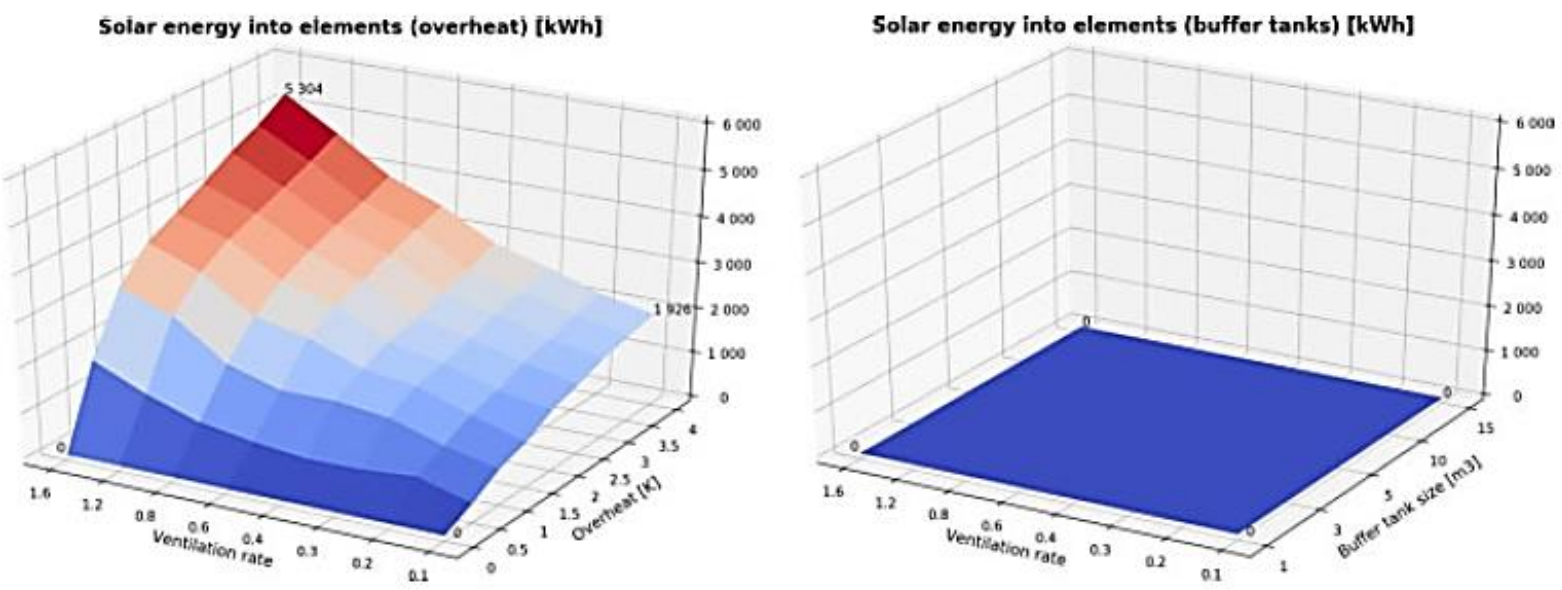

Fig. 6: Solar energy accumulated directly in activated building elements by overheating the building. Again values are plotted with respect to quality of building envelope and to overheat gap (left) and to buffer tank size respectively (right)

Fig. 6 displays the solar energy accumulated directly in activated building elements by allowing for the above mentioned "overheat" control strategy. With the "overheat" set of models (left-hand side) 
this makes up the major portion of the total solar energy displayed in Fig. 5. With the "buffer tanks" set of models there is no overheat thus there is no such accumulated solar energy.

Solar energy into buffer tank (overheat) [kWh]

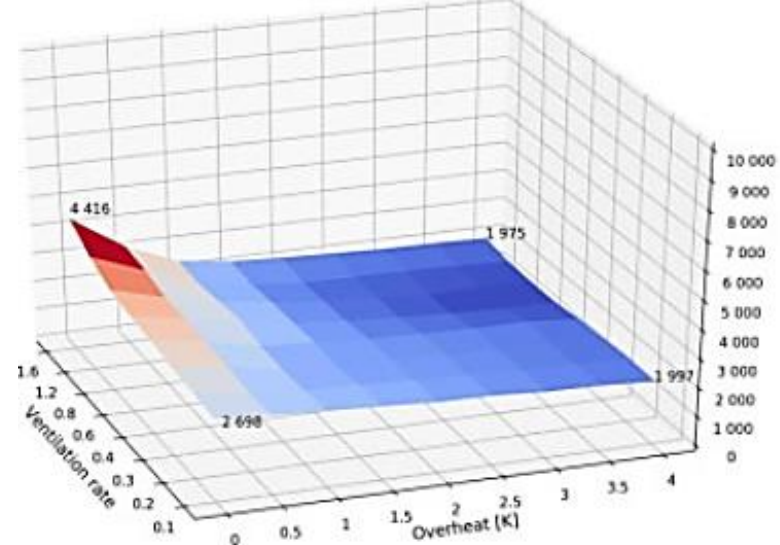

Solar energy into buffer tank (buffer tanks) [kWh]

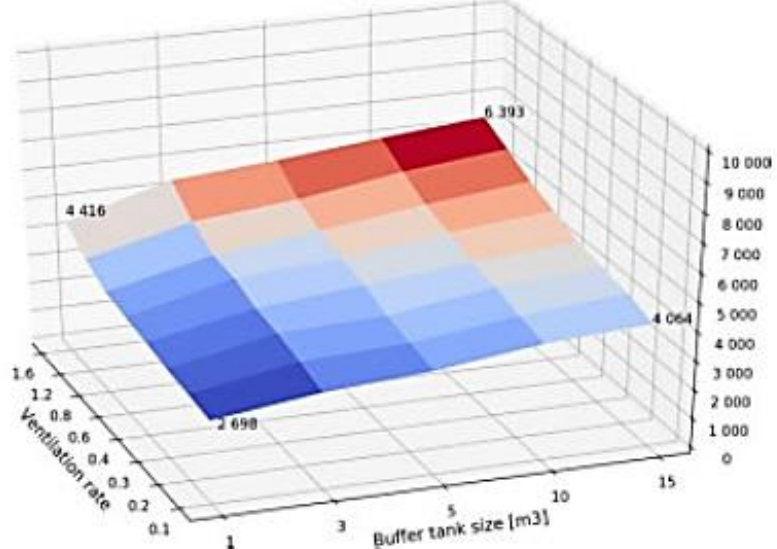

Fig. 7: Solar energy accumulated in storage tank with respect to quality of building envelope and to overheat gap (left) and to buffer tank size respectively (right)

Fig. 7 displays the solar energy accumulated in the storage tank. With the "overheat" set of models the amount decreases as the gap for overheat increases because most of the available solar energy is used for activating the relevant construction elements. (Fig. 6) The opposite is the case for the "buffer tanks" set of models. All available solar energy is accumulated in the storage tank.
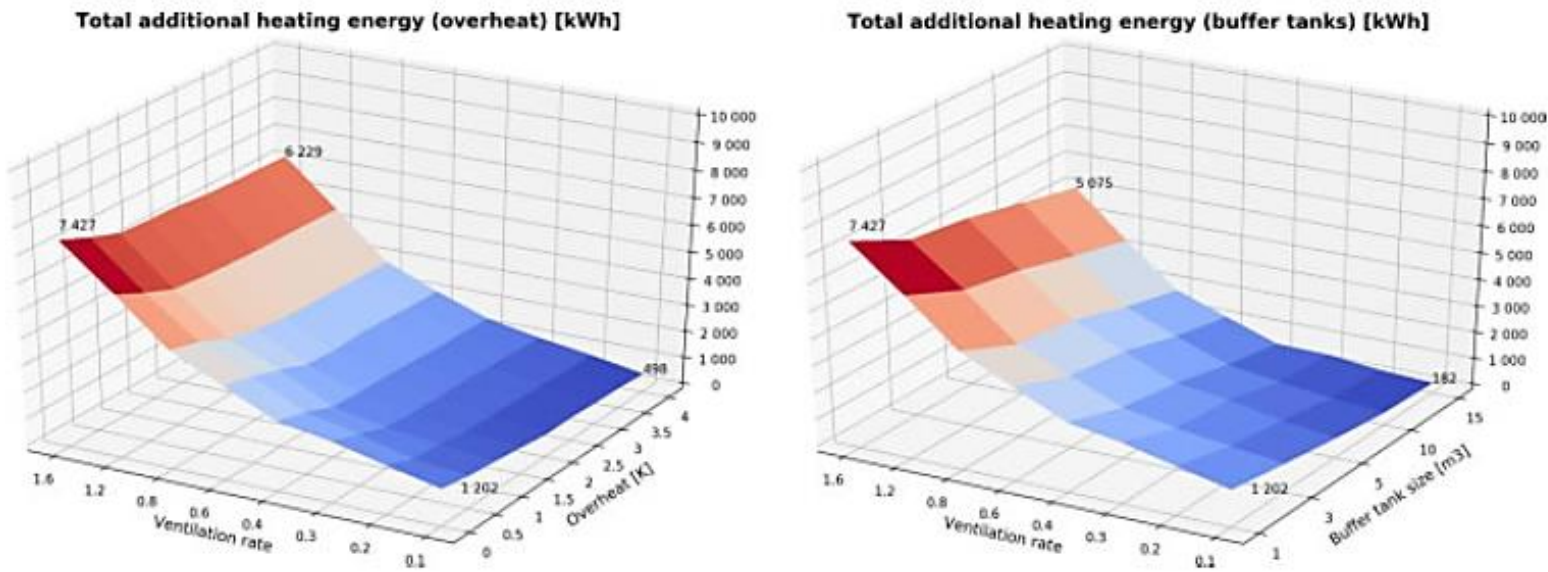

Fig. 8: Additional energy demand with respect to quality of building envelope and to overheat gap (left) and to buffer tank size respectively (right)

Fig. 8 displays the portion of the heating energy demand that cannot be covered with solar energy. As expected 150 the numbers shrink as the temperature gap for overheat is increased (left-hand side) and the size of the relevant buffer tank is increased respectively. (Right-hand side) 

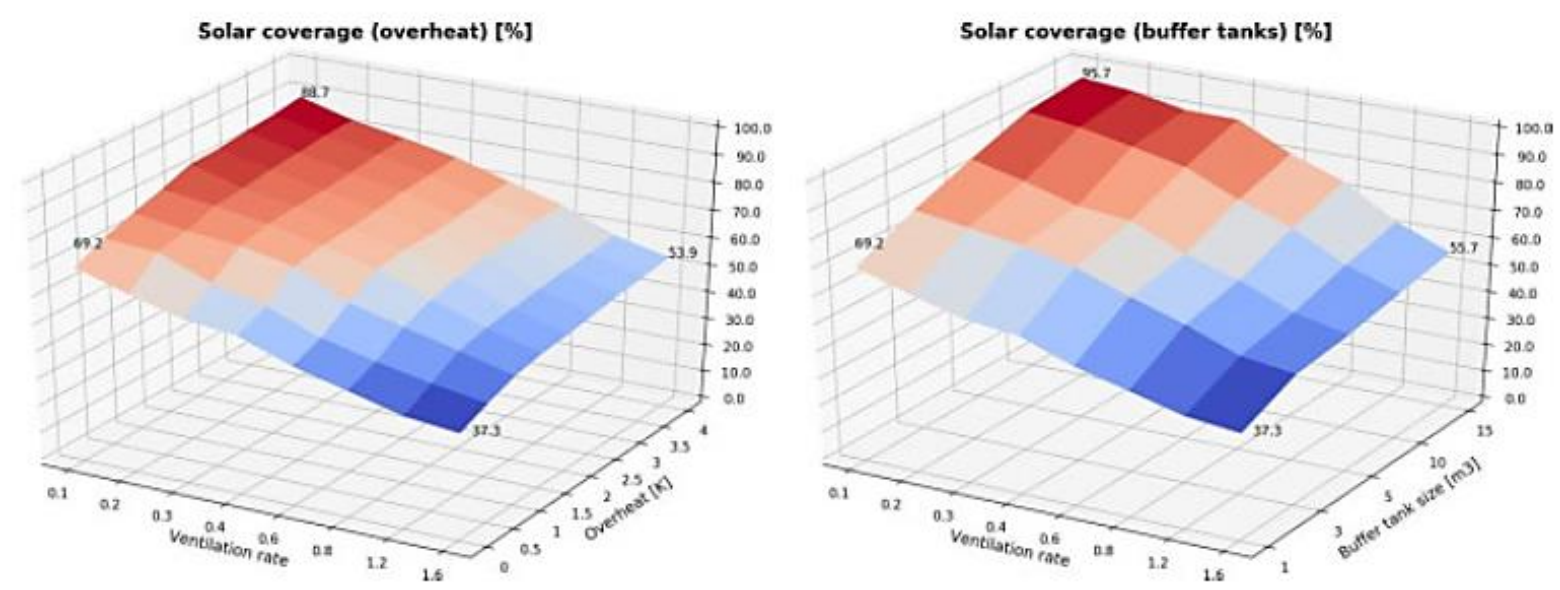

Fig. 9: Solar coverage with respect to quality of building envelope and to overheat gap (left) and to buffer tank size respectively (right)

Again the results from the "overheat" simulations match those from the "buffer tanks" set very well. (Fig. 9) One has to be aware though, that the simulations with higher solar coverage are not necessarily those with the lowest total additional heating energy demand.

\section{CONCLUSION}

A new method was presented to increase the amount of accumulated solar energy. The idea is to better exploit available solar energy by increasing the temperature level of the building rather than by increasing the thermal mass, i.e. the size of the buffer tank. The control strategy is not too complicated but needs to be supported by buffer tank manufacturers or heating installers in order to achieve the desired results.

The case study in the current work demonstrates that a control strategy allowing for overheating a building can produce similar results with respect to heating demand as buildings without the overheat strategy and bigger buffer tanks.

In fact quite a few more simulations were run following the traditional control strategy but with much bigger buffer tanks. However only the set of simulations was selected for generating the plots that best matched the overheat set of simulations.

Not only the fact that the heating installation needs to allow for the new control strategy but also uncertainties about user behavior can be considered as certain downsides of the idea presented in this work.

Are residents willing to accept an operational temperature that may well exceed the usual value by several K? may be one of the questions that need to be answered before the "overheat" control strategy is put into practice because residents that open windows when inside temperatures stagger too much can foil the best idea about the exploitation of renewable energy.

\section{ACKNOWLEDGEMENTS}

Research funded by Austrian Research Promotion Agency (FFG) the FFG, and the Federal Ministry for Transport, Innovation and Technology and Federal Ministry for Digital and Economic Affairs.

Further fundings were granted by the Bundesinnung Bau, Zement + Beton Handels- und Werbeges.m.b.H and

MA20 - Energieplanung.

\section{REFERENCES}

Achterbosch, G.G.J., de Jong, P.P.G., Krist-Spit, C.E., van der Meulen, S.F., Verberne, J., 1985. The development of a comvenient thermal dynamic building model. Energy and Buildings 8, 183-196. Dobbs, J.R., Hencey, B.M., 2012. Automatic model reduction in architecture: A window into building thermal structure. Fifth National Conference of IBPSA-USA. 
Dominković D.F., Gianniou P., Münster M., Heller A., Rode C., 2018. Utilizing thermal building mass for storage in district heating systems: Combined building level simulations and system level optimization. Energy, Volume 153, 2018, Pages 949-966, ISSN 0360-5442

Friembichler F., Bednar T., Handler S., Gladt M., Neusser M., Hofer R., Schöberl H., 2014. Thermische Bauteilaktivierung. Entwicklung eines Rechenkerns (Downloaded from https://nachhaltigwirtschaften.at/resources/hdz_pdf/berichte/endbericht_1411_thermische_bauteilaktiv ierung.pdf?m=1469661002, Aug. 22nd, 2018)

Gladt, M., PhD Thesis. Vienna University of Technology. 2014.

Gouda, M.M., Danaher, S., Underwood, C.P., 2002. Building thermal model reduction using nonlinear constrained optimization. Building and Environment 37, 1255-1265.

Hagentoft, C.-E., 2001. Introduction to building physics. Lund: Studentlitteratur, ISBN 91-44-018967.

Handler, S., PhD Thesis. Vienna University of Technology. 2014.

Kircher, K., J., Max Zhang, K., 2015. On the lumped capacitance approximation accuracy in RC network building models. Energy and Buildings, Volume 108, 2015, Pages 454-462, ISSN 0378-7788

Kramer, R., van Schijndel, J., Schellen, H., 2012. Simplified thermal and hygric building models: A literature review. Frontiers of Architectural Research 1, 318-325

Reilly, A., Kinnane O., 2017. The impact of thermal mass on building energy consumption. Applied Energy, Volume 198, 2017, Pages 108-121, ISSN 0306-2619 


\title{
BUILDING RETROFIT USING FAÇADE-INTEGRATED ENERGY SUPPLY SYSTEMS
}

\author{
Dagmar Jaehnig, Thomas Ramschak, David Venus, Karl Hoefler, Christian Fink \\ AEE - Institute for Sustainable Technologies (AEE INTEC) \\ Feldgasse 19, 8200 Gleisdorf, Austria \\ Phone: +43 (0) 3112 - 5886 - 0 \\ E-Mail: $\underline{\text { d.jaehnig@aee.at }}$
}

\section{SUMMARY}

A new approach of highly-efficient thermal refurbishments of multi-family buildings is presented that has a high potential to reduce costs and simplify the renovation processes significantly. This approach is based on prefabricated curtain wall elements that integrate components for the energy supply system such as heat pumps, PV panels and all the necessary pipework for supply and waste water lines. By using pre-fabricated curtain wall elements, scaffolding and the relocation of inhabitants can be avoided.

Three different system concepts have been evaluated and compared to a reference retrofit in terms of primary energy and life cycle costs. It has been shown that the new concepts can reduce the primary energy consumption significantly while reducing the costs over the lifetime of the system.

Finally, a functional mockup of such as pre-fabricated façade was constructed and successfully tested in the laboratory.

\section{INTRODUCTION}

There is a huge potential for highly efficient thermal refurbishments of multi-family buildings that would result in significant reductions of $\mathrm{CO}_{2}$-emissions. However, the renovation rate for existing buildings has been declining for the past few years. In the case of comprehensive thermal renovations in Austria, it was only $0.6 \%$ from 2004 to 2014 which is significantly lower than the goals set in the Austrian climate protection report. The current technical renovation standard as well as the renovation process have repeatedly proven to be insufficient in terms of providing the appropriate incentives. This applies not only to structural but also to HVAC refurbishments.

A comprehensive paradigm change is necessary in order to increase the refurbishment rate. There is a need not only for financial incentive mechanisms but also for technical solutions and business models which allow for a simplification of the renovation process. This, in turn, will result in lower costs for the owner and the users as well as in an increased property value which will be reflected in monetary terms.

Façade elements with a high level of insulation are a very promising technology for large-scale buildings. They can be prefabricated to a high degree which allows for quick installation and excellent quality of implementation. One possibility for reducing costs substantially and simplifying the prevailing renovation processes is the integrating of more functionality into traditional curtain walls, e.g. using the surfaces as energy conversion areas or for energy storage, integrating small-scale heat pumps and ventilation elements into the prefabricated component as well as distribution lines for heat, water, air or disposal of waste water. The recently completed research project "HVACviaFACADE" examined exactly this approach. 


\section{POTENTIAL}

In a first step, it was assessed which type of buildings from which period of construction offers the highest potential to introduce retrofitting with pre-fabricated curtain walls. Based on statistical data from Austria, multi-family buildings from the 1960s to 1970s were selected. The reason is that there is an especially large number of such buildings in Austria that have not yet been renovated. Typical floor plans of buildings from this period were used to define a reference building with 4 floor levels and 12 apartments (see Fig. 1). The total gross floor area is $687 \mathrm{~m}^{2}$. This reference building was used to define a reference retrofit and a few renovation concepts with pre-fabricated curtain walls that include different functionalities of the heat supply system for the apartments. These concepts were compared with the reference retrofit from the point of view of primary energy consumption and life cycle costs.
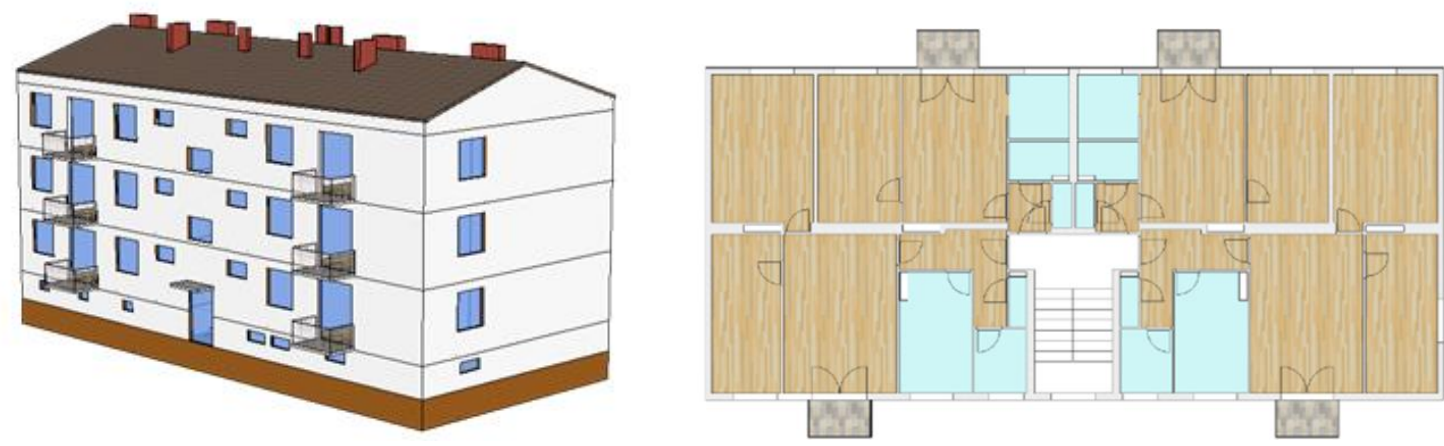

Fig. 1: Reference building

The reference retrofit consists of a thermal insulation composite system with supply and waste water lines all led inside the building. The energy supply system is a central gas boiler for space heating and electrical water heaters for domestic hot water in each apartment.

\section{EVALUATION OF CONCEPTS}

At the start of the project, 24 energy supply concepts were defined that allow for the integration of HVAC into pre-fabricated curtain walls. Their suitability as well as implementation was evaluated in a quantitative as well as qualitative way. The technologies evaluated are based on the five main technology groups: solar thermal, photovoltaics, heat pumps, ventilation technology as well as water heaters and heat transmission system. Most concepts consisted of a combination of technologies or of hybrid systems.

Three system concepts were selected based on this evaluation of concepts. They were simulated in detail for the reference multi-family building with 12 apartments and for 2 different thermal insulation standards (heating demand 30 and $15\left(\mathrm{kWh} / \mathrm{m}^{2} \mathrm{a}\right)$ ) implementing wood curtain walls. For this analysis, the dynamic simulation environment TRNSYS was used. Two system concepts use outdoor air heat pump solutions in combination with façade-integrated photovoltaic systems. One model has a central heat pump and the other one uses decentralized small-scale heat pumps which are integrated into the façade. The third concept is an electric heating system using infrared heating elements in combination with photovoltaics. All system concepts were simulated with different PV surface areas and rely on the same ventilation technologies depending only on the thermal insulation standard. For space heating demand 30 it is a controlled ventilation system with a central exhaust fan and air intake via an opening in the window structure. For space heating demand 15 it is a controlled ventilation system with heat recovery. 

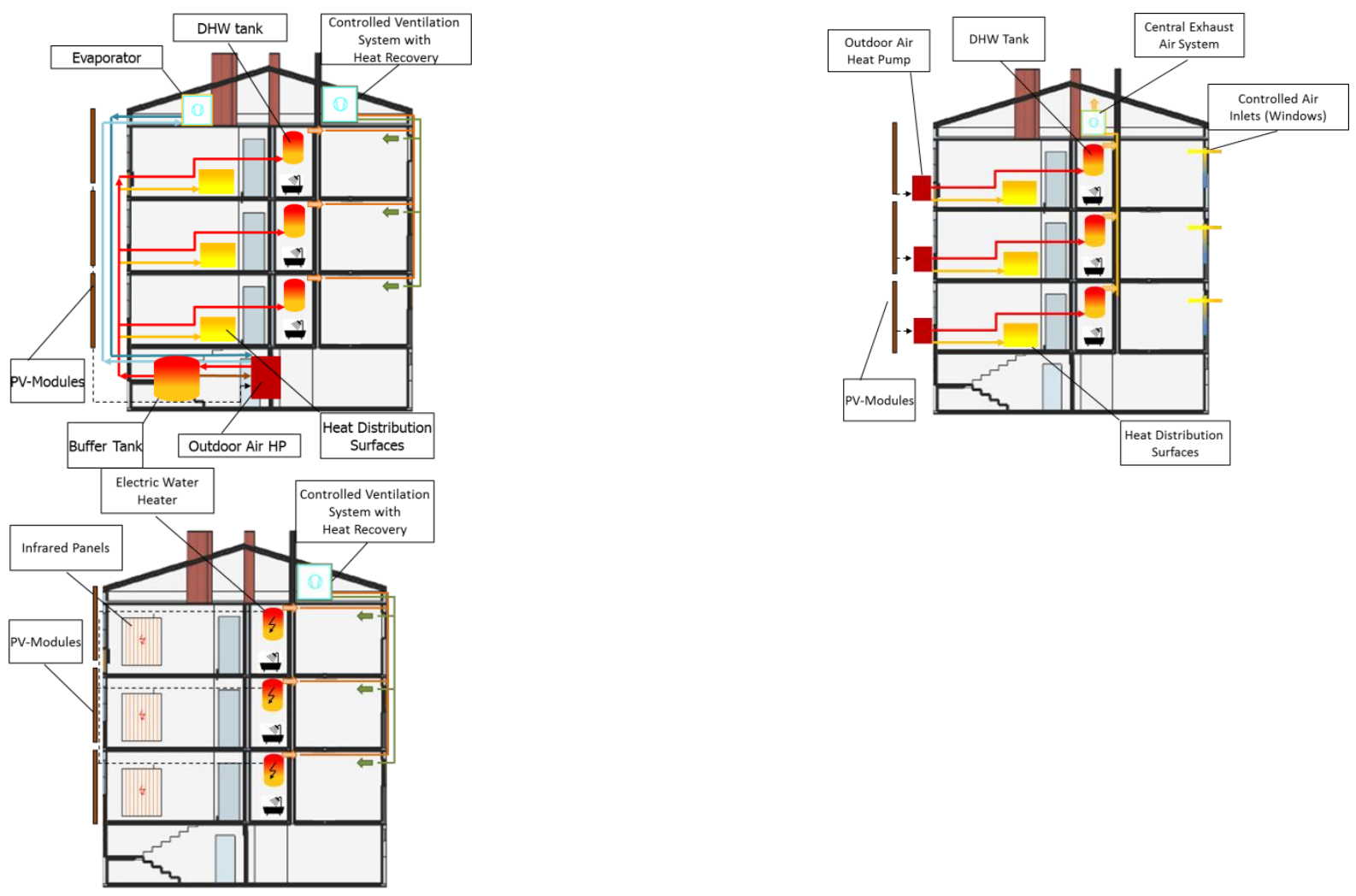

Fig. 2: Schematic representation of chosen system concepts (left: central heat pump, middle: decentralized smallscale heat pumps, right: electric heating system)

\section{ADVANCEMENTS OF THE PRE-FABRICATED CURTAIN WALL SYSTEM}

Based on the experiences from previous projects, the wood curtain wall system was further developed. The goal was to reduce the costs by minimizing the number of layers and by transferring simpler construction steps from pre-fabrication to the construction site. One main step was that the equalizing layer that is necessary to align the curtain wall with the existing wall was considered as a constructive element. The necessary insulation layer is used to equalize the existing wall (see Fig. 3). On the construction site, battens are mounted on the existing wall to equalize it. Then the pre-fabricated façade is mounted and insulation material is blown into the gap between the existing wall and the façade element. In addition, active as well as passive HVAC elements such as ascending pipes, descending waste water pipes, ventilation pipes etc. are integrated into the façade.

Thanks to all these measures, it was possible to optimize the current construction technique for wood curtain walls so that the costs could be reduced by $30 \%$. 


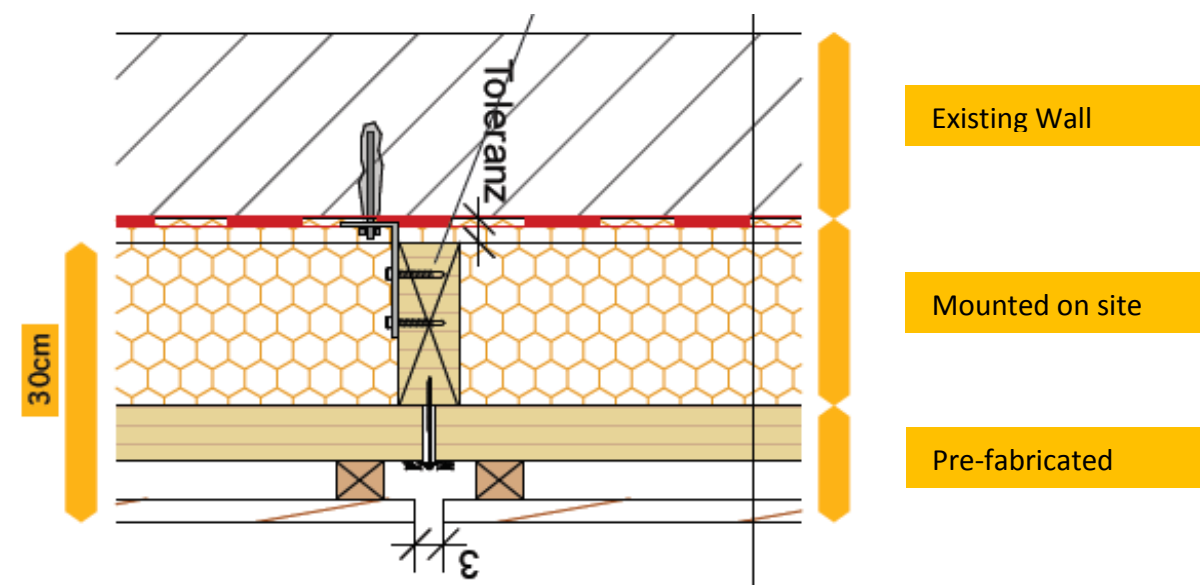

Fig. 3: Schematic representation of the newly developed concept for pre-fabricated wood curtain walls

\section{SIMULATION RESULTS}

The simulation results were analyzed based on the primary energy consumption of each innovative system concept compared to the reference retrofit. The primary energy factors 1.17 for gas and 1.91 for electricity (annual mean value) were used. For PV electricity no storage was assumed. That means the electricity that could be used directly by the system, was determined and deducted from the final energy consumption of the system. Excess PV electricity was assumed to be fed into the grid but not taken into account for the energetic or cost analysis.

The simulations showed that for the low energy building $\left(30 \mathrm{kWh} /\left(\mathrm{m}^{2}\right.\right.$ a) space heating demand) implementing heat pumps already reduces the primary energy demand by $40-50 \%$ compared to a reference scenario and a PV system increases this reduction to 65-70\% (see Fig. 4). Electric heating via infrared elements without photovoltaics does not lead to any advantages in terms of primary energy demand, compared to the reference renovation. In order to keep up with the primary energy demand of the heat pump systems, it requires huge PV areas which cannot fit onto the building anymore. The electrical heating system without photovoltaics consumes slightly more primary energy than the reference system due to the heat losses in the reference system.

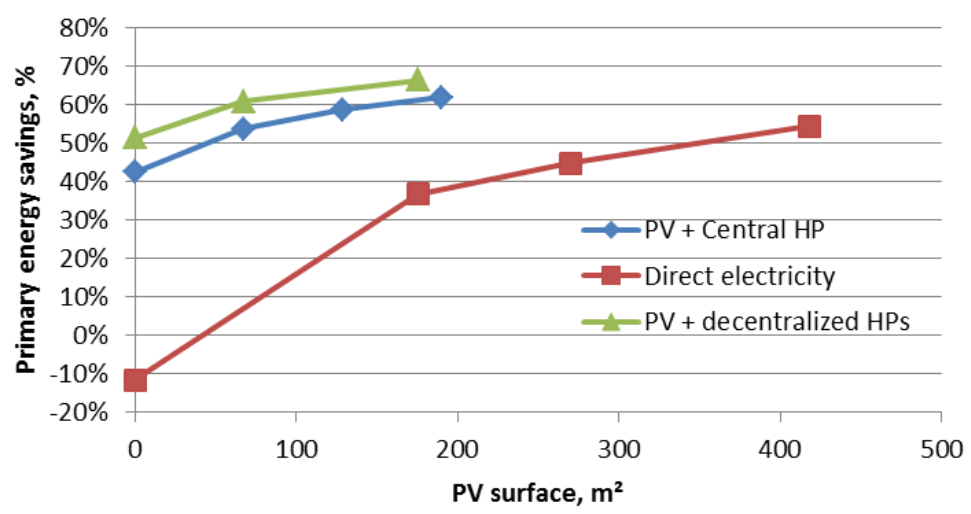

Fig. 4: Primary energy savings for the three system concepts compared to the reference retrofit $\left(30 \mathrm{kWh} /\left(\mathrm{m}^{2} \mathrm{a}\right)\right.$ space heating demand)

For the passive house retrofit (space heating demand 15), the primary energy savings are somewhat larger and the direct electricity system can reach the same primary energy savings of $70 \%$ with a PV surface of roughly $420 \mathrm{~m}^{2}$ which would mean that the entire east, west and south facades as well as the roof (except for the windows) would have to coverered with PV panels (see Fig. 5). 
The same analysis was also carried out including household electricity consumption. Of course some of the excess PV electricity can then be used to cover the consumption of the household appliances. However, the primary energy savings go down by about 10 percentage points for the same PV areas.

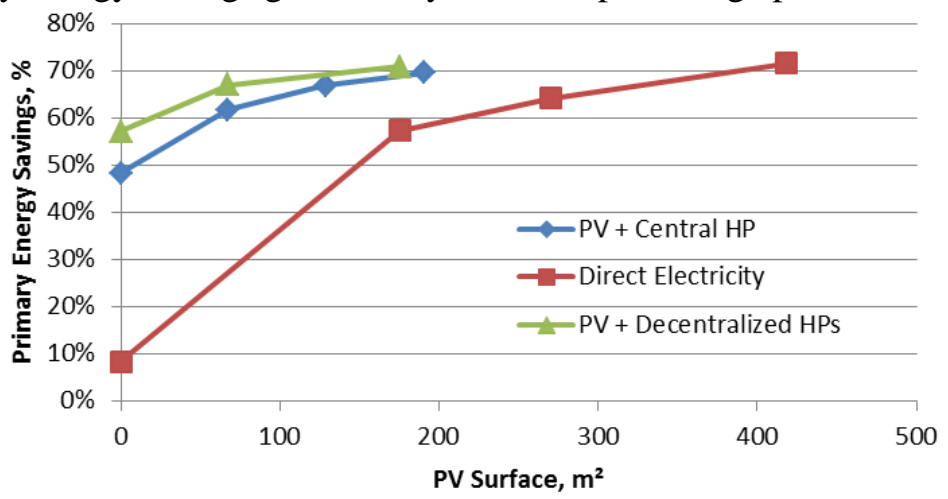

Fig. 5: Primary energy savings for the three system concepts compared to the reference retrofit $\left(15 \mathrm{kWh} /\left(\mathrm{m}^{2} \mathrm{a}\right)\right.$ space heating demand)

\section{LIFE CYCLE ANALYSIS}

Another important aspect of the project was the dynamic cost calculation of the entire renovation concepts and processes over the whole life cycle, from the construction to the demolition and removal of the building. The analysis was carried out using the present worth method that assesses all occurring costs during the lifetime of a building relating the costs that occur in the future to their present worth. For that purpose interest and inflation rates were defined (see Tab. 1). The investment costs are lowest for the reference retrofit: The direct electricity concept shows the lowest investment costs of the new concepts followed be the central heat pump system and the decentralized heat pump system. Important factors are the cost of the necessary PV system and the operating costs.

Tab. 1: Parameters for present worth method

\begin{tabular}{ll}
\hline Economic Factors & \\
\hline Period under consideration [a] & 40 \\
\hline Interest rate on capital [\%/a] & 4.0 \\
General inflation rate [\%/a] & 2.0 \\
Inflation rate on natural gas [\%/a] & 4.1 \\
Inflation rate on electricity [\%/a] & 4.0 \\
\hline
\end{tabular}

When combining energy-related as well as economic aspects and calculating the heat generation costs over the whole life cycle, it becomes clear that the heat pump systems not only achieve lower primary energy demand but also lead to lower life cycle costs than the reference renovation (using conventional thermal insulation systems and gas boilers). In Fig. 6, this is shown for the heat demand 15 building. The reduction of life cycle costs depends on the size of PV area included in the system. Highest life cycle cost savings can be achieved with no PV area, but primary energy savings are highest with roughly $180 \mathrm{~m}^{2}$ of PV i.e. it's a trade-off between life-cycle costs and primary energy savings. Life cycle cost savings are somewhat smaller or for some cases negative for the decentralized heat pump system. The reasons here are quite high investment costs for the small-scale heat pumps which may be reduced once they are mass-produced.

The cost reduction potential of the electric heating system via infrared heating depends heavily on the thermal insulation level. Heat demand 15 allows for cost advantages over the reference scenario while heat demand 30 does not (compare Fig.6 and Fig. 7). 


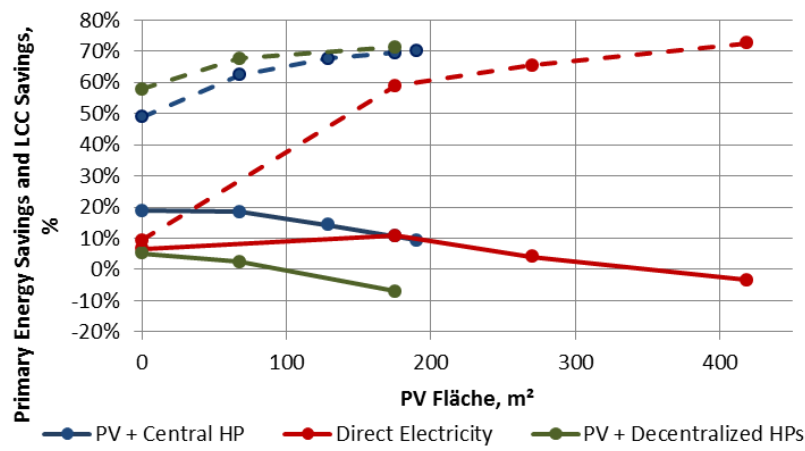

Fig. 6: Primary energy savings and savings in life cycle costs for the three system concepts compared to the reference retrofit $\left(15 \mathrm{kWh} /\left(\mathrm{m}^{2}\right.\right.$ a) space heating demand $)$

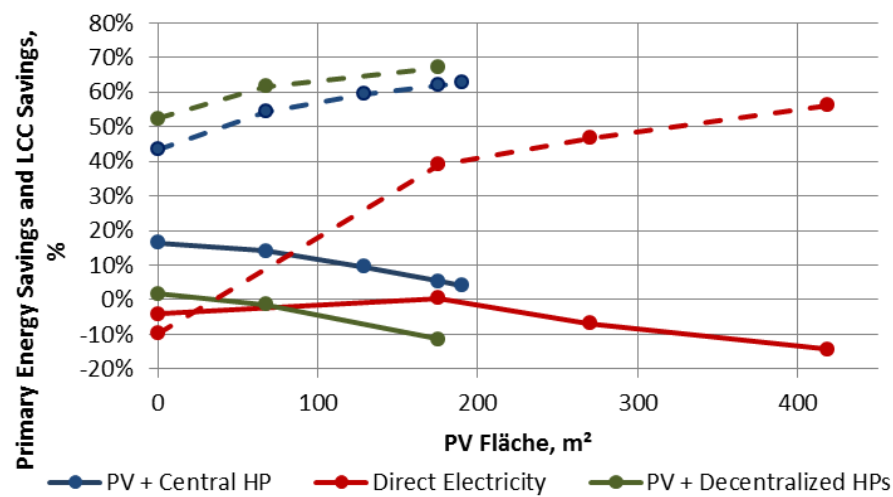

Fig. 7: Primary energy savings and savings in life cycle costs for the three system concepts compared to the reference retrofit $\left(30 \mathrm{kWh} /\left(\mathrm{m}^{2}\right.\right.$ a) space heating demand $)$

\section{DEVELOPMENT AND CONSTRUCTION OF FAÇADE ELEMENTS WITH INTEGRATED TECHNICAL COMPONENTS}

Due to the good performance of the heat pump systems, the concept using decentralized heat pumps for each apartment unit was selected for implementation into a curtain wall module as well as an $11 \mathrm{~m}^{2}$ test element. A functional mockup of such a façade was constructed that combines several technical elements (see Fig. 8): A window element, normal façade elements as well as a duct element that includes a small-scale heat pump and the necessary pipework as well as a PV element.

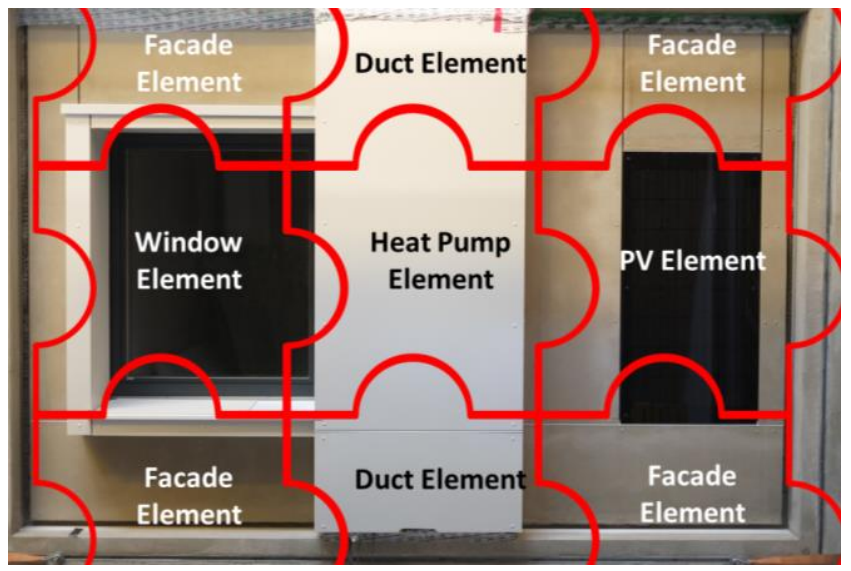

Fig. 8: Schematic representation of a curtain wall façade element built up from separate elements

The complete integration of the small-scale heat pump into the curtain wall proved to be very challenging. The heat pump concept had to be revised entirely so that the volume of the equipment could be reduced to only 60 liters, i.e. its size is comparable to a desktop PC while still producing up to $3 \mathrm{~kW}$ of heat. At the same time an intelligent solution for maintenance and repair work was 
developed by implementing a rolling system that allows for easy access to the heat pump when opening the windows. The heat pump is integrated into an HVAC duct together with all the other supply lines. This allows for easy prefabrication (can be done by HVAC contractors). For the integration of the photovoltaic system into the façade a mounting system was defined that follows the same sealing and mounting principles as the conventional mounting system for façade panels. This allows for aesthetic, simple and cost-effective integration of the PV system into the façade.
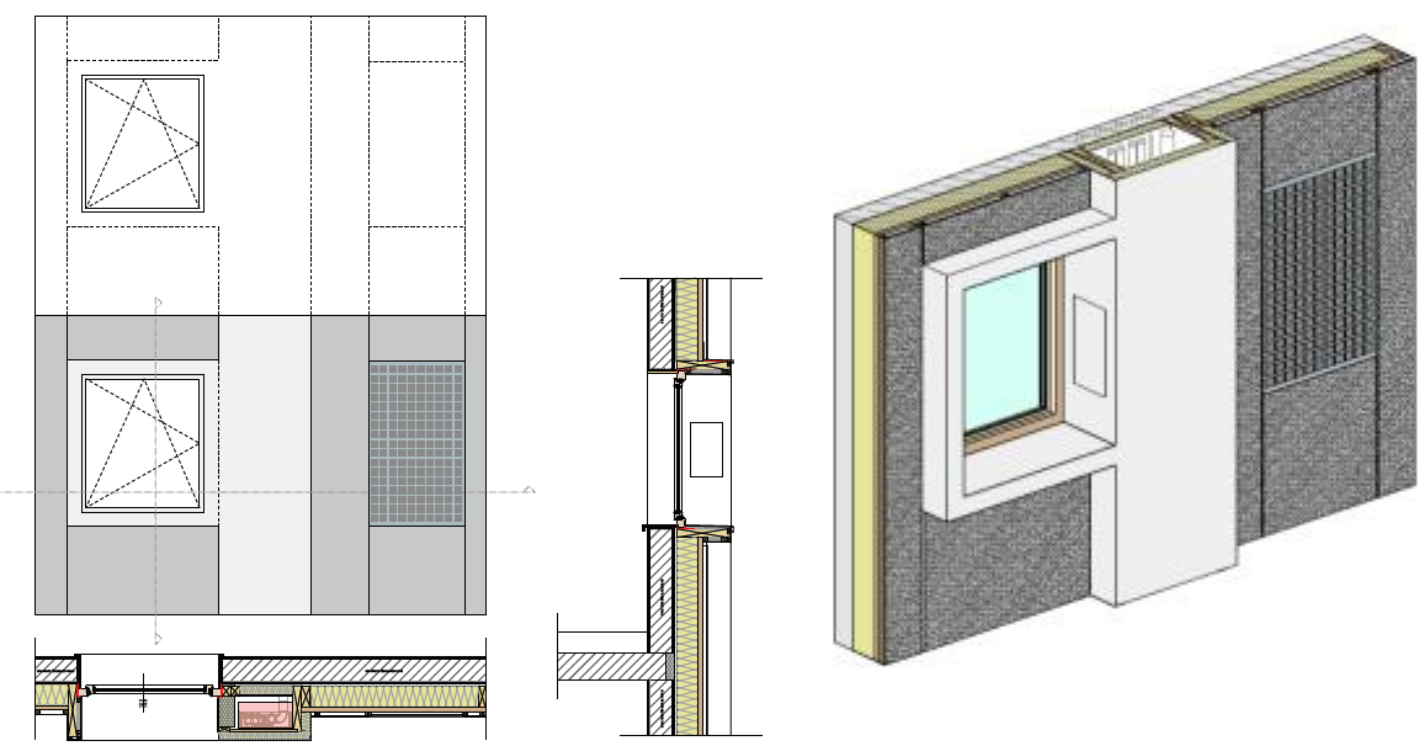

Fig. 9: Functional mockup - left: design drawing of the façade model with integrated small-scale heat pump in the duct element (in the horizontal plan the heat pump is shown in pink), right: design study of the façade model (picture source: Nussmüller Architekten ZT GmbH)

\section{LABORATORY TESTS OF FUNCTIONAL MODEL}

A number of laboratory test were carried out on the functional model to analyze the airtightness, the acoustic insulation and the sound intensity during operation of the heat pump. Also, a driving rain test was conducted.

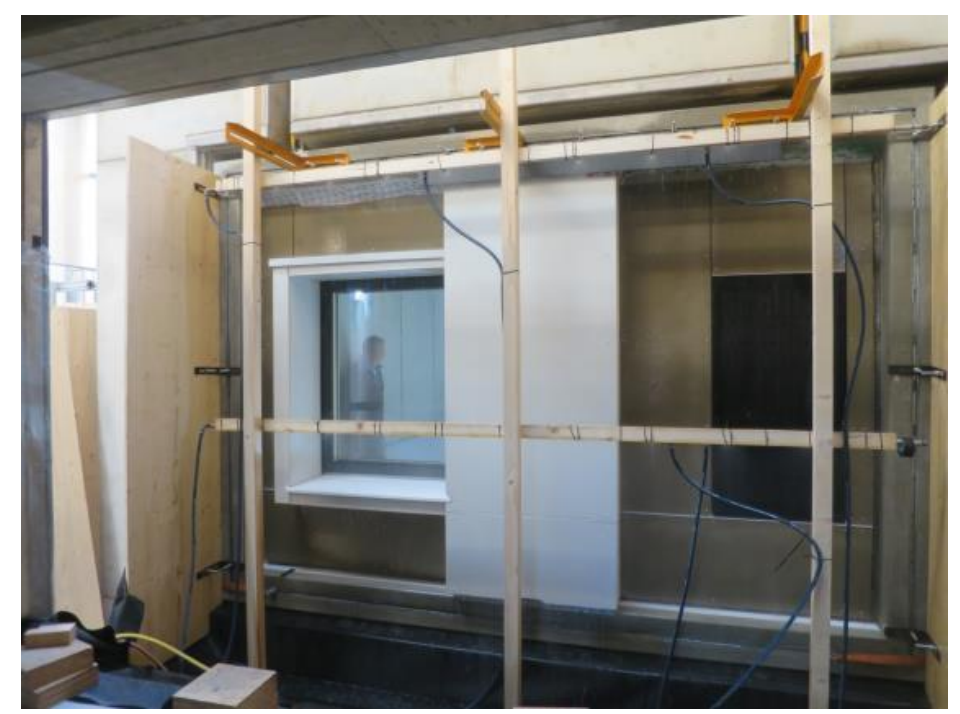

Fig. 10: Setup for the driving rain test (picture source: TU Graz)

During the driving rain test according to EN 12154 and EN 12155, a small water intake was detected at a pressure difference of $300 \mathrm{~Pa}$. After additional water proofing of the affected area, the test could 
be continued up to $600 \mathrm{~Pa}$ without problems. That means that the façade setup is well suited for the use as rain-proof barrier of a building.

An aspect that will need further attention during the continued development of the construction is the sound conduction from the heat pump in operation to other parts of the façade. In the tests, some vibration of the window element could be detected that need to be ruled out by constructive measures.

Overall, the metrological analysis of the functional model consistently showed positive results.

\section{ACKNOWLEDGEMENTS}

The project was funded within the program e!MISSION.at - Energy Mission Austria of the Austrian climate and energy fund. We would like to thank all project partners (Vaillant GmbH, Kulmer HolzLeimbau, Nussmüller Architekten ZT GmbH and TU Graz) for their contributions to the project.

\section{CONCLUSIONS}

There is a huge energy savings potential in the thermal retrofitting of multi-family buildings. However, the current retrofitting process is not suitable in order to reach the set climate goals in Austria and Europe. An entire paradigm change is necessary that includes new technical solutions, business models, incentive mechanisms, simplification of the retrofitting process as well as cost reduction.

The project presented in this paper suggests a technical solution that allows cost reduction and simplification of the retrofitting process by means of pre-fabricated highly insulated curtain walls that include functional elements such as heat pumps, PV panels and all necessary pipework. Thereby, retrofitting costs can be reduced by avoiding scaffolding and relocation of the inhabitants during retrofitting.

Three new system concepts were analyzed in detail by means of detailed system simulations and life cycle analysis. The results show large savings in primary energy consumptions as well as a reduction of life cycle costs.

Finally, a functional mockup of a façade element including PV and a small-scale heat pump was constructed. Laboratory tests of the façade show positive results that should lead to further development of the concept and first demonstration systems in the near future.

More details on the project HVACviaFacade can be found in [Fink et al., 2017]

\section{REFERENCES}

Fink et al. (2017): Final Project Report: Vorgefertigte Fassadenelemente mit maximal integrierten HVAC-Komponenten und -Systemen zur Bestandssanierung (in German), Austrian Klima- und Energiefond, Project number: 843945 


\section{DEEP RENOVATION OF MULTI-FAMILY-HOUSES WITH COMPACT HEAT PUMPS}

Fabian Ochs, Dietmar Siegele, Toni Calabrese, Georgios Dermentzis

Unit for Energy Efficient Building

Technikerstr. 13, 6020 Innsbruck, Austria

Phone: +43 51250763603

\section{SUMMARY}

E-Mail: fabian.ochs@uibk.ac.at

Within the framework of the Austrian FFG project "SaLüH!" concepts with high energy efficient and cost-effective decentral small scale heat pumps for heating and domestic hot water preparation for the renovation of small dwellings in multi-story buildings are developed and investigated. Very compact heat pumps are developed in order to enable the integration of these units into the window parapet or into the façade. The wall integration has a high potential in pre-fabrication and leads to an optimal solution for renovation of small apartments. The target is to create a complete renovation package with a decentralized (apartment size) exhaust-air heat pump (HP) for ventilation and heating installed in the kitchen and an air-to-water HP for domestic hot water (DHW) preparation installed in the bathroom. The solutions aim to be cost effective, involving components and technologies with high efficiency and minimum noise emissions. In addition, further aspects such as handling, compactness, attractiveness, maintenance, etc. are of high relevance to enable a minimum disruptive renovation.

Keywords: Renovation, Multi-Family House, heat pump, façade integration, modelling and simulation

\section{INTRODUCTION}

Experience shows that for multi-family houses (MFH), a complete renovation including conversion to central heating and DHW systems is hardly possible. Installation work inside the inhibited flats are often associated with number of complications (technical, legal, financial). Thus the centralization of ventilation, heating and DHW is often dropped. Unfortunately, energy and cost efficient decentralized (and less invasive) solutions are also not available. Hence, new innovative concepts for heating, ventilation and DHW preparation are investigated. Very compact heat pumps are developed in such a way that it will be possible to integrate these units into the window parapet or into a prefabricated timber façade. The wall integration has a high potential in pre-fabrication and leads to an optimal solution for the renovation of small apartments. The target is to create a complete renovation package with a decentralized (apartment size) exhaust-air HP for ventilation and heating installed in the kitchen and a DHW-HP in the bathroom, see Fig. 1.
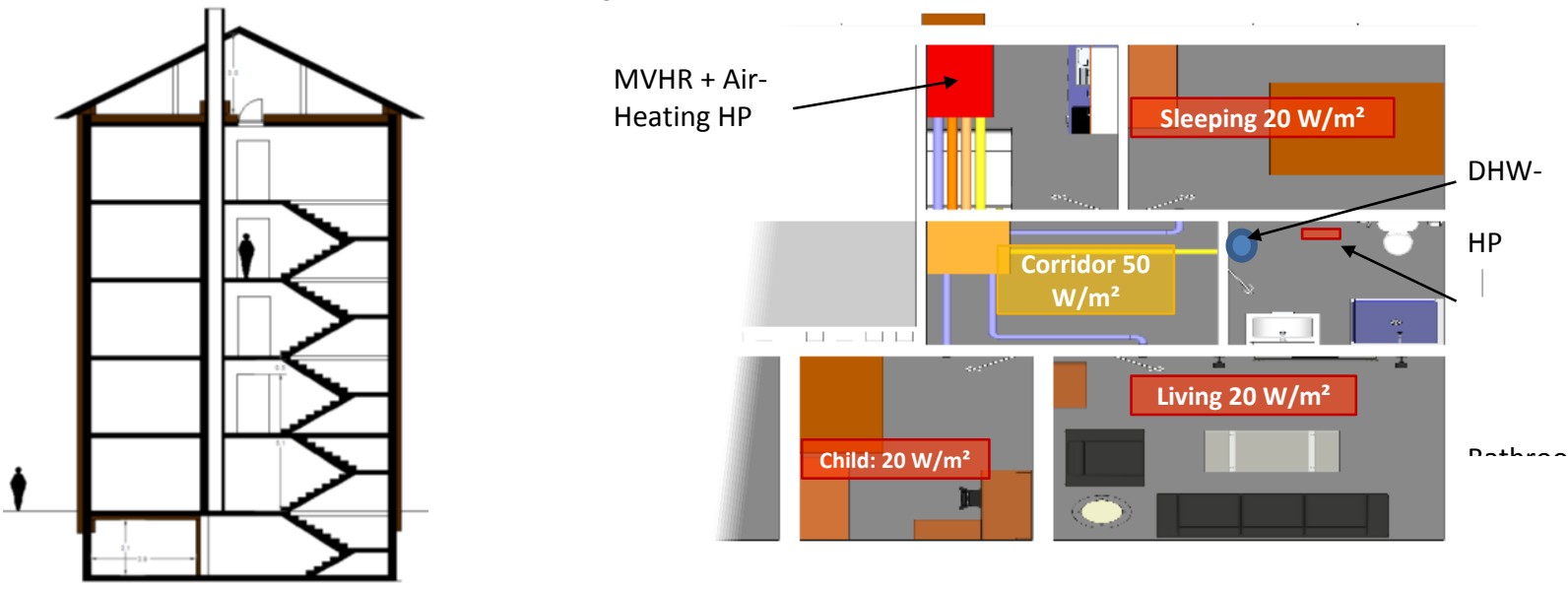

Fig. 1: (left) Typical MFH and (right) heat load (in $\mathrm{W} / \mathrm{m}^{2}$ ) of the thermal zones with optional recirculation air heating in the corridor; Ventilation and HP in the kitchen and DHW HP in the corridor 


\section{SUPPLY AIR HEAT PUMP WITH SECONDARY AIR RECIRCULATION}

\section{Supply Air Heat Pump}

A MVHR unit is, in many cases, one of the first steps for a deep energy renovation of a building in order to improve the IAQ, to reduce the ventilation losses and to maintain thermal comfort. The ducts, necessary for the ventilation system, can be used to distribute space heating power, using a supply air heat pump system. Fig. 2. shows the 3D view of the flat with the proposed system and a simplified scheme of the system Ochs et al. 2017.
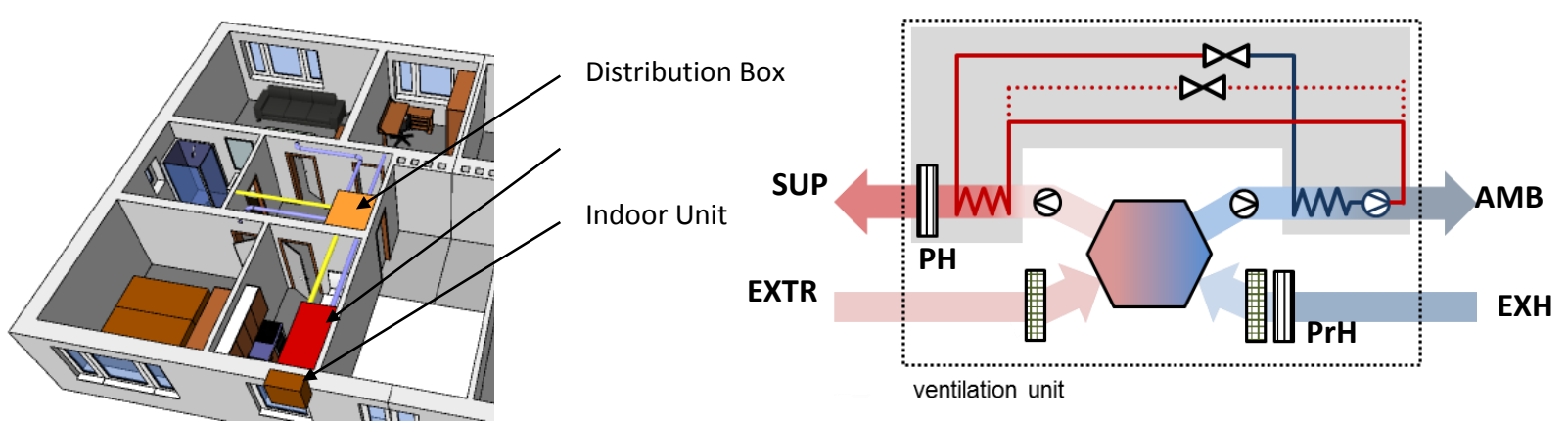

Fig. 2: 3D view of the flat with the supply air heat pump system (left) and scheme of the heat pump in combination with MVHR (right). "PH" and "PrH" represent the post-heater and the pre-heater, respectively

Siegele 2018

The unit can be installed in the façade or as a split system. The indoor unit (in which the condenser of the heat pump and the heat recovery system are placed) is installed in the kitchen (or alternatively could be integrated in the façade), while the outdoor unit contains the evaporator, the compressor and expansion valve of the heat pump. The air distribution system is placed in the corridor and supplies fresh air to the sleeping room, child room and living room. The air is extracted from bathroom and kitchen, cooled in the MVHR unit and its energy is used as source for the heat pump. An electric radiator is placed in the bathroom for comfort reasons. The heating system is controlled according to the operative temperature of a defined room (e.g. the corridor).

The evaporator of the heat pump is placed in the exhaust airflow after the heat recovery exchanger (HR) and uses the remaining energy of the exhaust air. The fresh supply air, pre-heated in a pre-heater and in the Heat Recovery Ventilation (HRV) or Energy Recovery Ventilation (ERV) exchanger, is heated in the condenser of the heat pump and, additionally, in a post-heater if the power of heat pump is insufficient, before to be supplied and distributed to the flat. The use of the ducts of ventilation system for the distribution of the heating power and the compact design represent the big advantages of this heating and ventilation solution. One of the limits of such a system is that air heating is coupled to the hygienic airflow rate and a room-wise control of temperature is possible only if electric radiator room-wise are additionally installed.

\section{Supply Air Heat Pump with Secondary Air Recirculation}

With an exhaust air-to-supply air heat pump (such as in a Passive House ( $\mathrm{PH}$ ) compact unit for ventilation, heating and DHW preparation) the heating power is limited by the hygienic flow rate and is usually $10 \mathrm{~W} / \mathrm{m}^{2}$ (the criterion for achieving PH Standard). If a renovation to PH Standard cannot be achieved (EnerPHit Standard for renovation allows $25 \mathrm{kWh} /\left(\mathrm{m}^{2}\right.$ a) corresponding to ca. $20 \mathrm{~W} / \mathrm{m}^{2}$ to 30 $\mathrm{W} / \mathrm{m}^{2}$ ), either additional room-wise post-heaters have to be used or the volume flow has to be increased. The use of energy recovery membrane (ERV) offers the possibility to increase the volume flow and thus the heating power without violating the lower limit for the relative humidity. For equal relative humidity in the flat, the air exchange rate can be increased with ERV compared to a heat 
recovery ventilation (HRV), see Ochs et al. 2017a, Siegele et al. 2018. Alternatively, recirculation air can be used allowing to increase the heating power and enabling a more individual room control. The exhaust air after the heat/energy recovery of the ventilation system is the source of the heat pump and its performance is directly connected to the effectiveness of the heat/energy recovery. The effectiveness of the energy/heat recovery depends on the operation and boundary conditions (in particular the average relative humidity in the exchanger and the volume flow), which are strongly coupled to the building use and operation. By means of simulation these complex dependencies are investigated (see also Ochs et al. 2017b). The potential increase of the heating capacity and the aspect of air distribution inside the flat is investigated with a transient multi-zone approach including moisture buffer. This contribution discusses the different heat pump and heat distribution concepts and compares the performance.
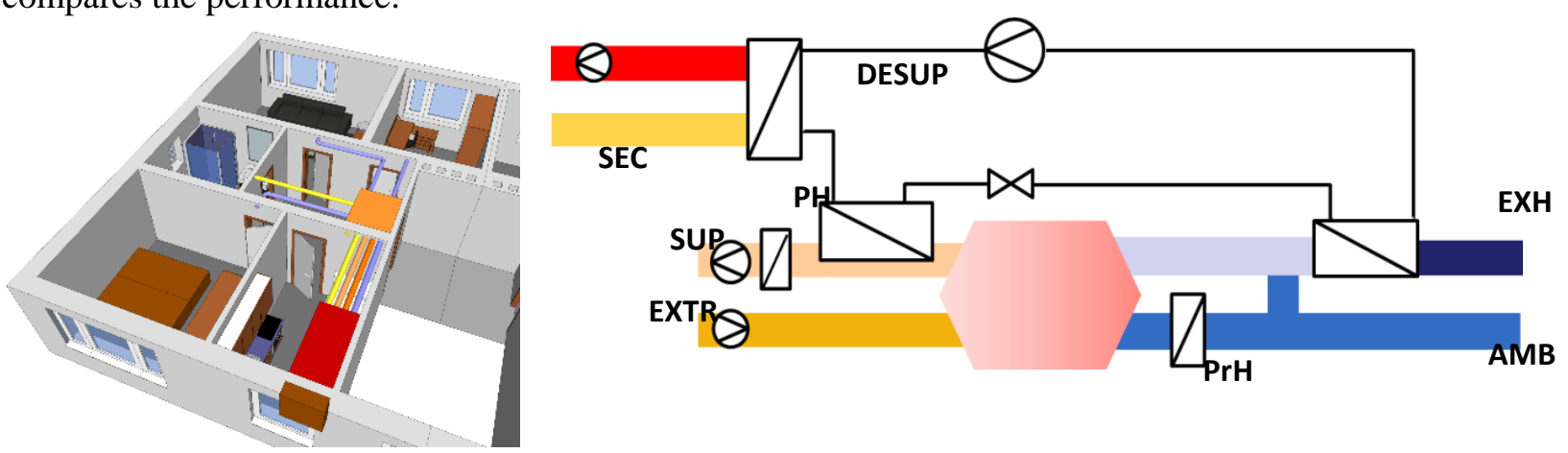

Fig. 3: 3D view of the flat with the supply air heat pump system with recirculation air in the corridor (left) and refrigerant cycle scheme (right). "PH" and "PrH" represent the post-heater and the pre-heater, respectively.

"DESUP" indicates the de-superheater that is used to heat the recirculation air in the corridor, (Siegele 2018)

\section{FUNCTIONAL MODEL OF SUPPLY AIR HP WITH SECONDARY AIR RECIRCULATION}

A functional model of an exhaust air heat pump with recirculation air was developed by the UIBK together with the company Siko Energiesysteme (see Fig. 4 and 5) and is investigated by means of laboratory measurements and building and HVAC simulations. A mock-up of a façade integrated outdoor unit was installed in the Passys test cell at UIBK for testing (see Fig. 5).
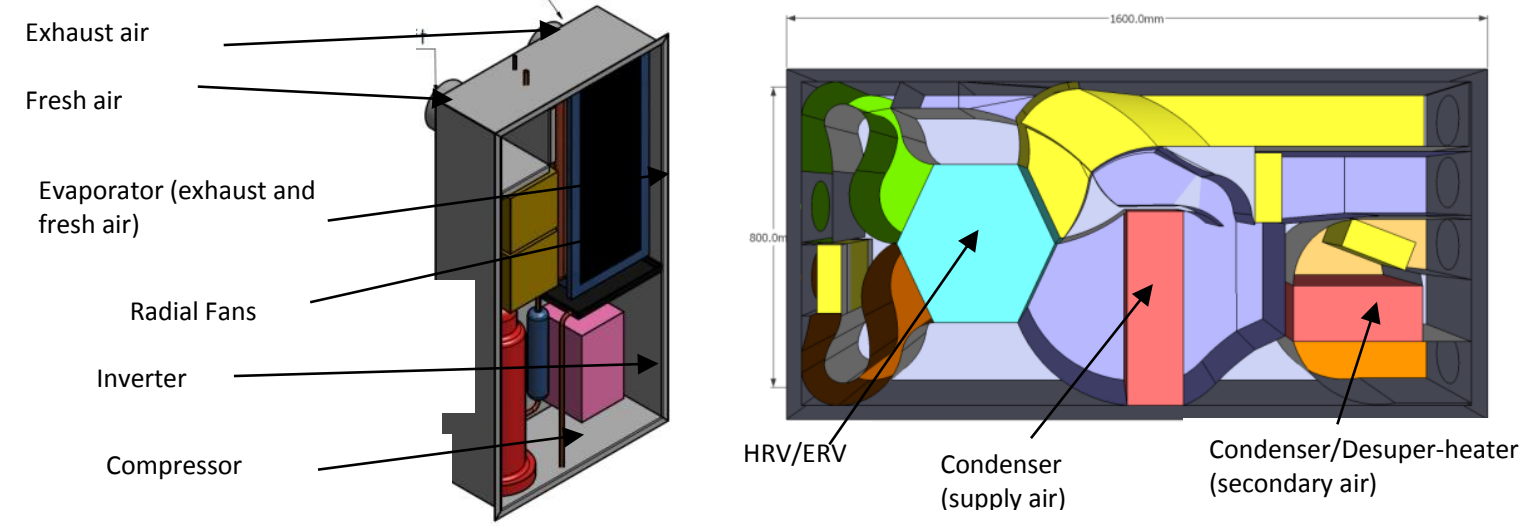

Fig. 4: 3D view of the outdoor unit with evaporator and compressor (left) and the indoor unit (right) with HRV/ERV, supply air condenser and secondary air condenser 

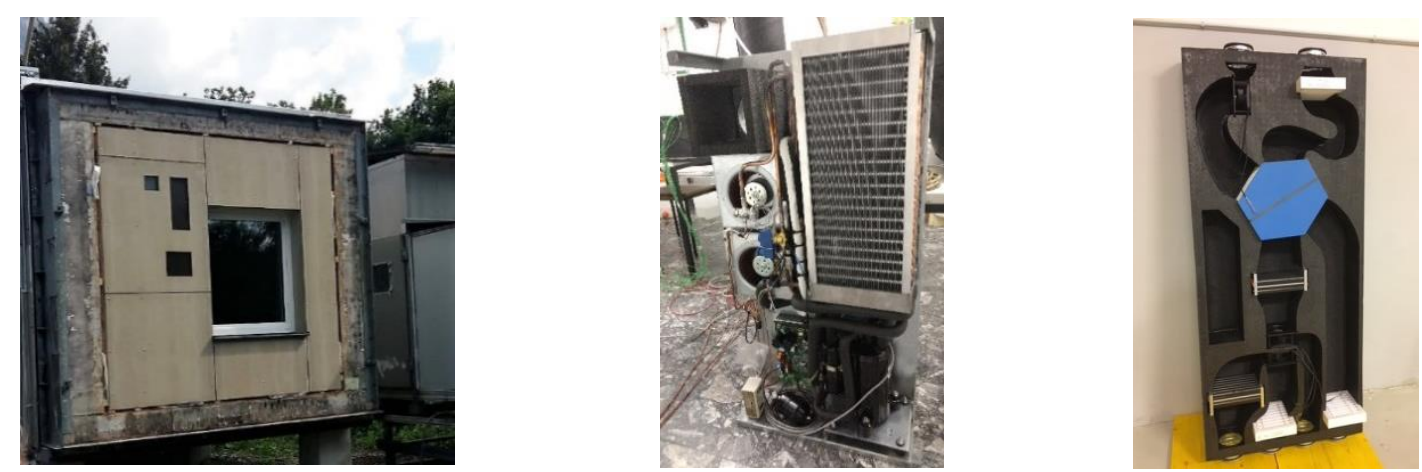

Fig. 5: Outside view of the outdoor unit integrated into the prefabricated timber frame façade in the Passys test cell (left), outdoor unit in the laboratory at the UIBK (center) and indoor unit

\section{MODELLING AND SIMULATION}

\section{Building Model}

The building under consideration is a typical multi-story building Fig 1.) located in Innsbruck (annual average ambient temperature of $9.1{ }^{\circ} \mathrm{C}$ ). It consists of five stories and two symmetrical flats for each storey. The staircase and the basement are not heated and a monthly average development for the ground temperature (annual average temperature of $14.9{ }^{\circ} \mathrm{C}$ ) in considered. The reference flat (area of $70.9 \mathrm{~m}^{2}$ ) considered here, is the flat of the first floor oriented to the Northeast side. The flat consists in six thermal zones (one for each room), while the others flats (below, above and adjacent the flat simulated), basement, staircase and ambient are boundary conditions in the simulation. Air exchange between two adjacent zones through the opening of the doors was modelled in Matlab/Simulink, based on an empirical model developed by Kopeinig et al. 2015.

A balanced mechanical ventilation unit with heat recovery (MVHR) supplies fresh air to the sleeping room (SL), child room $(\mathrm{CH})$ and living room (LI) and extracts air from the kitchen (KI) and bathroom (BA). An air change rate of $0.07[1 / \mathrm{h}]$ (constant throughout the year) due to infiltration through the façade is assumed.

Active cooling system is not considered. External movable shading blinds limit the solar gains during the summer and passive cooling of the flat is ensured by activation of night cooling ventilation and opening of the windows (see Ochs et al. 2018 for detailed information about the control strategies implemented).

The reference flat is occupied by three persons (ca. $24 \mathrm{~m}^{2} /$ person). The reference building is a residential building. It is assumed that the daily schedule of the occupation profile is valid also during the weekend. Additional information about the building model (e.g. internal gains, control strategies, wall constructions, etc.) can be found in Ochs et al. 2018.

\section{HP Model}

The performance of the supply air heat pump and supply air heat pump with secondary air recirculation were measured in the lab at UIBK. Based on these measurement results, in this study, performance maps of heating power and Coefficient of Performance (COP) of the heat pumps were simulated depending on the rpm of the compressor and ambient air temperature (see Figs. 6 and 7) . In case of supply air heat pump with secondary air recirculation system (Fig. 7), the heating power was split between the condenser (50\%) and the de-superheater (50\%) and an additional ambient air of 200 $\mathrm{m}^{3} / \mathrm{h}$ were used as source, in addition to the exhaust air. 

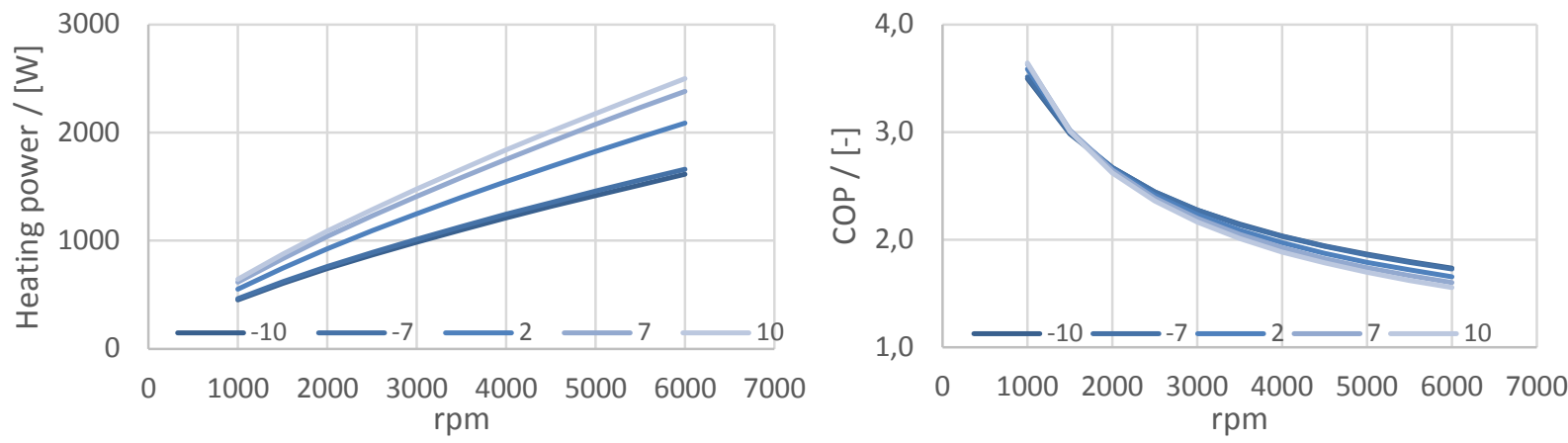

Fig. 6: Performance map of the thermal power of the heat pump (left) and the COP (right) depending on the rpm of compressor and ambient air temperature $\left[{ }^{\circ} \mathrm{C}\right]-$ Supply air heat pump system
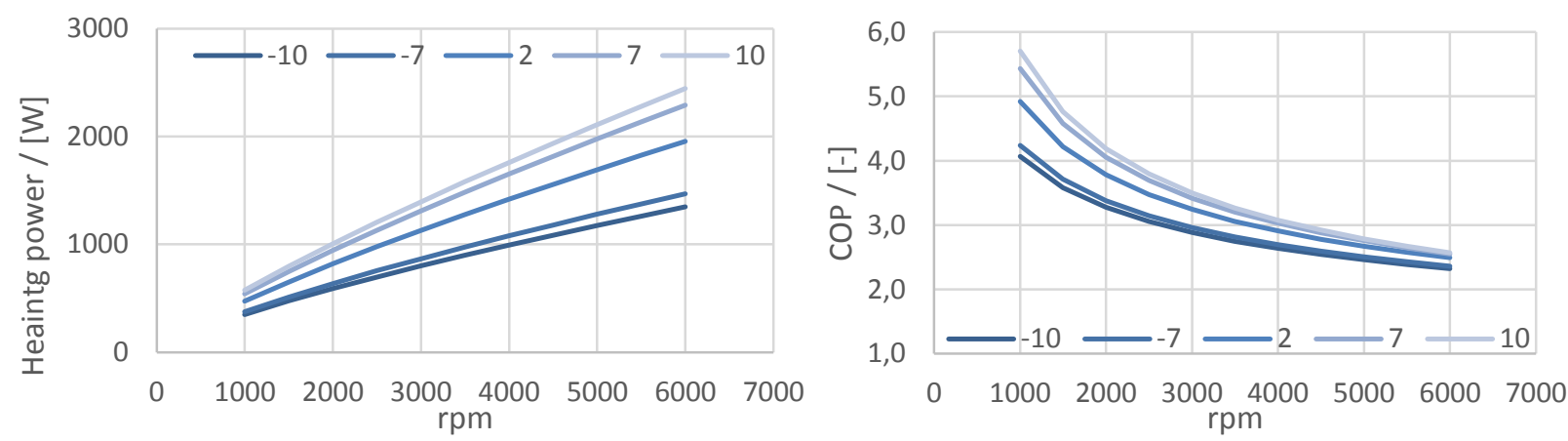

Fig. 7: Performance map of the total thermal power of the heat pump (left) and the COP (right) depending on the rpm of compressor and ambient air temperature $\left[{ }^{\circ} \mathrm{C}\right]$ - Supply air heat pump with secondary air recirculation system. Additional ambient air of $200 \mathrm{~m} 3 / \mathrm{h}$ was used as source. The total heating power was split between the condenser (50\%) for the supply air and the de-superheater (50\%) for secondary air.

\section{Reference Heating System}

\section{Electric radiator room-wise}

In the framework of project "SaLüH!" innovative solutions for heating and ventilation for the renovation of small flats of MFHs are investigated and compared with regard to energy performance, thermal comfort and indoor air quality. The different heating concepts are analysed and compared against a reference system. Electric radiator room-wise system is characterized by low investments costs, but has high operating costs due to the inefficient use of electricity. In such a system, the roomwise control of the temperature as well as the independence of ventilation and heating systems are one of the most important advantages. In the building model, six electric radiators (one for each room of the flat, see Fig. 8, left) are used to keep an operative room temperature of $21^{\circ} \mathrm{C}$.

\section{Split unit with recirculation air in the corridor}

A variant of the electric radiator room-wise system is presented in Fig. 8 (right hand side). Recirculation air in the corridor (CO) is heated with a heat pump split unit and allows indirect heating (through heat transmission and air exchange) of the other rooms of the flat. Electric post-heaters (with a maximum power of $1000 \mathrm{~W}$ ) are placed in all the rooms (except the corridor) to keep the operative temperature at the anticipated level, if necessary. Higher investment costs (compared to the direct electric heating system described above) due to the installation of the split unit must be considered, but a better performance can be expected and some cooling is possible in summer. In both systems heating and MVHR systems are separated and a room-wise control of temperature can be ensured. 

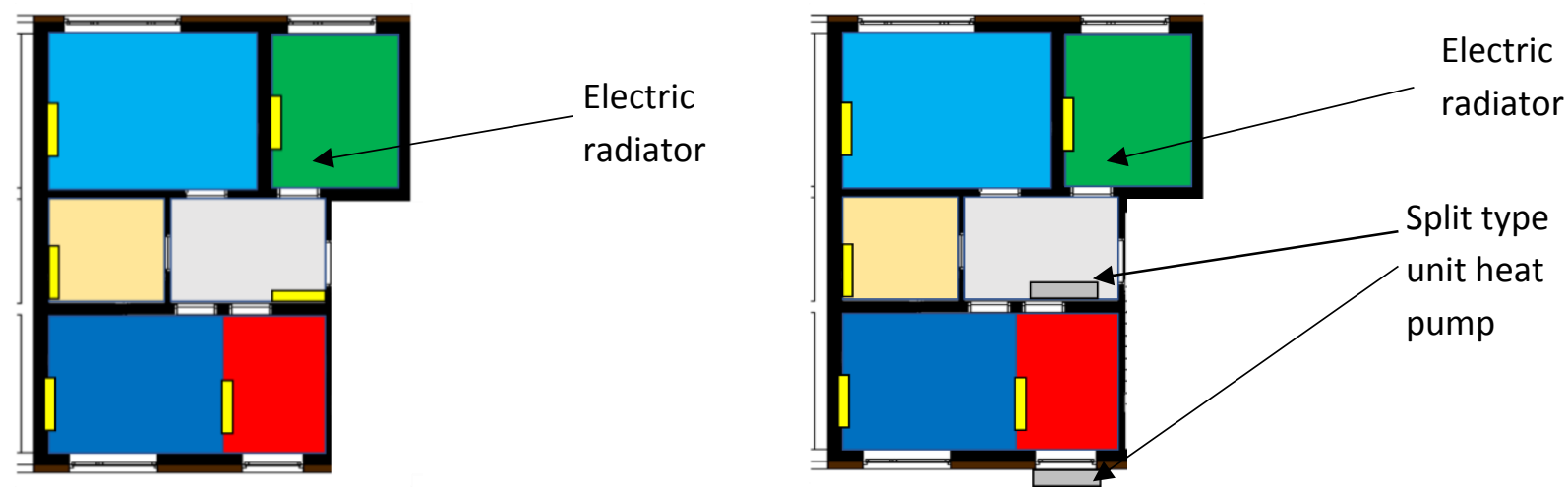

Fig. 8: 2D view of the flat with (left) electric radiators and (right) the HP split unit with recirculation of air in the corridor (Ochs et al. 2017). The indoor unit is installed in the corridor, while the outdoor unit can be integrated in the façade. All the rooms (except the corridor) are equipped with electric radiators

\section{PERFORMANCE INDICATORS}

Thermal comfort (T, r.H.), indoor air quality $\left(\mathrm{CO}_{2}\right)$ and performance of the proposed MVHR and HP systems are compared by means of simulation with the reference system. The following performance indicators are used:

$$
\begin{aligned}
S P F_{H P} & =\frac{Q_{\text {Condenser }}+Q_{\text {Desup }}}{\left(W_{\text {el,Compressor }}+W_{\text {el,Vsec }}+W_{\text {el,Vamb }}\right)} \\
S P F_{\text {sys }} & =\frac{\left(Q_{H P}+Q_{\text {Post-heater }}+Q_{\text {Radiator }}\right)}{\left(W_{\text {el, }, \mathrm{P} P}+W_{\text {el,Post-heater }}+W_{\text {el,Radiator }}\right)} \\
S P F_{H R V} & =\frac{\left(Q_{\text {Pre-heater }}+Q_{H X}\right)}{\left(W_{\text {el,Pre-heater }}+W_{\text {el,Fans }}\right)} \\
S P F_{\text {tot }} & =\frac{\left(Q_{H R V}+Q_{\text {sys }}\right)}{\left(W_{\text {el, }, \mathrm{HRV}}+W_{\text {el,sys }}\right)}
\end{aligned}
$$

\section{SIMULATION RESULTS}

\section{Comfort and indoor air quality}

Fig. 8 shows operative temperature of each room of the flat and the heating load versus the ambient temperature in the case of electric radiator room-wise system. This system is able to ensure optimal temperature distribution in each room because of the room-wise temperature control and can be assumed as base case to compare the four investigated systems. The flat requires a maximum hourly heating load of $1220 \mathrm{~W}\left(17.2 \mathrm{~W} / \mathrm{m}^{2}\right)$, while during the summer night cooling and opening of windows are able to avoid excessive overheating of the flat with no need of active cooling systems.

In order to ensure good thermal comfort and indoor air quality (IAQ), relative humidity should not fall below the recommended values of $30 \%$ and the concentration of $\mathrm{CO} 2$ should remain below to $1200 \mathrm{ppm}$, respectively. The four heating concepts ensure these conditions and there are no significant deviations between the concepts. Fig. 8 (right hand side) shows the box plot of relative humidity and $\mathrm{CO} 2$ concentration of each room in case of electric radiator room-wise system.

As the electric radiator room-wise system, the split unit system gives the possibility to control independently each room of the flat and this leads to a good temperature distribution (see Fig. 10). Due to the coupling of fresh air supply and heat supply, overheating of supply air rooms occurs in case of the supply air heating system. Overheating in SL, CH and LI is avoided in case of secondary air recirculation, but improvement of control is needed to avoid under-temperature. 

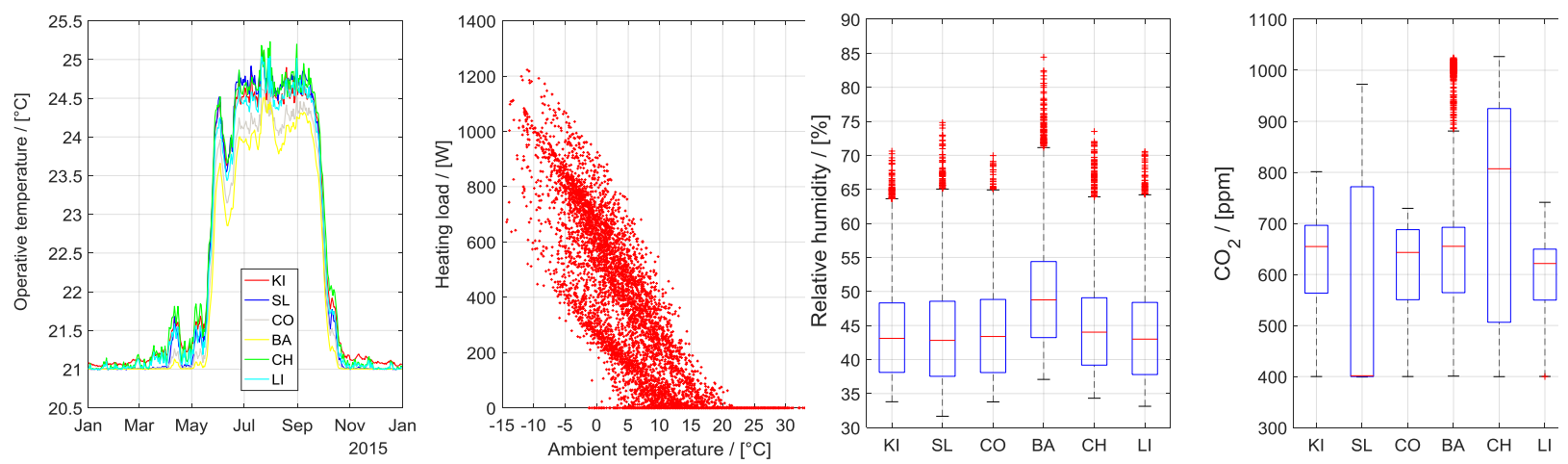

Fig. 9: Electric radiator room-wise system - daily average operative temperatures for each room of the flat (left) and hourly heating load versus ambient temperature (2nd from left); KI: Kitchen, SL: Sleeping Room, CO:

Corridor; BA: Batch room; CH: child room, LI: Living room; Box plot of relative humidity (2nd from right) and $\mathrm{CO}_{2}$ concentration (right) for each room of the flat; $\mathrm{CO}_{2}$ ambient concentration is $400 \mathrm{ppm}$
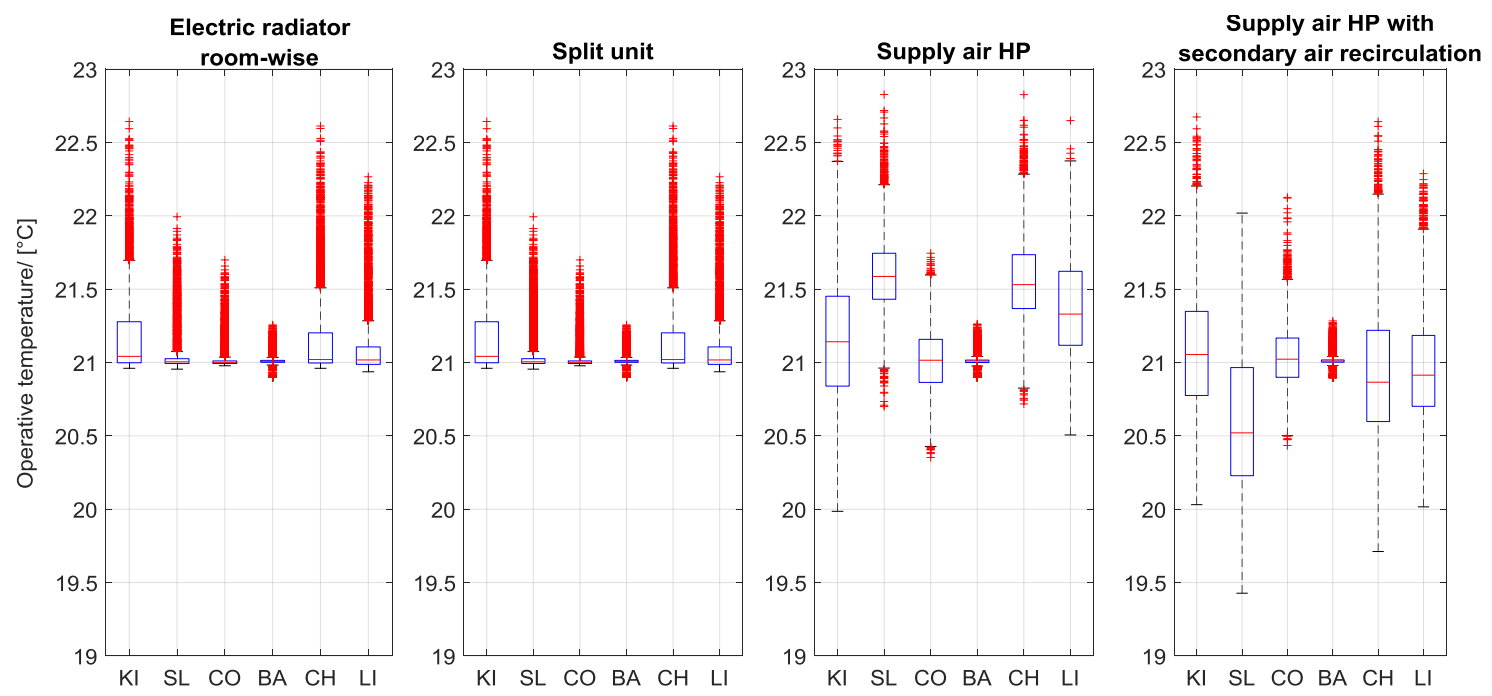

Fig. 10: Box plot of operative temperatures (hourly average) during the heating season for the four heating

\section{System performance}

concepts

Table 1 shows the heating demand and heating load of the flat and electricity demand of heating system for the four investigated cases. Three different coefficient of performance (COP) for the split unit heat pump (i.e. 2, 3 or 4) were assumed in this simulation study (see system "B" in Table 1). The overheating of the supply air rooms (see SL, CH and LI in Fig. 10) in case of system C explains the slight difference in the heating demand (+3\%) compared to system A. Similarly, system D presents lower heating demand $(-20 \%)$ because of the under-temperature of all the supply air rooms.

The performance evaluation of the four heating and ventilation concepts were performed through the definition of four SPFs according to the equations (1)-(4). In the definition of $S P F_{H P}$, the electricity consumption of the two additional fans for the secondary air (Specific Fan Power (SPF) of 0.093 $\left.\mathrm{Wh} / \mathrm{m}^{3}\right)$ and the additional ambient air $\left(\mathrm{SPF}=0.0565 \mathrm{Wh} / \mathrm{m}^{3}\right)$ were considered, while $W_{e l, F a n s}$ in the definition of $S P F_{H R V}$ indicated just the electricity consumption of the two fans for supply and extracted airflow rate $\left(\mathrm{SPF}=0.4 \mathrm{Wh} / \mathrm{m}^{3}\right)$. An additional electric power of $10 \mathrm{~W}$ was assumed for the cooling of the compressor of the heat pump through a fan.

The annual SPFs for the heating season for the four heating concepts are shown in $\mathrm{T}$. SPF $\mathrm{HP}_{\mathrm{H}}$ is slightly higher in system D (2.45) compared to system C (2.43) because of the lower supply temperature of the 
heat pump. System C presents the highest $\mathrm{SPF}_{\text {sys }}(2.07)$ and this value is higher compared to system $\mathrm{D}$ (1.93) because of the lower electricity consumption for the heating of the bathroom. SPF $_{\mathrm{HRV}}$ is slightly lower for systems A and B (5.6) compared to heating concepts C and D (6.05 and 6, respectively) because of the higher $W_{\text {el,Fans }}$ for system $\mathrm{A}$ and $\mathrm{B}$ due to the slight longer heating period. $\mathrm{SPF}_{\text {tot }}$ is 3.2 for system D, slightly higher compared to system C (3.15) and much higher of system B (2.03), even in case a COP for split unit heat pump of 4 is considered.

Table 1: Annual heating demand (HD), maximum hourly heating load (HL) and annual electricity demand (ED) of heating system for the four heating concepts investigated.

\begin{tabular}{|l|c|c|c|c|}
\hline & $\begin{array}{c}\text { Electric radiator } \\
\text { room-wise (A) }\end{array}$ & $\begin{array}{c}\text { Split unit } \\
(\mathbf{B})\end{array}$ & $\begin{array}{c}\text { Supply air HP } \\
\left(\mathbf{C}^{* * *}\right)\end{array}$ & $\begin{array}{c}\text { Supply air HP with } \\
\text { secondary air } \\
\text { recirculation }\left(\mathbf{D}^{* * *}\right)\end{array}$ \\
\hline $\mathrm{HD} /\left[\mathrm{kWh} /\left(\mathrm{m}^{2} \mathrm{a}\right)\right]$ & 29.7 & 29.7 & 30.5 & 23.8 \\
\hline $\mathrm{HL} /\left[\mathrm{W} / \mathrm{m}^{2}\right]$ & 17.2 & 17.2 & 17.3 & 14.8 \\
\hline $\mathrm{ED} /\left[\mathrm{kWh} /\left(\mathrm{m}^{2} \mathrm{a}\right)\right]$ & 29.7 & $27.1 / 26.3 / 25.9^{*}$ & 14.8 & 12.3 \\
\hline
\end{tabular}

$*$ in case of COP of 2,3 or 4 , respectively. ** The electric power of fan for compressor cooling (for system and D) and for secondary air (for system D) are taken into account in the calculation of electricity demand

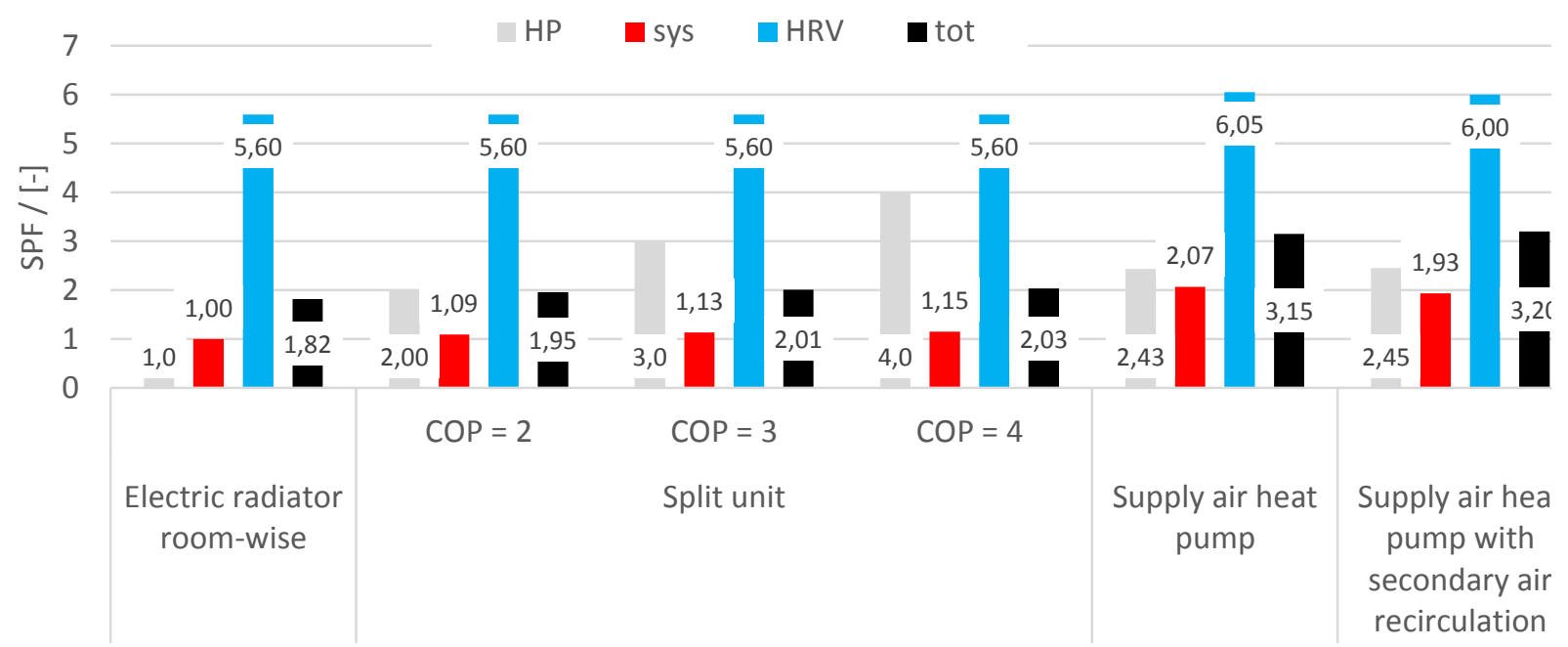

Fig. 11 Annual (heating season) SPFs of the heat pump ("HP"), heating system ("sys"), HRV and total HVAC ("tot"), according to equations (1)-(4)

\section{CONCLUSIONS}

In the framework of the Austrian project SaLüH!, four different heating and ventilation concepts were compared with regard to thermal comfort and energy performance to find efficient solutions for the renovation of small flats of MFH. All the systems investigated are able to ensure good indoor air quality in the flat and thermal comfort.

The innovative heating concept, based on a façade-integrated heat pump with secondary air recirculation, adds a new degree of freedom compared to the control of supply air heat pump system. The use of secondary air can limit the problem of overheating of supply air rooms during the heating season and allows to improve the energy performance of the heat pump $\left(\mathrm{SPF}_{\mathrm{HP}}=2.45\right)$ and of the whole system $\left(\mathrm{SPF}_{\text {tot }}=3.2\right)$ compared to all the others three heating concepts. Control strategies can be further improved in order to avoid under-temperature of supply air rooms during the heating season. 


\section{REFERENCES}

Ochs F., Hauer M., Bianchi Janetti M., Siegele DD., (2017a), Energy Performance of Membrane Energy Recovery Ventilation in Combination with an Exhaust Air Heat Pump, Building Simulation 2017, San Francisco, USA

Ochs F., Pfluger R., Dermentzis G., Siegele D., (2017b), Energy Efficient Renovation with Decentral Compact Heat Pumps, 12th International Heat Pump Conference 2017, Rotterdam, The Netherlands

Siegele D., Ochs F., Feist W., Simulation study on the use of enthalpy transducers in cold climates, $22^{\text {nd }}$ international Passive House Conference, Munich, 2018

SaLüH! (FFG), https://nachhaltigwirtschaften.at/de/sdz/projekte/salueh-sanierung-vonmehrfamilienhaeusern-mit-kleinen-wohnungen-kostenguenstige-technische-loesungsansaetze-fuer-

lueftung-heizung-und-warmwasser.php

Ochs F., Siegele D., Calabrese T., Dermentzis G., Venus D., System Simulation Models, Residential Buildings - Part B, IEA SHC TASK56 - Building Integrated Solar Envelope Systems, 2018

Rojas-K. G., Spörk-D. M., Venus D., Greml A., Krissmer L., Pfluger R., 2015. Lüften und Heizen in Passiv-häusern in Österreich-Variantenvergleich auf Basis von Behaglichkeit und Nachhaltigkeit,36/2015 (bmvit)

Siegele, D. Optimization and Appliance of small Air Exhaust Heat Pumps with Focus on Alpine Regions, PhD Thesis, University in Innsbruck, 2018, Work in progress, https://www.uibk.ac.at/bauphysik/forschung/projects/saluh/index.html.de

\section{ACKNOWLDEGEMENT}

This work is part of the Austrian research project SaLüH! Renovation of multi-family houses with small apartments, low-cost technical solutions for ventilation, heating \& hot water (2015-18); Förderprogramm Stadt der Zukunft, FFG, Project number: 850085. 


\title{
MULTI-BUILDING ENERGY RENOVATION FOR SOCIAL HOUSING
}

\author{
Rolf Bastiaanssen, Giulia Rinaldi \\ Bax\&Company \\ C/ Casp 118-120, 08013 Barcelona, Spain \\ Phone: +34934760444 \\ E-Mail: r.bastiaanssen@baxcompany.com, g.rinaldi@baxcompany.com
}

The DREEAM project aims to demonstrate an integrated renovation approach for energy efficiency at a multi-building scale that can reduce the net energy demand by the 75\%. DREEAM focuses on the deep renovation of social houses managed by a single owner with a large portfolio of residential buildings enabling EU-wide replication opportunities and with high energy saving potential. The project shows that multi-building energy renovations unlock the opportunity to benefit from economy of scale, to access to low-cost financing, and to better integrate innovative technology solutions, as well as decentralised renewable generation. This article describes the value of scaled investment planning for achieving building owners' long-term sustainability vision. Applying a "backcasting" approach, energy efficiency targets are considered the starting point for the development of investment strategies which realistically link the building owners' financial capacity with the renovation measures to be implemented.

\section{INTRODUCTION}

The European Commission estimates that $40 \%$ of the EU's total energy consumption and $36 \%$ of its $\mathrm{CO} 2$ emissions are related to the residential and commercial building sectors (1). The European housing stock consists of a large share of buildings older than 30 years which require refurbishment. Low energy efficiency makes them less comfortable and with greater heating/cooling demands. This leads to higher energy bills (around $€ 340$ bn per year) and GHG emissions. Standard renovations are usually tailored for a specific building and follow a "component" approach, such as improving roof and wall insulation, replacing windows and inefficient heating systems, achieving only $10-40 \%$ of energy consumption reduction. Associated investments are recovered in 5 to 10 years while more ambitious renovations, including renewable energy sources and better management, usually take more than 30 years to pay back.

Building owners and cities look to further reduce costs by enhancing the energy efficiency gains from renovations. In particular, social housing providers owning large portfolios of similar buildings aim to upscale renovation projects on a multibuilding scale in order to maximise the return of investment (ROI) while increasing the renovation rate of their building stock.

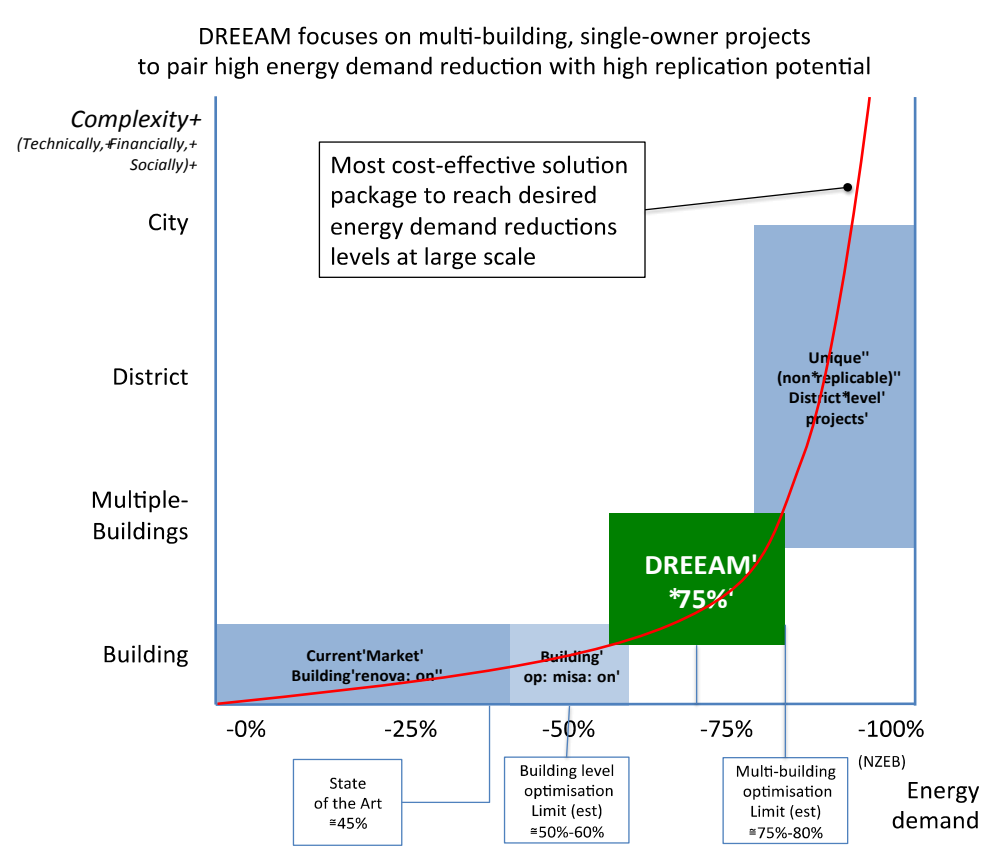

Figure 25. Relationship between scale and cost-effective renovation technologies

The EU H2020 project DREEAM (Demonstrating an integrated Renovation approach for Energy Efficiency at the Multi-building scale) provides housing companies with an integrated multi-building 
approach enabling a 75\% energy demand reduction and raising renovation ambitions to near Zero Energy Building (nZEB) standards.

\section{THE DREEAM APPROACH}

The DREEAM project is a collaboration between building owners, engineering firms, technology providers, researchers and experts in financing from across Europe. Using advanced analytical tools, DREEAM supports decision-making before, during, and after renovation by identifying the combination of technologies that deliver the most energy reduction for a set of buildings while taking into consideration building owners' financial capacity and tenants' preferences. What makes the DREEAM project unique and innovative is its end-to-end approach, addressing the complete renovation cycle by integrating all the key aspects of energy efficiency projects: investment planning, renovation design, quality assurance, energy

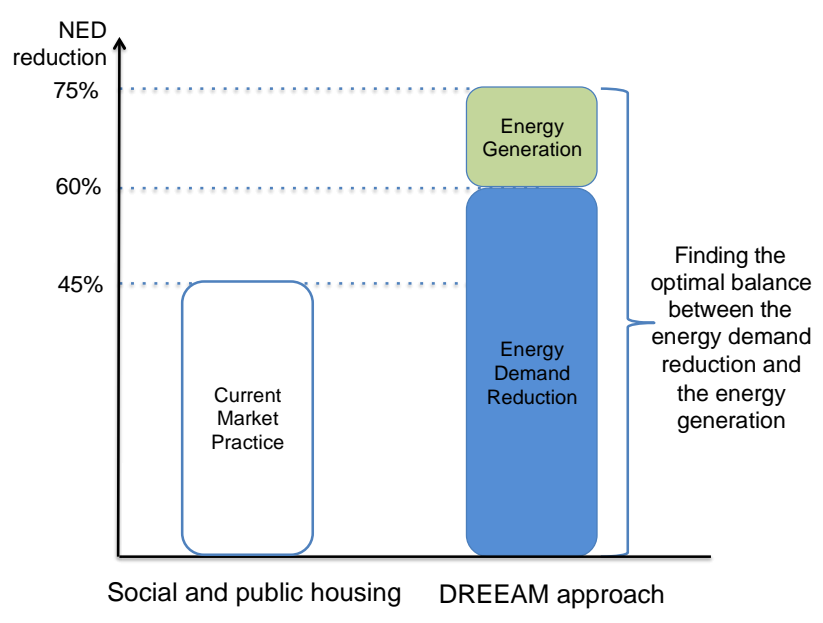

Figure 26. DREEAM approach for $75 \%$ NED reduction consumption monitoring, as well as tenant engagement. Deep energy renovations that achieve an energy consumption reduction of more than $75 \%$ are not financially feasible using a single technology focus. To cost-effectively achieve such demanding reduction targets, it is essential to consider energy systems packages that balance the implementation of both renewable energy generation and energy reduction measures.

District scale approaches tend to be highly tailored to local situations and difficult to replicate, as they require significant investment and joint decision-making by a large number of stakeholders.

By addressing the challenge of upscaling renovations from single to multi-building, social and public housing providers will benefit from reducing the energy demand of their properties, increasing the cost efficiency of a renovation per euro invested and providing a higher quality of life for their tenants.

Lastly, long-term financial and renovation planning at a building portfolio level is designed based on each organisation's individual sustainability strategies and ambitions.

\section{UPSCALING INVESTMENTS}

The average European renovation rate in the single owner housing sector is still low $(1,5 \%)$ and $80 \%$ of European renovations do not target energy performance improvements because of building owners' lack of interest and ambition in achieving high sustainability targets.

However, following the EU Energy Efficiency Directive and the Energy Performance in Buildings Directive (EPBD), housing providers are now subjected to regional and national renovation policies setting long-term energy efficiency targets to be achieved (1). Viable strategies and plans for largescale building renovation are still missing as are the decision-making tools which have an integrated perspective of cost-benefit evaluation encompassing complete renovation measures. Renovation programmes often start developing solutions for specific buildings, and asset owners usually follow a single technology approach to optimise their investments without strategically plan their renovation.

The DREEAM project helps cities and housing association in upscaling their sustainability ambitions and planning their investments at a portfolio level through an integrated approach considering casespecific technical, financial and social factors. Energy efficiency targets are considered as the starting point for developing alternative investment strategies in a manner that would lead the housing 
associations to reach their sustainability vision. This "backcasting" approach allows moving from a single-building perspective into scaled processes based on a data-driven analysis which project the impact of the alternative long-term investment strategies on both financial and energy indicators. Projection of the current financial practices results as a first exercise to link long-term ambitions with real investment options and to estimate the gap that stands between them.

Each investment pathway represents trade-offs of different renovation ambitions: on one side prioritising shallow renovation means maximising the number of units that can be upgraded but it leads to limited energy savings; on the other side, deep retrofit interventions significantly improve living standards and a dwelling's energy performance but entail high investment costs and hence, a reduced number of implementations.

\section{The approach}

The investment scenario analysis is characterised by four main phases whose outputs enable the housing associations to prioritise and strategically plan building stock energy efficiency interventions.

Figure 3 shows how the current status of the portfolio is assessed based on the crosscutting dataset built from operational, technical and financial data points at dwelling level. Building typologies (archetypes) and age are key tools for categorising the stock as well as a basis for the design of tailored renovation packages. Segmentation and data structure are based on a well-known and proved public tool (2) which provides the necessary framework for the construction of representative target groups for each European country. Based on standardised archetypes, the technical characteristics and distribution of energy performance or label are illustrated using graphs and maps which enable to identify opportunities for large-scale renovation of multiple buildings with similar needs where economies of scale can be achieved.

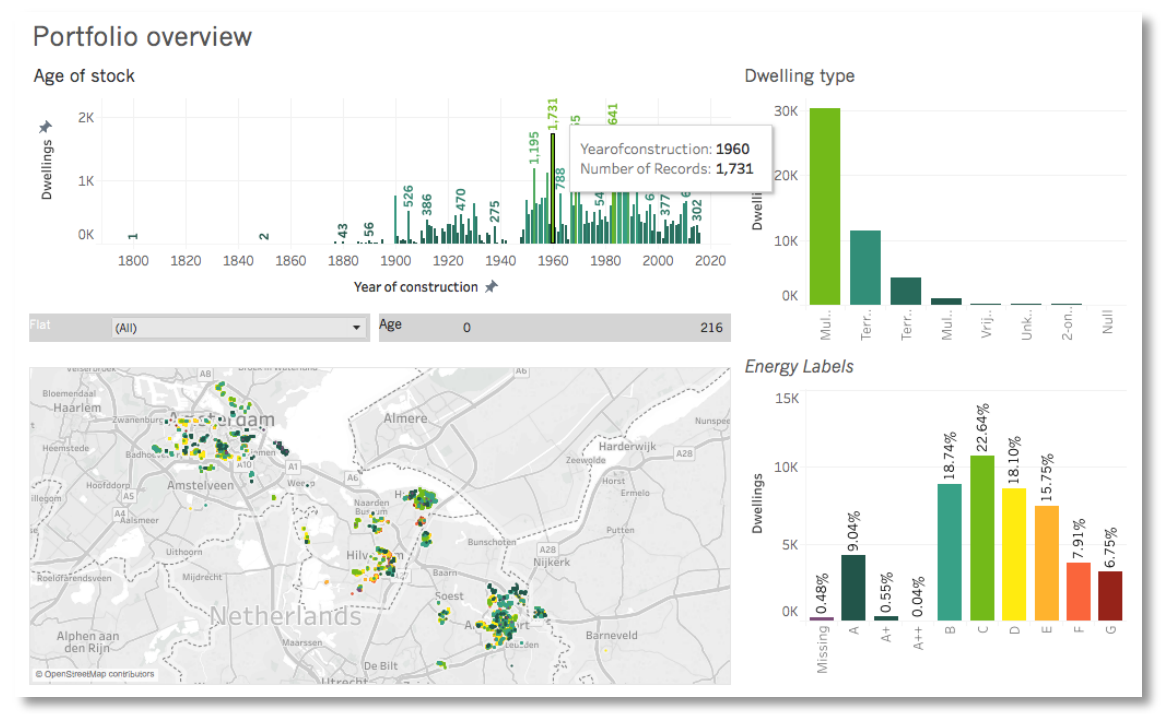

Figure 27. Example of building stock assessment and visualisation

For each target group consisting of buildings from the same archetype and construction period, standard renovation packages are designed to link investments with potential energy savings. As shown in the example of table 1, renovation packages are designed as a combination of measures that lead to a certain improvement of the energy performance which is reflected not only in a higher EPC label, but also in energy and $\mathrm{CO} 2$ savings. Shallow and deep retrofit options are priced and modelled based on energy efficiency and $\mathrm{CO} 2$ targets, financial constraints and operational concerns. According 
to the availability of pricing data and technical preference, renovation packages are designed with the housing associations' information or using academic and public studies.

Before proceeding with the development of alternative potential investment strategies, the business-asusual approach is projected to evaluate its capacity to achieve the policy targets across different timelines. Once the gap is identified, alternative renovation and investment pathways are developed to reach the selected long- term ambitions by using a custom-investment planning model.

Table 1. Example of renovation packages

\begin{tabular}{|c|c|c|c|c|}
\hline & \multicolumn{4}{|c|}{ FLATS } \\
\hline & \multicolumn{2}{|c|}{ 1. Shallow renovation } & \multicolumn{2}{|c|}{ 2. Deep renovation } \\
\hline & Before & After & Before & After \\
\hline Year built & $1980 \mathrm{~s}$ & & $1970 \mathrm{~s}$ & \\
\hline Investment cost ( $\epsilon /$ dwelling) & 11700 & & 14367 & \\
\hline Energy label & c & B & $\mathrm{F}$ & A \\
\hline \multicolumn{5}{|l|}{ Components } \\
\hline Windows & double glass & $\mathrm{HR}++$ glass & single glass & $\mathrm{HR}++$ glass \\
\hline Insulation & none & Very good & none & Very good \\
\hline Roof insulation & none & No change & none & No change \\
\hline Ventilation & Mechanical & Balanced & natural & natural \\
\hline Heating system & Gasverw.+geiser & HR Combi & VR-combi & HR Combit solar boiler \\
\hline Solar panels $(\mathrm{m} 2)$ & 0 & 0 & 0 & 0 \\
\hline \multicolumn{5}{|l|}{ Energy impacts } \\
\hline Energy costs ( $($ /dwelling/month) & 62 & 49 & 100 & 47 \\
\hline Energy index* & 1,574 & 1,301 & 2,529 & 1,078 \\
\hline KWH (year) & 4960 & 3920 & 8000 & 3760 \\
\hline $\operatorname{co} 2 \mathrm{~kg} *$ & 2403 & 2036 & 3848 & 1555 \\
\hline \multicolumn{5}{|l|}{ Financial impacts } \\
\hline Contract monthly rent* & 615,7 & 709,1 & 549,2 & 716,3 \\
\hline Market monthly rent* & 756,5 & 831 & 774,2 & 862,6 \\
\hline Unit Market value* & 162450 & 205473 & 190530 & 224488 \\
\hline
\end{tabular}

Proposed renovation and financial strategies to achieve policy goals optimise each of the different factors considered including the technological combinations given building energy renovation rules and investment capacity of the building owner.

Projection is based on the discussion with the portfolio managers about the typical decision rules for investment

planning currently in use and the relevant regional/national regulations or policy targets in force. The results of the scenario analysis give to the housing association insights on the value of the different financial approaches and on the indicative actions to be performed to meet the impact desired.

\section{Case study: UK housing association}

In the United Kingdom, national policies directly influence the social and public housing sector:

1) the minimum standards set out for the social housing sector (2006);

2) Health and Safety.

The Energy Efficiency regulations establish the minimum energy performance standards for new buildings and when "building work" is carried out to existing properties. Additionally, the Government has recently issued the "Clean Growth Strategy"(3) and the "Fuel Poverty Strategy"(4). Both policies aim to bring vulnerable houses to a minimum EPC label C by 2030 . 


\section{Building stock \& sustainability targets}

The housing association studied has a portfolio of buildings spread throughout the UK and compliant with 2015 Energy Efficiency Regulations setting the minimum standard for rented properties to EPC E (5). The $35 \%$ of the buildings were built after 1950 and the most common archetypes are flats, terraced houses, detached houses and bungalows. The large majority of the portfolio is rated with an EPC D (Figure 4). The housing association's long-term aim is to reach an overall portfolio energy label C, achieving the national standards for vulnerable houses.

\section{Renovation packages}

The dwellings in the portfolio have been grouped according to the most relevant archetypes (semidetached houses, flats, terraced houses) and construction periods. Thus, the renovation packages cover 8335 dwellings. For each target group two different set of renovation measures were developed in order to upgrade the dwellings from an average EPC label D to the minimum target (EPC C) and to a

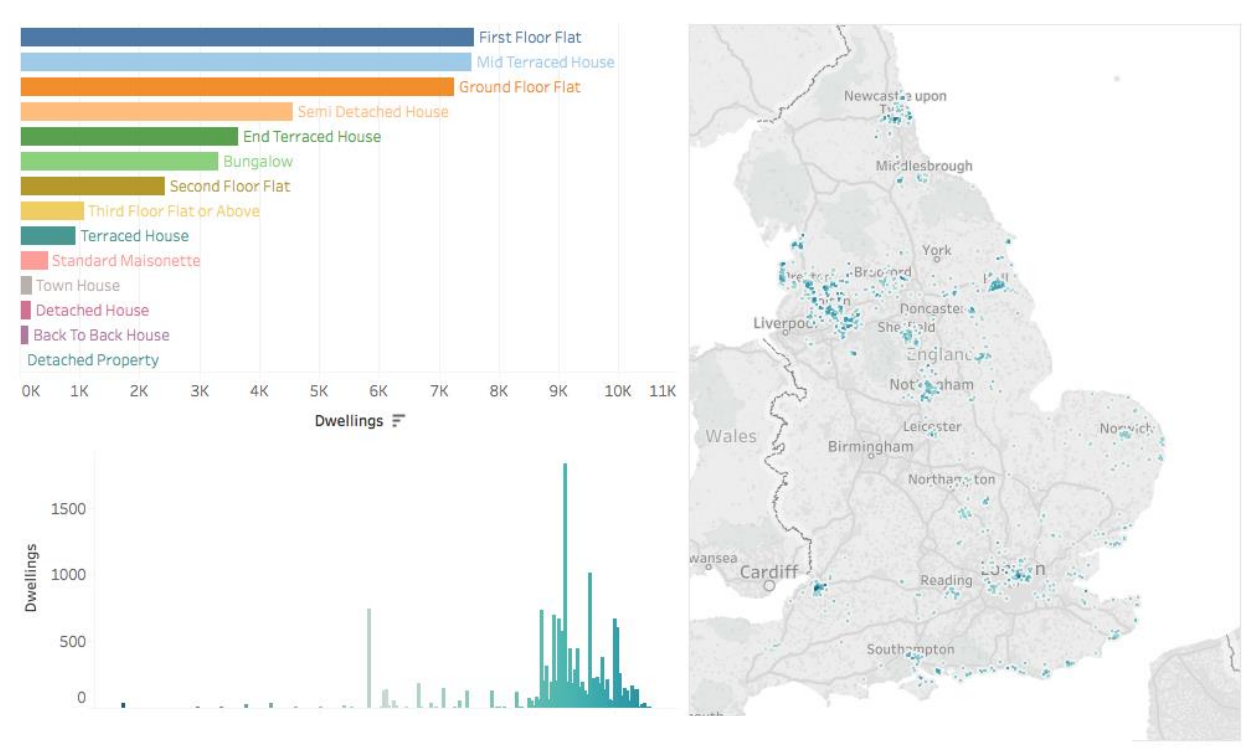

Figure 28. Archetypes and age of the stock

more ambitious performance.

\section{Gap to policy targets}

The current strategy is based on "shallow renovations" prioritising older dwellings over those built after 1945. The business as usual investment practices assume an annual budget of $€ 5 \mathrm{M}$ per year. If projected over 5 years, this would mean only $56 \%$ of the portfolio reach the targeted standard - thus is the need to explore different approaches. 


\section{Pathway analysis}

Two alternative strategies have been studied considering a higher budget and/or a longer timeframe to evaluate which would be the impact on the cost/energy savings and on the housing performance.

The pathways analysed (Figure 5) suggest that increasing the yearly budget for renovations over the same time period would allow the housing association to renovate $100 \%$ of the targeted stock to the EPC label $\mathrm{C}$ and increase the $\mathrm{CO} 2$ saving by $20 \%$. On the other side, following an integral deep renovation approach would increase the necessary cost per unit, decreasing the number of interventions performed within the available budget, but would allow for higher energy and CO2 savings (Figure 5).

\section{Results}

This scenario analysis gave to the housing associations a first insight into the current condition of its dwellings and on how to strategically prioritise their renovation. Visualisation of the building stock analysis enabled the identification of clusters of buildings with similar technical characteristics where multi-building renovations may be implemented. Additionally, the projection of current practices in energy efficiency renovation raised awareness among the housing association management team regarding the need to evaluate different financial strategies to achieve the established sustainability goals. In parallel to the development of alternative investment pathways, it has been possible to study the potential savings generated by a component-based renovation programme which optimises the replacement timing and cost-benefit impacts, offering opportunities for quick gains and bills reductions for tenants.

\section{The value of investment planning for portfolio renovations}

Housing associations recognise the value of the DREEAM scenario analysis as an instrument to support the decision-making process at board level with regards to energy efficiency financial strategies. Long-term planning of investments in energy efficiency renovation enables housing associations to achieve strategic sustainability targets. This analysis proved to be particularly relevant for those housing providers with novel sustainability push and/or under financial constraints who need to upscale investments for renovating their entire portfolio to higher energy standards. 


\section{Cost-effective integration of innovative technologies solutions}

It is currently common practice among building owners to base financial decisions on a single-technology perspective, focusing on minimising the ROI by selecting those options that pay back earlier at a single-building level. However, the DREEAM concept demonstrates that by applying an integrated approach that considers demand side, control and generation technologies, the overall performance of the energy system will significantly improve and the financial return horizon will shorten compared to each individual technology. By designing simplified renovation packages that optimise the balance between renewable energy generation and energy efficiency measures, innovative technologies that otherwise won't be considered by the housing associations are

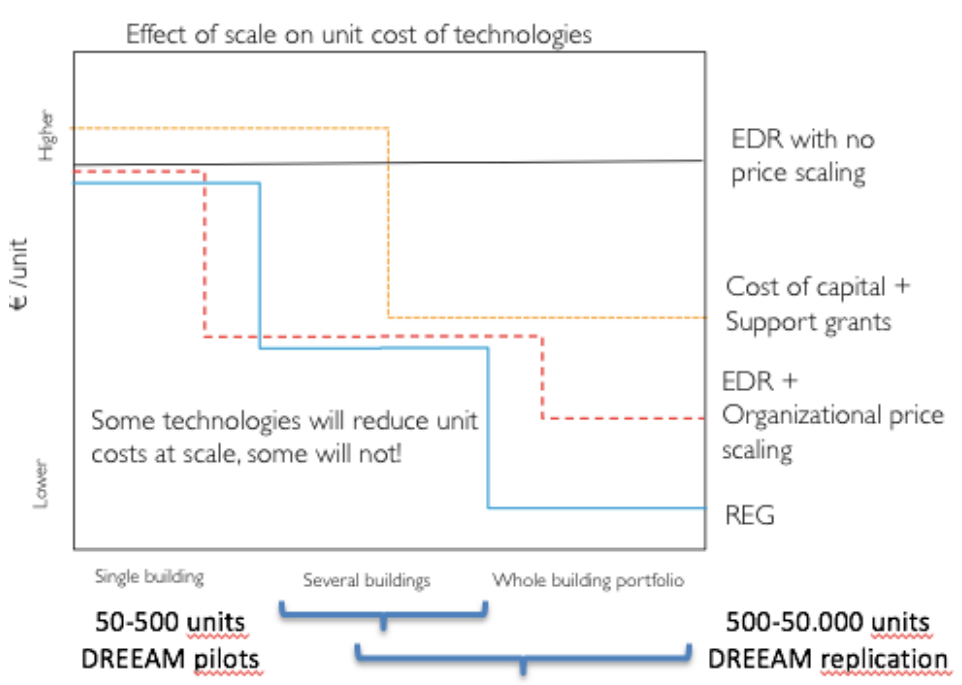

Figure 6. The benefit of scaling up energy efficeincy renovations evaluated and installed as part of complete renovation solutions.

\section{The economy of scale}

The DREEAM project focuses on scaling renovations and the economic benefits realised. By renovating multiple co-located buildings characterised by the same archetype, similar technical characteristics and energy performance, building owners might benefit from economy of scale. Indeed, portfolio renovation unlocks the opportunities for reducing the technology cost per unit, which can result in a payback period reduced by $30 \%$.

\section{Low-cost financing}

Housing associations could benefit from long-term planning versus the current short-term financing based on available cash-flow. Integration of renewable energy sources can be subsidised in most countries and different local grants are available to support residential energy improvements. Additionally, large-scale investment programmes aiming to improve energy efficiency in the residential sector can consider different European funding opportunities which can either finance the project and/or support the technical preparation of such investments. Furthermore, the green value of investments on a large scale can affect the cost of capital, influence decision makers, and lead to lower interest rates and more favourable conditions for investors.

\section{REAL-LIFE DEMONSTRATION AND REPLICATION POTENTIAL}

The scale of projects considered as optimal by the DREEAM approach is very useful for building owners, such as social and public housing associations, who own and/or manage thousands of units with high potential for energy performance improvements. This market segment accounts for $5 \%$ of the EU's total energy demand and covers the $12 \%$ of the total European housing stock, achieving 2.25B m2 floor area (6). Targeting this sector, characterised by a single ownership structure, DREEAM offers a unique opportunity for high replicability and effective scalability.

DREEAM aims to demonstrate its data-driven approach to interconnected energy systems in multibuilding renovation projects in social and public housing, because of 
the high potential for replicable energy demand reduction;

the large portfolio size allowing to validate economy of scale;

the low organisational decision-making barriers of single ownership properties.

The DREEAM approach is currently implemented in 3 pilot sites in the UK, Germany and Italy. Being applied in a real-life environment validates the DREEAM methodology across different climate, cultural and institutional configurations, unlocking opportunities for large-scale replication across Europe. The DREEAM project develops concepts that are specifically tailored to local and climatic conditions, as well as the characteristics of the building stock itself, the decision structure of the organisation involved and its specific sustainability goals.

Table 2. Quantified replication potential of the DREEAM approach

\begin{tabular}{|l|l|l|l|l|}
\hline Pilot site & Number of dwellings & Similar in the housing portfolio & Similar in the country & Similar in Europe \\
\hline Italy & 102 & 1,800 & 336,000 & $4,000,000$ \\
\hline Berlin & 164 & 3,000 & 500,000 & $1,500,000$ \\
\hline UK & 109 & 42,900 & $1,000,000$ & No data \\
\hline Total & 375 & 47,700 & $1,836,000$ & Min. 6 million \\
\hline
\end{tabular}

\section{THE DREEAM REPLICATION PROGRAMME}

DREEAM's overall objective is to raise the European social housing stock to nZEB standard. To facilitate a wider uptake of DREEAM solutions, the project offers to selected cities and housing associations support in developing an integrated renovation strategy. DREEAM partners have defined two key phases in the housing associations decision making process where the DREEAM approach brings an added value to the current practices unlocking the benefits of scale (Figure 7):

Strategy definition: long-term ambitions should be the starting point for the development of sustainable investment strategies at the beginning of the energy renovation decision process;

Exploration of renovation solutions: alternative options should be analysed before performing the renovation in order to select the optimal combination of solutions with higher benefits at multibuilding level.

Through the replication programme, DREEAM will work with 15 building providers to address the above challenges offering two different services through a voucher programme. Each voucher provides capacity support (20-25 days of work of the DREEAM team) free of charge to enhance investment strategies in energy efficiency renovations at a multi-building level, delivering data-driven analyses based on the DREEAM approach. The state-of-the-art process helps to inform decisionmakers on financial, technical and energy elements of integrated programme development. Candidates are owners/managers of large portfolios with annual budget allocated to energy efficiency renovation (7). 


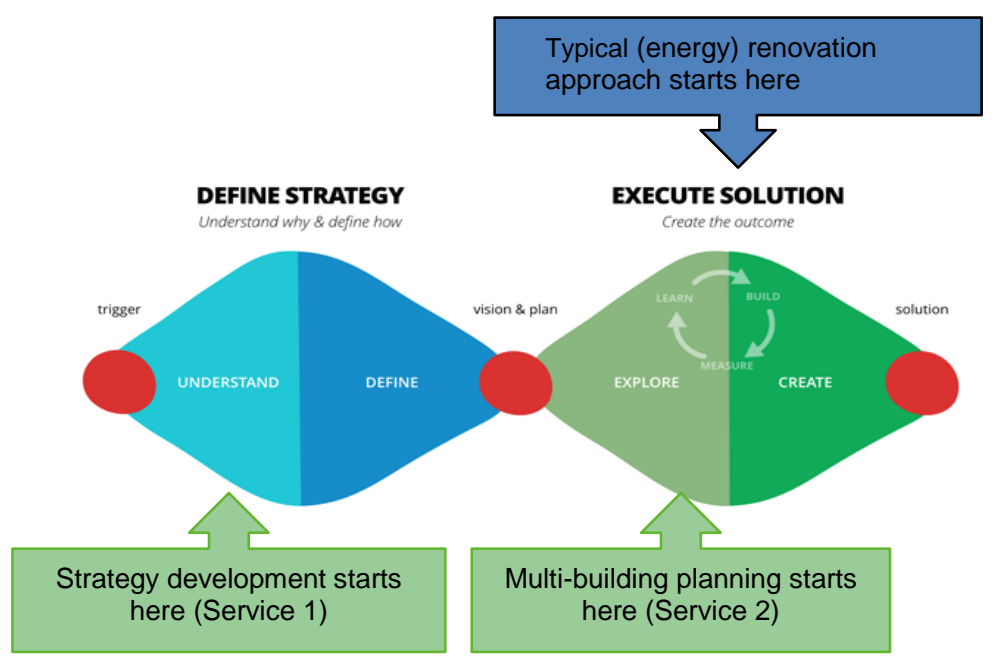

\section{CONCLUSIONS}

Figure 7. Decision making process for multi-building process.

The European H2020 project DREEAM aims to develop and demonstrate a systematic approach to design and implement cost-effective multi-building renovations achieving a total energy demand reduction of up to $75 \%$ and a high return of investments. The present paper briefly describes the DREEAM project concept and focuses on its innovative approach on long-term planning of investments for energy efficiency renovations in the social and public housing sector. Renovation programmes of housing associations start often from a "need" basis analysis, developing solutions for specific buildings without considering the benefit of long-term integrated renovation at scale. Within the DREEAM project, a new integrated approach is used to support cities and housing association in upscaling their sustainability ambitions and planning their investment at portfolio level. Alternative investment strategies are developed starting from the building owners' energy efficiency targets in a way that would lead to reach their sustainability vision. A backcasting analytical model is used to project the impact of the alternative investment pathways on both financial and energy indicators. By defining long-term integrated financial strategies, the DREEAM investment scenario analysis enable building owners to move to scaled processes, benefitting from higher return of investments, access to better financial instruments and lower cost of technology per unit.

\section{CONFERENCE TOPIC}

Innovation for the Decarbonisation of Buildings and Quarters

\section{REFERENCES}

(1) https://ec.europa.eu/energy/en/topics/energy-efficiency/buildings

(2) http://www.episcope.eu/

(3) BEIS (Oct 2017). The clean growth strategy - Leading the way to a low carbon future.

(4) HM Government (Mar 2015). Cutting the cost of keeping warm - A fuel poverty strategy for England.

(5) BEIS (October 2017). The domestic private rented property minimum standards

(6) Housing Europe (2015). CECODHAS.

(7) http://dreeam.eu/ 


\title{
QUALITY CONTROL FOR HVAC SYSTEMS IN RESIDENTIAL BUILDINGS WITH IOT BASED FDD - A STATE OF THE ART REVIEW
}

\author{
Stella Joos1,2, Daniel Buchmiller1, Andreas Gerber1, Sebastian Herkel2 \\ ${ }_{1}$ Hochschule Biberach \\ ${ }_{2}$ Fraunhofer Institute for Solar Energy Systems ISE \\ Heidenhofstraße 2, 79110 Freiburg, Germany \\ Phone: +49761 4588-5818 \\ E-Mail: stella.joos@ise.fraunhofer.de
}

\section{SUMMARY}

Installing Heating-, Ventilation and Air Conditioning (HVAC) systems correctly and operating them in their optimal operation point is a key factor regarding a high energy performance and thus can save costs. These days, HVAC systems are considered as high complex systems which contain many components and therefore even the fault of a valve might have a big impact on the performance of the system. The main differences of small, residential buildings to commercial and industrial buildings are the low costs and a high repetition of the systems. In addition the planning and installation process in small buildings is mostly done by the craftspeople while for big projects engineers and planners taking over the calculation with professional software, hence it needs attention on quality control. A low cost system for fault detection and diagnostics is a promising solution to tackle the before mentioned issues. Fault Detection and Diagnostics (FDD) as a tool of quality control is introduced and a review of applications for single HVAC components is done. A further focus is on Internet of Things (IoT) as promising cost effective solution for data handling and communication is given. The special needs of small HVAC systems are quality control of the installation and monitoring the system during the operation time for finding faulty operation modes. For reducing human-caused faults QR-Codes or RFID may be a helpful tool. For faults in the operation state IoT-based FDD for time-series analysis might be a promising tool. As a first example of IoT technology based FDD in a small HVAC system a prototype to identify faults in buffer storage shows promising results. With simple if-then rules it could be shown that the stratification of buffer storage is not guaranteed at any time of operation.

\section{INTRODUCTION}

Heating causes $30 \%$ of the $\mathrm{CO}_{2}$ production whole over the world [1]. The approach of nearly zero energy buildings as common European goal for new and existing building stock addresses the challenges of the climate change [2]. For decreasing $\mathrm{CO}_{2}$ emissions it is reasonable to optimize the heat production process of Heating-, Ventilation and Air Conditioning (HVAC) systems. Because of the complexity of the systems, faults of a single component may be compensated by another one, hence the craftspeople who installing the system or the occupant may not notice, that the heating system operate faulty. As a result, the system needs more primary energy than necessary or planned until a component showing malfunction and a technician is needed in order to check the system. Often the technician does not have enough information to be able to give a diagnosis and change the correct parameters of a device to run the system in its optimal mode of operation again. For residential buildings and small commercial buildings most of the processes in planning, commissioning and operation differ from that of large and non-residential buildings. In case of small residential buildings the craftspeople taking over the planning and installing process of the whole HVAC system, neither a planner nor an engineer is participating to the process. Therefore the technician considering the single components while for modern systems the considerations as a whole system is necessary. For high efficient systems many different involved actors have different targets and needs. For finding acceptance in the market the needs of all actors should be fulfilled. The occupants want a fault free and cheap running system; the craftspeople want a simple system where all components match each other and therefore installation is fast and easy; the operators want cheap systems with less maintenance as possible after installation; the industry wants to sell complete and in some cases closed systems with customer retention. For this reason the quality control should not be expensive and at the same time be trustable. With new digital inventions like the Internet of Things (IoT), Quick Response (QR) Codes etc. it will be easier to perform quality assurance in HVAC systems as a part of a "Smart-Home" and the user or technician is able to control and check the system remotely. To automate the 50 processes and quality control an IoT-based Fault Detection and Diagnostics (FDD) may be one solution. 


\section{STATE OF THE ART AND SCIENCE}

This section gives a brief overview of some work that has been done in quality of HVAC systems, Internet of Things and Fault Detection and Diagnostics.

\section{Quality in HVAC Systems}

Quality control in HVAC systems has a lot of different aspects. Instead of having a look to the single devices it is necessary to consider the quality of the life cycle of the whole system. Instead of dealing with single components the whole system has to be considered. Also it is necessary to look at the system behavior during time of operation.

Checking the quality of the devices is done by the industry manufacturer applying given rules like the ErP - No 60 1253/2014 and 1254/2014 for residential ventilation units [15]. Components are usually designed for operation within a product family. In case of using components of different manufacturer in a common system, due to a weak interoperability it may happen that the components are not working in an optimal point and therefore decreasing the efficiency of the whole system. Nevertheless in any case the quality of the installing and commissioning process, which is done for residential buildings by the technicians, should have a quality control. As Schmelzer et al. reported [9], many faults happening during installation caused by humans, while components, sensors or valves may be installed in wrong place, insulation may not be properly installed or the control is not set up correctly. All of this mentioned aspects are the quality of the design state. During whole the operation time, there will be some mandatory checkups for ensure the emissions are inside the determined area, but there are no checks to see if the quality is still good after some years of operation. By carry out a time series analysis of operation data like temperatures, pump states, volume and mass flows fault can be detected and therefore ensure the correct operation and quality of the system.

In an earlier work Jagnow et al. [10] found out, that the highest potential, in respect to total energy consumption, for energy saving is in new houses with low consumption. The main task of this project was to ensure the quality of HVAC systems and ensure the use of whole the potentially usable energy. They invented a tool to optimize HVAC systems in residential buildings with a minimum of information required.

In Austria Krempl et al. [11] tried to reduce faults that happen during the installation process by humans. For this reason they invented a guideline for ensure the quality of the installation process.

\section{IoT}

IoT connects physical devices in the internet and interlink them. The difference to standard building automation is the identification of each sensor or actor with a unique address as well as the connection over a gateway to the backend. In some systems the address space is quite limited while in others 32 bit (IPv4) or 64 bit (One-Wire) or even 128 bit (IPv6) is used [16].

IoT systems have the function to transport information gathered by small devices in a secure, cost effective way.

A systematic representation of common IoT communication protocols, ordered by their range and frequency of data transmission are shown in figure 1 [18].

To have the battery lifetime as long as possible the data range should be less or the frequency of which the data is send should be limited. LoRaWAN ${ }_{\circledast}[13]$ is one promising solution with low-power wireless standard coupled with the issues of low-power devices. Therefore it is supporting three different device classes. Class $A$ is the most efficient class, the device is receiving information only after it scheduled an uplink; Class B nodes has scheduled receive slots, at this scheduled time it can receive a beacon from the gateway; Class $C$ nodes are listening continuously and therefore have high energy consumption. 


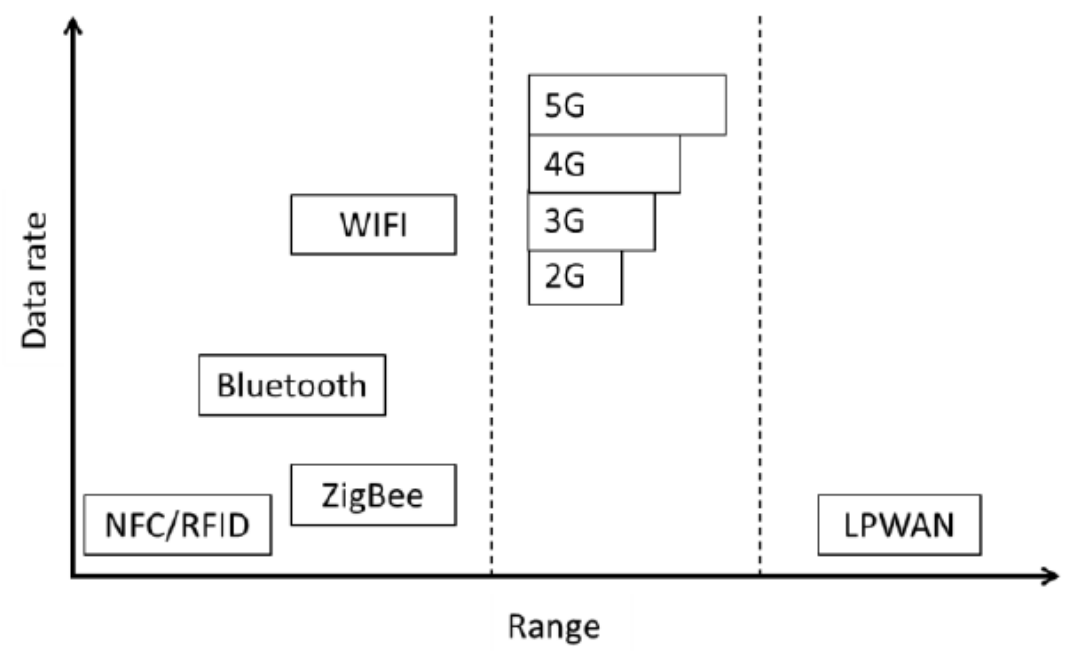

Fig. 1: Classification of the most popular wireless communication protocols with regard to their range and frequency 95 according to [18]

The LoRaWAN $\otimes_{\circledast}$ Gateways are grouped in the category LPWAN and are already wide spread in many cities and are cheap to install.

The costs for set up a LoRaWAN $\circledast$ IoT system are as followed [18]:

- base station $>1000 € 100$

- deployment gateway $>100 €$

- $\quad$ end device 3-5€

While all base stations are receiving the data, it is not necessary that everybody installing one, rather they can be shared.

The range capability is a key factor for choosing a technology. On the other hand the bandwidth or data rate and therefore the energy consumption is another factor which may influence the decision for one of these technologies. In the case of connecting sensors of a HVAC system a high range capability is required because of the obstacles inside the house and therefore decreasing the range of the signals. For getting an overview of the monthly consumption and the performance of the whole building, it is enough to get 3 or 4 measurements every month. For further analysis a higher frequencies and therefore a higher data transfer is needed, other solutions should be taken into account, like 4G or 5G which have a little bit less range than LPWAN but a much higher data rate. As a result of this two positive features it has a higher energy consumption and therefore can not operate on batteries. Three most common IoT network topologies are shown in fig. 2. It contains end nodes which are sensors and actuators, the gateways and the Sensor Nodes or Routers which are used just in the mesh topology as a broker. With the Point to Point topology (a) it is only possible to connect one end device to one Gateway; with the star topology (b) it is possible to connect several end nodes to one central gateway node; in the mesh topology (c) all nodes are connected to each other and one, or for reasons of redundancy, to several gateways [14]. The mesh topology is the most common topology in smart home applications because of the high density of nodes a long distance with low power is achieved.

For the communication several protocols with different features are available. For the Machine to Machine communication, protocols like Message Queuing Telemetry Transport (MQTT) can be used. It is a subscribe and publish protocol for light weight machine to machine communication [19]. The MQTT runs as a broker on the network server in the middle. The software as well as the hardware should be selected carefully in order they matching each other. With the implementation of loT a lot of opportunities for monitoring as well as quality control of the whole life cycle of a HVAC system can be done. 
a)

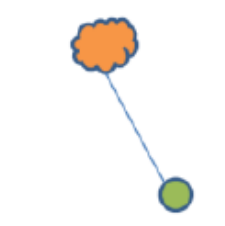

End Device / Sensor Node

b)

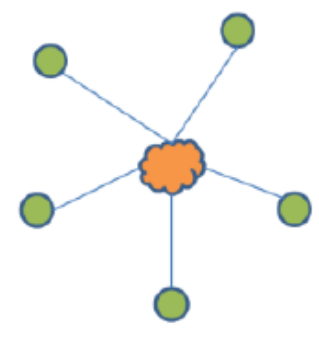

Sensor Node / Routing
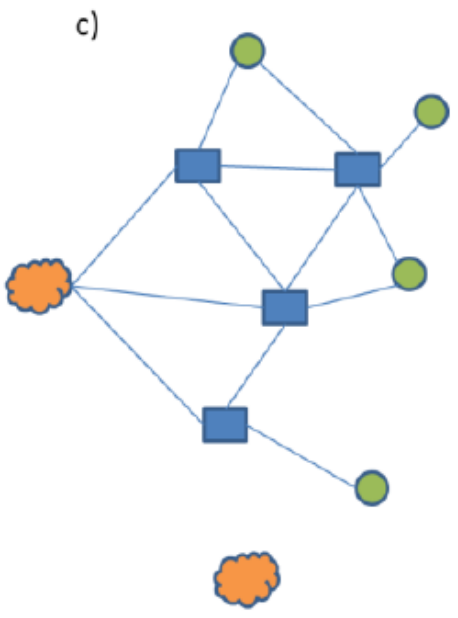

Gateway Node

Fig. 2: Schematic diagram of IoT network topologies: a) Point to Point topology b) Star topology c) Mesh topology

By tagging components with a QR Codes or RFID they can be easily identified and localized. QR Codes are already used in wide fields. Many manufacturers are tagging their products with a QR code to provide important information for users and installers (figure 3).

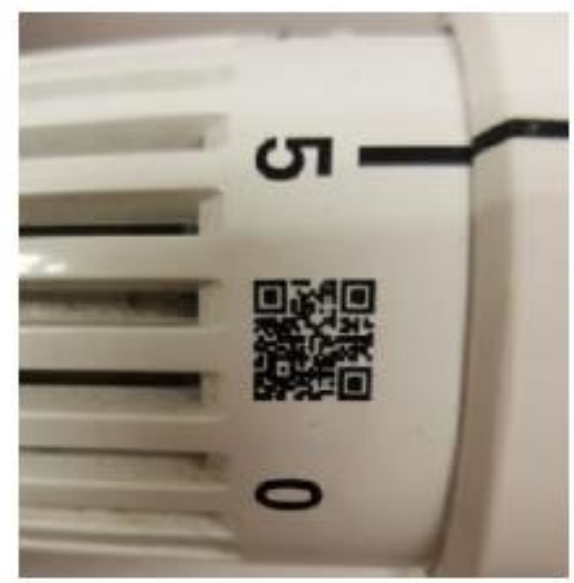

Fig. 3: QR Code for device properties on a Heating Thermostat of the company oventrop

\section{Fault Detection and Diagnostics}

According to D. Jacobs [17] the algorithms of Fault Detection and Diagnostics (FDD) can be divided in two different groups:

1) experts knowledge based algorithms

2) data based

The first category can be divided again in two groups of rule based FDD and simulation based FDD. The second category is based on artificial intelligence and therefore need a lot of data to train the algorithms.

Fault Detection and Diagnostics is easy to implement in large project, where a lot of sensors already monitoring the system and data is available. Furthermore FDD was applied in earlier work for single components like chiller [3], air handling units [4] and hydraulic faults [5] has been done. Recently W. J. N. Turner et al. [6] introduced a new data-driven FDD for HVAC systems by using a recursive least-squares model. 
FDD in closer look includes two main tasks, Fault Detection and Fault Diagnostics;

While fault detection can be done by different algorithms or simulations, diagnostics needs expert knowledge [16].

If the most common faults are known, fault detection based on expert-knowledge is based on simple If-Then rules. Because most of the systems in smaller buildings can be classified as repetitive systems, most of the faults can be known by the experience of similar systems and therefore this may be a good choice for residential buildings with small HVAC systems.

C. Schmelzer et al. [9] implemented a FDD algorithm for a solar thermal energy system on residential 155 buildings, which is divided in four steps:

1.) Measured value

2.) Characteristic

3.) Symptom

4.) Fault

For finding a fault the threshold is a significant important value. If the threshold is too tight faults are detected wrongly and if the range is too wide faults are not detected at all [9].

T. Müller et al. invented and successfully implemented a method of FDD which is working with real measured data to make the modelling effort small [7].

\section{REQUIREMENTS FOR SMALL SYSTEMS}

The main advantage of HVAC systems in residential buildings in respect to quality are the repetitiveness. Therefore some standard schemes can be derived for most of these systems. For small buildings the costs for quality control should be small in order to the low costs of the system acquisition costs and the installation. In most of these cases, technicians installing standard systems, where the quality of the components is already checked by the industry and no need of an exact calculation and planning process is necessary. Therefore the first and most common faults are happening during installation and commissioning. For solving this problem QR-Codes or RFID can help, while more information can be saved in these codes like R\&I Flowcharts, unique sensor id, all components of the system as well as the system size and others. This makes the installation of the components easier and may have the positive effect of fewer faults. Later on, in case of a revision or a fault during operation, technicians can get all important information of the system by simply scanning one code.

Because of the missing monitoring faults during operation are not noticed immediately. These days a lot of data is already measured by every component for the control technology. The first step to tackle the problem of quality control is to get access to the required data. Internet of Things is a promising concept/technology to digitalize and connect the components of a HVAC system of a building with each other and the Internet. With this technology it is possible to monitor the system and to change settings remotely. In future it can be even possible, that components connect to each other and find the optimal operation point by themselves. Because recent years the technicians not only install but also plan the system as well, the industry came to the point to sell closed island systems. Inside these island systems a lot of data is measured and saved but locked inside and therefore not useful for further analysis. To be able to connect all of components and sensors to the internet, it is practical to connect them wireless and powering them with long-lasting batteries.

Afterwards an automated FDD can help to decrease costs by operating the system in its optimal operation point while increase occupants satisfaction.

Some typical faults of HVAC systems are shown in table 1. 
Table 1: Typical faults of HVAC systems

\begin{tabular}{|c|c|}
\hline Fault Sensors & $\begin{array}{c}\text { Wrong position, damaged sensor, wrong calibrated, wrong } \\
\text { identification }\end{array}$ \\
\hline Fault Pumps & Wrong set up, wrong curve, wrong operation point \\
\hline Fault Storage & Faulty stratification \\
\hline Fault Controller & Faulty control settings of whole system or single devices \\
\hline Fault Valve & Wrong signals, wrong position, sticking valve, corrosion \\
\hline Fault Boiler & High energy consumption, wrong settings \\
\hline Fault Solar Thermal Energy Collector & Wrong settings, wrong set up, wrong connection \\
\hline Fault Heat Pump & Wrong installation, wrong settings \\
\hline Faulty hydraulic & Wrong pipes connected, missing hydraulic balancing \\
\hline
\end{tabular}

In the ongoing digitalization process like smart homes, the sizing effect of this technics will decrease costs in coming years. In this case, it will be less expensive and more feasible to install sensors and monitor the system and control the quality of HVAC systems in small residential buildings.

Not all of the FDD methods are applicable in residential buildings. The FDD methods which are based on measured data are not recommendable for these used-cases, because of the lack of many fault-free data and therefore it is cost intensive. Consequently here methods which are based on expertknowledge should be selected which is also needed for finding the source of the fault.

\section{FIRST RESULTS OF A SYSTEM WITH BOILER, HOT WATER TANK AND BUFFER STORAGE}

In a first field application one of the authors installed several temperature sensors in a 2001 Hot Water Storage which is supplied by a 8001 Buffer Storage to optimize the operation of the system. The buffer storage provides the hot water for the heating system and at the same time heating up the drinking hot water storage [8]. Because the data from the system was not accessible, seven temperature sensors were added as shown in figure 4. To ensure a good performance of the whole system, the stratification inside the buffer storage should work properly. Therefore the mass flow of the water should be moderate to not mix the water inside the tank and keep stratification with hot water on top and cold water at the bottom. To check the quality of this stratification two or three temperatures should be measured in different heights.

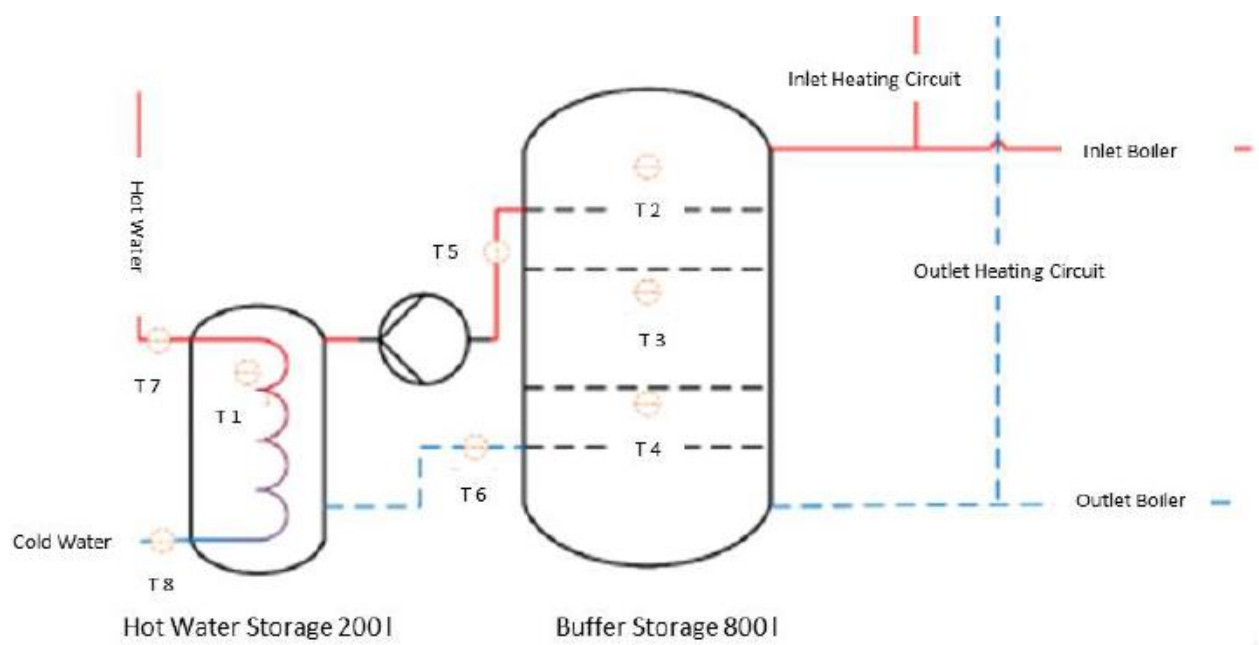

The hot water storage and the buffer storage were equipped with several digital temperature sensors (DS18B20 from Dallas Semiconductor) (Fig. 5, left). For getting access to this data a typical low-cost IoT approach was to connect sensors to an Arduino Uno and this furthermore to a Raspberry Pi 3 Model B as the main board. The network protocol which was chosen was the MQTT protocol. In Figure 5 on the right side, the temperatures in the hot water storage, the buffer storage top and buffer 
storage center are instructed for one day. The sensors show, that most of the time the temperature on top of the storage is, as highlighted in the red circles four degree less than in the center of the storage. This results in a faulty stratification of the storage and therefore a decrease of the efficiency of the whole system. Furthermore automated fault detection can be implemented by some simple rules:

- If $\mathrm{T}_{\text {top }}<\mathrm{T}_{\text {center }} \rightarrow$ fault

- If $\mathrm{T}_{\text {bottom }}>\mathrm{T}_{\text {center }} \rightarrow$ fault

As a next step not only temperatures should be measure, but also the state of pumps, valves and pressure sensors should be installed for keeping monitoring the quality of the system. By now the faults were only detected, but still it is not clear what is causing this fault. Therefore a fault diagnostic should be done afterwards. Sometimes a fault can be caused by several reasons or components, for finding the source some expert knowledge based rules should be implemented afterwards. In this case the fault can be caused by a too high mass flow or a wrong control setting.

As a first step, this example shows exemplary that with minimum expense a significant fault can be detected which could not be recognized by anyone.
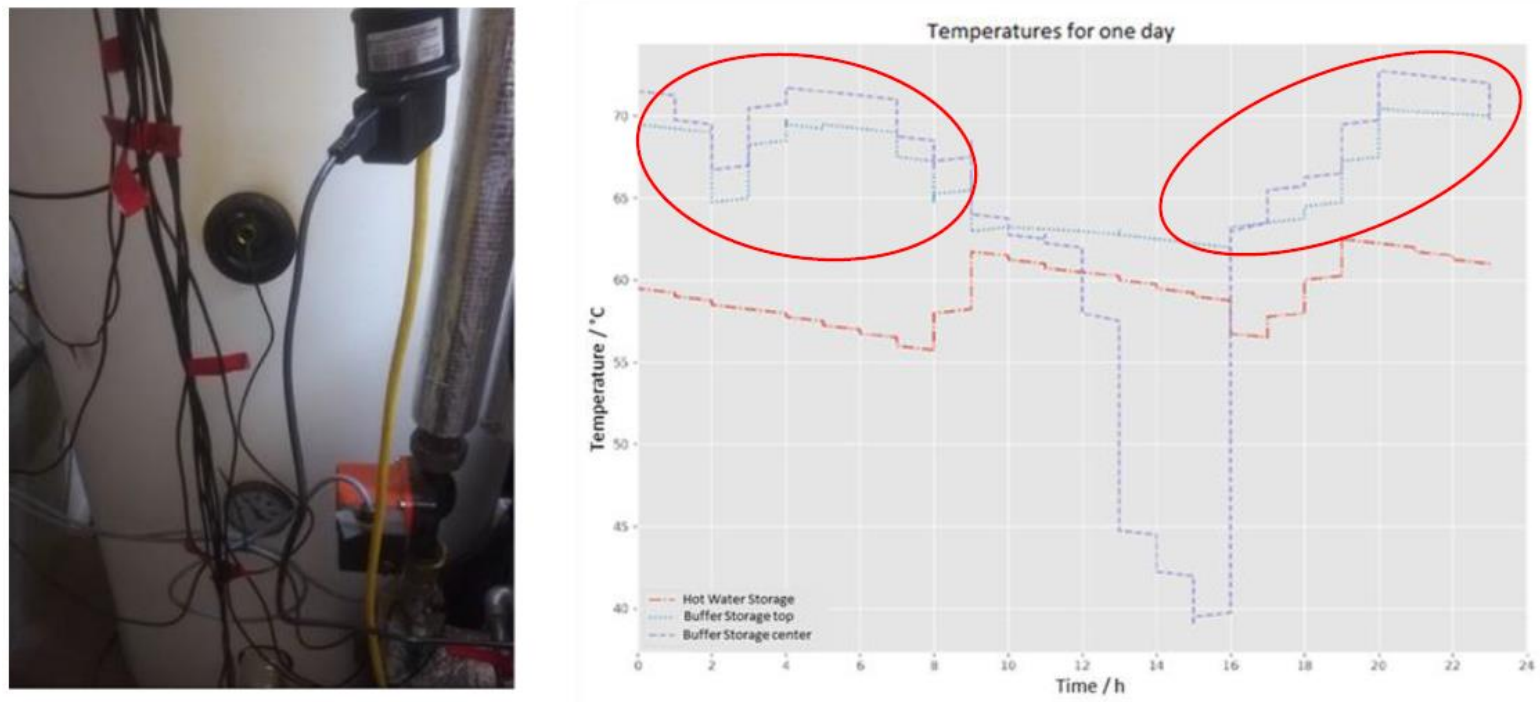

Fig. 5: Installed sensors (left) and results for one day (right) [10]

\section{CONCLUSION AND OUTLOOK}

For reducing $\mathrm{CO}_{2}$ emissions it is necessary to keep on optimizing HVAC systems. While in commercial buildings already a lot of research and application of new innovations has been done, in small residential buildings still the quality of the systems is not taken into account. The quality should be ensured in every part of the life cycle of a HVAC system. For big projects always some planers and engineers are involved in the planning, installing and commissioning process in order to calculate and check the quality with new technologies and software. In contrast, in small projects like residential buildings seldom an engineer or planner is involved and because of the costs just the mandatory technology for running the system is installed. The main differences of residential buildings to big projects are the low costs and the repetition of systems.

For controlling the quality in system level digitalization tools like QR-Codes and RFID can be a solution to prevent human-cause faults. For checking the quality of the running system time series analysis with IoT-based FDD can be done. FDD was already applied for single components of a HVAC system in field test and showed good results. As discussed, FDD based on expert-knowledge might be the easiest algorithm to tackle the problem of quality control of a whole system in residential buildings.

By a higher amount of used IoT technologies, integration of IoT devices in residential buildings will be more feasible in the next view years. In case of low frequency demand for data compared with low 
energy, the use of LoRaWAN ${ }_{\circledast}$ is a good choice. On the other hand for higher frequency data, some other technologies should be taken into account.

In further work data from three standard HVAC systems will be collected and evaluated. Likewise the work of Buchmiller, there should be a cheap and feasible solution for equip existing systems with additional sensors or take the data from new systems. An algorithm for automated fault detection will be developed and tested.

In addition some criteria for the quality of a standard system should be formulated in early state of installation as well as during operation.

Acknowledgements: This work is funded by the BMWi under Reference 03EGB0006B

\section{REFERENCES}

1. Global Alliance for Buildings and Construction, "Towards zero emission efficient and resilient buildings," Global Status Report, 2016.

2. https://ec.europa.eu/energy/en/topics/energy-efficiency/buildings.

3. Y. Zhao et al., Pattern recognition-based chillers fault detection method using support vector data description (SVDD), Appl. Energy 112, 2013.

4. Y. Yu et al, A review of fault detection and diagnosis methodologies on air-handling units, Energy Build. 82, 2014.

5. M. Bonvini et al., Robust on-line fault detection diagnosis for HVAC components based on nonlinear state estimation techniques, Appl. Energy 124, 2014.

6. Turner, W.J.N. et al., Residential HVAC fault detection using a system identification approach. Energy and Buildings, 2017.

7. T. Müller, K. Kruppa, G. Lichtenberg, N. Réhault, Fault Detection with Qualitative Models reduced by Tensor Decomposition methods, IFAC-PapersOnLine, 2015

8. D. Buchmiller, IoT-Technologien für die Automation kleiner Wohngebäude, Bachelorarbeit, Hochschule Biberach, 2018

9. C. Schmelzer, M. Georgii, K.Vajen, Entwicklung, Untersuchung und Anwendung von Methoden zur Langzeitüberwachung und automatisierter Fehlerdetektion großer, solarunterstützter

Wärmeversorgungssysteme, Abschlussbericht Förderkennzeichen 0325975A, Institut für Thermische Energietechnik Univerität Kassel, 2015

10. K. Jagnow, D. Wolff, Optimus, 2003

11. M. Krempl, C. Kuh, M. Grim, Zeitnahe Qualitätssicherung und Optimierung von Gebäuden, SpeedReg!, 2014

12. J. Schein, S. T. Bushby, N. S. Castro, J. M. House, A rule-based fault detection method for air handling units, Energy and Buildings, 2006

13. https://lora-alliance.org/about-lorawan

14. Ibrar Yaqoob, Ejaz Ahmed, Ibrahim Abaker Targio Hashem, Abdelmuttlib et al., Internet of Things Architecture: Recent Advances, Taxonomy, Requirements, and Open Challenges, IEEE Wireless Communications ( Volume: 24, June 2017

15. https://eur-lex.europa.eu/legal-content/EN/TXT/?uri=OJ:L:2014:337:TOC 285

16. S. L. Levin, S.Schmidt, IPv4 to IPv6: Challenges, solutions, and lessons, Telecommunications Policy, Volume 38, Issues 11, December 2014

17. D. Jacobs, Gebäudebetriebsoptimierung : Verbesserung von Optimierungsmethoden und Optimierung unter unsicheren Randbedingungen, Dissertation, Stuttgart: Fraunhofer Verlag, 2012

18. M. Mekki, E. Bajic, F. Chaxel, F. Meyer, A comparative study of LPWAN technologies for large 290 scale deployment, ICT Express, 2018

19. A. Schmitt, F. Carlier, V. Renault, Dynamic bridge generation for IoT data exchange via the

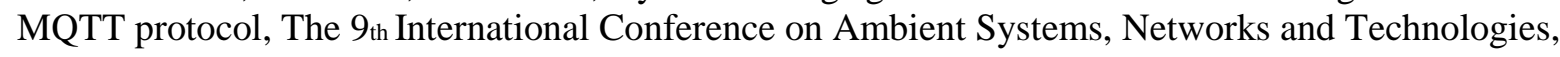
2018 


\title{
PEAR - ENERGY EFFICIENT AUTOMATION AND CONTROL OF BUILDINGS
}

\author{
Anita Preisler, MSc \\ teamgmi Ingenieurbüro $\mathrm{GmbH}$ \\ Schönbrunnerstrasse 44/10, 1050, Vienna, Austria \\ Phone: +43-(1)-5457489-0 \\ E-Mail: anita.preisler@teamgmi.com
}

\section{SUMMARY}

The efficient operation of buildings largely depends on an integrated design and interplay of building automation components: the energy demand calculated in the design phase often differs from measured values in the actual building operation. Therefore, quality assurance measures during commissioning and operation of buildings are required by thoroughly reviewing building automation and controls. This project presents a solution how to assess energy efficiency of control strategies in the fields of air conditioning systems, concrete core activation and free cooling. In order to validate results, control strategies are implemented in the demonstration building "Post am Rochus" as well as monitoring during the project duration.

This paper focuses on the results of the developed control strategies for the air-handling units in the office area using dynamic system simulations (TRNSYS, Version 17) in comparison to the real operation experiences according to monitoring data.

\section{INTRODUCTION}

The research project PEAR examines, evaluates and optimizes energy-efficient building systems in terms of their control strategies with the primary objective of significantly reducing the commissioning phase as well as guaranteeing a consistently energy-optimized operation while maintaining comfort. The acceptance of new technologies is largely dependent on user acceptance; however, user satisfaction needs to remain on the same level (or even increase) to ensure a successful deployment. Within the project a new hardware-in-the-loop method has been developed which is tested in the demonstration building "Post am Rochus" (see Figure 1). The goal was a hardware-in-theloop connects building automation hardware with a simulation environment in order to analyze and optimize the interaction between controls and (simulated) energy systems. Figure 1 and Figure 2 show the newly finished Head Quarter of the Austrian "Post" company in the 3rd district of Vienna with $47.300 \mathrm{~m}^{2}$ gross area.

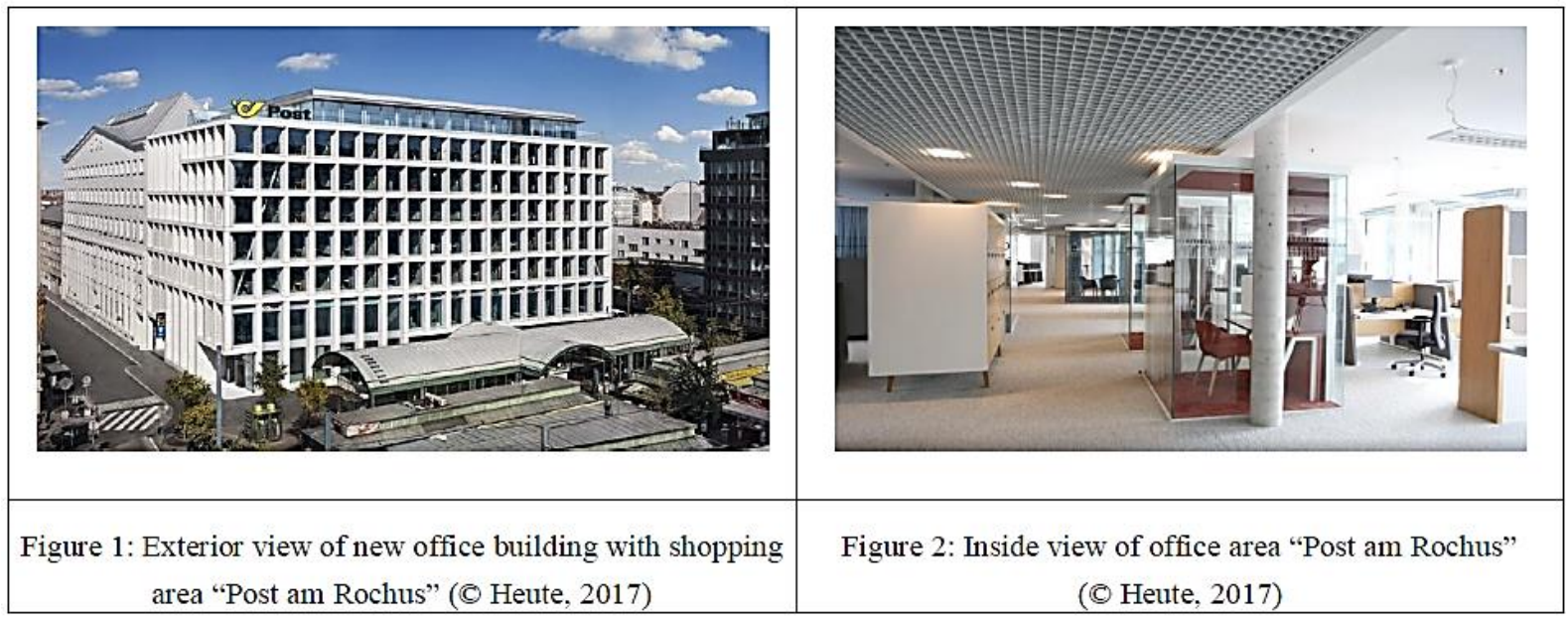

\section{RESULTS}

Figure 3 shows a scheme of one of the two identical air-handling units (AHUs) for the office area in the demonstration building with real-time data from the central building control system. The purpose of the AHUs is fresh air supply and humidity control. Heating and cooling of the office area is covered by other technologies. To increase the efficiency an enthalpy rotor for temperature and humidity recovery was chosen. AHU 01 has a design volume flow of $36.000 \mathrm{~m}^{3} / \mathrm{h}$ and AHU 02 has a design 
volume flow of $31.000 \mathrm{~m}^{3} / \mathrm{h}$ to supply fresh air for 1.000 employees. The configuration and control strategy is the same for both AHUs.

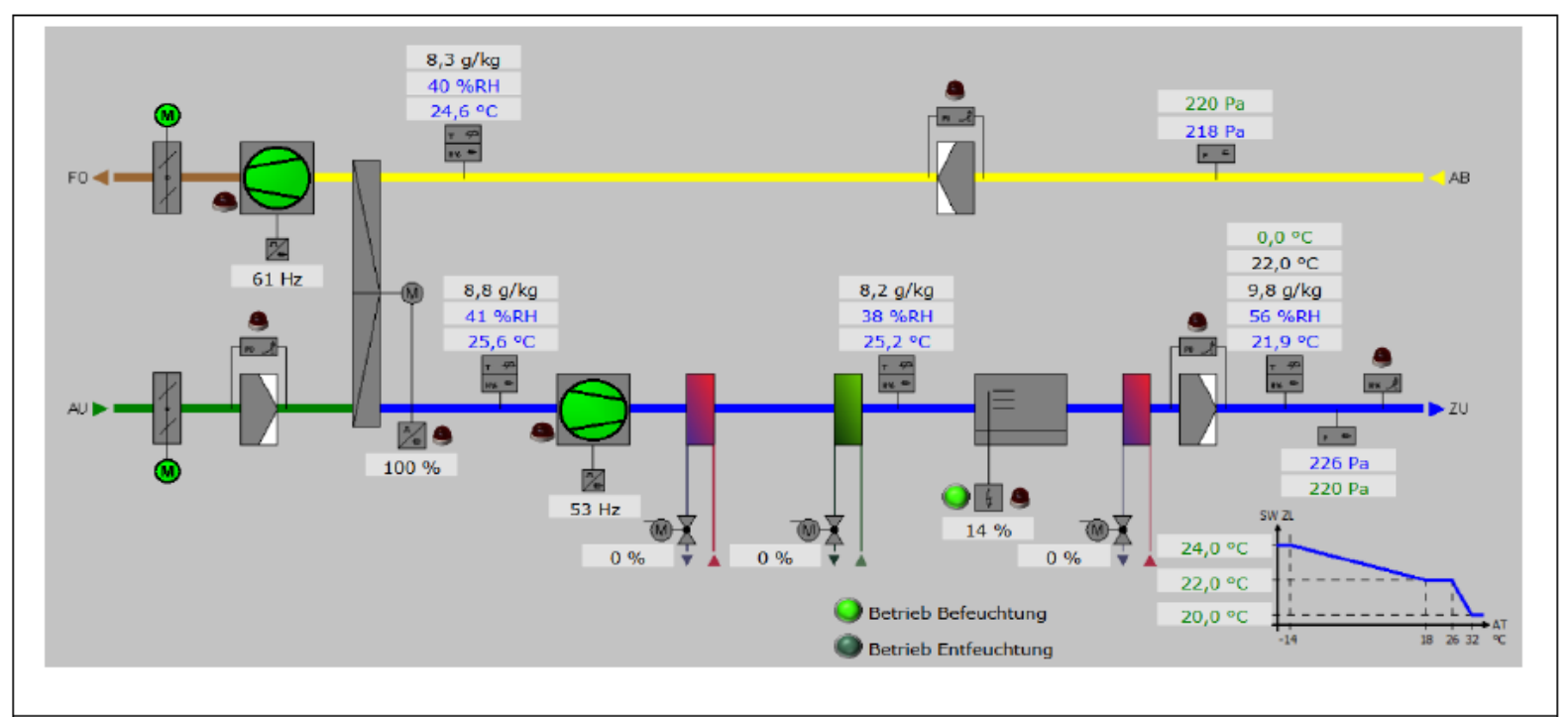

Figure 3: Scheme of air-handling unit office area with real-time data (source: demonstration building, 11.04.2018)

The monitoring evaluation shown in the following figures is based on 15 minutes recorded values. In October 2017 the users of the demonstration building moved in, the monitoring of the heating, ventilation and air-conditioning (HVAC) system started at the 14th of October 2017.

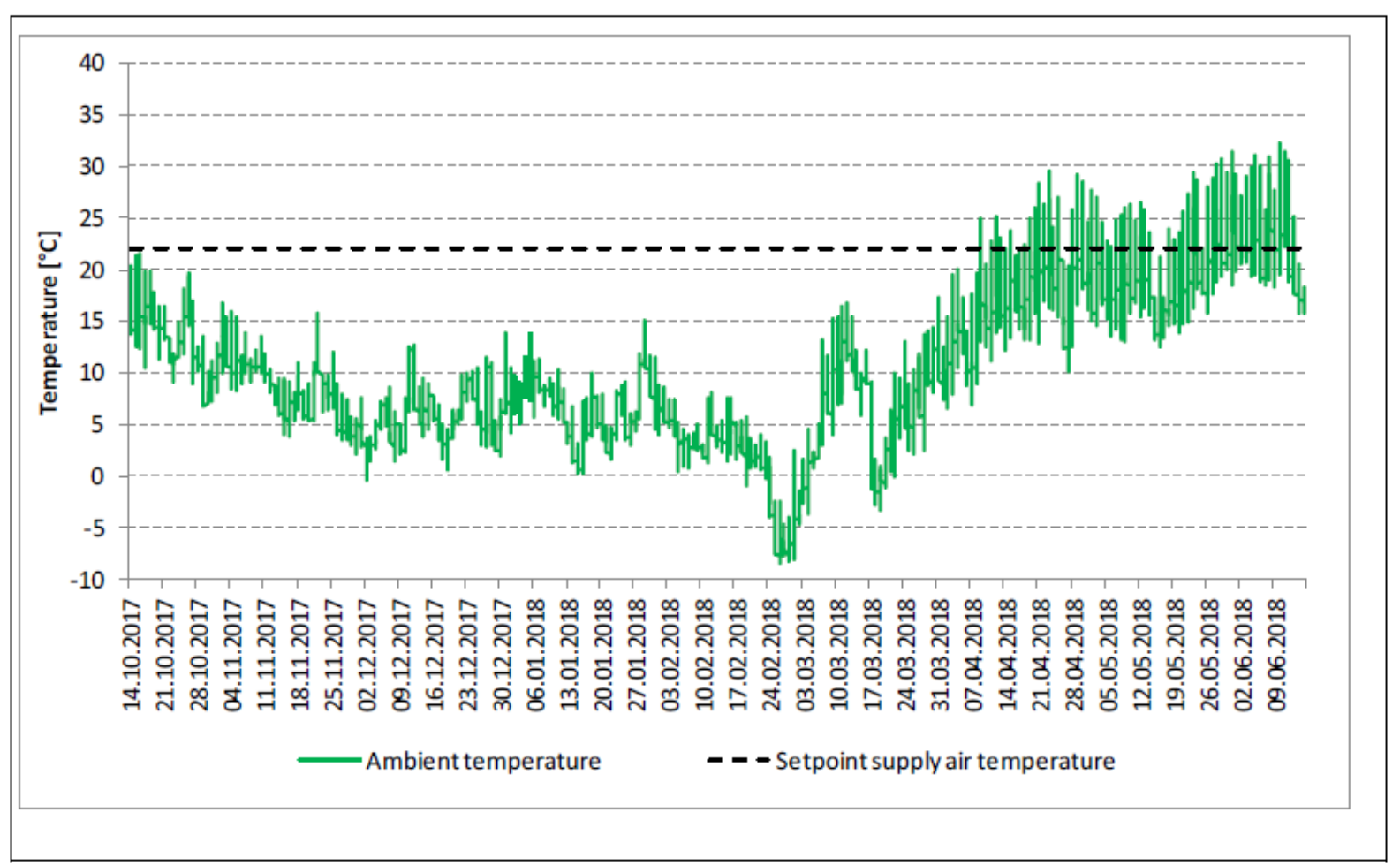

Figure 4: Outside temperature during monitoring period with supply air setpoint for AHUs (source: teamgmi 2018) 
In Figure 4 the outside temperature during the here analysed monitoring period is shown. The average setpoint of $22^{\circ} \mathrm{C}$ for the supply air temperature shows, that most of the monitoring period the AHUs had to heat the outside air. From April until mid of June the AHUs alternately had to heat and cool the air which is typical for the transition period. The outside humidity in Figure 5 shows the AHUs had to humidify the supply air for most of the monitoring period to reach the minimum humidity setpoint of $7 \mathrm{~g} / \mathrm{kg}$ measured in the return air. From April until mid of June the outside air was alternately too dry, or in the desired range, or too humid, which is typical for the transition period.

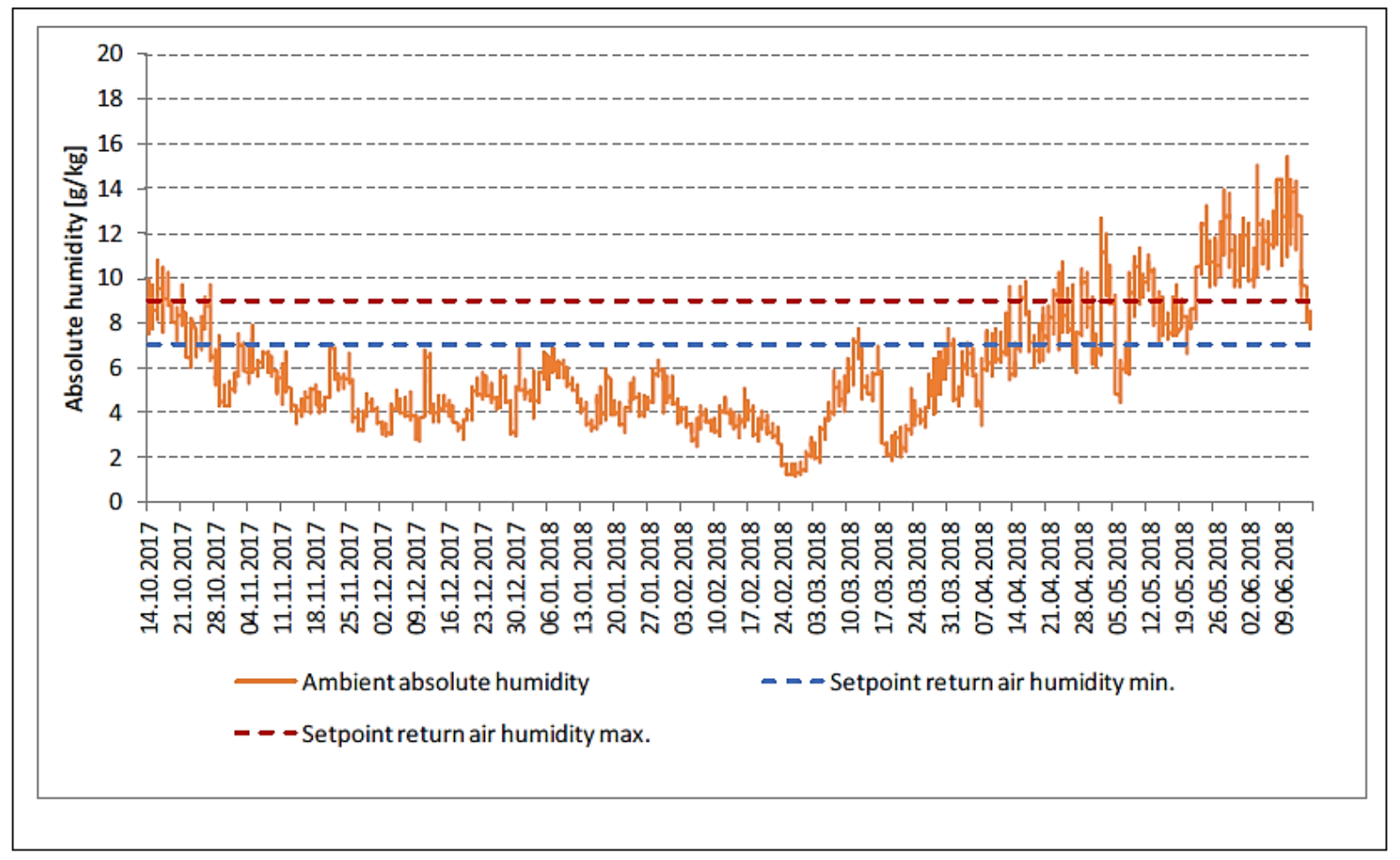

Figure 5: Outside absolute humidity during monitoring period with return air setpoints for AHUs (source: teamgmi 2018)

The measured electrical capacity of the AHUs in Figure 6 and Figure 7 shows, that the AHUs were not in full operation when the users moved in October 2017. According to the design a time schedule of 07:00 - 19:00 during the week and no operation on the weekend should be implemented. From the 19th of November on the monitoring data show a time schedule from 06:00 - 21:00 or even longer during the week and mostly no operation on the weekend. Figure 6 also shows that AHU 01 stopped operation at the $9_{\text {th }}$ of June 2018 and did not start again until the end of the here evaluated period. 


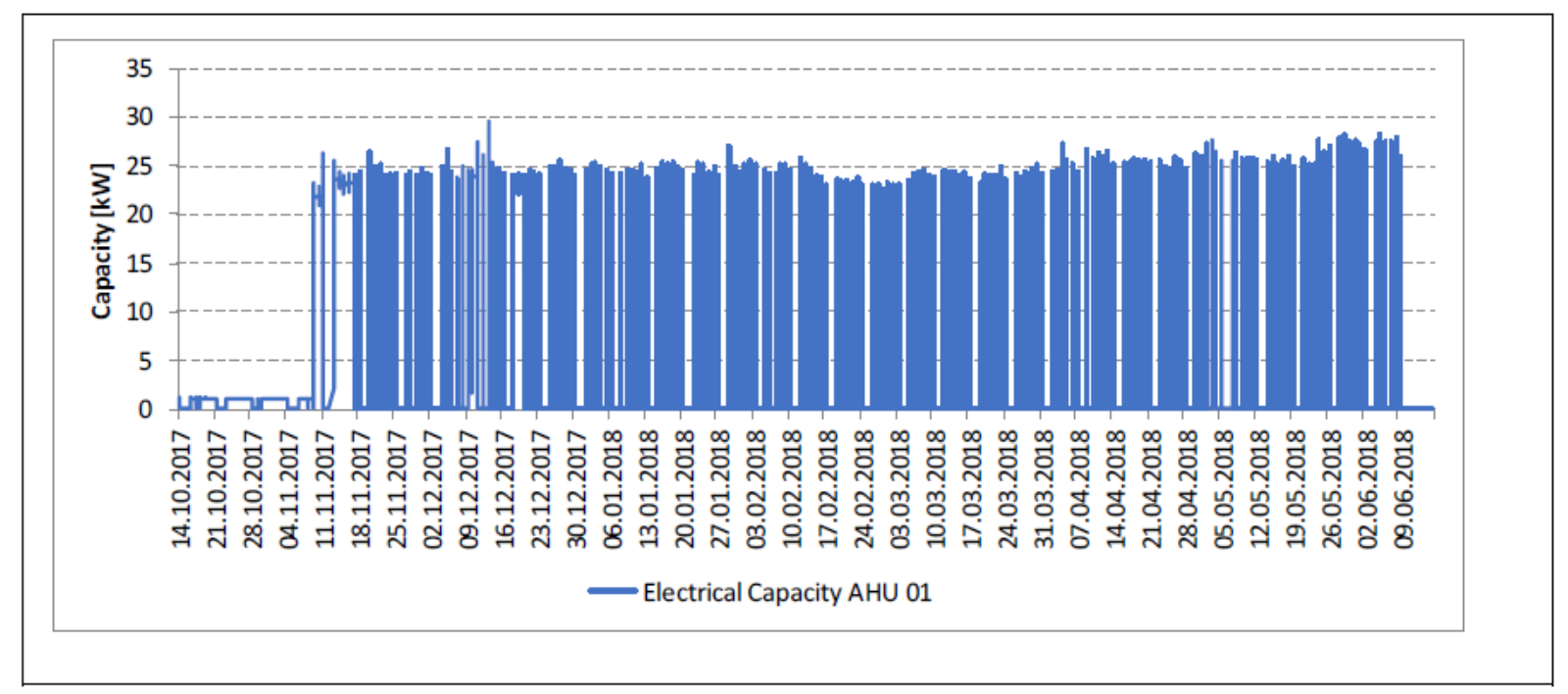

Figure 6: Measured electrical capacity of AHU 01 for office area (source: teamgmi 2018)

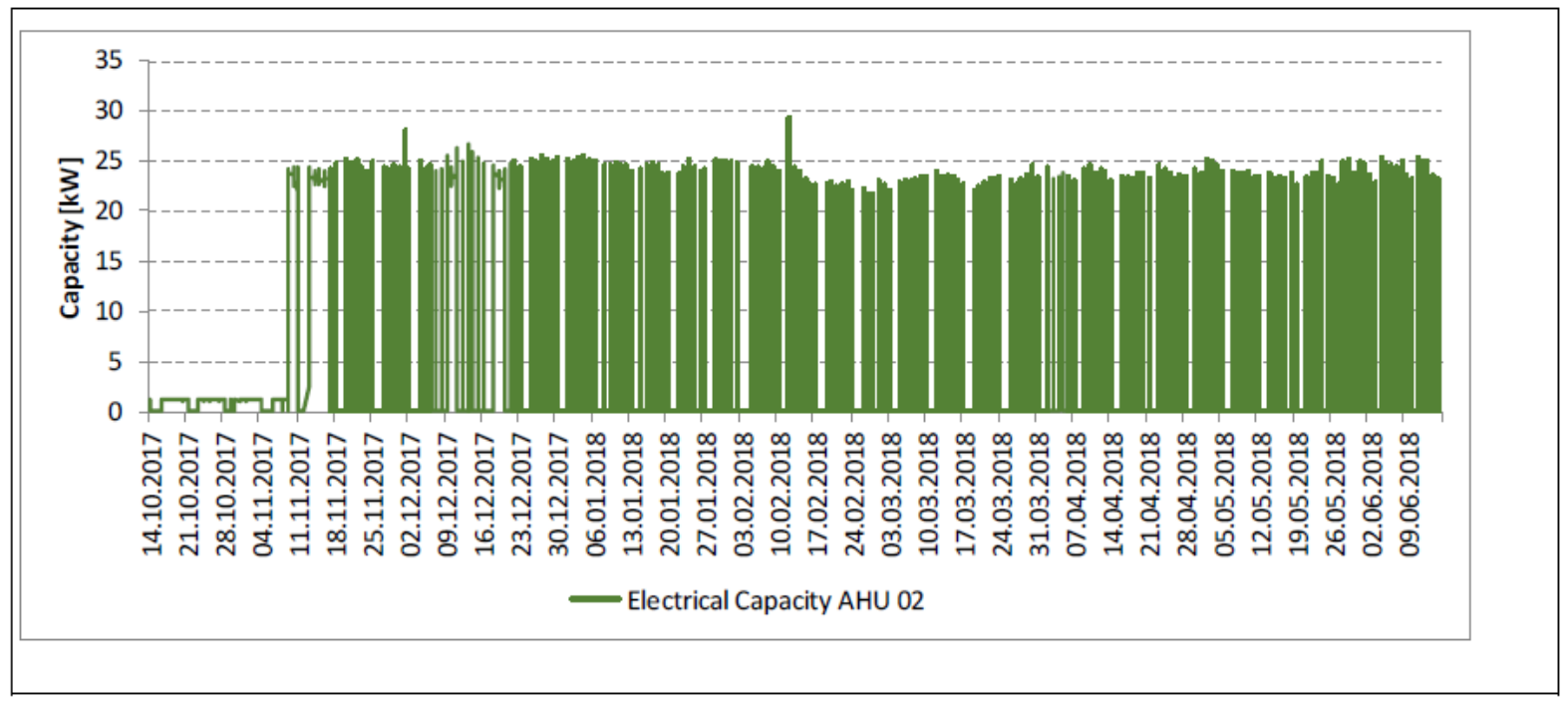

Figure 7: Measured electrical capacity of AHU 02 for office area (source: teamgmi 2018)

Table 1 shows a comparison of electrical values between design and monitoring. According to the monitoring data the specific fan capacity was always below the maximum design value for category 4 in both AHUs. Although, the electricity demand of AHU 01 is $33 \%$ and of AHU 02 is $37 \%$ higher than design values in the given period. This is probably due to the longer operation time of the AHUs than expected in the design phase. 


\begin{tabular}{|l|l|c|r|r|}
\hline \multicolumn{2}{|l|}{} & Unit & Design & Monitoring \\
\hline \multirow{4}{*}{ AHU 01 } & Volume flow & $\mathrm{m}^{3} / \mathrm{h}$ & 36.000 & - \\
\cline { 2 - 5 } & Specific fan capacity (SFP-4) max. & $\mathrm{Wh} / \mathrm{m}^{3}$ & 1,10 & 0,82 \\
\cline { 2 - 5 } & Electrical capacity max. & $\mathrm{kW}$ & 39,60 & 29,66 \\
\cline { 2 - 5 } & Electricity demand & $\mathrm{kWh} /(7 \mathrm{month})$ & 42.500 & 56.500 \\
\hline \multirow{3}{*}{ AHU 02 } & Volume flow & $\mathrm{m}^{3} / \mathrm{h}$ & 31.000 & - \\
\cline { 2 - 5 } & Specific fan capacity (SFP-4) max. & $\mathrm{Wh} / \mathrm{m}^{3}$ & 1,10 & 0,95 \\
\cline { 2 - 5 } & Electrical capacity max. & $\mathrm{kW}$ & 34,40 & 29,36 \\
\cline { 2 - 5 } & Electricity demand & $\mathrm{kWh} /(7$ month $)$ & 41.200 & 56.300 \\
\hline
\end{tabular}

Table 1: Performance of AHU 01 and AHU 02 from 19.11.2017 until 15.06.2018 (source: teamgmi 2018)

Figure 8 shows the performance of the AHUs according to their given setpoints for temperature and humidity. The monitoring data show, that the supply air temperature is for almost all the time in the desired range in both AHUs. The humidity - which is measured in the return air - is occasionally below the desired setpoint in both AHUs, but still in an acceptable range. In AHU 02 the humidity is below the minimum setpoint for a considerable time, due to a damaged humidifier in the given period. The AHUs are expected to operate according to the operation modes shown in Figure 8, which were developed before operation using the simulation environment TRNSYS (TRNSYS, Version 17). Figure 9 shows the simulated control modes for heating (red) and cooling (blue) and which of the components are "on" or "off" in each operation mode.

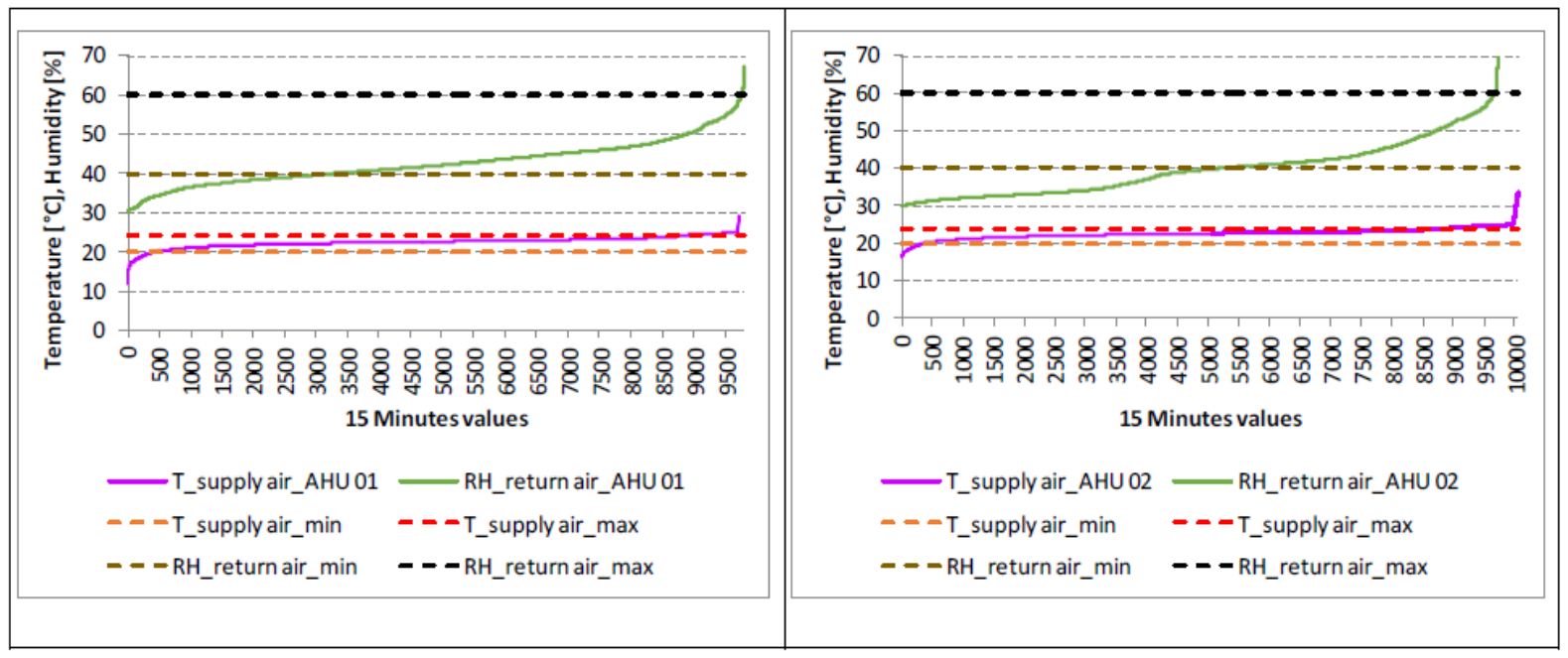

Figure 8: Sorted values of measured supply air and return air conditions for AHU 01 (left) and AHU 02 (right) from 19.11.2017 until 15.06.2018 with setpoints (source: teamgmi 2018) 


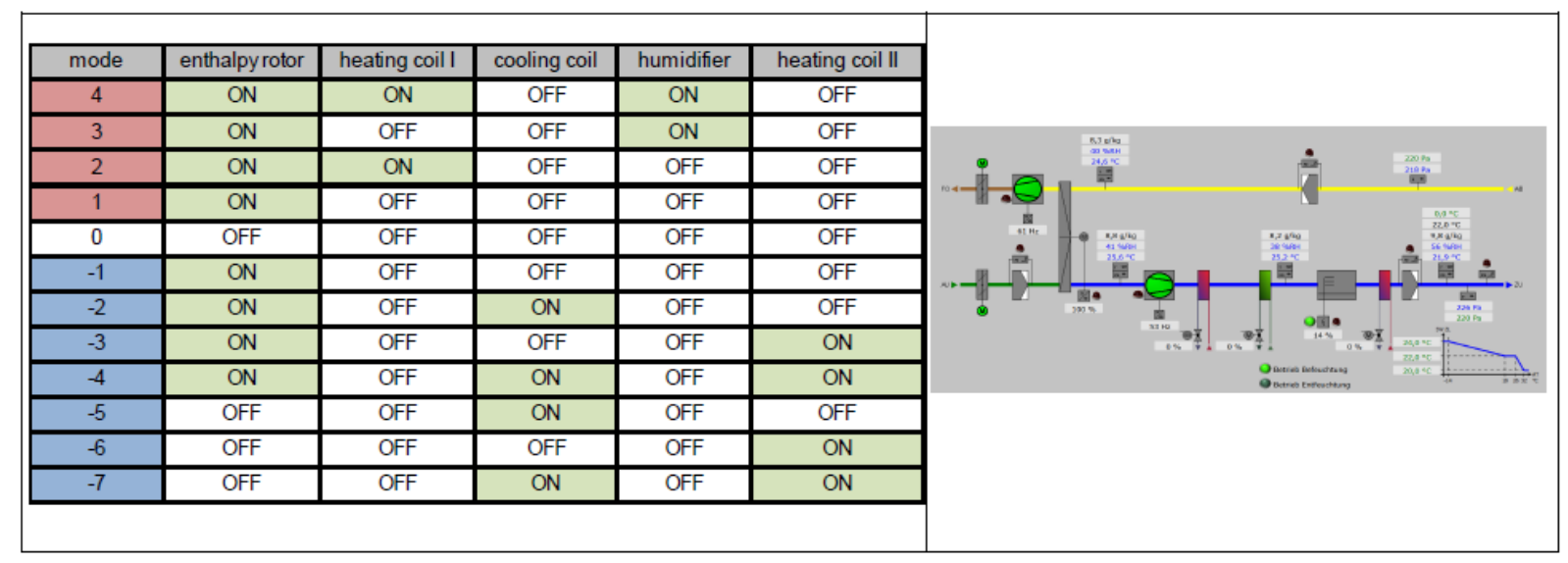

Figure 9: Simulated control modes (source: teamgmi 2017)

Figure 10 shows the operation modes according to the monitoring data. The allocation to the different modes might not be accurate, due to the 15 minutes values of monitoring data. It is quite interesting, that according to the monitoring data three additional modes are active in operation which were not considered in the simulations. The second heating coil (heating coil II) is not only in operation in the cooling modes but also in the heating modes (mode 2' and mode 4'). This might be due to the high maximum setpoint of $24^{\circ} \mathrm{C}$ of supply air when the outside air is very cold. As the AHUs have no heating purpose this setpoint could be reduced to generally $22^{\circ} \mathrm{C}$ and then the second heating coil might not be necessary any more for the heating modes. The humidifier was intended to operate only in heating modes. The monitoring data show, that the humidifier is also in operation when the cooling coil is needed (mode -2'). This mode was also not considered in the simulations. It would mean that the outside air temperature is over the desired supply air temperature setpoint, and the return air humidity is below the desired humidity setpoint, which might be possible in transition time.

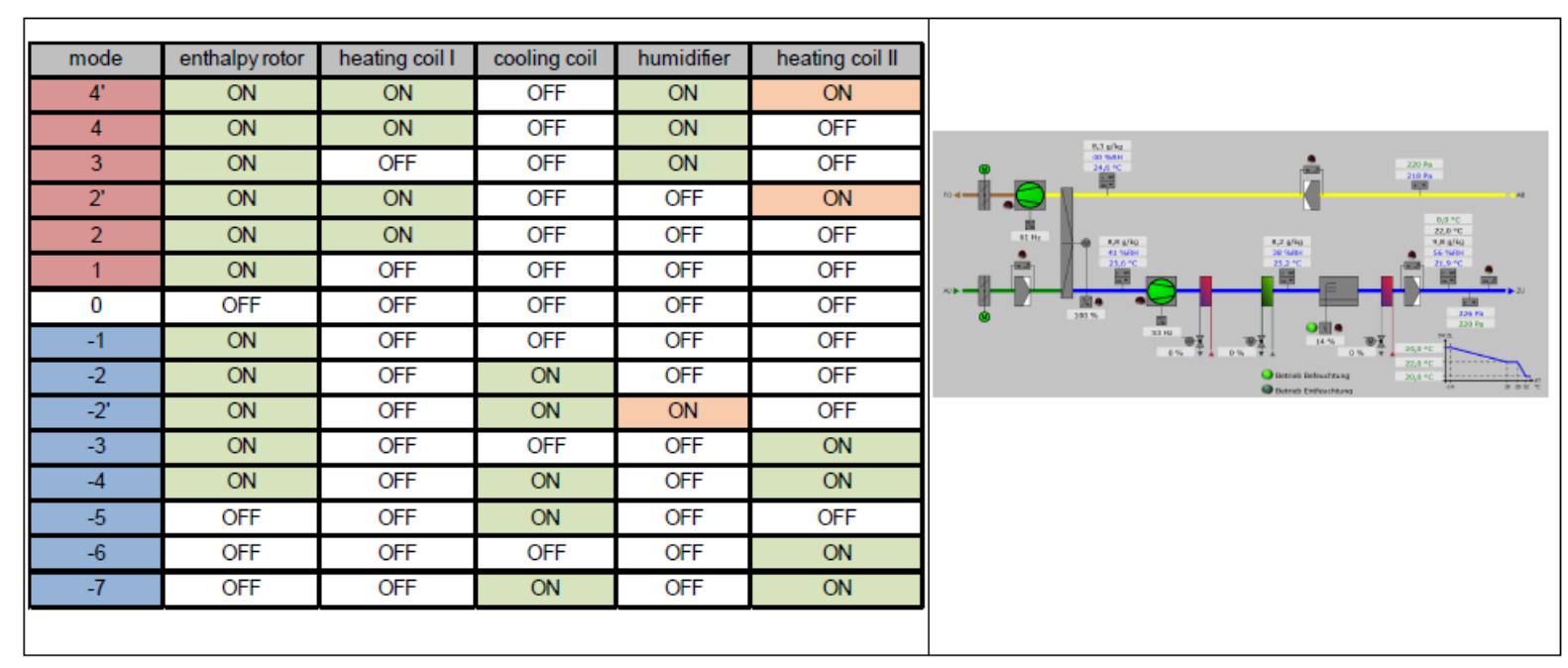

Figure 10: Measured control modes (source: teamgmi 2018)

Figure 11 shows a frequency distribution of the different modes for the measured control modes and the full year simulation. For heating "mode 4" was expected to be dominant which could be verified by the monitoring data. The hours of operation hours in "mode 2" are more than expected by the simulation results, but the measured return air humidity was often below the humidity setpoint, which means the control should have be in "mode 4". The results for cooling modes are only available from transition time. Here it is interesting that "mode -2 " is more often in operation than expected by the simulation results. As the monitoring period is now only 7 months, the sum of hours of operation show that the AHUs are more often in operation than it was intended in the design phase. 


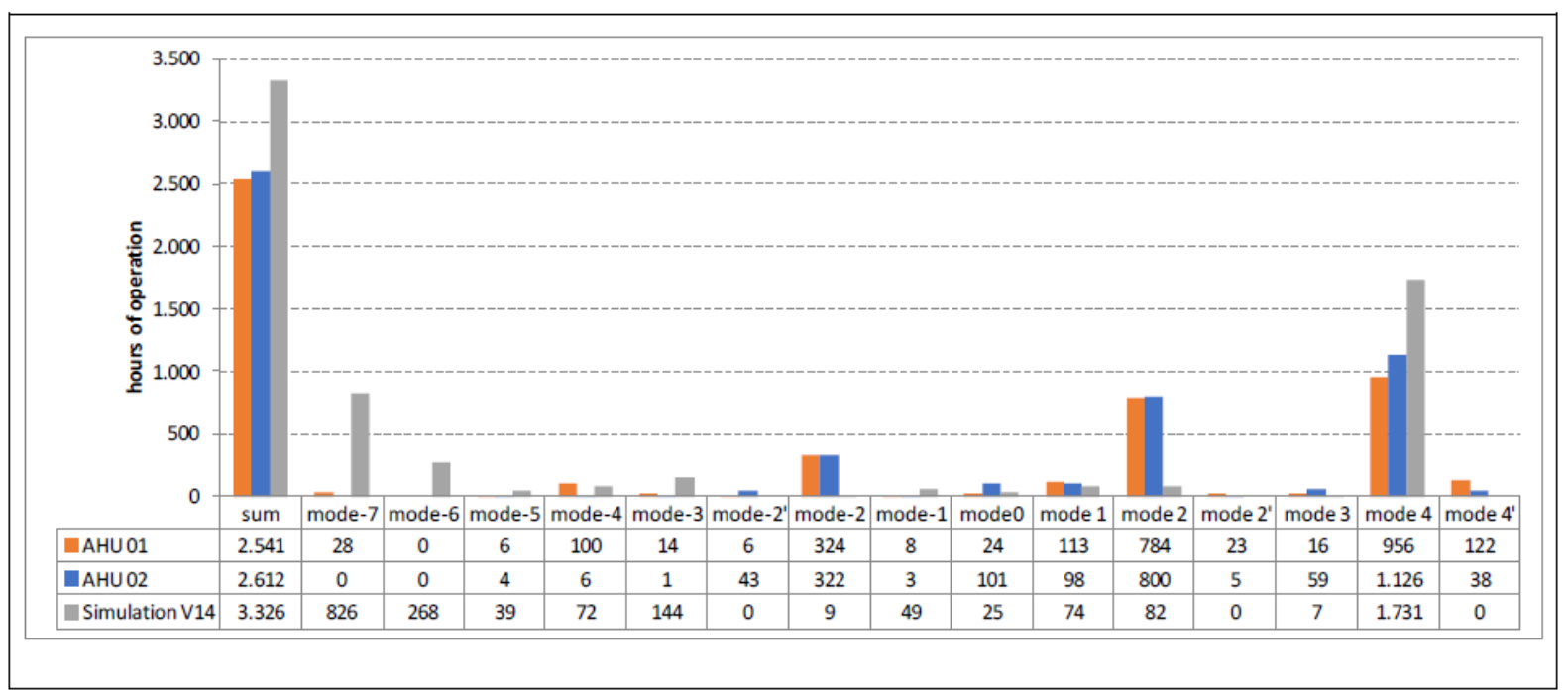

Figure 11: Comparison of measured modes from AHU 01 and AHU 02 from 19.11.2017 until 15.06.2018 with full year simulation (source: teamgmi 2018)

\section{CONCLUSION}

The air-handling units for the office area in the demonstration building show a quite good performance from the 19 th of November 2017 on. The necessary electrical capacity was always below the maximum design value, but due to longer runtimes the electricity demand is $33 \%$ to $37 \%$ higher than expected in the design phase. This runtimes should be reduced to the actual presence of people.

The desired setpoints were reached very well for the supply air temperature; the humidity is in an acceptable range, but often below the desired setpoint. Due to a damaged humidifier the humidity was quite low in AHU 02 for a considerable time of the monitoring period.

The high maximum setpoint of $24^{\circ} \mathrm{C}$ of supply air when the outside air is very cold should be reduced to generally $22^{\circ} \mathrm{C}$ as the AHUs have no heating purpose. Therefore, the second heating coil would probably not be necessary any more for heating modes, but only for cooling modes, as intended.

The evaluation of control modes is very difficult with 15 minutes monitoring data. A reduction to one minute values would increase the validity significantly.

Basic data of the project:

Funding programme: "Stadt der Zukunft", 2nd Call

Project partner:

- AIT - Austrian Institute of Technology (project lead)

- Österreichische Post AG

- teamgmi Ingenieurbüro GmbH

- IBO Österreichisches Institut für Bauen und Ökologie GmbH

- BPS Engineering Technisches Büro zur Planung Haustechnischer Anlagen GmbH;

Project run-time: 01.11.2015 - 31.10.2018

\section{REFERENCES}

TRNSYS. (Version 17). Transient System Simulation Tool, Version 17. Madison: Thermal Energy System Specialists, LLC. 


\section{ADVANCED SIMULATION AND CONTROL METHODS FOR OPERATION, PLANNING AND CONTROL OF DISTRICT HEATING SYSTEMS}

Ingo Leusbrock, Gerald Schweiger, Harald Schrammel, Keith O’Donovan, Basak Falay, Carles Ribas Tugores

AEE - Institute for Sustainable Technologies

A-8200 Gleisdorf, Feldgasse 19, Austria

phone: $+43(0) 31125886-261$

email: i.leusbrock@aee.at

\section{SUMMARY}

Cities are central elements and hotspots for a sustainable energy transition. Concepts for future, sustainable energy systems are largely characterized by a radical change of the entire system and thus also its planning and operation. District heating systems are considered in this regard as cornerstone for our future energy systems and sustainable heat supply, especially in urban areas. In order to investigate these challenges and opportunities for future systems, standard simulation tools and methods are unsuitable, because they often rely on simplified models, static relationships and singledomain approaches [1]. Based on several case studies, we will demonstrate how simulation and control can help to plan future district heating systems with increased share of renewables and high degree of flexibility, to develop advanced control strategies and unit commitment solutions and how we can reduce the complexity of these systems to allow for detailed simulations in short time periods without loss of information.

\section{INTRODUCTION}

Cities are central elements and hotspots for a sustainable energy transition. Concepts for future, sustainable energy systems are largely characterized by a radical change of the entire system and thus also its planning and operation. A key challenge is to match available energy from volatile sources with demand in terms of location, time and quantity. District heating systems are considered in this regard as cornerstone for our future energy systems and sustainable heat supply, especially in urban areas. A higher share of renewables and added flexibility measures such as storage and heat pumps represent a major challenge for future district heating systems, for both planners and operators. To design such systems that integrate large shares of fluctuating renewable energy sources while improving the overall efficiency and flexibility of district heating systems requires sophisticated simulation, optimization and control methods and tools. Several options exist for increasing energy system flexibility, including combining different energy domains, increasing supply and demand flexibility, integrating energy storage technologies and increasing the transmission capacity of the national grid as well as interconnections to other countries. In order to investigate these challenges and opportunities for future systems, standard simulation tools and methods are unsuitable, because they often rely on simplified models, static relationships and single-domain approaches [1]. One consequence of this is among others that they are frequently unable to capture the dynamic behavior of such complex systems. The conceptual planning and control of future district heating systems also leads to newer requirements for simulation and optimization.

- Multi-domain applications: Different sectors, coupling technologies and memories must be mapped in a model.

- Dynamic simulation: The dynamic simulation of detailed physical models enables an analysis of the interaction between sectors and individual components.

- Control and optimization: Sophisticated control strategies are required to enable optimum integration of non-controllable energies.

Recent advances in object-oriented, physical modeling of energy systems has led to potential for developing novel tools for system planning and operation control that focus specifically on these new challenges [2]. 


\section{RESEARCH OBJECTIVE}

Based on several case studies, we will demonstrate how simulation and control can help to plan future district heating systems with increased share of renewables and high degree of flexibility, to develop advanced control strategies and unit commitment solutions and how we can reduce the complexity of these systems to allow for detailed simulations in short time periods without loss of information.

We will showcase a case study where we upgrade an existing network to a 4th generation district heating system. The impacts of possible integration of e.g. large-scale solar units, prosumers, decentralized renewables, Power-2-Heat units, etc. on the overall system performance and the related challenges in simulation are presented. Furthermore, we will show how aggregation methods can help to reduce complexity of the system and simulation time without losing information. For our investigations, we will use Modelica. We will thus

highlight the differences between standard tools for planning and design of the systems with our approach and show the added value in terms of accuracy and representation of dynamics, which will become a vital feature of future $\mathrm{DH}$ systems.

\section{COMPLEXITY REQUIRES SIMPLIFICATION}

The efficient dynamic simulation and optimization of complex systems is the subject of current research. In order to analyze large networked systems dynamically, a simplification of the topology is necessary from a certain complexity. In the area of district heating networks, so-called aggregation processes are used. Previous work shows that networks can be aggregated to a very high degree without changing the basic characteristics of the network with regard to the dynamic behaviour of heat losses, pressure ratios or feed-in power [3]. Current research at AEE INTEC deals with these questions, for which various aggregation algorithms have been implemented and validated against real systems [4]. These methods are used in ongoing research to test new ideas and concepts. Networks themselves are used as storage to provide flexibility for the higher-level energy system. Here, a dynamic simulation allows the exact representation of temperature waves and thus better planning and control of this concept. When integrating seasonal storage systems into the simulations (for storing waste heat or renewables), it is necessary to simplify the topology in order to carry out seasonal simulations within a reasonable time. Dynamic system simulations can be used to analyze interactions between subsystems and, for example, to investigate the integration of short-term memories in order to avoid load peaks. In the case of innovative system concepts such as return/low temperature supply or other operation modes of subnetworks as part of a large overall network, the subnetwork to be evaluated is mapped in detail, while large parts that are of no interest for the questions are virtually hidden in the simulation. This saves simulation time.

\section{A (SIMULATED) VIEW INTO THE CRYSTAL BALL: MODEL PREDICTIVE CONTROL}

The use of conventional controllers, which are usually based on PID (proportional-integral-derivative controller) and time-controlled control, is no longer suitable for future systems as outlined above. Conventional regulations do not take into account information that must be taken into account for optimal control of the entire system: Weather forecasts, energy prices, user behaviour, etc.. Innovative control concepts such as model predictive control can take this information into account and thus significantly increase the efficiency of the entire system. Model Predictive Control is a class of algorithms in which a model of the system to be controlled is used to optimize operation. Available measured values are therefore used to continuously feed the simulation with current data which, with the aid of the simulation, historical data from the previous day / previous year and current weather and energy price data, then determines an optimised control for the next time step and, looking ahead, for example for the next 24 hours. Basically it should be pointed out that optimization is more complex than simulation and the demands on the user as well as on tool developers are correspondingly higher. Optimization problems in the field of (cross-domain) urban energy systems have the following characteristics:

some models are non-linear and some optimization variables can assume discrete values (boiler on/off) as well as continuous values (feed-in power). This leads to so-called mixed-integer nonlinear optimum control problems, for whose solution there are no robust algorithms. We will present a method for solving such problems, which is based on the separation of the discrete and continuous problem [3]. In energy technology, the discrete optimisation problem is often referred to as "unit commitment" (the 
order in which the various producers are used) and the continuous optimisation problem as "economic dispatch" (the mode of operation of the individual producers).

\section{REFERENCES}

[1] J. Allegrini, et al. 2015: "A review of modelling approaches and tools for the simulation of district-scale energy

systems" Renewable and Sustainable Energy Reviews, vol. 52, 1391-1404.

100 [2] Schweiger, G., Ribas Tugores, C. et al. 2017: "District heating and cooling systems - framework for Modelica-based

simulation and dynamic optimization, Energy, https://doi.org/10.1016/j.energy.2017.05.115.

[3] Larsen, Bøhm, Wigbels . A comparison of aggregated models for simulation and operational optimisation of district

heating networks. Energy Conversion Management 2004.

[4] Lund, Andersen, Østergaard, Mathiesen, Connolly. From electricity smart grids to smart energy systems - A market operation based approach and understanding. Energy 2012.

\section{CONFERENCE TOPIC}

Please indicate here to which of the Conference Topics this abstract refers:

- Urban District Heating and Cooling Technologies 


\title{
A NOVEL DISTRICT HEATING SOLUTION BASED ON ABSORPTION HEAT EXCHANGER(AHE) FOR DIFFERENT TYPES OF COGENERATION PLANTS
}

\author{
Tianle $\mathrm{Hu}^{1}$, Xiaoyun $\mathrm{Xie}^{1}$, Yi Jiang ${ }^{1}$, Alberto Coronas ${ }^{2}$ \\ Building Energy Research Center ${ }^{1}$ \\ Building Energy Research Centre, Tsinghua University, 100084 Beijing, China \\ Phone: +8618910673510 \\ E-Mail: hut115@mails.tsinghua.edu.cn \\ CREVER, Mechanical Engineering Department in Universitat Rovira i Virgili ${ }^{2}$ \\ Avinguda Països Catalans 26, Campus Sescelades, 43007 Tarragona, Spain
}

\section{SUMMARY}

A novel district heating (DH) solution based on absorption heat exchanger (AHE) is presented. Moreover, the corresponding optimized heating processes for two most common types of cogeneration plants are demonstrated and analysed. In general, the novel DH solution is able be recover low-grade heat $\left(30 \sim 50^{\circ} \mathrm{C}\right)$ in cogeneration plants, reduces heat transportation cost, and can expand heating capacity without extra fuel increase. Therefore, the novel DH solution presented in this paper provide an alternative sustainable solution for future DH design and optimization.

Key-words: District Heating; Absorption Heat Exchanger; Steam Turbine; Gas Engine; Cogeneration Plants

\section{INTRODUCTION}

Cogeneration systems and its district heating/cooling application has been proven to be a reliable, sustainable, and cost effective energy conversion solution(Çakir, Çomakli, \& Yüksel, 2012). In most of the cogeneration applications, the energy types produced simultaneously are electric and heat energy. Steam turbine and gas engine (internal combustion engine) are two common heat engines applied in cogeneration systems. In spite of the fact that many attempts were made in this field to optimize combustion process and cycle operation, there is still a great deal of thermal energy released around the environment as a result of heat engine operation limitations. In practice, exhausted steam from a steam turbine accounts for 35 60\% of input energy in a thermal power plant(Rattner \& Garimella, 2011), and exhaust gases and cooling system accounts for two-thirds of the thermal energy from the fuel in a gas engine(Seyedkavoosi, Javan, \& Kota, 2017). Consequently, waste heat recovery techniques for cogeneration systems have gained a lot of attention in recent years.

Waste heat recovery in:

Rankine cycle and heat pump are promising technologies for waste heat recovery in gas engine. Ge, Li, Liu, Duan, and Yang (2018) applied dual-loop organic Rankine cycle using zeotropic mixtures in gas engine system, and the system net power output relative increment rates of mixture systems are $2.5-9.0 \%$ and $1.4-4.3 \%$, respectively, compared to those of corresponding pure working fluids system with 573.15-623.15K engine exhaust gas temperature. Caf, Urbancl, Trop, and Goricanec (2016) applied high-temperature heat-pump in a gas engine system to exploitation of low-temperature energy, and total efficiency of the primary energy of natural gas is extended from $86.8 \%$ to $103.3 \%$.

Absorption heat pumps are usually used to recover waste heat in steam turbine systems. Sun, Fu, Sun, and Zhang (2014)proposed a complicated absorption heat pump system with the combination of a single-effect absorption heat pump, a double-effect absorption heat pump and a double-lift absorption heat pump for waste recovery heat from steam turbine cogeneration system. Hu, Xie, and Jiang (2017) presented a variable lift absorption cycle to recover waste heat in steam turbine cogeneration system, which can reduce the equivalent power consumption for space heating by $8.5 \%$ 15\% respectively when comparing to single effect cycle. 
Absorption heat exchanger (AHE) was presented a few years ago(Fu, Jiang, \& Zhang, 2008) to realize heat transfer between two fluids with mismatched flow rate. AHE has shown large energy saving capability in many actual district heating projects in China(Zhu, Xie, \& Jiang, 2016).

A novel DH solution based on AHE is introduced in this paper. Moreover, the resulting optimized heating process to recover wasted heat and increase energy efficiency for steam turbine and gas engine cogeneration systems are presented and analyzed.

\section{PRINCIPLE OF THE NOVEL DISTRICT HEATING SOLUTION}

AHE can serve as a replacement of conventional heat exchanger(HEX) in terminal buildings. Compared with conventional HEX, return water temperature of primary heating network(PHN) can be lower than the supply water temperature of secondary heating network (SHN), and accordingly AHE can expand heat transportation temperature (Xie \& Jiang, 2017). Scheme of the novel DH system based on AHE are shown as Fig. 1. The main benefits are as following:

1) The novel system is able be recover low grade heat $\left(30 \sim 50^{\circ} \mathrm{C}\right)$ in cogeneration plants;

2) The novel system reduces heat transportation cost;

3) The novel system can expand heating capacity without extra fuel increase.

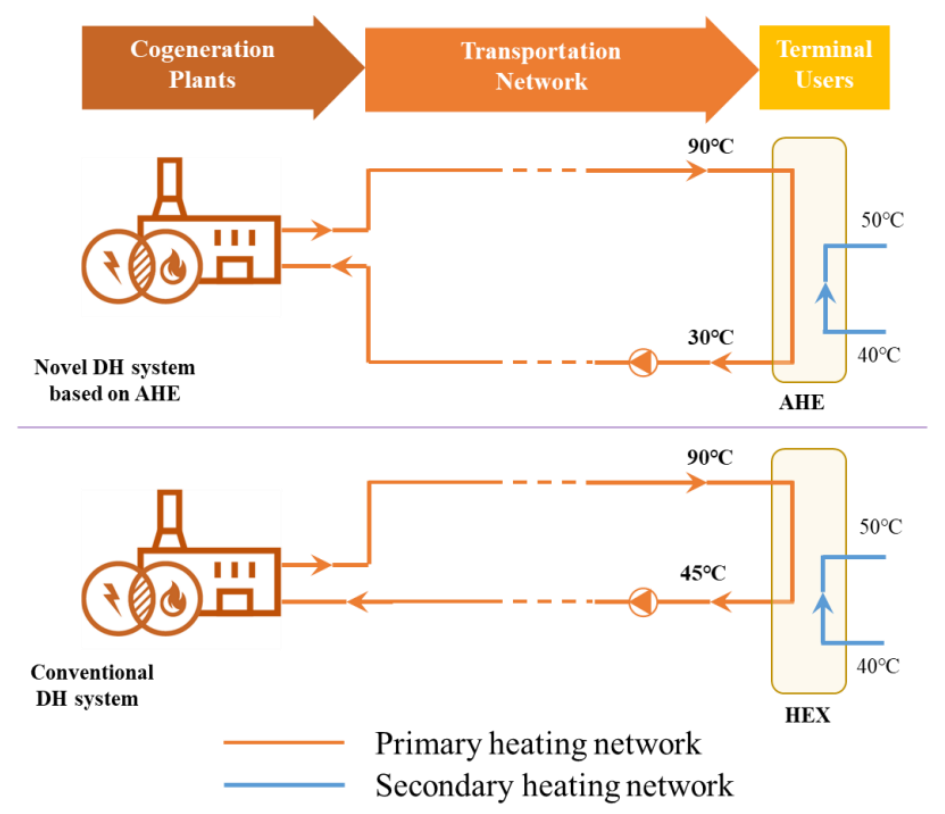

Fig. 1: Comparison of Principles of Novel DH system based on AHE and Conventional DH system

In addition, AHE has proved to be of high flexibility(Xie \& Jiang, 2017). Primary water return temperature can be maintain at $30^{\circ} \mathrm{C}$ by increase primary water supply temperature when secondary side temperature level is higher. It's feasible since heating sources in cogeneration plants are always much higher than $120^{\circ} \mathrm{C}$.

\section{A NOVEL HEATING PROCESS FOR A GAS ENGINE COGENERATION PLANT}

A novel heating process for a gas engine cogeneration plant are show in Fig. 3. Return hot water is preheated by low temperature cooling circuit (LTCC) of gas enginge, which is intecooler 2nd stage for the gas engine adopted in this paper, and then divided into two parts, one is heated by high temperature cooling circuit (HTCC), which includes intercooler 1st stage, lube oil, and cylinder jacket water for the gas engine adopted in this paper, and the other flows into absorber and condenser of an absorption heat pump (AHP). The AHP is driven by HTCC or flue gas. Outlet flue gas from AHP is continuously cooled by a circulation water between a gas/water HEX and the evaporator of the AHP. 
A gas engine cogeneration plant, which simultaneously produce electricity, chilled water, and hot water, generally consist a double effect AHP, and even a compression chiller for further refrigeration. Thus, three heat exchangers are the only initial investment when conduct the optimized heating process. Compared to conventional gas engine cogeneration plant, laten heat of the flue gas is deeply recovered in this novel heating process.

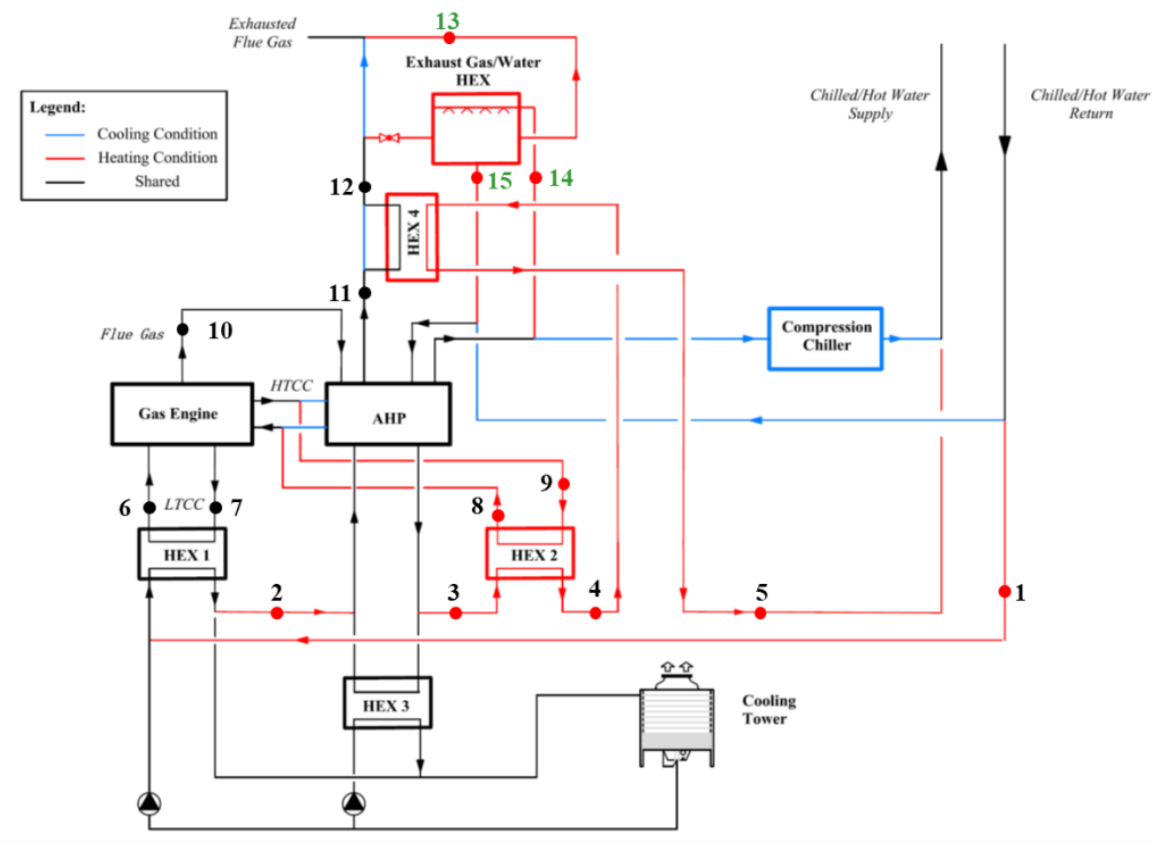

Fig. 3: A Novel Heating Process for a Gas Engine Cogeneration Plant

Energy conservation equation in HEX 1 is shown as follows:

$$
Q_{\text {LTCC }}=C_{p, w} m_{P H N}\left(T_{2}-T_{1}\right)
$$

Energy conservation equation in the absorber and condenser of the double effect AHP is shown as follows:

$$
Q_{A}+Q_{C}=C_{p, w} m_{P H N}\left(T_{3}-T_{2}\right)
$$

Energy conservation equation in the double effect AHP is shown as follows:

$$
Q_{H G}+Q_{E}=Q_{A}+Q_{C}
$$

High temperature flue gas is the heat source of high temperature generater in the double effect AHP, and energy conservation equation in the high pressure generator is shown as follows:

$$
Q_{H G}=m_{f g}\left(h_{f g, 10}-h_{f g, 11}\right)
$$

Latent heat of flue gas functions as heat source of the evaporator through the circulation water between the evaporator and the gas-water HEX, and energy conservation equation in the evaporator is shown as follows:

$$
Q_{E}=m_{f g}\left(h_{f g, 12}-h_{f g, 13}\right)
$$

Energy conservation equation in HEX 2 is shown as follows:

$$
Q_{H T C C}=C_{p, w} m_{P H N}\left(T_{4}-T_{3}\right)
$$

Energy conservation equation in HEX 4 is shown as follows:

$$
m_{f g}\left(h_{f g, 11}-h_{f g, 12}\right)=C_{p, w} m_{P H N}\left(T_{5}-T_{4}\right)
$$

To evaluate the exact performance of the novel heating process, Jenbacher engine JMS 620 was adopted. The technical data were obtained in a previous study as shown in Table 1. Moreover, the identical natural gas in Caf et al. (2016) was adopted for better comparison, and the enthalpy of flue 
gas was calculated using the same method the above study too. A double effect $\mathrm{LiBr}$ /water AHP was adopted in the simulation analysis.

Table 1. Technical data by the manufacturer of the cogeneration plant with a rated power of $3.3 \mathrm{MW}$ operating at $100 \%$ (Caf et al., 2016)

\begin{tabular}{lll}
\hline Natural gas LHV & 9.5 & $\mathrm{kWh} / \mathrm{Nm}^{3}$ \\
Energy input (LHV) & 7351 & $\mathrm{~kW}$ \\
Mechanical output & 3428 & $\mathrm{~kW}$ \\
Electrical output & 3349 & $\mathrm{~kW}$ \\
High Temperature cooling circuit: & & \\
$\quad$ Intercooler 1st stage & 883 & $\mathrm{~kW}$ \\
$\quad$ Lube oil & 290 & $\mathrm{~kW}$ \\
$\quad$ Jacket water & 463 & $\mathrm{~kW}$ \\
Exhaust gas cooled to $120^{\circ} \mathrm{C}$ & 1399 & $\mathrm{~kW}$ \\
Exhaust gas cooled to $25^{\circ} \mathrm{C}$ & 1100 & $\mathrm{~kW}$ \\
Intercooler 2nd stage & 197 & $\mathrm{~kW}$ \\
Surface heat dissipation & 202 & $\mathrm{~kW}$ \\
\hline
\end{tabular}

Table 2 shows the key point temperature value of the novel heat process at niminal design condition throuth simulation. The final exhaust flue gas temperature can achieve as low as $25^{\circ} \mathrm{C}$, which was $120^{\circ} \mathrm{C}$ since no latent heat can be recovered in conventianl system. Moreover, all intercooler $2 \mathrm{nd}$ stage heat, which was exhausted through cooling tower in conventional process, was recovered. Therefore, the novel heating process can recover $1297 \mathrm{~kW}$ more heat, and increases heat supply by $42.7 \%$ without additional energy input.

Table 2. Key point temperature value of the novel process at nominal conditon

\begin{tabular}{lll}
\hline Location & Fluid type & Temperature $\left[{ }^{\circ} \mathrm{C}\right]$ \\
\hline 1 & PHN water & 30 \\
2 & PHN water & 33 \\
3 & PHN water & 60 \\
4 & PHN water & 83 \\
5 & PHN water & 90 \\
6 & LTCC & 40 \\
7 & LTCC & 45 \\
8 & HTCC & 85 \\
9 & HTCC & 95 \\
10 & Flue gas & 365 \\
11 & Flue gas & 217 \\
12 & Flue gas & 120 \\
13 & Flue gas & 25 \\
14 & Circulation water & 20 \\
15 & Circulation water & 25 \\
\hline
\end{tabular}

For most of current gas engines cogeneration plant, hot water produced by cylinder water and flue gas are divided into two parts, one part of hot water supplies to users and the other part of hot water drives absorption chillers. In these cases, an optimized solution based on the novel heating process presented 
above is provided here: part of gas engines work at cooling condition and the part of gas engines work at heating condition.

\section{A NOVEL HEATING PROCESS FOR A STEAM TURBINE COGENERATION PLANT}

Since the temperature of PHN return water is low enough, the waste heat of exhausted steam from a steam turbine is recovered in two ways, which is shown in Fig.2. One is through circulating water between evaporator(s) in AHP and the condenser of the power plant, and the other is through preheating the PHN return water directly. PHN water firstly pre-heated by pland condenser and then flows into absorber and condenser in the AHP. If temperature of the PHN water out of the AHP is not high enough, then can be heated directly by extraction steam through a steam-water heat exchanger(SWHX).

The novel heating process presented above can be applied to various kinds of steam turbines (coal fired, gas fired, garbage burning, etc.). Especially, for a gas fired cogeneration plants, exhausted flue gas can be also deeply recovered as in gas engine.

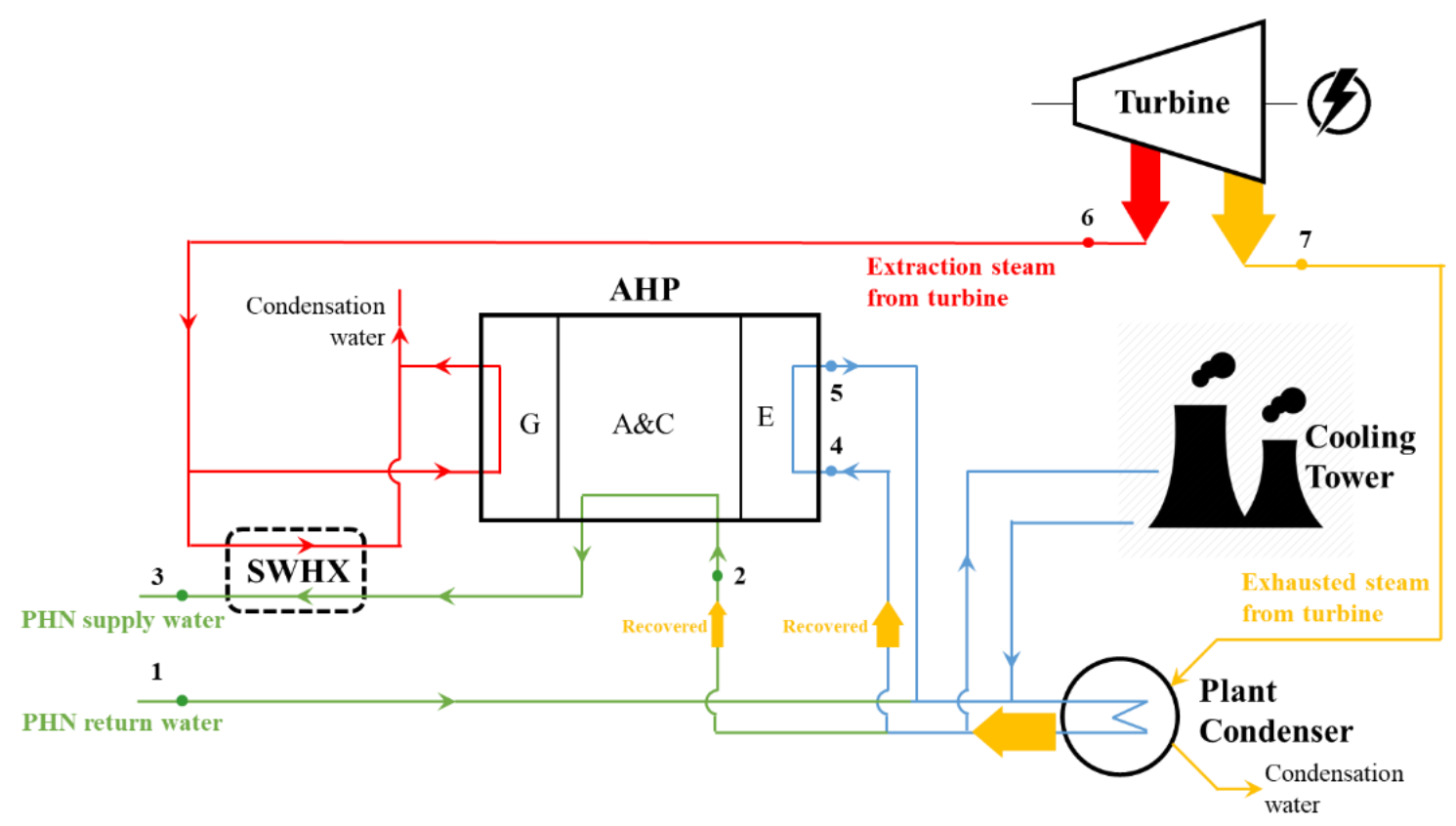

Fig. 2: A Novel Heating Process for a Steam Turbine Cogeneration Plant

When calculate the novel heating process at nominal condition, extraction steam ratio of the turbine $\alpha$ was given as $65 \%$, corresponding saturated temperature of extraction steam and exausted steam were given as $150^{\circ} \mathrm{C}$ and $45^{\circ} \mathrm{C}$ respectively. AHP is single lift single effect. In addtion, when variable lift AHP is applied, temperature level of extraction steam can be reduced(Hu et al., 2017). The simulation method was similar to our previous study(Hu et al., 2017). Other key point temperature values of the novel heating process at nominal conditon through simulation are shown as Table 3 . Through the simulation result, the SWHX is not needed at nominal condtion since the outlet water temperature of AHP can achieve $90^{\circ} \mathrm{C}$.

Table 3. Key point temperature value of the novel heating process at nominal condition

\begin{tabular}{lll}
\hline Location & Fluid type & Temperature $\left[{ }^{\circ} \mathrm{C}\right]$ \\
\hline 1 & PHN water & 30 \\
2 & PHN water & 40
\end{tabular}




\begin{tabular}{lll}
3 & PHN water & 90 \\
4 & Circulation water & 40 \\
5 & Circulation water & 30 \\
6 & $\begin{array}{l}\text { Extraction steam } \\
\text { (corresponding saturated temperature) }\end{array}$ & 150 \\
7 & $\begin{array}{l}\text { Exhausted steam } \\
\text { (corresponding saturated temperature) }\end{array}$ & 45 \\
\hline
\end{tabular}

From the simulation result. all latent heat in exhausted steam are recovered in nominal condition, which means thermal efficiency of the cogeneration plant achieved $100 \%$. Compared the conventional system, heat supply is increased by $73.1 \%$ by recovering the waste heat in exhauted steam.

\section{REFERENCES}

Caf, A., Urbancl, D., Trop, P., \& Goricanec, D. (2016). Exploitation of low-temperature energy sources from cogeneration gas engines. Energy, 108, 86-92. doi:10.1016/j.energy.2015.09.119

Çakir, U., Çomakli, K., \& Yüksel, F. (2012). The role of cogeneration systems in sustainability of energy. Energy Conversion and Management, 63, 196-202. doi:10.1016/j.enconman.2012.01.041

Fu, L., Jiang, Y., \& Zhang, S. (2008). District heating system based on Co-ah cycles in combined heating and power systems [J]. Journal of Tsinghua University (Science and Technology), 9, 001.

Ge, Z., Li, J., Liu, Q., Duan, Y., \& Yang, Z. (2018). Thermodynamic analysis of dual-loop organic Rankine cycle using zeotropic mixtures for internal combustion engine waste heat recovery. Energy Conversion and Management, 166, 201-214. doi:10.1016/j.enconman.2018.04.027

Hu, T., Xie, X., \& Jiang, Y. (2017). Simulation research on a variable-lift absorption cycle and its application in waste heat recovery of combined heat and power system. Energy, 140, 912921. doi:10.1016/j.energy.2017.09.002

Rattner, A. S., \& Garimella, S. (2011). Energy harvesting, reuse and upgrade to reduce primary energy usage in the USA. Energy, 36(10), 6172-6183. doi:10.1016/j.energy.2011.07.047

Seyedkavoosi, S., Javan, S., \& Kota, K. (2017). Exergy-based optimization of an organic Rankine cycle (ORC) for waste heat recovery from an internal combustion engine (ICE). Applied Thermal Engineering, 126, 447-457. doi:10.1016/j.applthermaleng.2017.07.124

Sun, F., Fu, L., Sun, J., \& Zhang, S. (2014). A new waste heat district heating system with combined heat and power (CHP) based on ejector heat exchangers and absorption heat pumps. Energy, 69, 516-524. doi:10.1016/j.energy.2014.03.044

Xie, X., \& Jiang, Y. (2017). Absorption heat exchangers for long-distance heat transportation. Energy, 141, 2242-2250. doi:10.1016/j.energy.2017.11.145

Zhu, C., Xie, X., \& Jiang, Y. (2016). A multi-section vertical absorption heat exchanger for district heating systems. International Journal of Refrigeration, 71, 69-84. doi:10.1016/j.jirefrig.2016.08.010

\section{CONCLUSION}

A novel district heating solution based on absorption heat exchanger(AHE) for different types of cogeneration plants is the subject of this paper. Two most common cogeneration plant types, gas engine and steam turbine, are simulated and analyzed in this paper. The main conclusions can be summarized as follows:

1) In the novel $\mathrm{DH}$ heating system based on $\mathrm{AHE}$, primary water return $\mathrm{T}$ can be reduced to $30^{\circ} \mathrm{C}$ without any change in terminal users side

2) Primary water return $\mathrm{T}$ can be maintain at $30^{\circ} \mathrm{C}$ by increase primary water supply $\mathrm{T}$ when secondary side $\mathrm{T}$ level is higher

3) Heat transportation cost (pumps power consumption) can be reduced since the temperature difference is expanded 
4) Low return temperature make it possible to recover low grade heat $\left(30 \sim 50^{\circ} \mathrm{C}\right)$ in cogeneration plants and then expand heat supply capacity and decrease the energy consumption. For gas engine cogeneration plant, heat supply can be increased by $42.7 \%$ without any additional energy consumption.For steam turbine cogeneration plant, heat supply can be increased by $73.1 \%$ without any additional energy consumption.

\begin{tabular}{|llll|}
\hline \multicolumn{2}{|l|}{ Nomenclature } & $\alpha$ & extraction steam ratio \\
AHP & absorption heat pump & \multicolumn{2}{l|}{ Subscripts } \\
PHN & primary heating network & & \\
SHN & Secondary heating network & A & absorber \\
C & specific heat at constant pressure, $\mathrm{J} /\left(\mathrm{kg} \cdot{ }^{\circ} \mathrm{C}\right)$ & $\mathrm{C}$ & condenser \\
Q & heat flux, $\mathrm{J} / \mathrm{s}$ & $\mathrm{E}$ & evaporator \\
$h$ & specific enthalpy, $\mathrm{J} / \mathrm{kg}$ & $\mathrm{HG}$ & High pressure generator \\
$m$ & mass flow rate, $\mathrm{kg} / \mathrm{s}$ & $\mathrm{LG}$ & Low pressure generator \\
$\mathrm{T}$ & temperature, ${ }^{\circ} \mathrm{C}$ & $\mathrm{W}$ & water \\
& & $\mathrm{fg}$ & Flue gas \\
Greek symbols & & \\
\hline
\end{tabular}

\section{ACKNOWLEDGMENTS}

The authors gratefully acknowledge the support from the National Natural Science Foundation of China (grant numbers 51306098, 51138005), the innovative Research Groups of National Natural Science Foundation of China (grant number 51521005), and Tsinghua University Initiative Scientific Research Program (No. 20151080470). 


\title{
SOLARTHERMAL ENERGY INTEGRATION ON A POWER PLANT SITE
}

\author{
Dr.-Ing. Sebastian Schramm, Andreas Thieme \\ GREENoneTEC Solarindustrie GmbH \\ Industriepark St. Veit, Energieplatz 1, A-9300 St. Veit/Glan \\ Phone: +4366488955611 \\ E-Mail: sebastian.schramm@greenonetec.com
}

\section{INTRODUCTION}

The solar system assembled by GREENoneTEC Solarindustrie GmbH, which is presented in this paper, stands out for extremely high system yields and for an impressive building implementation on a 68 meters high roof in the urbanized area of the Austrian capital city of Vienna. Speaking about solar district heating, typically moderate specific system yields with minimised installation costs - resulting from optimised system integration solutions and driven by scale factor - are the project's parameter. One of the main problems usually is the area availability in urbanized regions. As an example, Figure 29 shows the solar district heating plant nearby the downtown of Chemnitz with $2.230 \mathrm{~m}^{2}$ collectors split in two collector fields on a former car park.

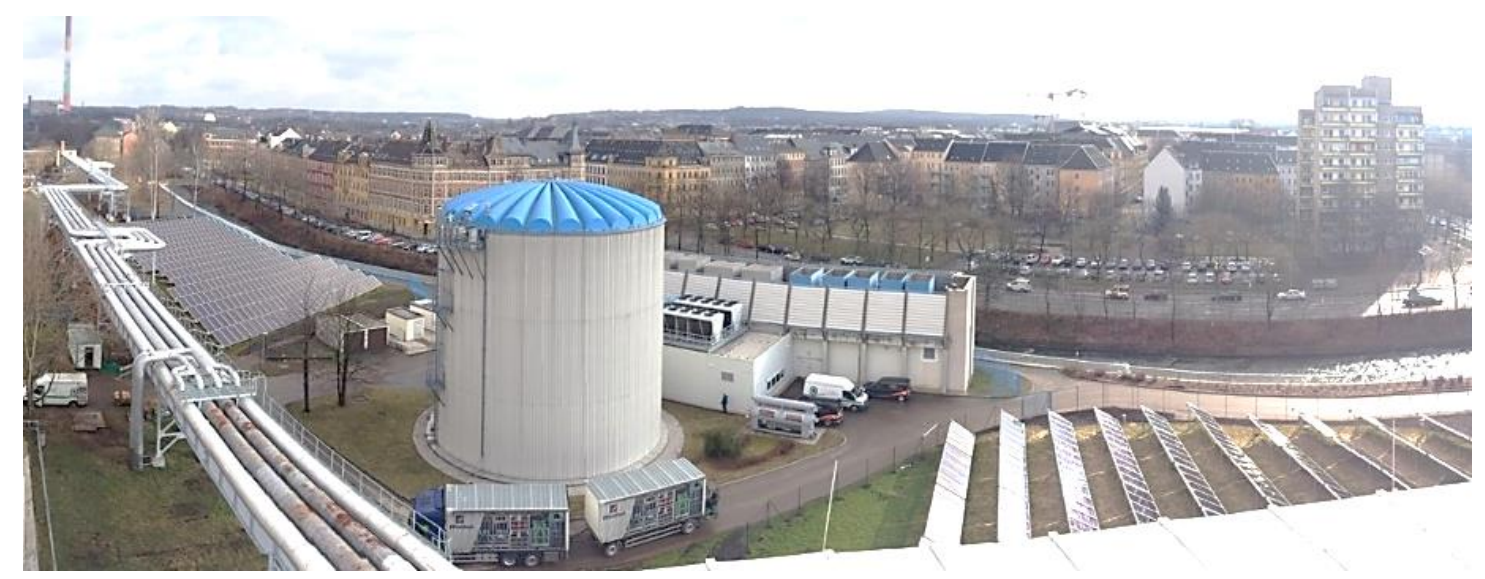

Figure 29: Solar district heating nearby downtown of Chemnitz (Germany)

The other way around works as well, as proven by this project: extremely high solar yields make the enormous effort of the collector mounting on the rooftop of a boiler house (Figure 14) economically feasible. Wien Energie made the decision of investing in the $656 \mathrm{~m}^{2}$ solar thermal installation at the power plant site Wien Simmering for a number or reasons, of which the important one was the solar heating price.

First measurement results, presented in this paper, prove the reliability and fulfil expectations of the solar thermal energy output. 


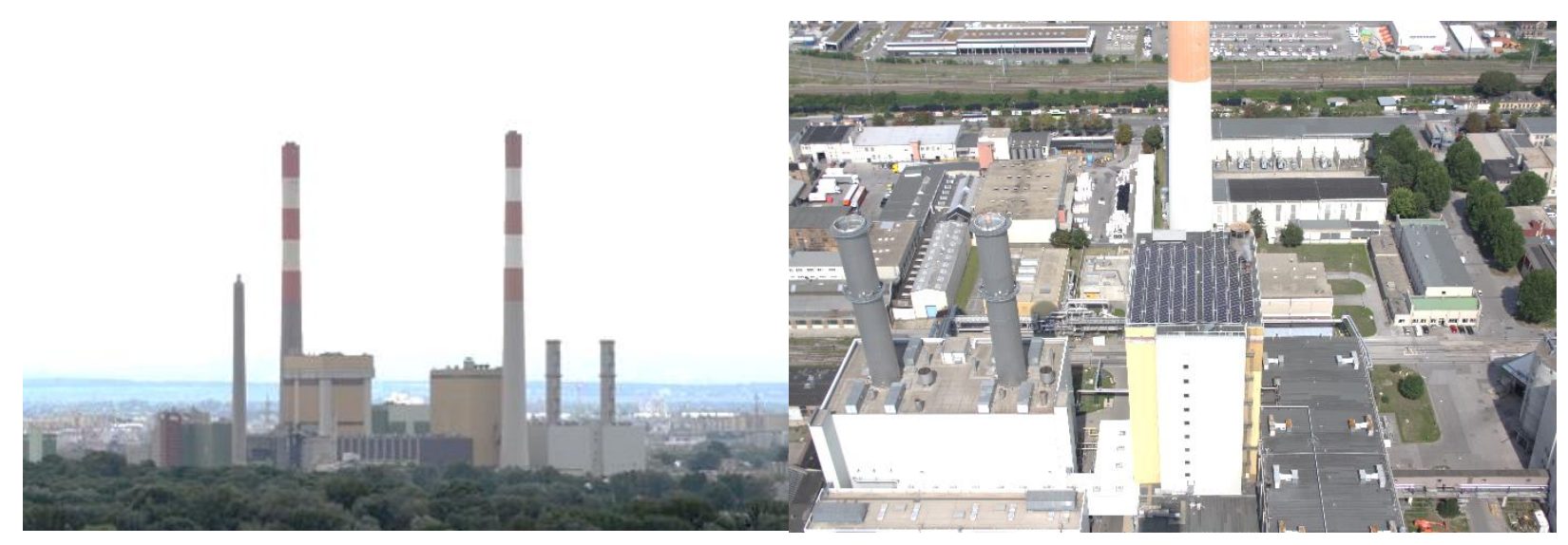

Figure 14: Collector field is on the top of the right boiler house (left)

Areal view of the collector field (right)

\section{PROJECT DESCRIPTION}

Placing a collector field at a $68 \mathrm{~m}$ high rooftop with the feed-in point in the basement (spot height $5 \mathrm{~m}$ ) is complicated and expensive - and therefore usually not the first choice - but in this case with no other available area it is the key to success. This is feasible because the temperature level and load profile of the heat sink is optimal in this situation and make enormous solar output possible. The expected specific system yield is at nearly $780 \mathrm{kWh} / \mathrm{m}^{2} \mathrm{a}-$ a maximum value, at least for Austria. The heat sink is the preheating of make-up water for a district heating network.

The challenge in this project is the load-bearing supporting construction and the weather resistance in regards to the strong winds that occur recurrently in Vienna. The static of the steelwork and the collectors has to withstand a wind speed of up to $240 \mathrm{~km} / \mathrm{h}$ in consideration of security surcharge. Another unique attribute is the distance of more than 70 meters between collectors and integration point - vertically. The static pressure, which works on the solar station, amounts to seven bar, in addition to the operating pressure of the collector field. GREENoneTEC built the solar system in July 2018.

\section{SYSTEM CONCEPT}

Figure 31 shows the simplified layout of the solar system with the primary loop on the left side, and the secondary loop right on the side of the heat exchanger. 


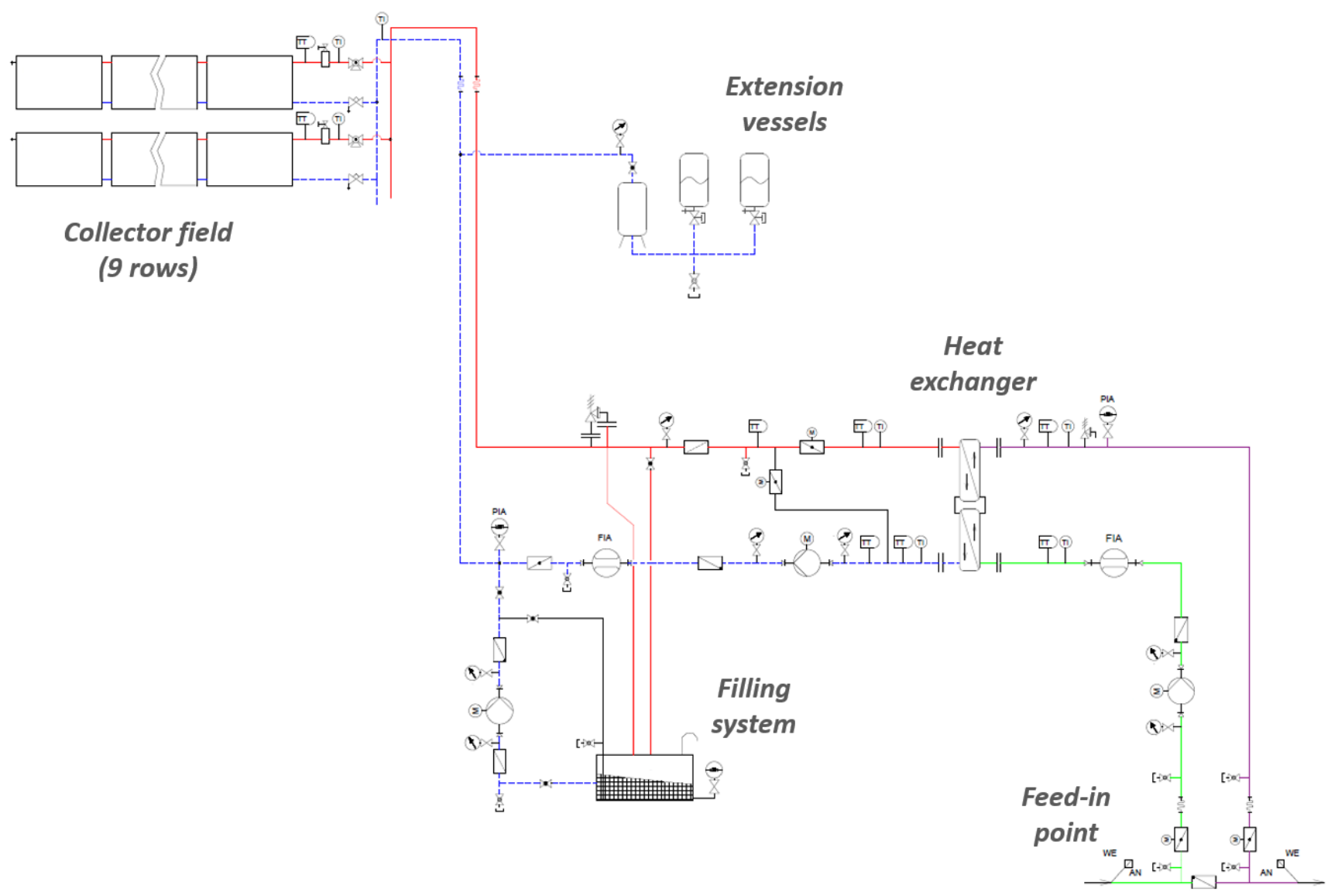

Figure 31: Layout plan

The collector field of $656 \mathrm{~m}^{2}$ single-glazed flat plate collectors (53x GK3003) consists of nine collector rows in total. Figure 32 shows the positioning of the collectors. Four to seven collectors installed per row. They are connected in parallel. The feed and return pipe are on the left side and hydraulically balanced. In the front and left corner of the main piping goes down to the basement nearby the solar station.

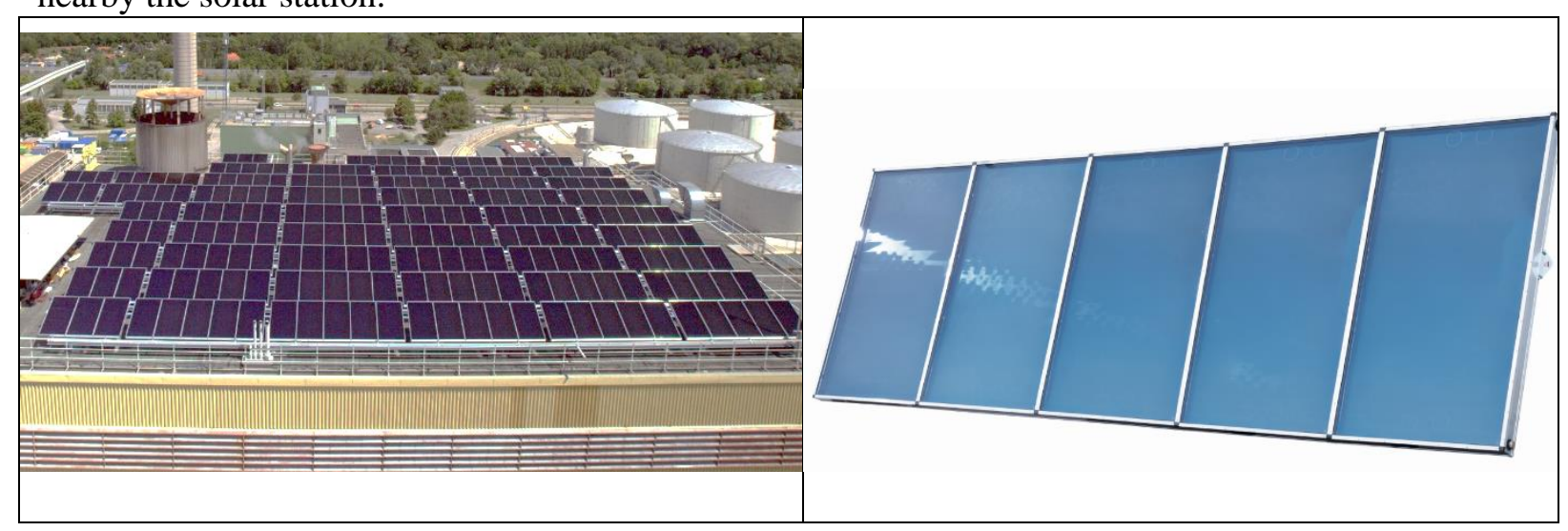

Figure 32: Collector field (left)

Large-scale high efficient collector GK 3003 (right)

This collector type is ideal for large-scale solar systems like those that the one presented in this paper. It ensures optimal stagnation and venting behavior thanks to the serpentine absorber tube and minimal 
external piping. Furthermore, GREENoneTEC's collector family GK3003 resists in conformity to test methods for assessing the durability, reliability and safety for fluid heating collectors (EN ISO 9806:2013) a pressure load of $3 \mathrm{kN} / \mathrm{m}^{2}$, what is necessary for this installation due to the wind conditions in Vienna.

The major advantage for the system implementation is that heat requirement exists almost continuously. Therefore, no storage considered in the plant design. The extension vessels are located just under the rooftop instead of nearby the solar station, because of the difference in altitude of more than 70 meters between collectors and solar station in the cellar. In this position no additional static pressure of seven bar (correspond to 70 meters altitude) works on the extension system, what would be the case were it located in the cellar. Due to this, a significantly smaller dimensioning of the tanks and cost saving were possible.

Demineralized water, which is used in the CHP system at the plant site Simmering, is partially taken out of the supply line with temperatures of about $20^{\circ} \mathrm{C}$ and after the solar pre-heating it is lead back again into the supply line a short way further.

Primary and secondary pumps are speed-controlled as a function of the collector field outlet temperature respectively inlet temperature. Therefore, the collector temperature is adjustable and condensation caused by low collector temperatures does not occur.

\section{MEASUREMENT RESULTS}

Figure 17 (left) shows the daily specific system output of the solar plant. The system output is equal to the supplied energy measured in the secondary loop. The corresponding allocation to the global radiations prove the reliable energy supply of the solar system in different operating states.

The right side of Figure 17 illustrates the efficiency of the solar system during the evaluated period: within a global radiation of more than $3 \mathrm{kWh} / \mathrm{m}^{2}$ day, the efficiency is about $70 \%$.

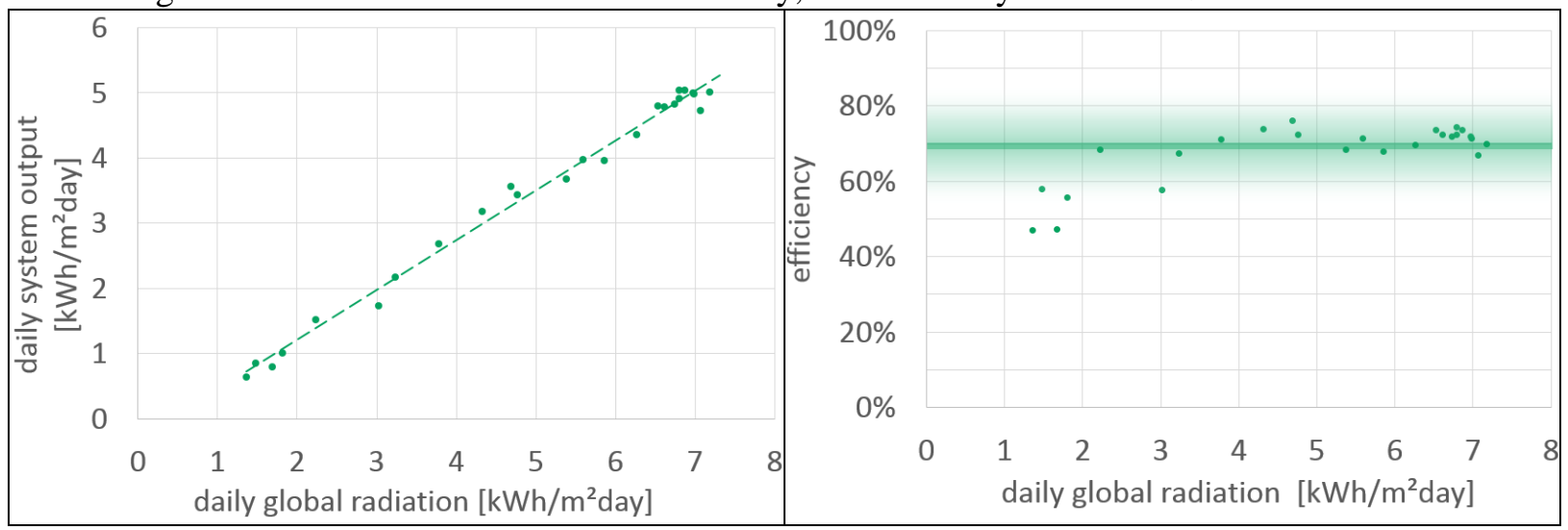

Figure 17: Daily system output; specific (left), efficiency (right)

The method by Nielsen (IEA SHC 45 task) for calculating a guarantee for the power output of large collector fields defines a performance evidence. The following equation (1) - applicable with hourly average values with solar radiation of more than $800 \mathrm{~W} / \mathrm{m}^{2}$ - gives this theoretical performance value:

$P_{g}=A_{c}\left[\eta_{0} G-a_{1}\left(T_{m}-T_{m}\right)-a_{2}\left(T_{m}-T_{m}\right)^{2}\right] \cdot f_{p} \cdot f_{u} \cdot f_{o}$

$\begin{array}{cl}P_{g}: & \text { Guranteed performance (thermal power output) } \\ A_{c}: & \text { Collector area } \\ \eta_{0}: & \text { Optical efficiency } \\ a_{1}: & 1^{\text {st }} \text { order heat loss coefficient }\end{array}$


$a_{2}: \quad 2^{\text {nd }}$ order heat loss coefficient

G: $\quad$ Solar irradiance on collector plane

$T_{a}$ : ambient air temperature

$T_{m}: \quad$ mean collector fluid temperature

$f_{p}: \quad$ Saftey factor taking into account heat losses from pipes etc.

$f_{u}$ : $\quad$ Saftey factor taking into account measurement uncertainty

$f_{p}: \quad \quad \quad$ Saftey factor taking for other uncertainties e.g. related to non-ideal conditions such as non-ideal flow distribution and unforeseen heat losses

This approach applied for the calculation of the theoretical energy supply by the solar system (system output). Therefore a safety factor $f_{p}=0,97$ is taken into account while the safety factors $f_{u}$ and $f_{p}$ remain 0,98 . The factor $f_{p}$ in this case represents the heat losses from external piping in the collector field as well as the connection pipes from the rooftop to the solar station in the cellar and the heat losses from heat exchanger and other installations. The solar system is fully meeting the expectations as is shown in Figure 34.

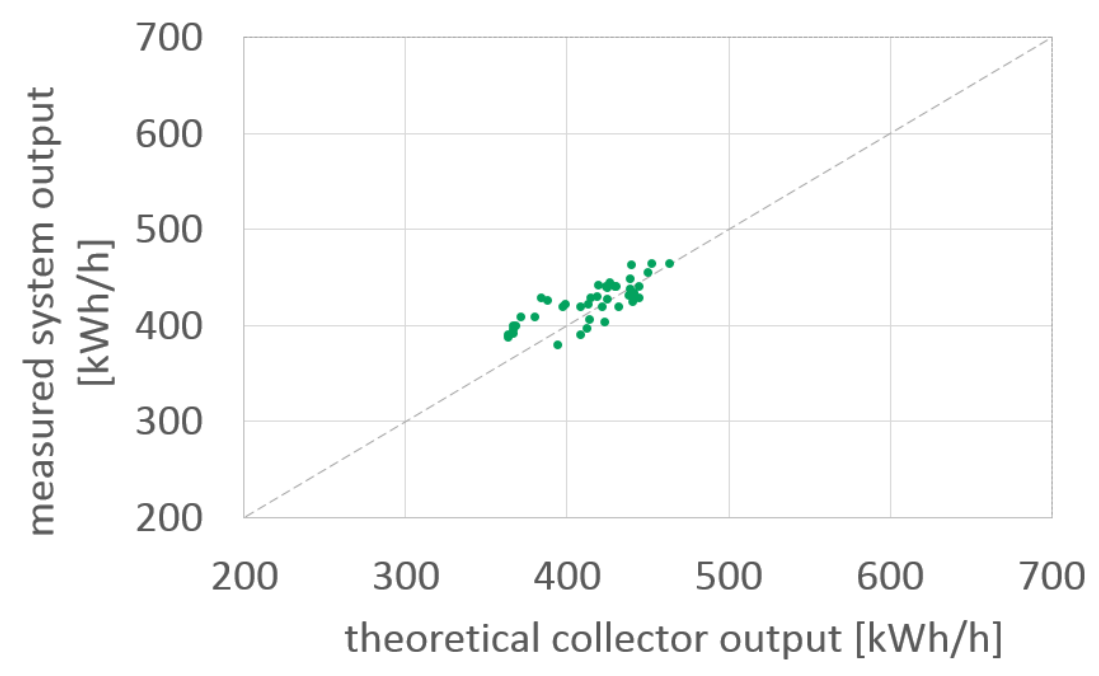

Figure 34: Theoretical and measured system output

Figure 35 represents two main aspects. On the one hand, it is evident that because of small temperature differences between collector and ambient air the collector efficiency curve comes into effect with a small proportion of thermal losses. Two days are shown - a hot summer day with air temperatures (grey dots, Figure 35 ) of about $30^{\circ} \mathrm{C}$ which leads to difference to the collector temperature (x-axis) of less than $10 \mathrm{~K}$ (19.08.) and a summer day with $20^{\circ} \mathrm{C}$ and temperature difference up to $20 \mathrm{~K}$ (27.08.). On these days the calculation results in a theoretical efficiency of about $79 \%$ respectively $75 \%$ (black dots) in consideration the safety factors as stated above. On the other hand, Figure 35 shows (similar to Figure 34) the satisfying system performance (green dots) in accordance to the theoretical derived expectations. 


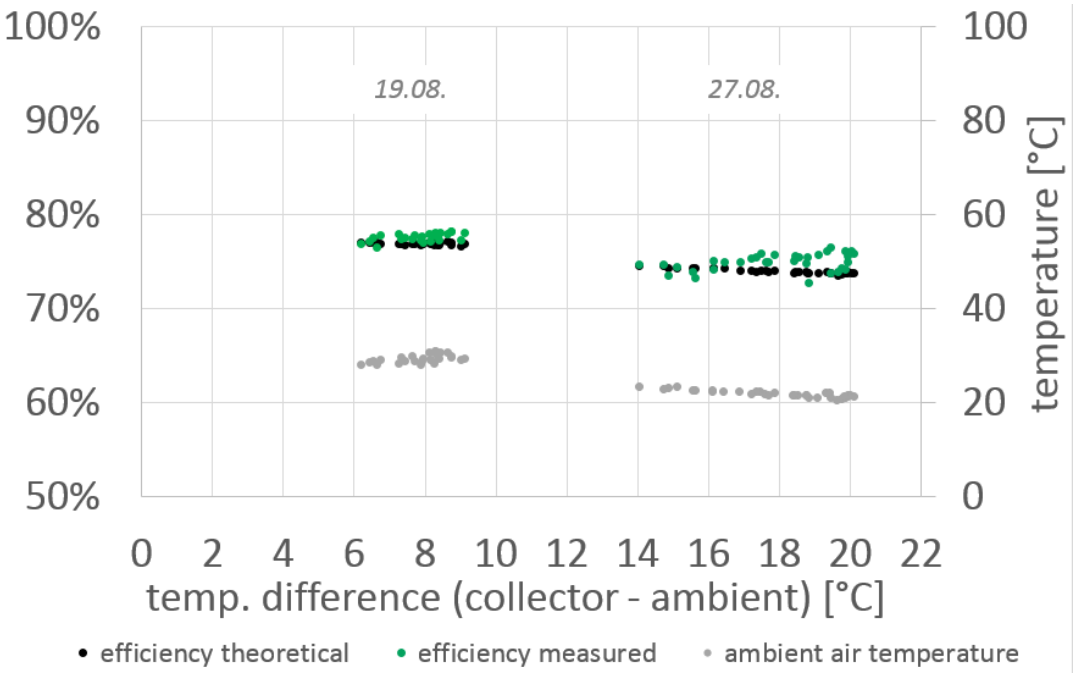

Figure 35: Theoretical and measured efficiency

\section{ENERGY COSTS}

The project costs are not comparable to usual solar systems because of the enormous effort regarding the mounting. The steelwork makes up $35 \%$ of the investment costs and contains the substructure of the collector field and the connection to the existing structures (Figure 36). This is the biggest part even before the collector field (including the lift on the roof and installation) with far less than one third of the total costs.

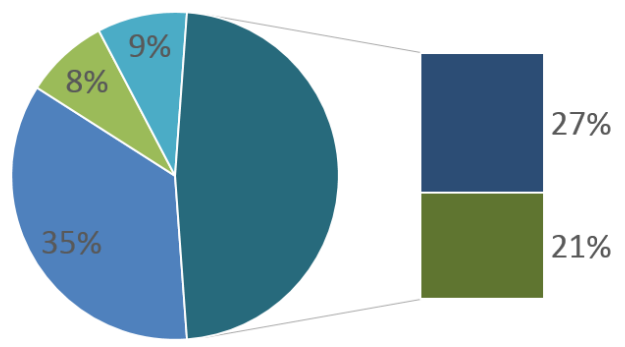

\footnotetext{
- steelwork, substructure control system

angineering collectors

ather installations
}

Figure 36: cost allocation

The solar heating costs are $42 € / M W h$ without funding and $24 € / M W h$ with funding, assuming an annuity loan and maintenance costs divided by the predicted annual solar output. Assumptions listed in Table 10.

Table 10: calculation assumptions

\begin{tabular}{|l|l|}
\hline investment costs & $600 € / \mathrm{m}^{2}$ \\
\hline calculation period & $25 \mathrm{a}$ \\
\hline interest rate & $2 \%$ \\
\hline maintenance costs & $1.200 € / \mathrm{a}$ \\
\hline system output & $510 \mathrm{MWh} / \mathrm{a}$ \\
\hline funding rate & $45 \%$ \\
\hline
\end{tabular}




\section{PROJECT PARTNER}

GREENoneTEC Solarindustrie GmbH is the world's largest manufacturer of thermal flat-platecollectors with an annual production capacity of more than 1.6 million $\mathrm{m}^{2}$ of panels. Eight highly automated, process-secured robotic production lines offer high quality products with excellent delivery performance and reliability - made in Austria, of course, and certified to ISO 9001 and ISO 14001 standards. The company looks back on 25 successful years of experience in the solar market. In addition, GREENoneTEC cooperates with local partners in order to offer solar thermal projects on EPC basis as well as heat contracting solutions around the globe and has been the general contractor of the project presented here.

Wien Energie, a subsidiary of Wiener Stadtwerke, is Austria's largest regional energy service provider supplying energy to over one million private customers. More than two million people, approximately 230,000 businesses, industrial facilities and public buildings, and about 4,500 farms in Vienna, Lower Austria and Burgenland supplied with electricity, natural gas and heat. 


\title{
PRESSURE REDUCTION IN HYDRAULIC SYSTEMS
}

\author{
$\underline{\text { Dr Tobias Sommer }}{ }^{1}$, Stefan Mennel ${ }^{1}$, Prof. Matthias Sulzer ${ }^{1}$ \\ Institut für Gebäudetechnik und Energie ${ }^{1}$ \\ Lucerne University of Applied Sciences and Arts \\ Technikumstrasse 21, 6048 Horw, Germany \\ Phone: +41413493847 \\ E-Mail: tobias.sommer@hslu.ch
}

\section{SUMMARY}

Piping costs are a major fraction of the initial investments for district heating and cooling networks. To reduce the maximum pressure in such networks, we present a novel technique based on alternating the connection of the expansion vessel. A pressure reduction is possible, when the pressure difference between the connection points is reversing during operation. The magnitude of the pressure reduction depends mainly on the pressure difference between the connection points of the expansion vessel.

\section{INTRODUCTION}

Piping costs are a major fraction of the initial investments for district heating and cooling networks and limit the dissemination of such networks. In this work, we present a new method for reducing the maximum pressure in such systems. Reducing the maximum pressure decreases the wall thickness for piping and thus decreases investments.

We focus on district heating and cooling networks of extremely low temperature below $20{ }^{\circ} \mathrm{C}$. Such systems are nowadays widely spread in Switzerland. At such low temperatures, direct cooling (without a chiller) is possible and low temperature environmental heat (lake water, ground water, geothermal sources) or waste heat from industrial processes serve as energy sources and/or seasonal storages. For heating purposes, heat pumps are required to raise the temperature level.

Low temperature heating and cooling networks typically consist of a warm line (with e.g. $10^{\circ} \mathrm{C}$ ) and a cold line (with e.g. $6^{\circ} \mathrm{C}$ ). All clients are equipped with their own circulation pumps and draw water from one of the two lines, depending on their heating or cooling demand. Seasonal storages (typically passive without circulation pumps) close the mass and energy balance. Distributed circulation pumps cause complex hydraulic dynamics that are difficult to predict and may result in cavitation at the circulation pumps. Cavitation occurs when the suction side pressures of the circulation pumps are below the evaporation pressure. To avoid cavitation, the current solution is to increase the system pressures by increasing the pressure of the expansion vessel. Large pressures require thicker wall thicknesses und thus result in costly piping.

In this work we use experiments carried out at the NODES ("New Opportunities for Decentralised Energy Systems")-Lab (Fig. 1) to present a method to decrease the pressure in low temperature networks by alternating the connection of the expansion vessel. 


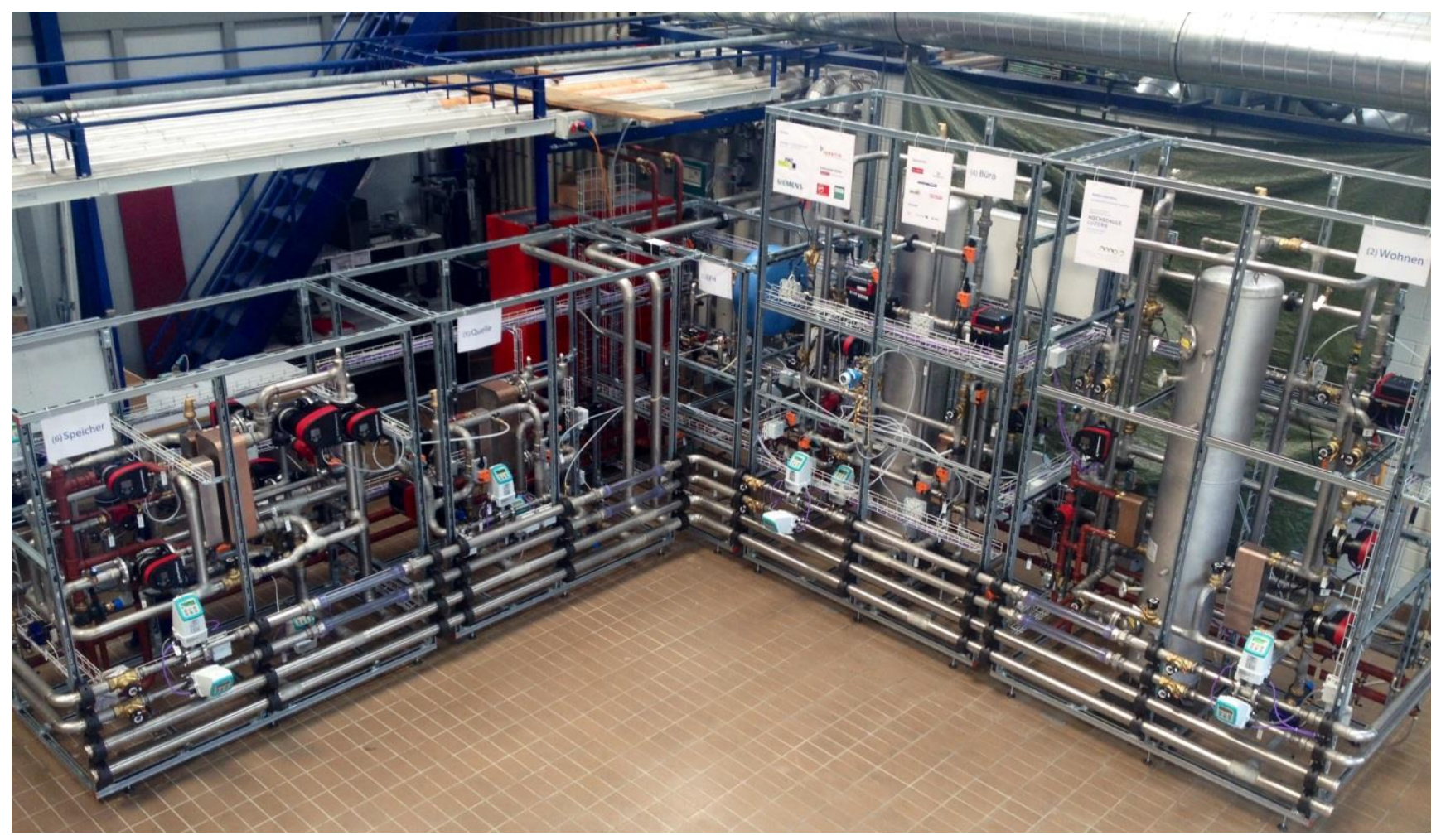

Fig. 1: NODES Lab, a district heating and cooling network at laboratoy scale.

\section{METHODS}

In conventional hydraulic systems, the expansion vessel is fixed to a single point. The primary goal of the expansion vessel is to absorb volume variations of the fluid caused by temperature variations. Because the expansion vessel also sets the system pressure at the connection point, the expansion vessel is typically connected at the suction side of the circulation pump to guarantee cavitation free operation. Cavitation occurs, when the pressure at the suction side of circulation pumps falls below evaporation pressure. If several circulation pumps operate in one system, a single position of the expansion vessel is not sufficient anymore to ensure cavitation free operation. Ideally, the connection point of the expansion vessel should switch between the suction sides of circulation pumps, always being connected to the point of lowest pressure. Because in low temperature networks, the pressure loss in the warm and cold line is small compared to the pressure losses in the clients, two connection points, one in the warm and one in the cold line, are sufficient.

\section{RESULTS}

If the expansion vessel is fixed to the warm line, independent of heating and cooling demand, the set pressure of the expansion vessel must be large enough to avoid cavitation, even if most clients have cooling demand and thus extract water from the cold line. The pressure of the expansion vessel is thus given by the minimum pressure to avoid cavitation plus the pressure difference between the warm and cold line. Our method switches the expansion vessel between the warm and cold line and is always connected to the line of lower pressure. If most clients have cooling demand, the expansion vessel is connected to the cold line and the pressure of the expansion vessel is thus given by the minimum pressure to avoid cavitation free operation. The system pressure can thus be reduced by the pressure difference between the warm and the cold line, without risking cavitation at all operational states. 


\section{REFERENCES}

[1] Lund H, Werner S, Wiltshire R, Svendsen S, Thorsen JE, Hvelplund F, et al. 4th Generation District Heating (4GDH). Integrating smart thermal grids into future sustainable energy systems. Energy 2014;68:1-11. doi:10.1016/j.energy.2014.02.089.

\section{CONCLUSION}

A simple method was introduced to reduce the pressure in district heating and cooling networks by switching the connection point of the expansion vessel. A pressure reduction is possible, when the pressure difference between the connection points is reversing during operation. The magnitude of the pressure reduction depends mainly on the pressure difference between the connection points of the expansion vessel. For low temperature networks, in particular with a large pressure difference between the warm and the cold line, a variable connection of the expansion vessel can significantly reduce piping costs, and thus initial investment. 


\section{DECARBONISATION OF THE SPACE HEATING AND HOT WATER SECTOR: PATHWAYS, CHALLENGES AND REQUIREMENTS FOR SECTOR COUPLING}

Lukas Kranzl, Andreas Müller, Michael Hartner Sebastian Forthuber, Gerhard Totschnig

Energy Economics Group, Institute of Energy Systems and Electrical Drives, Technische Universität Wien

Gusshausstrasse 25/370-3, 1040 Vienna, Austria

Phone: +43158801370351

E-Mail: lukas.kranzl@tuwien.ac.at

\section{SUMMARY}

Although the Paris COP 21 agreement calls for a strong and fast decarbonisation of the space heating and cooling sector, only a limited number of scenarios towards an (almost) complete decarbonisation is available. In particular, the role of sector coupling in such scenarios is not yet fully captured. In our paper, we deal with the question, how such feasible pathways towards decarbonisation can be characterized and what are policy implications, in particular regarding sector coupling and use of flexibility potentials. We will develop energy demand and supply scenarios as well as corresponding heat and electricity loads for the space heating, cooling and hot water sector based on a bottom-up building stock model. These results are fed into integrated energy system models to show the impact of sector coupling. We will discuss that the required policy package needs to include also strong regulatory measures as well as targeted policies for implementing effective sector coupling.

Keywords: decarbonisation, renewable heating and cooling, sector coupling, building refurbishment

\section{INTRODUCTION}

In previous publications (Kranzl et al., 2017), the authors showed that only a few studies so far have developed scenarios leading to (nearly) full decarbonisation of the heating / cooling sector. To overcome this gap we deal with the question: What are implications of strong decarbonisation scenarios (compared to e.g. 75\% decarbonisation)? Moreover, since the heating and cooling sector has to be understood as an integrated element of the whole energy system we draw particular attention to the question: What is the role of sector-coupling and which role can the building sector play in terms of flexibility options in strong decarbonisation scenarios?

\section{METHOD}

We build our analysis on the disaggreaged building stock model Invert/EE-Lab (see e.g. www.invert.at, (Müller, 2015) which has been applied in numerous projects and for several countries and EU-28 to develop policy driven scenarios of heating and cooling energy demand and supply. The model recently has been coupled with a series of supply side focused energy system models (Hartner et al., 2018)). In this paper, we focus on results of the model link to the energy system model HiREPS (Totschnig et al., 2017).

We will apply the models for selected EU countries and discuss detailed implications for a sub-set of them (in particular Germany and Austria). In particular, we discuss the role of flexibility options and the relevance of sector coupling by comparing model runs with and without sector coupling. In particular, we will discuss the implications of strong decarbonisation scenarios (in the range of -95\%) compared to moderate climate mitigation scenarios (based on literature and scenarios developed by the authors in previous projects, e.g. ...)

\section{RESULTS}

Figure 1 shows a feasible pathway for achieving a full decarbonisation of the space heating and hot water sector until the year 2050 for the example of Austria as a model result of Invert/EE-Lab. The long version of the paper will explain the required technology mix and assumed policy measures. Results from different considered countries will be compared: Building renovation, demolition and efficient new building construction lead to a reduction of final energy demand in the range of about $50 \%$ in all countries. However, the current state of heating systems has a strong impact on future energy carrier mix as well. While a phase out of oil and coal may be realisable with a mix of corresponding regulatory and economic measures in all considered countries until around 2040-2050, the complete phase out of natural gas would require much stronger regulatory changes, which seem hard to be implemented. A share of about $25 \%$ up to more than $50 \%$ of the heat demand (differing 
between countries) would be covered by heat pumps, decentral and in district heating. The resulting electricity load depend on the question how strong current electric resistance heaters can be replaced, whether renovation measures take up and last but not least how effectively heat pumps are integrated in the building stock. The results will show that under the assumed side conditions, the resulting electricity loads can be covered by the system. And we illustrate that using flexibility potentials in buildings is economically effective.

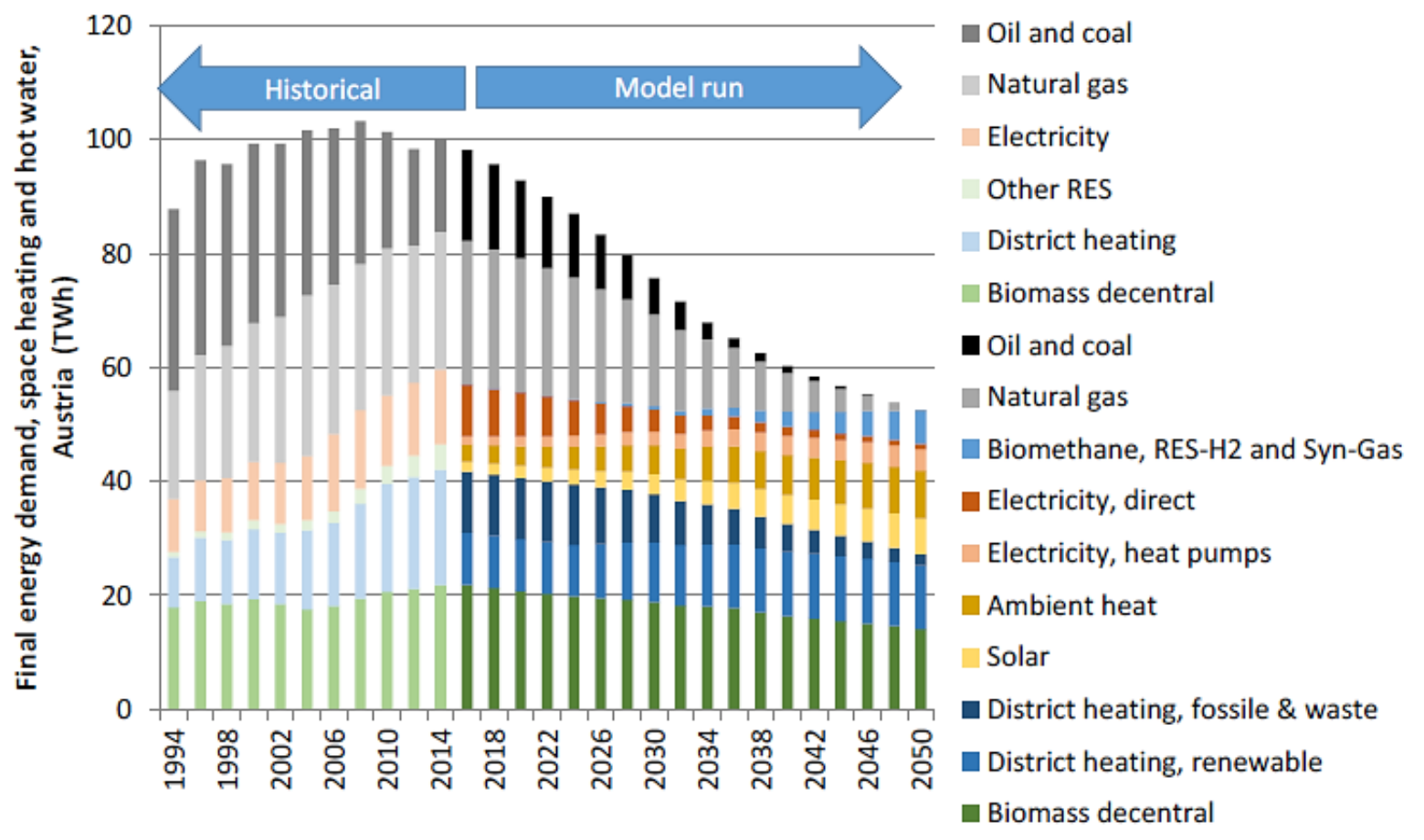

Fig. 1: Final energy demand for space heating and hot water in a strong decarbonisation scenario, Austria

\section{DISCUSSION AND CONCLUSIONS}

Even though we present feasible pathways towards decarbonisation of the space heating, hot water and cooling sector for various countries and the role of sector-coupling and decarbonisation, we emphasize that the results strongly depend on the economic and policy framework. We will discuss policy implications, in particular regarding the need for an ambitious policy package including regulatory measures like RES-H obligations and individual building renovation roadmaps with a mandatory component combined with a CO2-tax, subsidies, quality improvement and strategic heating and cooling approaches. Also sector coupling will require clear policy guidelines, e.g. regarding the mandatory installation of control devices and eventually thermal storage.

\section{REFERENCES}

Hartner, M., Forthuber, S., Kranzl, L., Fritz, S., Müller, A., Bernath, C., Sensfuss, F., MaranonLedesma, H., 2018. Summary report on case study: Energy demand and supply in buildings and the role for RES market integration, A report compiled within the H2020 project SET-Nav.

Kranzl, L., Aichinger, E., Forthuber, S., Hartner, M., Müller, A., Toleikyte, A., 2017. To what extent do "ambitious" scenarios of energy demand in the building stock reflect COP21 Paris targets? Presented at the eceee Summerstudy 2017, Hyeres, France.

Müller, A., 2015. Energy Demand Assessment for Space Conditioning and Domestic Hot Water: A Case Study for the Austrian Building Stock (PhD-Thesis). Technische Universität Wien, Wien.

Totschnig, G., Büchele, R., Fritz, S., Kranzl, L., Müller, A., Nagler, J., Ponweiser, K., Baumgartner, W., Postl, J., Adler, J., Brandmayr, J., Blarke, M.B., 2017. Potentiale, Wirtschaftlichkeit und Systemlösungen für Power-to-Heat, Berichte aus Energie- und Umweltforschung. Wien. 


\title{
THE POTENTIAL OF SMALL WIND TURBINE INTEGRATION IN RESIDENTIAL BUILDINGS COMPLEMENTING PV AND HEAT PUMP OPERATION
}

\author{
Marcus Brennenstuhl, Daniel Lust, Pirmin Boch, Malcolm Yadack, Ursula Eicker \\ Stuttgart University of Applied Sciences \\ Schellingstr.24 \\ 70174 Stuttgart, Germany \\ Phone: +49 71189262647 \\ E-Mail: marcus.brennenstuhl@hft-stuttgart.de
}

\begin{abstract}
Today the application of PV systems in the German residential building sector is wide spread. In contrast, small wind turbines are not much used for various reasons, despite offering a seasonal advantage when compared to PV for power-to-heat applications. At the same time, power-to-heat with a focus on efficient heat pumps (HP) is recognized as an important potential for offering demand response (DR) to counteract the fluctuations of renewable energy generation. Little research has been carried out to date for hybrid micro grids based on small wind turbines, PV and power-to-heat applications. This work investigates the combined operation of a heat pump system with thermal buffer storages and parallel electricity generation by PV and small wind turbines including a battery storage as well as an innovative seasonal chemical storage system in a residential building. Detailed white-box-simulation-models, already validated on real existing systems, are used to identify optimal strategies regarding self-consumption and grid infeed.
\end{abstract}

\section{INTRODUCTION}

Wind and PV are the most important and widest spread systems for renewable electricity generation. Independent of the system size, both are normally installed in separated contexts and systems both technically and in terms of locality. Little focus has been given to local system integration across PV and wind systems. Cost-saving potential exists, however, for small systems for example when the fixed costs of installation can be combined (Wagh \& Walke, 2017). In Germany the residential sector shows a growing share of heat pumps (Agora Energiewende, 2017) that is predicted to further increase in future. Thus, the combination of PV produced electricity and heat pump systems enables an increasing potential regarding self-consumption. Small wind turbines are nationally not supported by law (Schmelmer, Ramona; Denk, 2015) although they would offer a better seasonal electricity supply for heating applications. Concerning the combination of PV, small wind turbines and electricity storage (batteries, chemical storage) the research so far addresses the optimization of hybrid generation (Ahmed, Miyatake, \& Al-Othman, 2008) as well as optimized micro grid operations (Niknam, Golestaneh, \& Shafiei, 2013)(Mohammadi, Soleymani, \& Mozafari, 2014). Sector coupling, using power-to-heat applications, is rarely considered for this specific system combination. (Sichilalu, Tazvingza, \& Xia, 2016) examine a system with grid tied PV, wind and fuel cell together with heat pump operation in South Africa, but exclude the buildings dynamic thermal behaviour. In (Kaabeche, Belhamel, \& Ibtiouen, 2011) an optimization model for an integrated PV, wind and battery hybrid system is developed in Algeria integrating power-to-heat via electrical load profiles. (Arabali, Ghofrani, Etezadi-Amoli, Fadali, \& Baghzouz, 2013) analyse a heating, ventilation and air conditioning (HVAC) System combined with PV and wind generation as well as electricity storage. Here system sizing and smart load shifting strategies are optimized utilizing a genetic algorithm, also excluding thermal storages and thermal building dynamics. It shows that most of the studies do not consider the buildings dynamic behaviour that is relevant regarding overheating, undercooling and the resulting rebound effect. In addition, no conclusions are made for the German climatic and economic conditions, especially for the combined operation of PV and small wind turbines under those settings.

The work presented here addresses this specific issue. It is based on a plus energy district, which serves as pilot demonstration site in the German community of Wüstenrot. This district consists of 16 newly built, highly energy efficient residential buildings. The concept combines low depth geothermal systems, heat pumps and PV systems of sufficient size of $6 \mathrm{kWp}$ to $10 \mathrm{kWp}$ for each building. The low temperature energy source of the heat pumps is a central cold-water grid, which consists of a large innovative near surface "agrothermal" system (a variation of geothermal collectors). Six of the buildings are additionally equipped with battery storage systems and are monitored in detail. To this end, a cloud based monitoring and control system is implemented. For one of the buildings, a detailed white box computer model was developed and validated, including the building itself and the energy 
conversation systems consisting of heat pump thermal storage, battery system, PV system and wind turbine. Based on that model and on measured meteorological data (global radiation, ambient temperature, wind speed) the complementarity of parallel operation of hybrid electricity generation by PV and the wind turbine is investigated. The focus is on optimized operation regarding selfconsumption, self-sufficiency and grind infeed.

\section{The Case Study Building}

The building considered has a living area of $317 \mathrm{~m}^{2}$, a PV system with $13.7 \mathrm{kWp}$, a brine/water heat pump with $20 \mathrm{~kW}$ thermal power and approximately $4 \mathrm{~kW}$ electrical demand, two buffer storage tanks (1000 1 space heating, $3001 \mathrm{DHW}$ ) and a $5 \mathrm{kWh}$ battery storage, which is used for the optimization of PV self-consumption (see also Tab. 1). It is situated in the rural municipality of Wüstenrot in southwest Germany. Detailed monitoring data is available for all relevant thermal and electrical energy flows as well as for the relevant temperatures and weather data (ambient temperature, global radiation). Based on this building a detailed white box simulation model that is described below is created and validated. Wind speed and global radiation measurements are taken from a nearby weather station. The wind speed is measured in $2.5 \mathrm{~m}$ height. It is converted to the actual wind turbine height of $6.3 \mathrm{~m}$ according to (Kleemann \& Meliß, 1993).

Tab. 1: Considered building energy conversion systems

\begin{tabular}{|l|l|}
\hline System & Manufacturer / Type \\
\hline Heat Pump & Waterkotte Modell DS 5023.5Ai, $22.2 \mathrm{~kW}$ \\
\hline Thermal Buffer Storage & DHW 400l; Heating $1000 \mathrm{I}$ \\
\hline Battery Storage & Liacon; $5 \mathrm{kWh} ; 1 \mathrm{C}$ \\
\hline PV Orientation & $58.4 \mathrm{~m}^{2}\left(48\right.$ modules) orientation $180^{\circ}$, tilt $15^{\circ} ; 49.5 \mathrm{~m}^{2}$ (40 modules) orientation $0^{\circ}$, tilt $15^{\circ}$ \\
\hline PV Manufacturer and Model & Solar Frontier Typ SF155-L; $13,64 \mathrm{kWp}$ \\
\hline Wind Turbine Manufacturer and Model & Aeolos-V; $5 \mathrm{~kW}$ \\
\hline
\end{tabular}

\section{Energy Storage Options}

To increase the overall energy self-consumption an additional storage system is examined. As batteries are limited in their energy density, other options are considered for a seasonal energy storage. Powerto-gas (PtG) or Power-to-liquid (PtL) technologies seem to be a promising option. PtG means that a substance is converted to a substrate with a higher energy potential (in general a lower oxidation number) via electrical power. This process is called electrolysis. If the resulting substrate is present in a liquid state, the process is called Power-to-Liquid. The term PtG covers the electrolysis of water to hydrogen and oxygen as well as the further processing, for example the methanation of hydrogen and carbon dioxide (Sabatier process):

$4 \mathrm{H}_{2}+\mathrm{CO}_{2} \rightarrow \mathrm{CH}_{4}+2 \mathrm{H}_{2} \mathrm{O}(1)$

PtL results in liquid energy carriers with higher energy density as gases, for example synthetic longchain hydrocarbons (gasoline or kerosene) which could be formed via a Fischer-Tropsch process with renewable produced hydrogen. If the produced hydrogen from the electrolysis is not used directly it must be stored. State-of-the-art for hydrogen storage is the pressurization of the hydrogen gas up to 700 bar for mobile applications. Nevertheless, hydrogen pressurization comes along with losses. High pressure hydrogen storage systems are well described in literature and are not further regarded in this study. Another approach is to store hydrogen in chemical bonds. One suitable substance is formic acid. Although FA has a low hydrogen percentage of its overall mass (4.4\%), its non-toxicity and environmental harmlessness (Eppinger \& Huang, 2017) makes FA interesting as a hydrogen carrier. Its liquid character under ambient conditions makes FA easy to use with a good long-term stability, making it predestinated for seasonal storage.

FA could be formed catalytically from carbon dioxide and hydrogen (hydrogenation of carbon dioxide) under moderate reaction conditions (Hsu et al., 2014) with a ruthenium-based catalyst:

$\mathrm{CO}_{2}+\mathrm{H}_{2} \rightarrow \mathrm{HCOOH}(2)$ 
Offering a high variety for applications, FA could be used directly in so called Formic Acid Fuel Cells, which are currently subjects of research projects. On the other hand, hydrogen could be released from FA (dehydrogenation of FA) and used in conventional fuel cells:

$\mathrm{HCOOH} \rightarrow \mathrm{CO}_{2}+\mathrm{H}_{2}(3)$

For a utilization of the released hydrogen in PEM Fuel cells, it is important to prevent the dehydration of FA (reaction (4)) as carbon monoxide harms the cell membrane:

$\mathrm{HCOOH} \rightarrow \mathrm{H}_{2} \mathrm{O}+\mathrm{CO}(4)$

Instead of the direct usage, the hydrogen produced in the electrolyzing process could also be further processed, e.g. with the already mentioned methanation process. The production of methane has the advantage that it could be stored in the already existing gas grid. Also, methane could be used versatile e.g. in gas combustion-engines which leads to a good linking of the energy sectors electrical power, heat and mobility.

\section{METHODOLOGY}

\section{Building Model Description}

All systems and the thermal behaviour of the building are dynamically modelled in the simulation environment INSEL (www.insel.eu). The following Fig. 1 gives an overview over all components, their interaction and the data flow. All individual parts are described further below.

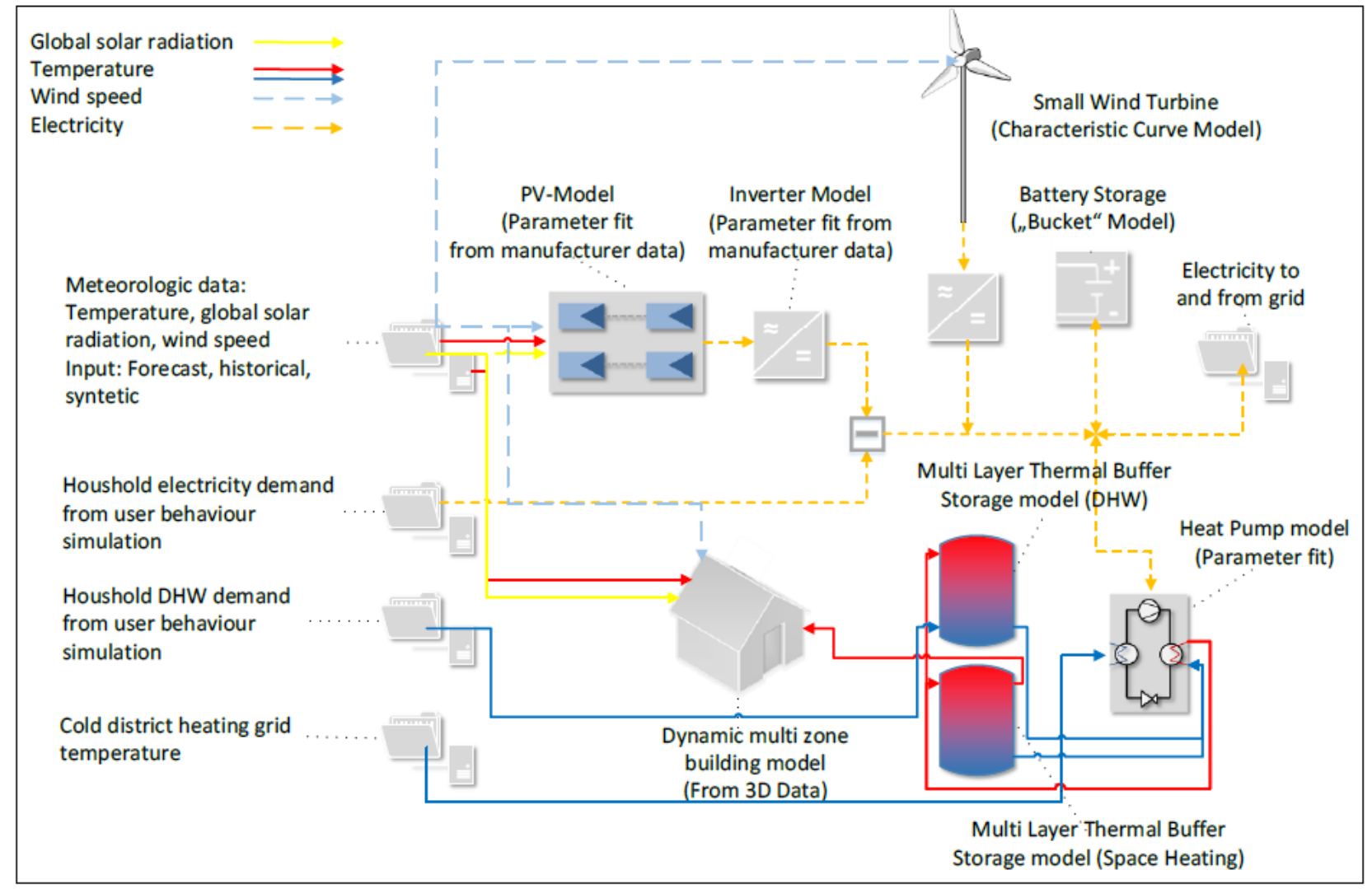

Fig. 1: Integrated white box INSEL model combining a dynamic building model and energy conversion systems

\subsubsection{Dynamic Building Model}

The utilized model provides a high level of detail while allowing for simplification at the data model level. It is based on the nodal method. This method is common for building simulations, especially when simulating multiple zone buildings. This approach also enables a good balance between level of detail in results and in calculation time. The model contains a one dimensional numerical solution of 
the heat conduction equation for each wall. Additional nodes contain room air and windows. Long wave radiative exchange between the room surfaces is also considered. Model inputs are external boundary conditions such as temperature and irradiance, which is converted to the different orientations.

\section{Heat Pump}

The applied model simulates the compressor's behaviour based on a polynomial function (according to norm DIN EN 12900). The heat exchangers on the evaporator and condenser side are resembled with the NTU method (Number of Transferred Units). This simplifies the calculation process because no complex calculations due to complicated streamlined shapes have to be carried out.

\section{Thermal Buffer Storages}

Within the thermal buffer storage model, each layer is modelled physically. Heat exchange in between the layers is taken into account. The heat is always fed into the appropriate layer. The thermal losses are credited to the suitable building zone.

\section{PV Modules and Inverter}

The modules are represented by a two diode model that is suitable for mono- and polycrystalline SI as well as for CIS modules. The parameters are created via a parameter fit according to module characteristic curves. The inverter is also represented through parameter fit by characteristic curves provided by the manufacturer. The PV modules are linked to a MPP (Maximum Power Point Tracking) loop.

\section{Battery Storage}

The battery storage model is kept simple. It does not simulate the electrochemical process of the battery. Charging and discharging efficiency and power, inverter efficiency and self-discharge are considered. The aging effect is considered dependent on number of cycles (linear aging coefficient).

\section{Wind Turbine}

The wind turbine is resembled by a characteristic curves model based on data provided by the manufacturer.

\section{Experimental Validation}

A validation was carried out with measured data collected from the real existing building on which the previously described model is based on. The monitoring data was collected from the time in between 18.02.2018 and 24.03.2018. In this period the simulated thermal heat pump energy output was 3998 $\mathrm{kWh}$ and the measured was $3595 \mathrm{kWh}$. In parallel the simulated heat pump electrical energy demand was $1034 \mathrm{kWh}$ and the measured was $1022 \mathrm{kWh} / \mathrm{d}$. The difference in thermal demand can be related to the variation of the heat pump COP according to varying set point temperatures. The comparison of the measured and simulated electrical demand indicates that the model is adequate for what is relevant for this study (see also Fig. 2). Fig. 3 shows that the heat pumps operation intervals also fit well. The correct operation times are hard to predict due to user behaviour. Fig. 4 presents a comparison of the measured and simulated building room temperature. It indicates an acceptable match, which is relevant for examinations on thermal building mass as storage. 


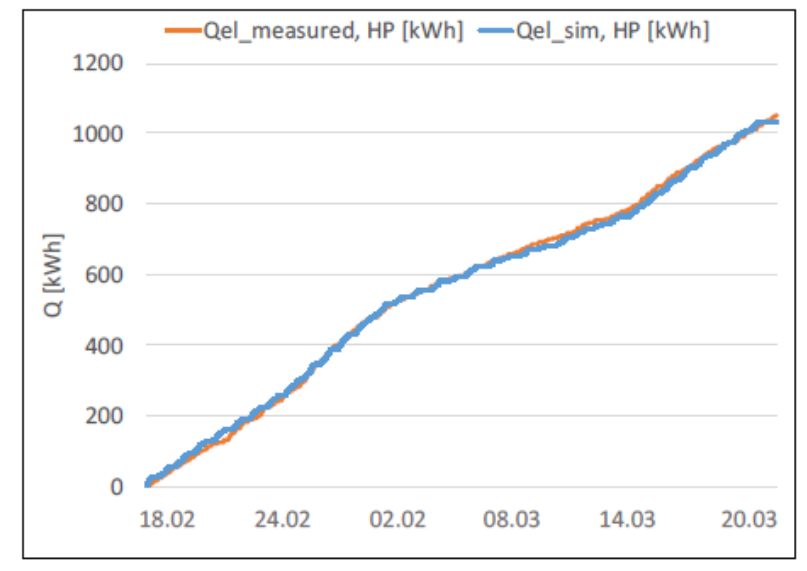

Fig. 2: Comparison of measured and simulated heating energy

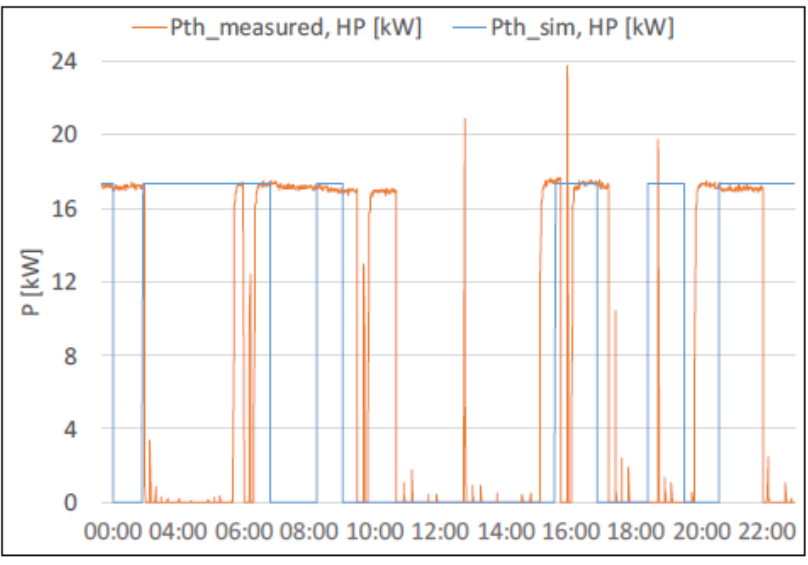

Fig. 3: Comparison of measured and simulated electrical power demand of the heat pump|

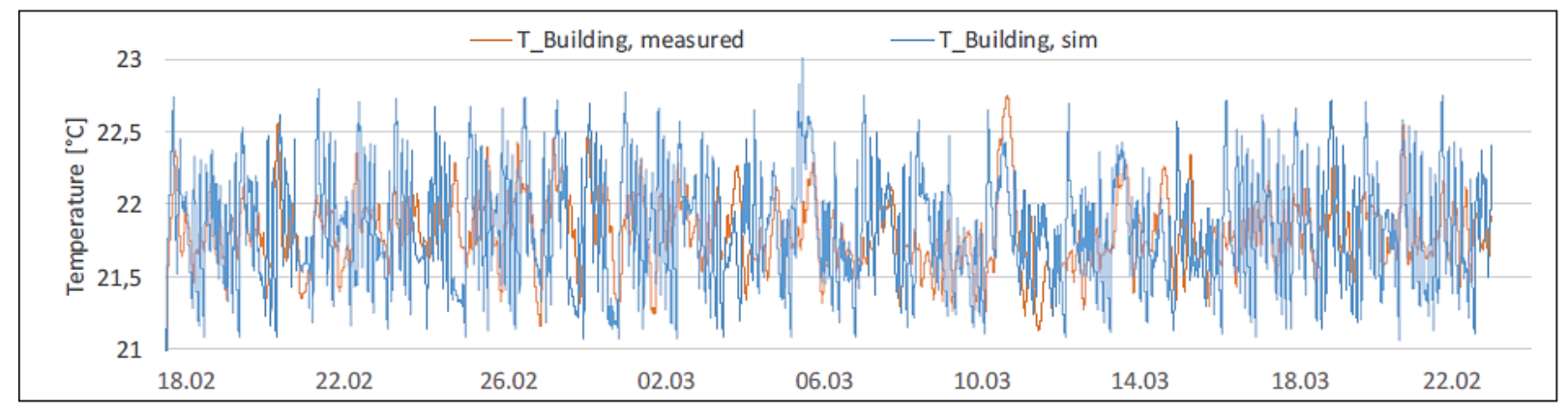

Fig. 4: Comparison of measured and simulated indoor room temperature

\section{RESULTS}

\section{Complementary Effect of PV and Wind Energy Generation}

The system self-consumption and autarky was examined for different battery sizes $(5 \mathrm{kWh}, 10 \mathrm{kWh}$ and $20 \mathrm{kWh}$ ) for solely PV electricity generation and for electricity generation from PV and a small 5 $\mathrm{kW}$ wind turbine. The rule is, that wind electricity is used first due to the fact, that the feed in tariff in Germany for July 2018 is 0.071 EUR/kWh for small wind turbines and 0.123 EUR/kWh for PV systems between $10 \mathrm{kWp}$ and $40 \mathrm{kWp}$ (Bundesnetzagentur, 2018). The detailed results can be found in Tab. 2. It can be seen, that the combined operation of the PV system and wind turbine has little impact on the PV system specific self-consumption ratio. The ratio drops from $36 \%$ to $31 \%$ with a wind system specific self-consumption ratio of $83 \%$ for a $5 \mathrm{kWh}$ battery storage, from $45 \%$ to $39 \%$ with a wind system specific self-consumption ratio of $87 \%$ for a $10 \mathrm{kWh}$ battery and from $53 \%$ to $46 \%$ with a wind system specific self-consumption ratio of $90 \%$ for a $20 \mathrm{kWh}$ battery. With that system combination up to $65 \%$ of building autarky can be reached including heating and household electricity demand. Fig. 5 clarifies this result. As drawback in the worst case ( $5 \mathrm{kWh}$ battery storage), the number of battery cycles would increase by $20 \%$ and thereby the battery life would decrease by the corresponding amount. Especially during the winter heating period, the wind turbine increases the electricity production, so that almost the entire heating demand can be provided by the wind and PV yield. The figure also indicates that there is a large excess production during the summer months mainly due to the seasonal differences in PV generation. Thus to reduce grid infeed stress theoretical electrical seasonal storage possibilities are examined. Thereby for full self-consumption $6120 \mathrm{kWh}$ and for full autarky $4330 \mathrm{kWh}$ of electricity have to be stored. 
Tab. 2: Simulation results with varying storage sizes

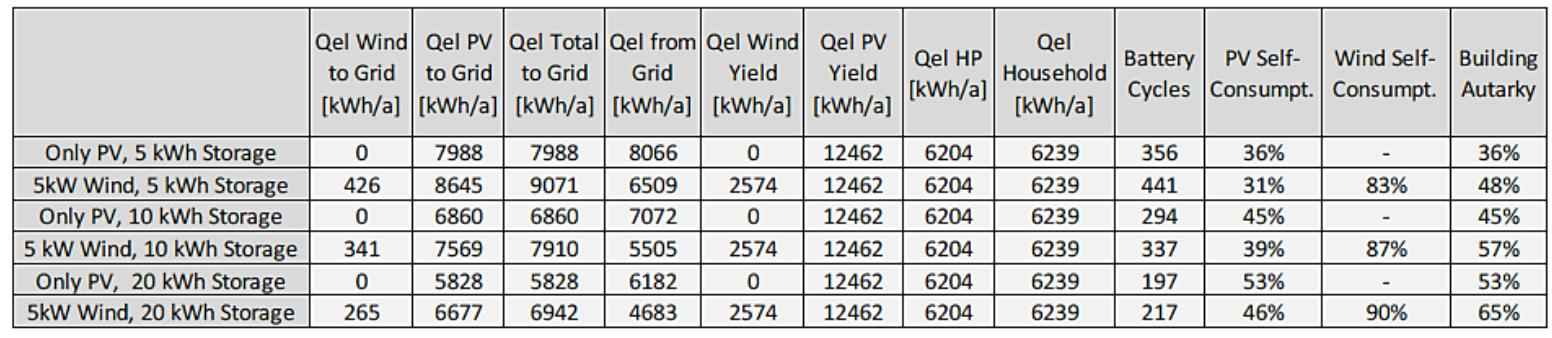

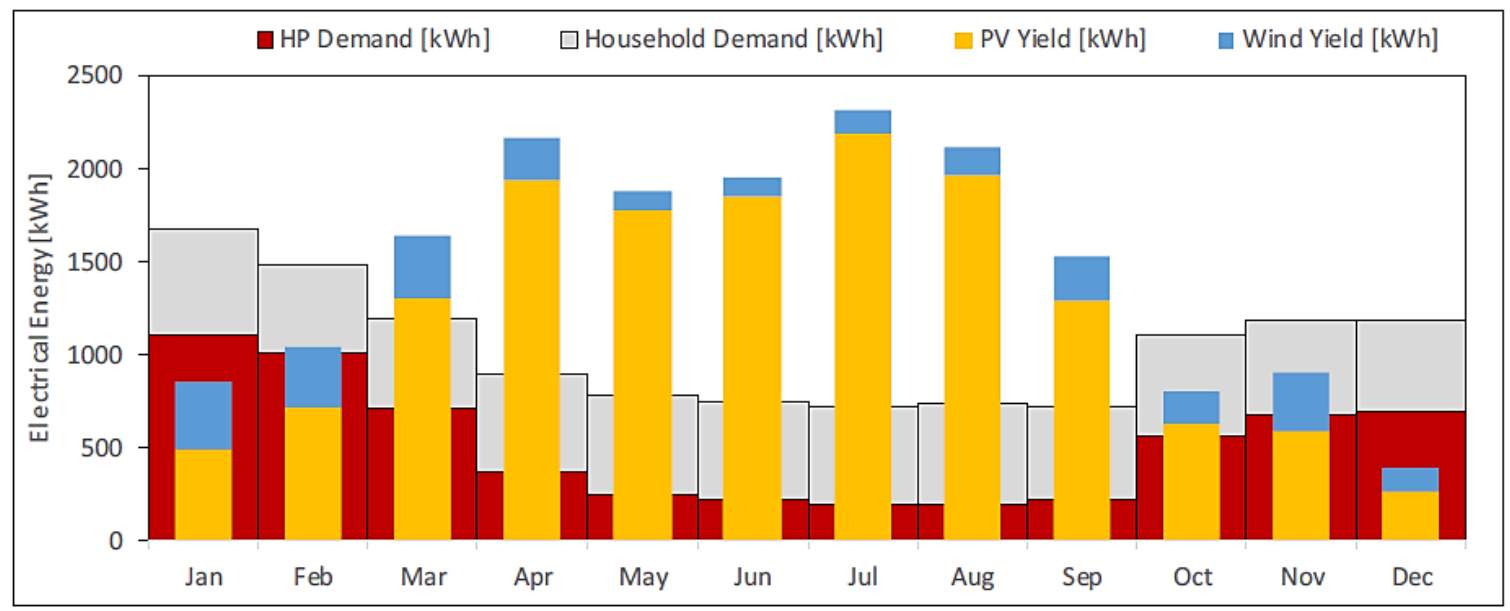

Fig. 5: Monthly wind and PV yield compared with heat pump and households electricity demand

\section{Chemical Seasonal Storage}

A combined storage system based on PtG and PtL technology is regarded. Therefore the electrical excess energy is assigned to an electrolyzer unit, where in a first step hydrogen is produced. Then boundary conditions (system size, amount of needed chemicals and needed energy supply) are determined. A full self-consumption of electrical energy could be achieved if the produced substance could be transformed back to electrical energy. $6120 \mathrm{kWh}$ of electrical energy has to be stored per year for the investigated building with a maximal power input of $15 \mathrm{~kW}$. Based on these numbers a chemical energy storage system with back-electrification consisting of a PEM-Electrolyzer, a FA hydrogen storage system and a PEM-Fuel cell is designed subsequently.

\section{PEM-Electrolyzer stack design}

To design the electrolyzer unit for a power input of $15 \mathrm{~kW}$, a current density of $1 \mathrm{~A} / \mathrm{cm}^{2}$ at an effective cell voltage of $1.7 \mathrm{~V}$ is required, which according to (Carmo, Fritz, Mergel, \& Stolten, 2013) lies in the middle of the performance range of currently published PEM polarization curves. A cell size of $10 \times 10 \mathrm{~cm}$, hence $100 \mathrm{~cm}^{2}$ cell area is assumed. This leads to a cell power of:

$P_{\text {cell }}=U_{\text {cell }} * i_{\text {cell }} *$ Acell $=1.7 \mathrm{~V} * 1 \frac{\mathrm{A}}{\mathrm{cm} 2} * 100 \mathrm{~cm}^{2}=170 \mathrm{~W}(5)$

To meet the specification of $15 \mathrm{~kW}$ input power, a stack of 89 cells is needed. The hydrogen production rate is calculated as follows:

$\dot{m} H 2=\frac{N * I}{z F} * 2.016 \frac{\mathrm{g}}{\mathrm{mol}} * \frac{1}{1000} * 3600=0.3347 \frac{\mathrm{kg}}{\mathrm{h}}(6)$

With $\mathrm{N}$ being the number of cells [-], I being the current [A], $\mathrm{z}$ being the number of transferred electrons [2], and F being the Faraday constant of $96485 \mathrm{C} / \mathrm{mol}$. With the lower heating value of hydrogen $(119.96 \mathrm{MJ} / \mathrm{kg}$ ), this means that $11.15 \mathrm{kWh}$ per hour could be stored as hydrogen with the above sized PEM-Electrolyzer. 


\section{PEM Fuel Cell design}

Just like the elecetrolyzer unit, the fuel cell is designed for a power of $15 \mathrm{~kW}$. For a good balance between performance and durability an average current density of $0.5 \mathrm{~A} / \mathrm{cm}^{2}$ with an effective cell area of $100 \mathrm{~cm}^{2}$ is assumed. This results in an operating current of $50 \mathrm{~A}$.

To keep the number of cells per stack at a reasonable amount, the fuel cell is designed with three stacks connected in parallel, thus increasing the overall current to $150 \mathrm{~A}$. The fuel stack voltage results from:

$U F C=\frac{P F C}{\mathrm{IFC}}=\frac{15000 \mathrm{~W}}{150 \mathrm{~A}}=100 \mathrm{~V}(7)$

Calculated with an average cell voltage of $0.7 \mathrm{~V}$ (Spiegel, 2008), 143 cells connected in series are needed per stack for a voltage output of $100 \mathrm{~V}$. To connect the fuel cell to the electrical grid and match the grid frequency and voltage, DC/DC boost converters and DC/AC inverters are needed.

\section{Hydrogen Storage}

According to (Eppinger \& Huang, 2017) the amount of needed catalyst for the dehydrogenation of FA could be estimated if the hydrogen consume rate $r F C$ of the fuel cell is known:

$n_{\text {cat }}=\frac{r F C * R F}{\text { TOFcat }}(8)$

With RF being the reserve factor [-] and TOF being the turnover frequency [s-1].

The hydrogen consumption rate $r F C$ for the above sized fuel calculates as follows:

$r F C=3 * \frac{143 * 50 \mathrm{~A}}{\mathrm{zF}}=0.11 \mathrm{~mol} \mathrm{~s}^{-1}(9)$

With a TOF of about 1800 for a Ru-based catalyst (Hsu et al.) and a RF of 2, the amount of needed catalyst results to:

$n_{\text {cat }}=\frac{0.11 \mathrm{~mol} \mathrm{~s}-1 * 2}{1800 \mathrm{~s}-1}=1.22 * 10^{-4} \mathrm{~mol}(10)$

If FA is considered as a seasonal storage, the overall needed volume of FA to store a specific amount of energy is calculated as follows:

$V F A=\frac{E}{\omega F C * \eta R C}(11)$

With the volumetric energy density of $\omega F A=1.77 \mathrm{kWh} l-1$ for hydrogen chemically bounded in FA (Eppinger \& Huang, 2017) and the efficiency of the reconversion of FA to electrical power $\eta R C$ The reconversion consists of the dehydrogenation of FA as well as the fuel cell process. For this study the efficiency of the fuel cell is considered to be $50 \%$ and the efficiency of the dehydrogenation $90 \%$. Experimental studies have to be carried out to confirm those values.

$\eta R C=\eta F A \rightarrow H 2 * \eta_{F C}=0,9 * 0.5=0.45$

Hence for full self-consumption (6120 kWh) 76831 and for full autarky (4330 kWh) 54361 of FA are needed.

\section{CONCLUSION}

A system of a German low energy residential building with heat pump, PV, small wind turbine and battery storage was studied for varying battery storage sizes based on validated white box simulation models and real measured data regarding ambient temperature, global radiation and wind speed. The results show that the additional wind turbine has a low impact on PV self-consumption with an average reduction of $16 \%$. Furthermore, up to $46 \%$ of PV and $90 \%$ of wind electricity could be 
consumed by the heating system and the household's electricity demand for a battery storage size of $20 \mathrm{kWh}$. This would also result in $65 \%$ of building autarky reducing feed in and demand grid stress. The study also shows that almost all the electricity demand for heating during winter months can be provided by self-produced wind and PV electricity. In addition, chemical seasonal storage possibilities based on a PEM-electrolyzer, hydrogen storage in the form of FA and a PEM-Fuel cell were examined. It shows that for full self-consumption of wind and PV electricity $6120 \mathrm{kWh}$ of electricity have to be stored, resulting in $100 \%$ electrical building autarky. This would result in 7683 liter of FA. For solely $100 \%$ of electrical autarky $4330 \mathrm{kWh}$ have to be stored, resulting in 54361 of FA.

To further investigate the possibility of a district energy storage system based on a chemical energy source that uses the benefits of different energy carriers (hydrogen, methane, liquid hydrocarbons) for an optimal sector coupling, further investigations will be carried out on the blocks of buildings level. Those will include a focus on power and electricity market interaction (flexible electricity tariffs, reserve power markets). It is also planned to include results regarding optimized building system operation into the buildings real system schedule.

\section{REFERENCES}

Agora Energiewende. (2017). Wärmewende 2030, (107/01).

Ahmed, N. A., Miyatake, M., \& Al-Othman, A. K. (2008). Power fluctuations suppression of stand-alone hybrid generation combining solar photovoltaic/wind turbine and fuel cell systems. Energy Conversion and Management, 49(10), 2711-245 2719. http://doi.org/10.1016/j.enconman.2008.04.005

Arabali, A., Ghofrani, M., Etezadi-Amoli, M., Fadali, M. S., \& Baghzouz, Y. (2013). Genetic-algorithm-based optimization approach for energy management. IEEE Transactions on Power Delivery, 28(1), 162-170. http://doi.org/10.1109/TPWRD.2012.2219598

Bundesnetzagentur. (2018). EEG-Registerdaten und EEG-Fördersätze. Retrieved July 18, 2018, from https://www.bundesnetzagentur.de/DE/Sachgebiete/ElektrizitaetundGas/Unternehmen_Institutionen/Erneuerbare Energien/ZahlenDatenInformationen/EEG_Registerdaten/EEG_Registerdaten_node.html;jsessionid=7BB18D09 $\underline{\text { 15C6BB11115B0AD523487A74 }}$

Carmo, M., Fritz, D. L., Mergel, J., \& Stolten, D. (2013). A comprehensive review on PEM water electrolysis. International Journal of Hydrogen Energy, 38(12), 4901-4934. http://doi.org/10.1016/j.ijhydene.2013.01.151

Eppinger, J., \& Huang, K.-W. (2017). Formic Acid as a Hydrogen Energy Carrier. ACS Energy Letters, 2(1), 188-195. http://doi.org/10.1021/acsenergylett.6b00574

Hsu, S.-F., Rommel, S., Eversfield, P., Muller, K., Klemm, E., Thiel, W. R., \& Plietker, B. (2014). Eine auf RuKatalyse basierende wiederaufladbare Wasserstoffbatterie. Angewandte Chemie, 126(27), 7194-7198. http://doi.org/10.1002/ange.201310972

Kaabeche, A., Belhamel, M., \& Ibtiouen, R. (2011). Techno-economic valuation and optimization of integrated photovoltaic/wind energy conversion system. Solar Energy, 85(10), 2407-2420. http://doi.org/10.1016/j.solener.2011.06.032

Kleemann, M., \& Meliß, M. (1993). Regenerative Energiequellen. Springer-Verlag.

Mohammadi, S., Soleymani, S., \& Mozafari, B. (2014). Scenario-based stochastic operation management of MicroGrid including Wind, Photovoltaic, Micro-Turbine, Fuel Cell and Energy Storage Devices. International Journal of Electrical Power and Energy Systems, 54, 525-535. http://doi.org/10.1016/j.ijepes.2013.08.004

Niknam, T., Golestaneh, F., \& Shafiei, M. (2013). Probabilistic energy management of a renewable microgrid with hydrogen storage using self-adaptive charge search algorithm. Energy, 49(1), $252-267$. http://doi.org/10.1016/j.energy.2012.09.055

Schmelmer, Ramona; Denk, P. (2015). Vertikale Kleinwindanlagen in Bayern. Wiesbaden: Springer Fachmedien.

Sichilalu, S., Tazvinga, H., \& Xia, X. (2016). Optimal control of a fuel cell/wind/PV/grid hybrid system with thermal heat pump load. Solar Energy, 135, 59-69. http://doi.org/10.1016/j.solener.2016.05.028 
Spiegel, C. (2008). PEM Fuel Cell: Modeling and Simulation using MATLAB. http://doi.org/10.1016/B978012374259-9.50006-9

Wagh, S., \& Walke, T. V. (2017). Review on Wind-Solar Hybrid Power System. International Journal of Research in Science \& Engineering IJRISE, 3(2), 71-76. 


\title{
OPTIMIZING EFFICIENCY OF BIOMASS-FIRED ORGANIC RANKINE CYCLE WITH CONCENTRATED SOLAR POWER IN DENMARK
}

\author{
Andreas Zourellis ${ }^{1}$, Bengt Perers ${ }^{2}$, Jes Donneborg ${ }^{1}$, Jelica Matoricz ${ }^{1}$ \\ Aalborg CSP A/S ${ }^{1}$ \\ Hjulmagervej 55, 9000 Aalborg, Denmark \\ Phone: +4588168836 \\ Technical University of Denmark, Department of Civil Engineering ${ }^{2}$ \\ Nordvej, Building 119, room 119, 2800 Kgs. Lyngby \\ E-Mail: jem@aalborgcsp.com
}

\section{SUMMARY}

A first of its kind concentrated solar power (CSP) installation has been integrated together with a biomass heat and power (CHP) plant using an organic rankine cycle (ORC) unit. The plant has been deployed in the northern part of Jutland in Denmark, right next to the city of Brønderslev. The plant has been supplying heat to the local district heating network since the end of 2016. Aalborg CSP has developed and built the CSP plant consisting of parabolic trough collectors with an aperture reflecting area of $26,920 \mathrm{~m}^{2}$. The CSP plant is able to deliver 16.6 MWth at its peak while it can supply the district heating or the ORC with approximately 16,000 MWh of heat annually. This paper serves as a description of the technical aspects of the system with specific focus on the CSP field as well as presents the first measured performance from the Summer of 2017.

Keywords: Solar Energy, Concentrated Solar Power, CSP, Concentrated Solar Heat, CSH, SolarThermal, Integrated Energy System, Organic Rankine Cycle, ORC, Biomass

\section{INTRODUCTION}

Solar energy is abundant and increasingly utilized in domestic systems to supply space heating and cooling. However, over the last couple of decades the world' energy demand has increased dramatically due to both industrial growth and population increase.

\section{Concentrated Solar Power in Denmark}

Concentrated solar power (CSP) plants have so far mainly been built to produce electricity for export to the grid, however, numerous advantages have been identified in industrial setting as well. Due to the technology's flexibility to produce high and mid temperature heat, it provides an ideal solarthermal solution for industrial purposes. Parabolic trough CSP plants are typically found in countries around the solar belt, but economic viability has also been proven in a place with limited solar resources, in Denmark, where efficiency of the system was monitored and compared with flat solarthermal panels. The report concluded that CSP produces more energy per square meter above $50{ }^{\circ} \mathrm{C}$, provides a better economy over the system's 25-years lifetime and ensures a year-round energy production even in Nordic climate conditions compared to flat panel systems [1]. It has also be seen that even when operating in higher temperature the parabolic trough collectors maintain a high heat yield per square meter or aperture area [2], [3]. That is because the heat is concentrated in a central receiver tube enclosed in a vacuum glass envelope. 


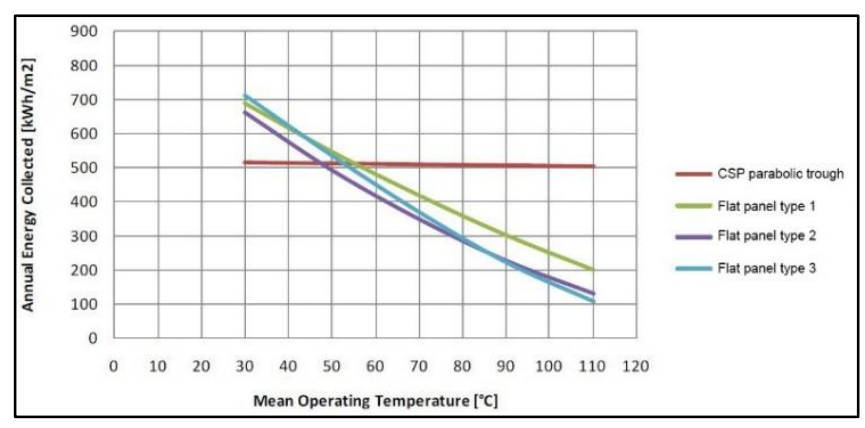

Figure 38: Parabolic trough collector performance vs. flat plate collectors in low heating temperatures

\section{TECHNOLOGY DESCRIPTION}

\section{Parabolic Trough Collectors (PTC)}
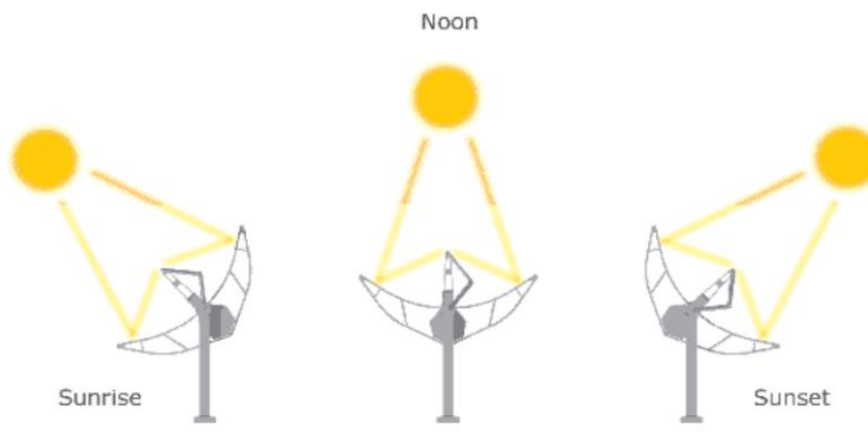

Figure 39: Sun tracking technology enabling the parabolic troughs to follow the sun's position throughout the day

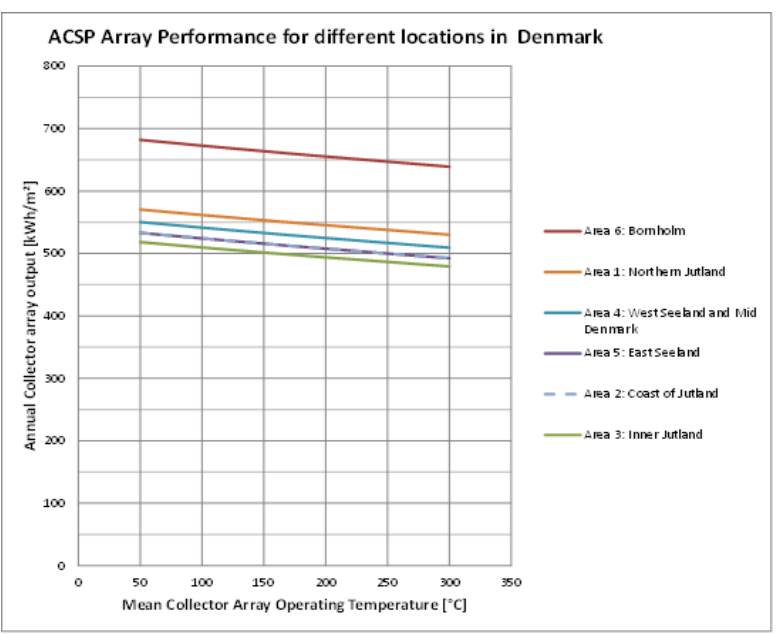

Figure 37: Parabolic trough collector performance in different Danish regions and high temperatures [3]

The parabolic troughs reflect the rays of the sun onto a receiver pipe filled with heat transfer fluid. The receiver pipe is located in the central focal point of the troughs. In the receiver pipe, the fluid is heated up and pumped into the heat consumer.

The CSP technology is the most effective solar heating method for high temperature ranges. The parabolic troughs has a special glass vacuum tube guaranteeing some of the markets lowest heat losses thus

providing stable energy production even at middle temperatures as the receiver pipe has very low heat losses to the ambient.

The parabolic trough uses a custom-made sun-tracking technology, where a computer calculates and calibrates the troughs into the required position to receive optimal radiation throughout the day. The sun-tracking technology achieves a very high efficiency per $\mathrm{m}^{2}$ of aperture mirror area, thereby optimizing the use of land intended for technology placement. The PTCs track and follow the sun in a tracking window of $\pm 0.15^{\circ}$ from the present solar position. The rotation is ensured by a gear and a hydraulic system.

\section{The system}

In December 2016, a world first CSP plant went into operation in the northern part of Denmark (town of Brønderslev). The plant's uniqueness lies in the fact that it was designed to be integrated with a biomass-fired Organic Rankine Cycle (ORC) which is currently still under the last stages of commissioning. This combined solution is the first large-scale system in the world to demonstrate how CSP, with an integrated energy system design can optimize efficiency of ORC even in areas with less sunshine, in this case Denmark. 


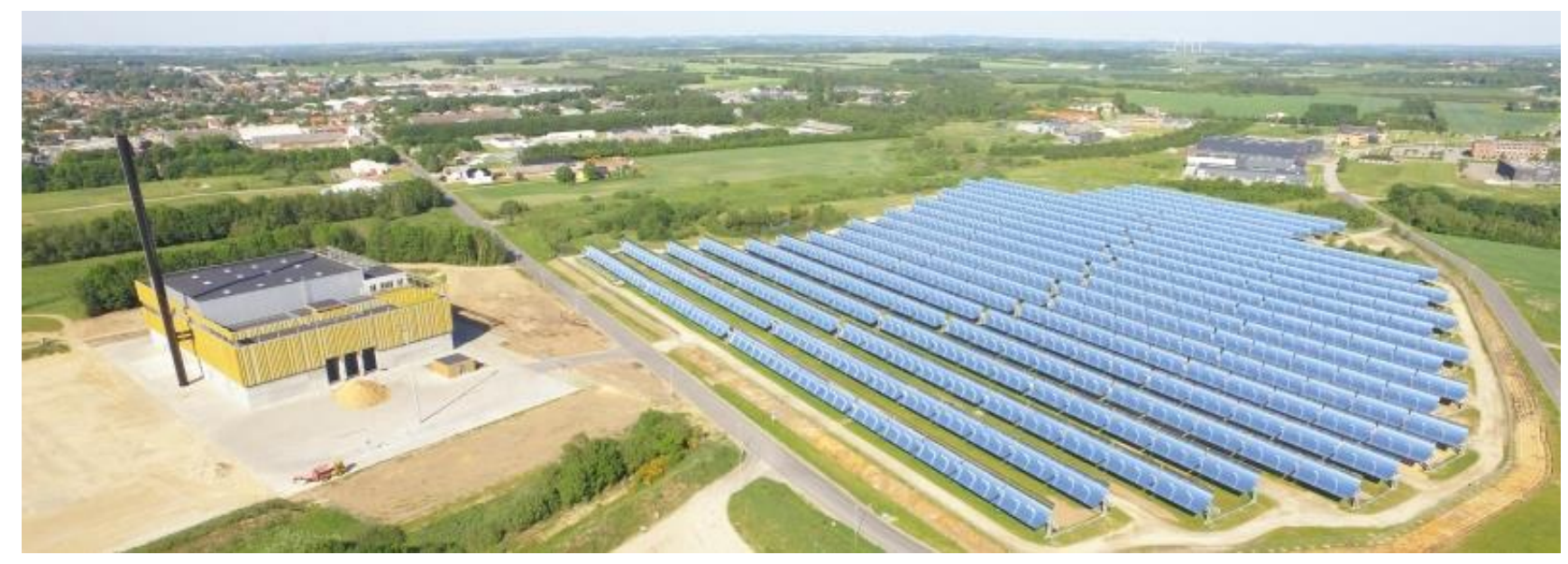

Figure 40: Image of the concentrated solar power plant in Brønderslev, Denmark

The solar energy plant was delivered by Danish renewable energy specialist Aalborg CSP, and it is based on the company's own CSP parabolic trough technology, also called the AAL-Trough ${ }^{\mathrm{TM}}$. The plant consists of 40 rows of $125 \mathrm{~m}$ U-shaped mirrors with an aperture area of $26,929 \mathrm{~m}^{2}$. These 40 rows are divided in 10 loops. Each loop has an inlet and outlet connection to the main pipe, meaning that the cold thermal oil will flow through 4 rows (i.e. $500 \mathrm{~m}$ ) until it gets the right temperature and finally leaves the loop. In order to fit within the land boundaries the loops were bended into half, saving cost for extra piping at the same time. As seen in Figure 5 the solar field is tilted $29^{\circ}$ from the North-South axis due to specific land availability.

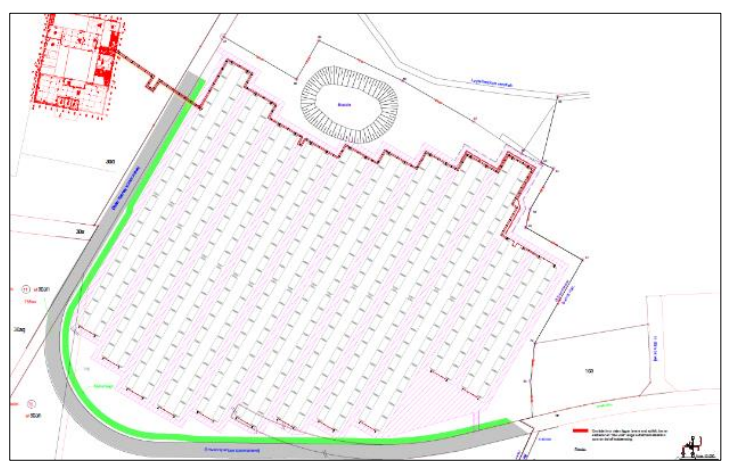

Figure 42: Top view of the solar field layout

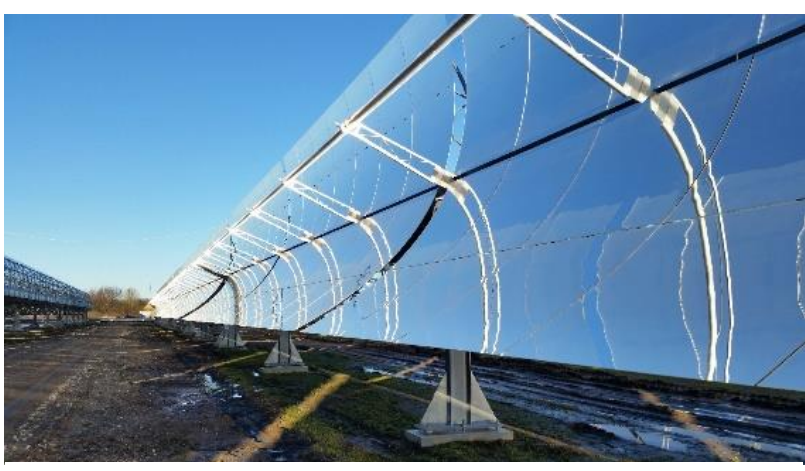

Figure 41: Photo of the AAL-TroughTM 3.0 model - third generation of Aalborg CSP's parabolic trough technology 
The curved mirrors collect the sunrays throughout the day and reflect them onto a receiver pipe, which sums up to 5 kilometer receiver tubes. This receiver pipe is surrounded by a special glass vacuum tube and inside this runs - only heated by the sun - thermal oil with temperatures up to $312{ }^{\circ} \mathrm{C}$. This high temperature is able to drive an electric turbine to produce electricity, but the flexibility of the system also allows production of lower temperatures for district heating purposes. The solar heating system can thus alternate between providing heat and power or deliver heat exclusively. To maximize yield of energy, the waste heat is utilized and sent to the district heating circuit whereas electrical power is generated at peak price periods.

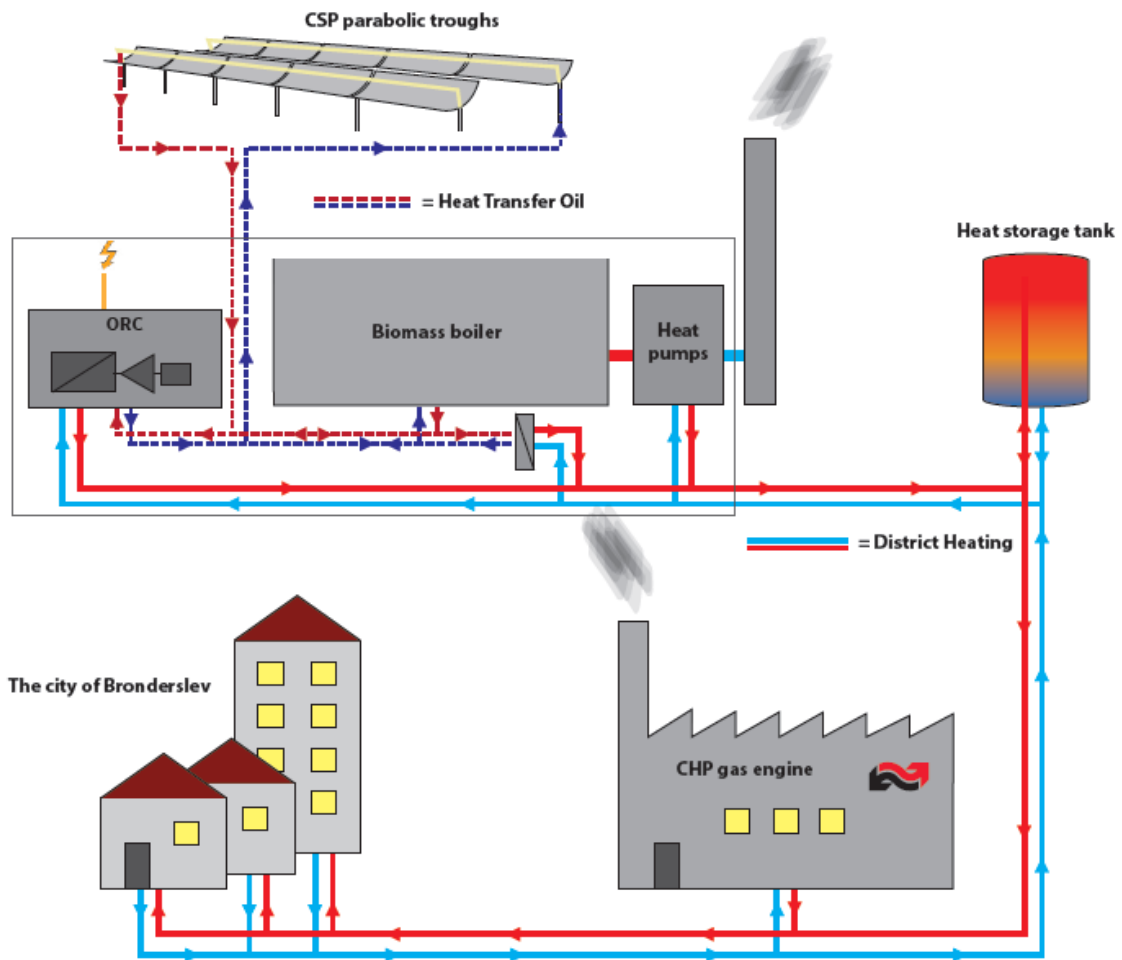

Figure 43: Flow chart of the energy distribution at the hybrid plant
In Figure 7, it can be seen that the CSP field is delivering hot thermal oil to both the ORC unit and the oil-to-water heat exchanger which delivers the heat to the district heating network to the city of Brønderslev. The CSP solar field has a thermal peak effect of 16.6 MWth and is expected to deliver approximately $16,000 \mathrm{MWh}$ of heat in an average weather year.

Apart from the solar field, there are two biomass boilers each of a maximum capacity of 10 MWth running with wood chips as fuel.

Both the CSP field and the biomass boilers are working in conjunction to supply with sufficient heat the ORC unit, which in full load is supposed to deliver 4MWe. The dissipated heat from the ORC condenser is used to provide additional heat to the district heating network. Therefore, one might notice that the hybrid system is designed in a sustainable and efficient way, avoiding to unnecessarily waste heat to the ambient. The biomass boiler house embeds a 2 MWth heat pump as well, to make use of waste heat from the biomass chimney and supply the district heating as supplementary source. In order to guarantee that the total energy demand is covered at any time, a CHP gas engine is placed as final backup.

The achievement of the world's first CSP system combined with a biomass-ORC plant is supported by the Danish Government's Energy Technology Development and Demonstration Programme (EUDP). 


\section{MONITORING AND VALIDATING PERFORMANCE DURING THE FIRST SOLAR SEASON}

As aforementioned, the peak performance of the plant is set to reach 16.6 MWth and the annual yield is expected to be $16,000 \mathrm{MWh}$ of thermal energy. Since the CSP-plant went into operation in the end of 2016, it has been meeting the expected operational goals.

In figure 8, the CSP performance in different seasons is illustrated. It is interesting to see that the time moves from Summer to Autumn, Spring and Winter the thermal power output curve shifts down. This is happening due to the lower solar altitude from month to month. As an example Figure 8 shows the expected thermal output for a day in January, April, July and October, based on an average weather year issued by the Danish Meteorological Institute [4]. Usually, when a PTC field tracking axis is not declining from the North-South axis then the profile of the thermal output curve has two symmetrical peaks (i.e. one in the morning and one in the afternoon). In the present case it should also be mentioned that the thermal peak in the morning hours is always higher than the one in the afternoon. This is explained from the tilted tracking axis in Brønderslev which deviates $29^{\circ}$ from the NorthSouth. Therefore, the optical losses are lower before solar noon, because of the lower incidence angle. The incidence angle is the angle in which the solar beams hit the surface of the reflecting mirrors in relation to the normal incidence. The lower the incidence angle is, the lower the optical losses are and thus the higher the thermal output is. The incidence angle of a day in May is seen in Figure 9.

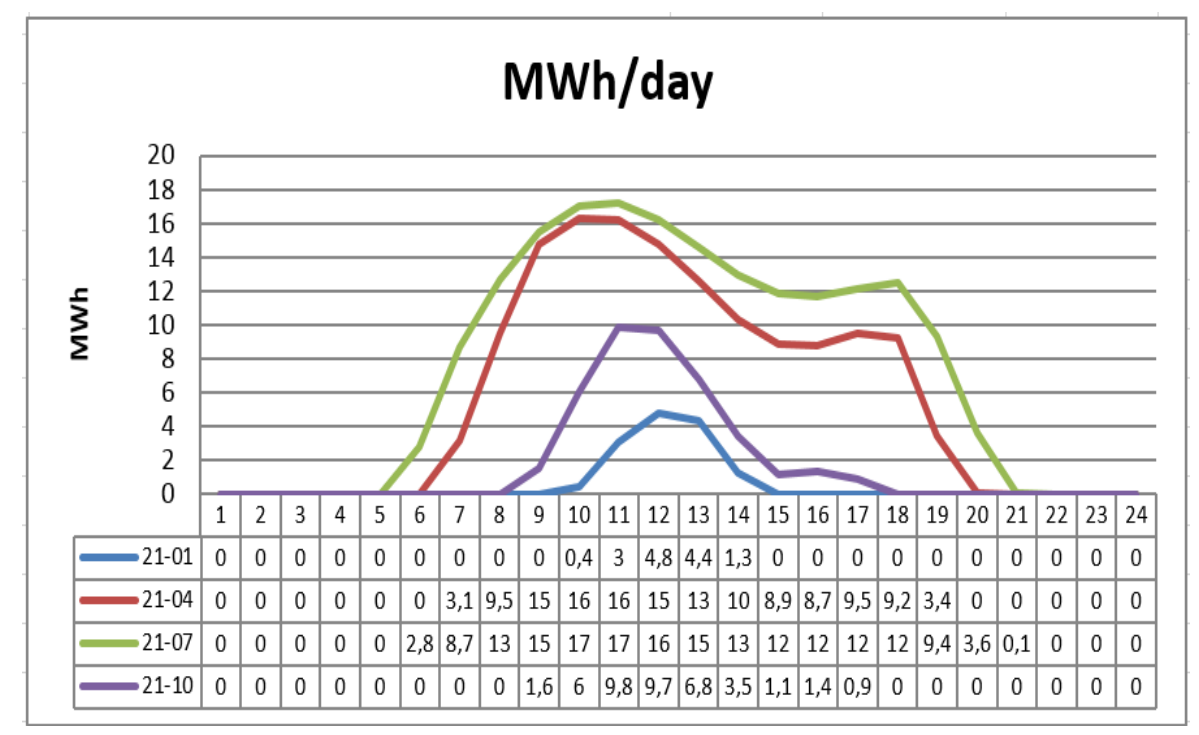

Figure 44: Seasonal variation on CSP thermal power output curve 


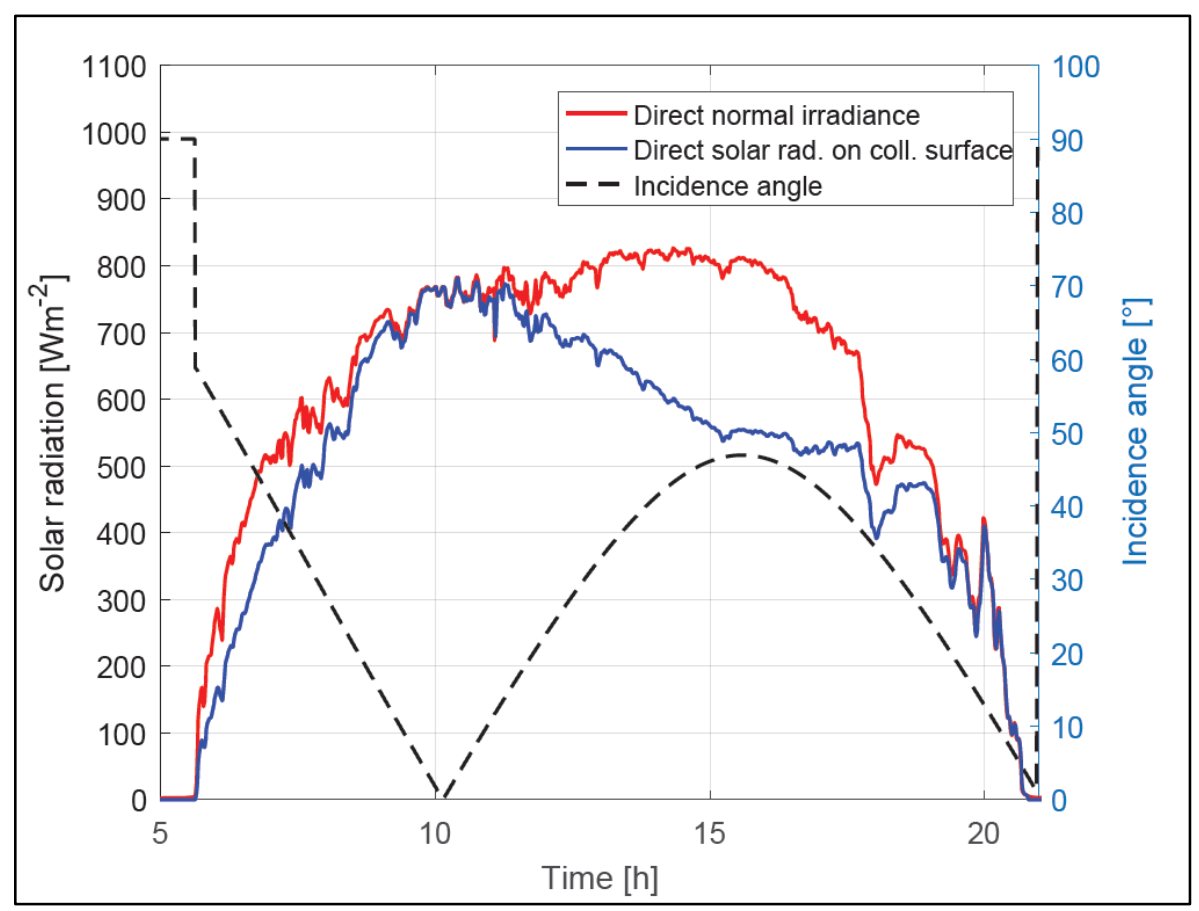

Figure 45: Incidence angle effect on thermal power output curve [2]

Figure 9 shows the effect of turning the tracking axis from exactly North South direction. The daily profile is then changed and may be adapted to the highest electricity price that at present often is in the mornings at around 8-10 [3].

The solar plant performance was monitored and cross checked during its first summer period from May 2017 to September 2017. Figure 10 illustrates the modeled versus the measured performance in $\mathrm{kWh}$ of heat produced per day and as it can be clearly seen the two series of data come in very good agreement when they get the same daily beam radiation as input.

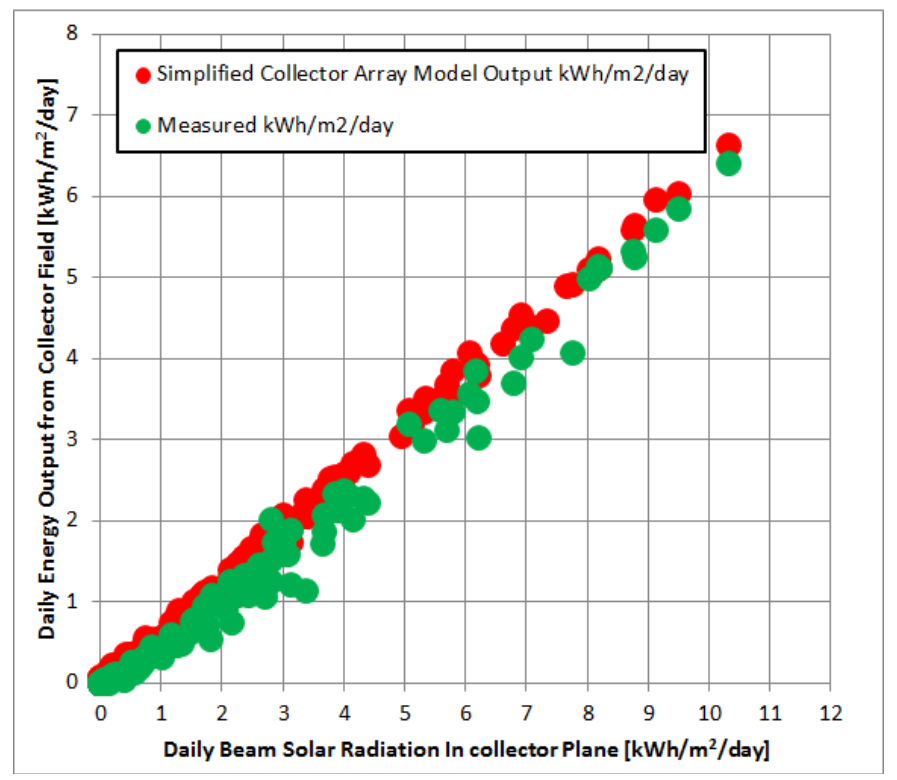

Figure 46: Validation of measured vs. computed performance for summer 2017, Perers et.al [3] 
The same procedure has been followed and is presented here for a whole day of solar heat production in May. In Figure 11 the measured and calculated thermal performance of the field are presented with a yellow and a black line respectively and it becomes apparent that they are almost identical. All the heat produced by the field at that moment was delivered to the district heating network. The monitored forward water temperature to the district heating grid was also in very good agreement with the calculated one and averaging at around $80^{\circ} \mathrm{C}$. With blue and red, the measured and calculated outlet temperature of the thermal oil is shown to be peaking at around $180^{\circ} \mathrm{C}$. At that moment the $\mathrm{ORC}$ unit was still under construction and there was not need to operate at a higher temperature than that.

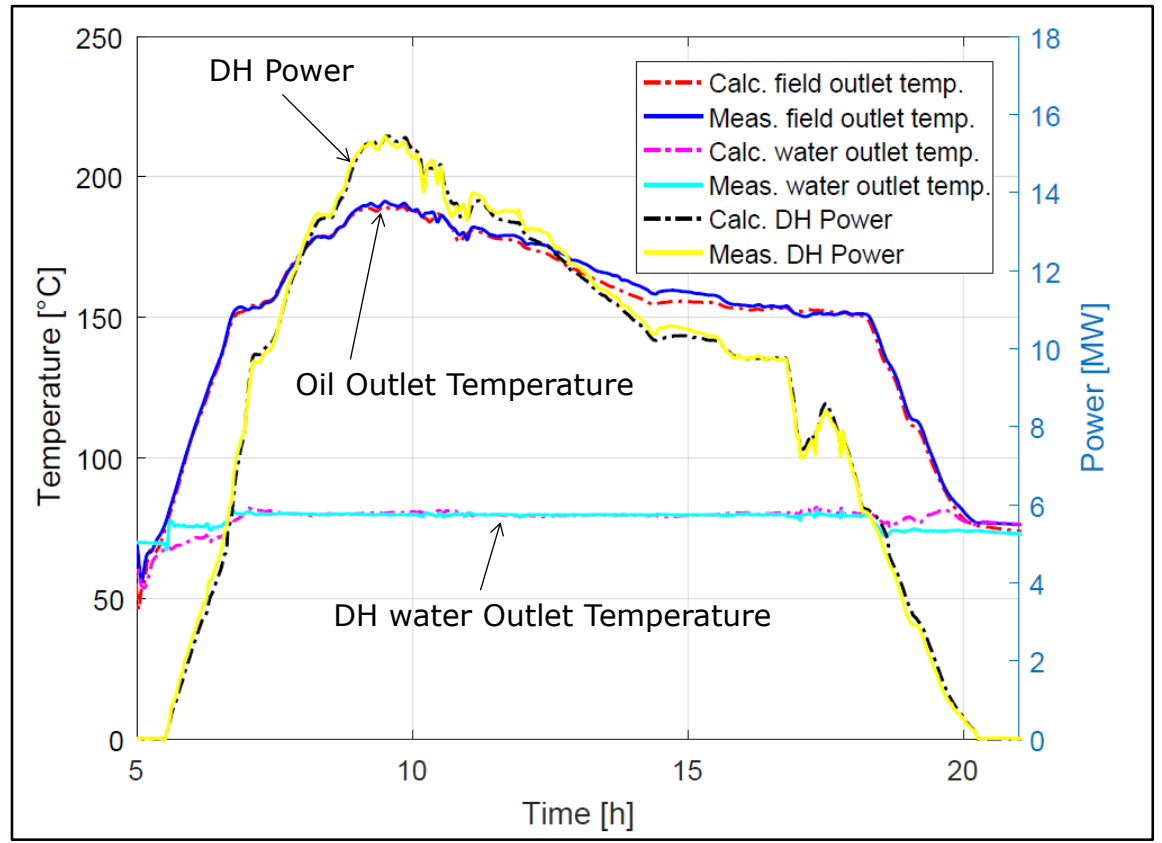

Figure 47: Measured and calculated performance of solar field [3].

\section{ACKNOWLEDGEMENTS}

The first author really appreciates the assistance from the Technical University of Denmark and especially Senior researcher Bengt Perers whose effort and continuous technical support was essential. Special thanks are expressed to Simon Furbo, Zhiyong Tian Alejandro de la Vega Fernandez for contributing in the performance analysis.

\section{REFERENCES}

[1] B. Perers, S. Furbo and D. Janne , "Thermal performance of concentrating tracking solar collectors," Technical Univeristy of Denmark (DTU) Civil Engineering Report R-292 (UK) Department of Civil Engineering, Denmark, 2013.

[2] Fernandez A. Analyses of Brønderslev solar energy plant by use of TRNSYS. DTU master thesis; 2017.

[3] B.Perers, S. Furbo, Z. Tian, J.H. Rothmann, J. Juul, T. Neergaard, P.V. Jensen, J.R. Jensen, P.A. Sørensen, N. From, "Performance of the $27000 \mathrm{~m} 2$ parabolic trough collector field, combined with Biomass ORC Cogeneration of Electricity, Brønderslev Denmark", Solar District Heating Conference 2018, Graz, Austria, 2018

[4] Danmarks Meteorologiske Institut, www.dmi.dk 


\title{
MUNICIPAL WASTEWATER TREATMENT SYSTEMS AND THEIR FUTURE ROLE IN EFFICIENT AND SUSTAINABLE ENERGY SYSTEMS
}

\author{
Mag. DI. Kerstin Schopf, Prof. Dr. Thomas Kienberger \\ Chair of Energy Network Technology, Montanuniversity Leoben \\ Franz-Josef-Straße 18, 8700 Leoben, Austria \\ Phone: +43 3842 402-5401 \\ E-Mail: evt@unileoben.ac.at
}

\section{SUMMARY}

Establishing efficient and sustainable energy systems is necessary to combat climate change. But it does not suffice to focus on central large-scale power plants or high voltage grids only. Also energy consumer and supplier integrated in medium and low voltage grids, like municipal wastewater treatment plants, are crucial components in the context of generating a sustainable future energy system. Wastewater treatment plants are responsible for up to $20 \%$ of the total energy consumption of municipalities, but the treatment process also leaves energy-rich residues, digester gas and sewage sludge, which represent significant bioenergy potentials. Thus, sewage plants can act as energy consumer and supplier. In order to estimate the energy demands and the energy production-potentials of different plant configurations a modular modelling tool for calculating non-time-resolved mass- and energy balances got designed. The tool enables to identify system configurations with a high electric and thermal energy self-sufficiency and builds the basis for further analysis concerning time-resolved load and production profiles as well as load shift potentials and production flexibilities.

Key words: wastewater treatment plants, mass balance, energy balance, energy recovery, nutrient recovery

\section{INTRODUCTION}

To combat climate change it is necessary to establish efficient and sustainable energy systems. But it does not suffice to focus on central large-scale power plants or high voltage grids only. Also energy consumer and supplier integrated in medium and low voltage grids, like municipal wastewater treatment plants, are crucial components in the context of generating a sustainable future energy system. By analysing municipal energy systems two main aspects can be observed. First a high energy consumption of municipal supply structures especially of wastewater treatment systems and second an increasing feed-in out of volatile renewable energy sources. With the focus on designing efficient and sustainable future energy systems it is important to take a closer look on both aspects. Therefore, the challenge is to analyse the state of the art municipal wastewater treatment system (MWWTS) configurations according their energy balances and their interaction with energy grids in order to identify possible future system configurations and their interaction possibilities.

\section{ENERGY-SYSTEMIC ANALYSIS OF MUNICIPAL WASTEWATER TREATMENT PLANTS}

A municipal wastewater treatment plant accounts for a proportion of about $20 \%$ of the total energy consumption of municipalities and is therefore a main energy consumer [1]. With an up-scaled total electric energy consumption of $650 \mathrm{GWh} / \mathrm{a}$, all Austrian sewage plants are responsible for approximately $1 \%$ of the total national electric energy consumption. Furthermore, about $350 \mathrm{GWh}$ of thermal energy are used annually [2]. But the treatment process also leaves energy-rich residues, digester gas and sewage sludge, which represent significant bioenergy potentials. Digester gas is already a widely-used energy source to cover parts of the internal power and heat demand of 
MWWTS. Sewage sludge offers due to the specific energy a potential input for thermal utilisation units.

The energy consumption as well as the mass and energy flows of digester gas and sewage sludge depends on numerous factors, like the chemical components of the wastewater or the applied treatment technology [3]. In order to determine the energy demands as well as the existing bioenergy potentials of a specific common or future MWWTS configuration, it is necessary to generate mass and energy balances including all influencing factors and process steps. Thus, the first step is to develop a modular modelling tool to simulate the whole energy system including all processes up to the dewatering unit as well as all relevant mass and energy flows on a non-time-resolved basis.

Building on this, the second step is to identify the influence of a system integration of the bioenergy potentials, digester gas and sewage sludge. This is necessary to estimate the possible decentralised energy production of different MWWTS configurations. Here, decentralised utilisation describes an on-site utilisation. Therefore, non-time-resolved mass and energy balances of on-site utilisation units for digester gas and sewage sludge like small-scale combustion or gasification units are integrated into the calculation model. With the extended modelling tool, optimal on-site utilisation paths for digester gas and sewage sludge can be determined through analysing different MWWTS configurations with the objective of increasing the electric and thermal energy self-sufficiency.

Here it should be mentioned that on-site sludge utilisation units (gasification or combustion units) are not a state of the art technology. The technological feasibility and economic efficiency of an integration into an existing MWWTS has to be analysed individually. In order to estimate the full energy potentials of these technologies operability ( 8,000 operating hours), compatibility with existing systems and optimal output quality are assumed.

\section{MUNICIPAL WASTEWATER TREATMENT PLANT AS DECENTRALISED ENERGY SERVICE PROVIDER IN MUNICIPAL ENERGY SYSTEMS}

The actual study focuses on on-site utilisation possibilities, to analyse the future role of wastewater treatment systems as decentralised energy service providers within municipal energy systems. MWWTS are necessary to guaranty a sufficient water purification and are therefore installed in numerous urban and rural areas. In Austria for example exist about 1,900 wastewater treatment plants [2]. Taking into account their energy intensive treatment processes, their bioenergy potentials and their storage possibilities, it gets clear, that MWWTS can provide energy services in low and medium voltage levels. Here the term energy services cover constant electric or thermal energy supply as well as providing short term frequency reserve [4] to stabilize the grid frequency. Especially the last point is gaining importance by observing the actual development of decentralised power supply units. An increasing volatile supply of renewable energy induces residual loads (temporal over- or undersupply of power) which lead to frequency deviations in low and medium voltage levels of the power grid. [5] Current studies already discuss the role of MWWTS as service providers but do not consider the effects of an on-site sludge utilisation [6,7,8]. Considering the results and taking into account the requirements of the pre-qualification procedure of the Austrian system operator APG [4] following characteristics enhance the feasibility of an interactive grid integration of a MWWTS:

\section{- High electric and thermal energy self-sufficiency}

A self-sufficiency shows the availability of energy production units like CHP plants (combined heat and power plants). A high parameter indicates, that units with considerable rated power are operated on-site which could lead to a higher flexibility.

- Minimal available power 
Here should be mentioned, that the system operator APG requires a minimal available power of +/-1MW or +/-5 MW depending on the type of control power. [8] This power supply can be reached through pooling strategies.

\section{- Deactivation time and responds speed}

According to the pre-qualification procedure the plant has to be able to cut the supply for 15 minutes and respond within 5 minutes. Furthermore, the plant must provide a specific supply for 45 minutes.

\section{- Full-load hours}

The full load hours influence the flexibility directly. For example, a CHP plant with 8,000 full-load hours can primary provide negative control power by reducing the energy production.

\section{- Storage possibilities}

Storage possibilities for digester gas and sewage sludge lead to higher flexibility.

As mentioned before the modular modelling tool enables the calculation of the energy production and consumption of different configurations of MWWTS. This builds the basis for the determination of optimal energy systems which can be integrated as energy service provider in future energy grids.

\section{MEHTODS}

The modular modelling tool is programmed in EXCEL and is based on modular non-time-resolved mass and energy balances. Here, different standardised calculation methods, thermodynamic basics and fluidic aspects are taken into account. [3,9] The tool is designed as a standalone application, the "OPTIEVLEX-Tool” (“Optimierte Energieverbunde kommunaler Abwasserbehandlungsanlagen als Flexibilitätsbautstein in den kommunalen Netzen der Zufkunft"). It enables users to define a specific wastewater treatment plant, by using selection- and input fields of an input screen. After the choice of the treatment system (aerobic or anaerobic wastewater treatment) as well as the specific characteristics of process units (e.g. equipment type, operating hours or rated power) the user can inter alia determine the influence of on-site integration of bioenergy potentials on the energy self-sufficiency of a specific MWWTS. The standalone application is realised via MATLAB.

The focus of this work lies on analysing the flexibility characteristic, electric and thermal energy selfsufficiency. The parameters reflect the energy consumption and supply of a MWWS and is the basis for identifying configurations which should be examined concerning their interaction with the energy grids. The initial analyses estimate the self-sufficiency of MWWTS with different capacities measured by the population equivalent (PE). [3] Due to the higher bioenergy potential caused by the production of digester gas the work considers only anaerobic treatment plants. [10] Here three configurations are analysed. Configuration 1 is a state of the art anaerobic treatment plant with a CHP unit for digester gas utilisation. Configuration 2 represents an expanded version of configuration 1 . Here, the plant gets in a first step upgraded by a low-temperature belt dryer. In configuration 3 a further expansion leads to the integration of a gasification unit for sewage sludge including a synthesis gas CHP unit.

\section{RESULTS}

Fig. 1 and Fig. 2 show the electric and thermal energy consumption, -production and -self-sufficiency of plants from 10,000 to 300,000 PE.

Fig. 1 considers electric energy. The results show, that on-site utilisation of digester gas as well as of sewage sludge enhance the electric energy self-sufficiency. The highest values can be reached by an optimal integration of a gasification unit and a CHP unit for the utilisation of synthesis gas. 

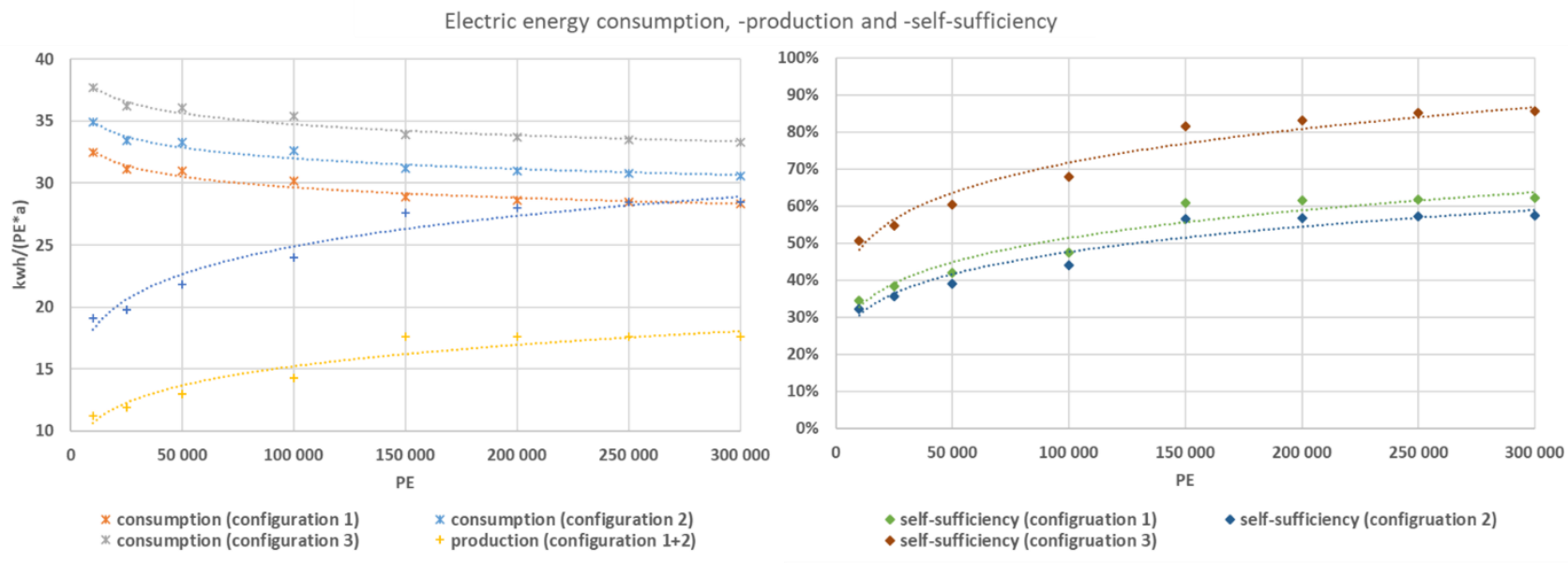

Fig. 1: Electric energy consumption, -production and -self-sufficiency of MWWTS configurations configuration $1=$ state of the art anaerobic plant; configuration $2=$ configuration $1+$ low-temperature belt tryer; configuration $3=$ configuration $2+$ gasification and synthesis gas CHP unit

Fig. 2 presents the thermal energy consumption, -production and -self-sufficiency of the MWWTS configurations. The results illustrate, that a drying system leads to a high thermal energy consumption which implies a sludge utilisation to produce a higher amount of thermal energy. This can also be seen by analysing the self-sufficiency of the different configurations.

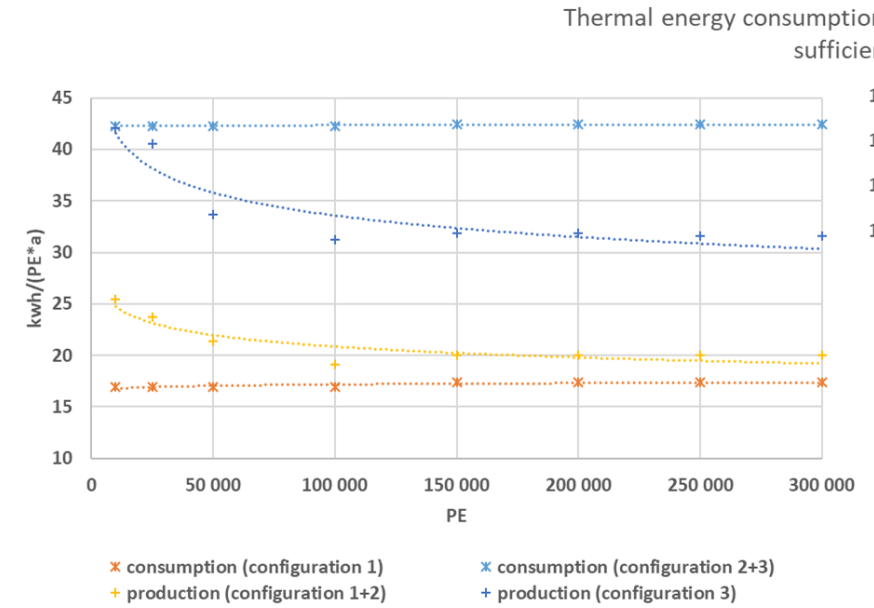
ufficiency

Fig. 2: Thermal energy consumption, -production and -self-sufficiency of MWWTS configurations configuration 1 = state of the art anaerobic plant; configuration $2=$ configuration $1+$ low-temperature belt tryer; configuration $3=$ configuration $2+$ gasification and synthesis gas CHP unit

The results in Fig. 1 and Fig. 2 show, that configuration 1 leads to a medium energy self-sufficiency from $34 \%$ to $62 \%$ and to a high thermal energy self-sufficiency from $150 \%$ to $115 \%$. Here it should be mentioned, that smaller CHP units lead to lower electric efficiency and higher thermal efficiency compared to bigger units. This configuration presents already a good option if sludge disposal is out of consideration. The oversupply of thermal energy can be used for drying as a further sludge treatment process. This enhance the storage and disposal opportunities. 
Therefore, the configuration 2 with a low-temperature belt dryer got analysed. Due to an approximately $8 \%$ higher energy demand and a more than doubled thermal energy demand this configuration shows the lowest values for both self-sufficiencies. The values for the electric selfsufficiency reach from $32 \%$ to $58 \%$. The range of the thermal efficiency lies between $60 \%$ and $47 \%$. The results clearly show, that the drying process leads to a higher energy consumption which may be covered by a sludge utilisation unit.

Thus, configuration 3 includes a gasification unit for sewage sludge and a CHP unit for the utilisation of the produced synthesis gas. This two processes lead to increasing self-sufficiencies compared to configuration 2. The electric self-sufficiencies with results between $51 \%$ and $86 \%$ are even higher than these of configuration 1 . This is caused by a higher electric energy production by the second CHP unit (up to $+70 \%$ ) in comparison to a small raise of the energy consumption (up to $+16 \%$ compared to configuration 1). The thermal energy self-sufficiency is ranked in an average level with values of about $100 \%$ to $75 \%$.

\section{DISCUSSION AND OUTLOOK}

The results show, that the on-site utilisation of digester gas as well as of sewage sludge should be taken into account by designing future MMWTS. Besides a higher energy self-sufficiency, a sludge gasification unit and a synthesis gas CHP unit lead to a higher flexibility potential. Some state of the art (only digester gas utilisation) and some feasible future configurations (digester gas and sewage sludge utilisation) with high electric and thermal energy self-sufficiency will be selected to further analyse the role of MWWTS in future energy systems. To identify the real interaction possibilities with local grids, it is necessary to estimate time dependencies. Therefore, time resolved load and production profiles of different treatment system configurations are needed. Moreover, it is necessary to identify the load shift potentials and production flexibilities of the systems to guaranty an optimal grid integration. The load and production profiles as well as the load shift potentials and production flexibilities will be estimated through the OPTIEVLEX-Tool. The basic tool including the non-time resolved mass and energy balances will therefore be expanded by a time-resolved inflow-model (dry weather day). [11] The expanded MATLAB-EXCEL-tool enables a time-resolved simulation of energy profiles and flexibilities options.

\section{REFERENCES}

[1] Fricke, K., (2009) Energieeffizienz kommunaler Kläranlagen (Energy-efficiency of Municipal Sewage Plants). https://www.umweltbundesamt.de/sites/default/files/medien/publikation/long/3855.pdf (accessed 26.07.2018).

[2] Überreiter, E., Lenz, K., Zieritz, I., Weber, K. and Eder, I. 2016 Kommunales Abwasser Österreichischer Bericht 2016 (Austrian Report - Municipal Wastewater 2016). https://www.bmnt.gv.at/dam/jcr:64403bf0-c625.../Lagebericht_20160628\%20gsb.pdf (accessed 26.07.2018).

[3] Metcalf and Eddy I AECOM. (2014) Wastewater Engineering: Treatment and Resource Recovery. 5th Edition, McGraw-Hill, New York.

[4] Austrian Power Grid AG (2018) - Market Information-Balancing. https://www.apg.at/en/markt/netzregelung (accessed 26.07.2018)

[5] Karl, J. (2012) Dezentrale Energiesysteme - Neue Technologien im liberalisierten Energiemarkt (Decentralised energy systems - New technologies in the liberalised energy market). Oldenbourg Verlag, München. 
[6] Schmitt, G. et al. (Projektleitung: TU Kaiserslautern, Fachgebiet Siedlungswasserwirtschaft) (2017) Abwasserreinigungsanlagen als Regelbaustein in intelligenten Verteilnetzen mit Erneuerbarer Energieerzeugung (Wastewater Treatment Plants as Flexibility Service Provider) https://erwasarrivee.de/userspace/EXT/arrivee/Dokumente/170821_Schlussbericht/170821_arrivee_Schlussbe richt_Final.pdf (accessed 26.07.2018)

[7] Reinhofer-Gubisch, M., Pucker, J., Frantes, B. and Rezania, R. (2015) LoadShift: Lastverschiebung in Haushalt, Industrie, Gewerbe und kommunaler Infrastruktur Potentialanalyse für Smart Grids (LoadShift; Load shift in households, industry, trade and municipal infrastructure - potential analysis for smart grids). https://nachhaltigwirtschaften.at/resources/e2050 pdf/reports/endbericht 201507h loadshift kom munale infrastruktur.pdf (accessed 26.07.2018)

[8] Müller, A.E., Graf, E., Kobel, B., Hurni, A., Wenger, R., Frei, U., Christen, C., Moser, R., Fritzsche, C., Mathys, O., Kernen, M., and Fahrni, J. (2013) Potential der Schweizer Infrastrukturanlagen zur Lastverschiebung (Load shift potential of Swiss infrastructure) http://www.infrawatt.ch/sites/default/files/2013_BFE\%20\&\%20InfraWatt_Potential\%20der\%20S chweizer\%20Infrastrukturanlagen\%20zur\%20Lastverschiebung_low_1_0.pdf $\quad$ (accessed 26.07.2018)

[9] DWA (2016) Arbeitsblatt DWA-A 131: Bemessung von einstufigen Belebungsanlagen (Worksheet DWA-A 131: Plant design of one-stage aeration plants). DWA, Hennef, Germany.

[10] Schopf, K., Judex, J., Schmid, B. and Kienberger, T. (2018) Modelling the bioenergy potential of municipal wastewater treatment plants. Water Science and Technology 77(11), S.2613-2622.

[11] Langergraber, G., Alex, J., Weissenbacher, N., Woerner, D., Ahnert, M., Frehmann, T., Halft, N., Hobus, I., Plattes, M., Spering, V., Winkler, S. (2008). Generation of diurnal variation for influent data for dynamic simulation. Water Science and Technology 57(9), 1483-1486. 


\section{EMERGING TECHNOLOGIES AT WASTE WATER TREATMENT PLANTS FOR NUTRIENT RECOVERY AND ENERGY NETWORK INTEGRATION}

Gruber-Glatzl, Wolfgang (1); Buchmaier, Judith (1); Brunner, Christoph (1); Garstenauer, Teresa (1); Obenaus, Christian (1); Mayr, Bernhard (2); Nowak, Otto (3)

Organisationen: (1) AEE INTEC, Austria (2) EnviCare Engineering GmbH, Austria (3) Nowak Abwasser

Beratung e.U., Austria

*Phone: +43(0)3112-5886-455

*E-Mail: w.gruber-glatzl@aee.at

\section{SUMMARY}

Waste water treatment plants (WWTP) are classical end-of-pipe systems. They use a high amount of energy to remove nutrients from sewage water in the process of purification. WWTPs have the potential to be an energy and nutrient hub for electricity, district heat, gas and sewage networks in local urban areas.

A Decision Support Tool (DEST) was developed to investigate scenarios with existing and emerging technologies at WWTPs, energy networks and hybridization technologies for their interconnection. Energy and nutrient recovery scenarios are evaluated from an energetical, economic and ecological point of view. The paper presents the analysis of the effect of a nitrogen $(\mathrm{N})$ recovery membrane distillation (N-MD) in a WWTP to recover ammonia $\left(\mathrm{NH}_{3}\right)$ from the waste water. Furthermore, other measures to increase the biogas yield for the delivery to a local district heating network are analyzed and discussed.

\section{INTRODUCTION}

The WWTP of the future has the potential to change its nature from having the sole purpose of purifying water to become a regional energy and nutrient hub for its urban area. New technologies, systems and business models are needed to achieve this goal. Tools are required to investigate, analyze and evaluate future scenarios where WWTPs apply such technologies and systems.

\section{PROBLEM STATEMENT}

The conventional municipal biological wastewater treatment is an energy intensive process. Vast amounts of potential energy (bound in carbon) and valuable nutrients (e.g. nitrogen and phosphorus) are destroyed by energy-intensive aeration. Electrical energy efficiency has received great attention but thermal energy efficiency not in the same scope since it has rather been seen as waste heat.

In order to increase the share of renewable energy resources in energy networks, new strategies must be considered between producers and consumers. The WWTP offers ideal internal infrastructural conditions to act as a hub between the energy networks (gas, heat, electricity) and the waste water system. The combined assessment of WWTPs and energy networks is necessary to investigate synergies and to decide which technologies and systems are best from an energetical, economic and ecological point of view.

It is estimated that between the years 2016-2021 a total of 2.25 billion Euro are needed to be invested in Austrians WWTP (ÖWAV, 2016).

\section{METHOD}

The project AR-HES-B 1 had the overall goal to assess the technological rearrangement of the WWTP in order to transform it from a high energy consumer towards an optimal integrated hybrid energy provider, energy storage and resource provider in an urban environment.

In order to jointly investigate WWTPs and energy networks, a new tool has been developed within the AR-HES-B project. The Decision Support Tool (DEST) is an openly available tool 2 that allows a detailed definition of an individual WWTP and the already connected or nearby energy networks (electricity grid, district heat network and gas grid). The tool requires input in 4 areas: (1) Definition of inlet data as daily average values, (2) Definition of applied technologies categorized in 6 clusters (pretreatment, main cleaning, sludge treatment, energy conversion, nutrient recovery, trace element elimination) including emerging technologies, (3) Definition of further specifications certain technologies may require, (4) Definition of all 3 energy networks in terms of geographical distances, available energy load or monthly demands as well as the purchase and selling price of all 3 energy 
types. For the electricity grid the EPEX (European Power Exchange) spot price of 2016 is included in the tool to consider flexibilization possibilities with a WWTP.

The waste water treatment is calculated based on the German guidelines for the dimensioning of activated sludge treatment plants (DWA, 2016). The energy conversion is based on energy balances of electricity, heat (considering temperature levels) and gas. Similarly, mass balances for carbon (using the parameter COD - chemical oxygen demand) and nitrogen (considering its different forms) are made. Economic analysis is done based on net annual costs. Investment costs are split to two types (construction and machines) and the annuity is calculated for both. Together with the yearly costs and revenues the net annual costs are calculated. For the ecological analysis, the greenhouse gas emissions (GHG) at the WWTP are considered $\left(\mathrm{CO}_{2}, \mathrm{CH}_{4}\right.$ and $\left.\mathrm{N}_{2} \mathrm{O}\right)$ but also the indirect emissions due to energy consumption.

The results of the current entry are presented in a number of charts and tables. Entries and results may be saved as a scenario. Up to 10 scenarios can be saved in the tool in order to compare the results, perform parameter studies and sensitivity analysis.

The DEST has been used for the results presented here in this paper: The application of the emerging technology nitrogen recovery membrane distillation (N-MD) has been examined in the framework of an existing WWTP which is considered to deliver biogas to the local district heat network. The paper presents the effects of the N-MD on the main goal of nutrient recovery, but also the additional benefits like reduced aeration needs (less electricity consumption by the WWTP), additional biogas produced (by increasing share of primary sludge removal) and decreased demand of the basin volume.

The scenarios presented in chapter 5 are defined for two purposes:

- Comparison of the baseline (scenario 1) with measures to increase the biogas yield (scenario 2) and the effect of the N-MD (scenario 3)

- Comparison of different external biogas utilization concepts (scenario 3a, 3b, 3c) where the waste water treatment technologies are the same for all scenarios.

The paper presents the effects of combined measures to increase the biogas yield and nutrient recovery for selling purposes and briefly outlines the business model considerations for this waste water treatment plant of the future. Finally, the paper presents the results of the ecological, economic and energetical analysis by means of the DEST.

\section{INVESTIGATED TECHNOLOGIES AND DEFINITIONS OF SCENARIOS}

The following scenarios (Table 1) were defined and investigated in detail. The aim was to investigate the effects of emerging technologies like alternative nitrogen removal (e.g. membrane distillation) to the potential of interlinking WWTPs to the energy grids (gas grid, electricity grid, district heating network).

The investigated WWTP has a capacity of 40.000 population equivalent, the waste water inflow is $8,000 \mathrm{~m}^{3} / \mathrm{d}$, the COD concentration is $600 \mathrm{mg} / \mathrm{l}$ leading to a daily load of $4,800 \mathrm{~kg} / \mathrm{d}$. The total nitrogen at the inflow is $55 \mathrm{mg} / \mathrm{l}$ and $440 \mathrm{~kg} / \mathrm{d}$. The WWTP is a conventional activated sludge treatment plant with denitrification before nitrification. Primary and secondary sludge is dewatered before entering a digestion tower. 
Table 1: Scenarios of emerging technologies and connection of WWTP to the energy grids

\begin{tabular}{|c|c|c|c|}
\hline \multicolumn{2}{|c|}{ Scenario Number and Name } & \multirow{2}{*}{$\begin{array}{l}\text { Technologies } \\
\text { Biogas-CHP ( } 33 \% \text { electrical efficiency; } \\
57 \% \text { thermal efficiency) }\end{array}$} & \multirow{2}{*}{$\begin{array}{l}\text { Description } \\
\text { Baseline scenario. Produced } \\
\text { biogas is converted in a CHP to } \\
\text { electricity and heat for internal } \\
\text { use. }\end{array}$} \\
\hline 1 & Baseline & & \\
\hline 2 & Biogas Increase & $\begin{array}{l}\text { Increased retention time in pre-treatment } \\
\text { basin, increased retention time digestion } \\
\text { tower, heat pump for WWTP thermal } \\
\text { demand } \\
\text { Biogas-pipeline }(600 \mathrm{~m}) \text { to district } \\
\text { heating }\end{array}$ & $\begin{array}{l}\text { Increased retention time leads to } \\
\text { increased biogas yield. Heat } \\
\text { pump allows the full external use } \\
\text { of biogas }\end{array}$ \\
\hline 3a & N-MD / Biogas selling & Scenario $2+\mathrm{N}-\mathrm{MD}$ & $\begin{array}{l}\text { Based on Scenario } 2 \text { but } \\
\text { including N-MD }\end{array}$ \\
\hline $3 \mathbf{b}$ & Biogas treatment & $\begin{array}{l}\text { Scenario 3a }+ \text { Biogas treatment } \\
\text { (biological desulphurisation, pressure } \\
\text { swing adsorption, adsorption dryer and } \\
\text { activated carbon), biogas storage ( } 420 \\
\mathrm{~m}^{3} \text { ) and gas pipeline (instead of biogas } \\
\text { pipeline) to gas grid }(600 \mathrm{~m})\end{array}$ & $\begin{array}{l}\text { Biogas is treated to achieve gas } \\
\text { grid quality. Gas is sold to gas } \\
\text { grid. }\end{array}$ \\
\hline $3 c$ & Power2Gas & $\begin{array}{l}\text { Scenario } 3 \mathrm{~b}+\text { electrolyser }\left(128 \mathrm{Nm}^{3} / \mathrm{h}\right. \\
\text { hydrogen) and methanisation plant }\end{array}$ & $\begin{array}{l}\text { Electrolyser designed to run } \\
4,000 \text { hours at times with lowest } \\
\text { EPEX spot price. Methanisation } \\
\text { to produce methane. Delivery to } \\
\text { gas grid together with treated } \\
\text { biogas (Scenario } 3 b \text { ) }\end{array}$ \\
\hline
\end{tabular}

Figure 1 shows some of the basic principles investigated in the scenarios defined above. The WWTP is connected to the energy grids (gas grid, district heating network and electricity grid) but of course also to the sewage network and the road-based networks like fertilizer and sludge transportation. Figure 1 shows scenario $3 \mathrm{a}$ and scenario $3 \mathrm{~b}$ as parallel options for biogas utilization.

In scenario 1 the produced biogas in converted in a CHP to electricity and heat for internal use. No heat pump and N-MD is applied. Scenario 2 is like scenario 3a but without N-MD.

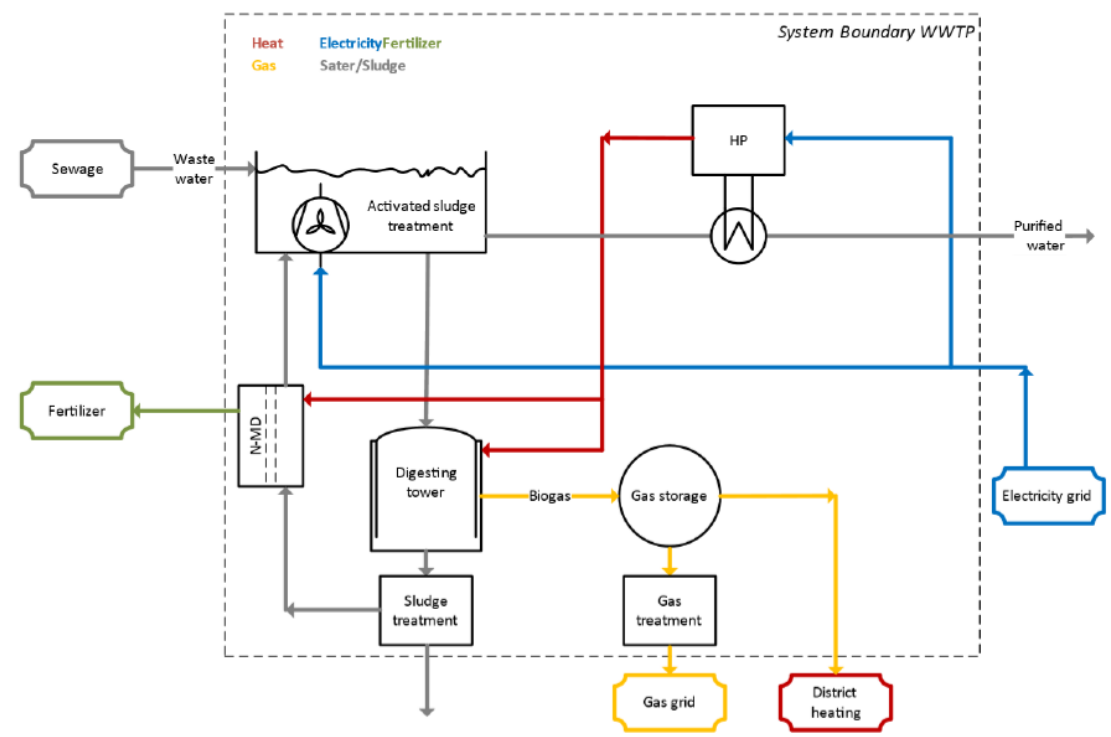

Figure 1: Simplified flowsheet of WWTP (Scenario 3a and 3b): N-MD (nitrogen membrane distillation), HP (heat pump)

Analysis of increasing biogas yield 
Figure 2 shows the changes in the energy balances of the WWTP for the different scenarios. The baseline (scenario 1) clearly indicates the aim to be self-sufficient in terms of energy. While the electrical energy demand is partly covered (46\% self-sufficiency) the produced thermal energy actually exceeds its demand (144\% self-sufficiency). This is the effect that has been indicated in the problem statement (chapter 3): Heat needs to be either released to the environment through an emergency cooler or biogas is flared.

In scenario 2 a heat pump ( $\left.159 \mathrm{~kW}_{\text {th }}\right)$ is used to cover the internal heat demand, thus the produced biogas (that has been increased by other measures described in Table 1) is fully available for external use. However, instead of covering part of the electricity demand internally, the heat pump requires additional electricity. The total balance shows that the outside electricity demand has increased significantly in comparison to scenario 1 (from 2,065 to $4,470 \mathrm{kWh} / \mathrm{d}$ ) but on the other hand 6,648 $\mathrm{kWh} / \mathrm{d}$ of biogas can be used externally). From this viewpoint the WWTP could be described as a power to gas system.

Scenario 3a is very similar to scenario 2 but has a N-MD installed. The effect on the energy balance in comparison to scenario 2 are the following:

- Recovery of $49 \mathrm{~kg} / \mathrm{d}$ nitrogen in N-MD. This equals to $229 \mathrm{~kg}$ of pure ammonium sulphate.

- $-135 \mathrm{kWh} / \mathrm{d}$ electricity demand for aeration in activate sludge treatment due to alternative nitrogen removal

- $\quad+126 \mathrm{kWh} / \mathrm{d}$ electricity demand for heat pump for providing heat to the N-MD (the heat pump is also bigger $181 \mathrm{~kW}$ th)

- $+10 \mathrm{kWh} / \mathrm{d}$ for operating the N-MD

- $+230 \mathrm{kWh} / \mathrm{d}$ biogas yield $(+3.5 \%)$ due to decreased COD demand in activate sludge treatment due to alternative nitrogen removal - COD now available for biogas production

- $\quad-660 \mathrm{~m}^{3}$ of main cleaning basin. The main cleaning basin can be reduced from 3,980 $\mathrm{m}^{3}$ to $3,320 \mathrm{~m}^{3}$

The main purpose of recovering nitrogen/ammonia is joined by the increase of biogas yield and a reduction of aeration demand. The latter balances out the energy demand of the N-MD. 
Scenario 1: Baseline

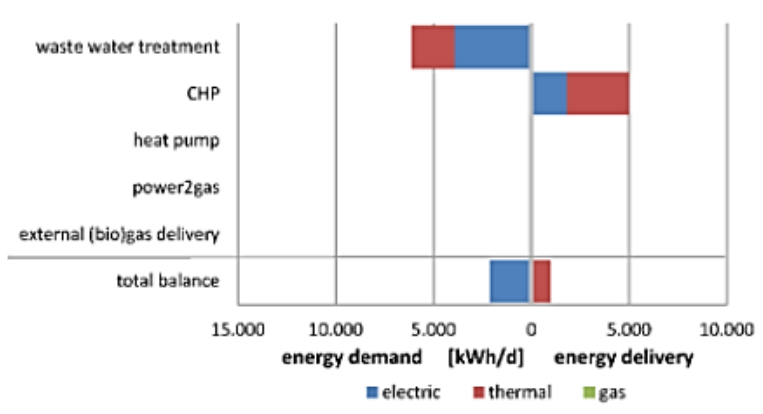

Scenario 3a: Selling of biogas / N-MD

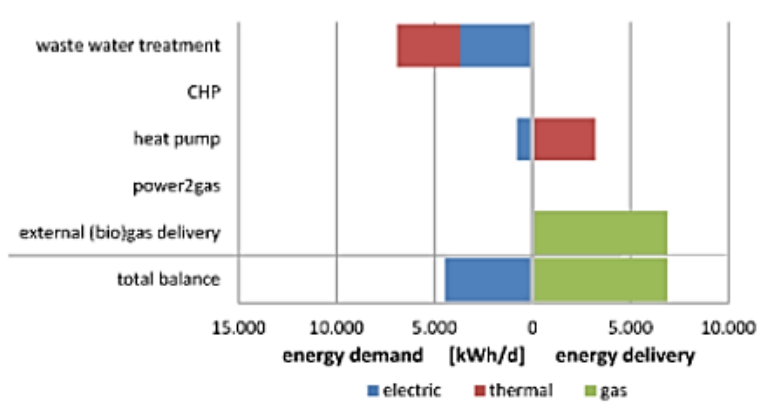

Scenario 3c: Power2Gas plant

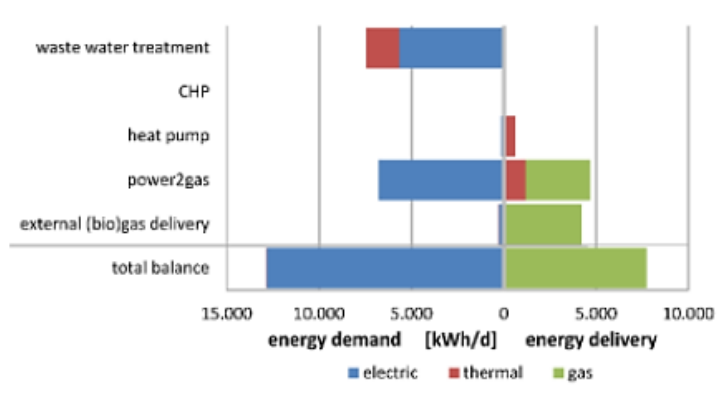

Scenario 2: Biogas Increase

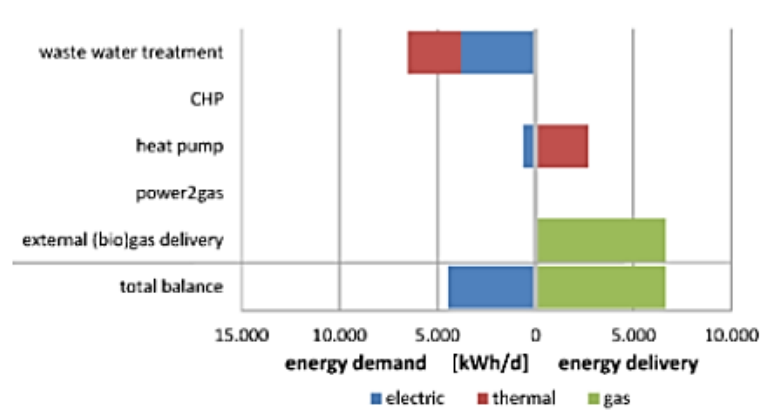

Scenario 3b: Biogas treatment

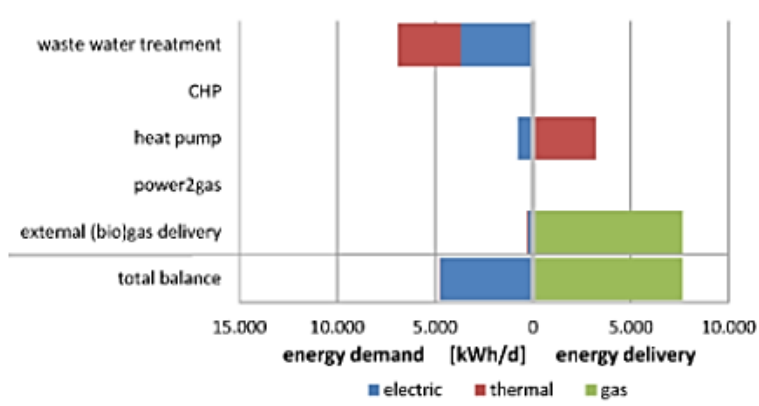

Figure 2: Energy balance (electric, thermal and gas) of WWTP. Each scenario shows the energy demand to the left and the energy delivery to the right. First line is the actual waste water treatment, line 2-5 are the energy conversion units, last line is the total energy balance of the WWTP

Figure 3 adds to the energetic analysis also the economic

and ecological evaluation of the scenarios 1, 2 and 3a. The energetic balance shows how scenario 1 has an overall energy demand ( $280 \mathrm{MWh} / \mathrm{a})$, while the scenarios 2 and 3a offer surplus energy. This is mainly due the utilization of waste water heat by the heat pump. It also shows that including the NMD results in an increase of energy surplus (991 MWh/a) compared to scenario 2 (907 MWh/a). Energy savings for fertilizer substitution outside of the system boundaries of the WWTP are not considered throughout this paper.

The net annual costs for baseline scenario is lower than for the new proposed scenarios. The baseline scenario has costs of $824 \mathrm{~T} €$ while scenario 2 has $896 \mathrm{~T} €$ and scenario $3957 \mathrm{~T} €$ of costs. This is mainly due to the increased size of pretreatment basin (from 330 to $1000 \mathrm{~m}^{3}$ ) and digestion tower $\left(1,330\right.$ to $\left.2,200 \mathrm{~m}^{3}\right)$ which effects the costs more than the reduction of the necessary size of the main cleaning basin $\left(4,120\right.$ to 3,980 respectively $\left.3,320 \mathrm{~m}^{3}\right)$. In these scenarios the revenue of biogas and fertilizer selling are not profitable. The different downstream biogas selling options are discussed in the next part of this paper. A detailed cost and benefit analysis of the N-MD is a future task.

In the annual costs method, no retrofit considerations are done but purely a full reinvestment in all equipment is considered. Thus, the actual investment costs may differ significantly if current basins can be reused and the actual capacity constraints are considered in the planning phase of a plant expansion. 
On the other hand, the greenhouse gas emissions (GHG) balance already shows the positive effect of the new 165 proposed systems. While scenario 2 (1,524 $\mathrm{tCO}_{2}$ equ/a) can reduce GHG by $22 \%$ from the baseline scenario $(1,946 \mathrm{tCO} 2 \mathrm{equ} / \mathrm{a})$, scenario 3 can even lower that value to $1,299 \mathrm{tCO}$ equ/a. The substitution of natural gas and the decrease of direct $\mathrm{N} 2 \mathrm{O}$ emissions are main positive factors and cover the negative effects of increased electricity consumption and increased release of methane in the bigger digestion tower.

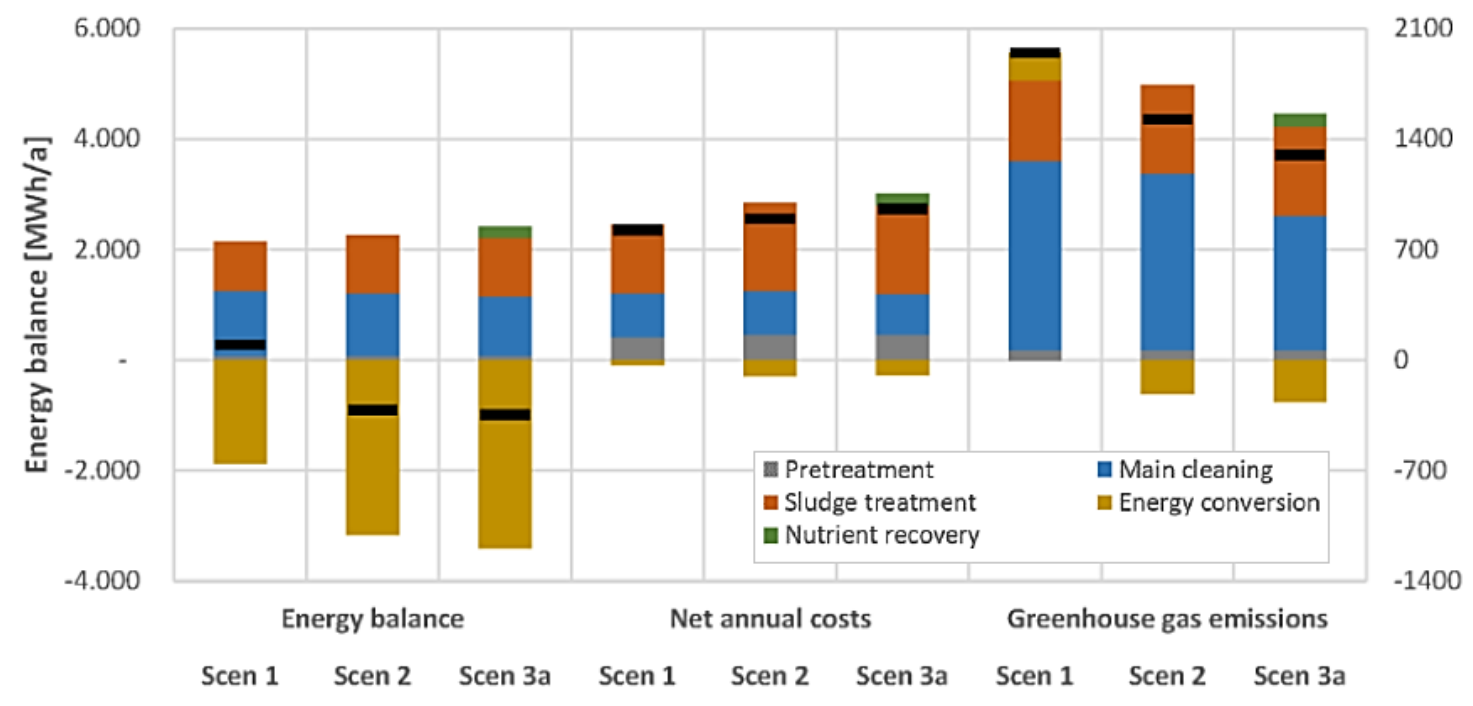

Figure 3: Energetic (left), economic (middle) and ecologic (right) comparison of scenario 1, 2 and 3a categorized in different clusters of a WWTP. Black lines indicate the value for the whole WWTP. Numbers are given in the text above.

\section{ANALYSIS OF BIOGAS UTILIZATION TO ENERGY GRIDS}

In this part, the different biogas utilization scenarios $3 \mathrm{a}, 3 \mathrm{~b}$ and $3 \mathrm{c}$ are analysed. The energetic analysis in Figure 2 has shown that - compared to the baseline scenario - in all scenarios the electricity consumption increases in order to utilize biogas externally. Besides the uncertainties in investment costs, the operating costs for electricity and the selling price of biogas or gas in grid quality are the main factors on the net annual costs of the scenarios.

Figure 4 shows that scenario 3c (Power2Gas) is the best if a gas price of more than $0,075 € / \mathrm{kWh}$ can be achieved. The selling of biogas to district heating (or other direct users of biogas) is best if a lower price for the gas is defined. In this comparison it is assumed that $50 \%$ higher prices can be achieved for methane (gas in grid quality) than for biogas. However, the selling of biogas is still preferable in all scenarios below from an economic point of view. The Power2Gas scenario profits in this analysis by assuming it operates using EPEX spot prices. So instead of $0.10 € / \mathrm{kWh}$ only $0.0183 € / \mathrm{kWh}$ (in average) has to be paid for electricity. 


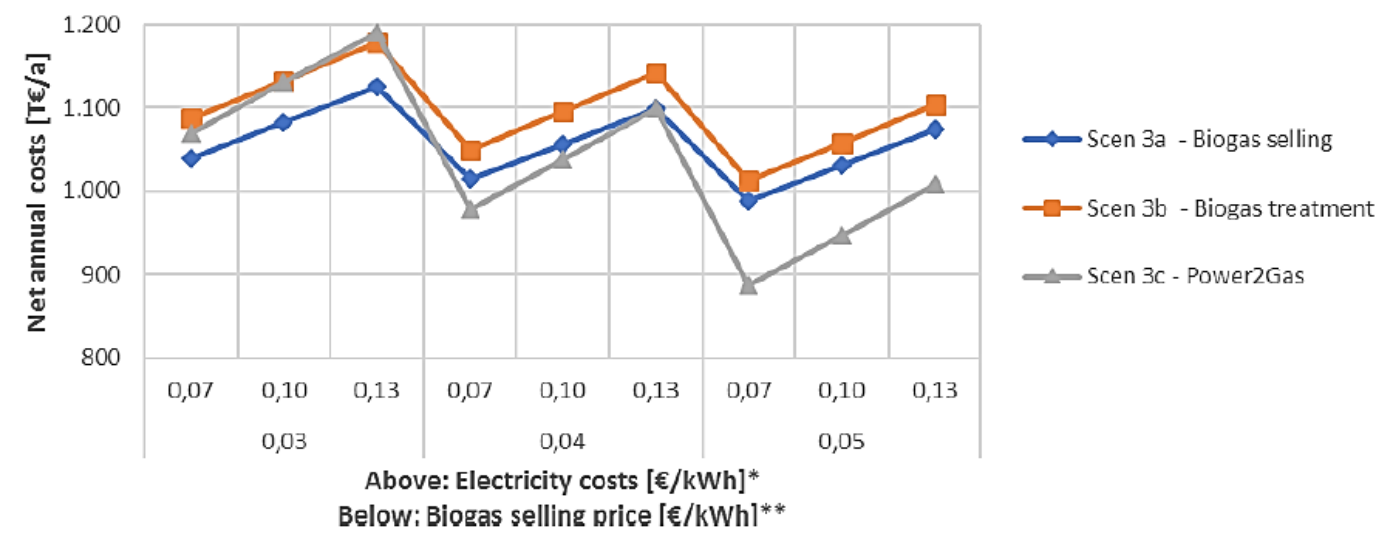

Figure 4: Economic analysis of biogas utilization to different energy grids.

* Electricity costs: EPEX spot price $0.0128|0.0183| 0.0237 € / \mathrm{kWh}$ for Scenario $3 \mathrm{c}$

$* *$ Gas selling price: $0.045|0.060| 0.75 € / \mathrm{kWh}$ for Scenario $3 \mathrm{~b}$ and $3 \mathrm{c}$

\section{CONCLUSIONS}

Selling biogas instead of using it within the boundaries of the WWTP has the advantage of using the full potential of biogas. It gives more incentives in investing in measures to increase the biogas yield. Biogas could be utilized in a nearby district heating network or other end users (e.g. industries) which is more profitable than other options if selling prices are low. Power2Gas is a profitable option if EPEX spot prices can be used and the plant is operated in a way to exploit low spot prices and thus also helping grid stability.

The N-MD has positive effects in nutrient recovery (ammonia), biogas yield (+3.5\%) and electricity consumption. The net annual costs are higher than the baseline scenario, but the greenhouse gas emissions are lower. The DEST which has been used for these analyses is verified and allows quick and easy comparison of different WWTP of the future.

\section{DISCUSSION AND OUTLOOK}

The paper presented an approach where a heat pump replaces the internal biogas utilization. Although this has positive effects on the thermal efficiency of the plant, it should be critically stated that this approach follows the overall trend in electrification of processes due to low electricity prices. Higher electricity prices would also favor an internal utilization of biogas to electricity and heat.

A detailed cost analysis of the N-MD is currently made to evaluate the potential in cost reductions both in investment and operation, but also in increasing the revenue like potential selling prices for fertilizer. Power2Gas has many synergy effects with other parts of a WWTP. Biogas treatment is one example (using $\mathrm{CO}_{2}$ off-gas for methanisation or using the same infrastructure to sell gas to the grid), while using $\mathrm{O}_{2}$ for aeration is another.

\section{REFERENCES}

DWA, (2016), DWA-A 131 Dimensioning of Single-Stage Activated Sludge Plants, Hennef, Germany: DWA.

ÖWAV, (2016), Branchenbild der österreichischen Abwasserwirtschaft, Wien

\section{CONFERENCE TOPIC}

- Hybridization of energy sectors

- Energy and Resource Recovery from Low-grade Sources

- Business models and financing schemes 


\title{
PCM STORAGE FOR INDUSTRY
}

\author{
Thomas Aigenbauer MSc., Dr. Gerald Steinmaurer, Dr. Bernhard Zettl \\ University of Applied Sciences Upper Austria \\ Ringstraße 43a, 4600 Wels, Austria \\ Phone: +4350804 / 46919 \\ E-Mail: thomas.aigenbauer@fh-wels.at
}

\section{SUMMARY}

The aim of the project was to find a phase change material as heat storage for a medium temperature cooling application. The variety of paraffin waxes and salt hydrates as medium temperature PCMs is very limited due to a limitation of the melting temperature to a range of $110-135^{\circ} \mathrm{C}$. Pure salts have significantly higher melting temperatures (above $250{ }^{\circ} \mathrm{C}$ ), so the first task of this project was, to find suitable PCM's between the temperature range of 135 and $190^{\circ} \mathrm{C}$ for an industrial process under vacuum conditions. Six promising materials were selected and examined in more detail. A cycling unit was built, which handled more than 60 samples of PCM at once. The cycling stability of six PCM candidates was determined up to 5000 cycles and also corrosion behaviour was determined. Four of six materials showed sufficient thermal and cycling stability and are suitable for this application.

Key-words: PCM, batch process, cycling stability, thermal stability, industrial process

\section{INTRODUCTION}

A major share of the thermal energy consumed in industry is in the medium temperature range between 100 and $300^{\circ} \mathrm{C}$ [1]. In different industry branches, thermal batch processes are very common and provide a huge energy saving potential, when using phase change material as thermal storage material.

First target of the project was to find and test possible PCM's, which fits the requirements for industrial applications. Beside the melting temperature also the material parameters heat of fusion, thermal and chemical stability, toxicity, separation behaviour and also the heat transfer coefficient were for important interest and were listed. The availability and material price was also examined for economic comparison. All in all, more than 40 PCM's were found like salt eutectics, carboxylic acids and phenols, sugar alcohols, polymers and metals. Under these consideration, and after some corrosion tests with possible heat exchange materials, six promising PCM's were studied in more detail and examined in certain intervals with Differential Scanning Calorimetry (DSC).

\section{METHODS AND RESULTS}

Three different methods were used to identify thermal and cycling stability of the materials. In the first method the mass signal during the DSC measurement was used as an indicator of the thermal stability of the PCM, which were overheated during the measurement to $200^{\circ} \mathrm{C}(10 \mathrm{mg}$ samples, $10 \mathrm{~K} / \mathrm{min}$, $40 \mathrm{ml} / \mathrm{min} \mathrm{N}_{2}$ ). The second was a cycling stability test (referring to the real batch process) with up to 5000 cycles and partial phase change. Partial phase change (i.e. partial melting - with remaining solid core material) was assumed as typical condition for this kind of cooling application in order to avoid sub-cooling of crystallite PCM. In the third method a constant thermal stress test $\left(180^{\circ} \mathrm{C}\right)$ without phase change (liquid) was investigated. Closed metal tubes (shown in Fig.1) were used as encapsulation and before sealing the fine thread with a PTFE band and a two-component epoxy adhesive, the samples were purged with argon to prevent oxidation reaction of the PCM. 

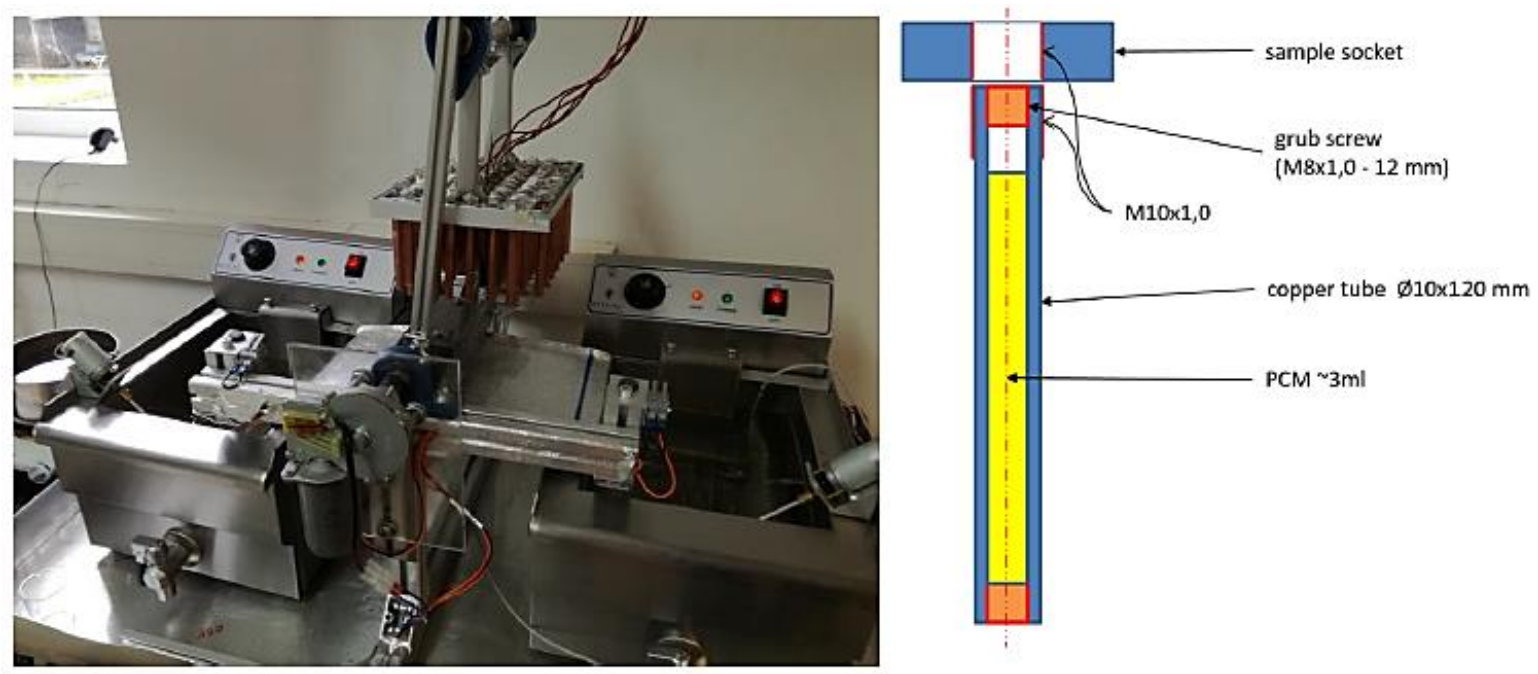

Figure 1: The cycling unit (left) with two oil bath (heating and cooling), a sample holder for specimen, and a mechanical construction for movement. Schematic of the sample tube (right) made of either Aluminium or

Copper;

All three methods attested four of six materials good thermal and cycling behaviour and contrary to other publications [2, 3] also the material D-Mannitol (shown in Fig.2) seems that it can be used as heat storage material for a longer time of period in the application field of industrial processes.

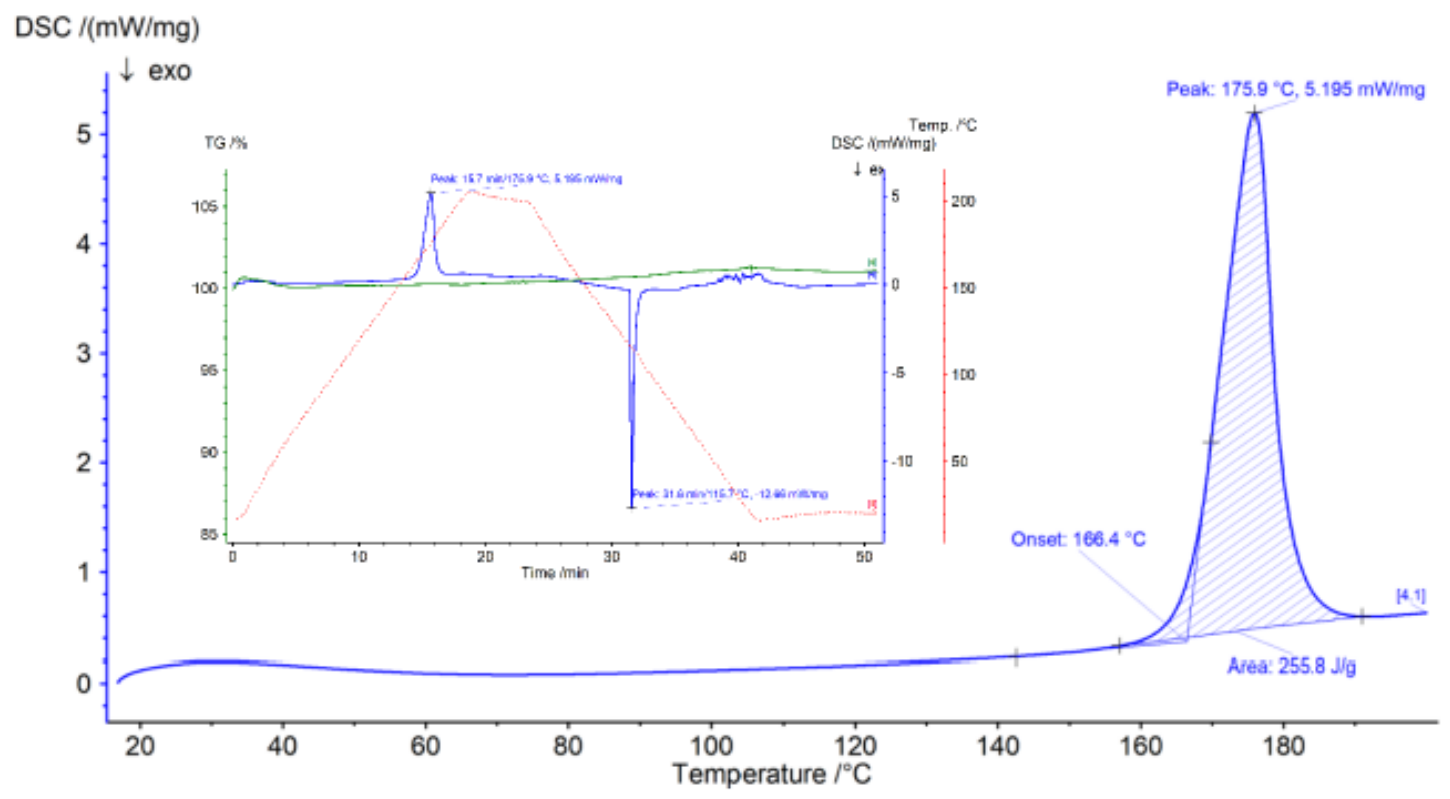

Figure 2: DSC result of D-Mannitol after 5000 cycles

Also the constant thermal stress test in a $180^{\circ} \mathrm{C}$ oven, tested with d-mannitol filled in aluminium and copper tubes, showed good values for the application in the industry process (shown in Figure 3 and Table 1). 


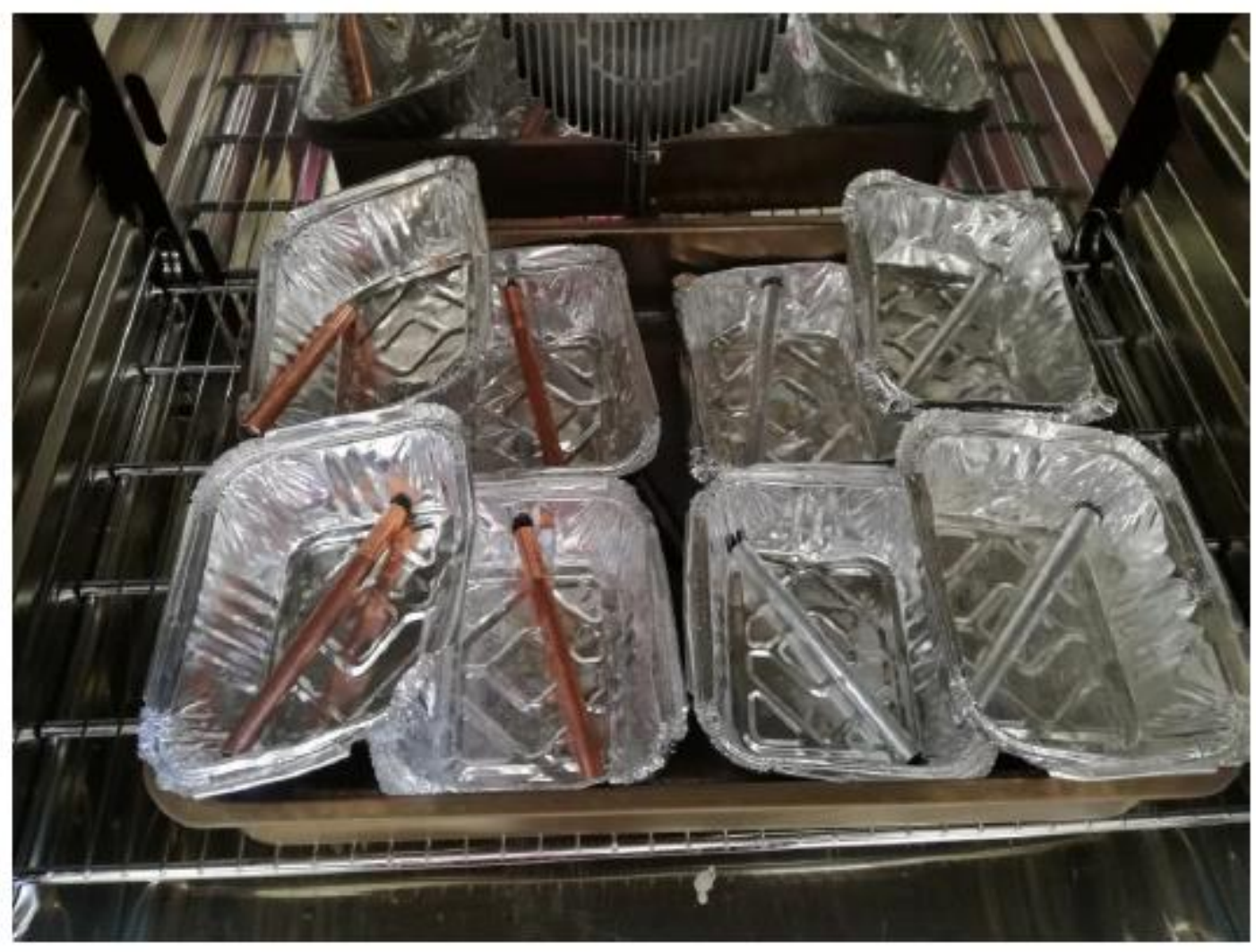

Figure 3: Prepared copper and aluminium D-Mannitol samples in the hot air convection oven $\left(180^{\circ} \mathrm{C}\right)$

Table 1: Results of the constant thermal stress test with aluminium and copper tubes

\begin{tabular}{|c|c|c|c|c|}
\hline & \multicolumn{2}{|c|}{ aluminium sample } & \multicolumn{2}{|c|}{ copper sample } \\
\hline PCM & $\begin{array}{l}\text { Melting Point } \\
\text { (Onset / Peak) }\end{array}$ & Latent Heat Capacity & $\begin{array}{l}\text { Melting Point } \\
\text { (Onset / Peak) }\end{array}$ & Latent Heat Capacity \\
\hline D-Mannitol (theory) & $167^{\circ} \mathrm{C}$ & $270 \mathrm{~kJ} / \mathrm{kg}\left(410,4 \mathrm{~kJ} / \mathrm{dm}^{3}\right)$ & $167^{\circ} \mathrm{C}$ & $270 \mathrm{~kJ} / \mathrm{kg}\left(410,4 \mathrm{~kJ} / \mathrm{dm}^{3}\right)$ \\
\hline pure material & $164,7 / 176,0$ & 291,6 & $164,7 / 176,0$ & 291,6 \\
\hline first time melted & $167,2 / 182,6$ & 266,1 & $167,2 / 182,6$ & 266,1 \\
\hline after $122 \mathrm{~h}$ & $164,6 / 174$ & 242,1 & $164,8 / 174,4$ & 247,2 \\
\hline after $290 \mathrm{~h}$ & $162,7 / 172,7$ & 228,7 & $164,0 / 173,7$ & 242,5 \\
\hline after $456 \mathrm{~h}$ & $161,5 / 170.9$ & 184 & $162,9 / 172,1$ & 208,2 \\
\hline
\end{tabular}

After $456 \mathrm{~h}$ and $13 \mathrm{~K}$ above the theoretical melting point of D-Mannitol, the material has lost about 1/3 of the initial heat of fusion. The melting temperature has also a decreasing tendency and it is more marked with the aluminium tube than with the copper tube samples.

Negative results are made with the commercial available product X165 (solid-solid phase change) and with the eutectic mixture $\mathrm{KOH}: \mathrm{NaOH}$. As it can be seen on Table 2, the DSC measurement with the pure material of X165 was more or less in the range of the theoretical values, the following measurements was examined with a mixture of solid X165 and paraffin, to increase the heat conduction in the copper tube. But the material combination seems to be unstable, so the latent heat capacity decrease while the melting temperature increase. 
Table 2: Negative result with the solid-solid PCM X165 mixed with thermal oil

\begin{tabular}{|c|c|c|}
\hline PCM & $\begin{array}{c}\text { Melting Point } \\
\text { (Onset bzw. Peak) }\end{array}$ & Latent Heat Capacity \\
\hline X165 & $165^{\circ} \mathrm{C}$ & $172,5 \mathrm{~kJ} / \mathrm{kg}\left(225 \mathrm{~kJ} / \mathrm{dm}^{3}\right)$ \\
\hline Pure material & $159,3 / 182,4$ & 190,2 \\
\hline First time melted & $160,2 / 182,8$ & 123,5 \\
\hline Zyklus 100 & $167,2 / 187,5$ & 105,8 \\
\hline Zyklus 300 & $177,2 / 189$ & 108,9 \\
\hline Zyklus 500 & $177 / 189,7$ & 112,0 \\
\hline Zyklus 1000 & $174,7 / 189,4$ & 125,7 \\
\hline Zyklus 1500 & $174,1 / 189,8$ & 122,6 \\
\hline Zyklus 2000 & - & - \\
\hline Zyklus 3000 & $177,2 / 190,2$ & 114 \\
\hline Zyklus 5000 & $179,4 / 190,7$ & 80,8 \\
\hline
\end{tabular}

The eutectic mixture $\mathrm{KOH}: \mathrm{NaOH}$ with a theotetical high latent heat capacity of $599,17 \mathrm{~kJ} / \mathrm{dm}^{3}$ and an melting point of $170^{\circ} \mathrm{C}$ was also a negative result. The material preparing was good and the expacted melting temperature correlated with the theoretical values in literature, but the results in the DSC measurements were not clear and showed no good latent heat capacity. Also the handling with the material was very problematic because of the highly caustic and corrosion behaviour, so we stopped the test with this material after a few measurements.

Parallel to the material testing, numerical finite element simulation were investigated, to identify good heat exchanger geometries. For the optimisation process the parameter temperature rising per second during the melting period was used to get the best design, to round over the use of PCM in industrial application.

\section{REFERENCES}

[1] Tamme, R., (2010), Optimized Industrial Process Heat and Power Generation with Thermal Energy Storage. IEA ECES Task 19 Final Report.

[2] Baýon, R., Rojas, E., (2017), Feasibility study of D-Mannitol as phase change material for thermal storage. AIMS Energy, 5(3): 404-424.

[3] Solé, A., et al., (2013), Thermal stability test of sugar alcohols as phase change materials for medium temperature energy storage application. SHC 2013, International Conference on Solar Heating and Cooling for Buildings and Industry.

\section{CONCLUSION}

The first DSC measurement tests showed, that the potential PCM materials react more or less sensitive by heating it up over the melting temperature. Where d-mannitol shows no significant mass loss up to $200^{\circ} \mathrm{C}$ during the DSC measurement, the material Puretemp $151 \mathrm{X}_{\circledast}$ lose $30 \%$ of the mass during the test, starting some Kelvin above the melting temperature. All in all, temperatures above the melting point should always be avoided if possible, because of the higher decreasing rate of heat of fusion.

After the cycling stability test, four of the six tested materials showed the potential to fulfil the requirements of the industry process and our project partner. Only a minimal degradation of about 5$10 \%$ of the melting temperature and latent heat capacity was measured. For D-Mannitol, the stability results shows a lower degradation than some literature expose, with an heat of fusion of 255,8 J/g and the onset melting point of $166,4^{\circ} \mathrm{C}$. Also the results in the constant thermal stress test showed acceptable values, in case of the copper tube sample, after $465 \mathrm{~h}$ exposure time D-Mannitol had a heat of fusion of $208 \mathrm{~J} / \mathrm{g}$ and an onset melting point of $162,9^{\circ} \mathrm{C}$. This high temperature time duration is much longer than in the cycling test (about 4 times) and tested also with higher thermal stress (because all the material is melted). So after this several material tests the conclusion is, that four PCM 
materials would fullfill 5000 cycles in the industrial process. Because of the relative cheap price per $\mathrm{kg}$ of the PCM's, it makes it possible to refill the heat exchanger during the annuity servicing.

The relevant link from the cycling test to the real cooling application in this paper was the percentage amount of melted PCM, similar geometry of the heat exchanger surface to PCM, but with very high heat flux to achieve an accelerated cycling test. For further research, a longtherm measurement test with one small segment but real dimensions and heat flux over a year, which simulate the industrial process, would be a recommended step before implementing it in the production line. 


\title{
INVESTIGATION OF THE CYCLING STABILITY OF SORBENT COMPOSITES FOR SORPTION THERMAL ENERGY STORAGE APPLICATIONS
}

\author{
Dr Elpida Piperopoulos1, Dr Vincenza Brancato2, Dr Larisa G. Gordeeva3,4, Dr Alexandra D. Grekova3,4, Dr \\ Candida Milone1, Dr Andrea Frazzica2 \\ Department of Engineering, University of Messina 1 \\ Contrada di Dio, 98166 Messina, Italy \\ Phone: +39 090/3977558 \\ CNR - Istituto di Tecnologie Avanzate per 1_Energia "Nicola Giordano"2 \\ Via Salita S. Lucia sopra Contesse 5, 98126 Messina, Italy \\ Boreskov Institute of Catalysis 3 \\ Ac. Lavrentiev av. 5, Novosibirsk 630090, Russia \\ Novosibirsk State University4 \\ Pirogova str. 2, Novosibirsk 630090, Russia \\ E-Mail: epiperopoulos@unime.it
}

\section{SUMMARY}

The present work deals with the characterization of an innovative sorbent composite LiCl/MWCNT/PVA, based on $\mathrm{LiCl}$ supported on multiwall carbon nanotubes (MWCNT), and shaped with PolyVinilAlkohol (PVA) as a binder, specifically developed for sorption thermal energy storage (TES) applications. The phase composition of the composite and its transformation during the ad/desorption cycles are studied. Particularly, the synthesized samples were investigated in terms of sorption capacity and characterized by temperature programmed X-ray diffraction analysis and scanning electron microscopy (SEM) operating under controlled humidity and temperature conditions.

Keywords: sorbent composite, sorption storage, stability, cycling, XRD, SEM

\section{INTRODUCTION}

Sorbent composites, often reported in literature as CSPMs (Composite "Salt in a Porous Matrix") [1], represent a family of materials suggested for heat transformation applications (i.e. cooling, heat pumping and heat amplification) about 20 years ago. They consist of an inorganic salt placed inside a porous structure (e.g. silica gel, vermiculite). Some research groups developed different compositions for sorption heat transformation and TES applications. These materials have demonstrated the ability to overcome (or alleviate) some of the constraints typical of the bulky systems "salt - salt hydrates" (namely adsorption/desorption hysteresis and poor mass-transport) and of pure porous matrix (small adsorption capacity toward water, alcohols and ammonia). Indeed, these materials are characterised by extremely high, water sorption capacity (e.g. higher than $0.5 \mathrm{~g} / \mathrm{g}$ ), low regeneration temperature (i.e. less than $95^{\circ} \mathrm{C}$ ) in line with solar thermal applications, and good potential to reach low cost, thanks to cheap raw materials and simple production process. Furthermore, due to their structure, the sorption capacity can be tailored by varying sorbent compositions (the chemical nature of the salt and the matrix, the salt content) and synthesis procedures, that positions them to an intermediate class of sorbents that combine advantages of absorbents, adsorbents and chemi-sorbents. Nevertheless, the stability of these materials under typical operating conditions of sorption is still an open issue and needs to be carefully investigated. This work aims to the characterization of the stability for a novel composite sorbent $\mathrm{LiCl} / \mathrm{MWCNT} / \mathrm{PVA}$ recently developed at the Boreskov Institute of Catalysis, based on carbon nanotubes as porous matrix and $\mathrm{LiCl}$ as embedded salt [2].

\section{COMPOSITE SORBENTS SYNTHESIS}

The composite sorbent LiCl/MWCNT/PVA was synthesized through a dry impregnation. The MWCNT were supplied by the Laboratory of Surface Compounds Synthesis of the Boreskov Institute of Catalysis. The composite sorbent was synthesized from the pristine MWCNT powder by using polyvinyl alcohol (PVA) as a binder. In order to prepare the composite, $3 \mathrm{~g}$ of MWCNT and $20 \mathrm{ml}$ of a $15 \mathrm{wt} . \%$ of PVA aqueous solution were mixed for $10 \mathrm{~min}$. Then the paste was extruded and dried at room temperature for $16 \mathrm{~h}$ and then at $90^{\circ} \mathrm{C}$ for $2 \mathrm{~h}$. The dried extrudate was calcinated in argon flow at $400 \mathrm{oC}$ for $4 \mathrm{~h}$ to carbonize the PVA binder. The calcinated MWCNT/PVA was grinded and sieved to get grains. The grains were impregnated with the alcoholaqueous solution of $\mathrm{LiCl}\left(\mathrm{H}_{2} \mathrm{O}: \mathrm{EtOH}=\right.$ $1: 1)$ of appropriate concentration and dried again at $160 \mathrm{oC}$ for $16 \mathrm{~h}$. The volume of the solution was 
equal to the pore volume of the MWCNT/PVA composite $V_{\text {com. }}$ The $\mathrm{LiCl}$ content in the $\mathrm{LiCl} / \mathrm{MWCNT} / \mathrm{PVA}$ composite was equal to $\_s=55 \mathrm{wt}$. $\%$. The texture characteristics (microand mesopores volume $V_{\mathrm{m}}$ and specific surface area $S_{\mathrm{sp}}$ ) of the MWCNT/PVA and the LiCl/MWCNT/PVA grains were measured by a low-temperature nitrogen adsorption and presented in Table. The total pore volume $V_{\text {com }}$ of the samples was measured as the maximum volume of ethanol, which can be caught by the composite without leaking out of its pores.

Table 1: The total pore volume $V_{c o m}$, meso- and micropore pore volume $V_{m}$, specific surface area $S_{s p}$ of the MWCNT/PVA and LiCl/MWCNT/PVA composites.

\begin{tabular}{cccc}
\hline Sample & $V_{\text {com }}, \mathrm{cm}^{3} / \mathrm{g}$ & $V_{\mathrm{m}}, \mathrm{cm}^{3} / \mathrm{g}$ & $S_{\text {sp }}, \mathrm{m}^{2} / \mathrm{g}$ \\
\hline MWCNT/PVA & 2.4 & 1.30 & 220 \\
\hline LiC1/MWCNT/PVA & 0.9 & 0.24 & 80 \\
\hline
\end{tabular}

\section{CHARACTERIZATION METHODS}

Isotherms of water vapor sorption on the synthesized LiCl/MWCNT/PVA were measured by a thermogravimetric technique on an automated vapor sorption analyzer (DVS Vacuum, Surface Measurement Systems Ltd.) in a range of the relative humidity $\eta=0.2-20.0 \%$ at temperatures 35,55 and $70^{\circ} \mathrm{C}$. Initially, the LiCl/MWCNT/PVA sample (about $20 \mathrm{mg}$ ) was loaded into the stainless steel crucible and placed into the instrument. Prior to being exposed to vapor flow, the sample was slowly heated up to $150^{\circ} \mathrm{C}$ under vacuum for $5 \mathrm{~h}$ (vacuum level: $0.1 \mathrm{~Pa}$ ), in order to degas the sample and determine its dry weight. Afterwards, the sample was cooled down to the adsorption temperature and then isothermally exposed to the desired relative pressure. The resulting vapor uptake was monitored under dynamic vapor flow. A series of equilibrium points was acquired by directly measuring the sample weight variation in response to several stepwise relative pressure changes under isothermal conditions. From each equilibrium point, the water uptake was calculated as

$w=m\left(P_{\mathrm{H} 2 \mathrm{O}}, T\right) / m 0$

(eq. 1)

where $m\left(P_{\text {H2о }}, T\right)$ and $m o$ are the equilibrium and dry weight of the sample, respectively. The obtained results were then used to calculate the achievable TES capacity under typical daily storage cycles.

LiCl/MWCNT/PVA sample was analyzed by means of X-Ray Diffraction (XRD, Bruker D8 Advance, Bruker, Billerica, MA, USA) and environmental scanning electron microscopy (ESEM, Quanta 450, FEI, Hillsboro, OR, USA) to determine morphology and crystal structure changes during the hydration/dehydration cycles. To study the stability of the composite sorbent crystalline structure during dehydration the diffractograms were acquired on the hydrated sample in a 2-theta range between 10 and 80 , in vacuum $\left(6 \times 10_{-2}\right.$ mbar) from room temperature up to $150^{\circ} \mathrm{C}$.

ESEM analysis was performed on $\mathrm{LiCl} / \mathrm{MWCNT/PVA} \mathrm{sample,} \mathrm{operating} \mathrm{with} \mathrm{an} \mathrm{accelerating} \mathrm{voltage}$ of $10 \mathrm{kV}$ at $35^{\circ} \mathrm{C}$ in controlled water vapor atmosphere, from $10 \mathrm{~Pa}$ to $800 \mathrm{~Pa}(0.1 \%$ to $14.3 \%$ of relative humidity), repeating the same conditions of hydration and vice versa those of the sample dehydration.

\section{RESULTS AND DISCUSSION}

The water adsorption isotherms presented as function of the Polanyi adsorption potential $\_F=-R T$ $\ln \left(P / P_{0}\right)$ are step-wise curves with two steps attributed to the formation of a mono-hydrate $\mathrm{LiCl} \cdot \mathrm{H}_{2} \mathrm{O}$ and di-hydrate $\mathrm{LiCl} \cdot 2 \mathrm{H}_{2} \mathrm{O}$ (Fig.1). The $\mathrm{LiCl} \cdot \mathrm{H}_{2} \mathrm{O}$ hydrate is stable over wide $\Delta F$-range. Similar steps were observed for the water sorption equilibrium on LiCl/MWCNT powder in [2]. Thus, shaping the grains with PVA does not change the character of water sorption equilibrium of the composite. Sorption/desorption hysteresis is observed at $N<2$. Indeed, the decomposition of the hydrates 
$\mathrm{LiCl} \cdot \mathrm{H}_{2} \mathrm{O}$ and $\mathrm{LiCl} \cdot 2 \mathrm{H}_{2} \mathrm{O}$ occurs at a larger $\Delta F$ than their formation. At $\Delta F<5.5 \mathrm{~kJ} / \mathrm{mol}$, the isotherms are smooth curves that indicate the formation of aqueous $\mathrm{LiCl}$ solution. Smooth parts of the isotherms almost coincide with each other, which gives one-to-one correspondence between the amount of the sorbed water and the adsorption potential $\Delta F$. The TES capacity of LiCl/MWCNT/PVA under conditions of the daily TES cycle [2] was estimated as 1.57-1.67 kJ/g at desorption temperature 75 and $85^{\circ} \mathrm{C}$, respectively.

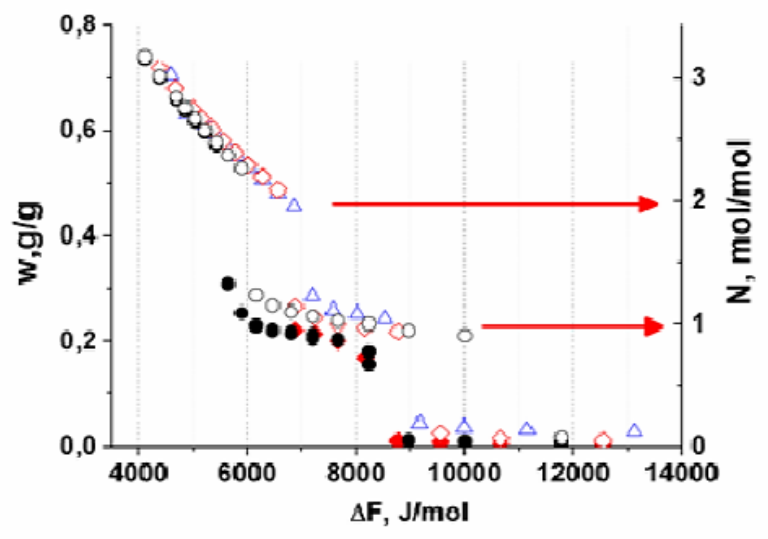

Fig. 1: Water sorption on $\mathrm{LiCl} / \mathrm{MWCNT} / \mathrm{PVA}$ as function of the adsorption potential $\Delta \mathrm{F}$ at various $\mathrm{T}: 35^{\circ} \mathrm{C}(\mathrm{O}), 55^{\circ} \mathrm{C}$ $(\diamond, \diamond)$ and $70^{\circ} \mathrm{C}(\Delta)$. Open symbols - desorption, solid symbols - sorption.

XRD patterns are shown in Fig. 2. At $25^{\circ} \mathrm{C}$ the peaks of tetragonal lithium chloride hydrate $\mathrm{LiCl} \cdot \mathrm{H}_{2} \mathrm{O}$ (2theta: $32.98^{\circ}, 47.38^{\circ}, 58.84^{\circ}, 68.88^{\circ}, 78.84^{\circ}$ ), in agreement with standard data (JCPDS: 22-1142) are present. The peaks appear sharp and narrow because of high degree of crystallization of the tetragonal $\mathrm{LiCl} \cdot \mathrm{H}_{2} \mathrm{O}$. Increasing the temperature up to $70{ }^{\circ} \mathrm{C}$ the peaks of anhydrous cubic $\mathrm{LiCl}$ (2theta: $30.09^{\circ}, 34.88^{\circ}, 50.16^{\circ}, 59.59^{\circ}, 62.53^{\circ}, 73.65^{\circ}$ ) in agreement with JCPDS 4-664, appear. The structure remains stable up to $150^{\circ} \mathrm{C}$, remaining unchanged until room temperature.

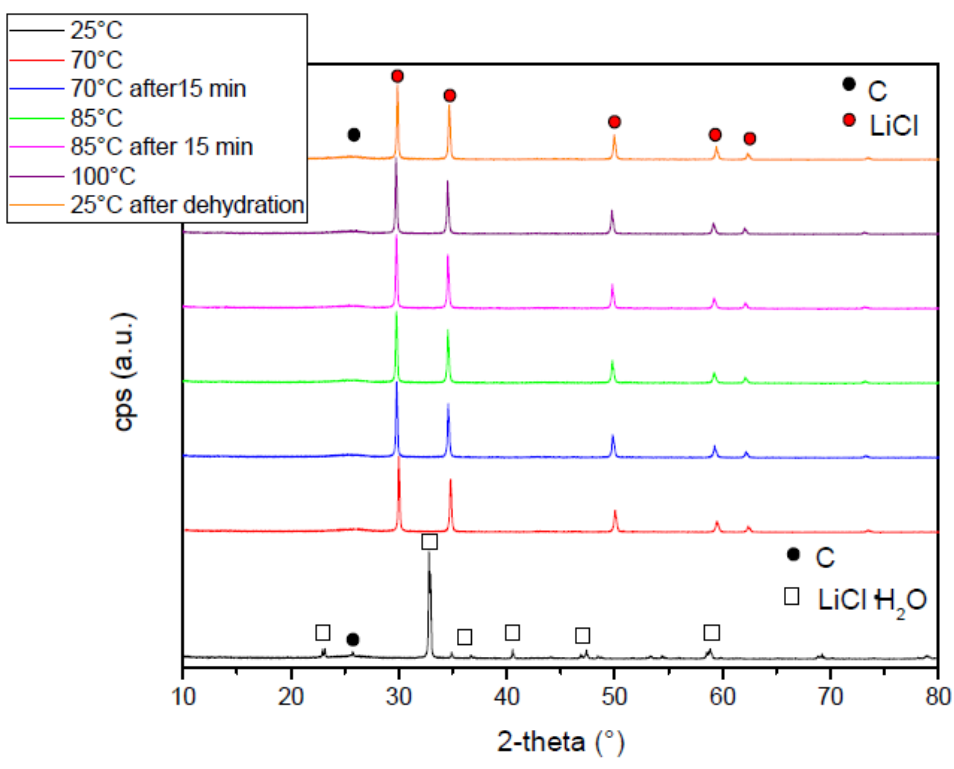

Fig. 2: Temperature programmed XRD analysis in vacuum

As shown in Fig. 3, the investigated sample undergoes a morphological transformation due to hydration of $\mathrm{LiCl}$ according to the reaction $\mathrm{LiCl}+\mathrm{H}_{2} \mathrm{O}=\mathrm{LiCl} \cdot \mathrm{H}_{2} \mathrm{O}$ and subsequently to the dehydration of $\mathrm{LiCl} \cdot \mathrm{H}_{2} \mathrm{O}$ when the water vapor pressure $(P)$ and consequently the relative humidity percentage on the sample changes, at $35^{\circ} \mathrm{C}$. At $400 \mathrm{~Pa}(4$ mbar) the sample still shows its morphology (Fig. 3c), even if after one hour (Fig. 3d), slight differences are perceptible, the salt seems to rise to the surface. While at $800 \mathrm{~Pa}(8 \mathrm{mbar})$, the hydration is evident, and deliquescence occurs (red arrows in 
Fig. 3e), remaining until the dehydration starts. During dehydration, salt dehydrates already at $400 \mathrm{~Pa}$, even if it does not reach the initial morphology at $10 \mathrm{~Pa}$ (compare Fig. 3a and Fig. 3j). In fact, a hysteresis in dehydration is easily identified by the morphology change. 


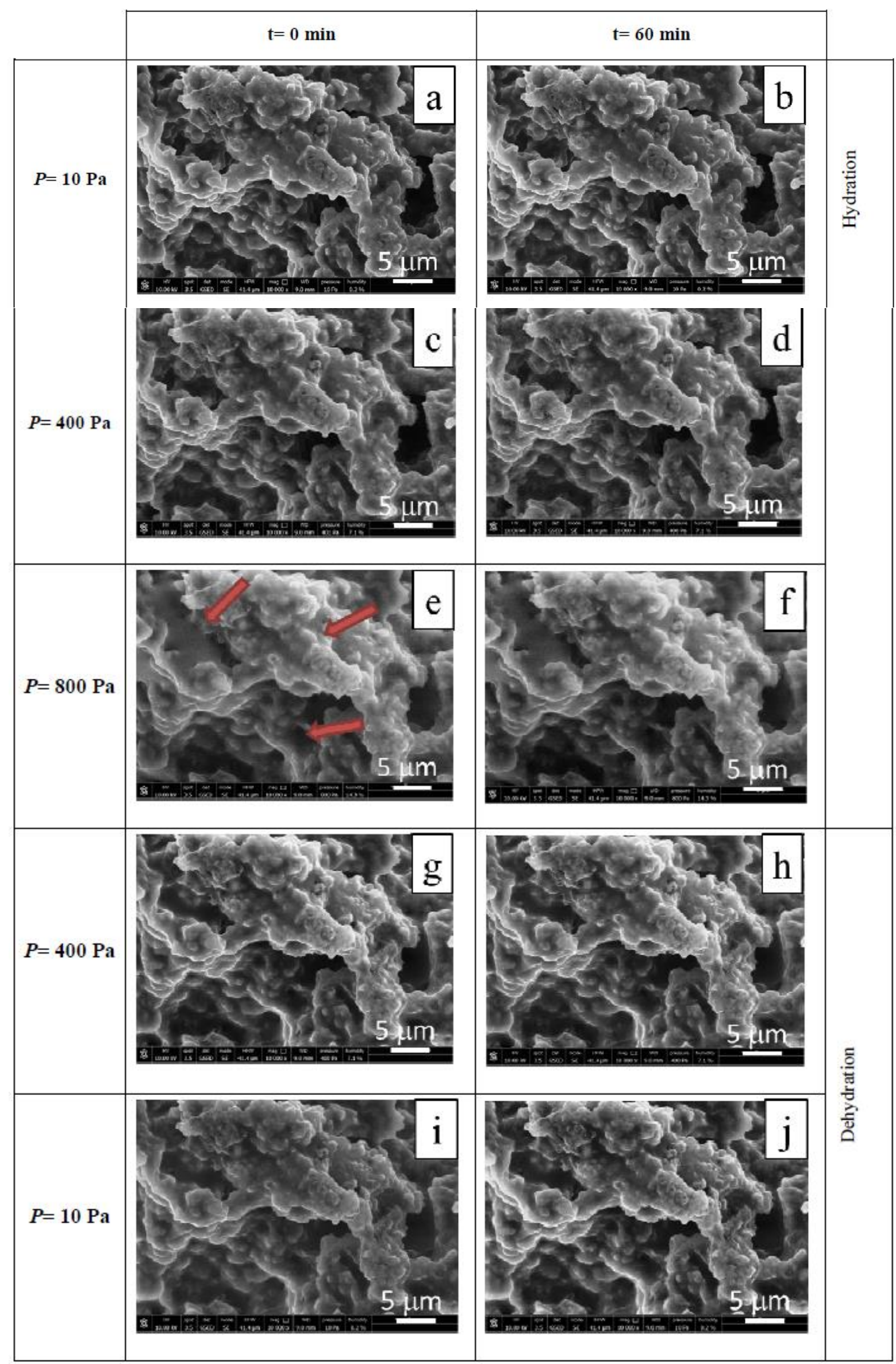

Fig. 3: ESEM analysis of investigated sample at $35^{\circ} \mathrm{C}$ at different water vapor pressure 
The formation of the crystalline hydrate is the probable reason of the adsorption/desorption hysteresis observed on the water sorption isotherms (Fig.1). The formation of crystalline solvates from salt is accompanied by the intense reorganization of the crystalline structure and proceeds with a certain inertia, especially in a lowtemperature range. The presence of inhibitions, i.e. a pronounced pressuretemperature region around equilibrium where the reaction is inhibited, is typical for such a reaction. Only outside this region, the reaction proceeds at a measurable rate [4]. This inhibition region reflects that the formation (adsorption) and decomposition (desorption) branches do not coincide on the theoretical salt sorption isobars, so a sorption/desorption hysteresis occurs.

\section{REFERENCES}

[1] Gordeeva, L. G., Aristov, Yu. I., (2012), Composites "salt inside porous matrix" for adsorption heat transformation: a current state of the art and new trends, Int. J. Low Carbon Technol., 7, 288-302.

[2] Grekova, D., Gordeeva, L. G., Aristov, Yu. I., (2016), Composites "Li/Ca halogenides 135 inside multiwall carbon Nano-tubes" for adsorptive heat storage, Solar Energy Materials and Solar Cells, $155,176-183$.

[3] Brancato, V., Gordeeva, L. G., Grekova, A. D., Frazzica, A, Aristov, Yu. I., Water adsorption equilibrium and dynamics of LiCl/MWCNT/PVA composite for adsorptive heat storage, Sol. Energy Mater. and Sol. Cells (submitted)

[4] Andersson, J. Y., (1986), Kinetic and mechanistic studies of reactions between water vapour and some solid sorbents, Department of Physical Chemistry, The Royal Institute of Technology, S-100 44, $27-44$.

[5] Frazzica, A., Freni A., (2017), Adsorbent working pairs for solar thermal energy storage in buildings, Renewable Energy, 110, 87-94.

[6] Grekova A.D., Gordeeva L.G., Aristov Y.I., (2017), Composite "LiCl/vermiculite" as advanced water sorbent for thermal energy storage, Applied Thermal Engineering, 124, 1401-1408.

[7] Scapino L., Zondag H.A., Van Bael J., Diriken J., Rindt C.C.M., (2017), Sorption heat storage for longterm low-temperature applications: A review on the advancements at material and prototype scale, Applied

Energy, 190, 920-948.

\section{CONCLUSION}

The phase composition and morphology of the composite LiCl/MWCNT/PVA, as well as their transformation during the ad/desorption cycles were characterised. Particularly, the synthesized samples were investigated in terms of sorption capacity and characterised by temperature programmed X-ray diffraction analysis and scanning electron microscopy (SEM) operating under controlled humidity and temperature conditions. It is shown that during water sorption the sample undergoes a morphological transformation due to hydration of $\mathrm{LiCl}$ towards crystalline hydrate $\mathrm{LiCl} \cdot \mathrm{H}_{2} \mathrm{O}$ and subsequently to the dehydration of $\mathrm{LiCl} \cdot \mathrm{H}_{2} \mathrm{O}$. The crystalline hydrate $\mathrm{LiCl} \cdot \mathrm{H}_{2} \mathrm{O}$ formation is likely to be a reason of the water adsorption/desorption hysteresis observed for the LiCl/MWCNT/PVA composite. 


\title{
SORPTION COLLECTOR - PERFORMANCE INCREASE OF CLOSED ADSORPTION STORAGES
}

\author{
$\underline{\text { Rebekka Köll }}{ }^{1}$, Waldemar Wagner ${ }^{1}$, Wim van Helden ${ }^{1}$, Harald Poscharnig ${ }^{2}$ \\ AEE INTEC \\ Feldgasse 19, 8200 Gleisdorf, Austria \\ Phone: +43 $3112 / 5886$ - 264 \\ GREENoneTEC Gmbh ${ }^{2}$ \\ Energieplatz 1, 9300 St. Veit/Glan, Austria \\ E-Mail: r.koell@aee.at
}

\section{SUMMARY}

The conventional storage system, consisting of a main storage and an evaporator/condenser heat exchanger connected to a water reservoir, was extended by a new innovative component. The so called sorption collector is used to increase the storage density based on the charge boost process. The collector prototype is a special designed vacuum tube collector filled with zeolite 13XBF. The charge boost process uses the pressure difference between two storages (main and sorption collector) at different temperature level to dry the material to a higher level compared to the conventional desorption. The sorption collector shows an achievable energy density of $>200 \mathrm{kWh} / \mathrm{m}^{3}$ at a temperature of $150{ }^{\circ} \mathrm{C}$ due to the repetition of the charge boost mode. The maximum measured bed temperature during experiments in a sunny week in January was $115^{\circ} \mathrm{C}$ in the sorption material and > $200{ }^{\circ} \mathrm{C}$ on the outside of the bed. The high temperatures measured show the potential of the sorption collector. The higher the temperature in the collector during day the higher energy density can be achieved. During night it shows a significant temperature and hence also a pressure drop in the collector which is necessary to have an efficient charge boost.

\section{INTRODUCTION}

Thermal energy storage is needed whenever there is a time discrepancy between energy production and energy demand. This time discrepancy is the main challenge for renewable heat sources, since their production is inconsistent or at periods with low heat demand there is a strong need for thermal energy storages, which store heat in times of overproduction and release the heat in times of heat demand. [Hauer A. et al, 2013]

Solar heat in combination with a seasonal storage has the potential to cover $100 \%$ of the heat demand of buildings with completely renewable energy. To store heat efficiently for such a long period of time there are following requirements for the storage technology to fulfil: high energy density to keep the system compact; low losses to store heat for a long period of time, a long lifetime and they need to be cost efficient to compete with alternative energy production.

A technology which can fulfil the mentioned requirements is the thermochemical energy storage. Compared to sensible storages, they have literally no heat losses during long storage periods and a 3times higher energy density [Köll R. et al, 2017a]. Nevertheless, the system size needs to be further reduced and at the same time there is a need to significantly reduce the investment costs of the system.

A new technique called "charge boost" was developed, which enables a further increase of the energy density and efficiency of the system at lower desorption temperatures [Mette B. et al, 2013]. This also contributes to a significant cost reduction of investment costs. The charge boost is applied after a complete desorption of two sorption storage vessels. Whereas the main storage vessel is on high 
temperature, the second storage vessel, the so called charge boost storage is on a lower temperature level. The resulting pressure difference is used to further desorb the main storage vessel by transferring the vapor from the main storage to the charge boost storage. The charge boost storage needs to be desorbed again afterwards and the charge boost process repeated. The result is a higher state of charge of the main storage, without increasing of the desorption temperature and the process can be repeated.

\section{DESIGN}

The charge boost mode enables new possibilities to make the sorption storage more efficient. To meet the new requirements of a suitable charge boost storage a new component, the so called sorption collector, was developed. Therefore, the sorption material zeolite 13XBF was filled into stainless steel pipes with a diameter of $97.8 \mathrm{~mm}$. In the center of the stainless steel pipe a perforated sleeve is placed to keep a free space to improve the vapor transfer within the zeolite bed. Five stainless steel pipes are welded to a vacuum manifold which is connected to the storage system. On the steel pipes, double copper pipes are placed which are connected to the hydraulic system and enable the conventional use of the collector. On the copper pipes a sydney-type glass pipe is placed and fixed on the top and bottom of the collector because of the relatively high weight. In addition the sorption collector is equipped with mirrors on the backside to increase collector performance [Köll R. et al, 2017b]. The design is shown in Figure 1. In total the collector has an aperture area of $3.05 \mathrm{~m}^{2}$ and contains $35 \mathrm{~kg}$ of zeolite $13 \mathrm{XBF}$ in the inner vacuum pipe, which is used for charge boost purposes.

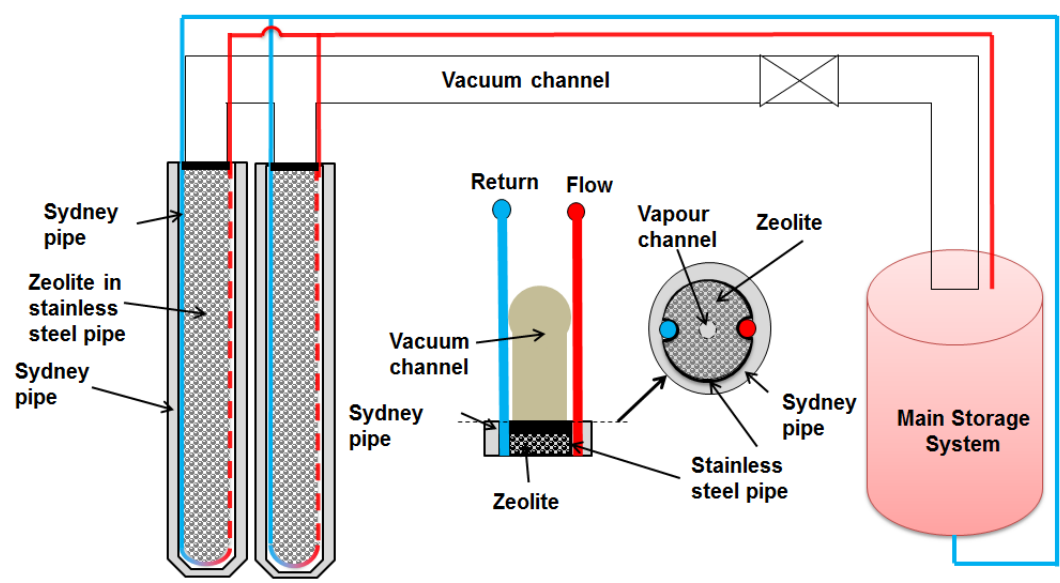

Figure 1: Concept of sorption collector applied as charge boost storage

The sorption collector is operated in a day/night cycle (see Figure 2). During the day the collector is used to heat up the main storage by transferring heat via the hydraulic system and the fixed bed heat exchanger in the sorption storage module. At the same time the sorption material in the collector is heated up and the released water vapor is condensed at the condenser heat exchanger. The main advantage during this step is that the collector sorption material is directly desorbed in the collector and therefore reaches the highest possible temperature. Additionally no extra containment is needed and the system can be built in a compact way. 


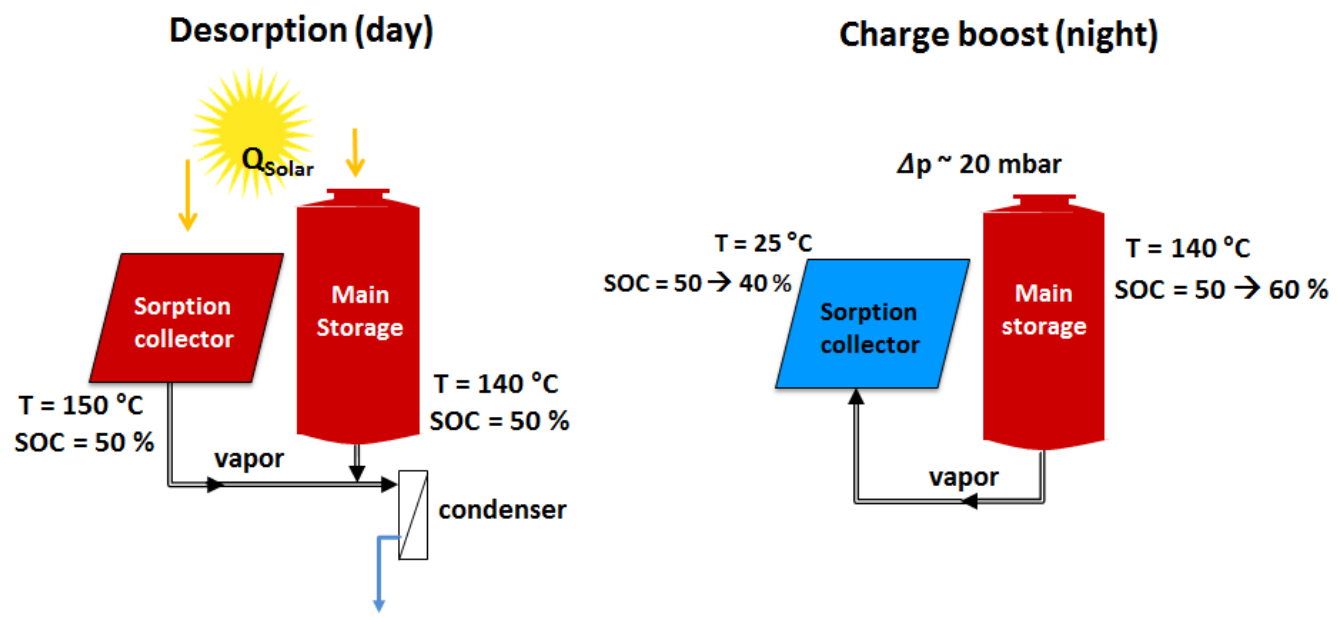

Figure 2: Working principle of the sorption collector under typical boundary conditions

During the night the charge boost mode can be applied. Therefore the high radiation losses of the collector are used to cool down the collector sorption material, which also induces a pressure drop. The resulting pressure difference between sorption collector and main storage is used to further desorb water vapor from the hot main storage to the cold sorption collector. This means that during the charge boost step the vapor is shifted to the sorption collector, but on the next sunny day it can be desorbed easily. The main advantage during this step is that the main storage can be desorbed to a higher state of charge, compared to a conventional desorption, without increasing of the desorption temperature. The sorption collector provides the ideal boundary conditions for this application because it enables high temperatures during day and cold temperatures during night.

\section{METHODE}

A prototype of a sorption collector was designed and built up in the laboratory. It is filled with zeolite $13 \mathrm{XBF}$ and implemented in the complete storage system. Experiments were carried out with the prototype to investigate and optimize the collector, as well as the system performance.

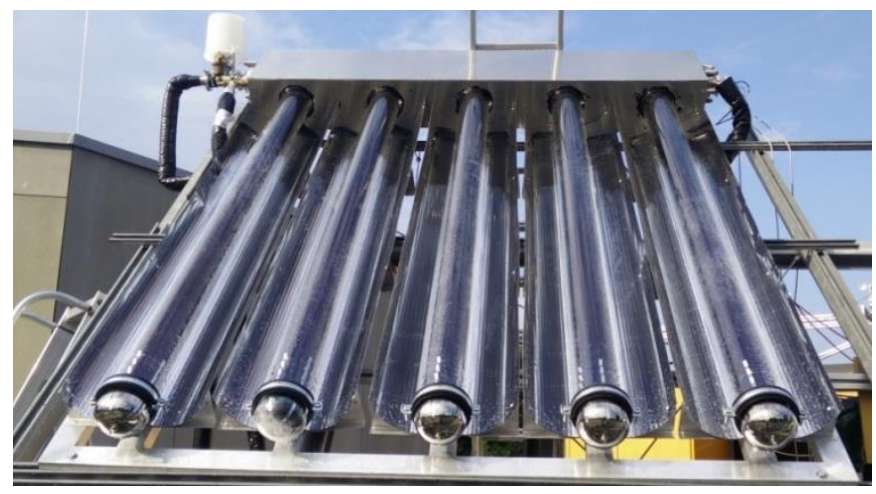

Figure 3: Developed prototype of sorption collector with glass tubes 


\section{RESULTS}

The performance of the sorption collector is tested experimentally under variation of the boundary conditions. First test results show that the thermal performance of the collector is not negatively influenced by the sorption material in the construction, but the storage system performance can be improved significantly. Due to the charge boost technique the storage capacity of the zeolite could be used more efficiently and hence the energy density can be improved significantly. In Figure 4 the potential of improvement of the energy density by repeating the charge boost is shown.

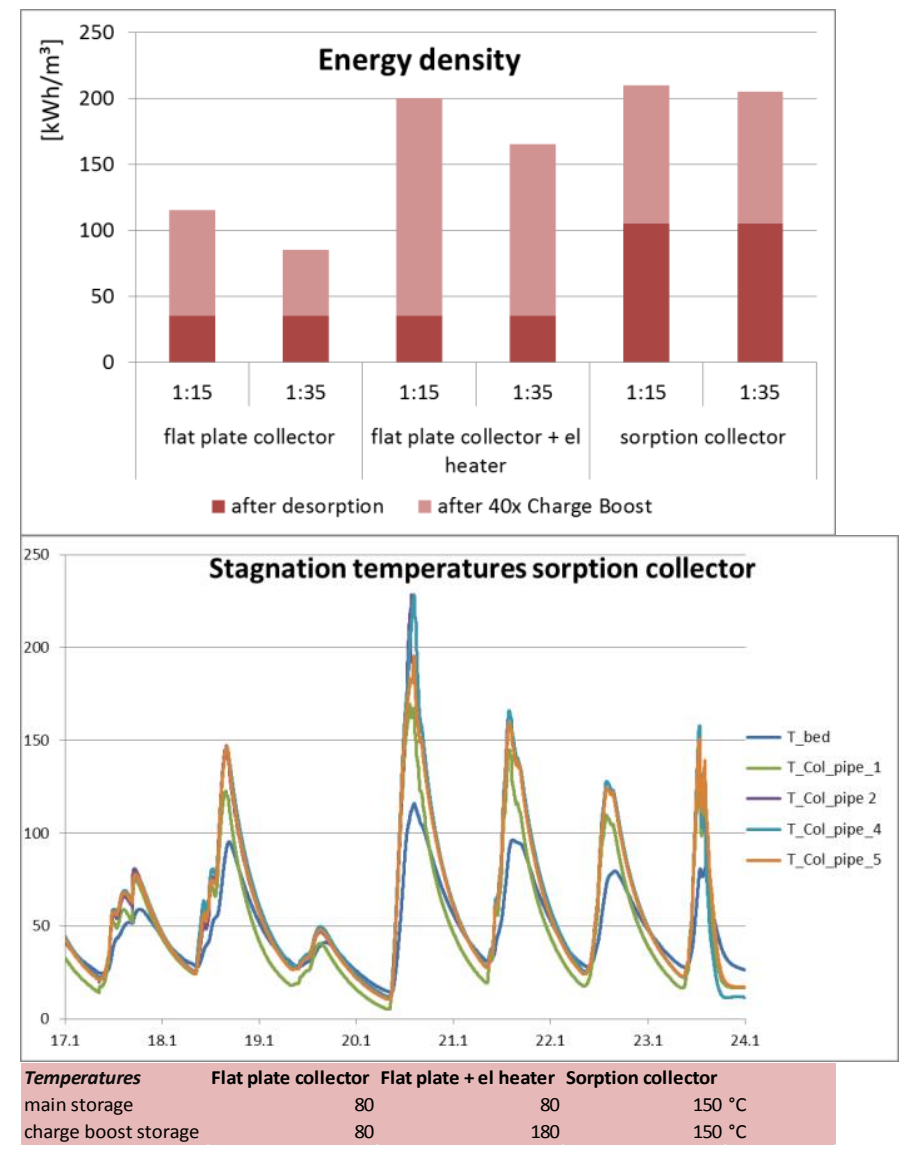

Figure 4: Left: Improvement of the energy density based on repeating the charge boost mode compared to the conventional desorption at the same temperature under different mass ratios between charge boost storage/sorption collector and main storage and different use cases (temperatures). Right: Measured stagnation temperature of the prototype sorption collector

In any use case the energy density can be doubled at least. The highest energy density can be achieved with the sorption collector combination, which achieves an energy density of $>200 \mathrm{kWh} / \mathrm{m}^{3}$. Also the combination of a flat plate collector and an electrical heater driven e.g. by PV electricity, is also a good alternative, achieving between $170-200 \mathrm{kWh} / \mathrm{m}^{3}$ (depending on the size).

The measured stagnation temperature show temperature peaks of $>220{ }^{\circ} \mathrm{C}$ on the outer side of the zeolite bed and $115{ }^{\circ} \mathrm{C}$ in the zeolite bed. The temperature difference between inside and outside is due to the low heat conductivity of the material and needs to be improved to achieve the highest possible temperatures in the sorption bed for a successful desorption. It can be also seen that due to the high radiation losses of the collector during the night the bed temperature drops to $20-30{ }^{\circ} \mathrm{C}$ during night and hence also a significant pressure drop is occurring, which is the ideal condition to perform the charge boost process. This way the day-night cycle can be used most efficiently. 


\section{CONCLUSION}

The energy density achieved with a sorption storage system depends on the temperatures available for drying. The charge boost technique enables a more efficient charging of the storage system at the same available temperature. The potential of the sorption collector is very high. At an assumed temperature of $150{ }^{\circ} \mathrm{C}$ in the collector material an energy density of $>200 \mathrm{kWh} / \mathrm{m}^{3}$ can be achieved. Compared to a conventional flat plate collector where only $110 \mathrm{kWh} / \mathrm{m}^{3}$ can be achieved. With the prototype a peak bed temperature of $115^{\circ} \mathrm{C}$ could be measured, but the temperature on the outside of the bed was above $200{ }^{\circ} \mathrm{C}$ which shows that with improvement of the heat transfer in the collector the temperature and hence also the energy density can be even higher than assumed for the calculation.

Due to the breaking of the glass during operation of the hydraulic system, only the stagnation temperatures could be measured. The reason for the breaking of the tubes during the operation of the hydraulic pump was that at higher temperatures in the pipes the thermal expansion caused some mechanical stress on the glass pipes and caused the breaking. The construction was reinforced to prevent the braking afterwards. Due to several changes in the construction to prevent the braking of the glass pipes, the sensors to measure the bed temperature were not positioned at a proper position any more. Therefore the bed temperature during the later experiments could not be measured precisely. Nevertheless, the measured temperatures on the outside of the bed show the potential of the bed temperature even in winter and therefore the potential of high energy density. The charge boost principle could be proven, but to achieve the full performance of the sorption collector the heat transfer in the fixed bed needs to be improved.

\section{REFERENCES}

Hauer A., Hiebler S. und Reuß M. (2013), Wärmespeicher, Karlsruhe: Fraunhofer IRB Verlag.

Köll R., van Helden W., Engel G., Wagner W., Dang B., Jänchen J., Kerskes H., Badenhop T., Herzog T. (2017), An experimental investiagion of a realistic-scale seasonal solar adsorption storage system for buildings, “ Solar Energy Vol.155, pp. 388-397, Gleisdorf.

Mette B., Asenbeck S., Kerskes H. und Drück H. (2013), „, Effizienzsteigerung thermochemischer Energiespeicher für solare Anwendungen durch Reduzierung der Regenerationstemperatur,“ in 23. Symposium "Thermische Solarenergie", Bad Staffelstein.

Köll R., Poscharnig H., Wagner W., van Helden W., Engel G., Rohringer C. (2017), „Erhöhung der Effizienz von geschlossenen Sorptionsspeichersystemen durch die Optimierung des Umladeprinzips und der Entwicklung eines Sorptionskollektors,“ in 27. Symposium Thermische Solarenergie, Bad Staffelstein. 


\title{
HUMIDIFIED AIR INJECTION FOR ZEOLITE BOILER IN THERMOCHEMICAL ENERGY STORAGE AND TRANSPORT SYSTEM UTILIZING UNUSED HEAT FROM SUGAR MILL
}

\author{
Shoma Fujii1, Yuichiro Kanematsu2, Yasunori Kikuchi2, Takao Nakagaki1 \\ Department of modern mechanical engineering, Waseda University 1 \\ 3-4-1 Okubo Shinjuku-ku, Tokyo, Japan \\ Phone: +8135286 2497 \\ E-Mail: shoma@fuji.waseda.jp \\ Presidential Endowed Chair for "Platinum Society", The University of Tokyo2 \\ 7-3-1 Hongo, Bunkyo-ku, Tokyo, Japan
}

\begin{abstract}
SUMMARY
Thermochemical energy storage and transport system utilizing zeolite steam adsorption and desorption cycle was considered to resolve spatial and seasonal heat mismatch between generating unused heat from sugarcane bagasse combustion and heat demand from fossil fuel in one island in Japan. Increasing both fuel reduction rate and heat recovery rate is an important key point to enhance the implementation of this system. The system mainly consists of heat charging device at the sugar mill and heat discharging device "Zeolite Boiler" at the heat demand, which generates more steam than absorbed steam from existing boilers. To increase the fuel reduction and heat recovery rate of the zeolite boiler, the combination system of injection of humidified air instead of steam generated by the existing boiler and economizing process was proposed and its performance was predicted by the quasi2-dimensional numerical simulation model. As a result, the fuel reduction rate and heat recovery rate increased by 11 and 10 percentage points, respectively comparing to the steam-injected zeolite boiler.
\end{abstract}

\section{INTRODUCTION}

In Tanegashima, where is an isolated island located in the southern part of Japan, a sugar mill produces raw sugar which is shipped to a domestic sugar refinery and a massive amount of bagasse. Bagasse is fibrous residue obtained from milled sugarcane after sugar juice has been extracted, and the boiler of sugar mill combusts the bagasse as a biomass energy. In this sugar mill employing backpressure steam turbine, more bagasse than requirement for their own heat and power is burned at the bagasse boiler because excess amount of bagasse cannot be burned in the off-season due to no heat duty of sugar evaporation. Hence, the temperature of flue gas increases and a massive amount of unused heat approximately at $200{ }^{\circ} \mathrm{C}$ is exhausted during the sugar mill operation from winter to spring. On the other hand, many other factories (Liquor, Starch, Cleaning, and so on) in this island use package boilers burning heavy oil or LPG which are imported from the outside the island to generate process steam at the temperatures up to $120{ }^{\circ} \mathrm{C}$ all year around. In order to resolve this spatial and seasonal heat mismatch, we propose a thermochemical energy storage (TES) and transport system using zeolite steam adsorption and desorption cycle, as shown in Fig. 1. In our previous work, material and heat flow analysis of the system, we concluded that there is a potential to distribute the unused heat from the sugar mill to many factories in this island (Fujii et al., 2016), and the heat discharging process determines the overall heat availability through the heat charge and discharge cycle. Many types of heat discharging devices have been reported (Krönauer et al., 2015) (Yu et al., 2013). The general problem of the packed bed reactor is low heat transfer between solid particle and the heat transfer fluid through the heat exchanger, and direct contact between solid particle and heat transfer fluid is one of the resolution to enhance the heat transfer (Oktariani et al., 2012). However, the heat demand in this island requires pressurized steam for their process and direct contact type reactor must be a pressurized chamber, leading expensive chamber cost. Also, the heat demand prefers continuous steam supply. To match these general requirements of the heat demand, we developed the moving bed and indirect contact type heat exchanger reactor as the heat discharging devices in this system. The conceptual design of heat discharging device, "Zeolite Boiler", employing moving bed and indirect heat exchanger was introduced in our previous study (Fujii et al., 2018). In this study, an advanced 
zeolite boiler employing humidified air injection and economizing process trying to increase the fuel reduction and heat recovery rate of existing boiler was evaluated.

\section{Nomenclature}

Symbols

$\begin{array}{lllll}c_{p} & \text { specific heat capacity } & \mathrm{kJ} /(\mathrm{kg} \cdot \mathrm{K}) & \text { ax } & \text { axial } \\ H & \text { enthalpy } & \mathrm{kJ} / \mathrm{h} & \mathrm{dec} & \text { decrease } \\ k_{L D F} & \text { overall mass transfer coefficient } & 1 / \mathrm{s} & \text { eq } & \text { equilibrium } \\ L & \text { length of heat exchanger } & \mathrm{m} & \text { eff } & \text { effective } \\ m & \text { mass flow rate } & \mathrm{kg} / \mathrm{h} & \mathrm{g} \quad \text { gas } \\ n_{\mathrm{bt}} & \text { number of tubes } & - & \mathrm{inj} & \text { injection } \\ p & \text { pressure } & \mathrm{kPa} & \text { max } & \text { maximum } \\ q & \text { adsorption amount } & \mathrm{kg} \text { water } / \mathrm{kg}_{\text {zeolite }} & \text { vap } & \text { vaporization } \\ r & \text { adsorption rate } & \mathrm{kg} \text { water } /\left(\mathrm{s} \cdot \mathrm{kg}_{z e o l i t e}\right) & \mathrm{w} & \text { water } \\ T & \text { temperature } & \mathrm{K} & \mathrm{z} & \text { zeolite } \\ u & \text { velocity } & \mathrm{m} / \mathrm{s} & 0 & \text { initial } \\ W & \text { adsorption volume } & \mathrm{mL} / \mathrm{kg} & 2 & \text { secondary } \\ x & \text { axial distance } & \mathrm{m} & & \\ \alpha & \text { heat transfer coefficient } & \mathrm{W} /\left(\mathrm{m}^{2} \cdot \mathrm{K}\right) & & \\ \Delta L & \text { evaporation heat } & \mathrm{kJ} / \mathrm{kg} & & \\ \Delta H & \text { adsorption heat } & \mathrm{kJ} / \mathrm{kg} & & \\ \varepsilon_{b} & \text { porosity of zeolite bed } & - & & \\ \eta & \text { Heat recovery rate } & \% & & \\ \lambda & \text { thermal conductivity } & \mathrm{W} /(\mathrm{m} \cdot \mathrm{K}) & & \\ \rho & \text { density } & \mathrm{kg} / \mathrm{m}^{3} & & \\ \phi & \text { relative humidity } & - & & \end{array}$

\section{PERFORMANCE PREDICTION OF NORMAL ZEOLTIE BOILER}

Figure 2 shows the schematic of the zeolite boiler. The zeolite boiler consists of a non-pressurized chamber and a multi-tubular heat exchanger, which is a vertically mounted counter-flow type reactor. Since zeolite needs steam to discharge the stored heat, zeolite boiler must be operated with fossil fuelfired boilers which have been already installed to supply heat for demands in this island. Zeolite is supplied into the chamber from the top and adsorbed steam is injected downwards from the top of heat exchanger. The zeolite adsorbs the injected steam and generates adsorption heat which is transferred to the heat exchanger settled into the chamber. Water is supplied from the bottom of the heat exchanger and flows upward with boiling. A quasi-2D model which has only axial distribution and radially heat exchange between the zeolite bed and the water through the tube wall, solving heat, mass and steam conservation equations was developed, leading to a performance characterization of this zeolite boiler. Mass balance of zeolite is given by (eq. 1).

$$
\frac{\partial}{\partial x}\left(\rho_{z} u_{z}\right)=\rho_{z 0} r
$$

Continuous equation is given by (eq. 2 ). 


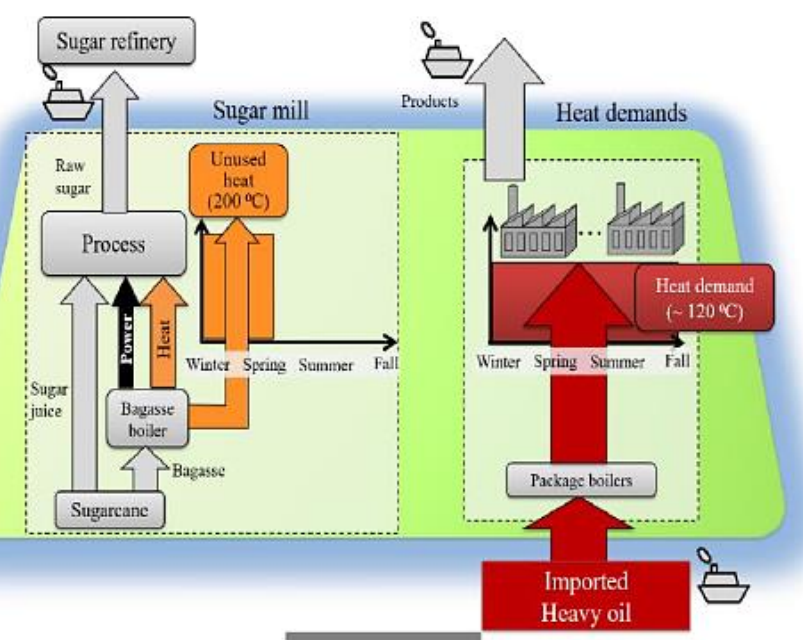

Applying TES

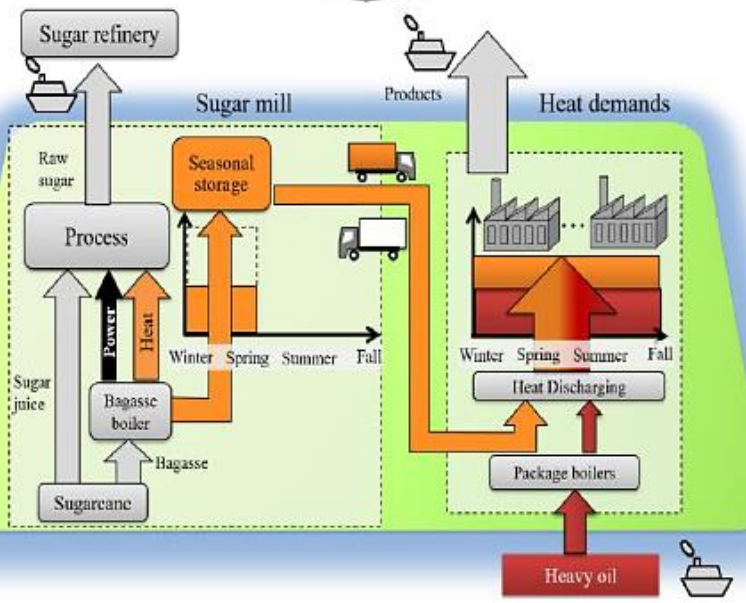

Fig. 1 Schematic of thermochemical energy storage and transport system in Tanegashima

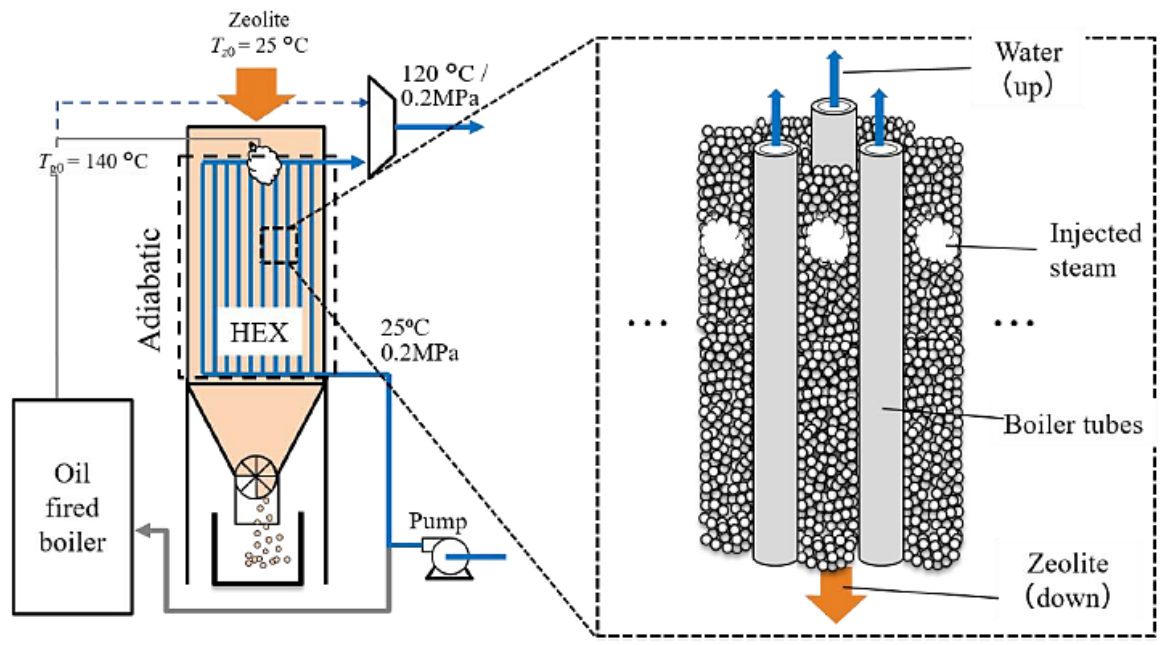

Fig. 2 Schematic of zeolite boiler 


$$
\frac{\partial}{\partial x}\left(\rho_{\mathrm{g}} u_{\mathrm{g}}\right)=-\rho_{\mathrm{z} 0} r
$$

Where $r$ is the adsorption rate determined by difference of equilibrium and current adsorption amount, and overall mass transfer coefficient based on Linear Driving Force model (Gorbach et al., 2004) given by (eq. 3).

$$
r=k_{\mathrm{LDF}}\left(q_{\mathrm{eq}}-q\right)
$$

Equilibrium adsorption amount data was obtained by fixed bed test and Sips equation was employed basically and Freundlich adsorption terms was added to simulate capirally effect simply in this study.

$$
W=\frac{W_{\max }(b \phi)^{1 / n}}{1+(b \phi)^{1 / n}}+K \phi^{m}
$$

Adsorption specific parameters ( $\mathrm{b}, \mathrm{n}, \mathrm{Wmax}, \mathrm{K}, \mathrm{m})$ were fitted to the experimental data utilizing a non-linear square fitting where $\mathrm{Wmax}=232.20 \mathrm{~mL} / \mathrm{g}, \mathrm{n}=1.27, \mathrm{~b}=85.4, \mathrm{~K}=91.4$ and $\mathrm{m}=3.23$.

Energy conservation equations for zeolite, gas and supplied water are given by (eq. 5), (eq. 6) and (eq. 7).

$$
\begin{aligned}
& \frac{\partial}{\partial x}\left(\rho_{\mathrm{z}} u_{\mathrm{z}} c_{p \mathrm{z}} T_{\mathrm{z}}\right)=\frac{\partial}{\partial x}\left(\lambda_{\mathrm{z} \_ \text {eff }} \frac{\partial T_{\mathrm{z}}}{\partial x}\right)-\frac{4 d_{\mathrm{bt}} n_{\mathrm{bt}} \alpha_{\mathrm{zw}}}{d_{c}{ }^{2}}\left(T_{\mathrm{z}}-T_{\mathrm{w}}\right)-\frac{6\left(1-\varepsilon_{\mathrm{b}}\right) \alpha_{\mathrm{gz}}}{d_{\mathrm{c}}}\left(T_{\mathrm{z}}-T_{\mathrm{g}}\right)+\rho_{\mathrm{z} 0} r \Delta H \\
& \frac{\partial}{\partial x}\left(\rho_{\mathrm{g}} u_{\mathrm{g}} c_{\mathrm{pg}} T_{\mathrm{g}}\right)=\frac{\partial}{\partial x}\left(\lambda_{\mathrm{axg}} \frac{\partial T_{\mathrm{g}}}{\partial x}\right)-\frac{4 d_{\mathrm{bt}} n_{\mathrm{bt}} \alpha_{\mathrm{gw}}}{d_{\mathrm{c}}{ }^{2}}\left(T_{\mathrm{g}}-T_{\mathrm{w}}\right)-\frac{6\left(1-\varepsilon_{\mathrm{b}}\right) \alpha_{\mathrm{gz}}}{d_{\mathrm{c}}}\left(T_{\mathrm{g}}-T_{\mathrm{z}}\right) \\
& \frac{\partial}{\partial x}\left(\rho_{\mathrm{w}} u_{\mathrm{w}} c_{\mathrm{pw}} T_{\mathrm{w}}\right)=\frac{4 d_{\mathrm{bt}} n_{\mathrm{bt}} \alpha_{\mathrm{zw}}}{d_{\mathrm{c}}{ }^{2}}\left(T_{\mathrm{z}}-T_{\mathrm{w}}\right)+\frac{4 d_{\mathrm{bt}} n_{\mathrm{bt}} \alpha_{\mathrm{gw}}}{d_{\mathrm{c}}{ }^{2}}\left(T_{\mathrm{g}}-T_{\mathrm{w}}\right)
\end{aligned}
$$

Heat transfer coefficient between zeolite and gas was calculated by Ranz Marshall model (Ranz and Marshall, 1952) and axial effective thermal conductivity of gas was calculated by the heterogeneous model (Dixon and Cresswell, 1979). Decrease in isosteric adsorption heat corresponding to adsorption amount increase (Hauer, A and Fischer, F., 2011) was considered in this model. Momentum equation is given by (eq. 8 ).

$$
\frac{\partial P}{\partial x}=\frac{150 \mu_{\mathrm{g}} u_{\mathrm{g}}}{d_{\mathrm{p}}^{2}} \cdot \frac{\left(1-\varepsilon_{\mathrm{b}}\right)^{2}}{\varepsilon_{\mathrm{b}}{ }^{3}}+\frac{1.75 \rho_{\mathrm{g}} u_{\mathrm{g}}{ }^{2}}{d_{\mathrm{p}}} \cdot \frac{1-\varepsilon_{\mathrm{b}}}{\varepsilon_{\mathrm{b}}{ }^{3}}
$$

The governing equations were solved by a commercial CFD program, COMSOL 5.3®, with boundary conditions which is described as follows;

Top of heat exchanger $(x=0)$

$$
T_{\mathrm{z} 0}=25^{\circ} \mathrm{C}, \quad T_{\mathrm{g} 0}=140^{\circ} \mathrm{C}, \quad \frac{\partial T_{(\mathrm{g}, \mathrm{z})}}{\partial x}=0, \quad q_{0}=0.05 \mathrm{~kg}_{\text {water }} / \mathrm{kg}_{\text {zeolite }}
$$

Bottom of heat exchanger $(x=L)$

$$
T_{\mathrm{w} 0}=25^{\circ} \mathrm{C}, \quad \frac{\partial T_{(\mathrm{g}, \mathrm{z})}}{\partial x}=0,
$$


The steam mass flow of $280 \mathrm{~kg} / \mathrm{h}$ is required for the rated heat demand cleaning factory. The diameter of chamber is set as $1.0 \mathrm{~m}$, which is roughly the same diameter as a small package boiler with 350 $\mathrm{kg} / \mathrm{h}$ steam mass flow. Heat exchange tubes with $15.9 \mathrm{~mm}$ of outer diameter and $1.2 \mathrm{~mm}$ thickness are assumed to form the tube bundle with $60^{\circ}$ and 1.25 pitch staggered array inside the zeolite boiler. Length of heat exchanger tested $0.5,1.0$ and $1.5 \mathrm{~m}$ and zeolite mass flow rate was adjusted to obtain super-heated steam from the outlet of boiler. Table 1 shows the zeolite mass flow rate minimally required, the temperature of exhausted zeolite and the ultimate adsorption amount of each length. Fig. 3 plots temperature distributions of zeolite and feed water, which are shown as normalized axis for 0.5 and $1.0 \mathrm{~m}$ heat exchangers. The temperature of zeolite rapidly increases around $220{ }^{\circ} \mathrm{C}$ at the top of the chamber and gradually decreases accompanying with heating and evaporating the supplied water. In the case of $0.5 \mathrm{~m}$ heat exchanger, zeolite is exhausted at $107^{\circ} \mathrm{C}$ from the bottom with $0.235 \mathrm{~kg} / \mathrm{kg}$ ultimate adsorption amount. In the both cases of 1.0 and $1.5 \mathrm{~m}$, zeolite is exhausted at $102{ }^{\circ} \mathrm{C}$ from the bottom with $0.244 \mathrm{~kg} / \mathrm{kg}$ ultimate adsorption amount, which means $10 \%$ of adsorption heat is exhausted as the sensible heat of zeolite from the bottom. In these cases, the fuel reduction and heat recovery rate defined as (eq. 11) stick to $19.9 \%$ and $16.7 \%$, respectively. The zeolite temperature exhausted from the bottom is governed only by the exchanging heat duty with cold water introduced from the bottom and less affected by the length with constant mass flow rates of zeolite and water. Adding a secondary heat exchanger giving functionality as an economizer of oil-fired boiler can recover the sensible heat of zeolite, leading to increase the fuel reduction and heat recovery rate of the zeolite boiler.

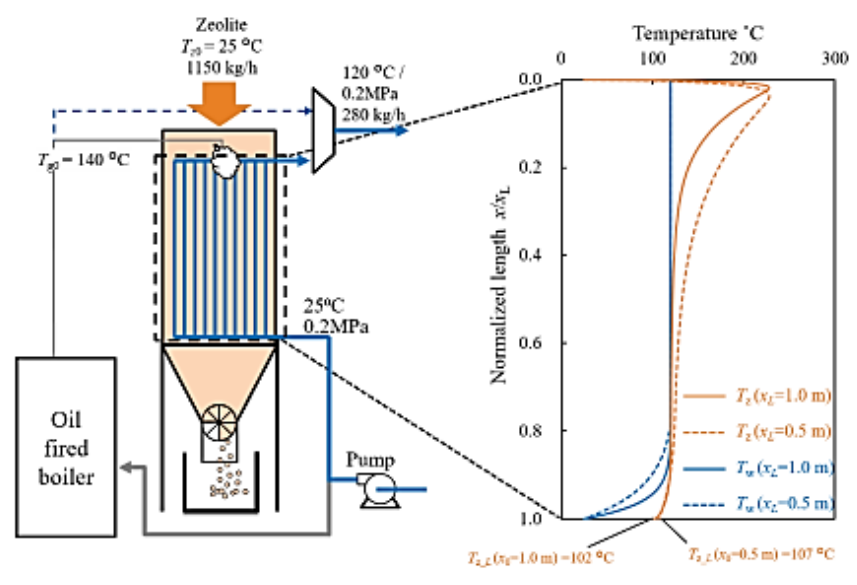

Fig. 3 Performance prediction of normal zeolite boiler

$$
\eta=\frac{H_{\mathrm{w}}-H_{\mathrm{inj}}}{\rho_{\mathrm{z}} \mathrm{u}_{\mathrm{z}} \Delta H\left(q_{\max }-q_{0}\right)}
$$

Table 1 : Zeolite mass flow rate and ultimate adsorption amount change at each length

\begin{tabular}{|c|c|c|c|}
\hline $\begin{array}{c}\text { Length } \\
\mathrm{m}\end{array}$ & $\begin{array}{c}\text { Zeolite mass flow rate } \\
\mathrm{kg} / \mathrm{h}\end{array}$ & $\begin{array}{c}\text { Exhausted zeolite temperature } \\
{ }^{\circ} \mathrm{C}\end{array}$ & $\begin{array}{c}\text { Ultimate adsorption amount } \\
\mathrm{kg} \text { water } / \mathrm{kg}_{\text {zeolite }}\end{array}$ \\
\hline $0.5 \mathrm{~m}$ & 1200 & 107 & 0.239 \\
\hline $1.0 \mathrm{~m}$ & 1150 & 102 & 0.244 \\
\hline $1.5 \mathrm{~m}$ & 1150 & 102 & 0.244 \\
\hline
\end{tabular}




\section{ZEOLITE BOILER WITH BUBBLING AND ECONOMIZING PROCESS}

To increase the both fuel reduction and heat recovery rate of the zeolite boiler, an advanced zeolite boiler employing humidified air injection and economizing process was proposed. The schematic of the advanced zeolite boiler is shown in Fig. 4. In this zeolite boiler, adsorption heat is transferred to the primary heat exchanger and evaporates supplied water in the same manner as the normal zeolite boiler. The sensible heat of zeolite is recovered by the secondary heat exchanger to heat up the water to a humidifier. Most of the water heated by secondary heat exchanger feeds into the oil-fired boiler and can save the oil consumption like an economizer. Air is blown into the humidifier and convects saturated steam. The humidified air is mixed with the steam from the existed boiler and injected from the top of the chamber. The pitch of primary and secondary tube bundles and the chamber diameter were the same as the normal zeolite boiler. Energy conservation for the secondary heat exchanger is given by (eq. 12). The length of the secondary heat exchanger tested 0.5 and $1.0 \mathrm{~m}$ and both outlet water temperatures of the secondary heat exchanger resulted in the same, which indicates $0.5 \mathrm{~m}$ is sufficient length to recover the sensible heat of zeolite.

$$
\frac{\partial}{\partial x}\left(\rho_{\mathrm{w} 2} u_{\mathrm{w} 2} c_{p \mathrm{w} 2} T_{\mathrm{w} 2}\right)=\frac{4 d_{\mathrm{bt}} n_{\mathrm{b} t 2} \alpha_{\mathrm{zw}}}{d_{\mathrm{c}}{ }^{2}}\left(T_{\mathrm{z}}-T_{\mathrm{w} 2}\right)-\frac{4 d_{\mathrm{bt}} n_{\mathrm{bt} 2} \alpha_{\mathrm{gw}}}{d_{\mathrm{c}}{ }^{2}}\left(T_{\mathrm{g}}-T_{\mathrm{w} 2}\right)
$$

In the humidifier, air is blown into the foamed metal and humidified with saturated steam. Due to the heat of vaporization of water, water temperature in the humidifier tank decreases corresponding the mass flow rate of vapor from the humidifier. Thus, the energy conservation in the humidifier is given by (eq.13), which expresses that the inlet enthalpy gained by a higher temperature than the humidifier tank compensates the heat of vaporization of water and steady temperature of the humidifier tank do not depend on the heat capacity ignoring the heat capacity of the blown air.

$m_{\mathrm{w} 2} c_{p \mathrm{w} 2} \Delta T_{\mathrm{dec}}=m_{\mathrm{vap}} \Delta L$

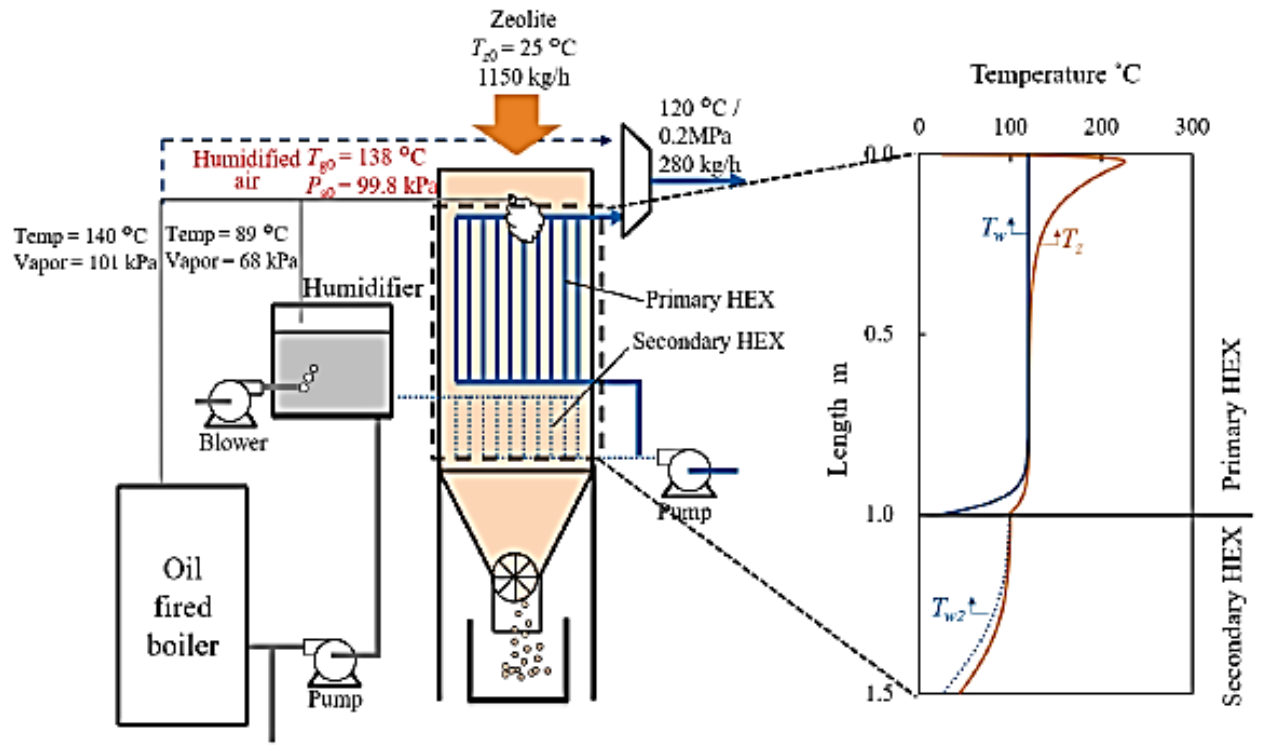

Fig. 4 Performance prediction of zeolite boiler with bubbling and economizing process 


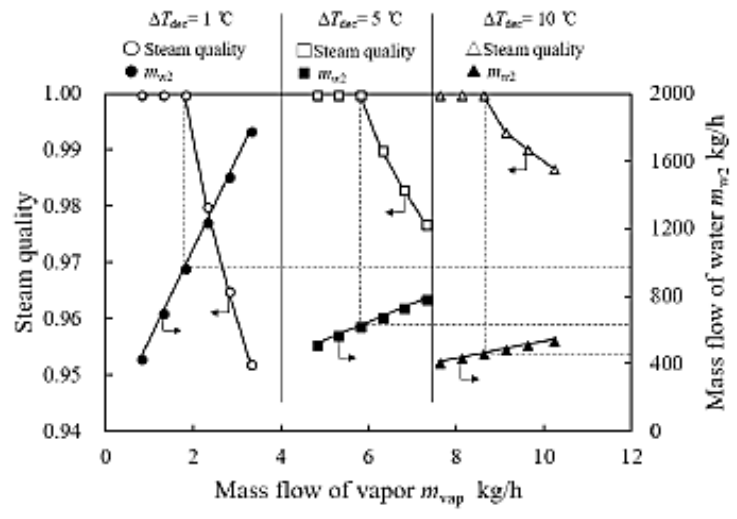

Fig. 5 Relationship between mass flow of vapor and steam quality, mass flow of water

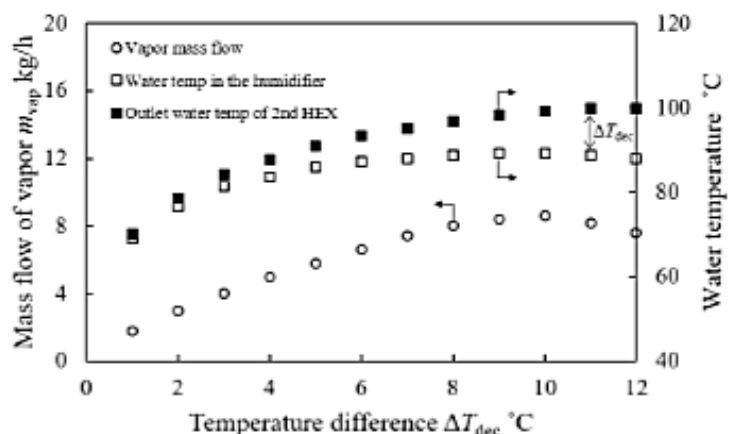

Fig. 6 Relationship between temperature difference and mass flow of vapor, water temperature

Where $\Delta T_{\mathrm{dec}}$ is the temperature difference between the outlet temperature of the secondary heat exchanger and the water temperature in the humidifier. Here, as show in Fig. 5, when determining a $\Delta T_{\text {dec }}$ first as the variable, correspondingly the relationship between $m_{\mathrm{vap}}$ and $m_{\mathrm{w} 2}$ is obtained to satisfy (eq.13). While the outlet enthalpy of the primary heat exchanger varies with $m_{\mathrm{vap}}$, the maximum value of $m_{\mathrm{vap}}$ is determined to obtain dry saturated steam and correspondingly the $m_{\mathrm{w} 2}$ is also determined. Figure 6 plots $m_{\mathrm{vap}}$ and outlet water temperature of the secondary heat exchanger and humidifier for each $\Delta T_{\text {dec }}$ from 1 to $12{ }^{\circ} \mathrm{C}$. Increasing $\Delta T_{\text {dec }}$ leads increasing the outlet water temperature of the secondary heat exchanger and decreasing $m_{\mathrm{w} 2}$. Also, increasing $\Delta T_{\text {dec }}$ leads increasing the water temperature of humidifier, which results increasing vapor pressure of humidified air and mass flow rate of vapor as shown in Fig. 6. However, the outlet water temperature of the secondary heat exchanger is limited below around $100{ }^{\circ} \mathrm{C}$ because the outlet zeolite temperature of the primary heat exchanger is almost constant at around $102{ }^{\circ} \mathrm{C}$ with $m$ vap. Therefore, $\Delta T_{\text {dec }}$ larger than $10{ }^{\circ} \mathrm{C}$ decreases the water temperature in the humidifier, which leads less $m_{\mathrm{vap}}$. Figure 4 shows the temperature distributions of zeolite and feed water in the case of $\Delta T_{\mathrm{dec}}=10^{\circ} \mathrm{C}$. In this case, $m_{\mathrm{vap}}=8.6 \mathrm{~kg} / \mathrm{h}$ was mixed to the steam from the existed boiler, and temperature and vapor pressure of mixed gas resulted in $138{ }^{\circ} \mathrm{C}$ and $99.8 \mathrm{kPa}$, respectively. The fuel reduction rate and heat recovery rate in this case resulted in $30.8 \%$ and $26.7 \%$, respectively.

\section{REERENCES}

Dixon, A, G., and Cresswell L David., (1979), Theoretical prediction of effective heat transfer parameters in packed beds, AIChE Journal, 25.4, 663-676.

Fujii, S., Kanematsu, Y., Kikuchi, Y., and Nakagaki, T., (2016), Material and Heat Flow Analysis in Thermal Energy Storage and Transport System Utilizing Unused Heat from Bagasse Boiler, Mechanical Engineering Journal, 3(5), 16-00334.

Fujii, S., Nakaibayashi, K., Kanematsu, Y., Kikuchi, Y., and Nakagaki, T., (2018), Development of zeolite boiler in thermochemical energy storage and transport system utilizing unused heat from sugar mill. 14th International Conference on Energy Storage, EnerSTOCK 2018.

Gorbach, A., Stegmaier, M., and Eigenberger, G., (2004), Measurement and modeling of water vapor adsorption on zeolite 4A-Equilibria and kinetics, Adsorption, 10(1), 29-46.

Hauer, A., and Fischer, F., (2011), Open Adsorption System for an Energy Efficient Dishwasher, Chemie Ingenieur Technik, Vol.83, No.1-2, pp.61-66.

Krönauer, A., Lävemann, E., Brückner, S., and Hauer, A., (2015), Mobile sorption heat storage in industrial waste heat recovery, Energy Procedia, 73, 272-280.

Oktariani, E., Noda, A., Nakashima, K., Tahara, K., Xue, B., Nakaso, K., and Fukai, J., (2012), Potential of a direct contact adsorption heat pump system for generating steam from waste water. International Journal of Energy Research, 36(11), 1077-1087. 
Ranz W. E., and Marshall W. R., (1952), Evaporation from drops, Chemical Engineering Progress, 48(3), 141-146.

Yu, N., Wang, R. Z., and Wang, L. W., (2013), Sorption thermal storage for solar energy, Progress in Energy and Combustion Science, 39(5), 489-514.

Green, C., (2018), Heating Systems for Houses, A Design Handbook. James \& James (Science Publishers) Ltd., London, UK.

\section{CONCLUSION}

In this study, the performance of the heat discharging device, "Zeolite Boiler" in the thermochemical energy storage and transport system was predicted numerically. To increase the fuel reduction and accompanying heat recovery rate of zeolite boiler, humidified air injection instead of steam generated by the existing boiler and economizing process was proposed and its performance was simulated by a numerical model. In the normal zeolite boiler, fuel reduction and heat recovery rate resulted in $19.9 \%$ and $16.7 \%$, respectively. By adding the secondary heat exchanger to heat up the water for humidifier, $8.6 \mathrm{~kg} / \mathrm{h}$ of vapor could be injected by the humidifier and water for the existed boiler could be preheated. Fuel reduction and heat recovery rate were increased to $30.8 \%$ and $26.7 \%$, respectively.

\section{ACKNOWLEDGEMENTS}

This work was supported by Shinko Sugar Mill Co., Ltd., Japan Boiler Association and JSPS Grantin-Aid for JSPS Research Fellow18J11316 Grant Number 18J11316. 


\title{
SUSTAINABLE HEATING AND COOLING IN INDUSTRY. SUSPIRE PROJECT CASE STUDY.
}

\author{
Iñigo Arrizabalaga ${ }^{1}$, Ane Sainz-Trapaga ${ }^{1}$ \\ ${ }^{1}$ Telur Geotermia y Agua \\ Aliendalde Auzunea, 6, 48200-Durango, Bizkaia, Spain \\ Phone: +34 946818916 \\ E-Mail: iarrizabalaga@ @elur.es, asainztrapaga@telur.es
}

\section{SUMMARY}

SuSPIRE project, granted by the European Community's H2020 framework program under the grant agreement 680169-SUSPIRE-H2020-EE-2014-2015/H2020-EE-2015-1-PPP, started in October 2015 and the completion is scheduled for September 2019.

This project aims to develop a demonstrator to recover up to a $70 \%$ of excess heat, currently wasted, in a casting factory placed in Barakaldo, Spain. In addition, savings of a $20 \%$ of the primary energy consumption of the plant should be reached.

The recovery and storage system, has been split in two different sub-systems named as high and low temperature. Telur, in charge of low temperature sub-system, is developing a recovery prototype, based on a Borehole Thermal Energy Storage (BTES) with a total drilling length of 3,750 m., to take advantage of recovered heat from process and space cooling.

According calculations recovering system will provide at least 3,000 MWh/y of heat to be used in steam production, indoor heating of the factory and SHW production. Excess recovered heat will be exported to third parties, a municipal swimming pool placed $150 \mathrm{~m}$ far from PCB (fig 1). A total amount of 2,500 MWh/y of cooling demand will be also supplied by the projected system mainly for process room conditioning.

System implementation will allow to save as much as 5,500 MWh/y of primary energy and associated $\mathrm{CO}_{2}$ emission will decrease in $1,100 \mathrm{t} / \mathrm{y}$.

Key-words: BTES, waste heat, thermal storage

\section{INTRODUCTION}

PCB, an ITP Aero company, casting facility in Barakaldo, Spain, spreads a 20,000 square metre space dedicated to the micro-fusion of super-alloys. PCB's goal is focused to manufacture top quality, high precision founded parts, specifically intended for the gas and industrial turbine market in general.

The factory is an intensive energy consumer generating a huge amount of excess heat. Heat, at very different temperatures, currently is dissipated in exhaust gas, cooling towers and air/water chillers. Heat is demanded mainly to high temperature for mould preheating or metal meting. Although factory also has a relevant lower temperature heat demand for steam production, space heating and sanitary hot water (SHW) currently supplied with NG or electricity.

The SuSPIRE project, granted by the European Community's H2020 framework program, aims to develop a demonstrator to recover up to a $70 \%$ of excess heat at PCB. A recovery and storage, R\&S, 
system split in two sub-systems, for high and low temperature, has been designed and currently is under implementation.

The high temperature sub-system is going to recover heat from furnace gases, with a maximum exhaust gas temperature of $850^{\circ} \mathrm{C}$, in order to use it mainly for steam generation

Telur, in charge of low temperature sub-system, is developing a prototype to take advantage of heat recovered, mainly, from melting furnaces cooling and process room space cooling.

\section{LOW TEMPERATURE RECOVERY SYSTEM}

Low Temperature recovery system core is formed by a BTES with 30 boreholes, $125 \mathrm{~m}$ deep, equipped with coaxial exchangers in PE-RT $\varnothing 63 \mathrm{~mm}$ PN16 SDR 11 for the outer pipe and $\varnothing 40 \mathrm{~mm}$ PN16 SDR 11 for the inner pipe. Piping and manifold have been installed with PE-RT and PPR rated for a maximal temperature use over $80{ }^{\circ} \mathrm{C}$. A pilot borehole was drilled in 2016 in order to get geological, hydrogeological and thermogeological characterization of the ground. Drilling starts with a superficial level, about 25 meters thick, of Quaternary alluvial and tidal silt and clay. At the bottom of this level a Lower Cretaceous limestone formation appears. Some fracturation and karstification can be found but the formation is laying in a marlstone massive formation decreasing the global permeability of the set.

Main design parameters have been calculated according results of a Pumping Test and a Thermal Response Test performed in the borehole. Groundwater level monitoring does not detect a ground flow, in coherence with the hydrogeological situation of the more transmissive formation under the regional water table basis level. Only a small tidal influence can be observed. For borehole field design commercial simulation software have been employed: FEEFLOW and EED. FEFLOW (Finite Element subsurface FLOW system) is an interactive finite-element simulation system for modelling 2D and 3D flow, age, mass and heat transport in the groundwater and the vadose zone. EED, Earth Energy Design, is a well known simulation software for Vertical Closed Circuit Borehole Heat Exchangers (BHEX).

Prior the borehole field construction a soil pollution assessment has been done in order to accomplish the legal requirements for the working site. BTES has been recalculated three times during the project due to space lake requirements and according the available HT excess heat to be stored. Drilling work started in late April and will be finished in July 2018 (fig. 2).

The BTES is going be connected to a high efficiency water-water chiller, of $550 \mathrm{~kW}$ of heating power and $500 \mathrm{~kW}$ of cooling power, in a 4-pipes hydraulic sketch in order to maximize simultaneous use as much as possible. Figure 3 depicts the main heat flow in the Low Temperature system. In base to monitoring and calculations during the project, and in order to assure the fulfil of the project goals, chiller power has been more than doubled from original proposal of $250 \mathrm{~kW}$ of heating power. Nowadays system design is ready and mechanical work has started inside the factory. System operation will start next November upon schedule. 


\section{ENERGY BALANCE AND SAVINGS EXCESS HEAT}

Total energy, natural gas (NG) and electricity, consumption of the plant is about $25.9 \mathrm{GWh} / \mathrm{y}$ in terms of aggregate primary energy. Calculation, according Spanish Government official conversion factors, provides a total amount of 4,400 t/y of $\mathrm{CO}_{2}$ associated emissions. An energy analysis of the productive process has been done inside the project in order to identify and monitoring all the thermal flows and the sources of residual heat in the factory. Fig. 4, depicts the excess heat sources of the factory, mainly:

\section{SPACE COOLING}

Mainly two process room: room and ceramics room, needing very precise climate conditions in order to keep temperature and humidity inside very narrow limits. These restrictive conditions, to fulfil process requirements, mean to heat and cool a wide space 24/7. Local climatic conditions and internal loads bring to a clear dominance of cooling demand. According chiller monitoring of electricity consumption done in this project, the annual cooling demand for process space conditioning has been estimated at $1,700 \mathrm{MWh} / \mathrm{y}$, with a dissipation of more than $2.400 \mathrm{MWh} / \mathrm{y}$.

A couple of air handler, linked to a NG set of boiler and two air-water chillers, are in charge to climate both process rooms. The offices building conditioning system has a dedicate reversible air-water heat pump to supply about $45 \mathrm{MWh} / \mathrm{y}$ of cooling.

\section{FURNACES}

Furnaces of the factory are currently using the exhaust gas to preheating the inlet air up to $300{ }^{\circ} \mathrm{C}$. Anyway there is still an interesting potential to recover the exhaust heat and the scope of the project includes to increase the heat recovered from one of the four existing furnaces. The mould cleaning furnace, in charge to remove the residual wax of the models, was chosen. Monitoring of exhaust gas temperature and combustion analysis, done during the first stage of SuSpire, revealed an available power of $140 \mathrm{~kW}$ at the maximum working temperature.

According the load profile of the furnace, and assuming that the exhaust gas stream temperature could drop until $150^{\circ} \mathrm{C}$, a total amount of $529 \mathrm{MWh} / \mathrm{y}$ would be recovered by the new heat exchanger and the HT system currently under developing and implementation.

\section{PROCESS COOLING}

A cooling loop, based on three evaporative towers Fig 5, is in charge to dissipate the thermal loads of the process, generated by:

- Windings of induction furnaces.

- Heat treatment furnaces.

- Wax injectors.

Monitoring information reveals that at least 3,000 MWh/y of process excess heat are currently dissipated and could be recovered in a relatively easy way. Regarding the the dissipated power distribution, a $49 \%$ of the working time, fig. 6 , ranges from 200 to $400 \mathrm{~kW}$ and a $23 \%$ of time is over $400 \mathrm{~kW}$. 


\section{THERMAL LOADS}

SuSpire Project is focused in the use of recovered heat in the same factory as much as possible. Obviously the biggest part of energy consumption of the factory is used in very high temperature processes as metal melting or moulds preheating, that are out of the scope of the project. Anyway, most of factories have a substantial demand of lower temperature heat and many of them have potential users, in very close places, able to be supplied by means of symbiotic projects. In addition, the production in conditioned spaces, due to dimensional requirements, the need to improve working conditions or more restrictive cooling conditions are getting cooling processes more and more energy consumer in modern industry. In this sense, all the factory LT thermal loads have been identified in order to be covered by the project at a first stage. Taking in account the existing imbalance, the LT heat excess, an exportation to a third party, the Sport Centre of Lasesarre, stands currently in an advanced stage of assessment.

\section{HEATING LOAD. SPACE CONDITIONING AND SHW}

The main LT heating loads of the plant are: space conditioning of wax and ceramic room, heating loop of the fan heaters of the factory and SHW production. Heating demand is currently supplied with a set of NG boilers placed in the plant roof. The boilers are connected to a manifold with a dedicated outlet to each circuit. Heating Peak load has been calculated in $550 \mathrm{~kW}$. The office building has also a smaller heating demand, about $100 \mathrm{~kW}$ of peak load, currently supplied by an air-water heat pump. Total heating demand has been quantified in $876 \mathrm{MWh} / \mathrm{y}$.

According simulations the recovered heat, after the implementation of the project SuSpire, will cover up to $95 \%$ of the space heating and SHW demand of the plant, meaning about $832 \mathrm{MWh} / \mathrm{y}$.

\section{HEAT LOAD. PROCESS}

The high temperature heat recovery system will be dedicated to the next uses:

- Fusion of wax of one injection line.

- Boiler-clave water preheating.

- Steam generation in potash boiler.

The High Temperature system, currently under construction, will supply at least a heat demand of 468 $\mathrm{MWh} /$ year after SuSpire implementation. System has been designed to cover high temperature uses but an estimated heat excess of about $60 \mathrm{MWh} / \mathrm{y}$ of HT system will be transferred to the Low Temperature System.

\section{COOLING LOAD. SPACE CONDITIONING}

Process rooms for moulding manufacture require very restrictive conditions of temperature and humidity, 24/7. Nowadays, a set of air-water chillers are in charge to supply a peak load of $900 \mathrm{~kW}$ and to cover a cooling demand of 1,670 MWh/y. Less of $60 \mathrm{MWh} / \mathrm{y}$ of additional cooling demand are supplied to the offices by the reversible heat pump. Taking in account the low chilling temperature, demanded for de-humectation, seasonal energy efficiency ratio of the conventional chillers can be more the doubled by the water-water chiller of the LT SuSpire system.

This system will cover about the $98 \%$ of the chilling demand of the plant. Instead of heat dissipation to the ambience the simultaneous production of heat and cool will allow to decrease the associated primary energy demand by an $86 \%$. SuSpire system will provide, as an additional advantage, an improvement of the reliability of the cooling system providing a system more redundant and less dependent of ambient conditions. 


\section{PROCESS COOLING}

As much as 3,000 MWh/y of heat are dissipated by the cooling loop of the factory at an average temperature of $21{ }^{\circ} \mathrm{C}$. This is a very efficient system with a seasonal performance over 30 . About a $30 \%$ of the energy will be recovered by SuSpire system employing the cooling loop as source for heat production and storage management of the BTES. More of 2,000 MWh/y will be still available for a hypothetical heating supply of new symbiotic consumers.

\section{HEATING LOAD. THIRD PARTY}

There is an important imbalance of thermal demand of the factory with a surplus of heat at low temperature. In the framework of the SuSpire an ESCO project to send heat to the Lasesarre swimming pool is being evaluated. On the one hand more than $70 \%$ of the sport centre could be supplied. On the other hand, chilling demand of the factory, covered by the SuSpire high efficiency system, is going to be increased up to $1,300 \mathrm{MWh} / \mathrm{y}$, meaning the $78 \%$ of the chilling demand.

A $150 \mathrm{~m}$ isolated double pipe, including $80 \mathrm{~m}$ length Horizontal Directional Drilling to cross the existing railway, has been projected to link both sites and nowadays agreement terms are under study in order to implement it in a next stage.

\section{ENERGY SAVINGS AND ENVIRONMENTAL INDICATORS}

The total energy savings after the implementation of the Recovery System is shown too in Table 1. The primary energy savings, of the Low Temperature System without the exportation to the sport centre, would be about 1,600 MWh/y. The HT system will add 1,250 MWh/y of energy saving, increasing the total primary energy savings up to $2,850 \mathrm{MWh} / \mathrm{y}$, a $11.1 \%$ of the total consumption of the plant. The energy supply to the sport centre would increase primary energy savings up to 5,500 $\mathrm{MWh} / \mathrm{y}$, reaching the $21.5 \%$ of the total primary energy consumption. The LT system will decrease about $900 \mathrm{tCO}_{2} / \mathrm{y}, 63 \%$ of the associated emission to the $\mathrm{H} \& \mathrm{C}$ system. The HT system will add around $175 \mathrm{tCO}_{2} / \mathrm{y}$ of additional saving.

\section{TABLES}

Table 1: Suspire LT Covered Demand and Environmental Savings

\begin{tabular}{|c|c|c|c|c|c|c|c|c|c|c|c|c|}
\hline \multirow{5}{*}{ 滛 } & \multirow{2}{*}{\multicolumn{2}{|c|}{ HEATING AND COOLING }} & \multicolumn{5}{|c|}{ HEATING } & \multicolumn{4}{|c|}{ COOLING } & \multirow{3}{*}{$\begin{array}{r}\begin{array}{r}\text { TOTAL } \\
\text { H\&C }\end{array} \\
\mathbf{8 , 0 2 2}\end{array}$} \\
\hline & & & \multirow{2}{*}{\begin{tabular}{|c|}
$\begin{array}{c}\text { OFFICES } \\
\text { CONDITIONING }\end{array}$ \\
80 \\
\end{tabular}} & \multirow{2}{*}{$\begin{array}{c}\text { PLANT SPACE } \\
\text { CONDITIONING } \\
774\end{array}$} & \multirow{2}{*}{$\begin{array}{l}\text { SHW } \\
23\end{array}$} & \multirow{2}{*}{$\begin{array}{l}\begin{array}{c}\text { SPORT } \\
\text { CENTRE }\end{array} \\
2,446\end{array}$} & \multirow{2}{*}{$\begin{array}{c}\text { TOTAL } \\
\mathbf{3 , 3 2 2}\end{array}$} & \multirow{2}{*}{\begin{tabular}{|c|}
$\begin{array}{c}\text { OFFICES } \\
\text { CONDITIONING }\end{array}$ \\
56 \\
\end{tabular}} & \multirow{2}{*}{\begin{tabular}{|c|}
$\begin{array}{l}\text { PLANT SPACE } \\
\text { CONDITIONING }\end{array}$ \\
1,672 \\
\end{tabular}} & \multirow{2}{*}{$\begin{array}{c}\begin{array}{l}\text { PROCESS } \\
\text { COOLING }\end{array} \\
2,972\end{array}$} & \multirow{2}{*}{$\begin{array}{l}\text { TOTAL } \\
\mathbf{4 , 6 9 9}\end{array}$} & \\
\hline & CONVENTIONAL & $\mathbf{M W h}_{\mathrm{t}}$ & & & & & & & & & & \\
\hline & PRIMARY ENERGY CONSUMPTION & MWh & 94 & 1,209 & 35 & 3,821 & 5,160 & 65.7 & 1,980 & 235 & 2,280 & 7,440 \\
\hline & EMISSIONS & tcO2 & 13.2 & 255 & 7.5 & 806 & 1,081 & 9.2 & 277 & 32.8 & 319 & 1,400 \\
\hline \multirow{4}{*}{ 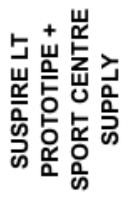 } & CONVENTIONAL & $M \mathrm{Mh}_{\mathrm{t}}$ & 0 & 3.0 & 11.4 & 710 & 724 & 0 & 26.4 & 2,139 & 2,166 & 2,890 \\
\hline & 500 KW GEOEXCHANGE BTES & $\mathrm{MWh}_{\mathrm{t}}$ & 80 & 771 & 11.4 & 1,736 & 2,598 & 56 & 1,646 & 832 & 2,534 & 5,132 \\
\hline & PRIMARY ENERGY CONSUMPTION & MWh & 47 & 479 & 6.7 & 2,136 & 2,669 & 8 & 280 & 199 & 487 & 3,156 \\
\hline & EMISSIONS & tco2 & 6.6 & 64 & 0.9 & 377 & 449 & 1.2 & 39.1 & 23.6 & 64 & 513 \\
\hline \multirow{4}{*}{ 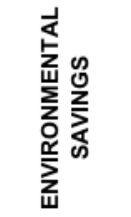 } & \multirow{2}{*}{ PRIMARY ENERGY } & MWh & 47 & 731 & 29 & 1,685 & 2,491 & 57 & 1,700 & 36 & 1,793 & 4,284 \\
\hline & & $\%$ & $50 \%$ & $60 \%$ & $81 \%$ & $44 \%$ & $48 \%$ & $87 \%$ & $86 \%$ & $15 \%$ & $79 \%$ & $58 \%$ \\
\hline & \multirow{2}{*}{ EMISSIONS } & tcO2 & 6.6 & 191 & 6.5 & 428 & 633 & 8.0 & 238 & 9.2 & 255 & 888 \\
\hline & & $\%$ & $50 \%$ & $75 \%$ & $87 \%$ & $53 \%$ & $59 \%$ & $87 \%$ & $86 \%$ & $28 \%$ & $80 \%$ & $63 \%$ \\
\hline
\end{tabular}




\section{FIGURES}

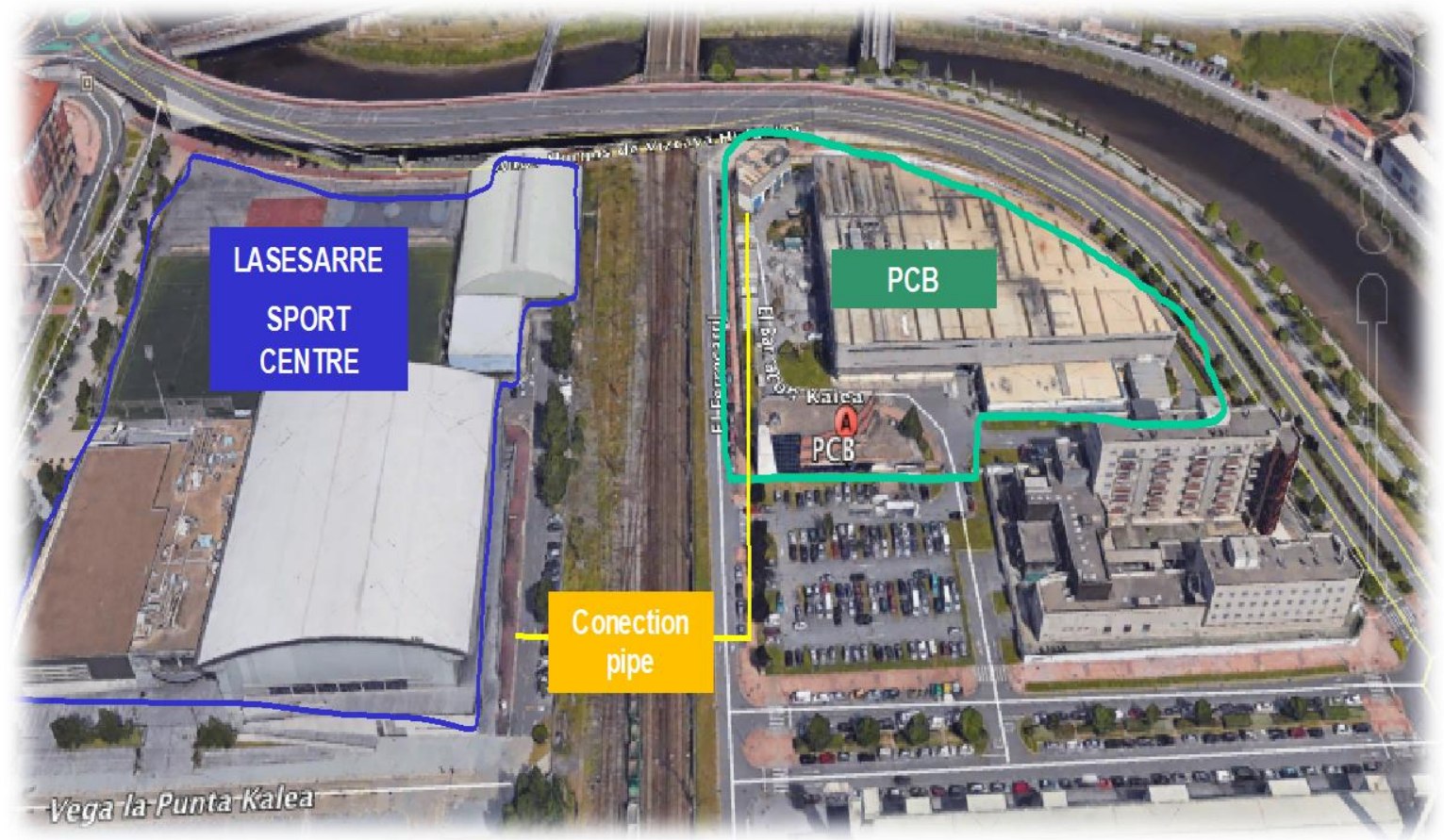

Fig 1. Factory and sport centre situation

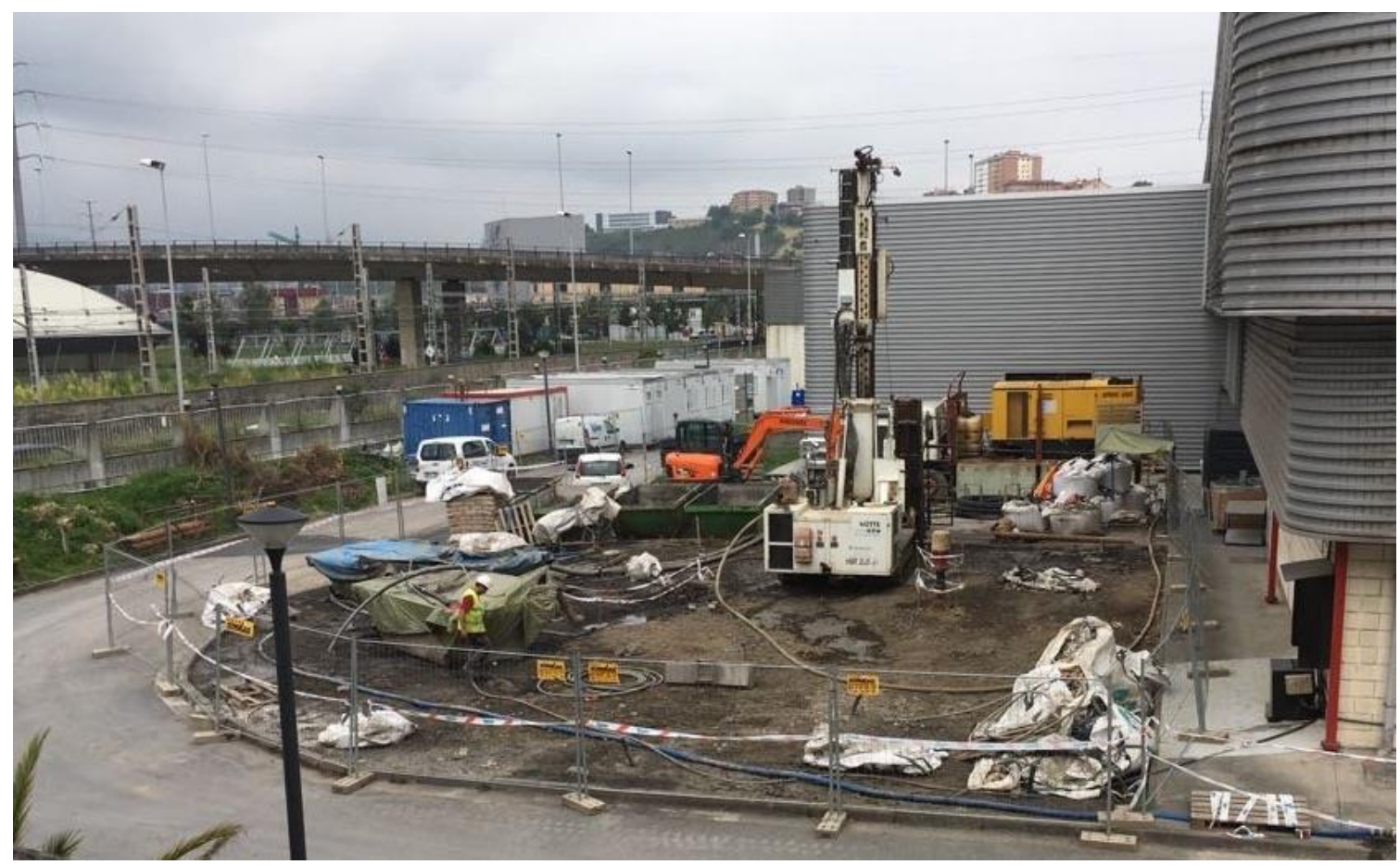

Figure 2. Drilling work to be finished in July 2018 


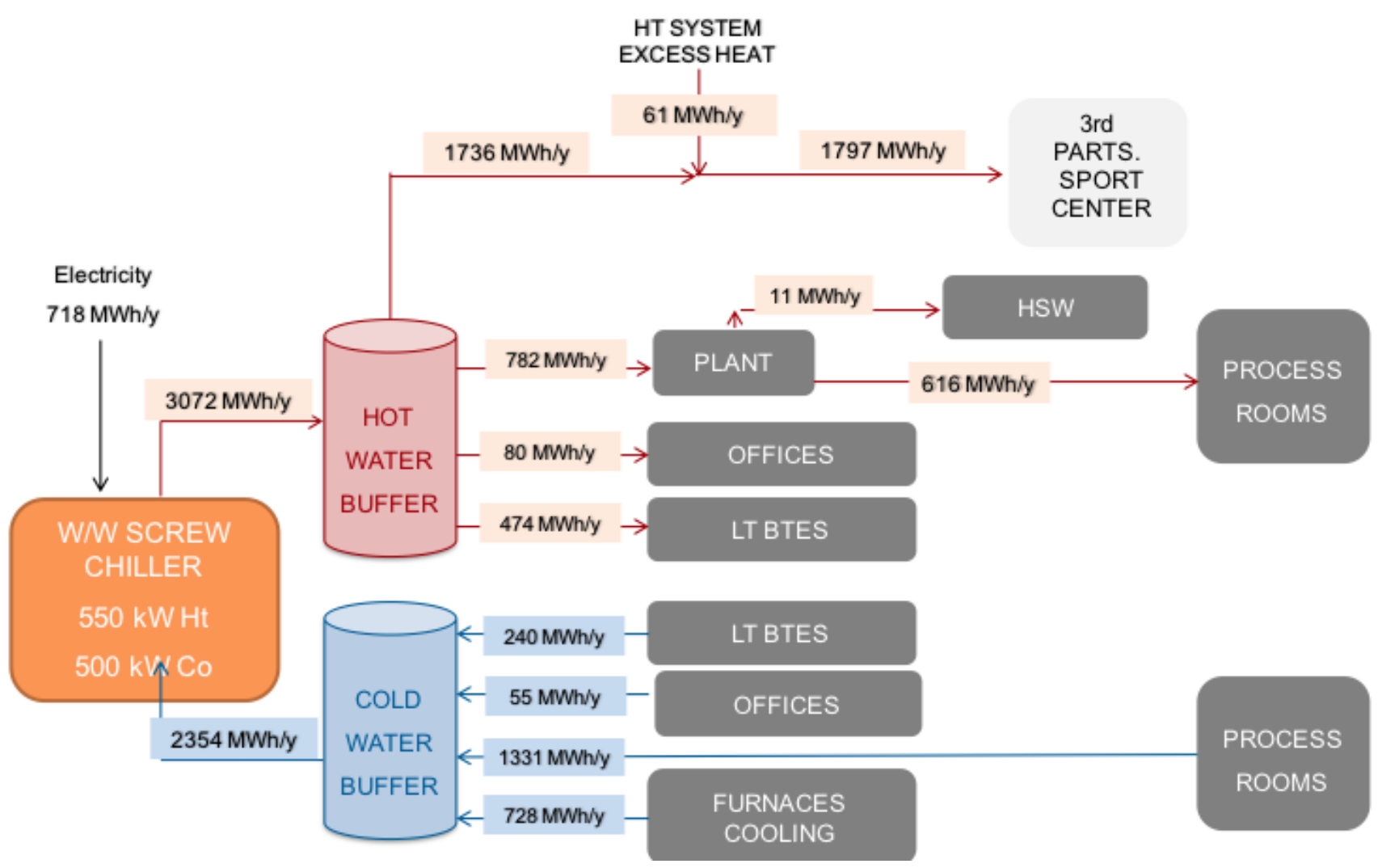

Fig 3. Low Temperature System

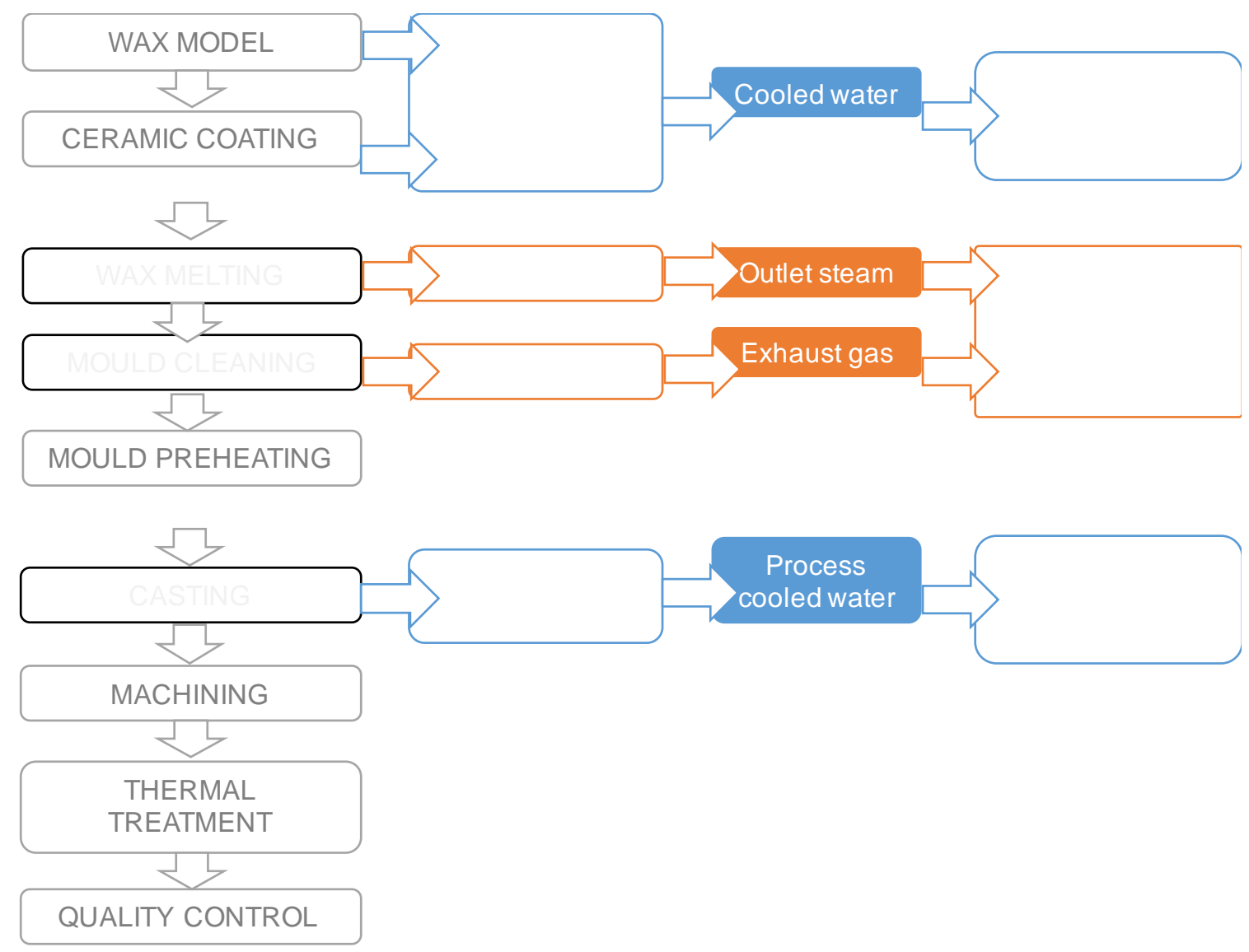




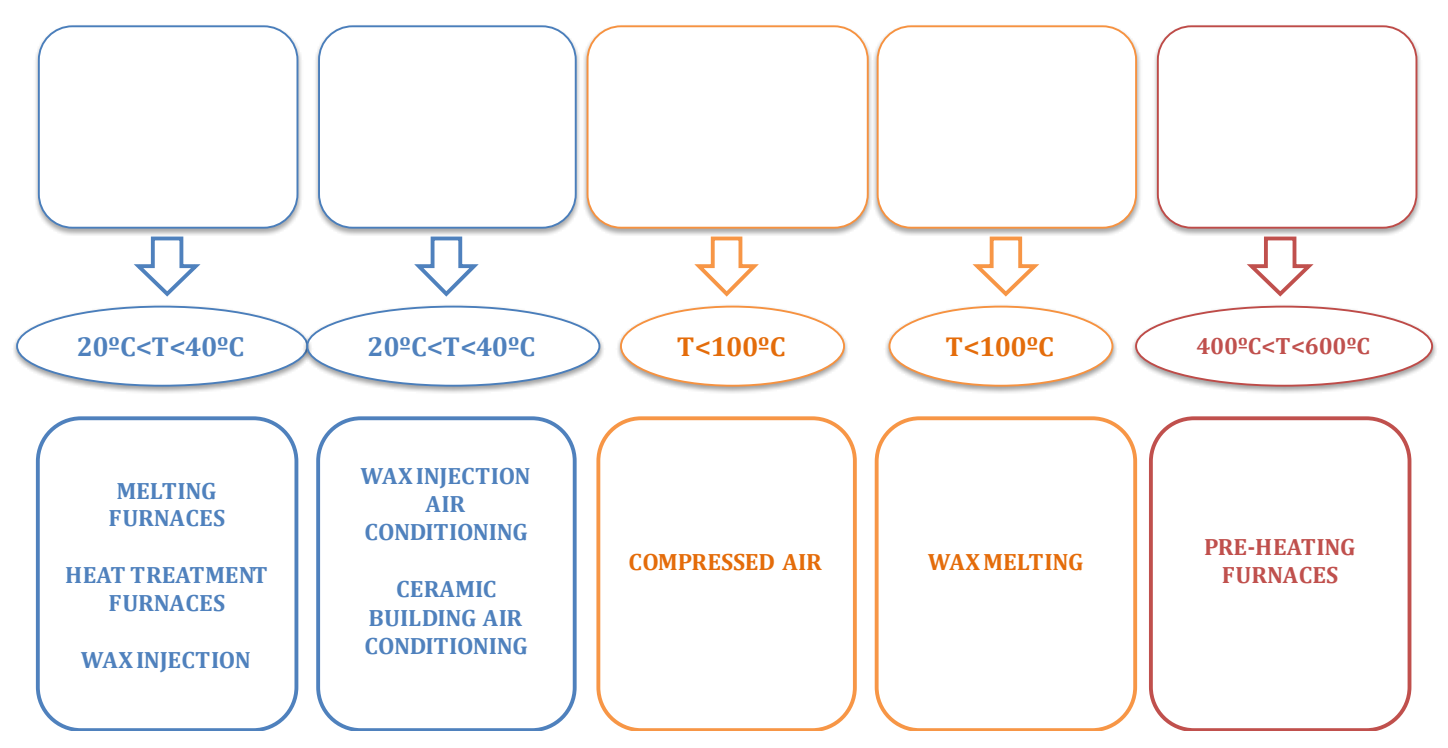

Fig. 4: Process and Excess Heat Sources

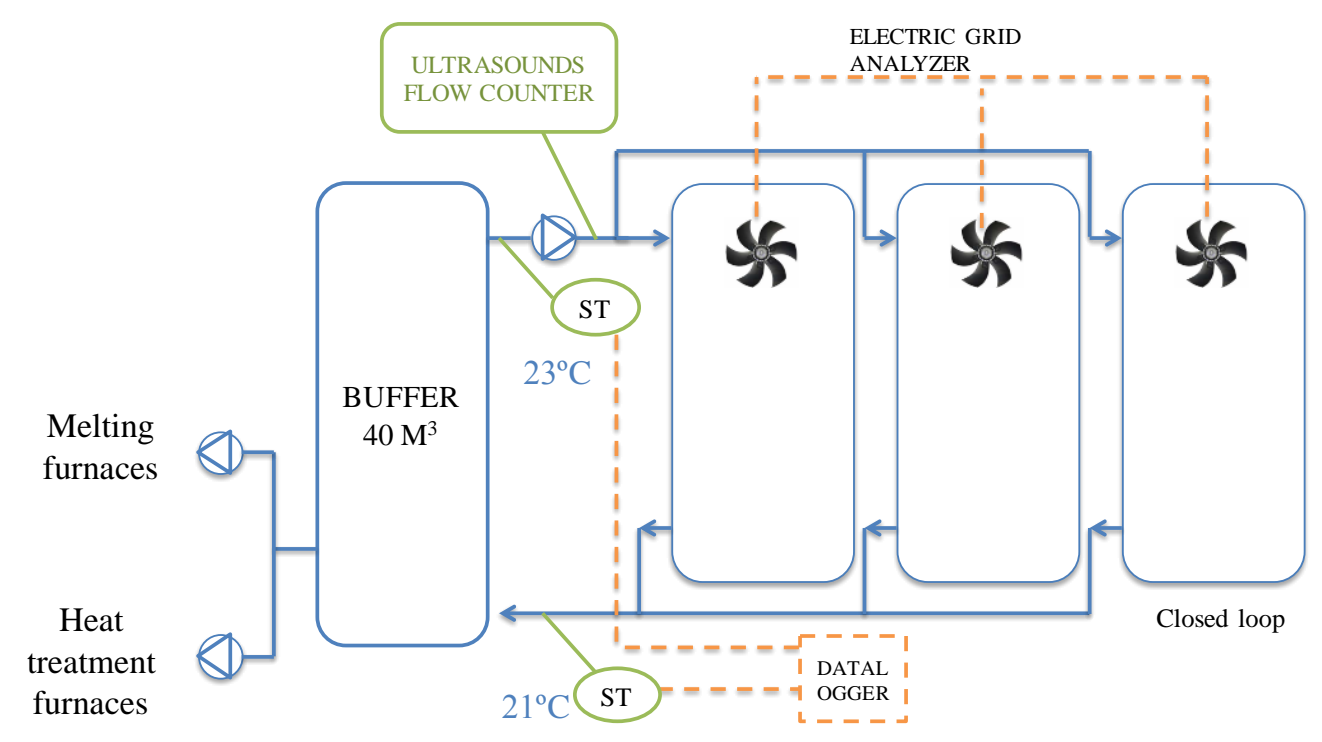

Fig. 5: Cooling loop

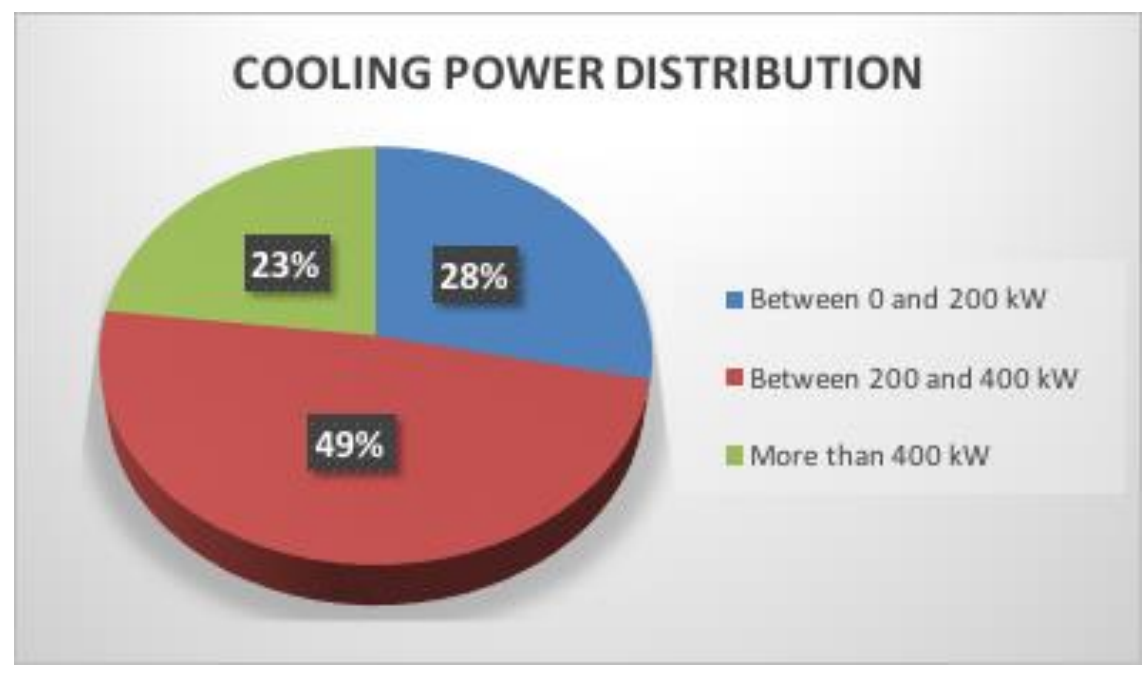

Fig. 6: Cooling power distribution 


\section{REFERENCES}

Sainz-Trapaga, A., Arrizabalaga, I.(2017), "Deliverable D 4.5 Theoretical Economical \& Technical study of energy recovery", Suspire project

\section{CONCLUSION}

The SuSpire Project aims to implement a demonstrator to recover up to a $70 \%$ of excess heat and to save a $20 \%$ of the primary energy consumption of a casting factory placed in Barakaldo, Spain. The recovery and storage system designed, has been split in two different sub-systems named as High Temperature (HTS) and Low Temperature (LTS).

Telur, in charge of LTS is developing a recovery prototype, based on a Borehole Thermal Energy Storage (BTES) and a screw chiller with a heating power of $550 \mathrm{~kW}$. LTS is currently under construction and upon schedule will be in operation next November.

According calculations more of 6,000 MWh/y of excess heat could be recovered inside the scope of SuSpire mainly, more than a $90 \%$, at LTS. The more relevant issues of the LTS, in order to reach project goals, are:

$\checkmark$ Recovered heat will cover the 98\% of the space heating and SHW demand of the plant saving more than $800 \mathrm{MWh} / \mathrm{y}$ of primary energy.

$\checkmark$ LTS will supply about 1,700 MWh for space cooling and de-humectation, up to a $98 \%$ of the demand and $800 \mathrm{MWh} / \mathrm{y}$ of process cooling. Performance improvement and simultaneity in the generation of heating and cooling will save about 1,800 MWh/y of primary energy.

$\checkmark$ Symbiotic project, involving a close Council Sport Centre, will to supply about 1,700 MWh/y of excess heat to the swimming pool. Primary energy savings have been estimated in 1,700 $\mathrm{MWh} / \mathrm{y}$.

$\checkmark$ Associated emissions will decrease in $900 \mathrm{tCO} 2 / \mathrm{y}$, over the $60 \%$ of related emission to the $\mathrm{H} \& \mathrm{C}$ system.

In the same way implementation of the SuSpire HTS system will recover $530 \mathrm{MWh} / \mathrm{y}$. HT heat use for steam production will save, at least, $1250 \mathrm{MWh} / \mathrm{y}$ of primary energy.

SuSpire project development has raised the primary energy savings from 4,222 $\mathrm{MWh} / \mathrm{y}$, of the project proposal, to 5,450 MWh/y. The energy symbiosis with the sport centre will allow to fulfil the project goal decreasing by a $21,3 \%$ nowadays primary energy consumption. 


\title{
AVAILABILITY OF EXCESS HEAT FROM THE SWEDISH KRAFT PULPING INDUSTRY
}

\author{
Elin Svensson ${ }^{1}$, Matteo Morandinㄹ Igor $\mathrm{Cruz}^{3}$, $\underline{\text { Simon Harvey }^{2}}$ \\ ${ }^{1}$ CIT Industriell Energi AB, Chalmers teknikpark, SE-412 88 Göteborg, Sweden \\ ${ }^{2}$ Energy Technology, Dept of Space, Earth and Engineering, Chalmers University of Technology, Sweden \\ Phone: +46 31772 8531, E-Mail: simon.harvey@chalmers.se \\ ${ }^{3}$ Energy Systems, Dept of Management and Engineering, Linköping University, Sweden
}

\section{SUMMARY}

An approach for estimating national targets for industrial excess heat recovery based on detailed analysis of data from case studies followed by regression analysis is proposed and applied to the Kraft pulping industry in Sweden. According to the resulting estimates, there is a large potential for increasing the excess heat utilization from the Swedish pulp and paper mills. The suggested methodology provides a more detailed picture of industrial excess heat availability in the Swedish pulp and paper industry and could be applied to other sectors and regions.

Key-words: industrial excess heat, pulp and paper industry, unavoidable excess heat, regression analysis

\section{INTRODUCTION}

Industrial excess heat is an important resource for meeting energy efficiency and climate goals. In Sweden, many industrial plants deliver excess heat to district heating systems and other external users. However, there is still a large potential for further utilization of industrial excess heat (see e.g. Broberg Viklund et al., 2012). Methods for estimating excess heat potentials can be characterized according to their approach (top-down or bottom-up), scale, and data acquisition (Brueckner et al., 2014). Furthermore, recovering heat from a process and exporting it competes with reusing the heat within the process itself. The most accurate way to estimate the excess heat potential of a specific site requires detailed process data of heating and cooling demands, and considers the distinction between avoidable and unavoidable excess heat (see e.g. Bendig et al., 2013). On a regional or national scale, however, it is not realistic to base estimations of excess heat potentials on detailed assessments of each individual plant. There is, consequently, a need for better tools to estimate the potential for increased excess heat utilization. This work proposes an approach for estimating national targets for industrial excess heat recovery. The approach is applied to the Kraft pulping industry in Sweden, and a detailed analysis of data from six case studies is used to estimate a sector-wide potential using regression analysis.

\section{ENERGY IN THE SWEDISH KRAFT PULPING SECTOR}

The pulp and paper sector is a major industrial sector in Sweden, representing about half of the national industrial energy use. Figure 1 (LEFT) shows the declared gross electricity production versus biofuel usage (black liquor, bark, and other wood residues) in Swedish mills in 2015, according to data provided by the Swedish Forest Industries Federation (Skogsindustrierna, 2003-2015). The linear trend between biofuel usage and electricity production is quite distinct, especially for Kraft mills.

Excess heat export from pulp and paper mills to district heating systems is common in Sweden. However, the correlation between sold excess heat and biofuel usage for different sizes and types of mill is less pronounced than for electricity, see Figure 1 (RIGHT). Possible reasons for this include geographical location, type and size of mill, and hot process water consumption. It is common that some excess heat from the pulp and paper process is exported to a nearby sawmill, but it is not always clear whether this excess heat export is reported as sold heat. 

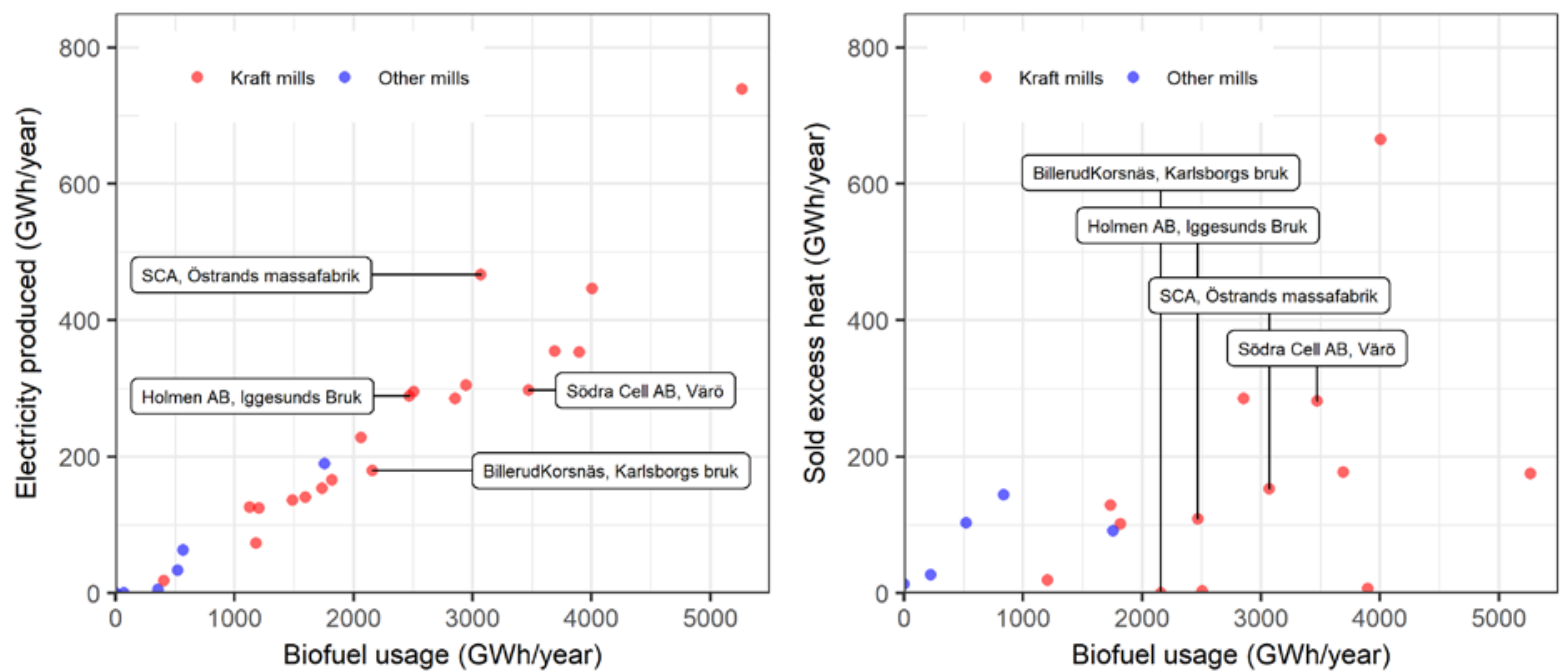

Figure 1: LEFT: Electricity production vs biofuel usage in Swedish mills 2015. RIGHT: Sold excess heat vs biofuel usage in Swedish mills 2015 (Skogsindustrierna, 2003-2015).

Table 1: Overview of Kraft mill study cases. Data taken from Miljödatabasen (Skogsindustrierna, 2003-2015) with the exception of the FRAM mills (Delin et al., 2005a,b)

\begin{tabular}{|l|c|c|c|c|c|c|}
\hline Mill & $\begin{array}{c}\text { Södra Cell } \\
\text { Värö }\end{array}$ & SCA Östrand & $\begin{array}{c}\text { Holmen } \\
\text { Iggesund }\end{array}$ & $\begin{array}{c}\text { Billerud- } \\
\text { Korsnäs } \\
\text { Karlsborg }\end{array}$ & $\begin{array}{c}\text { FRAM type } \\
\text { pulp mill }\end{array}$ & $\begin{array}{c}\text { FRAM type } \\
\text { int. mill }\end{array}$ \\
\hline Data year & 2012 & 2015 & 2013 & 2010 & - & - \\
\hline Pulp prod. (kt/y) & 419 & $900(+95)$ & 347 & 272 & 327 & 385 \\
\hline Market pulp (kt/y) & 419 & $900(+95)$ & 50 & 151 & 327 & 0 \\
\hline Paper/board (kt/y) & 0 & 0 & 381 & 151 & 0 & 512 \\
\hline Biofuel (GWh/y) & 3035 & na & 2327 & 1952 & 1975 & 2594 \\
\hline Fossil fuel (GWh/y) & 31 & na & 66 & 48 & 0 & 0 \\
\hline El. prod. (GWh/y) & 358 & na & 155 & 224 & 259 & 523 \\
\hline Sold heat (GWh/y) & 91 & na & 0 & 0 & 0 & 0 \\
\hline $\begin{array}{l}\text { Process thermal } \\
\text { data reference }\end{array}$ & $\begin{array}{c}\text { Bood and } \\
\text { Nilsson, 2013 }\end{array}$ & $\begin{array}{c}\text { Ah/ström and } \\
\text { Benzon, 2015 }\end{array}$ & $\begin{array}{c}\text { Isaksson et } \\
\text { al., 2013 }\end{array}$ & $\begin{array}{c}\text { Eriksson and } \\
\text { Hermansson, } \\
2010\end{array}$ & $\begin{array}{c}\text { Axelsson et } \\
\text { al., 2006 }\end{array}$ & $\begin{array}{c}\text { Axelsson and } \\
\text { Berntsson, } \\
\text { 2008 }\end{array}$ \\
\hline
\end{tabular}

\section{METHODOLOGY}

\subsection{Case studies - Pulp and paper mills}

Detailed process data collected in earlier studies was used to characterize the excess heat availability from four different Kraft mills, indicated by name in Figure 1. In addition, benchmark data about typical mills were obtained from the FRAM (Future Resource Adapted Pulp Mill) project. Benchmark mill specifications were extracted for bleached market Kraft pulp mills (Delin et al., 2005a) and integrated fine paper mills (Delin et al., 2005b).

Table 1 lists the main data for the case study mills. Some mills have undergone substantial revamping since the data were collected. The SCA Östrand mill planned for a new expansion in 2015, and the data were obtained by quotations and interaction with mill process experts, and therefore may be affected by a larger error compared to the other cases. 


\subsection{Targeting of power generation and characterization of excess heat availability}

Given the large amounts of high temperature excess heat from black liquor combustion in Kraft pulp mills, excess

heat availability was characterized considering that the mills are equipped with a steam turbine system designed for maximum power generation. The combined production targets for power and heat depend on the useful heat available from biofuel combustion and on the amount of steam required by the process. In this work, these targets were estimated using a mathematical programming framework, according to the steps described below.
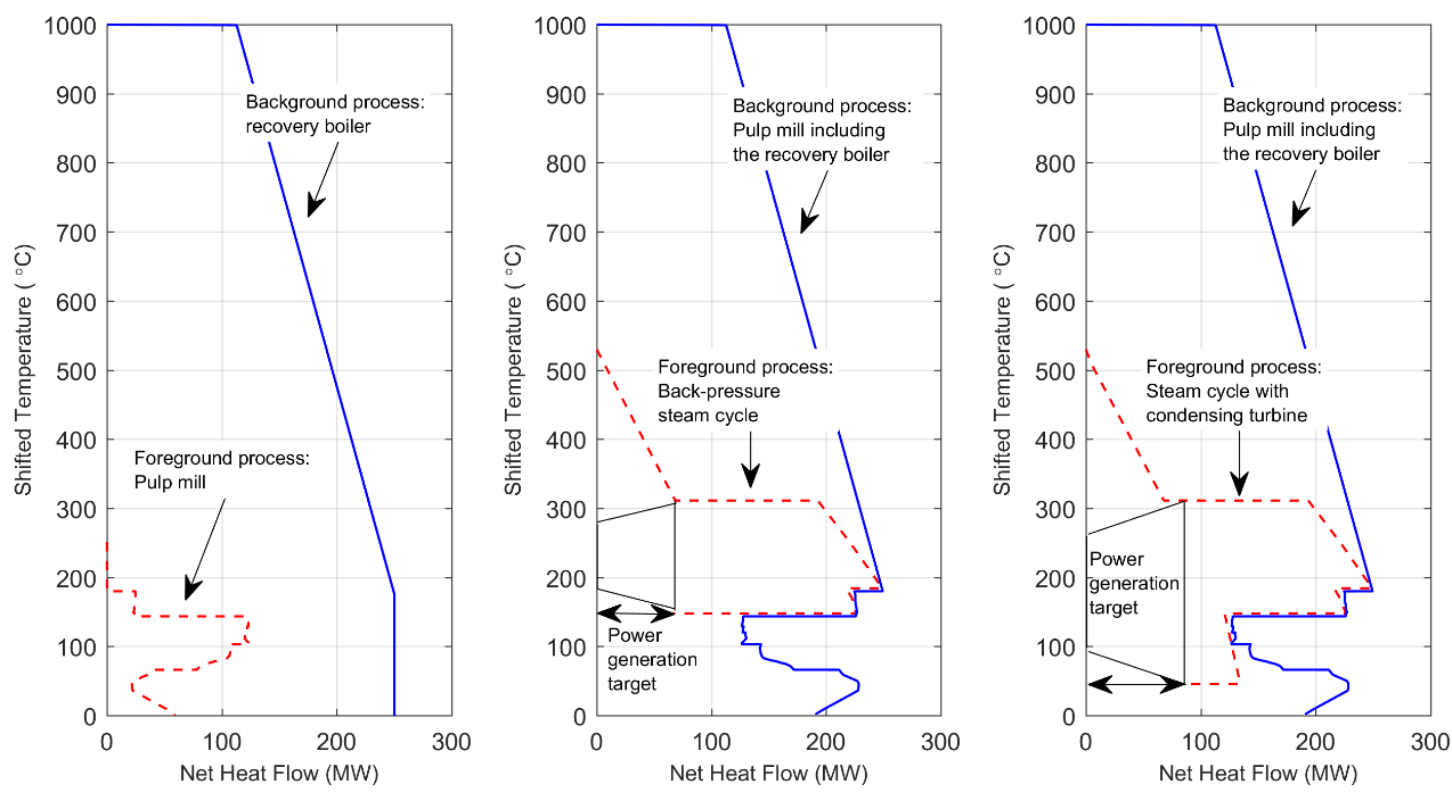

Figure 2. LEFT: Background/foreground analysis using split GCCs of the heat available from black liquor combustion in the recovery boiler and the net heating demand of the process. CENTER: Integration of a back-pressure turbine cycle with the pulp mill process using heat from the recovery boiler. RIGHT: Back-pressure and condensing turbine operation.

1. Characterization of high-temperature heat from black liquor combustion in the recovery boiler (blue line in Figure 2)

Black liquor is combusted in the recovery boiler for recovery of energy and regeneration of cooking chemicals. The boiler size is determined by the amount of black liquor being processed, which in turn depends on the production rate in the pulp digesters. Additional boilers, mainly fuelled with bark, are regarded as part of the utility system and not as necessary parts of the process itself.

2. Description of net process heating and cooling demands for the rest of the pulp mill processes

Ideal, maximized internal process heat recovery is estimated from the process Grand Composite Curve (GCC) assuming a minimum temperature difference for heat exchange of $0^{\circ} \mathrm{C}$ (blue line in Figure 3). When characterizing the current availability of excess heat, actual utility requirements of the process should be considered instead. In this case, the GCC is replaced by a representation of the current heating and cooling demands of the process at different temperatures.

3. Background/Foreground analysis using split GCCs for black liquor combustion heat and net process heating demand

This analysis shows if the heat content from the combustion of black liquor is sufficient to cover the heating demand of the process. When the split GCCs show that there is more heat available from the black liquor than is required by the pulping process, there is a potential for additional power generation without the use of additional fuel. This is the case for the example shown in Figure 2 (LEFT). 
4. Integration of a steam turbine cycle between the black liquor combustion heat from the recovery boiler and the net process heating demand (Figure 2)

Steam turbine cycle integration is illustrated in a background/foreground graph using split GCCs. In this case, the pulp mill process and the black liquor combustion heat from the recovery boiler are combined into a single background GCC. The steam cycle is represented by another GCC in the foreground.

Maximum fuel utilization can be achieved with a back-pressure turbine where the low-pressure outlet steam is sufficient to cover the process steam demand. In practice, steam is either available in excess, which opens the opportunity for a condensing turbine stage, or steam is directly reduced to lower pressure, by-passing the turbine. In the latter case, it might be justified to increase the steam production and back-pressure power generation, by firing additional bark in a boiler. This opportunity is considered for the assessment of excess heat availability under current utility requirements. However, for the theoretical heat integration case, minimized fuel use is prioritized.

For the example shown in Figure 2, steam is available in excess. The figure in the centre illustrates pure back-pressure turbine operation and an excess of low-pressure steam that could be delivered as excess heat to an external user. The figure to the right illustrates the case where a condensing turbine is added to the system. This way, the excess of steam is utilized for additional power generation. Note that this system design does not only limit the amount of excess heat available as steam from the steam turbine cycle, but also reduces the amount of excess heat available from the pulp mill process, since this heat is used for heating the condensate from the turbine condenser up to feedwater temperature.

5. Characterization of excess heat availability for processes with integrated steam cycle (Figure 3)

In this work, the availability of excess heat is characterized according to discrete temperature intervals. The resulting temperature profile is referred to as the excess heat temperature signature curve (XHT signature), which is constructed by aggregating the available excess heat at pre-defined temperature levels and temperature intervals. Two different signatures can be constructed: The Theoretical XHT signature represents the unavoidable excess heat, corresponding to theoretical, maximum internal process energy recovery, assuming a minimum temperature difference of $0^{\circ} \mathrm{C}$ for all heat exchangers. The Process Cooling XHT signature represents the current availability of excess heat.Figure 3 shows the estimated Theoretical XHT signatures for Södra Värö, assuming that a steam cycle with an additional condensing stage is integrated with the process.

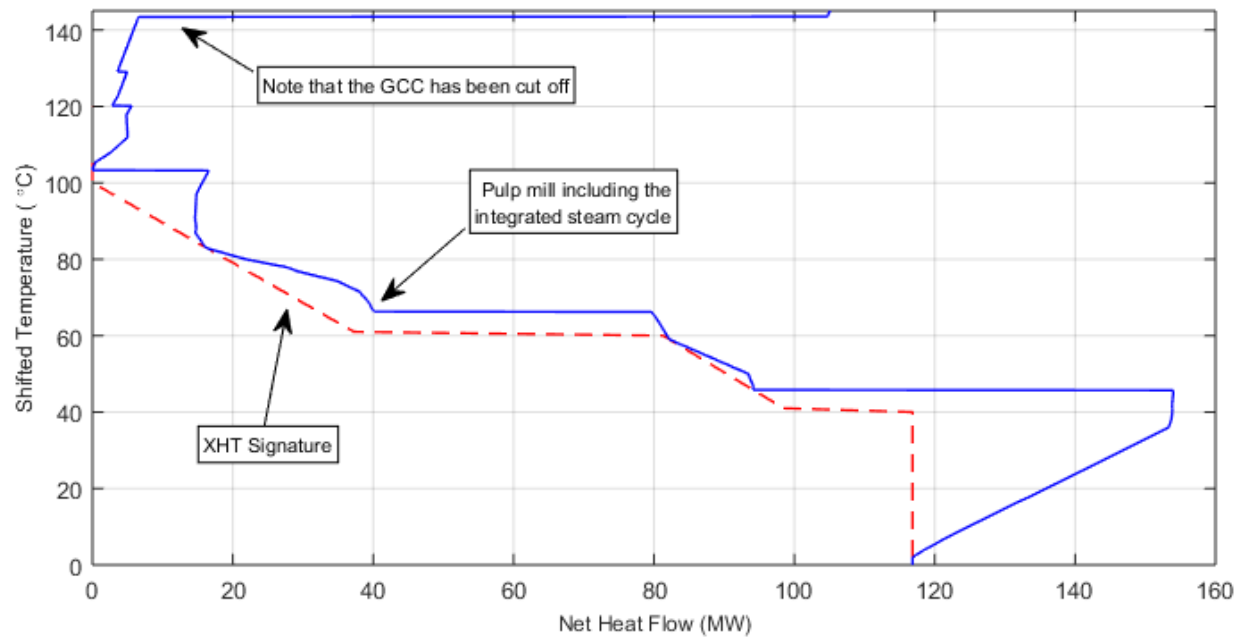

Figure 3. Estimated XHT Signatures based on the net cooling demand represented by the GCC of the integrated pulp mill process, recovery boiler and steam cycle. 


\subsection{Data and assumptions for the mill study cases}

The net useful heat available for steam production from black liquor combustion was estimated based on data for the FRAM mills, 6.046 MWh per air-dried tonne of pulp (Delin et al., 2005a). Bark feed to the bark boiler was estimated by subtracting the estimated black liquor from the reported value of total biomass fuel consumption (see Table 1). No steam was considered to be produced from fossil fuel. Theoretical maximum process heat recovery was estimated based on the thermal stream data for the case study mills. However, data about the actual heat exchanger network including process heaters and coolers are scarce and incomplete and it was not possible to define the actual cooling demand of each mill. Instead, the Process Cooling XHT signature curve was estimated based on assumptions about the current mill heat recovery as well as layout and operability limitations.

The heat generated by black liquor and bark combustion is used for power generation and low and medium pressure steam is used for process heating. The power generation depends on turbine inlet steam conditions, which are determined by the properties of the high-pressure (HP) boiler steam. In the theoretical case, the assumed steam properties for HP steam are representative of state-of-the-art recovery boiler technology (100 bar, $530^{\circ} \mathrm{C}$ ). For the estimation of the Process Cooling XHT signature, steam values corresponding to the Iggesund mill $\left(80 \mathrm{bar}, 480^{\circ} \mathrm{C}\right.$ ) were used. The back-pressure turbine isentropic efficiency was assumed to be 0.85 for the theoretical integration case and 0.75 for the assessment considering existing turbines. Low and medium steam pressure values were determined on a case by case basis with the objective of maximizing power generation. The condensing turbine option was considered only if the extra power produced by the condensing stage is greater than $10 \mathrm{MW}$. The assumed isentropic efficiency for the condensing stage was 0.9 and 0.85 for the theoretical and current systems, respectively. Steam reduction was modelled as separate steam production at 16 bar.

\subsection{Regression analysis}

Based on the analysis of the six mills, linear regression functions based on reported pulp and paper production rates were used to predict the power production targets as well as the availability of excess heat from the entire Swedish Kraft mill park. For excess heat, a regression analysis was conducted using eq. (1) for each of the temperature levels that were used to represent the XHT signature. Excess heat was assumed to be used primarily for district heating applications, which requires excess heat temperature levels above $60^{\circ} \mathrm{C}$. Constant mill production was assumed, reflecting the process conditions when thermal stream data were collected.

Excess heat $(\mathrm{GWh} / \mathrm{y})=k+m \cdot$ Market Pulp $(\mathrm{kADt} / \mathrm{y})+n \cdot$ Paper $(\mathrm{kADt} / \mathrm{y})$

\section{RESULTS}

\subsection{Theoretical heat integration in the studied mills}

When establishing the theoretical potential for internal heat recovery within the mill, no other sources of primary heat than the recovery boiler are considered, and ideal heat integration is considered with $\Delta \mathrm{T}_{\min }=0^{\circ} \mathrm{C}$. Furthermore, heat transfer is assumed to be possible directly between the recovery boiler flue gases and the pulp and paper process. The theoretical heat integration target is visualized for two of the studied mills in Figure 4. The split GCC analysis indicates that the heat available from black liquor combustion is substantially larger and at a sufficiently high temperature to cover the process heat demand without the need to use additional fuel. Furthermore, there is a much larger excess of heat from black liquor combustion in the market pulp mills than in integrated pulp and paper mills. Market pulp mills have a substantial potential for power generation, which highlights opportunities for a condensing turbine stage. Conversely, in the integrated pulp and paper mills, there might be the need for extra fuel combustion to obtain a well-balanced combined production of heat and power. 

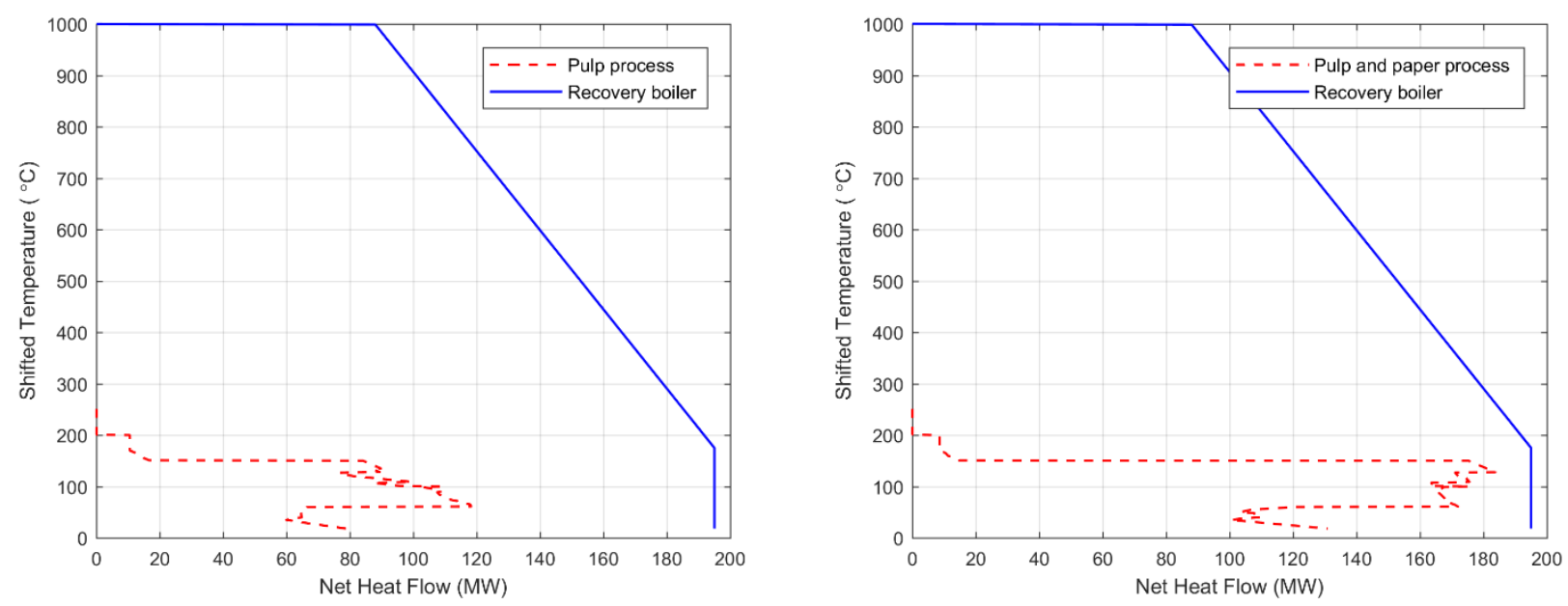

Figure 4: Split GCCs of the ideally integrated $\left(\Delta \mathrm{T}_{\min }=0^{\circ} \mathrm{C}\right)$ process (dashed line) and the heat from black liquor combustion in the recovery boiler (solid line). Combustion heat available above $1000^{\circ} \mathrm{C}$ is shown as a horizontal line.

LEFT: FRAM market pulp mill, RIGHT: FRAM integrated fine paper mill.

\subsection{Excess heat availability in the studied mills}

Figure 5 shows both XHT signatures for two of the studied mills. In most cases, the shape of the Process Cooling XHT signature is similar to that of the Theoretical XHT signature. However, the excess heat availability at medium to high temperature is lower for the theoretical case, due to increased heat recovery. This implies that excess heat from Kraft mills, e.g. for district heating application, can easily exceed the theoretical target which represents the limit of highly efficient excess heat utilization. Figure 6 presents a comparison between the six mills by showing the specific excess heat availability above $60^{\circ} \mathrm{C}$. The specific excess heat is evaluated as a ratio between the total excess heat and the biofuel consumption.
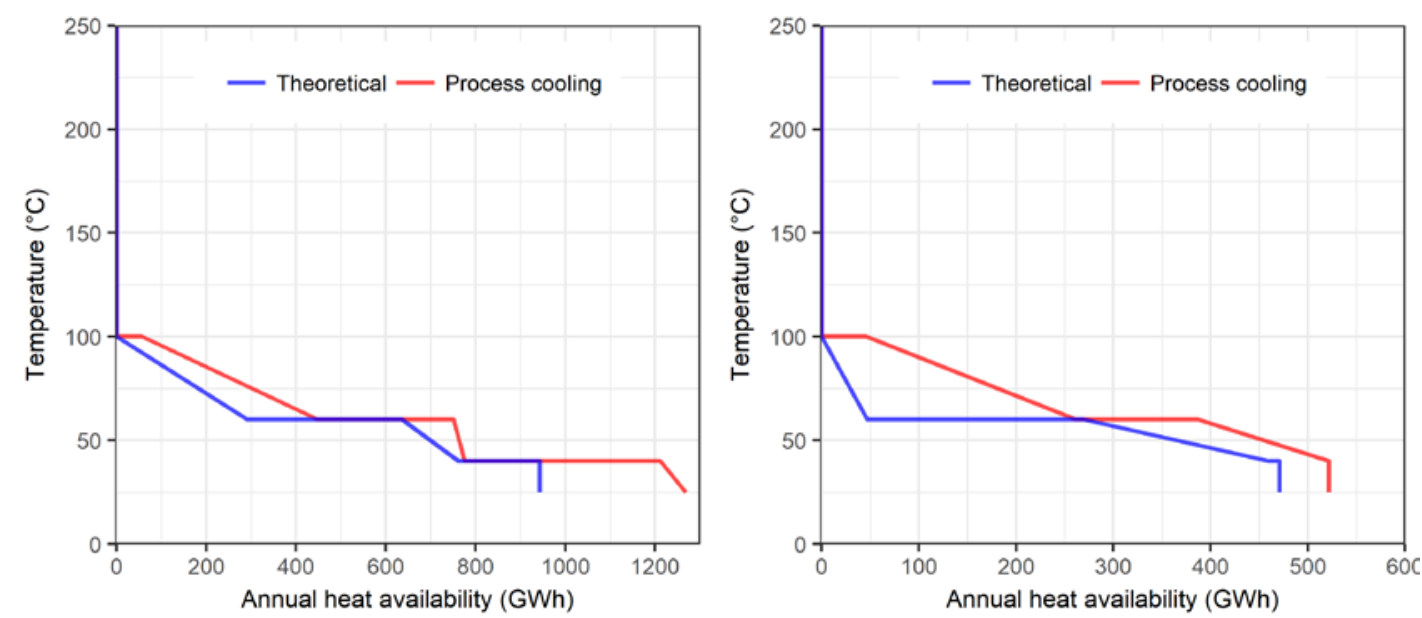

Figure 5. Estimated XHT signatures for Södra Cell Värö (LEFT) and Holmen Iggesund (RIGHT).

The specific availability of excess heat varies largely between the Kraft mills. However, the availability of excess heat for most of the cases is less than $15 \%$ of the biofuel energy supply, which is substantially below the $25 \%$ excess heat recovery level that has been suggested in generic studies assessing the potential of industrial excess heat levels for district heating applications (Persson et al., 2014). 


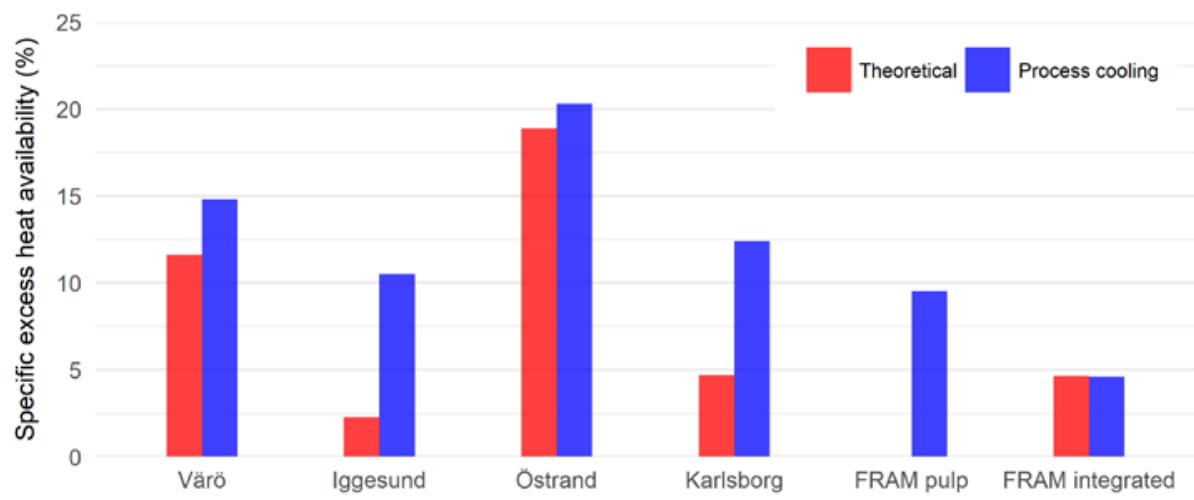

Figure 6: Estimated specific excess heat availability above $60^{\circ} \mathrm{C}$ in the six Kraft mills.

\subsection{Results from the regression analysis}

The availability of excess heat above $60^{\circ} \mathrm{C}$ was estimated for the six mills using (eq.1). The results are indicated in Figure 7 together with the standard deviation and the original estimates based on the detailed mill process data. Although the deviations are significant, the regression values agree quite well with the original values, at least for the real mills. The total excess heat above $60^{\circ} \mathrm{C}$ was extrapolated for the whole Swedish Kraft mill park using the above regression for all mills based on production data for 2015 (Skogsindustrierna, 2003-2015). The results indicate that there is a significant potential for increasing mill excess heat recovery, given that the theoretical target is about twice as much as the heat sold in 2015, and the 'process cooling' target is about three times larger than the sold heat in 2015. Figure 8 shows more details about each mill. There is no clear relation between amount of sold heat that is reported and the estimated excess heat potential. The theoretical excess heat potential is very small for small mills. This is an indication that the linear regression is not very accurate in this range. A more accurate prediction could be made with a more complex statistical analysis, provided that more data were available.

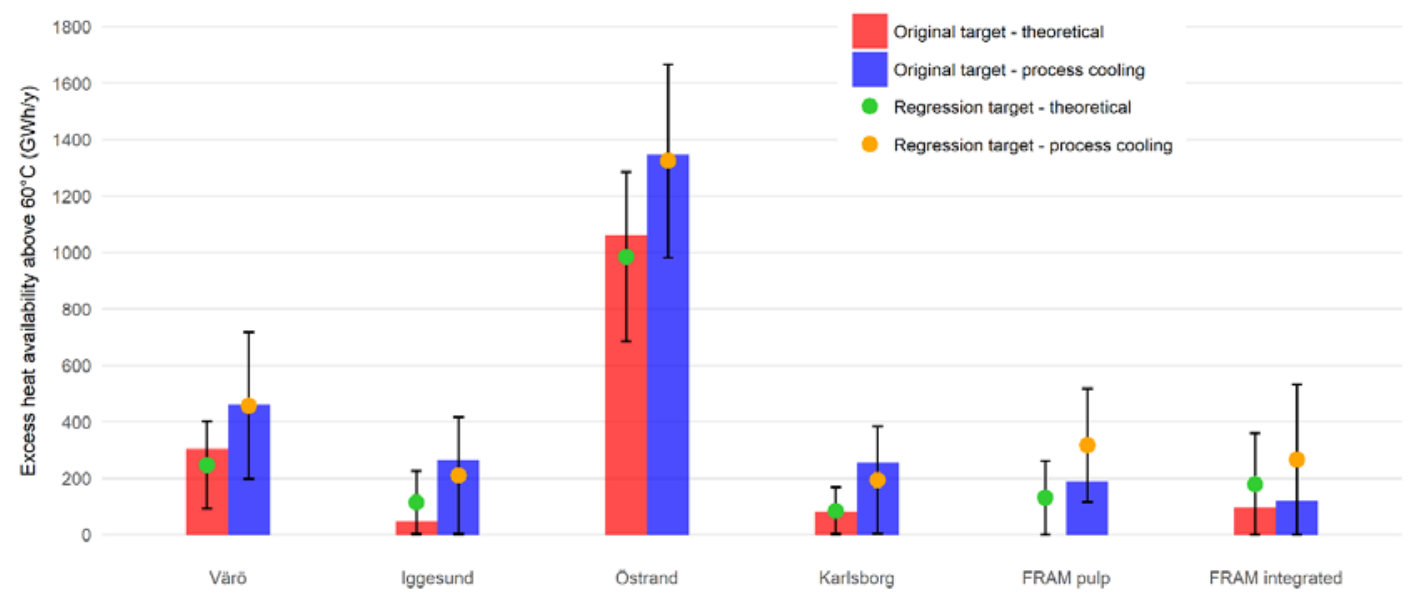

Figure 7: Estimated availability of excess heat above $60^{\circ} \mathrm{C}$ for the six Kraft mills (68\% confidence interval for regressed values).

Finally, the results from the prediction of power generation targets indicate that the reported electricity generation in the mills is close to the target corresponding to theoretical maximum internal process heat recovery. However, a potential for increasing the power production by 20 to 30\% is apparent when considering current heat recovery levels in the mills in which bark is used as a fuel for extra steam production above the theoretical minimum requirement. 


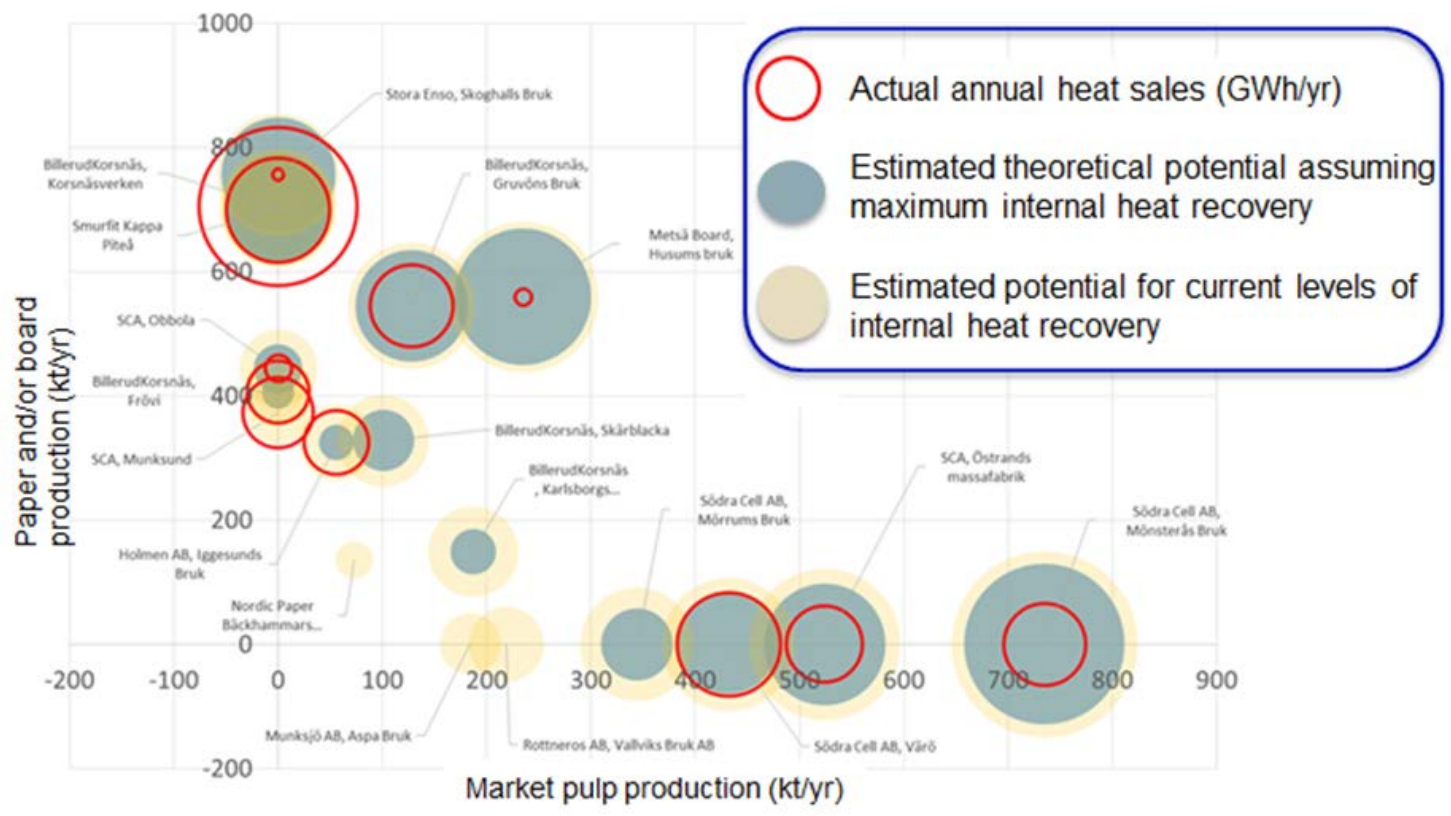

Figure 8: Availability of excess heat above $60^{\circ} \mathrm{C}$ from Swedish Kraft mills estimated based on 2015 production data, and sold heat according to declared data for the same year. Circle diameters proportional to heat deliveries and availability.

\section{CONCLUSION}

210 This paper proposed a bottom-up approach for estimating the availability and characteristics of excess heat from the Swedish pulp and paper industry. The results indicate that there is a large potential for increasing excess heat recovery in Swedish Kraft pulp and paper mills. The proposed approach based on detailed energy targeting studies for a selection of industrial plants should be possible to extend to other sectors and regions.

\section{REFERENCES}

Ahlström, J. and Benzon, M. (2015). Design of the heat recovery system at the reconstructed pulp mill Östrand [MSc thesis]. Chalmers University of Technology, Gothenburg, Sweden.

Axelsson, E. and Berntsson, T. (2008). Heat integration opportunities in an average Scandinavian fine paper mill: Model study and comparison with a market pulp mill. TAPPI Journal, 7, 19-25.

Axelsson, E., Olsson, M. and Berntsson, T. (2006). Heat integration opportunities in average Scandinavian kraft pulp mills: Pinch analyses of model mills. Nordic Pulp and Paper Research Journal, 21, 466-475.

Bendig, M., Maréchal, F., Favrat, D., (2013), Defining “Waste Heat” for industrial processes, Appl Therm Eng, 61, 134-142.

Bood, J. and Nilsson, L. (2013). Energy analysis of hemicellulose extraction at a softwood kraft pulp mill [MSc thesis]. Chalmers University of Technology, Gothenburg, Sweden.

225 Broberg, S., Backlund, S., Karlsson, M., Thollander, P., (2012), Industrial excess heat deliveries to Swedish district heating networks: Drop it like it's hot. Energy Policy, 51, 332-339.

Brueckner, S., Miró, L., Cabeza, L.F., Pehnt, M., Laevemann, E., (2014). Methods to estimate the industrial waste heat potential of regions - A categorization and literature review. Renew Sustain Energy Rev, 38, 164-171.

Delin, L., Berglin, N., Eriksson, T., Andersson, R., Samuelsson, Å., Lundström, A., et al. (2005b). Integrated fine paper mill. FRAM report no 10. FRAM R\&D program, Stockholm, Sweden.

Delin, L., Berglin, N., Lundström, A., Samuelsson, Å., Backlund, B. and Sivard, Å. (2005a). Bleached market kraft pulp mill - Reference and type mill. FRAM report no 09. FRAM R\&D program, Stockholm, Sweden.

Eriksson, L. and Hermansson, S. (2010). Pinch analysis of the Billerud Karlsborg, a partly integrated pulp and paper mill [MSc thesis]. Chalmers University of Technology, Gothenburg, Sweden.

235 Isaksson, J., Åsblad, A. and Berntsson, T. (2013). Influence of the drier type on the performance of a biomass gasification combined cycle co-located with an integrated pulp and paper mill. Biomass \& Bioenergy, 59.

Persson, U., Möller, B. and Werner, S. (2014). Heat Roadmap Europe: Identifying strategic heat synergy regions. Energy Policy, 74, 663-681.

Skogsindustrierna. (2003-2015). Miljödatabasen. Swedish Forest Industries Association, Stockholm, Sweden. 


\section{INTERACTIONS BETWEEN SOIL AND GEOTHERMAL HELICAL HEAT EXCHANGERS: AN OVERVIEW OF ITER PROJECT OUTCOMES}

Eloisa Di Sipio ${ }^{1}$, Martin Potten ${ }^{2}$, David Bertermann ${ }^{1}$

Department of Geology, GeoZentrum Nordbayern, FAU University Erlangen-Nuremberg, Germany ${ }^{1}$

Department of Engineering Geology, TUM University, Munich, Germany ${ }^{2}$

Schlossgarten 5, 91054 Erlangen, Germany

E-Mail: eloisa.disipio@gmail.com

\section{SUMMARY}

The thermal properties of soils can be considered one of the most important parameters for many engineering projects designing. In detail, the thermal conductivity plays a fundamental role when dimensioning ground heat exchangers, especially very shallow geothermal (VGS) systems, interesting the first $2 \mathrm{~m}$ of depth from the ground level. However, the determination of heat transfer in soils is difficult to estimate, because depends on several factors, including, among others, particle size, density, water content, mineralogy composition, ground temperature, organic matter. The performance of a VSG system, as horizontal collectors or special forms is strongly correlated to the kind of sediment at disposal and suddenly decreases in case of dry-unsaturated conditions in the surrounding soil. Therefore, a better knowledge of the relationship between thermal conductivity and water content is required for understanding the VSG systems behaviour.

Keywords: soil, water content, thermal conductivity, very shallow geothermal energy

\section{INTRODUCTION}

In the near future the shallow geothermal energy resource is becoming increasingly important as renewable energy resource for heating/cooling residential and tertiary buildings. Therefore, it is worthy of interest a better comprehension of how the different soil typologies (i.e. sand, loamy sand...) affect and are affected by the heat transfer exchange with heat collectors, especially when horizontal ones are adopted (Nagy and Körmendi 2012; Angelino and Sanner 2013).

In this paper part of the ITER European Project results (Improving Thermal Efficiency of horizontal ground heat exchangers) is shown. An overview of the complete laboratory and field test outcomes on different materials (coarse sand, loamy sand etc ...) and environmental conditions can be found in Di Sipio and Bertermann (2018a, 2018b, 2017a, 2017b). Key challenge of this project is to understand how to enhance the heat transfer of the sediments surrounding the pipes, taking into account the interactions between the soil, the horizontal heat exchangers and the surrounding environment. Changes of soil moisture content in the same climatic conditions and under the same thermal stress for five different soil mixtures have been monitored in the ITER test site (Di Sipio and Bertermann 2017a, 2018b). Since the available experimental data are often scattered, incomplete and do not fully support thermo-active ground structure modeling, here is provided an overview of physical-thermal properties variations under different moisture and load conditions for fine sand (fs) mixtures, based on laboratory and field test data collection (Di Sipio and Bertermann, 2017b, 2018a).

In this paper, the term 'soil' is used mainly in its geological and engineering acceptance, that is as the accumulation of disintegrated rock material, found in place or relocated, employed mainly as construction or foundation material, as also usually reported in the scientific literature devoted to the thermal properties study of unconsolidated material (Abu-Hamdeh et al., 2003; De Vries, 1963; Farouki, 1981; Nikolaev et al., 2013; Zhang et al., 2017).

Since 1980, soils have been studied and used also as heat reservoirs in geothermal applications, either as a heat source (in winter) or sink (in summer) coupled mainly with heat pumps (Ochsner et al., 2001; Chong et al., 2013). Soils are also a natural and valid renewable alternative for thermal energy supply for heating/cooling residential and tertiary buildings and agricultural greenhouses. On this regard, it is proved that the performance 
of VSG system rapidly decreases in case of dry-unsaturated conditions in the surrounding soil. For example, the coefficient of performance (COP), a technical parameter measuring the heat pump efficiency, suddenly decreases when the soil is dry and improves when an increase of the water content is noticed (Drefke et al., 2017; Farouki, 1981; Leong et al., 1998; Wu et al., 2015). Furthermore, in the first meters of depth below the ground surface, when groundwater flow is not present, conduction is the dominant process governing heat transfer in soil. This process is driven by temperature gradients and depends mainly on thermal conductivity, in turn, a property strictly related to water content (Gonzales et al., 2012; Leong et al., 1998; Schön J.H., 2011; Song et al., 2014). Therefore, a better knowledge of the relationship between thermal conductivity and water content is required for understanding the VSG systems behavior in saturated and unsaturated conditions. This constitutes the main focus of the research here presented.

\section{METHODOLOGY}

The laboratory activity here described focuses on analysing the physical-thermal properties of fine sand as pure and mixed material in dependence of the water content and the bulk density (Fig.1). Two additives are used to create the mixtures, consisting respectively of bentonite and clay powder, commercial products readily available on the market. They have been mixed separately to the coarse sediment in different weight percentages $(8 \%-15 \%-30 \%)$, as summarized in Table 1 . The fine sand (fs) used in this research belong to the Burgsandstein Formation (Trias), has a dominant quartz composition and is sieved up to $1 \mathrm{~mm}$. The bentonite (B) and clay powder (C) are, the former, a very fine non calcareous marine clay, belonging to the Holmehus Formation; the latter a grey marl-calcareous clay (Lias) collected near the city of Buttenheim (Bavaria, Germany). Quartz is the main component of fs and $\mathrm{C}$, while montmorillonite is the dominant mineralogical phase for B.

Table 1: The set of fine sand mixtures analysed in ITER laboratory test (modified by Di Sipio and Bertermann, 2018a)

\begin{tabular}{|l|l|l|}
\hline pure material & ITER mixtures & sample code* \\
\hline fine sand 0-1 mm & pure & fs \\
& with 8\% Bentonite & fs8B \\
& with 15\% Bentonite & fs15B \\
& with 30\% Bentonite & fs30B \\
& with 8\% Clay & fs8C \\
& with 15\% Clay & fs15C \\
& with 30\% Clay & fs30C \\
\hline
\end{tabular}

* $\mathrm{B}=$ bentonite and $\mathrm{C}=$ clay powder

The physical-thermal properties of the sediments selected have been analyzed both in laboratory and directly in situ (here not shown), in order to clarify their behavior respectively in a controlled environment or in unsteady conditions, affected by external factors (Di Sipio and Bertermann 2017a). The main parameters determined are:

- grain size by sieving the sand fraction $(<63 \mu \mathrm{m})$ of each mixture according to DIN 18123 and then using for the remaining fine particles the Sedigraph III Plus 5125;

- mineralogical content by X-Ray Diffraction (XRD) technique using the reference intensity ratio (RIR) methodology on powder samples (PanAnalytical X'Pert Pro instrument);

- thermal conductivity by thermal properties analyzer (KD2Pro apparatus, Decagon Devices, Inc.), operating according to the transient line source method (ASTM D5334-08);

- moisture content and bulk electrical conductivity (measured simultaneously) by time domain reflectometry (TDR) device (TRIME IMKO GmBH);

- bulk density on duly collected sample (for every moisture content step and each load) according to the DIN 52102; 
- volumetric water content on duly collected sample (for every moisture content step and each load) according to the DIN 18121;

At first, in laboratory, each mixture (about $60 \mathrm{~kg}$ ) is naturally dried in a ventilated room at standard temperature and pressure condition. Once dried, the material is collected and subjected to parameter acquisition. During each phase, measurements of bulk density and volumetric water content are performed together with acquisition of moisture content, bulk electrical resistivity and thermal properties values. The entire measurement procedure is repeated for each mixture under different water content and incremental load steps $(+0,+1,+3,+5$ tons). In fact, fresh water is added gradually to the anhydrous soil until its field capacity is overcome, while it goes from unconsolidated till consolidated condition. In this way a complete set of data is obtained, available to be compared with the values acquired in situ. Information about volumetric water content $(\theta)$, porosity $(\phi)$ and saturation (S) are calculated according to different German Standard Norms (DIN), as DIN 18121-1 (1998) and DIN 52102-02 (2006). The choice to perform measurements on large volumes of materials (about $56 \mathrm{dm}^{3}$ ) compared to the common laboratory scale is due to the attempt to make them comparable with the results given at local scale (i.e. field test), trying to minimize the scale effect (Di Sipio and Bertermann, 2018a; Bertermann and Schwarz, 2017; Moya et al., 1999; Kömle et al., 2007).
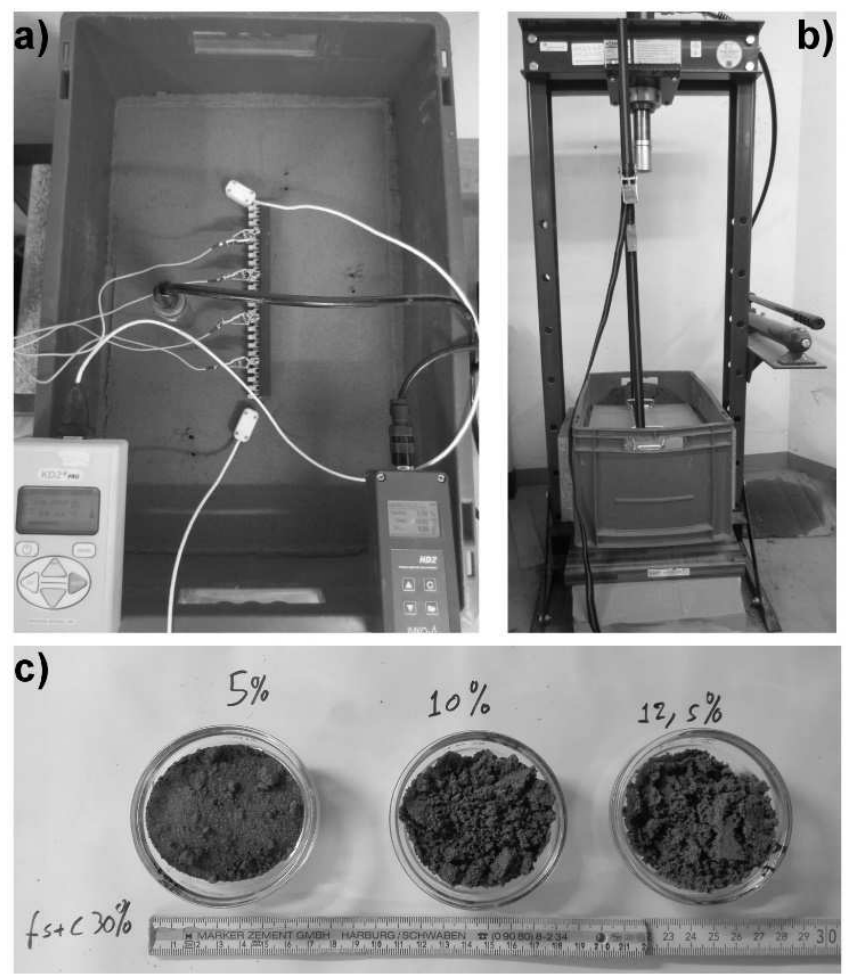

Fig. 1: Laboratory measurements: a) thermal conductivity, moisture content and bulk electrical resistivity acquisitions on a soil body; b) the workshop press to apply the incremental pressure steps; c) a detail of the thermal measurement samples collected to determine bulk density and volumetric water content of the mixture.

\section{RESULTS AND DISCUSSION}

Regarding the heat transfer capacity of the selected mixtures, the thermal conductivity $(\lambda)$ trend increases (range between 0.187 and $2.179 \mathrm{Wm}^{-1} \mathrm{~K}^{-1}$ ) as a function of the volumetric water content $(\theta)$ and the pressure loads, as expected from literature (Fig.2). In details, in completely anhydrous condition ( $\theta$ nearly $0 \%$ ), the fine sand mixtures show approximately a similar initial thermal conductivity value $\left(\approx 0.200 \mathrm{Wm}^{-1} \mathrm{~K}^{-1}\right)$, typical of a dry soil whose pores are filled with air. Then, increasing the $\theta$ amount, the heat transfer increases approximately linearly for each soil composition, showing a steep progression for the pure sands (fs), and a moderate trend for the sandy loam materials (additive B or C). In the latter case, for example, for each 
incremental pressure step, increasing $\theta$ the bentonite mixtures diverge from the pure material (fine sand) trend showing always a lower $\lambda$ value. However, the gap between pure material and bentonite compounds is reduced at $+5 \mathrm{t}$ step. As a matter of fact, increasing pressure on the soil, the heat transfer capacity of the mixtures comes close (Fig.3).

The volumetric water content ranges between 0.00 and circa $50.00 \%, \mathrm{~m}^{3} \mathrm{~m}^{-3}$, where the lowest values belong to fs and the highest to the fs30B mixture. For equal amount of additive (B or C) inserted in the same basic material ( $\mathrm{fs}$ ), the contribution of bentonite exceeds that of the clay powder in the ability to retain water: $\theta$ on average is about $23.84 \%$ and $15.31 \%, \mathrm{~m}^{3} \mathrm{~m}^{-3}$ for fsB and fsC mixtures respectively.

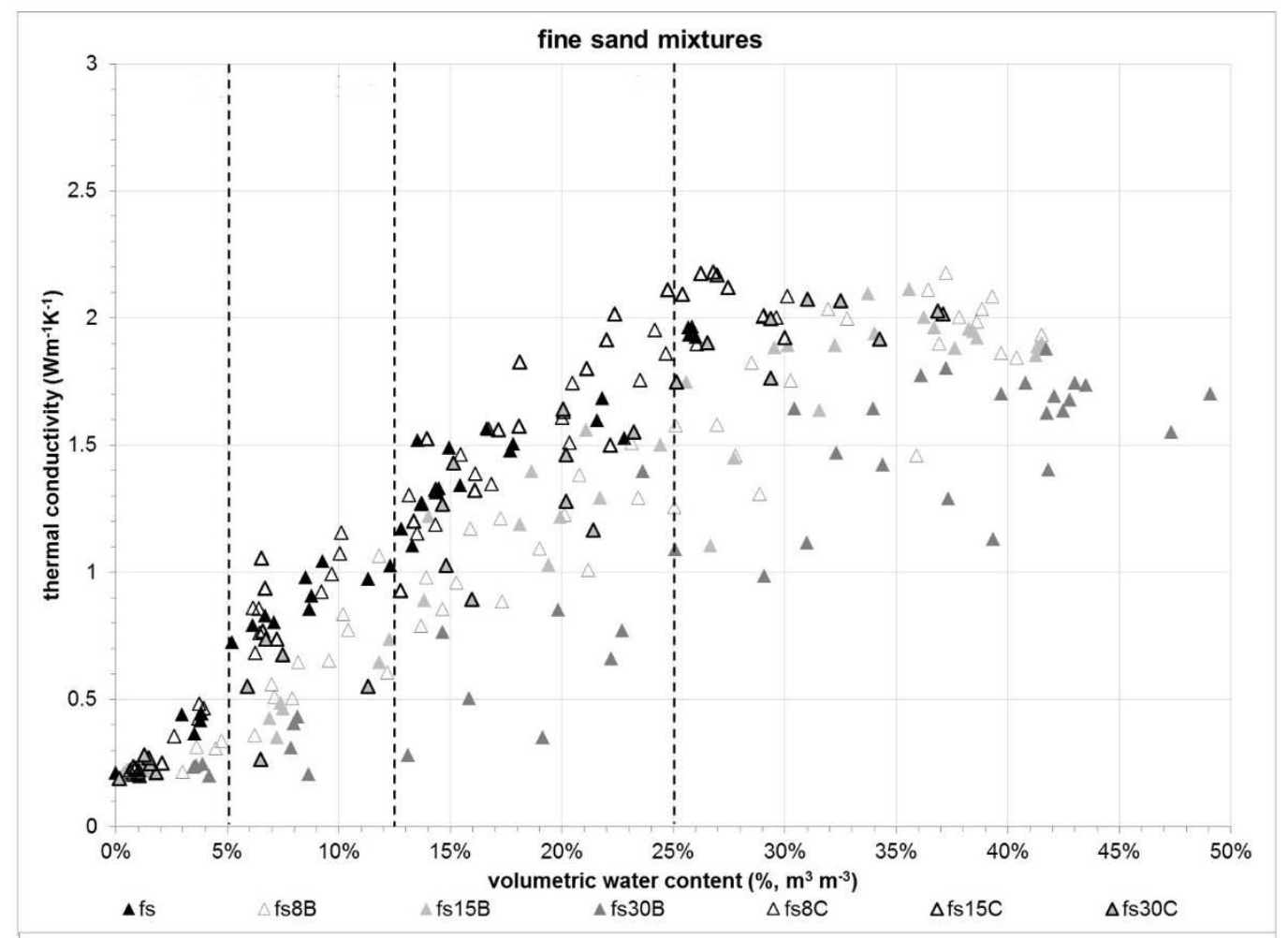

Fig. 2: Relationship between thermal conductivity and volumetric water content for ITER mixtures: graphical representation of the measurement performed on the fine textured material comprehensive of all the different pressure conditions and moisture content levels created in laboratory (modified by Di Sipio and Bertermann, 2018a)

At low degree of volumetric water content $\left(<5 \%, \mathrm{~m}^{3} \mathrm{~m}^{-3}\right)$, the $\lambda$ barely changes $\left(0.180<\lambda<0.500 \mathrm{Wm}^{-1} \mathrm{~K}^{-1}\right)$, for all fine sand mixtures at any pressure stage (Fig.2). The heat transfer here occurs mainly through contact points between soil particles and is hampered by the presence of air in the voids, while the water adheres to particle surfaces.

Then, a further increase of moisture $\left(5<\theta<12.5 \%, \mathrm{~m}^{3} \mathrm{~m}^{-3}\right)$ shows a rapid rise of thermal conductivity in all the mixtures, thanks to the creation of water bridges between soil grains, able to greatly enhance $\lambda$ at low water content $\left(<12.5 \%, \mathrm{~m}^{3} \mathrm{~m}^{-3}\right)$. The $\lambda$ evolution with changing degree of moisture content is steeper for pure fs compared to the mixed materials, reaching maximum values of about $1.2,1.3,1.1 \mathrm{Wm}^{-1} \mathrm{~K}^{-1}$ for fs, fsC, fsB mixtures respectively (Fig.2-3). In addition, the trend is even more dampened in soil characterized by a greater porosity, that is when bentonite instead of clay powder is added (Fig.2-3). In fact, in fine textured soils, characterized by particles with larger surface areas, more water is required to create the water bridges, so, on one hand, the $\lambda$ increase with $\theta$ is faster in the pure material and, on the other, greater $\theta$ values are obtained when bentonite and clay are added.

135 Further increasing the volumetric water content $\left(12.5<\theta<25.0 \%, \mathrm{~m}^{3} \mathrm{~m}^{-3}\right)$, a gradual and slower growth of $\lambda$ with $\theta$ is observed, due to the progressive replacement of air with water in the pore voids. In this case, the 
upper limit $\left(\theta=25.0 \%, \mathrm{~m}^{3} \mathrm{~m}^{-3}\right)$ has been derived by graphical observation, because after this threshold a further increase of water content does not affect the heat flow growth. However, in the mixture with a higher percentage of clay material or bentonite, this limit could be shifted towards greater moisture content value (i.e. $\theta>35 \%, \mathrm{~m}^{3} \mathrm{~m}^{-3}$ for $\left.\mathrm{fs} 30 \mathrm{~B}\right)$, because the finer grain size requires more water content to reach saturated condition.

The maximum values of thermal conductivity measured in laboratory for the fine sand mixtures belong to this domain and reaches values of about $2.2 \mathrm{Wm}^{-1} \mathrm{~K}^{-1}$ (Fig.2).

Finally, in the last water domain $\left(\theta>25.0 \%, \mathrm{~m}^{3} \mathrm{~m}^{-3}\right)$, the thermal conductivity is no longer influenced by the increase of pore water in the voids. Given that the air displacement in the voids is not more effective, drainage is possible, near saturation conditions are reached and $\lambda$ values in this regime are constant or can slowly decrease. For example, $\lambda$ trend for fine sand mixtures shows values decreasing slowly between $2.2-1.5 \mathrm{Wm}^{-}$ ${ }^{1} \mathrm{~K}^{-1}$ (Fig.2).

Moreover, it can be observed that adding an additive to a pure material enriched in quartz (i.e. fs) reduces its capacity to transfer heat: the greater the amount added (30\% of additive), the greater the observed reduction compared to the original soil (Fig.2-3). The increased number of grain contacts due to the presence of finer material, clay powder (C) or bentonite (B), is responsible for an increase of the thermal resistance between grains and consequently slightly diminishes the $\lambda$ values.

However, the mineralogical composition of the additive play also a role in the $\lambda$ variation. Thanks to its $45 \%$ quartz mineralogical composition (quartz has a good performance as heat transfer medium), the addition of clay powder, in any percentages, to fs does not result in a sharp decrease in values for any pressure steps considered. Instead, an increased amount of bentonite, characterized mainly by clay minerals, is responsible on one hand, for a lowering of the heat transfer values and, on the other, for a gain in the capacity for retaining moisture, compared both to pure material and to the clay compounds.

160 In addition, in the same moisture condition, an increase of the pressure load from +75 to $+5000 \mathrm{~kg}(0$ to $5 \mathrm{t})$ determines an improvement of the heat transfer ability in all the mixtures tested. The gap between the measurements performed at the lowest $(+75 \mathrm{~kg})$ and greatest pressure step $(+5000 \mathrm{~kg})$ increases as the percentage of clay powder or bentonite in the compounds increases too. A wider distribution of the results is observed when a $30 \%$ of additive is added to the pure material (Fig.3).
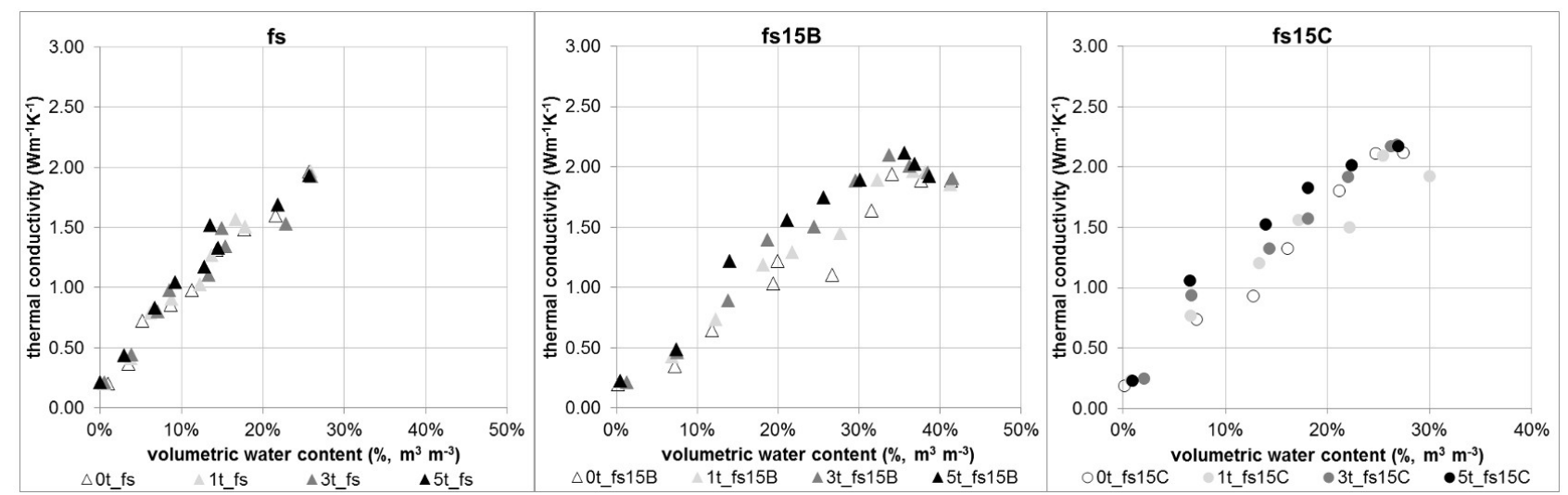

Fig. 3: Example of $\lambda$ vs $\theta$ relationship for specific ITER fine sand mixtures differentiated according to the pressure load exerted and the percentage of clay or bentonite additive used. In the caption, the pressure load steps are shown in tonnes and the first step $(75 \mathrm{~kg}$ ) is cited as $0 \mathrm{t}$ (modified by Di Sipio and Bertermann, 2018a)

The feature to retain water is really important if high and constant thermal conductivities are desired for a VSG system. Given the very shallow installation depth required by horizontal heat exchangers, if a soil, as fs, shows a change in $\lambda$ for a small water content variation, induced for example by atmospheric and/or environmental condition or operation condition of the heat exchanger itself, the efficiency of the system can be compromised. 
Therefore, a soil able to vary gradually its $\lambda$ content with a reduction of $\theta$ is preferable to ensure over time a constant performance of the VSG system.

\section{CONCLUSION}

The outcomes of ITER Project, at the forefront of very shallow geothermal research, are encouraging. In laboratory, under controlled and reproducible conditions, the bentonite mixtures specially created for the project shows a reduced ability to transfer heat compared with pure fine sand, but they can clearly carry a greater amount of water. This ability is essential to properly understand what could happen in a real case study. In fact, when dealing with in situ test site, both meteorological / environmental aspects and the operating conditions of the VSG systems can influence the volumetric water content distribution on surface, in depth, and in the heat collector surroundings, affecting in this way the heat transfer capacity of the soils. Therefore, strengthening the comprehension of the main factors able to affect the heat transfer exchange is essential, in order to improve the ability to preserve the soil thermal behavior over time.

On this regard, several soil mixtures characterized by different grain size distribution have been tested under different pressure, water content and mineralogical content allowing to create a detailed database of physicalthermal properties variations in soil bodies. The collected data constitute a valuable reference to support modelling for very shallow geothermal applications and to validate heat and water transport model in unconsolidated material. Due to the specific methodological approach selected, they can also be directly related to in situ field test measurements. In general, to summarize, bulk density and moisture content affect both the thermal conductivity of soil bodies: on one hand, an increase of soil density for the same moisture content lead to an increase of the thermal conductivity; on the other, for the same soil density, an increase in thermal conductivity is related to an increase of the moisture content. The measurements performed on different unconsolidated sediment typologies confirm the dominant influence of water content on the thermal conductivity of soils. In detail, in the fine sand mixtures here presented, the increase of $\lambda$ with an increase of $\theta$ is gradual and lead all mixtures to approaches an equilibrium at circa $2.0 \mathrm{Wm}^{-1} \mathrm{~K}^{-1}$ : bentonite and clay powder mixtures reach similar $\lambda$ but are characterized by different $\theta$ values $\left(\theta_{\text {bentonite }}>40 \%, \mathrm{~m}^{3} \mathrm{~m}^{-3}, \theta_{\text {clay powder }}<40 \%\right.$, $\left.\%, \mathrm{~m}^{3} \mathrm{~m}^{-3}\right)$.

\section{REFERENCES}

Abu-Hamdeh, N.H., (2003), Thermal properties of soils as affected by density and water content. Biosystems Engineering, 86(1), 97-102, doi:10.1016/S1537-5110(03)00112-0.

Angelino, L., and Sanner, B. (2013), A Strategic Research and Innovation Agenda for Renewable Heating and Cooling: Key Findings. Proceedings, European Geothermal Congress 2013 Pisa, Italy (2013), EGC-MA-05:16 .

Bertermann, D., Schwarz, H., (2017), Laboratory device to analyse the impact of soil properties on electrical and thermal conductivity. International Agrophysics, 31(2), 157-166, doi:10.1515/intag-2016-0048.

Chong, C.S.A., Gan, G., Verhoef, A., Garcia, R.G., Vidale, P.L., (2013), Simulation of thermal performance of horizontal slinky-loop heat exchangers for ground source heat pumps. Applied Energy, 104, 603-610, doi:10.1016/j.apenergy.2012.11.069.

DeVries, D.A., (1963), Thermal properties of soils. In Physics of Plant Environment, van Wijk WR, (ed.), Wiley: New York, 210-235.

DIN 18121-1, (1998), Soil, Investigation and Testing: Water Content Part 1. Determination by Drying in Oven, German Standard DIN, Berlin (Deutsches Institut für Normung).

215 DIN 52102-02, (2006), Test methods for aggregates - Determination of dry bulk density by the cylinder method and calculation of the ratio of density, German Standard DIN, Berlin (Deutsches Institut für Normung).

Di Sipio E., Bertermann, D. (2018a), Thermal properties variations in soil bodies for very shallow geothermal application: overview of ITER Project. International Agrophysics, 32, 149-164, doi: 10.1515/intag-2017-0002. 
Di Sipio E., Bertermann, D. (2018b), Soil thermal behavior in different moisture condition: an overview of ITER Project from laboratory to field test monitoring. Environmental Earth Sciences, 77: 283. https://doi.org/10.1007/s12665-018-7454-y.

Di Sipio E., Bertermann, D., (2017a), Factors influencing the thermal efficiency of horizontal ground heat exchangers. Energies (Open Access), 10(11), 1897, doi:10.3390/en10111897.

Di Sipio E., Bertermann, D., (2017b), Influence of Different Moisture and Load Conditions on Heat Transfer within Soils in Very Shallow Geothermal Application: An Overview of ITER Project. in ' PROCEEDINGS, 42nd Workshop on Geothermal Reservoir Engineering, Stanford University, Stanford, California, February 1315, 2017, pp- 1345-1353, SGP-TR-212GC 2016.

Drefke C., Schedel, M., Stegner, J., Balzer, C., Hinrichsen, V., Sass, I., (2017), Measurement Method of Thermal Properties of Cementitious Bedding Materials and Unsaturated Soils: Hydraulic Influence on Thermal Parameters. Geotechnical Testing Journal, 40(1), 160-170, doi:10.1520/GTJ20160027.

Farouki, O.T., (1981), Thermal properties of soils (No. CRREL-MONO-81-1). Cold Regions Research and Engineering Lab Hanover Nh.

Gonzalez, R.G., Verhoef, A., Vidale, P.L., Main, B., Gan, G., Wu, Y., (2012), Interactions between the physical soil environment and a horizontal ground coupled heat pump, for a domestic site in the UK. 235 Renewable Energy, 44, 141-153, doi: doi:10.1016/j.renene.2012.01.080.

Kömle, N.I., Bin,g H., Feng, W.J., Wawrzaszek, R., Hütte, E.S., He, P., Marczewski, W., Dabrowski, B., Schröer, K., Spohn, T., (2007), Thermal conductivity measurements of road construction materials in frozen and unfrozen state. Acta Geotechnica, 2(2), 127-138, doi: 0.1007/s11440-007-0032-1

Leong, W.H., Tarnawski, V.R., Aittomäki, A., (1998), Effect of soil type and moisture content on ground heat pump performance: Effet du type et de l'humidité du sol sur la performance des pompes à chaleur à capteurs enterrés. International Journal of Refrigeration, 21(8), 595-606.

Moya, R.E.S., Prata, A.T., Neto, J. C., (1999), Experimental analysis of unsteady heat and moisture transfer around a heated cylinder buried into a porous medium. International journal of heat and mass transfer, 42(12), 2187-2198.

245 Nagy, K., and Körmendi, K. (2012), Use of renewable energy sources in light of the "New Energy Strategy for Europe 2011-2020", Applied Energy, 96, 93-399.

Nikolaev, I.V., Leong, W.H., Rosen, M.A., (2013), Experimental investigation of soil thermal conductivity over a wide temperature range. International Journal of Thermophysics, 34(6), 1110-1129, doi:0.1007/s10765013-1456-5.

250 Ochsner, T.E., Horton, R., Ren, T., (2001), A new perspective on soil thermal properties. Soil science society of American Journal , 65(6), 1641-1647.

Schön, J.H., (2011), Physical Properties of Rocks. A workbook. In: Handbook of Petroleum Exploration and Production Volume 8 (J. Cubitt Ed.), Elsevier B.V., Amsterdam, The Netherlands.

Song, W.K., Cui, Y.J., Tang, A.M., Ding, W.Q., Tran, T.D., (2014), Experimental study on water evaporation 255 from sand using environmental chamber. Canadian Geotechnical Journal, 51(2), 115-128, doi:10.1139/cgj2013-0155.

Wu, R., Tinjum, J.M., Likos, W.J., (2015), Coupled Thermal Conductivity Dryout Curve and Soil-Water Characteristic Curve in Modeling of Shallow Horizontal Geothermal Ground Loops. Geotechnical and Geological Engineering, 33(2), 193-205, doi:10.1007/s10706-014-9811-2.

260 Zhang, T., Cai, G., Liu, S., Puppala, A.J., (2017), Investigation on thermal characteristics and prediction models of soils. International Journal of Heat and Mass Transfer, 106, 1074-1086, doi:10.1016/j.ijheatmasstransfer.2016.10.084. 


\section{AKNOWLEDGEMENTS}

ITER project (http://iter-geo.eu/) has received funding from the European Union's Framework Programme for 265 Research and Innovation Horizon 2020 (2014-2020) under the Marie Skłodowska-Curie Grant Agreement No.[661396-ITER]. 


\title{
LOW TEMPERATURE AND COLD DISTRICT HEATING AND COOLING SYSTEMS - TRANSITION, IMPLEMENTATION, PLANNING, LONG-TERM EVALUATION
}

Ingo Leusbrock, Harald Schrammel

AEE - Institute for Sustainable Technologies

A-8200 Gleisdorf, Feldgasse 19, Austria

phone: $+43(0) 31125886-261$

email: i.leusbrock@aee.at

\begin{abstract}
SUMMARY
District heating and cooling are recognized as key technologies for our future cold and heat supply. We present current efforts and results to enable a transformation of existing systems and implementation of new systems to Low - temperature district heating and cooling (LTDHC) systems (supply temperatures $50-70^{\circ} \mathrm{C}$ ) and Cold district heating and cooling (CDHC) systems (supply temperatures $30^{\circ} \mathrm{C}$ ). We will show their opportunities and overall applicability by establishing the necessary basics in conceptualization, planning and long-term evaluation. This is achieved by a) evaluation of possible system solutions for different configurations and boundary conditions, $b$ ) development of a multi-domain co-simulation framework for evaluation of technical and economic benefits, c) development of a stochastic approach for long-term system evaluation in regard of changing external and internal factors and d) development of economic evaluation method for LTDHC / CDHC including archetypal business models and new services.
\end{abstract}

\section{INTRODUCTION}

$51 \%$ of the primary energy demand of the EU is currently used for heat and cold supply with a major increase in cooling demand expected for the coming decades. To achieve the goals of the COP21 agreement, steps towards a complete decarbonization of our heat and cold supply are mandatory and have to be initiated now to become effective in due time.

District heating and cooling are recognized as key technologies in this regard as they enable a smart integration of renewables, waste heat, thermal storage and consumer while providing a cross-sectoral (with electricity and gas) and cross-infrastructural (with waste water and industrial waste heat) linkage, leading to increased overall efficiencies and effectiveness and reduction of primary energy demand.

\section{LOW - TEMPERATURE DISTRICT HEATING AND COOLING}

Low - temperature district heating and cooling (LTDHC) systems enable to lower supply temperatures to a minimum of the customer demands to guarantee the minimum domestic hot temperature required in accordance with hygiene regulations and comfort requirements. With LTDHC concepts, this results in supply temperatures between 50 and $70^{\circ} \mathrm{C}$ and target temperatures of 20 to $40^{\circ} \mathrm{C}$ in the return flow. For the medium-term future, a trend towards LTDHC is expected in newly constructed heating networks. These temperatures lead to educed heat network losses, easier feeding of waste heat, solar thermal energy and ambient heat from large heat pumps and efficient operation of (decentralised) CHP plants and large heat storage systems. Danish heating networks in particular are regarded as pioneers here (Voyens, Gram Brædstrup, Lystrup, Albertslund, Høje Taastrup, Aarhus). It also realised examples in GER (e.g. Stadtquartier Brühl (Chemnitz), Ohrberg-Siedlung, Emmerthal, Ackermannbogen-Munich) and AT (Salzburg-Lehen, Waldmühle Rodaun, Pettenbach, Danzermühle) and for many planned or under construction projects, LTDHC C solutions are being considered. In addition to the classification presented here, the literature contains various terms with different or not always clearly defined supply levels, e.g. LowEx networks $\left(45-60^{\circ} \mathrm{C}\right)$ or 4 th Generation DH (35$\left.60^{\circ} \mathrm{C}\right)$.

\section{COLD DISTRICT HEATING AND COOLING}

Cold district heating and cooling (CDHC) systems with a supply temperature of $<30^{\circ} 45 \mathrm{C}$ a) enable an easy integration of low-exergy sources like waste heat and renewables, b) minimize transport losses, c) significantly reduce primary energy demand compared to state-of-the-art solutions and d) are able to supply heat and cold with one infrastructure. Innovative grid topologies allow here for a high degree of flexibility in regard of supplying existing and newly built buildings, system extension as well as 
integration of new thermal sources respectively sinks and storages. First demonstration systems in Switzerland are proof for the potential of this technology and concept.

\section{CURRENT APPROACH}

To access the full potential of this concept and to establish it as the go-to solution for heat and cold solutions due to its inherent characteristics of easy integration of low-exergy sources, flexible expansion options and supply of older buildings, basic knowledge and methods on operation, planning and evaluation have to be extended to allow for a complete and holistic development and evaluation of such systems. Currently, a lack of knowledge exists here in regard of a) technical and economic evaluation methods, b) methods for the development of complete system solutions including suitable business models, c) minimal requirements, area of application and limitations and d) sound measures for the long-term evaluation of LTDHC / CDHC concepts. This paper will show the opportunities and overall applicability of innovative and sustainable heat and cold supply based on LTDHC / CDHC concepts by establishing the necessary basics in conceptualization, planning and long-term evaluation. This is achieved by a) evaluation of possible system solutions for different configurations and boundary conditions, $b$ ) development of a multi-domain co-simulation framework for evaluation of technical and economic benefits, c) development of a stochastic approach for long-term system evaluation in regard of changing external and internal factors and d) development of economic evaluation method for LTDHC / CDHC including archetypal business models and new services. Our findings and ideas are highly relevant for the further development and decarbonisation of Austrian district heating and cooling systems. Furthermore, they will support and enable the realization of innovative and sustainable thermal supply concepts based on low temperature sources.

\section{CONFERENCE TOPIC}

Please indicate here to which of the Conference Topics this abstract refers:

- Urban District Heating and Cooling Technologies

- Innovations for the Decarbonisation of Buildings and Quarters

- Spatial energy planning with focus on renewable energies 


\title{
AN ASSESSMENT OF CHALLENGES, OPPORTUNITIES AND MODEL FOR THE IMPLEMENTATION OF SOLAR THERMAL TECHNOLOGY ROADMAP FOR BOTSWANA AND IMPACT ON $\mathrm{CO}_{2}$ REDUCTION
}

\author{
Andrew Obok Opok \\ University of Botswana, Private Bag UB0022, Gaborone, Botswana \\ Phone: +2673554256 obokopok@ub.ac.bw or obokopok@gmail.com
}

\begin{abstract}
SUMMARY
The paper assesses the challenges and opportunities for implementing the Solar Thermal Technology Roadmap for Botswana. The roadmap was developed from a study undertaken between 2016 and 2017 and it is now a government strategy for implementing solar thermal technology in Botswana. The assessment covers policy and strategy, technology, economic and $\mathrm{CO}_{2}$ reduction, taking into account the lessons from international order relating to implementation of roadmaps. A multi-faceted approach was used for the assessment: stakeholder method using system dynamic model; policy and strategy, lessons from best international and regional practices, costs and efficiency of thermal technologies (SWH) for domestic applications and for industrial and institutional applications to be implemented through the roadmap. The evaluation of reduction of $\mathrm{CO}_{2}$ arising from the roadmap implementation is quantified and used as support for roadmap realization. The paper proposes a new model for implementation of SWH for Botswana and developing economies.
\end{abstract}

\section{INTRODUCTION}

The overarching rationale for the roadmap is to serve as a national strategy document for planning, development and deployment of solar thermal technology systems for application in Botswana. The roadmap aims to achieve installation of $910,000 \mathrm{~m} 2$ of solar thermal technology collectors between 2018 and 2030. That translates to target installation equivalent to $0.3 \mathrm{~m} 2$ of net solar thermal collector area for each person of the population by 2030 in Botswana. The challenges and opportunities of implementation to meet variable annual targets of collector (ranging for $500 \mathrm{~m} 2$ in 2018 to $349000 \mathrm{~m} 2$ in 2030) are the basis for the paper. The paper focuses on the question how these annual targets will be met given the current state of solar thermal systems In Botswana

\section{CHALLENGES}

A summary of key challenges to implementation of the roadmap include: High up front cost of SWH compared to electric water heaters currently overwhelmingly used in domestic and industriallinstitution applications; low and subsidized cost of electricity, which encourages use of electric water heaters; SWH and renewable energy systems are expensive and require subsidies; variability of solar energy; lack of trained personnel for installation and maintenance of SWH; concerns about reduction in demand by the utility when SWH are implemented in bulk through the roadmap; national energy policy focus is on rural electrification and away from SWH.

\section{APPROACH AND METHODS}

A mixed but integrated methods are applied to rationalise the opportunities for effective implementation of the roadmap. These consists of: (i) Survey data collected from a select group of stakeholders whose portfolios are relevant to the implementation of the roadmap. A system dynamic model based on the data collected was developed to answer the research question: How can the roadmap be effectively implemented given the diverse conditions, requirements and challenges that have to be met?. (ii) Evaluating Botswana's renewable energy policy with repect to SWH using International Renewable Energy Agency (IRENA) framework. (iii) Evaluating the costs and energy efficiencies of SWH systems recommended in the roadmap implementation, The rationale for cost analysis is to estblish economic factors such investment costs and payback periods - which are key factors for determining subsidy levels, loans and its conditions, (https://www.energy.gov). (vi) Using lessons from best international practices: market framework, business model, standards, financing, skills and capacities in the evaluation, for developing new model for implimentation of the roadmap. (v) Cost benefit analysis for $\mathrm{CO}_{2}$ emmissions which would be avoided if the roadmap is implemented. 


\section{OPPORTUNITIES}

From data collected, a system dynamic model is develoed, shown in Figure 1 based on causal loop diagram (CLD). The CLD articulates the interconnected nature for implementing the roadmap with the following loops and actions: the SWH technology loop; the policy and strategy loop; procurement loop. These in turn depend ownership loop which are driven by SWH potential market; SWH distributors and manufacturers; and solar water ownership.

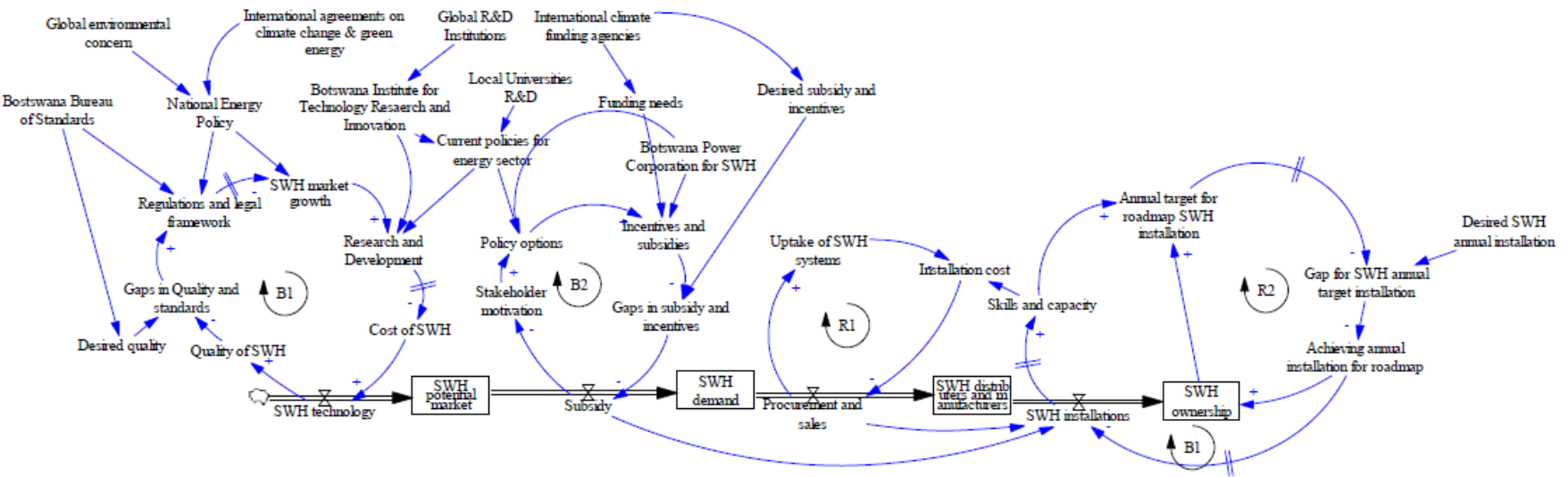

Figure 1: CLD of the for interelationship between factors needed for effective implimentation of the roadmap.

The evaluation of current policies and strategies in renewable energy and SWH identifies new opportunities in policy goals and strategy for the implementing the roadmap with criteria and indicators. Further opportunities for implementing the roadmap arising from the study include: to societal issues (environment impact)- raising awareness about lowering $\mathrm{CO}_{2}$ - this relates to an installed capacity of $637 \mathrm{MW}_{\text {th }}$ and annual electricity savings of $659,000 \mathrm{MWh}$, avoiding 182,000 tons of $\mathrm{CO}_{2}$; non technological aspects (market framework, business model, spatial planning, standards, financing, skills and capacities); technical aspects - effective and efficient solar thermal technogies. The paper concludes with a model proposed for implementation of the roadmap.

\section{REFERENCES}

1. F. Mauthner, W. Weiss, "Solar Heat Worldwide: Markets and Contribution to the Energy Supply 2012", International Energy Agency Solar Heating and Cooling Programme, 2014.

2. A. Robalino-L’opeza,b, A. Mena-Nietob , J.E. Garc'ia-Ramosa, “System dynamics modeling for renewable energy and $\mathrm{CO} 2$ emissions: A case study of Ecuador", Elsevier, Energy for Sustainable Development, Volume 20, June 2014, Pages 11-20

3. Epp, B. "The Barbados model: a success story in 3 Acts." Global Solar Thermal Energy Council. (2009).

4. http://www.irena.org

5. (https://www.energy.gov)

\section{CONFERENCE TOPIC}

Business models and financing schemes

Best practice policies 


\title{
EVALUATION OF CES-MED PROGRAM: OBJECTIVES, ACHIEVEMENTS AND RECOMMENDATIONS
}

\author{
Sabine Saad1,2, Dr Adel Mourtada1, Dr Marwan Brouche2, Dr Mazen Ghandour1 \\ Faculty of Engineering, Lebanese University, Hadath, Lebanon1 \\ Ecole Superieure d'Ingenierie de Beirut, Saint Joseph University, Lebanon 2 \\ E-Mail: sabinesaad@yahoo.com
}

\begin{abstract}
This impartial evaluation study allows a deep acknowledgement on the Cleaner Energy Saving Mediterranean Cities (CES-MED) program that cover the South-Mediterranean cities and municipalities to help them attend the same objectives and commitments of the Covenant of Mayors (COM) for mitigation and Green House Gas emissions reduction. Furthermore, this paper identifies gaps, difficulties and obstacles that face the establishment of an accurate Baseline Emission Inventory (BEI) and the elaboration of a feasible Sustainable Energy Action Plan (SEAP).

To contribute to sustainable urban development and meet the energy challenges in the Southern Mediterranean countries, the CES-MED project (Cleaner Energy Saving Mediterranean Cities) has the task of promoting the development of sustainable energy in Mediterranean cities. The objective of this program is to develop the capacity of local authorities in this region to formulate and implement more sustainable local policies, such as those related to the Covenant of Mayors (COM) and to the development of local Sustainable Energy Action Plans (SEAP). During 36 months, this program was funded by the "European Neighborhood and Partnership Instrument" (ENPI South) which covers the Southern Mediterranean countries. In each country, 3 authorities were selected. These authorities established the Green House Gas Baseline Emission Inventory (BEI), develop and start implementing sustainable policies, similar to the policies arising from adherence to the Covenant of Mayors. Each authority developed a SEAP including renewable energy solutions, energy efficiency measures to reduce GHG emissions, efficient management of water and waste, public transport that respects the environment, etc. Nevertheless, proposed tools of CES-MED have not always been sufficient as some cities that were selected in the beginning of the program and whose plans were expected by the end of 2015 haven't submitted their plans, yet the CES-MED authorization ended in June 2017. As for the methodology, it has been obstructed by several limitations such as the absence of values regarding the emission factors in the baseline carbon footprint specified to each country. To establish the BEI, local authorities had to rely on inaccurate data that generated inaccurate results. Furthermore, the methodology would not allow any authority wishing to join without any technical assistance from CES-MED to identify and determine the performance of each measure clearly listed by its Energy Efficiency priority and its related investment cost.

In this paper, CES-MED achievements, by authority, were collected from available sources and evaluated as well as obstacles and gaps are highlighted. In the end, many suggestions are recommended for both short and long terms to overcome difficulties.
\end{abstract}

\section{INTRODUCTION}

In the framework of the Covenant of Mayors (COM), being an EU funded initiative launched in 2008 in Europe, that gather local authorities to commit to the 2020's 20\% CO2 reduction objective by implementing climate and energy set of actions on their territories, more than 7000 cities, municipalities and regions across 57 countries have prepared, submitted and implemented a comprehensive Sustainable Energy Action Plan (SEAP) that sets the methodology to reach this target. Additionally, the global dimension of the CoM was first introduced through the establishment of Covenant of Mayors for East Europe (CoM EAST), as well as the CES-MED project for Maghreb and Mashreq Mediterranean countries, since early 2013. Recently, a "New and integrated Covenant of Mayors for Climate and Energy" or COM Global was launched in 2015 to extend the scope of the initial CoM, by setting a $40 \%$ reduction target by the 2030 to be reflected in a Sustainable Energy and Climate Action Plan (SECAP), which is a variation of the SEAP and by integrating adaptation and mitigation in the local authorities' actions.

The CES-MED project (Cleaner Energy Saving Mediterranean Cities) was initiated since January 2013 with a 36-month duration and regional offices in Rabat and Beirut. It was extended for the first time for 18 more months and then for the second time till April 2018. In the South Mediterranean region, 38 cities were selected from 9 countries: Algeria, Egypt, Jordan, Lebanon, Libya, Morocco, 
Palestine, Syria (suspended due to the political situation) and Tunisia. In each country, 3 authorities were selected. 23 cities have been directly assisted by CES-MED to prepare their SEAPs. Between 18 cities that have produced SEAPs, 15 have joined. The 5 last SEAPs are steadily underway and the rest is joining gradually. CES-MED provided training, technical assistance, capacity building seminars, help desk services and SEAP preparation manuals (in English, Arabic and French) to those municipalities. Another 44 cities have either applied to COM or/and are engaged in the preparation of SEAPs without any assistance of CES-MED, and more than 100 have requested to receive further support.

The CES-MED project has designed tools to prepare, elaborate and implement SEAPs. Nevertheless, these tools have not always been sufficient as some cities that were selected in the beginning of the program failed to submit their plans by the end of 2015, yet the CES-MED authorization ended in April 2018. As for the methodology, it has been obstructed by several limitations. This study will impartially evaluate the known and public project achievements to find the gaps in such applications, while highlighting on constraints and barriers. At the end, a list of recommendations will be provided to improve local sustainable development in South- Mediterranean.

\section{SEAP OVERVIEW}

As a top-down procedure, the SEAP (SECAP) [1] preparation to implementation is similar to the methodological and technical tools adopted in the framework of COM and it is described as following:

1. Initiation:

- Establish political commitment.

- Build stakeholders support.

\section{Planning:}

- Assess the current policy framework.

- Establish and conduct the Baseline Emission Inventory (BEI) that will assess its basic situation with respect to the calculation of GHG emissions. It quantifies the amount of $\mathrm{CO} 2$ emitted due to energy consumption in the territory of these local authority in a baseline year chosen that has data, to compile an inventory for 1990 (baseline year recommended by the COM), then it should choose the closest subsequent year for which the most comprehensive and reliable data can be collected. The collecting method is considered at the local level and is picked up from a mix of different sources as municipalities' services, existing database and archives and national statistics. Whenever data was not available, surveys and estimation were performed (survey of energy consumption in a representative sample of residential dwellings, counting cars in main roads, etc.). Once the samples were selected in the baseline emission inventories database, a reliable dataset will be produced through a statistical procedure in which calculation hypothesis were applied when necessary (energy costs, unitary consumption of buildings, etc.) to obtain the overall energy consumption.

- Develop the Vision: at least decrease 20 per cent of $\mathrm{CO} 2$ emissions in or across required and/or selected sectors by 2020 after analyzing the consumption per energy and sectors and so the emissions will identify the most consuming and/or emitting sectors and thus indicate the appropriate mitigation action. The environmental performance of each as part of a global approach should be studied to achieve by the determination of the energy performance, environmental benefit and the related investment cost for each measure. Quantitative indicators such as costs (in \$/year), energy savings (in MWh/year or Btu/year), clean energy production (in $\mathrm{MWh} / \mathrm{year}$ or Btu/year) and estimated $\mathrm{CO} 2$-eq emissions reduction (in tons/year or pounds/year) are the instruments to measure the impact of each action clarifying the economical/environmental benefits.

- Elaborate the plan (in the form of a priority action fiche).

- Secure the sustainable financial resources to support the implementations and the projects' funding. 


\section{Implementation:}

- Approve and submit the SEAPs (SECAPs).

- Implement the SEAPs (SECAPs).

\section{Assessment, Monitoring and Reporting:}

- Assess the results of the implemented SEAPs (SECAPs) that measure the actual annual energy consumption data (electricity, final energy (for fossils fuels) and primary energy (for RE generation/consumption) of the municipality.

- Monitor the SEAPs (SECAPs) actions.

- Report the SEAPs (SECAPs) results.

The SEAP (SECAP) focuses on Energy Saving through the following general Actions:

- Reduction of energy consumption in buildings (residential, municipal, tertiary and industrial).

- Enhance energy efficiency by adopting climate change adaptation measures.

- Electricity production and management at the local level (such as public street lighting).

- Sustainable and clean urban transport.

- Production of energy from non-conventional sources (renewable energy).

- Sustainable tourism and its facilities (Heritage and antiquities' sites, museums and ecotourism).

- Solid waste and wastewater management.

The benefits from implementing SEAPs (SECAPs) are numerous. Once a SEAP is accepted by the European Commission, this will increase the municipality national credibility and the international gain allowing itself to become a signatory of the COM. And once a SEAP is funded and implemented, this will allow the reduction of the energy use, the electrical bills and $\mathrm{CO} 2$ emissions on its territory, thus lowering its Carbon Footprint. Besides, this will ameliorate the quality of services of street lighting, buildings, waste collection, transportation etc., as well as enhancing the municipality's sustainable and green image, wherever relevant.

\section{METHODOLOGY AND TOOLS}

The CES-MED (Cleaner Energy Saving Mediterranean Cities) provides training, technical assistance, capacity building seminars, help desk services and SEAP preparation manuals (in English, Arabic and French) to the listed municipalities in the South-Mediterranean. The project's guidance or the "Information Kit to develop SEAPs" [2] is considered as a primary effective tool to improve policies and systemize the preparation and financing of SEAPs (SECAPS). It is a self-help guide designed by the CES-MED project for municipalities wishing to implement a sustainable energy policy. It is a stepby-step method that helps them achieve their sustainable energy goals without their full support. The BEI is the instrument allowing the local authority to measure the impact of its actions related to climate change. It will show where the local authority was at the beginning, and the successive monitoring emission inventories will show the progress towards the objective. The activity data that quantify the human activity occurring in the territory of the local authority is recommended to calculate the amount of $\mathrm{CO} 2$ emissions by multiplying the emission factor with corresponding activity data. The human activity for the baseline year is quantified in all the following sectors: municipal sector (buildings, equipment and facilities), non-residential sector (tertiary, commercial, service...), residential sector, municipal public lighting, transportation (public and private), local electricity production (electric generators and renewable energies locally produced) and is characterized by either electricity consumption or oil/fuel consumption [3].

To prepare a SEAP, a checklist of characteristics is required in the following topics:

A. Energy structure and $\mathrm{CO} 2$ emissions

- Level and evolution of energy consumption and $\mathrm{CO} 2$ emissions by sector and by energy carrier (global and per capita). 
B. Energy management

- Level and change in the energy consumption of the local administration by sector (buildings and equipment, public lighting, waste management, waste water treatment, etc.) and by energy carrier.

- Typology of the existing building stock: usage (residential, commerce, services, social etc.), age, thermal insulation and other energy-related characteristics, energy consumption and trends, protection status, rate of renovation, tenancy, etc.

- Existence of industries and its potentialities on energy saving and efficiency.

- Characteristics of the demand of mobility and modes of transport, the public transportation network. Management initiatives and mobility planning - initiatives to promote public transport, bicycle and pedestrian.

- Assessing the types of lamps, lighting and energy-related issues in public lighting.

- Characterization of the largest energy consumers among buildings and equipment/facilities, public lighting, transportation and industries.

- Established initiatives for improving energy saving and efficiency and results obtained to date.

- Identification of potentialities for improvement in energy savings and efficiency in buildings, equipment/facilities, public lighting, transportation and industries.

- Existence of electricity production plants, as well as district heating/cooling plants and characteristics, established initiatives for improving energy efficiency of those plants and identification of potentialities for improvement in energy efficiency.

C. Renewable energies

- Potentialities for renewable energy production: solar thermal and photovoltaic, wind, mini-hydraulics, biomass, others.

- Renewable energy production and trends and its self-supplying's degree.

- Use of agricultural and forest biomass as renewable energy sources.

D. Awareness

- Level of awareness of the population and stakeholders with reference to energy efficiency and potential savings.

- Existence of initiatives and tools to facilitate the participation of citizens and stakeholders in the SEAP process and the energy and climate change policies of the local authority.

E. Skills and expertise

- Existence of adequate skills and expertise among the municipal staff: technical expertise (energy efficiency, renewable energies, efficient transport...), project management, data management, financial management and development of investment projects, communication skills (how to promote behavioral changes etc.), green public procurement etc.

\section{ACHIEVEMENTS, CONSTRAINTS AND BARRIERS}

As an interpretation for the achievements listed in the Table 1, we can find the following:

- From 9 countries in South-Mediterranean, CES-MED gave assistance to 7 countries, due to the political situation of Libya and Syria.

- From 39 municipalities listed, 19 were supported by CES-MED (marked in yellow), and the other 20 did their SEAPs (SECAPs) by their own.

- From these 19 municipalities, 12 SEAPs (SECAPs) were submitted and 7 not submitted, 4 even not adhered yet till now, noting that the CES-MED project duration is expired.

- From the total GHG emissions calculated from the available data and considered 6.872376 $\mathrm{x} 10^{\wedge} 6$ tons of $\mathrm{CO} 2$-eq, if taking into consideration the authorities covered by CES-MED $5.178297 \times 10^{\wedge} 6$ tons of CO2-eq are emitted in a baseline year. After SEAP (SECAP) implementation $1.104856 \times 10^{\wedge} 6$ tons of $185 \mathrm{CO} 2$-eq will be reduced by 2030 . 
As for the methodology, it has been obstructed by several limitations such as the absence of values regarding the emission factors in the baseline carbon footprint specified to each country. To establish the Baseline Emission Inventory (BEI), local authorities had to rely on inaccurate data that generated inaccurate results. Furthermore, the methodology would not allow any authority wishing to join without any technical assistance from CES-MED to identify and determine the performance of each measure clearly listed by its EE priority and its related investment cost. Therefore, the tools designed and used by the CES-MED have not always been sufficient as some cities that were selected in the beginning of the program failed to submit their plans by the end of 2015, yet the CES-MED authorization ended in April 2018.

As final results:

- $63 \%$ of desirable SEAPs (SECAPs) prepared by CES-MED are submitted.

- If the project was successful, the amount of $\mathrm{CO} 2$ reduced will be estimated $1.511908 \times 10^{\wedge} 6$ tons of $\mathrm{CO} 2$-eq, $37 \%$ more reduction as per figure 1.

- Inaccurate results due to inaccurate data collection.

\section{CONCLUSION AND RECOMMENDATIONS}

As a conclusion, the CES-MED has partially accomplished his objectives in the South-Mediterranean region during his 5 years and the half duration task at local levels. In addition, this project has failed to contribute to mitigation ambitions and GHG emissions reductions at the national level during the last couple of years. His overall impact does not directly affect the national achievements in energy strategies and policies, as happened in Lebanon for example. This is particularly obvious in the absence of appropriate laws for mitigation and clean energy production as well as the poor awareness of such issues at the individual, collective and municipal levels.

Therefore, as suggestions, a developed tool is necessary. It shall include a specific database for the region to help municipalities calculate baseline emissions and assess the impacts of different SEAP measures. The new tool will take into account all the modules (sectors) contributing to the environmental impacts (buildings, infrastructure, transport, local production of energy / electricity, public market, industries ...), to study their environmental performance in the context of from a global approach to succeed by modeling the determination of Optimum Energy Performance / Environmental Benefit / Costs of energy efficiency measures in the various sectors of the municipality. Depending on the magnitude of the contribution of each sector (generating the greatest energy consumption and the largest emissions), the municipality will have the choice to decide on the measures to be implemented. This tool will assess the basic situation of a municipality in terms of calculating emissions, identify priority energy efficiency measures, determine the performance of each measure and the investment costs by a simple usage.

In addition, a regulation study must be done for the region to evaluate the existing environmental protection laws and energy conservation laws at both local and national levels to develop an overall regulation that protects these issues and supports the SEAP implementation. 


\section{CO2-eq Emissions and Reduction in tons $\mathrm{CO} 2$-eq}

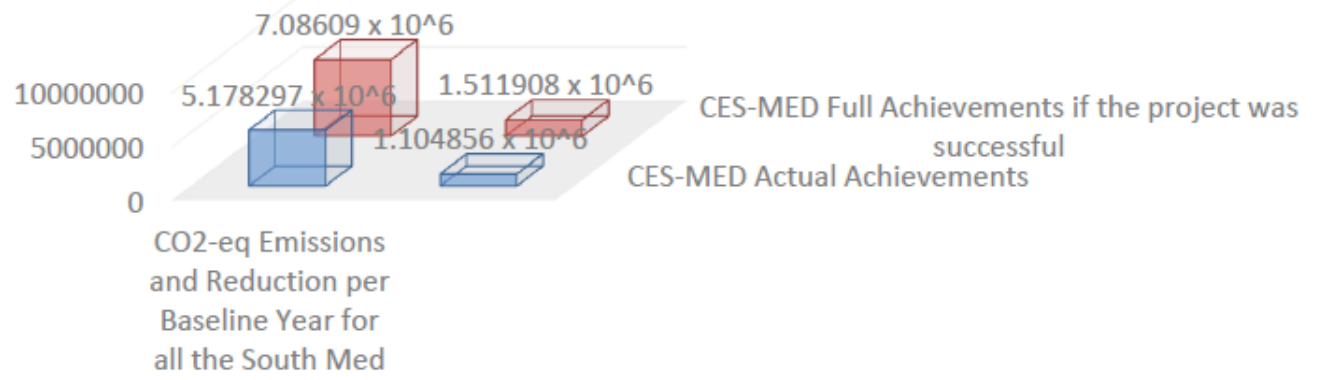

Figure 1. CO2 Emissions and Reduction before and after SEAP implementation - Actual CES-MED Achievements and Full Achievements if the project as successful. 
Tablel. Achievements of CES-MED

\begin{tabular}{|c|c|c|c|c|c|c|c|c|c|}
\hline 兽 & 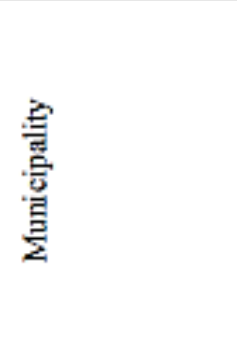 & $\frac{\text { 当 }}{\text { 总 }}$ & 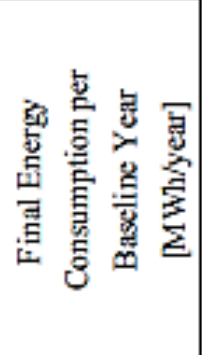 & 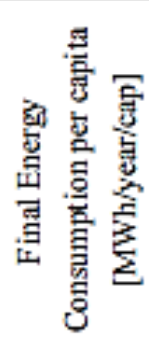 & 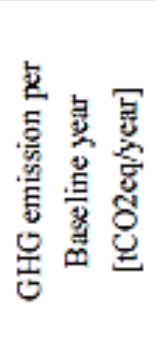 & 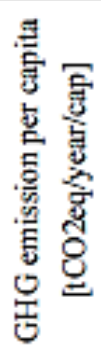 & 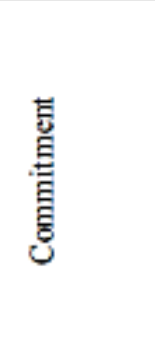 & 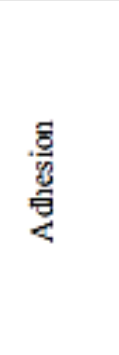 & 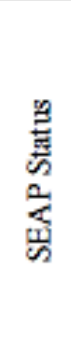 \\
\hline \multirow{3}{*}{ 墨 } & Batna & 2014 & 4676000 & 12.09 & 1190889 & $3.0 \mathrm{~s}$ & $20 \%-2020$ & Ad & Sub \\
\hline & Boumerdès & 2014 & 406000 & 7.44 & 122752 & 2.25 & $20 \%-2020$ & $\mathrm{Ad}$ & Sub \\
\hline & Sidi Bel Abbès & 2014 & 2671000 & 8.9 & 683128 & 2.32 & $20 \%-2020$ & Ad & Sub \\
\hline \multirow{3}{*}{ 窎 } & $\begin{array}{c}\text { Luxor } \\
\text { Governorate }\end{array}$ & 2015 & 4994000 & 8.71 & 1783000 & 3.1 & NA & Not Ad & $\begin{array}{l}\text { Not } \\
\text { sub }\end{array}$ \\
\hline & $\begin{array}{c}\text { Red Sea } \\
\text { Governorate }\end{array}$ & 2015 & NA & NA & NA & NA & NA & Not Ad & $\begin{array}{l}\text { Not } \\
\text { sub }\end{array}$ \\
\hline & Hurghada & 2015 & 3338000 & 11.9 & 1342000 & 4.8 & NA & Not Ad & $\begin{array}{l}\text { Not } \\
\text { sub }\end{array}$ \\
\hline \multirow{4}{*}{ 祃 } & Akaba & 2012 & 740292 & 6.27 & 339573 & 2.88 & $\begin{array}{c}40 \%- \\
2030+\mathrm{A} \\
\end{array}$ & $\mathrm{Ad}$ & Sub \\
\hline & Karak & NA & NA & NA & NA & NA & $\begin{array}{c}40 \%- \\
2030+A \\
\end{array}$ & $\mathrm{Ad}$ & $\begin{array}{l}\text { Not } \\
\text { sub }\end{array}$ \\
\hline & Madaba & NA & NA & NA & NA & NA & $\begin{array}{c}40 \%- \\
2030+A \\
\end{array}$ & Ad & $\begin{array}{l}\text { Not } \\
\text { sub }\end{array}$ \\
\hline & Sahab & 2014 & 25109 & 0.02 & 79563 & 0.01 & $20 \%-2020$ & $\mathrm{Ad}$ & Sub \\
\hline \multirow{10}{*}{ 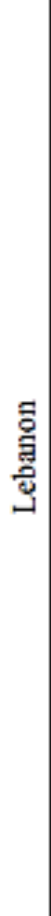 } & Baakline & 2013 & 76268 & 4.49 & 25635 & 1.51 & $25 \%-2020$ & $\mathrm{Ad}$ & Sub \\
\hline & Batloun Shouf & NA & NA & NA & NA & NA & $\begin{array}{c}40 \%- \\
2030+\mathrm{A} \\
\end{array}$ & $\mathrm{Ad}$ & $\begin{array}{l}\text { Not } \\
\text { sub }\end{array}$ \\
\hline & Hasbaya & NA & NA & NA & NA & NA & $\begin{array}{c}40 \%- \\
2030+A \\
\end{array}$ & $\mathrm{Ad}$ & $\begin{array}{l}\text { Not } \\
\text { sub }\end{array}$ \\
\hline & Jezzine & NA & NA & NA & NA & NA & $\begin{array}{c}40 \%- \\
2030+\mathrm{A}\end{array}$ & $\mathrm{Ad}$ & $\begin{array}{l}\text { Not } \\
\text { sub }\end{array}$ \\
\hline & $\begin{array}{c}\text { Kab Elias-Wadi } \\
\text { el Delm }\end{array}$ & 2013 & 165894 & 3.32 & 52987 & 1.06 & $20 \%-2020$ & $\mathrm{Ad}$ & Sub \\
\hline & Kawkaba & NA & NA & NA & NA & NA & $\begin{array}{c}40 \%- \\
2030+\mathrm{A}\end{array}$ & Not Ad & $\begin{array}{l}\text { Not } \\
\text { sub }\end{array}$ \\
\hline & Kherbet Rouha & NA & NA & NA & NA & NA & $\begin{array}{c}40 \%- \\
2030+A \\
\end{array}$ & Not Ad & $\begin{array}{l}\text { Not } \\
\text { sub }\end{array}$ \\
\hline & Menjez & 2013 & 1765 & 1.25 & 1307 & 0.93 & $\begin{array}{c}25 \%- \\
2020+\mathrm{A} \\
\end{array}$ & Ad & Sub \\
\hline & Moukhtara & NA & NA & NA & NA & NA & $\begin{array}{c}40 \%- \\
2030+\mathrm{A}\end{array}$ & Ad & $\begin{array}{l}\text { Not } \\
\text { sub }\end{array}$ \\
\hline & Beirut & NA & NA & NA & NA & NA & NA & Not Ad & $\begin{array}{l}\text { Not } \\
\text { sub }\end{array}$ \\
\hline
\end{tabular}




\begin{tabular}{|c|c|c|c|c|c|c|c|c|c|}
\hline 量 & NA & $\mathrm{NA}$ & NA & NA & NA & NA & NA & NA & NA \\
\hline \multirow{11}{*}{$\begin{array}{l}8 \\
8 \\
0 \\
\vdots\end{array}$} & Agadir & 2013 & 2418340 & 5.84 & 891857 & 2.15 & $20 \%-2020$ & $\mathrm{Ad}$ & Sub \\
\hline & Belfaa & $\mathrm{NA}$ & NA & NA & NA & NA & $\begin{array}{c}40 \%- \\
2030+A\end{array}$ & Ad & $\begin{array}{l}\text { Not } \\
\text { sub }\end{array}$ \\
\hline & Chefchaouen & 2014 & 235839 & 4.94 & 57648 & 1.21 & $45 \%-2020$ & $\mathrm{Ad}$ & Sub \\
\hline & Oujda & 2013 & 1706908 & 3.52 & 593835 & 1.22 & $20 \%-2020$ & $\mathrm{Ad}$ & Sub \\
\hline & Benslimane & 2013 & 191803 & 3.43 & 74326 & 1.33 & $20 \%-2020$ & $\mathrm{Ad}$ & Sub \\
\hline & Fam El Hisn & $\mathrm{NA}$ & NA & NA & NA & NA & $\begin{array}{c}40 \%- \\
2030+\mathrm{A} \\
\end{array}$ & Ad & $\begin{array}{l}\text { Not } \\
\text { sub }\end{array}$ \\
\hline & Figuig & NA & NA & NA & NA & NA & $\begin{array}{c}40 \%- \\
2030+\mathrm{A}\end{array}$ & $\mathrm{Ad}$ & $\begin{array}{l}\text { Not } \\
\text { sub }\end{array}$ \\
\hline & Mdig & $\mathrm{NA}$ & NA & NA & NA & NA & $\begin{array}{c}40 \%- \\
2030+\mathrm{A}\end{array}$ & Ad & $\begin{array}{l}\text { Not } \\
\text { sub }\end{array}$ \\
\hline & Salé & 2008 & 2148980 & 2.15 & 931453 & 0.93 & $20 \%-2020$ & $\mathrm{Ad}$ & Sub \\
\hline & Sefrou & $\mathrm{NA}$ & NA & NA & NA & NA & $\begin{array}{c}40 \%- \\
2030+\mathrm{A}\end{array}$ & Ad & $\begin{array}{l}\text { Not } \\
\text { sub }\end{array}$ \\
\hline & Tizmit & $\mathrm{NA}$ & NA & NA & NA & NA & $\begin{array}{c}40 \%- \\
2030+\mathrm{A} \\
\end{array}$ & Ad & $\begin{array}{l}\text { Not } \\
\text { sub }\end{array}$ \\
\hline \multirow{4}{*}{$\begin{array}{l}\text { 音 } \\
\text { 胥 }\end{array}$} & Nablus (WB) & 2013 & 705411 & 3.67 & $39 \$ 238$ & 2.07 & $20 \%-2020$ & $\mathrm{Ad}$ & Sub \\
\hline & Hebron (WB) & 2014 & 838559 & 4.15 & 448337 & 2.22 & $20 \%-2020$ & $\mathrm{Ad}$ & Sub \\
\hline & Tulkarem (WB) & 2014 & 314346 & 3.11 & 191509 & 1.9 & $20 \%-2020$ & $\mathrm{Ad}$ & Sub \\
\hline & $\begin{array}{c}\text { Abasan Al- } \\
\text { Kabira (Gaza) }\end{array}$ & 2010 & 76914 & 3.05 & 30273 & 1.2 & $\begin{array}{c}30 \%- \\
2030+\mathrm{A} \\
\end{array}$ & Ad & $\begin{array}{l}\text { Not } \\
\text { sub }\end{array}$ \\
\hline 悬 & & $\mathrm{NA}$ & NA & NA & NA & NA & NA & NA & NA \\
\hline \multirow{4}{*}{$\begin{array}{l}\text { 量 } \\
\text { 晋 }\end{array}$} & NA & $\mathrm{NA}$ & $\mathrm{NA}$ & NA & NA & NA & $\begin{array}{c}40 \%- \\
2030+\mathrm{A} \\
\end{array}$ & Ad & $\begin{array}{l}\text { Not } \\
\text { sub }\end{array}$ \\
\hline & Sfax & 2010 & 2449472 & 8 & 759066 & 3 & $20 \%-2020$ & $\mathrm{Ad}$ & Sub \\
\hline & Kayreouan & NA & NA & NA & NA & NA & $20 \%-2020$ & Not Ad & $\begin{array}{l}\text { Not } \\
\text { sub }\end{array}$ \\
\hline & Sousse & $\mathrm{NA}$ & NA & NA & NA & NA & $20 \%-2020$ & Not Ad & $\begin{array}{l}\text { Not } \\
\text { sub }\end{array}$ \\
\hline
\end{tabular}

Ad: Adhered; Not Ad: Not Adhered; Sub: Submitted; Not sub: Not submitted; A: Adaptation; NA: Not Available

\section{REFERENCES}

[1] JRC Science and Policy Reports, (2017), Covenant of Mayors in figures: 8-year assessment, JRC Science for policy report, Kona A., Melica G., Bertoldi P., Rivas Calvete S., Koffi B., Iancu A., Zancanella P., Janssens-Maenhout G. Dallemand J.F., Luxembourg: Publications Office of the European Union.

[2] CES-MED, (2017), Information Kit, how to prepare a Sustainable Energy Action Plan (SEAP), prepared by CES-MED.

[3] JRC Science and Policy Reports, (2014), How to develop a sustainable energy action plan (SEAP) in South Mediterranean cities guidebook. Saheb Y., Kona A., Maschio I., Szabo S. Luxembourg: Publications office 


\title{
DELIVERING HIGH QUALITY ENERGY EFFICIENCY PROJECTS WITH ICP EUROPE
}

\author{
DI Willibald Kaltenbrunner MBA, Mag. (FH) Andreas Lindinger \\ denkstatt GmbH / Investor Confidence Project (ICP) Europe \\ Hietzinger Hauptstrasse 28, 1130 Vienna, Austria \\ Phone: +4317868900, +436648118002 \\ E-Mail: willibald.kaltenbrunner@denkstatt.at, andreas.lindinger@denkstatt.at
}

\section{SUMMARY}

According to a recent IEA/IRENA study, average annual global investments into energy efficiency of more than $\$ 1,000$ bn are necessary to limit global warming with a $66 \%$ probability to $2^{\circ} \mathrm{C}$. This is five times the current amount of investments into energy efficiency. In the face of ambitious climate targets and decreasing public subsidies private investments need to play a more prominent role to tap the full economic and environmental potential of energy efficiency. But investors often don't find projects to invest in despite attractive returns and growing interest in energy efficiency, in particular because of a lack of standardisation.

The Investor Confidence Project (ICP) Europe developed a framework for investors, project owners and project developers to unlock financing for the energy efficiency markets by standardising how energy efficiency projects are developed, documented and measured. ICP's Investor Ready Energy Efficiency (IREE) certification ensures best practices, the right professionals and third-party validation are used to deliver high-quality building, industry, district energy and street lighting projects.

Keywords: Energy Efficiency, Project Development, Financing, Industry, Infrastructure

\section{INTRODUCTION}

According to a recent IEA/IRENA study, average annual global investments into energy efficiency of more than $\$ 1,000$ bn are necessary to limit global warming with a $66 \%$ probability to $2^{\circ} \mathrm{C}$. This is five times the current amount of investments into energy efficiency. In the face of ambitious climate targets and decreasing public subsidies private investments need to play a more prominent role to tap the full economic and environmental potential of energy efficiency.

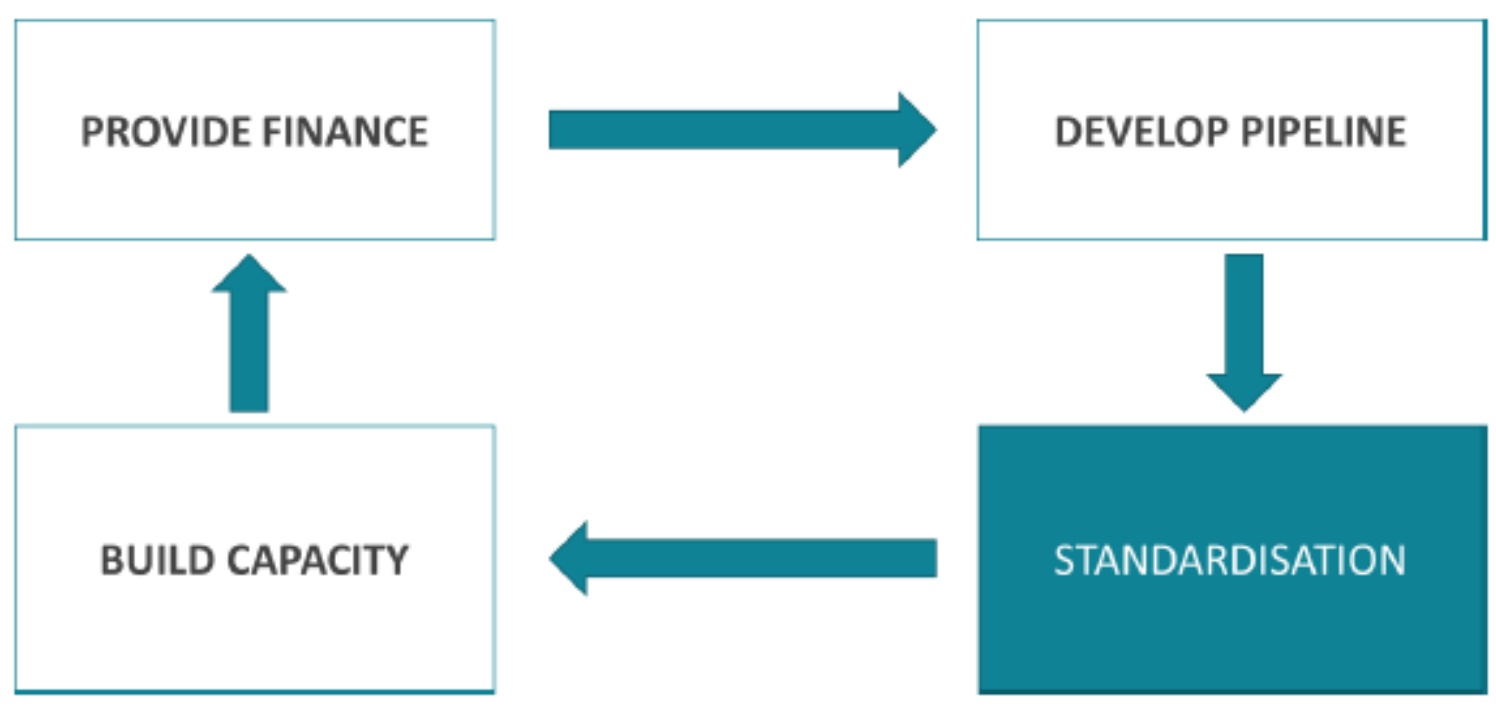

Fig. 1: The Energy Efficiency Finance cornerstones

However, investors often don't find projects to invest in despite attractive returns and growing interest in energy efficiency, in particular because of a lack of standardisation. This lack of standardisation in project development and documentation was identified by the Energy Efficiency Financial Institutions Group as one of the major barriers to increasing investment into energy efficiency and results in 
greater performance risk, higher transaction costs, uncertainty limiting demand as well as difficulties to aggregate projects in project portfolios and to build capacity.

Therefore, the Investor Confidence Project (ICP) Europe developed a framework for investors, project owners and project developers to unlock financing for the energy efficiency markets by standardising how energy efficiency projects are developed, documented and measured.

\section{METHODOLOGY}

ICP's Investor Ready Energy Efficiency (IREE) certification framework fosters transparent, consistent and trustworthy projects by standardising project development, the application of existing standards and best practices, and an independent verification during project development and operations. This allows for investment decisions with higher confidence in the technical fundamentals and the planned energy savings as well as lower transaction costs.

The ICP Protocols are at the centre of ICP Europe and were developed in a participatory approach together with technical experts from the buildings, industry, street lighting and district energy sectors. These Protocols and accompanying tools are published as open source documents on the ICP Europe website.

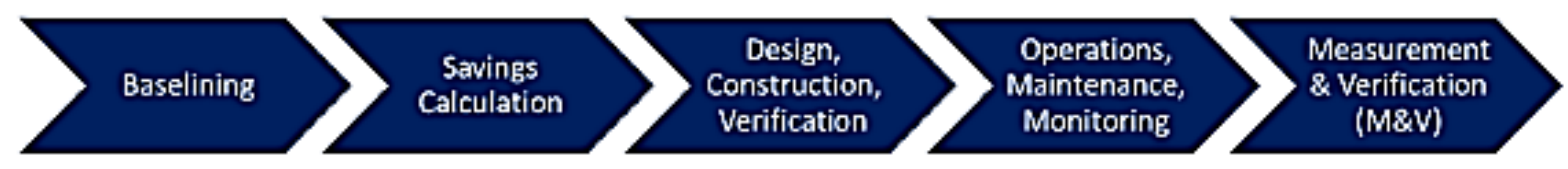

Fig. 2: Five phases of best practice project development according to ICP Protocols

These Protocols define a typical best practice process for energy efficiency project development and documentation that is based on existing standards and consists of five phases: The development of the energy baseline, the calculation of expected energy savings as well as the construction phase, the operations phase, and measurement and verification of energy savings. This covers all aspects of energy efficiency project development and documentation in a structured and consistent way.

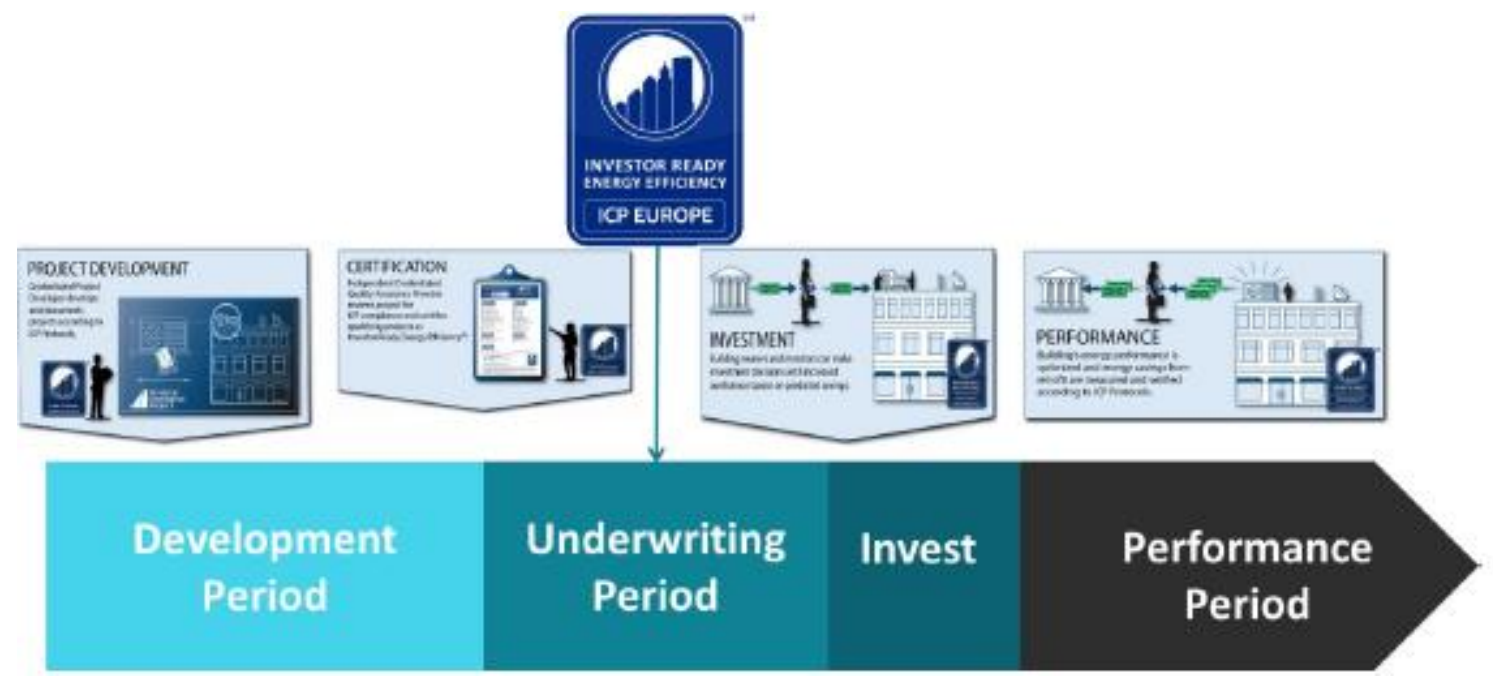

Fig. 3: IREE certification gives project owners and investors more confidence for their investment decisions

While a project is certified before its actual implementation, IREE guarantees that energy consumption is optimised after implementation and that energy savings are measured and verified according to the international IPMVP standard. Usually, a project is certified as Investor Ready at the end of the project development phase to give the project owner or investor in the underwriting phase more confidence for their investment decision. 


\section{APPLICATION AND RESULTS}

The IREE certification and ICP Protocols were developed with expert input in a collaborative, interdisciplinary process and successfully implemented in more than 30 pilot projects and programmes in the buildings sector across Europe in the first phase of ICP. In the second phase, ICP is currently applied to industry, street lighting and district energy projects across Europe.

Generally, before applying the ICP framework, a project developer has to pursue a free online training and register in the ICP Project Developer network. Particular focus in the development of an energy efficiency project according to the ICP approach lies on the first two phases of the ICP process:

- Baselining: Development of an energy baseline (usually using a regression model) and collection of all data for savings calculations, economic analyses, and implementation plans:

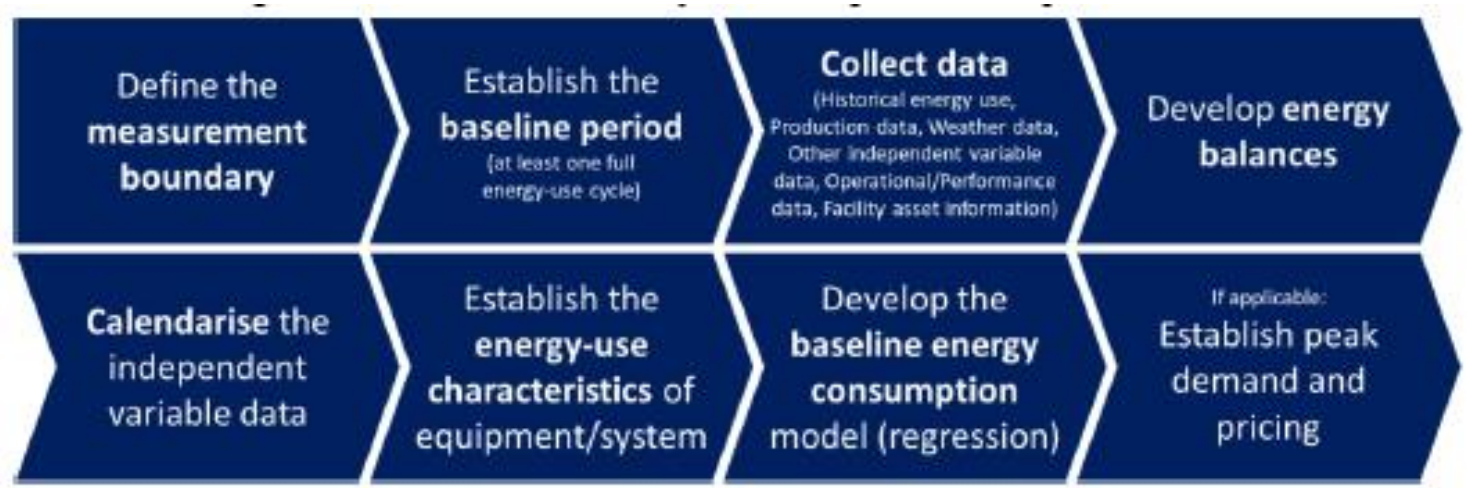

Fig. 4: Baselining process

- Savings calculations: Calculation of the projected energy savings on the basis of transparent calculation methods and tools and establishing the economics of the energy conservation measures:

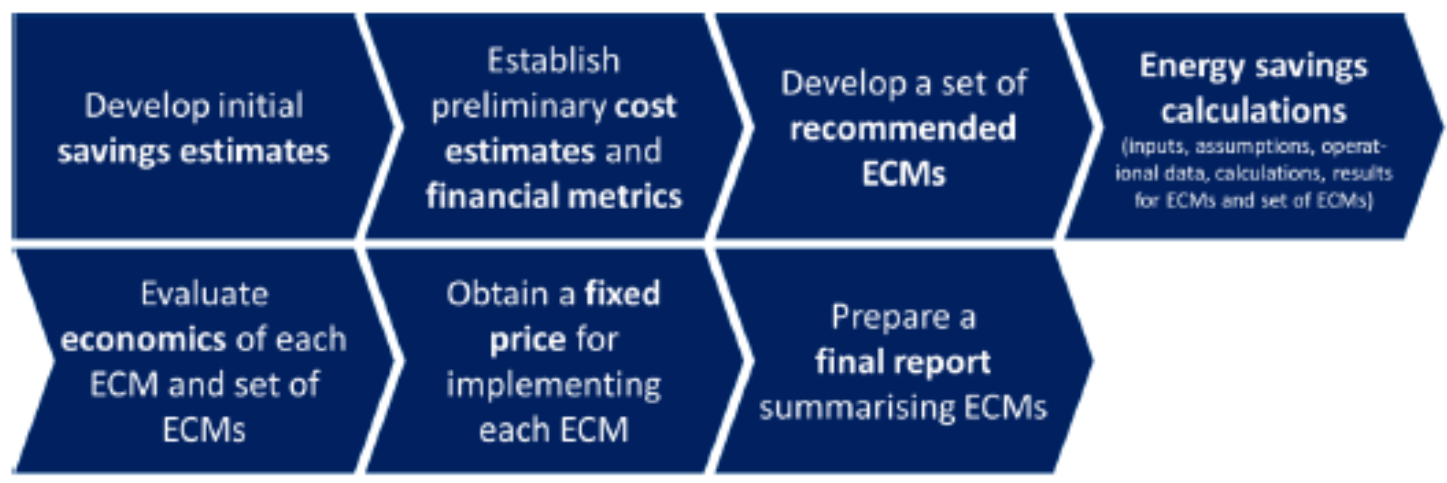

Fig. 5: Savings calculations process

With regards to the implementation phases, specific plans with the following objectives have to be developed:

- Operational Performance Verification (OPV) Plan: Establish a process which ensures that energy conservation measures are installed correctly (including training plans and systems manuals) and capable of achieving the predicted energy savings.

- Operations, Maintenance and Monitoring (OM\&M) Plan: Establish a systematic monitoring of energy system performance (including description of ongoing management regime, performance indicators, responsibilities, training plans and user manuals) including the implementation of corrective actions. 
- Measurement and Verification (M\&V) Plan: Develop an M\&V Plan according to the international IPMVP standard to compare the actual and predicted performance to calculate the energy savings.

The quality assurance process - which can usually be done in parallel to project development consists of a first review of the required information based on the respective ICP Protocol, followed by a gap analysis highlighting missing or non-compliant documents and information and further reviews of project documentation until a project fully complies with the requirements of ICP so that it can be certified as an IREE project.
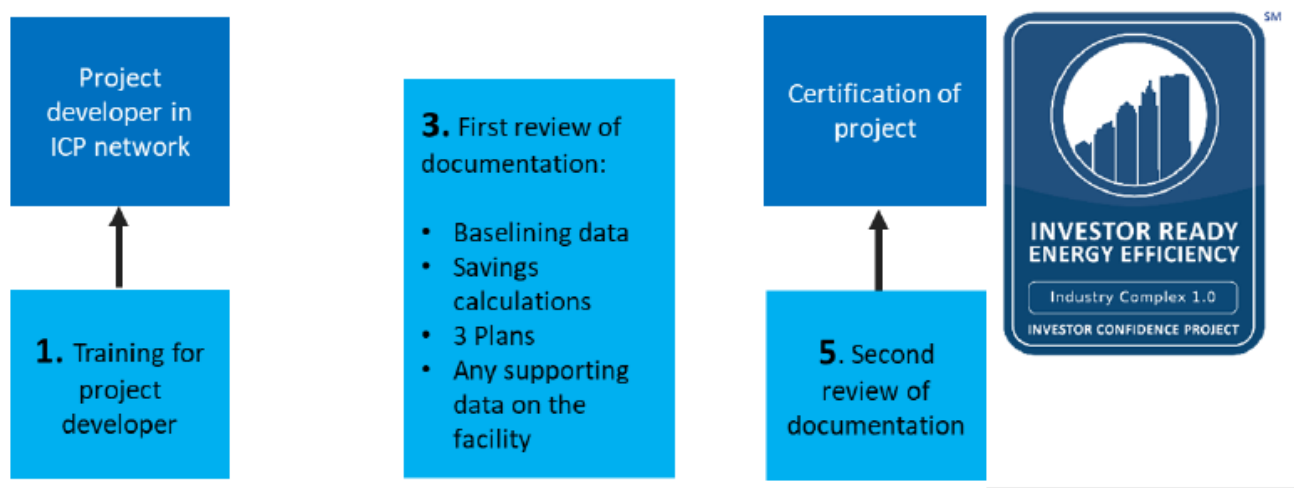

\section{Project development and certification}

Implementation
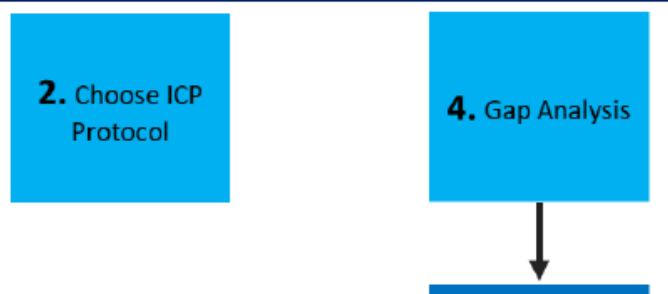

List of missing

or non-

compliant

documents

Fig. 6: ICP Quality Assurance process

This best practice project development and quality assurance process reduces risks and transaction costs of a technical due diligence, makes projects comparable and lays a foundation for successful project implementation. The integrated monitoring and verification of energy savings according to IPMVP increases the reliability of projected energy savings. In particular, ICP leads to better projects through:

1. Robust calculations: Higher confidence in the engineering fundamentals and the realisation of calculated savings projections

2. Best practices: Clear, transparent and consistent energy efficiency project based on industry best practices, with external measurement $\&$ verification

3. Qualified providers: Evidence of the qualification of professionals (project developers and quality assurance assessors)

4. Consistency: Consistent documentation enables comparability of projects across programmes

Finally, as the ICP approach builds on a broad stakeholder engagement process on a European and national level, ICP has developed a network of qualified project developers and quality assurance assessors, a global Investor Network of energy efficiency investors who recognize the benefit of industry best practice and standardised energy efficiency project delivery, and a European-wide ally network with more than 200 stakeholders (project/asset owners, industry, project developers, utilities, 
energy efficiency services businesses, investors, NGOs, public sector, universities, etc.) that support the development of ICP.

\section{CONCLUSION}

ICP Europe has collaboratively developed its Investor Ready Energy Efficiency (IREE) certification framework to ensure best practices, the right professionals and third-party validation are used to deliver high-quality building, industry, district energy and street lighting energy efficiency projects.

This market-oriented certification framework for developing, documenting and measuring energy efficiency projects aims at transforming the market for energy efficiency investments and thereby closing the gap between energy efficiency projects and investors. Moreover, it provides new market opportunities for project developers and other energy efficiency professionals.

More information on the Investor Confidence Project Europe as well as the open source ICP Protocols and other tools can be found on the project's website at http://europe.eeperformance.org. 


\title{
INNOVATIVE FINANCING AND EVALUATION OF ENERGY EFFICIENCY AND RENEWABLE ENERGIES IN INDUSTRY
}

\author{
DI Jürgen Fluch \\ AEE INTEC \\ Feldgasse 19, 8200 Gleisdorf, Austria \\ Phone: +4331125886 454 \\ E-Mail: j.fluch@aee.at
}

\section{SUMMARY}

Within the Horizon 2020 project TrustEE an innovative financing method linked to an automatic evaluation platform for energy efficiency (EE) and renewable energy projects (RES) was developed. On the platform the technical and economic data of the projects are submitted and automatically evaluated by a simulation model performing in the background. The result of the platform is a highly comprehensive project report serving as the basis for the financing with the securitisation vehicle "Sustainable Future TrustEE". By the service of TrustEE the transaction costs of energy efficiency and renewable energy projects are significantly reduced and become more attractive to investors, leading to a huge push of commissioning and implementations in Europe.

\section{INTRODUCTION}

Industrial process heat accounts for $74 \%$ of the industrial energy demand worldwide and half of it is low to medium-high temperature. (Philibert, 2017) Energy efficiency measures could be applied to reduce the energy consumption in general and renewable technologies such as solar thermal heat, heat pumps, biogas plants etc. could be integrated to produce heat for industrial processes and substitute fossil supply systems. Although the potential for the integration of energy efficiency measures and renewable energies is high, many technically and economically promising and well-developed projects - especially in SME - lack viable financing due to non-standardized project evaluation procedures, inadequate performance or credit guarantees, lack of guidelines for estimating project risks, unpredictable technical specifications of the sustainable concepts or due to other related financing challenges.

In the framework of the Horizon 2020 project TrustEE these issues and barriers are addressed. The aim is to promote the implementation of energy efficiency measures and the integration of renewable energies in the European industry. The development of an innovative financing model and a digital, web-based platform for automatically and standardized technical and economic evaluation of EE and RES projects aims to overcome these challenges. This paper will explain the methodology and operation of both tools, the financing model and the evaluation platform.

\section{METHODOLOGY}

The basis for the TrustEE project financing model are well evaluated projects. Therefore, a semiautomated platform for standardized technical and economic project evaluation is developed. On the TrustEE web-based platform (fulfilling maximal data security and protection requirements), equipment suppliers or industrial companies themselves can enter the technical and economic data for an energy efficiency or renewable energy project and undergo a three-stage evaluation. At level 1 and level 2 an automatic technical and economic evaluation of the parameters is carried out immediately on the platform. By means of automated background simulations based on high detailed calculation models, the queried parameters are validated, such as the economic viability or the expected yield, etc. If the project meets the criteria of level 1 and 2, options for further optimising the project - if necessary - will be evaluated together with the TrustEE manager and thus legal and insurance-related preparations for the re-financing of the investment will be made.

The financing model developed in TrustEE serves to refinance implementation projects for energy efficiency measures and renewable energies. The projects, e.g. the integration of solar thermal heat into a production process or the installation of a biogas plant, are planned, built and financed by an equipment supplier. In a conventional supply or service contracting model the equipment supplier is financing the plant and is taking both, the technical and economic risks. The envisaged energy savings or energy yield must be evidenced after a specified period of time after commissioning of the installation based on the contract. After providing evidence by successful acceptance, the customer makes the fixed payments specified in a payment plan to the equipment supplier. In the TrustEE 
financing model, the receivables from the payment plan are purchased from the technology supplier after successful acceptance of the project and securitized by a securitization vehicle. As a result, the equipment supplier receives the capital immediately after commissioning of the project and thereby improving the key figures that are relevant for the creditworthiness. The receivables are securitized by the securitization vehicle by issuing tradable bonds offered to investors in the capital market. To ensure that these securities meet the highest creditor protection standard, the securitization vehicle is incorporated under Luxembourg law under the name "Sustainable Future TrustEE".

\section{RESULTS}

The result of the platform is a standardized report, which contains a transparent description of the project including the technical and economic specifications of the project and thus forms the basis for the refinancing of the project. The service of the platform and the low technical risks remaining after the approval of the EE or RES project by the TrustEE management enable the financing of small and innovative projects whose risks are difficult to predict before commissioning. Also, the costs for complicated contract structure which are necessary for investors can be reduced by standardized procedures. The work of the TrustEE project will increase the implementation of energy efficiency measures as well as the integration of renewable energies into industry and contribute to the decarbonisation of the national and European industrial sector.

\section{DISCLAIMER}

TrustEE is a Horizon 2020 project to finance and realise energy efficiency and renewable energies in industry.

This project has received funding from the European Union's (EU) Horizon 2020 research and innovation programme under grant agreement No 696140. The sole responsibility for the content of this paper lies with the authors. It does not necessarily reflect the opinion of the European Union. The European Commission is not responsible for any use that may be made of the information contained therein.

\section{REFERENCES}

Philibert, C., (2017), Renewable Energy for Industry, From green energy to green materials and fuels, International Energy Agency, https://www.iea.org/publications/insights/insightpublications/Renewable_Energy_for_Industry.pdf assessed 13.03.2018

\section{CONFERENCE TOPIC}

Business models and financing schemes 


\title{
TRANSPARENT COSTING IN SMART THERMAL NETWORKS - A THERMOECONOMIC APPROACH
}

\author{
$\underline{\text { Stefano Coss }}$ \\ IMT Atlantique \\ 4 rue Alfred Kastler, 44307 Nantes, France \\ Phone: +33 / 769 / 408036 \\ E-Mail: stefano.coss@imt-atlantique.fr
}

\section{SUMMARY}

Energy transition and decarbonisation of the heating sector is one major challenge with regards to the actual environmental targets. The future role of district heating networks in future energy systems are referred to as smart thermal networks, which operate as a backbone for those systems, able to receive and distribute decentralized production of e.g. prosumers or renewable energy sources. The major barriers for this transition include the monopolistic design of thermal grids and the lack of a thermal market without clear third-party access for renewable energy or clean technologies such as waste heat integration. In order to solve this problem, a thermoeconomic model is developed, which can be applied to a dynamic simulation of networks of any size and amount of producers and consumers. A case-study of a large network shows, that the black-box costing approach applied by single utilities in today's networks deviates from the true cost of heating service and is not able to capture dynamic operation in future smart thermal networks.

Keywords: thermoeconomics, smart thermal networks, energy/exergy efficiency

\section{INTRODUCTION}

In order to promote decarbonisation and energy transition in the current energy sector, the development of a new paradigm and a new way of "thinking energy" can be observed. This new paradigm is called smart energy systems, which goes beyond the concept of "energy production" and "energy consumption" and aims in combining existing and newly developing technologies to one single system for the purpose of green urbanization. Heat networks which are able to perform these tasks are defined as smart thermal networks, which are able to "...connect the electricity and heating sectors" (Mathiesen et.al. 2015). In the field of energy services in DHN systems, producers face certain costs of the supplied energy based on the cost of their production. Industrial companies use black-box approaches to consider the unit costs of energy service supplied to the consumers. The problem of black-box (BB) approach is that it does not consider for dynamic behavior of the system which unavoidably occur in DHN operation when smart thermal networks are considered. Thus the dynamic behavior expressed through the change of thermal exergy in the system, is not taken into consideration. This leads to an estimation of losses which solely relies on the difference between inflow and outflow of exergy. Hence the problems related with BB costing approach are twofold:

1. Transient behavior of dynamic system operation is neglected

2. Only average unit costs, equal for every consumer, are possible to determine

In order to overcome these drawbacks to increase efficiency and cost transparency of smart thermal networks the development of a model for dynamic thermoeconomic simulation of DHN systems is carried out. More specifically, the formulation of exergetic costs according to (Lozano \& Valero 1993) is developed for general graph-based network models, which have initially been described in (Guelpa 2016). 


\section{METHODOLOGY}

\section{The problem of black-box costing}

Costing is a way of keeping track of "value" of certain goods. A certain good usually inherits some sort of value if it is used to supply some costumer's need. The initial value of that good can be defined through assignment of a certain "cost", which the one who deployed the good has to suffer. In economic terms, the value is determined based on the assignment of an economic cost, with the help of a monetary currency like Euros or Dollars. In the field of energy services in DHN systems, producers also face certain costs of the supplied thermal flows based on the cost of their production. This production cost is assigned to a certain amount of energy from which a specific cost can be determined. This specific cost is called unit energy cost and describes the amount of cost inherited by one unit of that energy. Based on the previous explanation, the unit energy cost is therefore a measure of value of a given energy flow.

According to the industrial experience of the author, costing approaches in real-exiting DHN systems are based on black-box costing due to several practical considerations. This leads to an accounting regime which assigns equal unit costs for each consumer in the system. This approach is easy to apply, since only the total energy supplied to- and from the system must be evaluated. This can be done through measurements at the production plant and at the substations connected to the DHN. However, this approach does not provide information of the cost generation in the network itself and is therefore not able to capture the value degradation of energy flow in DHN systems. Hence, this approach is not useful when considering smart thermal networks where producers and consumers are spread throughout the network with different exergetic supplies and demands, because their individual contribution to thermal losses and therefore the cost generation might vary considerably from the average black-box costing method.

Different costing approaches for energy services can be imagined in a DHN system. Those approaches can be either based on the energetic- or the exergetic value of the hot water flowing. Apart from energy or exergy considered, the approach can also be differentiated between a black-box approach, where the network behavior is unknown, and a white-box approach where detailed information on the thermodynamics of the network is available. A classification of those approaches is given in Table 1 .

Table 1: Classification of costing approaches in DHN systems

\begin{tabular}{l|cc}
\hline & Energy & Exergy \\
\hline Black-box (BB) approach & Traditional costing & Black-box exergy costing \\
White-box (WB) approach & Energy costing & Thermoeconomic costing \\
\hline
\end{tabular}

Apart from energy losses in DHN systems, the amount of exergy associated with an energy stream also suffers losses, called exergy destruction. On one hand side, a DHN can be seen as a black-box where a certain amount of energy enters, and a certain amount of energy exits the system, without information about the system behavior. This approach, in combination with energy as the "value asset", is defined as traditional costing. In that approach, only the global entering- and exiting flows from the DHN system are taken into account while losses or transient changes are globally accounted for. Traditional costing refers to the method used by today's industry using uniform pricing for thermal heat utilization. A black-box model using exergy as the value asset ( $B B$ exergy costing) is imaginable but does not exist in real practice according to author's knowledge. However, once the global exergetic behavior is known, black-box exergy costing can also be carried out.

On the other hand side, white-box costing approaches use the real system behavior to account for value loss in the system. Those could be based on energy (energy costing) or exergy (thermoeconomic 
costing). In order to understand the specific drawbacks between BB- and WB costing, the black-box costing approach is analyzed in detail according to Figure 1.

A DHN system as a black-box receives energy service, thus thermal energy $\left(\dot{\phi}_{P R}\right)$ or exergy $\left(\dot{\psi}_{P R}\right)$, and provides energy service, more precisely heating, expressed as energy $\left(\dot{\phi}_{C N}\right)$ and exergy $\left(\dot{\psi}_{C N}\right)$ to consumers. In this thesis, producers $(P R)$ and consumers $(C N)$ are considered as the sum of all producers $\dot{\phi}_{P R}=\sum_{i} \dot{\phi}_{p r_{i}}$ and consumers $\dot{\phi}_{C N}=\sum_{i} \dot{\phi}_{c n_{i}}$ (similarly for exergy), respectively.

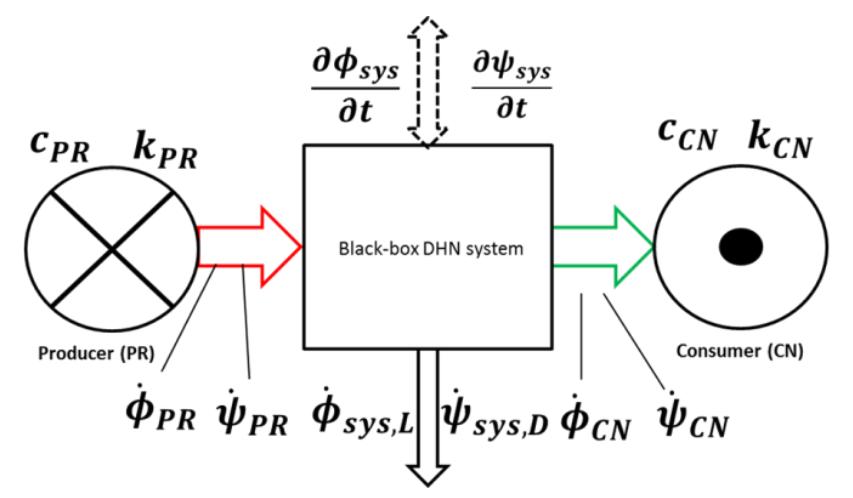

Figure 1: Black-box costing model

In the black-box approach, the difference between production and consumption is evaluated as the energy- and exergy losses occurring in the system. Those losses can be estimated on a system scale and might show variations of magnitude depending on the performance of the system. With knowledge of in- and outflows of thermal energy, it is instantly possible to apply black-box costing approach for both energy and exergy leading to equal unit costs for all consumers. This unit cost describes an average cost of utilization of energy from the system where the losses or destruction are accounted based on the global losses and can be used for pricing of the consumers.

This is done through applying a cost balances for the black-box model, leading to equations (eq. 1) (eq. 2).

$$
\begin{array}{ll}
\dot{\phi}_{P R} \pm\left|\frac{\partial \phi_{s y s}}{\partial t}\right|-\dot{\phi}_{s y s, L}-\dot{\phi}_{C N}=0 & \eta_{e n, s y s}=\frac{\dot{\phi}_{C N}}{\dot{\phi}_{P R^{+}}+\frac{\partial \phi_{s y s}}{\partial t}} \quad Y_{e n, s y s}=\frac{\dot{\phi}_{C N}}{\dot{\phi}_{P R}} \\
c_{C N}=\frac{c_{P R}}{Y_{e n, s y s}} & c_{C N^{*}}=\frac{c_{P R}}{\eta_{e n, s y s}}
\end{array}
$$

It must be noted, that $\eta_{\text {en,sys }}$ is valid in the case the transient term is positive (which refers to a network charge). When the network is charged (referring to cooling-down of the network) the transient term must be included in the numerator.

\section{Modeling approach for dynamic DHN simulation}

The definition of the DHN system focuses to connect producers and consumers through a thermal network, see Figure 2. 


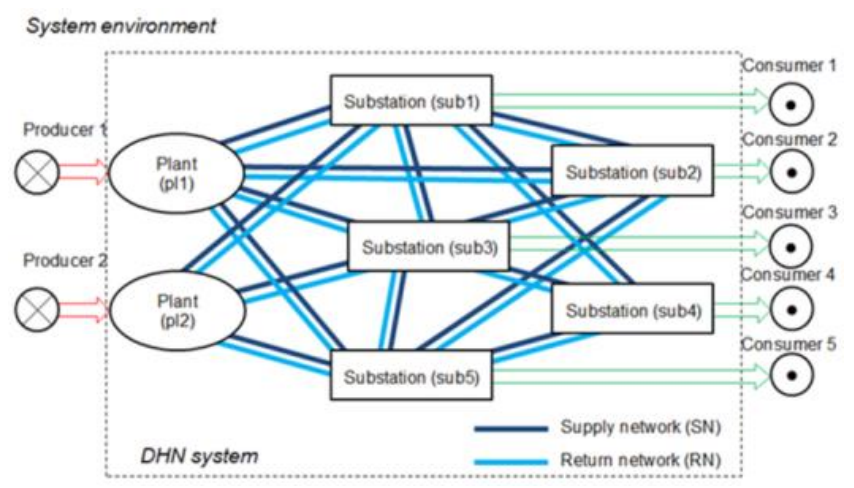

Figure 2: Representation of a DHN as smart thermal network

The focus hereby lies on production plants and consumers, which can be either heating plants, industrial plants or renewable energy plants. The supply network and the return network are described through a simple directed graph. The mathematical formulation of the graph includes two fundamental matrices, the adjacency matrix $(n \times n)$ and the incidence matrix $(\boldsymbol{A})(n \times b)$ (Wilson 1996). Both are used to relate nodes and branches in the graph, while only the incidence matrix is used in this work. The network is divided into control volumes (cv) which can be solved numerically. The resolution of the control volumes depend on the problem to be solved. In DHN modeling, control volumes are assigned at least to each single node, defined by the network topology. An upwind scheme is used (Patankar 1980) to connect the energy/exergy flows in the branch with the nodes in the control volume. When applying the upwind scheme to the control volumes, the branch variables are calculated on the basis of their upstream nodes, whether they are flows of exergy or flows of thermoeconomic costs. The temperature distribution is calculated based on a thermo-hydraulic model (Guelpa 2016).

\section{Matrix formulation for graph-based network topology}

In this section the matrix formulation is developed and shown in detail for thermal thermoeconomic costs. The aim is to provide an analytical matrix formulation to solve the control volume balances of every node. This offers a compact integration of the thermoeconomic analysis into the numerical architecture of graph-based network models. The matrix formulation must be able to integrate the thermoeconomic principles considering different costing approaches, like energy or exergy costing.

To represent the network topology, the incidence matrix $A(n \times b)$ is used (Wilson 1996). Every branch is associated with a flow of cost, energy or exergy. It is worth mentioning that to apply the upwind scheme integrated into the matrix, the real verses of the flow directions must be considered. This means that the matrix element $a_{n, b}$ of a flow $\dot{\psi}_{u, n}^{t h}$ is indicated as in (eq. 3).

$$
a_{n, b}=-\left|\dot{\psi}_{u, n}^{t h}\right| \quad \text { and } \quad a_{u, b}=+\left|\dot{\psi}_{u, n}^{t h}\right|
$$

Practically, A must be updated at each time step of the analysis once the direction of the flow is known by changing the signs in each column of A corresponding with negative mass flow rates. At first all necessary variables must be written in matrix form, see equations in (eq. 4).

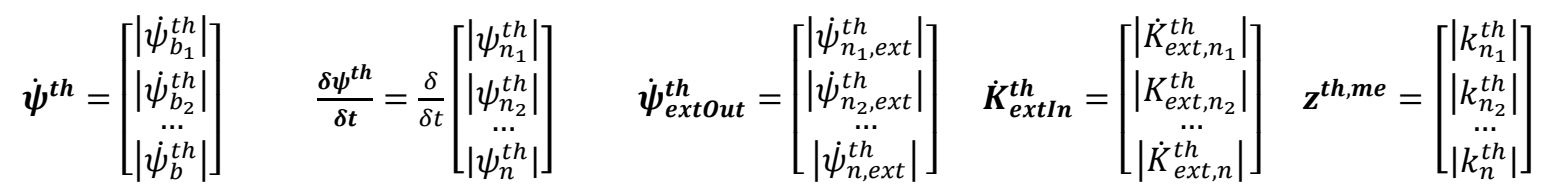

The exergy flow vector $\left(\dot{\psi}^{\text {th }}\right)$ contains the exergy flows of all branches in the network. The vector of transient flow of exergy contains all transient flows of the network nodes. The vector of external outflows $\left(\dot{\psi}_{\text {extout }}^{\text {th }}\right)$ contains all external outflows of the nodes and exit the control volumes at the 
given specific cost $\left(k^{t h}\right)$, while external inflows $\left(\dot{K}_{\text {extIn }}^{t h}\right)$ are considered as boundary conditions. Now the matrix formulation can be set up using the equations in (eq. 4), which leads to the matrix formation for thermal thermoeconomic costs in (eq. 5),

$$
\left\{\left[A \times I \dot{\psi}^{t h} \times\left[A^{+}\right]^{T}\right]+I\left[\dot{\psi}_{\text {extout }}^{t h}-\frac{\delta \psi^{t h}}{\delta t}\right]\right\} \boldsymbol{k}^{t h}=\dot{K}_{\text {extIn }}^{\text {th }}
$$

from which $k^{\text {th }}$ can be directly evaluated once matrix $A^{+}$is known. $A^{+}$is used to apply the exergy balance for every network branch in the $\mathrm{cv}$ and can be derived through applying (eq. 6) to every matrix element in $A$.

$a_{n, b}^{+}=\max \left(a_{n, b}, 0\right)$

This formulation can be used for any type of DHN topology, where every branch is connected to two nodes. The result is the calculation of unit costs at nodes which was described as the objective of the thermoeconomic model for the DHN. Based on (eq. 5), $k^{\text {th }}$ is calculated for every sampling time. Energy cost $\boldsymbol{c}^{\text {th }}$ can be similarly evaluated substituting the thermal energy flows in the respective equations. When analyzing the time-dependent behavior of the DHN, those matrix equations must be applied to each single timestep. In that case, each term must be evaluated based on current flows and the actual directions in the branches. Through these graph-based matrices any number of network nodes and branches can be simulated in the network.

\section{Introduction to the case study}

The case study is a DHN system located in Eastern Europe and supplies 138 substations which are connected to various different end-users; from residential buildings to commercial buildings, hospitals etc. Most of the substations are connection to subnetworks which supply those consumers, while some substations are direct users. The latter are directly connected to a building. However, a differentiation is not made upon them from a modeling perspective and the substation model is equally applied to each of them. An overview of the network topology based on the underlying QGIS data is given in Figure 3.

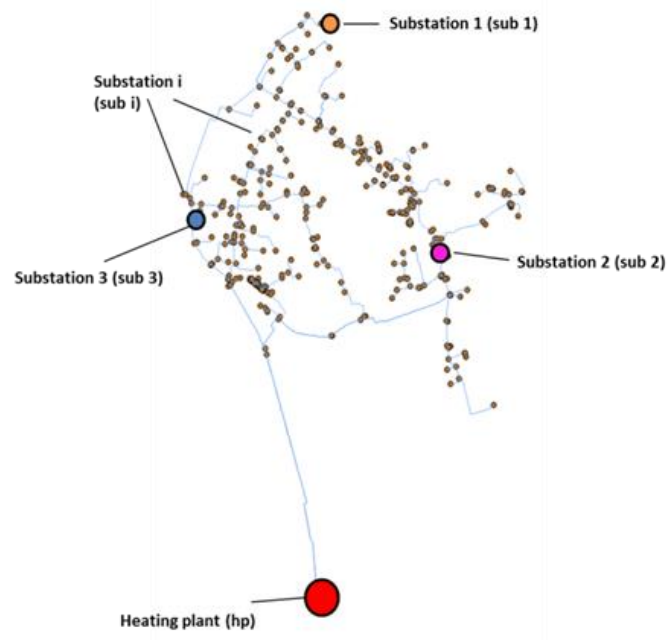

Figure 3: Case study network Rumania (taken from QGIS)

The network is operated by one heating plant $(h p)$ located to the south of the network indicated with a red circle. The network spans over the major part of the inner city, while the production plant is situated outside and is connected through a long pipe to the core part of the network.

The various points in the figure represent either internal nodes, such as connections/junctions in the network, or substations $\left(s u b_{i}\right)$. Three substations have been highlighted which are located close (substation 3) and far (substation 1+2) away from the plant. Those have been selected to investigate 
the individual impact of their operation on the cost generation of the system and are used to study the results of the thermoeconomic simulation on substation level.

The thermoeconomic model is applied to the case study network of 138 consumers and 1 heating plant during a timeframe of 4 days with an hourly resolution.

\section{RESULTS AND DISCUSSION}

In Figure 4, a comparison of the results obtained by the black-box costing and the thermoeconomic costing approach is shown for both energy and exergy costing.

On the left side of Figure 4, the unit costs of black-box costing and energy costing are compared. The unit energy cost obtained through energy $\operatorname{costing} c_{\text {sub } 3}^{\text {th }}$ shows a less fluctuating profile compared to the black-box results, where especially the peaks are less pronounced. In order to better compare the result, the black-box results are normalized to $c_{s u b 3}^{t h}$ to show the percentage of deviation. The normalized unit cost $c_{C N^{*}, \text { norm }}^{\text {th }}$, shows the relation between average dynamic unit costs to the unit energy cost of substation 3. It can be seen, that $c_{C N^{*}, \text { norm }}^{t h}$ is always lower then $c_{s u b 3}^{t h}$.
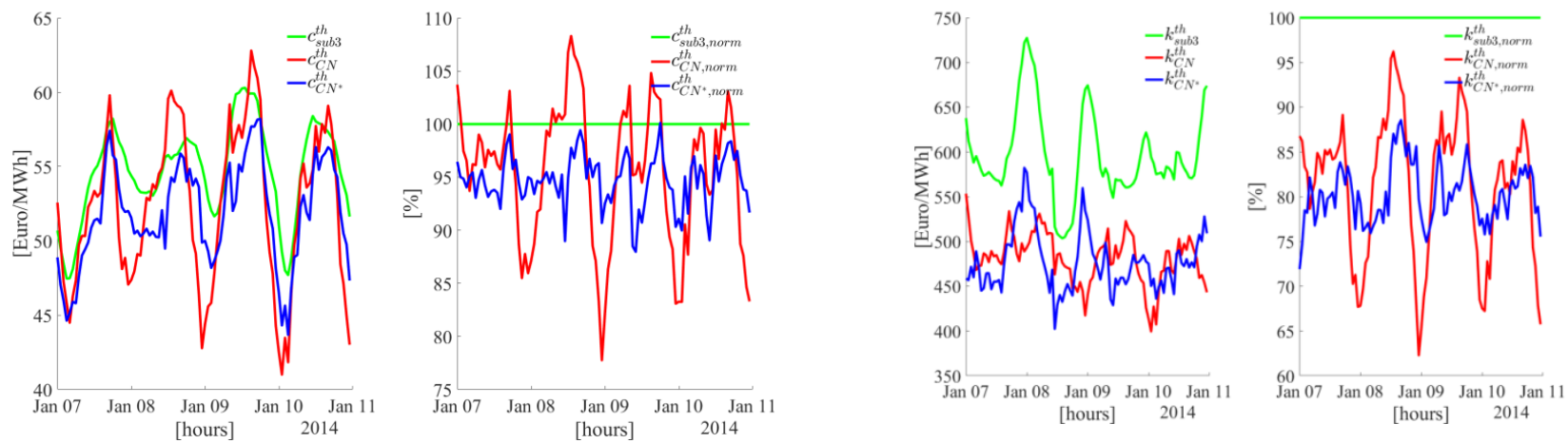

Figure 4: Comparison of costing approaches - energy (left) and exergy (right)

The point is that $c_{C N^{*}, \text { norm }}^{\text {th }}$ is in average about 5 to $10 \%$ lower than $c_{s u b 3}^{t h}$ during the whole timeframe, which indicates that the black-box costing method fairly underestimates the true unit energy cost of the substations. Since $c_{C N^{*}}^{t h}$ is averaging the thermal losses throughout the network, a lower relative value of $c_{C N^{*} \text {,norm }}^{t h}$ indicates that the individual costs are higher than average. This means, $c_{C N^{*}}^{t h}$ is underestimating the loss generation of substation 3 .

Furthermore, $c_{C N^{*}}^{t h}$ is much less fluctuating then $c_{C N}^{t h}$ because, $c_{C N}^{t h}$ also averages the transient behaviour of the network, leading to a unit cost highly inaccurate. This leads to a relative difference of $c_{C N, n o r m}^{t h}$ which is, first much more pronounced in terms of its peaks and second results also in an overestimation of the substation, during timesteps where $c_{C N, n o r m}^{t h}$ is greater than $100 \%$. In those cases, average unit cost result in higher unit cost compared to $c_{s u b 3}^{t h}$ and thus wrongly contributes more loss generation to substation 3 .

The errors obtained through black-box coting are significant and cannot be neglected. In the case, black-box energy costing is done; costing is mainly influenced by transient condition resulting in misleading result for substation 3. Even if transient behaviour is corrected through dynamic average costs, overall losses are averaged and underestimate the loss generation of substation 3 .

The results obtained by the comparison of energy costing do not reflect the thermodynamic behaviour of the substation. In order to compare the costing approaches including the thermodynamic behaviour of the substation, exergy costing is compared with the black-box approaches, see Figure 4 (right).

It shows that unit exergy costs of substation $3 k_{s u b 3}^{t h}$ are much higher then calculated based on average -and dynamic average unit costs. The differences can be investigated in the normalized plot, which 
shows that $k_{C N^{*}, \text { norm }}^{\text {th }}$ and $k_{C N, \text { norm }}^{\text {th }}$ are below $100 \%$. This means, that the black-box approach is highly underestimating the generation of exergy destruction of substation 3. Comparing $k_{C N^{*} \text {,norm }}^{\text {th }}$ and $k_{C N \text {,norm }}^{\text {th }}$ the same conclusion as for energy costing are true, with the difference that $k_{C N, n o r m}^{t h}$ is never above $100 \%$. This is due to the high exergy destruction in the substation, which causes $k_{\text {sub } 3}^{t h}$ to be always higher compared to the value obtained by energy costing.

It can be concluded, that the difference between black-box approach and exergy costing is even higher than for energy costing. The deviation is greater with $k_{C N^{*}, \text { norm }}^{\text {th }}$ of about $80 \%$ in average which is much larger than the deviation of $c_{C N^{*} \text {,norm }}^{t h}$ of about $5 \%$ in average. This means, that for exergy costing, the error between black-box and thermoeconomic result is with $20 \%$ far more substantial than for energy costing.

Furthermore, the three substations of Figure 3 are analysed in comparison with their black-box results in order to show the deviations of individual substations. Due to a lack of insights into DHN operation for real-exiting networks, it is assumed that in traditional approaches, only average unit costs for energy $c_{C N}^{t h}$ and exergy $k_{C N}^{t h}$ could be estimated. It is therefore interesting to study the difference between this black-box approach and the thermoeconomic results obtained for different substations. In order to compare energy- and exergy costing, the results of exergy costing, thus $k_{\text {sub }}^{\text {th }}$ for any substation, must be converted into unit cost of energy. This is done through using the energy $\Delta \dot{\phi}_{\text {sub }}^{\text {th }}$ and exergy $\Delta \dot{\psi}_{\text {sub }}^{\text {th }}$ flows associated with the thermal heating service to the substations, see (eq. 7),

$c_{\text {sub }}^{*, t h}=k_{\text {sub }}^{\text {th }} \frac{\Delta \dot{\psi}_{\text {sub }}^{t h}}{\Delta \dot{\phi}_{\text {sub }}^{t h}}$

where $c_{\text {sub }}^{* \text { th }}$ is the unit energy cost of the substation based on exergy costing. Thus the costing principles are based on exergy, while a direct comparison with $c_{\text {sub }}^{\text {th }}$ is possible. For simplification, $c_{\text {sub }}^{*, t h}$ is also referred as unit exergy cost even though it is in fact a unit energy cost based on exergy costing. Figure 5 shows the result obtained for the three substations.
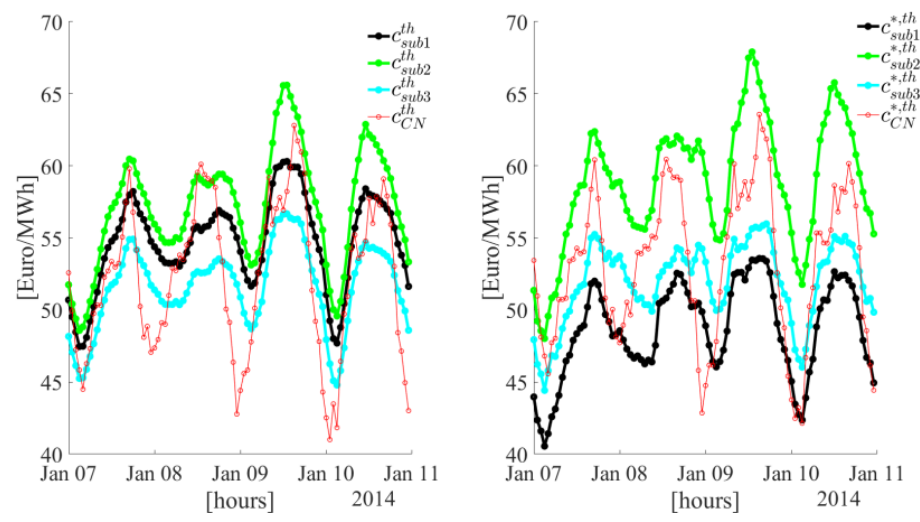

Figure 5: Comparison of energy- and exergy costing results for substations

The red line shows the black-box result $c_{C N}^{t h}$ and $c_{C N}^{*, t h}$ for unit energy and exergy cost. Substation 2 has the highest unit energy $\operatorname{cost} c_{\text {sub } 2}^{\text {th }}$ while substation 3 shows the lowest unit energy $\operatorname{costs} c_{\text {sub } 3}^{\text {th }}$. For a given timeframe, the black-box result either underestimates or overestimates the losses. This is equally true regarding exergy costing, with the difference that unit exergy costs of substation 1 and 3 are always lower compared to the average, while substation 2 shows higher peaks in exergy then energy costing. Substation 3 is closest to the plant compared to the others which is reflected by the relatively low unit energy. Interestingly, substation 1, which is located at the far north side of the network, shows lower unit exergy costs than substation 3, while for energy costs, the opposite is true. Through the conversion of unit exergy into unit energy cost, the amount of exergy compared to the amount of 
energy has significant influence on the cost estimation. This is the reason for the lower unit costs based in exergy compared to energy.

Thus, benchmarking of consumers highly depend on the costing technique applied, while the influence of the substation cannot be neglected and can even lead to a different benchmark between two substations (as seen for substation 1 and 3). This can be clearly seen when benchmarking the substations, when relative differences are plotted, see Figure 6.
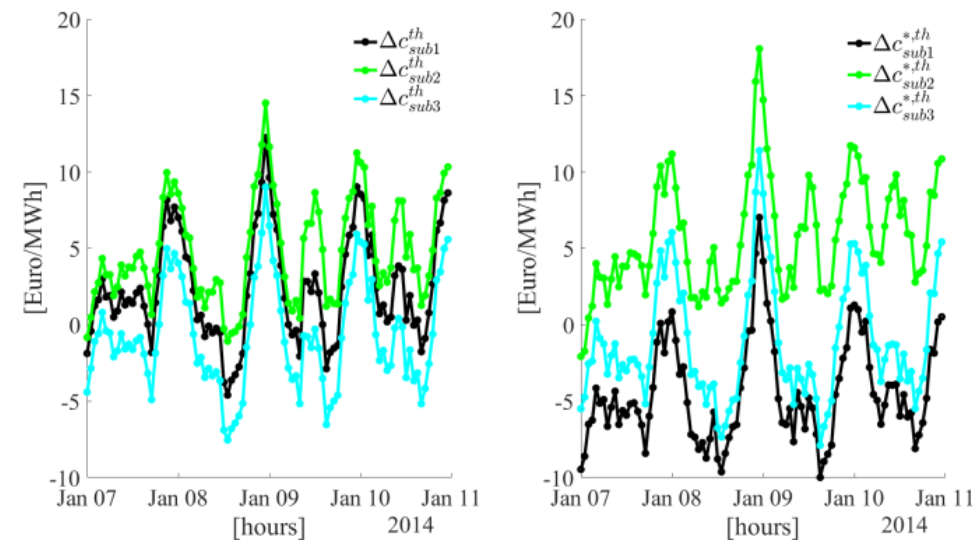

Figure 6: Thermoeconomic benchmark for substations

The benchmark uses the relative difference, such as $\Delta c_{s u b_{i}}^{t h}=c_{s u b_{i}}^{t h}-c_{C N}^{t h}$ for the results of unit energy and exergy cost. The benchmark shows the difference of unit costs applied from average costs compared to thermoeconomic results. Substation 2 is the most underestimated substation, with a peak difference of about 15 Euro/MWh. This means, the utilisation of one unit for energy on the 09.01 is 15 Euro/MWh more expensive, than estimated from a black-box analysis applied in industrial practise. Similarly for exergy, substation 2 is the most expensive with a peak difference of about 18 Euro/MWh. It can be seen that the peak is even more pronounced. Thus this result shows that thermoeconomics offers full transparency on the individual contribution of losses in the system, which, depending on the costing approach, vary considerably to the results which are obtained in traditional costing.

\section{CONCLUSIONS}

The formulation of thermoeconomics for graph-based network models can be applied to any DHN topology independently of the size of the network or the amounts of producers or consumers connected. The theoretical formulation provides a compact formulation for graph-based networks and is therefore especially suitable for large networks. External factors, such as boundary conditions of unit costs of external fuels or investment- and maintained costs can be comfortably integrated through the given matrix approach.

Furthermore, this approach is in accordance with the theory of exergetic costing. It assigns costs according to the loss of energy or exergy and is therefore able to capture the individual contribution of cost generation in DHN systems. The introduction of auxiliary equations is unnecessary, which avoids extra computational costs which would arise from traditional formulation. The formulation of thermoeconomic costs is an important piece of information for smart thermal network operation, because it enables the assessment of various measures such as waste heat and renewable energy integration or demand side measures such as temperature reduction of the consumers.

The case studies on the comparison of black-box costing vs. thermoeconomic costing revealed the drawbacks of black-box costing in the industrial context. The impacts of dynamic network behavior and individual contribution of the consumer are neglected which leads to wrong allocation of costs. 
Thermoeconomic provides a more transparent way of assigning the cost, namely individually at the place where inefficiency or thermal/mechanical losses occur. It was seen, that thermoeconomic provides a much clearer picture of the cost generating in network operation and offers the possibility for benchmarking between the consumers. Through that, the impact of consumer demand can be assessed and, if necessary, adapted through several measures such as dynamic pricing for demands with high costs etc.

\section{REFERENCES}

Guelpa, E. (2016), Modeling strategies for multiple scenarios and fast simulations in large systems: applications to fire safety and energy engineering. Politecnico di Torino. PhD thesis.

Lozano, M.A., Valero, A., (1993), Theory of exergetic cost. Journal of Energy, 18(9), 939-960.

Mathiesen, B.V., Lund, H., Connolly, D., Wenzel, H., Ostergaard, P.A., Moeller, B., Nielsen, S., Ridjan, I., Karnoe, P., Sperling, K., Hvelplund, F.K., (2015), Smart Energy Systems for coherent $100 \%$ renewable energy and transport solutions. Journal of Applied Energy, 145, 139-154.

Patankar, S.V., (1980), Numerical heat transfer and fluid flow. Series in Computational Methods in Mechanics and Thermal Sciences. ISBN 0-07-048740-5.

Wilson, R.J., (1996), Introduction to Graph Theory. 4th edition. Addison Wesley Longman Limited. ISBN 0-582-24993-7. 


\title{
HYDRAULIC SIMULATIONS OF LOW TEMPERATURE NETWORKS
}

\author{
Artem Sotnikov, Tobias Sommer, Christoph Stettler, Thomas Schluck \\ Institute of Building Technology and Energy \\ Lucerne University of Applied Sciences and Arts \\ Technikumstrasse 21, 6048 Horw, Switzerland \\ Phone: +41413493311 \\ E-Mail: artem.sotnikov@hslu.ch
}

\section{SUMMARY}

In low temperature networks (LTNs) with temperature level $10-20{ }^{\circ} \mathrm{C}$ thermal losses in the distribution system are very low, but because of the complex layout and low energy density the pump electricity consumption is high. Moreover, considering variable temperature of the network and continuous switching on/off of the heat and cold sources, the behavior of the LTN is very dynamic. In order to evaluate and to optimize LTN performance, dynamic hydraulic simulations are required. In the present study test cases of the LTN hydraulic are developed and then modelled in an in-house lab and in a simulation environment to verify the applicability of the simulation tool to model dynamic behavior of LTNs and to establish a basis for the further optimization studies. The simulation model shows a good agreement with the experiment setup.

Key-words: low temperature network, dynamic simulation, Modelica, Dymola

\section{INTRODUCTION}

A future sustainable district heating system (so called the 4 th generation of the district heating) operates at the network temperature $<50-60^{\circ} \mathrm{C}$ (Lund, et al., 2014). These systems allow integration of renewable energy sources as well as of seasonal heat storages, reduction of thermal losses in the distribution system, utilization of low temperature heat sources and drastic reduction of the fossil fuel consumption. Further reduction of the distribution system temperature to the level of $10-20^{\circ} \mathrm{C}$ combined with the low temperature seasonal heat storage (typically a borehole storage) additionally to aforementioned advantages allows also direct cooling of dwelling houses (Hangartner, Ködel, Mennel, \& Sulzer, 2017). A number of such LTNs have recently been built in Switzerland (Caratsch, Hangartner, Ködel, Sfeir, \& Sulzer, 2015). Due to the low temperature level, thermal losses in the distribution system are very small, in the same time the pump energy consumption is higher than in traditional systems. According to different sources the pump electricity consumption accounts for 2$10 \%$ and more of the total system energy consumption (Hangartner, Ködel, Mennel, \& Sulzer, 2017), (Vetterli, Thaler, Sulzer, \& Ryser, 2017). Therefore, understanding and optimization of the hydraulic behaviour of LTNs is an important task to improve system efficiency.

Most of the simulation tools consider the steady-state thermal and hydraulic behaviour of LTNs (Allegrini, et al., 2015). In order to compare different topologies and to estimate hydraulic losses and the pump electricity consumption as well as heat pump electricity consumption dynamic hydraulic simulations are required. The dynamic simulation of the hydraulic behaviour of the LTN is a complex task, which requires detailed models of hydraulic components (pipes, pumps and valves) as well as a robust solver.

\section{MAIN AIM AND METHODOLOGY}

For this study open-source component libraries, which are under development in the scope of IBPSA Project 1 (originally developed in Annex 60), are chosen (Wetter, et al., 2015). The libraries are developed in Modelica programming language (Modelica Association, 2014). The simulation environment is Dymola tool (Dassault Systèmes AB, 2017). The main aim of the current study is to evaluate applicability of Modelica language generally and IBPSA Project 1 libraries specifically for dynamic hydraulic simulation of LTNs.

Considering complexity of the LTN, namely, bidirectional grid, energy consumers are in the same time energy producers, integration of multiple heat (mostly renewables) and cold sources, which are typically at different temperature levels, decentralised design can improve the system efficiency and the pump control compare to centralised one (Vogelesang, 2009). Therefore, two simple test cases based on the decentralised system configuration are defined. Then, tests are conducted in the in-house test facility NODES ("New Opportunities for Decentralised Energy Systems")-lab (fig.1). 
The developed cases are modelled based on the components of IBPSA Project 1 libraries in Dymola environment. Then modeled results are compared with measured data of the physical experiments.

Later the developed test cases and models can be used as basis for an optimization study of the LTN hydraulic and the pump control.

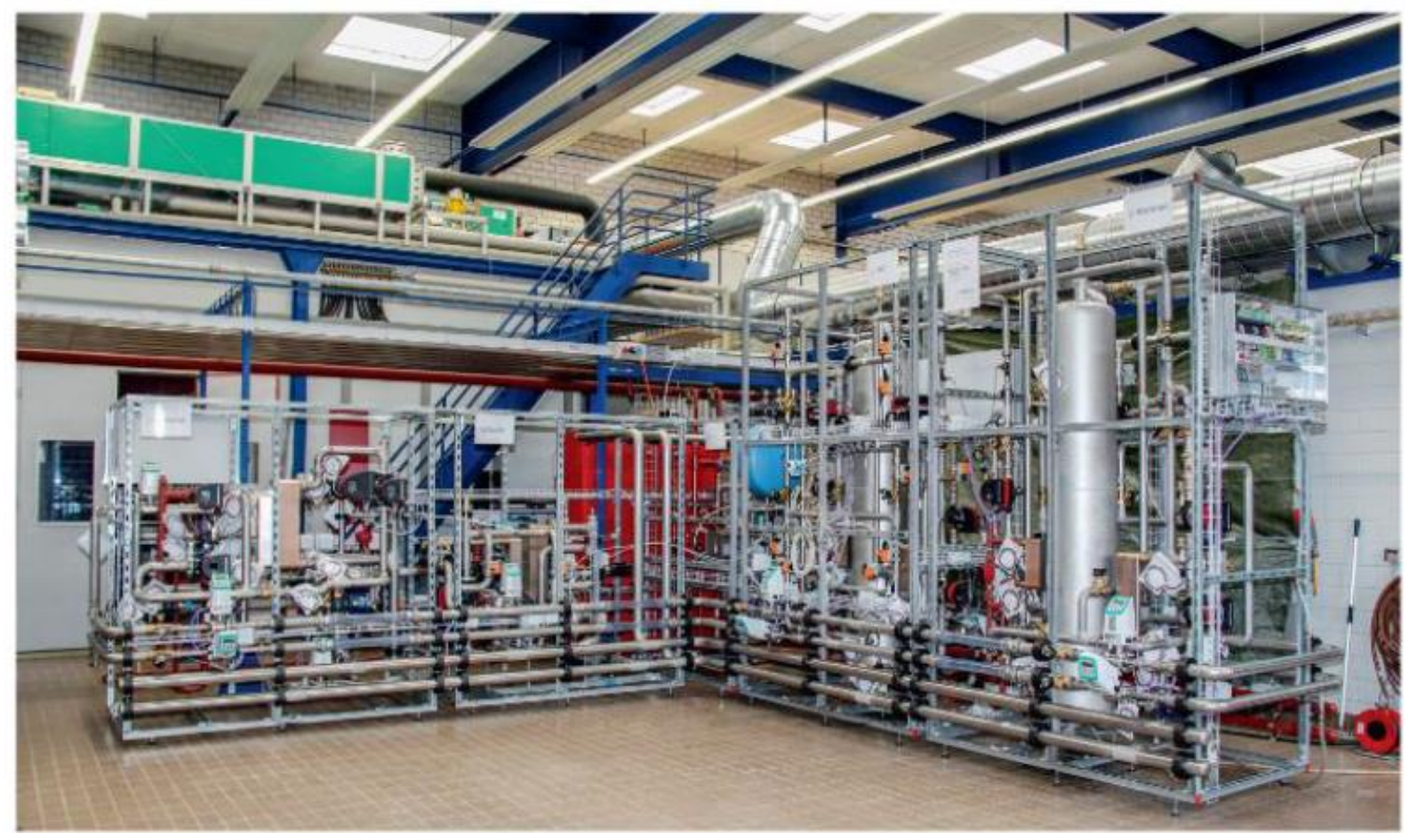

Fig. 1: "New Opportunities for Decentralised Energy Systems"-Lab (NODES).

\section{TEST DESCRIPTION}

The tested system consists of two producers/consumers (B2, B4) and a borehole storage (EWS). Each producer/consumers has a pump and a shut-off valve (fig.2 left). Hydraulic resistance represents the borehole storage. It is assumed that pressure losses in warm line and cold line are negligibly small. No thermal aspects are considered. There are two test cases. The first one is when both B2 and B4 are heat consumers ("Heating-Heating"), in this case pumps of B2 and B4 works in the same direction (fig.2 center). The second case is when B2 is a cold consumer but B4 is a heat consumer ("CoolingHeating") (fig. 2 right). Both test cases have the same time sequence (table 1).
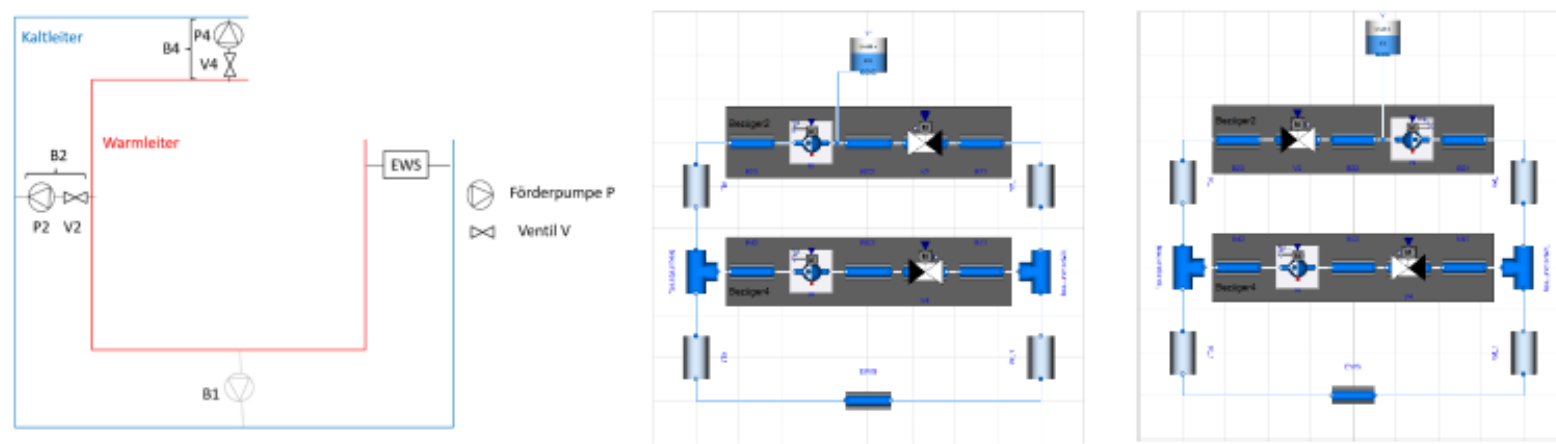

Fig. 2: Test setup scheme (left), case "Heating-Heating" (center) and case "Cooling-Heating" (right) in Modelica/Dymola environment. 
Table 1: Test sequence

\begin{tabular}{|c|c|}
\hline Time (minutes) & Component states \\
\hline 0 & Pumps P2 and P4 turned off. Valve V4 closed. Valve V2 opened. \\
\hline 3 & Pump P2 turned on and controlled at 1000 1/h. \\
\hline 6 & Valve V4 opened. \\
\hline 9 & Pump P4 turned on and controlled at 1000 1/h. \\
\hline 12 & Pump P4 turned off. \\
\hline 15 & Valve V4 closed. \\
\hline 18 & Pump P2 turned off. \\
\hline 21 & Valve V2 is closed \\
\hline
\end{tabular}

\section{RESULTS}

Experiment results are shown at figure 3. The top plot shows volumetric flow rate in $1 / \mathrm{h}$. The second plot from the top shows pump activity in \%. The second plot from the bottom shows pressure difference created by pumps in bar and the bottom plot shows pressure difference through EWS (hydraulic resistance) in bar. Time scale is given in minutes.
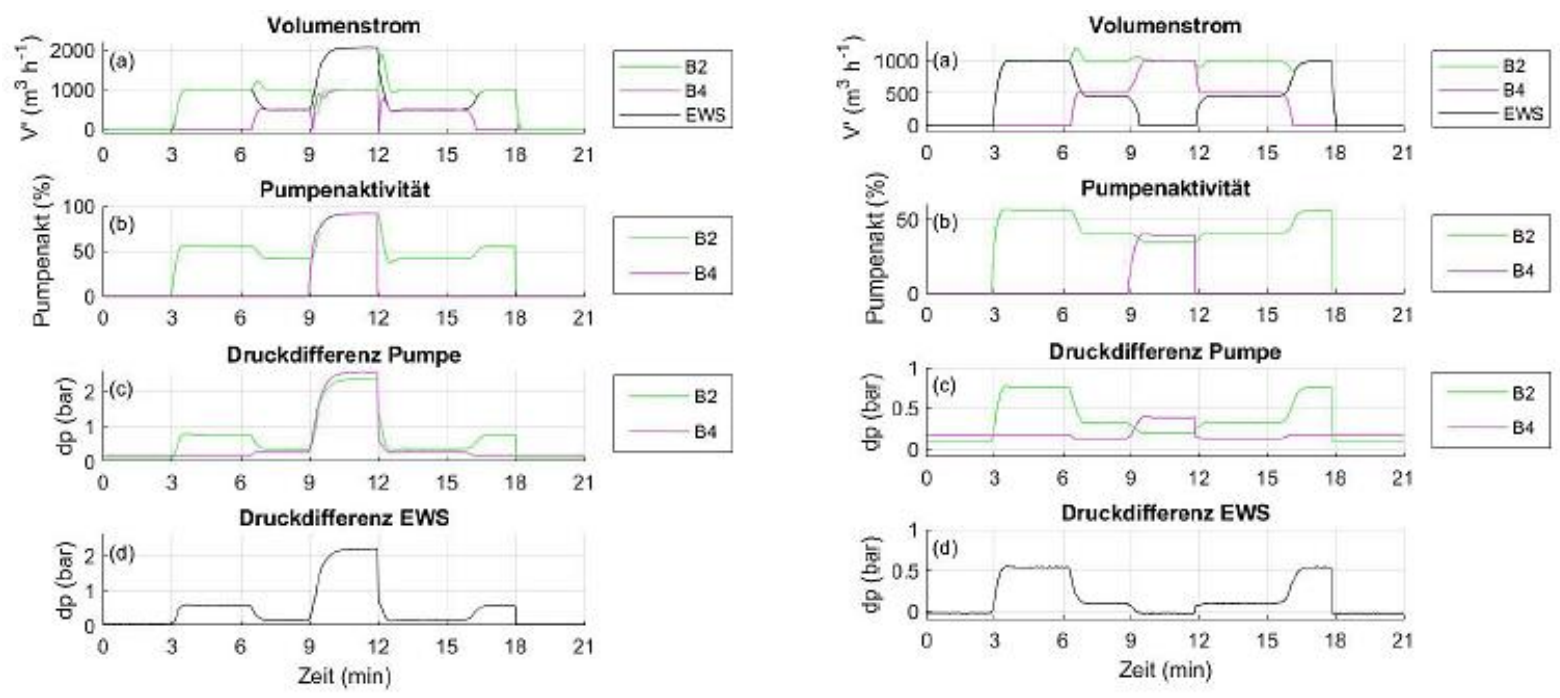

Fig. 3: Experiment (in NODES) results: "Heating-Heating" - left, "Cooling-Heating” - right.

Simulation results are shown at figure 4 . The top plot shows volumetric flow rate in $1 / \mathrm{h}$. The plot in the middle shows pressure difference created by pumps in bar and the bottom plot shows pressure difference through EWS (hydraulic resistance) in bar. Time scale is given in minutes. The system behaviour during the both tests is described in table 2 . 

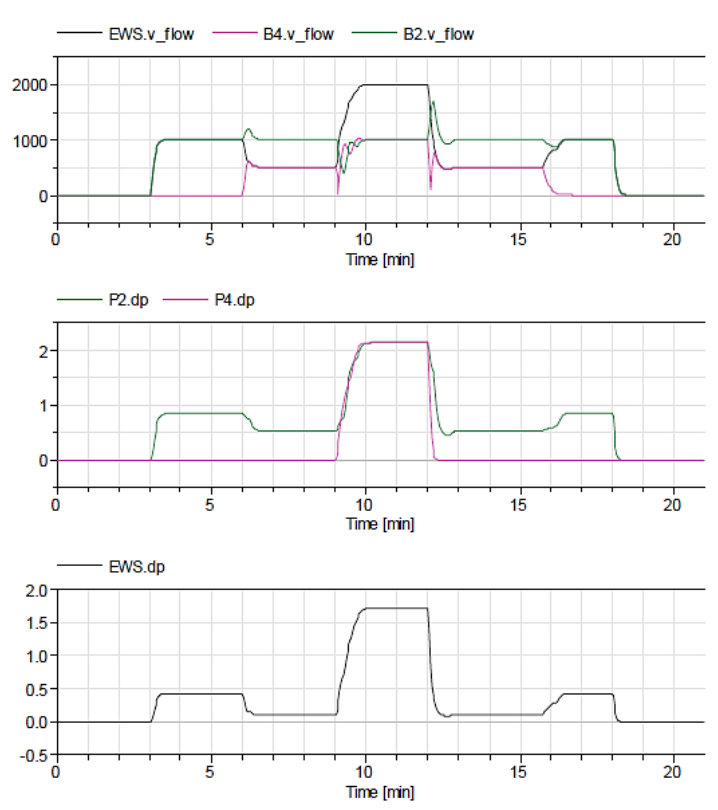
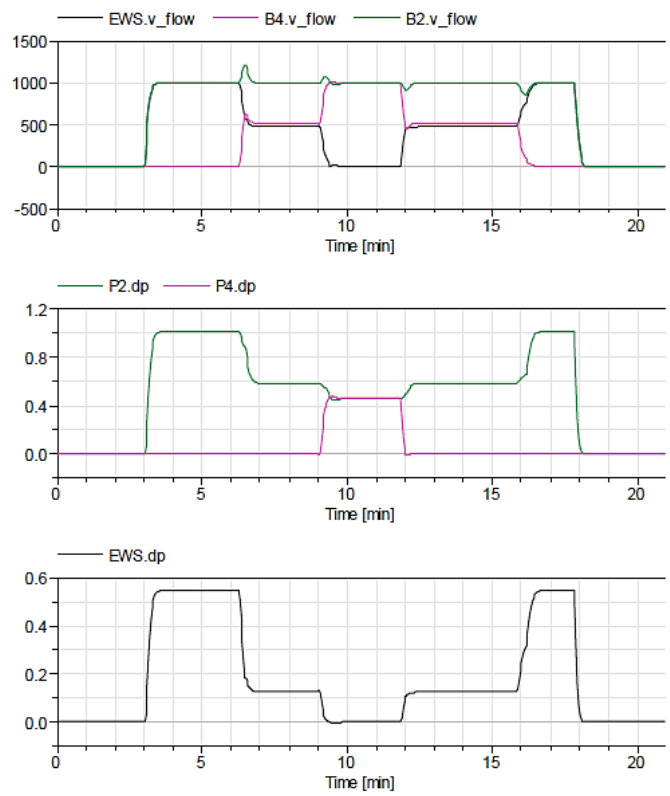

Fig. 4: Simulation results: "Heating-Heating" - left, "Cooling-Heating" - right.

Table 2: System behaviour

\begin{tabular}{|c|c|c|}
\hline Time (minutes) & "Heating-Heating" & "Cooling-Heating" \\
\hline 0 & There is no flow. & There is no flow. \\
\hline 3 & $\begin{array}{l}\text { Water flows through EWS with volumetric flow } \\
\text { rate } 1000 \mathrm{l} / \mathrm{h} .\end{array}$ & $\begin{array}{l}\text { Water flows through EWS with volumetric flow } \\
\text { rate } 1000 \mathrm{l} / \mathrm{h} .\end{array}$ \\
\hline 6 & $\begin{array}{l}\text { Water can flow also through B4. P2 has to } \\
\text { overcome smaller pressure difference. Therefore, } \\
\text { volumetric flow rate increases shortly until the } \\
\text { controller finds a new operation point. }\end{array}$ & $\begin{array}{l}\text { Water can flow also through B4. P2 has to } \\
\text { overcome smaller pressure difference. Therefore, } \\
\text { volumetric flow rate increases shortly until the } \\
\text { controller finds a new operation point. }\end{array}$ \\
\hline 9 & $\begin{array}{l}\text { For shot time period (about } 30 \mathrm{sec} \text { ) water flow } \\
\text { through B2 decreases drastically to } 370 \mathrm{l} / \mathrm{h} \text {, which } \\
\text { corresponds to } 63 \% \text { of the nominal value. When } \\
\text { pump controllers find their operation point water } \\
\text { flows } 1000 \mathrm{l} / \mathrm{h} \text { through } \mathrm{B} 2 \text { and } \mathrm{B} 4 \text { and } 2000 \mathrm{lh} \\
\text { through EWS. }\end{array}$ & $\begin{array}{l}\text { P2 has to overcome smaller pressure difference. } \\
\text { Therefore, volumetric flow rate increases shortly } \\
\text { until the controller find a new operation point. } \\
\text { Then, a hydraulic island is built and no water flows } \\
\text { through EWS. }\end{array}$ \\
\hline 12 & $\begin{array}{l}\text { Water flow through B2 increases shortly (about } 30 \\
\text { sec) up to } 1896 \mathrm{l} / \mathrm{h} \text {, which corresponds to } 90 \% \text { of } \\
\text { the nominal value. Then, a part of water flows } \\
\text { though B4 and the other part through EWS. }\end{array}$ & $\begin{array}{l}\text { P2 has to overcome higher pressure difference. } \\
\text { Volumetric flow rate falls down shortly, until a } \\
\text { new operation point is found. Then, a part of water } \\
\text { flows though B4 and the other part through EWS. }\end{array}$ \\
\hline 15 & $\begin{array}{l}\text { Volumetric flow rate falls down shortly, until a } \\
\text { new operation point is found. Then, water flows } \\
\text { through EWS. }\end{array}$ & $\begin{array}{l}\text { Volumetric flow rate falls down shortly, until a } \\
\text { new operation point is found. Then, water flows } \\
\text { through EWS. }\end{array}$ \\
\hline 18 & There is no flow. & There is no flow. \\
\hline 21 & There is no flow. & There is no flow. \\
\hline
\end{tabular}

\section{CONCLUSION AND OUTLOOK}

The simulation model shows a good agreement with the experiment setup. The system behaviour as well as absolute values of volumetric flow rates and pressure differences are very similar. The simulation model can reproduce very well volumetric flow rate deviations from nominal value in B2 depending on pressure difference fluctuations in the loop due to turning on/off pump P4 and opening and closing valve V4. 
The results show that the case "Heating-Heating" is more critical for hydraulic optimization than the case "Cooling-Heating", since in the case "Heating-Heating" volumetric flow rate falls down to 370 $1 / \mathrm{h}(63 \%$ from the nominal value) while in the case "Cooling-Heating" volumetric flow rate falls down only $20 \%$ from the nominal value. In the LTN the heating is realised by the heat pump. If the heat pump operates at the constant power, such a deep decrease of volumetric flow rate can even lead to the freezing of the water.

Considering a good agreement between of the simulation model results, the simulation environment can be used for the following hydraulic studies. The next step is the identification of other relevant cases as well as aspects, which influence hydraulic operation of the decentralized LTN. Then an enhanced controlling strategy is to be developed to ensure a stable operation of the LTN.

\section{ACKNOWLEDGEMENT}

This research project is financially supported by the Swiss Innovation Agency Innosuisse and is part of the Swiss Competence Center for Energy Research SCCER FEEB\&D.”

The simulation part of the study is performed in the scope of the IBPSA Project 1, an international project conducted under the umbrella of the International Building Performance Simulation Association (IBPSA). Project 1 will develop and demonstrate a BIM/GIS and Modelica Framework for building and community energy system design and operation.

\section{REFERENCES}

Allegrini, J., Orehounig, K., Mavromatidis, G., Ruesch, F., Dorer, V., \& Evins, R. (2015). A review of modelling approaches and tools for the simulation of district-scale energy systems. Renewable and Sustainable Energy Reviews 52, pp. 1391-1404.

Caratsch, M.-T., Hangartner, D., Ködel, J., Sfeir, J., \& Sulzer, M. (2015). Projekt "Thermische Vernetzung". Konzeptvorschlag.

Dassault Systèmes AB. (2017). Dymola. Dynamic Modeling Laboratory. User Manual. Volume 1.

Hangartner, D., Ködel, J., Mennel, S., \& Sulzer, M. (2017). Grundlagenpapier Thermische Netze. Thesen zu Fernwärme-Versorgungen und Erläuterungen zu Thermischen Netzen. Horw, Schweiz.

Lund, H., Werner, S., Wiltshire, R., Svendsen, S., Thorsen, J. E., Hvelplund, F., \& Mathiesen, B. V. (2014). 4th Generation Disctrict Heating (4GDH). Inegrating smart thermal grids into future sustainable energy systems. Energy 68, pp. 1-11.

Modelica Association. (2014). Modelica - a unified object-oriented language for system modeling. Language specification. Version 3.3 revision 1.

Vetterli, N., Thaler, E., Sulzer, M., \& Ryser, P. (2017). Monitoring Suurstoffi. Auswertung Okt.2013 Sep.2016.

Vogelesang, H. (2009, June). Delivering optimal energy savings. World Pumps, pp. 22-27.

Wetter, M., Fuchs, M., Grozman, P., Helsen, L., Jorissen, F., Lauster, M., . . . Thorade, M. (2015). IEA EBC ANNEX 60 Modelica Library - an International Collaboration to Develop a Free OpenSource Model Library for Buildings and Community Energy Systems. BS2015. Hyderabad, India. 


\section{A BOTTOM-UP METHODOLOGY FOR BUILDINGS ENERGY DEMAND CALCULAITON TO SUPPORT GRID BASED ENERY SYSTEMS IN URBAN AREAS}

Massimiliano Condotta ${ }^{1}$, Giovanni Borga $^{1}$, Tiziano Dalla Mora ${ }^{1}$, Alessandra De Angelis ${ }^{2}$, Onorio Saro $^{2}$

Università Iuav di Venezia ${ }^{1}$

Santa croce Dorsoduro 2196, Cotonificio veneziano, 30123 Venezia, Italy

Phone: +390412571271

E-Mail: condotta@iuav.it

Università degli Studi di Udine ${ }^{2}$

Via delle scienze 208, 33100 Udine

\section{SUMMARY}

The aim of the project IDEE is the development of a standard and shared procedure to support the evaluation of the better network energy system - based on centralized renewable energy plants or on heat recovered from energy loss - to be adopted at urban scale. The choice of the best solutions is affected by three main aspects: energy demand (amount of energy to be delivered to the buildings); energy supply (amount of energy that is possible to be recovered from industrial areas or centralized renewable energy power plants); district heating network configuration (distance from supply point to buildings, shape of network, ...).

In this paper, the focus is on the definition of a methodology and relative protocols for the calculation of energy demand of all buildings of a given urban environment.

Keywords: Buildings Energy demand, dynamic simulation, GIS.

\section{INTRODUCTION}

The EUROPA 2020 Strategy and the European Environment Action Plan highlight how territories competitiveness is closely linked to sustainability and resource-efficiency. Refurbishment of buildings, the use of new technologies such as low temperature district heating or heat pumps, recovery of waste energy of industry and the exploitation of biomass potential, will be crucial to making our urban energy systems more efficient and less polluting.

The project IDEE $^{13}$ concretizes a cross-border research network for integrated analysis and design of efficient and innovative energy systems in urban areas. This network combines the complementary competencies of research bodies and public authorities for developing a bottom-up framework for assessing city energy systems.

Through the definition of an integrated interpretation protocol for energy, environmental, building/technological and economic data - all geo-referenced at each single edifice - the IDEE network aims at developing a decision-making tool for local authorities. Thanks to this, the urban governance system can study and promote efficient investment based on the cost-benefit analysis of existing energy sources, on state-of-the-art cutting-edge technologies and their environmental impacts. The method is tested by applying it to the pilot areas of Maniago in Friuli Venezia Giulia, Feltre in Veneto and the Salzburger region Seenland in Austria. Subsequently, following the validation of the method, it will be possible to promote its transferability to other territories.

\footnotetext{
13 IDEE is a research project funded under the Interreg V-A Italy-Austria program; a cross border program under the European Territorial Cooperation Goal aiming to support a balanced and sustainable development and a harmonious integration of the border region between Italy and Austria.
} 


\section{OBJECTIVE OF THE RESERACH}

The integrated analysis and design of efficient and innovative energy systems in urban areas need a fully and deep knowledge of the city an of its energy behavior. This is the first fundamental step necessary for the assessment and the design of city energy systems. The object of the research described in this paper is therefore to set up a methodology and relative protocols for the calculation of buildings energy demand at urban level.

It is sometimes possible to reconstruct the energy behavior of a small suburb or of a small village through direct survey and analysis of each single building. In this way, it is possible to get a clear idea of what are the real necessity of each public or private building and describe the real necessities of the district. On the contrary, is almost impossible with this methodology to approach the analysis of the energy behavior of a whole city. The elevate number of buildings that would be necessary to audit, the difficulties to reach all the different social components and the impossibility to talk with all the householders, make this approach not applicable. The solution to break down this potential stalemate is a simulation-based approach.

The paper describes the methodology and the derived protocols that has been finalized to approach the study and the analysis of the energy behavior/energy demand of a city in order to support possible future studies of integrated urban energy systems. The methodology has been firstly developed using an Italian case study, but then it is planned to adapt it also at Austrian data sources in order to create a transnational methodology.

\section{METHODOLOGY: THE NEED OF SIMULATION STRATEGIES}

The methodology - proposed in the project IDEE - for the understanding of the energy behavior of a city, of a district or anyhow of a delimited territory with an elevate number of buildings is therefore made up of a chain of different simulations both at urban and building level.

For the purposes we have described above, a fully and deep understanding of the city and of its energy behavior starts from the knowledge of:

- Urban morphology (dimension, height and geometries of all the buildings that compose the city);

- Technological features (construction material of buildings, ...);

- Destination and uses of buildings.

These are the inputs necessaries to start a procedure that has the objective to calculate the energy behaviour of each building and then, consequently, of the whole city.

The process involves (1) simulations at urban level to reproduce the city morphology, the building/technological characteristics of edifices, buildings uses, and (2) simulations at building level to determinate the typical energy demand for each building category. Finally, a third simulation process at urban level is needed to reconstruct the energy behavior of the city.

In the pilot case of the project IDEE we have developed and tested an operational methodology (Figure 1) based on these three different phases.

The process starts with the collection of cadaster data, census data, cartography data and a questionnaire for householders. These are the input data that feed the first simulation by which determinate an urban catalogue. It is a selection (a sort of pattern book) of all the possible combination of buildings that can be present in the city.

The second phase involves a series of simulation at building level with the purpose of calculate the typical energy behavior of each type of building set in the first phase.

In the third phase, with a further simulation at urban level, the typical energy behaviors are applied at the respective type of buildings in a process that we call urban reconstruction. The urban reconstruction is a depiction of the real energy performance of the city. 


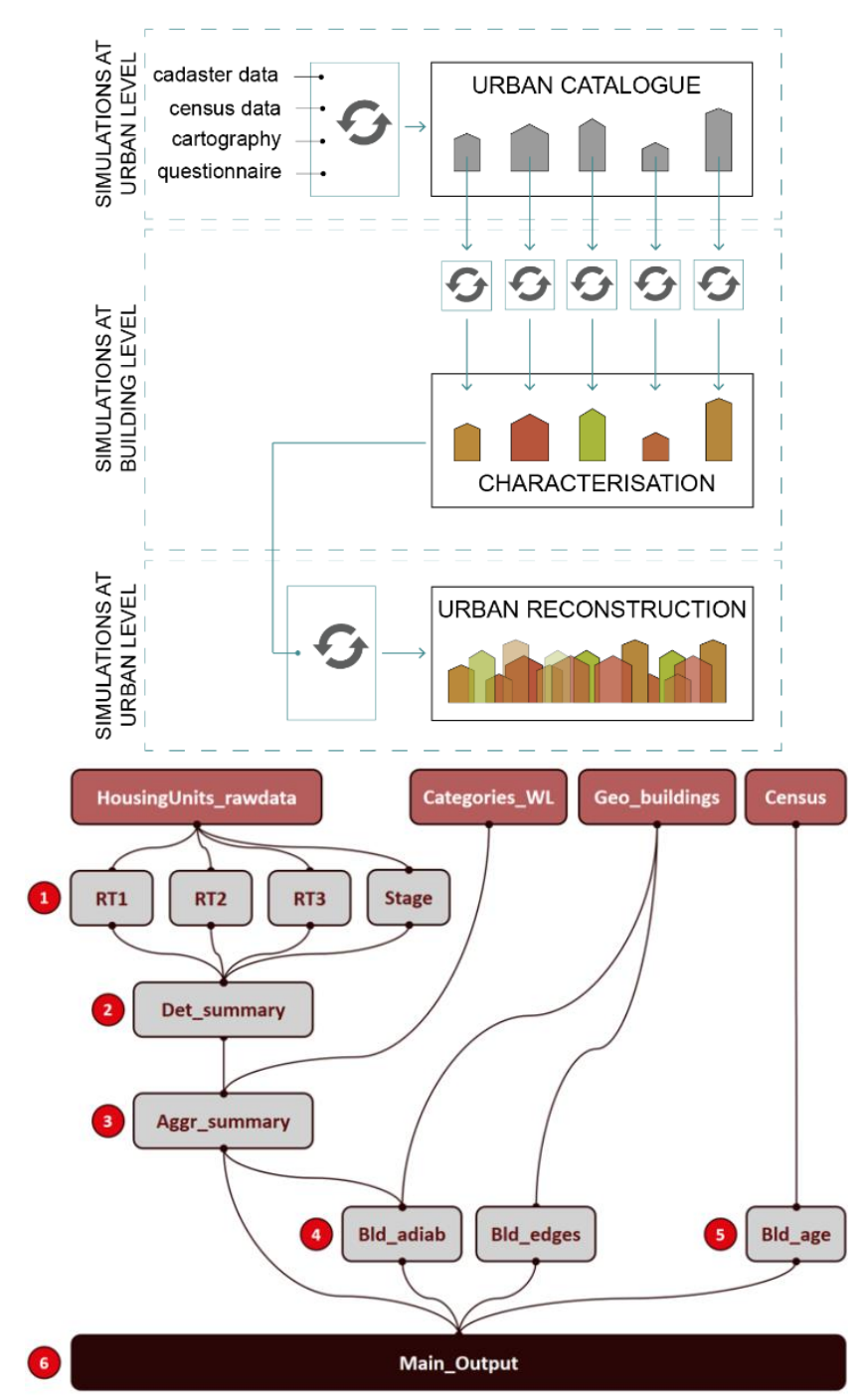

Fig. 1: Diagram of the methodology developed in the project IDEE with simulations at urban and building level. Fig. 2: Data processing workflow diagram.

\section{SIMULATIONS AT URBAN LEVEL}

In this research, in order to define a replicable methodology for the entire Italian national territory, the extraction and processing of the physical-morphological features of the buildings is based on the database provided by the national cadastral agency that has homogeneous characteristics for almost all Italian municipalities.

From a strictly technical point of view, unfortunately the Italian national cadastral Information System is not based on a traditional relational DBMS. As regards housing units' details data, they must be exported using a text format interchange file with fixed width field delimitation. The particularity of this format is that the field-set about each unit are spread on several lines instead of on one only, hence each line is provided with a code that tells the reference of the group of fields and it has its own set of field-widths. As for the geospatial data, the system instead provides a well-known topographicallycorrect SHP file that has simply to be re-projected on the needed Spatial Reference System (SRS).

Cadastral interchange text-files are provided in groups of three: one about housing units' data, one about owners' data and another about ownership data which contains all relationships between buildings and people. In this research, the processing model only needs housing units' data files which comes with a *.TAB extension and four files per year (one per three months). To set-up the first raw 
data table in the project database it has been necessary to develop a small piece of software to merges all text-files and perform some minor string processing.

Pre-processing of Feltre case study cadastral data (32 files) returned one raw data *.TFB text file of 238'331 rows which contains the cadastral history from 2001 to June 2017.

The project database is physically implemented in PostgreSQL with PostGIS spatial extension and, as shown in Figure 2 (upper 4 boxes), the core dataset is made of four entities: Housing units raw dataset; Categories White-List; Geospatial buildings layer; ISTAT census dataset.

Before explaining the whole processing model, we consider two basic assumptions: a) physical buildings and housing units are one-to-many related so we need a key to join them together; b) not all housing categories have to be considered in energy demand calculation, so we must filter-in the right ones using a sort of white-list. Below and in the diagram of Figure 2 we report a brief explanation of project database processing model structured in six stages.

Stage-1 of processing model is aimed at grouping housing units raw dataset per "row type" (RT) which is filtering rows by a code that defines the three main type of record we need: type-1) data about areas, floors, cadastral classes and categories; type-2) cadastral identification: sheet, parcel, sub-unit; type-3) street address. This is made by views "RT1", "RT2", "RT3". "Stage" view is a fourth procedure that select the ID number of the last registered situation of a housing unit among the cadastral history so to process only the latest one.

Stage- 2 of processing model performs a re-connection of the stage-1 datasets to output a detailed nonaggregated complete dataset of housing units. "Det_summary" view joins together the four previous views by housing unit primary key; moreover, it calculates the number of over-ground and underground floors and generate a one-field cadastral parcel primary key named "parc_key".

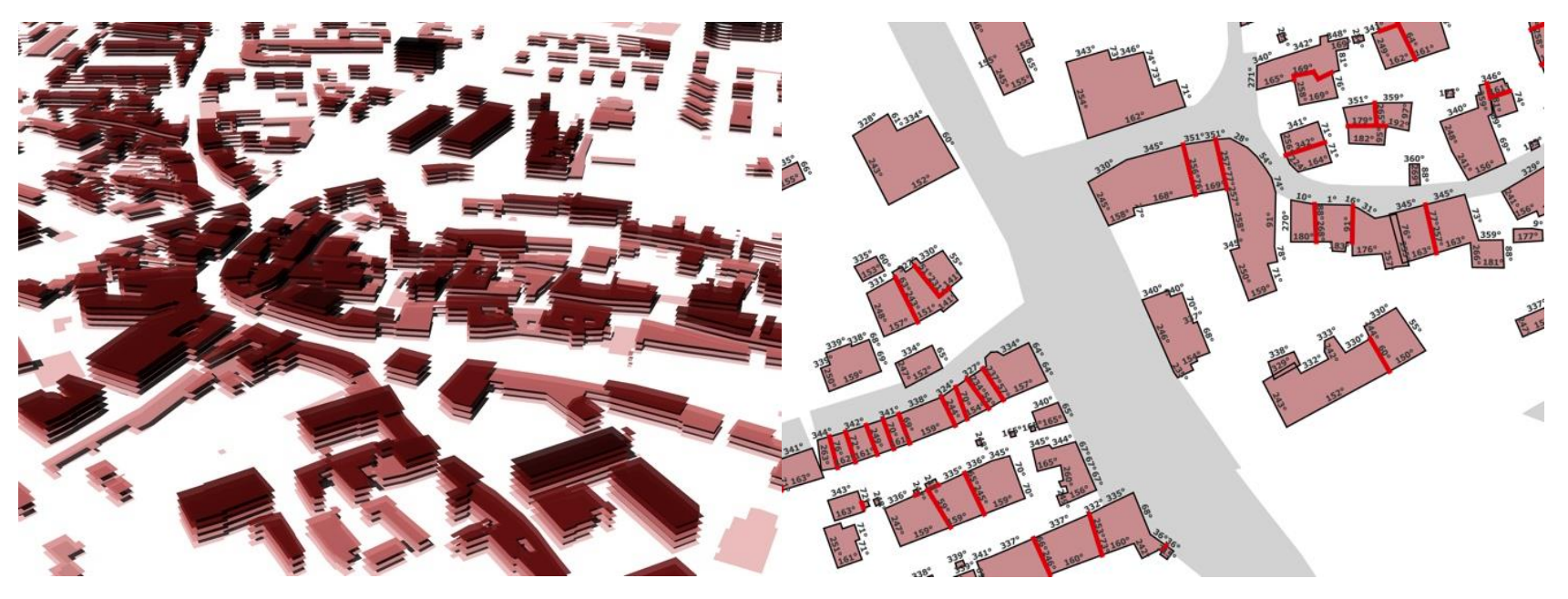

Fig. 3: Results of the simulation at urban level that calculates, for each building, the number of floors.

Fig. 4: Result map of \#4 processing stage.

Stage-3 of processing model performs a grouping of stage-2 dataset to get a summary of physical buildings features. "Aggr_summary" view joins together "Det_summary" view and categories whitelist and groups the record set by "parc_key". It calculates also heated floor area, number of housing units and total amount of over-ground and underground floors per building. Stage-3 data can be easily visualized on a map simply joining cadastral geospatial layer by "parc_key" field (see example in Figure 3).

Stage-4 of processing model carries out two geo-procedures aimed to perform geometric analysis of cadastral dataset. First geo-view, named "Bld_adiab", performs a self-intersecting overlay of buildings layer to extract base linestrings of adiabatic surfaces, then, joining to "Aggr_summary" selects number 
of floors to calculate the surface area of the facades. Second geo-view, named "Bld_edges" is a multinested complex geo-processing view that build a linestring layer of all edges of each building. "Bld_edges" has 4 level of nesting to generate a boundary for each building, extract all edges from boundaries and calculate the azimuth of each. Results of this stage can also be mapped as shown in Figure 4 in which we put in evidence adiabatic surfaces (red lines) and the orientation of each building façade expressed in degree (from $315^{\circ}$ to $45^{\circ}$ North, from $45^{\circ}$ to $135^{\circ}$ East, from $135^{\circ}$ to $225^{\circ}$ South, from $225^{\circ}$ to $315^{\circ} \mathrm{West}$ ).

In stage-5 of model, another geo-procedure named "Bld_age" assigns an "age class" to each building by overlaying census data provided by ISTAT national agency for each census zone. Age class is used to estimate construction techniques and materials in order to define envelope parameters (thermal transmittance, mass). To better check matching of Italian national statistical agency data and any changes in real situation, a questionnaire has been submitted to all householders with questions about their house and presence of retrofitting improvements.

Last stage 6 of the processing model returns the "main output dataset" (Table 1) where each calculated parameter is referred to every building of the city.

Table 1: GIS simulation outputs - Table of geometric parameters of buildings.

\begin{tabular}{|l|l|}
\hline Parameter name & Parameter description \\
\hline OverGroundFloors & Number of over-ground floors \\
\hline UnderGroundFloors & Number of under-ground floors \\
\hline TotalFloors & Total number of floors \\
\hline BuildingArea & Building base surface area \\
\hline TotalFloors SurfaceArea & Sum of each level surface \\
\hline SurfaceArea_N & North-facing surfaces \\
\hline SurfaceArea_E & East-facing surfaces \\
\hline SurfaceArea_W & West-facing surfaces \\
\hline SurfaceArea_S & South-facing surfaces \\
\hline AdiabaticSurfaceArea_N & Adiabatic North-facing surfaces \\
\hline AdiabaticSurfaceArea_E & Adiabatic East-facing surfaces \\
\hline AdiabaticSurfaceArea_W & Adiabatic West-facing surfaces \\
\hline AdiabaticSurfaceArea_S & Adiabatic South-facing surfaces \\
\hline
\end{tabular}

\begin{tabular}{|l|l|}
\hline Parameter name & Parameter description \\
\hline WindowArea_N & North-facing windows area \\
\hline WindowArea_E & East -facing windows area \\
\hline WindowArea_S & South-facing windows area \\
\hline WindowArea_W & West-facing windows area \\
\hline LossSurfaceArea_N & North-facing loss surfaces area \\
\hline LossSurfaceArea_E & East-facing loss surfaces area \\
\hline LossSurfaceArea_W & West-facing loss surfaces area \\
\hline LossSurfaceArea_S & South-facing loss surfaces area \\
\hline & \\
\hline & \\
\hline & \\
\hline & \\
\hline
\end{tabular}

\section{SIMULATIONS AT BUILDING LEVEL}

This phase consists on the definition of a methodology for a parametric calculation of the annual heating energy demand $(\mathrm{kWh})$ for existent buildings, meaning the quantity of heat required by a building during the year, referring both to space heating demand and domestic hot water demand.

A bottom-up approach is proposed. In fact, the estimated energy need is deduced by the values of different parameters given by the simulation of a set of typical buildings that compose the urban catalogue. The approach is based on the assessment of each single thermal zone of a building with a specific number of levels. The thermal zone is geometrically studied with $100 \mathrm{~m}^{2}$ of gross heated floor and a WWR (wall to windows ratio) of $10 \%$ for each orientation, and it is set up into three main types of buildings (L1, L2, L3) giving four different thermal zones (Ground, Basement, Roof, Medium) (see Figure 5). For example, a single level building corresponds to the thermal zone "Ground", that is different in respect to building with two levels where thermal zones are "Basement" and "Roof". In case of a building with three or more levels the method considers as many "Medium" thermal zone as 
intermediate levels (Figure 5). In this study, we assumed an average height for each thermal zone of $3 \mathrm{~m}$.
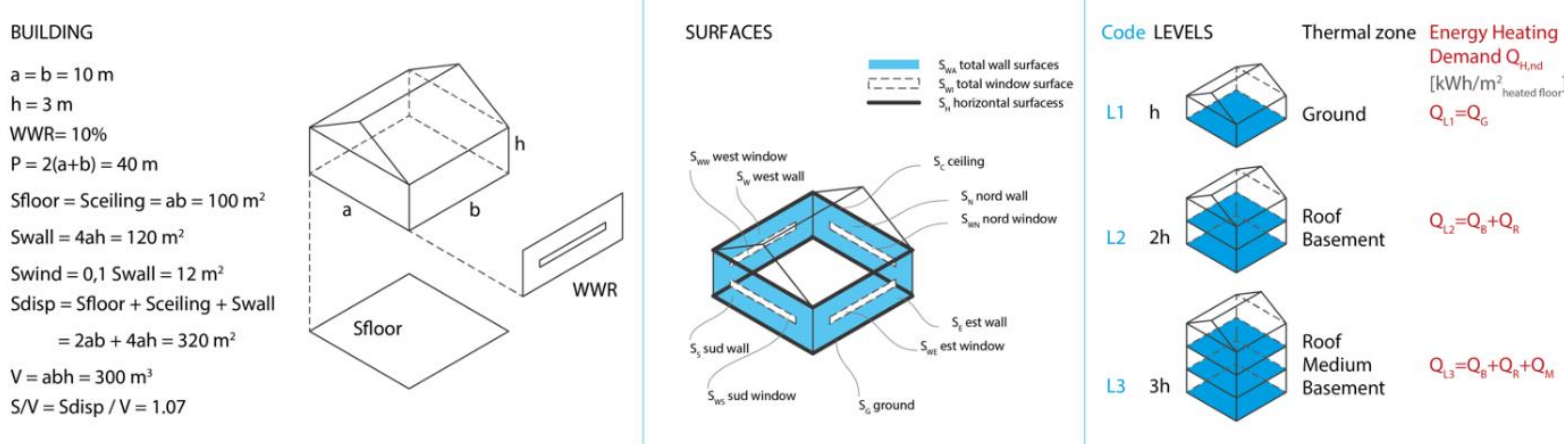

Fig. 5: Characteristics of typical buildings used in the simulations: geometry, surfaces and thermal zones definition.

National Census provides data to divide the building stock according to typology, use (residential, non-residential, office), and construction period (before 1919, 1919-1945, 1947-1970, 1970-1991, 1991-2005, 2005-2013). Assumed characteristics for building components are determined according to each construction period. U-values $\left(\mathrm{kWh} / \mathrm{m}^{2}\right)$ result from literature review and archive research (Peron, 2012).

The energy demands for space heating are evaluated by the methodology of the standard UNI TS 11300-1, which represents the Italian version of the EN ISO 13790. The evaluation assumes typical boundary conditions for winter season: an indoor air set point temperature equal to $20^{\circ} \mathrm{C}$, and the length of the heating season according to the Italian Law (starting from $15^{\text {th }}$ October to $15^{\text {th }}$ April). In this study, weather data for Belluno (pilot case location) are considered. The annual heating requirement $\left(Q_{H, n d}\right)$ of buildings is evaluated by the following equation:

$$
Q_{H, n d}=\left(Q_{H, t r}+Q_{H, v e}\right)-\eta_{H, g n}\left(Q_{i n}+Q_{s o l}\right)
$$

To calculate heating requirement, the equation subtracts, on a monthly basis, the thermal gains, that are, the sum of solar $\left(Q_{s o l}\right)$ and internal gains $\left(Q_{i n}\right)$, from the heat losses due to energy transmission $\left(Q_{H, t r}\right)$ and ventilation $\left(Q_{H, v e}\right)$. Heat gains are multiplied by an utilization factor $\left(\eta_{\mathrm{H}, \mathrm{gn}}\right)$, taking into account the dynamic behavior of the building. The assumed utilization factor is 0.80 (experience reveals that the index is usually within the range $0.7-0.9$ depending on thermal mass of the building and on the ratio between losses and gains). The parametric simulations to calculate typical primary energy demand are carried out using a software for energy dynamic simulation "Energy Plus" with "Design Builder" as graphic interface. Heating, cooling, ventilation, domestic hot water, lighting and auxiliary demands have been estimated in accordance with the Italian technical specifications UNI/TS 11300 implementing the European standards. The simulation is performed for each type of building according to number of thermal zones (levels) and construction age. Each simulation is then elaborated in order to obtain typical energy losses and gains for different geometric characteristics of the buildings. The data analysis results are summarized in Table 2 .

In order to define the size of the district heating system and to evaluate the suitable mixture of energy sources to feed the district heating network, three different values of required thermal load are needed: the maximum, the average and the minimum thermal load. Besides that, also the duration of each thermal load has to be known.

Starting from the annual heating requirement the value of average thermal load ( $\left.\Phi_{a v}\right)$ can be evaluated as: 
To calculate the maximum thermal load $\left(\Phi_{\max }\right)$ the proportional relationship between the thermal load and the temperature difference has been considered, obtaining the following expression:

$$
\Phi_{\max }=\Phi_{a v}\left(\Delta \vartheta_{\max } / \Delta \vartheta_{a v}\right) f_{s}
$$

In the evaluation of the maximum thermal load, also a safety coefficient $f_{s}$ has been added, that take into account that the maximum heat requirement has to be calculated considering the worse conditions (no gains contribution, intermittent operation of the heating plant, external air temperature exceptionally low).

Table 2: List of 25 energy loss/gain values $\left(\mathrm{kWh} / \mathrm{m}^{2} \mathrm{y}\right)$ calculated by the dynamic energy simulations.

\begin{tabular}{|c|c|c|}
\hline & Parameterization surface & Variable \\
\hline \multirow{22}{*}{$\begin{array}{l}\text { Transmission } \\
\text { loss: } Q_{\mathrm{H}, \mathrm{tr}}\end{array}$} & Ground Surface (1 storey) & $\mathrm{QH}, \operatorname{tr}(\mathrm{SG}) 11$ \\
\hline & Ground Surface (2 storeys) & $\mathrm{QH}, \operatorname{tr}(\mathrm{SG}) 21$ \\
\hline & Ground Surface (3 storeys) & $\mathrm{QH}, \operatorname{tr}(\mathrm{SG}) 31$ \\
\hline & Roof Surface (1 storey) & $\mathrm{QH}, \operatorname{tr}(\mathrm{SR}) 11$ \\
\hline & Roof Surface (2 storeys) & $\mathrm{QH}, \operatorname{tr}(\mathrm{SR}) 21$ \\
\hline & Roof Surface (3 storeys) & $\mathrm{QH}, \operatorname{tr}(\mathrm{SR}) 31$ \\
\hline & East wall Surface (1 storey) & $\mathrm{QH}, \operatorname{tr}(\mathrm{SE}) 11$ \\
\hline & East wall Surface (2 storeys) & $\mathrm{QH}, \operatorname{tr}(\mathrm{SE}) 21$ \\
\hline & East wall Surface (3 storeys) & $\mathrm{QH}, \operatorname{tr}(\mathrm{SE}) 31$ \\
\hline & North wall Surface (1 storey) & $\mathrm{QH}, \operatorname{tr}(\mathrm{SN}) 11$ \\
\hline & North wall Surface (2 storeys) & $\mathrm{QH}, \operatorname{tr}(\mathrm{SN}) 21$ \\
\hline & North wall Surface (3 storeys) & $\mathrm{QH}, \operatorname{tr}(\mathrm{SN}) 31$ \\
\hline & West wall Surface (1 level) & $\mathrm{QH}, \operatorname{tr}(\mathrm{SW}) 11$ \\
\hline & West wall Surface (2 levels) & $\mathrm{QH}, \operatorname{tr}(\mathrm{SW}) 2 \mathrm{l}$ \\
\hline & West wall Surface (3 or more levels) & $\mathrm{QH}, \operatorname{tr}(\mathrm{SW}) 31$ \\
\hline & South wall Surface (1 level) & $\mathrm{QH}, \operatorname{tr}(\mathrm{SS}) 11$ \\
\hline & South wall Surface (2 levels) & $\mathrm{QH}, \operatorname{tr}(\mathrm{SS}) 2 \mathrm{l}$ \\
\hline & South wall Surface (3 or more levels) & $\mathrm{QH}, \operatorname{tr}(\mathrm{SS}) 31$ \\
\hline & East windows Surface & $\mathrm{QH}, \operatorname{tr}(\mathrm{SWE})$ \\
\hline & Nord windows Surface & $\mathrm{QH}, \operatorname{tr}(\mathrm{SWN})$ \\
\hline & West windows Surface & $\mathrm{QH}, \operatorname{tr}(\mathrm{SWW})$ \\
\hline & South windows Surface & QH,tr(SWS) \\
\hline
\end{tabular}

\begin{tabular}{|c|c|c|}
\hline & $\begin{array}{l}\text { Parameterization } \\
\text { surface }\end{array}$ & Variable \\
\hline $\begin{array}{l}\text { Ventilation loss: } \\
\text { Q }_{\mathrm{H}, \mathrm{ve}}\end{array}$ & Total floor Surface & Qin(STF) \\
\hline Internal gain: $Q_{\text {in }}$ & Total floor Surface & Qin(STF) \\
\hline \multirow[t]{4}{*}{ Solar gain: $\mathbf{Q}_{\mathrm{sol}}$} & East windows Surface & Qsol(SWE) \\
\hline & Nord windows Surface & Qsol(SWN) \\
\hline & West windows Surface & Qsol(SWW) \\
\hline & South windows Surface & Qsol(SWS) \\
\hline & & \\
\hline & & \\
\hline & & \\
\hline & & \\
\hline & & \\
\hline & & \\
\hline & & \\
\hline & & \\
\hline & & \\
\hline & & \\
\hline & & \\
\hline & & \\
\hline & & \\
\hline & & \\
\hline & & \\
\hline
\end{tabular}

\section{RECONSTRUCTION OF THE URBAN ENERGY MODEL}

The final phase of the developed methodology is again a simulation at urban level through which reconstruct buildings' energy performance and energy demand of each single building and get a depiction of the real energy performance of the city. As mentioned earlier, the calculation of energy demand of buildings is based on the formula (eq. 1). This calculation is repeated inside the geographical database for each individual building and the typical values of energy loss (or gain) (Table 2) are matched with the geometrical dimension of the building (Table 2). The following formula (eq. 2) explain the query used in the geographical database that executes the formula (eq. 1) for each single building. The query initially calculate the values of $\mathrm{Q}_{\mathrm{H}, \mathrm{tr}}, \mathrm{Q}_{\mathrm{H}, \mathrm{ve}}, \mathrm{Q}_{\text {in }}$ and $\mathrm{Q}_{\text {sol }}$; and then sum these values according to formula (eq. 1). 


$$
\begin{aligned}
& \mathbf{Q}_{\mathrm{H}, \mathrm{tr}}=\mathrm{Q}_{\mathrm{Htr}}\left(\mathrm{S}_{\mathrm{G}}\right) \mathrm{xl} \cdot \mathrm{B}_{\text {uilding }} \mathrm{A}_{\text {rea }}+\mathrm{Q}_{\mathrm{Htr}}\left(\mathrm{S}_{\mathrm{R}}\right) \mathrm{xl} \cdot \mathrm{B}_{\text {uilding }} \mathrm{A}_{\text {rea }}+\mathrm{Q}_{\mathrm{Htr}}\left(\mathrm{S}_{\mathrm{E}}\right) \mathrm{xl} \cdot \mathrm{L}_{\text {oss }} \mathrm{S}_{\text {urface }} \mathrm{A}_{\text {rea_ }} \mathrm{E}+\mathrm{Q}_{\mathrm{Htr}}\left(\mathrm{S}_{\mathrm{N}}\right) \mathrm{xl} \cdot \\
& \mathrm{L}_{\text {oss }} \mathrm{S}_{\text {urface }} \mathrm{A}_{\text {rea_ }} \mathrm{N}+\mathrm{Q}_{\mathrm{Htr}}\left(\mathrm{S}_{\mathrm{W}}\right) \mathrm{xl} \cdot \mathrm{L}_{\text {oss }} \mathrm{S}_{\text {urface }} \mathrm{A}_{\text {rea_ }} \mathrm{W}+\mathrm{Q}_{\mathrm{Htr}}\left(\mathrm{S}_{\mathrm{S}}\right) \mathrm{xl} \cdot \mathrm{L}_{\text {oss }} \mathrm{S}_{\text {urface }} \mathrm{A}_{\text {rea_ }} \mathrm{S}+\mathrm{Q}_{\mathrm{Htr}}\left(\mathrm{S}_{\mathrm{WE}}\right) \cdot \\
& \left.\mathrm{W}_{\text {indow }} \mathrm{A}_{\text {rea }} \mathrm{E}\right)+\mathrm{Q}_{\mathrm{Htr}}\left(\mathrm{S}_{\mathrm{WN}}\right) \cdot \mathrm{W}_{\text {indow }} \mathrm{A}_{\text {rea }} \mathrm{N}+\mathrm{Q}_{\mathrm{Htr}}\left(\mathrm{S}_{\mathrm{WW}}\right) \cdot \mathrm{W}_{\text {indow }} \mathrm{A}_{\text {rea }} \mathrm{W}+\mathrm{Q}_{\mathrm{Htr}}\left(\mathrm{S}_{\mathrm{WS}}\right) \cdot \mathrm{W}_{\text {indow }} \mathrm{A}_{\text {rea_ }} \mathrm{S} \\
& \mathbf{Q}_{\mathrm{H}, \mathrm{ve}}=\mathrm{Q}_{\mathrm{H}, \mathrm{ve}}\left(\mathrm{S}_{\mathrm{TF}}\right) \cdot \mathrm{T}_{\text {otal }} \mathrm{F}_{\text {loors }} \mathrm{S}_{\text {urface }} \mathrm{A}_{\text {rea }} \\
& \mathbf{Q}_{\text {in }}=\mathrm{Q}_{\text {in }}\left(\mathrm{S}_{\mathrm{TF}}\right) \cdot \mathrm{T}_{\text {otal }} \mathrm{F}_{\text {loors }} \mathrm{S}_{\text {urface }} \mathrm{A}_{\text {rea }} \\
& \mathbf{Q}_{\text {sol }}=\mathrm{Q}_{\text {sol }}\left(\mathrm{S}_{\mathrm{WE}}\right) \cdot \mathrm{W}_{\text {indow }} \mathrm{A}_{\text {rea_ }} \mathrm{E}+\mathrm{Q}_{\text {sol }}\left(\mathrm{S}_{\mathrm{WN}}\right) \cdot \mathrm{W}_{\text {indow }} \mathrm{A}_{\text {rea_ }} \mathrm{N}+\mathrm{Q}_{\text {sol }}\left(\mathrm{S}_{\mathrm{WW}}\right) \cdot \mathrm{W}_{\text {indow }} \mathrm{A}_{\text {rea_ }} \mathrm{W}+ \\
& \mathrm{Q}_{\text {sol }}\left(\mathrm{S}_{\mathrm{WS}}\right) \cdot \mathrm{W}_{\text {indow }} \mathrm{A}_{\text {rea }} \mathrm{S}
\end{aligned}
$$

\section{REFERENCES}

CEN - European Committee for Standardization (2008). ISO - "International Organization for Standardization, Standard ISO EN 13790/2008: Energy performance of buildings - Calculation of energy use for space heating and cooling".

J. Dorfner, "GIS-based Mapping Tool of Urban Energy Demand for room heating and hot water", Mechanical and Building Industry Days Urban Energy Conference, Debrecen, 2011, 31-35.

European Committee for Standardization (CEN). EN 15243:2007. "Ventilation for buildings Calculation of room temperatures and of load and energy for buildings with room conditioning systems"; 2007.

European Committee for Standardization (CEN). EN 15316 (series). "Heating systems in buildings Method for calculation of system energy requirements and system efficiencies"; 2007-2011.

Modi Research Group, "Estimated Total Annual Building Energy Consumption at the Block and Lot Level for NYC" (Columbia University, 2012), http://modi.mech.columbia.edu/resources/nycenergy/about.

F. Peron, A. Righi, R. Romagnoni (2014). Politiche locali per il clima. Metodologie d'analisi e strumenti di intervento. "Migliorare l'efficienza energetica del patrimonio edilizio esistente". Pg. 60 104. ISBN 978-88-917-0579-2.

UNI - Italian Committee for Standardization (2014). Standard UNI TS 11300 Part 1: "Energy performance of buildings: Evaluation of energy need for space heating and cooling".

\section{CONCLUSION}

Many of the previous and existing experience on simulation of energy demand of cities are based on the urban morphology information such as dimension, height and volume of buildings [3,7]. In the IDEE approach some "new" factors are derived from urban morphology and become new parameters in the simulation process. They are the presence and extension of adiabatic surfaces and the orientation of the buildings surfaces.

The presence of adiabatic surfaces has a relevant influence in reducing the energy demand of a building, moreover in historic cities where edifices compose a continuous façade delimiting the streets. On the other side, we have realized that orientation of exposed facades has not a big influence on energy loss, but, on the contrary, has a relevant influence on the effects of solar gains. We can therefore conclude that the methodology developed has - considering the methodological aspect and protocols - the potentialities to return quite precise and affordable results in term of energy demand of buildings.

But a critical analysis must be done not only at methodology level but also at application level. Considering therefore the application of the methodology that we have done in our pilot case, the most relevant criticality is that the assignment of buildings to a particular construction category (energy performance of the building envelope) is based only on construction date. This approach - already 
used in many studies and approaches and considered valid for preliminary studies - can give back imprecise assumptions: not all buildings constructed in the same period share the same building technology. Moreover, with this approach, the presence of possible retrofitting actions is not considered. More specific studies - such as the planning of network systems at urban level nevertheless needs more precise outputs. For this reason, the solution adopted in IDEE to face this criticality has been to submit to all householders a questionnaire with specific questions about their house and presence of retrofitting improvements. Even if we will not get an answer from all the householders, the results will help us to calibrate and refine the urban catalogue and the attribution of each building at the correct category. 


\title{
OPTIMIZED METHOD TO PREDICT ENERGY IN A MICROGRID
}

\author{
Luc Dufour1, Dominique Genoud1, Bruno Ladevie2, David Wannier \\ Information System institute1, \\ TechnoPôle 3, 3960 Sierre, Suisse 1, \\ E-Mail: (luc.dufour, david.wannier,dominique.genoud)@hevs.ch 1 \\ CNRS UMR 53022 \\ Campus Jarlard, Route de Teillet, 81000 Albi, France 2 \\ bruno.ladevie@mines-albi.fr2
}

\section{SUMMARY}

Key Words: Optimized Machine learning, Data mining, Energy prediction, Microgrid

This work presents a data-intensive solution to predict the different energy flux in a microgrid. The ability to predict locally the energy considering meteorological uncertainty can play a key role in the management of microgrid. Our approach is to provide an easy implemented and flexible solution to predict the consumption and the production at the building level based on the machine learning technique and tested on the real use cases in a residential and tertiary sector. A new evaluation of the consumption is realized: the point of view is energy and not only electrical. The energy consumption is decomposed between the heating, the hot water, the electrical devices, the lighting and the ventilation consumptions. A prediction every hour is provided to create scenarios to pilot devices. $\mathrm{We}$ provide an original optimized method to limit the input data number. A classification and a prediction for the electrical consumption in a tertiary sector are provided for the next hour with an accuracy over the $98 \%+/-1 \%$.

\section{INTRODUCTION}

PV is the fastest-growing energy technology since 2002 with an average increase of $48 \%$ [COC-2012]. The majority plants are grid-connected systems and a high penetration of PV (like in the case of islands) raises issues for the grid-operators. Subsequently, forecasting the power output of the PV plant is necessary to assure the grid stability. A large amount of research studies in the domain lay emphasis on predicting solar radiation which is a key data to improve the results. Neural networks are widely used to this purpose and manage to reach a MAPE around 7\% on monthly and day-ahead solar radiation forecast [COC-2012]. We can cite the example of A. Mellit, a reference author in the subject who achieve a MAPE less than 6\% for day-ahead solar radiation in Algeria [MEL-2006]. Regression trees are not widely used but show a MAPE of 33\% for PV production prediction [Nom-2011]. The studies concern a wide choice of photovoltaic installations and data type, (study on 12 PV cells in the laboratory or $14,000 \mathrm{~m} 2$ of panels) with ARIMA models or neural networks [ZAM-2014] [GRA2016] [GUL-2016].

The consumption prediction models are very important, time scales are multiples and the predictions are made by the hour, the day, the month, the year, and so on. [REB-2015] [MOU-2016] [CHE-2016]. In [ZHA-2016], the consumption time series is predicted by a hybrid method with a regression followed by a learning method and the SVM algorithm. A disaggregation's of the global load curves are also performed to identify the electrical appliances [PAR-2011]. Other studies create simplify models and the building is modeled by a single equivalent electrical circuit ( $\mathrm{RC}, \mathrm{R} 2 \mathrm{C} 2, \mathrm{R} 3 \mathrm{C} 2, \mathrm{R} 6 \mathrm{C} 2)$ [JAZ-2002] [ARB-2015].

\section{METHODOLOGY}

\section{Energy problem Characterization}

We begin by a decomposition of the global energy need for a system without the heating, the hot water, the lighting, the ventilation and the specific electrical named EUSE :

$$
E t(i)=(\text { EHeating }+ \text { EHot_Water }+ \text { EUSE }+ \text { EVentilation }+ \text { ELighting }) t(i)
$$


The system can be connected at the decentralized production. With EenRe $t(i)$ the photovoltaïque solar pannel production and EenRT $t(i)$ the thermal solar pannel production, we have:

$E t(i)=($ EHeating + EHot_Water $+E U S E+$ EVentilation + ELighting $-E E N R E-E E N R T) t(i)$

A part of these energy can be controlled based on the different prediction. The system can have an electrical storage battery :

E $t(i)=(+/-$ EHeating $+/-$ EHot_Water $+/-$ EUSE+/-EVentilation +/- ELighting +/- EBattery-EENREEENRT $) t(i)$

(eq. 3)

\section{Transform Datas}

The data collected depends of the sensor selected to measure the consumption and the production which influence the data process. In the case of the identification of the electrical devices, a study per second is required for the electrical devices with short cycle times. In this paper, we apply a median filter of size 60 to remove high frequency at the global load curve level. In a case of no stable electrical network, it may ne possible to complete by a data process based on the voltage [HAR-1992].

\section{Events detection}

The power thresholds identify the short variations during a predetermined period. These techniques enable identification of the electrical events. It is between 40 Watts and 50 Watts 15 VAR for reactive power [HART-1992] to identify all electrical devices. This events identification can be done from a sliding average over a window of time to be defined according to the problem.

Events $t(i)=[$ Date $, \Delta P t(i), \Delta Q t(i)]$

(eq. 4)

\section{Statistics}

The statistics patterns are computed for the powers and the events. The mean, the minimum, the maximum, the standard deviation are computed each hour.

\section{Time characterization}

The different variables from the time variable can be extracted to have statistics per period with the month ,m ranging from 1 to 12, the days of the year from 1 to 365, the days of the week from 1 to 7 (Monday, Tuesday, Wednesday, Thursday, Friday, Saturday and Sunday), the hour h from 0 to 23 and the days corresponding to cantonal school holidays characterized by a Boolean variable 0 or 1 .

\section{Production planning integration}

the consumption prediction, In the tertiary and industrial sector, the arrival and departure times of staff and the days worked as input information help in the quality of the training set by creation of specific training set. The production planning enables to identify the people number adjusted with the production. For example, in the restaurant case, the restaurant may be open for the lunch and not for the dinner and open the weekday and not the week-end. These information's enable to create a specific training by period. In the residential case, a production planning associated with the heating and the hot water is added. pump is associated. This heat pump controls the heating and the hot water. A training model is created for days $\mathrm{k}$ from Monday to Friday (from 1 to 5) and another model for Saturday and Sunday (6 and 7).

\section{Meteo Data integration}

For the collection of meteorological data, we analyzed like World wea. ther online, Open Weather map, Meteoblue, Meteosuisse, Observations, Meteocentrale. The advantage of suppliers such as Meteoblue, Meteosuisse, Meteonews and Meteocentrale is the control of data by meteorologists which adjust them according to the changing meteorological context. The Meteosuisse is the solutions this project. the dataset contains historical real measures and forecast values of temperature and radiation. The forecasted weather values are a one day-ahead prediction in 2014. 


\section{Classification}

For the hot water and the heating, a supervised training is computed. If the energy is above a threshold, it is labeled ON, otherwise OFF. The same learning is done for the hot water per hour.

In the solar production and the others energy flux, the number of classes has not been predetermined and we are talking about unsupervised learning. These techniques determine the classes to characterize the electrical consumption and the solar panel production. In this paper, the EM (expectationmaximization) algorithm is proposed to decompose the time series [VIC-2015] and create the classes represented by a Gaussian.

\section{Train model}

Our objective is to provide the prediction in the next hour to manage the microgrid:

$$
\begin{aligned}
& \text { E } t(i+1)=(+/- \text { EHeating }+/- \text { EHot_Water }+/- \text { EUSE }+/- \text { EVentilation }+/- \text { ELighting }+/ \text { EBattery }- \\
& \text { EENRE-100 EENRT ) } t(i+1)
\end{aligned}
$$

The solutions are provided by the software KNIME with an industrial deployment. A connection between the database and the software is created and the data are proceeding each hour. The techniques predictions described in Introduction are tested with the linear method (ARIMA model and linear regression) and the no linear method (MLP, PNN, SVM, Random Forest and the GBT, Boosted Tree gradient).

We must ensure an equitable distribution among the different classes, especially that which requires a particular interest, such as the peak power consumption or production. A step called "boostsrap" can be necessary if classes are underrepresented. This step is particularly adapted to our problem of predictions of sudden variations of the consumption or the production and that algorithms like the AdaBoost or the Gradient Boosting Tree. Finally, different training model are represented by the planning production described in 3.2.3.

\section{Evaluation}

In our binary problem, the evaluation is realized by the Receiver Operating Characteristic (ROC) curve. It is a measure of the performance of a binary classifier. In no binary problem, the evaluation is realized by statistics computed. the coefficient of determination $\left(\mathrm{R}^{2}\right)$, the Mean Square Error (MSE), the RMSE (root mean square deviation), the Mean Absolute Error (MAE), the mean squared difference (MSD) [BER-2010].

\section{Optimization}

\section{Optimized the weather data predicted}

Meteorological data are essential in particular for the solar and the heating predictions. The prediction values provided by MeteoSwiss included extreme luminosity and temperature errors corresponding to local phenomena. An error is defined between the real and the predicted data for the temperature predictions and an error for the luminosity. A training model of the error associated with the time identification described in 3.2.2 is characterized by a non-parametric method.

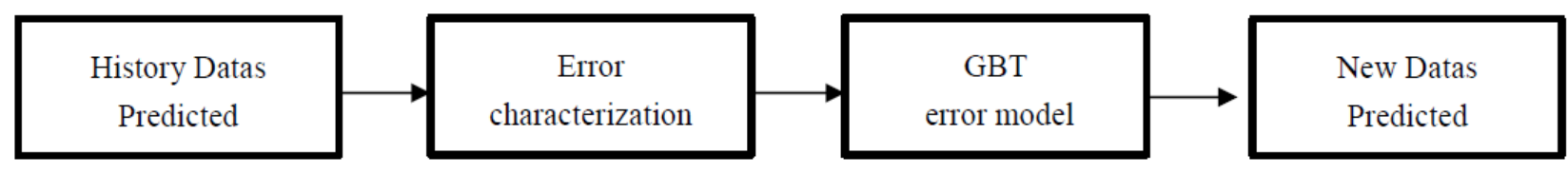

Figure 1: Method to optimize the weather data predicted

\section{Variables number impact}

The aim is to define a minimal inputs variable without decrease the prediction scored. Different algorithms are tested and evaluated in 2.4. At the output of the different implementations of the decision trees, the statistics provide the construction of the tree and enable a hierarchy in the input variables. The sum of statistics is computed for the construction of the three first levels. By iteration 
the number of the input variables is reduced based on their importance in the construction of trees. At each iteration, the scored of the prediction or the classification is computed describe in 3.4.

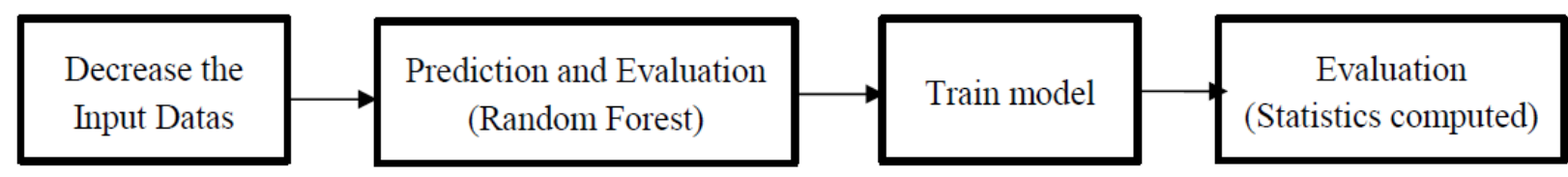

Figure 2: Method to optimize the weather data predicted

\section{Frequency impact}

The difference the prediction results is computed between the data at the second aggregate per minute and one data per hour to predict the next hour.

\section{USE CASES}

Techno-Pôles microgid is the use case contextualized in the I-BAT Swiss Project and ENTROPY European project. The data are displayed in real-time through: http://www.technopole-vert.ch. An Advanced Metering Infrastructure (AMI) based on the Internet of Things (IoT) has been implemented. This deployment provides energy-related parameters such as the overall building load curves and a wireless network of IoT-based smart meters to measure and control appliances. The Techno-Pôle of Sierre, the sunniest city in Switzerland as a $203 \mathrm{kWp} \mathrm{PV}$ plant that represents $1200 \mathrm{~m} 2$ of the roof surface. A weather station has been recently installed in 2015 and will provide more accurate weather data for the microgrids energy management. The site gathers 500 people working for 50 companies including private service providers as well as research institutes like HES-SO which carried out the microgrid project. The microgrid can also operate as an energy storage management demonstrator as batteries of $25 \mathrm{kWh}$ with a remote control of charge/discharge have been installed.

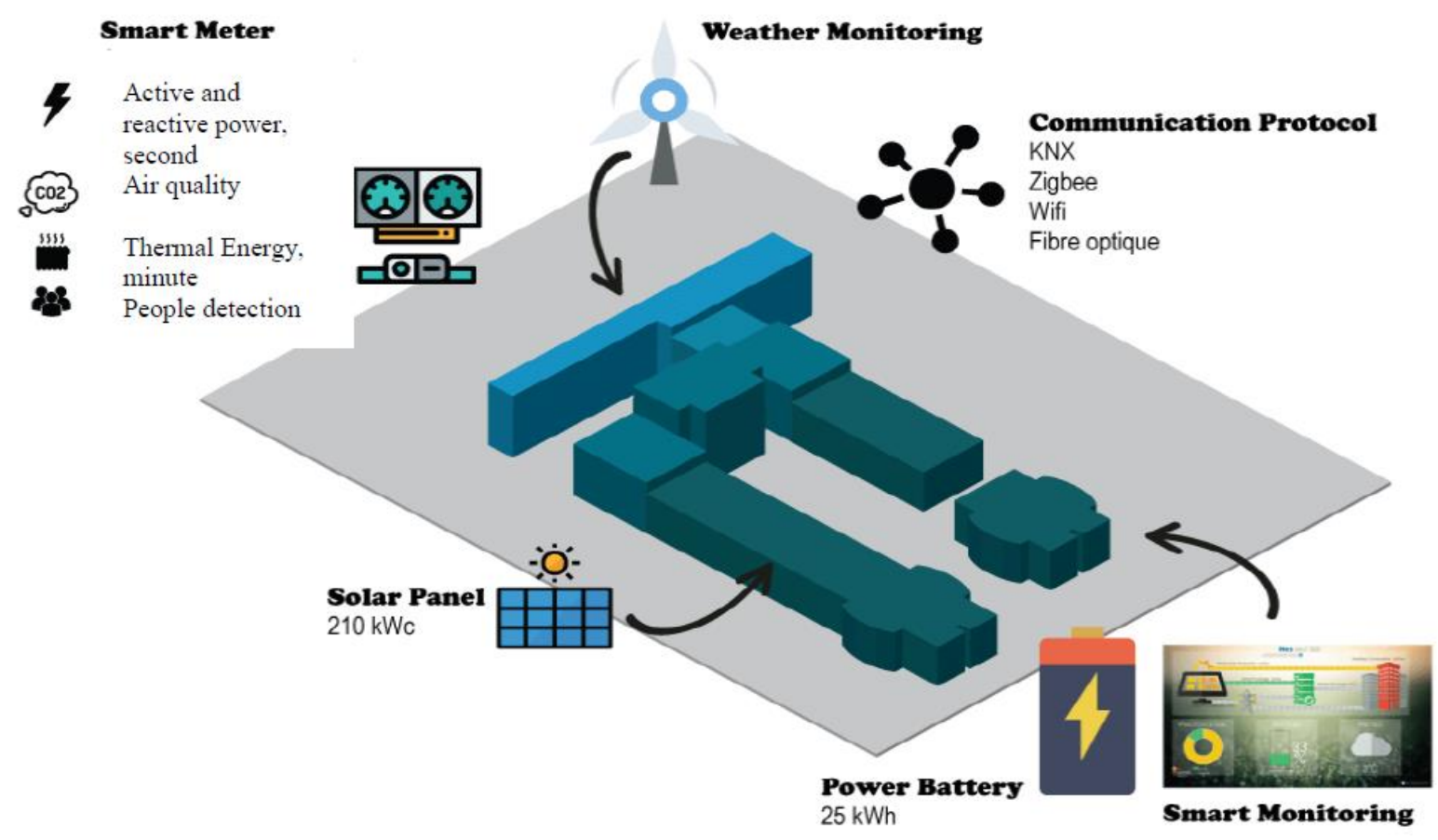

Figure 3: Statistical results at the output of the different algorithms for the prediction of overall electricity

The metering infrastructures provide at the one hertz frequency parameters (load curves from the photovoltaic plant provided by ELKO, and the grid consumption provided by Sierre-energy) and high frequency parameters (devices measures from the Ecowizz Zigbee smart meters). The information system contains the elements necessary for the storage of data via NO-SQL as the data is formatted in JSON which connected with Knime software for the prediction.

In this paper, we present our result through the restaurant of this technopole. In the tertiary sector, days and hours outside the production schedule are removed from our training and test data set. For the restaurant, the weekend is removed and only the hours between $6 \mathrm{am}$ and $5 \mathrm{pm}$ are represented in our 
training dataset corresponding to the production planning. For the restaurant, the electrical consumption includes the ventilation, the lighting, and the specific electrical devices like the oven (eq. 6 ). The heating and the hot water are provided by the radial panel.

$$
E t(i+1)=((+/-E U S E+/- \text { EVentilation }+/- \text { ELighting })-\text { EENRE }) t(i+1)+/- \text { EBattery }
$$

The dataset contains the data from September 1st 2013 to October 31st 2014 and the October 2014 is the test set. In output of the classification, each hour is labeled by a class c. A historic of each input variables for the last two days is created to identify one hour.

E t $(\mathrm{i})=[$ Date ; Statistics PowerPhase $1 \mathrm{t}(\mathrm{i}), \ldots$, Statistics PowerPhase $1 \mathrm{t}(\mathrm{i}-48)$,

Statistics Events $\mathrm{t}(\mathrm{i}), \ldots$, Statistiques Events $\mathrm{t}(\mathrm{i}-48)$

WeatherReal_Datas_CitySierre t(i),...., WeatherReal_Datas_CitySierre t(i) $\mathrm{t}(\mathrm{i}-48)$,

WeatherNew_Predicted_Datas_CitySierre t(i+1),..., WeatherNew_Predicted_Datas_CitySierre t(i-48)

Yearj ,Monthm ,Dayj,WeekDayjs ,Hourh,Jour fériés0.1,Vacances scolaires0.1, Classesc ]

(eq. 7)

\section{RESULTS}

\section{Evaluation of the classification}

At the end of unsupervised learning, the electricity consumption for the restaurant is represented by six classes (Table 38). The Class 6 represents the peak power of the restaurant during the considered period. The Gradient Boosted Tree best classifies the six classes representing power consumption (Table 1) with $98 \%+/-0.6$ accuracy.

Table 1 : Electricity consumption described by gaussian

\begin{tabular}{|c|c|c|c|c|}
\hline Energy & Clusters & Mean $(\mathrm{kWh})$ & Standard deviation & Repartition \% \\
\hline \multirow{2}{*}{$\begin{array}{c}\text { Electrical Energy } \\
\text { consumption, } \\
\text { tertiary Sector }\end{array}$} & 0 & 2.304 & 0.535 & 10 \\
\cline { 2 - 5 } & 1 & 4.448 & 1.29 & 24 \\
\cline { 2 - 5 } & 2 & 8.961 & 2.392 & 18 \\
\cline { 2 - 5 } & 3 & 16.622 & 2.539 & 18 \\
\cline { 2 - 5 } & 4 & 22.982 & 2.449 & 35 \\
\cline { 2 - 5 } & 5 & 28.246 & 1.927 & 2 \\
\hline
\end{tabular}

Table 2 : Classification results for the electricity load curve

\begin{tabular}{|c|c|c|}
\hline Models & Classification results & Standard deviation \\
\hline PNN & $84.6+/-1.2$ & 1.6 \\
\hline MLP & $88.8+/-1.3$ & 1.5 \\
\hline SVMI & $91.8+/-1.1$ & 1.6 \\
\hline Random Forest & $96.1+/-0.7$ & 1.5 \\
\hline Gradient Boosted Tree & $98.0+/-0.6$ & 0.8 \\
\hline
\end{tabular}

\section{Evaluation of the prediction}

For the electricity consumption, the decision tree and the Gradient Boosted Tree provides the best results. The average difference in absolute value is $1,162 \mathrm{kWh}$ for the GBT (Table 1). The precision of the models is explained by the repetition at specific times of stages to prepare the meals. A priori data collected by the restaurant as the number of customers planned or special orders (eg company parties) can improve the accuracy of our mathematical models. 
Table 3 : Comparison of the Statistical results per algorithms for the Electrical consumption prediction for the restaurant

\begin{tabular}{|c|c|c|c|c|c|}
\hline Models & MAE & MSE & RMSE & MSD & $\mathbf{R}^{2}$ \\
\hline MLP & 1.9 & 5.4 & 2.1 & 0.2 & 0.64 \\
\hline PNN & 1.9 & 5.4 & 2.1 & 0.2 & 0.64 \\
\hline Linear Regression & 2.3 & 5.4 & 2.1 & 0.2 & 0.64 \\
\hline ARIMA & 2.2 & 5.4 & 2.2 & 0.2 & 0.64 \\
\hline RF & 1.5 & 4.5 & 2.1 & 0.1 & 0.95 \\
\hline GBT & $\mathbf{1 . 2}$ & $\mathbf{4 . 3}$ & $\mathbf{1 . 9}$ & $\mathbf{0 . 1}$ & $\mathbf{0 . 9 8}$ \\
\hline
\end{tabular}

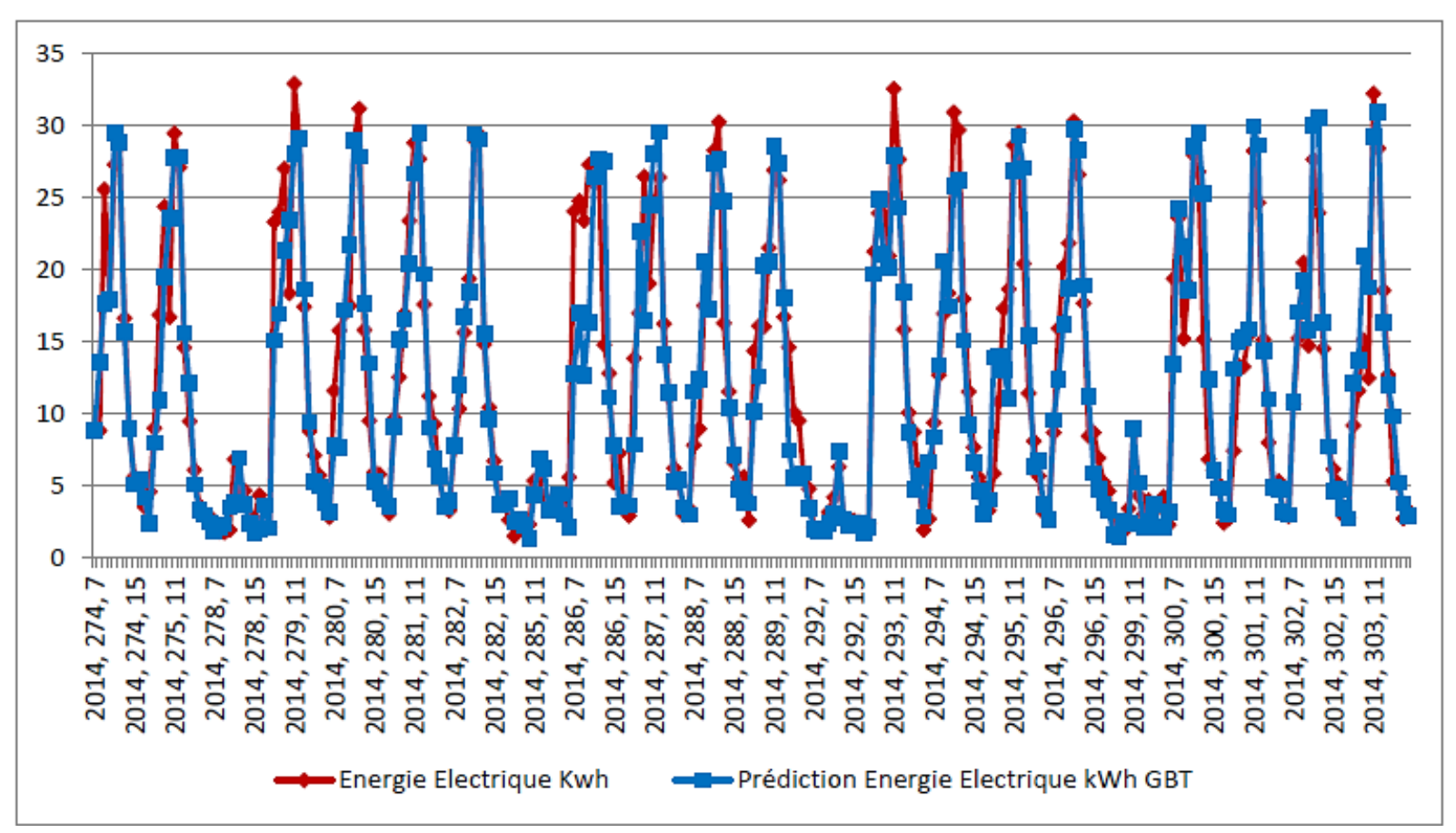

Figure 4: Electrical consumption prediction for the restaurant with the Gradient Boosting Tree (Year, Day, hour)

\section{Optimization results}

\section{Optimization meteo data}

To characterize the error, a history based on the actual and predicted data is created. This history represents the input data of our mathematical model. The training data correspond to measurements from January 1, 2010 to August 31, 2012. Test data are from September 1, 2012 to March 31, 2014.

Table 5 : Characterization of the errors for the luminosity before and after our method

\begin{tabular}{|c|c|c|c|c|c|}
\hline Variables number & Min & Mean & Median & Max & Std. Dev \\
\hline History error & -910 & 5.5 & 0.1 & 1116 & 304 \\
\hline New error & -550 & 7.1 & 0 & 510. & 203 \\
\hline
\end{tabular}

\subsubsection{Variables number optimization}

In this test, our objective is to determine the impact of the quantity of our input data in the variation of the result. The 15 best variables to predict the electrical consumption prediction for the restaurant is the standard deviation $\mathrm{t}(-1)$, the standard deviation $\mathrm{t}(-2)$, the minimum power $\mathrm{t}(-1)$, the median power $\mathrm{t}$ $(-1)$, the standard deviation Median Active power $\mathrm{t}(-1)$, the maximum events $\mathrm{t}(-1)$, the maximum 
Variations active power $\mathrm{t}(-2)$, the standard deviation events $\mathrm{t}(-1)$, the Minimum events $\mathrm{t}(-1)$, the Standard deviation Median events t (-1).

Table 6 : Variables number impact for the Electrical consumption prediction for the restaurant

\begin{tabular}{|c|c|c|c|c|c|}
\hline Variables number & MAE & MSE & RMSE & MSD & $\mathbf{R}^{\mathbf{2}}$ \\
\hline $\mathbf{4 1 6 0}$ & 1.4 & 4.4 & 2.1 & 0.1 & 0.94 \\
\hline $\mathbf{3 0 0}$ & 2.0 & 8.6 & 2.9 & 0.1 & 0.89 \\
\hline $\mathbf{1 5 0}$ & 1.9 & 7.5 & 2.7 & 0.1 & 0.90 \\
\hline $\mathbf{5 0}$ & 2.0 & 8.2 & 2.8 & 0. & 0.89 \\
\hline $\mathbf{1 5}$ & $\mathbf{2 . 2 2}$ & $\mathbf{1 0 .}$ & $\mathbf{3 . 2}$ & $\mathbf{0 . 1}$ & $\mathbf{0 . 8 6}$ \\
\hline
\end{tabular}

\section{Frequency optimisation}

We also test the difference in our results between the data at the second aggregate per minute and one data per hour to predict the next hour. The average error of the deviations increases by $0.5 \mathrm{kWh}$ (Table 7). The daily average electricity consumption curve follows the same pattern. In the tertiary sector, the companies follow a precise production schedule during the day, contrary in the residential sector [LUC-2017].

Table 7: Electrical consumption prediction results with different period for the restaurant

\begin{tabular}{|c|c|c|c|c|c|c|}
\hline Models & Period & MAE & MSE & RMSE & MSD & R $^{\mathbf{2}}$ \\
\hline Random Forest & $\mathbf{1}$ min & $\mathbf{1 . 4 8 8}$ & 4.494 & 2.12 & 0.124 & 0.946 \\
\hline Random Forest & $\mathbf{1}$ hour & $\mathbf{2 . 1 3 3}$ & 11.36 & 3.371 & -0.273 & 0.863 \\
\hline
\end{tabular}

\section{CONCLUSION}

Several methods and algorithms have been tested and we can conclude that the Combinatorial Random Forest and Gradient Boosting Tree is particularly adapted to our problem with predictions around 98\% $+/-0.6$. These techniques with the boosting are very useful in the context of the energy prediction for the consumption and the production peaks which represent only a few $\%$ in the training set.

From an information system point of view, the Combinatorial Random Forest and Gradient Boosting Tree analysis allows us to limit the number of data, create a simple model and guarantee the same level of precision, It is possible to interpret the prediction by analysis of the statistics in output of the random Forest algorithm.

The identification of the human presence is an essential parameter both for the piloting and the prediction of energy consumption. To create a non-intrusive system, it is easier to understand this problem in the residential and tertiary sector by integration of a production planning. This allows us to target model training on what interests us here, predict overall power consumption during business hours. We think that we should first focus on the microgrids in the tertiary and industrial sector, where the human variable is more predictable by the definition of business occupation schedules.

\section{REFERENCES}

[ARB-2015] Thomas Berthou, Développement de modèles de bâtiment pour la prévision de charge de climatisation et l'élaboration de stratégies d'optimisation énergétique et d'effacement, Ecole Nationale Supérieure des Mines de Paris, 2013

[BER-2010] Guide to Intelligent Data Analysis,How to Intelligently Make Sense of Real Data, Berthold, M.R., Borgelt, C., Höppner, F., Klawonn, F, 2010

[CHE-2016] Extended modeling procedure based on the projected sample for forecasting short-term electricity consumption, Che-Jung Chang, Jan-Yan Lin, Meng-Jen Chang, 2016 
[COC-2012] M. Cococcioni, E. DAndrea, B. Lazzerini, One day ahead forecastingof energy production in solar photovoltaic installations: An empiricalstudy, Intelligent Decision Technologies 6 , 2012

[JAZ-2002] S. JAZOULI et S. BARROIS : Calage d'un modèle thermique de bâtiment "r3c2" à partir de la courbe de charge et de la météo. Rapport technique, EDF, 2002

[HART-1992] G.W. Hart. Nonintrusive appliance load monitoring. Proceedings of the IEEE, vol. 80,no. 12, pages $1870-1891,1992$

[LUC-2017] Luc Dufour. Contribution à la mise au point d'un pilotage énergétique décentralisé par prédiction.

Génie civil. Ecole des Mines d'Albi-Carmaux, 2017.

[MEL-2006] A. Melli, M. Benghanem, S. A. Kalogirou. An adaptive wavelet-network model for forecasting daily total solar-radiation. Applied Energy, vol. 83, no. 7, p. 705-722, 2006.

[MOU-2016] Energy planning and forecasting approaches for supporting physical improvement strategies in the building sector: A review. Moulay Larbi Chalal , Medjdoub Benachir,Michael White,Raid Shrahily, 2016

[NOM-2011] F. Nomiyama, J. Asai, J Murata, A study on Global Solar Radiation Forecasting Using Weather Forecast Data, IEEE, 2011

[PAR-2011] Le perceptron multicouche et son algorithme de rétropropagation des erreurs Marc Parizeau Département de génie électrique et de génie informatique Université Laval, 10 septembre 2004

[REB-2015] Modeling and forecasting energy consumption for residential buildings in Algeria using bottom-up approach." Rébha Ghedamsi, Noureddine Settou, Abderrahmane Gouareh, Adem Khamouli, Nadia Saifi, Bakhta Recioui, Boubekker Dokkar, 2015

[VIC-2015] Victoria Moreno, Luc Dufour, Antonio F Gómez Skarmeta, Antonio J Jara, Dominique Genoud, Bruno Ladevie and Jean-Jacques Bezian, Big data: the key to energy efficiency in smart buildings, Soft computing manuscript, 2015

[ZAM-2013] "A benchmark of statistical regression methods for short-term forecasting of photovoltaic electricity production, part I: Deterministic forecast of hourly production." M. Zamo, O. Mestre, P. Arbogast, O. Pannekoucke, 2013

[ZHA-2016] Time series forecasting for building energy consumption usingweighted Support Vector Regression with differential evolution optimization technique." Fan Zhanga, Chirag Debb, Siew Eang Leeb, Junjing Yangb, Kwok Wei Shah, 2016 


\title{
MARKET OPTIONS FOR THE INTEGRATION OF HEAT PUMPS IN RURAL DISTRICT HEATING GRIDS IN AUSTRIA
}

\author{
Johanna Spreitzhofer1, Olatz Terreros1, Daniele Basciotti1, Tara Esterl1, Ralf-Roman Schmidt1, Manuel Ziegler2, \\ Marcus Pober, Max Kerschbaumer3, Ralph Huber3 \\ 1 AIT Austrian Institute of Technology, Energy Department, Giefinggasse 4, 1210 Vienna, Austria \\ 2 ENGIE Gebäudetechnik GmbH, Leberstraße 120, 1110 Vienna, Austria \\ 3 ENGIE Energie GmbH, Dresdner Straße 43, 1200 Vienna, Austria \\ Phone: +43664 / 88256109 \\ E-Mail: johanna.spreitzhofer@ait.ac.at
}

\begin{abstract}
SUMMARY
Heat pumps provide an ideal coupling point between district heating and electricity grids. They can help to increase the profitability of the heating grid, i.e. by using waste heat as a source for efficient heat production. Furthermore, the short-term electricity markets provide the possibility to profit from cheap or even negative energy prices. When thermal storages are available, they can offer their flexibility to the electricity grid to provide balancing energy and thus make additional revenues. The aim of the project fit4power2heat is to analyse those synergies between the heating and electricity domain, in order to create interesting business cases for heat pump integration in rural district heating grids.
\end{abstract}

Key words: district heating, flexibility, sector coupling, power-to-heat, heat pump pooling

\section{INTRODUCTION}

In Austria, many rural district heating grids are based on biomass plants, which are near the end of their technical lifetime and which often operate with low profitability. The idea of the project fit4power2heat is to equip those small heating grids with additional heat pumps, to make the heat production more economical by creating a link to the electricity domain. The schematics of this idea are shown in Fig. 1: The heat pump behaviour can be optimized to use cheap electricity prices on the day ahead spot market for its heat production. Additionally, it can participate in a pool to offer flexibility for the balancing market and get further revenues. A thermal storage and a biomass boiler as a backup heat source enable system flexibility.

This contribution gives an overview of the different market options for heat pumps in Austria. It analyses the advantages and drawbacks of different market segments. Potential use cases for heat pump integration are evaluated in a techno-economic analysis in order to create future business models.

\section{MARKET OPTIONS FOR HEAT PUMPS IN AUSTRIA}

There are several different options for heat pumps to be an active part of the electricity system in Austria, which differ both in potential revenues and in technical requirements:

First, they can adjust their consumption towards short-term prices on the day ahead and intraday spot market. By doing so, they cannot only avoid high peak prices, but also profit from negative prices on the spot market during some times of the year. In order to adjust its production flexibly, the heat pump either needs access to a thermal storage and/or alternative heating source like a biomass or gas boiler. Secondly, power-to-heat plants in Austria can also participate in the balancing markets, meaning they provide flexibility in case of an imbalance between production and demand in the electricity system. The currently most promising options for power-to-heat are the negative automatic and manual frequency restoration reserve. Here, the heat pump offers to switch on during times of excess electricity and therefore can get the electricity for free or even be paid for its consumption. Additionally, the availability itself is refunded as well and the grid costs are cheaper for balancing energy providers. The downside here are the strict technical preconditions, which require

fast reaction times and guaranteed availability. Additionally, there is a minimum size of 1-5 MW (electrical), which means, most heat pumps could only participate in the market by participating in a larger pool of several units. Furthermore, the frequent switching due to market calls might have negative effects on the efficiency of the heat pump.

Thirdly, heat pumps could also offer their flexibility to the distribution grid operator for local grid support or to their balancing group to reduce imbalance settlement costs. However, since the legal and 
regulatory framework for this option is not entirely clear in Austria, they are not considered further in this work.

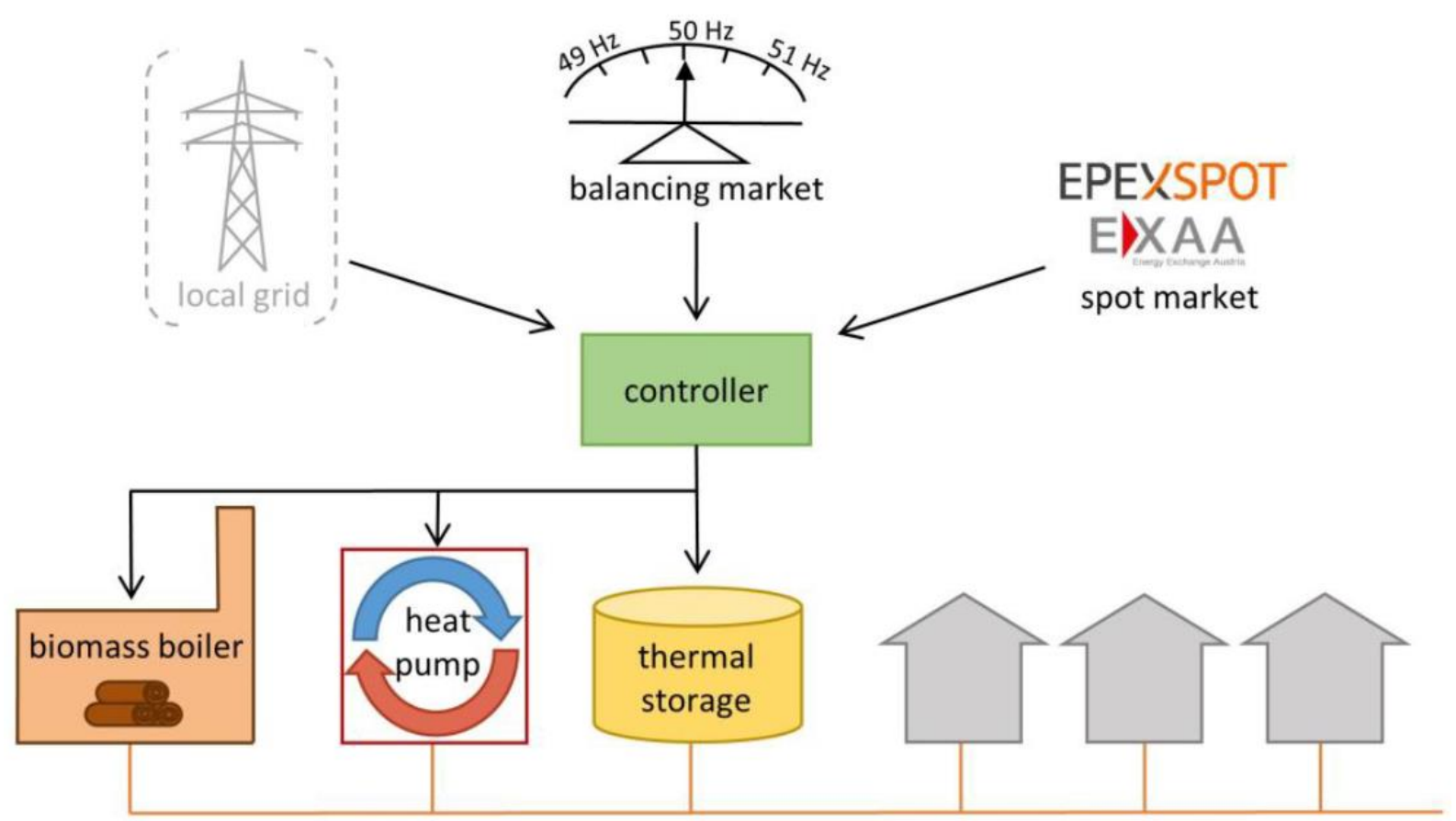

Fig. 1: Overview of the fit4power2heat idea

\section{TECHNO-ECONOMIC ANALYSIS}

In order to create possible business models, a detailed techno-economic analysis is applied. The basis for this are the configuration and measurement data from three typical rural heating grids in Austria. Different variations are considered, concerning the size and type of the heating demand (residential and non-residential), the temperature levels and size and integration concept of the heat pump. Investment and operating costs are compared to possible savings and revenues from the short-term market options. In a first pre-feasibility study, the most promising use cases are determined. Therefore, different market options as well as system configurations are taken into account. The most interesting cases are then analysed in more detail, using an operational optimization model. The resulting reduction of operational costs and achievable revenues act as inputs to develop future business models. Finally, the transferability of the solutions to other heating grids is evaluated.

\section{REFERENCES}

Landwirtschaftskammer Niederösterreich, (2016), Biomasse-Heizungserhebung 2015. Report, St. Pölten, Austria.

Esterl, T. et al., (2016), iWPP-Flex Intelligentes Wärmepumpen-Pooling als Virtueller Baustein in Smart Grids zur Flexibilisierung des Energieeinsatzes. Report, Vienna, Austria.

\section{CONFERENCE TOPIC}

Hybridization of energy sectors

Future role of buildings and industry for the flexibility and stability of thermal and electric grids 


\title{
PERFORMANCE OF SOLAR THERMAL - PV HYBRID SYSTEM
}

\author{
Mr Anadola Tsiu*, Dr N.B. Maliehe, Dr Mopeli Fabiane \\ National University of Lesotho \\ Physics \& Electronics Department, Roma, Lesotho \\ Phone: +266 62851721/22213905 \\ E-Mail: ajtsiu@gmail.com
}

\section{SUMMERY}

The project mainly focuses on designing and manufacturing of affordable solar thermal energy storage systems hybridized with solar PV. This combination presents modern and future energy technology systems for industry, rural and urbanized domestic energy demands. The work of this project aims to support Lesotho's National Strategy of Access to Clean Energy in Rural Areas. The energy storage capability of this technology makes it ideal as a reliable intermittent Renewable Electricity for industries, which they need during limited power transmission capability or during insufficient electrical loads. The idea is to substitute the use of imported battery energy storage devices with locally manufactured solar thermal energy storage systems. These improve sustainability and affordability. Lesotho does not optimally utilize its abundant solar energy resource, this being due to number of challenges. For example, limited life span of the currently available storage technologies. This project seeks to solve these challenges by exploring aternative green technologies which employ Organic Rankine Cycle generators or thermo electric generators. The approach eliminates dependency on batteries for storage at night and during wheather related minimum solar radiation periods.

\section{INTRODUCTION}

Lesotho Population is approximately 2,003 5469 and about $20 \%$ of these population has access to piped water [1]. The national overall energy dependency is on biomass (64\%), certain forms of imported oils, LPG gases and a small contribution from electricity (7\%) [1]. The use of solar energy technologies in Lesotho is very limited. Due to this insignificant contribution, it is not even shown in the comparative energy charts of the country. The collective documentation on existing solar installations in the country is also lacking. In an attempt to improve the situation, the National Energy Policy statement number four [2] stipulates that all new public buildings are to be equipped with solar geysers. The National target is to have installed solar collector area between $0.3 \mathrm{~m}_{2}$ to $0.5 \mathrm{~m}_{2}$ per capita by 2030 . This translates to $180,000 \times 2.5 \mathrm{~m}_{2}$ solar geysers target by 2030 [3]. The current project and its related studies are therefore much needed and critical.

\section{DELIVERABLE OUTPUTS}

Our prototype system provides traditional water heating used for general purposes, space heating with under floor heating, wall radiation. It also generates electricity using smart little known Organic Rankine Cycle (ORC) generator technology or using thermo-electric generators. This hybrid Thermal-PV system offers thermal energy Storage which is released during PV electricity supply limitations. It can also be deployed to serve as backup on Grid tied systems. Thus it eliminates need for unsustainable expensive storage batteries.

\section{Keywords : Solar Thermal ; Storage ; Sustainability}




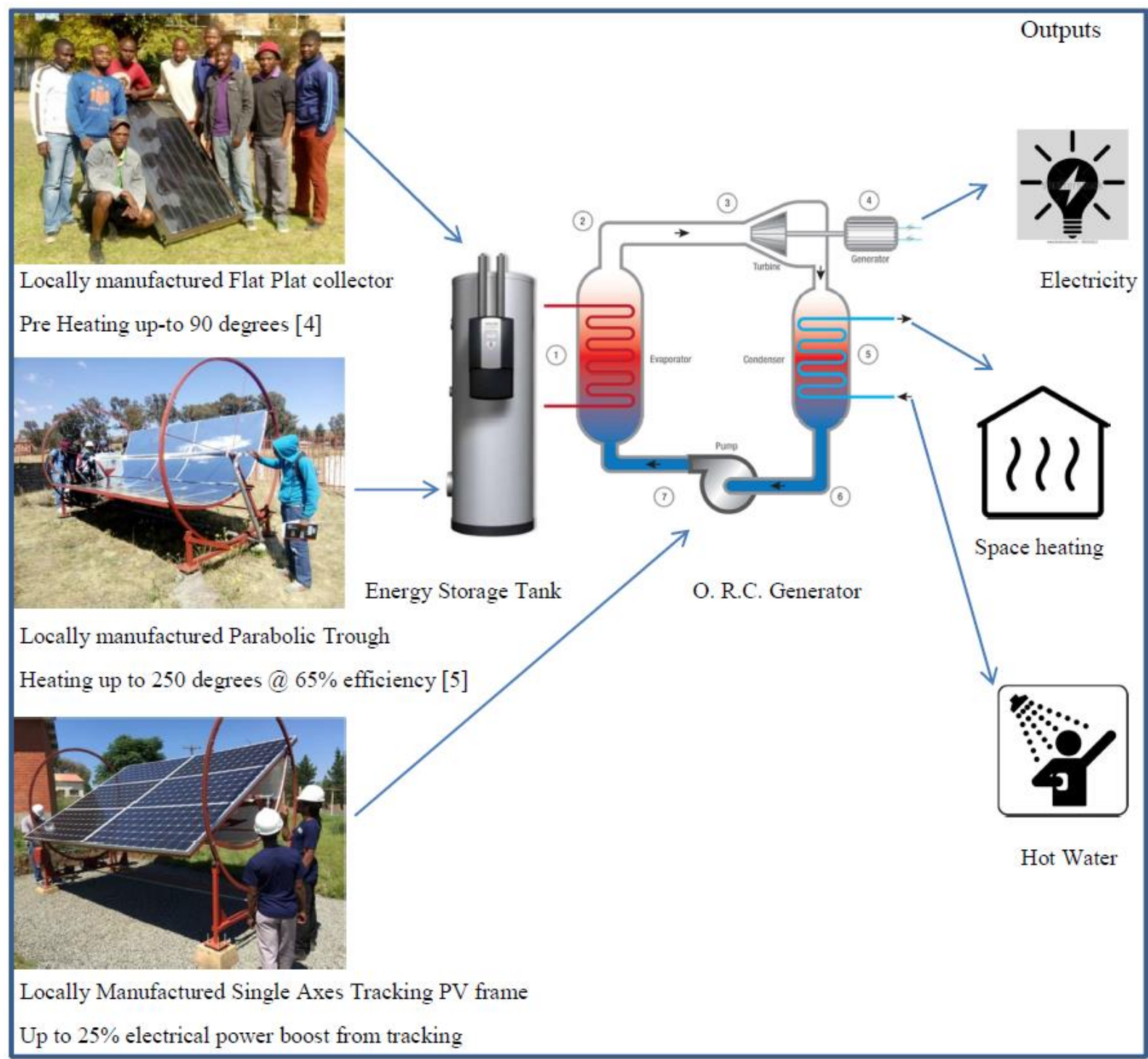

Figure 1 - Solar Thermal Energy Hybrid System

\section{REFERENCES}

[1] Bureau of Statistics Government of Lesotho

[2] Lesotho National Energy policy

[3] Lesotho Solar Thermal Roadmap

[4] Construction and performance Evaluation of a low-Cost Flat-Plate Solar Energy Collector) by A. Tsiu, Leboli Z. Thamae, I. Hapazari, and B. M. Taele - American Journal www.academicstar.us (Modern Environmental Science and Engineering (ISSN:2333-2581) January 2017, Volume 3, No1, Page 61-66

[5] Low-Cost Small Scale Parabolic Trough Collector Design for Manufacturing and Deployment in Africa by

Matthew Orosz, Paul Mathaha, Anadola Tsiu, et.al.

\section{CONFERENCE TOPIC}

Renewable Heating and Cooling in a future energy system 


\title{
DESIGN OF A HYBRID VAPOUR ABSORPTION MILK CHILLER (SOLAR AND BIOGAS) FOR SMALL SCALE DAIRY FARMS IN ZIMBABWE
}

\author{
1Gamuchirai Mutubuki, 2 Blessed Sarema, 3Samson Mhlanga \\ 123 National University of Science and Technology \\ Cnr Gwanda Road and Cecil Avenue P O Box AC 939, Ascot, Bulawayo, Zimbabwe \\ +263772679 234, +263773491518, +263772 250918 \\ E-Mail: gnmtubuki@gmail.com,blesarema@gmail.com,smhlanga126@gmail.com
}

\section{SUMMARY OF THE PAPER}

The Dairy sector today faces challenges and opportunities fuelled by rapidly rising energy costs and concerns about environmental impacts. Energy is used from milking, cooling to storage. Zimbabwe's $70 \%$ population is engaged in milk production, fruits and vegetables. Due to the lack of proper storage and transit facilities, the agricultural produce in remote areas loses its value. Milk should be stored at lower temperature of $5^{\circ} \mathrm{C}$ to prevent bacteria reproduction. Determining the best energy efficiency and energy management opportunities for dairy farms will help reduce energy costs, enhance environmental quality and increase productivity and profitability Henceforth raised the need for the design of a hybrid vapour absorption system which uses solar and biogas energy was designed in order to reduce energy costs. This paper presents the design of a milk chiller for not only rural Dairy farms but for the Dairy sector as a unit. Biogas was bottled, assembling the solar thermal system, experiments done during the day and night, system optimisation and commercialisation.

\section{Key words Absorption refrigeration cycle, Biogas, solar thermal}

\section{INTRODUCTION}

Energy is the backbone of all activities [1]. Dairy farms today face challenges fuelled by rapidly rising energy costs and concerns about environmental impacts. In a dairy, energy is used mainly in the milking, processing, cooling and storage [2]. A hybrid of solar and biogas will alleviate energy issues, increase production, profits and protect the environment. The designed system harness solar energy during the day and biogas overnight and days where the sun will be limited.

\section{PURPOSE}

Milk is harvested from cows at around $35-40^{\circ} \mathrm{C}$ and has to be maintained around $4-5^{\circ} \mathrm{C}$ to have good quality and low bacteria count. Dairies face challenges fuelled by rapidly rising energy costs. Cooling is amongst the processes that consume most energy other than pasteurisation thus need for renewable energy.

\section{THEORETICAL FRAMEWORK}

A milk chiller makes use of the refrigeration system. In this project design, a vapour absorption system was used with a hybrid of solar thermal and biogas. In this design solar thermal energy will be used for cooling the milk during the day time when there is plenty sunlight. Biogas energy will be used at night or when there is no sunlight. This system will be working supporting each energy source and at the same time having a backup of battery system. The heated water will be used to run the chiller and also for cleaning all the Dairy equipment. A backup system of a battery can be used. Biogas can be used to generate electricity using a biogas generator and the generated electricity can be either used directly or stored in a batter.

\section{RESULTS AND CONCLUSIONS}

The solar system, from $9 \mathrm{am}-4 \mathrm{pm}, 66-85^{\circ} \mathrm{C}$ maintained $4-5^{\circ} \mathrm{C}$ inside the chiller. Biogas was used from 5 pm- 9 am maintained $4^{\circ} \mathrm{C}$. A cost of $52.7 \%$ of US $\$ 7500$ total cost for the solar system was established, of which $20 \%$ is for the 4001 chiller system. A $20 \square \square$ biogas system will cost $33.1 \%$ of the total cost including piping. A lifetime of 15 years with close maintenance and $80 \%$ annual usage of the installed system, profit is realized after 5 years. The future of renewable energy is a good proposition and no doubt will find its place in future industrial applications. 


\section{REFERENCES}

1. Muneer, T., Asif, M. and Kubie, J., 2003. Generation and transmission prospects for solar electricity: UK and global markets. Energy conversion and management, 44(1), pp.35-52.

2. Edwin, M. and Sekhar, S.J., 2015. Thermal performance of milk chilling units in remote villages working with the combination of biomass, biogas and solar energies. Energy, 91, pp.842-851.

3. Demirbas, M.F. and Balat, M., 2006. Recent advances on the production and utilization trends of bio-fuels: a global perspective. Energy Conversion and Management, 47(15-16), pp.23712381. 


\title{
INTEGRATED PVT SOLAR SYSTEM
}

\author{
Dr Ilija Nasov \\ Camel Solar doo \\ Skopje, Macedonia \\ Phone: +38970 205635 \\ E-Mail: ilija.nasov@hotmail.com
}

\section{SUMMARY}

The solar energy is usually used separately; as photovoltaic (PV) panels for production of electricity and solar thermal collectors for production of hot water. The conversion rate of solar radiation into electricity by photovoltaic depends on cell type and it is usually between $12 \%$ to $14 \%$ per PV system yearly, for poly crystalline or mono crystalline cells. The greater part of the absorbed solar radiation by photovoltaic is converted into heat (that is about $70-75 \%$ ), increasing cell temperature. This effect reduces their electrical efficiency mainly to silicon cells. Solar hybrid PVT liquid collectors are the most widely studied configuration thanks to a large potential for the applications in buildings., hotels,... PVT liquid collectors show good heat usability in the low temperature applications like primary circuits of heat pumps $\left(0\right.$ to $\left.10{ }^{\circ} \mathrm{C}\right)$, pool water heating $\left(25\right.$ to $\left.35^{\circ} \mathrm{C}\right)$, domestic hot water and space heating (up to $60{ }^{\circ} \mathrm{C}$ ). (1) In this context, combination of PV and solar thermal systems is an ideal setup of the two systems for better utilization of solar energy. In this work is given an approach of their combination in a form of PVT (photovoltaic-thermal) systems.

Keywords: PV, solar thermal, PVT, efficiency

\section{INTRODUCTION}

PV panels can be instaled in parallel rows on horizontal plane, on the roofs and on the facades of the buildings.

In case of PV modules that are installed in parallel rows on horizontal plane at ground or building roof, the exposure of both PV module surfaces to the ambient permits their natural cooling.

On façades or inclined roof installation on buildings, the thermal losses are reduced due to the thermal protection of PV rear surface and PV modules operate at higher temperatures.

This undesirable effect can be avoided by applying a suitable heat extraction with a fluid circulation, air or liquid (water), keeping the electrical efficiency at a satisfactory level.

It means that PVT device give double effect to the end-user: around $20 \%$ more electricity /year compare with standard PV panel and several times more thermal energy from hot water compare with electricity produced from PV.

There are generally two types of PVT hybrid collectors: (I) water or glycol cooled and (II) air-cooled. The water-cooled collectors that are in operation are than divided as: glazed and unglazed. In last several years many scientists and experts are working in additional application : PVT solar assested HP.

The simulation results (2) show that at a fixed compressor speed and refrigerant flow, and with a condenser water supply of $30^{\circ} \mathrm{C}$, the PVT evaporator arrays showed an overall efficiency level in the range of $0.64 \sim 0.87$, thermal efficiency of $0.53 \sim 0.64$, and a PV efficiency range of $0.124 \sim 0.135$. With the cooling effect of refrigerant on PVT devices, (3) the PV efficiency can be improved. In addition, low-temperature refrigerant can directly extract ambient heat through the PVT array. This obviously enhances the thermal efficiency of PVT devices. The total PVT efficiency and the COP of the PVTA-HPWH system reach up to $86 \%$ and 7.09, respectively. Camel Solar started with production of glazed and unglazed PVT hybrid collectors. The power output from unglazed PVT collectors is higher than glazed but thermal output from glazed is higher than unglazed. Analyzing efficiency curve of the PVT unglazed collector, we realised that adequate temperatures of circulation fluid aimed to receive high power and thermal efficiency is max. $40^{\circ} \mathrm{C}$. Above this temperature, the efficiency is decreasing around $0,5 \% /{ }^{0} \mathrm{C}$. The additional benefit of cooled PV panel is longer tool life as a result of lower working temperature which means less diffusion process of defects in PV cells. 
Regarding the application requirements (more electricity or more thermal power), the end user could decide what type of PVT collector will be installed.

Camel Solar started with R\&D activities in PVT production process aimed for better efficiency: better heat transfer from PV EVA encapsulating to $\mathrm{Al}$ thermal absorber, better isolation material which will allow thinner isolation thickness. The self-cleaning and super hydro filling / hydro fobbing coating, which was result of a long term research, will be used for applying to glass of PV panel with aim to reduce the soiling of dust.

Almost every end user needs electrical and thermal energy.

The end users need several levels of heat temperatures: low, middle and some times high temperatures. Because of that, we created integrated solar system composed from PV, PVT, and flat plate collector in combination with concentrated collector. This system is producing more electricity than standard PV system, more than three times low temperature hot water from PVT panels compared with electricity and additional heat with higher temperature water from combined device (high efficient solar thermal collector plus new developed concentrating collector).

Our aim is to produce electricity and hot water with minimum $\mathrm{CO} 2$ emission and pollution, and minimum requirement of electricity from public network.

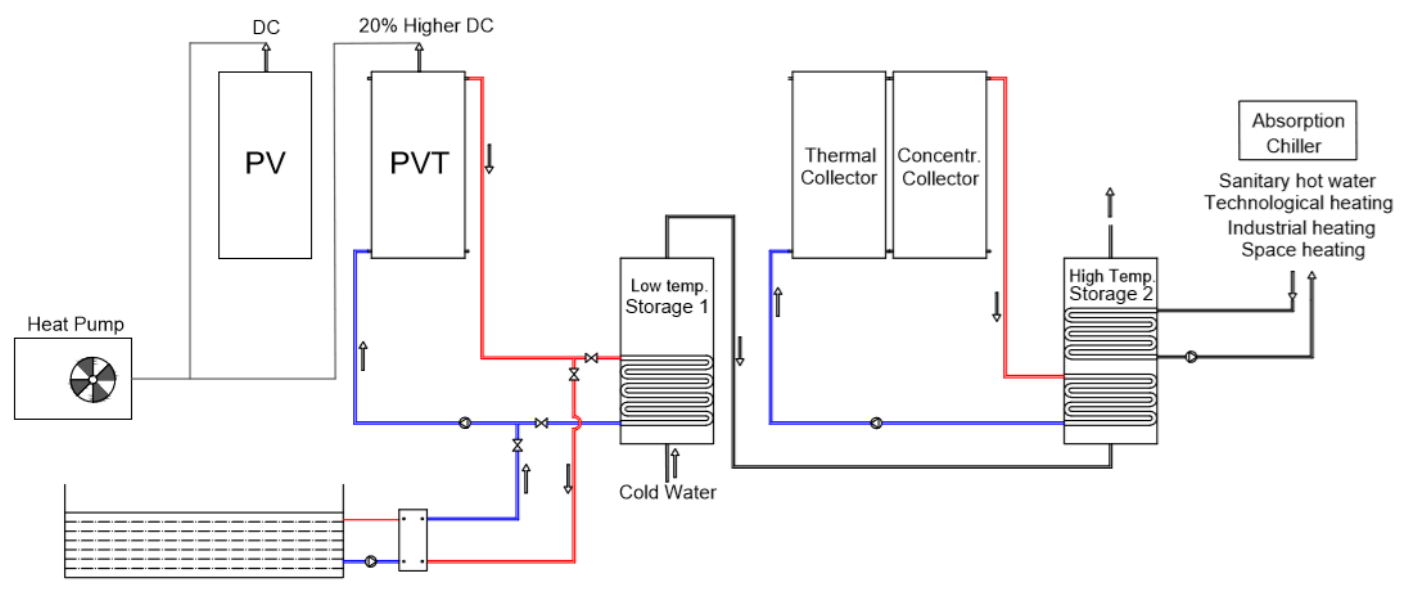

Fig. 1 Scheme of integrated PVT solar system .

From PVT device the hot water is going to a swimming pool ( this is one the best options for max.utilization of solar energy because the temperature of fluid in the PVT will be less than $30{ }^{\circ} \mathrm{C}$ which allows the highest efficiency of PV cells and in the same time the highest efficiency of PVT heat production. In a case where the end user doesn't have a swimming pool, than the hot water from PVT is going to first low temperature storage where the temperature is keeped to maximum $40^{\circ} \mathrm{C}$. The pre heated water from the first storage is going to the second storage as an input water and it is heated from the second combined device ( flat plate collector plus concentrating collector). The flat plate collector with very high efficiency is heating the water to around $70^{\circ} \mathrm{C}$ or more. That temperature is enough for nearly all end users, but if some end user needs even higher temperature, than additional heating is coming from the "new developed concentrating collector" where efficiency on higher temperatures is much higher, so it is possible to receive more than $100^{\circ} \mathrm{C}$ with satisfactory high efficiency.

The price of thermal energy depends of temperature. The lower temperature is cheaper than the higher temperature, so our concept include three types of solar devices: PVT ( low temperature water) flat plate (middle) and concentrating collector (higher temperature water) .

All above demands can be realazed from explained integrated system combined with air or solar assisted heat pump where COP will be increased as result of PVT pre heated water.

The system will be smart, monitoried and menaged by web based software .

Thanks to maximum efficiency for all steps, started from PVT, flat plate and concentrated collector ESCO model will be sustainable because integrated solar kombi system will produce very 
competitive both electical and thermal energy, long term efficiency, the most ecological with low $\mathrm{CO} 2$ emission/Kwh, and other pollutant as PM particles , SO2, CO....

\section{REFERENCES}

1. Thomas Matuska, 2013,Performance and economic analysis of hybrid PVT collectors in solar DHW system Energy Procedia 48,(2014)

2. Jin-Hee Kim Experimental performance of heating system with building- integrated PVT (BIPVT) collector Energy Procedia 48 (2014)

3. Huan-Liang Tsai , 2014,Design and Evaluation of a Photovoltaic/Thermal-Assisted Heat Pump Water Heating System Energies 2014 


\section{ENERGY RECIPES FOR REDUCED HOUSEHOLD ENERGY CONSUMPTION AND PEAK SHAVING}

Dr Francesco Reda, Dr Sami Karjalainen, Dr Riikka Holopainen, Ms Zarrin Fatima

VTT Technical Research Centre of Finland Ltd

P.O.Box 1000

FI-02044 VTT, Finland

Phone: +35820722111

E-Mail: Francesco.Reda@vtt.fi, Sami.Karjalainen@vtt.fi, Riikka.Holopainen@vtt.fi, Zarrin.Fatima@vtt.fi

\section{SUMMARY}

The fact that occupant behaviour has a large effect on buildings' energy consumption is well accepted. Occupants affect energy consumption by their behaviours: using lighting, appliances, thermostats etc. and interacting with envelope components such as windows and blinds. During the last decades, specific ICT solutions have been developed for addressing behavioural changes toward energy saving and consequently aiming to energy conscious occupant behaviours. This paper presents a mean of interaction between sensors and users for making behavioural changes toward energy saving in homes. This is based on premade rules and instructions (referred to as recipes), meant for tackling energy hungry everyday life actions and peak shaving. Besides decreasing household energy consumption and shaving the peaks, the adoption of energy recipes aims to generate useful knowledge for occupants about energy-related variables aiming to long-term benefits.

\section{INTRODUCTION}

Compared to the past decades, the number of electric appliances in homes is very large, which increases home electricity consumption. In addition, the constant population growth increases the need of the electric energy. Electricity is mainly produced with fossil fuels, contributing to the serious environmental hazard caused by climate change (Nejat et al. 2015). It is important to reduce the use of electricity and it has been estimated that residential sector can reduce wasteful electricity consumption by $48 \%$ by existing technologies and improved behaviour (De Almeida et al. 2011). The costs associated with electricity generated depend on time and it is advantageous to move demand from the peak hours to other hours. This concept is called peak load shifting or peak shaving. One way to achieve reduction in energy consumption and manage the loads during the periods of maximum demand is to provide the end users more specific information about their electricity consumption and enable interaction with appliances and devices.

\section{ENERGY RECIPES AS AN APPROACH TO INCREASE USER ENGAGEMENT}

Innovative use of advanced existing ICT devices can potentially increase user engagement in energy efficiency. An approach called recipes aims at engaging occupants with energy and creating new kind of interaction between occupants and household appliances and devices. As stated by Bjelica et al. (2010): "recipes define a sequence of action when a certain complex condition is met. The criteria based on which the condition is evaluated rely on the reception of events, coming from physical devices or virtual behavioural drivers."

The occupants are given a possibility to create recipes by themselves. Recipes can be also provided to the users. In this work, pre-formulated energy recipes were created by energy experts based on literature review, analysis of sensor data and own gained experience (Figure 1). 


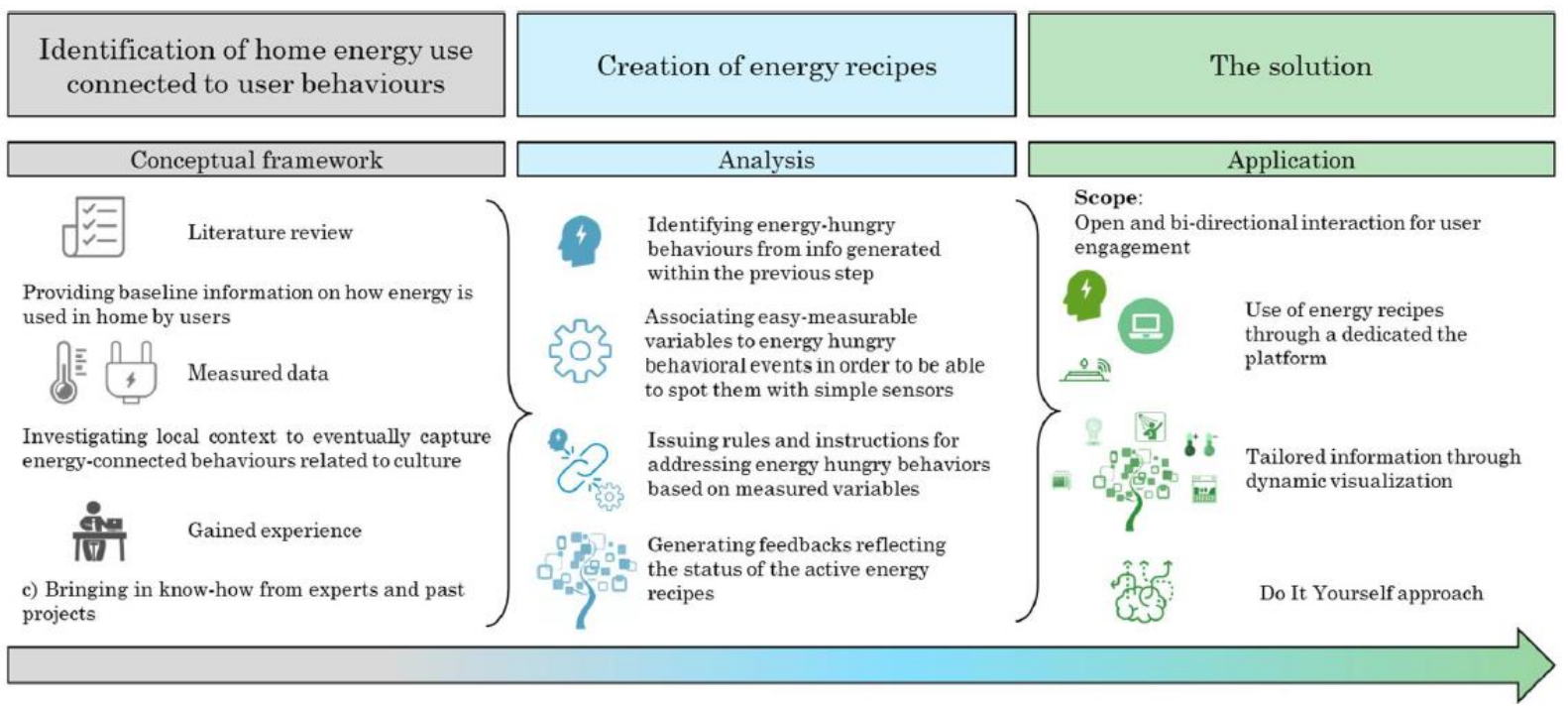

Fig. 1: Graphical representation of the methodology

\section{POTENTIAL OF ENERGY RECIPES FOR PEAK SHAVING}

The energy recipes can also be used for the purpose of peak shaving. The occupants can be given a possibility to choose which appliances and devices are used / not used during the periods of high price of electricity. The system could advice occupants when they should consider not using a specific appliance but postponing the use, e.g. for some hours. Figure 2 shows an example of peak shaving in which several household loads are moved to other hours. The maximum peak is decreased to less than $2.5 \mathrm{~kW}$ from more than $4 \mathrm{~kW}$.
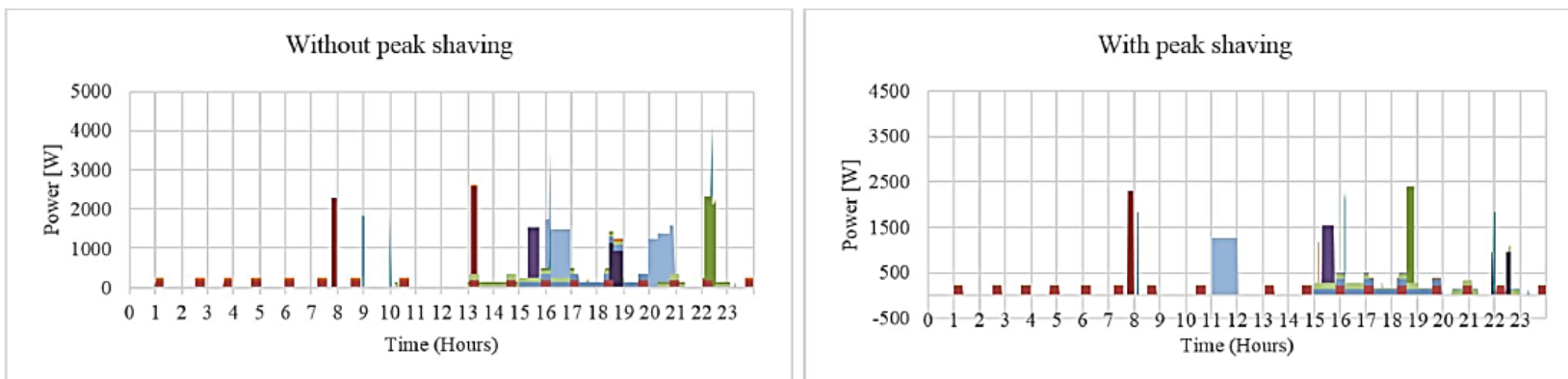

Fig. 2: An example of peak shaving during one day.

\section{REFERENCES}

Bjelica, M. Z., Papp, I., Teslic, N., \& Coulon, J.-M. (2010). Set-top box-based home controller. In IEEE

International Symposium on Consumer Electronics (ISCE 2010) (pp. 1-6). IEEE.

doi:10.1109/ISCE.2010.5523704

De Almeida, A., Fonseca, P., Schlomann, B., \& Feilberg, N. (2011). Characterization of the household electricity consumption in the EU, potential energy savings and specify policy recommendations. Energy and Buildings, 43(8), 1884-1894. doi:10.1016/j.enbuild.2011.03.027

Nejat, P., Jomehzadeh, F., Taheri, M. M., Gohari, M., \& Abd. Majid, M. Z. (2015). A global review of energy consumption, $\mathrm{CO} 2$ emissions and policy in the residential sector (with an overview of the top ten CO2 emitting countries). Renewable and Sustainable Energy Reviews, 43, 843-862.

doi:10.1016/j.rser.2014.11.066

\section{CONFERENCE TOPIC}

Smart building services and system operation 


\title{
EXPLORING SOLAR THERMAL INTEGRATION OPPORTUNITIES FOR THE TOURISM AND HOSPITALITY SECTOR IN ZIMBABWE
}

\author{
Guidence Muchengeti1, Blessed Sarema2, Samson Mhlanga3 \\ 123 National University of Science and Technology \\ Cnr Gwanda Road and Cecil Avenue P O Box AC 939, Ascot, Bulawayo, Zimbabwe \\ Phone: +2639282842 \\ E-Mail: muchengetiguidance@gmail.com, blesarema@gmail.com,smhlanga126@gmail.com
}

\section{SUMMARY OF THE PAPER}

The paper is a presentation of the work done to explore solar thermal integration opportunities for the tourism and hospitality sector in Zimbabwe. The project started with analysing the Zimbabwe tourism and hospitality map. Zimbabwe as a whole consists of vast tourist attractions of different forms and located all over the country. Figure 1 shows the tourism and hospitality map of Zimbabwe. These tourist resort areas have many hotels and lodges built around them so as to accommodate tourists from all over the world. Thus, in this research, the whole of Zimbabwe will be considered as the population and specific areas that are flocked by tourists for different reasons will be selected for analysis as the samples. The analysis will mainly be focused on the hotels surrounding the resort areas and specifically their water heating system to determine solar thermal integration potential for the provision of hot water at the facilities. Four research areas were selected and a survey was carried out on the hot water demand at the specific hotels. The existing heating systems being used at these facilities were assessed for optimal intergration design of the solar thermal heating system. One hotel was then used as a case study analysis in order to deeply understand the hotel operations. As the prices of energy sources rise and become more volatile in Zimbabwe, renewable sources become more attractive. Solar thermal water heating technology is becoming cheaper and is currently heavily incentivized in many areas except hotels. This project explored solar thermal integration opportunities in the tourism and hospitality sector in Zimbabwe and aims to close the gap of non-renewable energy consumption by at least $75 \%$ in the water heating sector.

\section{Key words Solar thermal, Intergration, Hospitality Industry, Zimbabwe}

\section{INTRODUCTION}

Energy is the backbone of all activities [1]. Water heating accounts for almost $30 \%$ of energy consumption in standard. In Zimbabwe, policy strategies that support renewable energy technologies are already there in the National Energy Policy. Solar Water Heating would reduce coincident electricity winter peak demand by $13 \%$ and reduce final energy demand by $27 \%$, assuming a $50 \%$ penetration rate of Solar Water Heating potential demand in Zimbabwe [2]. The demand of solar water heating systems in the hospitality sector was however unkown prior to this work.

\section{PURPOSE}

The aim of this project was to research on solar thermal integration opportunities in the tourism and hospitality sector in Zimbabwe. This was achieved by carrying out energy audits for the hot water heating section at selected hotels around the country. The average energy consumed by water heating systems in the tourism and hospitality sector was deduced and a cost benefit analysis of integrating solar thermal system and the payback period was determined for the case study hotel.

\section{RESULTS AND CONCLUSIONS}

In Zimbabwe hospitality industry water heating consumes a between $25 \%$ and $40 \%$ of the overal energy consumption. The study revealed that there is huge potential in the hospitality industry hence the need to unlock demand of solar water heating systems in the hospitality industry in Zimbabwe. A solar water heating system cost for pre-heating 20000 litre sytem costing USD 71648.61 would have a pay back period of close to 5 years. 


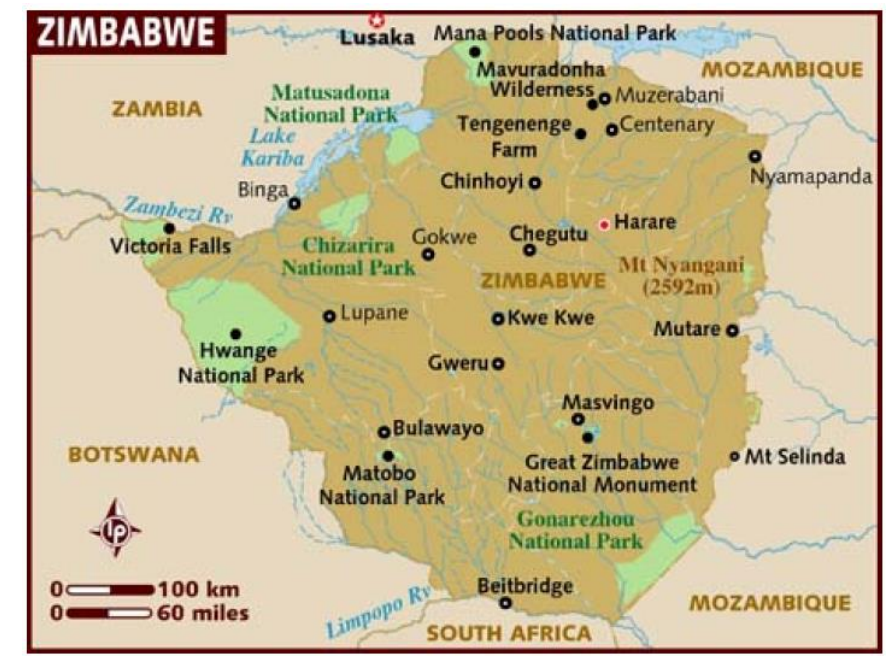

Figure 1: Tourism and Hospitality Map of Zimbabwe

\section{REFERENCES}

1. Muneer, T., Asif, M. and Kubie, J., 2003. Generation and transmission prospects for solar electricity:

UK and global markets. Energy conversion and management,44(1), pp.35-52.

2. Batidzirai, B. (2008). Potential for Solar Water Heating In Zimbabwe.

CONFERENCE TOPIC: RENEWABLE HEATING AND COOLING IN A FUTURE ENERGY SYSTEM 


\title{
UPGRADING THE PERFORMANCE OF DISTRICT HEATING NETWORKS IN EUROPE THE UPGRADE DH PROJECT
}

\author{
Dominik Rutz, Rainer Janssen, Cosette Khawaja \\ WIP - Renewable Energies, Sylvensteinstr. 2, D - 81369 Munich, Germany \\ Tel. +4989720 12743, Fax +498972012791 \\ E-Mail: dominik.rutz@wip-munich.de \\ Internet: www.wip-munich.de, www.upgradedh.eu
}

\section{SUMMARY}

This abstracts presents the EU project Upgrade DH (Upgrading the performance of district heating networks in Europe).

\begin{abstract}
According to the EU Strategy on Heating and Cooling (2016), the contribution of district heating (DH) in the EU accounts for 9\% and is mainly driven by fossil fuels such as gas and coal. District heating networks present a high potential for the transition of the heat sector, both technically and organizationally. They allow integration of renewable energies, improve the overall energy efficiency, as well as facilitate sector coupling (coupling between heating, electricity and mobility).

In order to use this potential, many district heating networks must first upgrade the existing distribution system, including the substations and consumer connections: reaching lower leakage rates and heat losses, reducing operation temperatures, adapting piping dimensions and hydraulic, introducing modern IT-based management systems and options for user control. This makes the heat distribution more efficient, but also improves the efficiency of the heat generation. Moreover, it allows the integration of renewable energies and waste heat. In order to have an optimized impact, the retrofitting measures should therefore begin with the retrofitting of the distribution network, including measures regarding substations and heating system of the connected buildings. In a further step, efficiency measures can be implemented on the generation side and the share of renewable and waste heat can be introduced and increased gradually. This must go hand-in-hand with predictions of future heat demand as well as with efficiency measures on the end use of heat.
\end{abstract}

Furthermore, many district heating systems face challenges such as lack of investment or unfavourable price regulation, low performance and negative consumer perceptions. In order to preserve existing heating networks, to increase their efficiency and public acceptance, the Upgrade DH project will widely promote and disseminate successful approaches to diagnosing and retrofitting inefficient networks, including sustainable business and organisational models.

The Upgrade DH project supports the upgrading and retrofitting process of DH systems in different climate regions of Europe, covering various countries. The target countries of the Upgrade DH project are: Bosnia-Herzegovina, Denmark, Croatia, Germany, Italy, Lithuania, Poland, and The Netherlands (Figure 1). In each of the target countries, the upgrading process will be initiated at concrete DH systems of the so called Upgrade DH demonstration cases (demo cases). The gained knowledge and experiences will be further replicated to other European countries and DH systems in order to leverage the impact.

The overall objective of the Upgrade DH project is to improve the performance of inefficient district heating networks in Europe by supporting selected demonstration cases for upgrading, which can be replicated in Europe.

Core activities of the Upgrade DH project include the collection of the best upgrading measures and tools, the support of the upgrading process for selected district heating networks, the organisation of capacity building measures about DH upgrading, financing and business models, as well as the development of national and regional action plans. In addition, an image raising campaign for modern DH networks will be carried out in the Upgrade DH project. The outcome will be the initiation of district heating upgrading process in the above mentioned target countries and beyond. 
The overall upgrading process to improve the efficiency of DH grids (Figure 2) is complex and sophisticated. It is time-consuming, long-lasting and implies high investments. Especially measures in connected buildings must be involved, for example when lowering the operation temperatures. It implies a direct cooperation with building owners and end consumers. Such a long and global process also has an impact on the city's or district's life that should not be underrated. That is why it should be very carefully planned in the long-term. Upgrade DH supports this.

A presentation at the ISEC Conference, will summarize the past, on-going and future activities of the three-years project Upgrade DH.

Figure 1 Upgrade DH target countries and demo cases

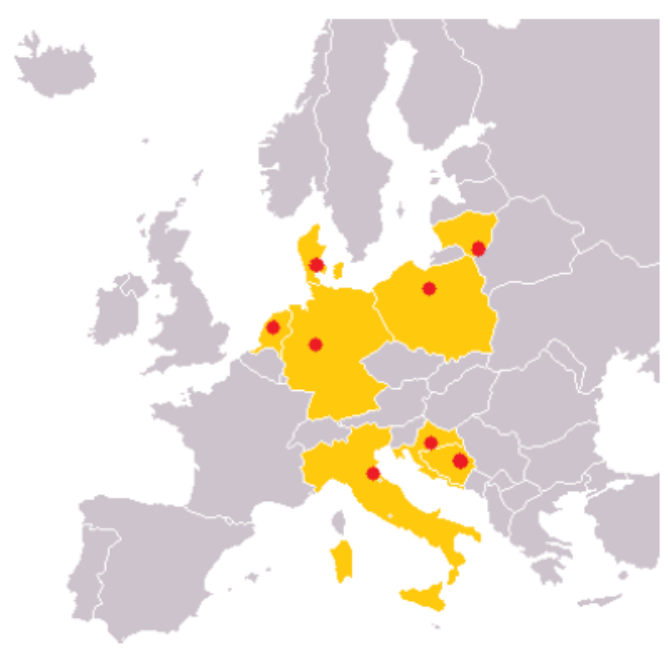

Figure 2: Upgrade DH upgrading process for existing district heating networks

\section{Overall upgrading process}

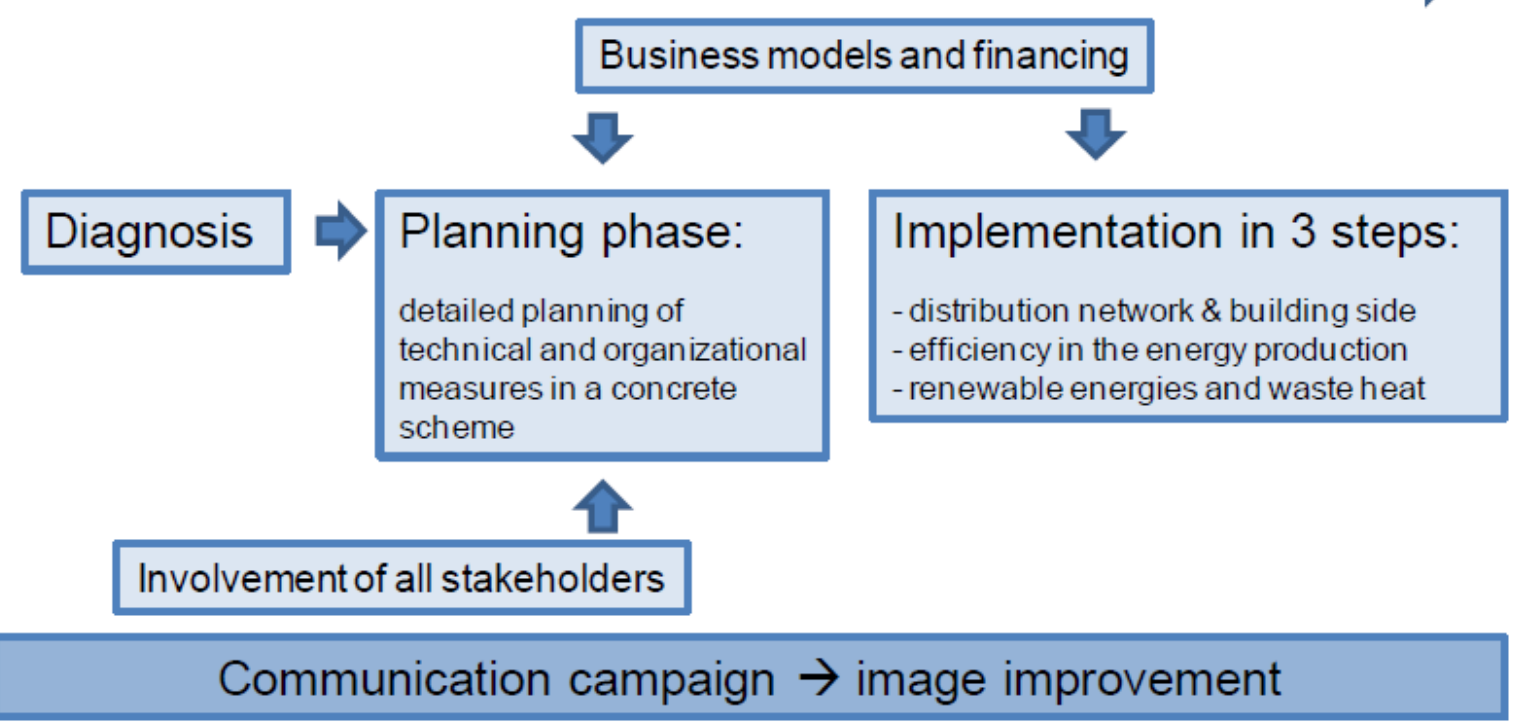

\section{CONFERENCE TOPIC}

Urban District Heating and Cooling Technologies 


\title{
HOUSEHOLD ENERGY CONSUMPTION: A STUDY OF MICRO RENEWABLE ENERGY SYSTEMS IN IRELAND
}

\author{
Michael Chesser \\ Dublin Institute of Technology \\ Aungier Street, Dublin, Ireland \\ Phone: +353851638070 \\ E-Mail: michaelchesser1@gmail.com
}

\section{SUMMARY}

Irelands Green Paper addresses how it will meet its commitments, it mentions that reductions in energy consumption rates can be assisted by its 'energy citizens' through the use of micro renewable energy systems(MRES). The purpose of this paper is, firstly to create a profile of households that have adopted MRES, which can inform policy makers. Secondly, to examine whether the presence of MRES in households have resulted in a reduction in energy consumption. To achieve these objectives, econometric analysis is performed on Irish household budget survey(HBS) data.

\section{INTRODUCTION}

Ireland's Green Paper (2014) highlighted increasing energy efficiency through the promotion and use of energy saving appliances and renewable energy systems in the residential sector, to accomplish its environmental goals. Irelands Department of Communications, Energy and Natural Resources, outlines the role that the households will play in integrating MRES which will allow them generate their own energy thus reducing energy demand. To support policy-makers' decisions about how to reduce energy use from the housing sector through the promotion of MRES, it is essential to know the profile of the average household that is currently adopting MRES as to better promote it. Also, it is worth investigating whether the adoption of MRES is successful in reducing domestic energy consumption. The purpose of this paper is to firstly reveal what are the common household characteristics exist among adopters of MRES using a logit regression model. Secondly this paper will provide a greater understanding of the main determinants of residential energy consumption and whether the growing number of MRES has had an impact on consumption levels for residential households in Ireland using an ordinary least squares (OLS) regression model. This paper uses anonymized microdata collected from the HBS 2010.

\section{RESULT TABLES}

\begin{tabular}{|c|c|c|}
\hline \multicolumn{2}{|c|}{ Logit MRES Coef. } & OLS Electricity Use Coef \\
\hline Micro Renewable Energy System & - & $0.211^{* * *}$ \\
\hline Log of Household Disposable Income & $0.4836^{* *}$ & $0.049^{* * *}$ \\
\hline Number of People & $0.0018^{* * *}$ & $0.126^{* * *}$ \\
\hline Urban \& Rural Household Location & $-1.0766^{* * *}$ & $0.022^{* *}$ \\
\hline Ownership or Rental Household & $2.0533^{* * *}$ & $-0.111^{* * *}$ \\
\hline Household with Children & $0.4893^{*}$ & $0.027^{*}$ \\
\hline Education Status of CES & & $-0.071^{* *}$ \\
Primary School, No Formal Education, Other & $-1.0794^{* *}$ & -0.022 \\
Secondary School & $-0.4701^{*}$ & (R.C) \\
Higher Institute & & \\
\hline
\end{tabular}




\begin{tabular}{|c|c|c|}
\hline House Construction Year. & & \\
\hline Pre 1918 & -0.5795 & 0.060 \\
\hline $1918-1945$ & $-1.1769 * *$ & -0.029 \\
\hline $1946-1960$ & $-1.8636^{* *}$ & 0.033 \\
\hline $1961-1970$ & $-1.2947^{* *}$ & (R.C.) \\
\hline $1971-1980$ & $-1.3410 * * *$ & 0.060 \\
\hline 1981-1990 & $-2.5067^{* * *}$ & $0.121^{* *}$ \\
\hline $1991-2000$ & $-0.9924^{* * *}$ & 0.038 \\
\hline 2001-2005 & $-1.3800 * * *$ & 0.054 \\
\hline $2006-2010$ & (R.C) & 0.030 \\
\hline Number of Bedrooms & $0.315^{* * *}$ & $0.044^{* * *}$ \\
\hline \multicolumn{3}{|l|}{ Appliances } \\
\hline Dishwasher & - & $0.138^{* * *}$ \\
\hline Tumble dryer & - & 0.039 * \\
\hline Fridge-freezer & - & -0.003 \\
\hline Microwave & - & 0.048 \\
\hline Console & - & $0.087^{* * *}$ \\
\hline Number of TVs & - & $0.025^{* * *}$ \\
\hline Constant & $-8.7955^{* * *}$ & $3.469 * * *$ \\
\hline No of Observations & 5,818 & 5489 \\
\hline $\mathrm{R}^{2}$ & 0.172 & 0.220 \\
\hline F-Stat & & $67.12^{* * *}$ \\
\hline
\end{tabular}

Table 1: Logit and OLS regression results for dependent variables MRES and Electricity Use, respectively.

\section{EQUATIONS}

In Ireland, the MRES sectors growth is quite slow and the possible reason for this may be ineffective governmental support mechanisms in comparison to other countries, even though the promotion of such systems is mentioned in Ireland green paper. The lack of incentives provided to Irish residents to adopt MRES may explained some of the results attained from our logit model. Where the average household that is most likely to adopt MRES is a house that has been constructed recently, large, situated in a rural area and is owner occupied. The owner is most likely highly educated and has a high weekly disposable income. Interpretation of these results would suggest that MRES is still a luxury purchase in Ireland. The second part of this study was to find the determinants of household energy use and whether MRES has had an impact on reducing energy use. It was found that the determinants of household energy consumption were in line of that of previous literature. Surprisingly, it was found that the presence of MRES is statically insignificant across all model's bar that of the electricity use model, where the presence of MRES increase electricity use.

\section{REFERENCES}

Irish Government. (2014). Green Paper on Energy Policy. https://www.dccae.gov.ie/enie/energy/topics/Energy-Initiatives/energy-policy-framework/green-paper/Pages/Stakeholders-

$\underline{\text { Seminars.aspx }}$ 


\section{MODELING AND SIMULATION OF A SOLAR THERMAL STORAGE COLLECTOR}

Harald Kirchsteiger, Patrick Kefer, Harald Dehner

FH Oberösterreich F\&E GmbH

Ringstraße 43a, Wels, Austria

Phone: +43 050804 / 46916

E-Mail: harald.kirchsteiger@fh-wels.at

\section{SUMMARY}

A novel solar thermal storage collector - an integrated solar collector-storage tank system with pipe heat exchanger - was modelled using first principles. The model was fitted to measurement data obtained at a hardware solar simulator and provides consistent results. Using the model, annual simulations on several locations were conducted to evaluate the usability of the system.

\section{INTRODUCTION}

Modeling of solar thermal collectors is a standard technique [1]. The modelling of integrated systems is, however, not so common to find in the literature and only a few results are known [2-4]. Here, we are considering an integrated collector as shown in Figure 1 with a storage of 150 liters of water.

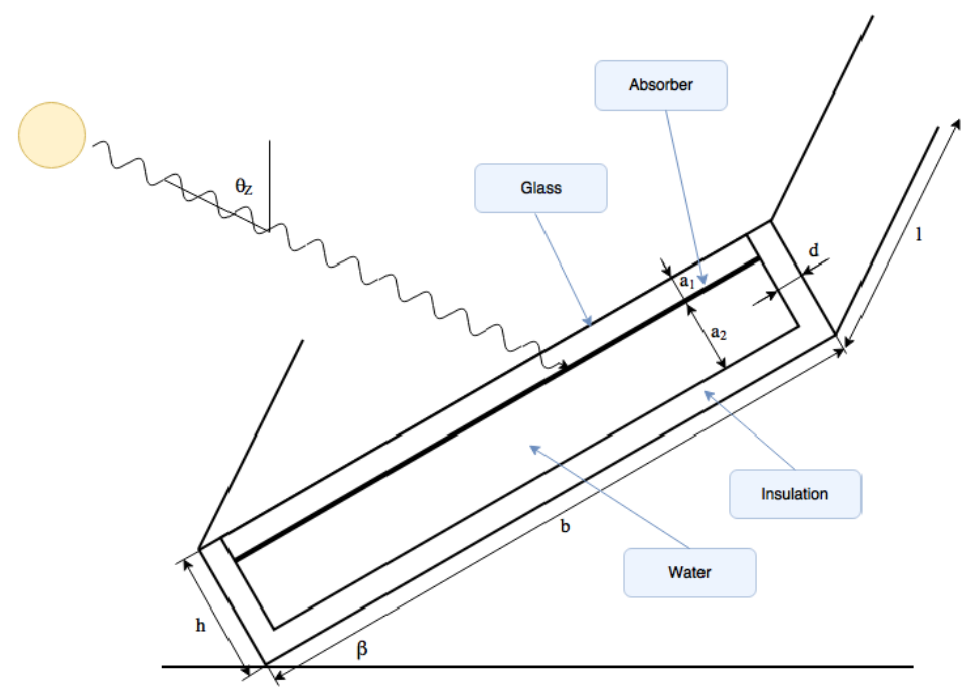

Fig. 1: Layout of the integrated collector system

\section{MATHEMATICAL MODEL}

A thorough description on mathematical modelling will be placed in the final paper. For the purpose of this abstract, we mention that:

The storage was considered as stratified tank, the individual heat flow rates were modeled

- Natural convection and radiation between 2 parallel plates

- Heat losses on the glass surface to ambient, convection and radiation

- Heat from absorber to the water through free convection

- Heat losses to ambient

- Heat gain on the absorber

\section{RESULTS}

The results in Fig. 2. show the good agreement between the mathematical model and the measurements. To obtain those results, three parameters of the collector model were adjusted to the measurements: the collector efficiency in order to fit the warm-up phase, the thermal conductivity of the insulation in order to fit the cool-down phase and the thermal conductivity of the heat exchanger in order to fit the water withdrawal phase. 


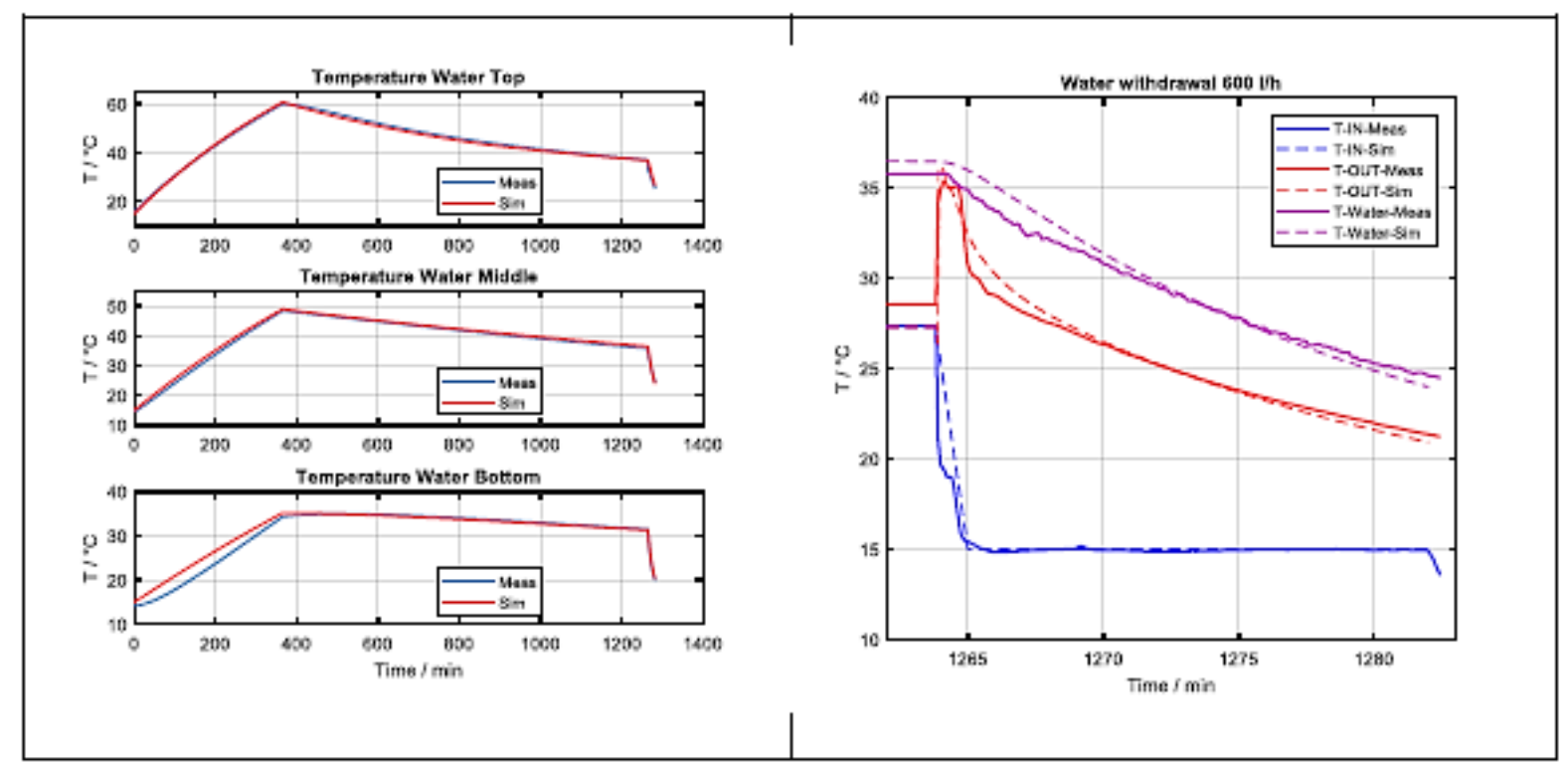

Fig. 2: Comparison of measurements from the hardware solar simulator (Meas) with the model (Sim). Left: constant exposure to $1000 \mathrm{~W} / \mathrm{m}^{2}$ (0-400 min), night phase with $0 \mathrm{~W} / \mathrm{m}^{2}$ (400-1300min). Right: water withdrawal following the night phase.

Using the developed model, annual simulations using real weather data from several locations were conducted. The goal was to simulate how often it is possible to operate a shower and use hot water for cooking. Showers are at 08:00, 08:15, 18:00, 18:15, cooking is at 12:00 and 19:00. Showering assumes a withdrawal of 30 liters within 5 minutes with $40^{\circ} \mathrm{C}$, cooking 10 liters within 1 minute with $40^{\circ} \mathrm{C}$. Table 1 summarizes how often within 1 year the desired temperatures could be reached when withdrawing water. In the final paper, results regarding the fulfillment of withdrawals according to EN-standards will be presented.

Table 1: Results of annual simulations: occurrences of successful withdrawals

\begin{tabular}{|c|c|c|c|c|c|c|}
\hline Location & Shower1 & Shower2 & Cooking & Shower3 & Shower4 & Cooking \\
\hline Brasilia & $52 / 365$ & $2 / 365$ & $202 / 365$ & $314 / 365$ & $287 / 365$ & $206 / 365$ \\
\hline Casablanca & $1 / 365$ & $0 / 365$ & $24 / 365$ & $300 / 365$ & $260 / 365$ & $167 / 365$ \\
\hline Dakar & $156 / 365$ & $65 / 365$ & $355 / 365$ & $364 / 365$ & $363 / 365$ & $359 / 365$ \\
\hline Damascus & $165 / 365$ & $156 / 365$ & $254 / 365$ & $313 / 365$ & $292 / 365$ & $248 / 365$ \\
\hline Los Angeles & $0 / 365$ & $0 / 365$ & $90 / 365$ & $258 / 365$ & $197 / 365$ & $66 / 365$ \\
\hline
\end{tabular}

\section{REFERENCES}

[1] J. A. Duffie and W. A. Beckman, Solar Engineering of Thermal Processes. John Wiley \& Sons, 2013.

[2] M. Souliotis, R. Singh, S. Papaefthimiou, I. J. Lazarus, K. Andriosopolos, Integrated collector storage solar water heaters: survey and recent developments, Energy Syst, vol.7 (1), pp. 49-72, 2016

[3] J. I. Currie et. al, Modeling bulk water temperature in integrated collector storage systems, Building Serv. Eng. Res. Technol., vol. 29 (3), pp. 203-218, 2008

[4] C. Garnier, T. Muneer and J. Currie, Thermal model for performance prediction of integrated collector storage systems, J. of Renewable and Sustainable Energy, vol. 3 (1), 2011

\section{CONFERENCE TOPIC}

Renewable heating and cooling in a future energy system 


\section{WASTE HEAT RECOVERY BELOW $80^{\circ} \mathrm{C}$ WITH THERMOMAGNETIC MOTORS}

Dr. Michael Maschek ${ }^{1}$, Prof. Dr. Ekkes Brück ${ }^{1}$, Dr. Nikolaus Vida ${ }^{2}$

Fundamental Aspects of Materials and Energy, Delft University of Technology ${ }^{1}$

Mekelweg 15, 2629 JB Delft, The Netherlands

Phone: +31 1527 81645, E-mail: m.maschek@tudelft.nl

Swiss Blue Energy ${ }^{2}$

Baslerstrasse 6, CH-5330 Bad Zurzach, Switzerland

\section{SUMMARY}

E-Mail: tmm@sbe-ag.ch

The growing energy demands in modern-day society and the influence of the related energy conversion processes on the global environment by greenhouse gasses require new technologies to efficiently utilize all available energy resources. Vast amounts of low-temperature waste heat below $80^{\circ} \mathrm{C}$, originating from large-scale industrial processes, are abundantly available. However, this form of waste heat is currently an untapped energy source, because commercially available energy conversion methods generally require temperatures of at least $100^{\circ} \mathrm{C}$. However, only in the past decade, it has been shown that magnetic conversion methods have the potential to effectively convert low-temperature heat below $80^{\circ} \mathrm{C}$ into electricity [1].

The first milestone towards waste-heat to power generation has been established by Swiss Blue Energy (SBE) [2], who successfully developed a demonstrator of a thermomagnetic motor (TMM) utilizing gadolinium $(\mathrm{Gd})$.

\section{INTRODUCTION}

The current TMM (K-2 prototype) of Swiss Blue Energy utilizes waste heat and produces a power output of about $1.3 \mathrm{~kW}$. It has been tested for long-term operation at an AXPO wood power plant in Switzerland ( $\sim 5000$ working hours). The power output of the TMM is continuous, which is a major advantage compared to most renewable energy technologies having an intermittent power output. With this technology it is possible to produce electricity with low cost by utilizing freely available heat that would otherwise be discarded. This may significantly increase energy efficiency of power plants and industrial processes resulting in lower costs of production. The compact and modular design allows a worldwide site independent and decentralized generation of electricity. Due to an emissionfree operation there is no negative impact on the environment making the TMM a valuable alternative to other green technologies. However, practical utilization is hampered due to the low optimal operation temperatures below $\approx 30^{\circ} \mathrm{C}$ for $\mathrm{Gd}$.

\section{PRINCIPLES OF MAGNETIC ENERGY CONVERSION}

The basic principle behind the magnetic energy conversion in a TMM is that a magnetic material strongly changes its magnetic response when heated or cooled in the vicinity of a magnetic transition.

(a)

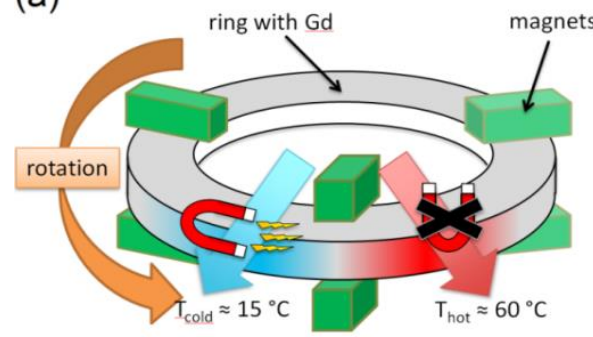

(b)

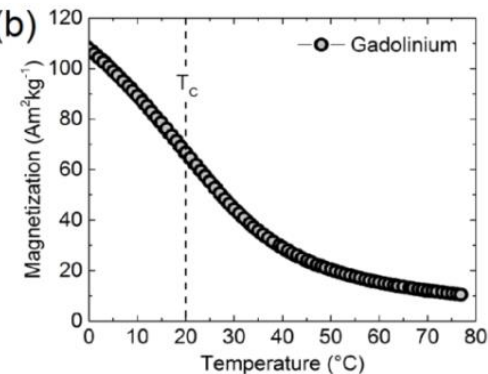

Fig. 1: (a) Schematic picture of the thermomagnetic motor (SBE). (b) Temperature dependent magnetization of $\mathrm{Gd}$.

When cooled, a segment of the magnetic material (ring) enters a state of high magnetization resulting 
in an attractive force towards the permanent magnets (Fig. 1a). In order to ensure that this segment is not slowed down after passing the magnet, the magnetic material is heated above the magnetic transition, defined by the Curie-temperature TC, where the material ideally enters a non-magnetic state. The resulting rotational movement of the ring is then further converted into electricity. The conversion of heat into electricity by magnetic fields can be strongly enhanced when Gd (Fig. 1b) is replaced by materials with a higher working temperature and a steeper change in magnetization with temperature.

\section{REPLACE GD BY NOVEL ADVANCED MAGNETOCALORIC MATERIALS}

Promising candidates for efficient magnetic energy conversion in thermomagnetic motors are $(\mathrm{Mn}, \mathrm{Fe})_{2}(\mathrm{P}, \mathrm{Si}, \mathrm{B})$ compounds [3], which have tunable Curie temperatures and large magnetization changes $(\Delta \mathrm{M})$ across the Curie temperature. The challenge in this system is to optimize the chemical composition in order to achieve a small thermal hysteresis to maintain reversibility during cycling between magnetic and non-magnetic state. Furthermore, volume changes across TC have to be minimized to avoid cracking i.e. damaging the magnetocaloric material. Our study involves tuning the chemical composition in $(\mathrm{Mn}, \mathrm{Fe})_{2}(\mathrm{P}, \mathrm{Si}, \mathrm{B})$ compounds towards magnetic energy conversion applications via changing $(\mathrm{Mn}, \mathrm{Fe})$ and $(\mathrm{P}, \mathrm{Si})$ ratios, varying heat treatments and insertion of additional

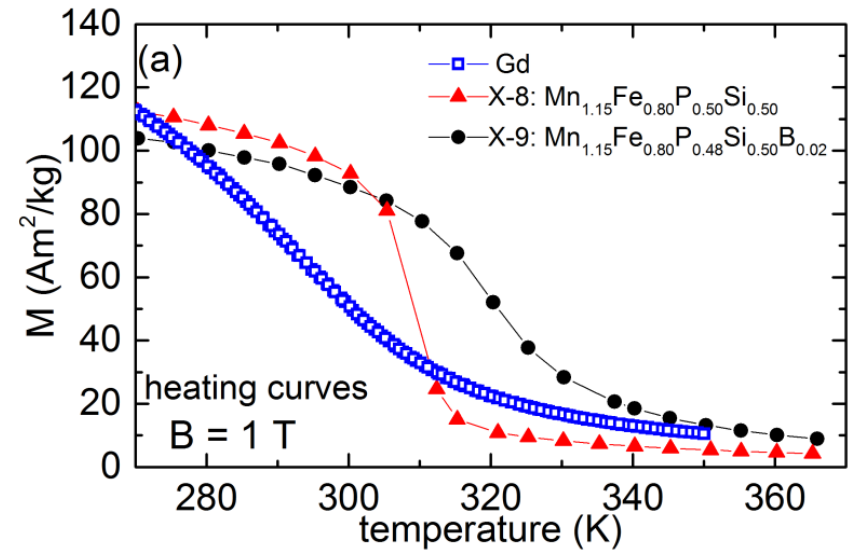

Fig. 2: Temperature dependent magnetization of $(\mathrm{Mn}, \mathrm{Fe})_{2}(\mathrm{P}, \mathrm{Si}, \mathrm{B})$ compounds in a magnetic field of $\mathrm{B}=1 \mathrm{~T}$ elements such as Boron

\section{REFERENCES}

[1] D. Vuarnoz, A. Kitanovski, C. Gonin, Y. Borgeaud, M. Delessert, M. Meinen, and P. W. Egolf, (2012), Appl. Energ. 100, 229.

[2] http://www.swiss-blue-energy.ch/.

[3] M. F. J. Boeije, M. Maschek, X. F. Miao, N. V. Thang, N. H. van Dijk, and E. Bruck, (2017),. J. Phys. D App.l Phys. 50, 174002

\section{CONCLUSION}

Magnetic energy conversion by a thermomagnetic motor has the potential to significantly improve the worldwide energy efficiency. In order to make this a viable technology, efficiency has to be improved by replacing Gd by magnetocaloric materials 


\title{
WINDOW OF THE FUTURE
}

\author{
$\underline{\text { Joe Kao }}$ \\ PHYSEE Group B.V. \\ Molengraaffsingel 10, 2629 JD, Delft, The Netherlands \\ Phone: +31653430622 \\ E-Mail:joe@physee.eu
}

\section{ABSTRACT}

Buildings consume $40 \%$ of the global electricity demand, and solar panels on the roof are not nearly sufficient to make these buildings energy neutral. We need the entire exterior of each building to generate power from the sun, including all windows. Although it is an inefficient material, glass has a huge potential and is widely used with increasing market demand every year.

Imagine all the glass in your building having the potential of generating sufficient power to cover the entire power demand of a glass-covered modern office building. PowerWindow + technology by PHYSEE enables this potential. Conventional glass reflects about $30 \%$ of the incoming light, we instead collect that light with our patented coating and transport it through the glass to convert it to electricity. Additionally, integrated smart sensors measure different weather conditions and are used to improve the user experience and productivity inside each room of the building resulting in the reduction of overall energy consumption. This will turn windows from an inert building material that costs energy and money, to the intelligent and sustainable building skin of the future generation.

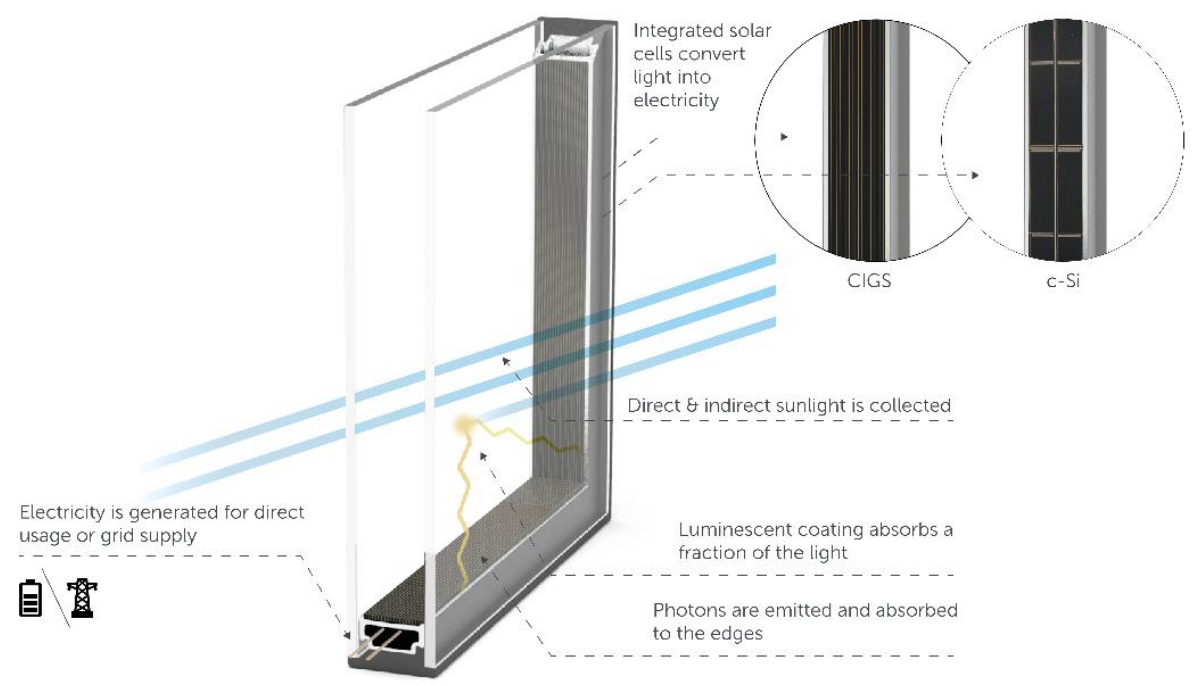

Fig. 1: An illustration of PowerWindow+ that uses the luminescent solar concentrator technology (LSC)

Our coating is made of an industry-standard inorganic material doped with a low concentration of the luminescent centres. The coating absorbs sunlight uniformly over a spectrum ranging from ultraviolet to visible light. It thereafter re-emits the light in the near-infrared region, resulting in zero overlap between the absorption and emission spectra. The result is a colourless coating with no selfreabsorption, making it the first scalable LSC without compromising on the aesthetics. 
The production methods used for the coating are magnetron sputtering and wet-coatings, which are standard in the global glass industry, serving industries ranging from commercial real estate to automotive. This is what makes our product scalable and applicable in various markets.

The collection of light in our coating happens independent of the window's orientation towards the sun. Consequently, PowerWindow+ will work both with direct and indirect light. The conversion of light into electricity happens at strips of solar cells in the frame, which only need to convert one specific wavelength, due to the characteristics of our coating. The result is minimal heat loss and high efficiency. Our coating is inert and has no risk of losing functionality due to exposure to solar irradiation or weather conditions. All other components are industrial standard and come with market conforming warranties of 10-15 years. As versatile as the deposition methods, the types of transparent surfaces are also unlimited; it can be curved, made of plastic or laminated, double and triple paned.

\section{REFERENCES}

Buildings - Energy - European Commission. (2018, August 30). Retrieved from https://ec.europa.eu/energy/en/topics/energy-efficiency/buildings 


\section{HOTSPOTS - HOLISTIC THERMOGRAPHIC SCREENING OF URBAN PHYSICAL OBJECTS AT TRANSIENT SCALES}

DI Dr Karl Höfler, DI Tobias Weiss

AEE - Institute for Sustainable Technologies

Feldgasse 19, 8200 Gleisdorf, Austria

Phone: +43 3112 / 5886-325

E-Mail: k.hoefler@aee.at

\section{SUMMARY}

The interdisciplinary team consisting of members from Siemens, Austrian Institute of Technology (AIT) and AEE INTEC has developed a procedure to capture energy losses of districts by aerial thermography. The gathered data allows identification of 'critical spots'. In addition, catalogues of measures and criteria were developed to support decisions on energy strategies and facilitate a transparent energy master planning process. The developed procedure was tested with success for the urban municipality of Gleisdorf and can be transferred to other towns. The project has been promoted by the Austrian Research Promotion Agency in the "Stadt der Zukunft" program.

\section{INTRODUCTION}

Up to now, data on buildings and energy in towns is available in varying quality and spatial resolution, and not always up to date. Therefore it is difficult to obtain a common database with harmonized data sets. This is however necessary, due to the increasingly important role of energy efficiency in modern urban and energy master planning.

Comprehensive aerial thermography ensures that space-resolved data on effective energy consumption can be gathered and promptly related to user behaviour and physical conditions. Subsequently, this knowledge forms the basis on which catalogs of criteria and measures for renovation and energy planning can be created and implemented.

\section{CONTENTS AND TARGETS}

The aim of HOTSPOTS was to offer municipalities scientifically founded tools and methods that allow investigating the present state of buildings regarding energy efficiency and thus enhance decisions to improve the state of buildings. In the framework of the project, an integrated procedure was developed and validated for Gleisdorf as a model town. This integrated procedure will help towns to discover and evaluate potentials for optimization. The selection of renovation measures for buildings and HVAC can thus be founded on a documentation based on measurement data. This will reduce the risk of incorrect decisions and misdirected investment.

\section{METHOD}

The data basis for the project was a 3D thermal cadastre, generated from aerial views. The task was the areawide collection of thermal data in the urban area. The single views were connected to a holistic database for the whole urban area and raised into the third dimension by deriving 3D building models from the image data. In this three-dimensional thermal cadastre, critical spots were identified. Critical spots define cells of infrastructure which show an outstanding potential for optimization. These critical spots were then analyzed and evaluated in more detail by image flights with drones. 


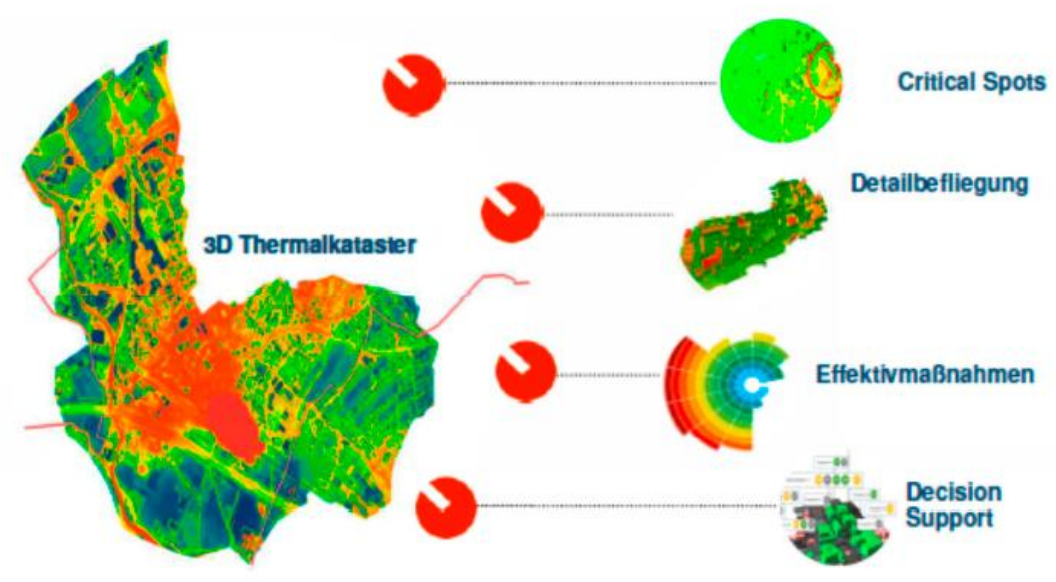

Fig. 1: A procedure to create a basis for energy master planning and optimization

The more detailed images were used to derive relevant geometrical parameters to generate 3D building models. Moreover, detailed thermal images allowed identification of refined optimization measures. The data of the critical spots was used to selectively analyze weak points. This resulted in a catalog of effective measures, which includes influencing factors for the critical spots identified in the town.

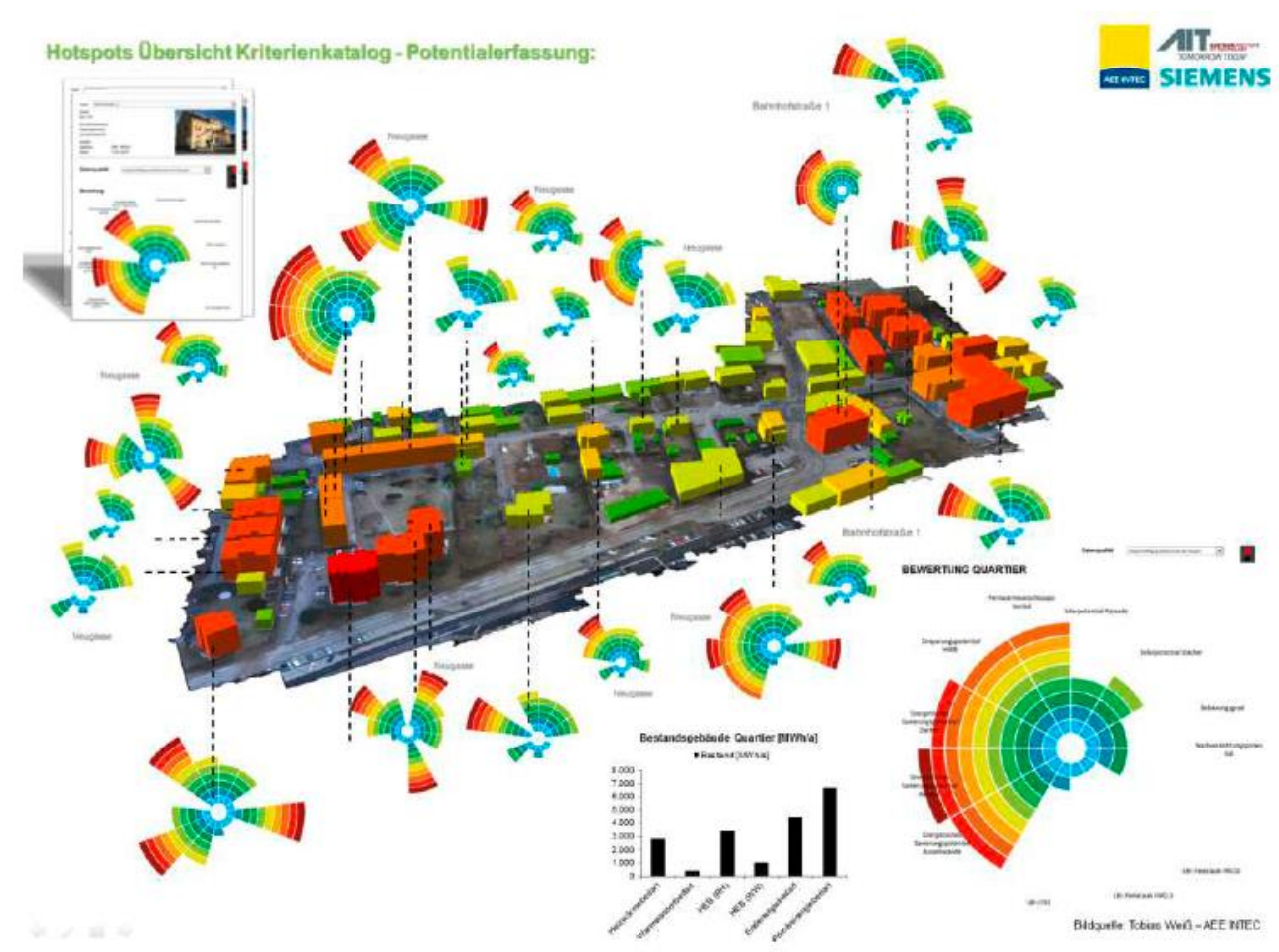

Fig. 2: Catalogue of criteria for effective measures and optimizations

\section{RESULTS AND OUTLOOK}

The developed procedure was tested in real settings and the results were successfully validated. Town quarters in need of renovation - so-called critical spots - were identified, and for these quarters, reasonable measures for the renovation of the thermal envelope and the building technology were derived. The identified catalog of effective measures for town quarters supports decisions of spatial planners and policymakers. The developed procedure can be transferred to other towns and municipalities at any time. 


\title{
DIRECT CONVERSATION OF WASTE HEAT FROM A SOLID-FUEL STOVE INTO ELECTRIC ENERGY USING A HIGH TEMPERATURE THERMOELECTRIC GENERATOR COMPARED TO BI2TE3 THERMOELECTRIC GENERATOR
}

\author{
Momir Tabakovic \\ Michal Masaryk \\ Slovak University of Technology in Bratislava \\ Institute of Energy Machinery \\ Phone: +4313334077573 \\ E-Mail: momir.tabakovic@technikum-wien.at
}

\section{SUMMARY}

Modern bioenergy plays an essential role in the International Energy Agency (IEA) $2^{\circ} \mathrm{C}$ Scenario. Modern technologies can convert this organic matter to solid, liquid and gaseous forms that can more efficiently provide for energy needs and replace fossil fuels. But the modern biomass needs electrical power for the heating pump, control system, regulation. A thermoelectric generator could work against this issue and produce the electric energy for the solid-fuel stoves. This work contributes to the research by characterizing, modeling and assessing the performance of a TEG, where low temperature thermoelectric modules with BI2TE3 materials are integrated on the chimney pipe chamber of a soildfuel stove, to keep structural changes minimal and the temperature difference large. Additional high temperature TEG used the Half-Heusler material, which is near to the market breakthrough is measured. Results from the measurement, calculation and testing will be presented.

Keywords: thermoelectricity, waste heat recovery, BI2TE3, Half Heusler, solid-fuel stove

\section{INTRODUCTION}

Energy crisis and environment deterioration are two major problems for 21 st century. Severe environmental issues, such as global warming, climate change, greenhouse gases emissions, as well as acid rain and ozone depletion, have arisen due to the excessive use of fossil resources. These crises raise a strong demand for development of efficient and clean energy technologies. Waste heat recycling or waste heat recovery offers a lot of opportunities to make a global contribution to these changes. Key concepts like waste heat recycling or waste heat recovery are the basic ideas in thermoelectricity so as to the design the newest solid-state sources of energy for a stable supply of electricity and environmental protection.

Thermoelectricity presents the possibility of harvesting any temperature grade heat; besides it also includes many other advantages which make thermoelectric generators perfect for generating electric power from waste heat. According to several theoretical and practical approaches that were done with solid-fuel stoves. In general, a modification of the stove is necessary. To keep the structural changes of the stove on a minimum and enable an easy installation, the thermoelectric generator is placed along the chimney of the solid-fuel stoves, which uses the waste heat of exhaust gas. A prototype series, named "BiomTEG" was developed, that represented the chimney of the stove. The most commonly used commercial TE module, Bismuth Telluride (Bi2 Te3), was used for these experiments. In the sense of a system approach the thesis deals on hand with development of the TEG system but also with the analysis of interaction between the TEG and the stove. The experimentation has been used to determine the parameters that influence the generation and to validate a generic computational model able to predict the thermoelectric generation where waste heat is harvested.

The simulation was validated through several experiments with different prototypes. The motto was “ check performance modify the design" . After improvements and findings solid- fuel stove from the company "KGH Engineering" was used to test the BiomTEG other real conditions. Fig. 1 shows the schematic set up of the thermoelectric generator with Bi2TE3 in the chimney of a real stove. 


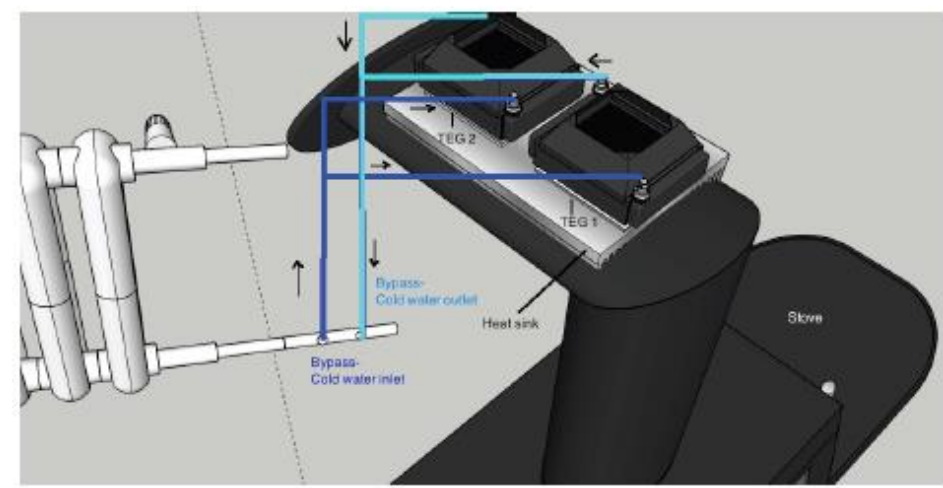

Figure 1 Schematic of the thermoelectric generator system in a real stove with two TE modules

New material classes could allow for waste heat recovery with better efficiency or use with higher temperature heat sources. These classes include skutterudites, clathrates, Half-Heuslers, and oxides such as cobaltites and perovskites. These new classes have been the subject of a great deal of research 50 but have had limited commercial use due to cost, reliability, efficiency, and processing issues that prevent them being selected over traditional materials. The newly developed TEG are based on HalfHeusler alloys, which can be deployed at temperatures of up to $550{ }^{\circ} \mathrm{C}$. With a size of $16 \mathrm{~mm} 2 \times 16$ $\mathrm{mm} 2$ they achieve a maximum output power of $2.8 \mathrm{~W}$. An experimental testing with the prototype " BiomTEG" and in the field test is done, to find out its performance in realistic conditions. This was not tasted in this kind of application till now.

\section{RESULTS}

The temperature difference is measured by using type $\mathrm{K}$ thermos couples. The measure is done by Data Acquisition/Switch Unit and transferred to the software Matlab via USB for further analysis. After several measurements and improvements of the prototypes, such changing the heat exchanger of the cold side and improving the insulation, etc. the thermoelectric system was integrated into the real stove. The results of the field test measurement with $\mathrm{Bi} 2 \mathrm{Te} 3$ modules is $8,6 \mathrm{~W}$ by a temperature difference of app. $150^{\circ} \mathrm{C}$ to $160^{\circ} \mathrm{C}$ as shown in Fig. 2. Further results with Bi2Te3 and Half-Heusler modules are done and will be illustrated. Additional an economical compression between the two thermoelectric generators with different materials will be presented.

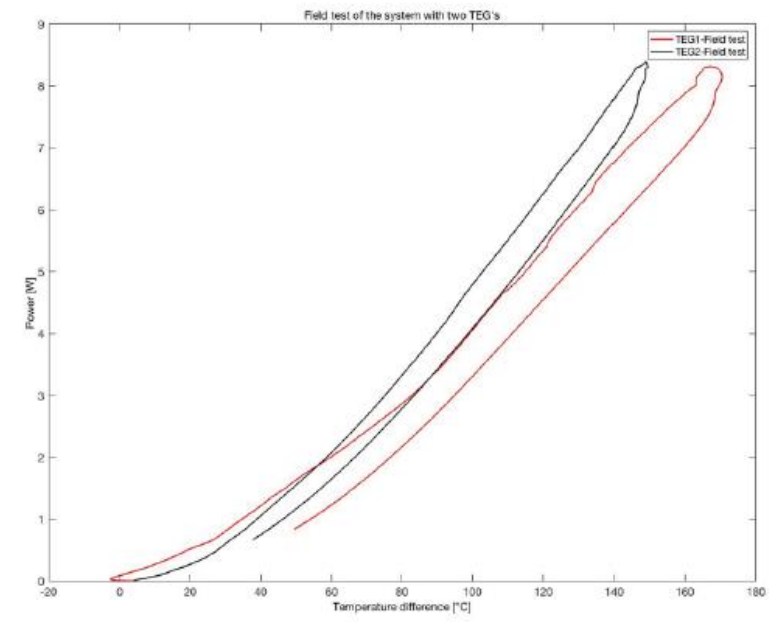

Figure 2 Power output vs. Temperature difference of the thermoelectric generator in the field test 


\title{
HEAT SUPPLY FROM WASTEWATER TREATMENT PLANTS - A METHODOLOGICAL APPROACH FOR INTEGRATED SUSTAINABILITY ASSESSMENT
}

\author{
F. Kretschmer ${ }^{1}$, G. Neugebauer ${ }^{1}$, F. Zach ${ }^{2}$, C. Loderer ${ }^{3}$, R. Farina ${ }^{4}$, D. Santi ${ }^{4}$, P. Jenicek ${ }^{5}$, Z. Varga ${ }^{5}$, P. \\ Lichtenwoehrer $^{1}$, G. Stoeglehner ${ }^{1}$, G. Langergraber ${ }^{1}$ \\ ${ }^{1}$ University of Natural Resources and Life Sciences, Vienna - BOKU, ${ }^{2}$ Austrian Energy Agency, Vienna - \\ AEA, ${ }^{3}$ Berlin Centre of Competence for Water - KWB, ${ }^{4}$ Italian National Agency for New Technologies, \\ Energy and Sustainable Economic Development, Bologna - ENEA, ${ }^{5}$ University of Chemistry and Technology \\ Prague
}

Corresponding author: Muthgasse 18, 1190 Vienna, Austria, Phone: +43 147654-81115,

E-Mail: florian.kretschmer@boku.ac.at

\section{SUMMARY}

Wastewater is usually considered a low-grade energy source, which is best used to supply wastewater treatment plants' internal demands. However, in many cases available quantities of low and high temperature heat can exceed self-sufficiency by far. Consequently, the questions arise, whether this excess heat could also be used to supply adjacent infrastructure. This article (abstract) introduces a methodological approach to assess the possibilities for integrating wastewater treatment plants into local heat supply systems from an integrated perspective.

\section{INTRODUCTION}

Global warming caused by the use of fossil energy sources is one of the grand challenges of our century. In the course of energy transition towards renewable energy sources even so far unconsidered resources come into view of discussion. In this context, wastewater can be considered an interesting option as it can serve as a basis for providing electricity as well as heat (FRIJNS et al., 2013, REMY et al., 2014). At wastewater treatment plants (WWTPs) the latter, which can basically be provided from digester gas combustion and wastewater heat recovery, is mostly available in surplus quantities (KRETSCHMER et al., 2016). Consequently, WWTPs might also serve as local thermal power plants. Integrating WWTPs into local energy/heat supply systems has already been addressed in international literature. KOLLMANN et al. (2017) describe a related assessment method considering spatial, economic and environmental evaluation criteria. GLATZL et al. (2017) present a decision support tool, which calculates mass and energy balances and estimates the economic efficiency of different WWTP external supply scenarios. These examples illustrate the interdisciplinary and thus rather complex character of the issue. To contribute to the development of knowledge and experience in this field, this article (abstract) introduces a methodological approach for integrated sustainability assessment (ISA), which can support feasibility judgement concerning heat supply from WWTPs from a multidisciplinary perspective.

\section{METHODOLOGICAL APPROACH}

The proposed methodology for integrated sustainability assessment supports decision making from a fourfold perspective: in a first step the (1) energetic context of the WWTP (potentials for energetic optimisation and provision of renewable energy) as well as its (2) spatial context (potentials for optimisation of settlement structures and identification of possible heat consumers as well as of other available location-bound energy sources) will be analysed. Based on these investigations objects (heat demands) can be defined, which appear suitable for being supplied from the WWTP. In a second step the (3) environmental as well as the (4) economic context of the designated supply systems will be analysed. In principle, these analyses are comparisons of current (mostly fossil) and future (renewable) 
$\mathrm{CO}_{2}$ emissions and energy prices, respectively. Finally, the assessment of the related environmental and economic benefits support the decision on the feasibility of the planned implementations. Fig. 1 displays this methodological approach for the integrated sustainability assessment in a simplified form.

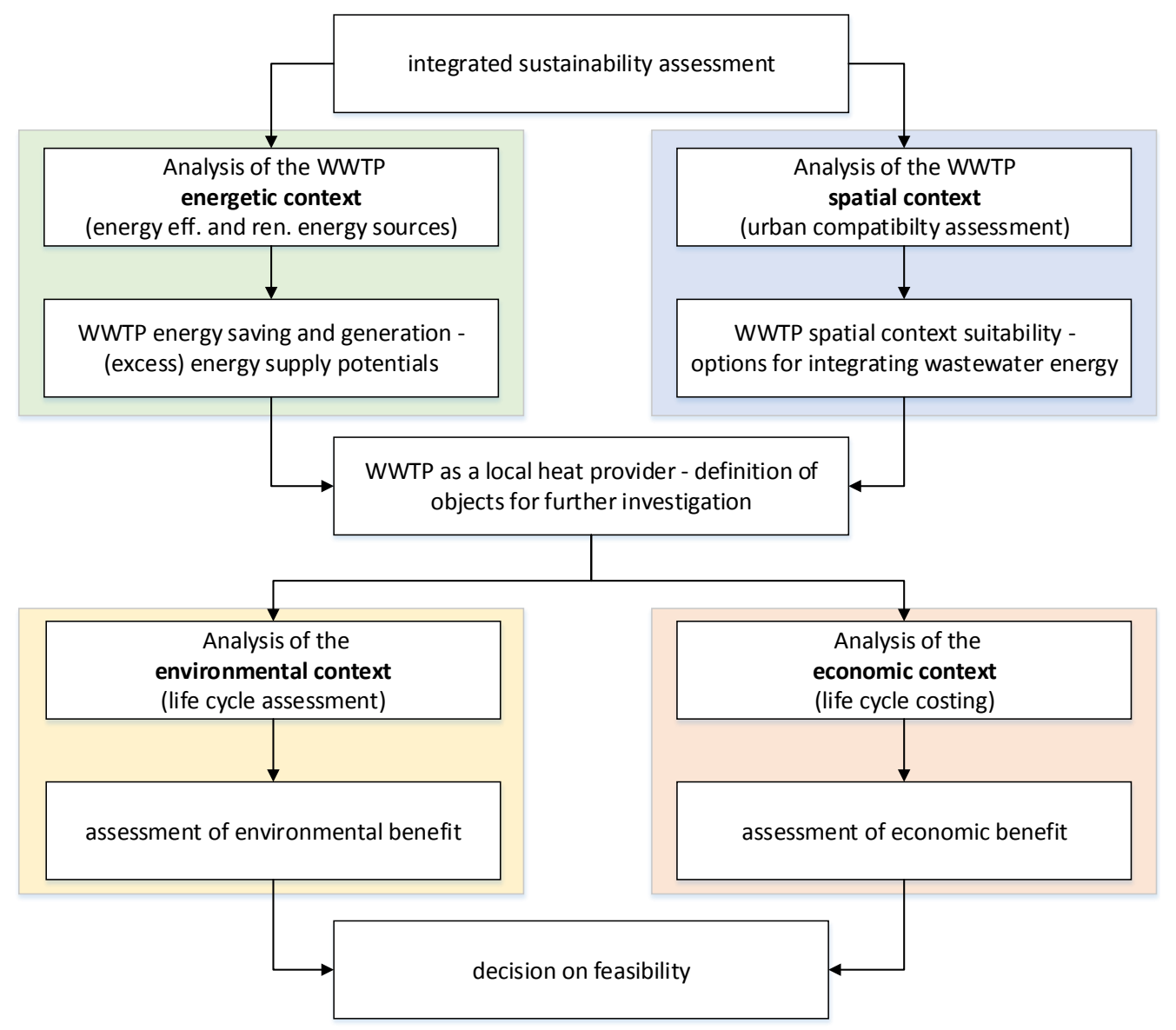

Fig. 1: Methodological approach for integrated sustainability assessment

\section{CONCLUSION AND OUTLOOK}

The authors consider the presented methodological approach an appropriate procedure to assess options for integrating a WWTP into local heat supply systems from an integrated perspective. Although currently still a theoretical concept, the presented approach will be applied and validated practically at five different case study sites across Europe during the next months (also considering WWTP borne electricity and biomethane supply).

The related work is being carried out within the framework of the ongoing INTERREG Central Europe project REEF $2 \mathrm{~W}$ - Increased renewable energy and energy efficiency by integrating, combining and empowering urban wastewater and organic waste management systems (http://www.interreg-central.eu/Content.Node/REEF-2W.html).

\section{REFERENCES}

Frijns, J., Hofman, J., Nederlof, M. (2013), The potential of (waste)water as energy carrier. Energy Conversion and Management 65, 357-363.

Glatzl, W., Meitz, S., Buchmaier, J., Hammerl, B., Schnitzer, H., Nowak, O., Mayr, B., (2017), Planungsleitfaden Energie und Wertstoffe aus Abwasser (planning guide energy and resources from wastewater). BMVIT, Vienna. 
Kollmann, R., Neugebauer, G., Kretschmer, F., Truger, B., Kindermann, H., Stoeglehner, G., Ertl, T., Narodoslawsky, M., (2017), Renewable energy from wastewater - Practical aspects of integrating a wastewater treatment plant into local energy supply concepts. Journal of Cleaner Production, 155, 119-129.

Kretschmer, F., Neugebauer, G., Kollmann, R., Eder, M., Zach, F., Zottl, A., Narodoslawsky, M., Stoeglehner, G., Ertl, T., 2016, Resource recovery from wastewater in Austria: wastewater treatment plants as regional energy cells. Journal of Water Reuse and Desalination, 06.3, 421-429.

Remy, C., Boulestreau, M., Lesjean, B., 2014, Proof of concept for a new energy-positive wastewater treatment scheme. Water Science and Technology, 70 (10) 1709-1716. 


\title{
EXPERIMENTAL STUDY OF COLOMBIAN COFFEE PARCHMENT PELLETS COMBUSTION
}

\author{
Raiza Manrique, Daniela Vásquez, Carlos Mario Ceballos, \& Farid Chejne* \\ Group of Applied Thermodynamics and Alternative Energies (TAYEA) \\ Facultad de Minas, Universidad Nacional de Colombia \\ Carrera 80 \# 65 - 223, M3-214. Medellín, Colombia \\ Phone: +5744255333 \\ E-Mail: fchejne@unal.edu.co
}

\section{SUMMARY}

Since Colombia is a coffee producer country in the world, there is a large amount of waste, one of which is coffee parchment. It represents about $12 \mathrm{wt} . \%$ of coffee grain. In the country, coffee parchment represented $1000 \mathrm{MJ}$ in 2016. However, this type of biomass presents several challenges related to moisture content, irregular shapes, and low-bulk density, which makes the use of current burners, for energy production, difficult. This study analyzes the combustion of coffee parchment pellets in order to design a high-efficiency burner to produce energy. The pellets were prepared in a pelletizing with $15 \%$ moisture and $20 \%$ yield. Additionally, an experiments with the pellet and separate material in a lateral reactor in bench scale was realized to understand the combustion process and the effects in the material under agglomerate. These results were allowed to determine the oxidation time, fouling and slagging factors and dimensions of a parchment coffee's pellets burner's proposal.

Key-words: Combustion, Biomass , Pellet, Energy Efficiency, Coffee Waste.

\section{CONFERENCE TOPIC}

Energy and Resource Recovery from Low-grade Sources 


\title{
FINAL RENOVATED SOCIAL HOUSING TO PH STANDARD WITH DISTRICT HEATING, CO2 EMISSIONS OF FUTURE ENERGY SYSTEMS
}

\author{
Søren Riis Dietz, Bjerg arkitektur \\ Kongensgade 7, Hjørring, Denmark \\ Phone: +45 2945 2854; sd@bjerg.nu
}

\section{Primary energy systems change to fossil free fuels - what are the $\mathrm{CO} 2$ emissions of heating and cooling of deep energy renovated buildings?}

European countries are to implement EU directive for NZB buildings in 2018. Amongst it's recommendations the directive points out that the primary energy needs to be considered. In Denmark approx. 2/3 of new housing are already heated with district heating. The State Building Research Institute recommends a level of $66 \%$ in order to make the building stock "Energy neutral" in 2050 [Energy-neutral Construction- Definition and future role in society, YEAR $2014^{14}$ ].

Thus the Top-down scenario to reach energy neutrality for buildings by district heating is in place in Denmark - or is it?

As the climate window to act narrows, it becomes increasingly important to check the top down scenario for a $\mathrm{CO}^{2}$ eq. low emission society for necessary crossover improvements to reach the overall climate $2{ }^{\circ} \mathrm{C}$ target of the Paris agreement. A investigation of real and future energyfactors based on deep energy renovated buildings shows potentiel for $\mathrm{CO} 2$ low emission society.

Also renovated buildings to PH standard use little energy for heating and the heating period is short approx. from Dec- Mar. Is a scenario with biomass supplying the primary energy for a future district heating system with a short heating peak period realistic?

The $2^{\circ} \mathrm{C}$ Climate Scenario limits emission to 2 tons/person in Denmark. As $40 \%$ of energy use is related to buildings roughly $800 \mathrm{~kg} \mathrm{CO} 2$ eq emissions could be a way to limit and to analyse the green potential of total emission from building related energy. When the heat demand drops by a factor $10-$ the emissions from the net energy mix, system and losses become relatively high compared to the demand and losses in the building.

In a bottum-up case these two aspects are analysed in PHPP with different Energy factors

\section{Fossil free energy systems}

In Toftebo, a social housing complex from 1950 has been renovated to meet PH standard, heated with district heating from two heating plants with a mix of 6 different energy fuels, $74,1 \%$ are fossil free. The PHPP calculated heating demand is reduced with $93 \%$ for. The primary energy is calculated using different factors to see how different factors influence the emissions. Results are listed in $\mathrm{CO} 2$ eq./person. In this way it is possible to question the primary factors and the total emission chain of energy system.

$20 \%$ net loss are included in the calculated future energyfactors listed In the rapport "Energy neutral" in 2050. Toftebo housing complex uses an energy mix of two heating plants. More than half of the fuels are "bio fuels". In this case PE factors for each fuel type are used to calculate a real PE factor for the district heating. The PHPP calculation shows an PE factor of 0,997 for biogas and 0,554 for firewood.

This significant influence of fuel-type on PE factor is not accounted for in the rapport "Energy neutral" in 2050.

\footnotetext{
${ }^{14}$ https://sbi.dk/Assets/Energineutralt-byggeri-Definition-og-fremtidig-rolle-i-samfundet/energineutralt-byggeridefinition-og-fremtidig-rolle-i-samfundet.pdf
} 


\section{Lessons learned}

In the future, District heating needs to be based on fossil free fuels. The case study shows that calculated primary factors are close to the future values for district heating with biomass. Still the definition of fuel based prim/end factors are not precise and information is lacking.

\section{Resume}

Results of End energy and Primary energy related CO2 eq. emissions differ with 59\%, with the highest emission using calculated PE factors from the energy fuel mix of the real district heat system. The future and the Danish Building Code primary factors for district heat is reduced from a factor of 0,8 in 2015 to a factor of 0,6 in 2020 (2018 EU directive). Future real factors for district heat on biomass fuels 2035 and 2050 are calculated to 0,88 and 0,97 . The 2050 value comes close to the calculated real value of 0,997 , but it seems that the needed extra primary energy to make up for $20 \%$ net loss is not taken into consideration in the building code values or even in the future values. The results of the rise in Prim/End factor for district heat biomass is more than taken up for by a reduction in the Prim/End factor for electricity. In the period from 2015 building code for Prim/End elec. drops from factor 2,5 to 1,13 for wind electricity in 2050. The ventilation energy related emissions in this way is reduced remarkably. But as the building 2015 and 2020 code is the foundation for final renovation of many projects, it should be discussed if they also lead to the strategic right solutions and decisions. It is argued in this paper that the net loss is not calculated in the future primary energy factors and therefor only End energy of the building demand should be taken into consideration for strategic energy reduction and renovation decisions.

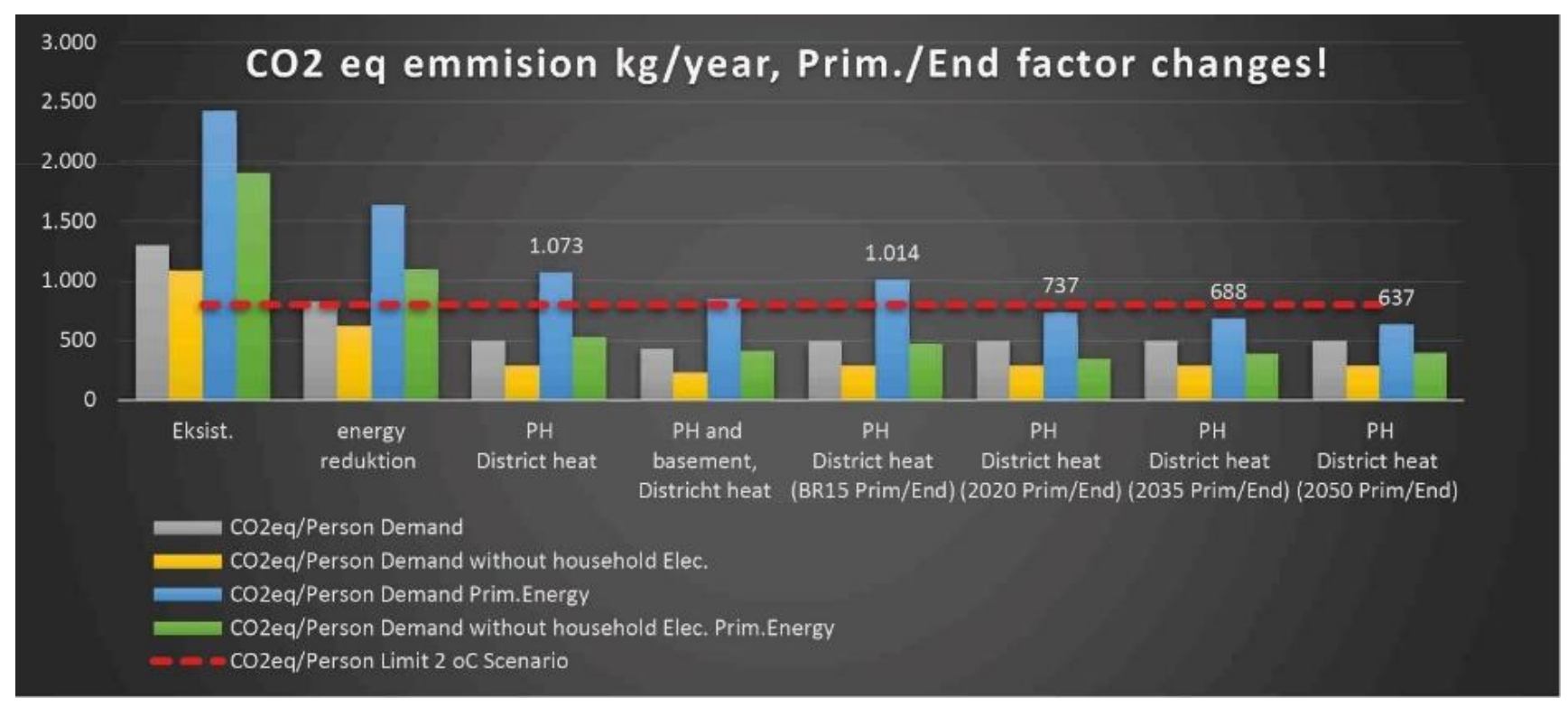

Figure 1 Result of $\mathrm{CO} 2$ eq. emission with real and future energy factors. 


\title{
ENERFUND - MAPPING THE ENERGY EFFICIENCY OF BUILDINGS TO ASSIST IN DECARBONIZING THE EUROPEAN BUILDING STOCK
}

\author{
Dr Susanne Geissler \\ SERA energy \& resources e.U. Consulting Engineers \\ Mariahilfer Str. 101/21, 1060 Wien, Austria \\ Phone: +4369910027427 \\ E-Mail: s.geissler@sera.global
}

\section{SUMMARY}

The European building sector accounts for $40 \%$ of energy consumption. It is urgently necessary to increase in the renovation rate in order to meet European energy efficiency and climate protection targets. Georeferenced building information supports the development of large-scale renovation projects and helps to exploit renewable energy potentials. The ENERFUND project has been developed to demonstrate the usefulness of georeferenced building information and to initiate discussions in participating Member States: https://app.enerfund.eu/.

\section{INTRODUCTION}

In Europe, buildings account for $40 \%$ of energy consumption. While recently constructed buildings have been targeted by stringent energy efficiency requirements imposed by European Directives, the major share of building stock needs to undergo renovation in order to improve energy efficiency as a condition to meet European energy efficiency and climate protection targets. However, in average, the renovation rate is still below $1 \%$ instead of $3 \%$, and thus dramatically lagging behind. (European Commission, 2016)

Renovation is often difficult and expensive due to ownership conditions, technical conditions, and the fact that projects are small in financial terms. European Directive (EU) 2018/844 amending European Directive 2010/31/EU (Energy Performance of Buildings Directive - EPBD) allows balancing energy consumption with renewable energy production under specific conditions and addresses the aggregation of small-scale projects to form packages which are more cost-efficient and easier accessible for investors. Georeferenced building information supports development of large-scale renovation projects and helps to exploit renewable energy potentials. This paper describes the ENERFUND tool developed to make use of building data generated by Energy Performance Certificates (EPCs) having been issued according to Directive 2010/31/EU and the previous Directive 2002/91/EC. Furthermore, the relevance of georeferenced EPCs for energy spatial planning is shown.

\section{POINT OF DEPARTURE FOR THE ENERFUND INITIATIVE: AVAILABILITY OF DATA}

The Energy Performance of Buildings Directive 2010/31/EU (EPBD) is one of the most important policy instruments aiming at improving the energy efficiency of the European building stock in order to achieve nearly zero energy standard which implies also the use of renewable energy technologies. Although not explicitly required by the current version of the EPBD, most countries have set up EPC databases to facilitate implementation of procedures in practice, such as setting up an independent control system according to article 18 EPBD. Databases do not only contain EPCs but also input data necessary for calculating the building energy performance, and interim calculation results, depending on the specific upload mechanism defined between energy performance calculation software and the database. This means that a large number of EPCs and building related data are available throughout Europe, although not all data are publicly accessible in each Member State. In some countries, strict data privacy rules apply, and EPCs are not published at all. In other countries, part of the EPC is considered public information accessible for everybody. 
It is normal to think about opportunities which might emerge from making use of EPC databases, in addition to showing compliance with the EPBD. (Geissler and Altmann, 2015) In Ireland, a method was developed how to aggregate EPC data to a level still compliant with data privacy rules and also appropriate to deliver information about energy related properties and the improvement potential of larger areas, in order to use this information for policy development (e.g. addressing energy poverty) and evaluation at the same time (see Fig. 1). Work was carried out in the EU-funded project EPISCOPE completed in 2016.

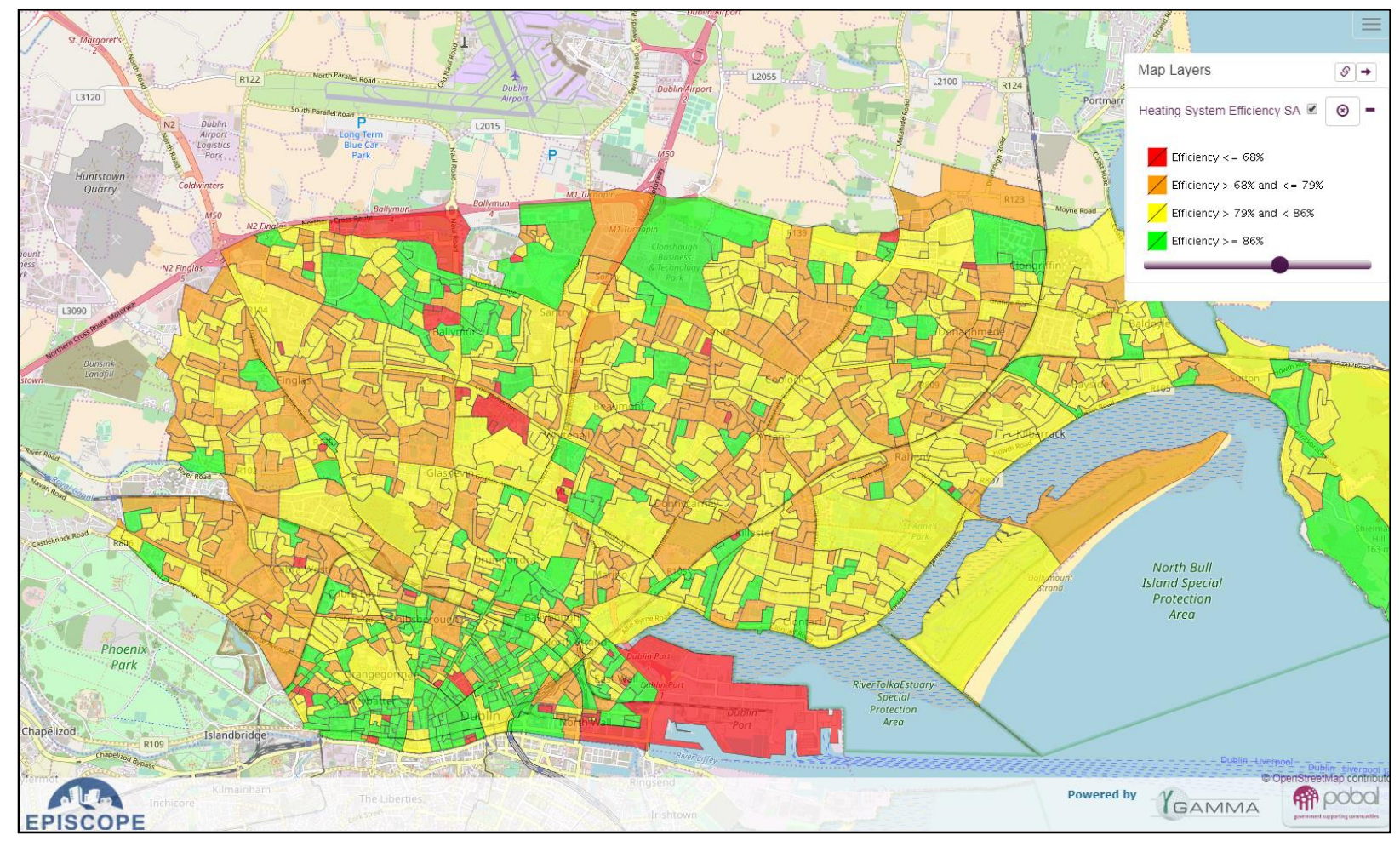

Fig. 1: EPISCOPE map, example from Ireland

However, EPCs represent only one source of building related energy data. Monitoring and collection of building energy consumption data has been spreading, there are open data initiatives on European and national level, data on social and economic aspects are publicly accessible, and developments such as the digitalization of the building sector and the increasing use of georeferenced data in general can be observed. There is the European INSPIRE framework to ensure that data formats are compatible, allowing for data analysis across Europe.

\section{ENERFUND TOOL: MAKING USE OF OPEN DATA TO IDENTIFY AREAS WITH HIGH POTENTIAL FOR IMPROVING BUILDING ENERGY PERFORMANCE}

The above said demonstrates that the combination of different data sources opens up entirely new possibilities to identify and assess building renovation opportunities, and to provide support for decision making to different target groups. Here, the ENERFUND project comes in. The project An ENErgy Retrofit FUNDing rating tool is funded under HORIZON 2020 Grant Agreement No 695873, started on 1 February 2016 and has a duration of 36 months. ENERFUND builds on national initiatives and the results of the above-mentioned EPISCOPE project to develop the ENERFUND tool (see Fig. 2). The tool is based on a GIS smart map of Europe where available, and compatible open source data are embedded. The methods of data selection, data aggregation and data presentation were developed in the first phase of the project. The tool is based on open data and specific interfaces allowing for automatic updates, in order to ensure smooth operation of the tool with a long-term 
perspective. In early June 2018, the tool included data from 10 European Member States, and more countries are in the process of being connected to the ENERFUND tool. The web application is available at http://app.enerfund.eu/ and allows the users to single out countries, regions or cities, in order to screen them for deep renovation opportunities. Filtering options make it easy for users to select buildings according to several parameters of their preferences, such as EPC rating, year of construction, $\mathrm{CO}_{2}$ emissions, building type or other building characteristics. In the light of the provisions of European Directive (EU) 2018/844 amending European Directive 2010/31/EU, the ENERFUND project demonstrates a promising approach how building related energy data can be processed in order to assist in developing large-scale renovation projects contributing to decarbonizing the European building stock.

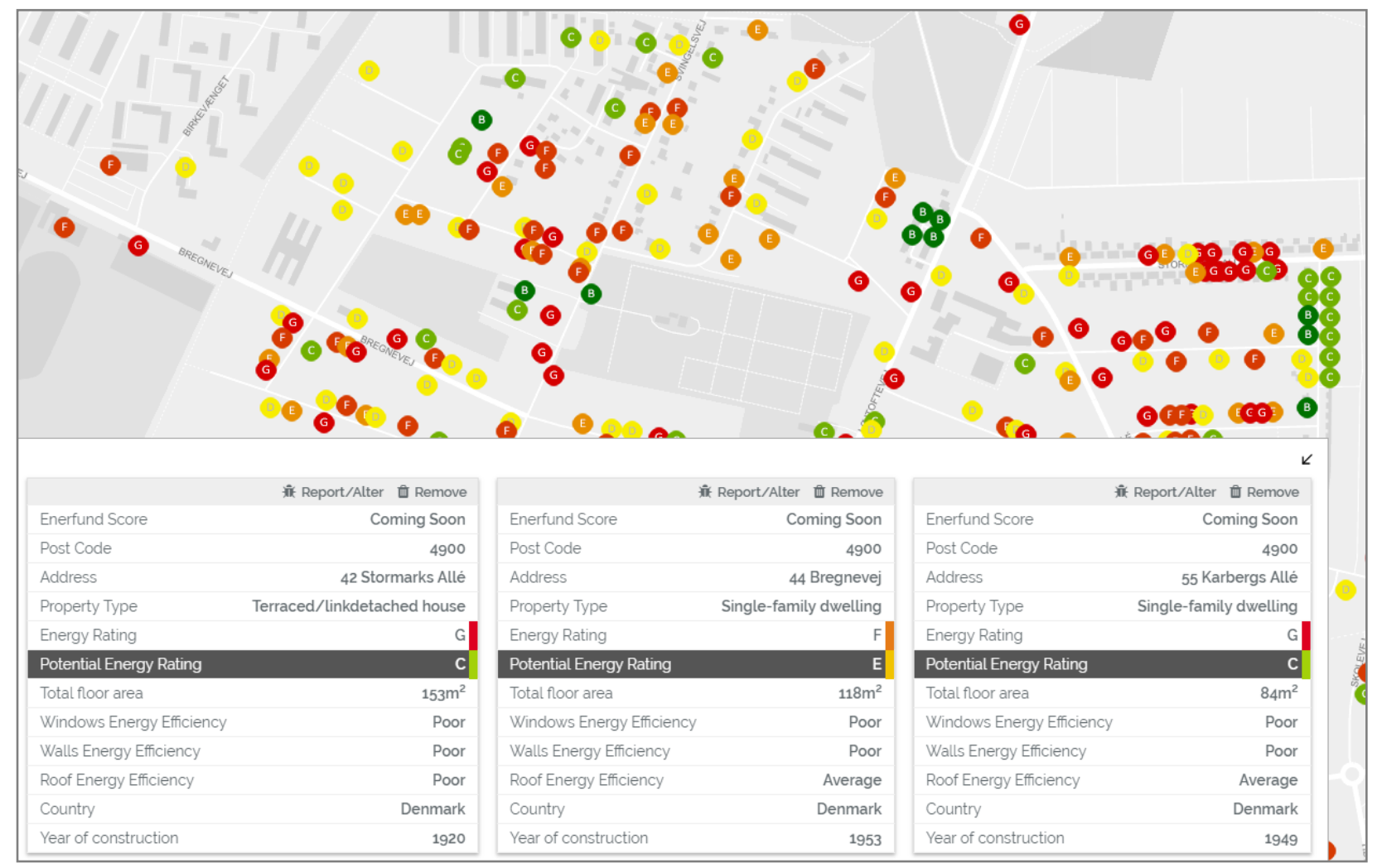

Fig. 2: ENERFUND map, example from Denmark

\section{ENERFUND TOOL SUPPORTS ENERGY SPATIAL PLANNING}

Strategies for decarbonizing the building sector rest on two pillars: first, increasing energy efficiency to reduce energy demand, and second, utilizing renewable energy sources to cover the remaining energy needs. In the light of the German Energiewende one could argue that energy efficiency strategies have lost importance. However, although the theoretical renewable energy potential is inexhaustible by human standards, the technical potential is certainly limited due to conflict of interests regarding land use. Spatial planning deals with land use tradeoffs, and it is obvious to pay specific attention to utilizing renewable energy sources depending on aspects such as the nature of the landscape, availability of geothermal energy, and meteorological conditions (see for example EU project SPECIAL). Energy spatial planning is based on various data sets to develop energy concepts tailored to the requirements of neighborhoods, districts, cities, or regions. Tools such as the ENERFUND App display georeferenced energy characteristics of buildings based on EPC data and thus provide valuable information about energy demand profiles. Among others, such tools have the 
potential to show in which areas energy demand is likely to decrease due to economic renovation opportunities, and this information supports supply-side decision making regarding investments in renewable energy systems and storage capacities. It is evident that the success of such a tool depends on three criteria: (1) quality of EPCs, (2) coverage of the building stock with EPCs, and (3) accessibility of data. While some Member States meet all these criteria, there is substantial room for improvement in Austria.

\section{CONCLUSION AND OUTLOOK}

The ENERFUND tool assists companies in identifying sound business opportunities, municipalities in promoting targeted incentives, and policy makers in strategic decision making. It is based on selected EPC data and other open data, and helps different types of users to make the first step towards largescale building renovation projects aiming at implementing energy efficiency measures and renewable energy technologies. It is evident that the ENERFUND tool cannot provide detailed investment-grade information for decision making. However, this was not the intention of ENERFUND because there are other tools available for this purpose. Current developments regarding energy spatial planning based on renewable energy technologies emphasize the importance of making georeferenced energy related building data accessible for decision making. The combination of up-to-date building data with natural resources data such as solar radiation and other information will contribute to the development of transparent and effective strategies targeting the reduction of $\mathrm{CO}_{2}$ emissions and increasing energy efficiency towards a decarbonized European building stock.

\section{REFERENCES}

ENERFUND - ENErgy Retrofit FUNDing rating tool: http://enerfund.eu/ (15.6.2018).

EPISCOPE - Energy Performance Indicator Tracking Schemes for the Continuous Optimisation of Refurbishment Processes in European Housing Stocks: http://episcope.eu/index.php?id=97 (15.6.2018).

European Commission, (2016), Fact Sheet, Putting energy efficiency first: consuming better, getting cleaner http://europa.eu/rapid/press-release MEMO-16-3986 en.htm (15.6.2018).

Geissler, S., Altmann, N., (2015), CT1 Thematic Report - How to make the best use of EPCs, available at: http://www.epbd-ca.org/ (15.6.2018).

INSPIRE - Infrastructure for spatial information in Europe: https://inspire.ec.europa.eu/ (15.6.2018).

SPECIAL - Spatial Planning and Energy for Communities In All Landscapes: http://www.specialeu.or g/ (15.6.2018). 


\title{
BUSINESS MODEL FOR SUSTAINABLE HEAT SUPPLY CONTRACTING OF QUARTERS
}

\author{
DI Gerhard Bayer/Dr. Katharina Sammer \\ Austrian Society for Environment and Technology (ÖGUT) \\ Project SEFIPA Sustainable Energy Financing in Austria \\ Hollandstrasse 10/46, 1020 Vienna, Austria \\ Phone: +4313156393 \\ E-Mail: gerhard.bayer@oegut.at ; katharina.muner-sammer@oegut.at
}

\section{SUMMARY}

Sustainable Energy Systems often require high investment costs, while they cause low operating costs during the life span. Thus in rented apartments there is a conflict of interest, as the investment cost must be borne by the landlord, while the tenant benefits from low operating costs. Based on this, sustainable energy systems are rarely implemented in renovated apartment houses and the cheapest solution for the landlord - often natural gas heating systems - are favoured. The project "smart block", financed by the Klima und Energiefonds and the City of Vienna as well as the EU-Project "SEFIPA" compared the overall costs of different heating systems for typical existing Viennese buildings. It appears, that a sustainable energy system with a heat pump (SPF 6), solar, geothermal storage and an anergy network shows about the same overall costs as a natural gas heating system.

In spring 2018 a pilot project has been started in which the first building phase of 2 houses (25 apartments) is already under construction. The business model, based on heat supply contracting provides a fair sharing of costs between landlords and tenants. Further 5 buildings will join the system within the next years.

\section{FULL COST ANALYSES OF HEATING SYSTEMS}

For retrofitted existing Viennese buildings, a full cost analyses for a heating system (heating and hot water) has been carried out. For an "average building" following data has been assumed:

- $1.000 \mathrm{~m}^{2}$ space floor

- 15 rented apartments

- 4 story building

- Thermal retrofitted, heating demand $50 \mathrm{kWh} / \mathrm{m}^{2} . \mathrm{a}$

- Natural gas network in the building and in all apartments, district heating connection at property line

The analyses included the costs for investment, service, maintenance and metering as well as the energy costs. In addition the expenditures of a moderate cooling system have been included in the comparison.

Many landlords as well as tenants are not aware of the amount of the overall costs, as they often only consider the direct energy costs (tenants) or only the investment costs (landlord). The cost analysis includes already existing subsidies for the energy systems.

Following heating systems have been analyses:

- Central natural gas heating system

- District heating

- Heat pump, hybrid solar collectors, geothermal drillings with seasonal heat storage, 
Furthermore, the system with heat pump and geothermal storage can also provide moderate cooling for the apartments. Considering the cooling service and the subsidy by the City of Vienna, the full cost analyses shows that the heat pump/geothermal/solar system is cheaper than the natural gas system within a period of 20 years.

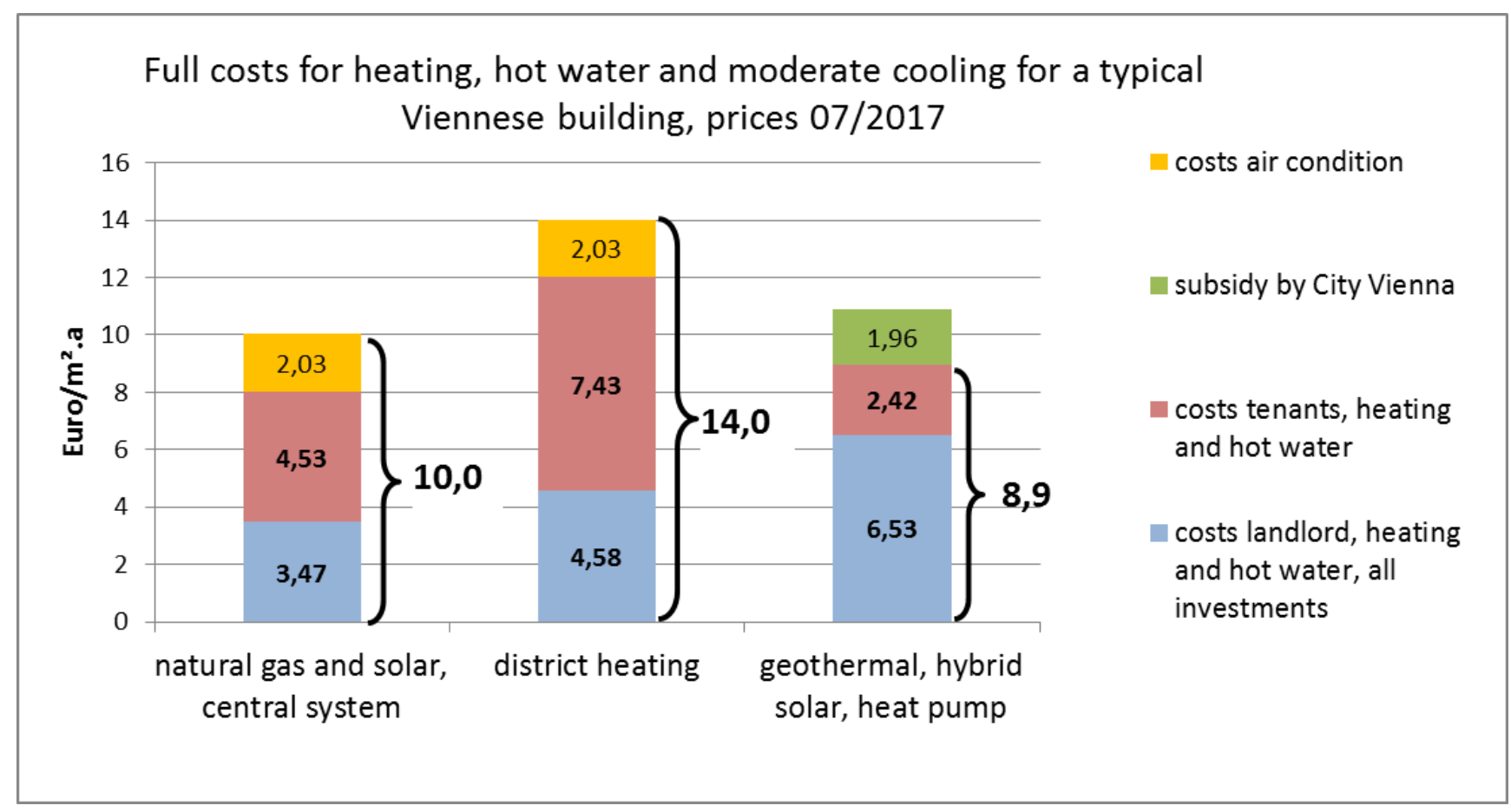

Fig. 1: Full cost analyses for heating and hot water, considering all investments and running costs within a period of 20 years, if the landlord covers all investment costs. Costs in Euro/ $\mathrm{m}^{2} \mathrm{floor}$ space and year,based on the prices $07 / 2017$

\section{ENERGY SYSTEM FOR A PILOT PROJECT IN 1170 VIENNA}

For a block of houses in 1170 Vienna a pilot project with an energy system and an anergy network (water with $5-25^{\circ} \mathrm{C}$ ) has been developed. Each building provides its resources as geothermal storage, solar surface or available space for technical facilities to the joint system. An anergy network connects all participants. Using the synergy effects the network leads to lower investment costs and higher energy efficiency (SPF) compared to a single building solution.

At one building there are already existing geothermal drillings for a heat pump since 15 years and the drillings have not been reloaded. Within the project it is planned to connect these drillings with the anergy network, to reload them and therefor to improve the seasonal performance factor (SPC).

Within the project also the opportunity of drillings at the pavement, inclined under the buildings has been surveyed, but drillings from the courtyards, partly inclining under the buildings have been preferred. 


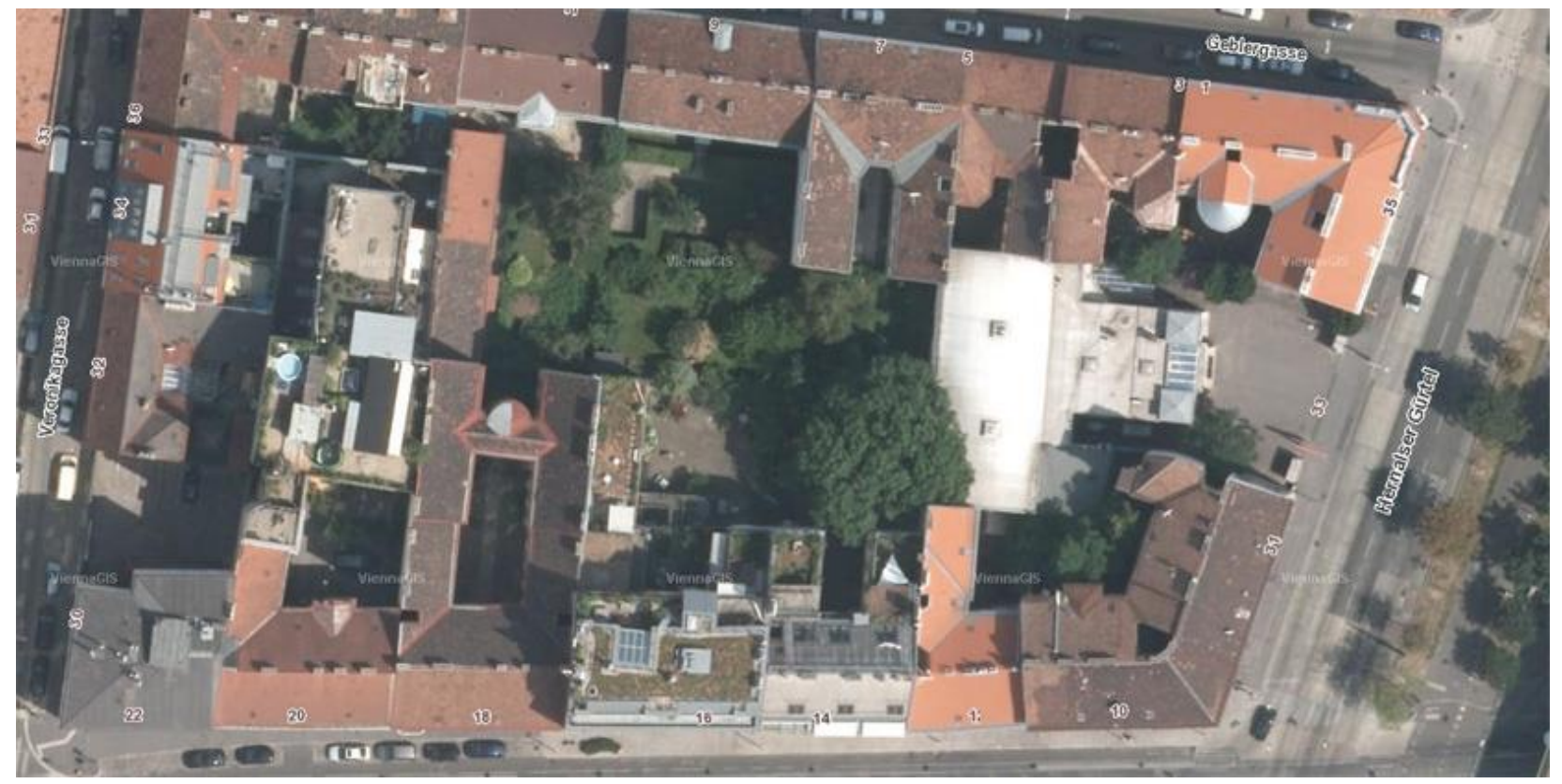

Graph 1: Aerial photo of the building block in 1170 Vienna, where the pilot project is being implemented

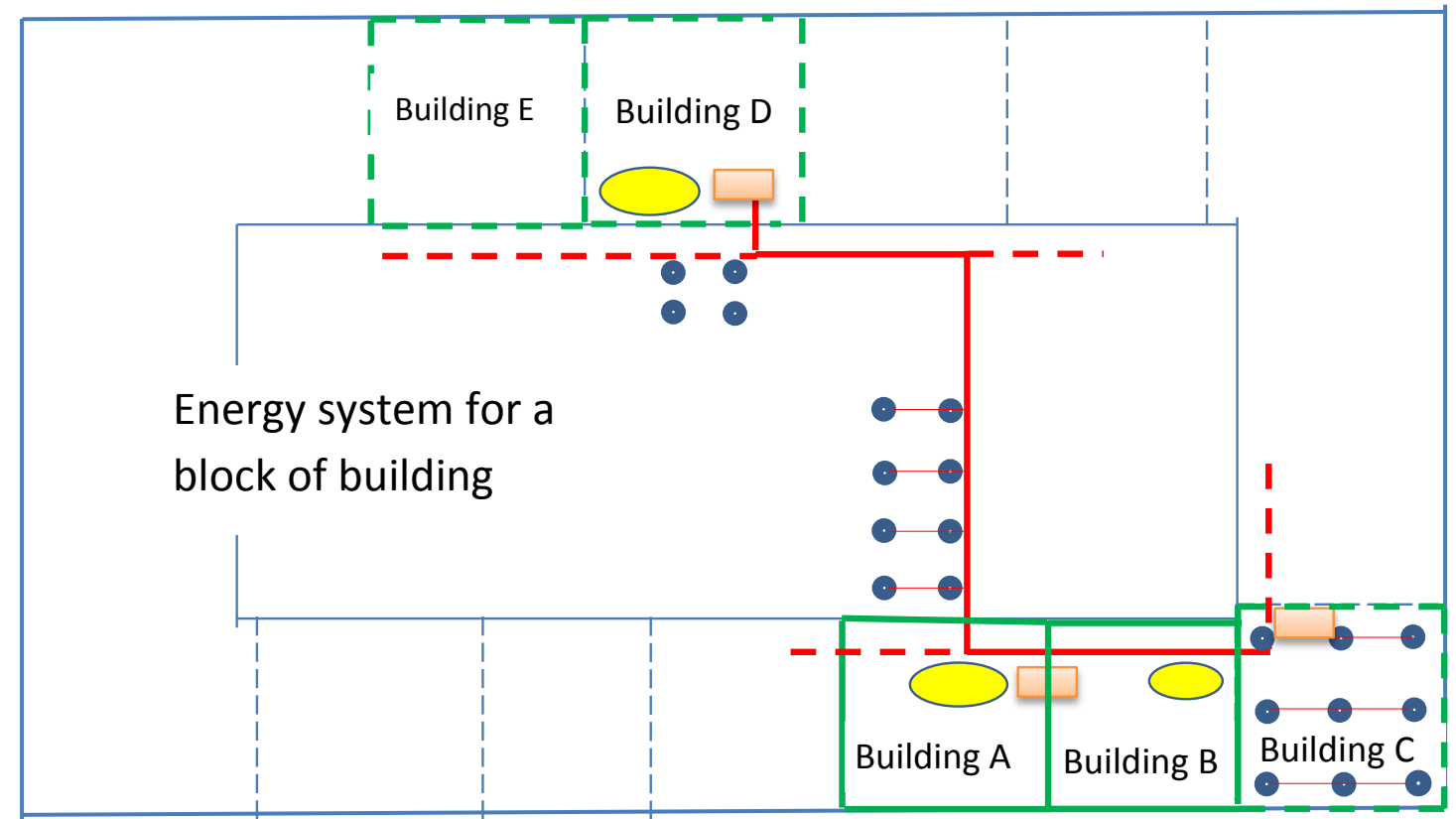

thermal solar

- - Anergy network $\left(5-25^{\circ} \mathrm{C}\right)$

geothermal - deep drilling

heat pump

Fig. 2: Overview of an energy system for a building block with geothermal drillings, heat pumps, thermal solar as well as an anergy net that connects the buildings.

A challenge for the implementation of the drillings is the constricted room in the courtyards and the narrow front doors of the buildings leading into the courtyard. Moreover in the Region of Vienna only 
a few companies with small scale drilling equipment are offering their services. In Western Austria as well as in Southern Germany these services are already widespread.

\section{Contact data for pilot Project "Heat Supply Contracting 1170 Vienna"}

\section{Architect: zeininger architekten}

Johannes Zeininger, A-1070 Vienna, Stuckgasse 3

T: 0043(0)1-5262600, E: office@ zeininger.at, www.zeininger.at

\section{Contractor: BauConsult Energy GmbH}

Franz Vogl, Vorgartenstraße 206 C, 1020 Vienna

M +43 66441274 51, M +43 190535 00, E: f.vogl@bauconsult.com, www.bauconsult.com

\section{HVACR planning: TB Käferhaus GmbH}

Jochen Käferhaus, Ybbsstrasse 29/1, A-1020 Wien

Tel.:+43 1 9686064, E: kaeferhaus@kaeferhaus.at, www.kaeferhaus.at

\section{BUSINESS MODEL SUPPLY CONTRACTING FOR FAIR COST SHARING}

In Austria, due to the Act on Tenancy Law (MRG), landlords may not transfer investment costs to the tenants, if they own the heating system. Therefore, until now, systems with high investment costs are implemented rarely in this sector. The business model ,heat supply contracting" can solve this problem. In this model, a private company invests in the energy system and compiles a contract with the landlords and the tenants to deliver them heat for the next 20 years at a certain price. After this period there is the option for a further contract period.

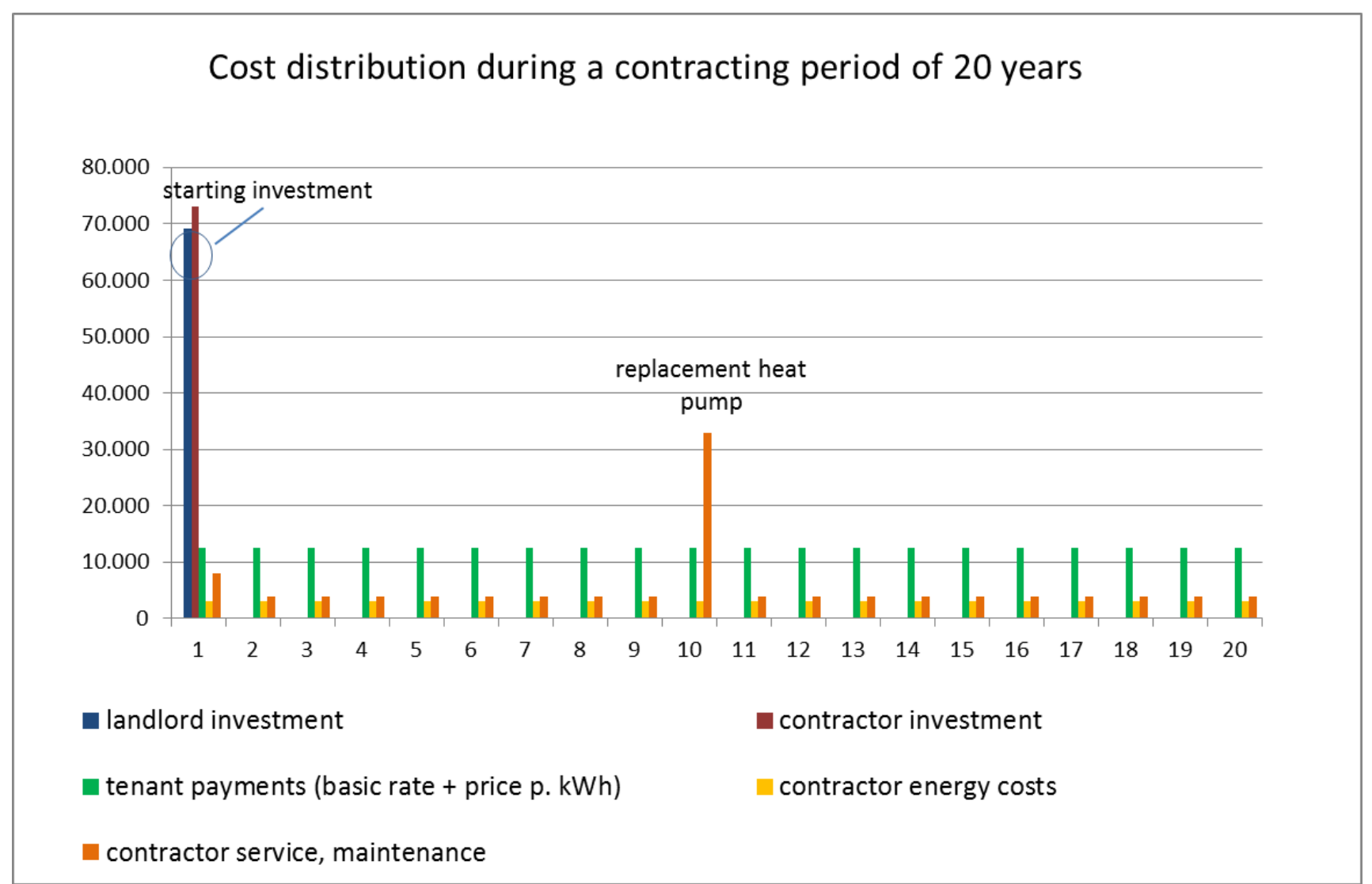

Fig. 3: Distribution of full costs between landlord, contractor and tenants during the contracting period of 20 years for a heat supply system with heat pump, geothermal drillings and hybrid solar.

Regarding to the MRG the contractor is allowed to distribute the full costs between landlord and 
tenant in a way which is fair to both parties. Usually the landlord takes over a share of the investment costs while the tenants pay annually energy costs, divided in basic costs per $\mathrm{m}^{2}$ floor space and variable costs per $\mathrm{kWh}$ heat. An agreement between landlord and contractor manages the financial risk in case of unoccupied flats.

\section{CONCLUSION}

The pilot project shows, that decentralized sustainable heat systems as heat pumps with seasonal geothermal storage are economically capable of competing with fossil systems like natural gas, oil or with district heating. If the heat system is realized on the size of a building block by using an anergy net, substantial synergies and cost decreases can by gained.

To implement a sustainable heat supply for existing urban regions following requirements must be fulfilled:

- Technical solution: Therm. Retrofitting, seasonal heat storage by deep drillings, solar and heat pumps, SPF $>6$

- Organizational solution: joint energy system for a block of building: economy of scale and gaining synergies

- Economic solution: Investment and operating by Supply contractor. High investment costs shared between landlord and tenant

- Fair share of investment costs between tenant and landlord

- Stable conditions, planning security for the heat supply contractor

$\circ$ Comparison of full costs of heating systems (e.g. 20 years period) 


\title{
AN ANALYSIS OF HEAT PUMPS FOR INDUSTRIAL APPLICATIONS
}

\author{
Alexander Arnitz1, René Rieberer1, Veronika Wilk2 \\ Institute of Thermal Engineering, Graz University of Technology 1 \\ Inffeldgasse 25B, $8010 \mathrm{Graz}$, Austria \\ Phone: +43 316873 7801, E-Mail: alexander.arnitz@tugraz.at \\ AIT Austrian Institute of Technology GmbH, Center for Energy 2 \\ Giefinggasse 4, A-1210 Vienna
}

\begin{abstract}
SUMMARY
Since the year 2000 the sales figures of heat pumps in Austria show a strong growth. However, the market is dominated by heat pumps for heating and hot water preparation in residential buildings. In contrast to this application industrial heat pumps seem to play a minor role. To emphasize the industrial heat pump market this paper highlights possibilities in a growing market. For some industrial applications absorption or compression heat pumps can be used. Therefore, a simple diagram is derived, which enables a rapid estimation of the operating cost ratio of both heat pump types using the $\mathrm{COPh}$ ratio and energy cost ratio as inputs. This estimation shows a substantial influence of energy carrier prices. In a next step the payback periods for the replacement of a natural gas heating boiler with a compression and an absorption heat pump are calculated. Finally, the main influencing parameters (efficiency, investment costs, energy prices, heating capacity and operating hours) are varied to determine their impact on the payback period.
\end{abstract}

Keywords: absorption, compression, operating cost estimation, sensitivity analysis

\section{INTRODUCTION}

Industrial heat pumps allow for utilization of waste heat and are therefore important energy efficiency measures. In industrial processes, different heat sources are available, ranging from cooling water streams to exhaust gas streams that otherwise are rejected to the environment. In this work both compression and absorption heat pumps are considered. Compression heat pumps are - in this work electrically driven, absorption heat pumps require a hot stream as driving energy, such as process steam, which is also commonly available in industrial processes. In this case an absorption or compression heat pump can be selected for a certain application. Therefore industrial heat pump applications are analyzed with regard to the expected operating costs and payback periods for both types of heat pumps to provide decision support.

\section{ENERGY PRICES AND INVESTMENT COSTS}

For the determination of the operating costs of a heat pump system the costs for the driving energy have to be known. Furthermore, a calculation of the payback period requires information about the investment costs. For this, the energy price ranges of electricity, biomass and natural gas are discussed and cost functions for the investment costs of compression and absorption heat pumps are shown.

\section{Energy prices}

The energy prices for different energy sources divided for small customers and large industry are shown in Table 1. The electricity prices have been taken from e-control (2018). For a small customer an annual electrical energy consumption of about 4-20 GWh/a is defined and for large industry the electrical energy consumption has to be higher than $150 \mathrm{GWh} / \mathrm{a}$. The prices for biomass have been taken from Biermayr et al. (2017). According to Biermayr et al. (2017) pellets are used as fuel for small capacity heating systems (heating capacity $<100 \mathrm{kWth}$ ), for this reason the prices of pellets are used as biomass energy prices of small customers. Wood chips are mainly used in medium (101 kWth $<$ heating capacity < $1000 \mathrm{kWth})$ and large capacity $(1001 \mathrm{kWth}<$ heating capacity) heating systems, therefore the prices of wood chips are used for the biomass prices of large customers (Biermayr et al., 2017). For the natural gas prices of small customers the prices of households and for large customers the prices of industry are used which were obtained from Statistik Austria (2018). 
Table 1: Energy prices of small and large customers for different energy carriers

\begin{tabular}{|c|c|c|c|}
\hline Energy carrier & $\begin{array}{c}\text { Price of small customers in } \\
\mathrm{ct} / \mathrm{kWh}\end{array}$ & $\begin{array}{c}\text { Price of large customers in } \\
\mathrm{ct} / \mathrm{kWh}\end{array}$ & Source \\
\hline Electricity & 9.82 & 5.41 & e-control (2018) \\
\hline Biomass & 4.77 & 3.34 & Biermayr et al. (2017) \\
\hline Natural gas & 6.7 & 3.5 & Statistik Austria (2018) \\
\hline
\end{tabular}

\section{Investment costs}

Information about investment costs of industrial heat pumps are not open to general public, therefore within this paper literature values are used for the calculation of the investment costs. Wolf et al. (2017) and Wolf (2017) give an overview about investment costs for industrial heat pumps including the costs for installation. They found specific investment costs Cic in a range of $250-700 \mathrm{EUR} / \mathrm{kW}$ th for compression heat pumps with heating capacities between 2 and $20000 \mathrm{~kW}$ th. For absorption heat pumps with heating capacities between 25 and $350 \mathrm{~kW}$ th they found specific investment costs in a

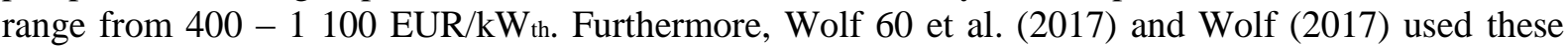
values to obtain cost functions for the specific investment costs of the heat pumps in dependence of the nominal heating capacity $Q_{t h, n o m}$ which is shown in eq. 1 for the compression heat pump and in eq. 2 for the absorption heat pump.

$$
c_{i c, c h p}=1521 \cdot \dot{Q}_{t h, n o m}^{-0,363}
$$

$$
c_{i c, a h p}=902.9 \cdot \dot{Q}_{t h, n o m}^{-0,172}
$$

According to eq. 1 and eq. 2 the investment costs of absorption heat pumps in the range from $25-350$ $\mathrm{kWth}$ are by a factor of 1.15 to 1.8 higher than the investment costs of compression heat pumps. The reason for this may be the higher number of components (heat exchangers, throttles) required to build an absorption heat pump and the additional installation costs for the external driving circuit to provide the driving energy for the absorption heat pump. Furthermore, the two typical working pairs ( $\mathrm{NH} 3 / \mathrm{H} 2 \mathrm{O}$ and $\mathrm{H} 2 \mathrm{O} / \mathrm{LiBr}$ ) require additional design work in comparison to compression heat pumps using synthetic safety refrigerants. For example when $\mathrm{NH} 3 / \mathrm{H} 2 \mathrm{O}$ is used as working pair of the absorption heat pump a useful heating temperature of $60{ }^{\circ} \mathrm{C}$ would lead to a pressure of about 30 bar. Furthermore, $\mathrm{NH} 3$ is toxic and hardly flammable therefore safety requirements have to be fulfilled which depend on the operating pressure and filling volume and increase the investment costs. When $\mathrm{H} 2 \mathrm{O} / \mathrm{LiBr}$ is used as working pair safety measures are not required but the absorption heat pump requires measures to prevent air in the system which also increase the investment costs. Finally, it has to be emphasized that the cost function of Wolf et al. (2017) and Wolf (2017) for the compression heat pumps does not distinguish between compression heat pumps using natural or synthetic refrigerants. A cost function for compression heat pumps using solely natural fluids may increase the investment costs of compression heat pumps and thus reduce the difference of the investment costs to absorption heat pumps.

\section{ECONOMIC COMPARISON OF BOTH HEAT PUMP TYPES}

For the economic comparison of the absorption and compression heat pump the operating costs and the payback period are investigated. In a first step only the operating costs are compared between the compression and absorption heat pump considering the efficiency and the energy prices. Then the payback periods of the heat pumps in comparison to a natural gas boiler are investigated to analyze the influence of the investment costs.

\section{Operating cost estimation}

For constant operating conditions the efficiency can be expressed with the $C O P h$. The operating cost estimation is based on the definition of the $C O P h$,ahp for absorption heat pumps according to eq. 4 and the definition of the COPh,chp for compression heat pumps according to eq. 5. The COPh can be obtained by the manufacturer or estimated for given heat sink and source temperatures. The efficiency 
of absorption and compression heat pumps varies with the operating conditions and behaves slightly different between both systems. In summary, compression heat pumps have advantages at low temperature differences between heat sink and source with regard to the efficiency.

$$
\begin{aligned}
& \operatorname{COP}_{h, a h p}=\frac{\dot{Q}_{c o n}+\dot{Q}_{a b s}}{\dot{Q}_{g e n}} \\
& \operatorname{COP} P_{h, c h p}=\frac{Q_{c o n}}{P_{e l}}
\end{aligned}
$$

The operating costs for $1 \mathrm{kWh}$ of heat Cheat assuming constant operating conditions can be estimated for an absorption heat pump using the thermal energy costs $c t h$ with eq. 6 and for a compression heat pump using the electrical energy costs $\mathrm{Cel}$ with eq. 7.

$$
\begin{aligned}
& C_{\text {heat }, a h p}=\frac{1}{\operatorname{COP}_{h, a h p}} \cdot c_{t h} \\
& C_{\text {heat }, \text { chp }}=\frac{1}{\operatorname{COP}_{h, c h p}} \cdot c_{e l}
\end{aligned}
$$

A division of eq. 7 by eq. 6 yields to the operating cost ratio of the compression to the absorption heat pump according to eq. 8. An operating cost ratio of 1 means similar operating costs between both heat pump types. An operating cost ratio $>1$ means the estimated operating costs of the absorption heat pump are lower and an operating cost ratio $<1$ means the estimated operating costs of the compression heat pump are lower.

$$
r_{C, \text { heat }, \text { chp } / a h p}=\frac{\operatorname{COP}_{h, a h p}}{\operatorname{COP}_{h, c h p}} \cdot \frac{c_{e l}}{c_{t h}}
$$

Fig. 1 shows the heat pump operating cost ratio of an absorption and an compression heat pump for equal heating capacities and operating hours with the COPh ratio (absorption to compression) as independent variable and the energy cost ratio (electrical to thermal) as additional parameter.

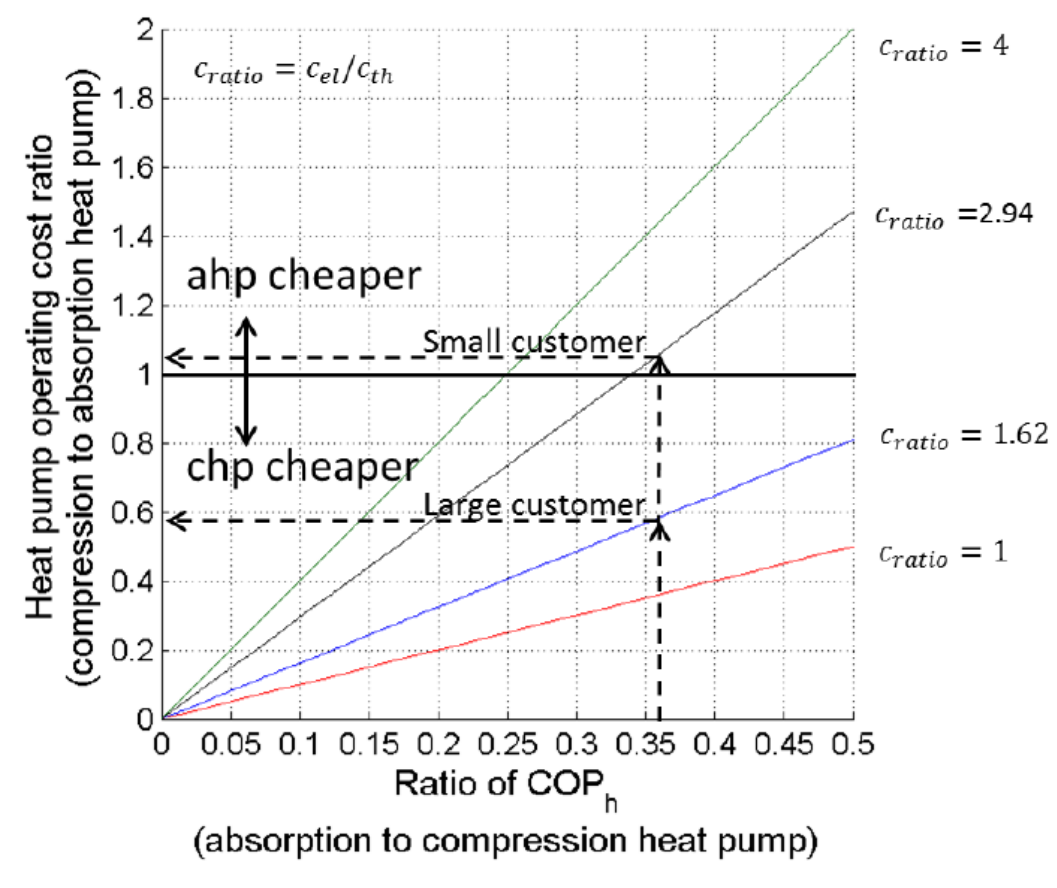

Fig. 1: Determination of the operating cost ratio (absorption (ahp) to compression (chp) heat pump) for a given efficiency and energy price ratio 
Furthermore, Fig. 1 illustrates an example for the estimation of the heat pump operating cost ratio. For a temperature difference between heat source and sink of about $25 \mathrm{~K}$, a COPh of about 1.8 for the absorption heat pump and a $\mathrm{COPh}$ of about 5 for the compression heat pump can be assumed, which leads to a $\mathrm{COPh}$ ratio of 0.36 . If the price of wood chips is used (neglecting the combustion efficiency) to determine the price for the driving heat of the absorption heat pump and the electricity price of a large customer is used to determine the energy price to drive the compression heat pump, the energy price ratio is about 1.62. At these conditions, the heat pump operating cost ratio is about 0.6 , which means heat production is more economic with the compression heat pump. If the electricity price of a small customer is used the energy cost ratio is about 2.94. In this case, the heat production cost ratio is slightly higher than 1 and the operation of the absorption heat pump is more economic. This example shows the substantial influence of the energy carrier prices on the expected operating costs.

\section{Payback period}

Investment decisions are mostly based on payback calculations, which require the investment costs and the expected return on investment as inputs. The payback period $\tau_{p b}$ is calculated according to eq. 9 using the investment costs $C_{i c}$ of each heat pump type and the difference of the operating costs $\Delta C_{o p}$ of each heat pump type to a natural gas boiler.

$$
\tau_{p b}=\frac{C_{i c}}{\Delta C_{o p}}
$$

The investment costs $C_{i c}$ are calculated with eq. 10 using the nominal heating capacity and the cost functions for the specific investment costs for the compression heat pump according to eq. 1 and for the absorption heat pump according to eq. 2 .

$$
C_{i c}=c_{i c} \cdot \dot{Q}_{t h, n o m}
$$

For the calculation of the difference of the operating costs $\Delta C_{o p}$ the operating costs are calculated for the heat pumps by the use of eq. 6 and eq. 7 and for the operating costs of the natural gas boiler an efficiency of 0.9 is assumed. The difference of the operating costs can then be calculated using the annual operating hours $\tau_{o p}$ and the nominal heating capacity according to eq. 11 .

$$
\Delta C_{o p}=\left(C_{\text {heat }, \text { gas }}-C_{\text {heat }}\right) \cdot \tau_{o p} \cdot \dot{Q}_{\text {th,nom }}
$$

The payback period is calculated for a compression and absorption heat pump using the reference conditions shown in Table 2. At these reference conditions the investment costs for the absorption heat pump are 72593 EUR and for the compression heat pump 44451 EUR. The operating costs with the absorption heat pump are about $2.27 \mathrm{ct} / \mathrm{kWh}$ th, with the compression heat pump about $1.52 \mathrm{ct} / \mathrm{kWh}$ th and with the natural gas boiler about $5.6 \mathrm{ct} / \mathrm{kWh}$ th. These values lead to a payback period at reference conditions for the compression heat pump $\tau_{p b, c h p, r e f}$ of 1.53 years and for the absorption heat pump $\tau_{p b, a h p, r e f}$ of 3.06 years. 
Table 2: Reference conditions for the payback calculations

\begin{tabular}{|c|c|c|}
\hline & Value & Unit \\
\hline$\dot{Q}_{\text {th,nom }}$ & 200 & $\mathrm{~kW}_{\text {th }}$ \\
\hline$\tau_{o p}$ & 3500 & $\mathrm{~h}$ \\
\hline$C O P_{h, a h p}$ & 1.8 & - \\
\hline$C O P_{h, c h p}$ & 5 & - \\
\hline$c_{\text {th }}$ & 4.1 & $\mathrm{ct} / \mathrm{kWh}_{\text {th }}$ \\
\hline$c_{e l}$ & 7.6 & $\mathrm{ct} / \mathrm{kWh}_{\text {th }}$ \\
\hline$c_{\text {gas }}$ & 5.1 & $\mathrm{ct} / \mathrm{kWh}_{\text {th }}$ \\
\hline
\end{tabular}

\section{Sensitivity analysis of the payback period}

To analyze the difference between the payback periods of the absorption and compression heat pump a sensitivity analysis is carried out. For this the normalized difference of the payback period $\Delta \tau p b$,norm between the compression and absorption heat pump is used which is calculated according to eq. 12 . This definition leads to a higher normalized difference of the payback period when the absorption heat pump is more expensive and to a lower normalized difference of the payback period when the compression heat pump is more expensive

$$
\Delta \tau_{p b, \text { norm }}=\frac{\tau_{p b, a h p}-\tau_{p b, c h p}}{\tau_{p b, a h p, r e f}-\tau_{p b, c h p, r e f}}
$$

Fig. 2 shows the results of a variation of the investment costs of the compression heat pump, the electricity price and the efficiency of the compression heat pump. These parameters show that the payback period of compression heat pumps can best be improved through a reduction of the investment costs followed by a reduction of the electricity costs. An increase of the efficiency of the compression heat pump has the slightest effect, but it should be noted that a high reduction has to be avoided as the nonlinear influence of the efficiency on the heat production costs would lead to a relevant decrease of the normalized payback period difference. 


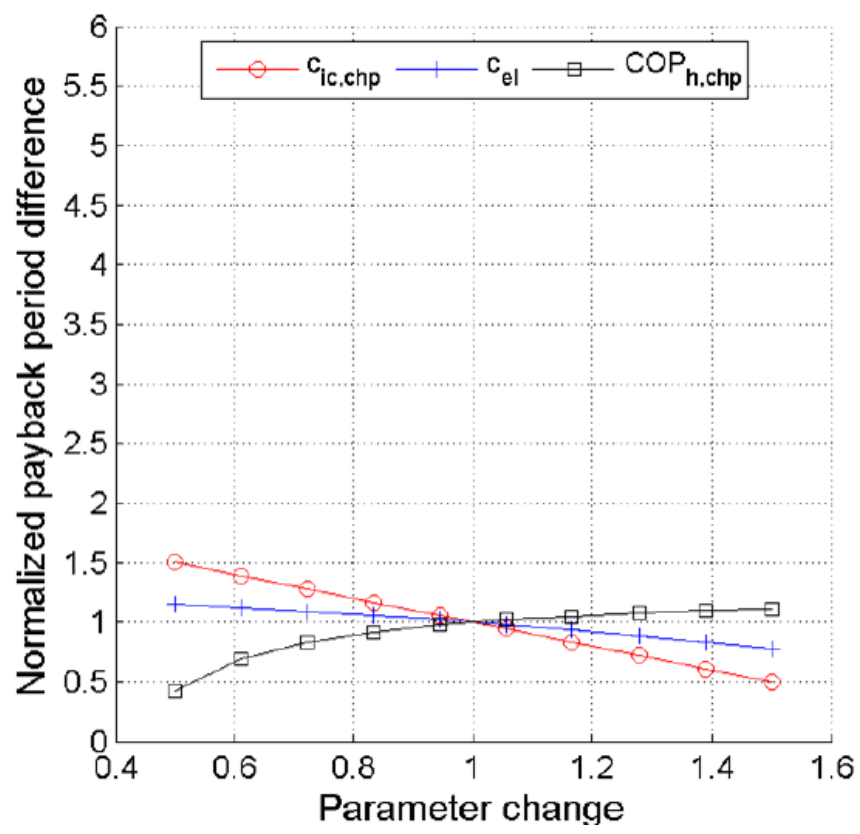

Fig. 2: Sensitivity analysis of the normalized payback period difference between absorption and compression heat pump investigating the price for electrical energy, the efficiency and the investment costs of the compression heat pump

Fig. 3 shows the results when the investment costs of the absorption heat pump, the price for thermal energy and the efficiency of the absorption heat pump are varied. The change of these parameters is investigated with regard to an improvement of the payback period of the absorption heat pump. The investment costs and the price for thermal energy have a similar impact near the reference point but the greater the fall below the reference point, the less the influence of the thermal energy price. The efficiency has a lower influence for an improvement of the payback period of the absorption heat pump in comparison to the investment costs and thermal energy price. However, a further improvement of the $\mathrm{COP}_{h, a h p}$ is not realistic due to the physical limits of a single stage absorption heat pump. Nevertheless, a lower efficiency than at the reference point should be avoided as the nonlinear influence of the $C O P h$,ahp leads to a significant increase of the normalized payback period difference.

Finally, in Fig. 4 the nominal heating capacity and the annual operating hours are varied. The variation of the nominal heating capacity leads to a slight improvement of the payback period of the absorption heat pump at lower heating capacities. This is mainly due to the parameters of the cost functions which are used in the calculations and may be different for real heat pumps. Though, the operating costs of the absorption heat pump at the reference point are higher than those of the compression heat pump a higher number of annual operating hours lead to a further reduction of the payback period for the absorption heat pump. This is due to the higher change of the payback period of the absorption heat pump in comparison to the payback period of the compression heat pump. 


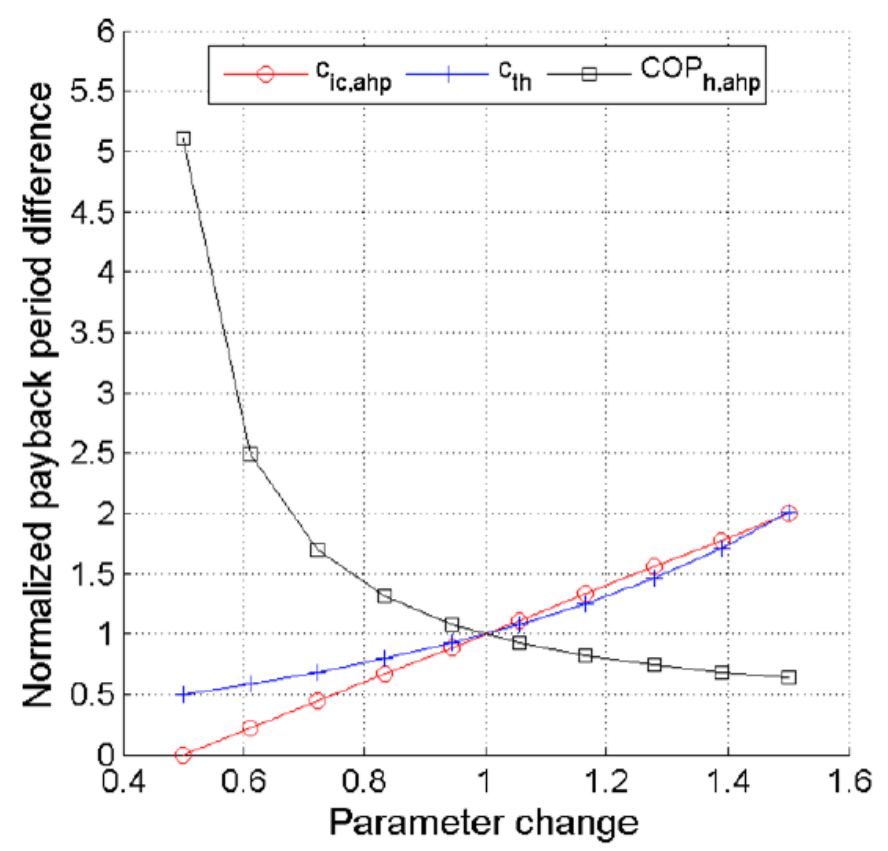

Fig. 3: Sensitivity analysis of the normalized payback period difference between absorption and compression heat pump investigating the price for thermal energy, the efficiency and the investment costs of the absorption heat pump

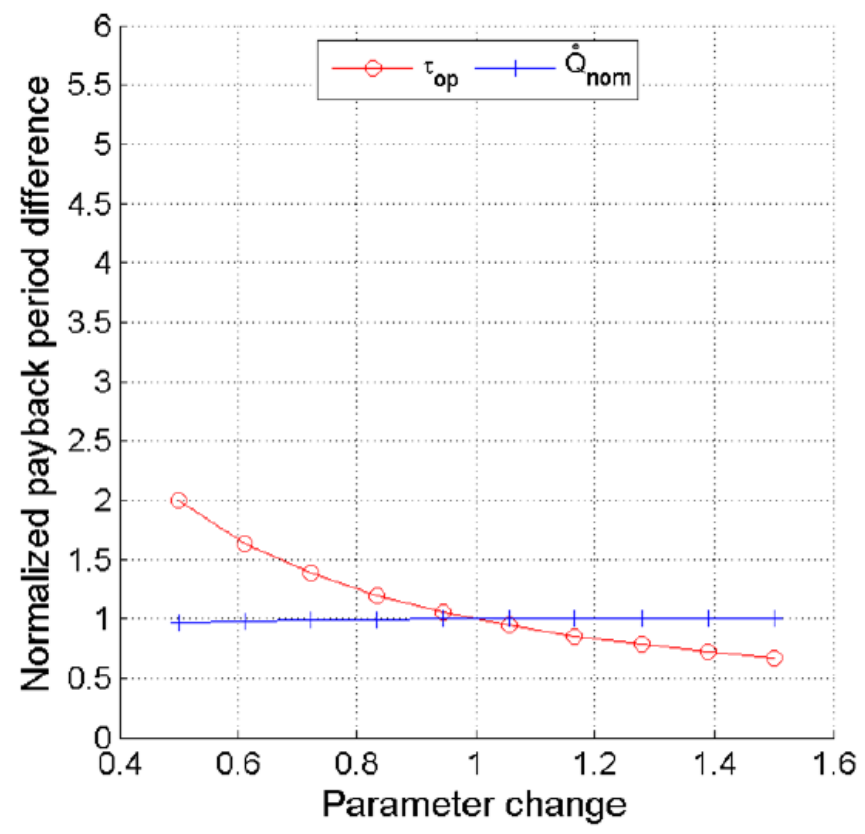

Fig. 4: Sensitivity analysis of the normalized payback period difference between absorption and compression heat pump investigating the annual operating hours and the nominal capacity

\section{CONCLUSION}

Within this paper an economic comparison of a compression and an absorption heat pump with regard to the operating costs and the payback period is carried out. To obtain realistic values for the energy prices and investment costs an overview about the current energy prices of electricity, biomass and natural gas and an overview about cost functions for the specific investment costs of compression and absorption heat pumps are given. 
A comparison of the operating costs assuming a typical efficiency for both heat pump types at a constant temperature difference between heat sink and heat source shows a significant impact of the energy carrier prices. A comparison of an absorption heat pump driven with thermal energy at the price of wood chips to a compression heat pump driven with electricity at the price of a large customer would yield to lower operating costs with the compression heat pump while the electricity price of a small customer would yield to a more economic operation with the absorption heat pump.

The investigations of the payback period in comparison to a natural gas boiler show reasonable values for both heat pump types. The payback period of the absorption heat pump is higher. This is mainly due to the higher investment costs of absorption heat pumps. Beside the investment costs a further reduction of the payback period of the absorption heat pump is possible through a reduction of the thermal energy prices and an increase of the operating hours.

\section{ACKNOWLEDGEMENT}

This project is part of the IEA research cooperation on behalf of the Austrian Ministry of Transport, Innovation and Technology. (“IEA HPP Annex IndHP2", FFG-No.: 853035)

\section{REFERENCES}

Biermayr, P., Dißauer, C., Eberl, M., Enigl, M., Fechner, H., Leonhartsberger, K., Maringer, F., Moidl, S., Schmidl, C., Strasser, C., Weiss, W., Wonisch, P., Wopienka, E., (2017), Innovative Energietechnologien in Österreich Marktentwicklung 2016, Bundesministerium für Verkehr, Innovation und Technologie, Austria.

Wolf, S., Flatau, R., Radgen, P., Blesl, M., (2017), Systematische Anwendung von Großwärmepumpen in der Schweizer Industrie, Endbericht, Institut für Energiewirtschaft und Rationelle Energieanwendungen (IER), Universität Stuttgart, Germany.

Wolf, S., (2017), Integration von Wärmepumpen in industrielle Produktionssysteme - Potenziale und Instrumente zur Potenzialerschließung, Dissertation, Institut für Energiewirtschaft und Rationelle Energienutzung (IER), Universität Stuttgart, Germany.

e-control, (2018), https://www.e-control.at/statistik/strom/marktstatistik/preisentwicklung, 27.03.2018. Biomasseverband, (2018), http://www.biomasseverband.at/en/service/energietraegervergleich/, 27.03.2018.

Statistik Austria, (2018), http://www.statistik.at/web_de/statistiken/energie_umwelt_innovation_mobilitaet/energie_und_umwel t/energie/preise_steuern/index.html, 27.07.2018 


\title{
EXPERIMENTAL EVALUATION OF A HYBRID SYSTEM FOR LOW-TEMPERATURE WATER HEATING INDUSTRIAL PROCESS
}

\author{
Daniela Vásquez, Carlos Mario Ceballos, Raiza Manrique \& Farid Chejne* \\ Group of Applied Thermodynamics and Alternative Energies (TAYEA) \\ Facultad de Minas, Universidad Nacional de Colombia \\ Carrera 80 \# 65 - 223, M3-214. Medellín, Colombia \\ Phone: +5744255333 \\ E-Mail: fchejne@unal.edu.co
}

\begin{abstract}
SUMMARY
A hybrid system was designed and built for water heating process by using solar-photovoltaic panels supplying energy to heat the water during the hours of solar radiation. This system is integrated with a natural gas Porous Media Burner (PMB) where the gases heat the water of a low-temperature industrial process $\left(<90^{\circ} \mathrm{C}\right)$. For this, a mathematical model was developed allowing to simulate different operation conditions of the PMB. Based on the model predictions, the prototype and the heat exchange system for heating the water were designed. This system was integrated into a solar panel system connected to an electrical resistance. The prototype was tested in a chocolate production plant in Colombia in order to maintain the chocolate temperature as constant while it is transported along the pipeline. From the evaluation of the prototype under real operation conditions, it was found that implementation of this decentralized system as a substitute to the existing centralized steam system leads to fuel savings of about $49 \%$ and a diminution of approximately $2100 \mathrm{CO}_{2}$ Ton/year.

Key-words: Porous Media, Combustion, Hybrid System, Industrial Process, Energy Efficiency.
\end{abstract}

\section{CONFERENCE TOPIC}

Energy efficiency, Process Intensification and Renewables in Industrial Processes 


\title{
DECARBONISATION BY RECYCLE AND REUSE FACADE COMPONENTS - GRIP FIXING INSTEAD OF ADHESIVE
}

\author{
Dr. techn. Dipl. Ing. Ferdinand Oswald \\ Institute for Architecture Technology \\ Graz University of Technology \\ Rechbauerstr. 12/I, 8010 Graz, Austria \\ Phone: +43699/19032022 \\ E-Mail: ferdinand.oswald@tugraz.at
}

\section{SUMMARY}

The status of Exterior Insulation Finishing Systems (EIFS) is, that there is no sustainability assessment of the entire life-cycle (including profitable separation, disposal and recycling) and no positive recycling behavior of building components at the end of the period of use (recovery of resources).

The paper describes the project facade4zeroWaste which includes the development, architectural design relevance, grants of patents, results of pre-certification testing's and the product publication in the time frame from 2009 till 2017. Aim of the research project facade4zeroWaste was the idea of a recyclable façade insulation system that can easily be dismantled after its lifetime and reused and recycled thanks to an innovative grip fixing system consisting of mushroom-shaped heads and loops Grip fixing instead of adhesive.

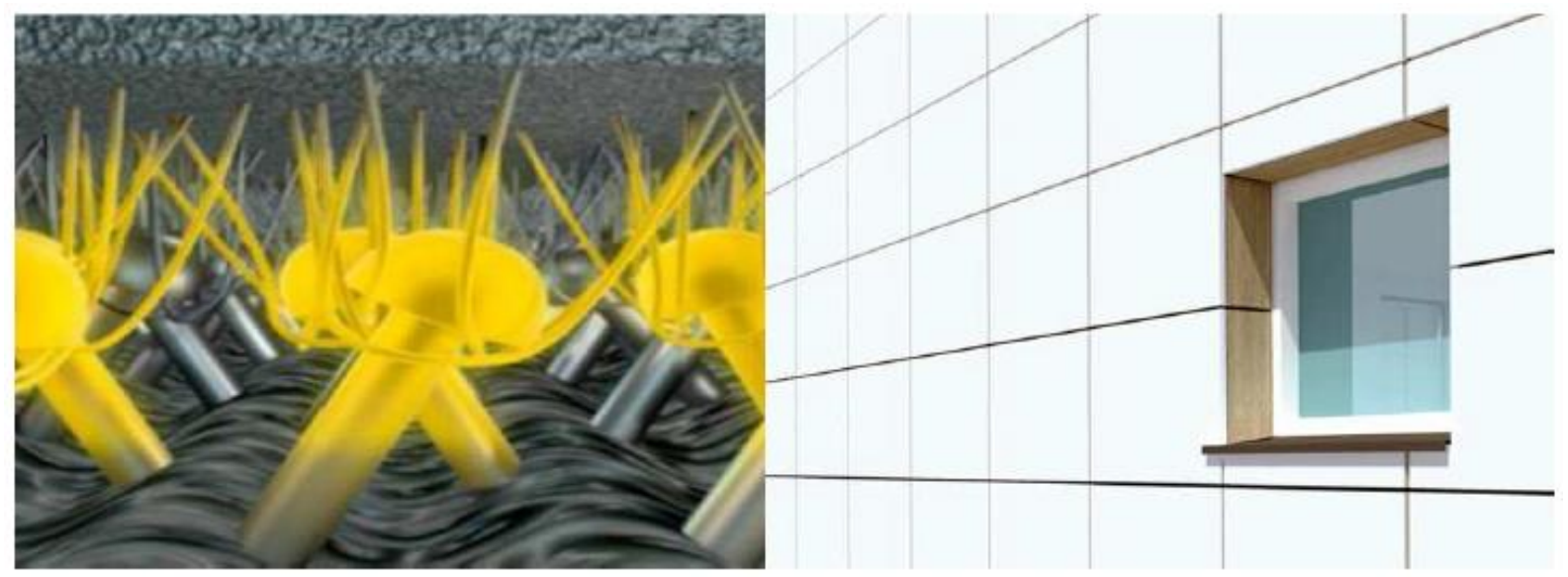

Fig. 1: Hook-and-pile fastener for the facade4zeroWaste system [1] (left)

Fig. 2: Architectonical relevance design opportunities façade design example with visible gaps [2]

(right).

\section{PRE-CERTIFICATION}

Under the leadership of Institute of Architecture Technology, TU Graz have been made several initial tests to become the pre-certification of the new façade system. It was the implementation of fire tests, construction statics tests, weathering-tests, building physics simulations and trial installations.

\section{EUROPEAN PATENT}

Different patents have been granted at the European Patent Office (EPO) for this project, like Fixing element, fixing system and facade system, it was published on 4th of April 2012 with the European patent - number: EP $2436851 \mathrm{~A} 2$. 

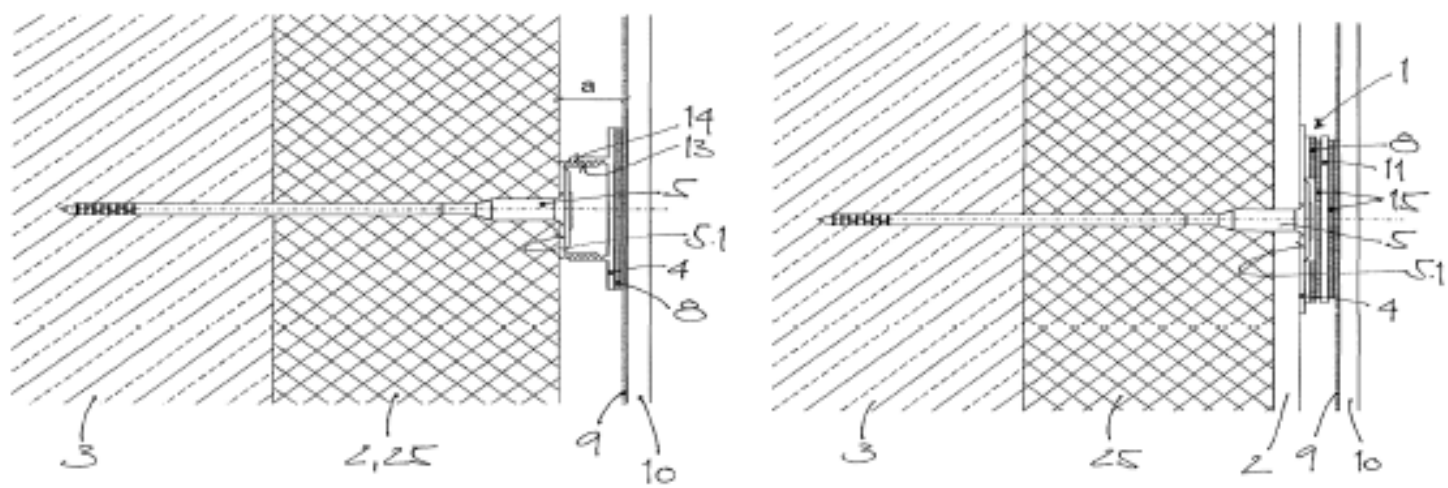

Fig. 3: Patent grants Fixing element, fixing system and facade system [3].

SORTING BY COMPONENT-TYPE FOR RECYCLING + REUSE AND TRANSPORTATION The dismantling is simple, dust- and noise-reduced and it has nearly unmixed separation as well as reuse and recycling of the system components.

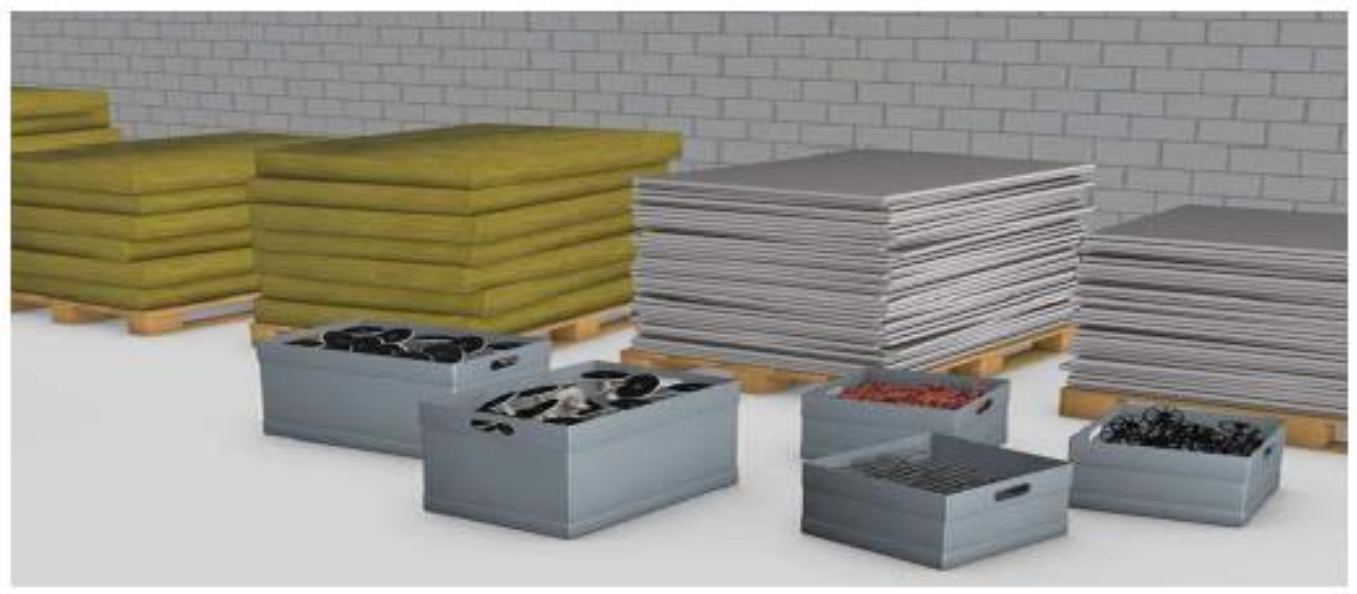

Fig. 4: Sorting by component-type for recycling + reuse and Transportation, StoSystain façade system [1].

\section{CONCLUSION}

The facade system facade4zeroWaste won the award of the EQAR - European Recycling Prize 2015. In January 2017 the façade system was presented from Sto SE \& Co. KGaA Germany as the product StoSystain R on the building fair BAU 2017 in Munich. There the façade system was getting the award: Innovation Award for Architecture and Building. The first 6 building projects with the StoSystain R system are realized already and in the near future more objects with StoSystain are planned.

\section{REFERENCES}

[1] W. Wiedenbauer, Sto GesmbH, Villach, StoSystain R: Kletten statt Kleben! Die Fassade der Zukunft, StoClimate, (2017) p. 8-11.

[2] F. Oswald, R. Riewe, Institute of Architecture Technology, Graz University of Technology, 2010. [3] Riewe, R., Oswald, F., Lueking, T., Hengel, K. M., Wiedenbauer, W., Weier, A., Rauter, E., Applicant: Sto AG: Method for creating a facade system and facade system, 2011, European Patent Agency, EP 2562320 (A1). 


\title{
MULTI-ACTIVE- FAÇADE - CLOSER TO A ZERO EMISSION BUILDING
}

\author{
DI Stefan Sattler, BSc. \\ University of Natural Resources and Life Sciences, Vienna \\ Peter-Jordan-Straße 82, 1190 Vienna, Austria \\ Phone: +43 47654-87536 \\ E-Mail: $\underline{\text { s.sattler@boku.ac.at }}$
}

\section{SUMMARY}

The Multi-Active-Façade is a prefabricated wooden pillar-beam façade with a main application in residential building refurbishments. It consists of a ventilated glass front with a honeycomb structure at the rear (see Figure 33) and an integrated heat recovery ventilation system. Because of the honeycomb structure, passive solar energy can be gained during winter whilst shading is provided during summer. The solar gains are used by the integrated photovoltaic cells in the glass on the outside of the ventilated façade. The generated energy is stored in batteries to power the ventilation system and to cover the electricity demand of the general areas of the building. Residents benefit from only one to two days on site refurbishment time per unit and an increased comfort, which can be compared to a passive house standard.

Photovoltaic, Refurbishment, Passive house, Passive- and active solar gains, residential buildings

\section{INTRODUCTION}

For the first time a housing complex of the City of Vienna with 54 units will be renovated to passivehouse standards by means of a special façade developed by the University of Natural Resources and Life Sciences (BOKU) and the research company alpS in the earlier COMET research project "B02 eNVELOP/ MULTIcover - Multifunctional envelop for thermally renovating façades and buildings." The façade layer serves two purposes: mounted onto the existing outer walls, it forms the new building envelope according to a passive-house standard and - at the same time - includes all building appliances needed for the passive-house standard. Therefore, no additional construction work has to be done inside the individual apartments.

A quick way to highly efficient refurbishments is crucial, as the construction and operation of buildings is responsible for about $40 \%$ of the $\mathrm{CO}_{2}$ emissions 0 and a higher refurbishment rate is needed to reach current and future environmental goals. Much of the energy used in buildings is still provided by fossil fuels, causing climate-related greenhouse gas emissions. Demographic changes following the move towards cities and the associated increase in housing demand require a special focus in the area of housing in urban areas. Reducing the energy demand, enhancing the efficiency and creating decentralised building integrated electricity supply by renewable energy, are strongly needed.

\section{FACADE SYSTEM}

The Multi-Active-Façade combines all these requirements. The reduction of heating energy demand is facilitated by thermal insulation and the honeycomb structure, which also uses the passive solar gains during the winter period due to its innovative design. The sunbeams can penetrate deep into the construction, heat up the building and reduce the building's energy requirements. During the summer, the honeycomb structure provides shading to the wall because the steep rays of sun cannot penetrate into the construction (see Figure 33). The Efficiency of the building is enhanced by the integrated ventilation system with heat recovery, which increases the comfort of the tenants because they do not need to rely on window ventilation. Open windows result in noise pollution at this location because the Hütteldorfer Straße is a very busy street with noise from car traffic and a tram line. 
In order to use the façade as an active element, there are transparent, frameless glass/glass photovoltaic modules integrated into the façade to generate energy for the general electricity demand of the housing complex (i.e. lighting for corridors) and the ventilation system. An energy management system in combination with a battery storage ensures operation when there is no solar radiation available. The storage system is designed to cover the energy demand for at least $24 \mathrm{~h}$. The Multi-Active-Façade was tested with different degrees of transparency with a computational fluid dynamic simulation and also in an experimental laboratory test carried out by the FH Technikum Wien 0 so that the best options, regarding passive and active solar yield is found.

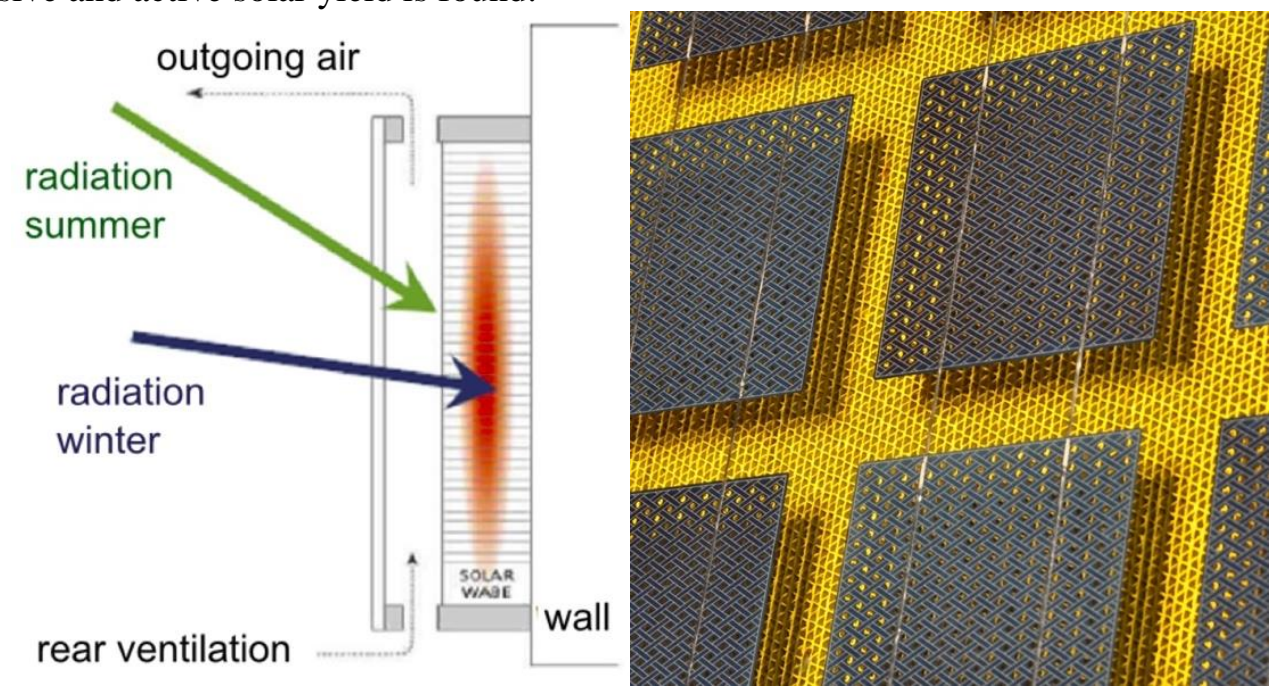

Figure 33 Functionality of the Muli-Active-Façade 0

The accompanying $\mathrm{LCC}$ and $\mathrm{CO}_{2}$ analysis will show how much energy compared to an ordinary façade insulation can be saved over the building's lifetime.

\section{CONCLUSIONS AND OUTLOOK}

Currently the construction of the refurbishment has just started and in the upcoming months, the Multi-Active-Façade will be installed for the first time in a future passive house.

In a next step, the potential for replication is examined and the energy and $\mathrm{CO}_{2}$ savings will be assessed to provide the City of Vienna a detailed analysis of the Multi-Active-Facade. It should prove, that the system can be highly beneficial in the application of residential refurbishment projects and thus add to a more environmentally friendly city, where less energy is needed, and energy is efficiently used. The project partner Stadt Wien - Wiener Wohnen is aiming at implementing this innovative, prefabricated façade system in future refurbishments, if the framework conditions are suitable and the building orientation allows on site solar energy generation.

\section{ACKNOWLEDGEMENTS}

The project "Multi-Active-Façade“ has been funded under the framework BUILDING OF TOMORROW by the Austrian Research Promotion Agency (FFG) under the project number 840645 .

\section{REFERENCES}

[1] World Business Council for Sustainable Development (WBCSD), Energy Efficiency in Buildings Transforming the market, WBCSD, Geneva, 2009

[2] B., Leitner, K., Leonhartsberger.: Experimentelle Untersuchung der GAP-Fassade. FH Technikum Wien 2017.

[3] GAP SOLUTION. „Gap Fassaden - Technische Unterlagen“. Linz, 2017. 


\title{
SOLAR SYSTEM WITH GLAZED PVT COLLECTORS FOR MULTIFAMILY BUILDING
}

\author{
Assoc. Prof. Tomas Matuska, Nikola Pokorny, Dr. Vladimir Jirka, Dr. Borivoj Sourek \\ University Centre for Energy Efficient Buildings, Czech Technical University in Prague \\ Trinecka 1024, 27343 Bustehrad, Czech Republic \\ Phone: +420224352481 \\ E-Mail: tomas.matuska@fs.cvut.cz
}

\section{SUMMARY}

The paper analyses the solar energy system with glazed solar photovoltaic-thermal collectors for hot water preparation and electricity supply for multifamily building. Glazed solar PVT collectors have been developed and experimentally tested. The mathematical model has been developed and validated for TRNSYS system simulations. The analysis shows the energy performance of the glazed PVT collectors in water heating application for multifamily building compared to separate installation of the photovoltaic and photothermal solar collectors. Despite the fact that energy gains per installed area are higher for the conventional technologies, the total energy savings for both heat and electricity are significantly higher for solar glazed PVT system. The heat gain is about $33 \%$ higher and electricity gain is about $35 \%$ higher than those for conventional system consisting of $50 \%$ of PV and $50 \%$ of photothermal collector area.

\section{INTRODUCTION}

The efforts to increase the energy performance of buildings and use of renewables for energy supply in buildings result in rational use of the envelope area for collection and conversion of solar energy. Solar photovoltaic-thermal (PVT) liquid collectors represent a new technology on the market which combines the electricity and heat generation from the same receiving surface in one device. Through the solar electricity and heat cogeneration, the total energy output per unit collector area can be higher than the outputs of conventional photovoltaic module and photothermal collector placed and operated separately with the equal total area. Such feature could be effectively used in the building applications with high and constant use of thermal energy during the year but with the limited roof area, e.g. DHW systems in the multifamily buildings.

There is already a number of commercial products available on the market as unglazed PVT collectors based on standard PV laminates from ethylene-vinyl-acetate (EVA) compound used in conventional PV modules. A significant drawback of the unglazed technology is a high heat loss and low heat output at real operation conditions (ambient temperature, wind convection) which lead mainly to lowtemperature applications combined with heat pumps.

Glazed PV-T collectors represent an alternative with significantly higher thermal output but lower electric energy generation because the additional glazing layer blocks a part of solar radiation which could be converted to electricity. Anyway, the total energy production balance is higher than in the case of combination of conventional technologies (PV modules and solar thermal collectors) and also than the unglazed PVT collectors (Matuska, 2014). On the other side, the concept of glazed PVT collectors restricts the use of conventional EVA compound as encapsulation material for PV cells. Glazed PVT collectors at stagnation conditions, i.e case with no heat removal from the collector, reach maximum absorber temperatures in the range of 120 to $180{ }^{\circ} \mathrm{C}$, while application of EVA is limited to operation temperatures up to $90{ }^{\circ} \mathrm{C}$ (Zondag, 2002). It has been experimentally proved that the longterm thermal load at higher temperature levels results in decomposition of EVA to acetic acid which causes the corrosion of PV cells contacts, delamination and also degradation of the encapsulation layer transparency (Poulek et al., 2012). 


\section{GLAZED SOLAR PVT COLLECTOR}

Glazed PVT collector with highly transparent low-e coating has been developed (see Fig. 1 left). The novel concept is based on PV cells encapsulated in polysiloxane gel. Polysiloxane gel offers several important advantages for glazed PVT collectors design like large range of operation temperature (from -60 to $+250{ }^{\circ} \mathrm{C}$ ) compared to conventional EVA material, high transparency for solar radiation, compensation of thermal dilatation stresses due to low modulus of elasticity (permanent gel), high physical adhesion to semiconductors, glass and most other materials without use of sub-layers and good heat transfer from PV cells to heat exchanger due to relatively high thermal conductivity (Matuska et al., 2014).

Strong accent has been put also to simplicity of the glazed PVT collector design and fabrication by means of reduced number of elements to compose the collector. A new concept of glazed solar PVT collectors has been introduced. Double glazing with a gap between glass panes $24 \mathrm{~mm}$ filled with argon has been used for encapsulation of PV cells with the copper heat exchanger by polysiloxane gel (see Fig. 1 right). The new design of spectrally selective PVT collector has been fabricated from double glazing with a low emissivity coating. The coating has a high transparency of solar radiation in the whole spectrum above $86 \%$ and infrared emissivity about $30 \%$ as a compromise between optical and thermal performance of the collector glazing. Antireflective coatings haven't been used. Plate and tube heat exchanger made of copper has been applied. Small distance between tubes $50 \mathrm{~mm}$ allows a good heat removal of the heat from the plate and thus also from PV cells. Photovoltaic part of the glazed PVT collector consists of monocrystalline PV cells at $125 \times 125 \mathrm{~mm}$ size, which are connected in 3 strings with 22 cells in series.

Gross size of the glazed solar PVT collector is $1600 \times 1000 \mathrm{~mm}$ to be easily combined with PV modules in case of need for electric power increase. PV cells are not placed into margins of the collector aperture to avoid shading by distance frame of the double glazing but they cover only about $75 \%$ of collector gross area. Absorber has been insulated by $30 \mathrm{~mm}$ of mineral wool on the back and $10 \mathrm{~mm}$ at the edge side. The collector box is made from aluminium.
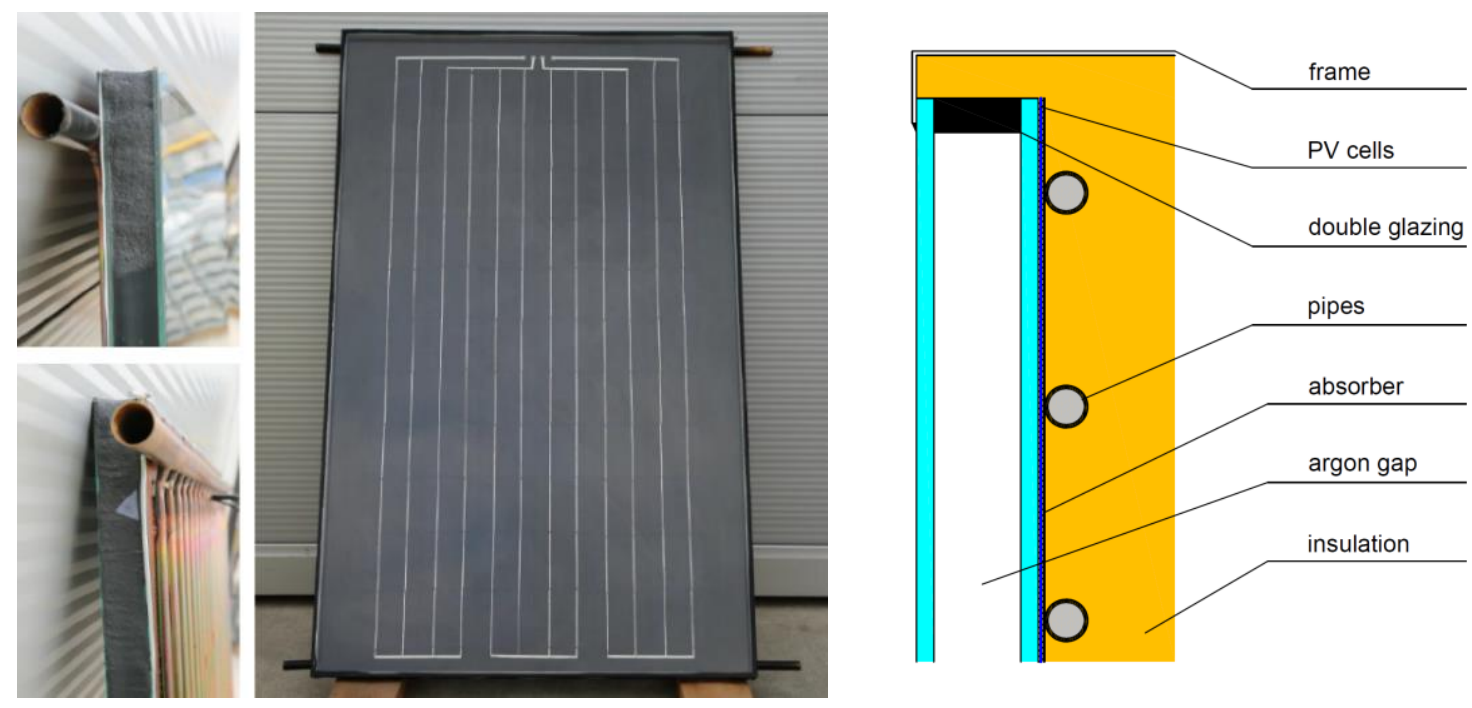

Fig. 1: PVT collector sandwich and construction layout

Glazed solar PVT collector prototype has been experimentally tested in Solar Laboratory under conditions of artificial sun at University Centre for Energy Efficient Buildings in Bustehrad. Tests have been performed in accordance with EN ISO 9806 for the open circuit mode and under MPPT electric load conditions. Electric and thermal part of the collector are mutually dependent. Graph at Fig. 2 shows the comparison of thermal and electric efficiency of the collector at testing conditions. It 
is evident that thermal performance is reduced when electricity load is applied. Electricity efficiency and production is reduced if high operation temperatures of the collector are achieved.

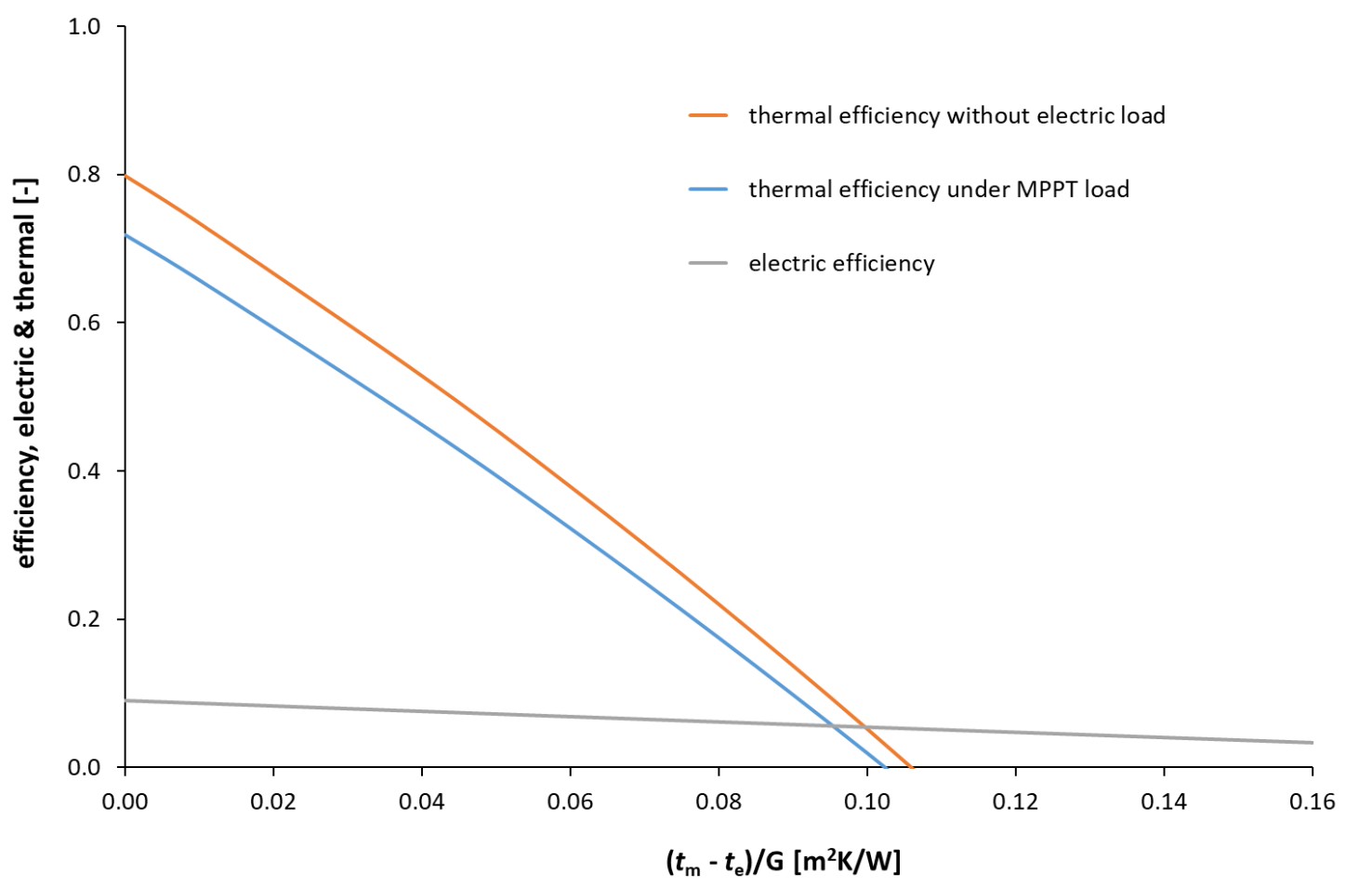

Fig. 2: Performance characteristics of PVT collector

\section{SOLAR SYSTEM FOR MULTIFAMILY BUILDING}

Glazed solar PVT collectors are suitable for applications with uniform heat and electricity load during the year and also with limited space for installation of solar collectors. Multifamily buildings are the typical case, where the advantages of PVT concept can be highlighted.

The performance analysis has been performed for combined solar heat and power system for multifamily residential building with 45 flats and 100 occupants. Solar heat is used for DHW preparation, solar electricity is used for the building appliances load. Heat demand for hot water use in the building is $116 \mathrm{MWh} / \mathrm{a}$. Electricity demand for the building is $96 \mathrm{MWh} / \mathrm{a}$. Analyses have been performed for four different climate zones in Europe with different annual solar irradiation level: Prague $\left(1096 \mathrm{kWh} / \mathrm{m}^{2} . \mathrm{a}\right)$, Stockholm $\left(1206 \mathrm{kWh} / \mathrm{m}^{2} . \mathrm{a}\right)$, Milan $\left(1367 \mathrm{kWh} / \mathrm{m}^{2} . \mathrm{a}\right)$ and Marseille $\left(1760 \mathrm{kWh} / \mathrm{m}^{2} . \mathrm{a}\right)$.

The solar system with glazed solar PVT liquid collectors has been compared with conventional solar heat and power system combining the state-of-art solar photothermal (PT) collectors and photovoltaic (PV) modules with identical polycrystalline cells as used in PVT collectors. Available solar collector field area $100 \mathrm{~m}^{2}$ on the building roof is the total collector area for all systems in the analyses. Conventional solar PV and PT system is considered in 5 alternatives with difference in the percentage ratio of PV and PT solar collector area applied (0/100, 25/75, 50/50, 75/25, 100/0).

Solar heat and power systems have been modeled in TRNSYS environment with use of the developed model of glazed PVT collector (Pokorny et al, 2015). Simplified scheme of the solar energy system is shown in Fig. 3. Solar thermal part of the investigated system variants consists of several main components: solar collectors with slope $45^{\circ}$ and orientation to south, insulated pipes of collector loop, heat exchanger and insulated solar DHW storage tank. Parameters of main components for the solar 
thermal systems alternatives are considered dependent on collector field area considered in the different variants. Dimension of the collector loop pipes is based on specific mass flow rate $15 \mathrm{~kg} / \mathrm{h} . \mathrm{m}^{2}$ of collector area (low flow solar system). Pipes length of solar collector loop in outdoor environment is $80 \mathrm{~m}$, length of pipes inside the building is $80 \mathrm{~m}$. Solar tank volume has been determined from

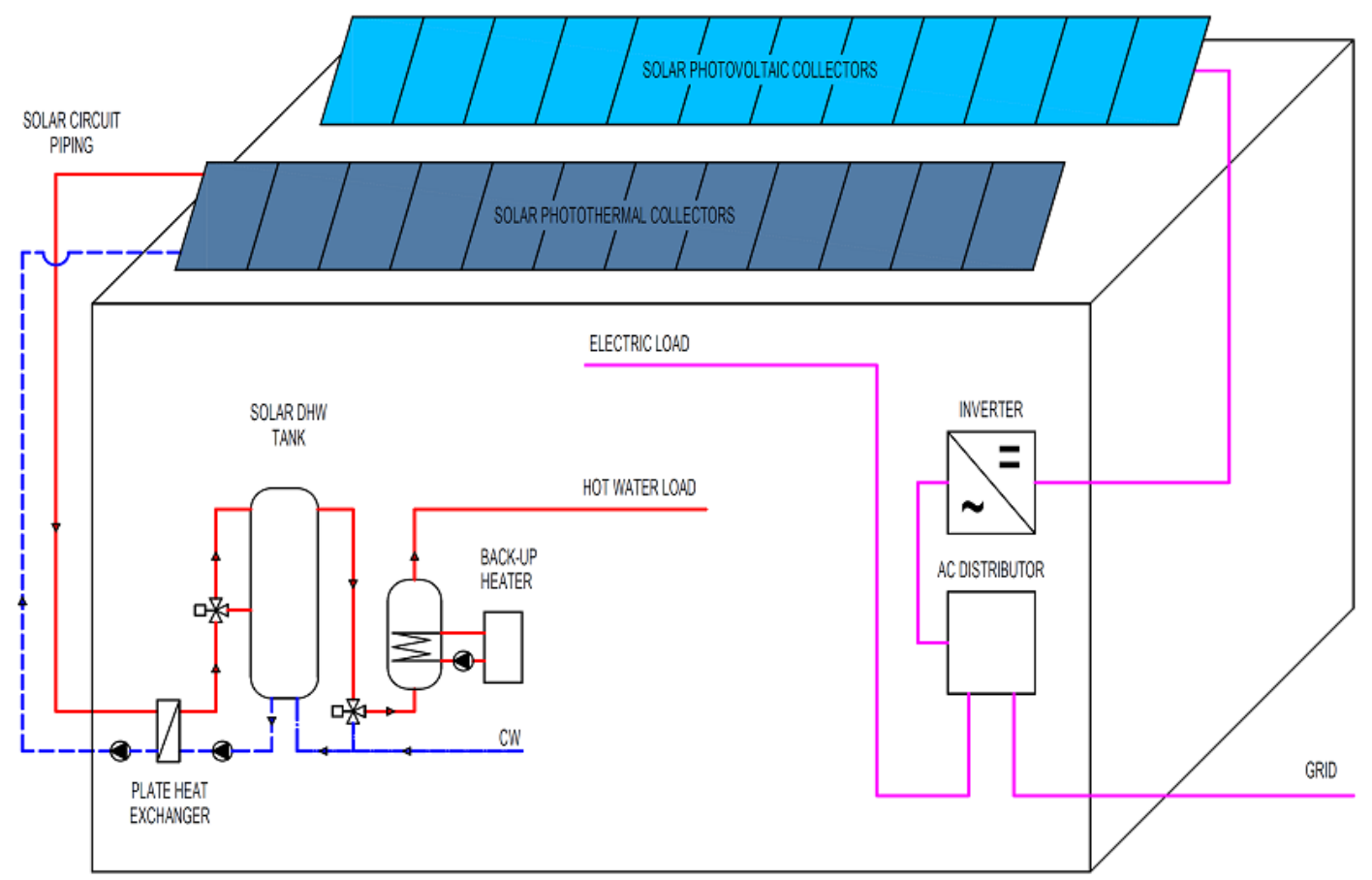

specific value $50 \mathrm{l} / \mathrm{m}^{2}$ of collector area (see Tab. 1). Solar plate heat exchanger has the efficiency 80 $\%$. PV power system is a conventional grid-on system with DC/AC inverter. Total system losses are considered $10 \%$. Whole PV electricity production has been assumed to be consumed for the building appliances load.

Fig. 3: Simplified scheme of solar energy system

Table 1: Solar thermal system parameters

\begin{tabular}{|c|c|c|c|c|}
\hline Alternative & $\begin{array}{c}\text { Solar thermal collector } \\
\text { area } \\
{\left[\mathrm{m}^{2}\right]}\end{array}$ & $\begin{array}{c}\text { Solar tank } \\
\text { volume } \\
{\left[\mathrm{m}^{3}\right]}\end{array}$ & $\begin{array}{c}\text { Collector loop } \\
\text { dimension } \\
{[\mathrm{mm}]}\end{array}$ & $\begin{array}{c}\text { Collector loop } \\
\text { insulation thickness } \\
{[\mathrm{mm}]}\end{array}$ \\
\hline $100 \% \mathrm{PV}$ & - & - & - & - \\
\hline $75 \% \mathrm{PV}-25 \% \mathrm{PT}$ & 25 & 1.25 & $22 \times 1$ & 19 \\
\hline $50 \% \mathrm{PV}-50 \% \mathrm{PT}$ & 50 & 2.50 & $22 \times 1$ & 19 \\
\hline $25 \% \mathrm{PV}-75 \% \mathrm{PT}$ & 75 & 3.75 & $28 \times 1.5$ & 25 \\
\hline $100 \%$ PT & 100 & 5.00 & $28 \times 1.5$ & 25 \\
\hline glazed PVT system & 100 & 5.00 & $28 \times 1.5$ & 25 \\
\hline
\end{tabular}

\section{RESULTS}

Results of TRNSYS simulations for different climates and systems are compared in Fig. 4 and 5. Comparison shows the general advantage of the glazed solar PVT collectors in energy yields. Despite the lower effectivity for heat production compared to conventional solar thermal collector and lower effectivity for electricity production compared to conventional PV modules the glazed selective PVT 
collector results in higher combined energy gains when compared with separate PV and PT collectors combination with a different ratio of area share between the technologies.

Comparing the glazed PVT installation with alternative with $50 \%$ of available area dedicated to PV modules and $50 \%$ to photothermal collectors (see Tab. 2), the glazed PVT collectors bring about $33 \%$ more heat and $35 \%$ more electricity in total than the separate installation. Solar fraction for heat here in 50/50 alternative differs from 22 to $39 \%$ (29 to $51 \%$ for PVT system), solar fraction for electricity ranges from 8 to $12 \%$ (10 to $16 \%$ for PVT system) both in dependence on climate.

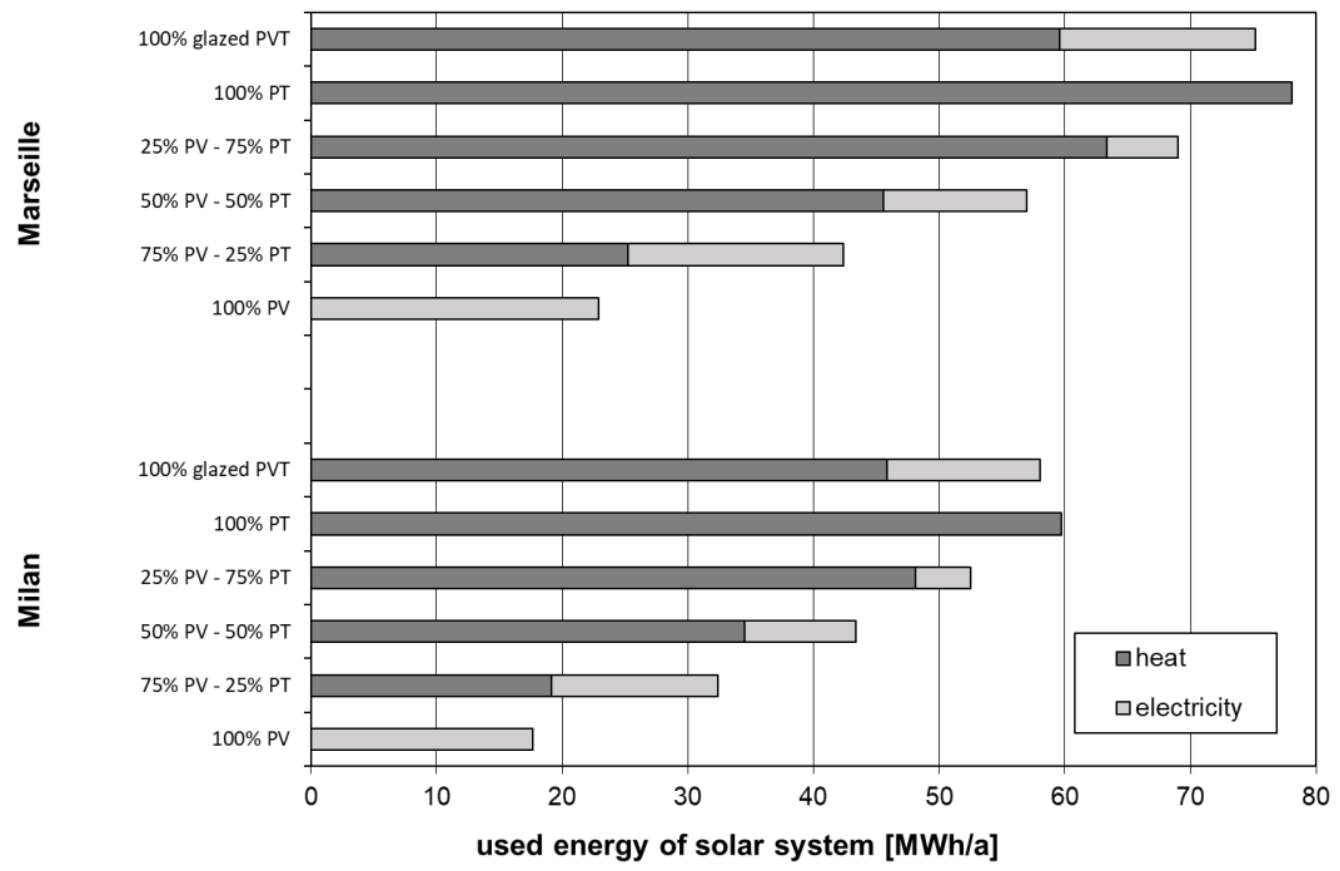

Fig. 4: Comparison of energy gains for solar system with PVT collectors and conventional combination (southern Europe)

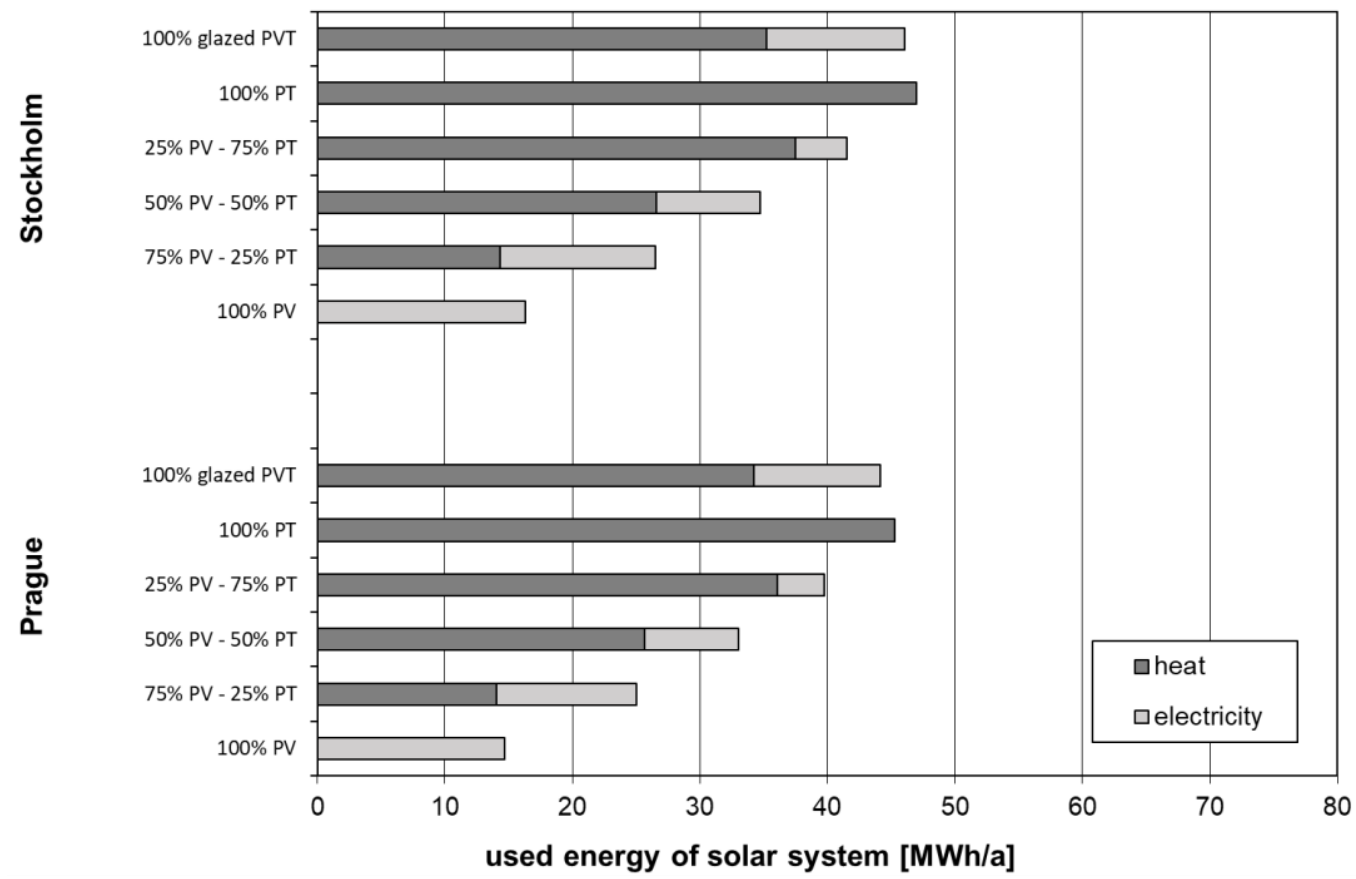

Fig. 5: Comparison of energy gains for solar system with PVT collectors and conventional combination (central and northern Europe) 
Table 2: Comparison of the results

\begin{tabular}{|c|c|c|c|c|}
\hline \multirow{2}{*}{ Alternative } & \multicolumn{4}{|c|}{ Usable heat / electricity [MWh/a] } \\
\cline { 2 - 5 } & Stockholm & Prague & Milan & Marseille \\
\hline $50 \%$ PV - 50\% PT & $26.5 / 8.2$ & $25.7 / 7.3$ & $34.5 / 8.8$ & $45.6 / 11.4$ \\
\hline glazed PVT system & $35.2 / 10.9$ & $34.2 / 9.9$ & $45.9 / 12.2$ & $59.5 / 15.6$ \\
\hline benefit of PVT & $+33 \% /+33 \%$ & $+33 \% /+35 \%$ & $+33 \% /+38 \%$ & $+31 \% /+36 \%$ \\
\hline
\end{tabular}

\section{CONCLUSION}

The new concept of glazed solar PVT collector with polysiloxane gel encapsulation of PV cells and selective coating has been presented together with the simulation analysis of potential application in domestic hot water for multifamily building. Simulation analysis has been made with a detailed model of glazed solar PVT collector experimentally verified with testing of prototypes. The results from simulation of hot water system and electric load in the given residential building have shown a large potential for achieving the higher coverage of the building demand than in the case of separate installation of conventional technologies regardless of the climate. Glazed PVT collectors can bring about $33 \%$ more heat and about $35 \%$ more electricity in total than the separate installation where $50 \%$ of the roof area has been used for photovoltaics and $50 \%$ for photothermal collectors. Thus, the solar fraction in multifamily building could be increased.

\section{REFERENCES}

Matuska, T., 2014. Performance and Economic Analysis of Hybrid PVT Collectors in Solar DHW System, Energy Procedia, Volume 48, pp. 150-156.

Zondag, H. A., Van Helden, W. G. J., 2002. Stagnation temperature in PVT collectors. PV in Europe, Rome, Italy.

Poulek, V. Strebkov, D. S., Persic, I. S., Libra, M., 2012. Towards 50 years lifetime of PV panels laminated with silicone gel technology. Solar Energy 86, pp. 3103-3108. http://dx.doi.org/10.1016/j.solener.2012.07.013

Matuska, T., Jirka, V., Poulek, V., 2014. Use of Polysiloxane Gel as Laminate for Solar PVT Collectors, Conference Eurosun 2014, Aix-les-Bains, France, doi:10.18086/eurosun.2014.16.15.

TRNSYS 17, 2012. TRaNsient SYstem Simulation program, Solar Energy Laboratory, University of Winconsin-Madison.

Pokorny, N., Matuska, T., Sourek, B., 2015. Modelling of Glazed Liguid PV-T Collector with Use of Detailed Model, Conference IBPSA 2015, Hyderabad. 


\title{
THERMAL ANALYSIS FOR THE DEVELOPMENT OF A SOLAR THERMAL ACTIVATED FACADE ELEMENT
}

\author{
Arch. Dipl.-Ing. Helmut Schober ${ }^{1}$, Dipl.-Ing. Dr.techn. Daniel Brandl ${ }^{2}$ \\ ${ }^{1}$ Institute of Building Construction, Graz University of Technology \\ Lessingstraße 25/III, 8010 Graz, Austria \\ Phone: +43 (0) 316 / 873 - 6243 \\ E-Mail: helmut.schober@tugraz.at \\ ${ }^{2}$ Institute of Thermal Engineering, Graz University of Technology \\ Inffeldgasse 25b, $8010 \mathrm{Graz}$, Austria \\ Phone: +43 (0) 316 / 873 - 7306 \\ E-Mail: daniel.brandl@tugraz.at
}

\section{SUMMARY}

Within an ongoing research project at Graz University of Technology the efficiency of different types of solar absorber layouts, all produced by using the "Roll-Bonding" fabrication method, was evaluated on the basis of Computational Fluid Dynamic (CFD) simulations. Fluid outlet temperature, energy output and pressure difference between inlet and outlet were compared. Based on the already completed and pending thermal analysis of the absorbers, prototypes of a solar thermal activated sandwich panel, the so called STAF-panel, were produced and measured under real climate conditions.

\section{INTRODUCTION}

Traditional sandwich panels are one of the cheapest and easiest solutions forming the thermal building envelope for industrial building constructions. They are pre-fabricated façade elements where millions of square meters were produced and mounted every year. A sandwich panel consists of an interior as well as an exterior metal plate with a thermal insulation between. The central idea of the Interreg project "ABS-Network SIAT 125" [1] is the solar thermal activation of a sandwich panel where solar radiation is transformed into thermal energy.

\section{DEVELOPMENT APPROACH}

In this case thermal activation means the conversion of solar energy in order to provide energy for the production of domestic hot water and/or heating and cooling applications. Due to a functionally convincing and creatively sophisticated revision of sandwich panels the field of application can be extended to office buildings, public buildings, residential buildings etc.

\section{PRODUCTION CONCEPT OF THE STAF-PANEL}

The metal plates of the Solar Thermal Activated Facade (STAF) -Panel have integrated fluid pipes which can be produced by the so called "Roll-Bonding" fabrication method. With this special sheet metal forming technique two metal sheets were combined to one steel plate whereby the fluid pipes are produced by inflation (one side or double side inflation). The exterior metal plate acts as an absorber of a solar thermal collector for conversion of solar energy into hot water whereas the interior metal plate can be used for heating and cooling of the interior rooms. The company Talum d.d. in Slovenia is using this technique in order to produce absorber plates for evaporators of refrigerators.

\section{THERMAL ANALYSES OF DIFFERENT ABSORBER PLATE GEOMETRIES}

Preliminary thermal analyses were performed at Graz University of Technology in order to understand and improve the efficiency of the participating heat transfer mechanism. This was done by means of Computational Fluid Dynamic (CFD) simulations with ANSYS Fluent [2]. With this method a various 
number of geometrical and operative parameters can easily be varied and compared to each other. For example, Fig. 1 shows the comparison of the temperature contours of the exterior absorber plate with different fluid pipe layouts. Additionally, this figure shows the fluid flow characteristic at the inlet region. The dimensions of the sandwich panel are $1.75 \times 0.5 \mathrm{~m}$ with a thermal insulation (PU foam) of $0.15 \mathrm{~m}$ between the interior and the exterior (aluminium) plate. The exterior temperature was set to 30 ${ }^{\circ} \mathrm{C}$, a solar radiation of $1000 \mathrm{~W} / \mathrm{m}^{2}$ at a solar angle of $45^{\circ}$ and an exterior heat transfer coefficient of 25 $\mathrm{W} / \mathrm{m}^{2} \mathrm{~K}$ were further boundary conditions in the simulation. The solar absorptivity of the solar coating at the exterior metal plate was assumed to $95 \%$. The interior temperature and heat transfer coefficient were assumed to $25^{\circ} \mathrm{C}$ and $5 \mathrm{~W} / \mathrm{m}^{2} \mathrm{~K}$. Finally, water was introduced to the exterior metal plate with a mass flow of $50 \mathrm{~kg} / \mathrm{h}$ and at a temperature of $15^{\circ} \mathrm{C}$.

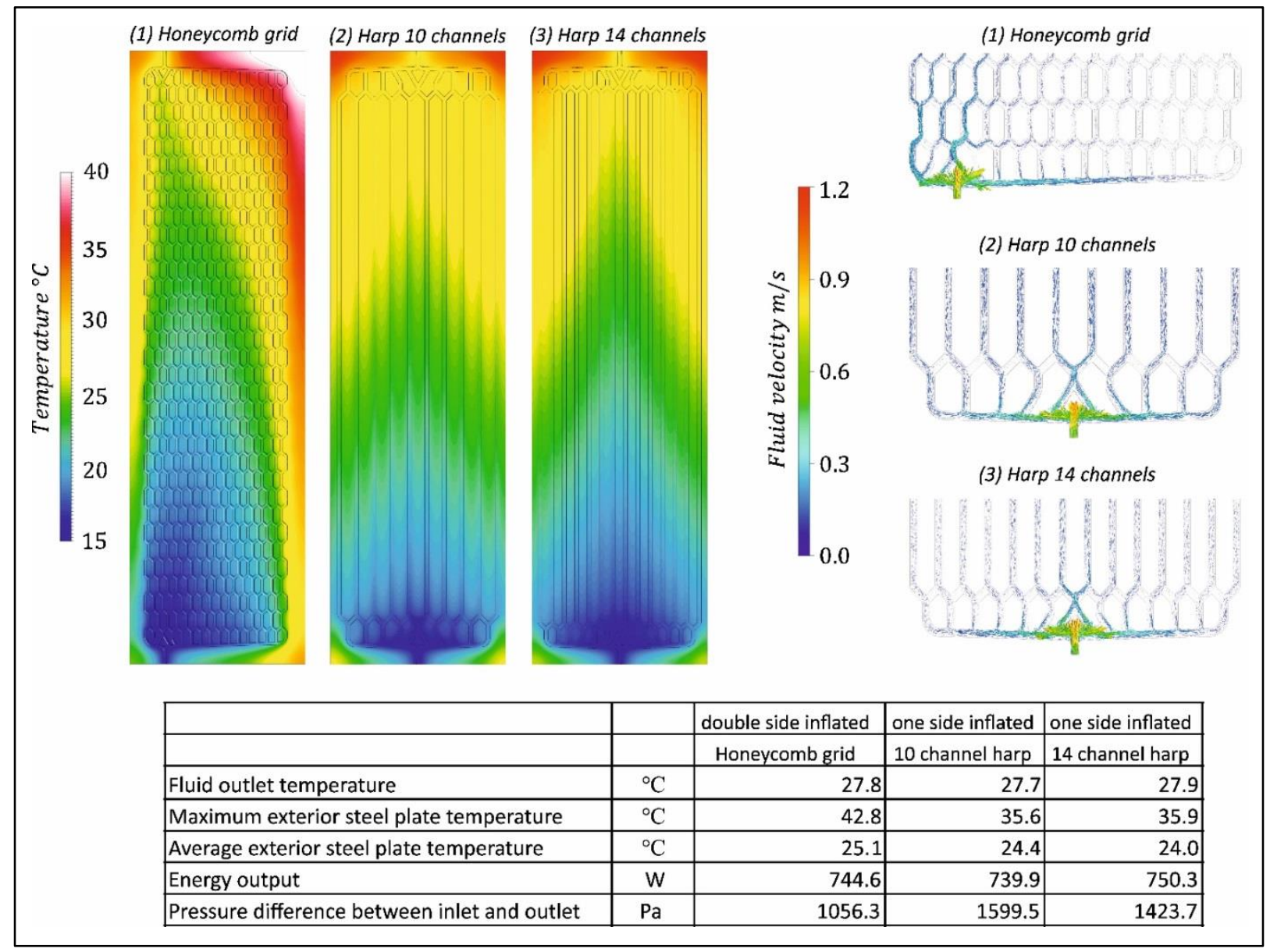

Fig. 1: Comparison of the temperature contours at the exterior metal plate and the fluid flow characteristic at the inlet region for a thermal activated sandwich panel with (1) a honeycomb grid, (2) a 10 channel harp and (3) a 14 channel harp fluid pipework.

\section{REFERENCES}

[1] Interreg-project "ABS-Network SIAT 125" (http://abs-network.eu/en)

[2] Fluid Dynamics software “ANSYS Fluent 18.2.0”,ANSYS Inc., Southpointe 275 Technology Drive Canonsburg, PA 15317, http://www.ansys.com, Release 18.2 (2018).

\section{ACKNOWLEDGEMENTS}

All contents of this publication are part of the ongoing Interreg-project "ABS-Network SIAT 125" at Graz University of Technology, Austria. Interreg V-A Slovenia-Austria is a cross-border cooperation programme between Slovenia and Austria in the programme period 2014-2020. An overview of the project and a list with all participants can be found under the following link: http://abs-network.eu/en 


\title{
ANALYTICAL STUDY ON A HEAT PUMP FOR $4^{\text {th }}$ GENERATION DISTRICT HEATING
}

\author{
Minwoo Lee ${ }^{1}$, Dongwoo Kim ${ }^{1}$, Yubi Kang ${ }^{1}$, Changuk Jo ${ }^{1}$, Yongchan $\mathrm{Kim}^{2}$ \\ Graduate School of Mechanical Engineering, Korea University ${ }^{1}$ \\ Seoul 136-713, Republic of Korea \\ qleldhwlq@korea.ac.kr; redadol@korea.ac.kr; eubi1127@naver.com; dnrckd16@gmail.com; \\ School of Mechanical Engineering, Korea University ${ }^{2}$ \\ Seoul 136-713, Republic of Korea \\ yongckim@korea.ac.kr
}

\section{SUMMARY}

District heating (DH), generating heat from central suppliers and distributing the heat to end-users, has been playing a major role in reducing gas emission and fuel consumption. However, there is a large amount of heat loss during the heat distribution process. The $4^{\text {th }}$ generation DH (4GDH) not only reduces the distribution heat loss effectively by lowering the supply temperature from the heat supplier below $60{ }^{\circ} \mathrm{C}$, but also makes it possible to use low-temperature heat sources, e.g. renewable energy and waste heat. However, in order to use these low-temperature heat sources, it is required to increase the temperature of the heat sources to usable and appropriate level. A heat pump is one of the most attractive solutions to exploit the low-temperature heat sources owing to its high efficiency. In this study, a simulation program predicting the performance of the heat pump for 4GDH was developed and validated by experimental results from a lab-scale apparatus. The variation in the heat pump performance for $4 \mathrm{GDH}$ was analyzed according to the operating condition, size, and working fluid.

Keywords: $4^{\text {th }}$ generation district heating $(4 \mathrm{GDH})$, renewable energy, heat pump, simulation program

\section{INTRODUCTION}

World has been putting a large amount of efforts into conserving energy and protecting environment. For a long time, fossil fuels have been used in various energy systems for heat and electircity. However, since it produces a large amount of gas emission and has limited reserves, other energy resources have to be used for replacing existing energy systems. Renewable energy is the most attractive and reliable alternative energy source becuase it has the nearly limitless reserves and does not produce harmful by-products against environment. District heating also has been focusing on exploiting these renewable energy sources. However, intermittence and low energy levels of the renewable energy make it hard to accomplish high share in district heating.

As the required supply temperature decreases below $60{ }^{\circ} \mathrm{C}$ in $4^{\text {th }}$ generation district heating (4GDH), the renewable energy can be more efficiently and stably used for district heating. Futhermore, the thermal stroage system makes this change in the energy consumption more feasible by alleviating the weakness of the renewable energy with intermittence and low energy level. Denmark, for example, set a target to make a $100 \%$ renewable energy system for not only heating but also eletricity before 2050 . However, in district heating, there are some challenges to overcome. First, although the 4GDH lowers the supply temperature below $60^{\circ} \mathrm{C}$, it can be used for low-energy buildings. Considering lifetime of buildings, the 4GDH also has to deal with existing buildings. Second, although the thermal storage system alleviates the effect of intermittence of the renewable energy, there is an anuual imblance between supply and demand. Therefore, in order to make supply correspond to demand, it is required to increase low energy level of the renewable energy up to usable and appropriate level for district heating. In order to overcome these challenges, the heat pump needs to be integated in the 4GDH. 
In this study, a simulation program for predicting the performance of the heat pump in the 4GDH is developed. Moreover, the performances of the heat pump under various operated conditions are analysed based on the simulation results.

\section{DEVELOPMENT OF HEAT PUMP SIMULATION MODEL}

As shown in Fig. 1, the heat pump simulated in this study consists of two plate heat exchangers, compressor, and electronic expansion valve (EEV). The input includes specific data for each component and data for water. The simulation starts with the compressor modeling, assuming the inlet and outlet pressures of the refrigerant passing through the compressor. Based on the compressor simulation, the mass flow rate of the refrigerant and the power consumption of the compressor are calculated. After the compressor simulation, the condenser is simulated by estimating the total heat transfer rate and pressure drop of the refrigerant and water. After the condenser simulation, the EEV is simulated by estimating the mass flow rate through the EEV. Accordingly, the mass flow rate through the EEV is compared with that through the compressor. If the error does not satisfy the convergence criterion, the simulation is repeated after adjusting the condenser pressure. If the error is within the convergence range, the evaporator is simulated by estimating the total heat transfer rate and pressure drop of the refrigerant and water. Afterwards, the enthalpy difference between the compressor inlet and evaporator outlet is estimated and compared with the convergence criterion. If the error diverges from the convergence criterion, the simulation is repeated after adjusting the evaporator pressure. If the error is within the convergence range, the simulation is terminated. The simulation is validated by comparing the predictions with the results from lab-scale experiments.

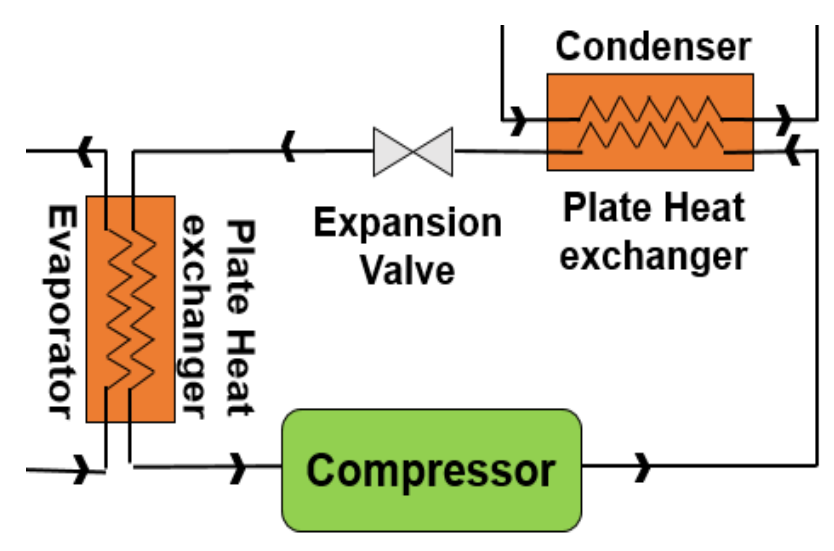

Fig. 1 : Diagram of the heat pump

\section{SIMULATION RESULTS AND DISCUSSION}

Figs. 2 and 3 show the variations in the outlet temperature of the supply water and COP of the heat pump according to the inlet temperature of the supply water in the condenser and compressor frequency. For preparing domestic hot water(DHW) whose temperature should be higher than $45^{\circ} \mathrm{C}$, heat pump has to increase the temperature of the supply water up to $53{ }^{\circ} \mathrm{C}$ to increase the temperature of the DHW up to $45{ }^{\circ} \mathrm{C}$. When the compressor frequency was relatively low, it was difficult to increase the temperature of the supply water up to $53{ }^{\circ} \mathrm{C}$. The temperture of the supply water increased with the increases in the inlet temperature of the supply water and compressor frequency. However, the COP of the heat pump decreased as the compressor frequency increased because less power was consumed at the lower compressor frequency. When the compressor frequency is $60 \mathrm{~Hz}$, the inlet temperature of the water should be higher than $40^{\circ} \mathrm{C}$ in order to increase the temperature of the supply 
water up to $53{ }^{\circ} \mathrm{C}$, which may yield the $\mathrm{COP}$ of 2.5 . When the compressor frequency is $120 \mathrm{~Hz}$, the inlet temperature of the water should be higher than $34.3^{\circ} \mathrm{C}$, which may yield the COP of 2.14.

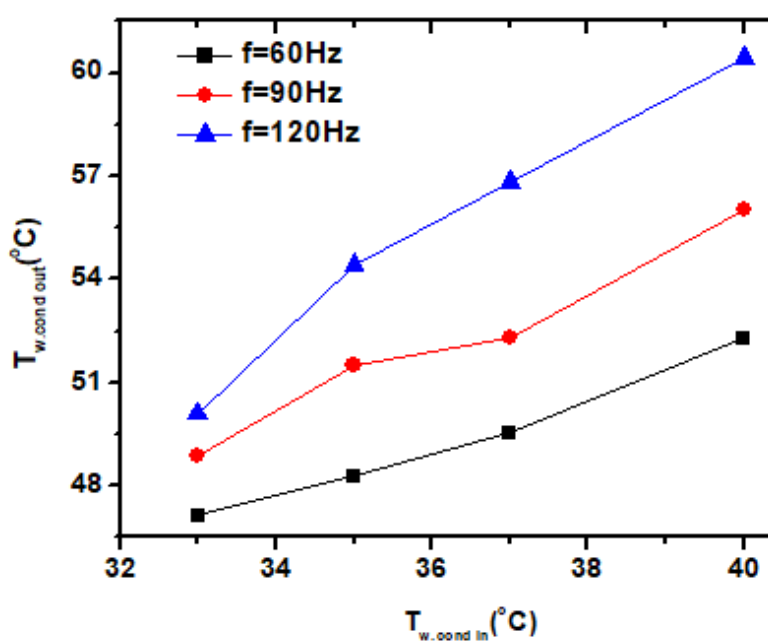

Fig. 2 : Variation in temperature of supply wate according to compressor frequency

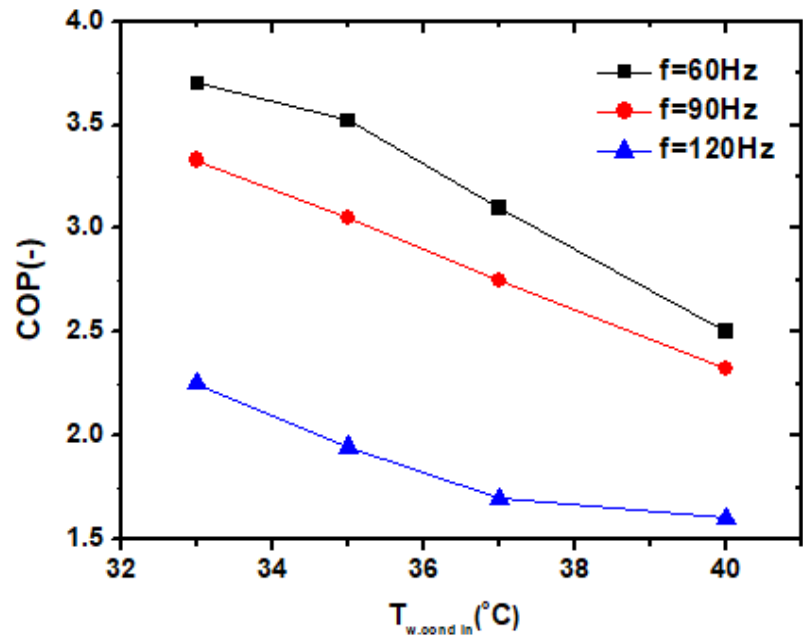

Fig. 3 : Variation in COP of heat pump according to compressor frequency

Fig. 4 shows the variation in the outlet temperature of the supply water and COP of the heat pump according to the mass flow rate of the supply water at the inlet temperature of $40{ }^{\circ} \mathrm{C}$ and compressor frequency of $90 \mathrm{~Hz}$. As the mass flow rate increaed, the outlet temperature decreased and the COP of the heat pump increased. For preparing the supply water whose temperature is higher than $53{ }^{\circ} \mathrm{C}$, the mass flow rate of the water should be lower than $1 \mathrm{~kg} \mathrm{~s}^{-1}$, which may yield the COP of 2.81 .

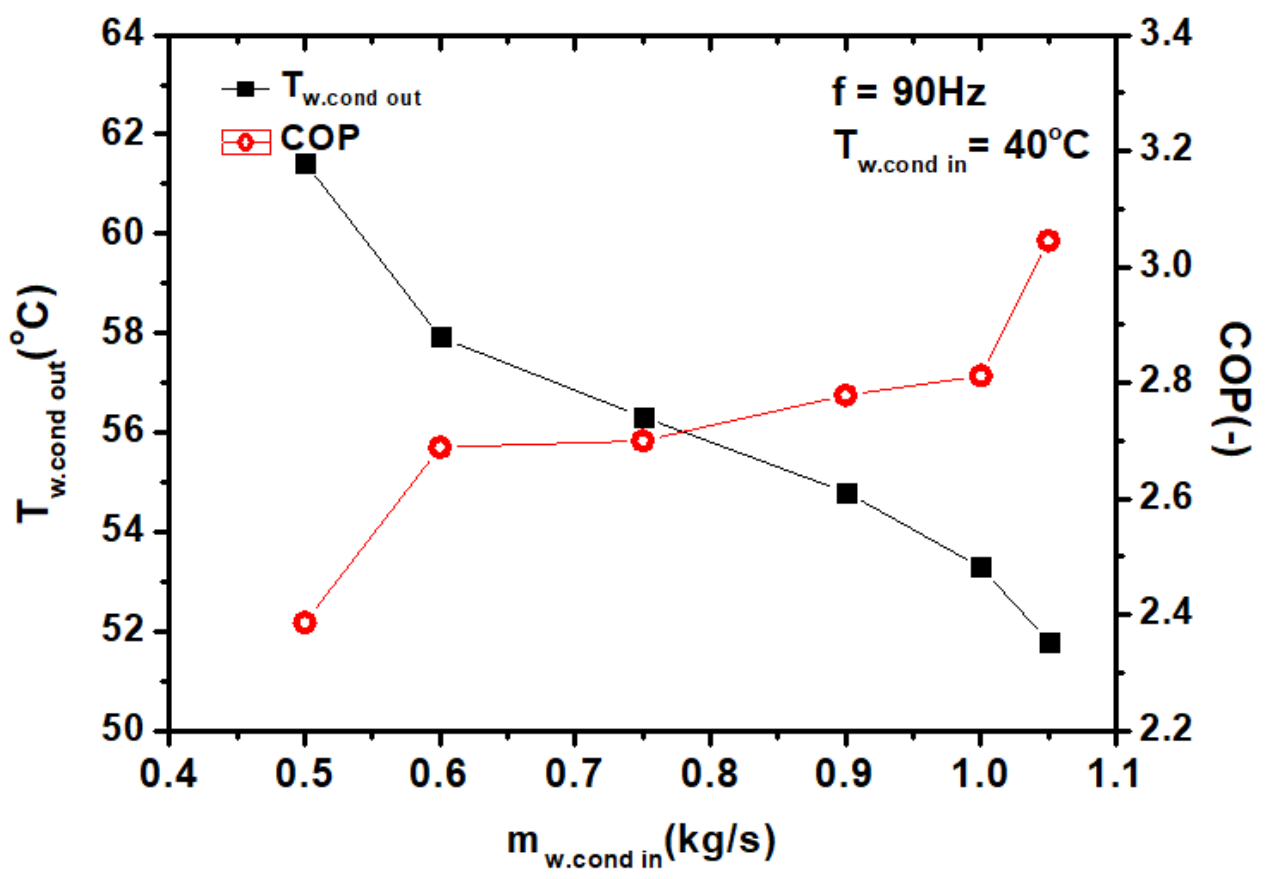

Fig. 4 : Variation in supply water and COP according to mass flow rate of supply water 


\section{CONCLUSIONS}

In this study, the simulation model for predicting the performance of the heat pump is developed. When the compressor frequency is relatively low, it is difficult to increase the temperature of the supply water up to $53{ }^{\circ} \mathrm{C}$. However, the COP of the heat pump decreases with the increase in the compressor frequency. For the $4 \mathrm{GDH}$ whose temperature is higher than $45^{\circ} \mathrm{C}$, it is required to increase the temperature of the supply water up to $53^{\circ} \mathrm{C}$. When the compressor frequencies are $60 \mathrm{~Hz}$ and 120 $\mathrm{Hz}$, the required inlet temperatures are $40{ }^{\circ} \mathrm{C}$ and $34.3^{\circ} \mathrm{C}$, respectively, which may yield the COP of 2.5 and 2.14 , respectively. For preparing the supply water whose temperature is higher than $53{ }^{\circ} \mathrm{C}$ at the inlet temperature of $40{ }^{\circ} \mathrm{C}$ and compressor frequency of $90 \mathrm{~Hz}$, the mass flow rate of the water should be lower than $1 \mathrm{~kg} \mathrm{~s}^{-1}$.

\section{REFERENCES}

Lund, H., et al., (2014), 4th Generation District Heating (4GDH). Energy, 68: p. 1-11.

Lund, H. and B.V. Mathiesen, (2009), Energy system analysis of $100 \%$ renewable energy systemsThe case of Denmark in years 2030 and 2050. Energy, 34(5): p. 524-531.

Zvingilaite, E., T.O.B. Elmegaard, M.L. Franck., (2018), Low temperature district heating consumer unit with micro heat pump for domestic hot water preparation, the 13th international symposium on district heating and cooling. 


\title{
POLICY IMPLICATIONS, MACROECONOMIC AND SYSTEMIC EFFECTS OF THE TRANSITION TO 100\% RENEWABLES IN INDUSTRY
}

\author{
$\underline{\text { Simon Moser }}^{1}$, Horst Steinmüller ${ }^{1}$ \\ Energieinstitut an der Johannes Kepler Universität Linz \\ Altenbergerstraße 69, 4040 Linz, Austria \\ Phone: +43732 24685656 \\ E-Mail: office@energieinstitut-linz.at
}

\section{SUMMARY}

The additional effort required to achieve the Paris or former EU emission targets implies an increase in the cost of greenhouse gas emissions. Industrial companies are striving for $\mathrm{CO}_{2}$-neutral process lines to reduce the $\mathrm{CO}_{2}$ emissions associated with product production. The project "Renewables4Industry", which was financed by the Austrian Climate and Energy Funds, aimed at identifying research and development needs, specific technologies and measures needed to support the transition to a carbonneutral energy supply. Being one part of the project, we assessed the macroeconomics of the transition to the $100 \%$ use of renewable energy sources (RES) in industry. This assessment includes the nonexclusive industrial use of cost-effectively provided renewables, i.e. we expect competitive demand for renewables by all sectors. The assessment is based on national energy balances and major technological trends (e.g. hydrogen-based steel production, e-mobility). We clearly find that all the paths of $\mathrm{CO}_{2}$ emission reduction must be applied in order to reach the $100 \%$ target.

\section{INTRODUCTION}

The project "Renewables4Industry" aimed at identifying R\&D needs, specific technologies and measures needed to support the transition of industry to be $100 \%$ carbon-neutrally supplied with all process adopted or still working. Securing Austria/Europe as the industries' site of choice has always been a central concern under which the transition has been analyzed.

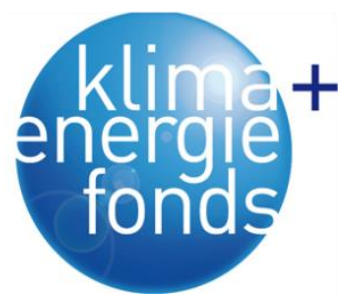

Fig. 1: The project "Renewables4Industry" was financed by the Austrian Climate and Energy Funds.

As part of the project, we assessed the macroeconomics of the transition to the $100 \%$ use of renewable energy sources (RES) in industry. In the beginning of the process, the renewables potential for Austria was assessed: For 2050, a renewable electricity potential of about 113 TWh is estimated for Austria. This consists primarily of hydropower (about $51 \mathrm{TWh}$ ), photovoltaic (about $33 \mathrm{TWh}$ ) and wind power (about $18 \mathrm{TWh}$ ). The renewable heat potential in 2050 amounts to about $106 \mathrm{TWh}$, which consists of the thermal use of biogenic fuels and the waste heat recovery from non-fossil thermal power plants and solar thermal energy. Thus, in total RES potential of about $220 \mathrm{TWh}$ is assumed (Renewables4Industry 2018). 


\section{MOTIVATION FOR DECARBONIZATION}

Industrial companies are striving for efficient and renewable energy-based production to reduce the $\mathrm{CO} 2$ emissions associated with product production. This is mainly due to the expected increase in the cost of $\mathrm{CO} 2$ emissions. Nonetheless, industrial enterprises are assumed to be subject to the condition of economic viability in the event of a process modification. This especially concerns the conversion of individual plants, which are currently design to be constantly supplied.

Among other things, the EU relies heavily on the market-based instrument of the Emissions Trading System to implement its climate change targets (Moser 2013). In the EU Emissions Trading Scheme (EU-ETS for short) plants with a capacity of more than $20 \mathrm{MW}$ were registered on the basis of EU Directive 2003/87/EC. According to Goers et al. (2010), the 11,500 installations account for 50\% of EU-wide $\mathrm{CO} 2$ emissions. Not only because of the many problems with other political instruments such as taxes and direct regulation, but also due to their theoretically cost-minimizing character, economists often advocate the use of certificate systems (Goers et al. 2010).

Even before the climate agreement of Paris, the main political pillars were fixed. The roadmaps published by the European Commission (which are not legally binding) for the transition to a competitive, low-carbon economy by 2050 and the Energy Roadmap 2050 have a long-term horizon, in line with the EU Council Decision on the $2{ }^{\circ} \mathrm{C}$ target to reduce greenhouse gas emissions of 80 95\% (European Commission 2011).

The Paris Agreement is an agreement of the 194 member states of the United Nations Framework Convention on Climate Change (UNFCCC) with the aim of protecting the climate in succession to the Kyoto Protocol. The differences between the Paris Convention and the 1997 Kyoto Protocol are significant. In the Kyoto Protocol, few countries have committed to achieving binding emission reductions, while now the Paris Convention is striking globally for 194 states, adapted to the different stages of development of each country.

The additional effort required implies an increase in the cost of greenhouse gas emissions (and in particular CO2) - either through appropriate adjustments in emissions trading (which is considered likely in the industrial sector) or through other policy instruments (especially outside the industrial sector).

\section{APPROACHES OF DECARBONIZATION}

The authors define "CO2 emission reduction" as a reduction of anthropogenic, fossil $\mathrm{CO} 2$ emissions. Thus, within the framework of the three solution strategies of "sustainability" for the provision of useful energy, the possibilities arise (Moser 2013) ...

- ... to use renewable energy sources instead of fossil fuels and as an alternative CCU (this corresponds in a broader sense to the consistency strategy of sustainability),

- ... by means of (energetic) energy efficiency, to make the primary and final use of energy (for example space heating) more energy-efficient, or

- $\quad .$. to reduce the amount of energy savings without compensation (e.g. intentional lower room temperatures, intentional denial of mobility services, etc) (sufficiency strategy).

The authors assume that the sufficiency strategy has limited political feasibility in the short term and moreover face a small literature basis. Consequently, this approach is disregarded. However, the necessity of a primarily non-technological basic research in this area is pointed out. On the basis of the 
consistency strategy and the efficiency strategy, the following approaches for a $\mathrm{CO} 2$ emission reduction result:

- Electrification: The majority of the final energy is provided by the energy source electricity, because private, industrial and transport processes are converted to electricity or electricitybased products such as hydrogen or synthetic methane.

- Thermal sources (solar and geothermal): The provision of the final energy is made to a maximum extent from the use of geothermal and solar thermal technology (without consideration of electricity generation).

- Biogenic energy sources (renewable hydrocarbons): The supply of final energy is made from a large proportion of biogenic energy sources (without consideration of electricity generation). Biogenic energy carriers are only suitable for reducing $\mathrm{CO} 2$ emissions if they have recaptured or previously bound the released carbon dioxide through their growth.

- Carbon Capture and Utilization. CCU is not treated separately in this paper.

- Energy efficiency: Avoiding CO2 emissions is achieved by using more efficient technologies. In this approach, a far-reaching fuel switch, as assumed in the other three approaches to $\mathrm{CO} 2$ emission reduction, only occurs if it implies an increase in efficiency.

\section{ENERGY BALANCE-BASED SCENARIOS}

Two scenarios have been prepared for the energy composition after the successful, complete transition to renewable energy sources. One scenario assumes a pure electrification (including hydrogen or renewable methane as storage) of all applications and processes, the other one assumes the equal application of all above-mentioned $\mathrm{CO} 2$ emission abatement approaches. It was taken into consideration ...

- ... the renewable energy potentials identified beforehand and

- $\quad .$. the uses that are possibly satisfied by an energy carrier with regard to energy balance's categories of energy applications (e.g. solar thermal will not satisfy mobility) and

- $\quad . .$. the energy trends that are currently to be estimated.

As energy trends to be realized we assume:

- Significantly higher energy efficiency in buildings in the long term; in addition to the final energy sources from bioenergy, solar and waste, the supply of space heating by high efficient heat pumps and the use of industrial and cogeneration excess heat is enforced.

- Transition of industrial processes to the usage of electricity or hydrogen and synthetic methane.

- Transition of mobility services to the usage of electricity or hydrogen and synthetic methane.

- Constant demand for ICT and lighting services, based on the assumption that higher electricity demand is accompanied by parallel increases in energy efficiency.

The following assumptions are made:

- We use the energy balance to derive the demand for renewable energy in the respective sectors or applications. As the energy balance does not indicate load profiles, we add usual storage demands.

- Note that in the pure electrification scenario, it is assumed that no other pathway of decarbonization is applied, not even in parts. In other words, residual lower-exergy potentials (for example, power plant and industrial waste heat) are not used and for example, solar thermal is not applied at all. Nevertheless, we assume efficient heat pumps to be applied instead of direct heating from electricity. 


\section{RESULTS}

The following statements can be derived from restructuring/recalculating the energy balance based on the above-mentioned assumptions:

Pure electrification cannot be achieved without imported electricity. Necessary imports can be described as "enormous" as they would equal three times the production. It can be deduced that electrification, as a stand-alone path of $\mathrm{CO} 2$ emission reduction, seems hardly feasible, especially if other countries pursue similar objectives and also apply the electrification pathway (and cannot provide such enormous electricity surpluses).

As a result, it can be further deduced that all the paths of $\mathrm{CO} 2$ emission reduction must be applied, since the others are not feasible on their own (usability of the energy carrier). A national energy autarky would require even greater efforts to reduce primary energy demand. These savings are nonspecifically required from all sectors. A national energy autarky is only realistic provided enormous efforts with regard to primary and end-use energy efficiency.

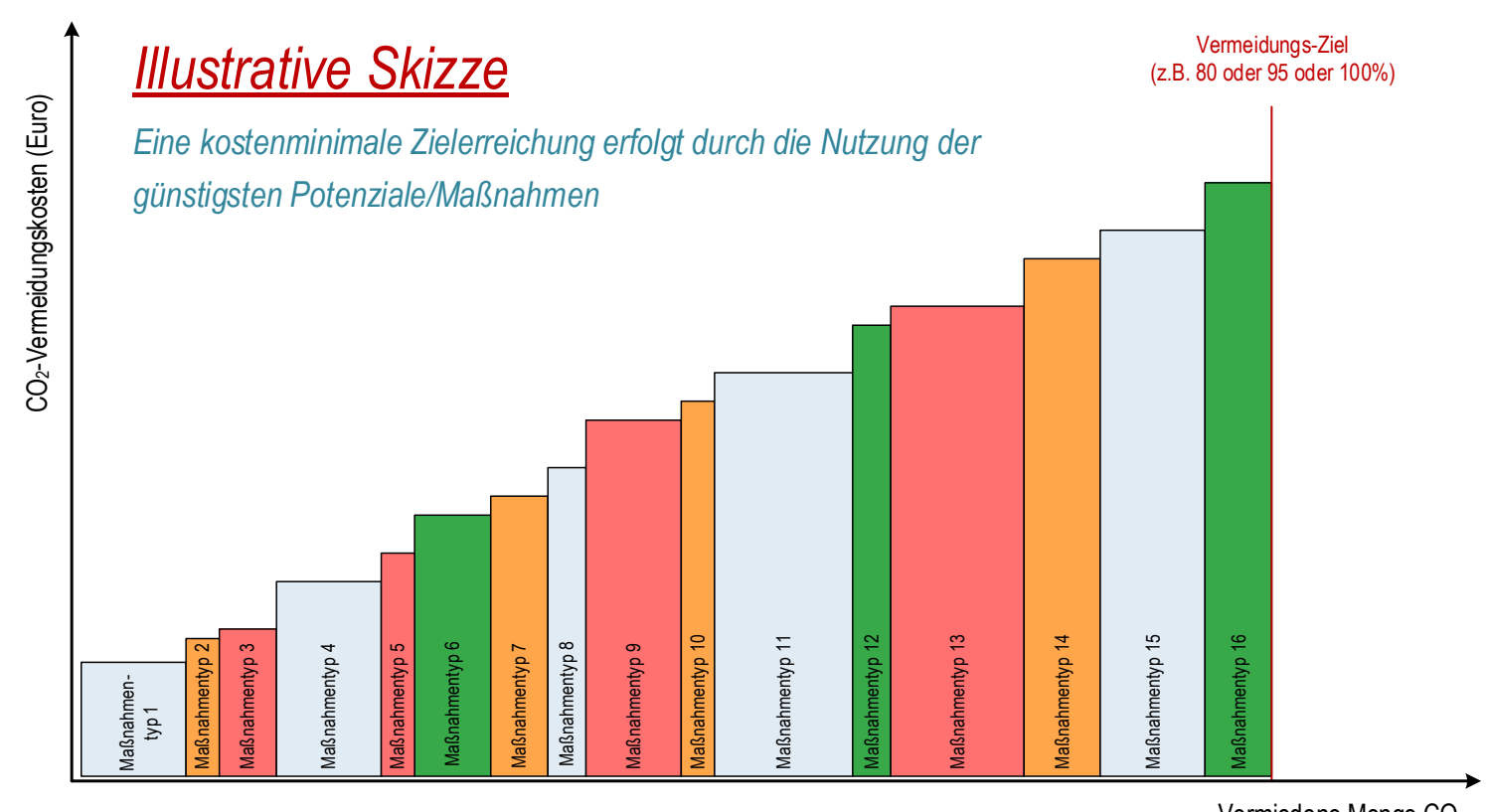

Vermiedene Menge $\mathrm{CO}_{2}$

\begin{tabular}{|c|c|}
\hline Energieeffizienz & Primärenergieträger Solar- oder Geothermie \\
\hline Primärenergieträger Elektrizität & Primärenergieträger Biomasse \\
\hline
\end{tabular}

Fig. 1: The results clearly indicate that there needs to be a political target definition instead of a political technology, pathway, or measure definition. Only cost-efficiency of measures will influence their applicability.

Source: Energy Institute at the JKU Linz.

From both scenarios it can be deduced that long-term storage and backup provision need to be provided by an renewable or renewables-based energy carrier which is easily storable in enormous amounts and easily transportable. Thus, only bioenergy and electrolytically produced hydrogen or renewable methane (eventually renewable liquids) are the first-best solution.

If the national potential of renewable energy sources is used to a large extent, the dependency on energy imports is reduced. An import dependency of about 30\% remains. However, if no further 
efforts on primary energy efficiency than those included in the energy trends described above are set, imports are required. Generally, imports must be at an exergetically appropriate level, i.e. in the form of electricity, hydrogen, renewable methane or bioenergy.

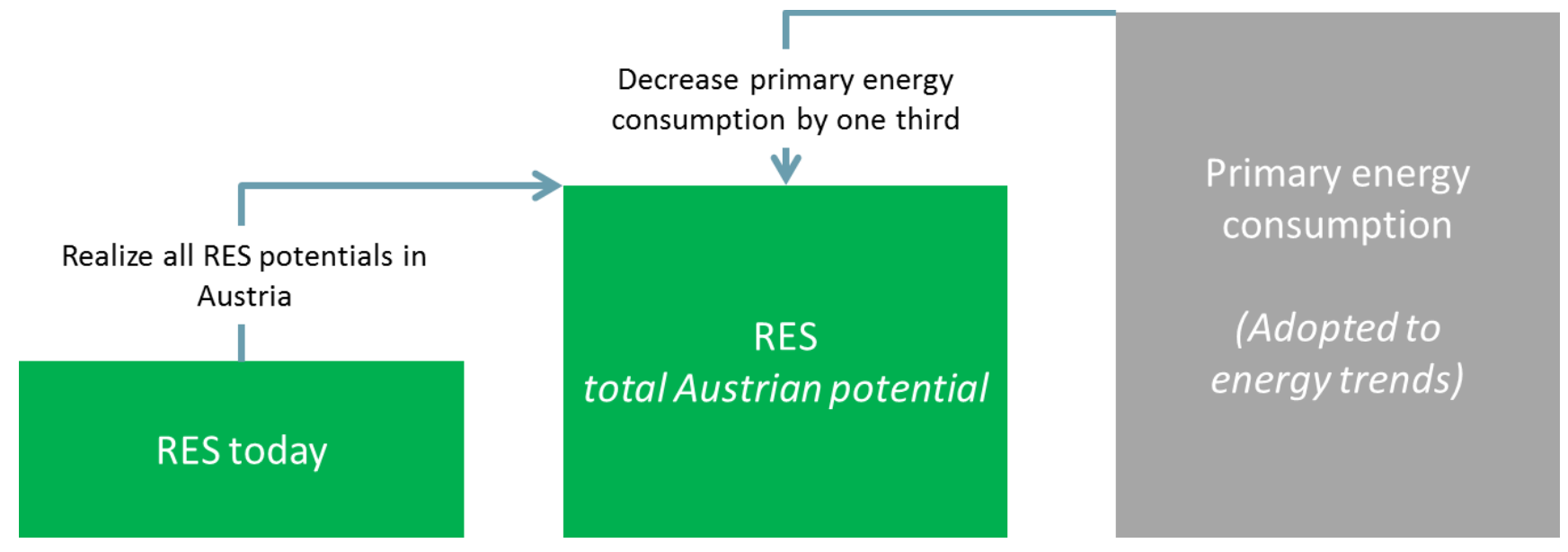

Fig. 2: The transition to $100 \%$ RES - totally covered by national supply - means to realize all renewable potentials and to decrease the already adopted primary energy consumption by about one third. Source: Energy Institute at the JKU Linz.

\section{CONCLUSIONS}

Based on the results, we derive essential fields of action. It is important to clarify, first, that the import of renewables is a trade-off to any decarbonization pathway implemented in Austria. Second, we want to emphasize that measures need to be concerted, i.e. stand-alone measures (e.g. support for single technologies) should be avoided and are possibly harmful with regard to the target.

Buildings must be designed so that they can be heated with low flow temperatures: Excess heat at low temperature levels from thermal power plants (least temperatures are necessary to achieve maximum electric efficiency) and industrial processes must be sufficient for space heating. Moreover, if buildings can be supplied with low temperatures, this implies that they do not compete for higherexergy heat-sources and leave the higher-exergy potentials to with processes needing higher levels of exergy. 


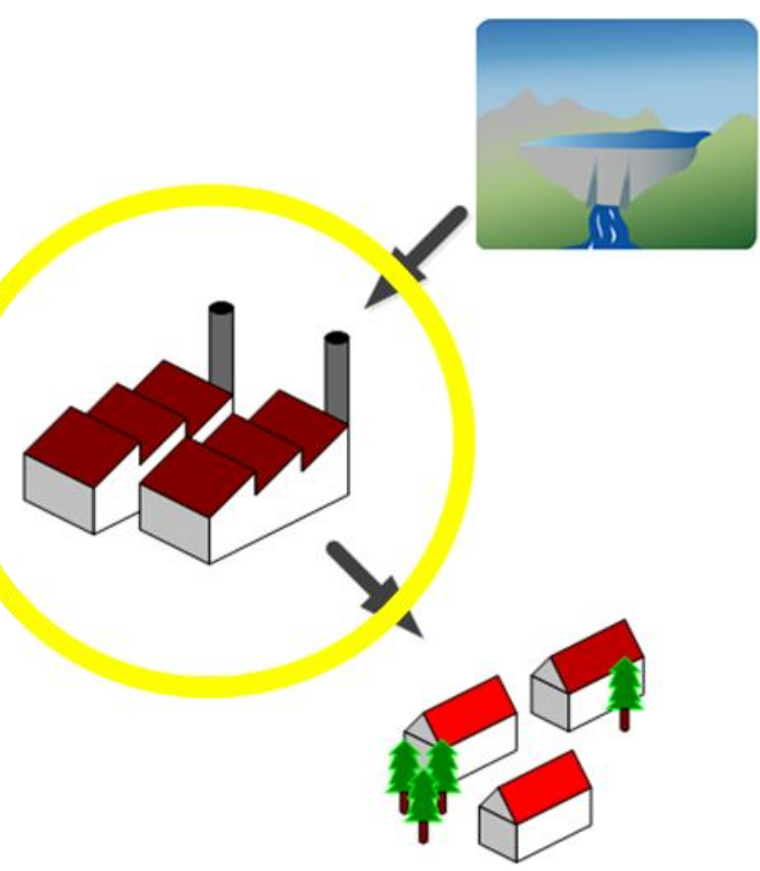

Fig. 3: One of the manifold needs in the transition is to supply buildings with waste heat from thermal power plants and from industry. But: due to the importance of primary energy efficiency building heating and hot water systems have to cope with a low-temperature supply. Source: Energy Institute at the JKU Linz.

A comprehensive expansion of excess heat recovery by feeding excess heat from industrial plants and thermal power plants into thermal grids is required. This again requires lower network temperature levels. In order to ensure the spatial feasibility of this low-temperature heat use and cost-efficiency of heating networks, requirements towards energy spatial planning are obvious.

The transition of industrial processes and the transport sector to electricity or renewable hydrogen/methane is an essential part of a comprehensive $\mathrm{CO} 2$ emission reduction. However, some of these potential modifications are already included in the adopted primary energy consumption as they constitute an energy trend. Thus, there is the need for further efficiency measures to decrease import dependency. To ensure a renewable energy system, it is necessary to ensure the import of renewable hydrogen or renewable gases. Biomass CHP plants and CHP plants supplied with renewable gases will make a major contribution to ensuring security of supply. Good storability of renewable gases in terms of quantity and duration, but also the use of natural gas as a bridging technology in the transformation of the energy system, underlines the need to preserve the high-ranking natural gas infrastructure and the gas-fired CHP plants.

\section{REFERENCES}

Renewables4Industry, (2018), Abstimmung des Energiebedarfs von industriellen Anlagen und der Energieversorgung aus fluktuierenden Erneuerbaren. Endberichtsteil 2 von 3, Diskussionspapier zum Projekt Renwables4Industry, F\&E-Dienstleistung für den Klima- und Energiefonds im Rahmen des Energieforschungsprogramms 2016.

Goers, Lindorfer, Moser, (2010), Emissionszertifikate, Ökostromzertifikate, Energieeffizienzzertifikate ... warum ausgerechnet Zertifikate? Energie-Info 7/2010 des Energieinstituts an der Johannes Kepler Universität Linz vom 19.10.2010.

European Commission, (2011), A Roadmap for moving to a competitive low carbon economy in 2050. European Commission COM(2011) 112 final 08.03.2011. 
Moser, (2013), Energieeffizienz-Verpflichtungen für Energieversorger - Optimierung eines österreichischen Verpflichtungssystems der endkundenseitigen Maßnahmensetzung. In: Priewasser, Steinmüller (Hrsg.): Energie- und Ressourcenmanagement, Band 1, NWV Verlag. 


\title{
Towards Giga-Scale Thermal Energy Storage for Renewable Districts in Austria
}

\author{
Wim van Helden ${ }^{1}$, Samuel Knabl ${ }^{1}$, Ingo Leusbrock ${ }^{1}$, Georg Engel ${ }^{1}$ \\ ${ }^{1}$ AEE-Institute for Sustainable Technologies \\ Feldgasse 19, 8200 Gleisdorf, Austria \\ Phone: +43 3112 / 5886-228 \\ E-Mail: w.vanhelden@aee.at
}

\section{SUMMARY}

The giga_TES project has as aim to develop materials and construction methods that are needed to realize the next generation of very large underground water storage, with a water volume from 200,000 to $2,000,000 \mathrm{~m} 3$, or in the order of a giga-litre. An industry-led international consortium works on the development of novel polymeric liner materials and concrete formulas, on building construction technologies for bottom, walls and lid of the storages, on simulation techniques for the thermal and hydraulic performance and integration into district heating systems and on the cost analysis of the system and its components. In the first half year of the project, novel polymers were produced in small batches for duration tests, hydrological mapping to the DH systems in Austria was started, first designs of floating lid elements made and construction technologies for deep constructions with and without groundwater influence inventoried.

\section{INTRODUCTION}

To reach the long-term goal of 100\% renewable energy supply, district heating (DH) networks require large-scale thermal energy storage (TES) technologies such as pit and tank storage. As these systems need to be implemented in an urban environment, the required surface area should be minimised to compensate for the relatively high land price in an urban environment. These minimized costs can be achieved by moving the system below surface level and further decreased by allowing usage of the surface area for recreation or installation of solar collector fields. DH systems call for TES volumes up from $50,000 \mathrm{~m}^{3}$ to as much as in the order of one billion litres, or 1 million $\mathrm{m}^{3}$. Presently, large-scale thermal storages have been built and are in operation in Germany and mainly Denmark, with recent storages having volumes of nearly $200,000 \mathrm{~m}^{3}$.

Experience with the existing plants is still limited due to the low number and short age of the storages. Improvements are needed on material performance and durability and on material and component development. Cost effectiveness and system integration call for higher storage density and thus higher temperatures, imposing even higher demands on the materials used. This and the requirements of vapour tightness, serviceability and durability of innovative solutions for lid, wall and liners call for novel materials and components as well as for improved durability testing methods. Additionally, the envisioned size of new giga-scale storage technologies and the construction in the subsurface require new construction methods.

The project giga_TES is an Austrian flagship- project targeting at the development of large-scale thermal storage concepts for renewable districts, with a focus on feasible Austrian implementations. The project consortium consists of key material and component industries, a major construction company, an engineering and two DH companies, backed by four Austrian and two foreign research institutes that have deep knowledge and experience in the field of materials, components and system technologies for very large thermal energy storages.

\section{METHOD}

The project pursues the following technical, economic and scientific goals:

(1) to develop a comprehensive overview with all requirements and relevant boundary conditions for the use and implementation of giga-scale storages and to implement a scientific decisionmaking tool for obtaining representative prospective application scenarios internationally and in Austria.

(2) to develop innovative and optimal construction methods for giga-scale TES with particular consideration of ground conditions. Based on five typical soil and rock profiles various ground engineering approaches for deep pit excavations will be assessed and their opportunities illustrated. 
(3) to elaborate economic and practicable solutions for critical storage components which are the bottom slab, walls and the cover.

(4) to develop novel polymeric and inorganic materials for the construction of large-scale thermal energy storages along with testing and lifetime assessment methods for faster and more realistic screening and pre-qualification of such materials.

(5) to develop simulation models with different modelling depths, and to test and apply them to optimize the storage design for relevant boundary conditions. Furthermore, a methodology to predict the ground and ground water temperature increase depending on the specific geohydrological conditions and the storage design as well as a co-simulation platform for optimization of the system configuration and control strategies will be developed.

to evaluate the added value and impact of large-scale storage in existing and future district heating systems and to analyse the sensitivities and mutual influence of system parameters on the overall performance, by that deriving operating windows and optimized system configurations for given boundary conditions, with separate attention to Austrian boundary conditions.

The project has a broad range of activities, ranging from geohydrological studies, design of floating cover elements, materials development for polymeric liners and functional concretes, building construction technologies for deep underground constructions with and without groundwater influences, numerical simulation of hydraulics and thermal performance of storage and its environment, numerical simulation of the district heating systems and cost evaluations.

\section{RESULTS}

In the first months of the project, work was performed on mapping the district heating grids in Austria to the geohydrology (Fig 1). A first numerical simulation was set up of a simplified model of the storage in a number of soil configurations in order to determine in first order the heat losses from the storage. Samples of novel liner formulas were produced and put in to long-term testing equipment, in order to determine the expected lifetime of the material under high temperature and elevated pressure conditions. First designs of floating lid elements, both in metal and in concrete, were made with special attention to the construction details, the thermal and water vapour diffusion properties and the operational aspects. And the different possibilities for deep constructions were inventoried (Fig.2) and used as a basis for the mock-up tests, to be prepared in the next period.

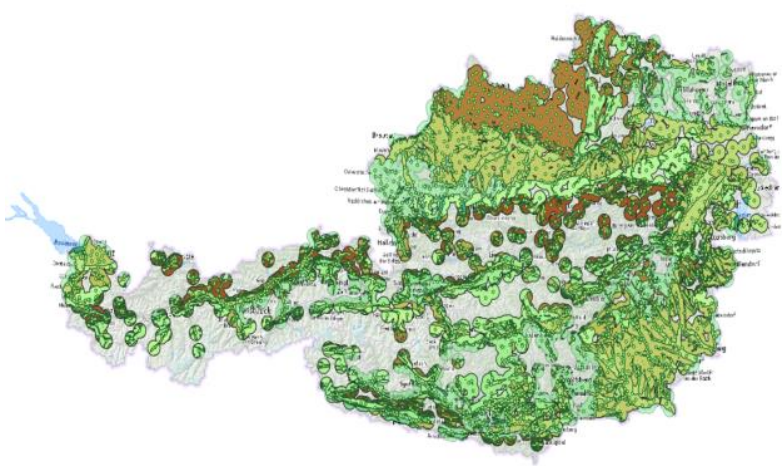

Fig. 1: GIS map of the lithology to be combined with the DH map.

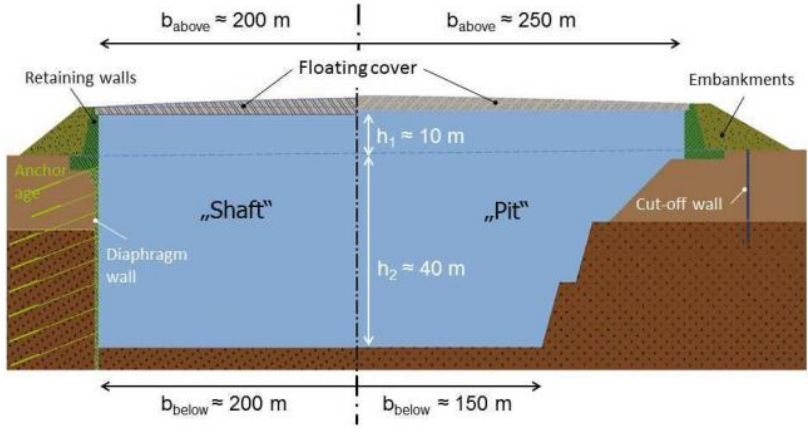

Fig. 2: Possible construction methods for a gigascale TES (source: ste.p)

At the conference, a more complete overview of the present results will be given. 


\title{
REDUCTION OF CO2-EMISSIONS WITHIN THE GAS SECTOR BY IMPLEMENTATION OF ENERGY EFFICIENCY MEASURES AND RENEWABLE PROCESS HEAT
}

\author{
Lukas Wimmer1, Dr. Bastian Schmitt 1, Prof. Dr. Klaus Vajen1, Roland Heinzen2, Detlef Grebe3 \\ 1 University of Kassel, Institute of Thermal Engineering, Kurt-Wolters-Str. 3, 34109 Kassel, Germany 5 Phone: \\ +49561804 3890, Email: solar@uni-kassel.de \\ 2 Enertracting GmbH, Altmüllerstr. 6-8, 34117 Kassel, Germany \\ 3 EnergieNetz Mitte GmbH, Johanna-Siegmund-Schuckert-Str. 2, 34225 Baunatal, Germany
}

\begin{abstract}
SUMMARY
The gas sector's yearly energy demand for transport equals almost $15 \%$ of the included energy of the transported gas mass [IWU, 2014]. Most of the energy is used in compressor stations to increase the pressure up to $10 \mathrm{MPa}$ for the long distance pipelines. For the delivery to end-users the pressure has to be reduced up to $0.1 \mathrm{MPa}$. Therefore, gas pressure regulating and metering stations (GPRMS) are used, which play a key role in urban gas supply. The gas throttling process within GPRMS are characterised by a constant heat demand. Thus, all German GPRMS consume nearly $1 \mathrm{TWh} / \mathrm{a}$. By analysing heating equipment, mode of operation, and control of GPRMS it can be found that many measures can be applied to reduce their energy consumption, such as optimized control strategies, optimization of hydraulics, implementation of efficient heat supply technologies like CHP or HP, or integration of large solar process heat plants. The paper gives an overview on potential measures that have already been implemented, are currently in planning and realisation, or within the conceptual design phase.
\end{abstract}

\section{INTRODUCTION}

Over $20 \%$ (3,022 PJ) of Germany's primary energy consumption is covered by natural gas [BMWi, 2018]. For the transportation of natural gas throughout the country, more than $510,000 \mathrm{~km}$ of pipelines are installed. These are divided in high pressure long distance pipelines (8.10 MPa) and low-pressure regional pipelines $(0.1 \mathrm{MPa})$. The associated pressure reduction between long distance and regional pipelines is ensured by so called gas pressure regulating and metering stations (GPRMS). Due to the Joule-Thomson-Effect the gas is cooled during the throttling. Since the gas throttling is endothermic, the natural gas has to be heated to prevent the freezing of the plant components and to ensure a steady gas supply. Typically, oversized natural gas boilers are used to preheat the gas, resulting in considerable costs and $\mathrm{CO}_{2}$-emissions. Due to the relatively low temperature level and an almost constant annual heat demand, GPRMS allow economic application of renewable heating systems such as solar heating plants or heat pumps. Based on different potential measures to reduce carbon emissions of GPRMS as well as past and ongoing R\&D activities, EnergieNetz Mitte GmbH (operation of GPRMS), University of Kassel (development of holistic concepts), and Enertracting $\mathrm{GmbH}$ (planning, realization, and financing) have set up a cluster of competence to develop, implement, and disseminate new energy concepts throughout the gas sector.

\section{ENERGY EFFICIENCY AND RENEWABLE PROCESS HEAT FOR GPRMS}

Based on very high requirements on safe and stable operation, GPRMS are typically characterised by redundant and oversized installations to handle the worst-case scenario in wintertime with high volume flows and low temperatures. Therefore, inefficient operating conditions can be detected throughout the year. Depending on the respective installation and mode of operation, different measures can be applied to increase the overall efficiency. Since the gas outlet temperature (GOT) heavily influences the preheating demand, a lowering of the GOT can significantly reduce the heat demand of a GPRMS. Often, this measure requires optimisation of hydraulic set-up and control within the GPRMS to enable a smooth and even operation.

Besides the operation with a constant and low GOT, it is possible to control this temperature according to the dew point of the air to avoid condensation outside pipes, valves, and accessories. Periods of low air dew point temperatures in winter are typically characterised by high natural gas flow and therefore significant energy consumption for preheating. As a result, a dew point control leads to large energy savings in winter and slightly higher energy consumption in summer, which is advantageous for the integration of solar heat. After identification and implementation of efficiency measures, the resulting heat demand can be provided by renewable or efficient heat sources. First, GPRMS turn out to be very 
suitable for integration of solar process heat, due to their constant heat demand in summer and low temperature level for integration. Therefore, it is possible to realise solar heating plants with high specific annual yields and very good economic feasibility. Besides solar heating plants, combined heat and power or heat pumps can be used, also in combination with solar heat to decrease the natural gas consumption for preheating.

\section{PLANNED AND REALIZED PROJECTS}

Up to now, the cluster of competence realised retrofits of three GPRMS. The first one was equipped with a large-scale solar heating plant of $360 \mathrm{~m}^{2}$ in combination with district heat from a nearby biogas CHP [Heinzen, 2014]. Thus, the renewable heating concept replaces over $90 \%$ of the initial natural gas demand for preheating. The other two GPRMS were equipped with a solar heating plant in combination with gas absorption heat pumps $\left(155 \mathrm{~m}^{2}\right.$ with $3 \times 41 \mathrm{~kW}$ and $420 \mathrm{~m}^{2}$ with $\left.3 \times 41 \mathrm{~kW}\right)$. The latter one has an additional own gas heat exchanger for solar and heat pump with an integration temperature of $20 . .30{ }^{\circ} \mathrm{C}$, resulting in specific solar yields over $600 \mathrm{kWh} / \mathrm{m}^{2} \mathrm{a}$ [Wimmer, 2016].

Besides these retrofitted GPRMS, two new efficient concepts will be implemented this year. One GPRMS will be equipped with an innovative drain back solar heating plant combined with improved hydraulics and new control of the gas heating process. The second GPRMS will be equipped with a solar heating plant in combination with a combined heat and power plant. Currently, both projects are in the planning phase, followed by the implementation in summer 2018.

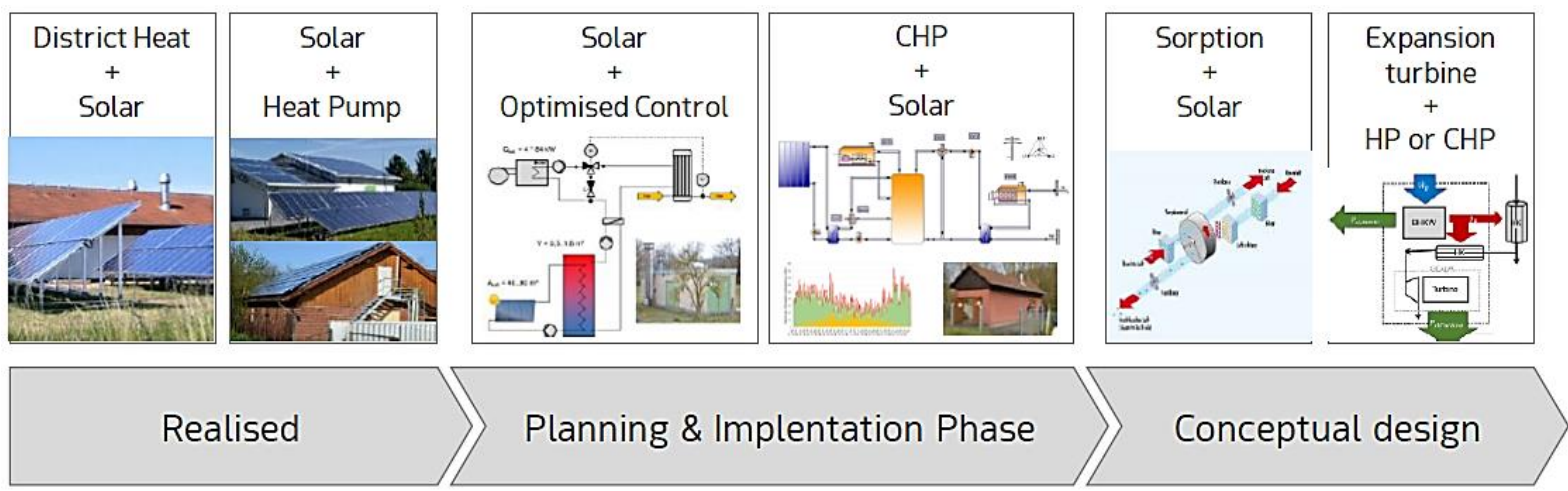

Fig. 1: Different measures for GPRMS being realized, implemented, or planned at the moment

Finally, new ideas for efficiency improvement and renewable heat are in conceptual design phase with the aim for realization by the end of 2019 , e.g. the combination of a solar heating plant with a sorption system including dew point control or the integration of an expansion turbine with a combined heat and power plant or a heat pump.

Next to the realisation of the mentioned concepts and their scientific monitoring, the cluster of competence will be active to disseminate their findings and operational experiences to other stakeholders such as gas network operators, energy consultants, and planners of GPRMS. Besides experiences and lessons learned from planning, realisation and operation of the named concepts, the paper will give insights on the economics and transferability of the installations.

\section{REFERENCES}

BMWi, (2018), Natural gas supply in Germany, Federal Ministry for Economic Affairs and Energy, Berlin, GER.

Heinzen, R., Zass, K., (2014) Solar heat contracting for multi-family houses and industry (in German language), IHKS Journal, Munich, GER.

IWU, (2014), Cumulative energy demand (CED), Institute for housing and environment, Darmstadt, GER.

Wimmer, L., Schmitt, B., Heinzen, R., (2016), Solar process heat seeking new fields of applications (in German language), Erneuerbare Energien, issue 01/2016, pp. 17-18 Gleisdorf, AT. 


\title{
EVALUATION OF ENERGY CONSUMTION AND ENVIRONMENTAL IMPACT OF LONG TERM HOT WATER THERMAL STORAGE CONSIDERING STRATIFICATION AND CONVECTIVE BEHAVIOUR
}

\author{
M.B. Rashevski*, Dr. H.D. Doan** and Prof. K. Fushinobu** \\ * Institute of Mechanics, Bulgarian Academy of Science \\ **Department of Mechanical and Control Engineering, Tokyo Institute of Technology \\ e-mail: mrashevski@hotmail.com
}

\section{EXTENDED ABSTRACT}

Energy consumption and energy saving have become priority concern in the $21^{\text {st }}$ century. About $40 \%$ of energy consumption in EU is generated by building consumption [1] - the highest demand compared to the rest of the sectors of the economy. Due to low efficiency of façade solutions and lack of proper architectural design abilities heating and cooling form about $65 \%$ of this demand [2] in the occupied residence buildings (including DHW and electricity for the stated purposes). One of the possible solutions of this problem is long term energy storage that can shift the excess of summer energy to the period of winter demand and thus equilibrate the system. Currently there is no best solution of long term storage, subject that remained open over decades with partial solutions varying from hot water accumulators, compressed air or reversible fuel cells, able to produce and store hydrogen over long periods and make the use of it post factum. This research deals with a specific problem - hot water storage over long periods, convective behaviour and stratification of thermal heat storage (THS). Applying mathematical model that takes into account fundamental thermo-dynamical relations, we would try to simulate an example of storage facility for a two storey office building next to Sofia, Bulgaria. This model consists of exact building data and should prove heating demand coverage or respectively heating demand insufficiency of the system, as well as longest period of coverage, highest $\Delta \mathrm{T}$ stratification its effect on the system and eventually environmental impact.
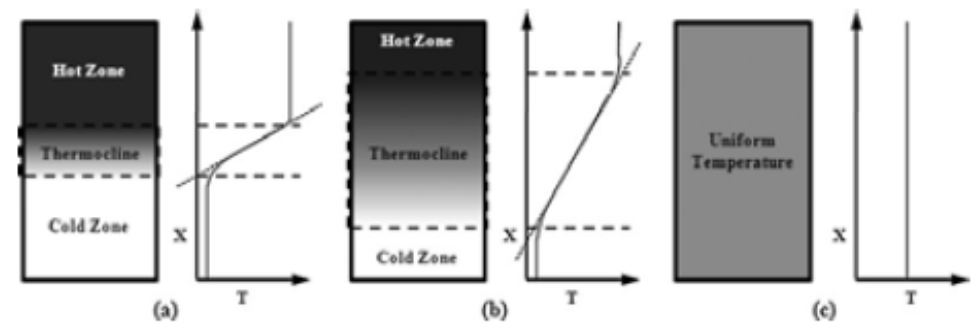

Figure 1 - Degrees of stratification within a storage tank with the same amount of stored heat (a) highly stratified, (b) moderately stratified and (c) showing fully mixed, unstratified storage. [3]

Initial model was completed using Maple 15 software with the finite differences method, however without taking into account convective behaviour of the water tank. Further analysis completed in Matlab, Microsoft Excel and other software products should help in the calculation processes - for more complicated simulations over long periods of time and for complicated algorithms relying on the computing abilities of the Supercomputer in Tokyo Institute of Technology. Temperature requierments for domestic hot water (DHS) use and low temperature surface heating is $40^{\circ} \mathrm{C}$. Further initial and boundery conditions are described. The building demand is calculated precisely taking into account climate conditions, construction details and effective applicable administration standards. 


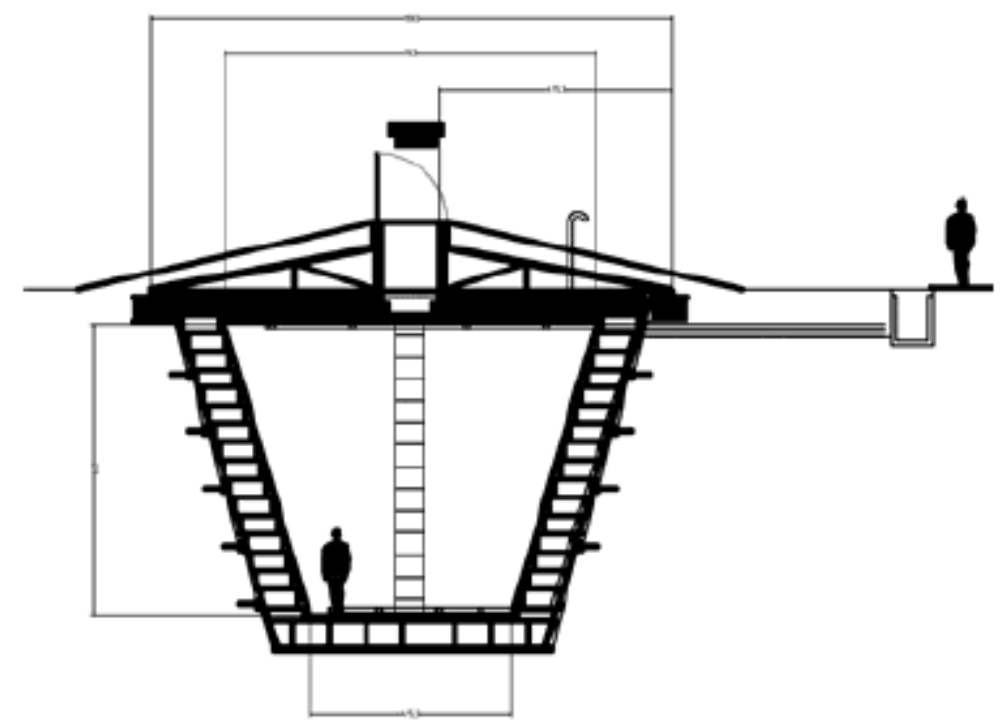

Figure 2 - Building data of investigated storage, including size and material properties [4]

\section{REFERENCES}

[1] Directive 2010/31/EU Of The European Parliament And Of The Council, pp.1, 19 May 2010.

[2] U.S. Energy Information Administration, Residential Energy Consumption Survey, 2009.

[3] Michel Y. Haller, Cynthia A. Cruickshank, Wolfgang Streicher a, Stephen J. Harrison, Elsa Andersen, Simon Furbo, Methods to determine stratification efficiency of thermal energy storage processes - Review and theoretical comparison, pp. 3, 26 August 2009

\section{ACKNOWLEDGEMENTS}

[4] Institute for Zero Energy Buildings (IZEB), Sofia, Bulgaria

We would like to acknowledge the members of Institute of Zero Energy Buildings in Sofia who kindly provided building data in form of plans and material properties data for the simulated mathematical model.

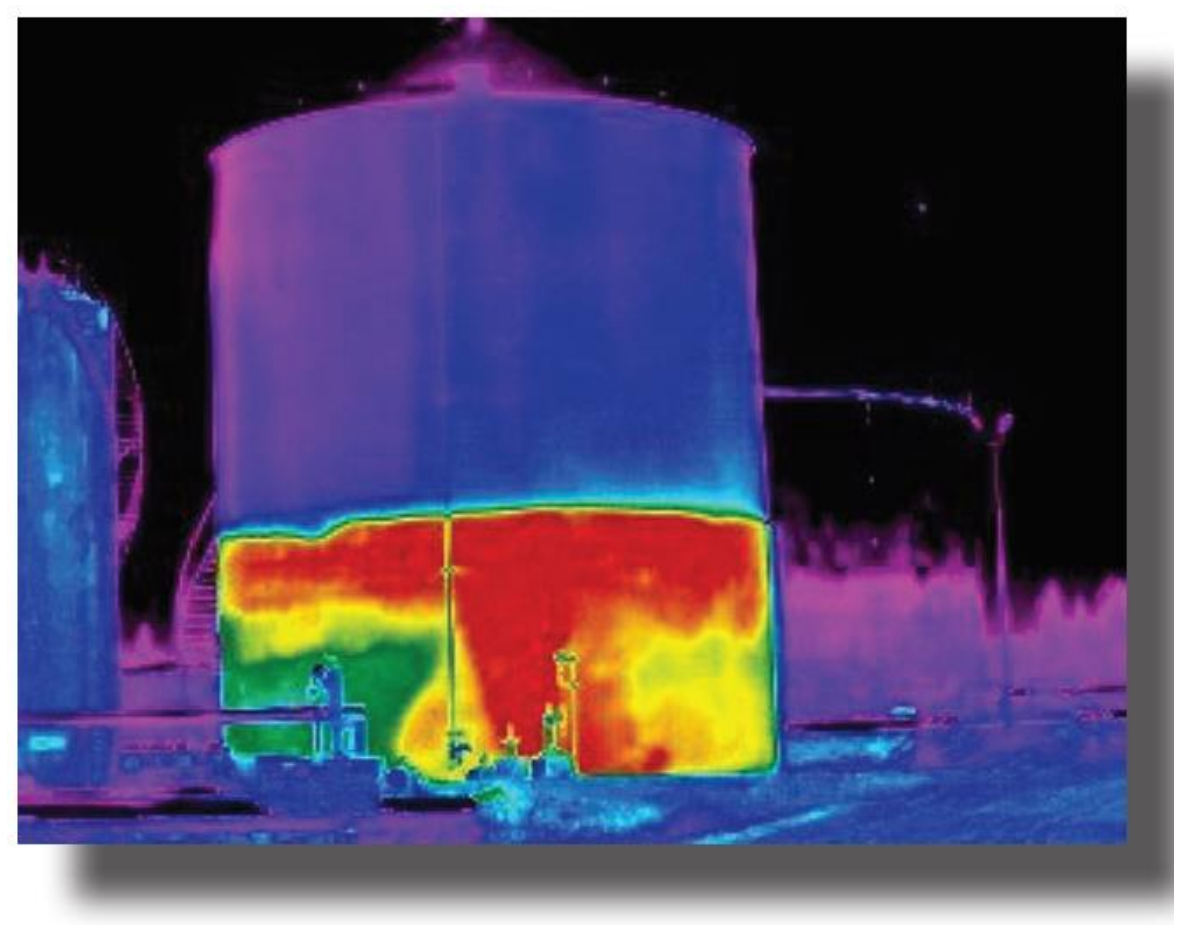

Long Term Energy Accumulation in Underground Hot Water Tanks.

Fluid Convective Behaviour and its Influence on the Thermal Losses 
Climatization Consumption Profiles in an Average Home
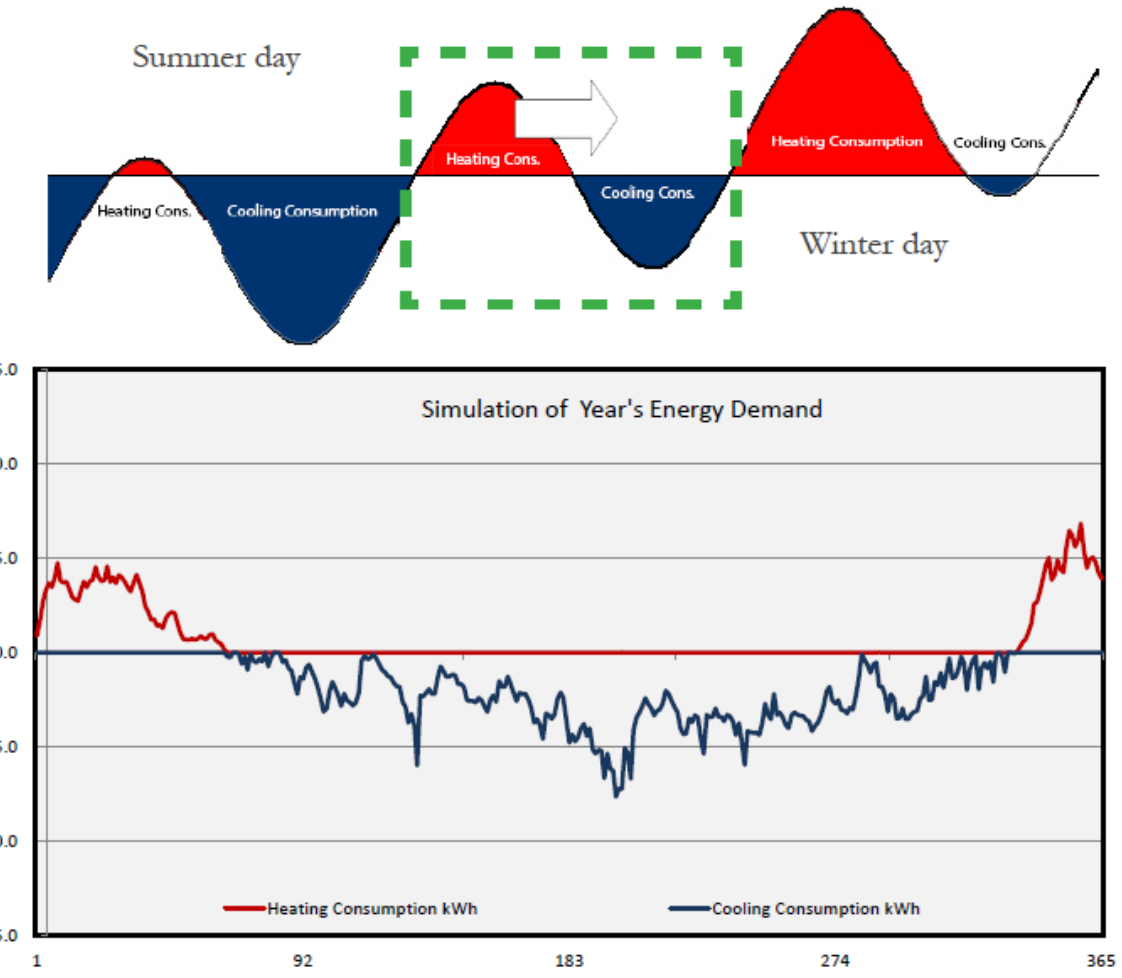

Need for shifting the excess of energy in time, thus

Equilibrating the system, through

Energy accumulation for

Short time periods

- less losses - easy ways, and

Long periods

- no absolute solution

Hot water thermal accumulator design

Construction materials

provided by Institute for Zero Energy Buildings in Sofia
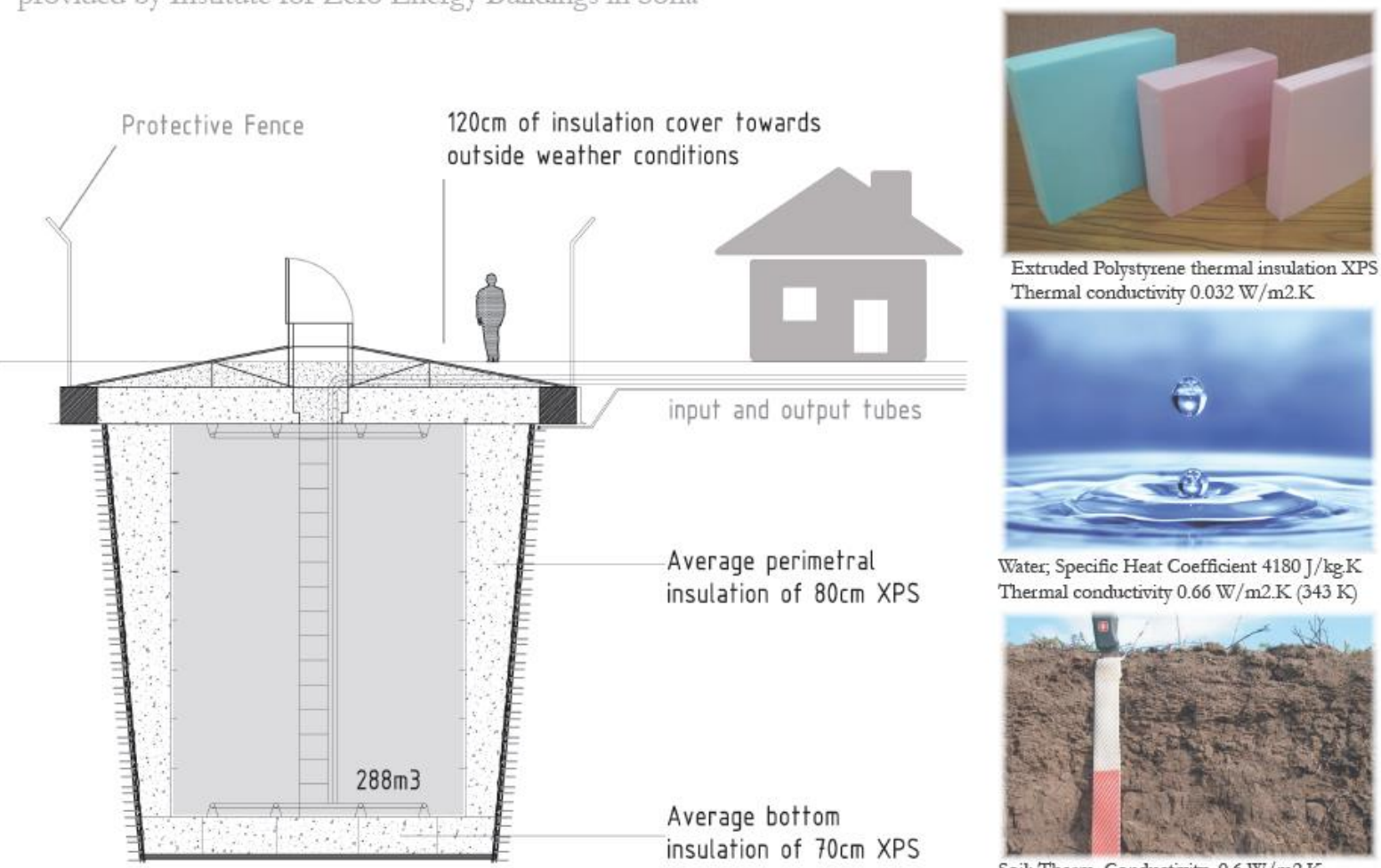

Water, Specific Heat Coefficient $4180 \mathrm{~J} / \mathrm{kg} . \mathrm{K}$ Thermal conductivity $0.66 \mathrm{~W} / \mathrm{m} 2 . \mathrm{K}(343 \mathrm{~K})$

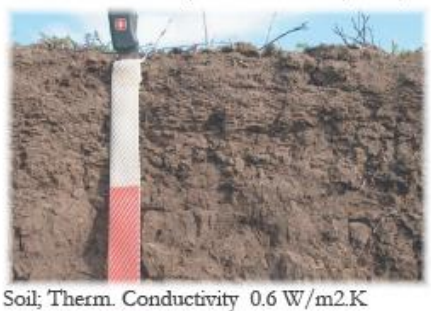




\section{Methods of solution}

\begin{tabular}{|c|}
\hline Resolution and non-dimensionalization for space and time \\
\hline Define internal $\mathrm{T}^{\circ} \mathrm{C}$ and outside air $\mathrm{T}^{\circ} \mathrm{C}$ in initial state \\
\hline Solving stream function equation (Successive Over Relaxation) \\
\hline Define vorticity on boundary \\
\hline $\begin{array}{l}\text { Solving the vorticity equation (Finite differences approach) } \\
\text { - } \text { stream function input } \\
\text { - temperature input for the buoyancy term }\end{array}$ \\
\hline $\begin{array}{l}\text { Solve temperature equation (Finite differences approach) } \\
\text { - stream function input }\end{array}$ \\
\hline Update boundary conditions for $\mathrm{T}^{\circ} \mathrm{C}$ and weather update \\
\hline Return to real values and plot \\
\hline
\end{tabular}

An Efficient Approach to Simulate Natural Convection in Arbitrarily Eccentric Annuli by Vorticity-Stream Function Formulation

C. Shu and K. S. Yeo

Department of Mechanical and Production Engineering, National University of Singapore, Singapore

Q. Yao

Institute of High Performance Computing, Singapore Science Park 1, Singapore

\section{GOVERNING EQUATIONS AND BOUNDARY CONDITIONS}

Based on the Boussinesq approximation, the nondimensional governing equations for a natural convection in the Cartesian coordinate system can be written

$$
\begin{aligned}
& \frac{\partial^{2} \psi}{\partial x^{2}}+\frac{\partial^{2} \psi}{\partial y^{2}}=\omega \\
& \frac{\partial \omega}{\partial t}+u \frac{\partial \omega}{\partial x}+v \frac{\partial \omega}{\partial y}=\operatorname{Pr}\left(\frac{\partial^{2} \omega}{\partial x^{2}}+\frac{\partial^{2} \omega}{\partial y^{2}}\right)+\operatorname{Pr} \operatorname{Ra} \frac{\partial T}{\partial x} \mid \\
& \frac{\partial T}{\partial t}+u \frac{\partial T}{\partial x}+v \frac{\partial T}{\partial y}=\frac{\partial^{2} T}{\partial x^{2}}+\frac{\partial^{2} T}{\partial y^{2}}
\end{aligned}
$$

Stream Function Equation

Vorticity Equation

Temperature Equation

where $\psi, \omega$, and $T$ are the stream function, vorticity, and temperature, respectively, and $\operatorname{Pr}=\mu C_{P} / k, \operatorname{Ra}=C_{p} \rho_{0}^{2} g \beta L^{3} \Delta T /(k \mu)$. Note that Eqs. (9)-(11) are normalised by reference length $L$, reference velocity $V_{\infty}=k /\left(\rho_{0} C_{p} L\right)$, reference density $\rho_{0}$, and reference temperature $\Delta T$. The velocity components can be computed from the stream function by $u=\partial \psi / \partial y, v=-\partial \psi / \partial x$ and the vorticity can also be computed from the velocity by $\omega=\partial u / \partial y-\partial v / \partial x$. 
Results - Temperature profiles after 30 and 180 days, variable Temp.Scale.Bar
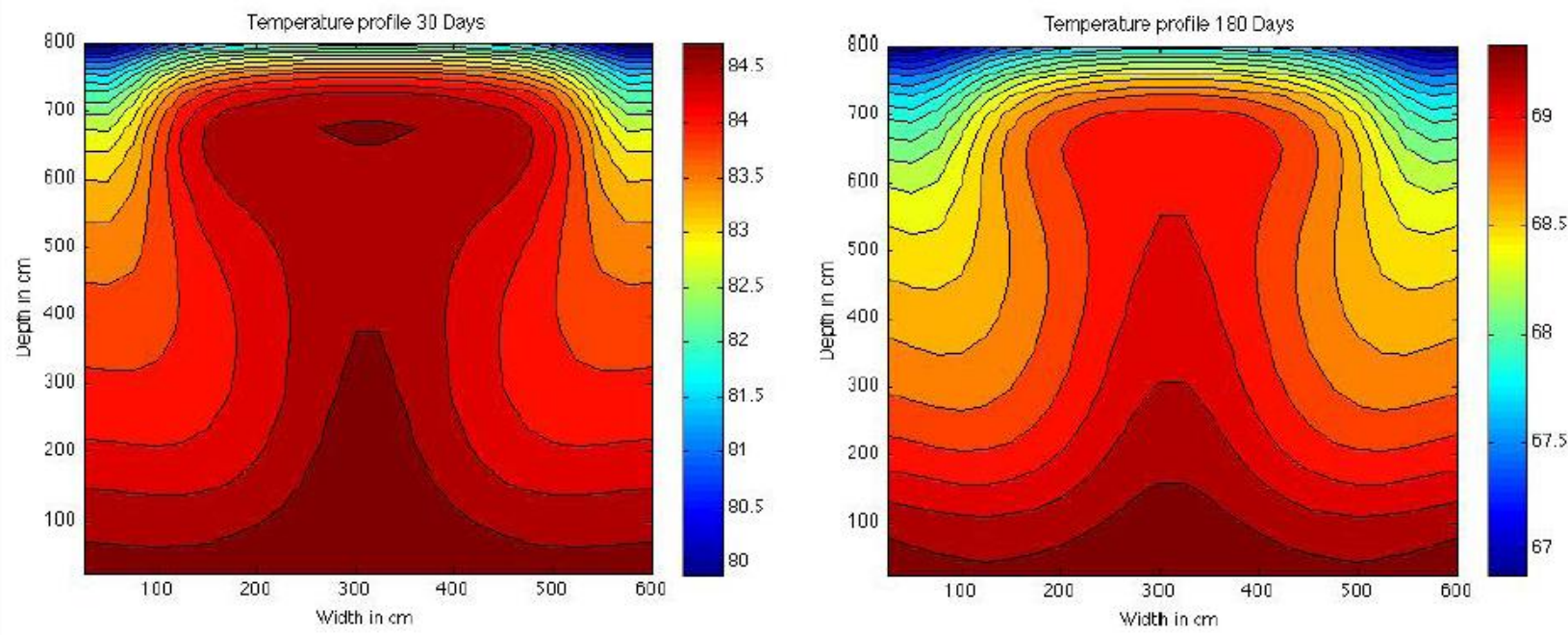

Results - Temperature profiles in 30 days step, fixed Temp.Scale.Bar

1 Month

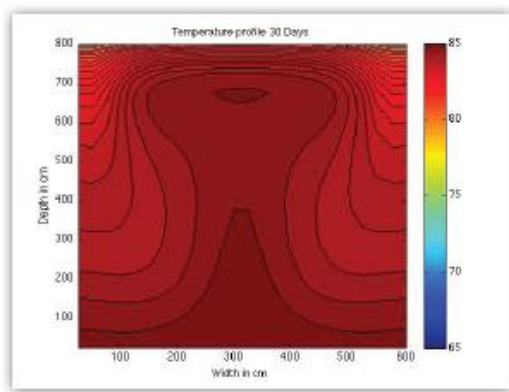

4 Month

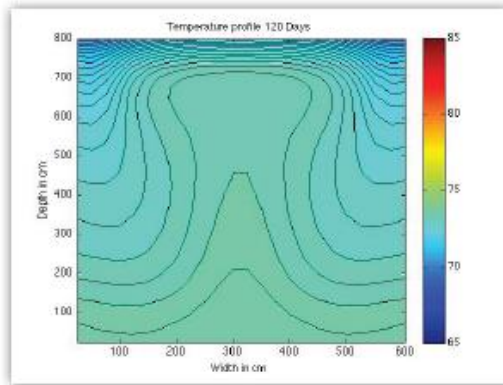

2 Month

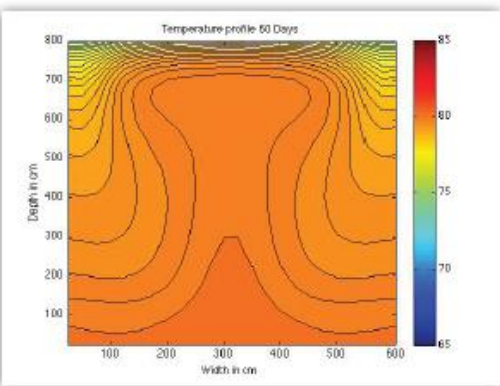

5 Month

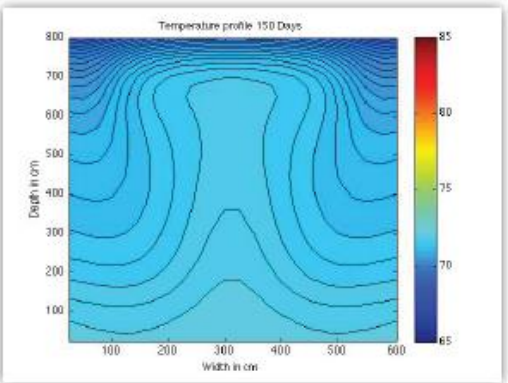

3 Month

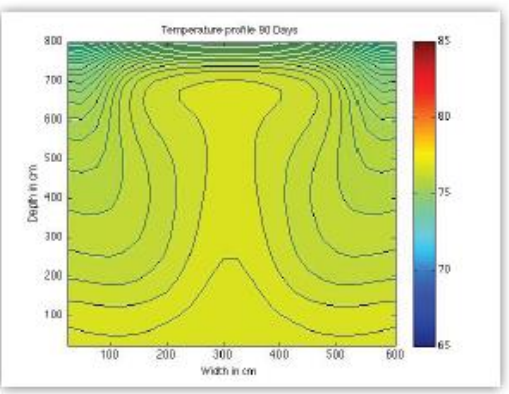

6 Month

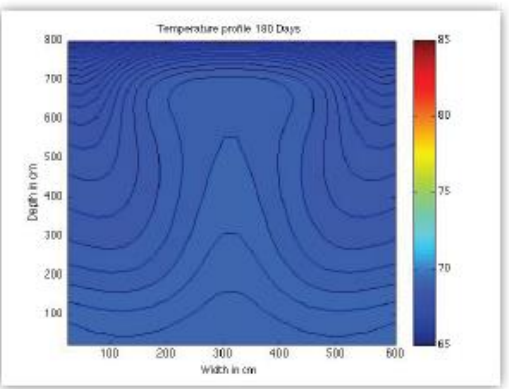




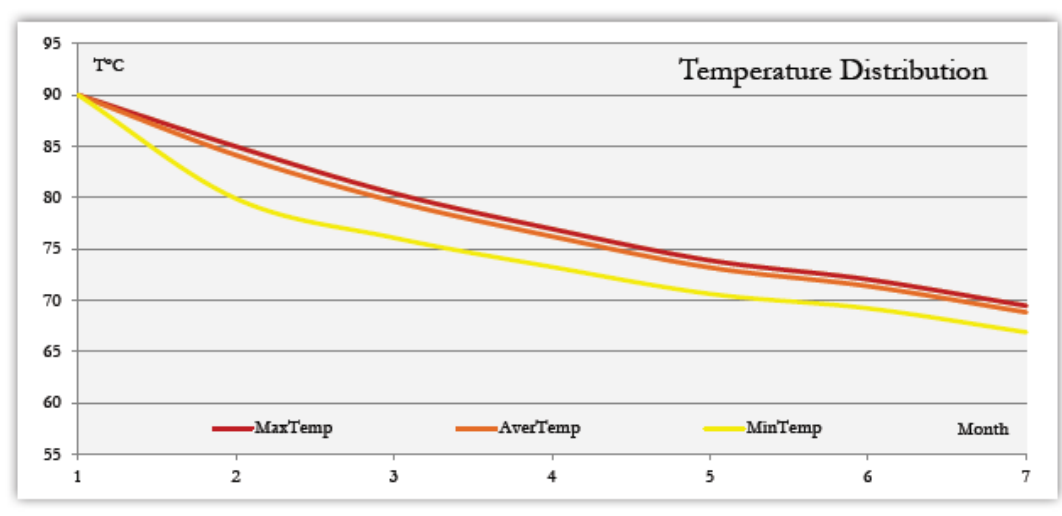

Calculation of disponible energy and heat losses after each month

$$
\mathrm{Q}=\left(\mathrm{T}_{\mathrm{AV}}-\mathrm{T}_{\mathrm{H}}\right) * \mathrm{CP}_{\mathrm{P}} * \varrho^{*} \mathrm{~V}
$$

\begin{tabular}{|c|c|c|c|c|c|c|}
\hline Period & \begin{tabular}{c|} 
Max Temp \\
$\left(1^{\circ} \mathrm{C}\right)$
\end{tabular} & $\begin{array}{c}\text { Aver Temp } \\
\left(T^{\circ} \mathrm{C}\right)\end{array}$ & \begin{tabular}{|c|}
$\begin{array}{c}\text { Min Temp } \\
\left(T^{\circ} \mathrm{C}\right)\end{array}$ \\
\end{tabular} & $\begin{array}{c}\text { Temp Differ } \\
\left(\mathrm{T}^{\circ} \mathrm{C}\right)\end{array}$ & \begin{tabular}{|c|} 
Disponible Energy \\
$(\mathrm{m} V \mathrm{Wh})$
\end{tabular} & $\begin{array}{l}\text { Energy Losses } \\
(\mathrm{mW})\end{array}$ \\
\hline Initial state & 90.000 & 90.000 & 90.000 & 0.000 & 16.720 & 0.000 \\
\hline 1 Month & 84.970 & 84.132 & 79.895 & 5.076 & 14.758 & 1.962 \\
\hline 2 Months & 80.412 & 79.631 & 76.077 & 4.335 & 13.253 & 1.505 \\
\hline 3 Months & 76.936 & 76.209 & 73.234 & 3.702 & 12.108 & 1.145 \\
\hline 4 Months & 73.870 & 73.177 & 70.613 & 3.257 & 11.094 & 1.014 \\
\hline 5 Months & 72.015 & 71.365 & 69.202 & 2.813 & - $10.488-$ & 0.606 \\
\hline 6 Months & 69.456 & 68.819 & 66.877 & 2.579 & $9.637 \mathrm{mWh}$ & 0.851 \\
\hline \multicolumn{5}{|c|}{ Efficiency of the system for an year cycle: } & $58 \%$ & 7.083 \\
\hline
\end{tabular}

\begin{tabular}{|l|c|c|}
\hline \multicolumn{2}{|l|}{ Thermal properties } & \multicolumn{2}{|l|}{${ }^{\circ} \mathrm{C}$} \\
\hline $\begin{array}{l}\text { Heating Mean } \\
\text { Temperature }\end{array}$ & 40 & $\mathrm{~J} / \mathrm{kgK}$ \\
\hline $\begin{array}{l}\text { Specific Heat Ca- } \\
\text { pacity of Water }\end{array}$ & 4180 & $\mathrm{~m} 3$ \\
\hline $\begin{array}{l}\text { Volume of the } \\
\text { Water Tank }\end{array}$ & 288 & $\mathrm{~kg} / \mathrm{m} 3$ \\
\hline $\begin{array}{l}\text { Density of the } \\
\text { Water }\end{array}$ & 1000 & \\
\hline
\end{tabular}

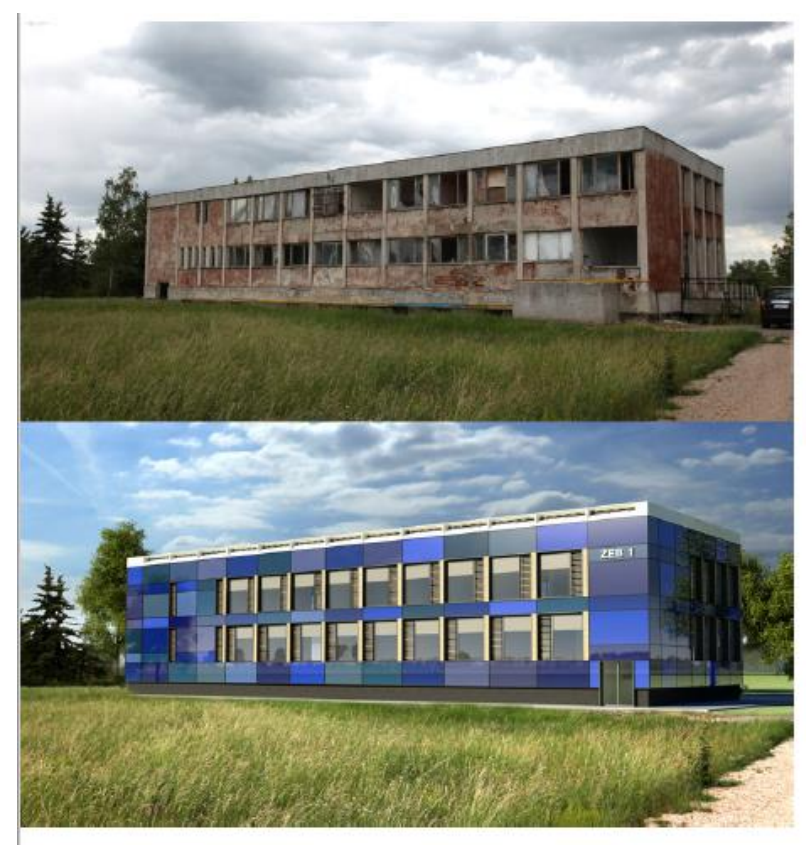

Energy recapitulation balance for the office building ZEB1 Sofia, Bozhurishte; Rehabilitation including the simmulated $288 \mathrm{~m} 3$ thermal accumulator

\begin{tabular}{|c|c|c|}
\hline Available energy ( $(\mathrm{kWh})$ & Design Surface (m2) & Maximal consumption (st/h/m $2 \mathrm{al}$ \\
\hline 9637 & 1150 & $8.4 \mathrm{kTVh} / \mathrm{m} 2 \mathrm{a}$ \\
\hline
\end{tabular}

\begin{tabular}{|c|c|c|c|c|}
\hline \multicolumn{3}{|c|}{ Disponible energy per $\mathrm{m} 3$ after 180d $(\mathrm{m}(\mathrm{m} / \mathrm{h})$} & \multicolumn{2}{|c|}{0.034} \\
\hline & 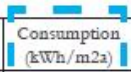 & $\begin{array}{c}\text { Usable } \\
\text { surface }(\mathrm{m} 2)\end{array}$ & $\begin{array}{l}\text { Required energy } \\
\text { for } 1 \text { year }(m W / 2)\end{array}$ & $\begin{array}{c}\text { Required } \\
\text { volume (m3) }\end{array}$ \\
\hline NearZeroEnergy house & 8 & 1150 & 9.20 & 275 \\
\hline Passive house & 15 & 1150 & 17.25 & 515 \\
\hline LowEnergy house & 40 & 1150 & 46.00 & 1373 \\
\hline
\end{tabular}

\begin{tabular}{|c|c|c|}
\hline \multicolumn{2}{|c|}{ Available energy in the hot water $\operatorname{tank}$ after $180 \mathrm{~d}(\mathrm{~m}(\mathrm{Th})$} & 9.637 \\
\hline & $x=-2=x_{1}$ & \\
\hline & Consumption $\mathrm{k} \pi \mathrm{h} / \mathrm{m} 2 \mathrm{a}$ & Max Usable Surface (m2) \\
\hline NearZeroEnergy house & I & 1205 \\
\hline Passive house & 15 & 642 \\
\hline LowEnergy house & 40 & 241 \\
\hline
\end{tabular}




\title{
THE EU HEATING AND COOLING TRANSITION: WHAT ARE THE PERSPECTIVES OF THE INDUSTRY SECTORY TOWARDS 2050
}

\author{
Tobias Fleiter, Andrea Herbst, Matthias Rehfeldt, Pia Manz \\ Fraunhofer Institute for Systems and Innovation Research ISI \\ Breslauer Str. 48, 76139 Karlsruhe, Germany \\ Phone: +49 721 6809-208 \\ E-Mail: Tobias.Fleiter@isi.fraunhofer.de
}

\section{INTRODUCTION}

Heating and cooling accounted for about 50\% of EU final energy demand in 2015. Industry contributes with about 2400 TWh or $38 \%$ to the total heating and cooling energy demand. At the same time, industry's energy supply is still strongly dominated by fossil fuels, mainly natural gas, but also coal and fuel oil have high shares. Process heating requires high temperature often above several hundred degrees and enormous capacities, which poses challenges to the use of renewable energies. In fact, for many process heating applictions, biomass is the only renewable source directly used, but also electricity or other renewable based secondary energy carriers like methane or hydrogene can play a role in industry decarbonisation. Besides the use of renewable energies, also increased energy efficiency is an important pillar of an industry sector transition. However, major industrial processes have been optimised considerably in the past and often, even the application of best available technologies only reveals efficiency improvements of 10-20\% compared to today's average technology. Further, industry can also facilitate the transition in other sectors, e.g. by providing excess heat to district heating networks. These are only selected examples for technical opportunities and restrictions in industry decarbonisation. The huge diversity poses a large challenge to efficient policy design. Long-term scenario analysis can support the development of an efficient policy-mix.

This paper explores the industry sector perspectives in the contect of a decarbonisation of the EU's heating and cooling sector based on a bottom-up simulation of the industrial energy system. We focus on the role and potentials of different technologies and look at the impact of policy instruments.

\section{METHODOLOGY}

We apply the well established bottom-up model FORECAST to simulate transition scenarios until 2050 taking into account specific development of technologies, energy prices, socio-economic drivers and the regulatory framework.

The FORECAST model is designed as a tool that can be used to support strategic decisions. Its main objective is to develop scenarios for the long-term development of energy demand and greenhouse gas emissions for the industry sector of entire countries. FORECAST considers a broad range of mitigation options combined with a high level of technological detail. Technology diffusion and stock turnover are explicitly considered to allow insights into transition pathways and speed. The model further aims to integrate policies and considers changes in the socio-economic framework.

The model shows a very high level of detail with regard to the diffusion of energy-efficient technologies as well as the fuel switch in heat supply. The latter is modeled based on combined technology stock and discrete choice models for steam generation, industrial furnaces and space heating taking into account the particular characteristics of these areas.

The definition of alternative scenarios based on specific policy levers allows conclusions on the impact of alternative policy-mixes on the transition speed, costs and effectiveness. 


\section{RESULTS AND CONCLUSION}

First results are available for a current policy scenario and an ambitious policy scenario. The first represents today's H\&C policy mix and the latter is an ambitious policy mix to decarbonise industrial $\mathrm{H} \& \mathrm{C}$.

It can be observed that in all countries biomass gains increasing shares in both scenarios until 2050 compared with 2012. However, a particularly strong growth is observed in the ambitious policy scenario, where it is the major energy carrier for $\mathrm{H} / \mathrm{C}$ in 2050 in all analysed countries. Other RES remain marginal (solar and ambient heat for heat pumps) although they increase substantially compared to 2012. Power-to-heat (Electricity) is also gaining importance until 2050 in the ambitious policy scenario (note that electricity in Figure 34 also includes process and space cooling). Still, even in the ambitious policy scenario it remains substantially lower than biomass, indicating the still relatively high price of electricity compared to biomass.

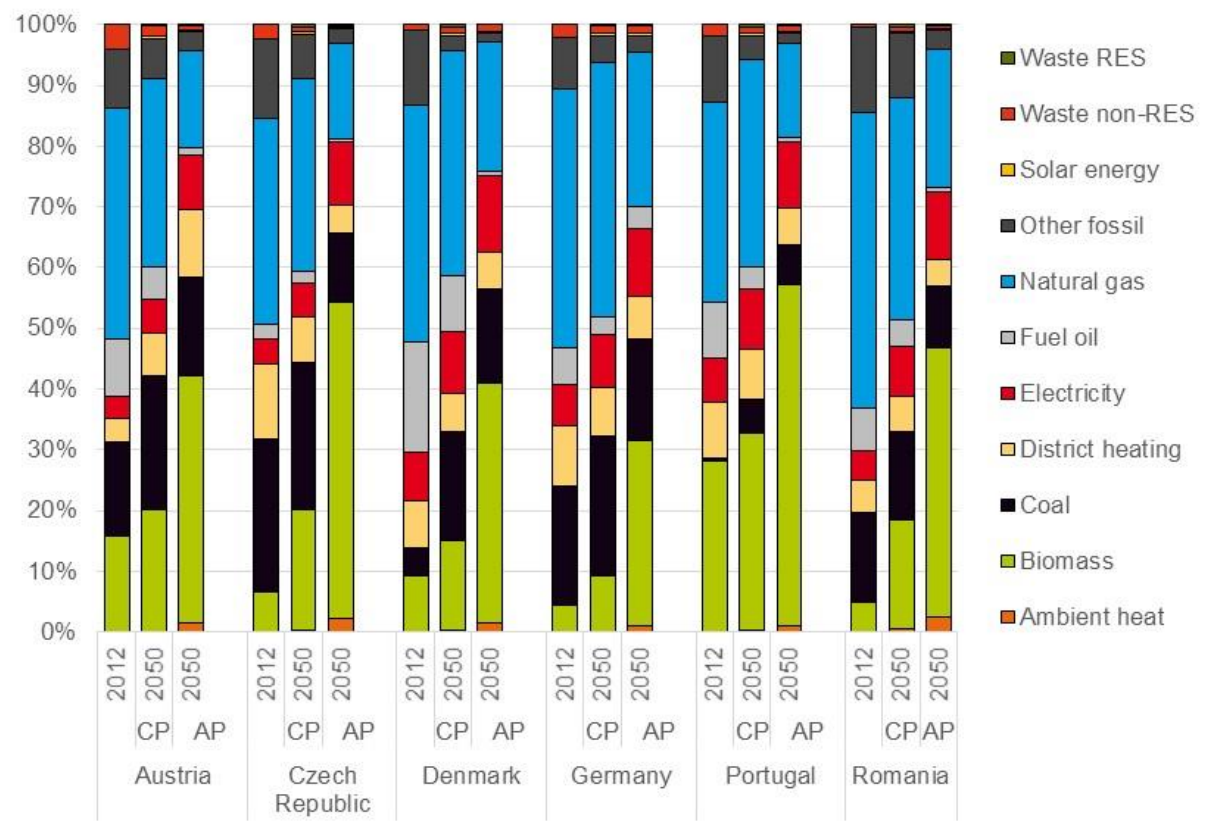

Figure 34: Energy carrier mix of industrial final energy demand for H/C by energy carrier for selected countries (CP: current policy scenario; AP: ambitious policy scenario)

Among others, the following cross-country recommendations can be derived from the country analyses.

- Current policy is not on track towards decarbonisation in all countries analysed, though, a slow decrease of industrial $\mathrm{CO}_{2}$ emissions is expected. Although, the ambitious policy scenario achieves substantial additional emission cuts, also this scenario is not in line with the Paris agreement in all countries analysed.

- Deep emission cuts require substantial changes in the iron and steel, cement and chemicals industries, but also support for RES and energy efficiency in other sectors and companies.

- Excess heat potentials are available and should be exploited. However, the resulting potentials in the analysed processes and with the assumptions taken (only heat $>100^{\circ} \mathrm{C}$ ) are limited.

- Biomass is the most important RES in industry, particularly in the medium term. In the longterm RES-based electricity (power to heat) can play a more important role, particularly if very low emission levels are reached in electricity generation, as (sustainable) biomass potentials are restricted.

- OPEX support of RES seems more effective than CAPEX support for steam and hot water generation. 


\title{
COST-EFFECITVE SOLUTIONS FOR THERMAL REGENERATION OF SAISONAL BOREHOLE HEAT EXCHANGERS IN URBAN RESIDENTIAL SETTLEMENTS
}

\author{
Gerhard Hofer \\ e7 Energie Markt Analyse GmbH \\ Walcherstraße 11/43, 1020 Wien \\ E-Mail: gerhard.hofer@e-sieben.at \\ Johannes Rammerstorfer \\ e7 Energie Markt Analyse GmbH \\ Walcherstraße 11/43, 1020 Wien \\ E-Mail: johannes.rammerstorfer@e-sieben.at \\ Paul Lampersberger \\ e7 Energie Markt Analyse GmbH \\ Walcherstraße 11/43, 1020 Wien \\ E-Mail: paul.lampersberger@e-sieben.at
}

\section{SUMMARY}

As soon as several borehole heat exchangers are combined into one geothermal field, there is a need for a thermal balance, so that the ground does not cool down steadily. In urban residential areas, there are too few actively-cooled areas in buildings, so that the waste heat of the cooling process can be used as heat of regeneration; free cooling of the apartments offers too little waste heat. The project examines various options (waste heat from commercial use in ground floor zones, targeted settlement of data centers, and additional installation of sustainable heat supply systems) within the settlement area, develops business models and assesses the heat generation costs of all solutions, so that a kind of "merit order" for regeneration heat can be developed.

Key words: spatial energy planning, urban settlement, neighbourhood, heat pumps, borehole heat exchanger, regeneration.

\section{BACKGROUND}

Due to urbanisation tendencies, larger cities are confronted with the necessity to develop new residential builings and new districts. This process includes the development of new energy supply systems. The objectives in terms of reducing greenhouse gas emissions require an extended use of local, renewable energy resources. On behalf of the City of Vienna a study about innovative energy systems in Donaufeld was carried out (Hofer 2016). The most promising heat supply concept contains the components of heat pumps and borehole heat exchangers for seasonal storage. To use these borehole heat exchangers over decades they have to be thermally regenerated, so that the temperature of the soil doesn't decrease constantly and deteriorates the efficiency of the system. Since in urban

residential area there is low cooling demand, which waste heat could be used to regenerate the borehole heat exchangers, additional solutions are required.

\section{METHOD}

The paper gives an overview of concepts for regeneration heat in urban residential areas. This includes a detailed technical examination, determination of cost data, development of business models, integration of appropriate stakeholders and the calculation of the "Merit-Order" for the following approaches of regeneration heat: Use of waste heat of industrial enterprises in the ground floor areas of residential buildings (eg supermarket), targeted settlement of data center in urban residential areas and use of the waste heat resources, technical assessment and economic evaluation of additional heat supply systems, such as hybrid collectors, solar absorbers, etc, know-how transfer and stakeholder process for the integration of asphalt collectors. By using a life cycle cost approach the heat generation costs of each concept or technology, calculated for four different building and neighbourhood scenarios, are determined and presented. 


\section{RESULT}

Results of the project are application guidelines for optimal early involvement of more cost-effective regeneration heat in urban residential areas. The guideline is targeted for municipalities as well as project developers and planners. The paper will show results of cost calculation of several solutions and scenarios and how to apply the most cost effective concepts during design phase.

\section{AKNOWLEDGEMENT}

The project was funded by the Austrian Ministry for Transport, Innovation and Technology (BMVIT) in the framework of the City of Tomorrow programme (https://nachhaltigwirtschaften.at/de/sdz)

\section{REFERENCES}

Hofer, G. et al. (2016), Energieversorgungsoptionen für das Stadtentwicklungsgebiet Donaufeld. MA 20 - Energieplanung, Vienna, Austria. 


\section{OPTIMISATION OF A SEASONAL THERMAL ENERGY STORAGE SYSTEM FOR SPACE HEATING IN COLD CLIMATE ZONES}

Sheikh Khaleduzzaman Shah, Lu Aye, Behzad Rismanchi*

Renewable Energy and Energy Efficiency Group, Department of Infrastructure Engineering, Melbourne School of Engineering, The University of Melbourne, Victoria 3010, Australia

*E-Mail: brismanchi@unimelb.edu.au

\section{SUMMARY}

One of the main concerns in cold climate zones is the space heating for thermal comfort, which is traditionally provided via direct use of the primary energy or in developed countries by electricity. In general, usage of renewable energies for space heating is limited due to the temporal availability. However, studies suggest that a hybrid system, which uses renewable source of energy coupled with long-term thermal storage, has overcome the limitations. A practical example is the seasonal thermal energy storage (STES) systems for space heating. For this investigation, a low-temperature thermal storage system coupled with double U-tube vertical borehole heat exchanger and evacuated tube solar collectors (ETSC) is selected to provide space heating. In order to minimise the net present value (NPV) of the life cycle cost (LCC), GenOpt is applied to determine the optimum system configuration (total solar collector area, number of borehole and depth of borehole).

Keywords: Solar energy, Cold climate, Space heating, Life cycle cost, Optimisation.

\section{METHOD AND KEY OUTCOMES}

In this investigation, a double U-tube borehole thermal energy storage (BTES) integrated with ground coupled heat pump (GCHP) and evacuated tube solar collectors (ETSC) system is considered for four selected cities in cold climate zones namely; Harbin (China), Ulaanbaatar (Mongolia), Sivas (Turkey) and Verkhoyansk (Russia). The locations and their maximum and minimum temperatures are presented in Table 1. A cluster of 30 residential buildings is considered for each city. In each building, the living room and the kitchen are considered heated during winter. The total heating areas are 1806 $\mathrm{m}_{2}, 2086 \mathrm{~m} 2,2133 \mathrm{~m} 2$, and $1618 \mathrm{~m} 2$ for a cluster of 30 residential buildings in Ulaanbaatar, Harbin, Sivas, and Verkhoyansk, respectively. TRNSYS - a transient systems simulation program (Klein et al. 2014) is used for simulating the heating load of the building in each city. Meteonorm software (Remund et al. 2016) was used to generate the TMY weather data file for all cities. The envelope characteristic, insulation materials, and dimensions of buildings were modelled in Type 56 base on the typical building in each city. The heating schedule of each cluster is determined based on the local occupant's daily schedule.

Table 1: Locations and temperatures of the cities selected

\begin{tabular}{lcccc}
\hline & Harbin & Ulaanbaatar & Sivas & Verkhoyansk \\
\hline Latitude $\left({ }^{\circ} \mathrm{N}\right)$ & 45.75 & 47.93 & 39.73 & 67.55 \\
Longitude $\left({ }^{\circ} \mathrm{E}\right)$ & 126.65 & 106.90 & 37.01 & 133.33 \\
Altitude $(\mathrm{m})$ & 198 & 1330 & 1336 & 270 \\
Maximum temperature $\left({ }^{\circ} \mathrm{C}\right)$ & 34.3 & 35.3 & 37.2 & 29.9 \\
Minimum temperature $\left({ }^{\circ} \mathrm{C}\right)$ & -28.0 & -37.4 & -19.0 & -56.4 \\
Mean annual temperature $\left({ }^{\circ} \mathrm{C}\right)$ & 5.48 & -0.68 & 9.72 & -14.1 \\
\hline
\end{tabular}

The proposed BTES-GCHP-ETSC system configuration is then optimised for the minimum NPV of LCC (for 20 years project life) by using TRNOPT (the dedicated TRNSYS interface for GenOpt optimisation program (Wetter 2003)). The optimisation variables considered in this investigation are total solar collector area, number of borehole and depth of borehole. The objective function LCC is determined by using Equation (1-3) (Lhendup, Aye, and Fuller 2014) and the assumed project life of 
20 years. Moreover, the annualised life cycle cost (ALCC) of the system (Equation (4)) and the unit heating cost (UHC) (Equation (5)) of the system are presented.

$$
\begin{gathered}
L C C=I C+(M C+O C) P_{a} \\
P_{a}=\frac{(1+d)^{n}-1}{d(1+d)^{n}} \\
d=\frac{(D-i)}{(1+i)} \\
A L C C=\frac{L C C}{P_{a}} \\
U H C=A L C C / Q_{H}
\end{gathered}
$$

where $I C$ is the initial cost (US\$), $M C$ and $O C$ refer to annual maintenance and operational cost (US\$), respectively, $P_{a}(-)$ is the present worth factor or the annual discount factor. The project lifespan is $n$ (year) and $d$ is the real discount rate (-) that is defined by Equation (3). $D$ and $i$ are referred to nominal discount rate (\%) and inflation rate (\%). $Q_{H}$ is the total annual heating load $(\mathrm{kWhh})$ of the buildings. It was found that the optimised system configuration is a viable solution to provide the required heating demand through the cold season for all selected cities.

\section{NOVELTY}

The parameter optimised for the proposed system based on LCC is a unique investigation. Although STES with GCHP and solar collector system have been verified and validated in other countries, the result cannot be used for particular cold climates because the performance of the system is highly climate sensitive. Therefore, this study intends to fill the knowledge gap by identifying optimum sets of system variables for four selected cities in cold climate zones.

\section{REFERENCES}

Klein, S, Beckman, W, Mitchell, J, Duffie, J, Duffie, N, Freeman, T, Mitchell, J, Braun, J, , Evans, B, Kummer, J, Urban, R, Fiksel, A, Thornton, J, Blair, N, Williams, P, Bradley, D, McDowell, T, Kummert, M, Arias, D \& Duffy, M (2014), TRNSYS 17: a TRaNsient SYstem Simulation program.

Lhendup, T, Aye, L \& Fuller, RJ (2014), Performance Analysis of Ground-coupled Heat Pump Integrated with Unglazed Solar Collector, Proceedings of the 52nd Annual Conference, Australian Solar Energy Society (Australian Solar Council) Melbourne, Australia.

Remund, J, Müller, S, Kunz, S, B, H-L, Studer, C \& Cattin, R (2016), Meteonorm Software.

Wetter, M (2003), GenOpt (R), generic optimization program, User Manual, Version 2.0.0. 


\title{
SYNTHESIS AND CHARACTERIZATION OF CARBOXYLIC ESTERS AS NOVEL PHASE CHANGE MATERIALS (PCM) FOR LATENT HEAT STORAGE (LHS) APPLICATIONS
}

\author{
Rebecca Ravotti1, Oliver Fellmann1, Prof. Dr. Ludger J. Fischer 1, Dr. Anastasia Stamatiou 1, Prof. Dr. Jörg \\ Worlitschek 1 \\ Lucerne University of Applied Sciences and Arts1 \\ Technikumstrasse 21, 6048 Horw, Switzerland \\ Phone: +41413493311 \\ E-Mail: rebecca.ravotti@hslu.ch
}

\section{SUMMARY}

In this work, we propose fatty esters derived from carboxylic fatty acids as new bio-based organic Phase Change Materials (PCM). The goal of this study is to evaluate the thermal properties of different fatty esters for their suitability as PCM, and to individuate proper applications for the phase change ranges observed. As such, esters derived from palmitic acid (hexadecanoic acid) coupled with alcohol of various chain length (methanol, 1-pentanol, 1-decanol) have been synthesized through Fischer esterification and their thermal properties characterized through differential scanning calorimetry and thermogravimetric analysis. The dependency between thermal properties, such as melting temperatures and enthalpies of fusion, and chemical structure of the esters was studied to allow for easier identification of potential esters as PCM in future works.

Keywords: Phase Change Materials, Latent Heat Storage, Esters, Fatty Esters, Sustainability

\section{INTRODUCTION}

Latent Heat Storage systems (LHS) with Phase Change Materials (PCM) present several advantages compared to established Sensible Heat Storage (SHS) systems, such as a more compact thermal storage and higher energy efficiency due to nearly constant temperatures during charging and discharging (Mehling and Cabeza, 2008). In order to be considered suitable for LHS applications, PCM materials need to fulfil certain key requirements among which high enthalpy of fusion, high chemical and thermal stability and low to no toxicity. While several studies are currently being performed on both inorganic PCM (e.g. salt hydrates) and organic PCM (e.g. paraffins and carboxylic acids), little is known about esters as PCM. Esters are chemical compounds derived from carboxylic acids and alcohols, and can be commonly found in nature as animal fats and vegetable oils. However, the scarcity of easily available highly pure commercial products and the elevated costs made it difficult to collect experimental thermal data so far. Regardless, preliminary studies on esters derived from renewable feedstock and bio-sourced fatty carboxylic acids conducted by Aydin et al. (2011), Stamatiou et al. (2017), Sari et al. (2009) and Ravotti et al. (2018) show promising results with high chemical and thermal stability, broad melting temperatures, no toxicity, no flammability and endless of combinations between carboxylic acids and alcohols. This, in addition to the environmental sustainability and potential biodegradability makes esters increasingly interesting candidates as new PCM.

The scope of this project is to investigate different classes of esters (e.g. fatty esters, lactones, diesters) to collect experimental thermal data that allows for the creation of a database and for a better understanding of the relations between thermal behaviour and chemical structure of the compounds. Some studies have been conducted in regards of this matter, and in particular an odd-even effect was observed by Nöel et al. (2017), who state that melting points can be seen increasing with increasing molecular weight, but with different trends between odd and even-numbered carbon chains. Still, further studies have to be performed in order to gain a clear understanding and to have extensive knowledge.

As such, an experimental database of the esters' thermal behaviours would not only consent to individuate the most suitable candidates for different applications, but ideally also to predict which chemical structure should the compound have in order to achieve determined phase transitions required by specific applications.

In order to be able to correctly produce unavailable or costly esters, in the previous work by Ravotti et al. (2018) the synthesis procedure was first optimized by synthesizing a commercial ester (methyl 
palmitate, or methyl hexadecanoate, abbr. MEPA) in the laboratory and comparing its properties with the analogue product purchased. The various synthesis parameters were then adjusted until no significant difference was observed between the two samples. After correct validation of the synthesis procedure, esters of palmitic acid coupled with 1-pentanol (pentyl palmitate or pentyl hexadecanoate, abbr. PEPA) and 1-decanol (decyl palmitate or decyl hexadecanoate, abbr. DEPA) were formed. Therefore, the relation between thermal properties and chemical structures were considered, in order to assess whether some trends that would allow to predict thermal behaviour based on chemical structure exist. In addition to the esters mentioned above, so as to study the effect of the carboxylic acid chain length on the final thermal properties, and to compare it with the influence of the alcohol chain length, the ester of behenic acid (docosanoic acid) and methanol was formed (methyl behenate or methyl docosanoate, abbr. MEBE). All chemical structures, IUPAC names and abbreviations for the esters produced are observed in Figure 1 below.

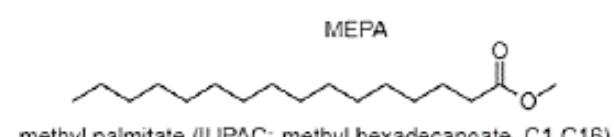

methyl palmitate (IUPAC: methyl hexadecanoate, C1-C16)

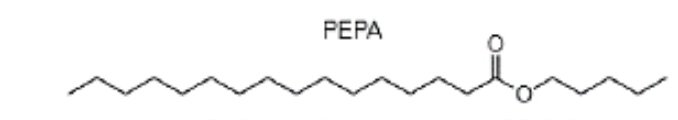

pentyl palmitate (IUPAC: pentyl hexadecanoate, C5-C16)

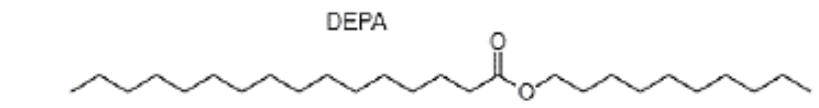

decyl palmitate (IUPAC: decyl hexadecanoate, C10-C16)

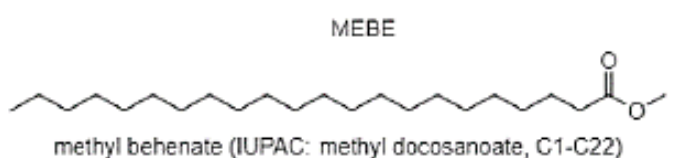

methyl behenate (IUPAC: methyl docosanoate, C1-C22)

Fig. 1: Chemical structures, trivial and IUPAC names and shortenings of the esters produced. The carbon length shortening is derived from the number of carbons in the alcohol, and subsequently the number of carbons in the carboxylic acid chain. For example, methyl palmitate derives from methanol (C1) and palmitic acid (C16), therefore it is indicated as C1-C16.

While methyl palmitate has been extensively produced and reported in literature, and is easily purchasable, the thermal properties of both the decyl esters of palmitic acid and methyl behenate produced hereby have been studied and reported for the first time by Ravotti et al. (2018) to the best of the authors' knowledge. On the other hand, no clear data on the thermal properties of pentyl palmitate has been encountered in the literature. Therefore, characterization of PEPA produced hereby helps gaining a broader view on the thermal behaviour of fatty esters and on the trends correlating the phase change transition and the chemical structure.

\section{METHODOLOGY \& RESULTS}

The esters were synthesized by Fischer esterification, which comprises the attack from the nucleophilic alcohol group on the carboxylic acid group after the latter has been protonated in acid catalytic conditions. Generally, since the reaction is governed by Le Châtelier principle, it is performed in great excess of either alcohol or acid, in presence of a Dean-Stark water trap in order to favour the product formation under heating and reflux conditions. In the work of Ravotti et al. (2018), after investigating the optimal ratios between reagents, the highest product conversion was obtained with a 5:1 ratio of alcohol:acid, 3.5\% w/w of $\mathrm{H}_{2} \mathrm{SO}_{4}$ as acid catalyst, $5 \mathrm{~h}$ heating time and without any water trap since no significant difference was observed in regards of the matter. The esters were separated from the reaction mixture in a separating funnel with consecutive ethyl acetate and deionized water washings, dried first on $\mathrm{Na}_{2} \mathrm{SO}_{4}$ and subsequently in rotary evaporator. In the case of esters deriving from longer alcohols (1-pentanol and 1-decanol), one or more additional crystallizations in 10:1 excess of 
methanol (at $-20^{\circ} \mathrm{C}$ for 1-pentanol and $2-5^{\circ} \mathrm{C}$ for 1 -decanol) had to be performed to obtain esters with purities $\geq 85 \%$. Attenuated total reflectance infrared spectroscopy (ATR-IR), nuclear magnetic resonance (NMR) and gas

chromatography coupled with mass spectroscopy (GC-MS) were used with the same methods as used by Ravotti et al. (2018) to estimate the purities of the compounds produced and to confirm the chemical structures.

Figure 2a shows a comparison of ATR-IR of self-produced esters (MEPA, PEPA, DEPA and MEBE) with commercial MEPA. Here no residual unreacted acid at $1705 \mathrm{~cm}^{-1}$ and no significant differences in the spectra of the esters were observed, indicating high purity of the samples and that the synthesis was successful. Only a slight shift in the ester peak at $1730 \mathrm{~cm}^{-1}$ instead of $1735 \mathrm{~cm}-1$ for esters derived from longer alcohols was noted, supposedly due to the influence of the alkyl chain. Figure $2 b$ shows the increase in purity from samples prior to crystallization to samples crystallized two times in methanol respectively. The peak from the ester group at $1735 \mathrm{~cm}^{-1}$ is seen increasing after crystallizations in methanol, while the peak from unreacted carboxylic acid at $1705 \mathrm{~cm}-1$ decreases, indicating a gain in the purity of the sample. At the same time, the disappearance of the unreacted alcohol peak at $3300-3600 \mathrm{~cm}^{-1}$ is observed for purified samples. While ATR-IR was considered an efficient technique to detect impurities on a qualitative level, it did not allow to quantify the purity of the sample and to discern between different esters.
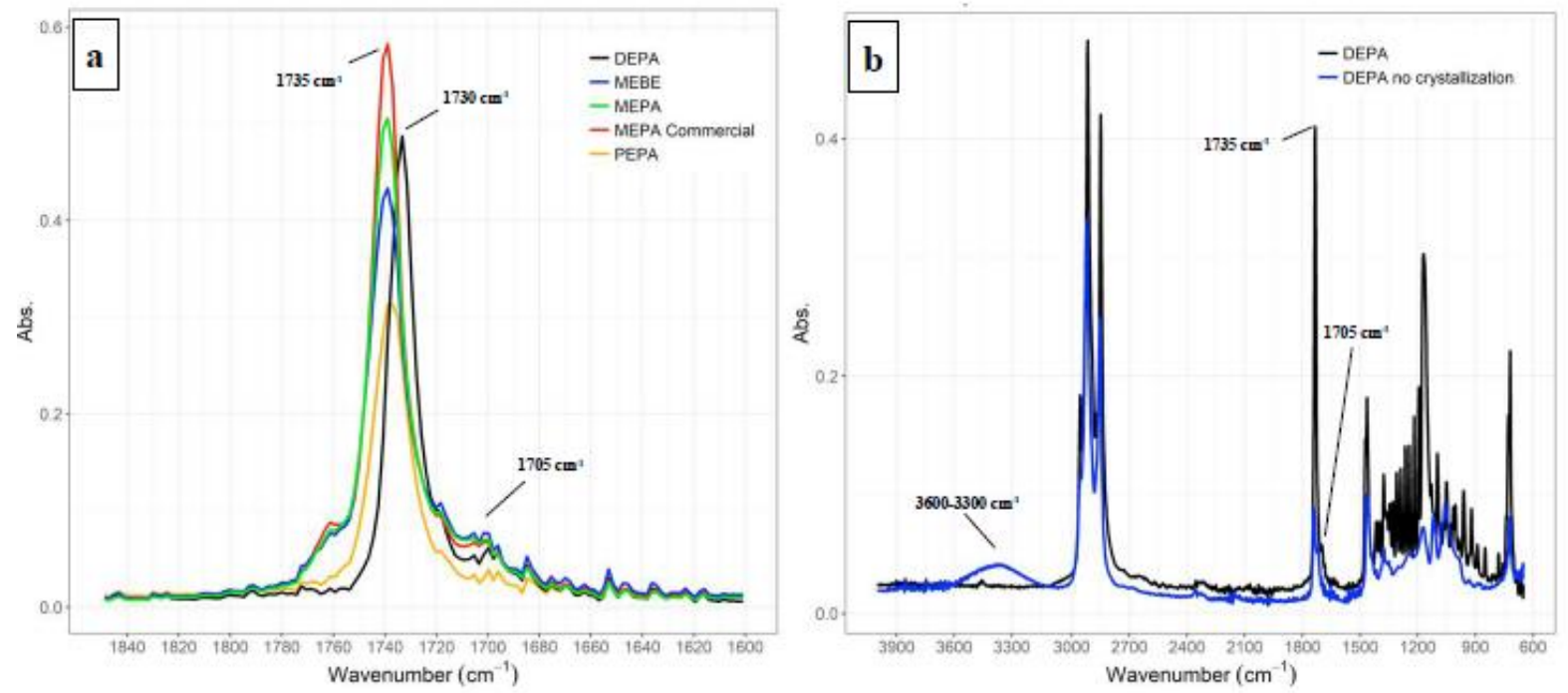

Fig. 2: a) Comparison between ATR-IR spectra of commercial MEPA and produced esters. No significant differences are observed between commercial MEPA and self-produced MEPA, and no impurities from the unreacted acid peak at $1705 \mathrm{~cm}-1$ are visible for all samples, therefore the products are considered pure. A slight shift in the ester peak for PEPA and DEPA is observed. b) Spectra of DEPA before and after crystallization in methanol. A gain in the ester peak at $1735 \mathrm{~cm}-1$ and the disappearance of the unreacted alcohol peak at $3300-3600 \mathrm{~cm}-1$ is seen, thus confirming the efficiency of the purification procedure.

Both GC-MS and NMR, unlike ATR-IR, served to quantify the purity and confirm the identity of the samples produced. In Table 1 below the main peaks from NMR spectra for the different types of esters are reported. In the case of MEPA and MEBE differentiation is possible through the normalized values of the integrals, which account for the different chain length and number of hydrogens, while esters derived from longer alcohols (PEPA, DEPA) present a triplet peak around 4.07-4.04 ppm instead of the singlet at $3.66 \mathrm{ppm}$ observed in methyl esters due to longer alcohol chain. In the case of alcohol impurities a triplet at 3.66-3.62 ppm arising from the $-\mathrm{CH}_{2}$ in $\alpha$ to the hydroxyl groups in the free unreacted alcohol molecules was observed and allowed to estimate the purity of the sample. 
Table 1: List of 1H NMR peaks recorded in CDCl3 from MEPA, PEPA, DEPA, and MEBE which allow to correctly identify the compounds. TMS was used as reference and is assigned the value of $0 \mathrm{ppm}$. The peak position is reported in the peak section and the coupling in between brackets ( $\mathrm{s}=\mathrm{singlet}, \mathrm{t}=$ triplet, $\mathrm{m}=\mathrm{multiplet}$ ). For each peak the corresponding normalized value is reported in the integral column in the same order as the peak column, while the corresponding groups forming the signals are reported in the interpretation column.

\begin{tabular}{|c|c|c|c|c|}
\hline Compound & $\begin{array}{c}\text { Chemical } \\
\text { Structure }\end{array}$ & Peak (ppm/TMS) & Integral & Interpretation \\
\hline MEPA (C1-C16) & $\mathrm{C}_{17} \mathrm{H}_{34} \mathrm{O}_{2}$ & $\begin{array}{c}3.66(\mathrm{~s}), 2.32-2.28(\mathrm{t}), 1.65- \\
1.58(\mathrm{~m}), 1.26(\mathrm{~m}), 0.90-0.86 \\
(\mathrm{t})\end{array}$ & $3,2,2,24,3$ & $\begin{array}{c}-\mathrm{CH}_{3} \text { (alcohol chain), }-\mathrm{CH}_{2} \text { in } \alpha,-\mathrm{CH}_{2} \text { in } \beta,- \\
\mathrm{CH}_{2} \text { (aliphatic chain), }-\mathrm{CH}_{3} \text { (end carboxylic } \\
\text { chain) }\end{array}$ \\
\hline PEPA (C5-C16) & $\mathrm{C}_{21} \mathrm{H}_{42} \mathrm{O}_{2}$ & $\begin{array}{c}4.07-4.04(\mathrm{t}), 2.31-2.27(\mathrm{t}), \\
1.64(\mathrm{~m}), 1.25(\mathrm{~m}), 0.91-\end{array}$ & $2,2,4,28,6$ & $\begin{array}{c}-\mathrm{CH}_{2} \text { in } \alpha \text { (alcohol chain), }-\mathrm{CH}_{2} \text { in } \alpha \text { (carbonyl), } \\
\text { chain), }-\mathrm{CH}_{3} \text { (end carboxylic chain) }\end{array}$ \\
\hline DEPA (C10-C16) & $\mathrm{C}_{26} \mathrm{H}_{52} \mathrm{O}_{2}$ & $\begin{array}{c}4.07-4.04(\mathrm{t}), 2.30-2.27(\mathrm{t}), \\
1.65-1.58(\mathrm{~m}), 1.25(\mathrm{~m}), 0.90- \\
0.86(\mathrm{t})\end{array}$ & $2,2,4,38,6$ & $\begin{array}{c}-\mathrm{CH}_{2} \text { in } \alpha \text { (alcohol chain), }-\mathrm{CH}_{2} \text { in } \alpha \text { (carbonyl), } \\
-\mathrm{CH}_{2} \text { in } \beta \text { (aliphatic chain), }-\mathrm{CH}_{2} \text { (aliphatic } \\
\text { chain), - } \mathrm{CH}_{3} \text { (end carboxylic chain) }\end{array}$ \\
\hline MEBE (C1-C22) & $\mathrm{C}_{23} \mathrm{H}_{46} \mathrm{O}_{2}$ & $\begin{array}{c}3.66(\mathrm{~s}), 2.32-2.28(\mathrm{t}), 1.65- \\
1.58(\mathrm{~m}), 1.25(\mathrm{~m}), 0.90-0.86 \\
(\mathrm{t})\end{array}$ & $3,2,2,36,3$ & $\begin{array}{c}-\mathrm{CH}_{3} \text { (alcohol chain), }-\mathrm{CH}_{2} \text { in } \alpha,-\mathrm{CH}_{2} \text { in } \beta,- \\
\mathrm{CH}_{2} \text { (aliphatic chain), }-\mathrm{CH}_{3} \text { (end carboxylic } \\
\text { chain) }\end{array}$ \\
\hline
\end{tabular}

Concerning MS spectra, the molecular ion of the ester was always clearly visible, and together with the different fragmentation corresponding to the loss of the alcohol group (fragment at $239 \mathrm{~m} / \mathrm{z}$ for all palmitate esters, and at $323 \mathrm{~m} / \mathrm{z}$ for behenate esters) it allowed to distinguish between methyl, pentyl and decyl esters. In addition, all four types of esters always showed a progressive fragmentation of the alkane chain from the acid side with consecutive loss steps of $14 \mathrm{~m} / \mathrm{z}$ each (loss of a - $\mathrm{CH} 2$ group).

Differential scanning calorimetry (DSC) and thermogravimetric analysis (TGA) served to study the phase change transition temperatures, degrees of supercooling, enthalpies of fusion and thermal stability. No significant difference was observed between the onset melting and crystallization temperatures and enthalpies of fusion of self-produced MEPA at both $2 \mathrm{~K} / \mathrm{min}$ and $10 \mathrm{~K} / \mathrm{min}$ heating rates and commercial MEPA (not shown hereby), thus confirming the validity of the synthesis procedure (Ravotti et al., 2018). Since no variations between the different heating rates were detected (Figure 3a), only one heating-cooling cycle at $10 \mathrm{~K} / \mathrm{min}$ for each sample is shown in Figure 3b. All samples proved to be stable over three cycles with high enthalpies of fusion above $190 \mathrm{~J} / \mathrm{g}$ and a constant low degree of supercooling $\left(<10^{\circ} \mathrm{C}\right)$, therefore proving to be interesting PCM candidates. No impurities, which would appear in the form of lower enthalpies of fusion and side peaks at the melting temperatures of the alcohols, were observed. Therefore, it could be concluded that the synthesis was successful and gave high purity products, as confirmed by NMR and GC-MS. The cause for the splitting of the peak in the crystallization curve of MEBE is to be assigned to the small volume of the DSC pan, which for waxy or dense samples can cause onset crystallization in different spots simultaneously. 

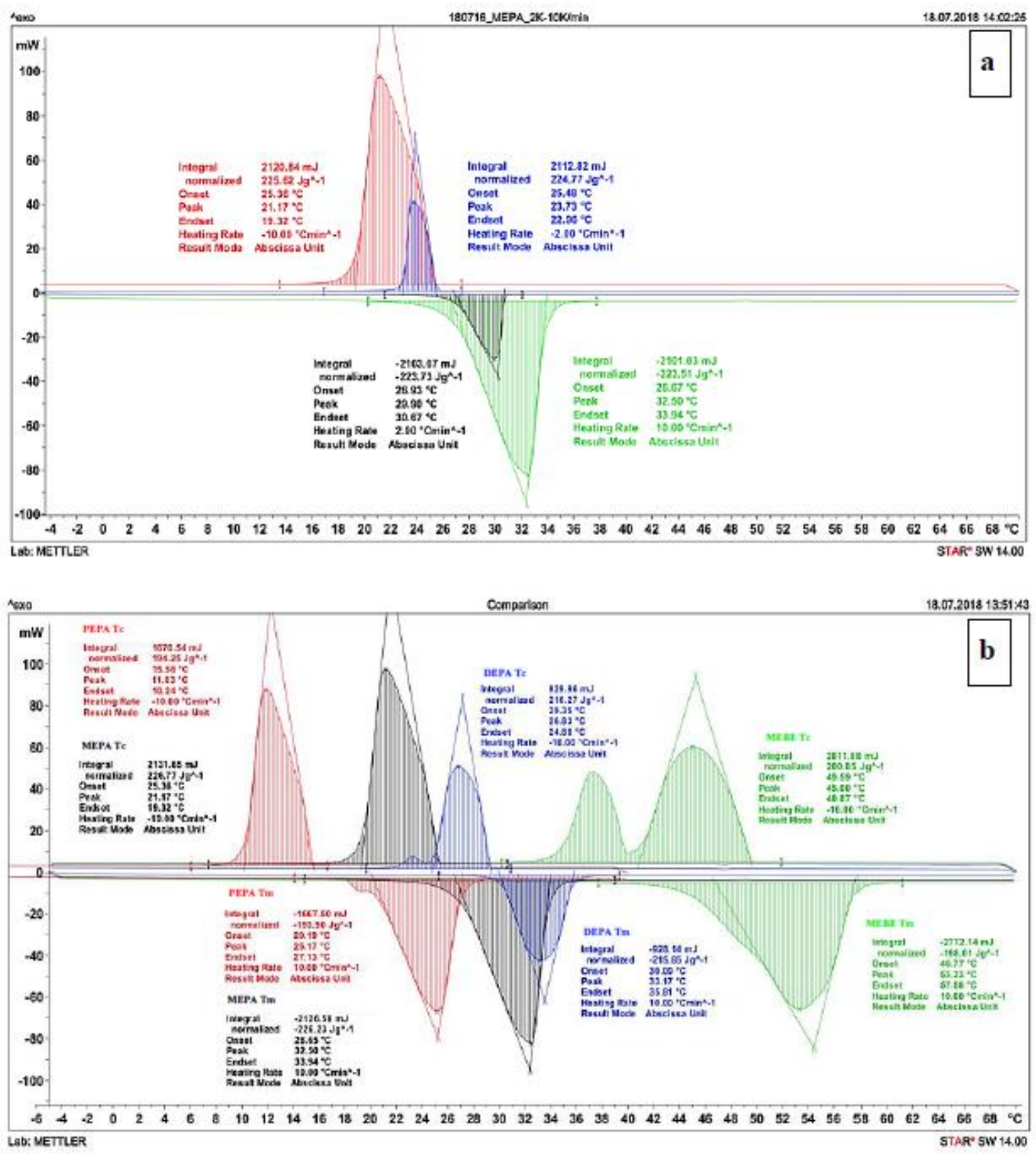

Fig. 3: a) DSC measurements at $2 \mathrm{~K} / \mathrm{min}$ and $10 \mathrm{~K} / \mathrm{min}$ for self-produced MEPA. No differences in the onset melting and crystallization points as well as enthalpy of fusion are observed at both heating rates compared to commercial MEPA (not reported hereby), thus the two samples are considered equivalent and the heating rate is supposed to not affect the experimental data. b) Comparison between DSC measurements at $10 \mathrm{~K} / \mathrm{min}$ of MEPA, PEPA, DEPA and MEBE. All samples show high enthalpies of fusion above $190 \mathrm{~J} / \mathrm{g}$. The degree of supercooling (intended as the difference between onset melting and crystallization temperatures) never amounts over $10^{\circ} \mathrm{C}$ for all samples. Such a behavior remained constant over three heating-cooling cycles for all samples. The melting temperatures retrieved range from $15^{\circ} \mathrm{C}$ to $50^{\circ} \mathrm{C}$.

The main thermal properties measured for the samples produced are summarized in Table 2. The uncertainties reported are calculated based on the experimental results of three consecutive heatingcooling cycles. 
Table 2: Summary of the thermal properties registered for the esters produced (onset crystallization temperature $\mathrm{Tc}$ and melting temperature $\mathrm{Tm}$, enthalpy of fusion $\Delta \mathrm{H}(\mathrm{J} / \mathrm{g})$, and range of degradation temperatures $\left({ }^{\circ} \mathrm{C}\right)$. The chemical formulas are reported to allow for easier individuation of correlations between the chemical structure and the thermal properties for each ester. In general, a strong odd-even effect is observed with PEPA being the ester with the lowest Tm and earliest degradation interval. A stronger influence on the final Tm of the ester is observed for longer carboxylic acid chains (comparison MEPA-MEBE) compared to longer alcohol chains (comparison MEPA-DEPA).

\begin{tabular}{|c|c|c|c|c|c|}
\hline Compound & $\begin{array}{c}\text { Chemical } \\
\text { Formula }\end{array}$ & Tc (onset, ${ }^{\circ} \mathrm{C}$ ) & Tm (onset, $\left.{ }^{\circ} \mathrm{C}\right)$ & $\Delta \mathrm{H}(\mathrm{J} / \mathrm{g})$ & $\begin{array}{c}\text { Degradation } \\
\text { temperature }\left({ }^{\circ} \mathrm{C}\right)\end{array}$ \\
\hline MEPA (C1-C16) & $\mathrm{C}_{17} \mathrm{H}_{34} \mathrm{O}_{2}$ & $24.2 \pm 0.2$ & $26.3 \pm 0.1$ & $201 \pm 1$ & $\begin{array}{c}119 \pm 10 \\
-272 \pm 4\end{array}$ \\
\hline PEPA (C5-C16) & $\mathrm{C}_{21} \mathrm{H}_{42} \mathrm{O}_{2}$ & $15.2 \pm 0.5$ & $19.6 \pm 0.5$ & $194 \pm 14$ & $\begin{array}{c}80 \pm 5 \\
-240 \pm 10\end{array}$ \\
\hline DEPA (C10-C16) & $\mathrm{C}_{26} \mathrm{H}_{52} \mathrm{O}_{2}$ & $28.9 \pm 1.2$ & $29.0 \pm 1.9$ & $193 \pm 6$ & $\begin{array}{c}145 \pm 7 \\
-310 \pm 14\end{array}$ \\
\hline MEBE (C1-C22) & $\mathrm{C}_{23} \mathrm{H}_{46} \mathrm{O}_{2}$ & $41.5 \pm 1.0$ & $47.9 \pm 0.7$ & $200 \pm 5$ & $\begin{array}{c}155 \pm 7 \\
-305 \pm 20\end{array}$ \\
\hline
\end{tabular}

As can be observed, phase change temperatures ranged in between circa $15^{\circ} \mathrm{C}$ and $50^{\circ} \mathrm{C}$. Upon close observation of possible trends within the series and of the results from previous studies conducted by Ravotti et al. (2018) on saturated fatty esters, it was noticed that for esters derived from the same carboxylic acid (palmitic acid) a slight increase in the melting temperature was registered when coupled with longer alcohols (1-decanol, DEPA) compared to methanol (MEPA). However, by adding PEPA to the class this trend is contradicted, since PEPA showed the lowest melting temperature in the whole series. Upon comparison between MEPA and MEBE, it was deduced that a steep increase in the melting temperature arises for esters derived from the same alcohol (methanol) and a longer carboxylic acid such as behenic acid (MEBE). In the case of MEPA and MEBE, an increase in the carbon length of 6 units due to longer carboxylic acid had a much higher impact on the final melting temperature than an increase of 10 units due to longer alcohol chain of 1-decanol (MEPA and DEPA). This indicates that the carbon length of the carboxylic acid has a higher influence on the phase change transition than the alcohol. Such trends are visible in Figure $4 \mathrm{a}$ and $4 \mathrm{~b}$. 

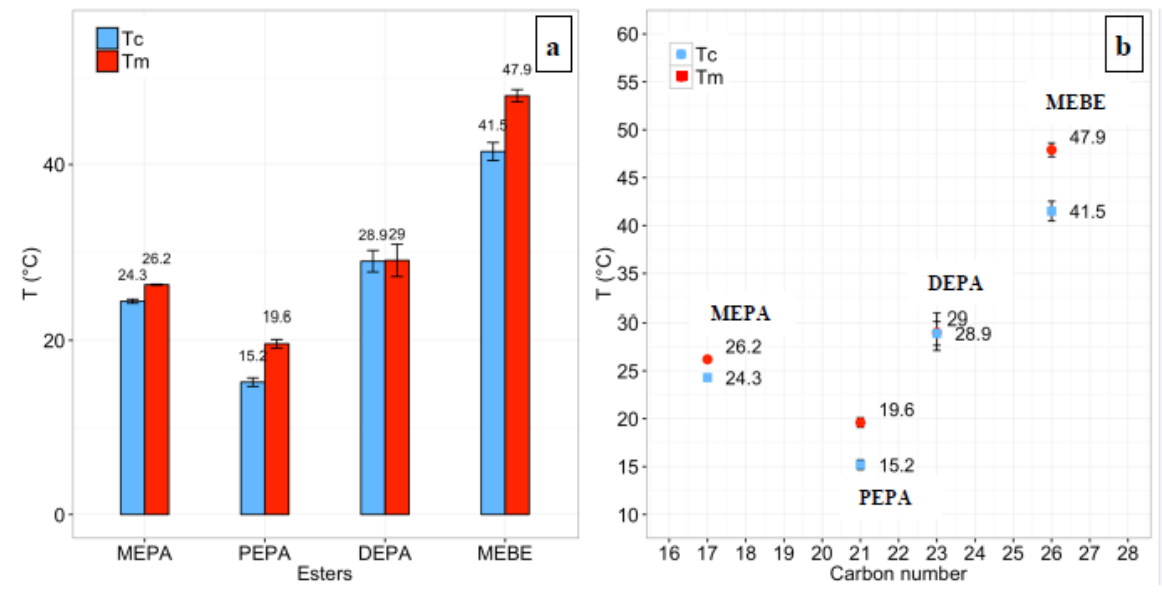

Fig. 4: a) Bar plot showing the different crystallization and melting temperatures produced with uncertainty derived from each ester being produced three times. b) scatter plot correlating the phase change temperatures and the carbon number of the compounds, for better investigation on possible relations between the chemical structure and thermal behavior. In synthesis, the melting temperature is seen increasing for increasing carbon chains both on the alcohol and the acid side, with the exception of esters derived from 1-pentanol which register the lowest melting temperature in the series. It can be deduced that the increase of carbon number from the acid chain (MEPA to MEBE) has a higher impact on the phase change temperature compared to the increase of carbon number from the alcohol chain (MEPA to DEPA).

A possible explanation to the behavior discovered previously in regards of the esters produced can be derived from the odd-even effect theory explained in the work of Noël et al. (2017). Here is reported that odd-numbered and even-numbered carbon chain lengths present different trends in regards of the melting point due to the fact that even numbered structures have higher occurrence in nature. The melting point of a molecule is the direct consequence of the crystal packing the compound is able to form and of the strength of its intermolecular bonds. Therefore, compounds which are able to form crystal structures with high symmetry will present higher melting points. Concerning this, Bunn (1955) observed a similar behavior in aliphatic polymeric chains and attributed it to the fact that the end bonds of an odd - $\mathrm{CH} 2$ sequence are inclined to each other while those at the ends of an even sequence are parallel, therefore the latter are able to form more tightly packed structures and give rise to higher melting points. However, other parameters can influence the melting point of a compound, such as degrees of freedom and its ability to rotate around its bounds. This could explain the fact that the carboxylic acid chain length is thought to a higher influence on the final melting point compared to the alcohol chain length. Two separate effects are supposed to generate this behavior: on one hand, a longer aliphatic chain gives rise to stronger intermolecular forces and to a more tightly packed structure, however on the other hand it also introduces a higher rotation of the structure, creating discrepancies and being more prone to disrupt the crystal lattice structure. Therefore, both effects level each other, ending up increasing the melting temperature of the final ester less than esters with shorter alcohol chains such as methyl esters.

From the point of view of the energy, no clear trend was observed between the values of the enthalpies of fusion ( $\mathrm{J} / \mathrm{g})$ and the chemical structure of the compounds. No mentions of existing trends that correlate the enthalpy of fusion $(\mathrm{J} / \mathrm{g})$ to the chemical structure of a compound have been found in the literature as well. When calculating the molar enthalpies of fusion $(\mathrm{J} / \mathrm{mol}$ or $\mathrm{KJ} / \mathrm{mol})$ a linear increase for increasing molecular weight is observed. This is due to the fact that while all samples showed nearly constant enthalpies of fusion oscillating between 190 and $200 \mathrm{~J} / \mathrm{g}$, the carbon number and consequently the molecular weight increased from MEPA to MEBE, therefore the molar enthalpy of fusion increased as well in a proportional manner (Equation 1). 


$$
\Delta H\left(\frac{J}{m o l}\right)=\Delta H\left(\frac{J}{g}\right) * M w\left(\frac{g}{m o l}\right)
$$

Equation 1: The molar enthalpy of fusion $(\mathrm{J} / \mathrm{mol})$ of a substance can be calculated from the enthalpy of fusion $(\mathrm{J} / \mathrm{g})$ multiplied for the molecular mass $(\mathrm{g} / \mathrm{mol})$ of that substance.

Nevertheless, enthalpies of fusion over $190 \mathrm{~J} / \mathrm{g}$ indicate the suitability as PCM for LHS applications for all esters presented in this work and in the work of Ravotti et al. (2018).

Concerning degradation temperatures, a similar trend to the molar enthalpies is observed, with increasing onset degradation temperatures for increasing carbon chain length. Esters with longer carbon chain length are seen degrading completely at higher temperatures $\left(305 \pm 20{ }^{\circ} \mathrm{C}\right.$, MEBE) than esters with shorter carbon chains $\left(272 \pm 4{ }^{\circ} \mathrm{C}\right.$, MEPA). Once again, the only ester showing a different trend is PEPA, with earlier onset of degradation. This could be explained once again with the oddeven effect theory, and with the fact that, as reported previously, odd-numbered carbon chains have the methylene $-\mathrm{CH}_{2}$ groups inclined to each other, which lowers the symmetry of the crystal lattice structure.

\section{CONCLUSIONS}

In the present work, esters derived from carboxylic acids of different chain length (palmitic acid C16 and behenic acid C22) coupled with alcohols of different lengths (methanol C1, 1-pentanol, C5, 1decanol C10) were synthesized, purified and their thermal properties presented. At first, in order to validate the synthesis procedure, a commercial ester (methyl palmitate) was reproduced and its properties compared with the homologue produced in the laboratory by the authors (Ravotti et al., 2018). Once no significant difference was observed between the two esters, the synthesis procedure was considered as suitable for the production of new esters (pentyl palmitate, decyl palmitate and methyl behenate). While high enthalpies of fusion above $190 \mathrm{~J} / \mathrm{g}$ were registered for all

samples, marking their suitability as PCM, no clear trend was observed that correlates the molecular structures of the esters to the enthalpies of fusion $(\mathrm{J} / \mathrm{g})$ measured. A linear increase in the molar enthalpies of fusion $(\mathrm{J} / \mathrm{mol})$ was noticed for increasing molecular weight of the samples, due to the fact that the enthalpies of fusion are nearly constant (oscillating between 190-200 J/g) for the whole series.

A correlation was found in regards of the melting points and chemical structures. The carbon length of the carboxylic acid is deduced to have a higher influence on the final melting point than the carbon length of the alcohol. This is thought to be due to the higher degree of rotation of longer alcohol chains, which disrupts the symmetry of the compound's crystal packing, therefore dampening the increasing effect it would have on the melting point value. On the other hand, pentyl palmitate was found to have the lowest melting point and degradation temperature of the whole series of esters produced. This could be explained with an odd-even effect, where not only odd-numbered carbon chains have lower occurrence in nature, but they also have less symmetric crystal packing due to the fact that the methylene - $\mathrm{CH} 2$ groups are pointed to each other instead of being parallel.

Except from pentyl palmitate, the degradation temperatures were found to increase for increasing molecular weight, which confirms the theory that more rigid crystal structures have higher energy due to tighter packing and are harder to break.

Although several trends have been identified which allow for easier prediction of the thermal properties of different esters, further studies on a broader range of esters are required to confirm what stated above. As such, new studies on fatty esters as well as on different classes of esters (diesters, lactones, etc.) are planned and will be performed by the authors.

\section{ACKNOWLEDGMENTS}

The Authors would like to thank the Swiss National Science Foundation (SNSF, project number PZENP2_173636) and Swiss Competence Center for Energy Research Storage of Heat and Electricity (SCCER HaE) for the financial support. The authors wish to thank the University of Zürich (UZH, Zürich, Switzerland) and Fachhochschule Nordwestschweiz (FHNW, Basel, Switzerland) for the GCMS and NMR measurements conducted. 


\section{REFERENCES}

Mehling, H.; Cabeza, L.F. Heat and Cold Storage with PCM. An up to Date Introduction into Basics and Applications; Springer: Berlin, Germany, 2008, ISBN 978-3-540-68556-2.

Aydin, A.A.; Okutan, H., High-chain Fatty Acid Esters of myristic Alcohol with even carbon number: Novel organic phase change materials for thermal energy storage-1. Sol. Energy Mater. Sol. Cells, 2011, 95, 2752-2762, doi:10.1016/j.solmat.2011.04.015.

Stamatiou, A.; Obermeyer, M.; Fischer, L.J.; Schuetz, P.; Wortlischek, J. Investigation of unbranched, saturated, Carboxylic Esters as phase change materials. Renew. Energy 2017, 108, 401-409, doi:10.1016/j.renene.2017.02.056.

Sari, A.; Biçer, A.; Karaipekli, A. Synthesis, characterization, thermal properties of a series of stearic Acid Esters as novel solid-liquid phase change materials. Mater. Lett. 2009, 63, 1213-1216, doi:10.1016/j.matlet.2009.02.045.

Ravotti, R.; Fellmann, O.; Lardon, N.; Fischer, L.J.; Stamatiou, A.; Wortlischek, J. Synthesis and Investigation of Thermal Properties of Highly Pure Carboxylic Fatty Esters to Be Used as PCM. Appl. Sci. 2018, 8, 1069, doi: 10.3390/app8071069

Noël, J.A.; Samer, K.; White, M.A. Molecular Structure and Melting: Implications for Phase Change Materials. Can. J. Chem. 2017, 1-8, doi:10.1139/cjc-2017-0578.

Bunn, C.W.; The Melting Points of Chain Polymers. J. Polym. Sci. 1955, 16, 323-343, https://doi.org/10.1002/pol.1955.120168222. 


\title{
LOW-TEMPERATURE LATENT HEAT STORAGE BASED ON SALT HYDRATES
}

\author{
Christoph Rathgeber, Stefan Hiebler \\ Bavarian Center for Applied Energy Research e.V. (ZAE Bayern) \\ Walther-Meissner-Str. 6, 85748 Garching, Germany \\ Phone: +498932944288 \\ E-Mail: christoph.rathgeber@zae-bayern.de
}

\section{SUMMARY}

Latent heat storage provides thermal energy with high storage density. Salt hydrates are appropriate storage materials for applications up to $100{ }^{\circ} \mathrm{C}$. To apply salt hydrates over a long period of time, a latent heat storage with immersed capillary tube heat exchanger has been developed. This concept has been installed as support of the dry recooling of sorption chillers in solar heating and cooling systems and as part of a space cooling system. Novel salt hydrate mixtures for further energy-relevant applications are currently being investigated.

Keywords: energy efficiency, energy storage, latent heat storage, phase change material, salt hydrate

\section{INTRODUCTION}

Phase change materials (PCM) are applied as thermal storage materials in latent heat storage systems. During the phase change, large amounts of thermal energy can be stored in the PCM at nearly constant temperature. In applications, especially the phase change from solid to liquid and vice versa is relevant. Salt hydrates are appropriate PCM for low-temperature applications between approx. 0 and $100{ }^{\circ} \mathrm{C}$. Salt hydrates provide high energy densities at comparably low costs. To ensure long-term stability, corrosion and phase separation of salt hydrates have to be taken into account. Based on the results of three research projects, the possibilities and challenges of low-temperature latent heat storage using salt hydrates are outlined.

\section{APPLICATIONS: RECOOLING OF SORPTION CHILLERS AND SPACE COOLING}

In the first project, a latent heat storage was part of the recooling circuit of a sorption chiller (Fig. 1, left).
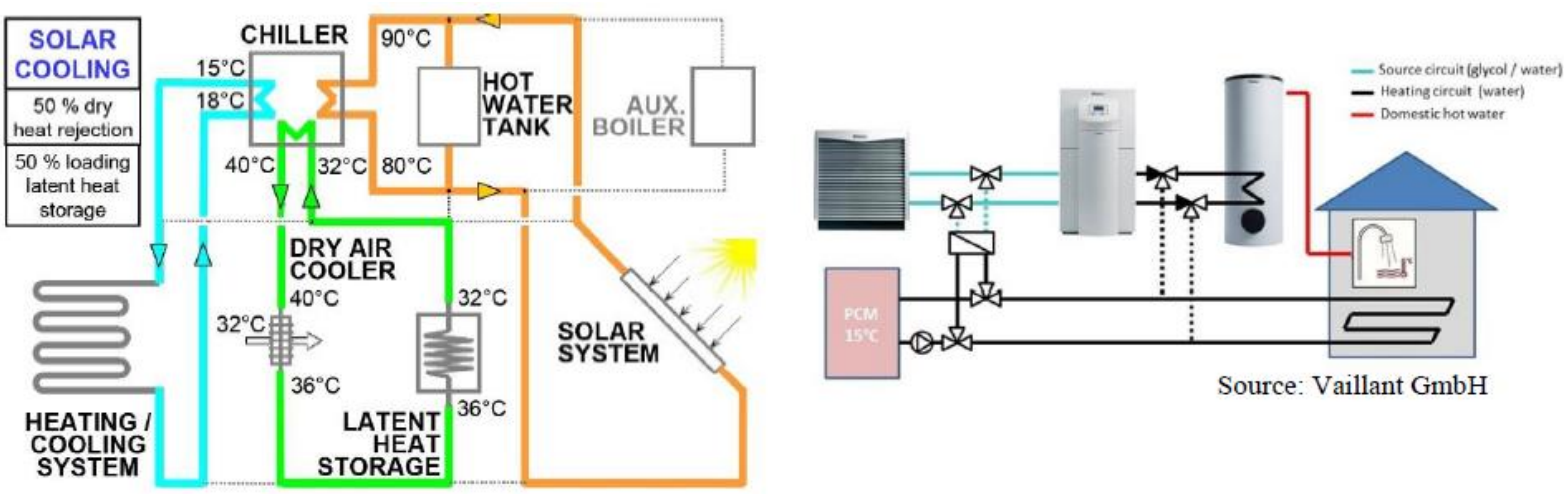

Fig. 1: Recooling of sorption chiller (left) and space cooling together with heat pump (right)

Solar thermal collectors supply heat at $90{ }^{\circ} \mathrm{C}$ to the sorption chiller which produces cold water at 15 ${ }^{\circ} \mathrm{C}$ to cool the building. For a continuous process, heat at $40{ }^{\circ} \mathrm{C}$ has to be dissipated to the ambient. Supporting a dry cooler, the PCM storage is integrated in the recooling circuit. Throughout the day, the PCM storage takes up part of the reject heat and the PCM melts. At night, in the case of low ambient temperature, the PCM storage is regenerated again and the PCM solidifies. Calcium chloride hexahydrate $\left(\mathrm{CaCl}_{2} \cdot 6 \mathrm{H}_{2} \mathrm{O}\right)$ with a melting temperature of approx. $29{ }^{\circ} \mathrm{C}$ is an appropriate PCM for this application. To obtain a constant power output during the phase change, an immersed capillary tube heat exchanger has been constructed. A state-of-charge device has been developed to improve the integration of the PCM storage in the cooling system. Since unmodified $\mathrm{CaCl}_{2} \cdot 6 \mathrm{H}_{2} \mathrm{O}$ shows phase separation after thermal cycling, an anti-separation unit is necessary to ensure long-term stability. 
In the second project, this storage design has been evolved to use the storage together with a heat pump to provide domestic hot water and space cooling. For space cooling applications, a PCM melting around $15{ }^{\circ} \mathrm{C}$ is energetically favourable compared with ice or cold water. A mixture of calcium chloride hexahydrate and calcium bromide hexahydrate $\left(\mathrm{CaBr}_{2} \cdot 6 \mathrm{H}_{2} \mathrm{O}\right)$ was identified as a promising candidate for this application. In collaboration with Vaillant $\mathrm{GmbH}$, a latent heat storage was constructed and tested. During the hot water preparation in summer, the reject cold of the heat pump is stored thereby solidifying the PCM. Then, the cold is delivered to the building, e.g. via an existing floor heating system, and the PCM melts again (Fig. 1, right).

\section{DEVELOPMENT OF NOVEL SALT HYDRATE MIXTURES}

In several energy-relevant temperature ranges, there is lack of suitable salt hydrate based PCM. Mixtures of two or more salt hydrates could fill these gaps. Solely relying on experimental techniques, solid-liquid phase diagrams of multicomponent mixtures are difficult to determine. In a current research project, the so-called modified BET-model is applied to calculate solid-liquid phase diagrams of salt hydrate mixtures. For applications, mixtures are interesting that do not change their composition (and thereby their thermal properties) during repeated melting/crystallization cycles. Such a cycling-stable phase change behaviour can be expected for eutectic mixtures, corresponding to local temperature minima of solid-liquid phase diagrams.

\section{CONCLUSIONS}

Salt hydrates or salt hydrate mixtures are suitable storage materials for latent heat storage systems in low-temperature applications due to their high energy densities at comparably low costs. The following development steps are recommended to apply salt hydrates as PCM: first, the identification of the optimum composition of the material based on the corresponding solid-liquid phase diagram; second, the design of the heat exchanger to meet the power requirements of the application without impairing the long-term stability of the PCM; third, an application-oriented testing of functional models of the PCM together with the heat exchanger and additional components like a state-of-charge device or an anti-separation unit.

\section{ACKNOWLEDGEMENTS}

The responsibility for the content is with the authors. The corresponding research projects received national funding under the grant numbers 0329605D, 0329605O, 03ESP138D, and 03ET1342A.

\section{REFERENCES}

Rathgeber, C., Helm, M., Hiebler, S., Energiespeicher für die Energiewende: Salzhydratbasierte Niedertemperatur-Latentwärmespeicher, Chemie Ingenieur Technik, 90 (2018), 193-200 


\title{
MODELING AND VALIDATION OF THE ICE GROWTH IN AN ICE STORAGE SYSTEM
}

\author{
Stefanie Paulini, M.Sc.1, Prof. Dr.-Ing. Tobias Plessing1, Prof. Dr.-Ing. Dieter Brüggemann2 \\ Institute for Water and Energy Management at Hof University1 \\ Kulmbacher Straße 76, 95213 Münchberg, Germany \\ Phone: +49 9281/4098455 \\ Department of Engineering Thermodynamics and Transport Processes (LTTT), University of Bayreuth2 \\ Universitätsstr. 30, 95440 Bayreuth \\ E-Mail: stefanie.paulini@ @of-university.de
}

\section{SUMMARY}

This work deals with the optimization of the icing process in an ice storage system. It is focused on the improvement of the icing behavior, which is to be achieved by different heat exchanger geometries in the ice storage tank. Therefore, CFD simulations were implemented to acquire and visualize the flow conditions, the temperature behavior and the growth of the ice layer during the cooling process. The results are compared and validated with model experiments on an experimental ice storage. It could be shown that the heat extraction of current technologies can be increased by more than $50 \%$ by using geometries that are more efficient.

Keywords: CFD simulation, ice storage, model validation

\section{INTRODUCTION}

Ice storage systems enable to supply heat pumps with temperatures above $-10{ }^{\circ} \mathrm{C}$ for a longer time. To increase the annual operating efficiency and the overall efficiency of the heat pump system, the ice storage has to be fully exploited. Therefore, the flow conditions in the ice storage tank have to be analyzed. A homogeneous mixing during the icing process enables a longer sensible heat extraction and temperatures above $0{ }^{\circ} \mathrm{C}$ for a longer time. In addition, the $4{ }^{\circ} \mathrm{C}$ sump could be avoided by homogeneous mixing.

In the presented project, the heat input into a solar ice storage with a helix and a star-shaped heat exchanger is examined. In addition to model experiments, CFD simulation is used for the acquisition and visualization of the flow conditions and ice build-up. The aim is to optimize the heat input and the icing process by adapting the heat exchanger geometry.

\section{BOUNDARY CONDITIONS}

The main aim is to enhance the overall icing process in the ice storage tank. The convective flow with in the water tank should be improved in order to achieve a more homogeneous mixing in the ice storage system. The aim of the further investigation is to optimize the ice building process in the ice storage tank along the heat exchanger surface. By keeping the ice built up parallel to the heat exchanger plates as long as possible, the temperature of the return flow of the heat pump decreases slowly and the heat pump can be supplied with temperatures above $-10{ }^{\circ} \mathrm{C}$ for a longer period. This increases the annual operating efficiency and thus the overall efficiency of the heat pump system. An adapted ice growth on the heat exchanger plates increases the proportion of the water volume which could be iced. The influence of the heat exchanger geometry on the flow, the temperature layering and the ice growth is pictured and analyzed by CFD simulations. Therefore, 2D and 3D models of the ice storage and heat exchangers are built with ANSYS Fluent. The simulation models are validated by model experiments on a horizontally divided hemispherical experimental ice storage on a scale of $1: 2$ to the original ice storage.

\section{SETUP AND RESULTS}

Based on former CFD simulations, a model test bench was created for the visualization of the icing process in the ice storage tank. The test bench consists of an $8 \mathrm{~kW}$ heat pump with a refrigerating capacity of $6 \mathrm{~kW}$, a $2.5 \mathrm{~m}^{3}$ horizontally divided hemispherical ice storage tank and small storage with electrical heating rod as shown in figure 1. 


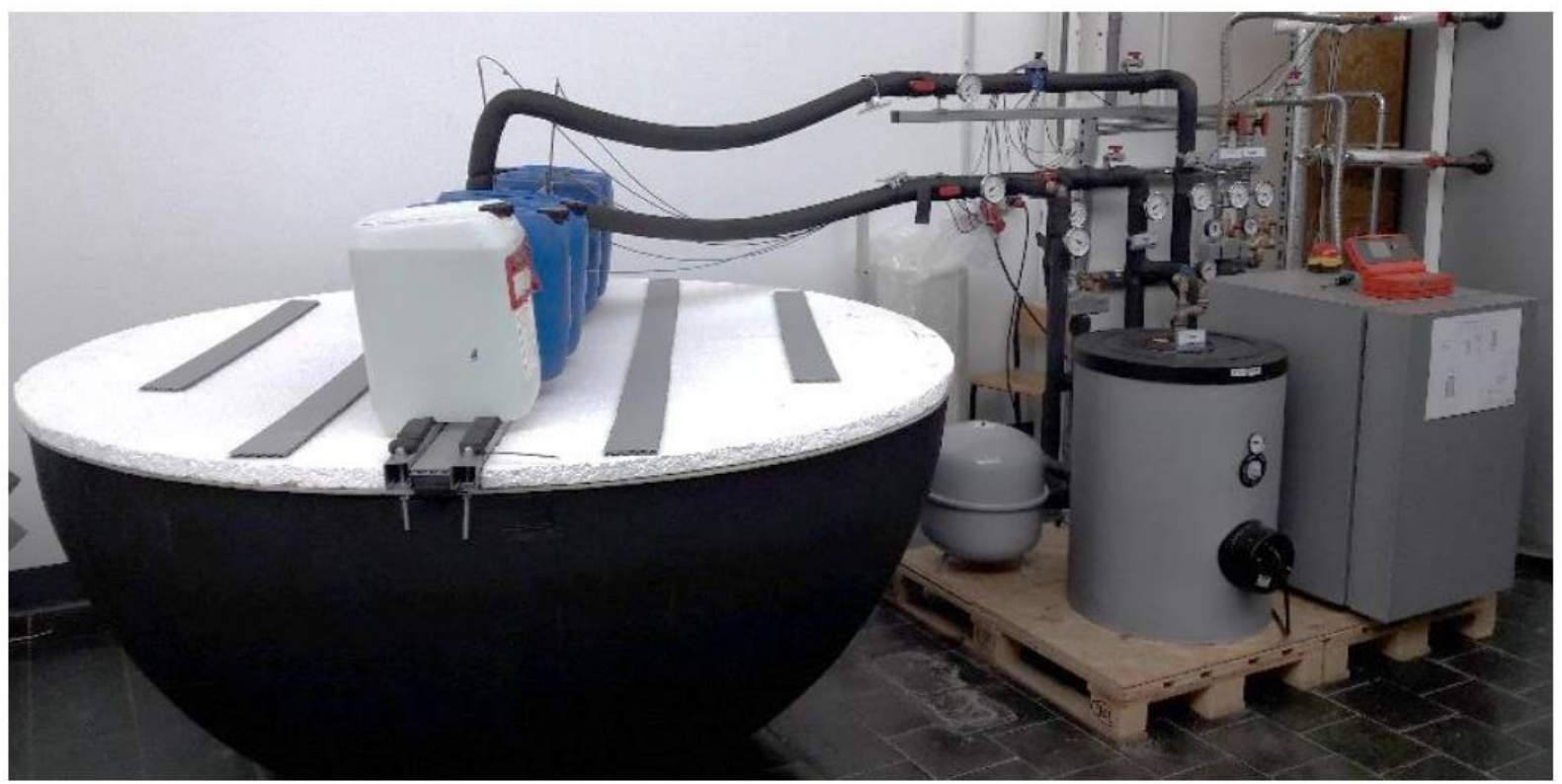

Fig. 1: Test bench with isolated ice storage (left), storage with heating rod (middle) and heat pump (right)

The ice storage tank was isolated to reduce the heat input from the surrounding area. The small storage with the heating rod was installed to reheat the ice storage after the icing experiments. The water-filled tanks on the ice storage counteract the rising buoyancy force of the ice during the icing process. As a basis for the first model experiments, a helix heat exchanger was chosen. The ice storage was iced until the return flow temperature of the heat pump reached the switch-off temperature of $-10{ }^{\circ} \mathrm{C}$. Afterwards the heat exchanger was replaced by a star-shaped geometry with an arrangement of 24 heat exchanger plates. Thereafter, the number of the heat exchanger plates was halved from 24 to 12 plates to improve the icing process. Six temperature sensors in the ice storage and one temperature sensor in each case in the flow and return flow of the primary circuit of the heat pump documented the icing process.

The simulation of the phase change was realized by the ANSYS Fluent solidification and melting tool. The solver settings were designed according to Abduljalil A. Al-abidi et al. (2013) and Amrit Om Nayak, G.Ramkumar, T.Manoj, and R.Vinod (2011). The geometry was transferred to a 1:1 scale CFD model, simulated with boundary conditions of the model experiments and then validated with the data from the model experiments. The helix heat exchanger was realized with 2D-CFD simulation. The model was simulated in horizontal and vertical cut through the heat exchanger and the ice storage as seen in figure 2.

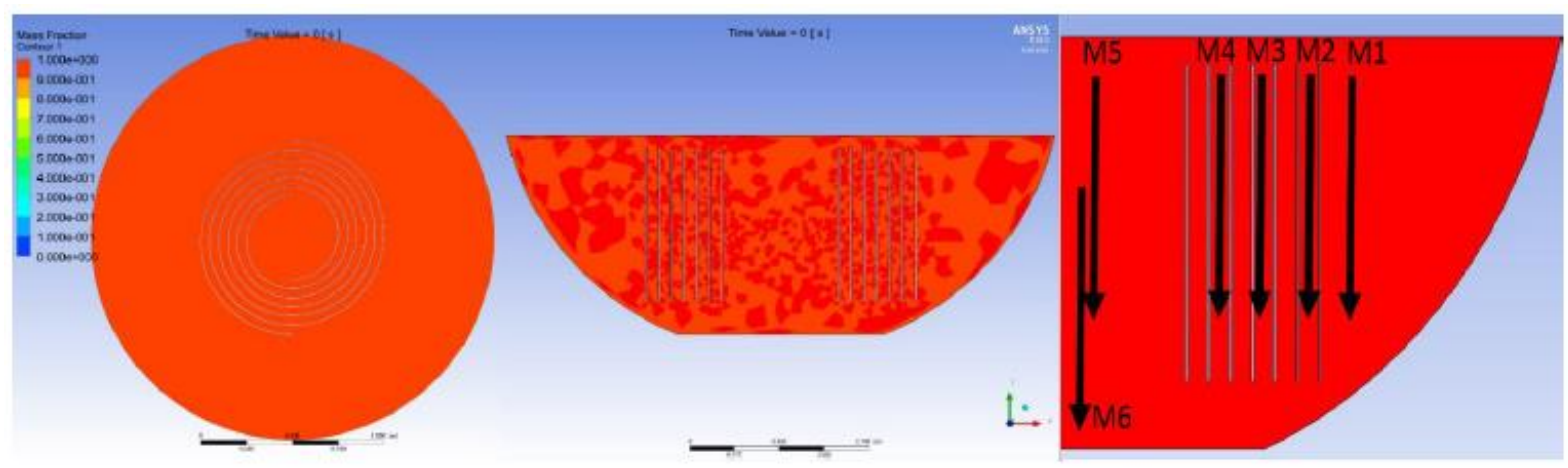

Fig. 2: Horizontal (left) and vertical (middle) cut through the helix heat exchanger and ice storage with points of temperature measurement (right)

The points of temperature measurement were set to the same places as the temperature sensors in the model test bench. Five sensors were set in a horizontal row to picture the temperature behavior 
through the horizontal cut of the ice storage and one was set to the ground of the ice storage to picture the $4{ }^{\circ} \mathrm{C}$ sink at the bottom of the tank. This sink is due to the anomaly of water. The heat exchanger temperature from the model experiments was implemented as a boundary condition for the plate temperature for the simulations. The simulation models were validated and adjusted with the measurement results from the model experiments with the helix heat exchanger.

The star shaped heat exchanger was simulated with 3D-CFD simulation. Due to the radial symmetry of the model, only $1 / 12$ of the ice storage was transferred to a simulation model. The modelled geometry with two heat exchanger plates is shown in figure 3 .

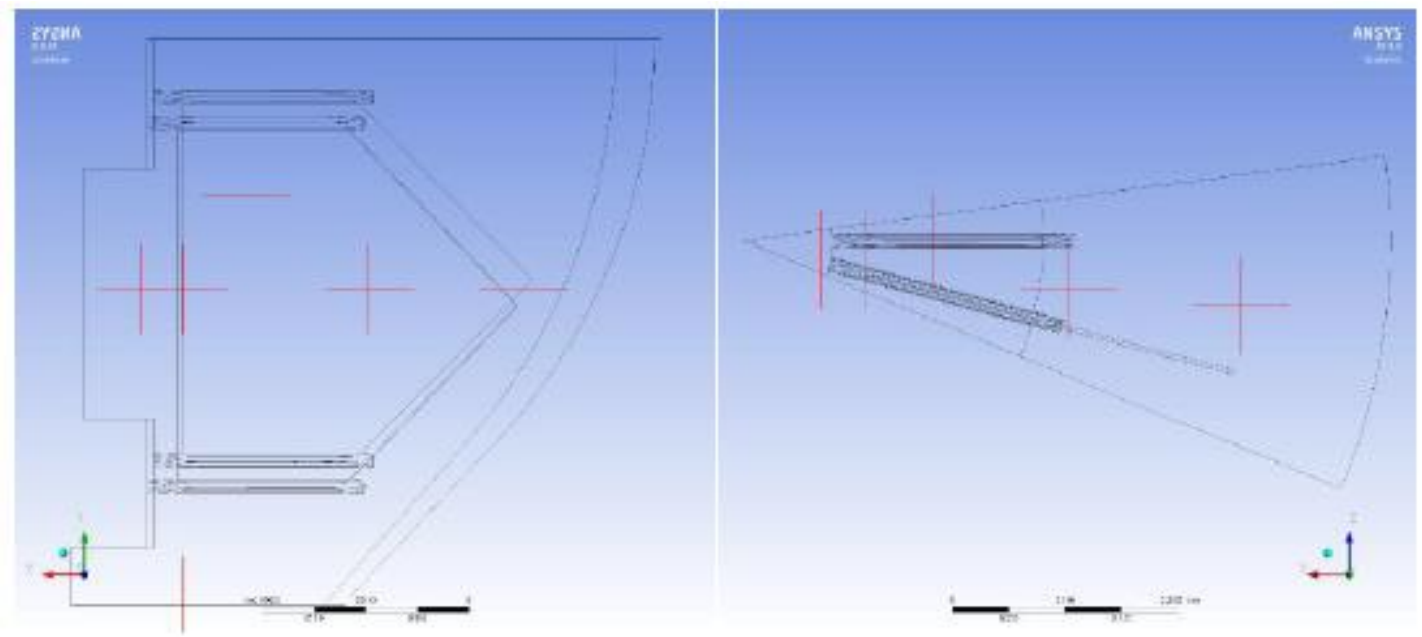

Fig. 3: Arrangement of the temperature sensors in the simulated part with two heat exchanger plates

The red crosses represent the six points at which the temperature is logged during the simulations. They are similar with the six temperature sensors in the ice storage volume at which the temperature is measured during the experiments at the model test bench. Similar to the 2D-CFD simulation the heat exchanger temperature from the model experiments was implemented as a boundary condition for the plate temperature for the simulations. The simulation models were also validated and adjusted with the measurement results from the model experiments with the star-shaped heat exchanger.

The results of the 2D-CFD simulation of the helix heat exchanger and the measurements at the test bench are shown below. The simulation showed good accordance with the results of the model experiments from the test bench. The temperature profile of the temperature sensor 2 until the switchoff point of the heat pump is pictured in figure 4 .

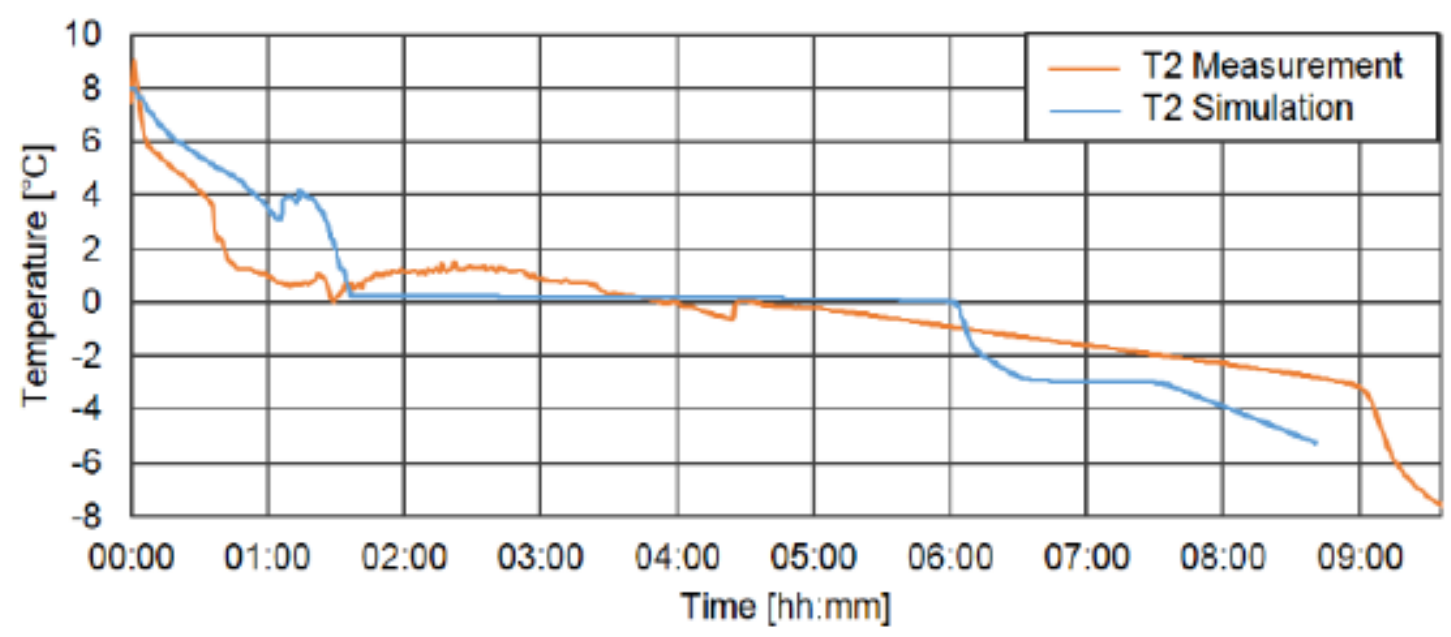

Fig. 4: Temperature profile ofthe model experiment (orange) and the simulation (blue) during the icing process

The latent heat transfer could be mapped very well in the simulation (blue line) by the long temperature stability during the ice build-up between 1:30 and 6:00 of the simulation time. The measured temperature (orange line) shows, that some effects like supercooling could only be described 
inadequate by the simulation. Nevertheless, the overall temperature behavior of the simulations fits with the experimental values. The decreasing temperature line of the model experiment (orange line) pictures the dipping of the temperature sensor into the ice. The temperature drop at the end of the time line describes the point at which the ice walls grow together. Several model experiments on the helix heat exchanger showed the reproducibility of the individual temperature behavior. Slight divergences could be explained by the different starting temperatures in the ice storage volume. The simulation clearly shows the temperature stratification in the ice storage with the $4{ }^{\circ} \mathrm{C}$ sink at the bottom of the ice storage tank. Although the heat exchanger plates are already freezing, half of the fluid volume is still warmer than $1{ }^{\circ} \mathrm{C}$. These two circumstances lead to the fact that the heat exchanger plates begin to freeze in the upper third and the lower part of the plates are not yet frozen at the end of the simulation. This also shows the unfavorable shape of the heat exchanger compared to the surrounding fluid in the simulation. Due to the geometry, only a fraction of the existing fluid can really be iced. This results in a latent heat extraction of $50.59 \mathrm{kWh}$ in $8 \mathrm{~h} 42 \mathrm{~min}$ by a heat exchanger surface of $34 \mathrm{~m}^{2}$.

Based on these basic experiments and simulations, a preferred geometry is to be determined, which represents the basis for a subsequent development. A star shaped heat exchanger with 24 plates was chosen for the further analyses. The geometry was reduced to 12 plates after the first experiments. The number of heat exchanger plates influences the point on which the ice walls on the plates grow together and the temperature in the return flow of the heat pump drops off. The arrangement of the star-shaped heat exchanger with 12 and 24 plates with the temperature sensor 1 (red dot) is shown in figure 5 .

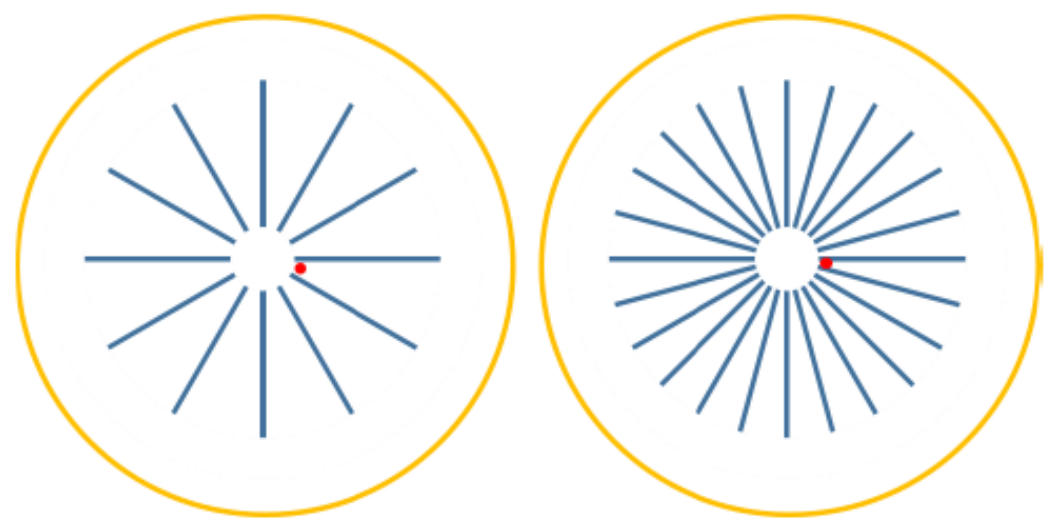

Fig. 5: Arrangement of the heat exchanger plates and temperature sensor 1 in the star-shaped model with 12 plates (left) and 24 plates (right)

The temperature sensor 1 is the point, where the ice grows together first. When the temperature at sensor 1 falls below $0^{\circ} \mathrm{C}$, it is dipping into the ice. Due to the reduction of heat exchanger plates, the specific extraction of heat of each heat exchanger plate is doubled compared to the star-shaped heat exchanger with 24 plates.

The results of the star-shaped heat exchanger are shown below. The star shaped heat exchanger with 24 plates has a theoretical ice thickness of $13.3 \mathrm{~cm}$. During the icing experiments at the ice storage test bench, this value could not be reached. One of the reasons was the small distance between the backs of the plates in the middle of the star geometry. That leaded to a fast growing together of the ice walls, what reduced the overall ice surface. Therefore, the temperature at the heat pump return flow decreased and the heat pump stopped working. The ice buildup after one icing cycle until the heat pump shut-off at $-10{ }^{\circ} \mathrm{C}$ is pictured in figure 6 . 


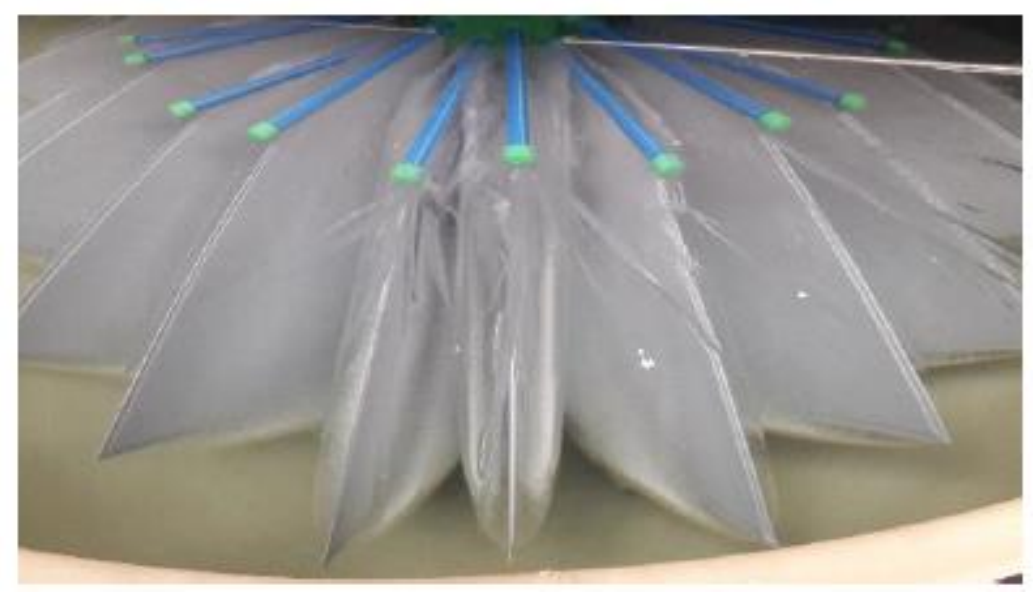

Fig. 7: Ice built-up after one icing cycle of the heat pump at the star shaped heat exchanger with 24 plates

The picture shows the small ice layer of only about $6 \mathrm{~cm}$. The back surfaces of the plates are grown together, what can also be seen in the temperature profile of the temperature sensor 1 and the return flow temperature of the primary circuit of the heat pump. The ice built-up results in a latent heat extraction of $105.14 \mathrm{kWh}$ in $15 \mathrm{~h} 52 \mathrm{~min}$ by a heat exchanger surface of $34 \mathrm{~m}^{2}$, which is double the value of the helix heat exchanger. The comparison of the return flow temperatures of the primary circuit of the heat pump of the helix heat exchanger and the star shaped with 24 plates is illustrated in figure 7.

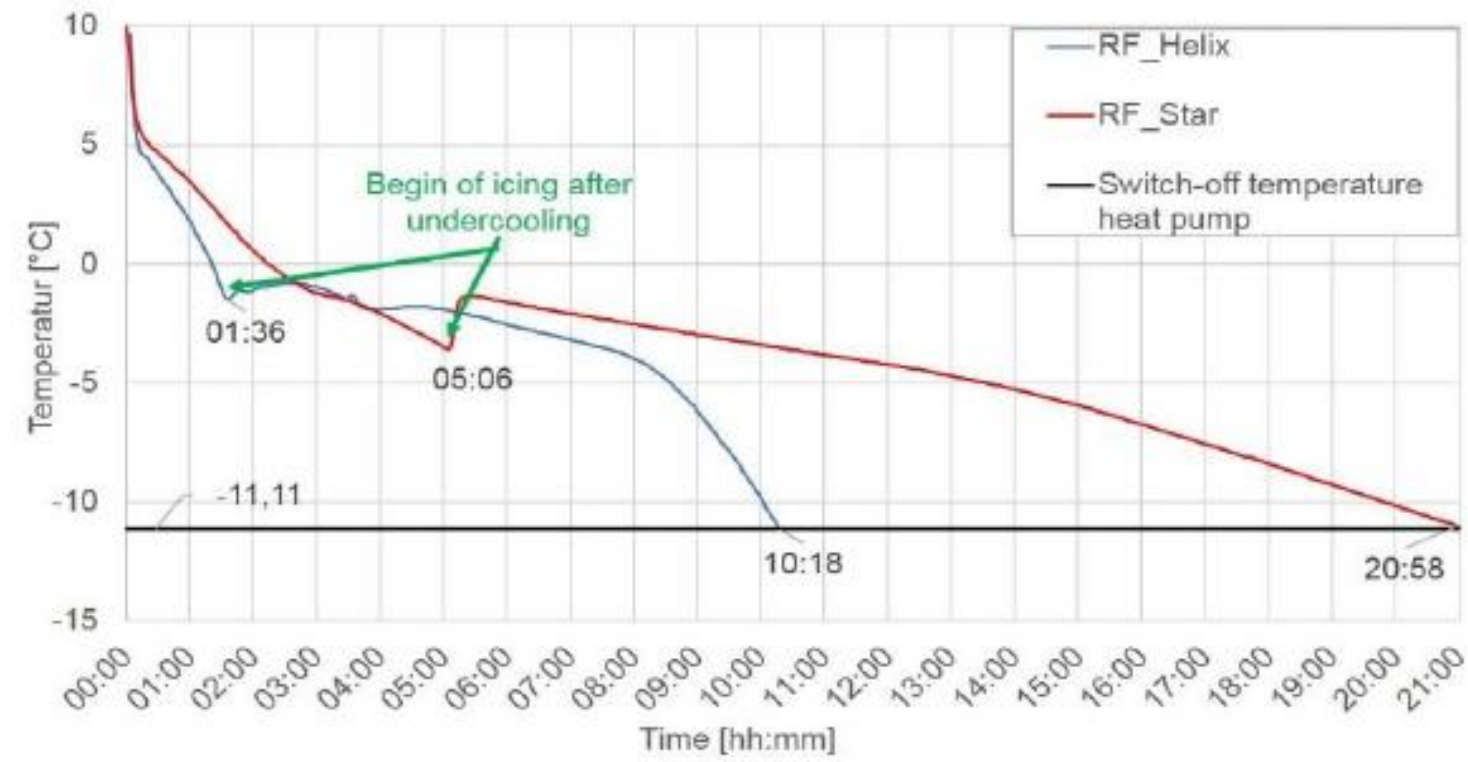

Fig. 6:Difference of the return flow temperatures of the primary circuit of the heat pump with helix (blue) and star-shaped (red) heat exchangerwith 24 plates

The improved flow through the ice storage with star-shaped heat exchanger significantly delayed the onset of icing and increased the amount of sensible heat extracted by $50 \%$. The rapid decreasing in the return flow temperature of the helix structure after 8 hours of experimentation is attributable to the coalescing ice layers between the helical turns and thus to a rapid reduction of the heat transfer surface. In the star-shaped geometry, the heat transfer surface is reduced only slowly, which is reflected in the gradually decreasing temperature curve of the return flow temperature. In both cases, it comes to a significant supercooling of the heat transfer medium before the icing of the heat exchanger surfaces starts. In the helical geometry, the supercooling is $-1.8^{\circ} \mathrm{C}$, in the star-shaped structure even - 
$3.7^{\circ} \mathrm{C}$. Since the sensitive heat depends on the flow, the amount of water and the start temperature of the water volume in the ice storage tank, it is not considered in this work.

The icing experiments with the star-shaped heat exchanger with 12 plates showed good accordance to the theoretical icing behavior. The theoretical calculated ice thickness of $6.6 \mathrm{~cm}$ per plate surface could be reached during the icing process as pictured in figure 8 .

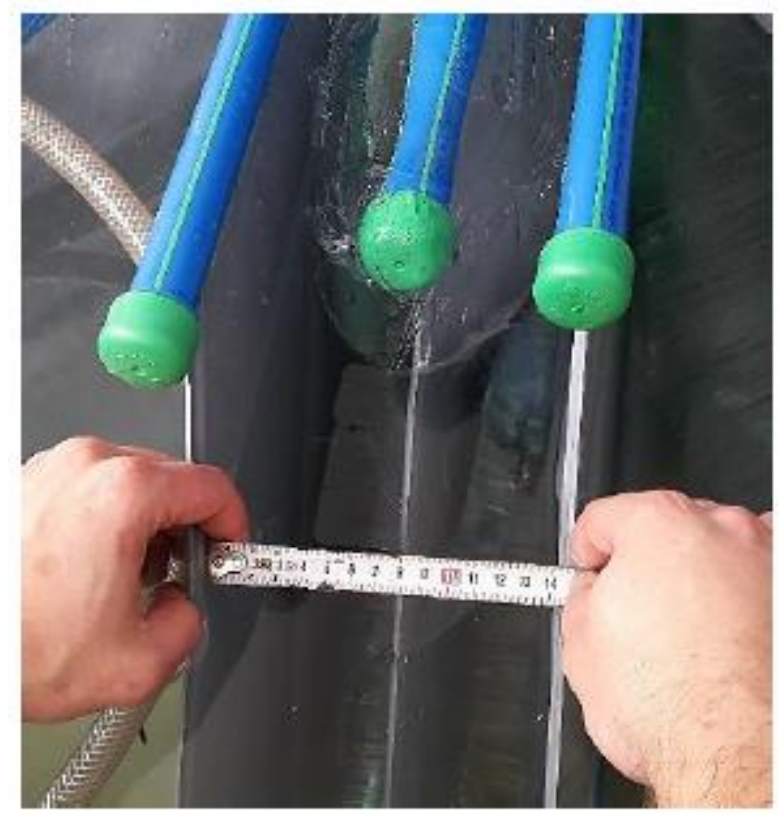

Fig. 8: Thickness of the ice walls of $14 \mathrm{~cm}$ from ice surface to ice surface with plate in the middle after one icing circuit with the star-shaped heat exchanger with 12 plates

Due to the big distance between the individual plates, the ice built-up could proceed parallel during the whole icing process. This shows that the reducing heat exchanger surface was the limiting factor for the ice built-up with 24 plates and helix heat exchanger in the former experiments. This restriction could also be outlined by the temperature profiles of temperature sensor 1 during the icing experiments with the helix heat exchanger and the star-shaped heat exchanger with 24 and with 12 plates in figure 9.

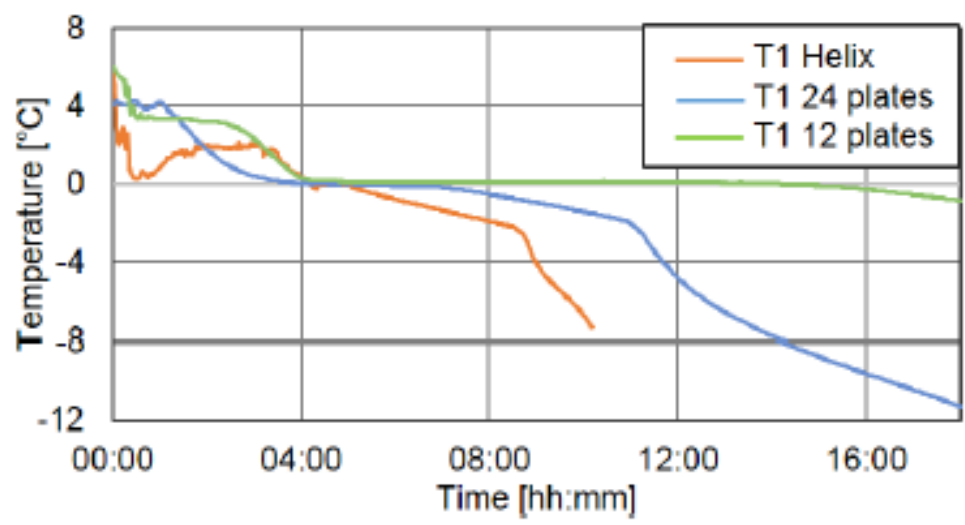

Fig. 9: Difference of the temperature profileof temperature sensor 1withhelical heat exchanger (orange), 24 plates (blue) and 12 plates(green) heat exchanger during the icing process

The drop off in the orange and blue temperature profile of the helical and the 24 plates heat exchanger is owed to the coalescing ice layers and the rapid reduction of the heat transfer surface. The heat exchanger with 12 plates starts icing together at the end of the icing experiments. This shows, that there is a parallel icing during the whole process. 
The difference of the icing behavior of the heat exchanger with 24 and with 12 plates is clearly shown in figure 10 below. It illustrates the difference in the return flow temperatures of the primary circuit of the heat pump for 12 and 24 plates of the star-shaped heat exchanger and the temperature sensor 1 in both cases on the model test bench.

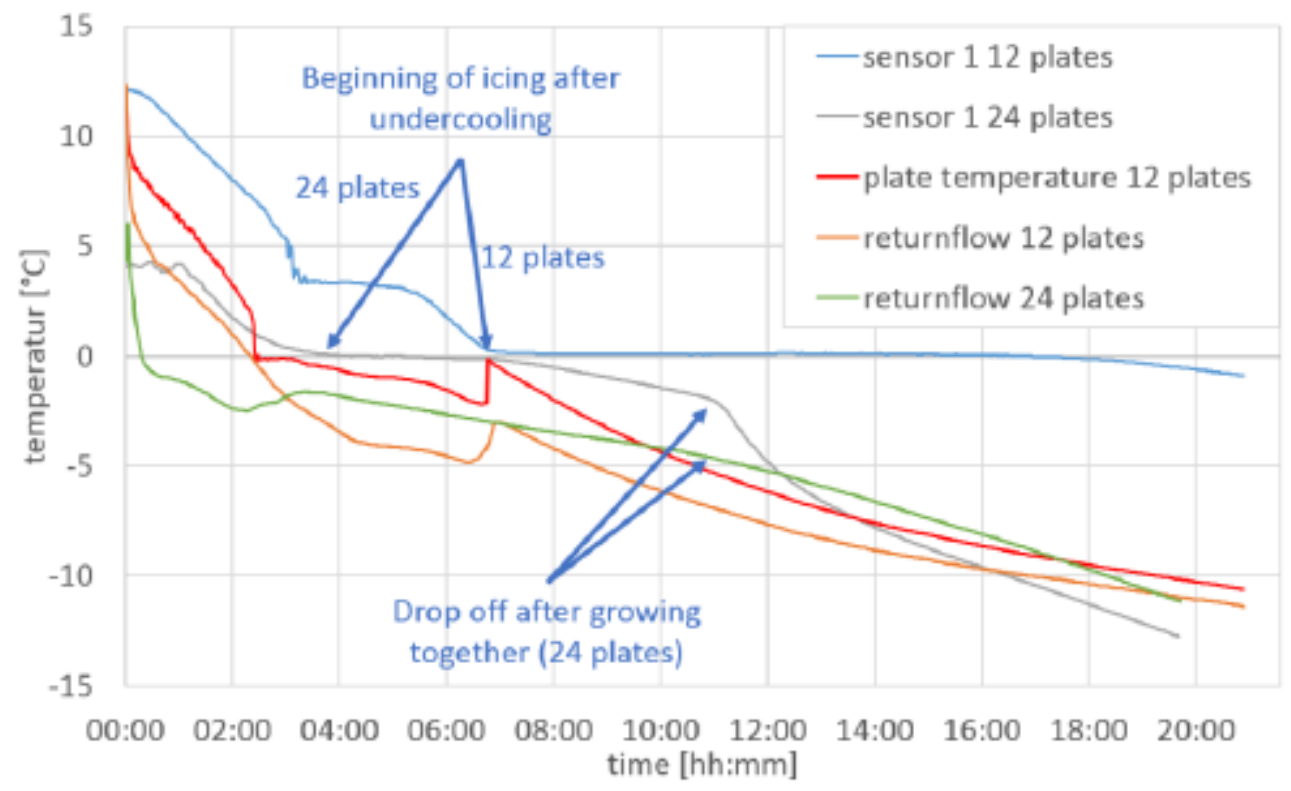

Fig. 10: Difference ofthe return flow temperatures of the primary circuit of the heat pump with 12 (yellow) and 24 (green) heat exchanger and of the temperatures between the plates (blue and grey)

The plate temperature (12 plates) clarifies the beginning of the icing process after the undercooling process.

The time of the parallel icing from the beginning of icing after undercooling until the temperature drop-off is much longer at the heat exchanger with 12 plates than with 24 plates. The undercooling of the return flow temperature with 24 plates is $-2.5^{\circ} \mathrm{C}$, while the undercooling of the return flow temperature with 12 plates reaches $-4.8{ }^{\circ} \mathrm{C}$. One of the reasons for this effect is the improved flow conditions at the geometry with 12 plates.

Though the 3D simulation of the star-shaped heat exchanger with 24 plates took several months to calculate, it is only considered for a qualitative statement. The ice built-up of the 3D simulation and the icing experiment with the star-shaped heat exchanger with 24 plates is shown in figure 11. 


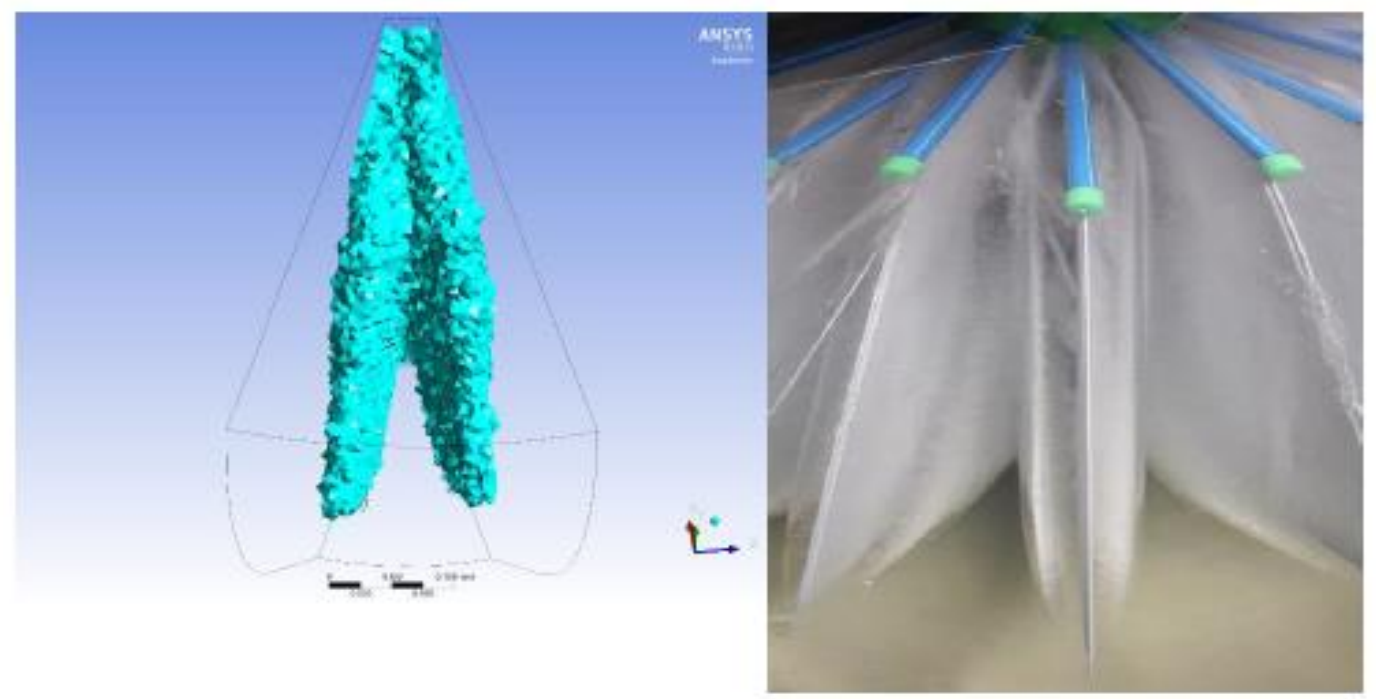

Fig. 11: Ice built-up in the simulation (left) and icingexperiment (right) for the star-shaped heat exchanger with 24 plates

Due to the coarse grid, the surface of the simulated ice layer is irregular. The simulation resulted in an ice volume of $0.381 \mathrm{~m}^{3}$ or $350 \mathrm{~kg}$, while the experiment resulted in a calculated ice layer of about 360 $\mathrm{kg}$.

\section{CONCLUSION AND FUTURE WORK}

The modeling of an ice storage system with 2D- and 3D-CFD simulation with ANSYS Fluent and the validation of this simulations with model test bench experiments is shown in this work. The temperature behavior during the cooling and icing process in the ice storage could be mapped very well despite a time gap by 2D-CFD simulations. The ice build-up could be predicted well by the 2Dand 3D-CFD simulations. The simulations and the experiments showed the increasing of the heat extraction by using a star-shaped heat exchanger geometry.

The accuracy of the 2D simulations should be further improved by adapting the parameters and boundary conditions. The 3D simulation should be improved by model reduction.

\section{REFERENCES}

Abduljalil A. Al-abidi et al., 2013. CFD applications for latent heat thermal energy storage: a review. Renewable and Sustainable Energy Reviews. 20, 353-363.

Amrit Om Nayak, G.Ramkumar, T.Manoj, and R.Vinod, 2011. Comparative Study between Experimental Analysis and CFD Software Analysis of PCM material in Thermal Energy Storage System. International Journal of Chemical Engineering and Applications. Vol. 2, No. 6.

\section{ACKNOWLEDGEMENT}

This work has been funded by the Bavarian State Ministry of Education, Science and the Arts within the framework "TechnologieAllianzOberfranken (TAO)" and the Federal Ministry for Economic Affairs and Energy within the framework "Zentrales Innovationsprogramm Mittelstand (ZIM)". The authors gratefully acknowledge this support. 


\title{
HOW EFFICIENT IS A CLOSED SORPTION THERMAL ENERGY STORAGE (TES) SYSTEM BASED ON SODIUM HYDROXIDE?
}

\author{
Dr. Mihaela Dudita, Dr. Xavier Daguenet-Frick, Lukas Omlin, Dr. Paul Gantenbein \\ Institut für Solartechnik SPF \\ Oberseestrasse 10, CH-8640 Rapperswil, Switzerland \\ Phone: +41552224169 \\ E-Mail: mihaela.dudita@spf.ch
}

\begin{abstract}
SUMMARY
A compact chemical heat storage prototype based on water absorption/desorption in a high-energy density and low cost sorbent is presented. The heat and mass exchangers (HME) from this $1 \mathrm{~kW}$ prototype are based on the tube bundle and falling film models. Improvement of the HME efficiency is done by performing small-scale experiments with different tube bundle surface textures and by optimizing the sorbent wetting and residence time. A modular design was chosen for the HME from the absorber/desorber unit with the aim of studying several types of tubes and sorbent modifications. First experimental results and heat coefficient calculation are presented.
\end{abstract}

\section{INTRODUCTION}

Thermal Energy Storage (TES) is a promising solution for providing renewable heat all over the year, not only during summer time. TES can also be used in combination with other heat sources, for example waste heat or excess electricity. Charging is done when the energy generation is more efficient and convenient, while discharging is done based on consumer needs.

Thermal energy can be stored as sensible heat by using high heat capacity materials like water, as latent heat by running a constant temperature process like in phase change materials or as chemical heat storage. The chemical or sorption storage uses reversible processes in which the fluid/vapour is absorbed and heat is released or heat is absorbed and vapour is released, depending on the process stage and entropy/enthalpy. Sorption heat storage has the highest theoretical energy density and it can be used to store large quantities of energy over a relatively long period, with insignificant heat losses. Sorption thermal energy storage involves the physical and chemical bonds between at least two components for storing the thermal energy. Heat is added during charging process to separate the sorbent and the sorbate (desorption phase). For discharging, the sorbent is combined with the sorbate, which is an exothermic process. Different materials like adsorbents, salts hydrates and hydroxides are investigated for thermal sorption storage. The materials characteristics (e.g. water vapour uptake, crystallization) are influencing the system's efficiency, and also the operating parameters like temperature for charging, discharging, condensation and evaporation, mass flow rate (Hadorn, 2005). Research is still needed to solve different materials instability issues and increase the low technology readiness (Scapino, Zondag, Van Bael, Diriken, \& Rindt, 2017).

A first TES closed sorbtion prototype based on sodium hydroxide was developed in the EU COMTES project. Investigations have shown a lower efficiency compared to the theoretical value during the absorption phase (storage discharging) (Daguenet-Frick et al., 2014). This was explained by a poor wetting of the A/D (Absorber/Desorber) tube bundle (AISI 316L) with the concentrated, viscous sodium lye and its short residence in the water vapor. Thus, a $1 \mathrm{~kW}$ prototype is developed by Institut für Solartechnik SPF in a consecutive Swiss national R\&D project (ABSTOREX) to optimize the reaction zone unit for the tube bundle case. Another type of HME is investigated in parallel in the frame of SCCER Heat and Electricity by Empa in Dübendorf $(\mathrm{CH})$.

\section{DEVELOPMENT OF A 1KW THERMOCHEMICAL STORAGE PROTOTYPE USING SODIUM HYDROXIDE.}

The $\mathrm{CAD}$ view of the sorbent and sorbate loops: $\mathrm{A} / \mathrm{D}$ and $\mathrm{E} / \mathrm{C}$ units together with the optimized $1 \mathrm{~kW}$ laboratory test rig is represented schematically in Fig. 1. The reaction zone consists of two main units: $\mathrm{A} / \mathrm{D}$ (Absorber/Desorber) and E/C (Evaporator/Condenser). Both the A/D and E/C units are based on a vacuum tight container comprised of a fluid distribution manifold and a falling film tube bundle as HME. The heat and mass transfer and thus the exchanged power during charging and discharging is strongly depending on the sorbent and sorbate materials properties, sorbent residence time in sorbate vapour and the wetting properties of the heat transfer fluid over the tube bundle surface. Based on our previous investigations (Dudita, Daguenet-Frick, \& Gantenbein, 2017) about surface wetting and use 
of ceramic foams for residence time improvement, different tube modifications are implemented in the new A/D tube bundle unit (Fig. 2).

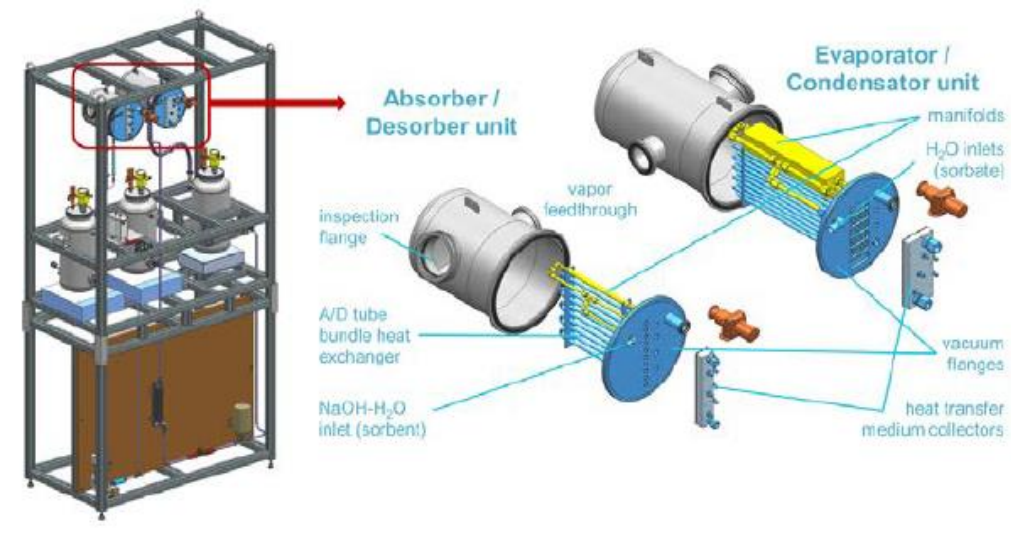

Fig. 1 View of the $1 \mathrm{~kW}$ laboratory test rig with the heat and mass exchanger unit as well as the storage tanks

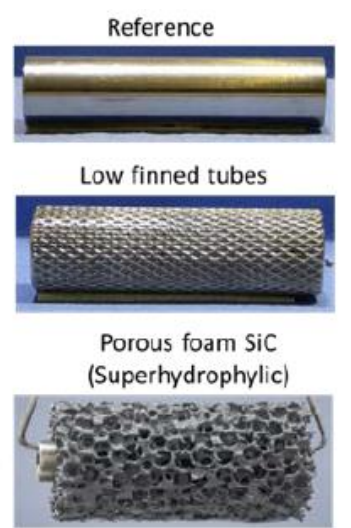

Fig. 2 Tube modifications to be implemented in the new $A / D$ unit

First experimental results obtained with the $1 \mathrm{~kW}$ prototype are reported, including calculation of the heat coefficient and comparison with theoretical models for the three tube bundles.

\section{ACKNOWLEDGEMENTS}

This research was supported by the Swiss Federal Office of Energy in the frame of the ABSTOREX project, by the Commission of Technology and Innovation in the frame of SCCER Heat and Electricity Storage as well as by the HSR University of Applied Sciences of Rapperswil.

\section{REFERENCES}

Daguenet-Frick, X., Gantenbein, P., Frank, E., Fumey, B., Weber, R., \& Williamson, T. (2014). Reaction Zone Development for an Aqueous Sodium Hydroxide Seasonal Thermal Energy Storage. Energy Procedia, 57

Dudita, M., Daguenet-Frick, X., \& Gantenbein, P. (2017). Closed Sorption Seasonal Thermal Energy Storage with Aqueous Sodium Hydroxide. In Nearly Zero Energy Communities: Proceedings of the Conference for Sustainable Energy (CSE) 2017 (pp. 239-246). Springer International Publishing. Hadorn, J.-C. (2005). Thermal energy storage for solar and low energy buildings: state of the art Scapino, L., Zondag, H. A., Van Bael, J., Diriken, J., \& Rindt, C. C. M. (2017). Energy density and storage capacity cost comparison of conceptual solid and liquid sorption seasonal heat storage systems for low-temperature space heating. Renewable and Sustainable Energy Reviews, 76, 1314-1331. 


\title{
STUDY OF HYBRID DRY COOLING SYSTEMS FOR STE PLANTS BASED ON LATENT STORAGE
}

\author{
Rocío Bayón1*, Mario Biencinto1, Esther Rojası, Nerea Uranga2 \\ CIEMAT-PSA 1 \\ Av. Complutense 40, 28040 Madrid, Spain. \\ Phone: +34 913466048 \\ IK4-TEKNIKER2 \\ C/ Iñaki Goenaga 5, 20600 Eibar, Guipuzkoa, Spain \\ E-Mail: rocio.bayon@ciemat.es
}

\section{SUMMARY}

This work focuses on the study of a hybrid cooling system for a solar thermal electricity (STE) plant composed by a latent storage module and an air-cooled condenser. In a first approach various commercial products have been studied in order to determine their feasibility as PCMs for the latent storage module. Differential scanning calorimetry (DSC), heat capacity (Cp) measurements and daily thermal cycling tests have been performed in order to determine the thermal properties of the selected PCMs and their melting/freezing behavior. Form these tests the product RT35HC has been chosen as PCM for the latent module. In order to evaluate the advantage of using a hybrid versus a conventional dry cooling system, the annual performance in terms of electricity production of a STE plant located in Ouarzazate (Morocco) has been simulated with both kinds of systems. Simulation results show that $0.31 \%$ increase in electricity could be obtained per year if a hybrid cooling system was used. However, the feasibility of this concept strongly depends on economic issues like electricity prices along the plant lifetime and both materials and equipment cost for the latent storage system.

\section{INTRODUCTION}

A Solar Thermal Electricity (STE) plant, as any other thermal power plant, requires large amounts of water (from 2000 to $3000 \mathrm{~m} 3 / \mathrm{GWh}$ ) for its operation and maintenance. The majority of this water, about $82 \%$ to $94 \%$, is consumed in power-block cooling whereas the rest is used for steam generation and solar field cleaning. Taking into account that areas with high direct solar irradiation and hence most appropriate for STE plants may be deserts with low water availability, the implementation of water saving strategies in a power plant to be constructed in those locations is major issue. The WASCOP project aims to overcome such challenge by offering different solutions for reducing water consumption in STE plants. Some of the water saving strategies included in WASCOP project are focused on the power-block cooling system since, as said above, a high percentage of water consumption in these plants is devoted to it. The Task 2.2 of this project evaluates the feasibility of a dry cooling concept, initially proposed by Pistocchini and Motta, 2011. This concept consists on using an air-cooled condenser (ACC) combined with a latent storage module as a hybrid cooling system. The latent module accumulates all or part of the exhaust heat from the turbine during the day for releasing it during the night, when lower ambient temperatures are expected. This should not only reduce ACC fan power consumptions but also improve the overall performance of the power block. With this approach, the condensation temperature during turbine operation can be maintained in most cases at lower values than with a conventional ACC cooling system and hence the efficiency of the power cycle can be increased. The heat stored in the latent module is released to the environment at night-time with the help of the ACC by taking advantage of the lower ambient temperature. The selection of the most adequate phase change material (PCM) to be implemented in the latent storage module has to take into account both the temperature of the exhaust heat released by the power block and the ambient conditions of the locations in which the STE plant may be constructed. These requirements impose that $\mathrm{PCM}$ should have a phase change temperature in the range of $25{ }^{\circ} \mathrm{C}$ to 40 ${ }^{\circ} \mathrm{C}$. In this work some commercial PCMs have been considered in order to ensure their availability in the market and also with the hope that problems related to material stability are already solved by the manufacturer. Prior to selecting the most appropriate PCM, the thermal properties required for the storage module design were measured for the selected candidates. Also, daily melting/freezing cycles under air were performed in a temperature controlled oven in order to study the PCMs under conditions similar to the operating ones and hence assessing their feasibility as storage materials for this specific application.

After selecting one PCM, annual simulations of performance for a typical STE plant located in a deserted location with this kind of cooling system have been carried out. Similar simulations have 
been done for the same STE plant with a conventional ACC cooling system and the results have been compared and discussed.

\section{EXPERIMENTAL}

In this work four commercial PCMs, two organic (RT35HC and RT28HC) and two inorganic (SP31 and SP26E), were tested since this manufacturer can supply small amounts of materials $(1 \mathrm{~kg})$ and also offers the possibility of PCM encapsulation, which would facilitate product handling, especially because melting temperatures are too close to room temperature. The thermophysical properties of these PCMs provided by the manufacturer (Rubitherm, 2017) are recorded in Table 1.

Table 1: Thermophysical properties of PCMs tested (Rubitherm, 2017).

\begin{tabular}{|c|c|c|c|c|c|c|}
\hline Product & Type & $\mathbf{T}_{\text {melt }}\left({ }^{\circ} \mathrm{C}\right)$ & $\Delta \mathrm{H}_{\text {melt }}(\mathrm{kJ} / \mathrm{kg})$ & $\rho\left(\mathrm{kg} / \mathrm{m}^{3}\right)$ & $\mathbf{k}(\mathrm{W} / \mathrm{mK})$ & $\mathbf{T}_{\max }\left({ }^{\circ} \mathrm{C}\right)$ \\
\hline RT35HC & Organic & 35 & 240 & $\begin{array}{l}860(\mathrm{~s}) \\
770(1)\end{array}$ & 0.2 & 70 \\
\hline SP31 & Inorganic & $31-33$ & 220 & $\begin{array}{l}1350(\mathrm{~s}) \\
1300(1)\end{array}$ & & 60 \\
\hline RT28HC & Organic & 28 & 245 & $\begin{array}{l}880 \text { (s) } \\
770 \text { (1) }\end{array}$ & 0.2 & 50 \\
\hline SP26E & Inorganic & $25-27$ & 190 & $\begin{array}{l}1500(s) \\
1400(1)\end{array}$ & 0.6 & 60 \\
\hline
\end{tabular}

Thermal characterization of these PCMs was performed by using a Mettler Toledo DSC1/500 apparatus. Temperature was calibrated with both $\mathrm{In}$ and $\mathrm{Zn}$ standards and enthalpy was verified with the In standard. DSC scans for obtaining phase change enthalpies and temperatures (Tmelt, $\Delta \mathrm{Hmelt}$ ) were carried out at $10^{\circ} \mathrm{C} / \mathrm{min}$, under $\mathrm{N} 2$ flux at $50 \mathrm{ml} / \mathrm{min}$ and samples were contained inside $40 \mu \mathrm{l}$ aluminium crucibles with micro-perforated covers. Heat capacity $(C p)$ measurements were performed in the same DSC apparatus under similar experimental conditions in terms of calibration standards, N2 flux and sample containers. However, the specific method for obtaining $C p$ implies to perform a base line, then a calibration with a sapphire standard and finally the measurement of the corresponding sample. This measurement consisted in two 10 min isothermal steps at both minimum and maximum temperature and a dynamic test at $10{ }^{\circ} \mathrm{C} / \mathrm{min}$.

In order to prove the viability of these PCMs as storage materials, tests under conditions as close as possible to the service ones should be required. In this way daily heating/cooling cycles were performed in an oven under air for samples of about 4-30 g contained in a ceramic crucible. Temperature interval and heating/cooling rate depended on the kind of PCM tested. During the cycles, sample temperature was monitored for detecting any change in PCM behaviour.

\section{PCM CHARACTERIZATION}

\section{DSC measurements}

DSC scans for the organic PCMs (RT35HC and RT28HC) were performed twice in order to homogenize the samples. The curves corresponding to the second heating/cooling cycle are displayed in Fig. 1. As we can see, none of these PCMs shows supercooling although during freezing they show two transitions. For RT35HC the two transitions are clearly distinguished whereas for RT28HC they are not so good resolved and only a shoulder is observed. However, if cooling rate was lower, probably two peaks would probably be obtained as well. For both materials phase change temperatures agree quite well with the values recorded in Table 1, however enthalpies are higher than the ones provided by the manufacturer (see Table 1), especially for RT35HC. Soupart-Caron, 2015, also performed DSC measurements and obtained phase change enthalpies around $234 \mathrm{~kJ} / \mathrm{kg}$ and hence also higher than the value given by the manufacturer (see Table 1). 

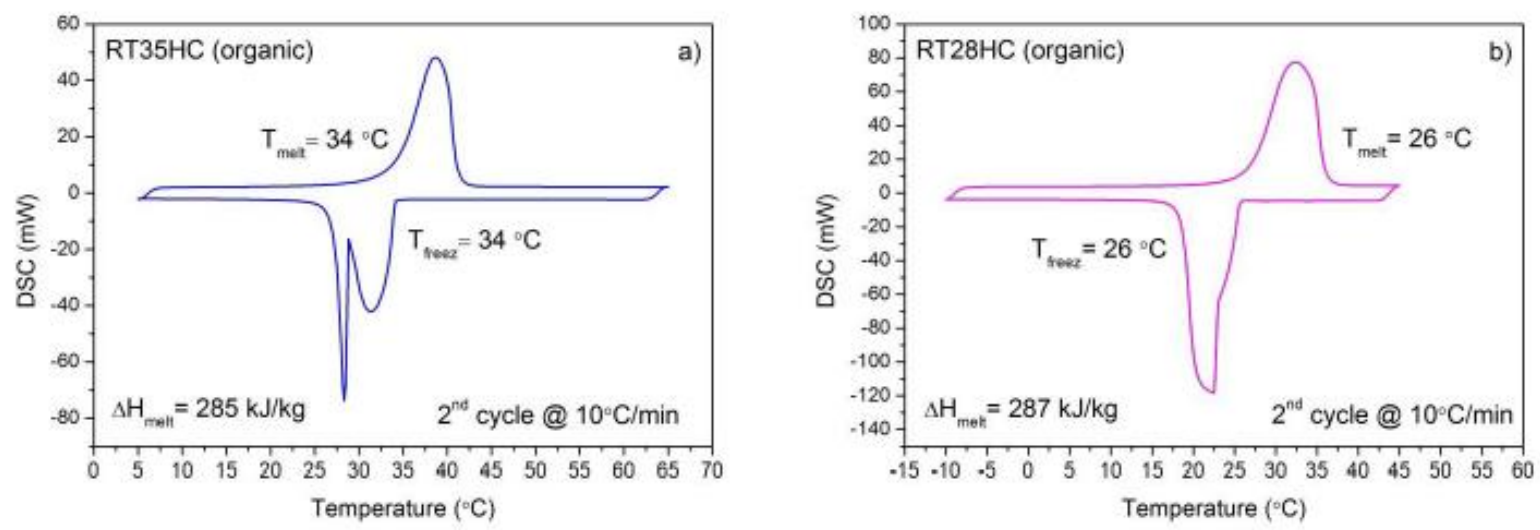

Fig. 1: DSC measurements performed for the organic PCMs RB35HC a) and RB28HC b).

When DSC measurements were performed for the inorganic PCMs (SP31 and SP26E), which are hydrated salts, some problems were encountered. For the case of RT31SP the initially solid sample was heated at $10{ }^{\circ} \mathrm{C} / \mathrm{min}$ and a melting peak at $34{ }^{\circ} \mathrm{C}$ was observed with an enthalpy of $277 \mathrm{~kJ} / \mathrm{kg}$. However, when this sample was cooled down, no freezing peak was observed even if a final temperature of $-40^{\circ} \mathrm{C}$ was attained. Therefore, a second sample was prepared but this time SP31 was initially in liquid estate. This time DSC measurements were performed at $0.5{ }^{\circ} \mathrm{C} / \mathrm{min}$ starting with a cooling cycle down to $-25^{\circ} \mathrm{C}$ and a subsequent heating run up to $55^{\circ} \mathrm{C}$. These cycles are displayed in Fig. 2 a) and, as we can see, both melting and freezing peaks are observed at $26{ }^{\circ} \mathrm{C}$ and $17{ }^{\circ} \mathrm{C}$ respectively. However the enthalpies obtained in these runs are about $37 \mathrm{~kJ} / \mathrm{kg}$ and hence much lower than the value reported by the manufacturer.
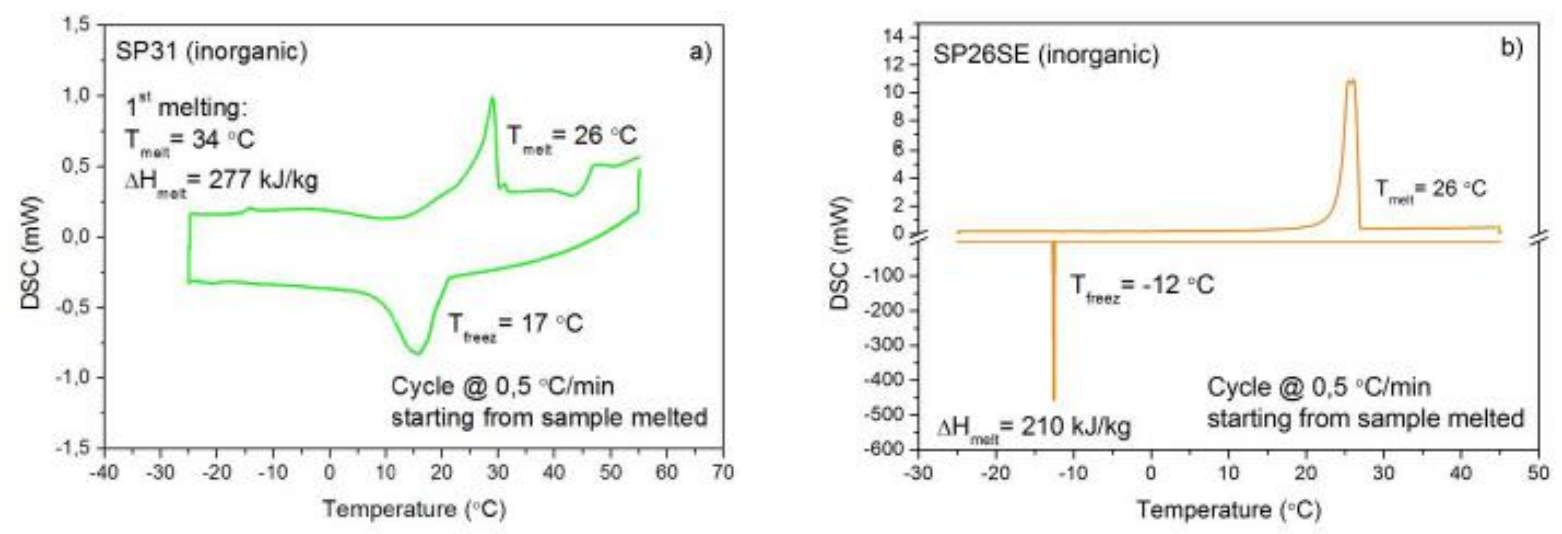

Fig. 2: DSC measurements performed for the inorganic PCMs SP31 a) and SP26E b).

The other inorganic PCM (SP26E) shows a better behavior in DSC scans with quite a high enthalpy, but it has a strong supercooling since it melts at $26{ }^{\circ} \mathrm{C}$ but it does not solidify until temperature reaches $-12{ }^{\circ} \mathrm{C}$ (see Fig. 2 (b). Apart from supercooling the reason for the behavior of these inorganic PCMs (hydrated salts) can be due to the occurrence of incongruent melting/freezing phenomena.

\section{$C_{p}$ measurements}

In general, $C p$ measurements are much more difficult to perform than DSC scans since they are not so straight forward. Actually, $C p$ values for the inorganic PCMs (SP31 and SP26E), which displayed strong supercooling and maybe incongruent melting/freezing, could not be carried out by applying the conventional methods developed in the laboratory for such measurements. Only the organic PCMs (RT35HC and RT28HC) could be measured and the variation of $C p$ with temperature was obtained, as displayed in Fig. 3. For both PCMs we observe that in solid state increases $C p$ as temperature 
approaches the melting point and it shows a constant value afterwards. $C p$ values given by the supplier for both PCMs are $2 \mathrm{~kJ} / \mathrm{kgK}$ (see Table 1), which is the mean value we observe for the solid phase of these two PCMs. However, as displayed in Figure 3, in liquid state they show slightly higher values of around $2.2-2.3 \mathrm{~kJ} / \mathrm{kgK}$.

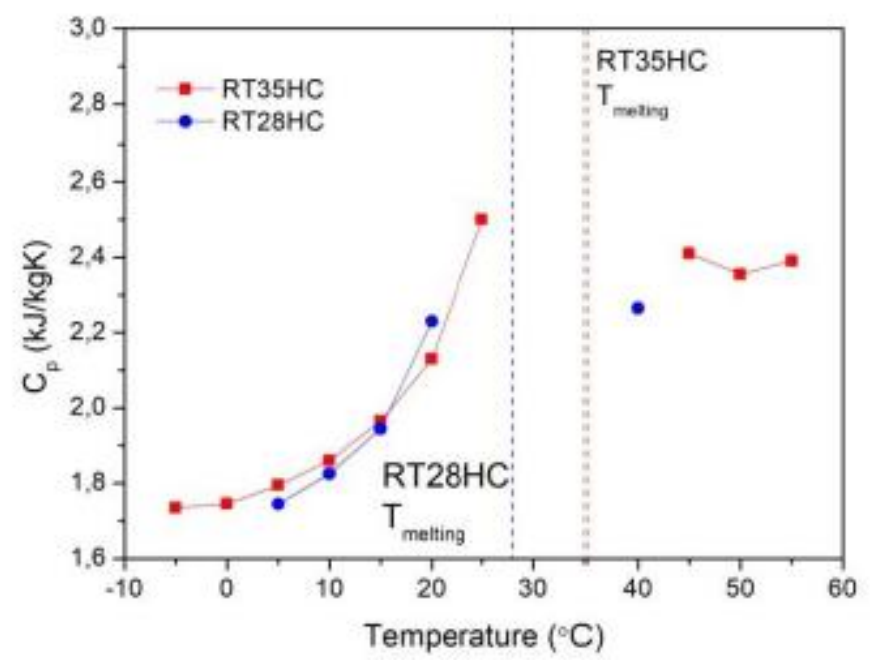

Fig. 3: Cp measurements performed for the organic PCMs: RB35HC and RB28HC

\section{Daily heating/cooling cycles}

Daily heating/cooling cycles were performed for the selected commercial PCMs in order to reproduce test conditions closer to the service conditions in comparison with DSC measurements. Temperature interval for the cycles was $\pm 10-20{ }^{\circ} \mathrm{C}$ around Tmelt taking care that working temperature limit recomended by the manufacturer (see Table 1) was not exceeded. In Fig. 4 temperature-time curves for RT35HC a), SP31 b) and RT28HC c) are recorded and the curves corresponding to different cycles are compared. 

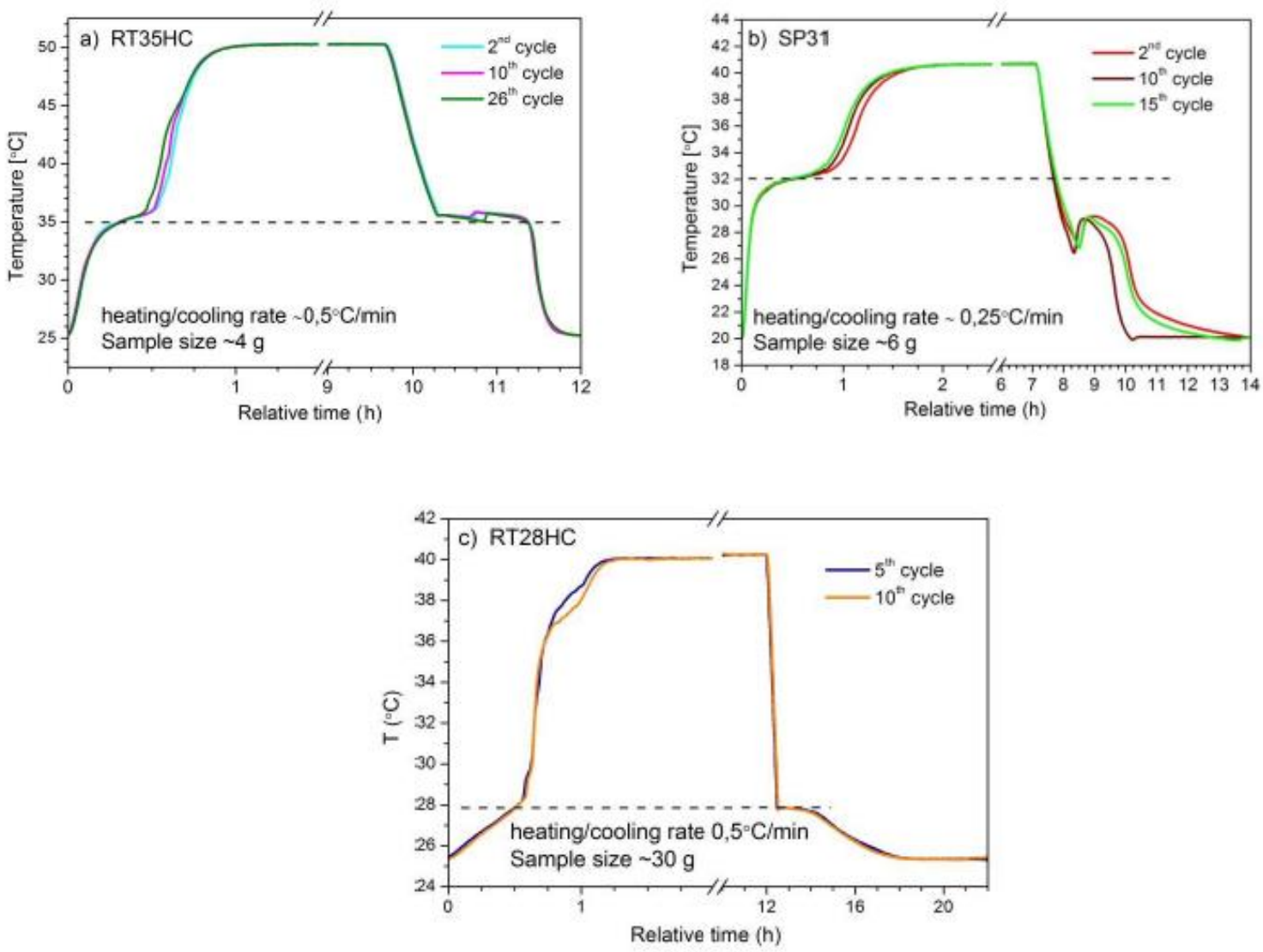

Fig. 4: Temperature-time curves of daily heating/cooling cycles performed in the oven under air for $\mathrm{RT} 35 \mathrm{HC}$ a), SP31 b) and RT28HC c).

For the three PCMs tested the temperature-time curves remain almost the same not only for the first cycles (2nd and 5th) but also for the last cycles performed (10th, 15th or 26th). In this kind of tests a flat plateau should be observed during both heating and cooling processes around melting temperature. RT35HC behaves this way not only in the 2nd cycle but also in the 26th cycle. RT31SP also displays a plateau during heating around melting temperature, whereas during cooling, temperature has to go down to $26^{\circ} \mathrm{C}$ until solidification occurs, which means that this compound presents supercooling. The latent heat produced during the freezing is able to heat up the sample and this is why the temperaturetime curve has this shape. SP31 behaves much better when cycled in the oven than in DSC measurements (see Fig. 2 (left)). In oven test this SP31 also displays supercooling, but the extent of this phenomenon is smaller since for this test a sample of about $5 \mathrm{~g}$ was used in comparison to the sample sizes used in DSC measurements which are normally in the order of 10-20 mg. This shows the importance of performing heating/cooling tests for PCMs under conditions as similar as possible to the service ones. RB28HC does not display a flat plateau during heating but a slope ending at Tmelt=28 ${ }^{\circ} \mathrm{C}$. However, it does show a plateau during cooling at this temperature. Finally, SP26 could not be tested since lab temperature is practically the same as melting temperature which makes it difficult the testing of this PCM even in an oven with forced ventilation. Moreover, if we take into account DSC results, some supercooling should be expected which means that freezing temperature would be lower than $26^{\circ} \mathrm{C}$.

Taking into account both DSC results and cycling test in the oven we can say that the RT35HC is the most reliable of the three commercial PCMs tested for being implemented in the latent storage module of the hybrid cooling system.

\section{IMPLEMENTATION OF THE LATENT STORAGE MODULE IN THE DRY COOLING SYSTEM}

For implementing a latent storage module in a hybrid dry cooling system of a STE plant, similar to the one proposed by Pistocchini and Motta, 2011, it is necessary to establish the operating conditions of the plant and this will depend on its geographical location. The chosen STE plant is a conventional one 
with a 55 MWe gross rated power block, a solar field of parabolic troughs with thermal oil as heat transfer fluid and 2-tank molten-salt thermal storage of $8 \mathrm{~h}$ capacity. A STE plant of this kind in a good summer day is expected to operate about $12 \mathrm{~h}$ with solar-only support plus $8 \mathrm{~h}$ from the storage system, which implies $20 \mathrm{~h}$ operation of the power block. This leaves a window of $4 \mathrm{~h}$ for discharging the latent storage system because, for simplicity, we assume that discharging process should take place when turbine is not under operation. Therefore, we have chosen $3 \mathrm{~h}$ for the latent storage capacity for which we can arrange charging and discharging processes whenever it is convenient.

Taking into account that RT35HC displays a good thermal behavior, we chose this PCM for a preliminary design of a hybrid dry cooling system for a STE plant that includes an air-cooled condenser (ACC) combined with a latent storage module. In Fig. 5 the scheme of such dry cooling system in a direct configuration is displayed for both charging (a) and discharging processes (b). During charging process, exhaust heat from turbine output is delivered to the latent storage and/or to the ACC by means of a three-way control valve, depending on ambient temperature and latent storage state. Due to the melting temperature of the PCM and considering a pinch point of $3{ }^{\circ} \mathrm{C}$, the temperature of the exhaust steam leaving the turbine outlet should be, at least, $38{ }^{\circ} \mathrm{C}(0.0663 \mathrm{bar}$ condensing pressure). Therefore, this would be the minimum charging temperature for the latent storage module.

a) Charging process

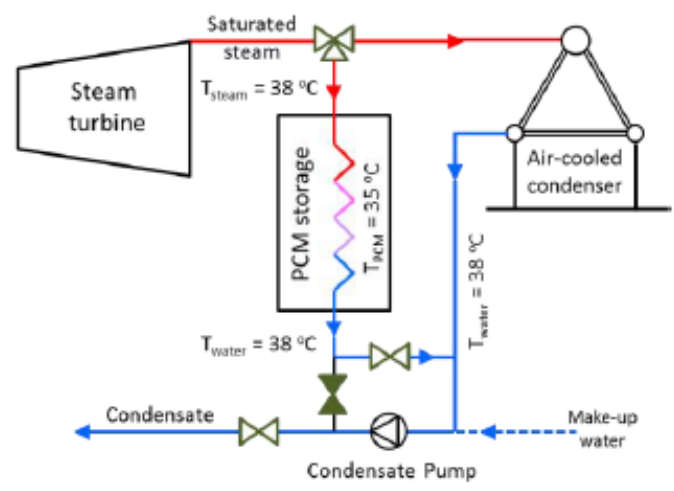

\section{b) Discharging process}

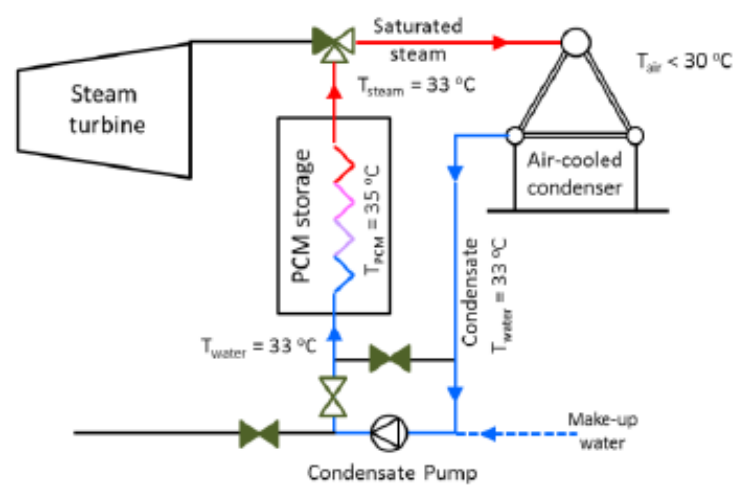

Fig. 5: Schemes of charging (a) and discharging (b) processes of a direct cooling system for a STE plant that combines a latent storage module with an air-cooled condenser.

During the night-time, when steam turbine is not working, latent storage can be discharged by producing low temperature steam which is condensed with the help of the ACC. Hence, for discharge processes $2{ }^{\circ} \mathrm{C}$ pinch point is assumed in the latent storage module and at least an additional pinch point of $3{ }^{\circ} \mathrm{C}$ in the ACC has to be considered as well. This implies that the ambient temperature required to condense the steam coming from the storage should be well below $30^{\circ} \mathrm{C}$.

\section{Daily heating/cooling cycles of RT35HC between real operating temperatures}

From the schemes of Fig. 5 it is clear that the latent storage containing RT35HC will be charged and discharged at $38{ }^{\circ} \mathrm{C}$ and $33{ }^{\circ} \mathrm{C}$ respectively. This means that this PCM should be able to melt and freeze completely within such a narrow temperature range $\left(5^{\circ} \mathrm{C}\right)$. In order to study RT35HC behavior under these conditions additionally daily heating/cooling cycles were performed in the temperature intervals $38{ }^{\circ} \mathrm{C}-33{ }^{\circ} \mathrm{C}$ and $37{ }^{\circ} \mathrm{C}-34{ }^{\circ} \mathrm{C}$. The resulting temperature-time curves for both the sample are displayed in Fig. 6. The plots show that RT35HC undergoes melting not only when oven temperature is $3{ }^{\circ} \mathrm{C}$ above Tmelt but also when it is $2{ }^{\circ} \mathrm{C}$ above, since in both cases a shoulder is observed. Obviously, the lower the temperature gradient inside the oven the slower the melting process is. Actually, if we compare with the temperature-time curves of Fig. 4 a), for which oven temperature is $50^{\circ} \mathrm{C}$, we can see that the melting process is faster for the same amount of sample $(4 \mathrm{~g})$. Note that time scales of Fig. 4 a) and Fig. 5 are different. Sample state (either liquid or solid) was checked by visual inspection with a quick open-close of the oven. We could assess that PCM was completely 
melted when the flat constant temperature segment was attained (either $38{ }^{\circ} \mathrm{C}$ or $37^{\circ} \mathrm{C}$ ) and that it was completely solid during the flat segments at $34{ }^{\circ} \mathrm{C}$ and $33{ }^{\circ} \mathrm{C}$.

These results prove that $\mathrm{RT} 35 \mathrm{HC}$ undergoes full phase change even within a very narrow temperature interval (Tmelt $\pm 2{ }^{\circ} \mathrm{C}$ ) although we have to take into account that melting/freezing time could be long and hence storage power could be lower than expected.

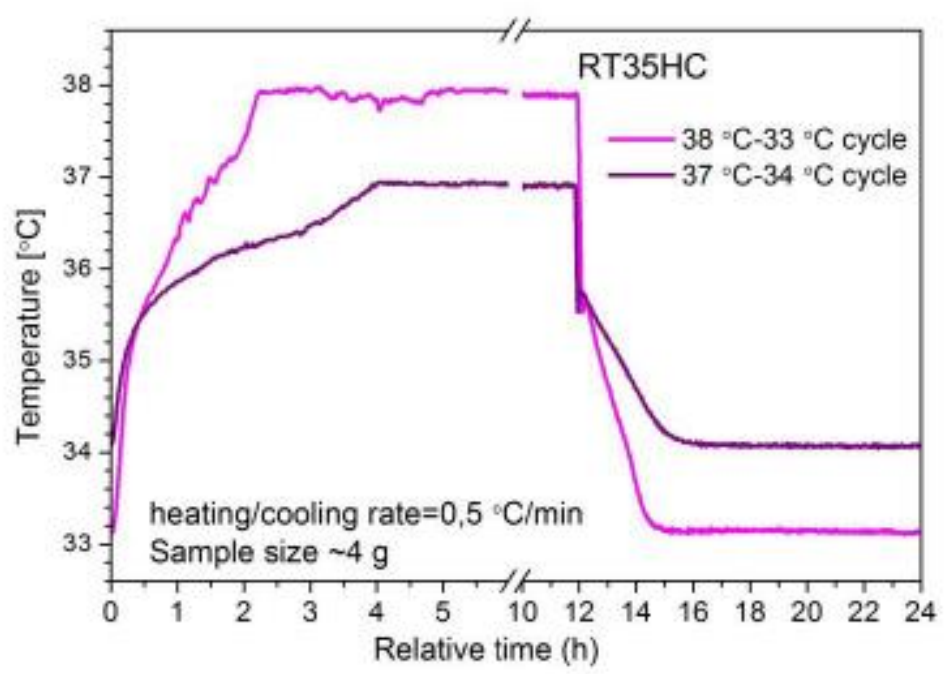

Fig. 6: Temperature-time curves of daily heating/cooling cycles performed for RT35HC in the oven under air in the temperature intervals: $38^{\circ} \mathrm{C}-33^{\circ} \mathrm{C}$ and $37^{\circ} \mathrm{C}-34^{\circ} \mathrm{C}$.

\section{Desert selection and results of annual simulation of the STE plant}

After evaluating the yearly ambient temperatures of various deserted locations, we chose Ouarzazate (Morocco) because it shows very high diurnal temperatures (above $35^{\circ} \mathrm{C}$ ) and low enough night-time temperatures (down to $17^{\circ} \mathrm{C}$ ). In Fig. 7 a) daily temperature variation in the hottest month (July) for this desert is displayed. As we can see, the most appropriate time window for discharging the latent storage is between 3 a. m. and 6 a. m. since ambient temperature is the lowest. In Fig. 7 b) the daily mean temperature of this time window for the summer months (June-September) has been plotted. In this graph we see that July and August have the highest minimum mean temperatures and for both of them a mean temperature value of about $20^{\circ} \mathrm{C}$ is obtained for the whole month.
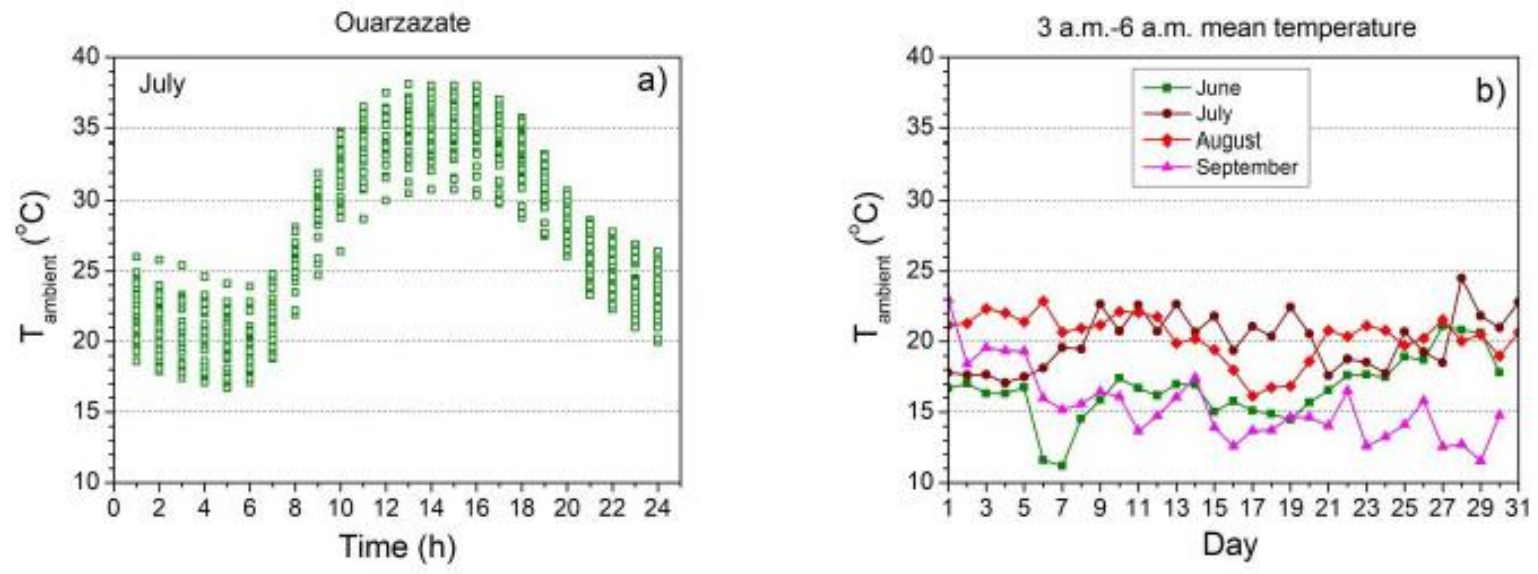

Fig. 7: Daily temperature variation in Ouarzazate (Morocco) in July (a) and mean temperature between $3 \mathrm{a}$. $\mathrm{m}$. and $6 \mathrm{a}$. $\mathrm{m}$. for the summer months in the same location (b). 
The simulation model for evaluating the STE plant annual performance was developed in TRNSYS software environment (Klein et al., 2013) and is basically composed by a solar field, a thermal storage system and a power block. Nevertheless, the implementation adopted for this study allows the possibility of including either a dry cooling system based on a conventional ACC or a hybrid system that combines ACC and latent storage so that both options can be compared. An overall description of the STE plant model can be found in Biencinto et al., 2014.

Annual simulations of the two STE plant options mentioned above were performed by using as input file a typical meteorological year (TMY) of Ouarzazate $(30.933 \mathrm{o}$ N, 6.9o W) provided by MASEN (MASEN, 2017).

For a STE plant with a conventional direct dry cooling system (only with ACC) the model yields an annual net electricity production of 180.35 GWhe, whereas for the plant with a hybrid cooling system (ACC combined with latent storage) the annual net production obtained is 180.91 GWhe. Hence by using the hybrid cooling system a net electricity gain of $560 \mathrm{MWhe}$ is obtained. This corresponds to $0.31 \%$ of the expected annual production for the proposed STE plant in this location. Actually, the electricity production can be further increased if strategies for plant integration are optimized. However, we have to take into account that apart from the increase in annual production, the costeffectiveness of the hybrid cooling system will strongly depend on both electricity prices and PCM costs.

\section{CONCLUSIONS}

In this work four commercial products (RT35HC, RT28HC, SP31 and SP26E) have been characterized and tested in order to prove their feasibility as PCMs for a latent storage module that, combined with an air-cooled condenser (ACC), should work as dry cooling system for a STE plant. Taking into account both DSC results and daily heating/cooling cycles test performed in an oven we can conclude that the RT35HC is the most reliable material from the original candidate list.

In order to evaluate the advantage of using a hybrid (latent storage+ACC) versus a conventional direct dry cooling, annual simulations of a STE plant located in Ouarzazate (Morocco) with either one or other system have been performed. For a plant in this location it has been obtained that by using a hybrid cooling system based on RT35HC, the expected annual electricity production can be $0.31 \%$ increased. However, the economic feasibility of this cooling concept will depend not only on electricity prices and PCM cost but also on the expected incomes along the useful lifetime of the plant, which should be able to overcome the total cost of both materials and equipment for the latent storage system.

On the other hand, although RT35HC fits temperature conditions of Ouarzazate desert, PCMs with higher melting temperatures might be more suitable for other deserted locations with higher night-time temperatures allowing higher gains in terms of electricity production.

\section{FUNDING}

This work has been developed thanks to the financial support of WASCOP project from H2020 Program (Grant agreement: 654479) and of ALCCONES Project (S2013/MAE-2985) from the Community of Madrid Program of R\&D activities between research groups in Technologies 2013, cofinanced by structural funds.

\section{REFERENCES}

Biencinto M., Bayón R., Rojas E., González L. (2014) Simulation and assessment of operation strategies for solar thermal power plants with a thermocline storage tank. Sol. Energy, 103, 456-472. doi:10.1016/j.solener.2014.02.037.

Klein S.A. et al. (2013) TRNSYS 17: A Transient System Simulation Program. Solar Energy Laboratory, University of Wisconsin-Madison: http://sel.me.wisc.edu/trnsys

MASEN: Moroccan Agency of Sustainable Energy (2017): http://www.masen.ma/en/

Pistocchini L., Motta M. (2011) Feasibility Study of an Innovative Dry-Cooling System With PhaseChange Material Storage for Concentrated Solar Power Multi-MW Size Power Plant. ASME. J. Sol. Energy Eng.,133(3), 031010-031010-8. doi:10.1115/1.4004268.

Rubitherm web site (2017): http://www.rubitherm.eu 


\section{DETAILED 3-D MODELS OF A LARGE-SCALE UNDERGROUND THERMAL ENERGY STORAGE WITH CONSIDERATION OF GROUNDWATER CONDITIONS}

Abdulrahman Dahash, Michele Bianchi Janetti and Fabian Ochs

Unit of Energy-Efficient Buildings, Institute of Structural Engineering and Material Science, University of Innsbruck

Technikerstraße 13, 6020 Innsbruck, Austria

Phone: +4351250763601

E-Mail: abdulrahman.dahash@uibk.ac.at

\section{SUMMARY}

Seasonal thermal energy storage (STES) systems are key components for district heating as they offer the dispatchability and flexibility for integrating renewables into those systems. Therefore, thermal behaviour of such systems is of interest. It can influence the surroundings causing a violation to the hydro geological standards (e.g. groundwater's temperature exceeding $20^{\circ} \mathrm{C}$ to $25^{\circ} \mathrm{C}$ ). In this work, an underground tank and pit thermal energy storage are numerically modelled. The model considers the storage system and the surroundings around the storage. Then, the temperature distribution in the storage and the ground is investigated. In particular, thermal stratification in the storage is examined and, finally, heat storage's interaction with the surrounding ground is illustrated.

Keywords: TTES, PTES, Groundwater, Numerical modelling, Stratification.

\section{INTRODUCTION}

Nowadays, the energy demand in the buildings sector (i.e. space heating and domestic hot water) accounts for more than one-third of the total energy demand in the European countries. Thus, the European Union has supported several research projects to improve the buildings' energy efficiency. As a principal part, district heating (DH) approach has been often used to meet the buildings' heating demand as it enhances the transition to sustainable energy utilization, thus, developments of these systems have grown rapidly in the last decade (Sartor, 2017). Additionally, EU has sponsored a series of policies, plans and actions to promote the European energy scheme. One of the crucial objectives is to enhance the exploitation of renewables in DH systems to substitute the fossil fuels and, thereby, many goals can be realized (e.g. efficient utilization of renewables, less $\mathrm{CO}_{2}$ production) (Tulus et al., 2016). Out of all renewables, the solar energy appears to be the most promising alternative energy source compared to the fossils and, therefore, central solar heating plants have received a great attention in literature (Guadalfajara et al., 2015).

Yet, it is widely evident that the heat availability from renewables and buildings' heating demand vary mostly with asynchronous pattern, which is often observed as a result to the large variation in the outdoor temperatures between summer and winter (Xu et al., 2018). Take the solar energy as an example, the mismatch is observed between the solar heat availability in summer and the high space heating demand in buildings during winter season. Thus, the major drawback of renewables is the nondispatchability as they fluctuate daily, weekly and seasonally. As a result, a significant amount of heat might be lost during the summer season, when the buildings' heating demand is commonly minimal. Accordingly, large-scale thermal energy storage (TES) represents a good opportunity for compensating the seasonal mismatch observed between energy supply and demand (Stutz et al., 2017).

\section{SEASONAL THERMAL ENERGY STORAGE IN DH SYSTEMS}

In order to bridge the gap between solar heat abundance in summer and the space heating demand in winter, a seasonal thermal energy storage (STES) is required (Sarbu, I. and Sebarchievici, C., 2017). Nevertheless, STES systems are frequently seen challenging, and this is due the large volume and space availability required for the storage (Xu et al., 2014). For instance, if a seasonal tank TES has a size more than 100,000 $\mathrm{m}_{3}$, then more efforts are needed to build a free-standing tank (Ochs, F., 16 19 September 2014) and, accordingly, those systems are mostly buried either fully or partially under the ground forming the so-called underground TES (UTES) systems (Ochs, F., 2009).

The most common types of UTES following construction criterion are: 1) Aquifer thermal energy storage (ATES) system, 2) Borehole thermal energy storage (BTES) system, 3) Tank thermal energy storage (TTES) system, 4) Pit thermal energy storage (PTES), and 5) Cavern thermal energy storage (CTES) system (Novo et al., 2010). 
In DH applications, water is commonly used as heat carrier and, subsequently, it is the storage medium for UTES systems integrated in DH systems. Its availability, low cost, chemical stability, high heat capacity and the operative temperature range make all together water as a suitable storage medium in UTES (Heier et al., 2015). Some kinds of UTES systems rarely employ the ground (e.g. rock, soil/sand) as storage media but they are not considered further in this study. Therefore, TTES and PTES systems are the focus of this study as they employ hot water as storage medium.

\section{LITERATURE REVIEW}

Large-scale TES systems are often seen as viable means for energy conservation and, therefore, research has been ongoing to address their modelling. This is also because construction of large-scale TES tends to be costly and, accordingly, the importance of modelling is strongly highlighted as an effective approach to achieve the economic and technical feasibilities.

Thermo-hydraulic modelling of large-scale TES systems is an extensive work that requires high computation efforts. However, it is important to understand how these systems firstly work. TTES and PTES systems usually operate utilizing stratification that is mostly driven by thermal buoyancy. In stratification, the hot water, which flows into the tank, eventually gathers at the top of the tank due to thermal buoyancy, whereas the cold water gathers at the bottom of the tank because of its higher density. This natural physical process generates a thermocline region that is situated between the hot and cold regions $(\mathrm{Li}, 2016)$. The importance of the thermocline region is that it works as a dynamic natural barrier preventing the hot water from mixing with the cold one. Therefore, the smaller the thermocline region, the less the mixing effect is and, accordingly, better stratification.

Therefore, thermo-hydraulic modelling of tanks has been widely investigated in literature reporting stratification and its influence on system performance, tank design, thermal losses etc. Yet, there have been poor efforts to investigate such a phenomenon in large-scale TTES and PTES systems and its relation directly and/or indirectly with geometry of large-scale storage considering the surroundings (soil, groundwater).

For instance, Panthalookaran et al. (Panthalookaran et al., 2008) presents numerical CFD models that are experimentally validated for charging/discharging against monitored data from two buried storage tanks in Germany. One is located in Hannover-Kronsberg with a total volume of 2,750 m3, whereas the other is the existing underground storage in Friedrichshafen-Wiggenhausen with a volume of ca. $12,000 \mathrm{~m}$ 3. Later, a new characterization method for performance evaluation of various boundary designs during storage mode large-scale stratified hot water tanks was developed by utilizing these two models (Panthalookaran et al., 2011).

The simulation of CFD models requires large computation efforts in order to solve the partial differential equations for large-scale tanks and, currently, this is often seen not feasible and also in the near future (Ochs et al., June 14-17, 2009). Therefore, assumptions are frequently made in geometry, material properties and boundary conditions for the simulation, which produces a notable reduction of the computation efforts forming the so-called "coarse models" (Ochs, F., 2009). Yet, this reduction has a cost that yields sometimes a defect in the depiction of thermal hydraulic behaviour and, accordingly, coarse models do not accurately account thermal losses. Yet, research has been ongoing reporting coarse models for largescale TES. For example, Ochs (Ochs, F., 16 - 19 September 2014) presented a dynamic numerical model based on finite element discretization. The model is able to represent various construction shapes (cylinder, cone, or pyramid stump) for underground hot water TES in Matlab/Simulink environment. Then, the model is further coupled to a finite difference model for the ground. Nevertheless, Ochs concluded that there are some difficulties observed during the simulations. Thus, the authors found a gap in numerical modelling of TTES and PTES with consideration of surroundings. The importance of this consideration arises from the fact that in several countries in Europe (e.g. Austria) there are several hydro geological standards. These standards state on preventing the groundwater's temperature from increasing above $20^{\circ} \mathrm{C}$ to $25^{\circ} \mathrm{C}$. This increase in temperature is usually seen due to the long storage period and, thus, higher amount of lost heat that increases the temperature. Therefore, numerical modelling approach is important to investigate the thermal behaviour and to quantify the heat lost to the ground.

This paper presents a detailed axial symmetrical model for a circular cross-sectional systems (i.e. conical pits and tanks) with its surrounding environment, which is able to predict the surroundings 
temperature with low computation efforts. In addition, the paper depicts the temperature profiles in the storage and the ground.

\section{NUMERICAL MODELLING}

Modelling of thermal hydraulic behavior hot water tanks is a challenge. The few available models are appropriate for the rough sizing of the tank system, whereas the aforementioned models require large computation efforts beside some additional works to include the surroundings in the modelling. Therefore, a new numerical axial symmetric model is developed using COMSOL Multiphysics in which the model is discretized in a finite element fashion as shown in Figure 1. It is worthy to mention that the overall model consists of compiling two component-level models. One component-level model is the storage model, which is developed as 1-D model, whereas the other one is an axial symmetrical 2-D model that is used to represent the surroundings.

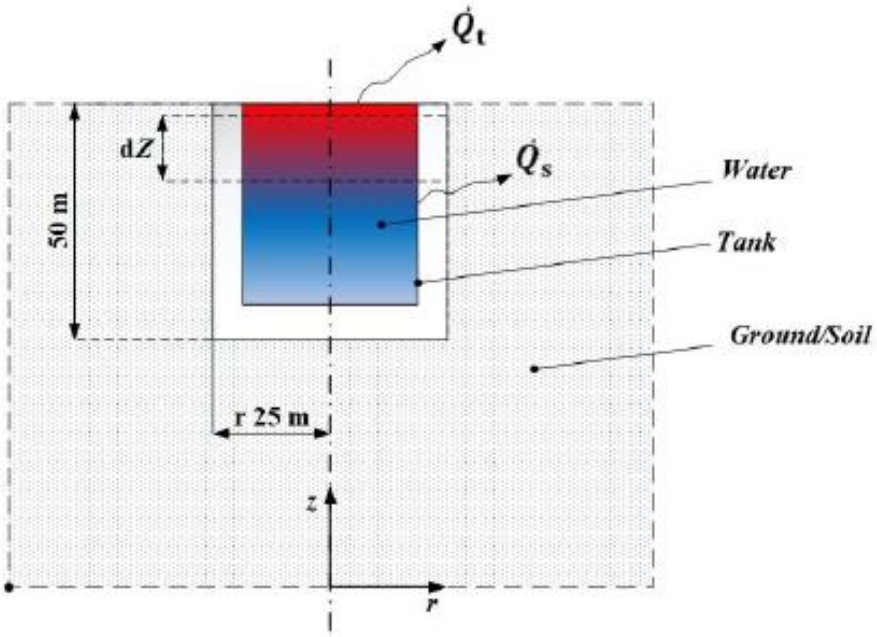

(a): Underground tank with surroundings

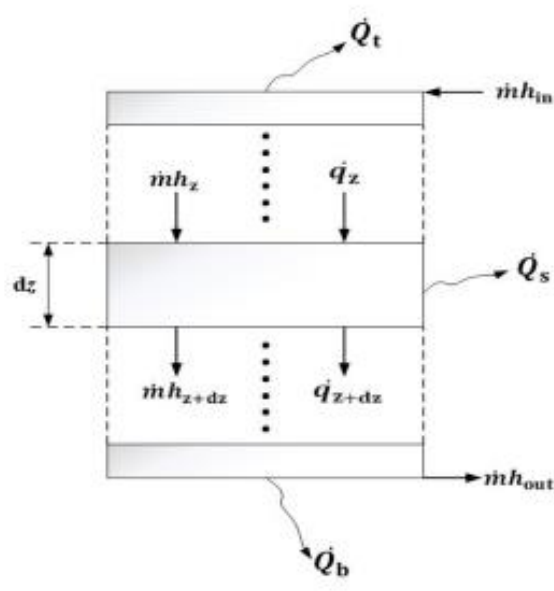

(b): Finite differential element of the tank

Figure 1: Schematic overview of an underground tank with its surroundings

The model is suitable only for axial symmetric geometries (e.g. truncated cones or cylinders) for the time being. However, there are ongoing efforts to develop the model into a parameterized model that simulates different geometries (e.g. pyramid stump). The impact of the soil and the groundwater on the thermal losses from the tank and the stratification can be investigated and, accordingly, the thermal behavior and the water temperatures can be depicted. Therefore, the model can perform simulationbased optimizations to determine the optimum distribution of insulation around the storage to minimize the thermal losses. Moreover, the model depicts the temperature of the ground, which helps in return in determining whether regulations with respect to the ground (temperature below $20^{\circ} \mathrm{C}$ to $25^{\circ} \mathrm{C}$ ) are violated. Accordingly, many configurations can be proposed to insulate the tank and its impact on the groundwater resulting in keeping the temperature below $20^{\circ} \mathrm{C}$ to $25^{\circ} \mathrm{C}$.

In the 1-D tank model, it is imposed that the mass of the water flowing into/from the tank is conserved and, thus, the steady-state continuity equation for the water is given as follows:

$m$ in $=m$ iout $=m$

Whereas the energy stored in a one of the central volume elements can be described by the following equation: 


$$
\begin{aligned}
& \frac{\partial E(t)}{\partial t}=\dot{m} \cdot\left(h_{\mathrm{z}}-h_{\mathrm{z}+\mathrm{dz}}\right)+\left(\dot{q}_{\mathrm{z}}-\dot{q}_{\mathrm{z}+\mathrm{dz}}\right)-U_{\text {wall }} \cdot A_{\text {side }}\left(T(t)-T_{\text {ground }}(t)\right) \\
& \left(\rho A c_{p}\right) \frac{\partial T(t)}{\partial t}=-\left(\rho A c_{p} v\right) \frac{\partial T(t)}{\partial z}+A \frac{\partial}{\partial z}\left(\lambda_{\mathrm{w}} \frac{\partial T(t)}{\partial z}\right)-U \cdot(\pi d) \cdot\left(T(t)-T_{\text {ground }}(t)\right)
\end{aligned}
$$

In equation (3), $v$ denotes the mean velocity of the fluid, while $\rho$ and $c p$ represent the density and specific heat capacity of the fluid, respectively. $U$ wall stands for the overall heat transfer coefficient of the storage envelope (fluid to ground), Aside is the mantle area of the segment, whereas $A$ is the cross section area of the segment. It is important to mention that other heat loss terms $\left(Q \dot{\mathrm{t}}\right.$ and $\left.Q_{\mathrm{b}}{ }^{*}\right)$ are accounted for. Therefore, Atop and Abot are used to include the top and bottom surface areas of the first and last segments, respectively, in calculations. Also, it is assumed that the tank volume is divided into a finite number of segments. Moreover, the heat transfer equation in the 2-D ground model can be described as follows:

$$
\begin{aligned}
& \left(\rho_{\mathbf{g}} c_{p g}\right) \frac{\partial T_{\text {ground }}(t)}{\partial t}=\frac{1}{r} \frac{\partial \dot{q}_{\mathrm{r}}}{\partial r}+\frac{\partial \dot{q}_{\mathrm{z}}}{\partial z} \\
& \left(\rho_{\mathbf{g}} c_{p g}\right) \frac{\partial T_{\text {ground }}(t)}{\partial t}=\frac{1}{r} \frac{\partial}{\partial r}\left(r \cdot \lambda_{\mathrm{g}} \frac{\partial T_{\text {ground }}(t)}{\partial r}\right)+\frac{\partial}{\partial z}\left(\lambda_{\mathbf{g}} \frac{\partial T_{\text {ground }}(t)}{\partial z}\right)
\end{aligned}
$$

Table 1 shows a list of parameters used for the simulations and Figure 2 displays the values used for the charging and discharging variables (velocity, temperature) as well as ambient temperature.

\begin{tabular}{|c|c|}
\hline \multicolumn{2}{|c|}{$\underline{\text { Value }}$} \\
\hline Cylindrical tank & Conical tank \\
\hline $50 \mathrm{~m}$ & $50 \mathrm{~m}$ \\
\hline $50.5 \mathrm{~m}$ & $20 \mathrm{~m}$ \\
\hline $50.5 \mathrm{~m}$ & $75.7 \mathrm{~m}$ \\
\hline 90 & 60.9 \\
\hline \multicolumn{2}{|c|}{$100,000 \mathrm{~m}^{3}$} \\
\hline \multicolumn{2}{|c|}{$0.6 \mathrm{~W} /(\mathrm{m} . \mathrm{K})$} \\
\hline \multicolumn{2}{|c|}{$0.15 \mathrm{~W} /\left(\mathrm{m}^{2} \cdot \mathrm{K}\right)$} \\
\hline \multicolumn{2}{|c|}{$0.3 \mathrm{~W} /\left(\mathrm{m}^{2} . \mathrm{K}\right)$} \\
\hline \multicolumn{2}{|c|}{$0.3 \mathrm{~W} /\left(\mathrm{m}^{2} . \mathrm{K}\right)$} \\
\hline \multicolumn{2}{|c|}{$1.5 \mathrm{~W} /(\mathrm{m} . \mathrm{K})$} \\
\hline \multicolumn{2}{|c|}{$880 \mathrm{~J} /(\mathrm{kg} . \mathrm{K})$} \\
\hline \multicolumn{2}{|c|}{$1000 \mathrm{~kg} / \mathrm{m}^{3}$} \\
\hline
\end{tabular}

Table 1: Model parameters and its corresponding values and description

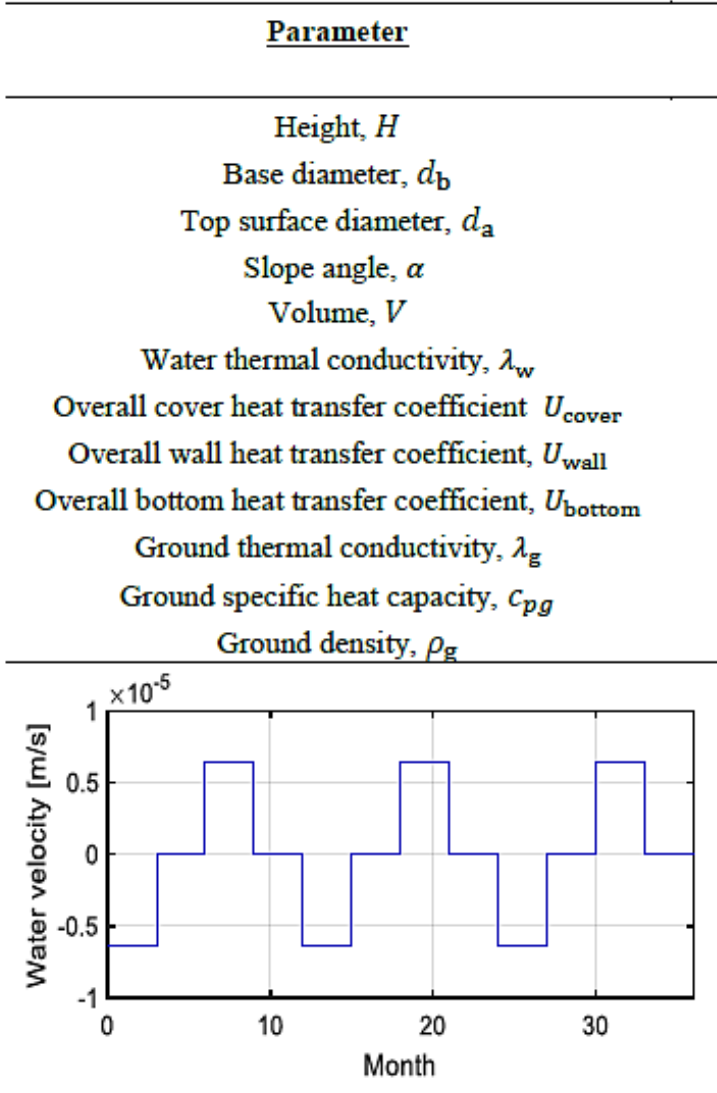

(a): Water inlet velocity

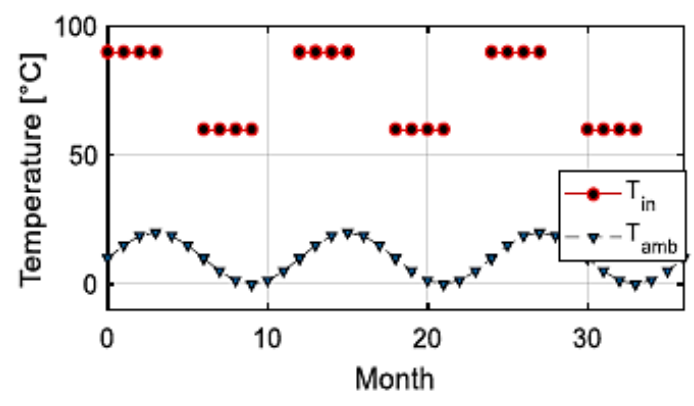

(b): Ambient and water inlet temperatures

Figure 2: Exemplary model input parameters over the simulation period ( 36 months) 


\section{RESULTS}

In order to avoid complex simulations, simplified charging/discharging scenarios were chosen (see Figure 2) and this allows also to evaluate the stratification in the storage. During charging, the inlet temperature is always set to $90^{\circ} \mathrm{C}$, whereas set to $60^{\circ} \mathrm{C}$ during discharging. Here only the results for long-term storage will be presented. Also, the investigation period is set to 36 months (3 years) in which one cycle is performed within a year.

Figure 3 reveals the amount of energy stored in the storage (tank or pit) within the investigation period (36 months). At the beginning of simulations, the storage is assumed to contain initial energy, which means water is stored at $60^{\circ} \mathrm{C}$. Then, the energy content starts to increase with time as the charging phase takes place until the maximum energy content is reached after 3 months. Next, the energy is stored for 3 months (half a year). It is important to mention that some heat is obviously lost during the storing phase as the stored energy decreases until the point at which the discharging phase starts. This is also confirmed by temperature profiles for water in the tank and the pit (see Figure 4 (a-f)).

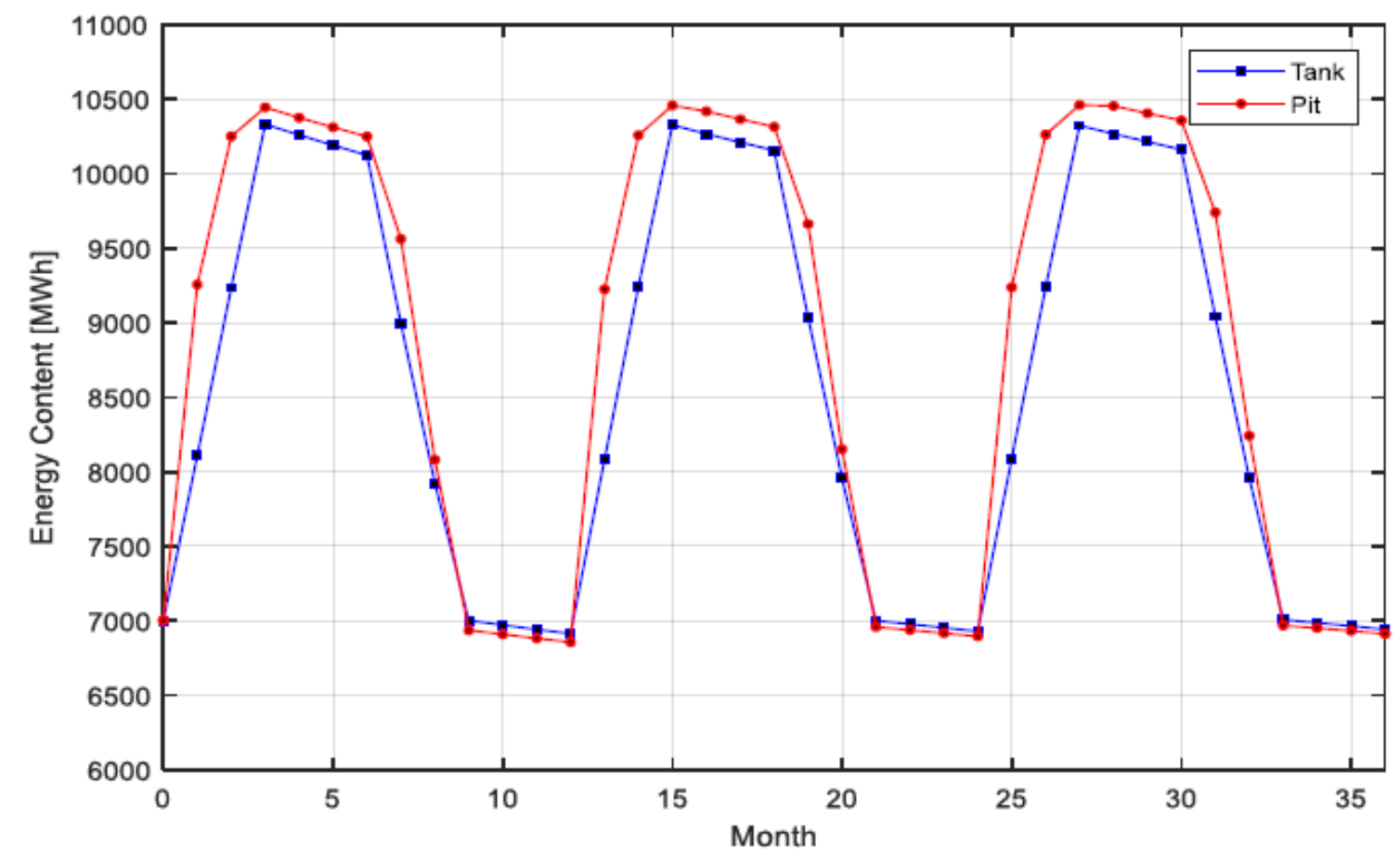

Figure 3: Energy stored in the underground storage over 36months

In Figure 4 (a-c), it is clearly proven that charging temperature reaches $90^{\circ} \mathrm{C}$ and, then, the storing phase takes place as the tank is fully charged. Whilst the discharging phase starts at a temperature below $90^{\circ} \mathrm{C}$. This demonstrates that thermal losses are accounted in the model. Whereas Figure 4 (d-e) emphasizes that the temperature in the pit tends to be higher than that in the tank and, therefore, better stratification profile during storage phase can be obtained as shown in Figure 4 (b, e). Yet, the pit discharges water with a temperature during almost similar to that of the tank (see Figure $4 \mathrm{c}, \mathrm{f}$ ). Under the considered conditions in simulation, Figure 4 (c, f) reveals that the pit tends to discharge faster than the tank as proven by the black line. Moreover, Figure $4(\mathrm{e}, \mathrm{f})$ depict an oscillation for the temperature distribution profile in depth from 0 to $5 \mathrm{~m}$ and the reason for this behavior is not clear at present and has to be investigated in future works. 


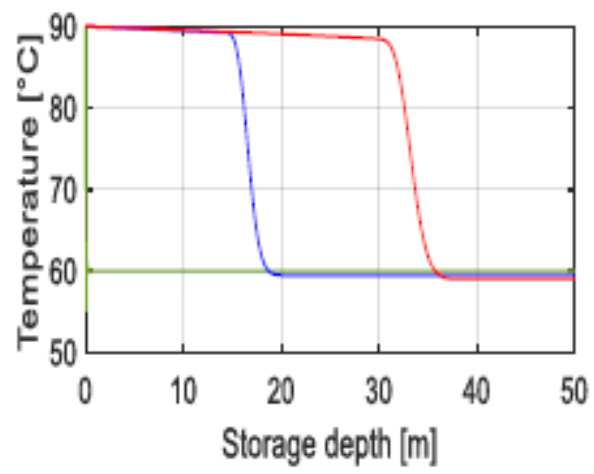

(a): Charging mode in tank

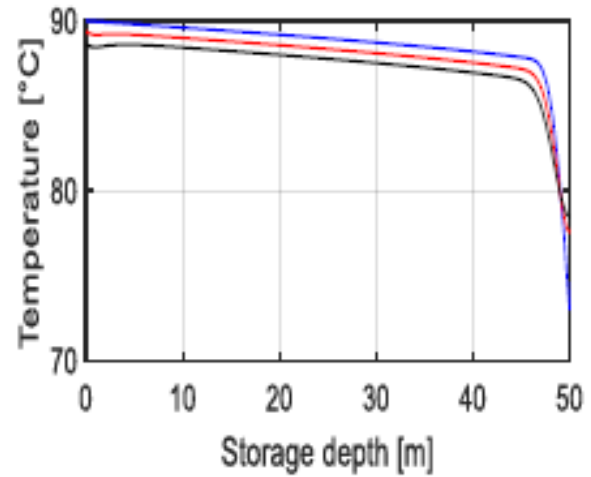

(b): Storage phase in tank

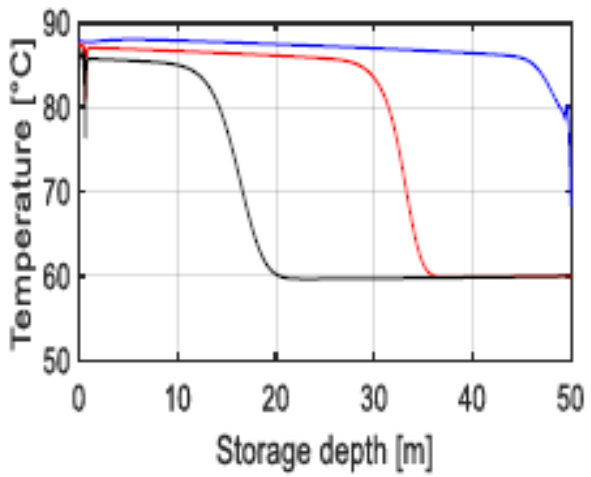

(c): Discharging phase in tank

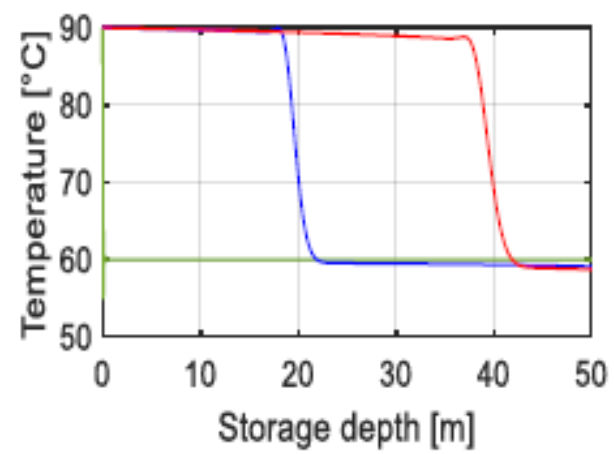

(d): Charging mode in pit

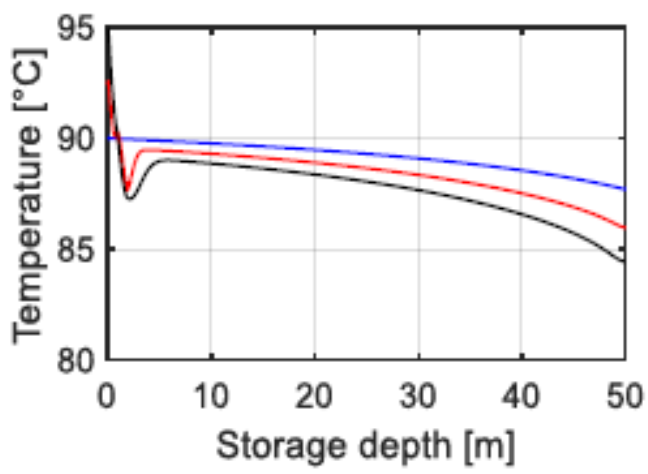

(e): Storage phase in pit

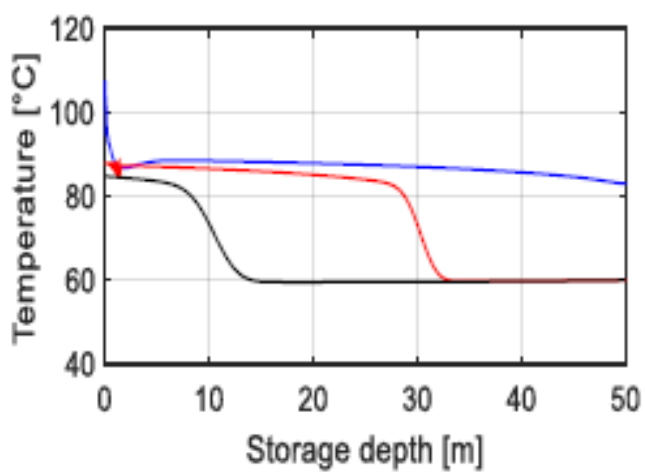

(f): Discharging phase in pit

Figure 4: Temperature profiles of water in the tank and the pit during the three operating phases of a tank storage (charging, storing and discharging)

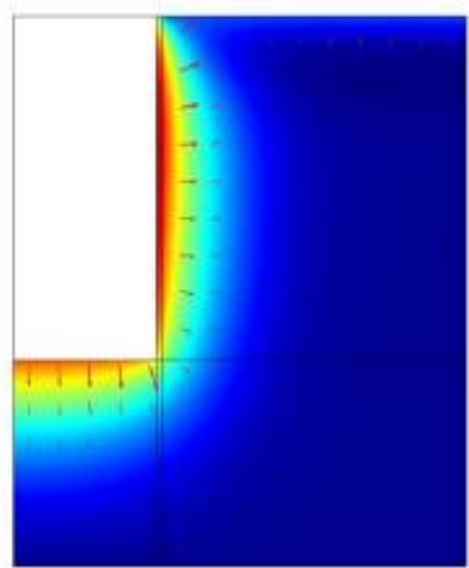

(a): Tank

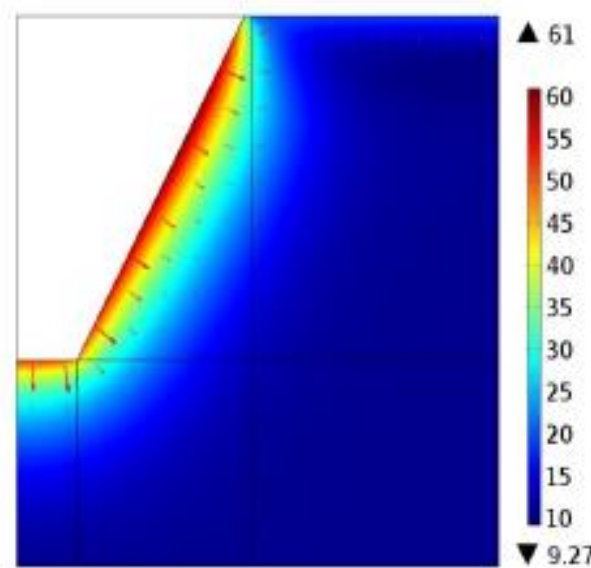

(a): Pit

Figure 5: Contour plots for the surroundings of the storage during the storage phase at the $3^{\text {nd }}$ year 


\section{CONCLUSION}

Large-scale TES systems are increasingly in demand for solar-assisted DH applications. Therefore, research has been ongoing to address those systems and their benefits for the overall energy scheme. Thus, an axial symmetrical 1-D tank model and an axial symmetrical 2-D ground model were developed and coupled, then, the models were tested with exemplary charging/discharging profiles (flowrates and temperatures) to examine the stratification in the tank and the pit, respectively. The models are able to examine underground axial symmetric structures (e.g. TES systems with truncated conical or circular geometries) and, therefore, it provides a thermal analysis for such systems, which makes it possible to perform optimization with regard to thermal losses. The results depict that stratification takes place inside the tank and the pit storage over time and this implies that the thermohydraulic behavior of the storage medium is correctly implemented (see Figure 4 a-f). Also, the results reveal that the ground is highly influenced during the storage phase in which the surroundings temperature exceeds $50^{\circ} \mathrm{C}$ (see Figure 5). Therefore, it can be said that an amount of energy is stored in the ground and it is difficult to retain it back. Hence, better insulation system is required to prevent this loss of energy as well as to protect the ground from violating the hydro- geological standards. Also, the model experiences low computation efforts as it simulates an underground storage system over 36 months within a duration of 22-25 minutes for the tank, whereas it costs 26-29 minutes for the pit due to more edges and complex boundaries. Yet, the results impose imposes that the models are reliable.

Future works will primarily focus on validation process to test the reliability of the model. Also, parametrizing the model through LiveLink feature that couples COMSOL Multiphysics with Matlab is one of the milestones in the near future works. This could help in realizing different geometries (e.g. truncated pyramid) not only the cylindrical ones. Moreover, future developments will examine the influence of different aspect ratios $(\mathrm{H} / \mathrm{d})$ on thermal losses from the storage and how the losses can be effectively minimized, particularly in presence of groundwater.

\section{ACKNOWLEDGMENT}

This work is part of the Austrian research project "Giga-Scale Thermal Energy Storage for Renewable Districts" (giga_TES, Project Nr.: 860949) and funded by Austrian Research Promotion Agency (FFG).

\section{REFERENCES}

Guadalfajara et al. (2015). Simple calculation tool for central solar heating plants with seasonal storage. Solar Energy, 120, 72-86. doi:doi.org/10.1016/j.solener.2015.06.011

Heier et al. (2015). Combining thermal energy storage with buildings - a review. Renewable and Sustainable Energy Reviews, 42, 1305-1325. doi:10.1016/j.rser.2014.11.031.

Li, G. (2016). Sensible heat thermal storage energy and exergy performance evaluations. Renewable and Sustainable Energy Reviews, 53, 897-923. doi:10.1016/j.rser.2015.09.006.

Novo et al. (2010). Review of seasonal heat storage in large basins: Water tanks and gravel-water pits. Applied Energy, 87(2), 390-397. doi:doi.org/10.1016/j.apenergy.2009.06.033 185

Ochs et al. (June 14-17, 2009). Modeling Large-Scale Seasonal Thermal Energy Stores. Effstock 2009, Thermal Energy Storage for Efficiency and Sustainability: 11th International Conference on Thermal Energy Storage. Stockholm, Sweden. Retrieved April 23, 2018, from https://talon.stockton.edu/eyos/energy_studies/content/docs/effstock09/Session_8_2\%20Models_and_ Design\%20tools/67.pdf

Ochs, F. (16 - 19 September 2014). Large-Scale Thermal Energy Stores in District Heating Systems Simulation Based Optimization. In D. E. Papillon (Ed.), EuroSun 2014: International Conference on Solar Energy and Buildings. Aix-les-Bains, France: International Solar Energy Society (ISES). doi:http://proceedings.ises.org/paper/eurosun2014/eurosun2014-0080-Ochs.pdf

Ochs, F. (2009). Modelling Large-Scale Thermal Energy Stores. Stuttgart: Shaker Verlag.

Panthalookaran et al. (2008). Calibrated models for simulation of stratified hot water heat stores. International Journal of Energy Research, 32(7). doi:10.1002/er.1423

Panthalookaran et al. (2011). The Effects of Boundary Design on the Efficiency of Large-Scale Hot Water Heat Stores. Journal of Solar Energy Engineering, 133(4). doi:10.1115/1.4004472 
Sarbu, I. and Sebarchievici, C. (2017). Solar Heating and Cooling Systems: Fundamentals, Experiments and Applications. Oxford, UK: ELSEVIER. doi:doi.org/10.1016/B978-0-12-8116623.01001-X

Sartor, K. (2017). Simulation Models to Size and Retrofit District Heating Systems. Energies, 10(12). doi:doi:10.3390/en10122027

Stutz et al. (2017). Storage of thermal solar energy. Comptes Rendus Physique, 18(7-8), 401-414. doi:doi.org/10.1016/j.crhy.2017.09.008

Tulus et al. (2016). Enhanced thermal energy supply via central solar heating plants with seasonal storage: A multi-objective optimization approach. Applied Energy, 181, 549-561. doi:doi.org/10.1016/j.apenergy.2016.08.037

$\mathrm{Xu}$ et al. (2014). A review of available technologies for seasonal thermal energy storage. Solar Energy, 103, 610-638. doi:doi.org/10.1016/j.solener.2013.06.006

$\mathrm{Xu}$ et al. (2018). Application of large underground seasonal thermal energy storage in district heating system: A model-based energy performance assessment of a pilot system in Chifeng, China. Applied Thermal Engineering, 137, 319-328. doi:doi.org/10.1016/j.applthermaleng.2018.03.047 


\title{
AN OPEN SORPTION HEAT STORAGE APPLICATION
}

\author{
Dipl Ing. Dr. Bernhard Zettl, Dr. Harald Kirchsteiger \\ University of Applied Sciences Upper Austria \\ Dpt. Research and Development \\ Ringstr. 43A, 4600 Wels, Austria \\ Phone: +43 50804 / 46918 \\ E-Mail: bernhard.zettl@fh-wels.at \\ Dr.-Ing Henner Kerskes, Dipl.-Ing. Sebastian Asenbeck \\ University Stuttgart, Institute of Thermodynamics and Thermal Engineering \\ Pfaffenwaldring 10, 70550 Stuttgart, Germany
}

\section{SUMMARY}

The actual state of a sorption process development using zeolite for a long-term heat storage application is showing advantages and drawbacks of the open sorption technology. While the prototype can be constructed with standard components from bulk material process technology material handling needs special solution to avoid abrasion, dust extraction and heat losses of desorption furnace and adsorption reactor. Modelling of the adsorption reactor is based on a modified fixed bed model together with the assumption of complete homogenized material bed due to rotation movement.

\section{INTRODUCTION}

The development of thermo-chemical heat storage is of key importance for high solar fraction in the application of solar thermal and waste-heat-recovery systems. The technology exhibit the potential for high amounts of savings of greenhouse gases due to high energy-storage densities. Several areas for application of this heat storage technology are possible including domestic heating, industrial production routes, vehicles, etc., all of them based on the need for long-period heat conservation (> weeks) or other possible advantages (air dehumidification, for example).

Several varieties of sorption systems are under development: open and closed systems, fixed bed and agitated bed, fluid or granular sorbents, each showing specific opportunities and drawbacks. Open sorption systems can be realized with relative little effort of construction materials (stainless steel) compared to other systems like closed sorption (working in vacuum), but desorption (dehumidification) of the storage material requires much higher temperatures.

The main goal of the actual project is to build a laboratory prototype (thermal power downsized by factor three compared to real application) with automatic control for mass and heat transfer for both, adsorption and desorption part. Molecular sieves (zeolites) are used actually and composite material (salt impregnated structures) are planned for demonstration as storage material.

The goal of modelling and numerical simulation is to develop a sufficiently simplified strategy for controlling the storage procedure on several levels: 1. operation control of ad-, and de-sorption under certain conditions, 2 . control of daily cycles dominated by solar radiation and user profiles, 3 . strategic use of stored energy (seasonal forecast and year-around-control).

\section{EXPERIMENTAL SETUP}

The concept of the prototype is based on findings of several earlier projects (see also Zettl et. al (2014) and (2015), Zettl and Lachner (2015)). The rotating heat drum was found to be an appropriate reactor to reach sufficient temperatures for domestic applications with the utilization of zeolite or salt containing granular flow heat storage material. Both, zeolite 4A (Linde-type) and composite material 
(salt impregnated natural zeolite Clinoptilolite) have been used for earlier evaluation. Until now, only single functions with manual material transfer has been realized like adsorption as batch process with manual material handling or desorption with hot air, infrared, microwave in smaller laboratory setups. The present prototype should highlight the feasibility of a fully controlled automatic workflow that means the automated material transfer, the controllability of thermal power and temperature, and high reliability in the presence of corrosive materials and dust (abrasion of the storage material). Nevertheless, the prototype was not optimized in the sense of space-saving or cost-savings. Both of these are subject to further development.
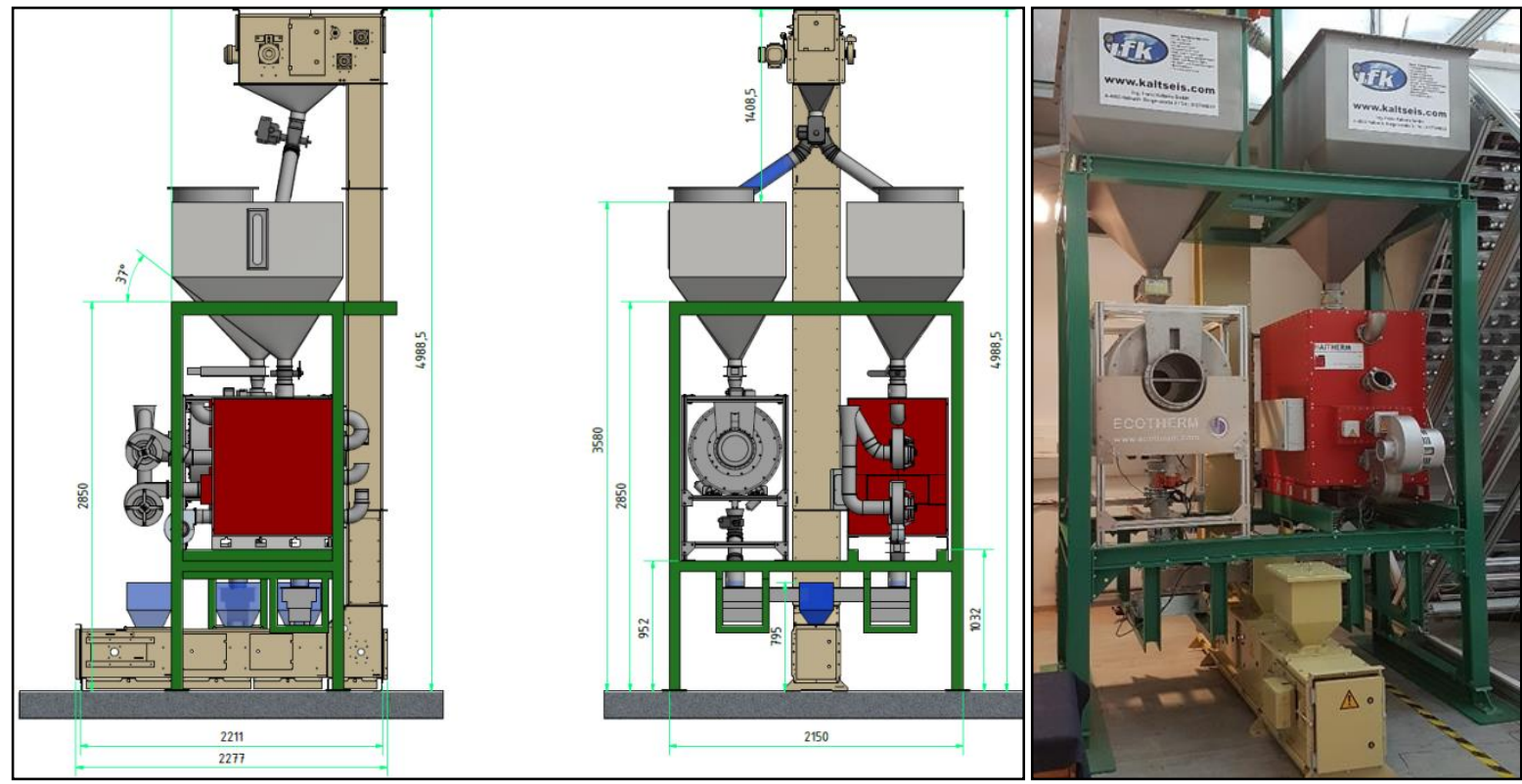

Figure 35: Concept of the prototype with dimensions (left), as built in the laboratory (right)

The prototype concept was developed together with company partners specialized on specific industrial applications like bulk material treatment, industrial furnace construction, and thermal engineering with functional components commonly used in process technology.

The main components of the Prototype are shown in Figure 36. The prototype construction consist of rigid steel frame (green), two containers for dry and humid material (on the top), a desorption reactor (furnace, in red), a cylindrical adsorption reactor (rotating heat drum, left of furnace), and a conveyor used to lift the material from bottom to top (brown). In addition, we realized a solar thermal installation at the top of the University building (Figure 36). The aim of that hot air solar installation was to get a data source for measuring the maximal reachable summer temperatures and thermal power (for desorption), the temperature gains during winter used to humidify process air (for adsorption), and to demonstrate the achievable cost per unit of the (relative simple) hot air-collector technology. 

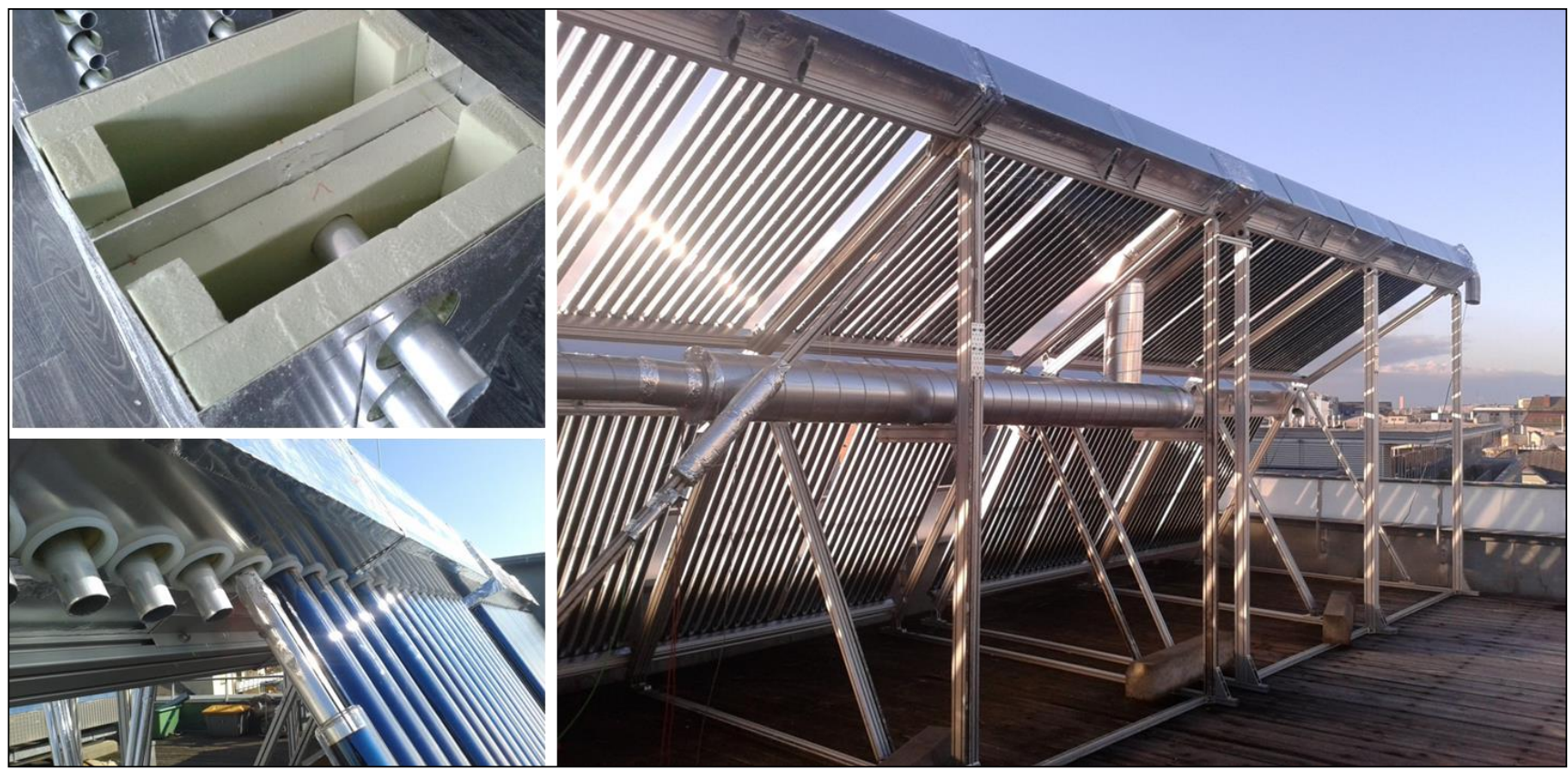

Figure 36: Solar thermal hot air installation on top of the university-building (right), with details of the tube in tube air channel (lower left) and manifold construction (upper left).

Details of the rotating adsorption drum (without storage material) are shown in Figure 37. The function principle works as follows: an external ventilator blows process air into the right opening of the central (inner) cylinder. The inner cylinder is fixed, while the outer (together with the material) is rotating. The process air is entering the material bed via the openings at the bottom of the inner cylinder (supported by mesh-material), and flowing through the moving grain bed to the top. Then the air is leaving the material bed at the upper part of the volume where an air channel is formed since only about $80 \%$ (approx. 150 liter) of the inner volume is filled. The upper air channel is connected to the left opening of the cylinder by a (chimney like) tube, enabling the air to leave the reactor.

The reactor is filled and emptied by an installed valve (electrical connection via brush contacts) and slowly driven (1 turn per minute) by a AC motor.

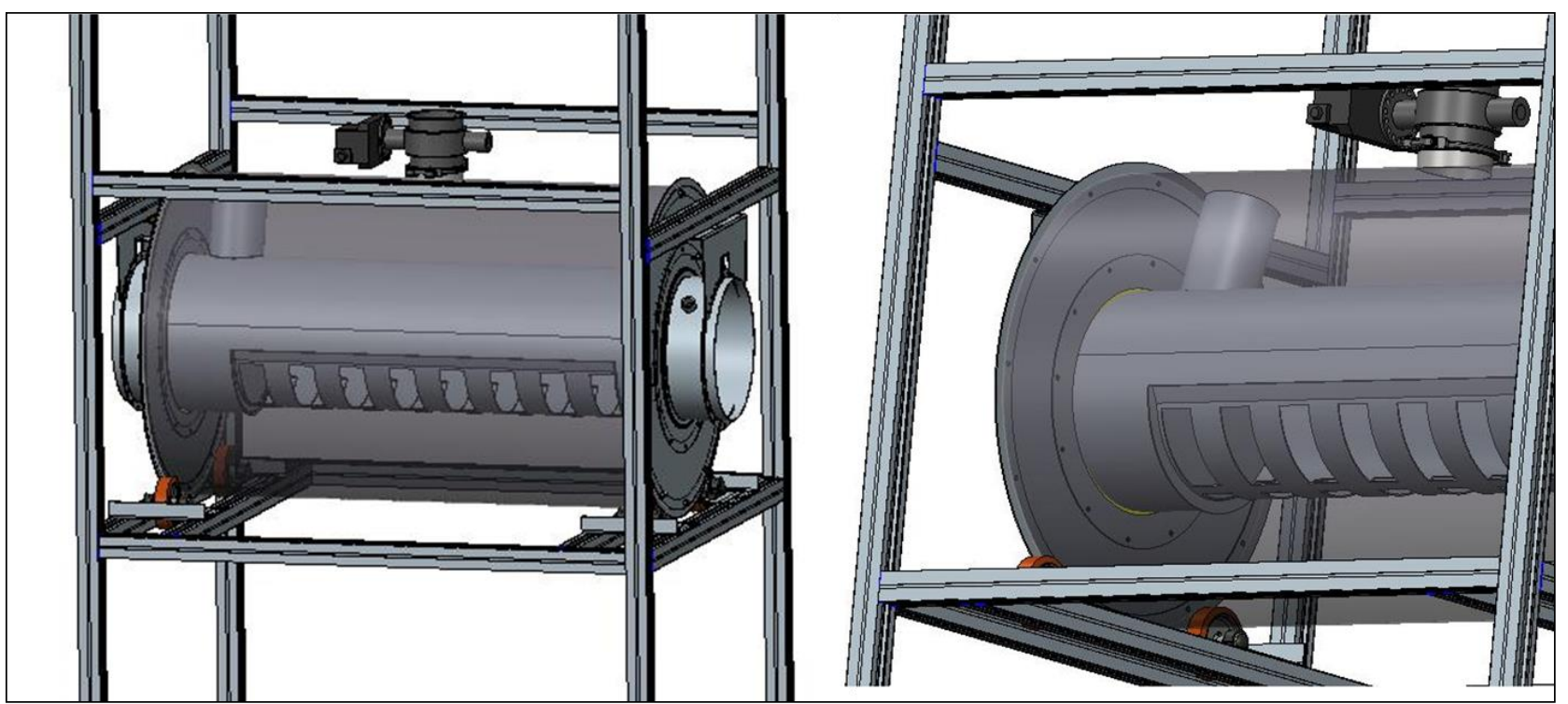

Figure 37: Construction details of the rotating cylindrical adsorption reactor 
While passing the grain bed the humidity of the incoming air is transferred to the storage material. This process of adsorption generates adsorption heat, released by the water molecules bound at the inner surface of the pores of the zeolite (or inside salt crystals) and their transition from gaseous to liquid. The process temperature mainly depends on the air water content and temperature of the incoming air, while the air mass flow determines thermal power. For the aim of warm water generation an internal heat exchanger realised as double wall construction of the inner cylinder can be used, while building heating power would be extracted by an air/air heat exchanger.

The reactor is designed to release a certain thermal power (determined by air mass flow) an energy determined by the amount of material inside the drum. Once the material is discharged (typically after $6 \ldots 8 \mathrm{~h})$ it has to be replaced by new one; the discharged material is transferred to the respective container by the conveyor and new material is fill into the drum from beyond.

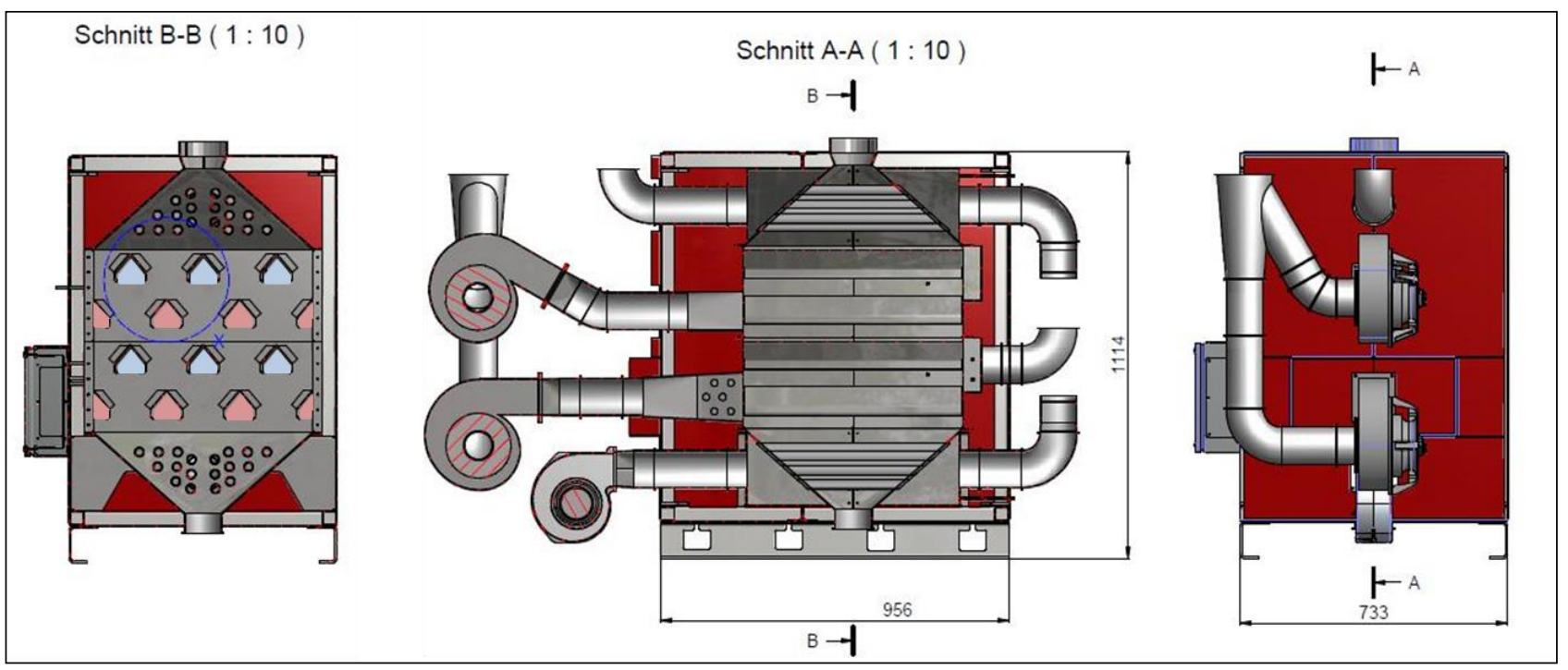

Figure 38: Construction details of the desorption furnace from different points of view

Details of the function of the desorption furnace are shown in Figure 38. The middle schematic illustrates the passage of the four different air flows through the furnace: the most upper one is connected to the lowest one via a hose. The lowest is used to cool down the hot material at the bottom before it leaves the furnace. The air is guided to the top were it preheats the incoming material (both with tube registers- see Figure 38 left schematic). Both of the middle air channels guide the air into the material bed where heating and dehumidification takes place. The air channels (indicated as light red and blue in Figure 38 left schematic) are connected with the hot front end (red) or with the cool back end (blue). The air is forced from the hot end into the red channels, then into the grain bed, through the grain bed towards the blue channels, and then out again. This principle is known from much bigger drying application in agricultural production (for drying crop or corn, for example).

The thermal power for desorption should be supplied from the solar thermal hot air installation and eventually supported by an electrical heater (integrated in the lower heater channel). The furnace and blowers are designed for temperatures of $200^{\circ} \mathrm{C}$, thermal power is about $6 \mathrm{~kW}$ that should be sufficient to dehydrate approx. $20 \mathrm{~kg} / \mathrm{h}$ of storage material. A shutter at the bottom opens every few seconds and controls the material mass flow. 
Every material movement especially the drum rotation induce wear of the zeolite grains that results in dust. This fine durst is carried out by the air mass flow and, even it is harmless to the ambient, it may produce problems in the hoses, heat exchangers or near the air outlet. An efficient way to remove the fine dust, is a simple box -trap, an opening of the air channel to a wider diameter to decelerate air velocity, so the dust can sink to the bottom. The experimental thermo-physical characterization of the storage cycle takes place with a set of sensors to measure temperatures, humidity and air velocities. The material behaviour of the used model material in the storage prototype will be used as a measure to compare results of numerical modelling and simulation of the process based on standard material characterization technique by calorimetry (DSC). In the following section, we describe the methodology for modelling a rotating sorption storage and present simulation results.

\section{MATHEMATICAL MODELING}

\section{Purpose and setup}

The purpose of the developed model is its use in a process control algorithm. Based on the underlying model, informed decisions can be made on the best possible operating condition of the storage. The model tracks the loading $x_{S}(t)$ of the sorption material with water inside the drum and can also forecast this quantity given the future input signals over time. As inputs to the model, the characteristic values of the gas (air) stream, i.e. mass flowrate $m_{G}(t)$, absolute humidity $x_{G}(t)$, and the temperature $T_{G}(t)$ have to be used. Based on the mass of the adsorbed water, the released energy can be derived when assuming a constant adsorption enthalpy parameter, which depends on the specific material used. In this way, the released and the potentially still useable energy of the material is known and can be used in an advanced process control algorithm.

There are some results on fixed bed sorption storage in the literature (see for example Mette et. al (2014), Engel et. al (2017), Krese et. al (2018)), however, a revolving drum reactor was not treated yet. The fundamental difference in the process is that in elongated fixed beds, a reaction front is moving from one end of the reactor towards the other causing a significant variation of the loading of the sorption material with water. Therefore, in the modelling strategy a temporal as well as a spatial dimension needs to be considered. In the revolving drum, a portion of the material is exposed to the inlet gas stream, but then immediately mixed with the rest of the material as the drum continues to rotate. Consequently, the fundamental modelling assumption here is to consider identical loading for all individual particles inside the drum. This may not be true at a particular instant in time, but since the purpose of the model is to evaluate total energies and average temperatures, it is a sufficient approximation.

\section{Model equations}

The loading $x_{S}(t)$ in $\mathrm{kg}$ water per kg material is governed by the equation

$\frac{d x_{S}(t)}{d t}=\frac{m_{G}(t)}{M_{S}}\left(x_{G}^{\text {in }}(t)-x_{G}^{\text {out }}(t)\right)$

where $M_{S}$ is the mass of the sorption material in the storage in dry conditions and $x_{G}$ represents the absolute humidity in $\mathrm{kg}$ water per kg dry air mass. The saturation of the sorptive material is given by adsorption isotherms which can be found in the literature for common material types (for example in Kim et. al (2016)) or obtained from manufacturers. The equilibrium state is a function of the provided humidity and the temperature of the material in the storage:

$x_{S, e q}=f\left(x_{G}^{i n}(t), T_{\text {store }}(t)\right)$ 
The equation for the air humidity is

$\frac{d x_{G}(t)}{d l}=\frac{\rho A}{m_{G}(t)} k\left(x_{S}(t)-x_{S, e q}(t)\right)$

Where $\rho$ is the density of the sorption material, $A$ is the cross section at which the material is exposed to the inlet air stream and $k$ is a parameter describing the speed of the reaction. The equation relies on the assumption of a force proportional to $x_{S}(t)-x_{S, e q}(t)$ which drives $x_{S}(t)$ towards $x_{S, e q}(t)$. This type of models are known as linear driving force models (Do 1998). The length $l$ is a virtual quantity which would be the total length in case of a fixed bed reactor and is here such that the total volume $l \mathrm{~A}$ can hold the sorption material $M_{S} / \rho$.

The temperature of the storage is then

$\frac{d T_{s t o r e}(t)}{d t}=\frac{1}{M_{S} c_{p 0}+M_{S} x_{S}(t) c_{p 1}}\left(q_{a d s}(t)+q_{G}(t)+q_{a m b}(t)\right)$

where $c_{p 0}$ and $c_{p 1}$ represent the specific heat capacities of the dry adsorbent and the adsorbed water, respectively. The heat fluxes are given by

$$
\begin{aligned}
& q_{a d s}(t)=m_{G}(t)\left(x_{G}^{\text {in }}(t)-x_{G}^{\text {out }}(t)\right) h_{\text {ads }} \\
& q_{G}(t)=m_{G}(t) c_{p G}\left(\left(1+x_{G}^{\text {in }}(t)\right) T_{G}(t)-\left(1+x_{G}^{\text {out }}(t)\right) T_{\text {store }}\right) \\
& q_{a m b}(t)=U A_{a m b}\left(T_{a m b}-T_{\text {store }}(t)\right)
\end{aligned}
$$

where $h_{a d s}$ is the heat of adsorption released per $\mathrm{kg}$ of water adsorbed, $c_{p G}$ is the specific heat capacity of the humid air, $U A_{a m b}$ is an effective heat transfer rate from the storage to ambient which will depend on the specific insulation, and $T_{a m b}$ is the ambient temperature, which is assumed to be constant throughout the simulation.

\section{Simulation results}

The simulation considers a revolving drum sorption storage filled with $55 \mathrm{~kg}$ Zeolite $13 \mathrm{X}$ material. The parameter $k$ (linear driving force) is assumed to be $810^{-3} 1 / s$ and the heat of adsorption for Zeolite $13 \mathrm{X}$ is approximately $3400 \mathrm{~kJ}$ per $\mathrm{kg}$ water adsorbed. In initial condition, the storage is at ambient temperature, which was chosen to be $20^{\circ} \mathrm{C}$. The initial loading of the Zeolite material is assumed $0.1 \mathrm{~kg}$ water per $\mathrm{kg}$.

Simulations are done under different operating conditions: the air stream has a constant temperature of $20^{\circ} \mathrm{C}$, mass flowrate of $250 \mathrm{~kg} / \mathrm{h}$, and the absolute humidity takes the values $2,4,6,8$, and $10 \mathrm{~g} / \mathrm{kg}$.

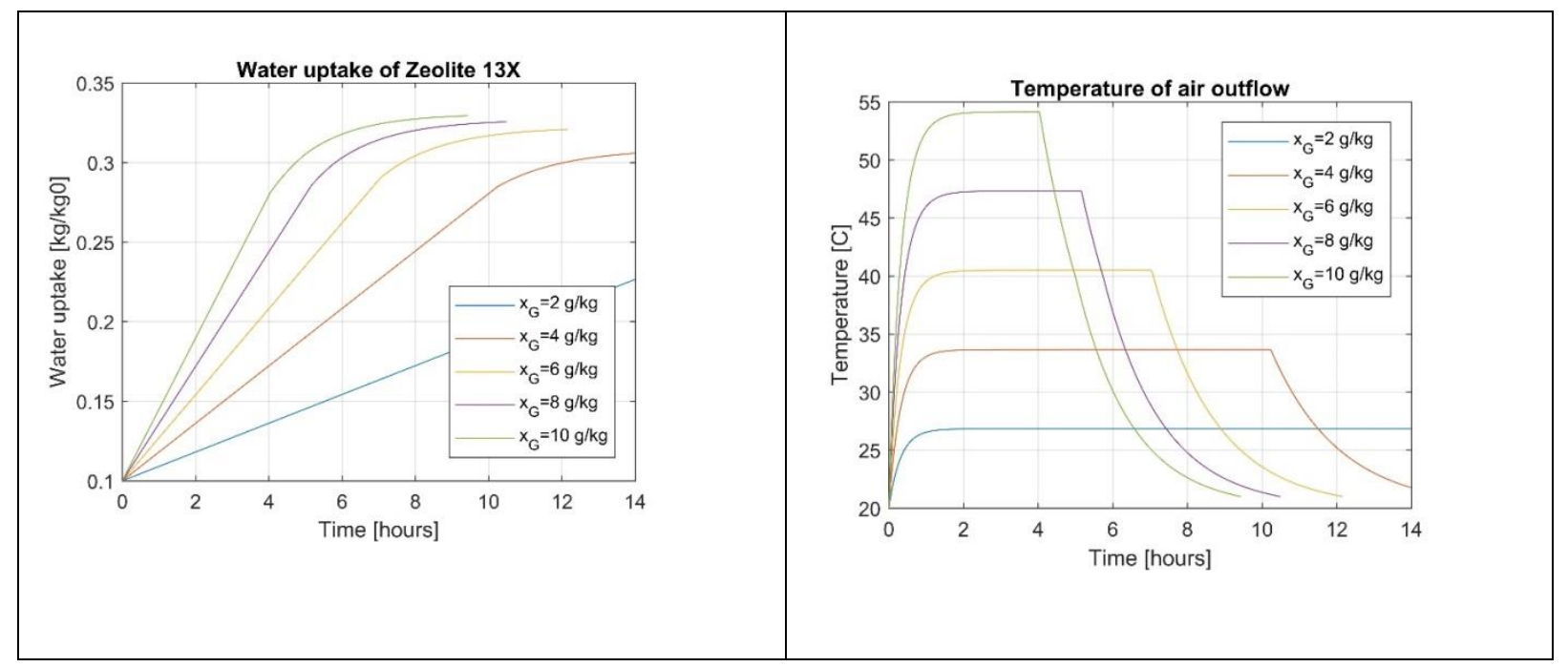

Figure 39: Water uptake and temperature evolution for a range of absolute air humidity $\mathrm{x}_{\mathrm{g}}=2 \ldots 10 \mathrm{~g} / \mathrm{kg}$ 
The simulation results in Figure 39 show the expected behaviour: the loading of the Zeolite with water increases faster if the provided air is more humid and saturates at the material specific values given by the adsorption isotherms. Similarly, the temperature rises to higher values with higher air humidity. The simulation suggests that under the given assumptions, for a period of approximately 10 hours, an outlet air temperature of $34^{\circ} \mathrm{C}$ (a temperature increase of $14^{\circ} \mathrm{C}$ with respect to ambient temperature) can be achieved.

The next step in model development will be the adjustment of some parameters in the model equations to fit the experimental setup. For this purpose, measurements of the real system will be used in a parameter optimization algorithm with the goal to minimize the quadratic error of the deviation from simulated and measured temperatures and loading.

\section{REFERENCES}

Do, D. D. (1998), “Adsorption Analysis: Equilibria and Kinetics” Imperial College Press, London

Engel, G. et. al (2017), "Simulation of a seasonal, solar-driven sorption storage heating system", in Journal of Energy Storage, vol. 13, pp. 40-47

Kim, K. et. at (2016), "Adsorption equilibria of water vapour on Zeolite 3A, Zeolite 13X, and dealuminated Y Zeolite”, in Journal of Chem. Eng. Data, vol. 61(4), pp. 1547-1554

Krese, G. et. al (2018), "Thermochemical seasonal solar energy storage for heating and cooling of buildings" in Energy and Buildings, vol. 164, pp. 239-253

Mette, B. et. al (2014), "Experimental and numerical investigations on the water vapour adsorption isotherms and kinetics of binderless zeolite 13x", in International Journal of Heat and Mass Transfer, vol. 71, pp. 555-561

Zettl B. et. al (2014), "Development of a revolving drum reactor for open-sorption heat storage processes", in Applied Thermal Engineering 70 (2014) 42-49

Zettl B. et. al (2015), „An open sorption heat storage concept and materials for building heat supply” in Proceedings of the 9th International Renewable Energy Storage Conference, IRES 2015, Düsseldorf

Zettl B. and Lachner M. (2015), "An Advanced Desorption Concept for TES-Materials", in Proceedings of SHC 2015, International Conference on Solar Heating and Cooling for Buildings and Industry, Istanbul

\section{ACKNOWLEDGES}

The author thanks the Austrian Research Promotion Agency (FFG) for financial support of the project. Thanks also to the project partners (in alphabetical order): AIT- Austrian Institute of Technology (Vienna), ECOTHERM Austria GmbH (Hartkirchen), HAITHERM Industrieofenbau und Wärmebeh. GmbH (Walding), IFK-Schüttgut-Technik GmbH (Haibach o.d. Donau), ITW- Institute of Thermodynamics and Thermal Engineering (Univ. Stuttgart), SOLAR- LAUS (Linz). 


\section{COMBINED SHORT- AND LONG-TERM HEAT STORAGE WITH SODIUM ACETATE TRIHYDRATE FOR SOLAR HEAT SUPPLY IN BUILDINGS}

Gerald Englmair1, Christoph Moser2,3, Simon Furbo1, Hermann Schranzhofer2, Jianhua Fanı

1Department of Civil Engineering, Technical University of Denmark Brovej 118, 2800 Kgs. Lyngby, Denmark; E-Mail: gereng@byg.dtu.dk

2Institute of Thermal Engineering, Graz University of Technology

3Now at: AEE- Institute for Sustainable Technologies, Gleisdorf, Austria

\section{SUMMARY}

Because of its ability to preserve latent heat of fusion at room temperature in supercooled state, the phase change material (PCM) sodium acetate trihydrate (SAT) can be utilized for combined short and long-term heat storage in buildings. This was experimentally proved with a novel solar combisystem demonstrator including a segmented heat storage prototype, consisting of 4 flat units containing 200 $\mathrm{kg}$ of SAT composites each. A numerical model of the demonstrator was developed in TRNSYS environment. Ongoing system simulation indicates potential for more efficient domestic hot water and space heating supply. Further, based on experience with flat units, an inexpensive, cylindrical heat storage unit of the same PCM volume as one flat unit was designed and tested. Results showed that 27 $\mathrm{kWh}$ of heat can be stored. After cooling to room temperature, $11 \mathrm{kWh}$ (long-term storage potential) were discharged during SAT composite solidification.

\section{HEAT STORAGE WITH STABLE SUPERCOOLING OF SAT}

SAT has a melting point of $58^{\circ} \mathrm{C}$ and a latent heat of fusion of $264 \mathrm{~kJ} / \mathrm{kg}$ (Zalba et al., 2003). It was identified as suitable phase change material for heat storage in households for space heating and domestic hot water supply. After melting above $78^{\circ} \mathrm{C}$ (Araki et al., 1995) SAT can cool down to the ambient temperature without solidifying and remain stable in supercooled state. The solidification can be initiated and the heat of fusion released later when heat is in demand. This concept was successfully applied to flat prototype heat storage units (Dannemand et al., 2016). Additives are used to prevent phase separation when SAT is supercooled below its melting temperature. Kong et al. (2016) investigated different stabilizers and concluded that SAT composites with HD 310, a liquid polymer prototype solution, had high heat of fusion after supercooling periods.

\section{NOVEL SOLAR COMBISYSTEM DEMONSTRATOR}

Englmair et al. (2018) reported the design and the functionality of a solar heating system with $22.4 \mathrm{~m} 2$ solar collectors, a heat storage prototype consisting of four $200 \mathrm{~kg}$ PCM storage units and a $735 \mathrm{~L}$ water tank (Fig. 1). It was built (part of the former EU-COMTES project) in a prototype test facility at Technical University of Denmark to apply domestic hot water and space heating demand patterns of a Passive House in Danish climate.

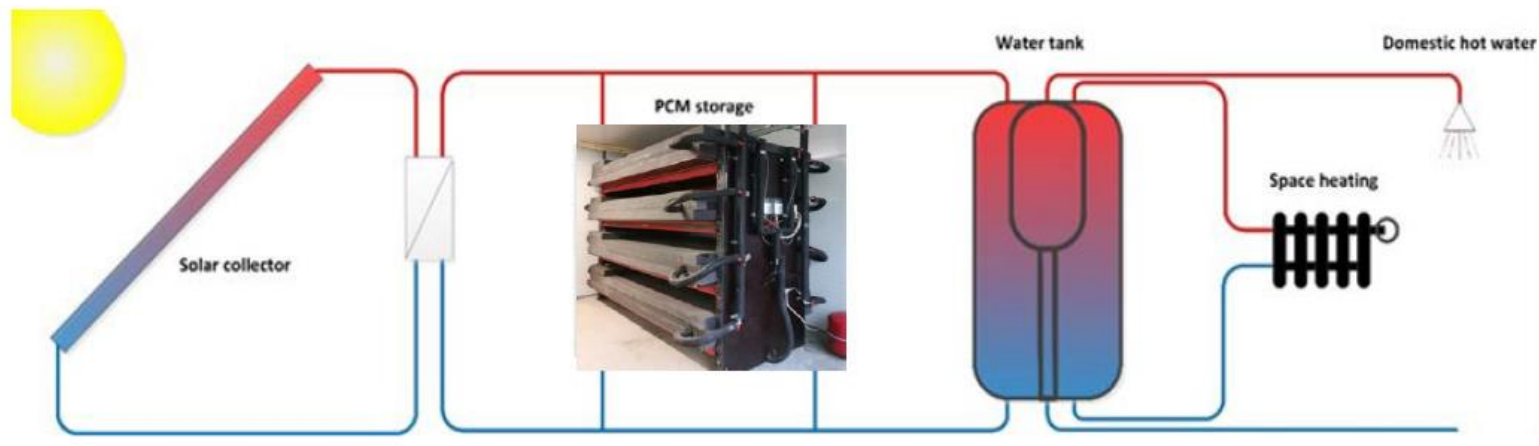

Fig. 1: Diagram of the demonstrator system layout

Fluctuating heat transfer rates from the solar collectors peaked at $16 \mathrm{~kW}$. An automated sequence for combined operation of the water tank and a variable number of PCM units were set by a LabView control system. Fast charge of a single PCM unit (250 minutes) was possible. 
During discharge heat transfer rates reached $4 \mathrm{~kW}$. TRNSYS types of the segmented PCM storage, the water tank and of the system controller were developed and validated. Preliminary results on system simulation showed higher solar fractions of building heat supply with more than 4 PCM units.

\section{TESTING OF ECONOMIC HEAT STORAGE UNITS}

A cylindrical heat storage prototype based on standard components of water heat stores (Fig. 2a) was designed as a more economic storage unit. It was manufactured by the Danish company NILAN A/S and contained $200 \mathrm{~kg}$ of SAT composite and $70 \mathrm{~L}$ of water. Performance evaluation of a test sequence is presented in Fig. 2b:
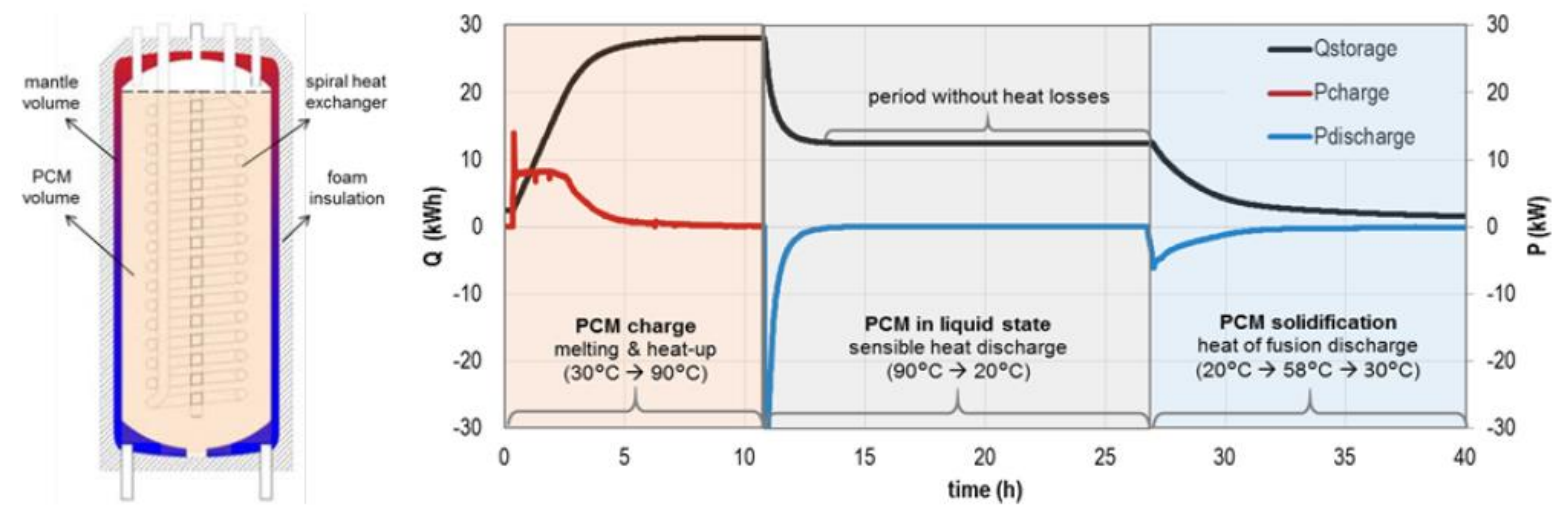

Fig. 2: a) Diagram of cylindrical heat storage; b) Development of heat transfer rates and heat content during test.

During 10 hours charge from initially solid PCM state $27 \mathrm{kWh}$ of heat were stored. Then it was discharged and the storage cooled down to ambient temperature within 2 hours. After a heat-loss-free storage period, $11 \mathrm{kWh}$ (long-term storage potential) were discharged during SAT composite solidification.

\section{CONCLUSIONS}

The heat storage concept of utilizing stable supercooling of SAT for combined short- and long-term heat storage was proved in system scale. A storage unit with inexpensive industrial components showed a significantly higher effective storage capacity than water stores of the same volume. In general, storage units containing melted PCM worked like water vessels in the temperature span from 20 to $90^{\circ} \mathrm{C}$ with the additional feature to bridge energy supply gaps over weeks or months. This potentially enables more efficient solar heating or heat pump applications in domestic buildings. TRNSYS simulation of the novel solar combisystem will elucidate its potential for more efficient solar heat supply of a Passive House in Danish climate.

\section{REFERENCES}

N. Araki, M. Futamura, A. Makino, and H. Shibata, "Measurements of Thermophysical Properties of Sodium Acetate Hydrate," Internaltional J. Thermophys., vol. 16, no. 6, pp. 1455-1466, 1995.

M. Dannemand, J. Dragsted, J. Fan, J. B. Johansen, W. Kong, and S. Furbo, "Experimental investigations on prototype heat storage units utilizing stable supercooling of sodium acetate trihydrate mixtures," Appl. Energy, vol. 169, pp. 72-80, 2016.

Kong, W., Dannemand, M., Johansen, J. B., Fan, J., Dragsted, J., Englmair, G., \& Furbo, S., 2016. Experimental investigations on heat content of supercooled sodium acetate trihydrate by a simple heat loss method. Solar Energy, 139, 249-257.

G. Englmair, C. Moser, S. Furbo, M. Dannemand, and J. Fan, "Design and functionality of a segmented heat-storage prototype utilizing stable supercooling of sodium acetate trihydrate in a solar heating system.”, Applied Energy, vol. 221, pp. 522-534, 2018

B. Zalba, J. M. Marín, L. F. Cabeza, and H. Mehling, "Review on thermal energy storage with phase change: materials, heat transfer 65 analysis and applications," Appl. Therm. Eng., vol. 23, no. 3, pp. 251-283, 2003. 


\title{
BREAK THE DEPENDENCY ON FOSSILE FUELS IN INDUSTRIAL PROCESSES WITH AN INDUSTRIAL HEAT PUMP THAT CAN PROVIDE CLEAN ENERGY PRODUCTION UP TO $160^{\circ} \mathrm{C}$
}

\author{
Mattias Nilsson, Dr Tim Hamacher, Harald Nes Rislå and Torunn Hansen-Tangen \\ Viking Heat Engines Germany GmbH \\ Walter-Freitag Str. 1, 42899 Remscheid, Germany \\ Phone: +49 1706942185 \\ E-Mail: mattias.nilsson@vikingheatengines.com
}

\section{SUMMARY}

The paper describes the potential use and system boundaries of the HeatBooster system in industrial processes, such as the brewing, sugar and automotive industries as well as the building sector. It also describes the HeatBooster's key features and includes measured system performance with the working fluids HFC-245fa and HFO-1336mzz(Z).

Keywords: (1) High-temperature heat pump (2) Piston compressor for heat pump system (3) Renewable heating and cooling, (4) Solution for decarbonization, (5) Energy efficiency, (6) Electrification of industrial processes, (7) Environmental-friendly refrigerants

\section{INTRODUCTION}

Traditionally, heat pumps have been limited to output temperatures in the 70 to 80 -temperature range. Moving away from oil and gas boilers has thus been difficult. But this is about to change with the introduction of the HeatBooster. This industrial heat pump turns waste heat into steam or usable heat up to $160^{\circ} \mathrm{C}$, saving hundreds of tons of $\mathrm{CO}_{2}$ emissions per year and drastically reduces a company's energy bill. The HeatBooster is based on a piston expander/compressor technology, which is equally capable of operating in organic-rankine-cycles (ORCs) [1] as well as vapor compression heat pump systems.

A significant amount of industrial energy is wasted as low-temperature heat (e.g. dryer vent gases and cooling water) that could be lifted by the HeatBooster to the required temperatures needed for several industrial processes and heating of buildings. The list of possible applications is endless, and the chance to make a real difference is great. The HeatBooster system can minimize energy use across the entire manufacturing industry - from paper, steal and plastics to milk, beer and chocolate and in the building sector.

\section{DEVELOPED BY PISTON AND ORC-ENGINE SPECIALISTS}

The HeatBooster is based on a tried and tested engine technology developed by AVL Schrick, part of the world's leading engine design company AVL, and Viking Heat Engines, a green tech company from Norway. This keeps costs and any technological uncertainties to a minimum. More specifically, the HeatBooster stems from a piston expander developed for the ORC market, which has been extensively tested to handle temperatures up to $200^{\circ} \mathrm{C}$. The HeatBooster compressor was developed by adjusting the expander valve system and adding well-known heat pump components. The commercial unit is virtually maintenance free and produced at a low cost.

The HeatBooster uses environmental-friendly refrigerants, such as the HFC-245fa and HFO$1336 \mathrm{mzz}(\mathrm{Z})$ working fluids with a global warming potential of 858 and 2, respectively [2]. These fluids have attractive safety and thermodynamic properties as well as a high chemical stability at high temperatures. 
The performance of the HeatBooster has improved since the start of testing in March 2016. Today, the heat pump produces $200 \mathrm{~kW}$ at a COP of 4 with temperature lifts as big as $40^{\circ} \mathrm{C}$. This confirms the nominal capacity of the current heat pump at $200 \mathrm{~kW}$ with the tested working fluids at input temperatures from $70^{\circ} \mathrm{C}$ and above.

A low-carbon future will require a very effective use of energy. The HeatBooster produces heat up to $160^{\circ} \mathrm{C}$ with current working fluids at very high efficiencies and operational flexibility. The test results show that the HeatBooster has an operating range that is perfectly suited for many processes in the manufacturing industry and building sector. Additionally, the HeatBooster is a compact and easy-toinstall system, which further improves the economic savings for a company interested in installing it.

The HeatBooster has five main components (as outlined in figure 1): The evaporator, condenser and recuperator are plate-type heat exchangers. The compressor is a piston engine, fully hermetically designed, and the expansion valve is an electronically controlled device with a high operating temperature specification. There is also a working fluid reservoir in the form of a tank, which ensures that there is always enough fluid available to keep the process going. Beyond these components, there are pipes, safety valves, electrical cabinet and a control system to ensure safe and autonomous operation of the system.

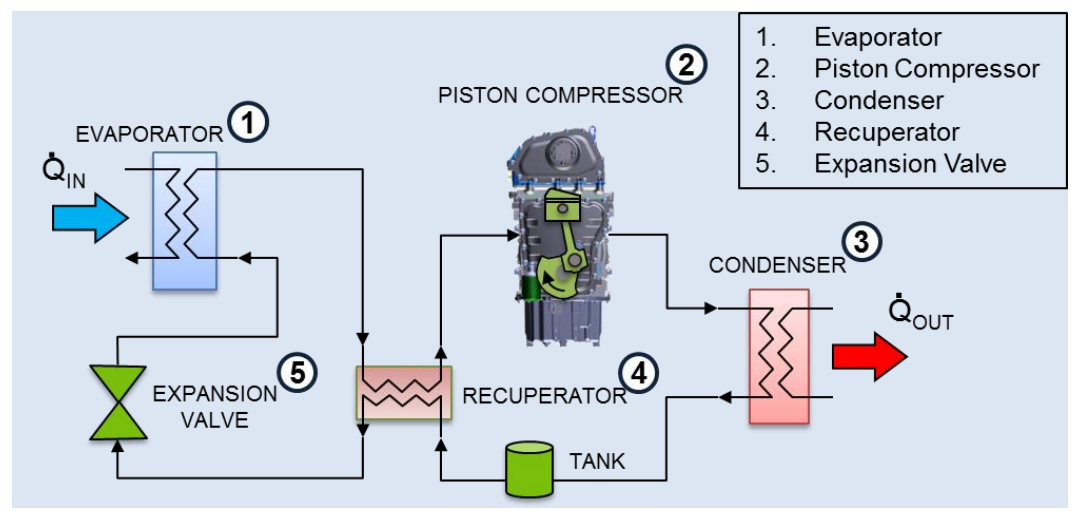

Fig. 1: Main HeatBooster system components and simplified flow diagram

\section{BOOSTING THE COP WITH A RECUPERATOR}

A special feature of the HeatBooster design is the recuperator. When a recuperator is used, the refrigerant is subcooled before entering the expansion valve which lowers the $\mathrm{x}$-fraction (i.e. the dryness fraction) in the evaporator. This process increases the evaporation point and improves both the capacity and the COP. Furthermore, the recuperator allows the refrigerant to get more superheat before entering the piston compressor which augments the enthalpy states and increases the maximum output temperature. Controlling the superheat is key to avoiding compression into the wet zone of the socalled dry refrigerants, such as the HFO- $1336 \mathrm{mzz}(\mathrm{Z})$. When a recuperator is used, superheating is done without sacrificing the heat transfer area in the evaporator or using heat from the heat source loop that doesn't give any extra output to the heat sink loop. A basic overview of the HeatBooster principle is presented in figure 2 . 


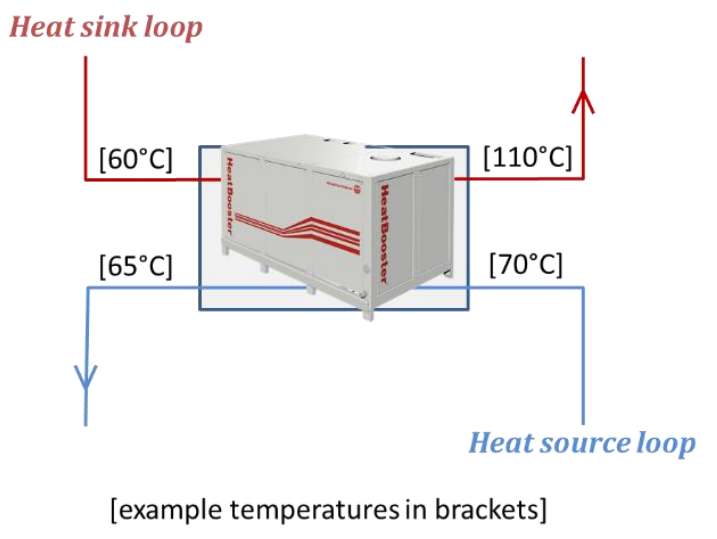

Fig. 2: Example of taking waste heat at $70^{\circ} \mathrm{C}$ to provide $110^{\circ} \mathrm{C}$ useful heat

\section{PERFECTLY SUITED FOR INDUSTRIAL AND RESIDENTIAL APPLICATIONS}

The HeatBooster is ideal for drying, evaporation, distillation, sterilization and pasteurization as it takes processed waste heat and a bit of electricity to reach the required temperatures, drastically reducing the overall energy efficiency of these processes. One HeatBooster alone can save $25 \mathrm{~m}^{3}$ of natural gas per hour, which is equal to 430 tons of carbon dioxide per year. This allows applications such as drying, stripping and juice concentration to be conducted in a climate-friendly way.

The building sector is another area where the HeatBooster can make a big difference. The building sector worldwide uses approximately 32 percent of all energy generated today and more than one-third of that is for heating and cooling [3]. The HeatBooster can, for example, be used for distributed steam production in district heating. This would reduce the infrastructure investments and improve the energy efficiency of district heating in many European countries, such as Germany and the Netherlands, and American cities, such as New York and Boston. The HeatBooster would enable residential areas to use existing heating solutions without having to make major changes to the buildings or investments into new biomass or fossil fuel-fired power plants, which are normally used for residential heat production.

\section{MEASURED SYSTEM PERFORMANCE}

Tests are currently ongoing with two different refrigerants, namely HFC-245fa and HFO-1336mzz(Z). HFC-245fa is used to reach temperatures up to $140^{\circ} \mathrm{C}$ and $\mathrm{HFO}-1336 \mathrm{mzz}(\mathrm{Z})$ is used to reach temperatures up to $160^{\circ} \mathrm{C}$. The two refrigerants have not yet been tested at the same pressures, which explains why the COP and heat output are higher for HFC-245fa compared to HFO-1336mzz(Z). Figure 3 shows the latest test results in two graphs.
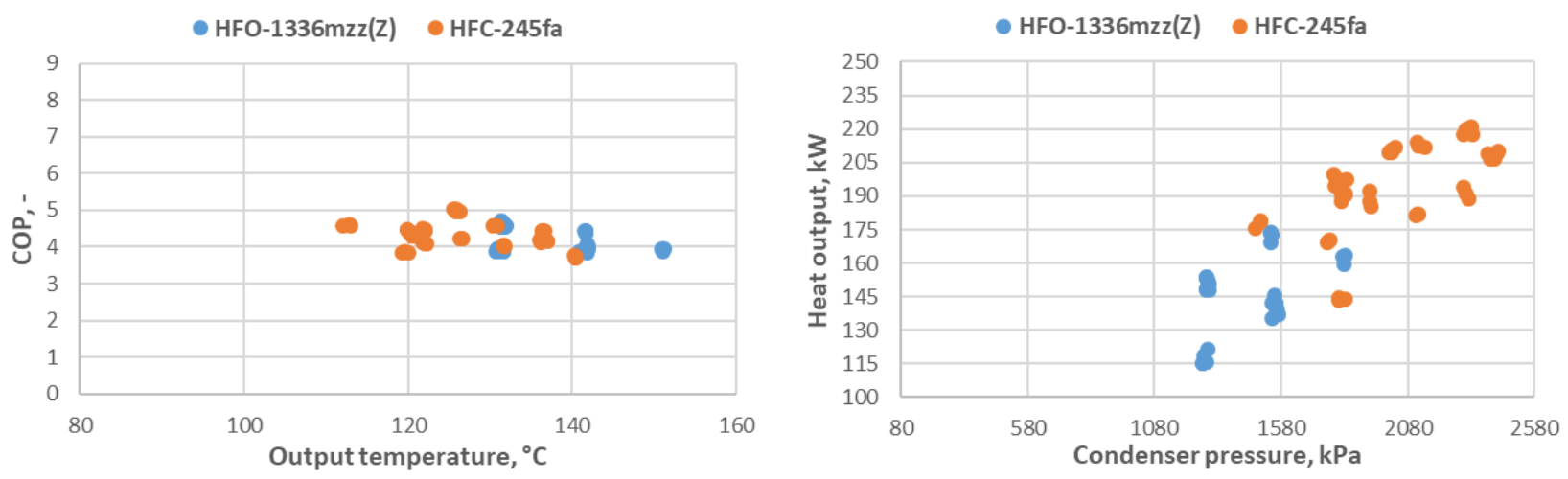

Fig. 3: Performance overview of recorded measurements 


\section{CONCLUSIONS}

The HeatBooster can operate at unprecedented temperature levels for closed-loop heat pump systems by using piston compressors. The heat pump showed stable COP between 4 to 5 at different temperature lifts in this system configuration. HFO-1336mzz(Z) has not been tested at the same pressures as HFC-245fa, which explains the difference in heat output. The results show that the HeatBooster is ideal for industrial processes such as drying, distillation, pasteurization, sterilization as well as the building sector in the form of distributed steam production and heat storage as it provides the required temperatures by recirculating process waste heat, adding the adequate amount of electricity and subsequently increase the overall energy efficiency of these processes drastically.

\section{REFERENCES}

[1] Kontomaris, Konstantinos, et al. Combined Heat and Power From Low Temperature Heat: HFO$1336 m z z(Z)$ as a Working Fluid for Organic Rankine Cycles. Purdue : 21st International Congress of Refrigeration, 2016.

[2] Palm, Björn. Low GWP refrigerants for high temperature heat pumps. [Online] KTH Royal Institute of Technology, September $\quad 2014 . \quad$ [Cited: $\quad$ March 20,2 2018 https://www.kth.se/en/itm/inst/energiteknik/forskning/ett/projekt/ koldmedier-med-lag-gwp/low-gwpnews/koldmedier-med-lag-gwp-for-hogtemperaturvarmepumpar-1.501185.

[3] Project Drawdown. Buildings and cities. Heat pumps. [Online] July 2017. [Cited: March 21, 2018] http://www.drawdown.org/solutions/buildings-and-cities/heat-pumps. 


\title{
DEVELOPMENT OF AN ALL-IN-ONE SOLAR THERMAL COLLECTORS AND SYSTEMS TESTING FACILITY FOR WATER HEATING, ROOM HEATING AND INDUSTRIAL APPLICATIONS
}

\author{
Dr. Edward Rakgati, Mr. Ronnie Phuthego \\ Botswana Institute for Technology Research and Innovation (BITRI) \\ Maranyane House, Plot 50654, \\ Machel Drive, Private Bag 0082 \\ Gaborone, Botswana
}

\section{SUMMARY}

Testing of solar thermal systems and components requires various standards and thus different testing facilities. Usually each facility is individually designed and constructed for tests according to a specific test method either in accordance to the Botswana National Standards (BOS) or International Standards (ISO). For performing tests of solar thermal systems and collectors in accordance to the most popular standards, test laboratories or manufacturers have to invest a huge amount of staff time, resources and capital to set up the required testing facilities. A way to overcome this problem is the development of an all-in-one solar thermal testing facility (STTF) in which Botswana Institute for Technology Research and Innovation (BITRI) envisaged to put in place, in cooperation with the SWTTechnologie from Stuttgart, German. The complete testing facility is designed and installed inside a conventional 12 foot $(3.65 \mathrm{~m})$ porter cabin. This all-in-one solar thermal testing facility (STTF) is custom designed and delivered to the operation site as a turn-key product that can be put into operation within one single day. Performance testing of thermal solar collectors includes the rain penetration test, impact resistance test, pressure tests, fluid capacity test whereas the performance testing of thermal solar systems includes system configuration, storage tank heat losses, pressure test, and circulation test. Even more, the STTF is a three-in-one testing facility, since it is possible to perform tests according to three different standards and test methods identified. The performance of solar collectors can be tested according to the European standard EN 12975 or the international standard ISO 9806, respectively. The performance of solar thermal systems can be determined according to the international standards ISO 9459-2 (Continuous System Testing Group [CSTG]-method) and ISO 9495-5 (Dynamic System Testing [DST]-method). For data acquisition, STTF comprises of comprehensive set of data capturing system which has the central data logger connected to the computer and a mobile data logger built-in into the meteorological station and the STTF for effective data collection and analysis. This paper describes the STTF in details and its applications and relevance to households and industrial applications.

\section{KEY WORDS:}

Solar Thermal Testing Facility (STTF), Solar Collectors, Solar Systems, Performance Test, Reliability Test, Data Acquisition, ISO 9459-2, ISO 9806 \& EN 12975

\section{TESTING FACILITY SET UP}

One aim of the newly developed test facility is to combine all three test methods in a one testing facility for convenience purposes. This testing facility must be able to fulfil all requirements and qualifications resulting from the above mentioned and articulated standards.

The housing of the test facility is a conventional 12 foot $(3.65 \mathrm{~m})$ office container or porter cabin. In this cabin the hydraulics for the temperature unit are located as well as measuring equipment, data logging instruments and computing gadgets. In order to operate the facility independent from a cooling network, a chiller combined with a 600 Liter cold water store is installed in this all in one testing facility. With the exception of the chiller, all components are located inside the porter cabin.

The facility is designed in such a way that it allows for parallel testing of;

- Four collectors (according to EN 12975 / ISO 9806) or

- Four systems according to ISO 9495-2 (CSTG-method) or

- Two systems according to ISO 9495-5 (DST-method) 
To realize these different test configurations, the hydraulic arrangement consisting of one main loop that can be divided into six smaller loops by using several valves is designed. These six loops are required to test two solar thermal systems according to the DST-method at the same time. The hydraulics for testing according to ISO 9459-2 and ISO 9806 / EN 12975 consist of only one test loop, which can be used to test four systems (CSTG-method) or four collectors simultaneously.

\section{TESTING OF THE DURABILITY AND RELIABILITY}

In addition to the equipment required for thermal performance testing, the test facility is delivered with the complete equipment required for durability and reliability testing of solar thermal collectors according to EN 12975-2. This comprises test facilities for outdoor exposure, external and internal thermal shock, rain penetration, mechanical load test and internal pressure test.

\section{CONCLUSIONS AND FURTHER PERSPECTIVES}

The development and the set-up of a mobile, stand-alone test facility based on a 12 foot $(3.65 \mathrm{~m})$ porter cabin was described. For the future it is intended to develop a mobile, stand-alone solar thermal test facility which, additionally, allows for testing of hot water stores according to ENV 12977-3.

\section{REFERENCES}

The European Standards (EN)

International Standards (ISO)

Botswana National Standards (BOS) 


\title{
SONNENHAUS 4.0: SOLAR SELF-SUFFICIENT BUILDINGS IN CITIES
}

\author{
DI Roger Hackstock \\ Verband Austria Solar \\ Mariahilferstr. 89/22, 1060 Wien, Austria \\ Phone: +431581132712 \\ Mobil: + 436642521645 \\ E-Mail: roger.hackstock@austriasolar.at \\ DI Hilbert Focke \\ Initiative Sonnenhaus Österreich \\ Anastasius-Grün-Straße 20, 4020 Linz, Austria \\ Phone: +436641475440 \\ E-Mail: h.focke@ sonnenhaus.co.at
}

\section{SUMMARY}

The Sonnenhaus concept covers thermal energy demand of buildings by using the sun over the whole year. The innovative concept combines low energy buildings with different storage options as water tank and building components. Several built Sonnenhaus already prove the concept as a cost efficient way to fulfil the Energy Performance of Buildings Directive which will be in force after 2020. In future also photovoltaic and e-mobility is an integrated part of the energy concept, as recent buildings show.

\section{ENERGY SUPPLY OF CITIES MAINLY BASED ON FOSSIL FUELS}

In Vienna, renewable energy currently covers only 13 percent of total energy demand, in Frankfurt seven percent, in Berlin merely three percent. In order to reach the paris climate agreement for 2050, cities will play a crucial role. According to OECD nearly 70 percent of world population will live in urban areas at midcentury. Thus the Energy Performance of Buildings Directive was launched by the EU Commission in 2016 to help promote the use of smart technology in buildings. Under the Directive all new buildings must be nearly zero energy buildings by 31 December 2020 (public buildings by 31 December 2018).

In Austria the directive can be accomplished not only by efficiency measures like additional thermal building insulation and airtightness, but also by using local renewable energy sources like solar energy. So the Austrian regulation favours a dual approach in order to help the concept of Sonnenhaus to disseminate.

\section{SONNENHAUS 4.0 - THE NEARLY ZERO ENERGY BUILDING OF THE FUTURE}

In Austria annual solar radiation in total is several times higher than the annual energy demand for heating and domestic hot water in residential buildings. Since solar energy in winter is low, different storage options are applied in Sonnenhaus 4.0 to help covering the thermal energy demand by using the sun over the whole year. 


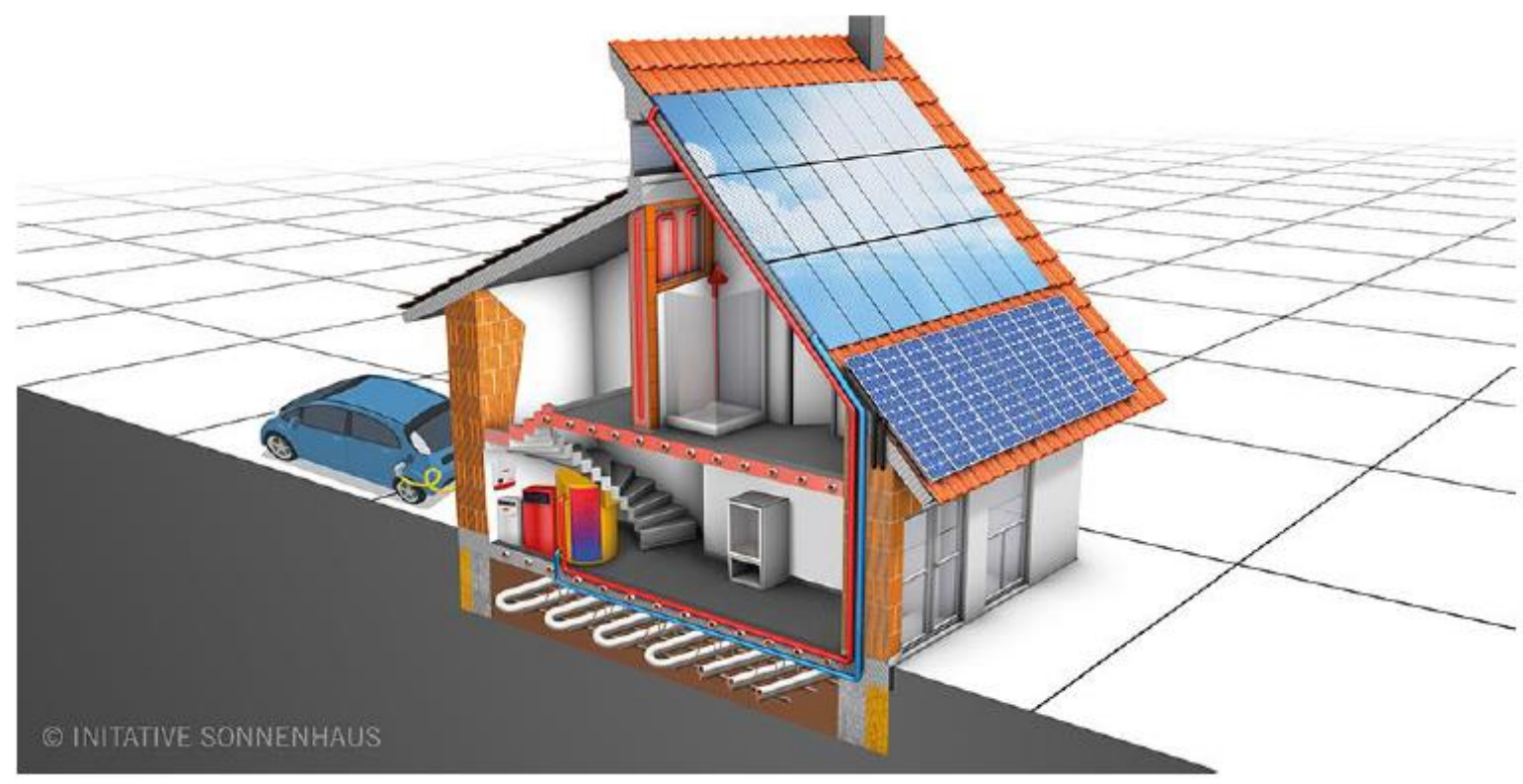

Sonnenhaus 4.0 Concept: Solar thermal system with building components as heat storage and small water storage. Heat pump or Biomass boiler and solar regenerated probes. PV for electricity demand and mobility.

For many years, the building concept of Sonnenhaus was connected to rather large solar collector areas and storage two floors high in a single family house. To overcome the concept of Sonnenhaus comprehensive studies and developments have been conducted in the last decade. As result Sonnenhaus 4.0 today can cover up to 100 percent of heating demand by solar in using building components like basement and ceiling as massive heat storage. Exemplary calculations of existing Sonnenhaus will show actual results of economic benefits of solar supplied houses. The concept of Sonnenhaus 4.0 also includes photovoltaic and e-mobility as integrated part of the energy concept, as will be shown.

\section{EXAMPLE BUILDINGS}

\section{EXAMPLE 1: SINGLE-FAMILY “E4 ZIEGELHAUS 2020“ LOCATED IN THE CITY} ZWETTL (AT)

The Sonnenhaus of the Lugauer family in Zwettl is heated $60 \%$ with the sun. A solar thermal system with a heat output of $34 \mathrm{~kW}\left(48 \mathrm{~m}^{2}\right.$ collector area) directs the sun's energy into a very well insulated 9.580 liter storage tank. Thus, the solar house is heated even in cloudy and cold weather over several days and weeks with stored solar energy. In extreme cold periods, a wood boiler with $40 \mathrm{~kW}$ heating power supplies the energy for heating and hot domestic hot water preparation. By this concept, only about 3 to 5 cubic meters of wood are needed per heating period. Due to the low energy consumption of the brick building, the wood boiler only has to be heated about a dozen times a year. The electricity in the house is also generated from renewable energy sources. A $6.5 \mathrm{kWp}$ photovoltaic system $\left(49 \mathrm{~m}^{2}\right.$ module area) on the garage roof supplies enough electricity for all household appliances and building services. 


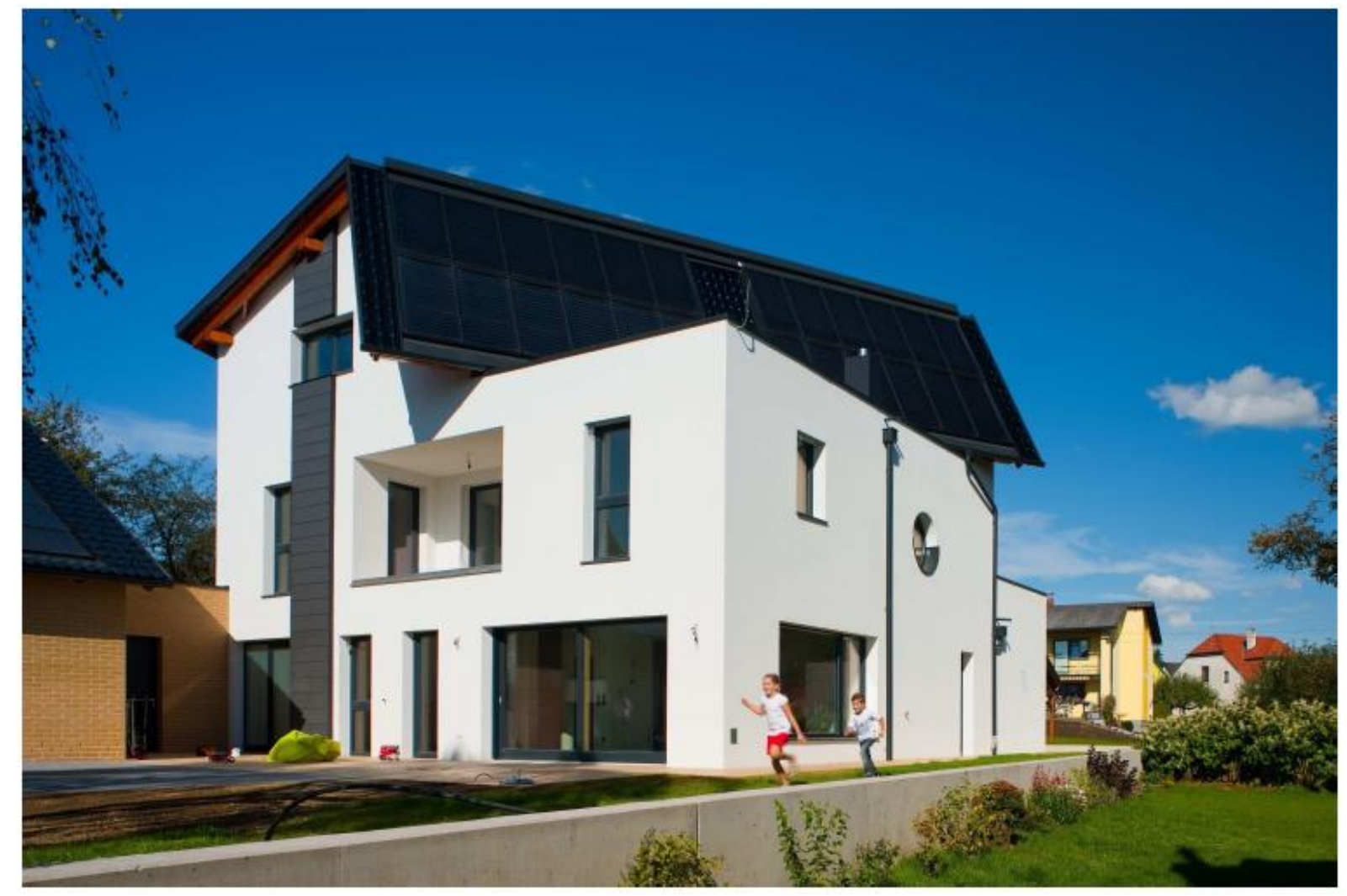

Fig. 2: Single family "e4 Ziegelhaus 2020 " with $60 \%$ solar supply annually, using $34 \mathrm{~kW}\left(48 \mathrm{~m}^{2}\right)$ solar thermal, 9,6 mз water tank and partially brick wall components as heat storage (Source: Wienerberger AG)

\section{EXAMPLE 2: EVENT LOCATION AND LIVING HOUSE „VITAL SONNENHAUS PRO“ LOCATED IN THE CITY SCHWERTBERG (AT)}

The $150 \mathrm{~m}^{2}$ large house of the builder Boris Maier designed for events and overnight stays is heated 70 $\%$ with energy from the sun. The heat of the $56 \mathrm{~kW}\left(80 \mathrm{~m}^{2}\right)$ solar thermal system in the south facade is delivered into two 4.500 liter storage tanks. Also the cellar ceiling, the ceiling of the ground floor and the northern outer wall are used as component storage. For longer cold periods in winter, a $9 \mathrm{~kW}$ pellet boiler supplies the remaining heat, as well as a stove with $7.8 \mathrm{~kW}$ heating power. The well-insulated and mainly solar heated house requires only $1.600 \mathrm{~kg}$ of pellets per year, the annual heating demand is $24 \mathrm{kWh} / \mathrm{m}^{2}$. For power supply, photovoltaic modules with a total capacity of $9.5 \mathrm{kWp}$ are installed on the roof and in the facade. Together with a $9.6 \mathrm{kWh}$ electricity storage, the system covers the entire power consumption in the house and feeds surplus energy into the grid. 


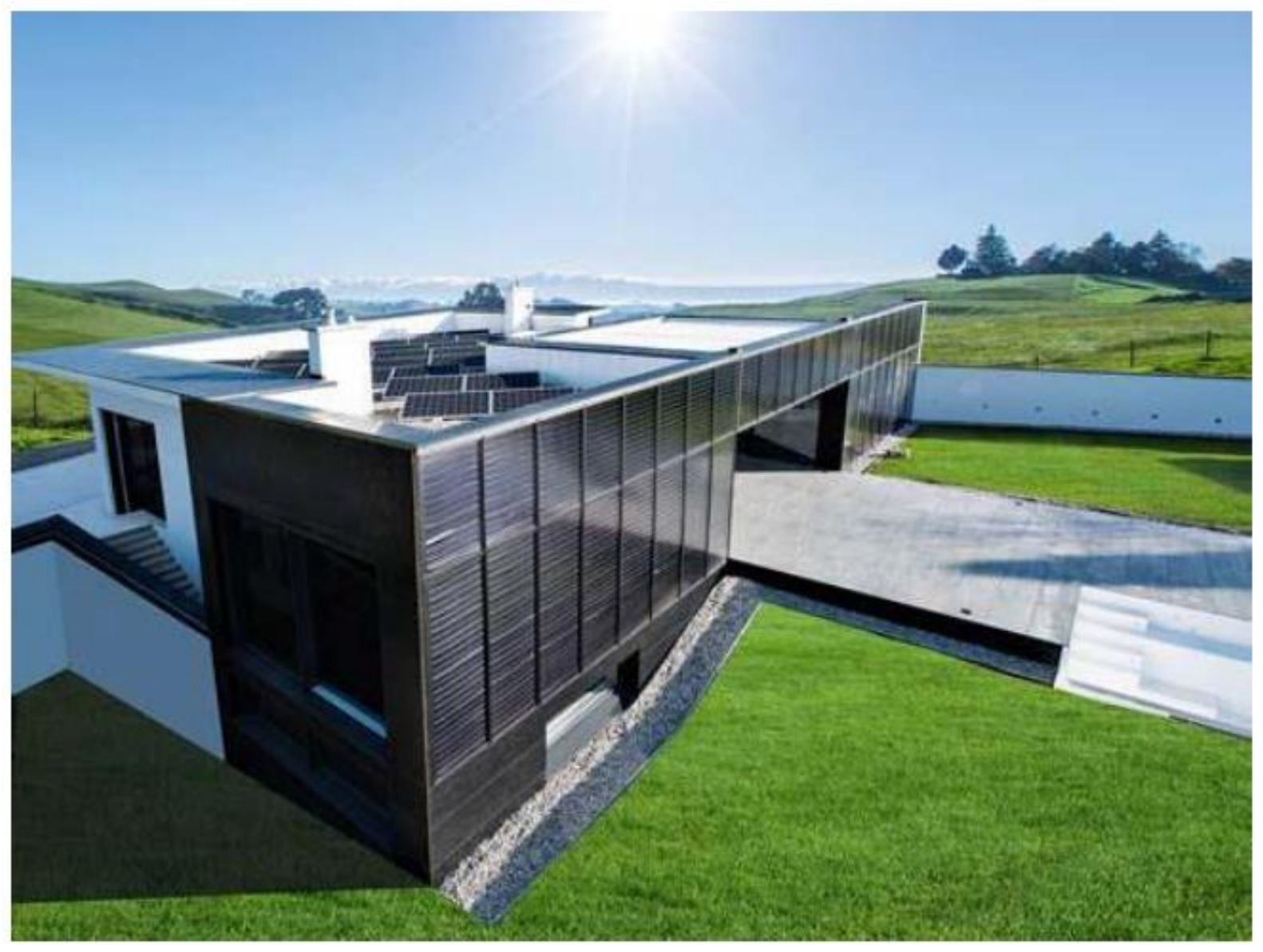

Fig. 3: Event Location and living house with $70 \%$ solar supply annually, using $56 \mathrm{~kW}\left(80 \mathrm{~m}^{2}\right)$ solar thermal, $4.5 \mathrm{~m}^{3}$ water tank and building components as heat storage (Source: Bauhütte Leitl-Werke $\mathrm{GmbH})$

\section{EXAMPLE 3: MULTI-FAMILY SONNENHAUS LOCATED IN THE CITY CHEMNITZ (DE)}

Not only single-family houses can be heated with the sun, also multi-family houses can be built as solar houses in the future. The house with 30 apartments in Chemnitz is heated up to $50 \%$ with the sun. The energy of the $222 \mathrm{~kW}\left(317 \mathrm{~m}^{2}\right)$ solar thermal system in the south and east facade is consumed for hot water and heating in the house, surplus solar energy in summer is stored in a $200 \mathrm{~m}^{3}$ long-term storage for winter. What the solar system does not deliver is provided by a condensing gas boiler with $80 \mathrm{~kW}$. The construction costs of the building were about 100 euros per $\mathrm{m}^{2}$ living space higher than in conventional buildings, the price for a $100 \mathrm{~m}^{2}$ apartment increased therefore only by about 10.000 euros, with extremely low heating costs over decades. 


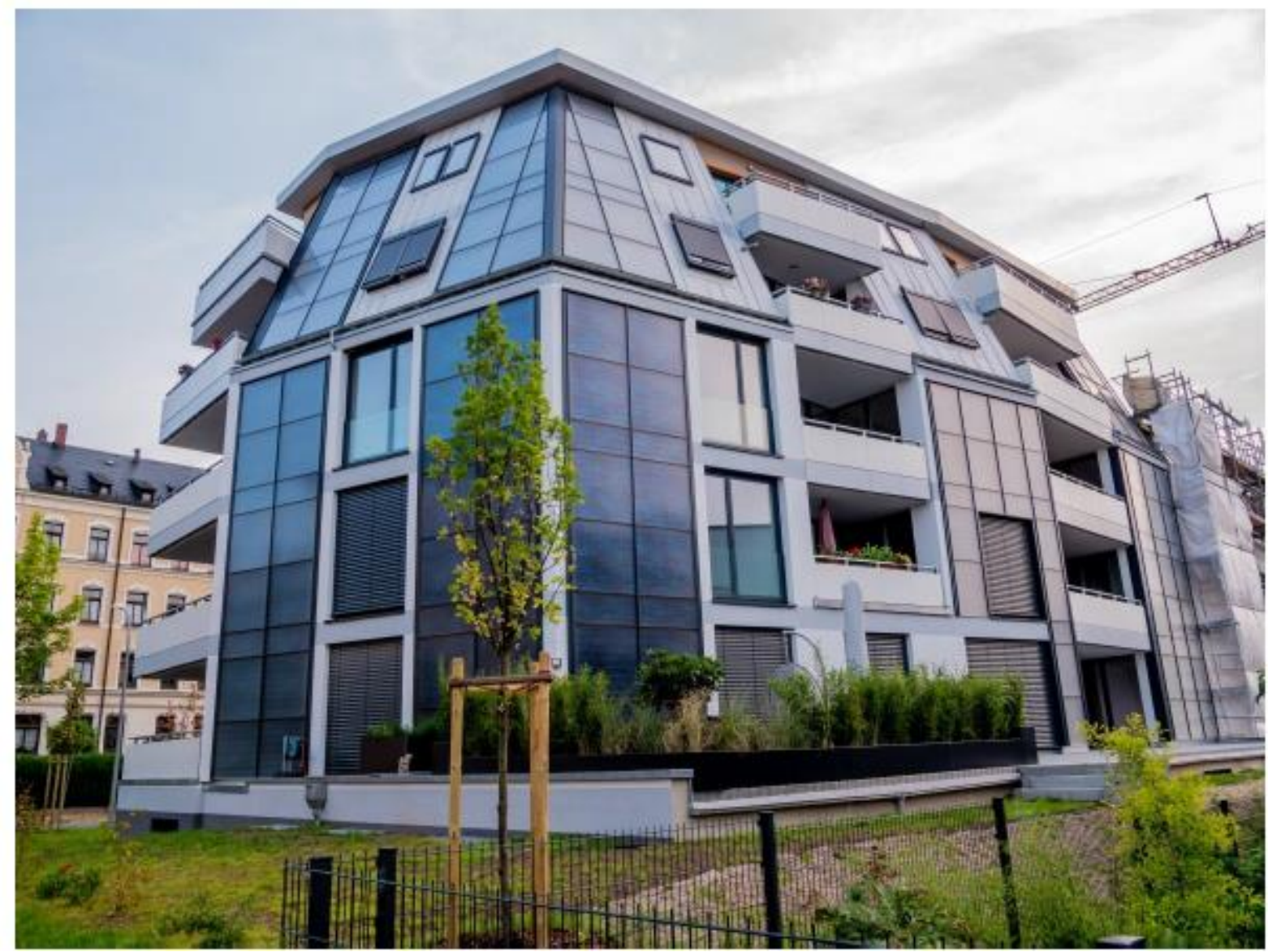

Fig. 4: Multi-family Sonnenhaus with $50 \%$ solar supply annually, using $222 \mathrm{~kW}\left(317 \mathrm{~m}^{2}\right)$ solar thermal and a $200 \mathrm{~m}_{3}$ long term storage tank (Source: FASA AG)

\section{AUSTRIAN SUBSIDY FOR SOLAR SELF-SUFFICIENT BUILDINGS}

Since 2014, Sonnenhäuser have been generously funded by the Climate and Energy Fund in Austria. In total, more than 70 solar houses were approved, the prerequisite is a solar coverage of the total heat demand of a one or two-family houses of at least $70 \%$. At selected funded houses, data is collected and scientifically evaluated over one year in order to improve the concepts of solar houses and to strengthen Austria's pioneering role in its dissemination in Europe. The measurement results show that intelligent planning of Sonnenhäuser can reach up to $100 \%$ solar coverage in best case. In addition to the solar system, renewable additional heating systems and photovoltaic systems up to $5 \mathrm{kWp}$ are supported in the funding programme.

\section{REFERENCES}

Initiative Sonnenhaus Österreich (2016), DAS SONNENHAUS - Ein Bau- und Heizkonzept der Gegenwart und der Zukunft, 2. Auflage, Linz, Austria.

Hackstock R. (2014), Energiewende - Die Revolution hat schon begonnen, Verlag Kremayr \& Scheriau, Wien, Austria.

Hackstock R. (2017), Flexibel und frei - Wie eine umfassende Energiewende unser Leben verändert, oekom Verlag, München, Germany.

Subsidy Programme for Solar Houses by Climate- and Energy Fund - all information at https://www.klimafonds.gv.at/ausschreibungen 


\section{CONTROLLING OF A DISTRIBUTED SOLAR DISTRICT HEATING PLANT IN DENMARK}

Jes Donneborg and Andreas Zourellis

Aalborg CSP A/S

Hjulmagervej 55, 9000 Aalborg, Denmark

Phone: +4588168847

E-Mail: jem@aalborgcsp.com

\section{SUMMARY}

This paper presents a unique "distributed" system of a solar district heating plant consisting of flat panels. Located in Havdrup (Solrød Municipality), the 1.9MWth plant is directly feeding the district heating grid with hot water. Due to the integration of a large hot water storage and its complex control philosophy, the solar plant can supply heat to the grid 24/7 for several weeks especially during summertime. This is a unique approach in Denmark achieving substantial savings.

\section{SOLRøD SOLAR DISTRICT HEATING PLANT, A UNIQUE "DISTRIBUTED” SYSTEM}

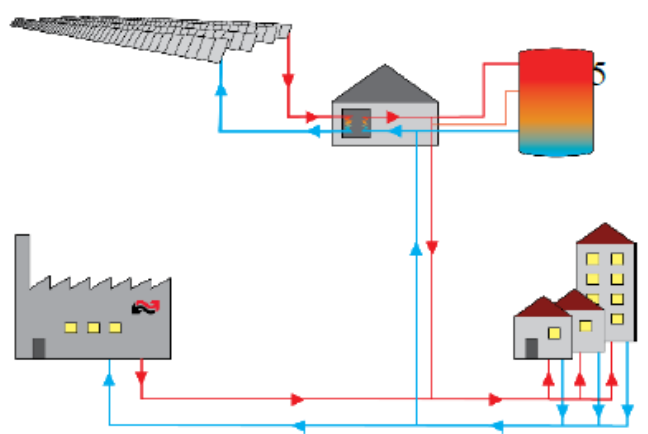

Figure 1: Havdrup distributed solar district heating plant

By the end of September 2016 Denmark has reached a milestone regarding its solar thermal activity. More than one million square meters of solar collectors had been installed by that time [1]. Among those hundreds of thousands of flat plate collector square meters was the solar district heating plant of Solrød Kommune (Solrød Municipality) at the small town of Havdrup. Although the solar district heating plant is relatively small in size compared to the rest of Denmark, it has a unique characteristic. The plant in Havdrup is a distributed solar district heating system. This means that the solar plant is directly feeding the district heating grid with hot water. Normally "these plants use the grid as storage (as long as they provide a minor amount of heat in comparison to the total load of the district heating system" [2]. However, the solar plant in Havdrup due to the integration of a large hot water storage and its complex control philosophy can supply the heat to the grid 24/7 for several weeks especially during the summer. That results in substantial savings for the district heating plant (Solrød Fjernvarme) as they are capable to completely shut down their gas boilers for a long period.

\section{DESCRIPTION OF THE SOLAR DISTRICT HEATING PLANT}

Delivered by Aalborg CSP A/S, the solar plant in Havdrup consists of $2,569 \mathrm{~m}_{2}$ of GREENoneTEC flat plate collectors from the GK3003 product line, a technical house with all the technical equipment, instrumentation and heat exchanger and a hot water accumulation tank with a capacity of $1,250 \mathrm{~m}_{3}$. The GREENoneTEC panels have a $13.3 \mathrm{~m}_{2}$ collector area and a mix of single- and double glazed collectors are used in the solar field. These panels embed serpentine risers which helps the circulation as the water rises from the bottom footer to the top header when it gets warmer. The solar field includes 11 parallel rows of up to 20 solar panels connected in a series. Due to space limitation the top two rows had to be bended in half. The first half of each row contains single glazed collectors while the second half consists of double glazed collectors in order to minimize the heat losses as the operating fluid (i.e. water $30 \%$ glycol) gets heated up by the sun. At the north-west corner of the plant, the hot water accumulation tank and the technical building are located. The plant is able to supply heat to approximately 350 households (i.e. 1200 inhabitants) covering as much as $28 \%$ of their annual heat demand during an average weather year. That way Solrød Fjernvarme is saving 233 tons of $\mathrm{CO} 2$ yearly. 


\section{CONTROLLING A DISTRIBUTED DISTRICT HEATING PLANT}

The SCADA system gives an overview of the different system components as well as various operational modes. The operational control philosophy of the plant in Havdrup has two main goals. It maintains a stable solar field outlet temperature not only in clear sunny days but in cloudy periods too. Additionally, it supplies the district heating network with an accurate forward temperature, in the range of $65-75^{\circ} \mathrm{C}$ depending the season. The control of the plant allows preheating early in the morning by recirculating the flow both in the primary and the secondary side, through the respective by-pass valves. Furthermore, it embeds two anti-freezing modes. The first one starts when the ambient temperature gets a few degrees lower than $0^{\circ} \mathrm{C}$. For more aggressive cold weather with ambient temperatures around $-10^{\circ} \mathrm{C}$ or below, the secondary anti-freezing mode kicks in, using heat either from the top of the tank or from the city grid. During normal operation, the plant has the possibility to supply directly the heat to the grid through the assistance of a so called "city pump".

5

Since the plant is intentionally overdimensioned there are instances especially during summer, that there is surplus of heat produced. This heat excess is directed to the top of the accumulation tank. As the top of the tank becomes hotter and hotter as the days pass by, it is important to maintain the stratification of the different temperature levels of the tank. Specifically, during morning hours when the plant has just completed preheating and the temperature of the hot water is still lower than both the top of the tank and the district heating, the flow is driven to the middle diffuser of the tank. These "middle" temperatures cannot be utilized and occupy significant space on the tank. For that reason, there is a shunting procedure. In cases, when the temperature coming from the secondary side of the heat exchanger is very high or at least higher than the desired temperature in the grid, the small shunt pump withdraws water from the middle of the tank to cool down the forward stream towards the city grid and get rid of these middle temperatures at the same time. For instances, when more than half of the tank is full with very high temperatures, the shunt pump withdraws cold water from the bottom of the tank and/or the return flow of the city. Last but not least, when the tank gets more than $3 / 4$ full of hot water of around $90^{\circ} \mathrm{C}$, then the operator gets a notification to manually operate the night cooling mode during the following night. In that way, significant part of the tank's hot content circulate at the solar field and eventually lose is energy to the surroundings. As a result there will be enough space to store the expected heat the coming day.

\section{CONCLUSION: THERMAL PERFORMANCE OF SOLRØD PLANT}

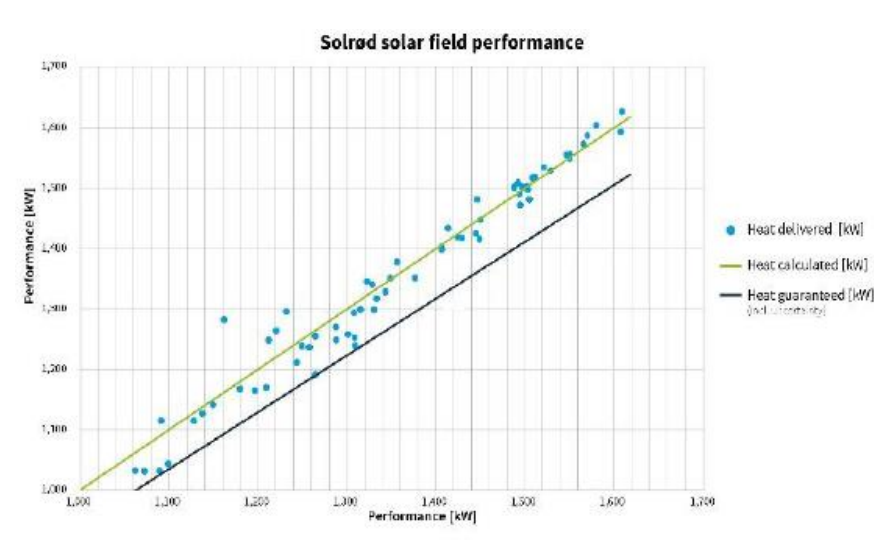

The solar plant is regulated to maintain an outlet temperature of around $95-97^{\circ} \mathrm{C}$ and has proven to do so with excellent precision, even in unstable weather conditions. The plant has a peak thermal output of 1.8 MWth and an estimated yearly production of approximately 1,300 MWh. In a clear summer day the solar field can produce up to $12 \mathrm{MWh}$ of heat.

The plant has been delivered to the client at the end of spring 2017 and as shown on Figure 2, it has been over-fulfilling its guaranteed performance. Until the end of

June 2017 the plant was performing 5\% better than the expected calculated performance.

\section{REFERENCES}

[1] 1 Million Square Meters Solar Thermal Collectors in Danish District Heating Plants. (2016, September 28). News and Articles.[URL: http://solar-districtheating.eu/NewsEvents/News/tabid/68/ArticleId/477/1-Million-Square-Meters-Solar-ThermalCollectors-in-Danish-District-Heating-Plants.aspx]

[2]. O. Miedaner, T. Pauschinger, J.O. Dalenbäck, "Categories of solar district heating systems" Solar District Heating guidelines, Solites, CIT Energy Management, Chalmers University, April 2012. 


\section{DEVELOPMENT OF OPTIMIZED CONTROL STRATEGIES FOR LARGE-SCALE SOLAR THERMAL PLANTS WITH ABSORPTION HEAT PUMPS AND SEASONAL PIT STORAGE \\ Christoph Moser \\ AEE - Institute for Sustainable Technologies \\ Feldgasse 19, 8200 Gleisdorf, Austria \\ Phone: +43 3112 / 5886241 \\ E-Mail: c.moser@aee.at}

\section{SUMMARY}

The aim of the project "sBSc - scaled BIG SOLAR control" is the development of an optimized control strategy for a cost-efficient operation of a large-scale solar thermal plant in combination with an absorption heat pump and seasonal pit storage for urban district heating support. A test setup will be set up in laboratory size to learn from a combined real system and simulation approach.

\section{INTRODUCTION}

The cost-efficient operation of big solar concepts like "BIG SOLAR Graz" (Reiter, Poier, \& Holter, 2016) with large-scale solar thermal plants with an absorption heat pump and seasonal pit storage is a major challenge. (Reiter et al., 2016) showed in a feasibility study a high potential for providing heat for the district heating network of Graz. Compared to other realized projects like SUNSTORE 3 (Jensen, 2014), the scale of "Big Solar Graz" is far greater even in the smallest considered size. The next challenge compared to SUNSTORE 3 is the higher needed flow temperature in Graz. It is necessary to minimize the risk of outage and malfunction and to optimize the control strategy for energy provision in an economical and technical point of view. The integration of an absorption heat pump and a large number of interconnections with other system areas represents a potential source of uncertainty. To minimize the implementation risks for "BIG Solar" projects and to guarantee an optimum operation it's essential to gain the appropriate control technologies beforehand.

Absorption heat pumps are mainly driven by thermal power compared to the electricity demand of the compressor of compression heat pumps, which allows a direct usage of renewable energy (biomass, biogas or waste heat).

\section{METHOD}

The system layout and the used components of the project "sBSc" are based on the proposed "BIG Solar Graz" system layout. This project arranges a large-scale solar thermal plant, seasonal pit storage, an absorption heat pump and auxiliary heating. Therefore a test facility with a small-scale setup with the main components and the hydraulic layout of "BIG Solar Graz" will be installed at the energy lab of AEE GmbH in Gleisdorf. A Hardware in the Loop (HiL) approach is used to model the seasonal thermal energy storage, the auxiliary heating, and the solar thermal plant. The scaled absorption heat pump has an equal working fluid $(\mathrm{LiBr}-\mathrm{H} 2 \mathrm{O})$, like in the desired heating pumps in the BSG concept. This heating pump is connected to the heating, cooling and electrical grid of the laboratory. According to the HiL-approach, the inlet temperatures and the mass flows of the heat pump is set to model the other main components.

Starting from the secured manufacturer operation temperatures of the components, the temperatures of the generator heat input and the cooling source will be varied to optimize the efficiency of the whole system. This setup has the ability to identify critical operational states and the operating and thermal limits of the absorption heat pump and find optimal operation strategies for the combined system.

\section{RESULTS}

In the laboratory of AEE GmbH, a test setup is equipped with an absorption heat pump. The other components of the BSG concept are modeled with the HiL-approach and give the input temperatures, and mass flows of the heating and cooling grid to supply the absorption heat pump. First test results are shown. 


\section{REFERENCES}

Jensen, M. V. (2014). Seasonal pit heat storages - Guidelines for materials \& construction, 31. Retrieved from http://task45.iea-shc.org/fact-sheets

Reiter, P., Poier, H., \& Holter, C. (2016). BIG Solar Graz: Solar District Heating in Graz - 500,000 m2 for 20\% Solar Fraction. Energy Procedia, 91, 578-584. http://doi.org/10.1016/j.egypro.2016.06.204 


\title{
INTELLIGENT CONTROLLING OF POWER DRIVEN SOLID BIOMASS CHP PLANTS IN FLEXIBLE DISTRICT HEATING WITH A SEASONAL HEAT STORAGE AND A POWER-TO-HEAT COMPONENT
}

\author{
Katharina KOCH, M.Sc. \\ Technical University of Munich \\ Associate Professorship of Regenerative Energy Systems \\ Schulgasse 16, 94315 Straubing, Germany \\ Phone: +49 9421 187-125 \\ E-Mail: katharina.jh.koch@tum.de
}

\section{SUMMARY}

The main work task is the simulation and optimization of an existing district heating system with a solid biomass CHP plant, by the intelligent integration of a power-to-heat component and a seasonal heat storage. For an efficient energy system, the flexible operation mode of the solid biomass CHP plant in order to be able to contribute in the energy markets is essential. In the focus of the simulation model is the optimized dimensioning and regulation of the different components in the energy system, in order to rise the resource efficiency (ecology, economy, efficiency) of the model. Concepts for a flexible heat supply with a high share of renewable energies will be developed and analysed in different scenario simulations. Based on input data from the metering of the modelled heating grid (district heating Grassau, Biomassehof Achental, Germany) and under the consideration of the energy prices (controlling market, SPOT Market), the program decides between different operation modes of the solid biomass CHP plant and determines the charging management of the seasonal heat storage.

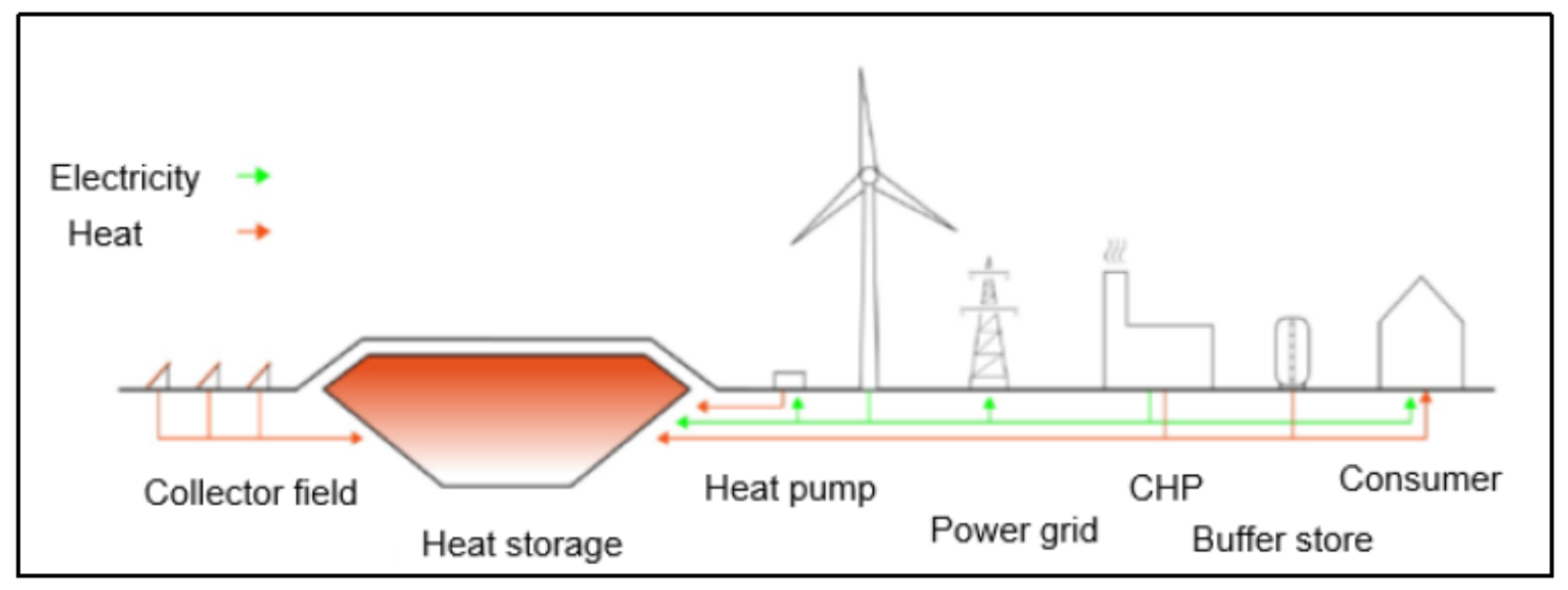

Fig. 1: Model concept for flexible district heating [1]

\section{PURPOSE OF THE WORK}

The goal of this work is to enlarge the simulation model of an existing district heating system with a seasonal heat storage and a power to heat component, in order to drive the CHP plant according to the prices of the energy markets. An optimized dimensioning and controlling of the different components in the mode, should lead to a resource efficient combined heat and power system.

\section{APPROACH}

The simulation model is generated with MATLAB. The input data for the validation of the model is removed from a metering system of the simulated district heating (district heating Grassau, Biomassehof Achental, Germany). For the optimization of the resource efficiency, numerical optimization algorithm like the monte carlo algorithm and the simulated annealing, will be used.

\section{SCIENTIFIC INNOVATION AND RELEVANCE}

With power-to-heat components and the seasonal heat storage it is possible to cut the overload in the power grid and to use this energy (power-to-heat) for the heat supply in district heating systems. The sector coupling reduces frequency and voltage fluctuations and ensure therefore the security of supply. 
This lead to lower costs for the TSO (Transition system operators) and reduces therefore the costs for the end costumers. The scientific relevance lies in the complex connection and controlling of different equipment components in order to obtain a resource optimized energy system.

\section{RESULTS AND CONCLUSIONS}

The simulation results should give recommendations for a practical, resource efficient realization of the described district heating system. Essential are the cost-optimized integration of energy price signals, the flexible operation mode of the CHP plant and the charging management of the seasonal heat storage.

\section{CONFERENCE TOPIC}

This abstract refers to the Conference Topic "Renewable Heating and Cooling in a future energy system"

\section{REFERENCES}

[1] Berberich, M., Deschaintre, L., Mangold, D., Schmidt, T., Willwerth, L., (2015), SOLAR-KWK Entwicklung multifunktionaler Systeme zur solar unterstützten Kraft-Wärme-Kopplung - solare Fernwärme und saisonale Wärmespeicher für die Energiewende, Forschungsbericht, SOLITES 


\title{
APPROACHES TOWARDS LOW ENERGY RESILIENT NEIGHBORHOODS - CASE STUDIES
}

\author{
DI Dr Anna M. Fulterer \\ AEE - Institute for Sustainable Technologies \\ Feldgasse 19, 8200 Gleisdorf, Austria \\ Phone: +43 3112 / 5886-325 \\ E-Mail: a.m.fulterer@aee.at
}

\section{SUMMARY}

The target is to analyse the role of energy master planning in the transition towards an efficient and renewable energy supply by studying specific cases of neighbourhoods that have undergone the transition to low energy. This is done by identifying the drivers of the process, the reasons for choosing specific technological solutions and the defined targets. Also the results of the process, enabling factors and identified obstacles are investigated. One important aspect will be whether resilience was a target and how resilience it has been addressed in the planning process. The results show how the successful integration into the existing infrastructure and energy systems depends on municipal energy master planning and how this supports the design process.

\section{INTRODUCTION}

It is now well known how to realize low energy buildings. This does however not mean that all buildings are being brought to this level. Renovation of buildings depends on many factors, like renovation costs and energy expenditures, financing schemes and decision processes. One important factor is also the energy supply system into which the building is integrated. Knowledge on options for energy supply, and local renewable energy potentials can allow reaching very low consumption levels or even plus energy in new buildings and enhance renovation processes. This data is however often not available to planners, just like an energy master plan is often missing. Thus, planners rely on standard solutions or aim at autarky to reduce the dependence from municipality and local energy providers. On the other hand, an energy master plan is expected to motivate towards high-level solutions and to even enable the creation of new energy networks that can also include buildings adjacent to the ones that are developed.

\section{CONTENTS AND TARGETS}

The case studies show how depending on data availability and existence of master plans resilient energy supply systems can be established in neighbourhoods. The target is to show what enhances and what hinders these processes, which tools are available for the design phase and which gaps have been identified during the processes.

\section{METHOD}

The data basis for this paper is the investigation of case studies on low energy neighbourhoods. Many of these cases are well studied since they were part of funded research programmes. Literature and data on them can be accessed easily. The second step will be the analysis of other aspects that are expected to have had an influence on the development of the low energy neighbourhood, as shown in Figure 1. A comparative analysis of the case studies and their context will lead to the aspired results. The aspect of resilience will be considered by identifying critical processes and analyse how they need to be supplied by energy. Moreover, we will analyse whether and how resilience and autarky have been addressed in the planning process. 
Fig. 1: The Figure shows how the initial case study of the low energy neighbourhood is analysed relating it to its

\section{RESULTS AND OUTLOOK}

Results will focus on the effect of the existence of a local energy master plan on the outcome of the development. Moreover we will lay out which obstacles have been identified in the specific development processes. 


\section{ADVANCED SHALLOW GEOTHERMAL ENERGY PRODUCTION AN INTODUCTION TO THE PROJECT GEOTHERMIE - MODELLREGION FÜRSTENFELD}

Nikolaus Petschacher1, Robert Gether2, Christian Luttenberger3, Wolfgang Weber4 \& Bernd Böchzelts 1Institute of Applied Geosciences, University of Technology, Rechbauerstraße 12/EG, A-8010 Graz 2Impulsregion Fürstenfeld, Augustinerplatz 1, A-8280 Fürstenfeld 3 Geosys, Grazerstraße 20, A-8200 Gleisdorf

4Energieregion Oststeiermark GmbH, St. Margarethen an der Raab 163, A-8321 St. Margarethen an der Raab 5Technisches Büro Böchzelt, Ludersdorf 69, A-8200 Gleisdorf

Phone: $+43 / 676 / 3871458$

E-Mail: petschacher@hydro.or.at

\section{SUMMARY}

By the means of an early data acquisition in combination with a prospective company settlement the near-surface geothermal potential of the region of Fürstenfeld shall be explored and made accessable. Based on extensive literature research, areas of interest were selected in which consequently subsurface explorations were carried out. The underground data serve as a base for a numerical model, which simulates the procedure of a well system in respect of sustainable and most efficient utilization of near-surface groundwater heating and cooling use.

\section{INTRODUCTION}

The main ambitions of the "Geothermie-Modellregion Fürstenfeld" are to identify the potential of geothermal energy in the region of Fürstenfeld, to build a concept of using an unused natural resource in the most reasonable way and to promote this geothermal potential to prospective companies. Especially in combination with existing infrastructure, for example a new highway is currently buildup through the region, the supposed geothermal potential could be a big factor and help develop the region in a long-term sustainable way.

Especially geothermal energy use of shallow groundwater is one of the most efficient ways to cool and heat buildings sustainably. However, the usage is strongly dependent on the sufficient availability of groundwater. Therefore, geological/hydrogeological examinations are necessary for the construction of bigger facilities. The project Geothermie-Modellregion Fürstenfeld is also focused on examining the advantage of data acquisition at an early stage in combination with a possible settlement of companies. Therefore, a numeral simulation of the facility process was created. The coupled flow and heat transport model should also clarify if it is possible to increase the efficiency of the well system, consisting of a production well and a slug well. Thereby, the focus will be on a possible positive use of the heat- and coldplume, which diffuses in the underground due to the operation of the groundwater well system (groundwater heat and cold storage).

\section{METHODOLOGY}

The data collection is based on the evaluation of 20 underground profiles as well as on an extensive web and archive inquiry. In order to obtain useful results, areas of interest were filtered out and subsequently underground examinations were taken trough trail pits and analyzed in order to get useful underground parameters.

The simulation of the geothermal well system was carried out by the means of a software (Visual MODFLOW Pro Version 2011.1 (2011) provided by Schlumberger Water Services Company (Waterloo, Canada).

\section{RESULTS}

In the range of the project area quaternary sediments as well as two different shallow aquifers (GWK Festritztal und GWK Ilz- und Rittscheintal) were located (Amt der Steiermärkischen Landesregierung, 2018). A first estimation of these aquifers in the region of Fürstenfeld showed that the existing aquifers do not feature homogenous underground conditions and thus cannot be considered for a largescale geothermal groundwater use. Due to this reason, areas with favourable hydrogeological underground conditions in connection to the already existing company settlement plan were determined. The assessment of an existing underground profile (fig. 1) showed that an aquiferous gravel layer at a depth of 2 to 6 meters below ground level in the region of the new S7 highway is apparent. Theses gravels are expected to be strongly permeable with hydraulic conductivity between 
$1 \mathrm{E}-3$ and $5 \mathrm{E}-3 \mathrm{~m} / \mathrm{s}$. Under the assumption that the groundwater is taped and the drawdown of the water table is 2 to $4 \mathrm{~m}$ it is possible to calculate the groundwater production rate as a function of the hydraulic conductivity (cited in Hölting \& Coldewey, 2004) by Darcy's law. (eq. 1). Figure 2 illustrates the groundwater production rate as a function of the hydraulic conductivity. Depending on the real hydraulic conductivity, flow rates between 6 and 30 1/s can be expected. Subsequently this results in thermal output between 125 and $627 \mathrm{~kW}$ by a temperature spread of $5^{\circ} \mathrm{C}$.

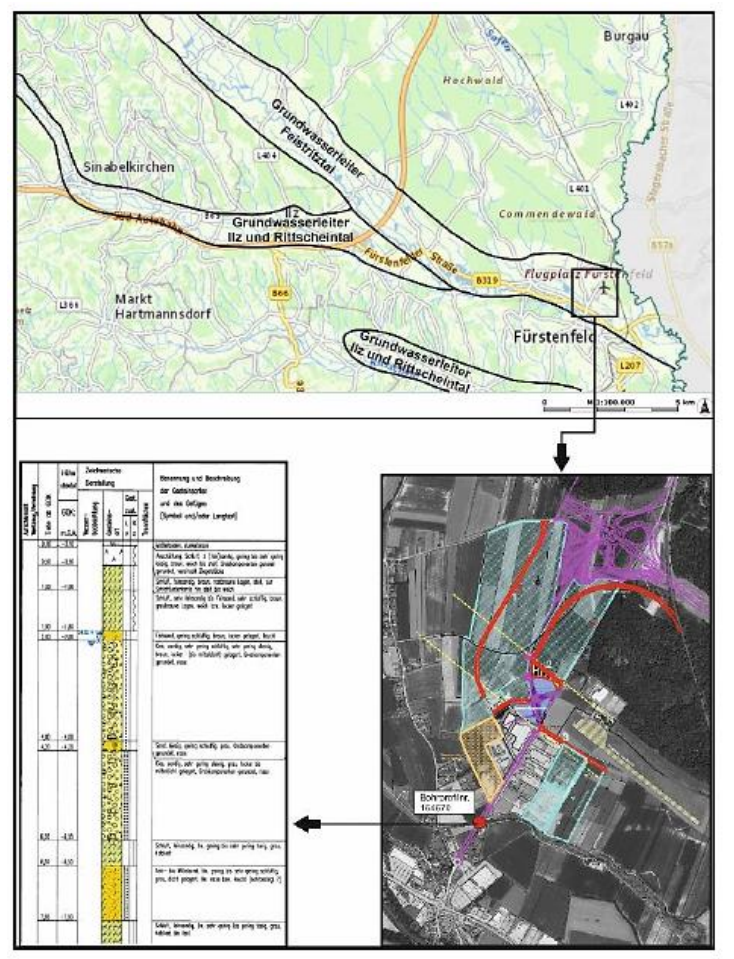

Figure 1: Areas with favorable hydrogeological underground conditions in connection to the already existing company settlement incl. underground profile.

As depicted in figure 2 the groundwater production rate and subsequently the thermal output are strongly dependent on the hydraulic conductivity of the underground. Due to this reason and in order to define the hydraulic conductivity the next step was to take rock samplings and a grain size analysis of the aquifer in the area of interest.

Also trail pits were carried out and samples were taken. (Fig. 3).

The sediment in the depth of the aquifer can be described as silty, gravelly, strong stony, coarse sand with an expected groundwater thickness of $5,5 \mathrm{~m}$. (Fig. 3). Because of bad sorting of the sediment, it was not possible to get a value of the hydraulic conductivity by grain size analysis. According to experience, it was supposed that the sediment has a hydraulic conductivity of around $1 \mathrm{E}-3 \mathrm{~m} / \mathrm{s}$.

$$
\begin{array}{ll}
Q=k f * h m * s & \begin{array}{l}
\text { (eq. 1; Hölting \& } \\
\text { Coldewey, 2004) }
\end{array} \\
& \\
Q & \text { Groundwater production rate }[1 / \mathrm{s}] \\
k f & \text { Hydraulic conductivity of the underground }[\mathrm{m} / \mathrm{s}] \\
s & \text { Groundwater drawdown under static water level[m] } \\
h & \text { Drawn down water level over bottom }[\mathrm{m}]
\end{array}
$$

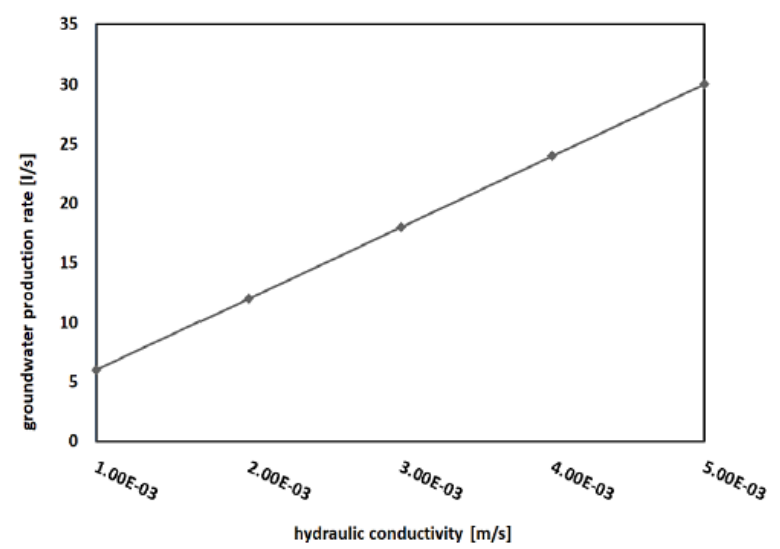

Figure 2: Groundwater production rate $[1 / \mathrm{s}]$ as a function of the hydraulic conductivity $[\mathrm{m} / \mathrm{s}]$ at drawdown of the water table from 2 to $4 \mathrm{~m}$ below ground level. Calculated by Darcy's law.

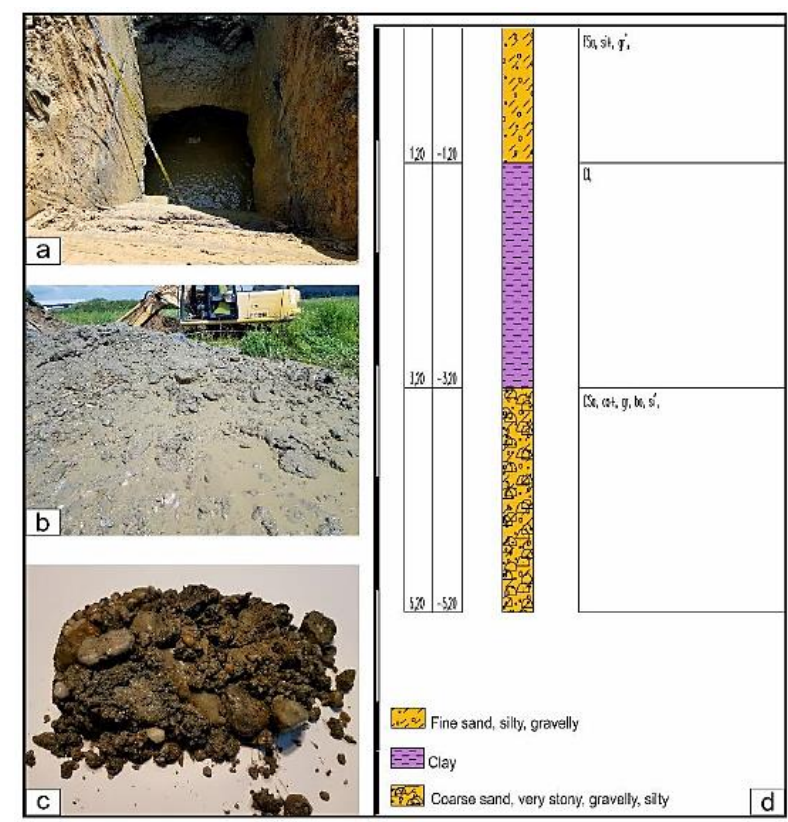

Figure 3: a, trail pit with line of sight to the aquifer. b, excavation material. c, silty, gravelly, strong stony, coarse sand form the aquifer. d, lithological profile 


\section{The numerical model}

The setup of the numerical flow model according to the method of finite differences was carried out by the means of the software Visual MODFLOW Pro Classic Interface (2013) from the company Schlumberger Water Services (Waterloo, Canada).

The total model area was defined as rectangle by the following corner points:

$\mathrm{X} 1=729927 \mathrm{Y} 1=213535$ (bottom left)

$\mathrm{X} 2=659670 \mathrm{Y} 2=213650$ (top right)

Consequently, the area of the total active model area is (1000 $\mathrm{m} \times 1500 \mathrm{~m}) 1,5 \mathrm{~km} 2$.

On the basis of available hydrogeological data the aquifer was represented by a model layer in which vertical homogenous hydraulic attributes were assumed. The cell size of the grid is $5 \times 5 \mathrm{~m}$. In consideration of the size of the model region, the amount of available data and the predicted assumptions about the groundwater conditions the cell width offers an adequate resolution. The overlaid grid of the model region was approximately positioned in direction of WSW-ENE.

Under stationary hydraulic boundary conditions for average groundwater conditions, the unsteady groundwater

flow due to groundwater extraction and infiltration as well as the transient thermal spread of the temperature plume due to introduction of heated and cooled groundwater were calculated over two years.

The two interfaces of the model area in vertical direction are the ground level and the surface of the aquiclude. Due to the schema model the ground level was constantly defined with $252 \mathrm{~m}$. a. s. 1 .

\section{Input data:}

The assumed hydraulic and thermal aquifer parameters (aquifer material: gravel) which were used for the simulation calculations are summarized in Table 1 and 2.

Table 1: hydraulic parameter

\begin{tabular}{|c|c|c|}
\hline Parameter & Value & Unit \\
\hline hydraulic conductivity, $\mathrm{k}_{\mathrm{f}}$ & $1,0 \times 10^{-3}$ & $\mathrm{~m} / \mathrm{s}$ \\
\hline effectiv porosity $\mathrm{n}_{\mathrm{e}}$ & 0,18 & - \\
\hline total porosity $\mathrm{n}$ & 0,21 & - \\
\hline bulk density $\rho_{\mathrm{b}}$ & 1940 & $\mathrm{~kg} / \mathrm{m}^{3}$ \\
\hline longitudinal dispersivity $\mathrm{a}_{\mathrm{L}}$ & 5 & $\mathrm{~m}$ \\
\hline transversal dispersivity $\mathrm{a}_{\mathrm{T}}$ & 0,5 & $\mathrm{~m}$ \\
\hline thermal distribution coeffivient $\mathrm{K}_{\mathrm{d}}$ & $2,035 \times 10^{-7}$ & $1 /(\mathrm{mg} / \mathrm{L})$ \\
\hline thermodiffusion $\mathrm{D}_{\mathrm{Th}}$ & $6,204 \times 10^{-7}$ & $\mathrm{~m} / \mathrm{d}$ \\
\hline
\end{tabular}


Table 2: material parameter

\begin{tabular}{|l|c|c|c|c|c|}
\hline \multirow{2}{*}{ Property } & $\begin{array}{c}\text { bulk density } \\
\mathbf{\rho b}\end{array}$ & $\begin{array}{c}\text { Thermal conductivity } \\
\lambda\end{array}$ & $\begin{array}{c}\text { sp. heat capacity } \\
\mathbf{c}\end{array}$ & $\begin{array}{c}\text { total porosity } \\
\mathbf{n}\end{array}$ & $\begin{array}{c}\text { effectiv porosity } \\
\mathbf{n}_{\mathbf{e}}\end{array}$ \\
\hline material & {$\left[\mathbf{k g} / \mathbf{m}^{3}\right]$} & {$[\mathbf{W} / \mathbf{m} \cdot \mathbf{K}]$} & {$[\mathbf{J} / \mathbf{k g} \cdot \mathbf{K}]$} & {$[-]$} & 0,18 \\
\hline gravel & $1900-2300$ & $1,6-2,0$ & $840-864$ & 0,21 & \\
\cline { 2 - 5 } chosen: & 1940 & 1,8 & 850 & & - \\
\hline water & 999,4 & 0,63 & 4180 & & - \\
\hline
\end{tabular}

The heat retardation factor indicates how much slower the heat plume moves compared to the groundwater flow rate due to the heat exchange with the surrounding rock (heat storage). The factor is expressed through the ratio of the two velocities.

$$
\mathrm{R}=1+\frac{(1-n) \cdot c_{G} \cdot \rho_{G}}{n \cdot c_{W} \cdot \rho_{W}}=1+\frac{c_{G} \cdot \rho_{b}}{n \cdot c_{W} \cdot \rho_{W}}=1+\frac{850 \cdot 1940}{0,18 \cdot 4180 \cdot 999,4}=2,19
$$

To represent the thermal equilibrium between water and rock, the thermal distribution coefficient is defined and calculated as follows:

$$
\mathrm{K}_{\mathrm{d} \text { temp }}=\frac{\mathrm{c}_{\mathrm{G}}}{\mathrm{c}_{\mathrm{w}} \cdot \rho_{\mathrm{w}}}=\frac{850}{4180 \cdot 999,4}=2,035 \cdot 10^{-4} \frac{\mathrm{m}^{3}}{\mathrm{~kg}}
$$

The heat conduction in the aquifer (thermal diffusion) is taken into account by the heat diffusion coefficient due to temperature gradients. Thereby the thermal conductivity of the aquifer (rock and water) is calculated as a geometric mean from the thermal conductivity of the rock and the water.

$$
\begin{aligned}
& D_{\mathrm{Th}}=\frac{\lambda_{\mathrm{V}}^{n} \cdot \lambda_{\mathrm{G}}^{1-n}}{(1-n) \cdot P_{\mathrm{G}} \cdot \mathrm{c}_{\mathrm{G}}+\mathbf{n} \cdot \mathrm{P}_{\mathrm{V}} \cdot \mathrm{c}_{\mathrm{W}}}=\frac{\lambda_{\mathrm{W}}^{n} \cdot \lambda_{\mathrm{S}}^{1-n}}{P_{\mathrm{b}} \cdot \mathrm{c}_{\mathrm{G}}+\mathrm{n} \cdot \mathrm{PW}_{\mathrm{W}} \cdot \mathrm{c}_{\mathrm{W}}}=\frac{0,63^{0,18} \cdot 1,8^{1-0,18}}{1940 \cdot 850+0,18 \cdot 999,4 \cdot 4180}= \\
& =\frac{0,920 \cdot 1,619}{1649000+751948}=6,204 \cdot 10^{-7} \mathrm{~m}^{2} / \mathrm{s}
\end{aligned}
$$

The outer boundary conditions of the model are fixed potential margins of the groundwater level in WNW at $-1.25 \mathrm{~m}$ below ground level and in OSO at $-2.75 \mathrm{~m}$ below ground level. Thus, for the WSW potential border a height of $250.75 \mathrm{~m}$ above sea level and for the ONO potential border $249.25 \mathrm{~m}$ above sea level were assumed. The $\mathrm{N}$ and $\mathrm{S}$ boundaries of the model area were supposed to be streamlines and therefore act as no flow boundary (no flow boundary). The thickness of the aquifer was assumed to be constant at $5.5 \mathrm{~m}$. In the model area, only the injection wells with the associated injection rate were considered.

If the formulation of the Darcy law describing the filtration velocity assumes that the permeability is depending of the kinematic viscosity and the standpipe height is depending on the density of the water, the reformulation of Darcy's law is required in the following form: 
$k_{f}=-\frac{k_{0}}{\rho_{W} \cdot v_{W}}\left(\nabla p-\rho_{W} \cdot g\right)$

eq. 4

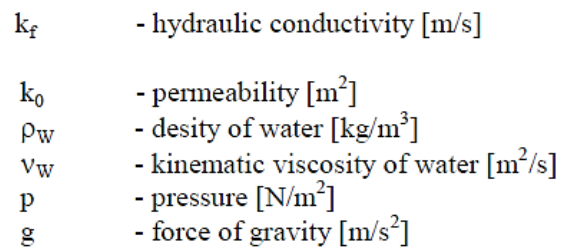

The simulation is based on the coupled solution of the differential equations of the groundwater flow and the heat transport in the aquifer. It was assumed that groundwater from WNW direction flows at constant temperature of $8.0^{\circ} \mathrm{C}$ over the whole year. Therefore, a fixed thermal potential at $8.0^{\circ} \mathrm{C}$ was defined the WNW. This groundwater temperature was constantly assumed for a 2 year period of the simulation. An internal boundary condition represents the variable temperature of the removed process water. The cooling and heating periods with their infiltration rates and temperatures are shown in Table 3. The seasonal temperature changes were defined as a point source in the cell of the slug well.

Table 3: Groundwater production rate and the thermal utilization due to the heat pump over 2 years.

\begin{tabular}{|c|c|c|c|c|c|}
\hline month & $\begin{array}{l}\text { days } \\
\text { from }\end{array}$ & $\begin{array}{l}\text { days } \\
\text { to }\end{array}$ & infiltration rate (l/s) & $\begin{array}{l}\Delta \mathrm{T} \\
\left({ }^{\circ} \mathrm{C}\right)\end{array}$ & $\begin{array}{l}\text { T water } \quad\left({ }^{\circ} \mathrm{C}\right) \\
\left(\text { Tref }=8.0^{\circ} \mathrm{C}\right)\end{array}$ \\
\hline 1. heating period & 0 & 182.5 & 7,0 & -4 & 4 \\
\hline 1.cooling period & 182.5 & 365 & 7,0 & 4 & 12 \\
\hline 2. heating period & 365 & 547.5 & 7,0 & -4 & 4 \\
\hline 2. cooling period & 547.5 & 730 & 7,0 & 4 & 12 \\
\hline
\end{tabular}




\section{Results of the numerical model}

Figure 4 and 5 shows the expansion of the thermal plume over a time period of 2 years.

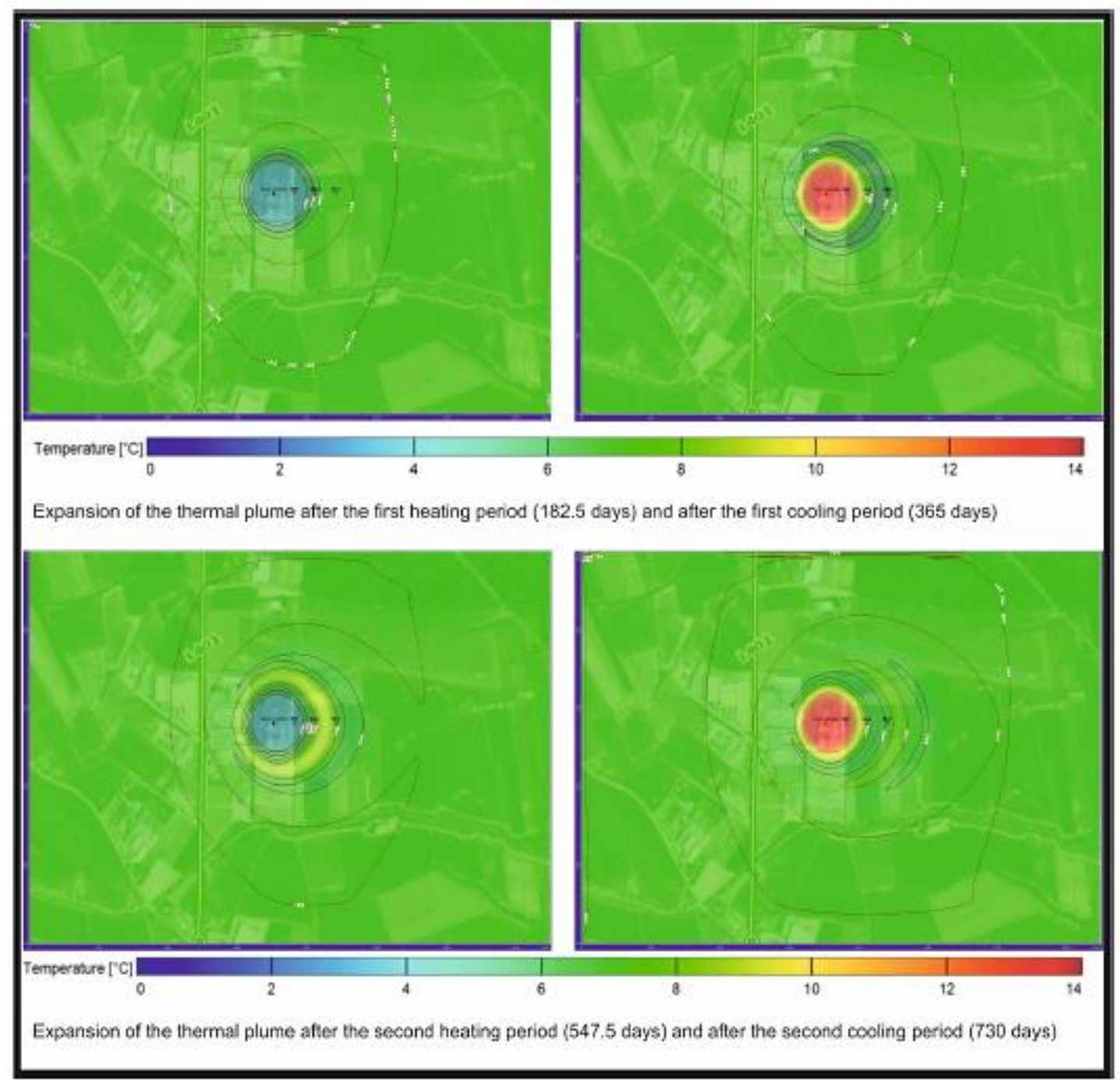

Figure 4: Expansion of the thermal plume over a time period of 2 years.

It is visible that the maximum heating or cooling takes place at a distance of $60 \mathrm{~m}$ from the infiltration site at the end of each heating or cooling period (maximum temperature spread after half a year). These results in temperatures of $12{ }^{\circ} \mathrm{C}$ in $60 \mathrm{~m}$ distance from the infiltration site after the cooling periods and $4{ }^{\circ} \mathrm{C}$ after the heating periods at initial temperature of $8{ }^{\circ} \mathrm{C}$. The further one moves away from the infiltration site, the more abated and delayed is the temperature change (see figure 5). At 120 meter distance from the infiltration site,

temperatures of $10.5^{\circ} \mathrm{C}$ after cooling periods and of $5^{\circ} \mathrm{C}$ after heating periods in 270 days are calculated. At a 225 distance of $180 \mathrm{~m}$, the maximum heating is only $9^{\circ} \mathrm{C}$ and the maximum cooling only $7^{\circ} \mathrm{C}$ in 400 days. 


\section{GIS BASED ANALYSIS OF POTENTIAL FOREST RESIDUES FOR ENERGY IN ALENTEJO, PORTUGAL}

$\underline{\text { Paulo Mesquita }^{1}}$, Ricardo Nepomuceno Pereira $^{1}$, Isabel Malico ${ }^{1,2}$, Ana Cristina Gonçalves ${ }^{3}$, Adélia O. M. Sousa ${ }^{3}$ Universidade de Évora, Escola de Ciências e Tecnologia ${ }^{1}$

R. Romão Ramalho, 59, 7000-671 Évora, Portugal

Instituto Superior Técnico, Universidade de Lisboa, LAETA, IDMEC ${ }^{2}$

Av. Rovisco Pais, 1, 1049-001 Lisboa, Portugal

Universidade de Évora, Instituto de Ciências Agrárias e Ambientais Mediterrânicas (ICAAM) ${ }^{3}$

Apartado 94, 7002-554 Évora.

Phone: (+351) 266760823

E-Mail: pmesquita@uevora.pt

\section{SUMMARY}

The present work evaluates the quantity and spatial distribution of potential forest residues in Alentejo, the largest geographic region in Portugal. Forest residues from the five main species in Portugal, tasmanian blue gum, cork oak, maritime pine, holm oak and stone pine, were assessed. The characterization of the biomass residues was performed in a Geographical Information System environment using the Portuguese land cover map and residues production rates from the available literature sources. The calculations were executed for every cell of a $250 \mathrm{~m}$ cell size grid. The total potential residues obtained was around 330,000 dry ton/year, with 172,000 dry ton/year being from cork oak. The residues spatial distribution occurs mainly in the western and north-western parts of Alentejo. This area was also the only one to achieve values of residual biomass above 80,000 dry ton/year for a $35 \mathrm{~km}$ radius collection area.

Keywords: GIS, solid biomass, forest residues, energy.

\section{INTRODUCTION}

\section{Solid biomass energy}

Global energy dependency has been satisfied through the use of fossil energy sources such as coal, oil and natural gas. The choice for the use of a particular energy source is still mainly driven by its price, although the climate framework is becoming a critical factor. Among the priority actions to carry out the energy transition driven by the increasing need for decarbonization, energy leaders reported that the use of renewable energy sources is the measure with the highest impact on decision-making (World Energy Council, 2017). Since the beginning of the millennium, policies have been established to support renewable energy sources, but they have been mainly directed towards power generation (REN21, 2017). Integration of renewables into the remaining sectors needs to be done under a broader concept of renewable-based energy system, which brings together the relevant aspects when planning energy systems and infrastructures, financing schemes and policy development (REN21, 2017).

World renewable energy share on global final energy consumption was estimated in 2015 to be $19.3 \%$. $73.1 \%$ of this contribution came from biomass ( $66.3 \%$ was consumed in the heating sector, $2.1 \%$ in the power sector and $4.7 \%$ in the transport sector (REN21, 2017). The global primary energy supply of biomass was almost entirely from solid biomass resources such as fuelwood, charcoal, forest, agriculture and industry residues, municipal solid wastes and pellets, which together contributed with $92 \%$ of the total biomass supply; the remaining came from biogas (2\%), biodiesel (2\%) and bioethanol (4\%) (World Energy Council, 2017). In EU28, renewable energy share on total gross inland consumption (TGIC) of energy was 13\% in 2016, of which 65\% came from biomass (Eurostat, 2018). 
According to AEBIOM (2017), in Europe in 2015, $74 \%$ of biomass was consumed for heating, $14 \%$ for power generation and $12 \%$ for transport. The contribution of solid biomass (including charcoal and renewable municipal wastes) to TGIC of biomass energy was 77\% (Eurostat, 2018).

In Portugal, renewables play a very important role since they represented, in 2016, 97\% of the total primary energy production (TPEP), $5823 \mathrm{ktoe}$, and the remaining came from non-renewable wastes (Eurostat, 2018). Portugal does not have primary production of fossil fuels and nuclear energy is not exploited. The biomass role in Portugal is significantly relevant, with a 51\% share on TPEP from renewables. Solid biomass primary production was 2.7 Mtoe in 2016, representing $87 \%$ of the biomass production. The solid biomass fuels mostly produced in the country were fuelwood, wood residues, agriculture and forestry residues, briquettes and pellets (53\% of the TPEP). Black liquor was also relevant (39\% of the TPEP) and renewable municipal solid wastes accounted for $4 \%$ of the TPEP from solid biomass (Eurostat, 2018; DGEG, 2018).

The TGIC of solid biomass in Portugal was 2.5 Mtoe in 2016, representing about $15 \%$ of the Portuguese TGIC of energy (transport sector not included) (DGEG, 2018). Figure 1 presents the TGIC of solid biomass in Portugal per type of end-use, sector and source. It can be seen that almost half of the consumption occurs in combined heat and power (CHP) generation systems used in the pulp and paper and wood products industries. It should be noted that the black liquor share on solid biomass consumption was $42 \%$ and as a waste from pulp and paper manufacture it is produced and consumed inside the own industry. In stand-alone systems, solid biomass is mainly consumed in the domestic sector for heating purposes and in the electric sector.

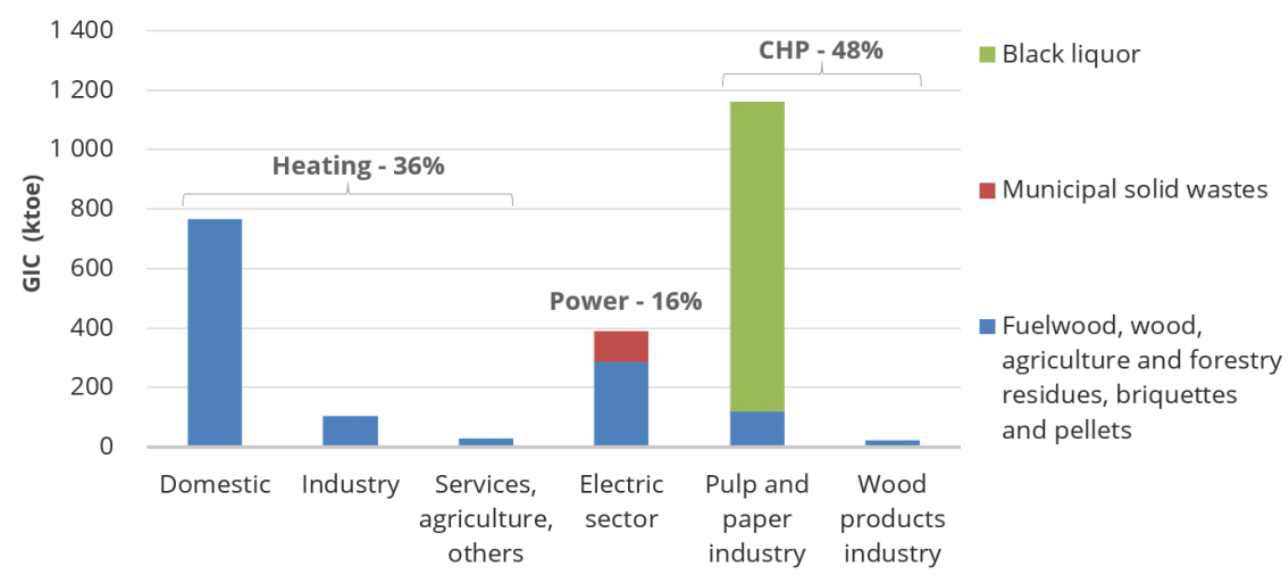

Figure 1: Portuguese gross inland consumption of solid biomass per sector/end-user in 2016. (DGEG, 2018).

\section{Portuguese forest}

According to the preliminary results of the $6^{\text {th }}$ Portuguese National Forest Inventory (ICNF, 2013), $35 \%$ of the land use in Portugal in 2010 was forest, with 3,154,800 ha. The same report presents a net loss of forest area of 5\% since 1995. Despite the reduction of forest area and due to the several disturbances, it is highlighted that this value represents a strong resilience of the Portuguese forest. Forest fires that consumed around 2,500,000 ha (between 1990 and 2012), and pine wilt disease that impacted severely maritime pine forest. This assessment allows to define the Portuguese forest as a relatively stable resource in the last two decades.

Tasmanian blue gum (Eucalyptus globulus) is the main forest species in area with 811,943 ha, followed by cork oak (Quercus suber) with 736,775 ha, maritime pine (Pinus pinaster) with 714,445 ha, holm oak (Quercus rotundifolia) with 331,179 ha, and stone pine (Pinus pinea) with 175,742 ha (ICNF, 2013). These five species constitute around $90 \%$ of the total Portuguese forest area.

\section{GIS and biomass residues studies}


Some studies have been conducted worldwide with the objective of quantifying, for a given geographic unit, the potential of biomass residues and their possible allocation to energy production. For example, Vasco and Costa (2009) used thematic cartography and forest growth data to quantify the forest biomass residues in a province of Mozambique (Maputo); López-Rodríguez et al. (2009) quantified and mapped the bioenergy potential of the most representative species in a region of Spain (Cáceres); Gómez et al. (2010) assessed the energy potential of agricultural and forest residues in Spain.

In Portugal, Viana et al. (2010) quantified at a national scale, with discriminated data for smaller geographic units, the forest residues of maritime pine and tasmanian blue gum; Fernandes and Costa (2010) and Lourinho and Brito (2014) quantified the agricultural and forest residues for smaller administrative regions.

Although these studies have different degrees of complexity and follow different approaches regarding the biomass residues that are considered, and the scale and detail of the geographical region of interest, all of them use Geographical Information Systems (GIS) based methodologies to quantify biomass residues potential to energy. Voivontas et al. (2001) state that GIS provide the means to identify and quantify the parameters related with the available and technological biomass potential. Lourinho and Brito (2014) point that the possibility of data manipulation and analysis with a spatial component allowed in GIS environments can be considered one of the principal characteristics of such systems.

\section{Current study}

This study focused on the primary residues, residues derived from forest management activities, of the five most common forest species in Portugal.

The study area, the Alentejo region, is the largest province in Portugal and although there are assessments of biomass residues of particular forest species for the entire region (Viana et al., 2010), or for smaller regions (Fernandes and Costa, 2010; Lourinho and Brito, 2014), no studies exist with high spatial detail regarding forest biomass residues for energy of all main forest species for the entire region.

The methodology to determine the potential production of forest residues was based on the studies of Lourinho and Brito (2014) and Fernandes and Costa (2010), where land cover information was used in conjunction with residues production rates for different forest species to evaluate the annual residues production for a part of Alto Alentejo region in Portugal.

The available biomass as a resource for energy is usually calculated for a collection area, around the geographical location of the biomass consumption facility. The collection area is related with biomassassociated costs (Viana et al., 2010). This author determined in the same study a radius of $35 \mathrm{~km}$ for a 9 MW power plant that would use maritime pine and tasmanian blue gum. In this study the same radius was used to assess the potential biomass available across the study area.

The objectives of this study are 1) to assess, for a square grid with $250 \mathrm{~m}$ cell size, the potential of the forest residues for the five main forest species (holm oak, cork oak, tasmanian blue gum, maritime pine, stone pine) in Alentejo; and 2) to map the availability of these residues at a radius of $35 \mathrm{~km}$ for each one of the grid cells.

\section{STUDY AREA}

Alentejo is the largest province in Portugal with $27,000 \mathrm{~km}^{2}$, which represent around $30 \%$ of the country continental area, and has a forest area superior to $11,000 \mathrm{~km}^{2}$ (DNGF, 2010). It is divided in four NUTSIII regions: Alto Alentejo, Alentejo Central, Alentejo Litoral, and Baixo Alentejo. Figure 2 presents the location of Alentejo, its sub-regions and terrain. The maximum elevation in the region is around $1000 \mathrm{~m}$, in the north-eastern part of Alto Alentejo, this region and Alentejo central have the 
highest elevations and the most irregular terrain. Alentejo Litoral and Baixo Alentejo have the lowest elevations and more regular and plain terrain. The climate is Mediterranean, with hot and dry summers in the majority of the region.

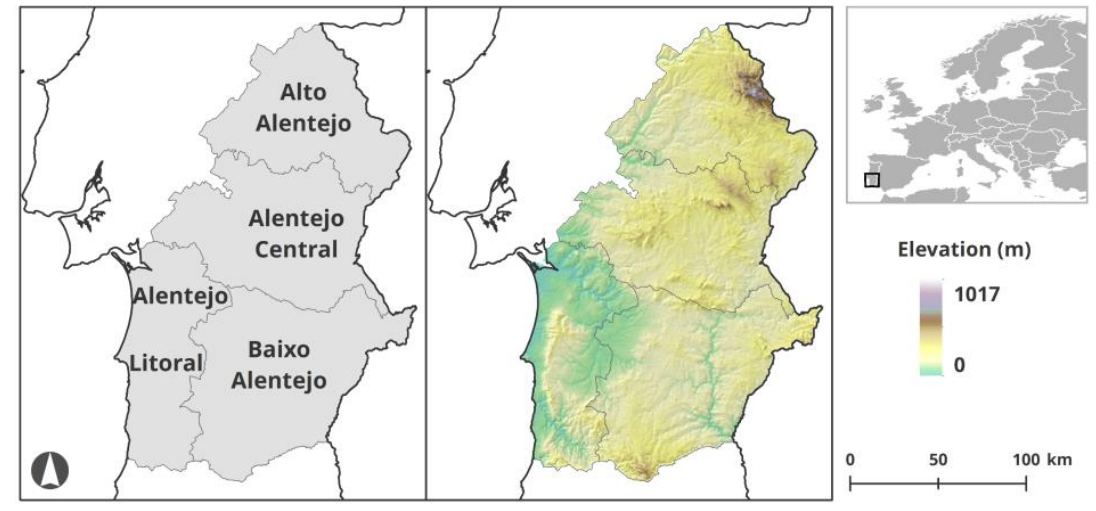

Figure 2: Study area location, sub-regions and terrain.

\section{METHODS}

The biomass residues potential was estimated in a GIS environment using spatial analysis techniques. The land cover map of Portugal from 2010 (COS2010V1.0), (Direção-Geral do Território, 2016) was used as the source of the geographic information about the forest species in evaluation. This information has an hierarchical nomenclature with five levels with 255 classes in the last level, the minimal cartographic unit is 1 ha, the thematic accuracy is better than $85 \%$ and the geometric accuracy is better than $5.5 \mathrm{~m}$ (Sarmento et al., 2016). As identified by Lourinho and Brito (2014), the polygon areas obtained from the land cover map do not translate directly to the effective area occupied by each forest species. This occurs because land cover classes can contain multiple species with variant degrees of vegetation cover within and between classes. The effective cover area for each species was determined as a function of the forest types and species vegetation cover. With this purpose, 56 land cover classes related with the forest species of interest were identified and reclassified into 17 forest types that aggregate information about the species type (hardwood for both the oak species and tasmanian blue gum, and softwood for both pine species), composition (e.g., pure, mixed), and structure (e.g., open forest). Table 1 presents the considered forest types and the respective values used in the calculations. The horizontal projection of the vegetation (HPV) represents the percentage of vegetation cover in a polygon of a given forest type, the percentage of the first species represents the percentage of the vegetation cover attributed to the first species, and the percentage of the second species represents the vegetation cover attributed to the second species (Lourinho and Brito, 2014).

Table 1: Forest types and respective parameters. (Lourinho and Brito, 2014).

\begin{tabular}{|l|c|c|c|}
\hline \multicolumn{1}{|c|}{ Forest type } & HPV & $\begin{array}{c}\text { \% First } \\
\text { Species }\end{array}$ & $\begin{array}{c}\text { \% Second } \\
\text { Species }\end{array}$ \\
\hline Agroforest system with pastures & 55 & 25 & 25 \\
\hline Agroforest system with permanent crops & 55 & 25 & 25 \\
\hline Agroforest system with temporary irrigated crops & 55 & 25 & 25 \\
\hline Agroforest system with temporary non-irrigated crops & 55 & 25 & 25 \\
\hline Clear cuts and new plantations & 5 & 100 & \\
\hline Hardwood forest & 65 & 100 & \\
\hline Hardwood open forest & 20 & 100 & \\
\hline Mixed forest of hardwood and softwood & 65 & 75 & 25 \\
\hline Mixed forest of softwood and hardwood & 65 & 75 & 25 \\
\hline Mixed hardwood forest & 65 & 62.5 & 37.5 \\
\hline Mixed hardwood open forest & 20 & 75 & 20 \\
\hline
\end{tabular}




\begin{tabular}{|l|c|c|c|}
\hline Mixed open forest of hardwood with softwood & 20 & 75 & 25 \\
\hline Mixed open forest of softwood with hardwood & 20 & 75 & 25 \\
\hline Mixed softwood forest & 65 & 62.5 & 37.5 \\
\hline Mixed softwood open forest & 20 & 62.5 & 37.5 \\
\hline Softwood forest & 65 & 100 & \\
\hline Softwood open forest & 20 & 100 & \\
\hline
\end{tabular}

These values are multiplied by the area of each forest type for each cell to obtain the effective cover area. A conservative approach was used, using minimal values for the percentages of vegetation cover of agroforestry systems that can be very heterogenous regarding composition and vegetation cover.

The potential quantity of residues was determined with the residues productivity rates presented in Table 2. These values were multiplied by the effective vegetation cover areas for each species present in a given cell. The land cover map contains information about the main forest species present in a given class, but sometimes the secondary species is not discriminated. A conservative approach was also used when calculating the biomass residues production of secondary species, unknown secondary species were not considered.

The biomass availability was calculated for a collection area of $35 \mathrm{~km}$. For each cell the sum of all residues at that radius was determined.

Table 2: Management conditions and residues productivity for each forest specie. (Lourinho and Brito, 2014).

\begin{tabular}{|l|l|}
\hline Forest species & \multicolumn{1}{|c|}{ Residue productivity } \\
\hline Holm oak & $\begin{array}{l}\text { Pruning of a tree every 20 years and thinning of } 0.20 \text { trees/ha every year with a biomass } \\
\text { yield of 5 dry t/ha and 0.22 dry t/ha/year; total biomass yield of } 0.48 \text { dry t/ha/year }\end{array}$ \\
\hline Cork oak & $\begin{array}{l}\text { Moderate pruning of } 70 \text { trees/ha every } 9 \text { years and thinning of a tree/ha every year with a } \\
\text { respective biomass yield of } 2.50 \text { dry t/ha and } 0.75 \text { dry t/ha/year; total biomass yield of } 1.0 \\
\text { dry t/ha/year }\end{array}$ \\
\hline $\begin{array}{l}\text { Tasmanian blue } \\
\text { gum }\end{array}$ & $\begin{array}{l}\text { Selection of logs after the first revolution with biomass yield of } 14 \text { dry t/ha; total biomass } \\
\text { yield of } 0.88 \text { dry t/ha/year }\end{array}$ \\
\hline Maritime pine & $\begin{array}{l}\text { Pruning and thinning at } 10 \text { and } 15 \text { years of age with biomass yield of } 12 \text { dry t/ha and } 5.2 \text { dry } \\
\text { t/ha; trimming at next term with biomass yield of } 20 \text { dry t/ha; total biomass yield of } 1.0 \text { dry } \\
\text { t/ha/year }\end{array}$ \\
\hline Stone pine & Biomass yield of 0.85 dry t/ha/year \\
\hline
\end{tabular}

\section{RESULTS AND DISCUSSION}

Figure 3 presents the potential biomass residues spatial distribution. Cork oak had the largest amount of potential residues with around 172,000 dry ton/year distributed mainly in Alentejo Litoral and in the north-western limits of Alentejo Central and Alto Alentejo. Holm oak and tasmanian blue gum had similar total values of potential residues, around 54,000 dry ton/year each, however the residual biomass potential distribution between the two species is very different. Holm oak biomass is extensively distributed in the region (except in Alentejo Litoral) and tasmanian blue gum is concentrated in fewer locations but with higher residues potential. Stone pine and maritime pine had the lowest residues values, respectively around 28,000 dry ton/year and 21,000 dry ton/year. Both these species potential biomass is mainly located in the northern part of Alentejo Litoral, with maritime pine being in the western part near the ocean and stone pine in the interior.

The combined amount of forest residues obtained in Alentejo was 330,000 dry ton/year, with only around 6,000 dry ton/year from holm oak as secondary species. This very low value can be attributed to the very conservative approach used in the calculations of secondary species residues. The totals per sub-region are: Alentejo Litoral 123,000 dry ton/year; Alto Alentejo 78,000 dry ton/year; Alentejo Central 75,000 dry ton/year; Baixo Alentejo 53,000 dry ton/year. The map with the potential biomass for all species shows that the distribution of the residues occurs mainly in the west and northwest areas of Alentejo. 
The obtained results are concordant with the results of other studies for smaller areas within Alentejo or for fewer species. Viana et al. (2010) estimated, with a different methodology, the residues for maritime pine and tasmanian blue gum in Alentejo between a minimum of 25,795 dry ton/year, a maximum of 85,551 dry ton/year, and mean value of 55,658 dry ton /year. Our results for both those species was around 75,000 dry ton/year, therefore within the expected interval. The values for each of the four geographic divisions were also very similar to the results obtained by Viana et al. (2010). Lourinho and Brito (2014) estimated around 33,500 dry ton/year of residues for the five species in analysis, in their study for some municipalities of Alto Alentejo. Our results in the same municipalities were 33,000 dry ton/year. Fernandes and Costa (2010) for Marvão municipality, obtained around 2,200 dry ton/year of forest residues, our study obtained for the same region 2,030 dry ton/year.

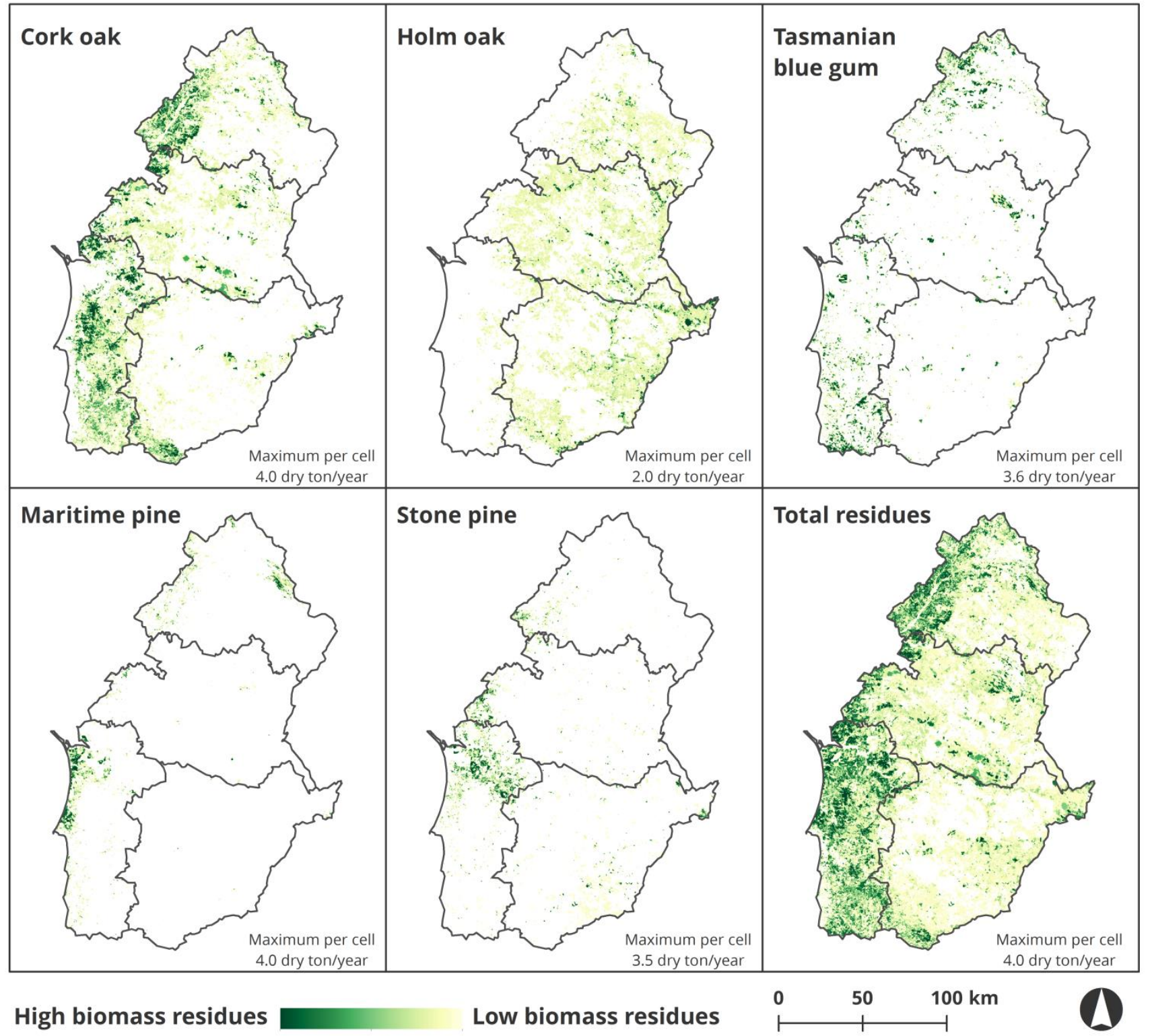

Figure 3: Potential forest biomass residues distribution.

Figure 4 shows the result of the cumulative biomass residues assuming a collection area of $35 \mathrm{~km}$ around each cell. The region with more biomass available is located in northern Alentejo Litoral. This is the only zone that reaches cumulative values of biomass above 80,000 dry ton/year for a collection area of $35 \mathrm{~km}$. This value is near the amount of forest biomass needed to feed a $8 \mathrm{MW}$ dedicated biomass power plant (lower heating value of $15 \mathrm{GJ} /$ ton (Fernandes and Costa, 2010), electrical 
conversion efficiency of 18\% (IEA, 2012) and $7200 \mathrm{~h} /$ year of operation). The higher residues potential in Alto Alentejo is related to the presence of multiple species: cork oak, maritime pine and stone pine. These species have a big presence in the region with high values of potential residues production, and although the pine species had the lowest residues potential for the entire study area when compared with the other species the large majority of those residues potential is concentrated in zones of the northern part of Alto Alentejo. On the other extreme is the eastern part of Alentejo, mainly Baixo Alentejo and Alentejo Central, with cumulative values below 40,000 dry ton/year. This is expected because these are areas of intensive agriculture production.

The use of $35 \mathrm{~km}$ supply radius can create false cut off effects in the final map, imposed by the limit of the study area. Whereas the western border of the study area represents a true physical limitation due to the presence of the ocean, and the eastern border represents an international border. The northern and southern borders of the region may impose false limitations on the biomass availability, which is probably the case in the western part of Alto Alentejo. In this region the biomass is concentrated on a stripe of around $20 \mathrm{~km}$ from the region border. This means that many cells will have a reduced supply when using a $35 \mathrm{~km}$ radius. It's very likely that this area would have more cumulative residues potential, for the same radius, if the study area were extended at that location.

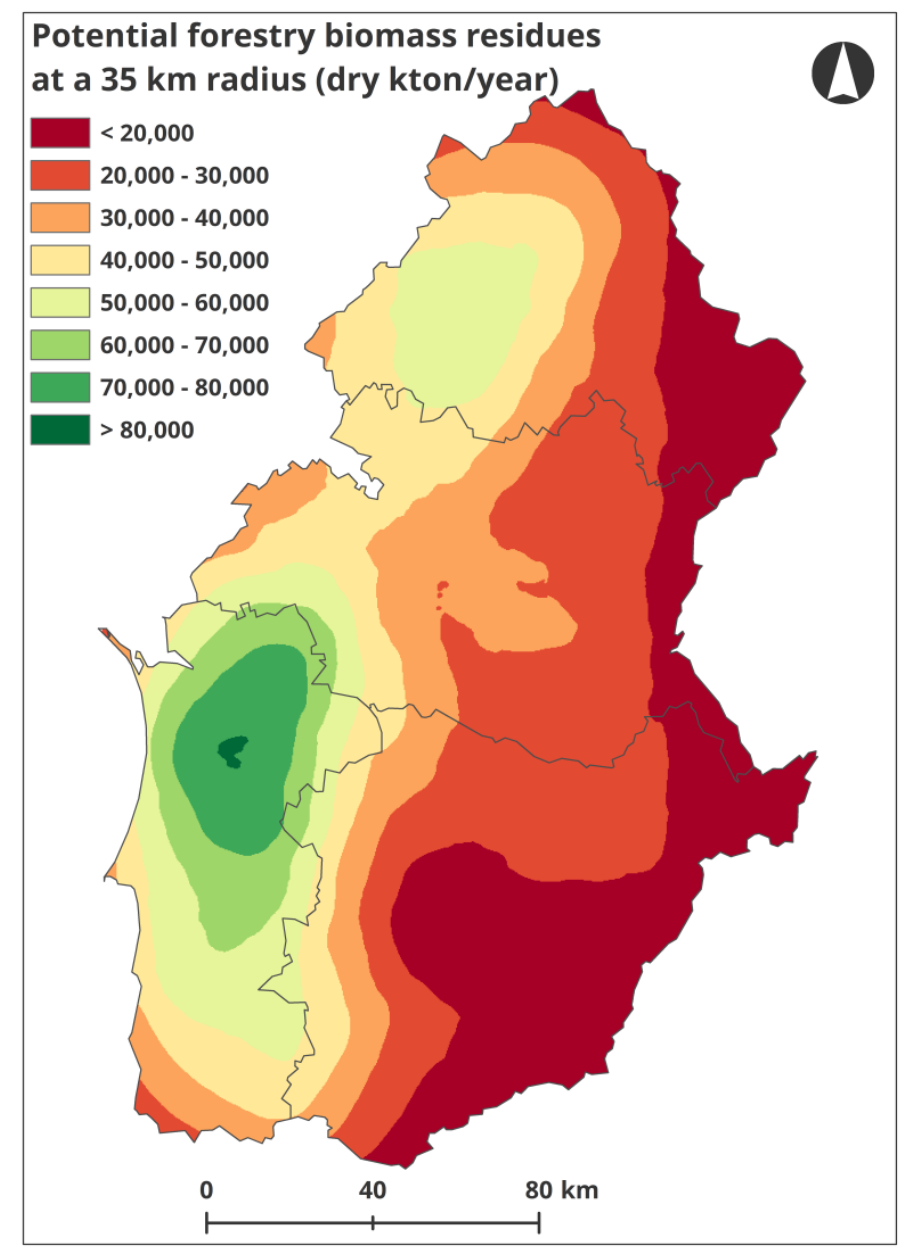

Figure 4: Potential forest residues available for a $35 \mathrm{~km}$ radius collection area. 


\section{REFERENCES}

AEBIOM, 2017, Statistical report 2017 Key Findings.

DGEG, 2018, http://www.dgeg.gov.pt/, Last accessed in 28 June of 2018.

Direção-Geral do Território, 2016, Especificações técnicas da Carta de uso e ocupação do solo de Portugal Continental para 1995, 2007 e 2010.

DNGF, 2010, Aplicação para consulta dos resultados do $5^{\circ}$ Inventário Florestal Nacional.

Eurostat, 2018, http://ec.europa.eu/eurostat, last accessed in 28 June of 2018.

Fernandes, U., Costa, M., 2010, Potential of biomass residues for energy production and utilization in a region of Portugal. Biomass and Bioenergy 34, 661-666.

Gómez, A., Rodrigues, M., Montañés, C., Dopazo, C., Fueyo, N., 2010, The potential for electricity generation from crop and forestry residues in Spain. Biomass and Bioenergy 34, 703-719.

ICNF, 2013, Inventário Florestal Nacional 6 - Áreas dos usos do solo e das espécies florestais de Portugal continental em 1995, 2005 e 2010. Lisboa.

IEA, 2012, Technology Roadmap. Bioenergy for Heat and Power. Paris: IEA Publications, p. 62.

López-Rodríguez, F., Atanet, C.P., Blázquez, F.C., Celma, A.R., 2009, Spatial assessment of the bioenergy potential of forest residues in the western province of Spain, Caceres. Biomass and Bioenergy 33, 1358-1366.

Lourinho, G., Brito, P., 2014, Assessment of biomass energy potential in a region of Portugal (Alto Alentejo). Energy 81, 189-201.

REN21, 2017, Renewables 2017 Global status report. REN21 Secretariat, Paris.

Sarmento, P., Monteiro, G., Marcelino, F., Igreja, C., Caetano, M., 2016, Avaliação da exatidão temática das cartas de uso e ocupação do solo para Portugal Continental COS1995v1.0, COS2007v2.0 e COS2010v1.0.

Vasco, H., Costa, M., 2009, Quantification and use of forest biomass residues in Maputo province, Mozambique. Biomass and Bioenergy 33, 1221-1228.

Viana, H., Cohen, W.B., Lopes, D., Aranha, J., 2010, Assessment of forest biomass for use as energy. GIS-based analysis of geographical availability and locations of wood-fired power plants in Portugal. Appl. Energy 87, 2551-2560.

Voivontas, D., Assimacopoulos, D., Koukios, E.G., 2001, Aessessment of biomass potential for power production: a GIS based method. Biomass and Bioenergy 20, 101-112.

World Energy Council, 2017, World energy issues monitor 2017.

\section{CONCLUSION}

The main conclusion of this study is that most of the theoretical potential of forest residues are located in Alentejo Litoral and in the western part of Alto Alentejo. Alentejo Litoral represents around 37\% of the total residues obtained. The central part of northern Alto Alentejo has the largest cumulative values for a $35 \mathrm{~km}$ radius collection area, around 80,000 dry ton/year. The species with the largest sum of residues potential is cork oak with 172,000 dry ton/year, representing more than $50 \%$ of the total potential residues in the region.

The used GIS based method can be a relevant mechanism to assess the spatial distribution of potential forest residues with high spatial detail for large geographic areas. It allowed to draw preliminary conclusions on potential residues spatial distribution. This information can be helpful to develop more comprehensive and efficient analysis by identifying regions and species of interest that could be targeted with more detailed models. Sensitivity analysis can also be made varying residues rates and vegetation cover (globally, regionally or locally) allowing to better understand the resilience of these types of models to different possibilities of realities. 


\title{
ENERGY PLANNING AT NATIONAL AND COMMUNITY LEVEL IS THE KEY TO INTEGRATE COST EFFECTIVE RENEWABLE ENERGY
}

\author{
Anders Dyrelund and Thomas Rønn \\ Ramboll Energy \\ Hannemanns Alle 53, 2300 Copenhagen S, Denmark \\ Phone: +45 $51618766 /+4551615562$ \\ E-Mail: $\underline{\text { ad@ramboll.com / tsr@ ramboll.com }}$
}

\section{SUMMARY}

Unlike the fossil fuels, renewable energy is of low quality and fluctuating. Almost no efficient and renewable energy source can be used at the building level in a cost effective and environ friendly way to meet the demand. However, at campus, city and national level, there is an opportunity to identify and utilize a wide range of sources benefitting of economy of scale. Careful energy planning is the key to harvest these sources considering the campus, the local community or the state as one profit centre optimizing the energy system including the power, gas, district heating and district cooling system. The integrating energy system opens for an opportunity for storing and utilizing energy, which else would be wasted and it improves the local environment, not least the air quality. We will show three cases on how the energy planning of district heating and cooling (DH\&C) which is obvious for any campus owner also is of interest for local communities and governments.

\section{INTRODUCTION}

There are many renewable energy sources as well as free or efficient energy to consider in the energy planning, e.g. biomass, waste, biogas, deep geothermal heat, wind energy, solar heat, solar PV, industrial surplus heat, surplus heat from power generation (CHP), free cooling, surplus heat from cooling processes and surplus cooling from heat pumps. These sources are typically of low quality and fluctuating and can only be utilized and stored in a cost-effective way in large scale, in large hot- and cold-water systems in campuses or in DH\&C systems.

For campus owners or district energy utilities it is therefore possible to react fast on the price signals and the national energy policy and plan for integrating the most cost-effective combination of efficient and renewable energy, e.g. CHP plants, electric boilers, large heat pumps for combined heating and cooling, large-scale solar heat, thermal storages and cold storages.

But what if there is no thermal infrastructure? International experience shows that it is very difficult to establish the optimal thermal infrastructure in urban areas unless the city council or the state plan for $\mathrm{DH} \& \mathrm{C}$ as a natural part of the urban infrastructure like water, sewage and electricity. Campus owners and urban developers however, who are responsible for all the buildings and energy infrastructure in their district, can plan and implement least cost solutions. They can even do it better than any utility, as they also own the buildings.

We will show case studies who are implemented already or being implemented, which demonstrate how campuses and developers can plan and implement least cost solutions for their districts. An interesting case is the large-scale heat pumps for combined heating and cooling. Due to economy of scale the integrated solutions save both operating costs and investments and improve the environment as well compared to building level solutions.

\section{ENERGY PLANNING AT THE NATIONAL LEVEL}

According to our EU directives, all national and local communities and cities should plan for heating and cooling in order to pave the way for utilizing surplus heat from power plants and renewable energy. In Denmark it has been a national priority for many years. Since 1976 all power plants have 
been situated with respect to the heat market and all local communities have had the responsibility for heat supply planning since 1979. Today, there is almost no heat wasted from power generation and all waste, which can-not be recycled efficiently is used for heat and power. The market share of DH has increased from around 30\% to $60 \%$ and the $\mathrm{CO} 2$ emission from heating has been reduced significantly. The graph below shows the historical development of heat supply forms from 1980 to 2015 and a prognosis up to 2050. Thus, we can see that individual boilers have been replaced with district heating. Therefore, the $\mathrm{CO} 2$ emission in $\mathrm{kg}$ per. MWh heat has been reduced by a factor 3 from 1980 to 2010. This has opened for surplus heat and renewable energy, so far from waste and biomass, but in the years to come more and more solar heat and fluctuating wind energy.

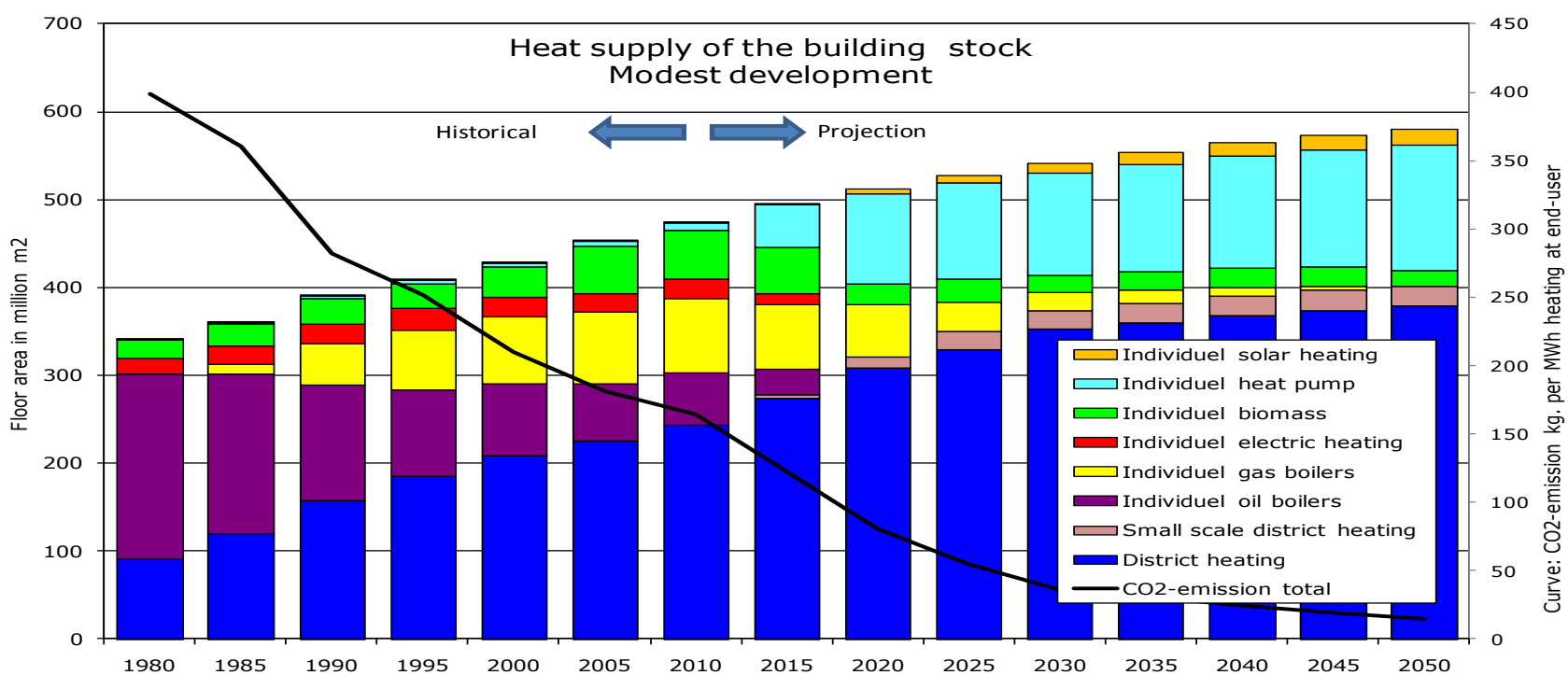

Fig. 1: Heat supply in Denmark, Heat Plan Denmark 2010 [1]

The figure below shows the market share of heat supply forms in Denmark for new buildings since year 2000. Besides, the financial crisis we see that district heating is the dominating heat supply form for new buildings.

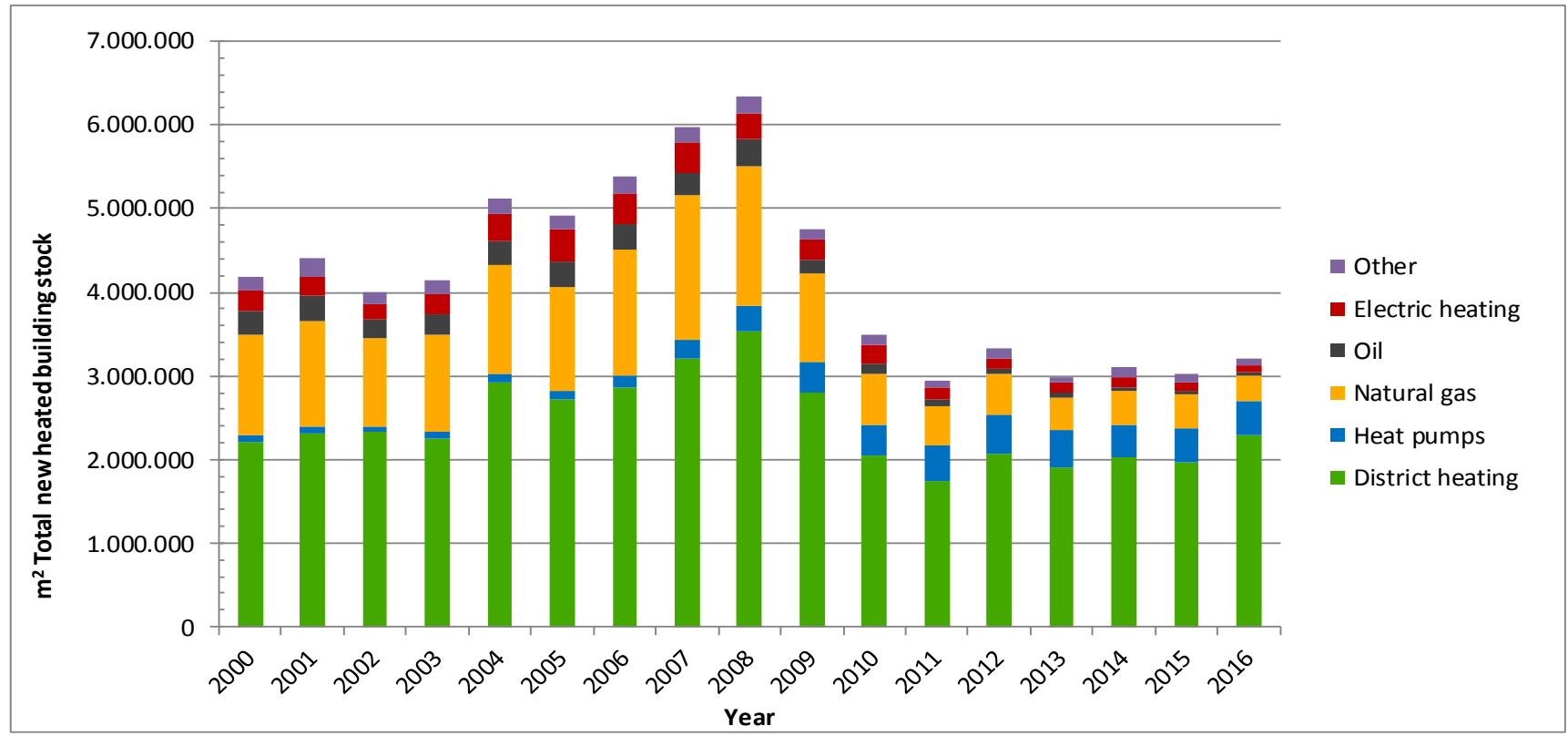

Fig. 2: Heat supply to new buildings in Denmark, Source: BBR register edited by Ramboll 
In a recent strategic study "Cooling Plan Denmark" [2], we have analyzed the potential for DC in Denmark and found - surprisingly- that there is a huge potential for many cost-effective DC systems in city centers and business districts, mainly in symbiosis with the DH. In fact, the DC market in Denmark is growing significantly, and we have noticed that there is an untapped potential in most EU countries.

\section{DEVELOPERS AND CAMPUSSES PLAN FOR ENERGY}

Campus owners and developers have a natural interest in energy planning. The more cost effective and environmental friendly energy supply, the more value is created for their property. Carlsberg Properties had around 2010 the opportunity to plan for energy to all new buildings at their old industrial $300.000 \mathrm{~m} 2$ site of Carlsberg in Valby, Copenhagen. The challenge was to identify the most sustainable supply of energy to $600.000 \mathrm{~m} 2$ of floor area, of which around $50 \%$ also would have a cooling demand. The criteria for sustainability we used had 3 criteria: economic, environmental and social sustainability. The solutions, which are best for the society taking into account cost of emissions and for the local community should get the largest score. The energy solutions, which got the largest score in our comparison of relevant alternatives were:

- DH being a part of the integrated DH system in Greater Copenhagen

- local DC including chilled water storage, a centralized chiller and in the longer term a heat pump for combined production of heating and cooling

- utilization of electricity from off-shore wind, which is much more cost effective and environmental friendly than local generation of electricity from micro wind turbines or solar PV

The plan has almost been fully implemented:

- The DH has been established by HOFOR as a further development of the network Copenhagen

- A new DC network in PEH pipes, a large underground chilled water concrete tank and a chiller has been established by Frederiksberg Forsyning, and Carlsberg City ensures via commercial contracts that all buildings which have a cooling demand will be connected, thereby reducing the costs for all and improving the local environment.

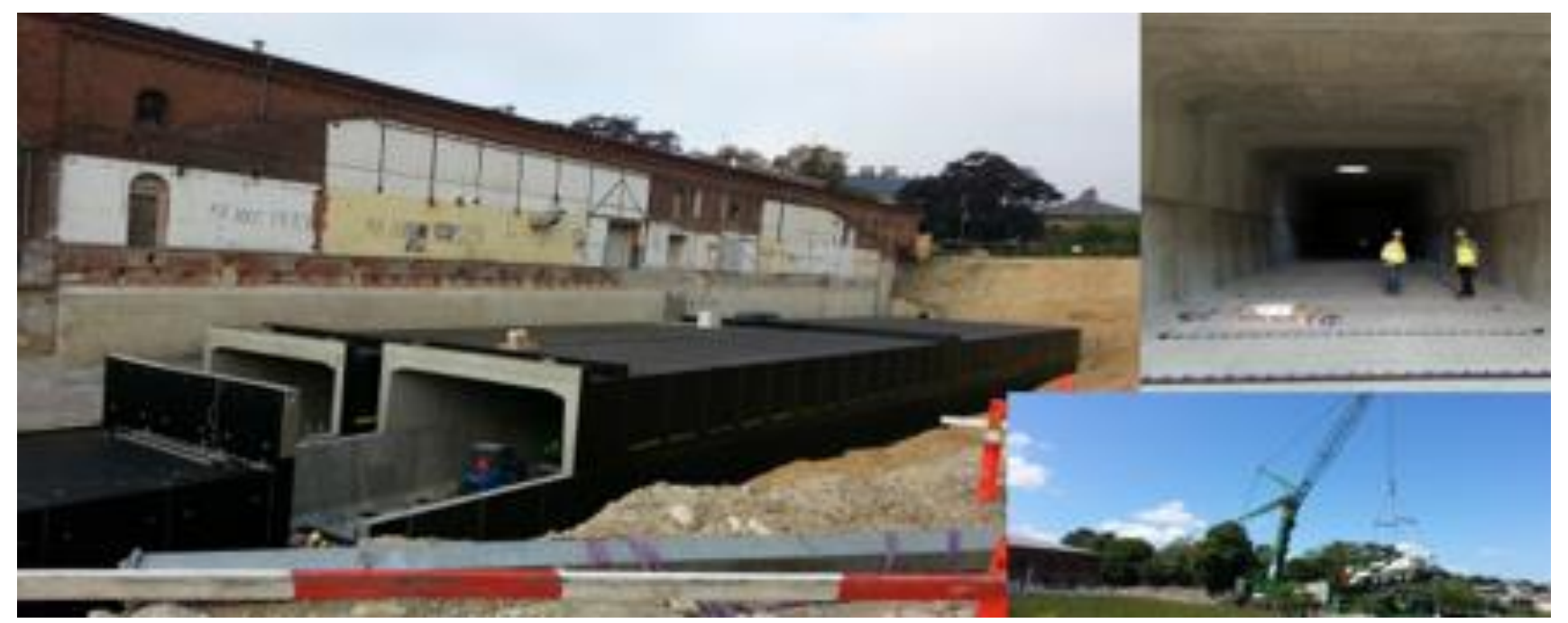

Fig. 3: Chilled water storage under construction 
Due to constrains in the building code, it has however not been possible for the developer to replace solar PV on some of the roof tops with a share of a more sustainable off shore wind turbine.

\section{LOCAL COMMUNITY PLANS FOR DH\&C}

Local communities acting on behalf of all residents and land-owners should have the same interest in energy planning as campus-owners. Moreover, according to the EU directives and the Danish Heat Supply Act local authorities have the obligation to plan for energy. Hillerød Municipality have had the opportunity to plan for the energy supply to a new urban development area Favrholm, [3]. The plan is to establish around $600.000 \mathrm{~m} 2$ of floor, including a new hospital and a business district close to a new train station. The average building density of the whole area will be around $50 \%$. There will be a mix of districts for business, institutions, apartment buildings and terraced houses - in other words a variety of building density in the districts. In our approach we looked at the planning in two dimensions. The most important was to identify the most cost-effective zoning of DH\&C networks against individual heat pumps and chillers. We could rank the districts with respect to profitability of the DH\&C. Gas boilers were not an option to new buildings, as the overall energy policy is to reduce the dependency of fossil fuels. The second dimension was to identify the most cost-effective production of heat and cold to the DH\&C grids in the long term taking into account the local opportunities. This was an iterative process. Taking into account local conditions for heat storage, peak capacity and ground source cooling lowered the cost of production, which increased the optimal market for the networks, which again lowered the cost of production due to economy of scale.

In the first step in the iteration we assumed that there was no district heating next to the district, and we divided the area in uniform districts with respect to density and use. The best solution for all was to establish DH in most districts, and DC in the business districts and to benefit from the symbiosis between DH and DC. The same heat pump could e.g. generate cooling in summer and heating in winter in combination with ground source cooling (so-called ATES system), and the existing DH system could provide peak capacity and heat storage capacity.

Interesting is, that the key parameter for analyzing the zoning of both DH and DC is economy of scale for investing in heat pumps and chillers. It turned out that the total investment of the integrated DH\&C system was almost equal to the total investment in the individual solution. Thus, we proved that the integrated solution with thermal storages and large heat pumps, which compared to individual heat pumps and chillers can integrate fluctuating renewable energy and act like an electric battery - a socalled virtual battery - can be established at no additional cost.

To conclude, research in energy storages should not only focus on electric batteries (more suitable for traffic), but also on integrated energy planning. 


\begin{tabular}{|c|c|c|c|c|c|c|c|c|}
\hline Optimized DH\&C to the district & & DH & DC & & & & & \\
\hline Length og network and branch lines & $\mathrm{km}$ & 35 & 10 & Investment in base line & & Heating & Cooling & Total \\
\hline DH storage tank, rough estimate & $\mathrm{m}^{3}$ & & 3500 & Individual heat pumps / schillers & mio.Euro & 20 & 19 & 39 \\
\hline Capacity demand to network & MW & 12,0 & 11,0 & & & & & \\
\hline Capacity leveling of storage & MW & & 3,0 & Investment in DH\&C system & & DH & $D C$ & $\mathrm{DH} \& \mathrm{C}$ \\
\hline Ground source cooling & MW & & 3,0 & DH\&C networks & mio.Euro & 20,0 & 7,9 & 27,9 \\
\hline Gas boiler for peak & MW & 5,0 & & DH\&C storages & mio.Euro & 1,6 & 0,8 & 2,4 \\
\hline Total installed heat pump for DH\&C & MW & 7,0 & 5,0 & DH\&C boiler / ground source cooling & mio.Euro & 0,7 & 1,1 & 1,7 \\
\hline Total installed capacity & MW & 12,0 & 11,0 & DH\&C heat pump for $\mathrm{DH} \& \mathrm{C}$ & mio.Euro & 5 & 5 & 5,5 \\
\hline $\begin{array}{l}\text { Necessary electric capacity } \\
\text { Total COP for cogen of DH\&C }\end{array}$ & $\begin{array}{c}\text { MW } \\
\text { MW/MW }\end{array}$ & $(7+5$ & & Total DH\&C & mio.Euro & & & 37 \\
\hline
\end{tabular}

Fig. 4: Optimized DH\&C in symbiosis, Total investments base-line and DH\&C system

The implementation of the plan is in progress driven by the construction of the new hospital and a new business district.

\section{FROM GAS BOILERS TO DH}

There is a huge untapped potential for saving fossil fuels by shifting from gas boilers to DH, based on renewable and efficient heat sources. As DH is an expensive natural monopoly network careful energy planning and business planning is vital for the success. Even in Denmark there was after year 2000 a huge potential in some local communities in which natural gas so far had got first priority. Vestforbrænding, who is a part of the integrated DH system in Greater Copenhagen operating a waste for energy plant and a local DH network, started a process a decade ago of strategic energy and business planning in co-operation with the local communities, one of them Lyngby-Taarbæk Municipality [4]. We identified how the existing DH grid could be extended from 300 to $900 \mathrm{GWh}$ heat supply in 5 local authorities and besides transmit surplus heat to two other heat transmission systems north of Vestforbrænding. The result of the planning was a zoning and ranking of the districts with respect to the profitability for DH. In 2018 the plan has almost been implemented and DH is supplied to most larger buildings, but not to small buildings (yellow color on the figure below). The successful strategy for connection $90 \%$ of all buildings to the network the first year has been a competitive offer including installation of building level substation and no connection fee. 


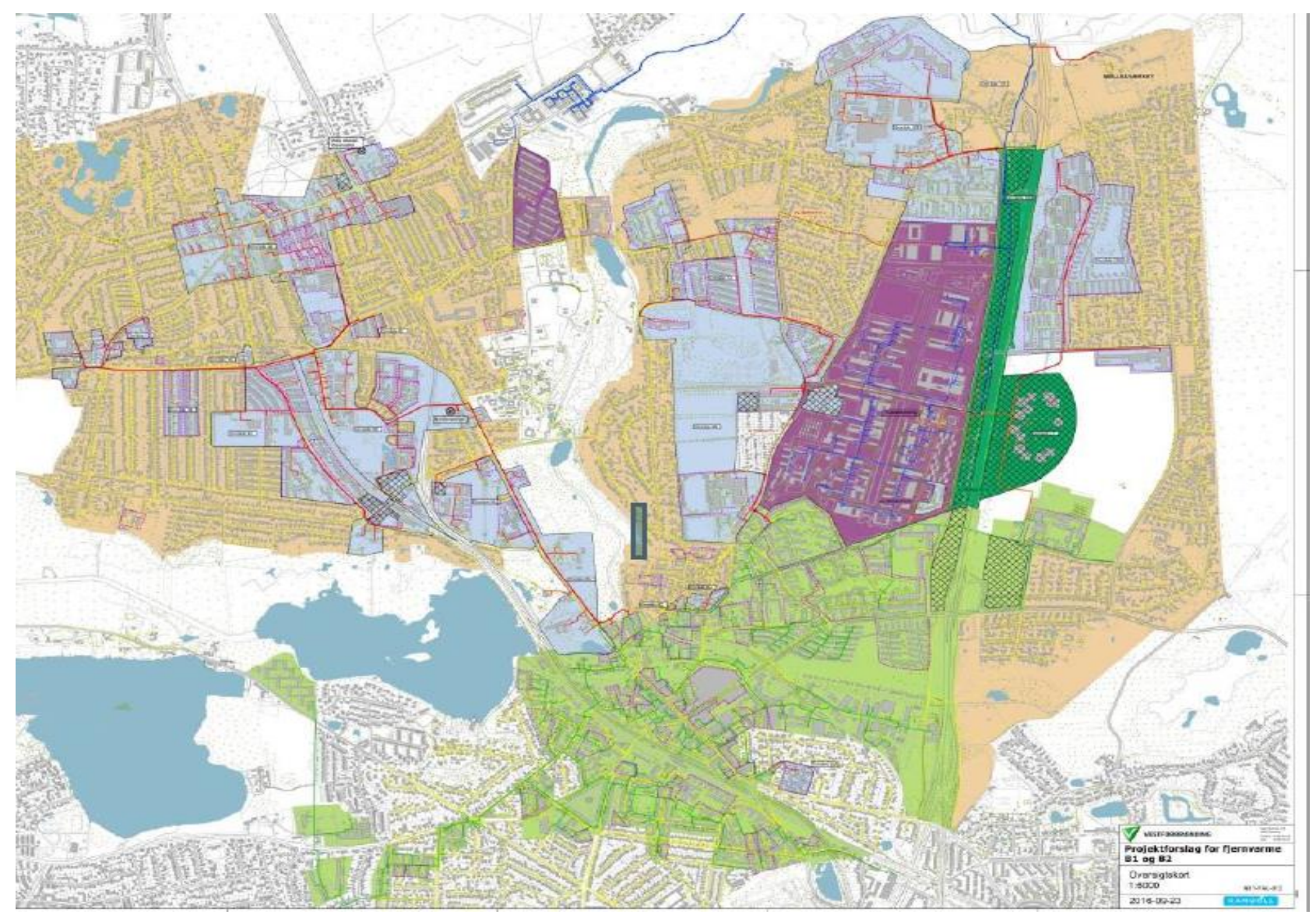

Fig. 5: From gas boilers to DH from Vestforbrænding in Lyngby-Taarbæk Municipality

In a recent study from EU, JRC [5], the district heating system in Greater Copenhagen, including Vestforbrænding is described as an interesting case to replicate.

\section{REFERENCES}

[1] Varmeplan Danmark 2010

http://www.danskfjernvarme.dk/viden-om/f-u-konto-subsection/rapporter/2010-02-varmeplandanmark-2010

[2] Køleplan Danmark, April 2016

http://www.ramboll.dk/projects/rdk/koleplan-afslorer-10-mia-kroners-besparelse

[3] Strategisk energiplan for Favrholm, Hillerød Municipality, December 2016

http://www.favrholm.dk/nyheder/nyheder/strategisk-energiplan/

[4] Strategisk energiplan for Lyngby-Taarbæk Kommune, June 2013

http://www.ltk.dk/sites/default/files/uploads/public/user_uploads/CMP/Byggesag/strategisk_energipla n_2013.pdf

[5] Efficient district heating and cooling systems in the EU, JRC, December 2016

https://ec.europa.eu/jrc/en/publication/efficient-district-heating-and-cooling-markets-eu-case-studiesanalysis-replicable-key-success 


\section{CONCLUSION}

Campus owners and local authorities, who serve the interest of the land-owners and residents can benefit from energy planning, in which we can identify the most cost-effective and sustainable energy solutions for low carbon communities. Due to economy of scale and the ability to store the fluctuating renewable energy as if it was a battery, DH\&C often turns out to be the most cost-effective solution compared to individual building level solutions. The implementation is not a problem for the campus owner, who owns all buildings or controls the land-use. For the local community, serving the interest of many stakeholders, it is possible to demonstrate the total benefit for all and how it could be divided on all stakeholders, but often a challenge to implement, as the major stakeholders have to agree on how to share the benefit. 


\section{POTENTIAL ASSESSMENT FOR THE USE OF NEAR SURFACE GEOTHERMAL ENERGY IN THE ALPINE REGION}

Magdalena Bottig, Doris Rupprecht, Hoyer Stefan

Geological Survey Austria, Neulinggasse 38, 1030 Vienna,

Phone: +4317125674341

E-Mail: magdalena.bottig@geologie.ac.at

\section{SUMMARY}

The project GRETA (Geothermal REsources in the Territory of the Alpine space) aims to foster NearSurface Geothermal Energy (NSGE) in the territory of the Alpine space. Main goals are to assess potentials of NSGE, exchange knowledge and best practices on a transnational basis and to integrate NSGE into policy instruments. Twelve partners from Germany, France, Italy, Switzerland, Slovenia and Austria are participating in this project, co-financed by the European Regional Development Fund through the Interreg Alpine Space programme. In Austria, the focus within the project is set on the potential of NSGE in the Alpine space, thus, mainly addressed to winter tourism in high altitude regions. The focus region comprise the communities Leogang and Saalbach-Hinterglemm. For a sustainable use of NSGE in heating and cooling, it is important to know the potential in its spatial extent. Therefore, potential maps for groundwater heat pump systems and for borehole heat exchangers were calculated. These maps shall serve as tools for spatial energy planning.

\section{ASSESSMENT OF NSGE POTENTIAL USING BEST PRACTICE EXAMPLES}

In the course of the assessment on the status of high altitude NSGE installations, so called "best practice examples" were identified. These examples show the broad applicability of NSGE systems in the Alpine space. Examples include different types of NSGE systems like horizontal collectors, groundwater heat pumps or borehole heat exchanger fields. They show how operators run a valuable NSGE installation in high altitudes (e.g. the Hotel Crystal, Ötztal, $1905 \mathrm{~m}$ altitude). They also show how in areas with so-called rough climate, conditions can be unexpectedly well (e.g. the company Euroclima, Sillian, $1083 \mathrm{~m}$ altitude). From these examples, challenges as well as relevant operational criteria and constraints were derived in a qualitative approach.

In the first year of the project, challenges of high altitude regions and remote areas were assessed in in the course of this best- but also "bad" practice example evaluation. Challenges identified are mainly related to underground temperatures - e.g. cold underground temperatures at very high altitude locations or below-average temperatures of groundwater aquifers in low altitude locations due to drainage from mountainous areas.

\section{GROUND TEMPERATURE AS KEY PARAMETER FOR A SUSTAINABLE USE OF NSGE}

The abovementioned assessment made clear that underground temperature is a key parameter for NSGE potential assessment. This parameter is usually calculated based on outside air temperature under consideration of elevation. It can be assessed more precise under consideration of e.g. solar radiation, snow cover and underground properties. This method is not used as a standard because multiple parameters are needed which are not easily available. Thus, calculations tend to get more complex and special software is required. The University of Natural Resources and Life Sciences in Vienna has developed a software called SoilTempSim, allowing complex simulations of underground temperatures (Grabenweger, 2015).

For validation of these simulated values, measurement stations are necessary - those were missing in the Austrian case study area (Leogang and Saalbach-Hinterglemm). That is why, in autumn 2016, monitoring stations were installed in the municipality of Leogang. Two stations were realized in the valley at about $800 \mathrm{~m}$, two were installed further up the mountain at $1250 \mathrm{~m}$ (south-slope) and $1400 \mathrm{~m}$ (north-slope). Dependent on underground properties, the drillings reached depths of one to three meters. The measurement chains are in-house developments and consist of single digital thermometers (Ds18b20) measuring the underground temperature. They are attached to a data cable in depths of 10 $\mathrm{cm}, 20 \mathrm{~cm}, 50 \mathrm{~cm}, 1 \mathrm{~m}, 1.5 \mathrm{~m}$ and $3 \mathrm{~m}$ below surface. The data loggers connected to the measurement chains are based on an Arduino Micro controller. Lead accumulators and a solar panel supply power and data is transmitted on a daily basis via SMS protocol.

The analysis of the monitoring data show interesting results. Different than expected not the sensors in the valley ( $800 \mathrm{~m}$ altitude) show the highest underground temperatures but the sensors located up the 
mountain at an altitude of $1250 \mathrm{~m}$ (station Sonnberg, heading southwards). This might be due to an early snow cover acting as thermal insulation. The earliest and thickest snow cover is at the northfacing station "Bergbahn". Here, the temperature drop is the least significant. These first results show, how much influence parameters like the snow cover or the exposition do have on the underground temperature and that altitude as such is not reliable to predict underground temperatures.

\section{FIRST RESULTS}

Based on the ground temperature studies in the focus region, a workflow for an improved ground temperature map for the region was elaborated. The calculation is based on multiregression analysis. In the first attempt, the measured annual mean underground temperatures are plotted vs elevation and the first linear regression is calculated. The second step is the linear regression of the temperature deviation vs the mean solar exposure of the particular site. Using this approach, the mean underground temperature can be reproduced within satisfactory limits and further be calculated using gridded data. The resulting map serves as key input for the potential calculation for borehole heat exchangers.

The start of this three years lasting project was in December 2015, current results are accessible via the project homepage http://www.alpine-space.eu/projects/greta/en.

\section{REFERENCES}

Grabenweger, P. (2015): SoilTempSimV3C - A modelt o calculate soil temperatures accounting for frozen soil

conditions (user manual). University of Natural Resources and Life Sciences, Vienna 


\section{A EUROPEAN HEAT DENSITY MAP}

Andreas Müller, Mostafa Fallahnejad

Energy Economics Group, Institute of Energy Systems and Electrical Drives, Technische Universität Wien

Gusshausstrasse 25/370-3, 1040 Vienna, Austria

Phone: +43158801370362

E-Mail: mueller@eeg.tuwien.ac.at

\section{SUMMARY}

Heat density maps can be a useful tool for the spatial energy planning and the assessment of decarbonization options for the building sector. In this article, we present the methodology applied for the Hotmaps European Heat Density Map (HEHDM), developed in the course of the Hotmaps project, funded by Horizon 2020 program. The developed HEHDM covers the EU-28 countries as well as Norway, Iceland and Switzerland at the level of $100 x 100 \mathrm{~m}$. Builds on publicly available data on the building stock, population soil sealing and climate data, statistical data on the delivered energy for space heating and domestic hot water preparation on the NUTS0 level, as well as simulation results for the energy needs of different building categories and construction periods. A special feature of the developed heat density map and underlying background data is that the dataset has been published at the GitLab platform and can be freely assessed and used by anyone for non-commercial as well as commercial purposes.

Keywords: heat density map, spatial analysis, building stock, renewable heating and cooling,

\section{INTRODUCTION}

Reaching the goals of the Paris COP 21 agreement requires the deep decarbonization of the energy supply for space heating and domestic hot water preparation. To effectuate such a decarbonization, the analysis of decarbonization measures on the national level (e.g. see Kranzl et al., 2017) needs to be translated into local circumstances and possibilities. A first step into this direction is the development of heat density maps, with which the spatial distribution of the heat demand can be assessed. They can help to assess the potential usage and associated heat distribution costs for district heating, indicate the electricity loads caused by the electrical heat production or the compare the energy needs and or delivered energy with locally available renewable energy resources such as biomass, solar energy or excess / waste heat from industrial or commercial sites.

The Heat Roadmap Europe project and it's predecessor projects derived such a heat density (PETA4 atlas) on a hectare level $(100 \times 100 \mathrm{~m})$ for 14 European countries and presents the results at their website (PETA4, 2017). As valuable as the presented data are, the heat demand data are only depicted in four different categories, of which one category represents no heat demand or less than $50 \mathrm{TJ} / \mathrm{km}^{2}(\sim 14$ $\mathrm{GWh} / \mathrm{km}^{2}$ ) and the tool lacks the possibility to really assess the calculated heat demand for a given area. Furthermore, since the map is a first of its kind and regional scope, the data quality is difficult to judge.

In this work, we present a new heat density map (HEHDM) for the countries of the European Union on a hectare level.

\section{METHOD}

In order to derive local heat maps, we collected and assessed spatial data on the European building stock. This includes raster data for the EU-28 (and Iceland, Norway and Switzerland) at different grid resolutions levels, starting from $25 \times 25 \mathrm{~km}$ (daily outdoor temperature profiles for the period of 1950 2014) and 1x1 km for population data, 100x100 m for data such as soil sealing for 1975, 1990, 2000 and 2014 and land cover classes up to a grid resolutions level of 30x30m for the elevation and 10x10m for settlement areas. Furthermore vector data (e.g. population and value added for $~ 110.000$ LAU2 regions) and the building layer of the openstreetmap project were used. The later source, even if the regional coverage is poor for some regions and the shapes tend to overestimate the actual building footprint in discontinously populated areas, proved to be a valuable data source.

It contains the perimeter of about 500 million buildings in the region of scope, of which about 6 million buildings shapes also include information on the building height.

We then developed a Python-based model which we used to derive top-down heat maps on the level of 1 hectare, as well as all necessary sub-datasets such gross floor area, heating and cooling degree days, 
share of heated area for different construction periods, population, value added, heated building volume etc.

\section{RESULTS}

The heat demand data (on the hectare level) as well as derived background data such as heated floor area, heated building volume, heating degree days or population (all at the hectare resolution) have been made freely available via the GitLab platform (open data-policy) in the widely used geotif format, allowing interested users to assess and use data to for their own needs. Furthermore, the data are integrated into a web platform currently under development, which does not only depict the data but also allows spatial calculations on those data.

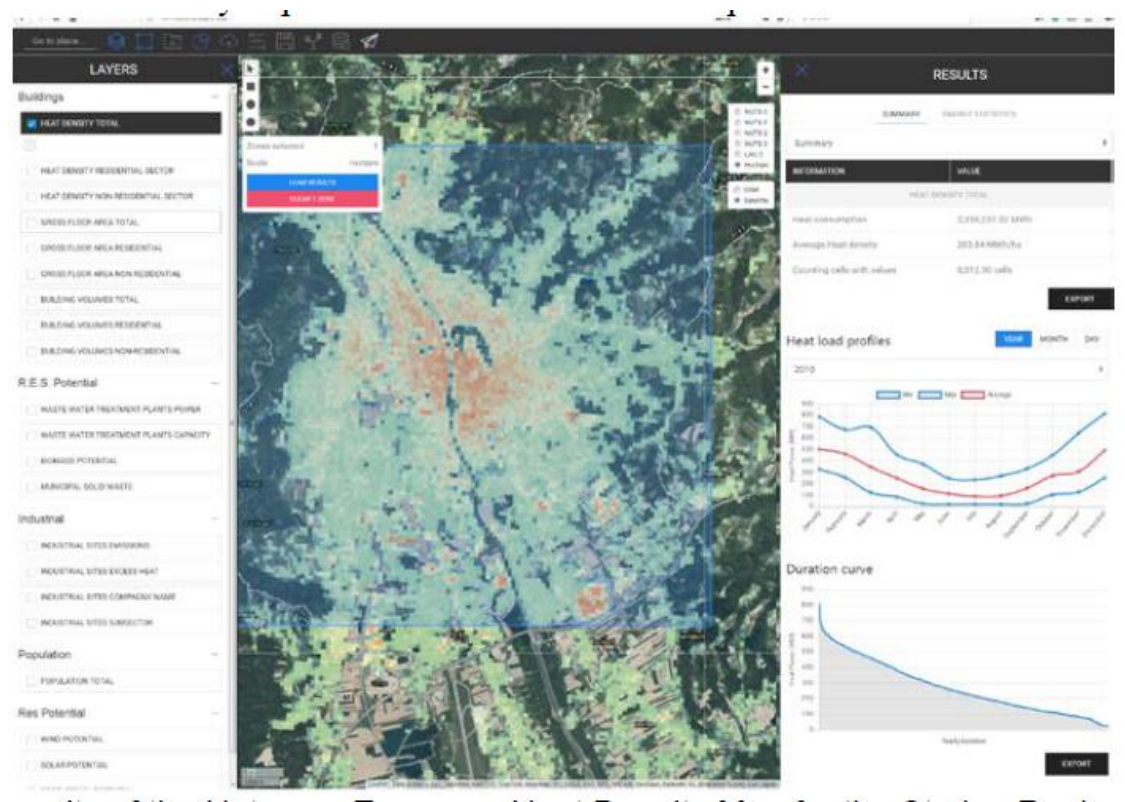

Fig. 1: Results of the Hotmaps European Heat Density Map for the Styrian Region of Graz

\section{DISCUSSION AND CONCLUSIONS}

Even though the developed data set does build in available, generic data on the local building stock rather than on actual measurements, it can be used as a valuable starting for regions, for which no such assessment is available or accessible. Furthermore, since the methodology is independent (yet partly similar) from that, applied for the PETA4 atlas, it offers the possibility to assess the results quality and data uncertainty for those countries, which are also covered by the PETA4 atlas.

\section{REFERENCES}

Kranzl, L., Aichinger, E., Forthuber, S., Hartner, M., Müller, A., Toleikyte, A., 2017. To what extent do "ambitious" scenarios of energy demand in the building stock reflect COP21 Paris targets? Presented at the eceee Summerstudy 2017, Hyeres, France.

PETA4. 2017. Pan-European Thermal Atlas 4 (Peta4) [Online]. Europa-Universität Flensburg, ArcGIS Online. Heat Roadmap Europe - A low-carbon heating and cooling strategy for Europe. Available at (2018-03-31): http://heatroadmap.eu/Peta4.php [Accessed]. 


\title{
Spatial correlation of heating supply and demand - GIS mapping for Energy planning
}

*T.Novosel, T. Pukšec, A. Lovrak, N. Duić

Faculty of Mechanical Engineering and Naval Architecture, Department of Energy, Power Engineering and Environment

University of Zagreb, Zagreb, Croatia

e-mail: tomislav.novosel@ fsb.hr

\begin{abstract}
Due to its high energy demand and inefficiency, the building sector of the European Union presents a crucial segment for the decarbonisation of its energy system. A lot of the potential for this relates to the heating and cooling sector, pushing district energy systems much higher in priority for a lot of national and European strategies and regulation. District heating, alongside the increase of efficiency and potential for the utilization of renewable and excess energy, also provide synergy to the power sector with increased flexibility of the overall system with the use of power to heat technologies. Due to the high upfront investment costs related to such systems, detailed spatial analysis of their economic potential is crucial. Heat demand and potential supply mapping is therefore becoming an essential tool in the analysis of the feasibility of new and the expansion of existing district heating systems. This fact is emphasized even further as more and more advances are made in the utilization of excess and renewable heat sources making the knowledge regarding the spatial distribution of supply and demand even more vital. This research presents the creation of an energy atlas which presents the energy demands, potential supplies as well as various demographic and macroeconomic data useful for energy planning purposes and the analysis of the potential for the utilization of district energy systems based on renewable and excess energy. Croatia has been used as a case study for this purpose.
\end{abstract}

\section{KEY WORDS}

District heating, energy atlas, energy supply and demand analysis. 


\title{
LARGE-SCALE HEAT PUMPS - THE KEY TECHNOLOGY IN EFFICIENT URBAN HEATING AND COOLING
}

\author{
Anders Dyrelund and Troels Hansen \\ Ramboll Energy \\ Hannemanns Alle 53, 2300 Copenhagen S, Denmark \\ Phone: +4551618766/+4551618118 \\ E-Mail: ad@ramboll.com / trha@ ramboll.com
}

\begin{abstract}
SUMMARY
Large-scale heat pumps in the district heating (DH) and district cooling (DC) systems are becoming a key-technology in the energy system, as they integrate three of the four energy carriers. Moreover, due to economy of scale they will normally be connected to heat and cold storages and back-up capacity, which allows it to react on the fluctuating electricity prices. They can use large amounts of electricity for heating in a smart way without over loading the power system.
\end{abstract}

\section{INTRODUCTION}

The integrated energy system, including the 4 energy carriers: electricity, DH, DC and gas, is vital for cost-effective and efficient integration of renewable energy. The electric heat pump technology is one of the key-technologies in this energy system, as it integrates three of the energy carriers. Electric chillers for air conditioning, who waste the heat, and electric heat pumps, e.g. geothermal heat pumps, who waste the cooling, are wide spread technologies, mainly in small scale at the building level. Typically, they can-not respond on the fluctuating electricity prices from wind and solar, they are relatively expensive and can be difficult to integrate in the local environment in densely urban areas. Large heat pumps, however, e.g. from $1 \mathrm{MW}$ and above, which can be connected to the campus or to the city-wide DH and DC grids benefit from economy of scale. That includes typically connection to both grids, to hot and cold storages and to aquifer thermal energy storages (ATES). Thereby they can generate heat and cold in co-generation, which is more energy efficient and not least more cost effective, as they generate two useful and valuable products - cooling capacity in summer and heating in winter. Moreover, it will normally be cost effective also to operate them either for heat-only or for cold-only production in case there is no need for both products at the same time. They are flexible and can respond on the market prices in the grids for electricity, heat and cold during the year, as they are integrated with thermal storages and back-up capacity. Like-wise, the absorption heat pump can generate cold and low temperature heat from a high temperature heat source, e.g. boilers or deep geothermal energy in case these sources are available when needed.

We will present the state-of the art technology and interesting and spectacular cases, which have been implemented recently or are in the pipe-line.

\section{THE ELECTRICAL HEAT PUMP}

Electrical heat pumps for heating homes, like ground source heat pumps is a well-known, but expensive technology, which is far more efficient than direct electric heating. However, it can be a problem to eject the cooling in case there is not space for tubes in the ground and in densely urban areas, the noise from fans can be an environmental problem.

Therefore, due to economy of scale it will often be cost effective to interconnect the buildings and establish a centralized heat pump, e.g. one large heat pump in the range of 5-10 MW heat instead of 10-100 small individual heat pumps. 
Case: 100 buildings in a district, in total 30,000 MWh/a and $15 \mathrm{MW}$ heat installed capacity at the building level

Baseline:

- 100 heat pumps, in average $150 \mathrm{~kW}$ each, total $15 \mathrm{MW}$ heat

- investment in heat pumps 2 mill.Euro/MW heat, in total 30 mill.Euro

DH system:

- $10 \mathrm{MW}$ max. to the network, 1 heat pump 4x1,5 MW= $6 \mathrm{MW}$ heat

- Back-up from storage and/or peak boiler $4 \mathrm{MW}$

- Investment in heat pump 1 mill.Euro/MW heat, in total 6 mill.Euro

- The base load from the heat pump covers at least $95 \%$ of the annual production

This reduced investment can justify even large investments in DH network and storages.

The COP is typically 3 for these heat pumps, but it depends on the range from the ambient temperature to the temperature in the heating system. As regards the electricity price the large heat pump could be connected to a transmission grid and avoid distribution fee from the low voltage network.

Moreover, as the large heat pump will normally be combined with a heat storage tank and back-up capacity to allow the heat pump to stop in case of power shortage or high prices. This will reduce the cost of electricity in the market, ant the distribution tariff for electricity should be reduced as the plant can be interrupted. In total, the large plant in the DH system will be more efficient to integrate the fluctuating renewable energy than the small plants.

\section{THE DISTRICT COOLING POTENTIAL}

According to the UN Climate Panel, IPCC, there is a growing need for cooling capacity, due to the urbanization, the industrialization and the increasing welfare. Moreover, according to IPCC, global warming is expected to increase the need by $25 \%$. In some cities we already can observe load shedding, due to uncontrolled electricity consumption from many small chillers, who overload the power system. Even in a mild and coastal climate like in Scandinavia, there is a need for active cooling in modern commercial buildings to maintain a good indoor climate. This warm summer 2018 there was even a market for active cooling in many new residential buildings.

A district cooling infrastructure in campuses and city districts is the smartest way to solve this problem. Due to economy of scale, simultaneity and the opportunity to store the cold in large scale, DC reduces the need for cooling capacity and opens for demand response, e.g. to generate the cold in off peak hours only.

Campus owners can easily identify this opportunity and coordinate replacement of many small chiller with a DC grid, a cold storage tank and a large chiller. In cities, which are not used to energy planning it is difficult as there are many individual building owners, who have difficulties to co-operate and find the best common solution for all.

Case: A campus has 15 buildings and a total cooling capacity of $10 \mathrm{MW}$ cold. 


\section{Baseline:}

- 15 chillers, in theory in total $10 \mathrm{MW}$, but often more, e.g. $15 \mathrm{MW}$ cold

- Total investment 2 mill.Euro/MW cold, in total 20-30 mill. Euro

DC:

- The total observed capacity demand to the network typically would be reduced to around 7 MW due to simultaneity and as the real total demand of all buildings can be measured and adjusted year by year.

- By installing a chilled water tank to level the daily fluctuations, the maximal capacity demand could be further reduced to around $5 \mathrm{MW}$

- The cost of one $5 \mathrm{MW}$ chiller plant is only around 1 million USD/MW cold, in total 5 mill.Euro

Thus, the total investment in chillers can be reduced by at least a factor 4 which will compensate for investments in the DC grid and chilled water storage. Besides, the centralized plant opens for opportunities for ground source cooling, free cooling and not least combined heating and cooling.

\section{COMBINING DISTRICT HEATING AND COOLING}

There is a strong symbiosis between DH and DC. One of them is that the large heat pumps can generate useful heat and cold, either one or the other or in co-generation.

- In case the baseline is heat production based on ambient heat, e.g. ground water at 10 dgr.C, then the heat pump automatically delivers the cooling capacity in co-generation with the heat, and we can consider the cooling energy as a waste product.

- In case the baseline is cold production from a chiller (or a heat pump), which delivers cold at 10 dgr.C and eject the thermal heat at the ambient temperature in summer of around 30 dgr.C, then we can upgrade this chiller to a useful heat pump, which can generate cold at 10 dgr.C and heat at e.g. 80 dgr.C by adding one more heat pump in serial connection with the first or by installing a two-step heat pump. The additional cost of upgrading the chiller to such a heat pump will only be around $\mathbf{0 , 3}$ mill.Euro/MW heat, and the marginal COP of generating heat at 80 dgr.C compared to a chiller, which waste heat at 30 dgr.C will be around $\mathbf{6}$. Thus, compared to a "heat-only" heat pump the investment is reduced by a factor 3 and the efficiency is increased by a factor 2 .

Due to this symbiosis between heat and cold it is a good idea for the DH utilities consider DC as a new business.

Case: Grundfos campus and local DH 


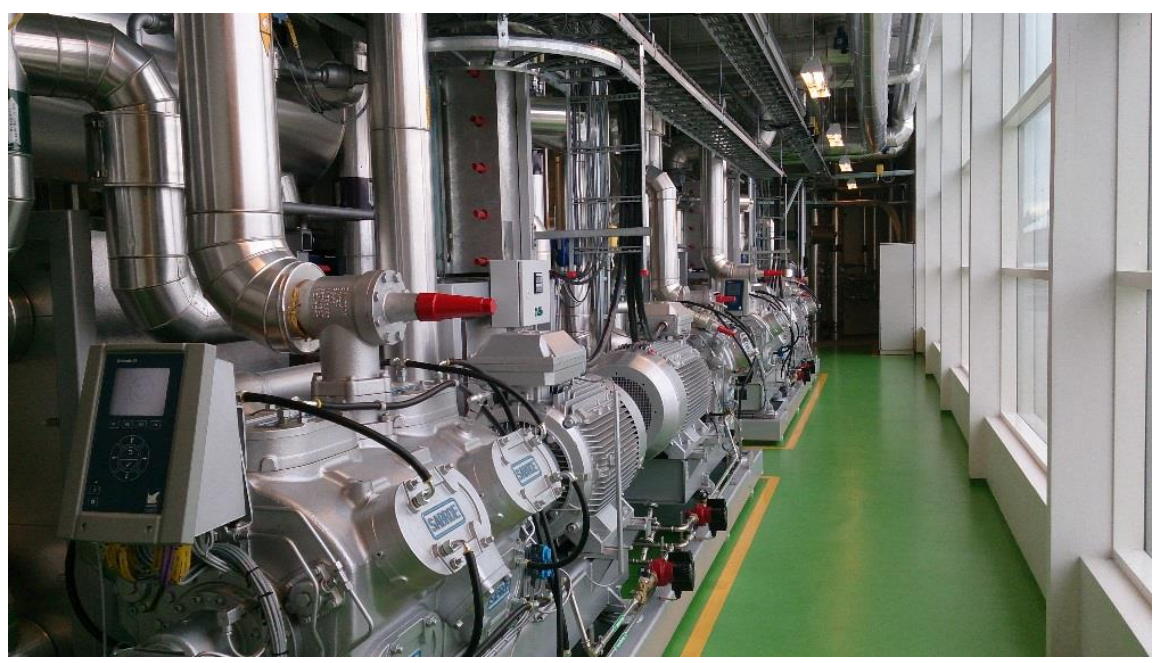

Fig 1. Heat pump, Grundfos, Gudenådalens Energiselskab

Grundfos has installed a DC grid to supply cooling at the campus. The cooling is generated by heat pumps, which deliver heat to the local district heating system in combination with ground source cooling.

\section{Case: Tårnby DH company in Greater Copenhagen}

The DH company establish a heat pump installation to deliver cooling to an urban development area. The heat pump will deliver heat in two steps from 6 dgr.C to around 75 dgr.C to the DH network. The cold side will be connected to a new DC grid, to a chilled water tank, to a ground source cooling (ATES) and to the treated waste water. The max load hours for combined heating and cooling will be around 3,000 max load hours, and the heat only production, "wasting the cooling in the waste water" will be around 4,000 max load hours.

\section{DH AND DC TEMPERATURE LEVELS}

In case the end-users for heating and cooling have significantly variations in temperature demand we can use decentralized heat pumps or supply the temperatures in steps.

Case. Decentralized additional cooling for ice

In case a DC network is designed for comfort cooling, e.g. supply 6 dgr.C and return 15 dgr.C, the cooling can be used to feed decentralized heat pumps, which reduce the temperature further to e.g. -8 dgr.C or even -20 dgr.C. In case there are several end-users for the ice-water in a district, it could be cost effective to establish a local ice-water network with glycol and supply it from a heat pump which is connected to the DC network. In Carlsberg City in Copenhagen chillers are not allowed for environmental reasons and therefore the refrigerators and freezers in the super market are connected to the DC network in Carlsberg City.

Case: Central production of DH and ice water and end-users in cascade

In Copenhagen Markets, for whole sale of vegetables and flowers, there is installed a DC network to around 70 end users, of which the first group need -8 dgr.C and the rest are supplied from the return pipe of the first group. The centralized heat pump installation, which is established by Høje Taastrup DH company next to Copenhagen Markets, includes three steps going from -8 dgr.C up to 75 dgr.C to the DH network. 


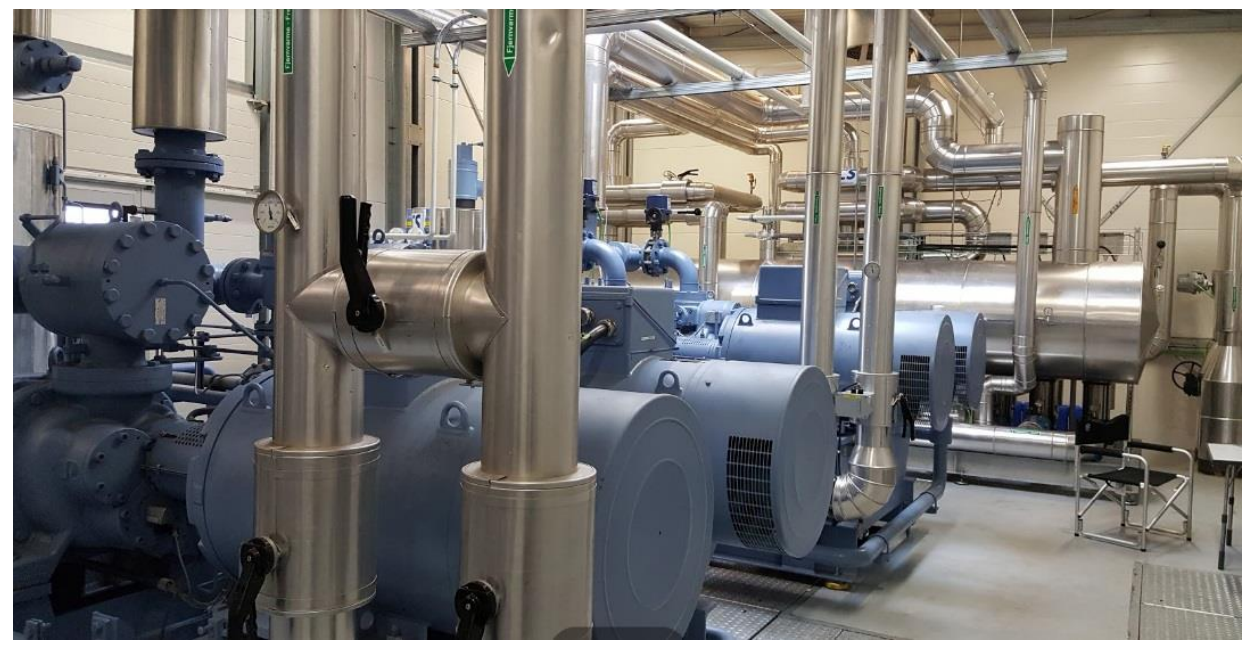

Fig.2 Heat pump, Copenhagen Markets, Høje Taastrup Fjernvarme

\section{ABSORPTION HEAT PUMPS}

In case there is a surplus of high temperature heat available at the same time there is a cooling demand, an absorption heat pump can be an interesting alternative to the electric heat pump or chiller, e.g. in the following cases:

Case Absorption heat pump for district cooling

In case a cheap heat source is available at a temperature of more than around 110 dgr.C or more, e.g. deep geothermal, this heat can be distributed in a DH system for super-heated water, it can be stored in steel tanks and distributed to buildings for heating and for generating cooling by an absorption chiller at the building level. In case the building need low temperature heat e.g. for hot tap water, the return temperature can be used for heating. Such a system is installed to deliver part of the cooling to a new hospital in Aarhus Denmark.

Case 2 Absorption heat pumps for flue gas condensation

For installation of efficient flue gas condensation, e.g. at a waste incinerator, at a biomass boiler or even at a gas boiler, which operates as base load, an absorption heat pump could be an alternative to an electric heat pump. In case the plant is a CHP plant, it has to be taken into account that the steam for the heat pump reduces the power production, which has to be compared with the electricity consumption for an electric heat pump. In case it is a heat only boiler, the absorption heat pump can use the exergy potential in case the boiler is designed for super-heated water, e.g. up to 160 dgr.C. Vestforbrænding in Copenhagen has at one of the waste incinerator CHP units installed an absorption heat pump, which reduces the return temperature to condensate the flue gas and generate around 12 MW heat, which else would be wasted as vapor in the flue gas.

\section{CONCLUSION}

The heat pump technology will be one of the key technologies in the future energy system in line med CHP plants, as it integrates three of the four energy carriers and has a vital role to make surplus energy sources for heat and cold useful for the end-users.

The technology can be used even in small scale in apartments, but due to economy of scale for the heat pump itself for the related technologies like thermal storages, which are important for integrating 
fluctuating and low-quality energy sources, heat pumps will also be a driver for more integrated DH and DC systems.

However, due to the technologies of heat pumps there is not much to gain by increasing the capacity from e.g. $20 \mathrm{MW}$ heat to $100 \mathrm{MW}$ heat. Therefore, the heat pumps will be distributed around in the network and located at with respect to the best sources for heat and cold and where it fits into the urban planning.

This complexity opens for many advanced integrated combinations of heating and cooling. We have only presented an appetizer. 


\title{
ON DESIGN PROCESS FOR INTEGRATING RENEWABLES INTO EXISTING DISTRICT HEATING SYSTEMS
}

C. Ribas Tugores1, I. Leusbrock1, M. Moser2, H. Poier2, H. Schranzhofer3

\author{
1AEE - Institute for Sustainable \\ Technologies \\ Feldgasse 19, A-8200 Gleisdorf \\ Phone: +43 (0) 31125886229 \\ E-Mail: c.ribastugores@aee.at \\ 2SOLID - Gesellschaft für \\ Solarinstallation und Design $\mathrm{mbH}$ \\ Puchstrasse 85, A-8020 Graz \\ Phone: +43 (0) 316-292840 \\ E-Mail: m.moser@solid.at \\ ${ }_{3}$ Graz University of Technology, \\ Institute of Thermal Engineering \\ Inffeldgasse $25 \mathrm{~B}, \mathrm{~A}-8010 \mathrm{Graz}$ \\ Phone: +43 (0) 3168737314 \\ E-Mail: hermann.schranzhofer@tugrat.at
}

\section{SUMMARY}

The optimal design of flexible energy concepts with high share of renewables is a complex and timeintensive task. The workflow followed in the Urban-DH-extended project, which aim is to add flexibility and increase renewable share of existing district heating systems, is here depicted with help of a case study. The different steps are described, pointing out their main aim, obstacles and necessary level of detail.

key-words: renewables, district heating system, energy concept design

\section{INTRODUCTION}

District heating in dense urban environments possesses significant $\mathrm{CO}_{2}$-reduction potential. It provides multiple possibilities for the hydraulic integration of renewable energies, storage technologies and waste heat utilization. Thus, fossil fuels are substituted and the flexibility of the energy system increases. Aim of the Urban-DH-extended project is to add flexibility and increase renewable share of existing district heating systems. This will be reached by integrating innovative energy concepts such as the combination of large solar thermal plants with long term thermal energy storages and if applicable compression or thermal heat pumps

to three existing district heating networks in Austria. The optimal design of these flexible energy concepts with high share of renewables is a complex and time-intensive task. The workflow followed in the project, is depicted here with help of a case study. The different steps are described, pointing out their main aim, obstacles and necessary level of detail.

\section{ENERGY CONCEPT DESIGN}

There are many possible technical feasible energy concepts. Though, just some will show themselves as economically feasible. Despite innovative energy concepts, such as the combination of large solar thermal plants with long term thermal energy storages and heat pumps, are gaining interest in several countries such as Denmark (e.g., Silkeborg, Marstal) and Austria (e.g., BigSolarGraz) [2, 1], the feasibility of the concept is strongly determined by the local system situation and its boundary conditions. In this regard, the first step is to gather the necessary information to carry out an status quo analysis. E.g. - district heating system related information such as operating temperatures, energy demand and energy

production units

- local information such as weather data and potential residual heat sources

- economic data regarding production unit cost and feed-in tariff 
Notice that the exact needed information cannot be fully prescribed since it is related to the energy concepts and technologies that can be considered. The gathered information is analysed to gain of a good understanding of the current situation. Based on available knowledge of existing technologies and the local boundary conditions different interesting energy concepts can be defined. With these energy concepts, the pre-feasibility analysis can be carried out. For that purpose, different freeavailable tools are available [3]. In this stage of the project, there are still many unknowns and uncertainties. Therefore, the aim focus is to elucidate under which conditions the different energy concepts are economically viable and, very important, to emphasize the critical points of the energy concepts as well as the disregarded aspects during the pre-feasibility analysis. In this sense, results are not definitive numbers, but rather a set of conditions under which the energy concept fulfills certain goals, e.g., maximal allowed heat production costs. Results provide a basis for discussion and the energy concepts can be refined if needed. The challenge remains in obtaining reliable results by doing a rough analysis of the system. In this regard, a key aspect here is to recognize which are the most relevant aspects to be taken into account and which aspects can be considered in a more approximative way. In the last step, a detail simulation of the energy system is carried out to obtain an optimal system design including a control strategy as well as to analyze specific situations in detail (e.g. summer heat supply). This is usually a high time-consuming task for which rarely all required data is available. Thus, many assumptions have to be taken adding more uncertainty to the results. In this regard, it is important to understand the implications of the undertaken simplifications (e.g. during the modeling) and assumptions. Therefore, communication with the network operator during the whole design process; from the early stages, gathering the necessary data and the energy concepts definition, until the final stage, discussing and evaluating the results of the detailed simulations, is crucial for the achievement of the desired results of the project; i.e. the

achievement of an optimal district heating system with high share of renewables.

\section{REFERENCES}

[1] Patrick Reiter, Hannes Poier, and Christian Holter, (2016), BIG SOLAR GRAZ: Solar district heating in Graz - $500.000 \mathrm{~m} 2$ for $20 \%$ solar fraction, in 4th International Solar District Heating Conference, Billund, Denmark.

[2] Solarthermalworld, Overview largest solar thermal installations worldwide, http://www.solarthermalworld.org/installations.

[3] Carles Ribas Tugores and Ingo Leusbrock, (2018), Integration of solar thermal installations into existing district heating systems - an overview of feasibility analysis tools and necessary improvements, in $5^{\text {th }}$ International Solar District Heating Conference 2018, Graz, Austria. 